In cooperation with the Texas Department of Transportation

\title{
Statistical Characteristics of Storm Interevent Time, Depth, and Duration for Eastern New Mexico, Oklahoma, and Texas
}

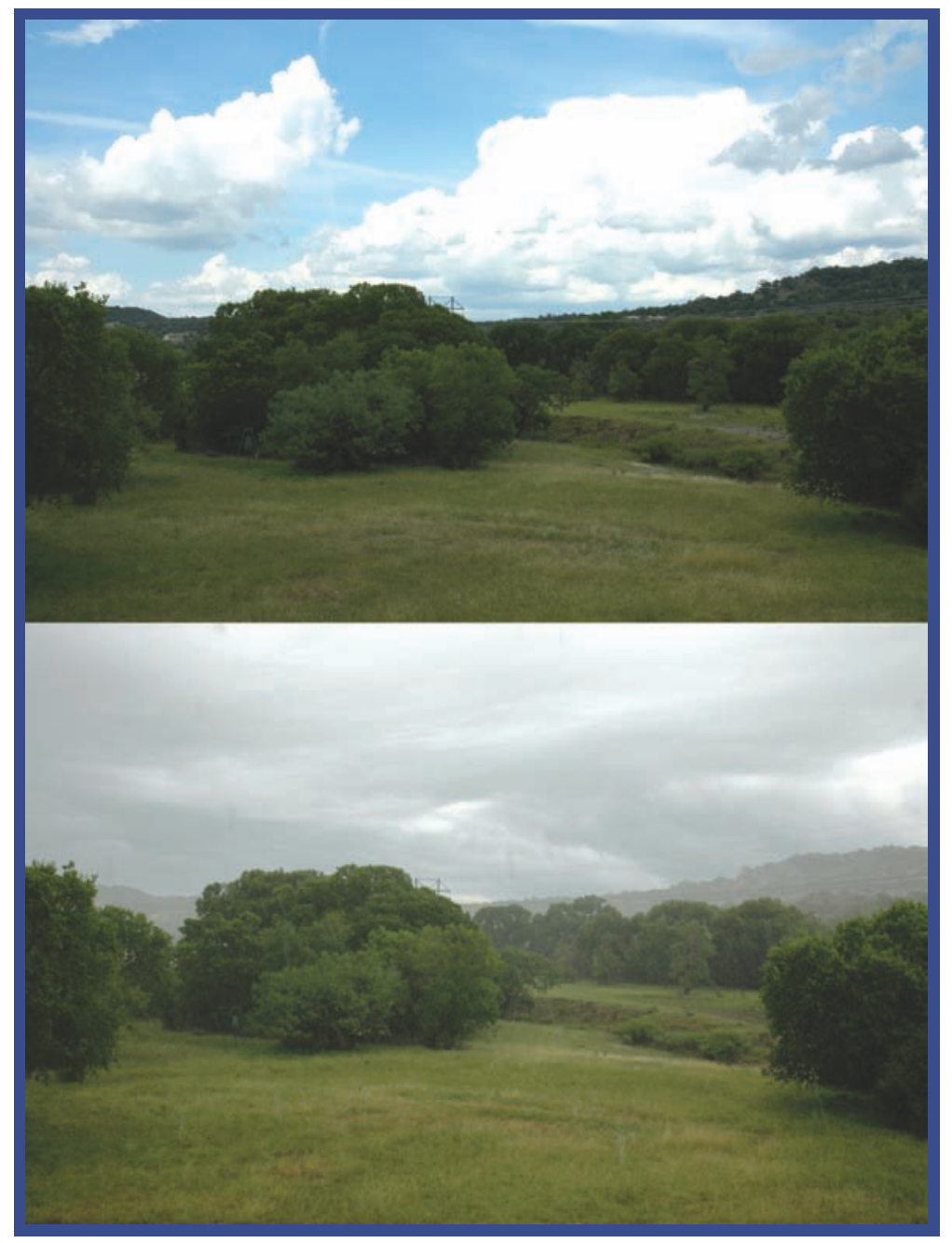

Professional Paper 1725 


\begin{tabular}{|c|c|c|c|c|}
\hline $\begin{array}{l}\text { 1. Report No. } \\
5-4194-01-1\end{array}$ & 2. Government Accession No. & \multicolumn{3}{|c|}{ 3. Recipient's Catalog No. } \\
\hline \multicolumn{2}{|c|}{$\begin{array}{l}\text { 4. Title and Subtitle } \\
\text { STATISTICAL CHARACTERISTICS OF STORM INTEREVENT } \\
\text { TIME, DEPTH, AND DURATION FOR EASTERN NEW MEXICO, } \\
\text { OKLAHOMA, AND TEXAS }\end{array}$} & \multicolumn{3}{|c|}{$\begin{array}{l}\text { 5. Report Date } \\
\text { July } 2006\end{array}$} \\
\hline \multicolumn{2}{|c|}{$\begin{array}{l}\text { William H. Asquith, Meghan C. Roussel, Theodore G. Cleveland, Xing Fang, } \\
\text { and David B. Thompson }\end{array}$} & \multicolumn{3}{|c|}{$\begin{array}{l}\text { 8. Performing Organization Report No. } \\
\text { U.S. Geological Survey } \\
\text { Professional Paper } 1725\end{array}$} \\
\hline \multicolumn{2}{|l|}{$\begin{array}{l}\text { 9. Performing Organization Nar } \\
\text { U.S. Geological Survey } \\
\text { Water Resources Discipline } \\
8027 \text { Exchange Drive } \\
\text { Austin, TX } 78754\end{array}$} & \multicolumn{3}{|c|}{$\begin{array}{l}\text { 11. Contract or Grant No. } \\
\text { Project 5-4194-01-1 }\end{array}$} \\
\hline $\begin{array}{l}\text { 12. Sponsoring Agency Name and Address } \\
\text { Texas Department of Transportation } \\
\text { Research and Technology Implementation Office } \\
4000 \text { Jackson Ave., Bldg. } 1 \\
\text { P.O. Box } 5080 \\
\text { Austin, TX } 78731\end{array}$ & $\begin{array}{l}\text { U.S. Geological Survey } \\
\text { Water Resources Discipline } \\
8027 \text { Exchange Drive } \\
\text { Austin, TX } 78754\end{array}$ & \multicolumn{3}{|c|}{$\begin{array}{l}\text { 13. Type of Report and Period Covered } \\
\text { Implementation of Research } \\
\text { Sept. 2005-July } 2006\end{array}$} \\
\hline \multicolumn{5}{|c|}{$\begin{array}{l}\text { 15. Supplementary Notes } \\
\text { Project conducted in cooperation with the Texas Department of Transportation. }\end{array}$} \\
\hline \multicolumn{5}{|c|}{$\begin{array}{l}\text { The design of small runoff-control structures, from simple floodwater-detention basins to sophisticated best-management } \\
\text { practices, requires the statistical characterization of rainfall as a basis for cost-effective, risk-mitigated, hydrologic engineering } \\
\text { design. The U.S. Geological Survey, in cooperation with the Texas Department of Transportation, has developed a framework to } \\
\text { estimate storm statistics including storm interevent times, distributions of storm depths, and distributions of storm durations for } \\
\text { eastern New Mexico, Oklahoma, and Texas. The analysis is based on hourly rainfall recorded by the National Weather Service. } \\
\text { The database contains more than } 155 \text { million hourly values from } 774 \text { stations in the study area. Seven sets of maps depicting } \\
\text { ranges of mean storm interevent time, mean storm depth, and mean storm duration, by county, as well as tables listing each of } \\
\text { those statistics, by county, were developed. The mean storm interevent time is used in probabilistic models to assess the } \\
\text { frequency distribution of storms. The Poisson distribution is suggested to model the distribution of storm occurrence, and the } \\
\text { exponential distribution is suggested to model the distribution of storm interevent times. The four-parameter kappa distribution } \\
\text { is judged as an appropriate distribution for modeling the distribution of both storm depth and storm duration. Preference for the } \\
\text { kappa distribution is based on interpretation of L-moment diagrams. Parameter estimates for the kappa distributions are } \\
\text { provided. Separate dimensionless frequency curves for storm depth and duration are defined for eastern New Mexico, } \\
\text { Oklahoma, and Texas. Dimension is restored by multiplying curve ordinates by the mean storm depth or mean storm duration to } \\
\text { produce quantile functions of storm depth and duration. Minimum interevent time and location have slight influence on the scale } \\
\text { and shape of the dimensionless frequency curves. Ten example problems and solutions to possible applications are provided. }\end{array}$} \\
\hline \multicolumn{2}{|c|}{$\begin{array}{l}\text { 17. Key Words } \\
\text { Precipitation, Storm statistics, Storm runoff, Urban runoff, L-moments, New } \\
\text { Mexico, Oklahoma, Texas }\end{array}$} & \multicolumn{3}{|c|}{$\begin{array}{l}\text { 18. Distribution Statement } \\
\text { No restrictions. }\end{array}$} \\
\hline $\begin{array}{l}\text { 19. Security Classif. (of report) } \\
\text { Unclassified }\end{array}$ & $\begin{array}{l}\text { 20. Security Classif. (of this pa } \\
\text { Unclassified }\end{array}$ & & $\begin{array}{l}\text { 21. No. of pages } \\
299\end{array}$ & $\begin{array}{l}\text { 22. Price } \\
\$\end{array}$ \\
\hline
\end{tabular}

Form DOT F 1700.7 (8-72)

Reproduction of completed page authorized

\section{Front Cover:}

Top: A partly cloudy day with developing rain showers north of Comfort, Texas, June 24, 2006.

Bottom: An afternoon rain storm north of Comfort, Texas, June 24, 2006.

\section{Back Cover:}

Top left: A small runoff-detention basin in northeast Austin, Texas, June 30, 2006.

Top middle: A small runoff-detention basin in northeast Austin, Texas, June 30, 2006.

Top right: A large runoff-detention basin under construction in north Austin, Texas, July 1, 2006.

Middle back: Fair-weather clouds over ranchland in southwestern Medina County, Texas, June 23, 2006.

Middle front: A late afternoon sea breeze thunderstorm viewed east from central Austin, Texas, June 21, 2006.

Bottom left: A cloudy afternoon at the Gray County, Texas, Interstate 40 rest area on August 12, 2005.

Bottom right: An evening thunderstorm along U.S. Highway 84 near Roscoe, Texas, on May 26, 2004. 


\section{Statistical Characteristics of Storm Interevent Time, Depth, and Duration for Eastern New Mexico, Oklahoma, and Texas}

By William H. Asquith, Meghan C. Roussel, Theodore G. Cleveland, Xing Fang, and David B. Thompson

In cooperation with the Texas Department of Transportation

Professional Paper 1725 


\title{
U.S. Department of the Interior DIRK KEMPTHORNE, Secretary
}

\author{
U.S. Geological Survey \\ P. Patrick Leahy, Acting Director
}

\section{U.S. Geological Survey, Reston, Virginia: 2006}

For sale by U.S. Geological Survey, Information Services

Box 25286, Denver Federal Center

Denver, CO 80225

For more information about the USGS and its products:

Telephone: 1-888-ASK-USGS

World Wide Web: http://www.usgs.gov/

Any use of trade, product, or firm names in this publication is for descriptive purposes only and does not imply endorsement by the U.S. Government.

Although this report is in the public domain, permission must be secured from the individual copyright owners to reproduce any copyrighted materials contained within this report.

Suggested citation:

Asquith, W.H., Roussel, M.C., Cleveland,T.G., Fang, Xing, and Thompson, D.B., 2006, Statistical characteristics of storm interevent time, depth, and duration for eastern New Mexico, Oklahoma, and Texas: U.S. Geological Survey Professional Paper 1725, $299 \mathrm{p}$.

Also available at http://pubs.water.usgs.gov/pp1725/

\section{Library of Congress Cataloging-in-Publication Data}

Statistical characteristics of storm interevent time, depth, and duration for eastern New Mexico, Oklahoma, and Texas / by William H. Asquith ... [et al.].

p. cm. — (Professional paper ; 1725)

Includes bibliographical references.

ISBN 1-411-31041-1

1. Rainstorms-New Mexico-Statistics. 2. Rainstorms-Oklahoma-Statistics. 3. Rainstorms-Texas—Statistics. I. Asquith, William $\mathrm{H}$.

0C925.1.U8N6 2006

$551.57 ' 72789-\mathrm{dc} 22$ 


\section{Contents}

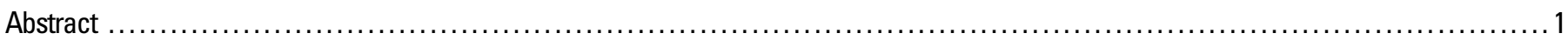

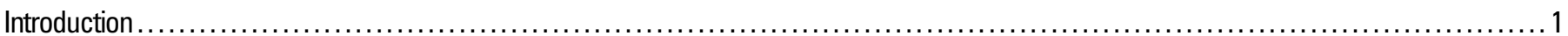

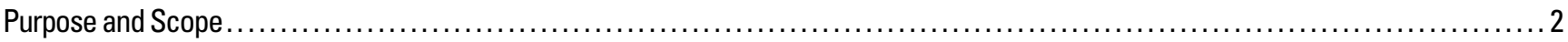

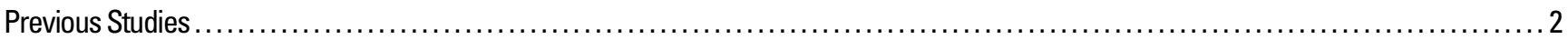

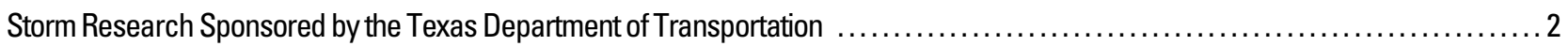

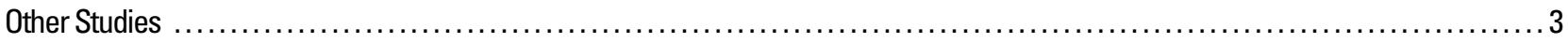

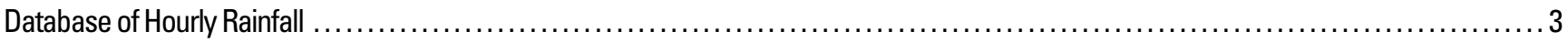

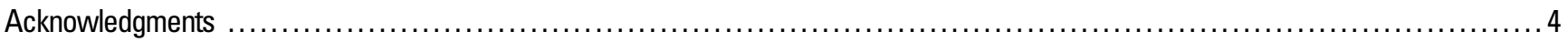

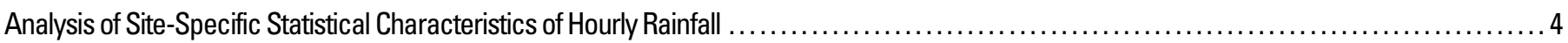

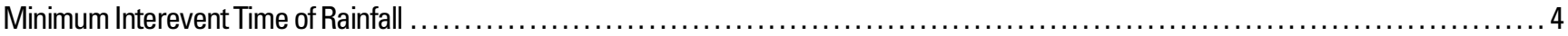

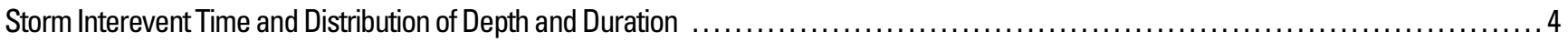

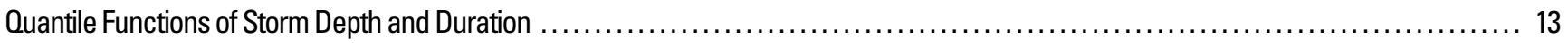

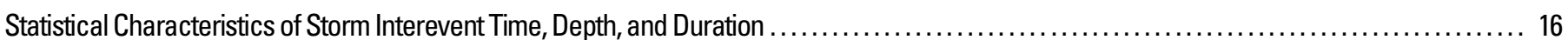

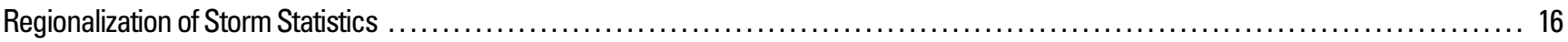

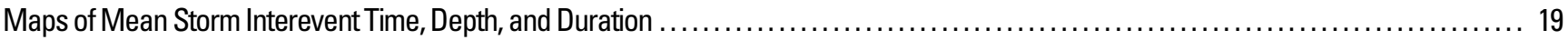

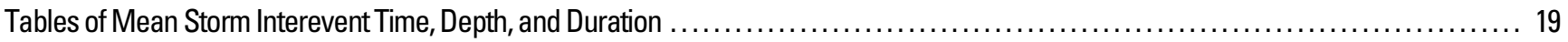

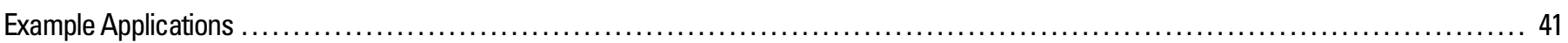

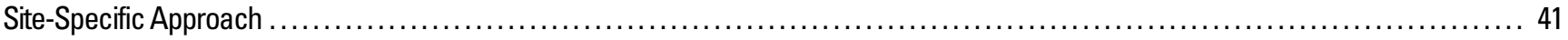

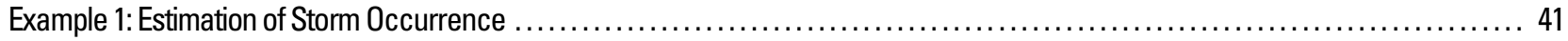

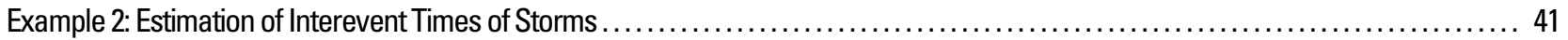

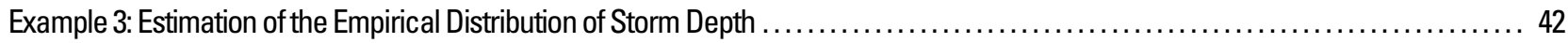

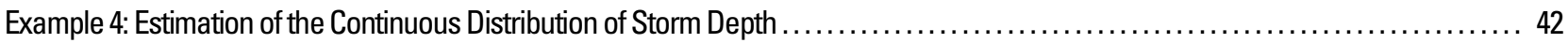

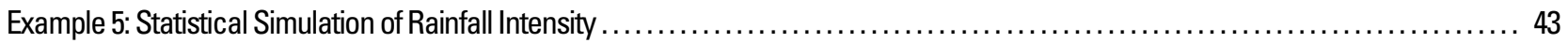

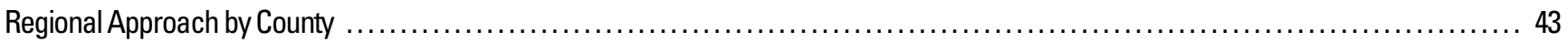

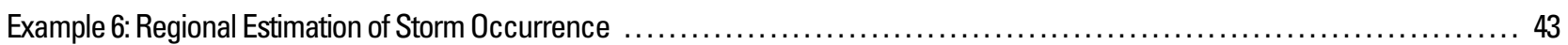

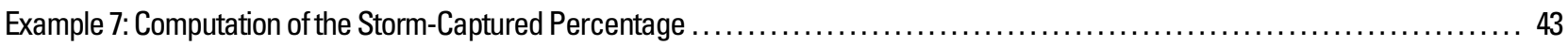

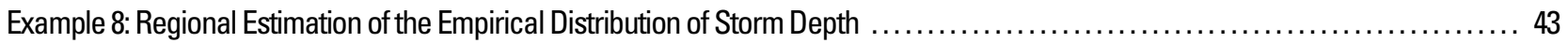

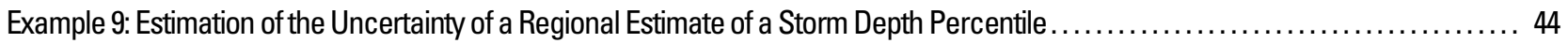

Example 10: Estimation of the Expected Storage in a BMP Using Mean Storm Interevent Time, Depth, and Duration ............... 44

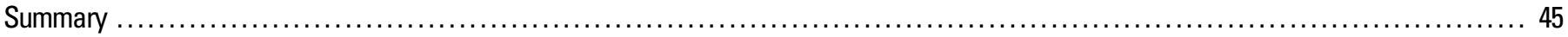

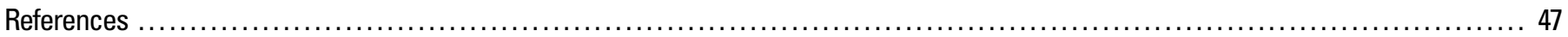

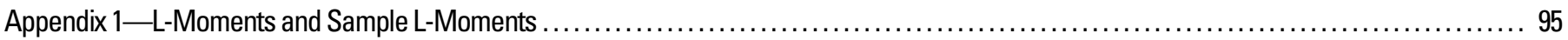

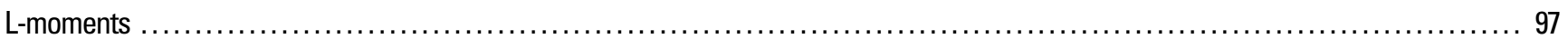

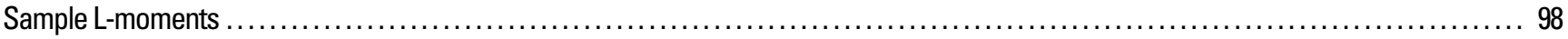

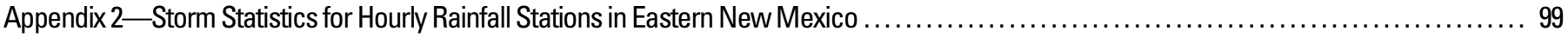

2-1.1 - 2-1.7. Number of storms, total duration, and mean storm interevent time defined by:

2-1.1. 6-hour minimum interevent time for hourly rainfall stations in eastern New Mexico . ...................... 101

2-1.2. 8-hour minimum interevent time for hourly rainfall stations in eastern New Mexico . ....................... 101

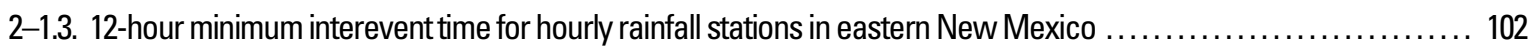

2-1.4. 18-hour minimum interevent time for hourly rainfall stations in eastern New Mexico ...................... 102

2-1.5. 24-hour minimum interevent time for hourly rainfall stations in eastern New Mexico $\ldots \ldots \ldots \ldots \ldots \ldots \ldots \ldots \ldots \ldots$

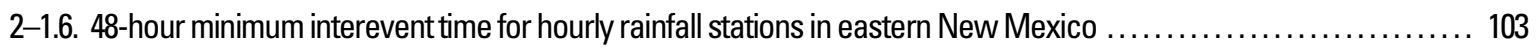

2-1.7. 72-hour minimum interevent time for hourly rainfall stations in eastern New Mexico ...................... 104 
2-2.1 - 2-2.7. L-moments of storm depth defined by:

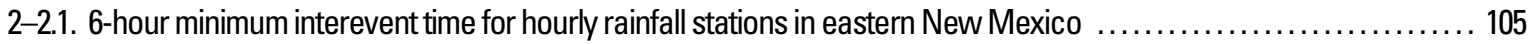

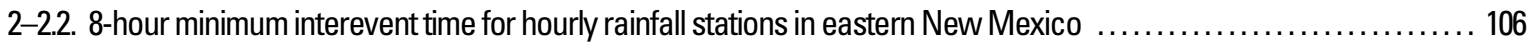

2-2.3. 12-hour minimum interevent time for hourly rainfall stations in eastern New Mexico . ....................... 107

2-2.4. 18-hour minimum interevent time for hourly rainfall stations in eastern New Mexico ........................ 108

2-2.5. 24-hour minimum interevent time for hourly rainfall stations in eastern New Mexico . ....................... 109

2-2.6. 48-hour minimum interevent time for hourly rainfall stations in eastern New Mexico . ..................... 110

2-2.7. 72-hour minimum interevent time for hourly rainfall stations in eastern New Mexico ....................... 111

2-3.1 - 2-3.7. L-moments of storm duration defined by:

2-3.1. 6-hour minimum interevent time for hourly rainfall stations in eastern New Mexico $\ldots \ldots \ldots \ldots \ldots \ldots \ldots \ldots \ldots \ldots \ldots \ldots \ldots \ldots \ldots \ldots \ldots$

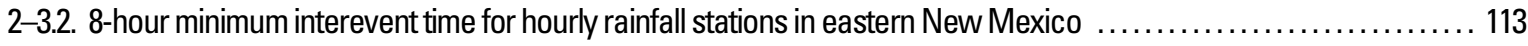

2-3.3. 12-hour minimum interevent time for hourly rainfall stations in eastern New Mexico ....................... 114

2-3.4. 18-hour minimum interevent time for hourly rainfall stations in eastern New Mexico ....................... 115

2-3.5. 24-hour minimum interevent time for hourly rainfall stations in eastern New Mexico ...................... 116

2-3.6. 48-hour minimum interevent time for hourly rainfall stations in eastern New Mexico . ...................... 117

2-3.7. 72-hour minimum interevent time for hourly rainfall stations in eastern New Mexico ...................... 118

2-4.1 - 2-4.7. Empirical distribution of storm depth defined by:

2-4.1. 6-hour minimum interevent time for hourly rainfall stations in eastern New Mexico ....................... 119

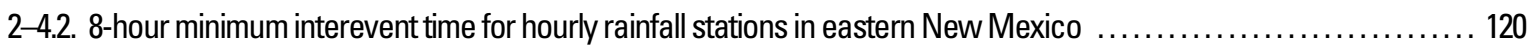

2-4.3. 12-hour minimum interevent time for hourly rainfall stations in eastern New Mexico ........................ 121

2-4.4. 18-hour minimum interevent time for hourly rainfall stations in eastern New Mexico ...................... 122

2-4.5. 24-hour minimum interevent time for hourly rainfall stations in eastern New Mexico ...................... 123

2-4.6. 48-hour minimum interevent time for hourly rainfall stations in eastern New Mexico ....................... 124

2-4.7. 72-hour minimum interevent time for hourly rainfall stations in eastern New Mexico ...................... 125

2-5.1 - 2-5.7. Empirical distribution of storm duration defined by:

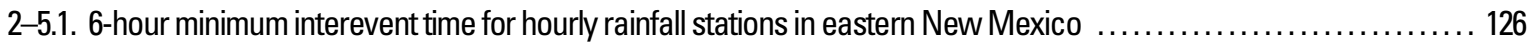

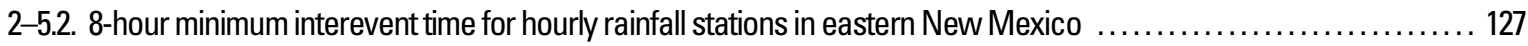

2-5.3. 12-hour minimum interevent time for hourly rainfall stations in eastern New Mexico ...................... 128

2-5.4. 18-hour minimum interevent time for hourly rainfall stations in eastern New Mexico . ....................... 129

2-5.5. 24-hour minimum interevent time for hourly rainfall stations in eastern New Mexico ...................... 130

2-5.6. 48-hour minimum interevent time for hourly rainfall stations in eastern New Mexico ...................... 131

2-5.7. 72-hour minimum interevent time for hourly rainfall stations in eastern New Mexico ...................... 132

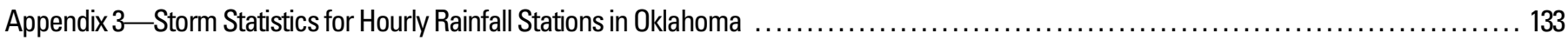

3-1.1 - 3-1.7. Number of storms, total duration, and mean storm interevent time defined by:

3-1.1. 6-hour minimum interevent time for hourly rainfall stations in Oklahoma .............................. 135

3-1.2. 8-hour minimum interevent time for hourly rainfall stations in Oklahoma .............................. 136

3-1.3. 12-hour minimum interevent time for hourly rainfall stations in Oklahoma . ............................. 137

3-1.4. 18-hour minimum interevent time for hourly rainfall stations in Oklahoma ............................ 138

3-1.5. 24-hour minimum interevent time for hourly rainfall stations in Oklahoma ............................... 139

3-1.6. 48-hour minimum interevent time for hourly rainfall stations in Oklahoma . ............................ 140

3-1.7. 72-hour minimum interevent time for hourly rainfall stations in Oklahoma ............................. 141

3-2.1 - 3-2.7. L-moments of storm depth defined by:

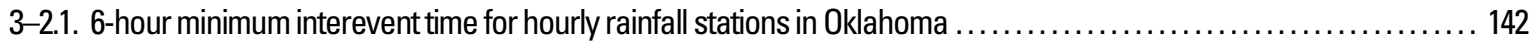

3-2.2. 8-hour minimum interevent time for hourly rainfall stations in Oklahoma .............................. 143

3-2.3. 12-hour minimum interevent time for hourly rainfall stations in Oklahoma ............................ 144

3-2.4. 18-hour minimum interevent time for hourly rainfall stations in Oklahoma .................................. 145

3-2.5. 24-hour minimum interevent time for hourly rainfall stations in Oklahoma ............................. 146

3-2.6. 48-hour minimum interevent time for hourly rainfall stations in Oklahoma .............................. 147

3-2.7. 72-hour minimum interevent time for hourly rainfall stations in Oklahoma .............................. 148 
3-3.1 - 3-3.7. L-moments of storm duration defined by:

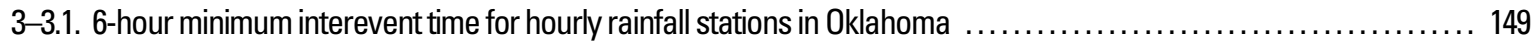

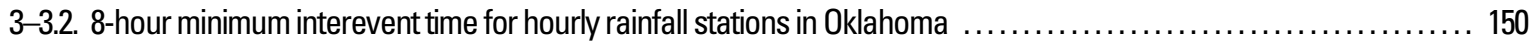

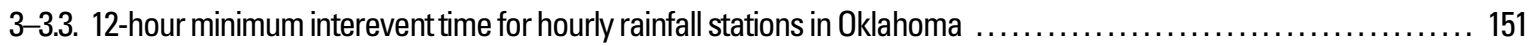

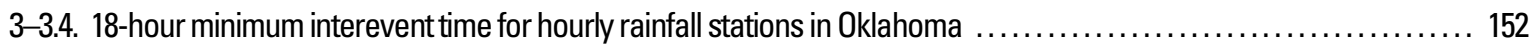

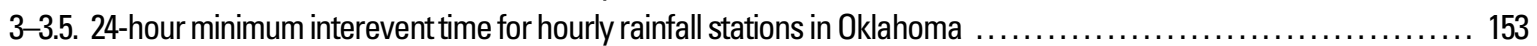

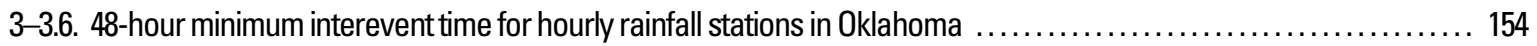

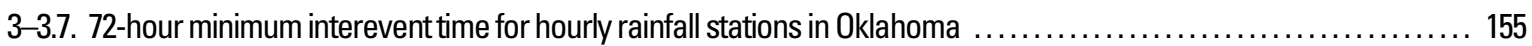

3-4.1 - 3-4.7. Empirical distribution of storm depth defined by:

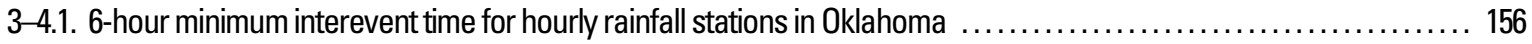

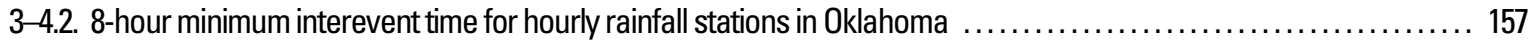

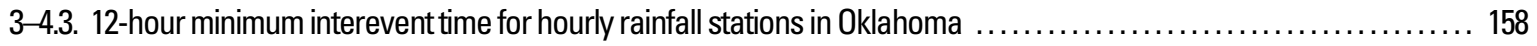

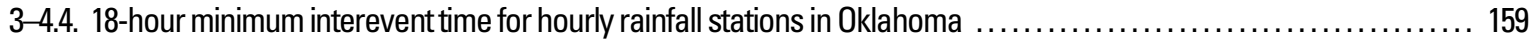

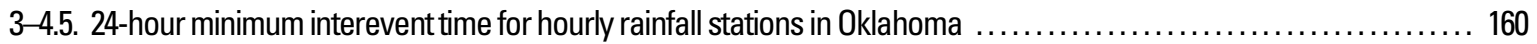

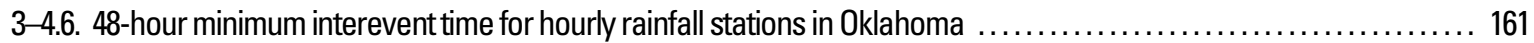

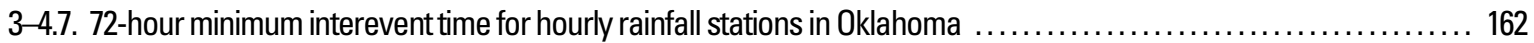

3-5.1 - 3-5.7. Empirical distribution of storm duration defined by:

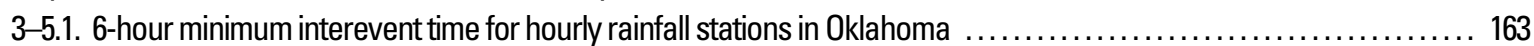

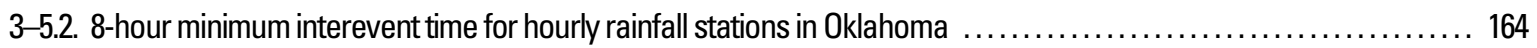

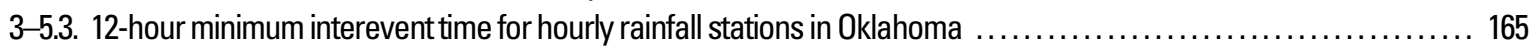

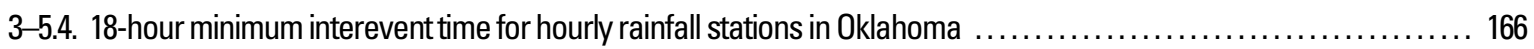

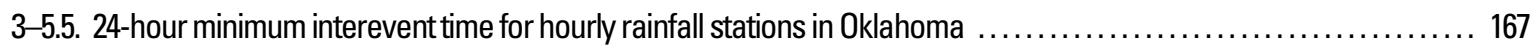

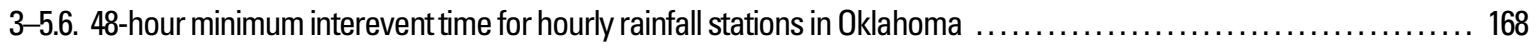

3-5.7. 72-hour minimum interevent time for hourly rainfall stations in Oklahoma $\ldots \ldots \ldots \ldots \ldots \ldots \ldots \ldots \ldots \ldots \ldots \ldots \ldots$

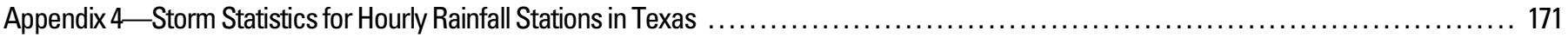

4-1.1 - 4-1.7. Number of storms, total duration, and mean storm interevent time defined by:

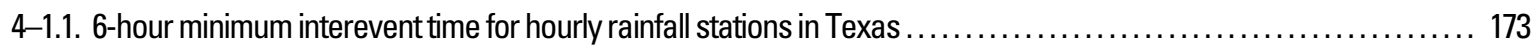

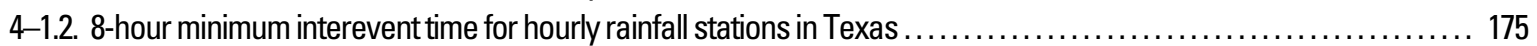

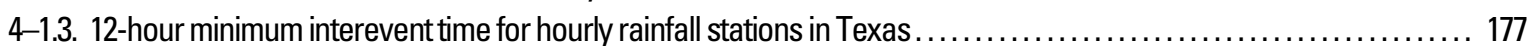

4-1.4. 18-hour minimum interevent time for hourly rainfall stations in Texas ................................. 179

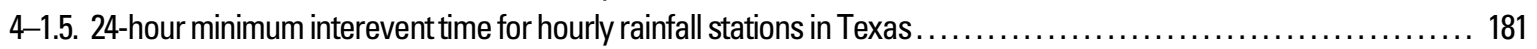

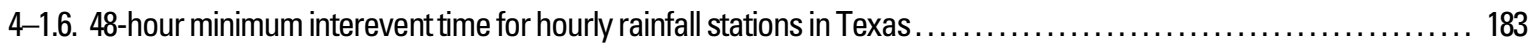

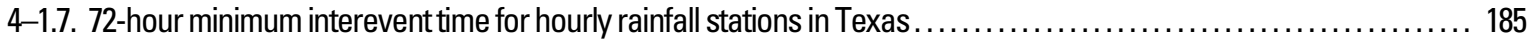

4-2.1 - 4-2.7. L-moments of storm depth defined by:

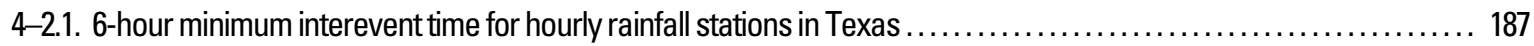

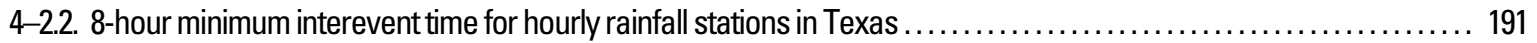

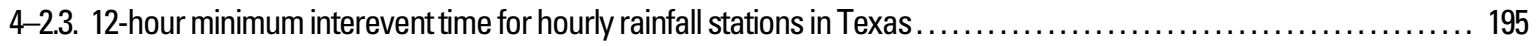

4-2.4. 18-hour minimum interevent time for hourly rainfall stations in Texas ................................ 199

4-2.5. 24-hour minimum interevent time for hourly rainfall stations in Texas ................................... 203

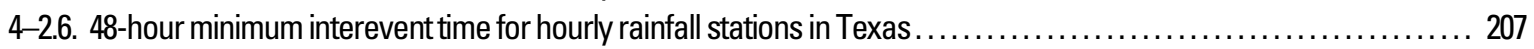

4-2.7. 72-hour minimum interevent time for hourly rainfall stations in Texas ................................ 211

4-3.1 - 4-3.7. L-moments of storm duration defined by:

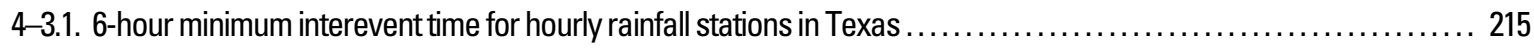

4-3.2. 8-hour minimum interevent time for hourly rainfall stations in Texas .................................. 219

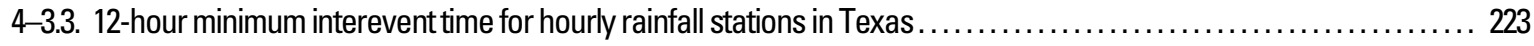

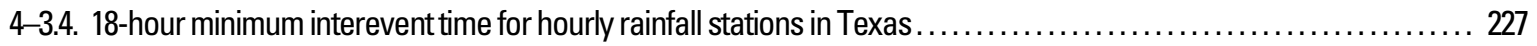

4-3.5. 24-hour minimum interevent time for hourly rainfall stations in Texas ................................... 231

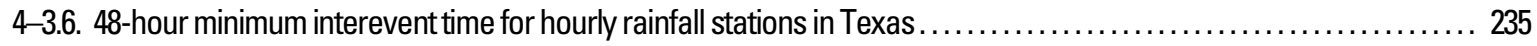

4-3.7. 72-hour minimum interevent time for hourly rainfall stations in Texas ................................ 239 
4-4.1 - 4-4.7. Empirical distribution of storm depth defined by:

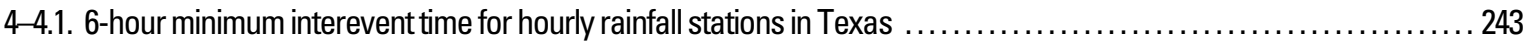

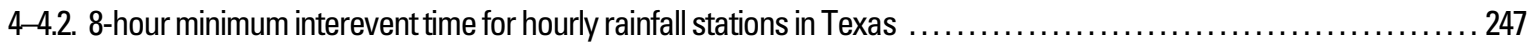

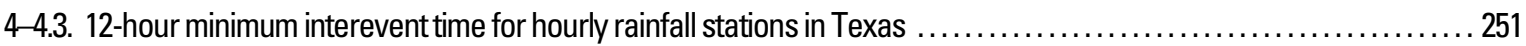

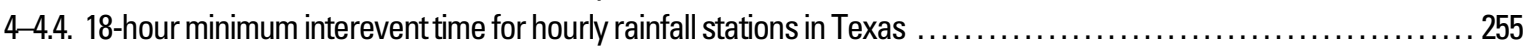

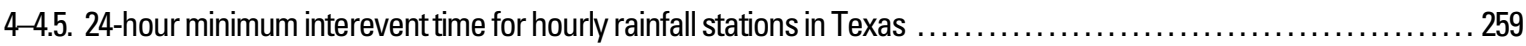

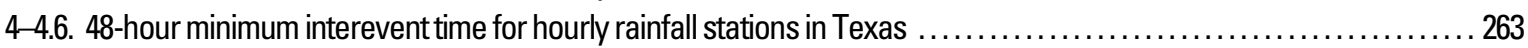

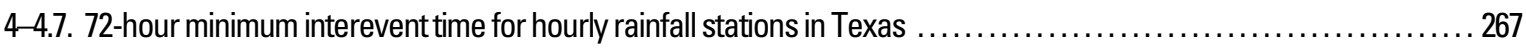

4-5.1-4-5.7. Empirical distribution of storm duration defined by:

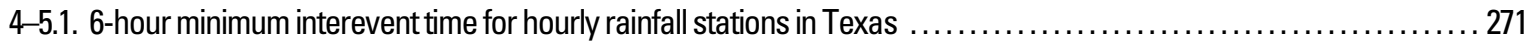

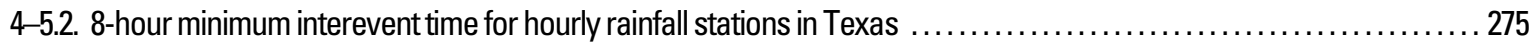

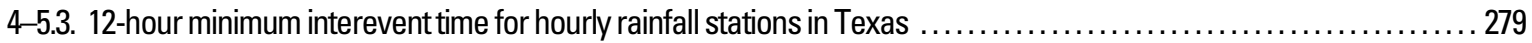

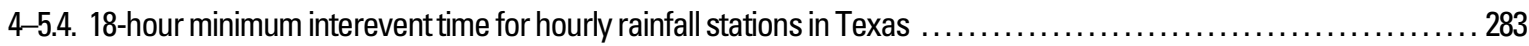

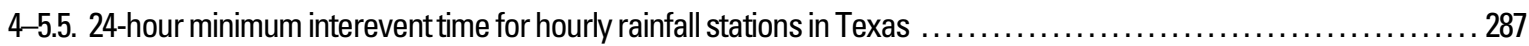

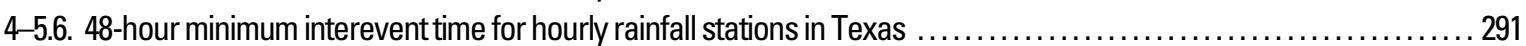

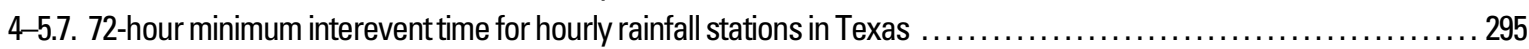

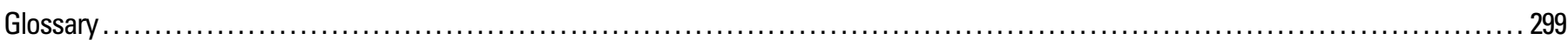

\section{Figures}

1-3. Maps showing locations of National Weather Service hourly rainfall stations in:

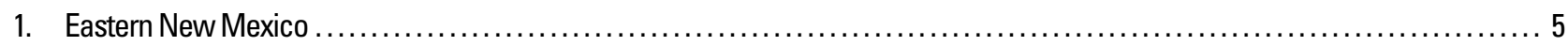

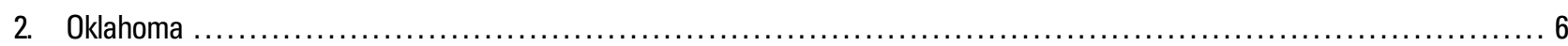

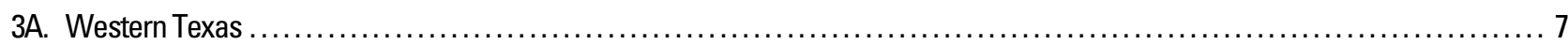

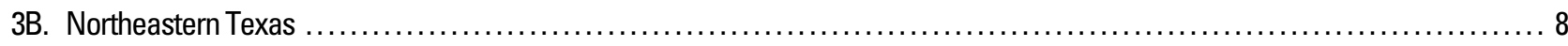

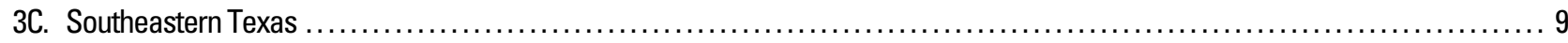

4. Graphs showing empirical distributions of $(A)$ storm depth and $(B)$ storm duration for 3,331 storm events defined by an 8-hour

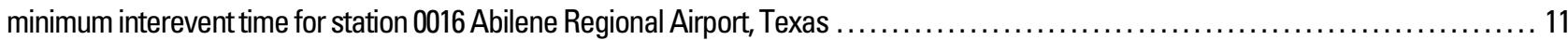

5. L-moment diagram at two scales ( $A$ and $B$ ) showing relation between $L$-skew and L-kurtosis of the distributions of storm depth and storm duration defined by the 8-hour minimum interevent time and theoretical relations for selected probability distributions

6-9. Graphs showing:

6. Dimensionless kappa distribution frequency curves for $(A)$ storm depth and $(B)$ storm duration for the selected

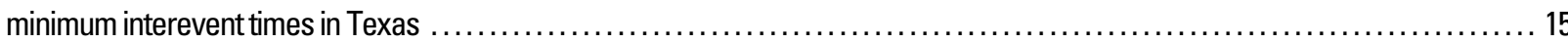

7. Dimensionless kappa distribution frequency curves for storm depth and storm duration for 24-hour minimum interevent

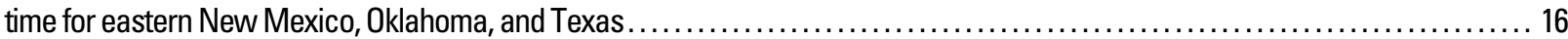

8. Dimensionless exponential gamma and kappa distributions of storm depth for 24-hour minimum interevent time in

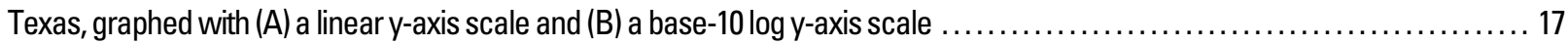

9. Storm depth and storm duration for 3,331 storm events defined by an 8-hour minimum interevent time for station 0016 Abilene Regional Airport, Texas

10-16. Maps showing mean storm interevent time defined by:

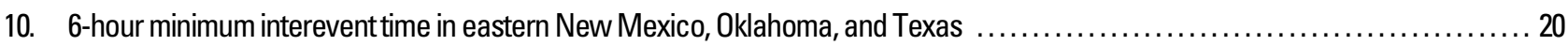

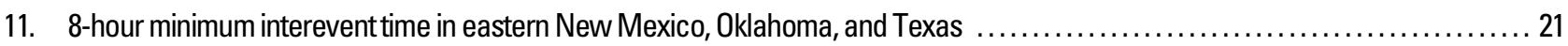

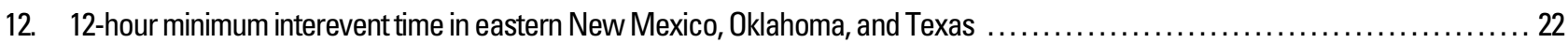

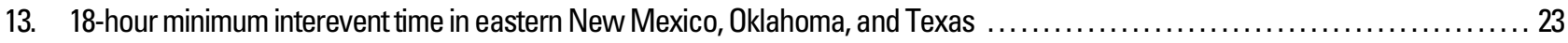

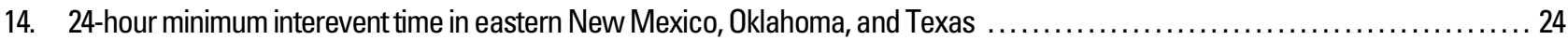

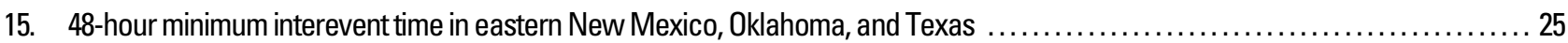

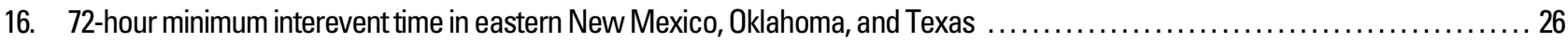


17-23. Maps showing mean storm depth defined by:

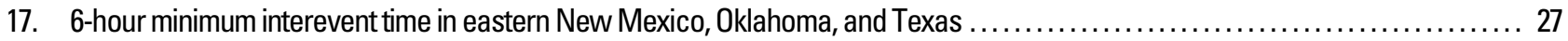

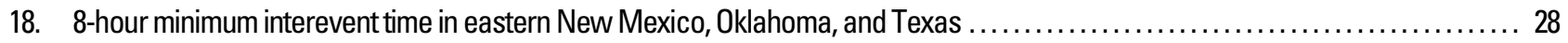

19. 12-hour minimum interevent time in eastern New Mexico, Oklahoma, and Texas .................................. 29

20. 18-hour minimum interevent time in eastern New Mexico, Oklahoma, and Texas .................................... 30

21. 24-hour minimum interevent time in eastern New Mexico, Oklahoma, and Texas ................................... 31

22. 48-hour minimum interevent time in eastern New Mexico, Oklahoma, and Texas ................................... 32

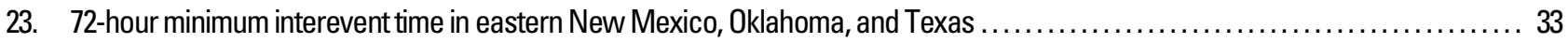

24-30. Maps showing mean storm duration defined by:

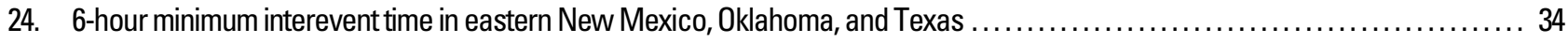

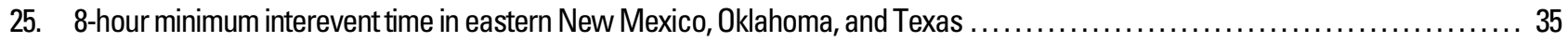

26. 12-hour minimum interevent time in eastern New Mexico, Oklahoma, and Texas .................................. 36

27. 18-hour minimum interevent time in eastern New Mexico, Oklahoma, and Texas .................................... 37

28. 24-hour minimum interevent time in eastern New Mexico, Oklahoma, and Texas ................................... 38

29. 48-hour minimum interevent time in eastern New Mexico, Oklahoma, and Texas ..................................... 39

30. 72-hour minimum interevent time in eastern New Mexico, Oklahoma, and Texas ................................... 40

\section{Tables}

1-3. National Weather Service hourly rainfall stations in:

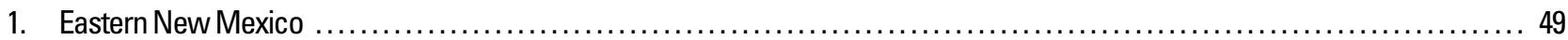

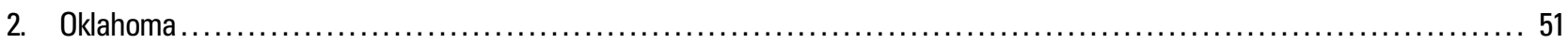

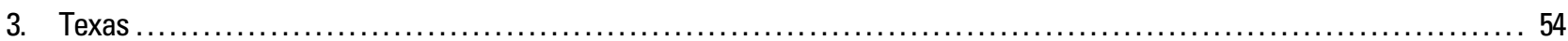

4. Selected storm statistics for station 4570 Jayton, Texas, illustrating influence of minimum interevent time on statistics .............. 64

5-6. Regionwide or statewide record-length, weighted-average:

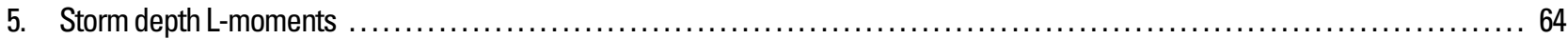

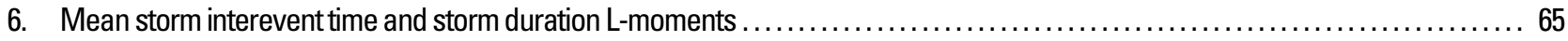

7-8. Dimensionless gamma and kappa distributions fit to record-length, weighted-average storm:

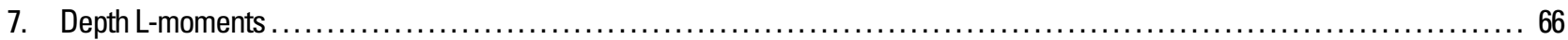

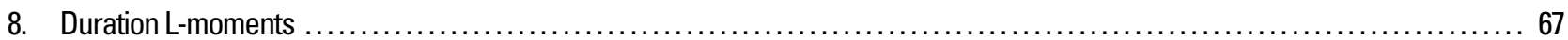

9-11. Summary statistics and diagnostic statistics of mean storm:

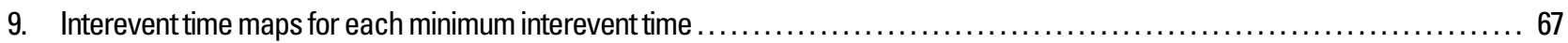

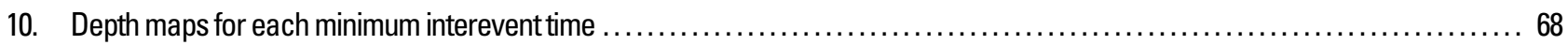

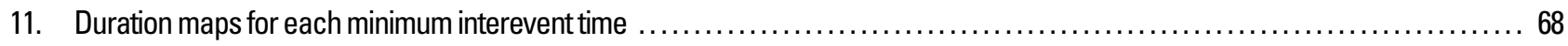

12-20. Mean storm:

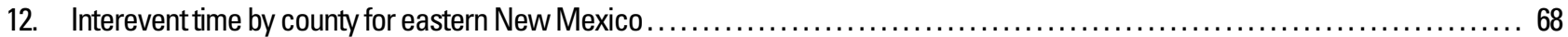

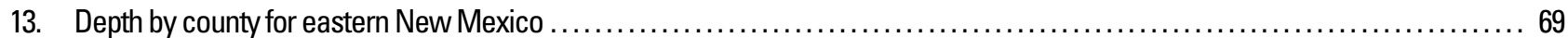

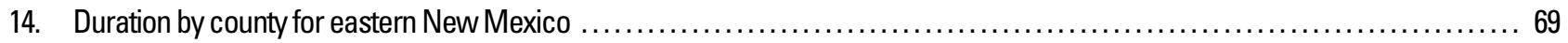

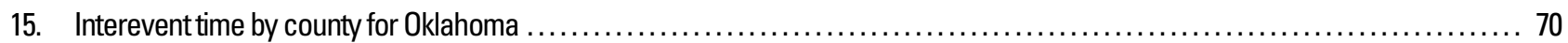

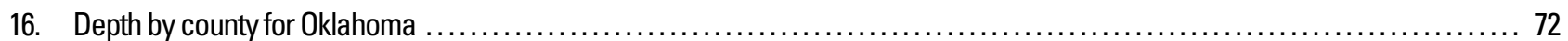

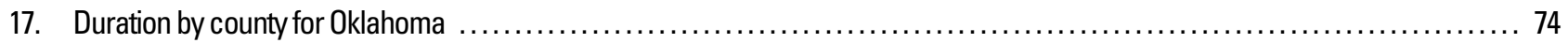

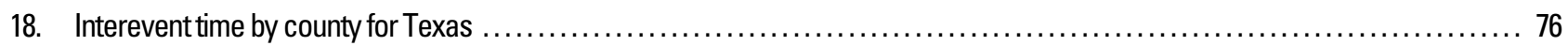

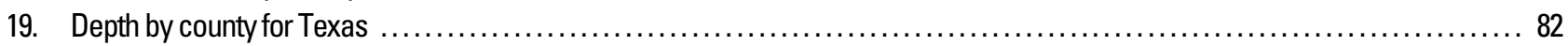

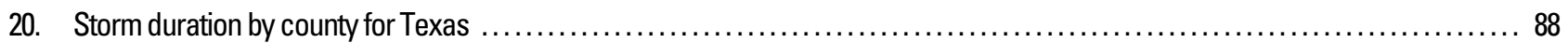

21. Example simulations of storm interevent time from example 2 using 24-hour minimum interevent time for station 0211 Amarillo

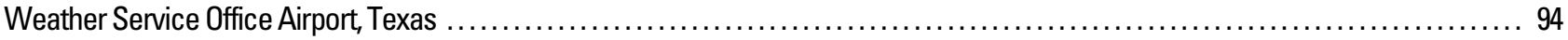

22. Distribution of storm depth for examples 3 and 4 based on site-specific statistics for station 4311 Houston Alief, Texas ............. 94

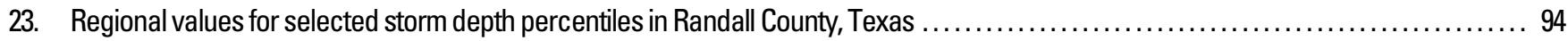


viii

\section{Conversion Factors}

\begin{tabular}{lcl}
\hline Multiply & \multicolumn{1}{c}{ By } & To obtain \\
\hline inch (in.) & 25.4 & millimeter $(\mathrm{mm})$ \\
foot (ft) & 0.3048 & meter $(\mathrm{m})$ \\
mile (mi) & 1.609 & kilometer $(\mathrm{km})$ \\
\hline
\end{tabular}

Vertical coordinate information is referenced to the National Geodetic Vertical Datum of 1929 (NGVD 29). 


\title{
Statistical Characteristics of Storm Interevent Time, Depth, and Duration for Eastern New Mexico, Oklahoma, and Texas
}

\author{
By William H. Asquith', Meghan C. Roussel ${ }^{1}$, Theodore G. Cleveland ${ }^{2}$, Xing Fang ${ }^{3}$, \\ and David B. Thompson ${ }^{4}$
}

\section{Abstract}

The design of small runoff-control structures, from simple floodwater-detention basins to sophisticated best-management practices, requires the statistical characterization of rainfall as a basis for cost-effective, risk-mitigated, hydrologic engineering design. The U.S. Geological Survey, in cooperation with the Texas Department of Transportation, has developed a framework to estimate storm statistics including storm interevent times, distributions of storm depths, and distributions of storm durations for eastern New Mexico, Oklahoma, and Texas. The analysis is based on hourly rainfall recorded by the National Weather Service. The database contains more than 155 million hourly values from 774 stations in the study area. Seven sets of maps depicting ranges of mean storm interevent time, mean storm depth, and mean storm duration, by county, as well as tables listing each of those statistics, by county, were developed. The mean storm interevent time is used in probabilistic models to assess the frequency distribution of storms. The Poisson distribution is suggested to model the distribution of storm occurrence, and the exponential distribution is suggested to model the distribution of storm interevent times. The fourparameter kappa distribution is judged as an appropriate distribution for modeling the distribution of both storm depth and storm duration. Preference for the kappa distribution is based on interpretation of L-moment diagrams. Parameter estimates for the kappa distributions are provided. Separate dimensionless frequency curves for storm depth and duration are defined for eastern New Mexico, Oklahoma, and Texas. Dimension is restored by multiplying curve ordinates by the mean storm depth or mean storm duration to produce quantile functions of storm depth and duration. Minimum interevent time and loca-

\footnotetext{
${ }^{1}$ U.S. Geological Survey.

${ }^{2}$ University of Houston.

${ }^{3}$ Lamar University.

${ }^{4}$ Texas Tech University.
}

tion have slight influence on the scale and shape of the dimensionless frequency curves. Ten example problems and solutions to possible applications are provided.

\section{Introduction}

The design of runoff-control structures, from simple floodwater-detention basins to sophisticated best-management practices (BMPs), such as engineered sand-filtration ponds, requires the statistical characterization of rainfall as a basis for cost-effective, risk-mitigated, hydrologic engineering design. BMPs and similar structure types (collectively referred to in this report as BMPs) are present in many suburban and urban areas, usually in small watersheds (less than about 10 square miles). BMPs can be a substantial component of public and private drainage infrastructure; the characteristics of rainfall for a given location strongly influence the hydrologic and hydraulic function and, to a lesser degree, the water-quality performance of these structures. However, to date (2006), there has not been a comprehensive procedural framework in Texas for analysis of the probabilistic, or expected, performance of these structures in the context of rainfall inputs to a watershed.

In 2000, the U.S. Geological Survey, in cooperation with the Texas Department of Transportation, and in collaboration with University of Houston, Lamar University, and Texas Tech University, initiated a research program of Texas rainfall characteristics to enhance hydrologic engineering design. One major objective of the program is to provide comprehensive statistics of hourly rainfall in support of BMP design in Texas. To better define rainfall characteristics near the borders of Texas, the study area for this report was expanded to include eastern New Mexico and Oklahoma. The expansion also was made so that the study area has a more rectangular boundary to enhance geostatistical analysis of storm statistics near the borders of Texas. 


\section{Statistical Characteristics of Storm Interevent Time, Depth, and Duration for Eastern New Mexico, Oklahoma, and Texas}

Information on mean storm interevent time enables computation of the expected number of storms in a specified period of time. Information on rainfall depth for storms (hereinafter, storm depth) facilitates cost-benefit analysis of BMP performance efficiency. Hydrologic engineers and decision makers use storm depth information in reviewing, revising, or establishing guidelines for BMP design. For BMP design, the distribution of storm depth is important because it is related to runoff pollutant mass (Wanielista and Yousef, 1993, p. 221). The distribution of storm duration is useful in assessing storm average rainfall rates and other aspects of hydrologic engineering design. Storms are defined by a minimum interevent time-a time in which no rainfall occurs. The minimum interevent time, which is appropriate for specific applications, can be determined by the drawdown time, infiltration time, or treatment time for a given BMP design.

\section{Purpose and Scope}

This report presents a framework for analysis of the probabilistic, or expected, function of BMPs in the context of rainfall inputs to a watershed. Specifically, the report documents three statistics of storms recorded in hourly rainfall data by the National Weather Service in eastern New Mexico, Oklahoma, and Texas. The three statistics are (1) mean storm interevent time, measured in days of no rainfall between successive storms, (2) distribution of storm depth, measured in inches, and (3) distribution of storm duration, measured in hours. These statistics are provided for each of seven selected minimum interevent times: $6,8,12,18,24,48$, and 72 hours.

The primary products or results reported here are

1. Maps depicting ranges of mean storm interevent time, mean storm depth, and mean storm duration, by county;

2. Tables listing mean storm interevent time, mean storm depth, and mean storm duration, by county; and

3. Numerous dimensionless frequency curves for storm depth and duration to be used in conjunction with the maps and tables of mean storm depth and duration.

Secondary results are extensive tables listing site-specific storm statistics. These statistics include the number of storms, total duration, mean storm interevent time, L-moments (mean, L-scale, coefficient of L-variation, L-skew, L-kurtosis, and Tau5), and percentiles (1st, 2nd, 10th, 25th, 50th, 75th, 90th, 98th, and 99th) for both storm depth and duration for each of the seven minimum interevent times. Finally, 10 example problems and solutions using site-specific and regional (by county) approaches to possible applications are provided.

\section{Previous Studies}

\section{Storm Research Sponsored by the Texas Department of Transportation}

The Texas Department of Transportation has sponsored a multifaceted research program through several research projects on rainfall characteristics in Texas from the mid-1990s through 2005. A chronological list with brief description of results follows:

1. Asquith (1998)_-Defines the depth-duration frequency (DDF) of rainfall annual maxima in Texas by providing an atlas of the parameters of probability distributions. DDF values commonly are used in hydrologic engineering design. An example of a DDF value is the depth of rainfall for the 50-year, 6-hour storm.

2. Lanning-Rush and others (1998)_Provides envelope curves for extreme storms in Texas showing the relation between areal storm depth and storm extent. The report also provides a bibliography of large and historically important storms in Texas.

3. Asquith (1999)—Defines areal-reduction factors (ARF) for the 1-day design storm in the Austin, Dallas, and Houston areas. ARF are used in conjunction with DDF values to adjust DDF for the influence of watershed area.

4. Asquith and Famiglietti (2000)_Documents the annualmaxima-centered approach used by Asquith (1999) to define ARF.

5. Al-Asaadi (2002)_-Provides detailed analysis of dimensionless hyetographs for 204 runoff-producing storms for 12 watersheds in the San Antonio area. The report also provides analysis of the burst characteristics of the storms. The rainfall data considered are summarized in Asquith and others (2005).

6. Asquith (2003) —Provides a comprehensive analysis of L-moments and other statistics of hyetographs for runoffproducing storms in Texas. The rainfall data considered are summarized in Asquith and others (2005).

7. Asquith and Roussel (2003)_-Provides an atlas of mean interoccurrence intervals of selected thresholds of daily rainfall in Texas. Interoccurrence intervals can enhance the planning and construction of infrastructure as well as runoff-control structures by providing hydrologic engineers with information on the frequency of daily rainfall.

8. Asquith and others (2003)_-Provides two separate equation pairs based on a triangular model of the expected hyetograph for runoff-producing storms having more than 0.5 inch of rainfall in Texas for two ranges of storm duration ( 0 to 24 hours and 24 to 72 hours). The report augments the research of Asquith (2003). 
9. Asquith and Thompson (2003) — Provides an alternative hyetograph model (L-gamma) to the triangular model (Asquith, 2003; Asquith and others, 2003). Provides three distinct hyetograph equations for three storm duration ranges for Texas. These models are more sophisticated than the triangular models and might be preferable to the triangular models in some applications.

10. Asquith and Roussel (2004)_Provides a directly interpretable atlas of DDF in Texas on the basis of research results of Asquith (1998). The report contains 96 maps of the depth of rainfall for 12 storm durations and eight annual nonexceedance probabilities (recurrence intervals). More information regarding the report is available in Strand (2003).

11. Williams-Sether and others (2004)_-Provides documentation of the empirical dimensionless hyetographs for selected durations of runoff-producing storms in Texas. The report augments the research of Asquith (2003).

12. Asquith and others (2005)_Provides numerous dimensionless rainfall hyetographs for Texas, describes the analytical approach used, and provides information regarding distribution of storm depth essentially identical to that presented in this report.

\section{Other Studies}

The U.S. Environmental Protection Agency (1986, fig. A-2) provides a map of nine regions encompassing the conterminous United States and a table showing the mean and coefficient of variation for storm interevent times, storm depths, and storm durations for storms defined by a 3- to 4-hour minimum interevent time. The area east of 96 degrees $\left({ }^{\circ}\right)$ west longitude (region 4 ) is coincident with part of the study area (eastern Oklahoma and eastern Texas) and has a mean storm interevent time of about 4.1 days, a mean storm depth of about 0.58 inch, and a mean storm duration of about 7.3 hours. The area west of $96^{\circ}$ west longitude (region 5) is coincident with the remaining part of the study area and has a mean storm interevent time of about 4.5 days, a mean storm depth of about 0.33 inch, and a mean storm duration of about 4.0 hours. (The results in this report from rigorous analysis show that these statistics have a more complex pattern of variation than shown by the U.S. Environmental Protection Agency [1986], but specific comparison is difficult.)

Schueler (1987, p. 3.9) describes six rules for sizing extended detention BMPs (basins) in the Washington, D.C., area. For example, rule 4 states that the BMP should be sized to accommodate the "runoff volume generated from a one inch storm released [within the BMP area] over 24 hours."
Wanielista and Yousef (1993, p. 221) state that 4- to 5hour minimum interevent times have been used by previous authors for urban BMP design and also report that a minimum interevent time for a given BMP design "should be long enough to ensure that runoff events are independent of one another." Contaminant removal effectiveness (when measured in terms of runoff diversion) is based on the number of storms per year or the percentage of storms captured by a BMP. Wanielista and Yousef (1993, table 7.1) report that the 90th-percentile storm for Austin, Tex., for a 4-hour minimum interevent time is about 1.0 inch $^{5}$.

Adams and Papa (2000) discuss the probabilistic functioning of small-watershed BMPs and characterize the statistical distributions of mean storm interevent times, depths, and durations. Adams and Papa (2000) also discuss minimum interevent times and derive extensive formulas to estimate various aspects of BMP function, including the expected capture or expected spillage of a BMP. They use a runoff-coefficient initial-abstraction model of the rainfall-runoff process. Their derivations are based on an assumption that storm interevent time, depth, and duration each are exponentially distributed ${ }^{6}$.

\section{Database of Hourly Rainfall}

This study, although done specifically for Texas, includes data from eastern New Mexico and Oklahoma and therefore is applicable to these areas as well. Data from these areas were included to enhance the reliability of statistical interpretation near the Texas borders with New Mexico and Oklahoma.

National Weather Service hourly rainfall data for stations in the study area were obtained from Hydrosphere (2003). All hourly data for the period of record for all stations, including number, name, latitude, and longitude, were compiled for the study. The first calendar year of data is 1947 for eastern New Mexico, 1947 for Oklahoma, and 1940 for Texas; the last calendar year of data is 2002 for the three states. The database contains more than 155 million values of hourly rainfall (zero values included) from 774 hourly stations. Of these, 92 stations and more than 18 million values are available for eastern New Mexico; 149 stations and more than 33 million values are available for Oklahoma; and 533 stations and more than 103 million values are available for Texas. For perspective, for an 8-hour minimum interevent time, 97,491 storms in eastern New Mexico, 206,646 storms in Oklahoma, and 584,159 storms in Texas are identified. Stations used in the analysis are listed in tables 1-3 (at end of report) and are shown in figures 1-3 for eastern New Mexico, Oklahoma, and Texas, respectively.

\footnotetext{
${ }^{5}$ For comparison, the 90 th-percentile storm depth for a 6-hour minimum interevent time at station 0428 Austin Camp Mabry, Tex., is computed as 1.14 inches for this report (see section "Analysis of Site-Specific Statistical Characteristics of Hourly Rainfall” in this report).

${ }^{6}$ A demonstration of a subset of the results of Adams and Papa (2000) is provided in example 9 in section "Example Applications" in this report. The statistical results for eastern New Mexico, Oklahoma, and Texas are used in some of the techniques developed by Adams and Papa (2000).
} 


\section{Acknowledgments}

The authors acknowledge the following colleagues of the Texas Department of Transportation for their considerable professional and technical support of this work and their critical feedback on project direction: Amy J. Ronnfeldt, 5-4194 Project Director; George R. Herrmann, 5-4194 Project Advisor; and David Stolpa (retired), 5-4194 Project Advisor. The authors also acknowledge David J. Holtschlag, U.S. Geological Survey, for his thoughtful review comments and supporting numerical simulations of an early draft; and J. Curtis Weaver, U.S. Geological Survey, for a thorough review that greatly enhanced the report.

\section{Analysis of Site-Specific Statistical Characteristics of Hourly Rainfall}

Statistical analysis of storms for each station includes the computation of site-specific values of mean storm interevent time, selected L-moments of storm depth, percentiles of storm depth, L-moments of storm duration, and percentiles of storm duration. On the basis of the site-specific statistics, regional analysis was used to develop a method to estimate the statistics for arbitrary locations in the study area. These statistics provide for detailed assessment of BMP function for specific rainfall characteristics.

\section{Minimum Interevent Time of Rainfall}

Before the site-specific statistics of rainfall can be computed, it is necessary to establish a method to define distinct storms from the hourly time series of rainfall data. Typically, distinct storms are defined using a minimum interval of no rainfall, which is referred to as a minimum interevent time.

Time series of hourly rainfall data are described as sequences of nonzero rainfall depths separated by sequences of zero rainfall depths, both of varying lengths. As the lengths of zero rainfall sequences increase, it is natural to categorize the intervals of rainfall into distinct storms. Brief periods of zero rainfall (intrastorm zero values) can be, and often are, present within a particular storm.

One approach to distinguish between short and long time intervals is to analyze the rainfall time series using autocorrelation (not presented in this report). Another approach, the focus of this report, is to distinguish long time intervals according to the drawdown or drainage time of a BMP. BMP design is influenced by requirements for the drawdown time, infiltration time, or treatment time - a structural minimum interevent time.

To clarify the concept of structural minimum interevent time, consider a hypothetical city ordinance that requires BMPs, which start from full storage conditions and no additional runoff input, to drain completely in 48 hours. If storm statistics are defined by a 48-hour structural minimum interevent time associated with storms, the BMP in the context of rainfall input is said to be memoryless. The term "memoryless" refers to expected absence of BMP storage before the runoff from the next storm arrives. Specifically, the 48-hour minimum interevent time ensures that storage in the BMP is zero prior to the arrival of runoff from the next storm. Without so naming, Wanielista and Yousef (1993, p. 222-223) discuss a structural minimum interevent time being set by the infiltration time so that the infiltration pond (a BMP) will be empty before the next storm begins.

Seven structural minimum interevent times were selected: $6,8,12,18,24,48$, and 72 hours. These interevent times are expected to provide flexibility for a wide range of applications. From minimum interevent times in this sequence, users of this report can interpolate statistics to minimum interevent times not explicitly considered here.

Minimum interevent time has great influence on storm statistics. For the mean storm interevent time, mean storm depth and depth percentiles, and mean storm duration and duration percentiles, the magnitude of each statistic increases with increasing minimum interevent time. The influence of minimum interevent time is illustrated using selected site-specific statistics for station 4570 Jayton, Tex. (table 4, at end of report). The mean storm interevent time increases from about 8.5 days to almost 15 days; whereas the mean depth increases from about 0.4 to about 0.8 inch. Increasing minimum interevent time also affects BMP design. For example, the 90th-percentile depth for storms with an 8-hour minimum interevent time is about 1.2 inches. However, if a 72-hour drawdown time BMP is to have memoryless performance- that is, to perform without regard to previous storage conditions of the structure from earlier storms-then about 2.1 inches of storage is required to capture the runoff from 90 percent of all storms on the watershed. (For simplicity, it is assumed that all rainfall is converted to runoff.) An additional 0.9 inch (2.1 minus 1.2) or 1.75 (2.1 divided by 1.2) times more storage thus is required when the minimum interevent time increases from 8 to 72 hours.

\section{Storm Interevent Time and Distribution of Depth and Duration}

For each of the minimum interevent times, the time series of hourly rainfall for each station was separated into sequences of storms for subsequent statistical analysis. As part of the statistical analysis, assumptions were made about the rainfall data, extracted storms, and computed statistics. The following assumptions extend discussion by Adams and Papa (2000, p. 60) and are not mutually exclusive:

1. Storms defined by the minimum interevent time are samples from a single underlying population.

2. Storms are homogeneous - that is, generated from the same population. 

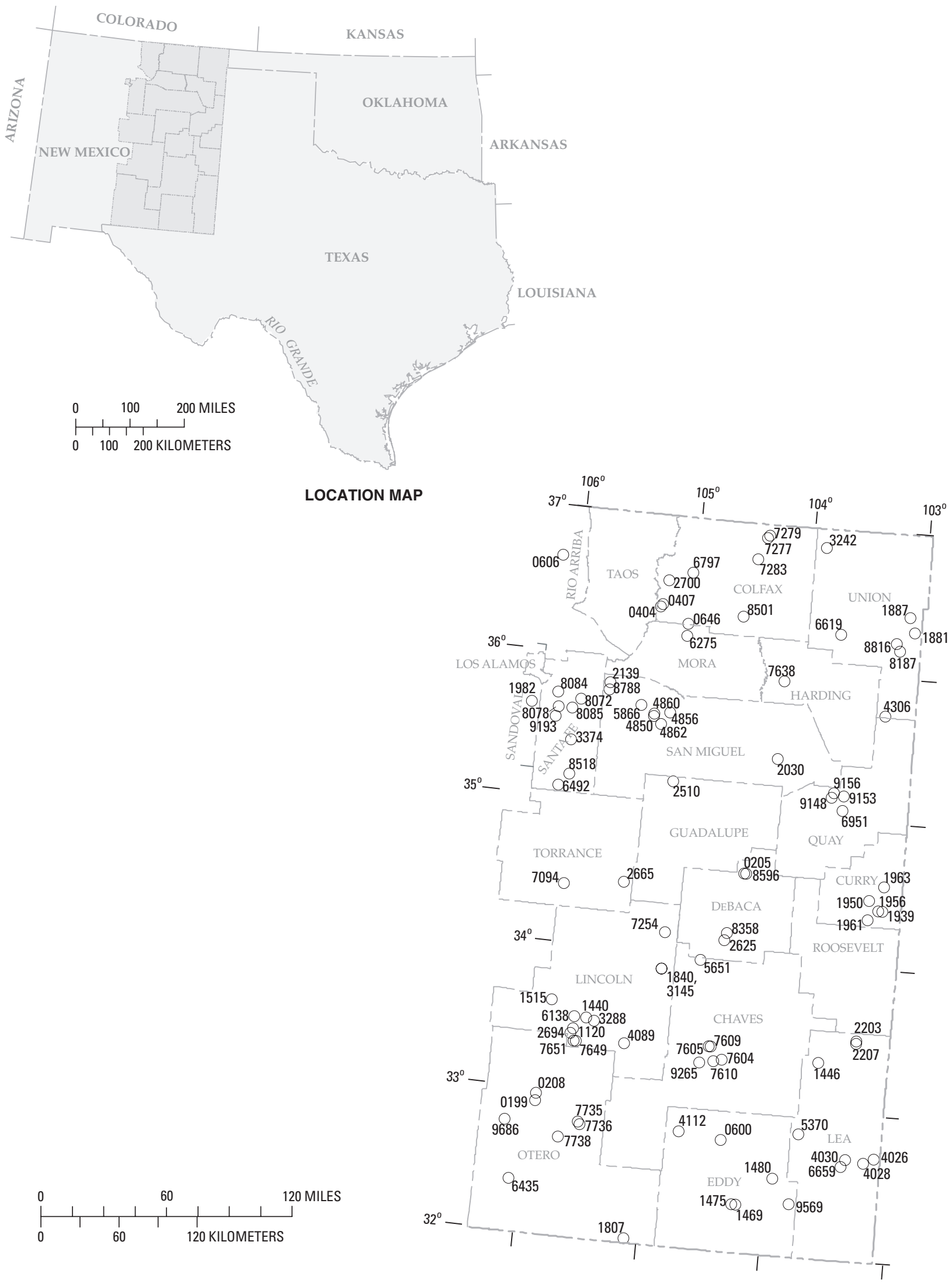

Figure 1. Locations of National Weather Service hourly rainfall stations in eastern New Mexico. 


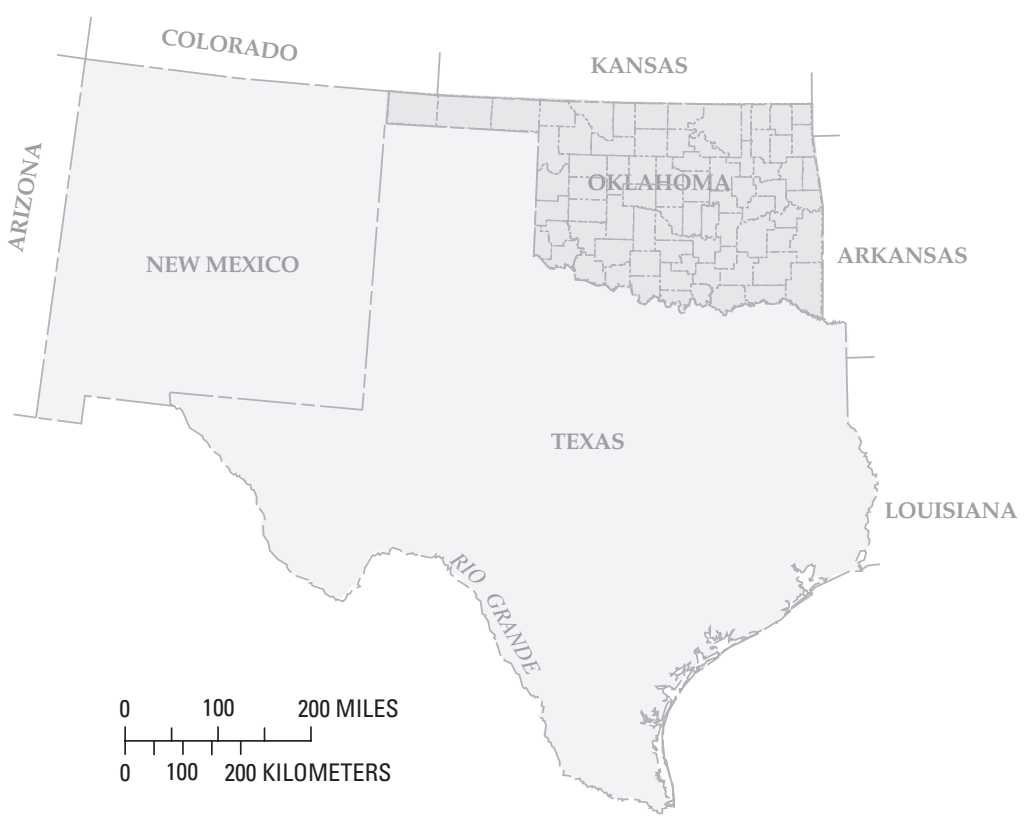

LOCATION MAP

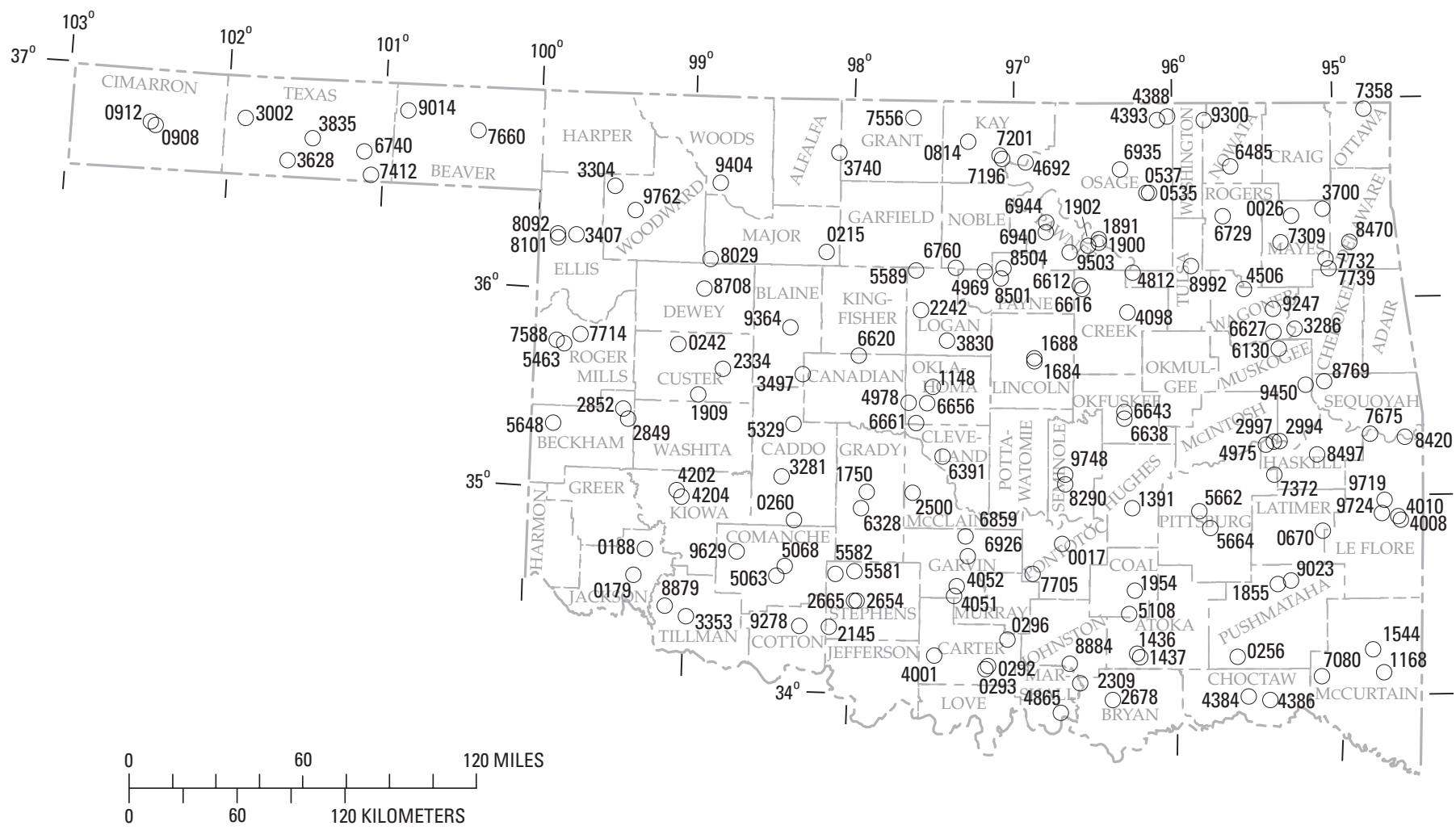

Figure 2. Locations of National Weather Service hourly rainfall stations in Oklahoma. 

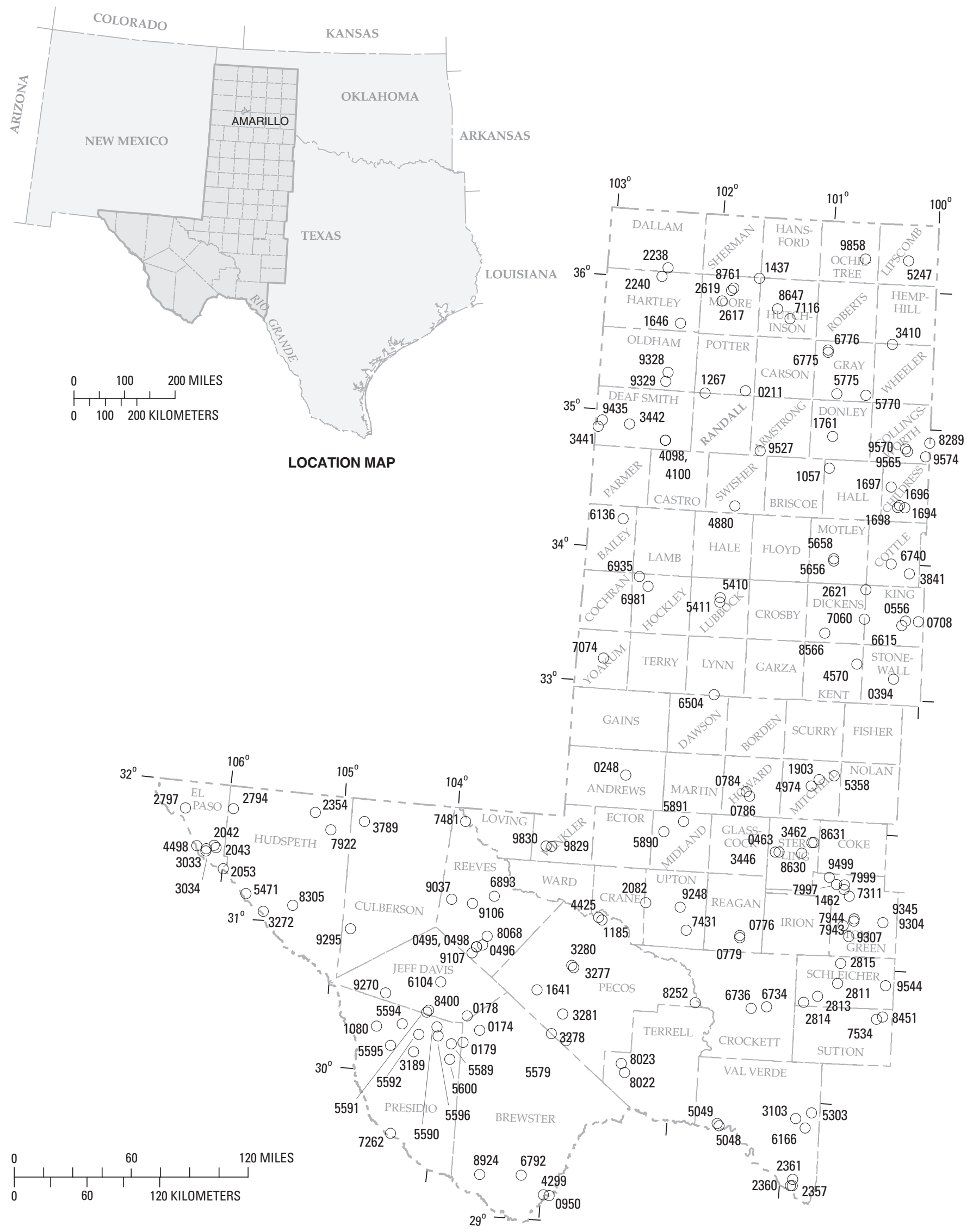

Figure 3A. Locations of National Weather Service hourly rainfall stations in western Texas. 


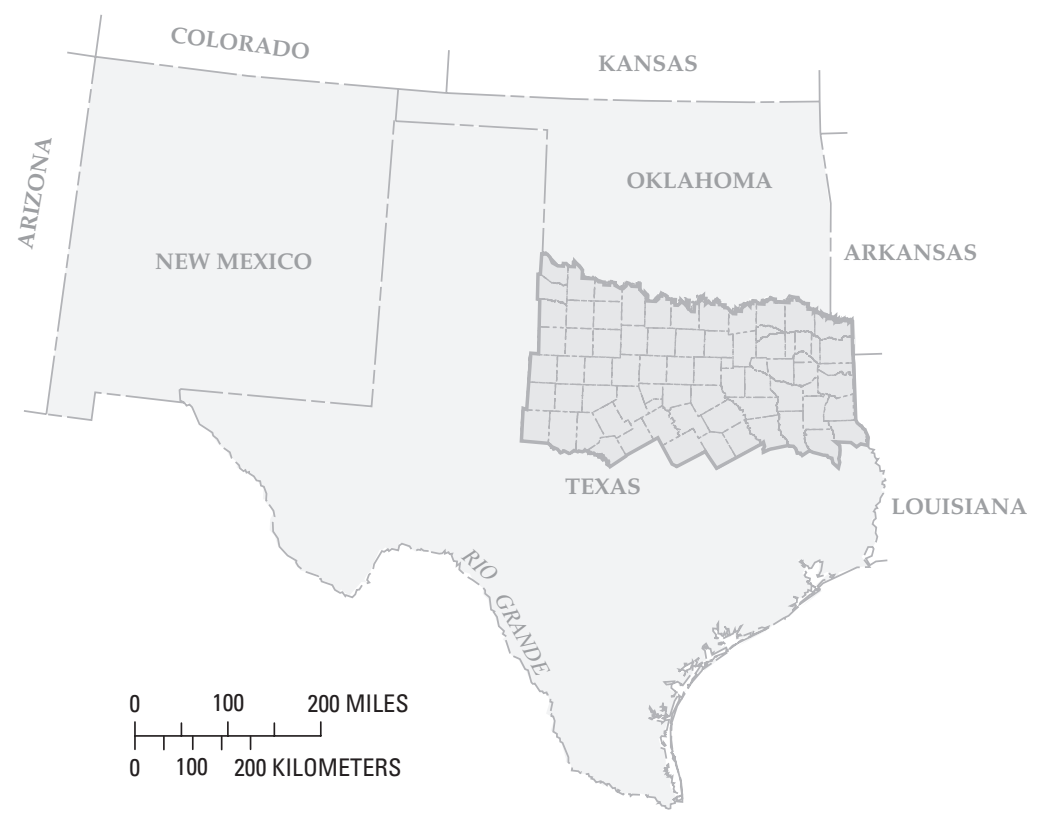

LOCATION MAP
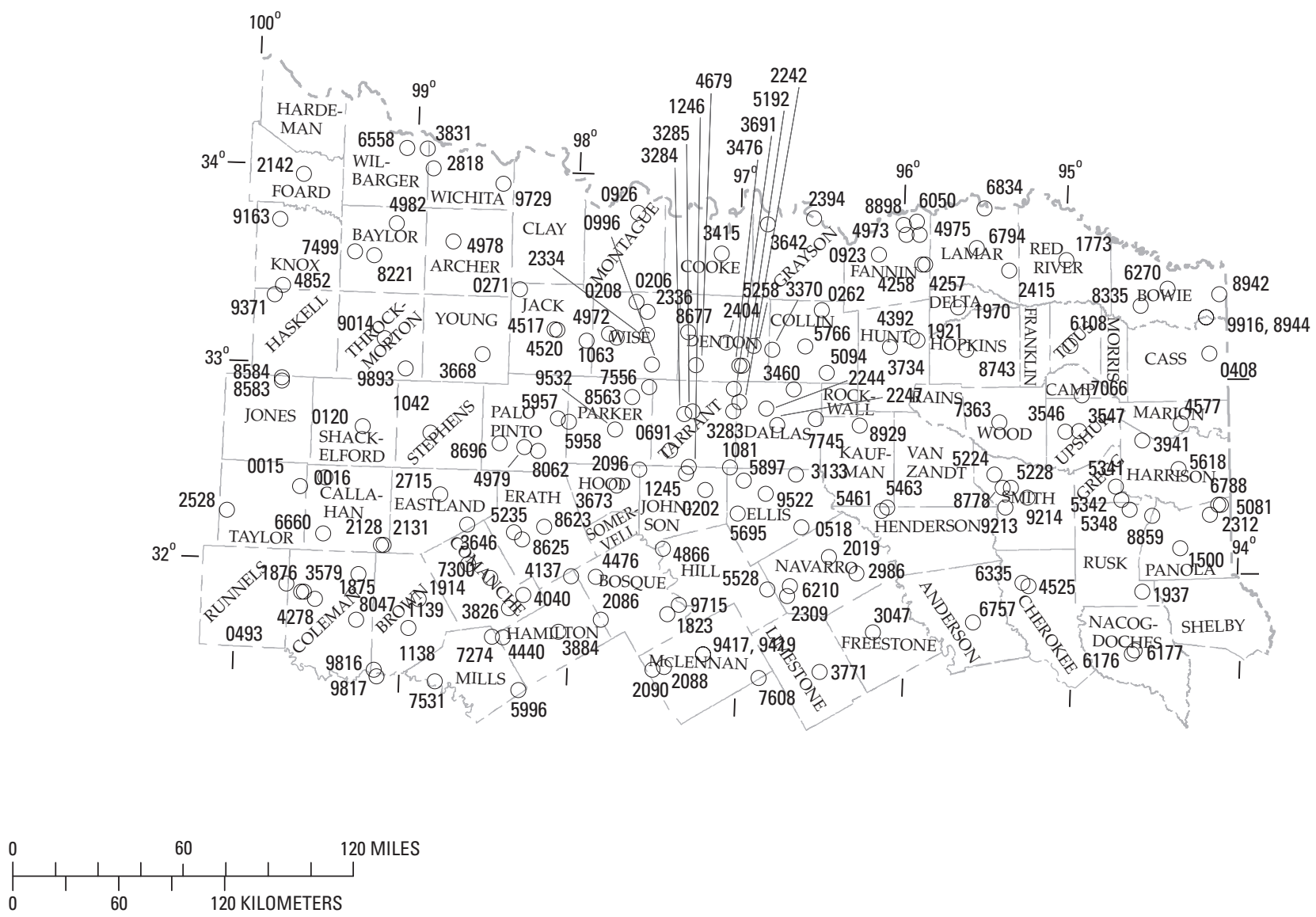

Figure 3B. Locations of National Weather Service hourly rainfall stations in northeastern Texas. 


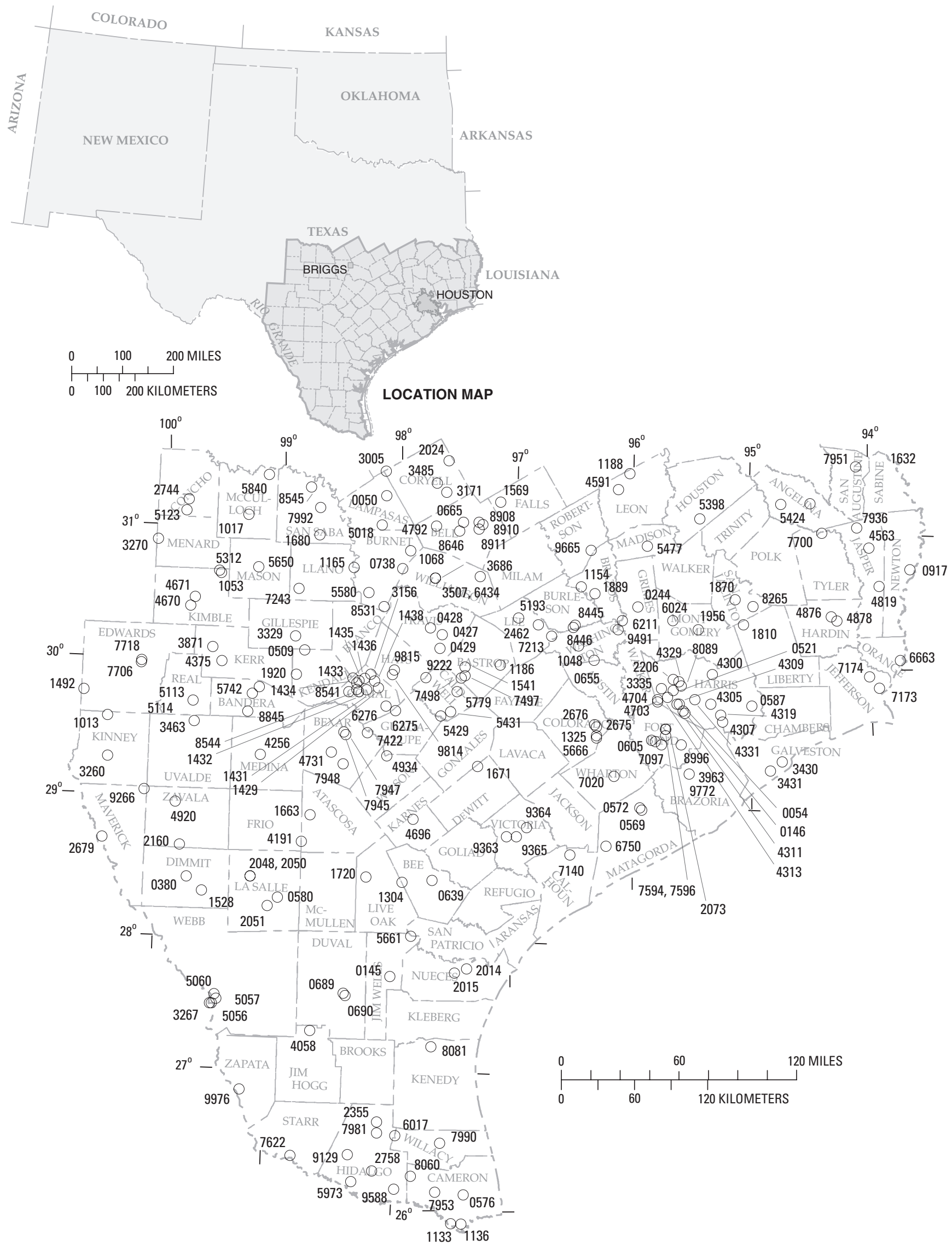

Figure 3C. Locations of National Weather Service hourly rainfall stations in southeastern Texas. 
3. The processes that generated the storms throughout the record for each rainfall station do not change with time (assumption of stationarity). There are no historical changes to the frequency of storms or the distributions of depth and duration.

4. The processes that generated the storms throughout the year for each rainfall station do not change with time. Seasonal differences in storm statistics are ignored.

The mean storm interevent time was computed as the total length of record (hours) divided by the number of observed storms minus the mean storm duration (hours). The mean storm interevent time then was converted to units of days per storm. Storm interevent time is a mean or average rate (time per storm); however for brevity, hereinafter that rate is expressed as time, although the term "interevent" implies the per-storm concept. Nine stations have at least one missing interevent time value: one in eastern New Mexico, one in Oklahoma, and seven in Texas. Whether there is a missing interevent time for the nine stations varies with the minimum interevent time. A missing interevent time can occur when no rainfall or only one sequence (storm) of rainfall is in the data record. An estimate of mean storm interevent time requires at least two storms in the time series.

The mean storm interevent time can be used in probabilistic models to assess the distribution of storm occurrence. The Poisson distribution is suggested to model the distribution of the number of storms in a given time period (see "Example 1: Estimation of Storm Occurrence" in the section "Example Applications"). The exponential distribution is suggested to model the distribution of the storm interevent times of successive storms.

The depth and duration of rainfall for each storm for each station were computed. Subsequently, the 1st, 2nd, 10th, 25th, 50th, 75th, 90th, 98th, and 99th percentiles of storm depth and duration were computed for each station as sample size permitted. For example, a sample size of 10 storms is insufficient to empirically estimate the 1st, 2nd, 98th, and 99th percentiles. Missing storm depth and duration percentiles are symmetrical about the 50th percentile (median). The authors identify missing percentiles rather than extrapolate into the tails of the distribution.

The nine selected percentiles characterize the empirical distribution of storm depth and duration. Examples of these two observed distributions for station 0016 Abilene Regional Airport, Tex., are shown in figure 4. The distribution, when characterized by the percentiles, is referred to in this report as the empirical distribution; the selected percentiles of the distribution also are shown in figure 4 .

The observed distributions are graphed on a normal probability graph. Hourly rainfall data are reported to the nearest $0.01 \mathrm{inch}$, and duration resolution is reported to the nearest hour. The step pattern shown on the left side of the curves in figure 4 therefore occurs because of the small resolution of the data in depth and duration. L-moment statistics of the two observed distributions were computed and are annotated in the figure. L-moment statistics (Hosking, 1990; Stedinger and others, 1992; Hosking and Wallis, 1997; Gilchrist, 2000; and Dingman, 2002) are used in this paper in lieu of product or central moment statistics. L-moment statistics are mathematically described in appendix 1. L-moment statistical analysis of rainfall distributions currently (2006) is an accepted state-of-thepractice technique.

L-moments provide a useful theoretical framework because L-moments have well-documented statistical advantages over product moments. Specifically, L-moments are less sensitive to outliers in the data, show less bias, are more accurate in small samples, and do not require logarithmic or other power transformations of the data. L-moments also provide more secure inferences of distributional form than do product moments through the use of L-moment (ratio) diagrams. The primary concept is that L-moments are exact analogs of product moments; that is, the interpretations of L-moments are similar to interpretations of the mean, variability, skew, kurtosis, and higher measures. Although L-moments are analogs of product moments and have similar interpretations, except for the mean, they do not have numerical values similar to those of product moments.

The L-moments considered in this report are the mean, L-scale, coefficient of L-variation (L-CV), L-skew, L-kurtosis, and Tau5. L-CV is dimensionless and is defined as the ratio of $\mathrm{L}$-scale to the mean. L-skew, L-kurtosis, and Tau5 also are dimensionless. The sample L-moments were considered missing values unless five or more storms were observed in the data record. Also, the storm duration L-moments were considered missing values if all of the storm duration values were equal, even though five or more storms were observed. This situation occurred for stations where all observed storms were recorded as 1-hour long; L-moments cannot be computed if all data values are equal.

Regionwide (eastern New Mexico) or statewide (Oklahoma and Texas) record-length, weighted-average storm depth L-moments consisting of the mean depth, L-CV, L-skew, L-kurtosis, and Tau5 are listed in table 5 (at end of report). Similarly, weighted-average mean storm interevent time and storm duration L-moments are listed in table 6 (at end of report). The L-moments listed in tables 5 and 6 are called regional L-moment statistics. For the record lengths listed in tables 5 and 6 , the record lengths for storm duration (table 6) often are less than those for storm depth (table 5) because all storm durations were equal-hence no computed L-moments—for one or more stations.

An increase in mean storm interevent time, depth, and duration with minimum interevent time is evident in tables 5 and 6. Some patterns in the L-moments are apparent, such as decreasing L-skew of the storm depth distribution with increasing minimum interevent time. Two critical characteristics of the tabulated data are (1) the regionwide or statewide mean values for the dimensionless L-moments (L-CV, L-skew, L-kurtosis, and Tau5) are all of the same general order of magnitude as minimum interevent time increases; and (2) the dimensionless 

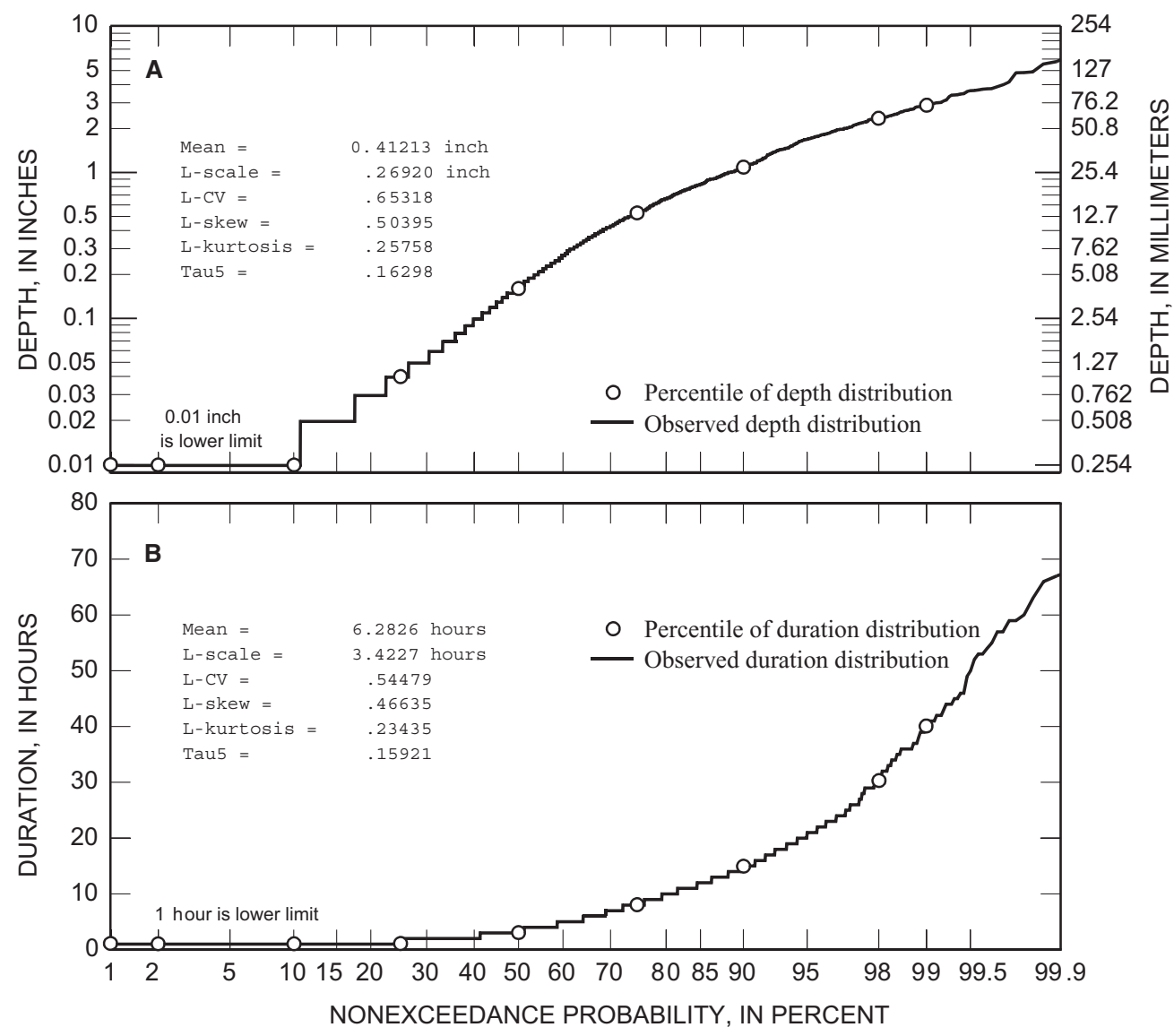

Figure 4. Empirical distributions of (A) storm depth and (B) storm duration for 3,331 storm events defined by an 8-hour minimum interevent time for station 0016 Abilene Regional Airport, Texas.

L-moments for eastern New Mexico, Oklahoma, and Texas are of the same general order of magnitude.

The two characteristics are critical because they are interpreted to indicate that a single dimensionless frequency curve can be used to represent the scale (slope) and shape (curvature) of the distribution in eastern New Mexico, Oklahoma, and Texas, and only an estimate of the mean depth or duration for a given location is required to construct a continuous distribution of either storm depth or duration. Specifically, the first characteristic implies that the general appearance of a dimensionless frequency curve is relatively invariant with minimum interevent time. The second characteristic implies that the general appearance of a dimensionless frequency curve is relatively invariant with location in the study area. Dimensionless frequency curves are discussed and presented in the section "Quantile Functions of Storm Depth and Duration;" but first an analysis and discussion of appropriate forms for modeling the distributions of storm depth and duration are appropriate.

An L-moment diagram comparing L-skew and L-kurtosis of depth and duration for Texas storms defined by the 8-hour minimum interevent time is shown in figure 5 . Superimposed on the diagram are the theoretical relations of L-skew and
L-kurtosis for six distributions. Hosking (1990), Vogel and Fennessey (1993), Hosking and Wallis (1997), and Dingman (2002) provide details of L-moment-diagram construction and interpretation. L-moment diagrams are used to evaluate the suitability of candidate distributions for modeling the distribution of data.

The curves in figure 5 , with the exception of the theoretical limits of the L-moments, represent three-parameter distributions. (The one-parameter exponential distribution plots as a single point - an asterisk in the figure.) The theoretical limits of the L-moments are $0.25\left(5 \tau_{3}^{2}-1\right) \leq \tau_{4}<1$, where $\tau_{3}$ and $\tau_{4}$ are L-skew and L-kurtosis, respectively. Several observations about suitable probability distributions in which to model the storm depth and storm duration can be made. First, the trajectory of the three-parameter Pearson Type III distribution (not the log Pearson Type III distribution familiar to many engineers and hydrologists) passes near the centers of the storm depth and storm duration data-point clusters. However, the majority of the storm depths have slightly larger L-kurtosis than the Pearson Type III distribution; this is indicated in figure 5 by the majority of open circles above the Pearson Type III line. The L-kurtosis values for storm duration are more consistent with the Pearson 

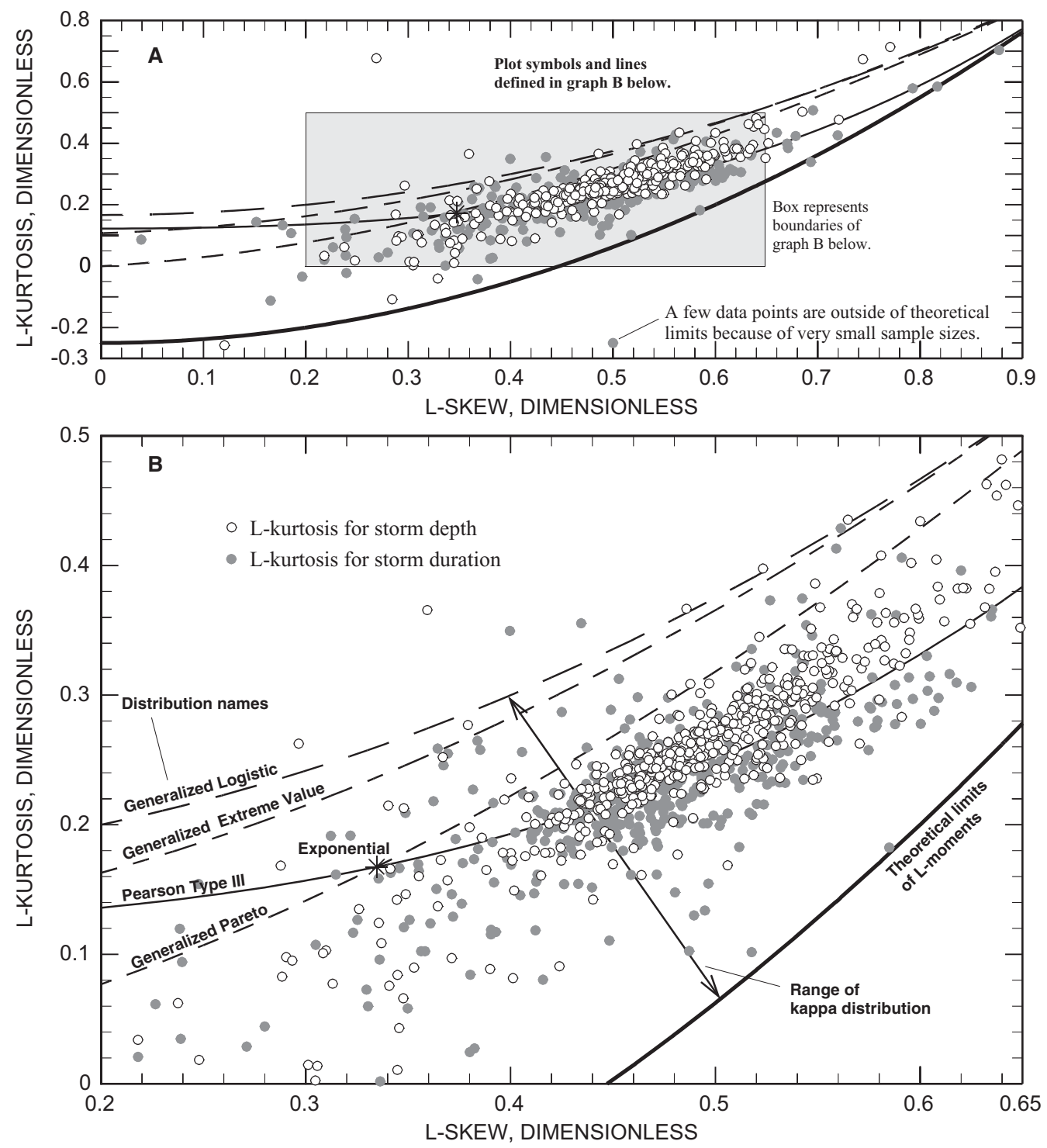

Figure 5. L-moment diagram at two scales ( $\mathrm{A}$ and $\mathrm{B}$ ) showing relation between L-skew and L-kurtosis of the distributions of storm depth and storm duration defined by the 8-hour minimum interevent time and theoretical relations for selected probability distributions.

Type III distribution than are the L-kurtosis values for storm depth. The distribution of storm duration, in other words, is more Pearson Type III-like than the distribution of storm depth.

The range of the four-parameter kappa distribution also is shown in figure 5 . The kappa distribution can accommodate any L-skew and L-kurtosis combination between the theoretical L-moment limits and the generalized logistic distribution. Thus, the kappa distribution can acquire a wide range of values and in general fits the L-skew and L-kurtosis of the site-specific samples of storm depth and duration.

Adams and Papa (2000) use the exponential distribution to model the frequency of storm interevent time, depth, and duration to develop closed-form solutions for various operational characteristics of small-watershed BMPs. Wanielista and
Yousef (1993, p. 52 and 221) suggest, and provide citations stating, that the one-parameter exponential or two-parameter gamma distributions can be used to model the distributions of storm depth and duration. Clarke (1998, p. 56) suggests the gamma distribution for modeling the distribution of daily rainfall.

The gamma distribution is a special case of the Pearson Type III distribution with positive L-skew (Stedinger and others, 1992, p. 18.19) and the location parameter of the Pearson Type III distribution set to zero. The L-kurtosis and L-skew values of the gamma distribution follow the curve for the Pearson Type III distribution. However, because the gamma distribution is a special case of the Pearson Type III 
distribution, the gamma distribution is not actually fit to the L-skew of the data as a third parameter is not available.

The U.S. Environmental Protection Agency (1986) uses the gamma distribution to approximate the distributions of storm interevent time, depth, and duration. The exponential and gamma distributions are readily implemented. However, on the basis of the relations shown in figure 5, the authors suggest that the Pearson Type III and kappa distributions would be more representative models than the exponential or gamma distributions with a modest increase in analytical complexity. Furthermore, because the kappa distribution has an additional parameter, can fit the L-kurtosis of the data, can mimic the Pearson Type III, and is expressible as a quantile function (see next section), the kappa distribution is preferred by the authors for the distributions of storm depth and duration.

\section{Quantile Functions of Storm Depth and Duration}

The exponential, gamma, and kappa distributions are considered for modeling the distribution of storm depth and duration in this report. Both the simpler exponential and gamma distributions have precedence in analytical solutions to BMP function. In terms of quality of distribution fit, the analysis in the previous section indicates that the kappa distribution is preferable. The authors consider all three distributions and compute the parameters of the gamma and kappa distributions to facilitate use of this report.

A quantile function, which is the inverse of a cumulative distribution function, or frequency curve for a random variable $X$ (either storm depth or duration) can be written as

$$
X(F)=\mu \times x(F),
$$

where $X(F)$ is the variable for nonexceedance probability, $F$; $\mu$ is the arithmetic mean (first L-moment) of the variable; and $x(F)$ is the dimensionless quantile function (a dimensionless frequency curve). The dimensionless frequency curve represents constant multipliers or frequency factors, which are applicable to mean storm depth. Uncertainty in the distribution of the variable $X(F)$ is assessed through uncertainty in the mean $(\mu)$. Uncertainty in the estimation of the mean is described in the section "Maps of Mean Storm Interevent Time, Depth, and Duration."

A dimensionless frequency curve is fit to the data using the method of L-moments by setting the mean equal to unity and L-scale equal to the L-CV. The higher L-moments (L-skew, L-kurtosis, Tau5) remain unchanged. (In terms of the product moments, a dimensionless distribution is fit to the data by setting the mean to unity, the standard deviation equal to the coefficient of variation, and all other moments are unchanged.) This technique for dimension removal is useful in statistical hydrology (Hosking and Wallis, 1997, and references therein).
The quantile function of a dimensionless exponential distribution is

$$
x(F)=-\ln (1-F),
$$

where $x(F)$ is the dimensionless frequency curve for nonexceedance probability, $F$. There is no parameter to estimate. The L-CV of the dimensionless exponential distribution is 0.5 (Hosking, 1990, p. 112). Values for L-CV for storm depth and duration are all greater than 0.5 in tables 5 and 6 . As previously discussed, the exponential distribution is extensively used by Adams and Papa (2000) in a BMP design context. The exponential distribution also is discussed by Ross (1994, p. 223-230), Evans and others (2000, p. 77-81), and Bhat and Miller (2002, p. 205).

The cumulative distribution function of the dimensionless gamma distribution is

$$
F(x)=\frac{\beta^{-\theta}}{\Gamma(\theta)} \int_{0}^{x} t^{\theta-1} e^{-t / \beta} d t,
$$

where $F(x)$ is the nonexceedance probability, cumulative percentage, for dimensionless value $x$ (see eq. 1); $\theta$ and $\beta$ are parameters; and $\Gamma(\theta)$ is the gamma function for $\theta$. There is no explicit solution for $x$ in terms of $F$. The parameters can be computed using the mean and L-CV. Hosking (1996) provides algorithms, with the mean of the distribution computed as

$$
\mu=\theta \beta,
$$

and the L-CV (L-scale divided by mean, or $\lambda_{2} / \mu$ ) of the distribution computed as

$$
\mathrm{L}-\mathrm{CV}=\frac{\beta \Gamma(\theta+0.5)}{\sqrt{\pi} \times \Gamma(\theta) \times \mu} .
$$

Because the gamma distribution is dimensionless in the context here, the following conditions apply: $\theta \beta=1$ and $\mathrm{L}-\mathrm{CV}=[\beta \Gamma(\theta+0.5)] /[\sqrt{\pi} \times \Gamma(\theta)]$.

Properties of the gamma distribution are listed in Evans and others (2000, p. 98-105). This distribution in the context of atmospheric statistics and rainfall is described in Wilks (1995, p. 86-93) and in the context of rainfall stochastics in Clarke (1998). Although much of their work is based on the exponential distribution, Adams and Papa (2000, p. 72-73) also describe the gamma distribution. Use of the gamma distribution in the context of estimation of BMP function has precedent (U.S. Environmental Protection Agency, 1986).

The quantile function of the dimensionless kappa distribution (Hosking, 1994) is

$$
x(F)=\xi+\frac{\alpha}{\kappa}\left[1-\left(\frac{1-F^{h}}{h}\right)^{\kappa}\right],
$$

where $x(F)$ is the value for a nonexceedance probability, $F$; and $\xi, \alpha, \kappa$, and $h$ are parameters. The four parameters can be computed using the mean (set to unity), L-CV, L-skew, and L-kurtosis. However, kappa parameter estimation is not 


\section{Statistical Characteristics of Storm Interevent Time, Depth, and Duration for Eastern New Mexico, Oklahoma, and Texas}

manually tractable. Hosking and Wallis (1997, p. 202-204) report that there are "no simple expressions for the parameters [of the kappa] in terms of the L-moments." Newton-Raphson iteration can be used for parameter estimation and is described by Hosking (1996).

For rapid implementation of the results of this report, the parameters for both gamma and kappa distributions, which correspond to the L-moments of storm depth and duration (mean set to unity and L-scale set to L-CV) listed in tables 5 and 6, respectively, have been computed. The parameters for gamma and kappa distributions of dimensionless storm depth frequency curves are listed in table 7 (at end of report), and similarly, the parameters of dimensionless storm duration frequency curves are listed in table 8 (at end of report).

The quantile function of the storm depth or duration is restored to the dimensionless frequency curves of storm depth or duration by multiplying a mean for storm depth or duration according to equation 1. For example, from equation 6 the 90th percentile of dimensionless storm duration in Texas for a kappa distribution model using the 18-hour minimum interevent time (parameters from table 8) is

$$
\begin{aligned}
& x(0.90)=-2.073+\frac{2.224}{0.0896}\left[1-\left(\frac{1-0.90^{2.794}}{2.794}\right)^{0.0896}\right] \text { or } \\
& x(0.90)=2.72 .
\end{aligned}
$$

Thus, the 90th-percentile storm duration for a particular location in Texas is 2.72 times the mean storm duration. For this report, the mean storm duration for the 18-hour minimum interevent time for a location preferably is estimated using the mean for a county.

From the previous section, dimensionless frequency curves of both storm depth and duration are relatively insensitive to minimum interevent time and to location in the study area. This conclusion is based on the fact that, because the dimensionless L-moments (L-CV, L-skew, L-kurtosis, and Tau5) in tables 5 and 6 are similar, the parameter estimates for the dimensionless distributions in tables 7 and 8 for storm depth and duration, respectively, also are similar.

To illustrate the distribution similarity for the selected minimum interevent times, the dimensionless kappa distribution frequency curves from tables 7 and 8 for storm depth and duration are graphed in figure 6 . The curves for storm depth (fig. 6A) are similar as expected. Likewise, the curves for storm duration (fig. 6B) also are similar. In both graphs, the largest intercurve differences are on the left side of the distribution; however, use of a base-10 log scale for the ordinate accentuates the differences. To illustrate the spatial insensitivity of the dimensionless frequency curves, the storm depth and storm duration curves for the 24-hour minimum interevent time for eastern New Mexico, Oklahoma, and Texas are shown in figure 7. It is evident from the figure that the curves for storm depth (gray lines) and storm duration (black lines) are fairly similar. Further, the shape of the depth and duration curves are comparable, although there is no apparent reason to expect such similarity between dimensionless storm depth and storm duration distributions.

A comparison of exponential, gamma, and kappa distributions of storm depth for 24-hour minimum interevent time for Texas indicates the differences between the distributions. There is no parameter of the dimensionless exponential distribution. The exponential and gamma distributions have precedence as models of storm depth and storm duration distributions in BMP design. The quantile function of a dimensionless exponential distribution is defined in equation 2 . The cumulative distribution function of the dimensionless gamma distribution (parameters from table 7) is

$$
F(x)=\frac{1.579^{-0.6333}}{\Gamma(0.6333)} \int_{0}^{x} t^{0.6333-1} e^{-t / 1.579} d t .
$$

The quantile function of the dimensionless kappa distribution (parameters from table 7) is

$$
x(F)=-0.5790+\frac{1.115}{-0.1359}\left[1-\left(\frac{1-F^{1.747}}{1.747}\right)^{-0.1359}\right] .
$$

These three distributions are shown in figure 8. In figure $8 \mathrm{~A}$, the distributions are graphed with a linear y-axis scale; whereas in figure $8 \mathrm{~B}$, the distributions are graphed with a base$10 \log \mathrm{y}$-axis scale. The kappa distribution bends upward more steeply than the other distributions (see right side of fig. 8A); this demonstrates that the kappa distribution is capable of producing larger "outliers" of storm depth, which are known to occur in rainfall data. The exponential distribution is the flattest of the three distributions. The flattening of the kappa distribution on the left side (fig. 8B) shows that the kappa distribution has a lower limit of about 0.09 . Thus, if the mean storm depth is about 0.5 inch, the lower limit is about 0.045 inch $(0.09$ multiplied by 0.5$)$. The lower limit of the exponential and gamma distributions is zero. The resolution of the data is 0.01 inch; therefore, the minimum storm depth recorded is $0.01 \mathrm{inch}$. The left-tail differences (nonexceedance probability less than about 0.05) between the distributions become substantial; the choice of distribution thus becomes more problem-specific.

The association between storm depth and storm duration is important. An example of the association for station 0016 Abilene Regional Airport, Tex., is shown in figure 9. Clearly, the larger storm depths are associated with storms of longer duration. However, no discernible relation between depth and duration is evident for durations between 1 and about 20 hours, and there is considerable variability in depth for a given duration. Similar assessments were made for a geographically disperse subset of stations (results not reported here).

The proportional association between large storm depths and durations implies that storm depth and duration are weakly dependent random variables. Thus, conditional probability theory - depth is conditional to duration - is required when analyzing the joint probability of storm depth and duration. However, Adams and Papa (2000, p. 120-121, and references therein) comment extensively on the dependence of storm depth and duration in the context of small-watershed drainage-design 

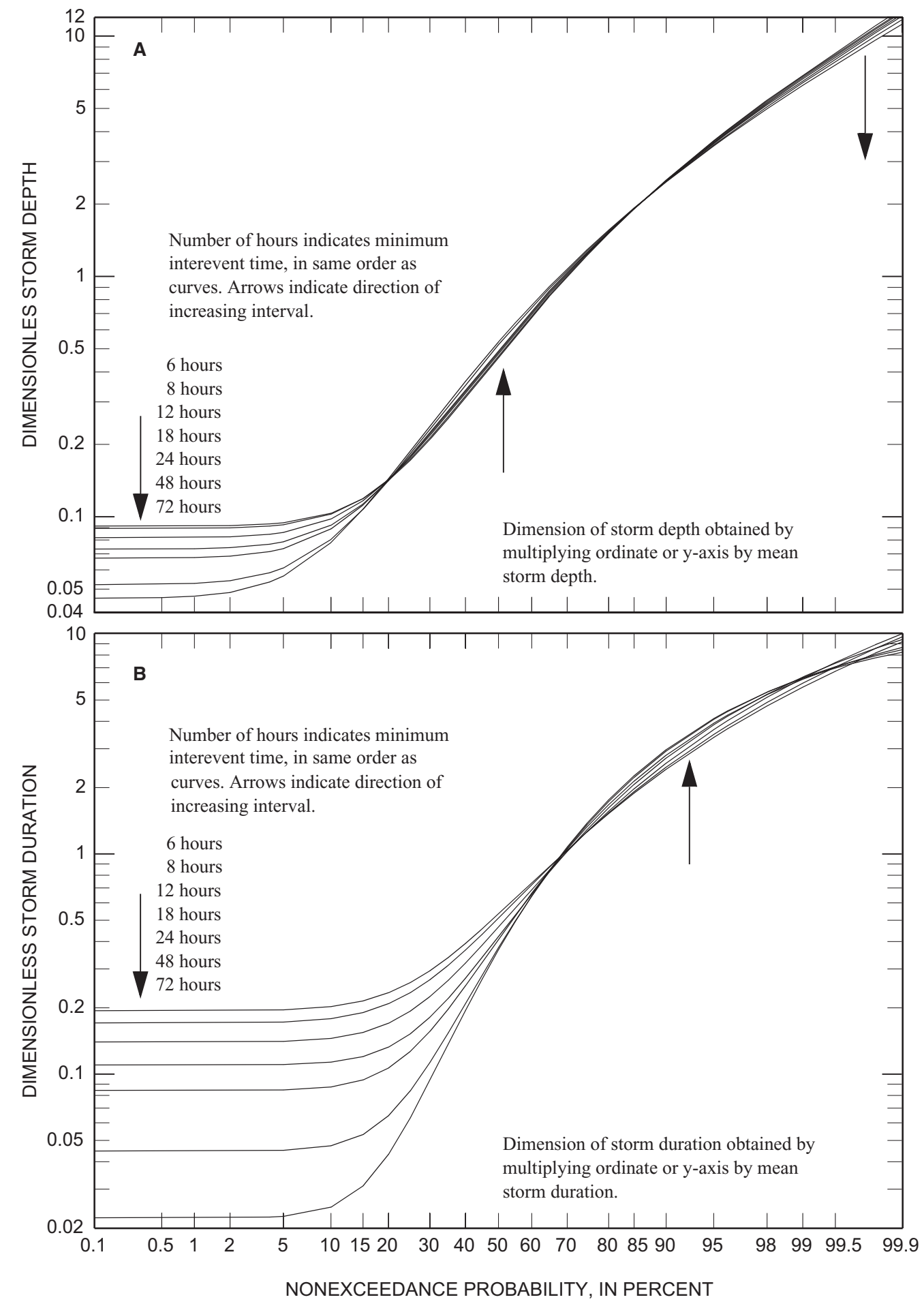

Figure 6. Dimensionless kappa distribution frequency curves for (A) storm depth and (B) storm duration for the selected minimum interevent times in Texas. 


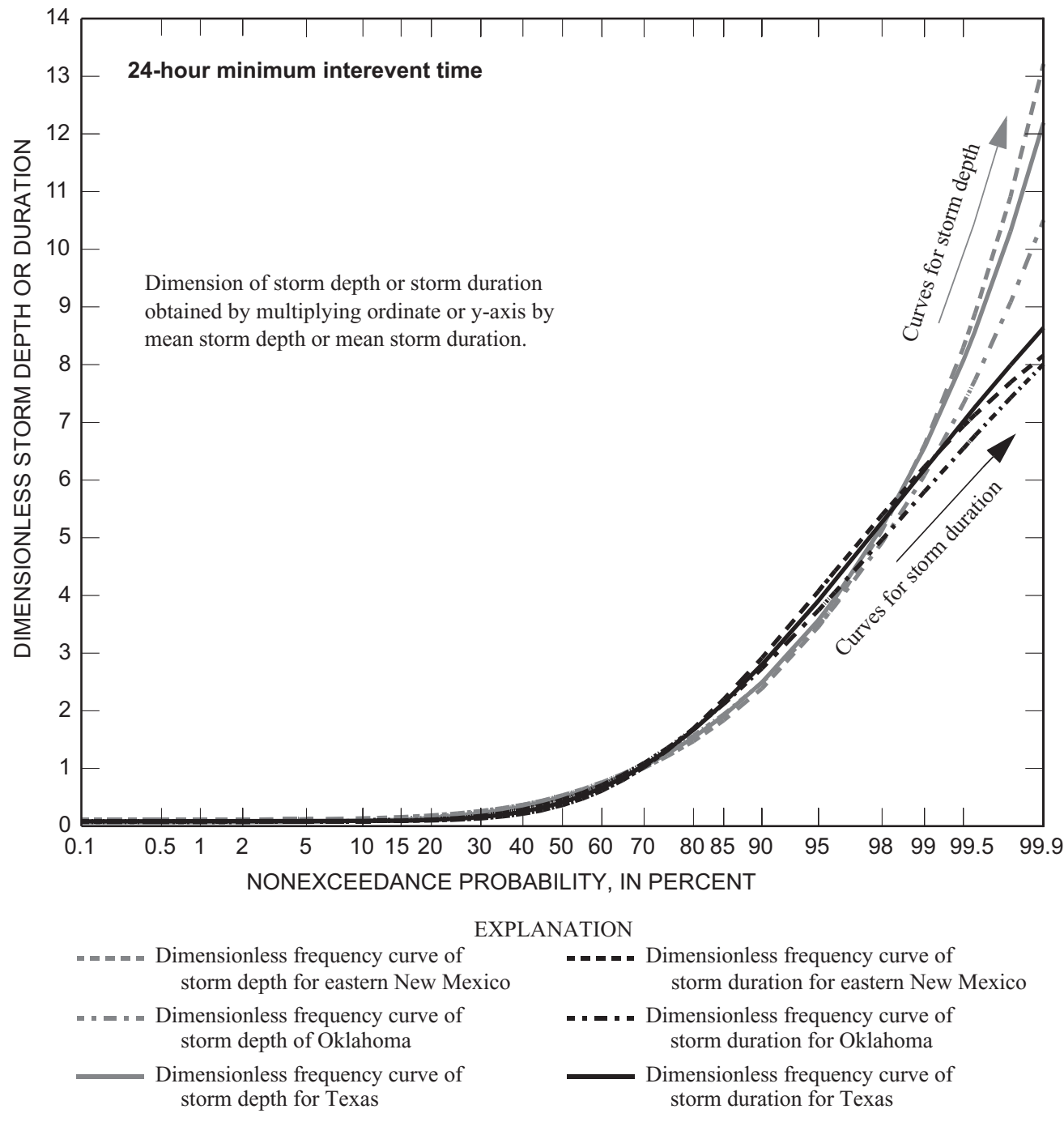

Figure 7. Dimensionless kappa distribution frequency curves for storm depth and storm duration for 24-hour minimum interevent time for eastern New Mexico, Oklahoma, and Texas.

practices including BMPs. For many applications, such as the example applications in this report, it often is assumed that the distributions of storm depth and duration are independent. No further analysis of the relation between storm depth and duration is provided in this report.

\section{Statistical Characteristics of Storm Interevent Time, Depth, and Duration}

Twenty-one maps show mean storm interevent time, mean storm depth, and mean storm duration by county for the study area. For each of the three statistics, there is one map for each of the seven minimum interevent times. Important summary and diagnostic statistics for the maps also are tabulated. Nine tables (three each for eastern New Mexico, Oklahoma, and Texas) list the three statistics by county for the seven minimum mean interevent times. Before introduction of the maps and tables, a description of the regionalization approach is informative. Regional analysis is important because storm statistics are highly variable in space and time; many rainfall stations have short records, which implies more error for estimates of sitespecific rainfall statistics. The regional analysis provides a method to estimate more reliable statistics.

\section{Regionalization of Storm Statistics}

Regionalization in this report is a two-step process. First, a spatial analysis, or neighborhood smoothing, of the mean storm statistics for a particular minimum interevent time, such as the mean storm depth for storms defined by the 8-hour minimum 


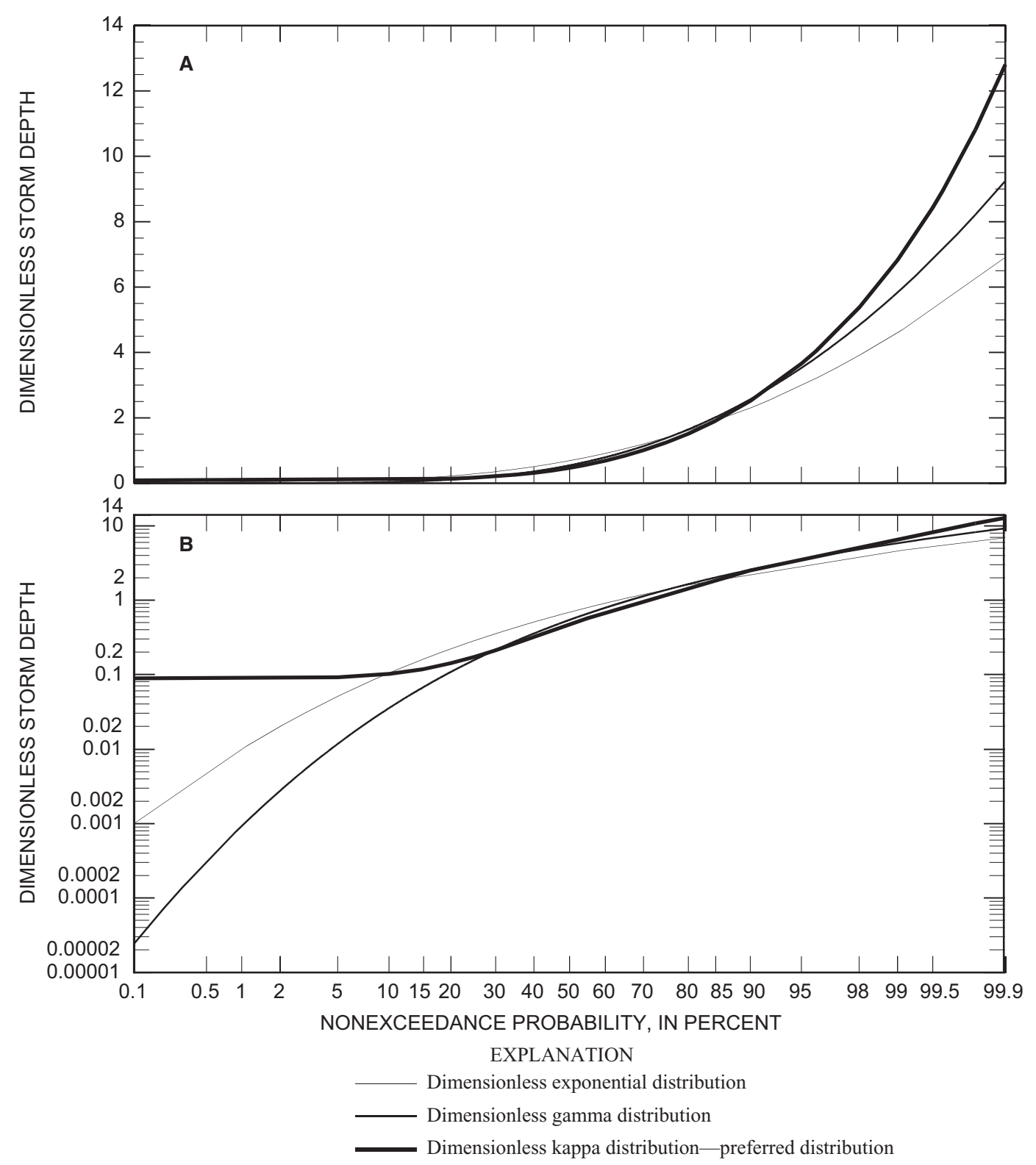

Figure 8. Dimensionless exponential gamma and kappa distributions of storm depth for 24-hour minimum interevent time in Texas, graphed with (A) a linear $y$-axis scale and (B) a base-10 log y-axis scale.

interevent time, is done for each station in the study area. Second, geostatistical analysis of the smoothed statistics for the stations is done to produce a continuously varying map of the statistic. The map is used to estimate countywide means for the mean storm interevent time, mean storm depth, and mean storm duration.

Neighborhood smoothing is the process by which a particular statistic at a particular station is combined, or pooled together, with the corresponding statistics at surrounding stations to develop a more reliable estimate of the statistic for each particular station than can be derived from the data for the station alone. In other words, the neighborhood of $m$ stations surrounding a particular station contains more informationmore hours of record-about the characteristics of rainfall in that area than the particular station.

Neighborhood smoothing for each station consisted of computing the smoothed statistic through a weighted average of the statistic for the station and the statistics for the four nearest stations. Five stations thus constitute the neighborhood. Record length, as measured by the total number of hours of rainfall record, provided the weights. Occasionally a value for a particular statistic, such as the mean storm duration, was missing for one or more of the neighboring stations. When that occurred, the station with a missing value was dropped from the 


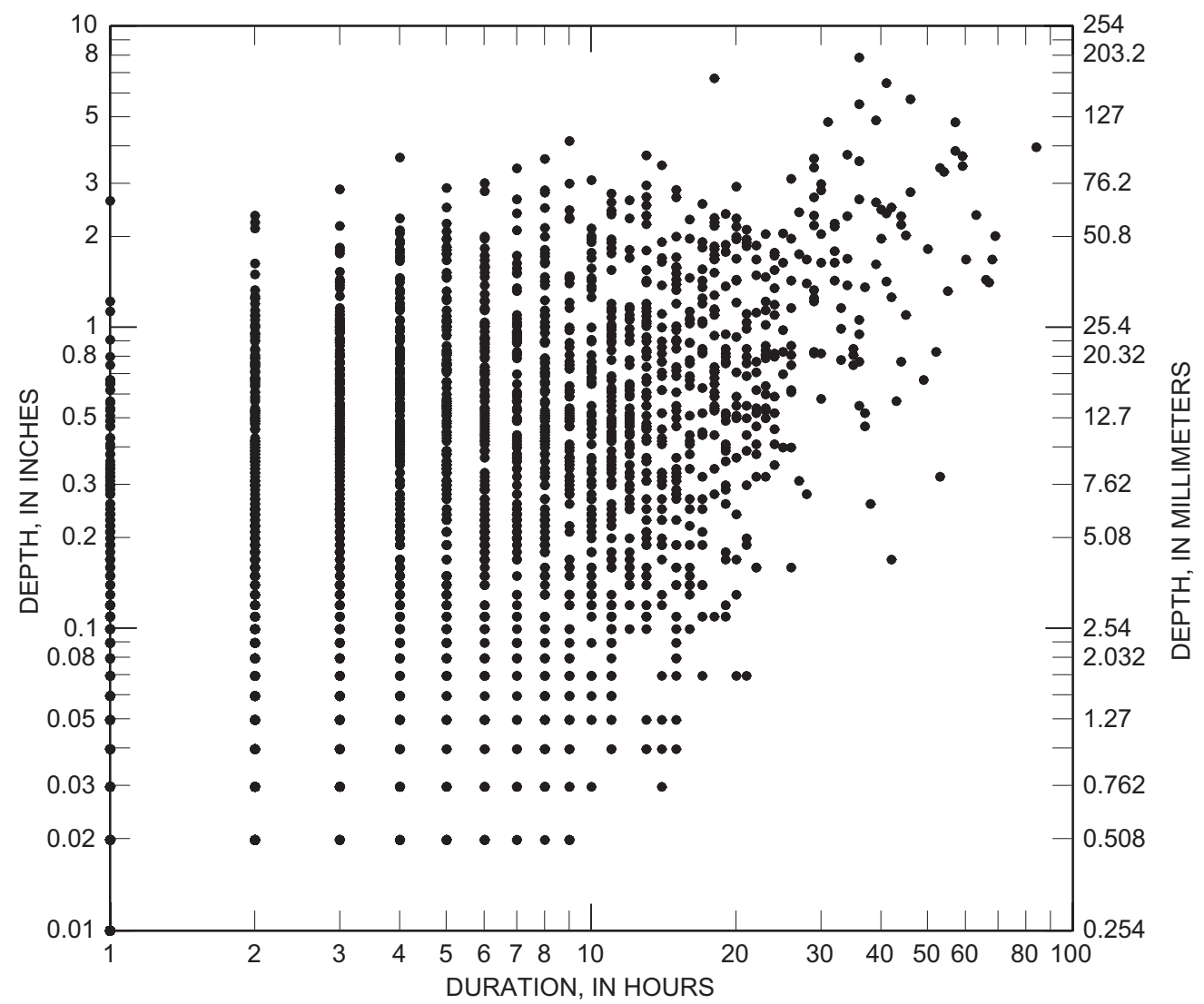

Figure 9. Storm depth and storm duration for 3,331 storm events defined by an 8-hour minimum interevent time for station 0016 Abilene Regional Airport, Texas.

neighborhood; no additional stations were sought. Thus, the number of stations in the neighborhood was reduced by the number of stations with missing values. No cases occurred in which an entire neighborhood had missing values; the minimum number of stations in a neighborhood was two.

Smoothing reduces the point-to-point variability of a particular statistic through the incorporation of record length and facilitates more reliable regionalization of the statistic. Smoothing is considered necessary because of large differences in record lengths. The authors' decision not to remove stations from the regional analysis because of short record length resulted in a trade-off: Additional spatial information was gained at the expense of increased station-to-station variability.

After smoothing, regionalization of storm statistics included geostatistical-based mapping using the method of kriging (Isaaks and Srivastava, 1989). Kriging was done using a spherical model of the semivariogram. That semivariogram model was selected on the basis of intermediate spatial analysis (not presented here) as part of the kriging operation in an integrated software system (Environmental Systems Research Institute, Inc., 2002). The semivariogram was automatically fitted by the software. The fit was verified through the graphical interface provided by the integrated software system. Other semivariogram models were evaluated in the intermediate anal- ysis; most of the maps are relatively insensitive to the choice of semivariogram model. The neighborhood for the kriging operation used a search radius of 12 stations in conjunction with a circular search method. The output cell size for the kriging operation was 4,799.55 meters, which translates to a 263-row by 251-column orthogonal grid encompassing the study area. The grid then was clipped to the external boundaries of the study area to form a grid map.

Subsequent to regionalization of the storm statistics, the grid maps of the storm statistics were evaluated through computation of selected summary and diagnostic statistics. The evaluation documents the error of the grid maps to facilitate assessment of the uncertainty in the storm statistic regionalization.

The summary and diagnostic statistics for each grid map were computed. These statistics are weighted values based on record length. Summary statistics include the number of stations without missing values for each minimum interevent time, the mean of a particular storm statistic (mean storm interevent time, mean storm depth, and mean storm duration) for the study area, and the weighted standard deviation (wSD) of the particular statistic for the study area. Diagnostic statistics computed are weighted mean bias of the grid map, root-weightedmean-square error (RwMSE) of the grid map, and the percentage difference between wSD and RwMSE of the grid map. The 
mean bias is the weighted average of the station residuals. A residual is defined as the value of the observed statistic at each station minus the statistic predicted by the grid map. The mean biases are expected to be about zero. The RwMSE is the square root of the mean square difference of the observed statistic minus the statistic predicted using the grid map. Percentage difference is defined as $100 \times(\mathrm{RwMSE}-\mathrm{wSD}) / \mathrm{wSD}$. Negative percentage difference implies that the grid map provides a more accurate estimate of a particular statistic than the weighted mean of the statistic for the study area.

Another component of the evaluation was an analysis of the spatial variation of the station residuals. The residuals should have little or no spatial dependency; for example, a residual map of the study area should show no systematic change from one side to another. The results of the residual analysis for the statistics (not reported here) indicated essentially no spatial dependency.

Finally, to provide consistency between graphical and tabular presentation of results, the means of storm interevent time, depth, and duration were computed using the grid map of each statistic and the spatial extent of each county. These means were used to produce the maps and associated tables described in the next two sections.

\section{Maps of Mean Storm Interevent Time, Depth, and Duration}

Maps depicting mean storm interevent times by county for each of the seven minimum interevent times are presented in figures 10-16. Maps depicting ranges of mean storm depth by county and mean storm duration by county are presented in figures 17-23 and figures 24-30, respectively.

Summary statistics and diagnostic statistics for the mean storm interevent time maps are listed in table 9 (at end of report). The summary statistics and diagnostic statistics for the storm depth and storm duration maps are listed in tables 10 and 11 (at end of report), respectively. The mean biases are approximately zero, as expected.

Comparison of the percentage differences between wSD and RwMSE in tables 9-11 shows that the differences are largest for mean storm depth and smallest for mean storm interevent time, which indicates that the mean storm depth maps explain comparatively more variability in storm depth across the study area than the mean storm interevent time maps or mean storm duration maps. Hence, relatively less uncertainty is associated with the storm depth maps than with the maps of the other two statistics.

The mean storm interevent time maps (figs. 10-16) show considerable east-to-west difference in the length of time between successive storms for a given minimum interevent time. Storms are most frequent in eastern Texas and least frequent in far western Texas. Storms along the Rio Grande in an easterly direction from about $101^{\circ}$ longitude are less frequent than the east-west location of that segment of the Rio Grande might indicate. A region of relatively more frequent storms is in the northeastern Texas Panhandle. Storms also are relatively more frequent along the northwestern side of the eastern New Mexico region. In general, similar patterns are evident among the storm interevent time maps as minimum interevent time changes.

From the storm depth maps (figs. 17-23), there is a clear tendency for smaller storm depths to occur in the western part of the study area. The changes in storm depth are influenced far more by east-west location than north-south location. A notable exception is that changes in storm depth in southern Texas (east-west along the Rio Grande) are relatively smaller than east-west changes across other parts of the study area. Another observation about the storm depth maps is that the patterns vary more smoothly than the patterns for the storm interevent time or storm duration maps; this is consistent with the greater percentage difference between wSD and RwMSE (table 10) for the storm depth maps. In general, similar patterns among the storm depth maps is evident as minimum interevent time changes.

The storm duration maps (figs. 24-30) show a tendency for the shortest-duration storms to be in western Texas; however, much more variation is in the storm duration maps than in the storm depth maps. In general, similar patterns among the storm duration maps is evident as minimum interevent time changes; however, as the minimum interevent time increases, the region of highest mean storm duration becomes more spatially restricted to the southeastern corner of Texas. A region of locally large storm duration is centered on Hays County, Tex. ( $30^{\circ}$ latitude and $98^{\circ}$ longitude); this region of large storm duration persists with changing minimum interevent times. Asquith (1998, figs. 33-37) indicates that the scale parameter (an expression of variability) of the generalized extreme-value distribution for rainfall annual maxima for durations of 1 to 7 days shows a region of locally increased value in Central Texas. The two local regions - that of large storm duration in this report and of increased scale parameter in Asquith (1998) — are approximately coincident.

\section{Tables of Mean Storm Interevent Time, Depth, and Duration}

Tabulated mean storm depth and duration could be used with the dimensionless frequency curves discussed in the section "Quantile Functions of Storm Depth and Duration" in this report. The mean storm interevent time, depth, and duration by county for eastern New Mexico are listed in tables 12, 13, and 14 (at end of report), respectively; for Oklahoma in tables 15, 16, and 17 (at end of report), respectively; and for Texas in tables 18, 19, and 20 (at end of report), respectively. Because of the large database analyzed, the regionalization of storm statistics as represented by the dimensionless kappa distribution of storm depth and duration (table 7) and the tables of mean storm interevent time, depth, and duration (tables 12-20) are preferable to other methods described here for statistical characterization of storms in eastern New Mexico, Oklahoma, and Texas. 


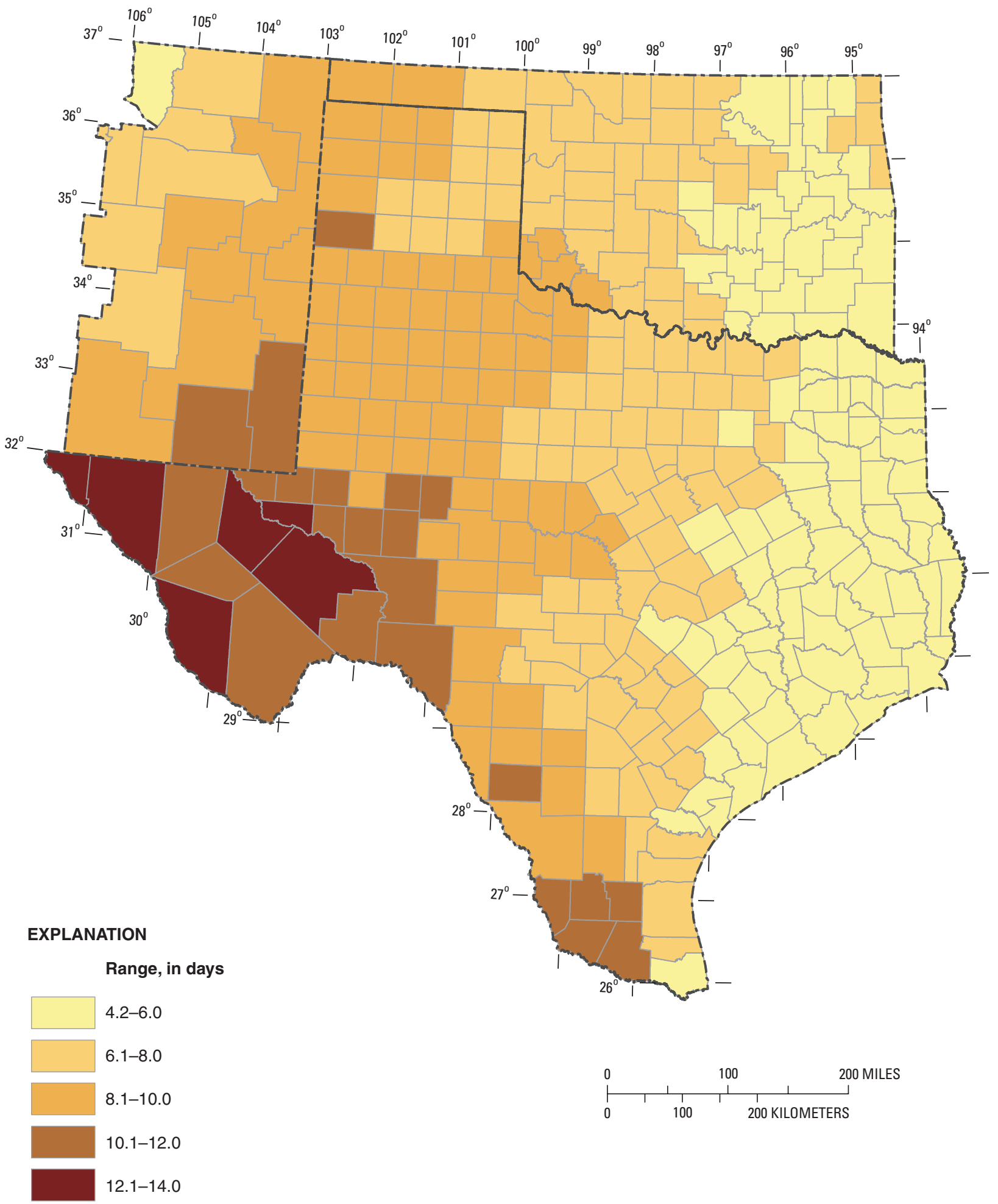

Figure 10. Mean storm interevent time defined by 6-hour minimum interevent time in eastern New Mexico, Oklahoma, and Texas. 


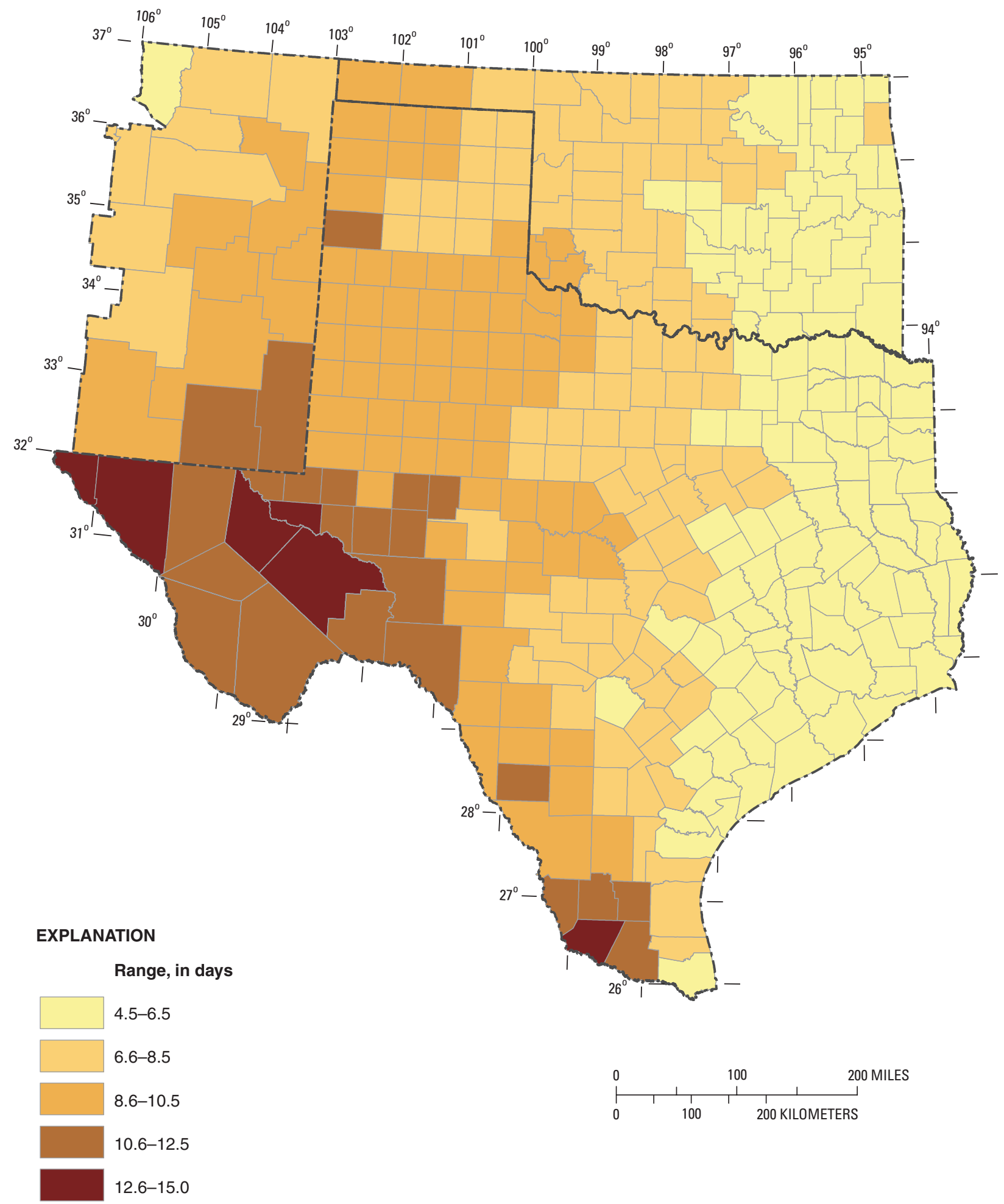

Figure 11. Mean storm interevent time defined by 8-hour minimum interevent time in eastern New Mexico, Oklahoma, and Texas. 


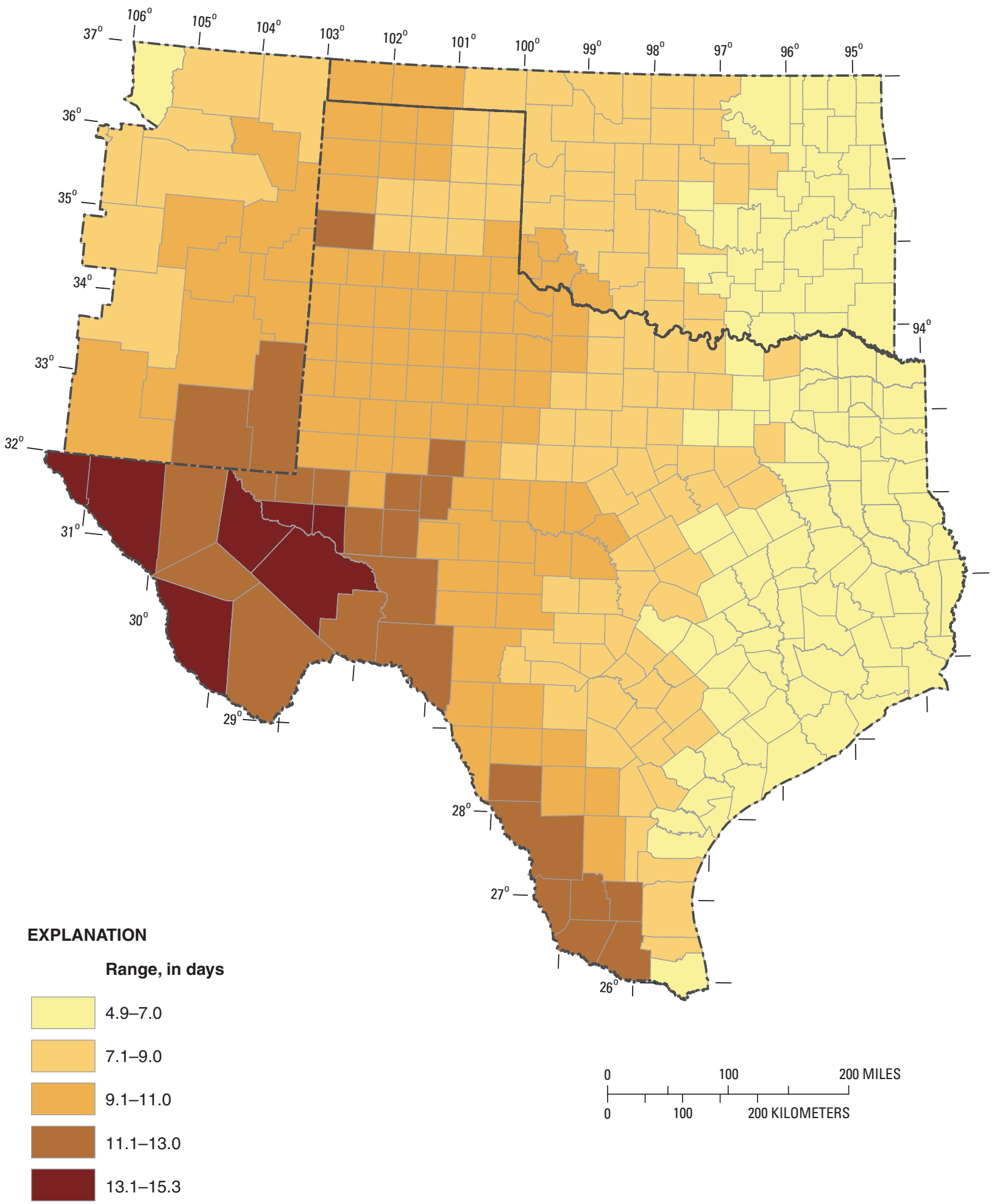

Figure 12. Mean storm interevent time defined by 12-hour minimum interevent time in eastern New Mexico, Oklahoma, and Texas. 


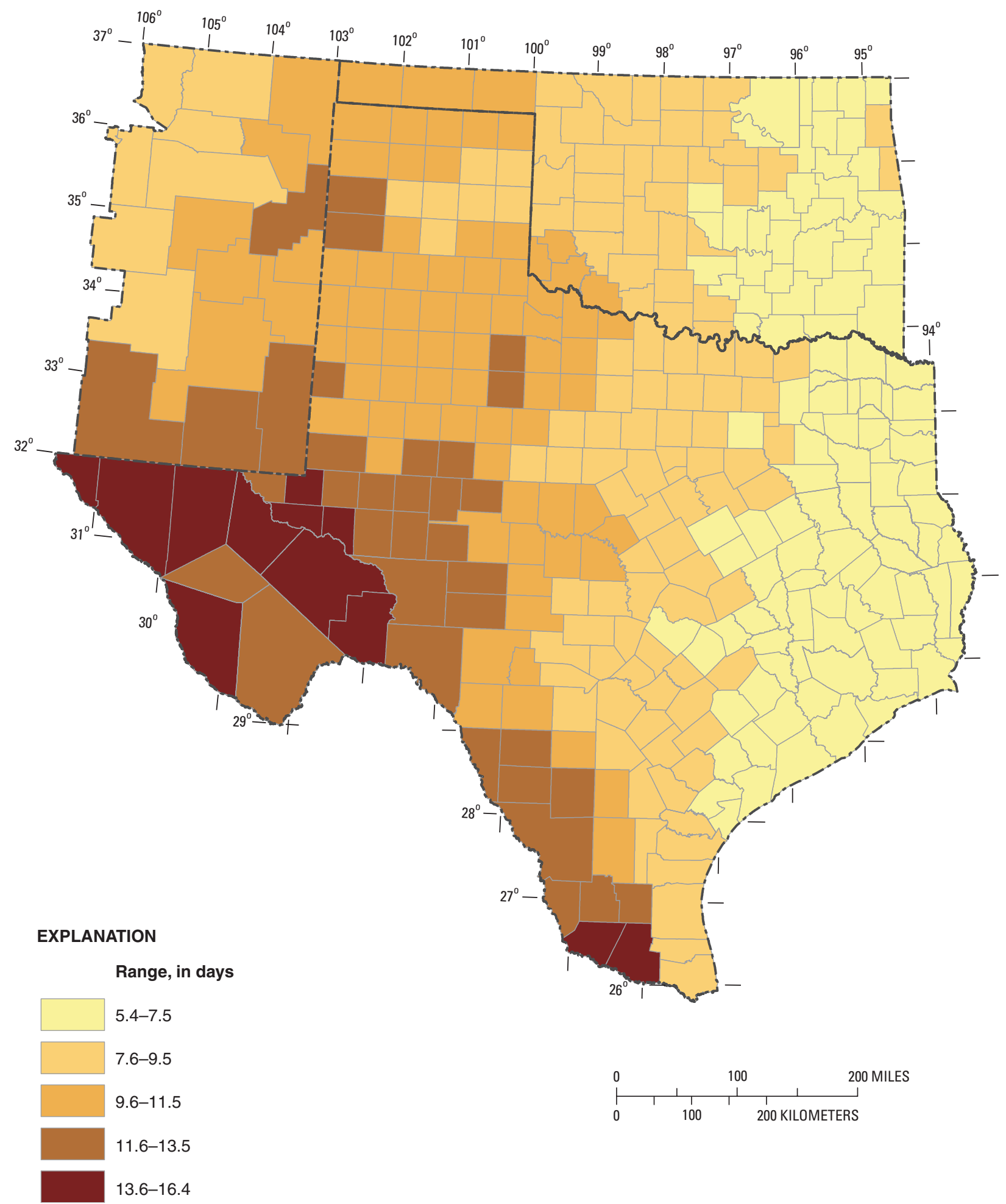

Figure 13. Mean storm interevent time defined by 18-hour minimum interevent time in eastern New Mexico, Oklahoma, and Texas. 


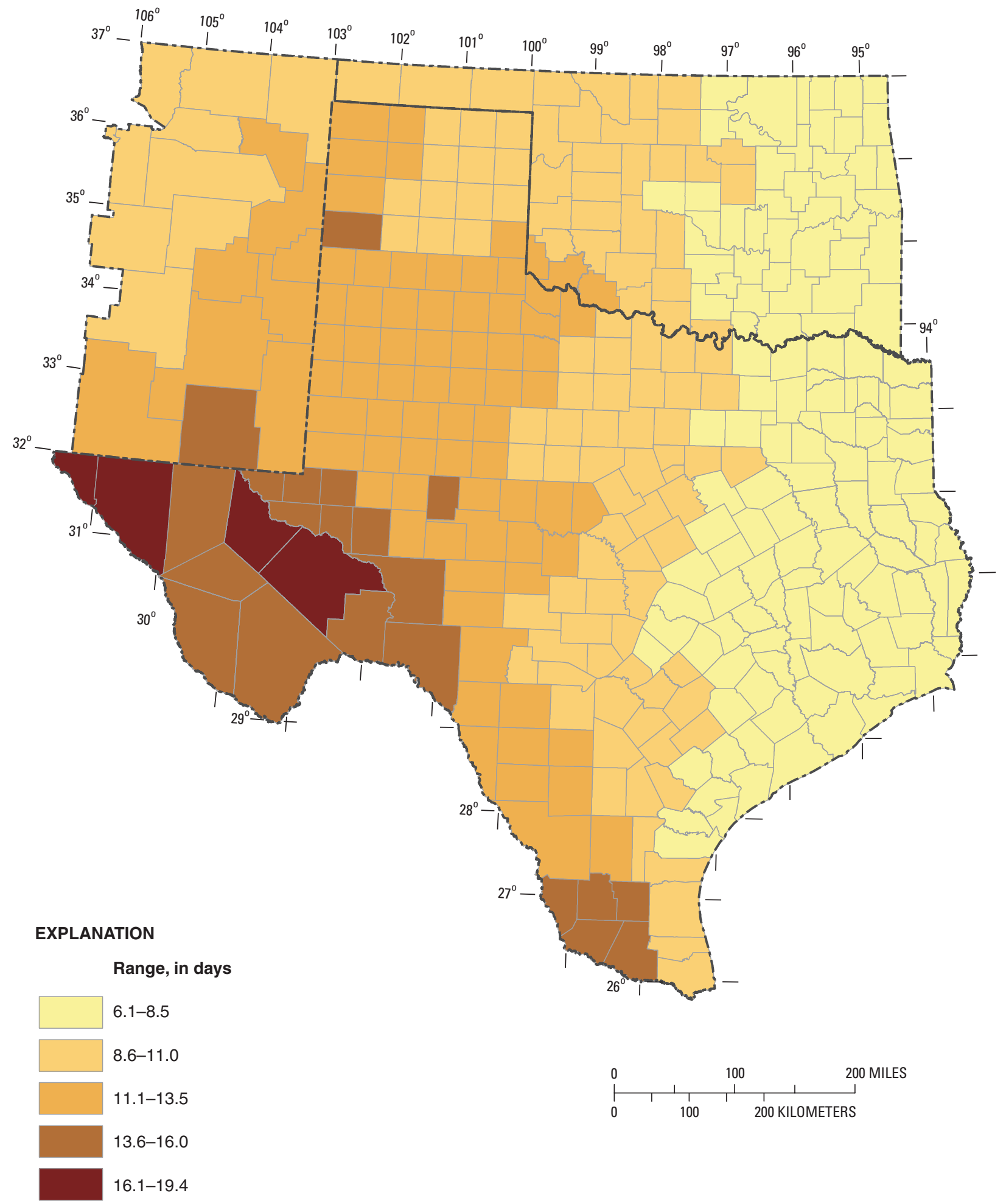

Figure 14. Mean storm interevent time defined by 24-hour minimum interevent time in eastern New Mexico, 0klahoma, and Texas. 


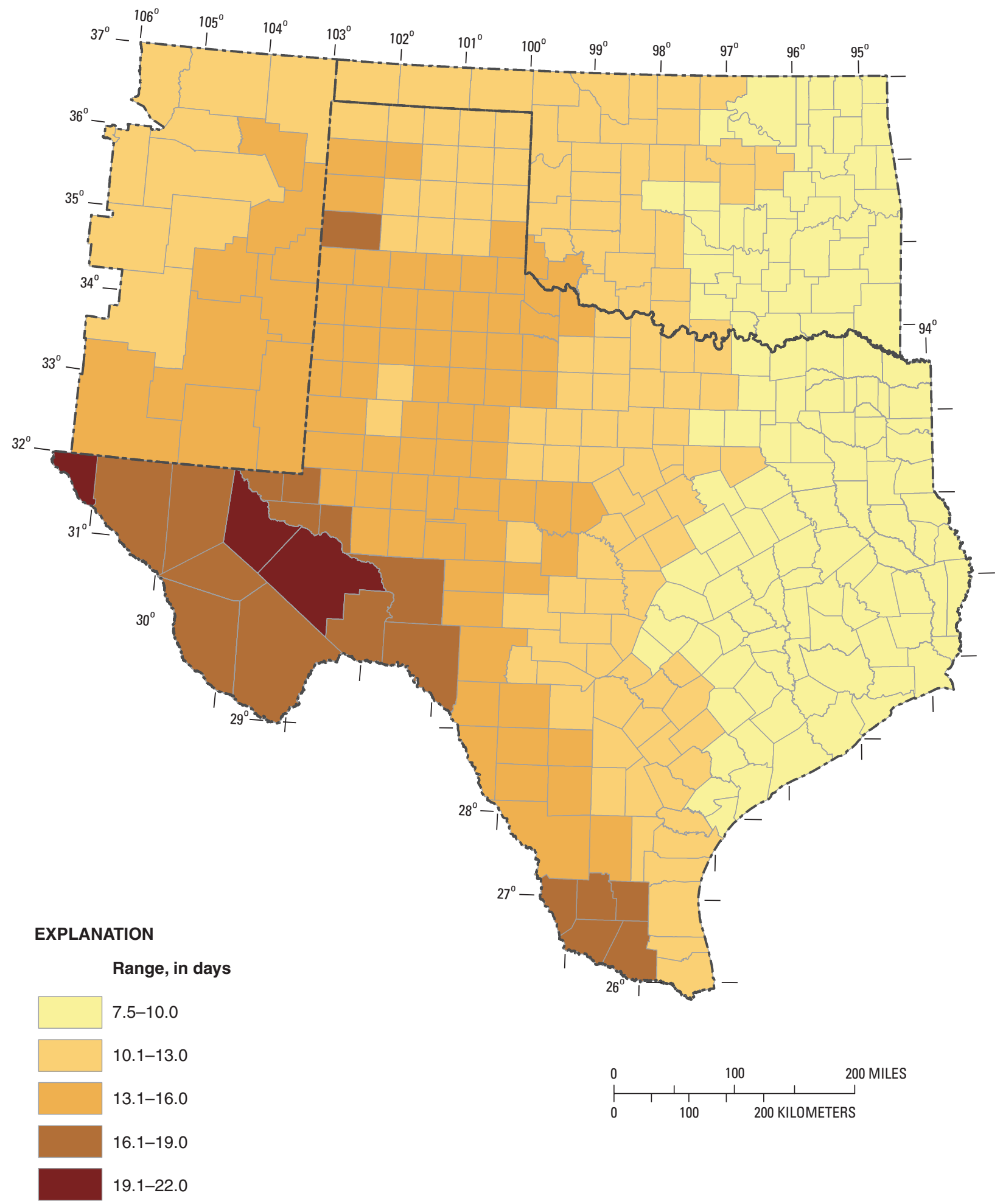

Figure 15. Mean storm interevent time defined by 48-hour minimum interevent time in eastern New Mexico, Oklahoma, and Texas. 


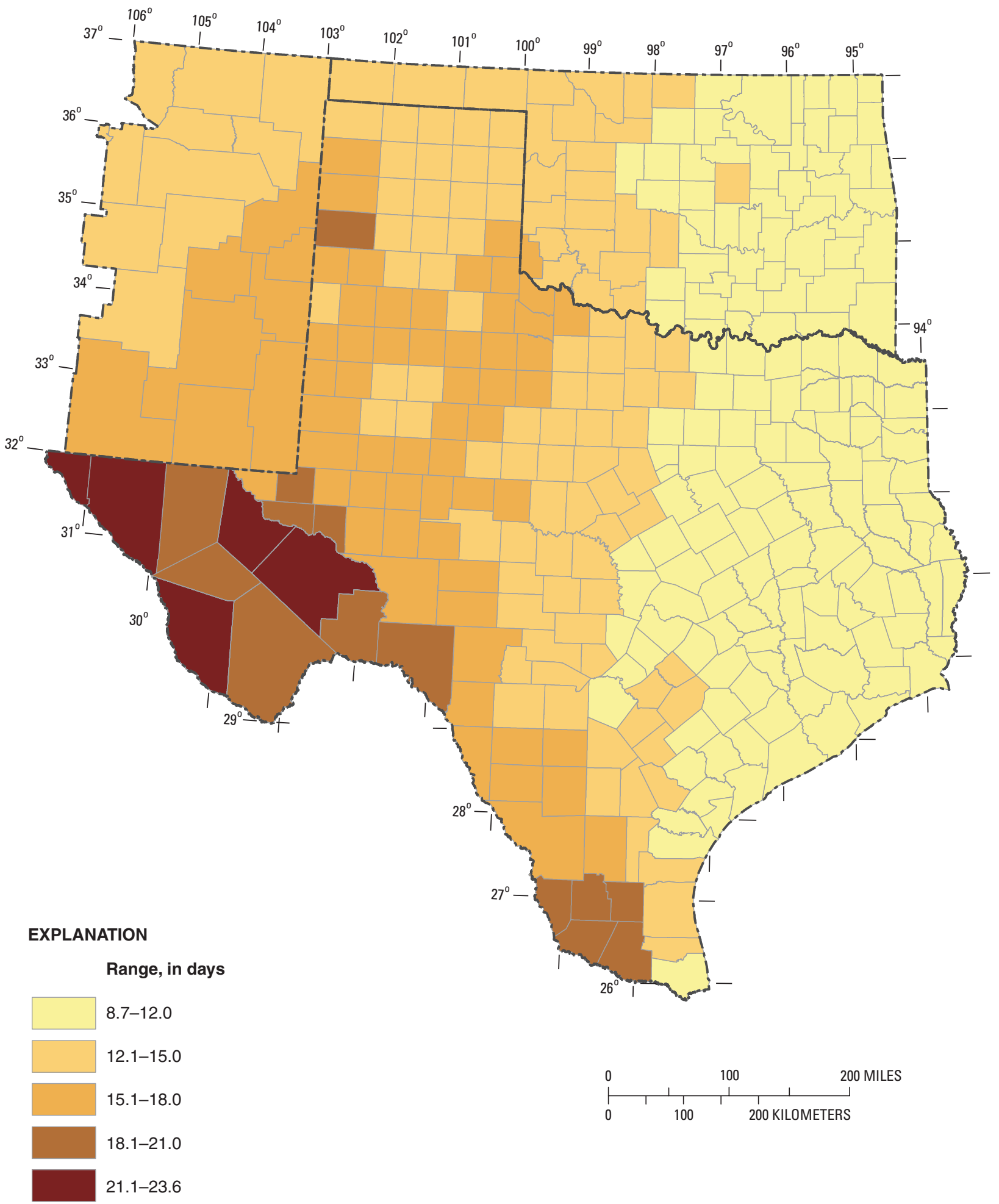

Figure 16. Mean storm interevent time defined by 72-hour minimum interevent time in eastern New Mexico, 0klahoma, and Texas. 


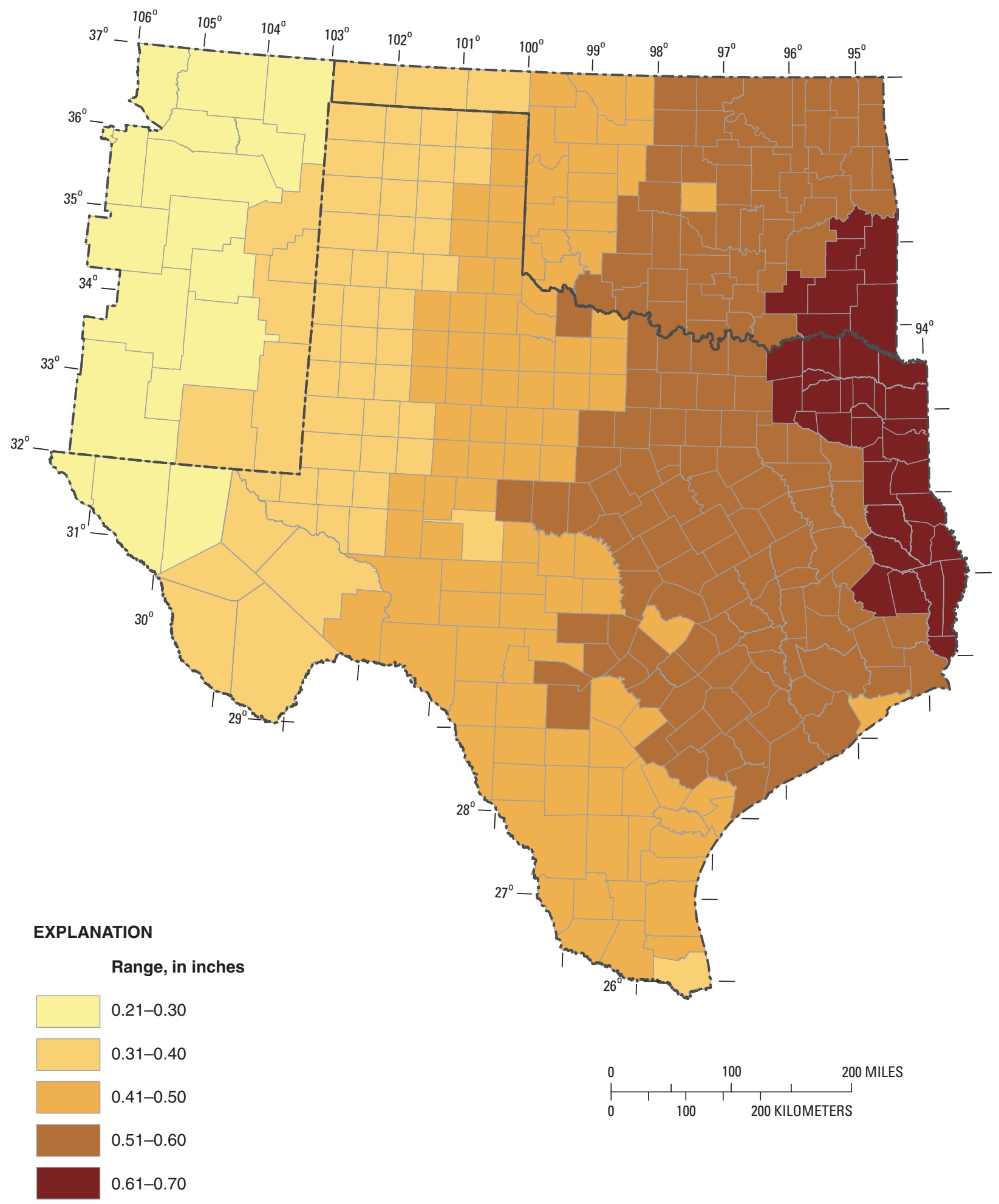

Figure 17. Mean storm depth defined by 6-hour minimum interevent time in eastern New Mexico, Oklahoma, and Texas. 


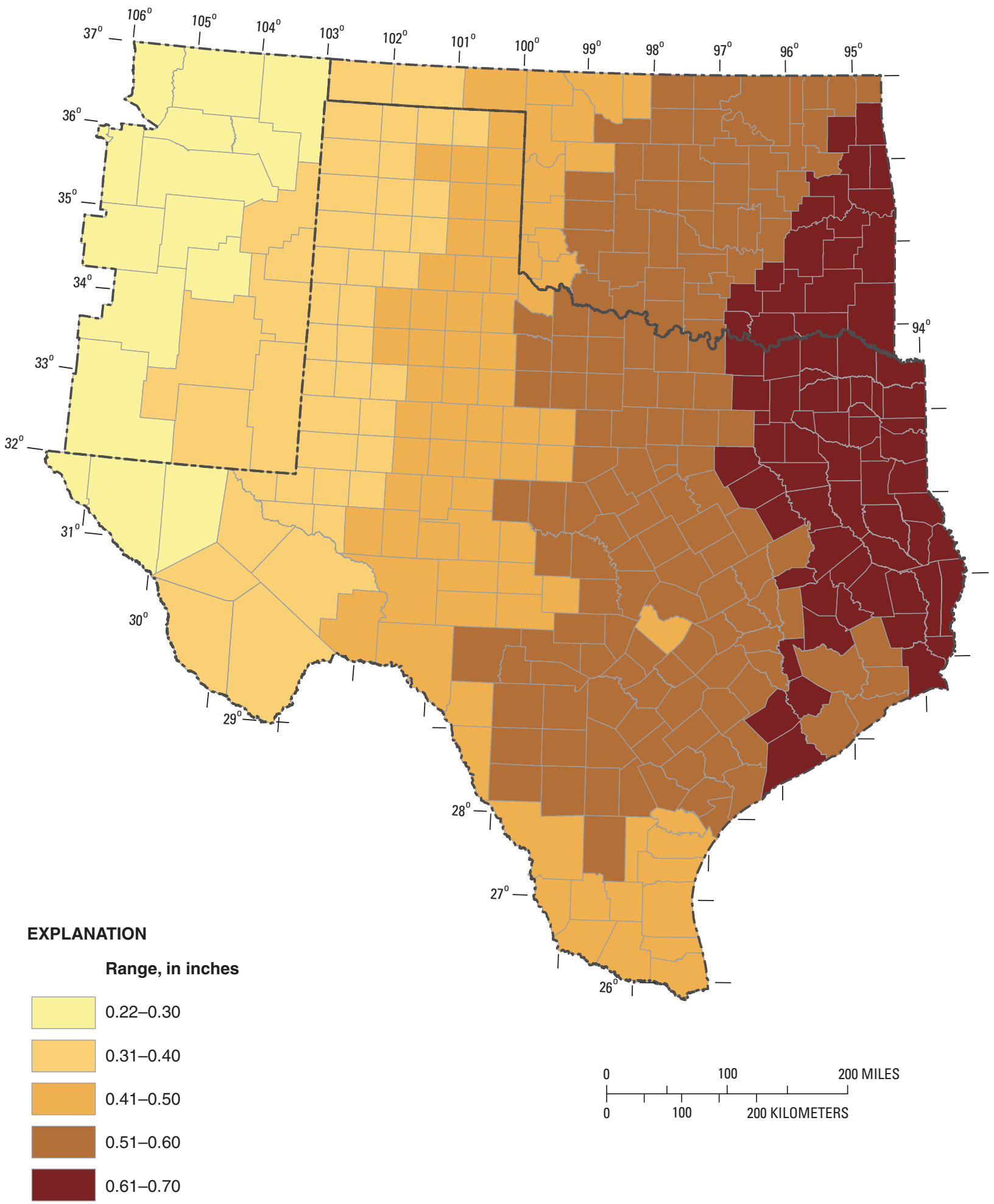

Figure 18. Mean storm depth defined by 8-hour minimum interevent time in eastern New Mexico, Oklahoma, and Texas. 


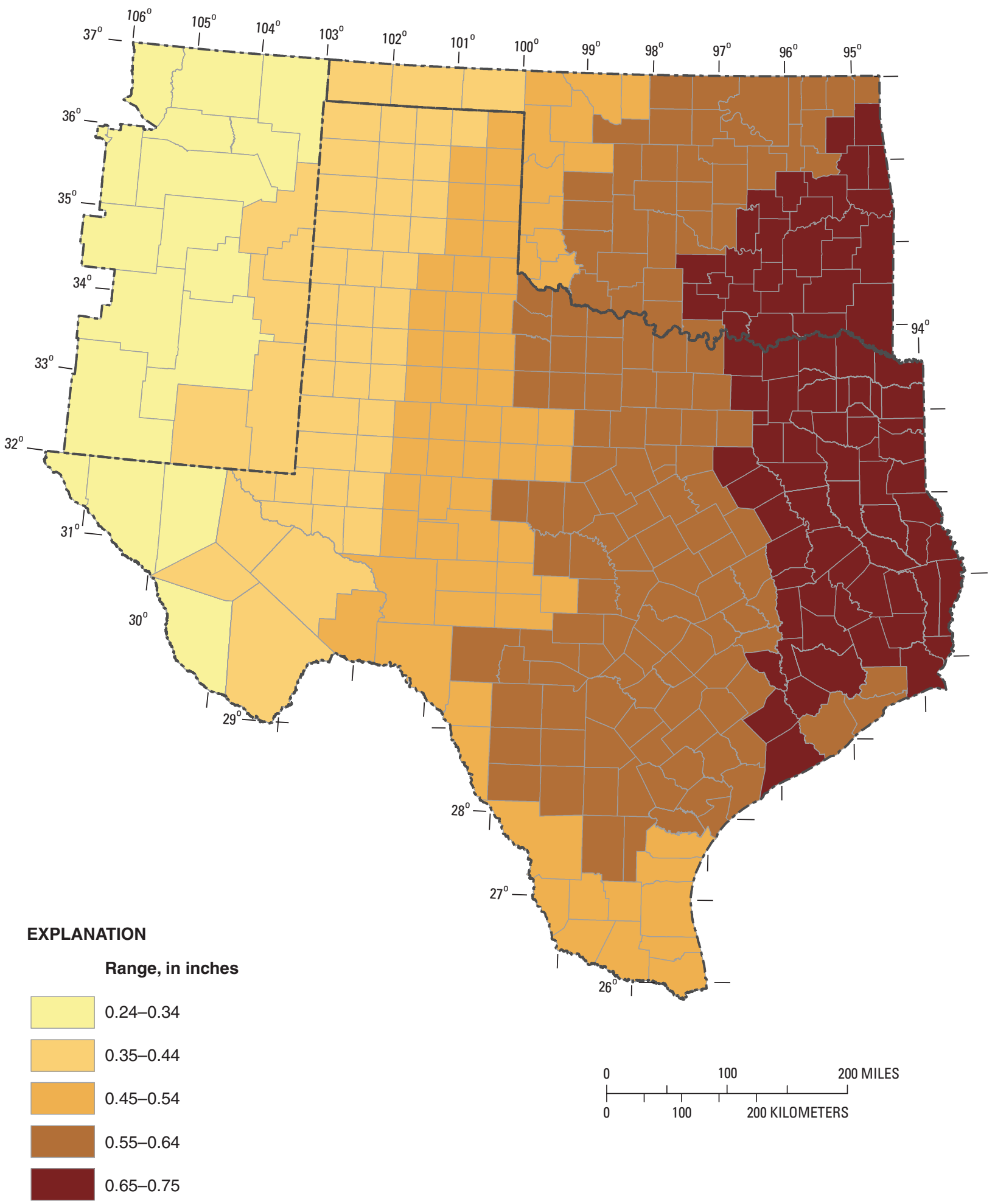

Figure 19. Mean storm depth defined by 12-hour minimum interevent time in eastern New Mexico, Oklahoma, and Texas. 


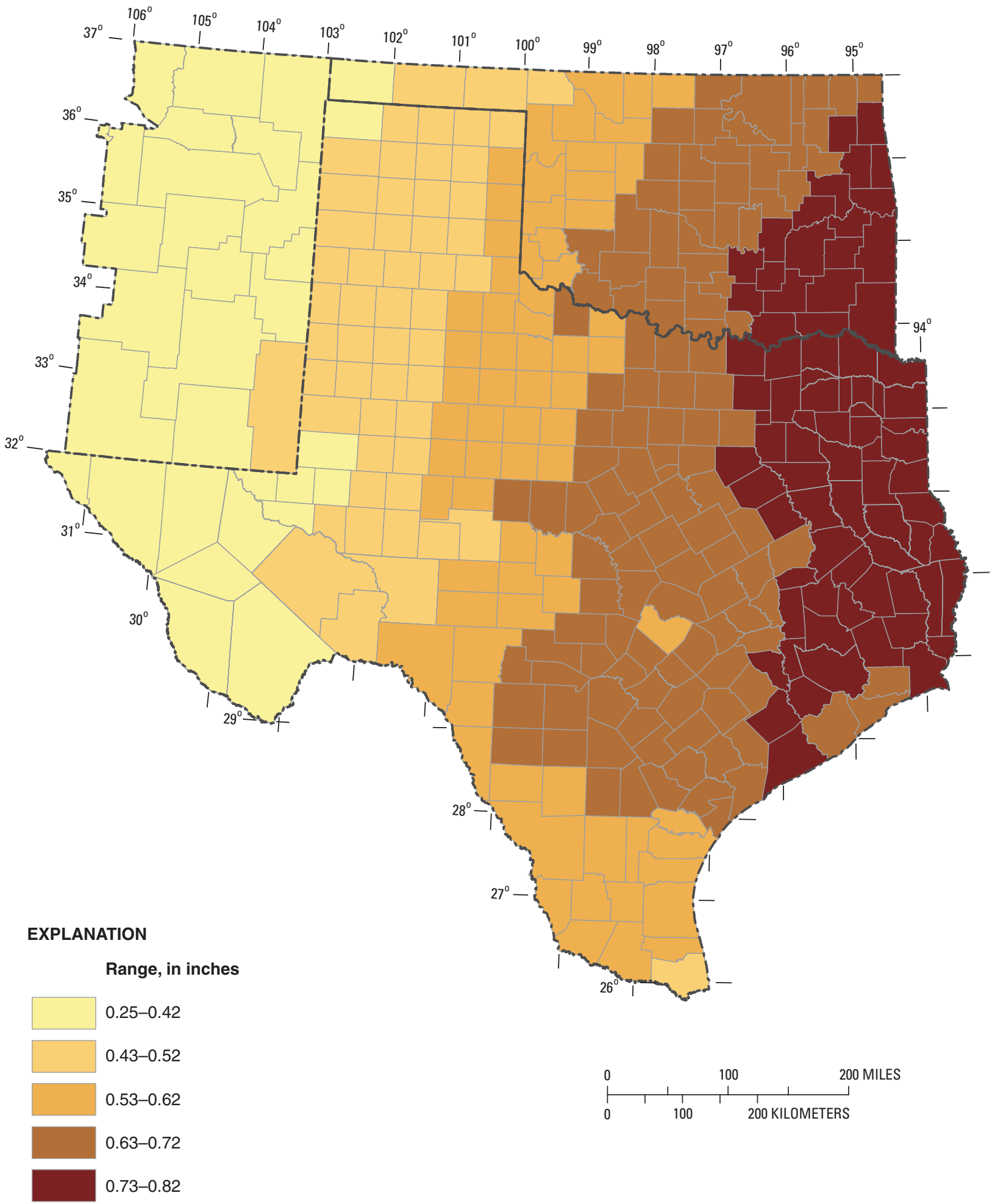

Figure 20. Mean storm depth defined by 18-hour minimum interevent time in eastern New Mexico, Oklahoma, and Texas. 


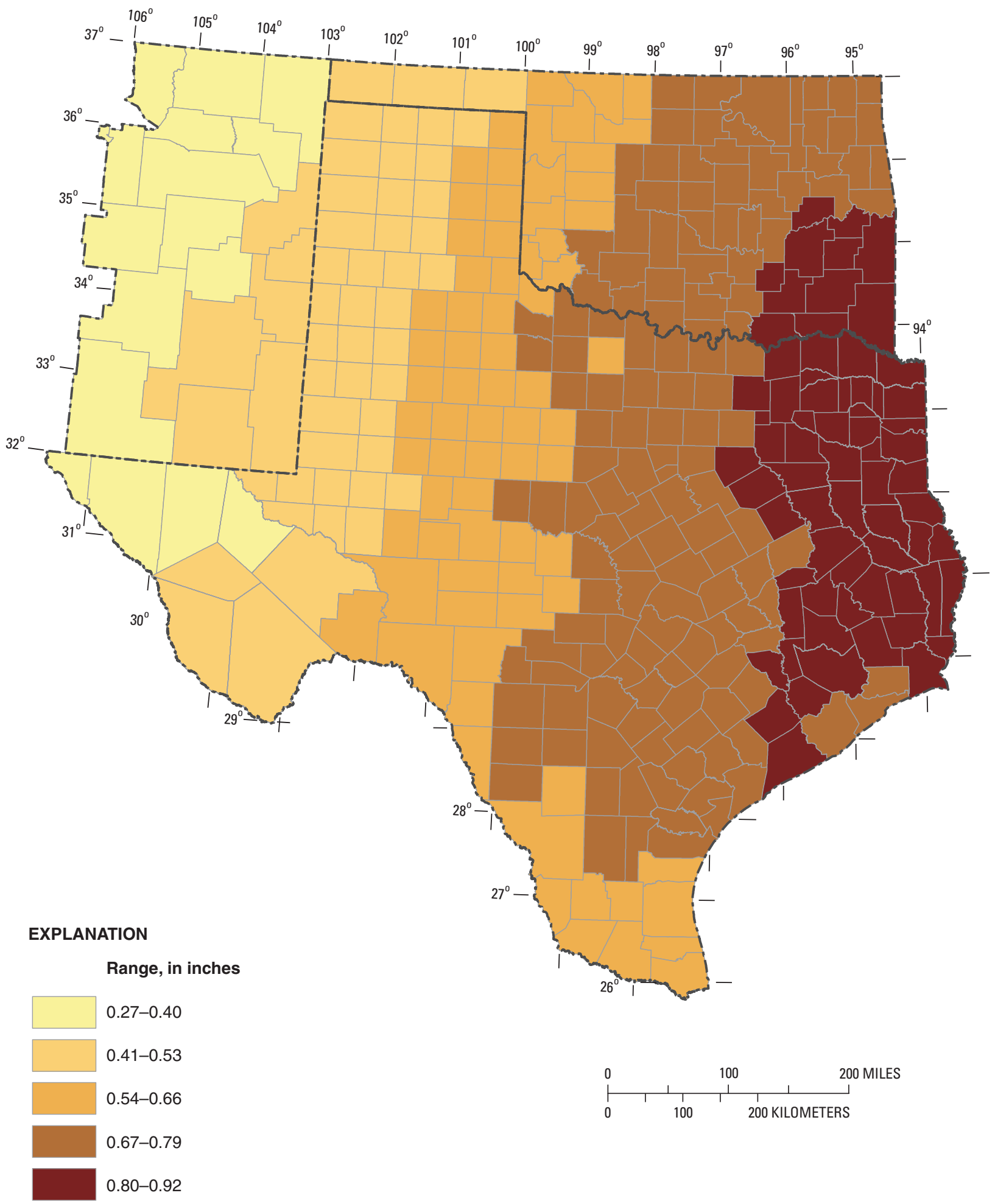

Figure 21. Mean storm depth defined by 24-hour minimum interevent time in eastern New Mexico, Oklahoma, and Texas. 


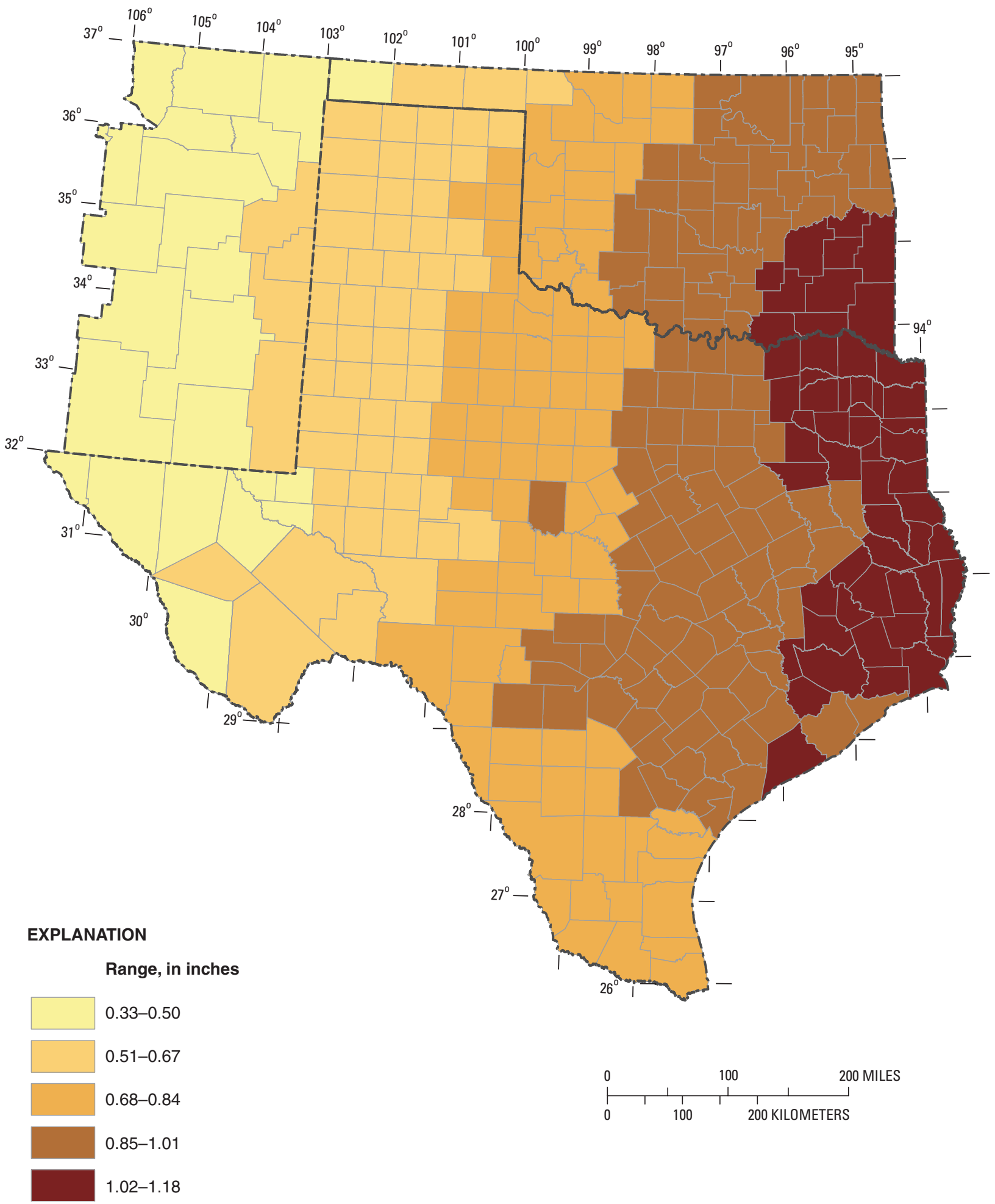

Figure 22. Mean storm depth defined by 48-hour minimum interevent time in eastern New Mexico, Oklahoma, and Texas. 


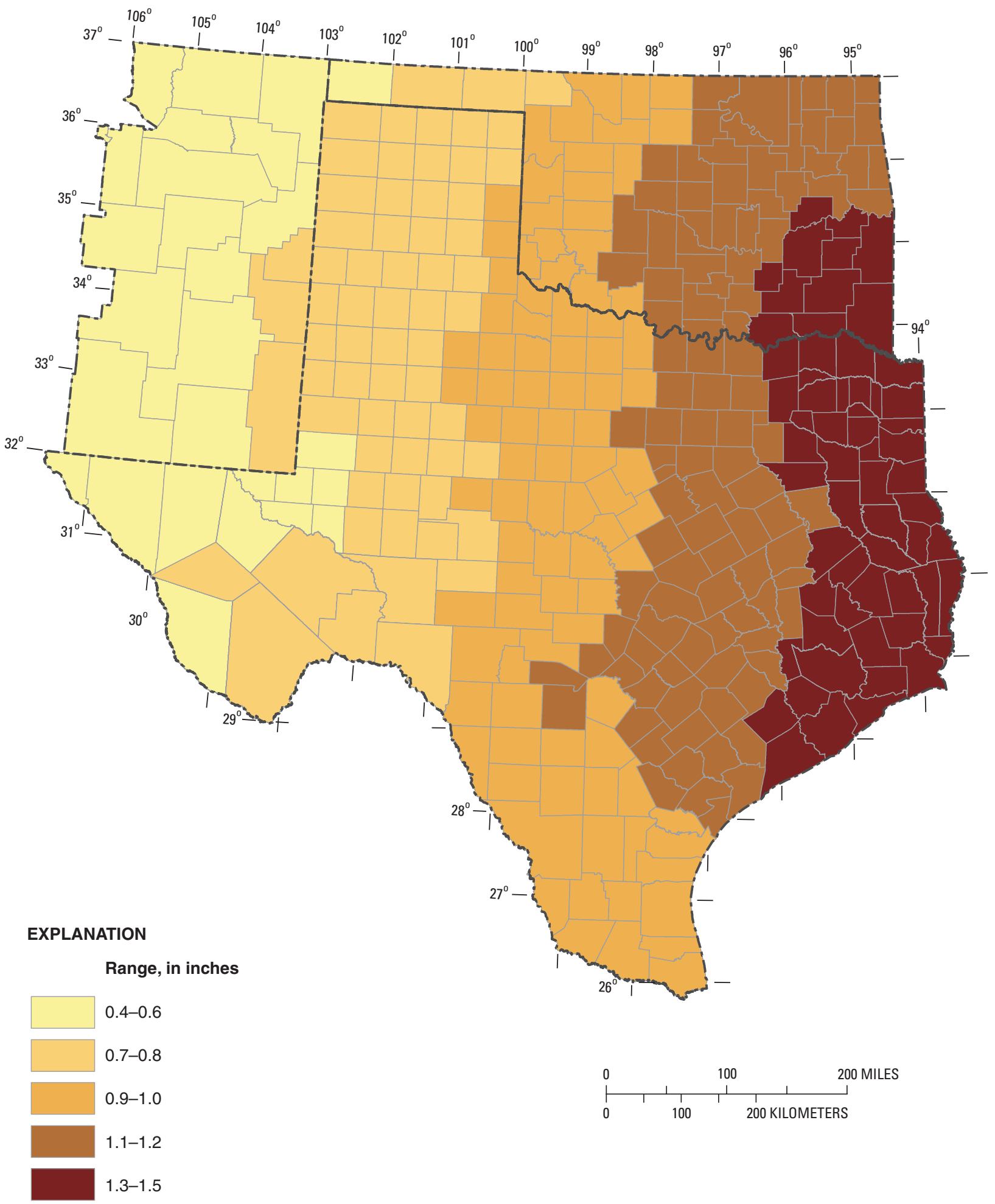

Figure 23. Mean storm depth defined by 72-hour minimum interevent time in eastern New Mexico, Oklahoma, and Texas. 


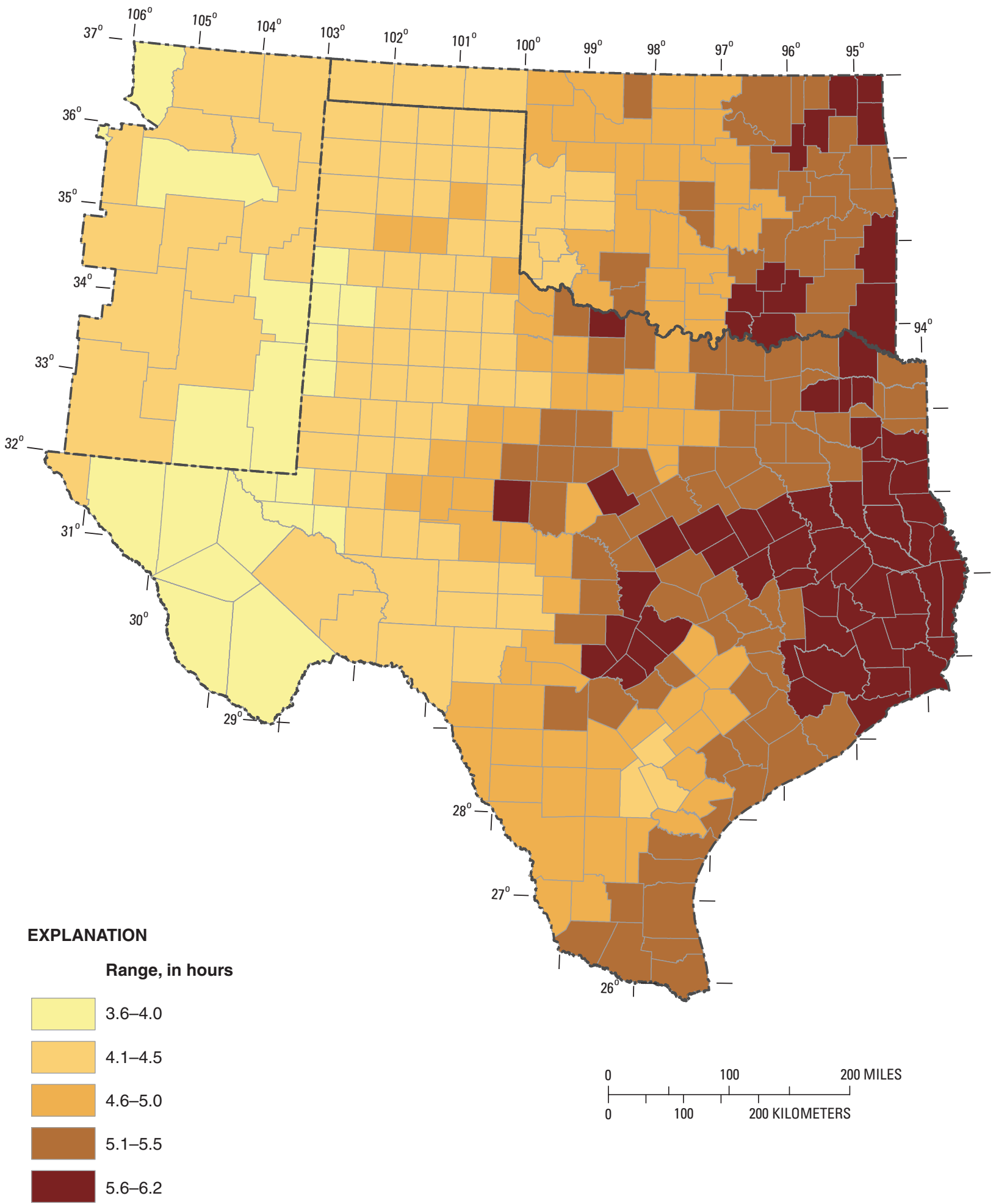

Figure 24. Mean storm duration defined by 6-hour minimum interevent time in eastern New Mexico, Oklahoma, and Texas. 


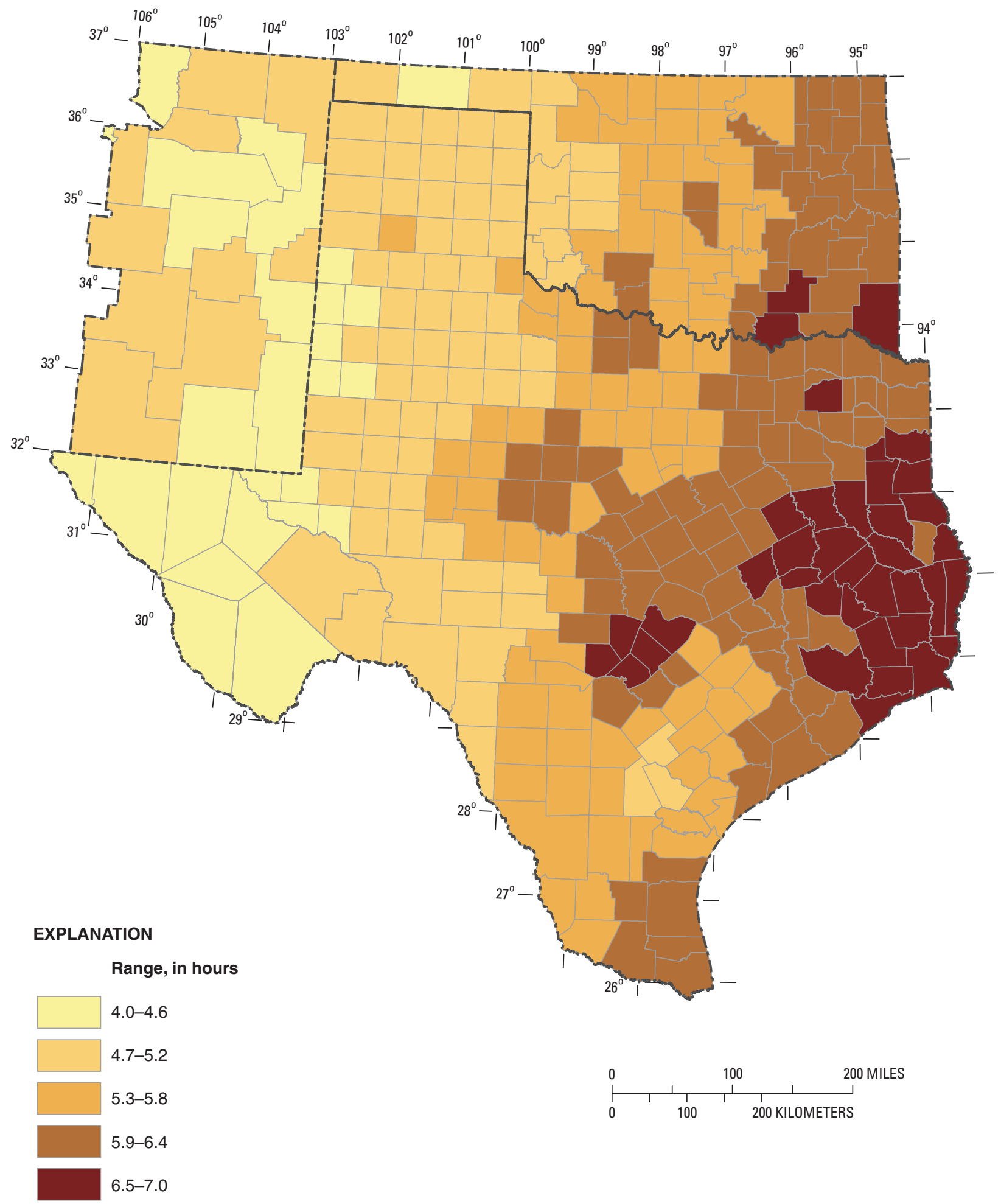

Figure 25. Mean storm duration defined by 8-hour minimum interevent time in eastern New Mexico, Oklahoma, and Texas. 


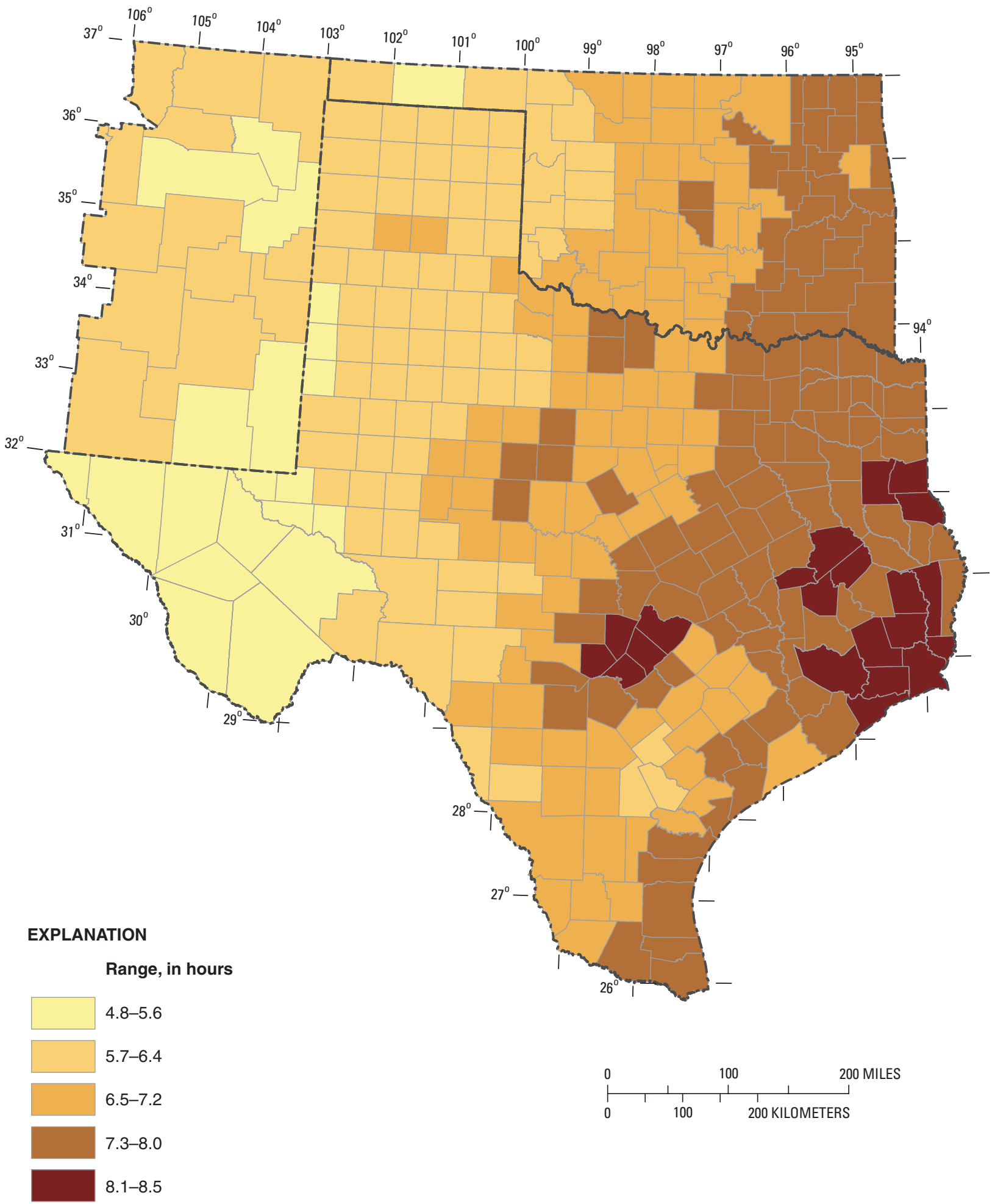

Figure 26. Mean storm duration defined by 12-hour minimum interevent time in eastern New Mexico, Oklahoma, and Texas. 


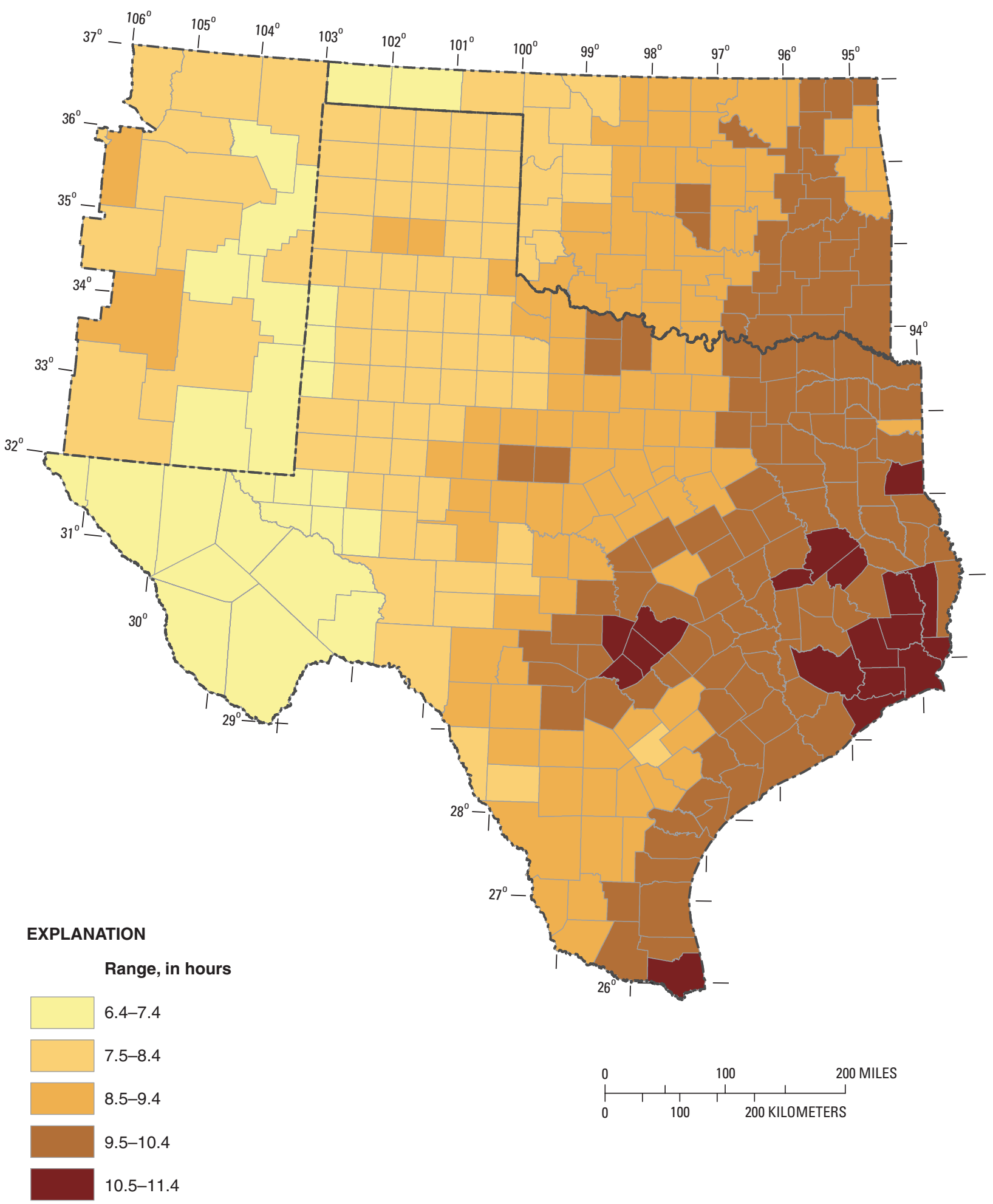

Figure 27. Mean storm duration defined by 18-hour minimum interevent time in eastern New Mexico, Oklahoma, and Texas. 


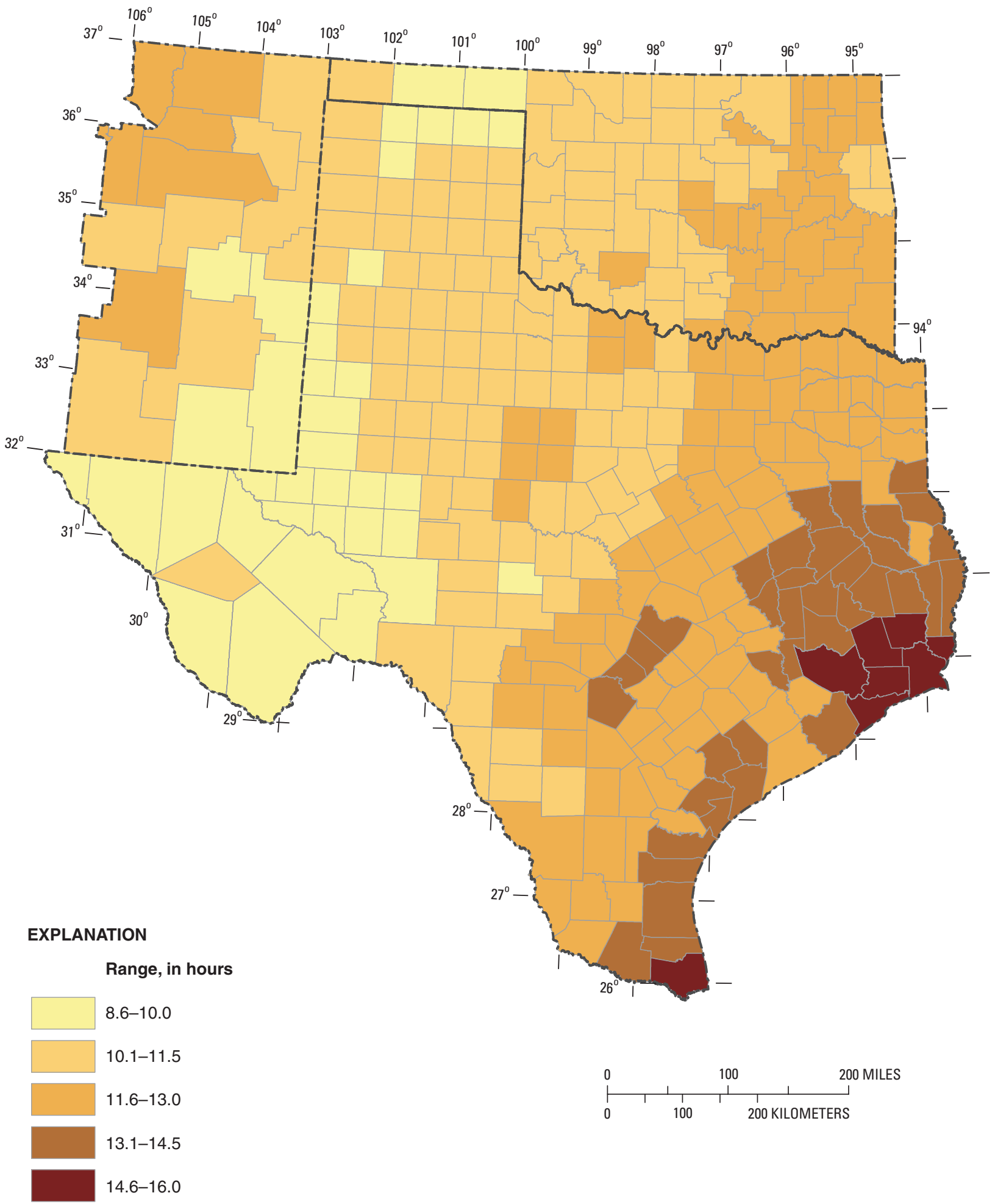

Figure 28. Mean storm duration defined by 24-hour minimum interevent time in eastern New Mexico, Oklahoma, and Texas. 


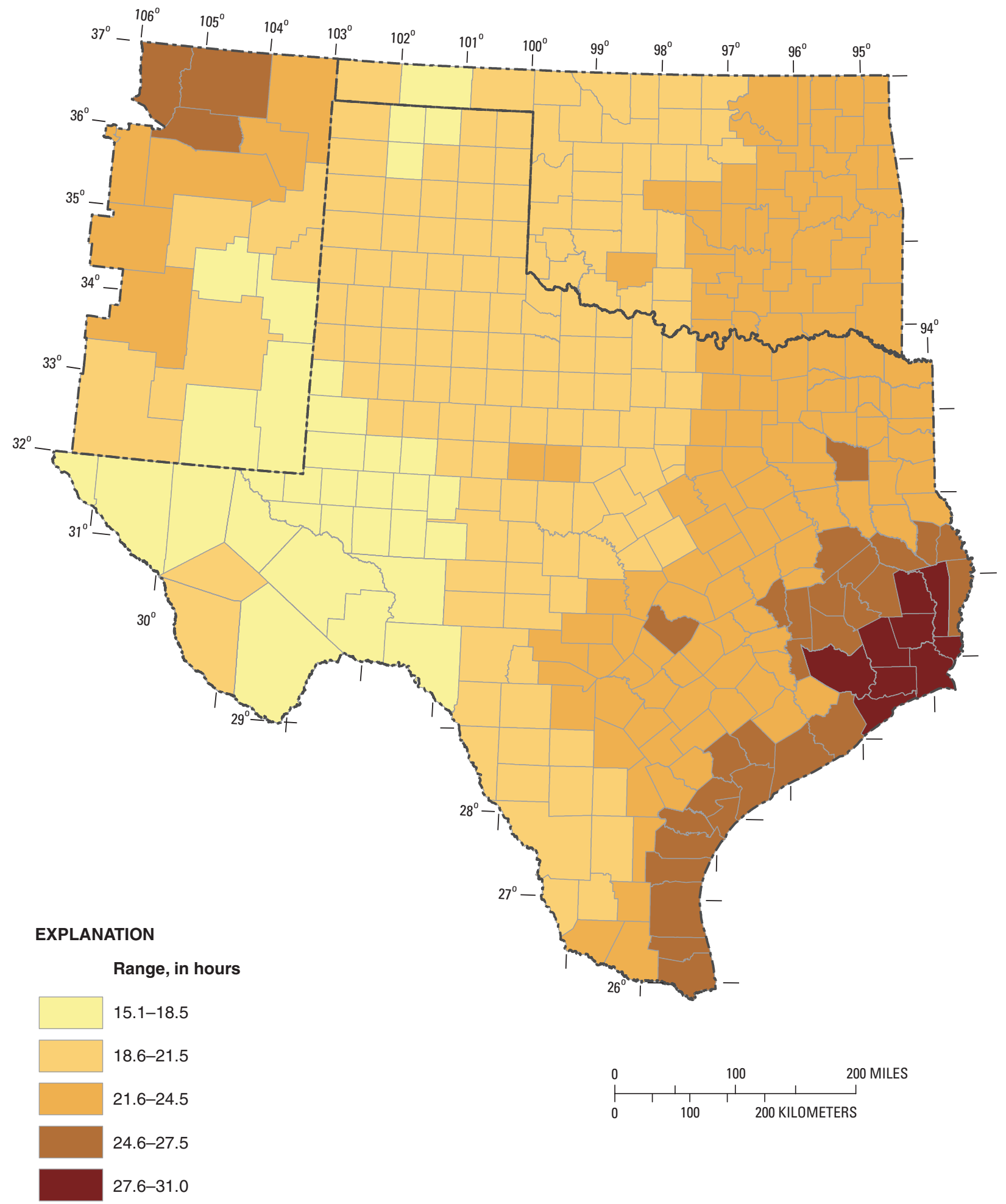

Figure 29. Mean storm duration defined by 48-hour minimum interevent time in eastern New Mexico, Oklahoma, and Texas. 


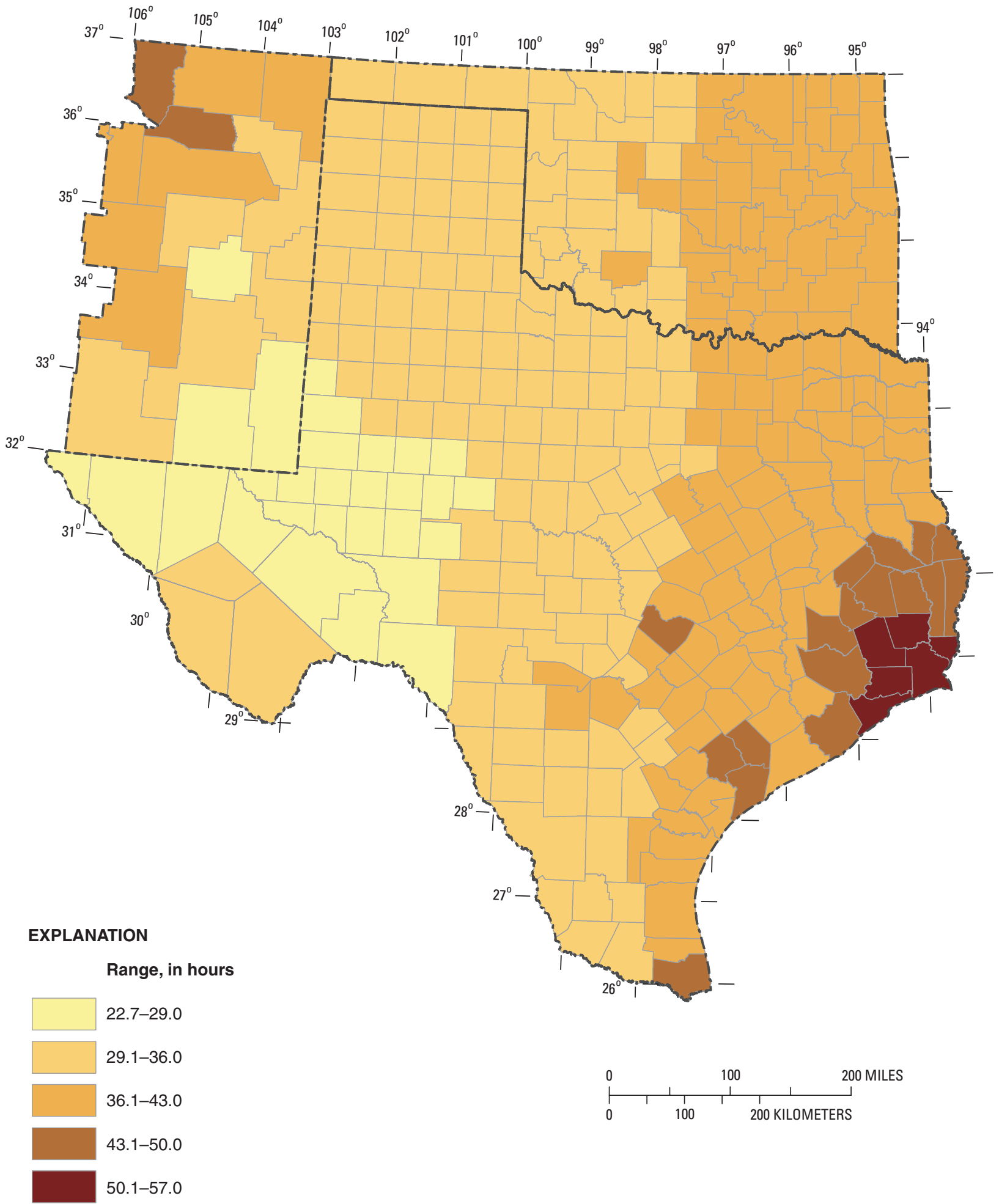

Figure 30. Mean storm duration defined by 72-hour minimum interevent time in eastern New Mexico, Oklahoma, and Texas. 


\section{Example Applications}

The three statistical components that describe storms are storm interevent time, distribution of storm depth, and distribution of storm duration. The components facilitate a wide range of sophisticated BMP function and other analyses (for example, Wanielista and Yousef, 1993; and Adams and Papa, 2000). In this section are 10 example problems and solutions that illustrate applications of the statistics presented here; this section is intended as a guide for general application of this report. Storm statistics for hourly rainfall stations in eastern New Mexico, Oklahoma, and Texas are listed in appendixes 2, 3, and 4, respectively.

Before the example problems and solutions are presented, additional discussion to further clarify the intent of this section is warranted. The comprehensive statistical characterization of storms in this report facilitates application of the results to a diverse group of problems. The setup, computation, and interpretation of the solutions to the problems presented here ranges from simple to complex.

Examples in which a star $(\star)$ precedes the problem statement (examples 4,5 , and 10 ) are sufficiently complex that complete background, context, or steps of the solution are beyond the scope of this report. It is anticipated that most design engineers and decision makers will be more informed by the less complex (no star) problems. For the more complex problems, integrated mathematical or statistical software packages or custom computer programs are required for some solution steps. The intended audience for the more complex problems are those with considerable knowledge of univariate statistical theory, magnitude and frequency analysis, and probabilistic numerical modeling of rainfall processes. Recent publications useful in the context of these problems are Stedinger and others (1992), Wanielista and Yousef (1993), Wilks (1995), Hosking and Wallis (1997), Clarke (1998), Adams and Papa (2000), Evans and others (2000), and Bhat and Miller (2002).

Two classes of approach for application of the storm statistics are possible: site-specific and regional. The sitespecific approach uses storm statistics recorded from a single station independent of the statistics from nearby stations. In practice, the site-specific approach typically is limited to long-record stations. It is not unusual for site-specific statistics from long-record stations to be transferred to a nearby ungaged site. The regional approach differs from the site-specific approach in that statistics from numerous neighboring stations are combined or regionalized to produce alternative and often more reliable estimates for storm statistics than possible from the site-specific data record. The statistics by county (tables 12-20) and the dimensionless frequency curves (table 7) are products of the regional approach. Both approaches are demonstrated here.

\section{Site-Specific Approach}

\section{Example 1: Estimation of Storm Occurrence}

PROBLEM: The 75th percentile of the number of storms in a 1-year time interval for an ungaged site near Briggs, Tex. (fig. 3C), is needed for construction scheduling and planning because construction activities stop when storms occur.

Because no time scale for a watershed is specified, a minimum interevent time of 8 hours is assumed. It is useful to assume that storm occurrence follows a Poisson process (Clarke, 1998; Bhat and Miller, 2002, and references therein).

SOLUTION: Station 1068 Briggs, Tex., is the nearest long-term station. The station has 59 years of record. From appendix 4-1.2, this station has recorded 2,882 storms in 512,393 hours. Therefore, the Poisson parameter $(\Lambda)$ is 7.408 days ([512,393 hours/2,882 storms] $\times$ day/24 hours). The cumulative distribution function $F_{n}(T)$ of the Poisson distribution is

$$
F_{n}(T)=e^{-T /(\Lambda)} \sum_{i=0}^{n} \frac{(T / \Lambda)^{i}}{i !},
$$

where $F_{n}(T)$ is the cumulative probability for $n$ events within a $T$-day time period, and $\Lambda$ is in days.

Because the 75th percentile $(F=0.75)$ is needed, the number of storms in a 365-day time period can be solved by setting the left side of the equation to 0.75 and solving for the integer $n$ that best satisfies the equality. The integers that best satisfy the equality are 53 at an $F$ of 0.732 and 54 at an $F$ of 0.775 . The 75th percentile of the number of storms per year is about 54 by rounding to the highest integer. Therefore, 54 or fewer storms can be anticipated with a cumulative probability of about 0.75 . The probability of exactly 54 storms in 1 year is only about 0.043 .

\section{Example 2: Estimation of Interevent Times of Storms}

PROBLEM: As part of a numerical soil-zone study in the Amarillo, Tex. (fig. 3A) area, a soil scientist is interested in simulating interevent times for storms. The 24-hour minimum interevent time will be used. The mean interevent time for the study is assumed equal to the mean interevent time at station 0211 Amarillo Weather Service Office Airport, Tex.

SOLUTION: Station 0211 has an observed mean interevent time of 7.60 days (appendix 4-1.5). For the simulation, the scientist assumes that storms follow a Poisson process (Clarke, 1998; Bhat and Miller, 2002, and references therein). The exponential distribution describes the cumulative distribution of the interevent times of a Poisson process, with an adjustment for the minimum interevent time (Adams and 
Papa, 2000, p. 74). The cumulative distribution of storm interevent times is

$$
F(x)=1-e^{-1\left(\frac{M I T-x}{\Lambda-M I T}\right)} \text { for } x \geq M I T,
$$

where $F$ is the cumulative or nonexceedance probability for the $x$ interevent time, and $M I T$ is the minimum interevent time in days. The parameter $\Lambda$ is the mean interevent time in days. The inclusion of the minimum interevent time adjusts the exponential distribution because interevent times less than the minimum interevent time are not possible. Equation 10 can be solved in terms of $x$. The resulting equation is the quantile function of interevent time and is

$$
x(F)=M I T-(\Lambda-M I T) \ln (1-F) \text { for } x \geq M I T .
$$

When random numbers between 0 and 1 are substituted for $F$ in equation 11 with $\Lambda$ equal to 7.60 days and MIT equal to 1 day (24 hours), a random sequence of interevent times is generated. Five simulations based on a random sequence of five interevent times are listed in table 21 (at end of report). The mean of the simulations is 7.19 days - the mean approaches 7.60 as the number of simulations becomes large.

It is illustrative to compare the 7.60 days mean interevent time to the results of Asquith and Roussel (2003, fig. 4). Asquith and Roussel (2003, fig. 4) shows that the interoccurrence of daily rainfall (not hourly) of 0.05 inch or more is, on average about 8 days for the Amarillo area. The two interevent times are of the same order as expected, but the values should not be equal.

\section{Example 3: Estimation of the Empirical Distribution of Storm Depth}

PROBLEM: The 98th-percentile storm from the empirical distribution of storm depth for a site very close to station 4311 Houston Alief, Tex. (fig. 3C) (62 years of record), is required by an environmental consulting firm working on a project proposal in a watershed where BMPs are to have a 24-hour drawdown time. Hence, the statistics of storms with a 24-hour minimum interevent time are appropriate.

SOLUTION: The 98th percentile and other selected percentiles of storm depth are listed in appendix 4-4.5 and in column two of table 22 (at end of report). The 98th-percentile storm has a depth of 4.55 inches. (Column three of table 22 is a component of example 4.) The median storm depth is 0.44 inch and the interquartile range is 1.03 inches (1.18 minus 0.15$)$ for station 4311.

\section{Example 4: Estimation of the Continuous Distribution of Storm Depth}

$\star$ PROBLEM: As part of a city ordinance, a BMP for a small urban watershed in the city is believed to accommodate 90 percent of all storms when 2 inches or less of runoff is cap- tured. The temporal distribution of runoff (outflow rate) from the BMP is to be ignored. Engineering firm $\mathrm{A}$ is to design a $\mathrm{BMP}$ for a given watershed in which the ordinance applies. The ordinance states that the BMP is to have a 24-hour drawdown time; hence an analysis of storms with a 24-hour minimum interevent time is required. Engineering firm $\mathrm{A}$ is questioning whether a 2-inch design runoff would accommodate the 90thpercentile storm as reflected by the ordinance or instead would accommodate approximately the 95th-percentile storm. Thus, firm A believes that the ordinance might contribute to overdesign of BMPs. The scientific credibility of the ordinance hence is in question; the results of this report can be used to evaluate the ordinance. Assume, for the purpose of illustration, that near the planned BMP is long-term station 4311 Houston Alief, Tex. (station considered in example 3).

SOLUTION: The first step toward the solution is to compute the depth of rainfall that produces 2 inches of runoff on the watershed. A simple runoff model (Adams and Papa, 2000, p. 121, eq. 6.28) used for illustration is

$$
R=\phi\left(P-S_{D}\right)
$$

where $R$ is runoff in inches, $\phi$ is the runoff coefficient, $P$ is rainfall in inches, and $S_{D}$ is depression storage or an initial abstraction in inches. It is widely accepted that a typical initial abstraction for the watershed is 0.25 inch and the runoff coefficient is about 0.8 . Upon variable substitution, the rainfall producing 2 inches of runoff is 2.75 inches.

The L-moments of storm depth for a 24-hour minimum interevent time for this station are $0.88849 \mathrm{inch}, 0.52954 \mathrm{inch}$, 0.45778 , and 0.23879 for the mean, L-scale, L-skew, and L-kurtosis, respectively (appendix 4-2.5). A four-parameter kappa distribution (see section "Quantile Functions of Storm Depth and Duration" in this report) can be fit by use of these L-moments using an algorithm such as in Hosking (1996) (data not shown in this report). The fitted kappa distribution corresponding to these L-moments is

$$
P(F)=-0.4990+\frac{1.028}{-0.1117}\left\{1-\left[\frac{\left(1-F^{1.650}\right)}{1.650}\right]^{-0.1117}\right\} \text {, }
$$

where $P$ is storm depth and $F$ is nonexceedance probability. Substituting 2.75 inches for the left side of the equation and solving the equation for $F$ yields 0.932 or 93.2 percent. In other words, a rainfall depth of 2.75 inches is about the 93rdpercentile storm depth. Therefore, a statistical estimate of the storm percentage associated with 2 inches of runoff for the watershed is 3 percentage points larger than 90 percent. The 90th percentile for the distribution $(F=0.90)$ is 2.24 inches.

Thus, the ordinance reflects a depth of 2.75 inches; whereas, the statistical estimate of the 90th-percentile storm is 2.24 inches using the Hosking (1996) algorithm. Therefore, the question of engineering firm $A$ that a storm associated with 2 inches of runoff would accommodate approximately the 95th-percentile storm is questionable. The depth for the 95thpercentile storm is 3.18 inches by substituting $F=0.95$ into 
equation 13. The runoff from the 95th-percentile storm is about 2.34 inches from equation 12 .

To further illustrate the application of this report, from equation 13 the quantiles for each of the selected percentiles or nonexceedance probabilities $(0.01,0.02,0.10,0.25,0.50,0.75$, $0.90,0.98$, and 0.99 ) are listed in column three of table 22. As seen in the table, the empirical storm depth percentiles and storm depth percentiles from the kappa distribution are similar for each percentile as expected.

\section{Example 5: Statistical Simulation of Rainfall Intensity}

$\star$ PROBLEM: An analyst wants to construct synthetic temporal distributions of average rainfall intensity for station 4311 Houston Alief, Tex., to investigate the influence of rainfall rates on the spill volume of a numerical model of a particular BMP design.

SOLUTION: The kappa distribution of storm depth $P$ for nonexceedance probability $F$ is given as equation 13 in example 4. The L-moments of storm duration for the station are listed in appendix 4-3.5. The mean, L-scale, L-skew, and L-kurtosis are 13.434 hours, 8.1389 hours, 0.46763 , and 0.20844 , respectively. Fitting a kappa distribution to these L-moments using the Hosking (1996) algorithm (data not shown in this report) results in the following equation for the storm duration $D$ in terms of nonexceedance probability, $F$ :

$$
\begin{aligned}
D(F)= & -23.466+\left(\frac{28.137}{0.093897}\right) \\
& \left\{1-\left[\frac{\left(1-F^{2.4775}\right)}{2.4775}\right]^{0.093897}\right\} .
\end{aligned}
$$

It is convenient to assume that storm depth and duration are independent random variables, which is supported by the scattered relation in figure 9. Under this assumption, storm depth is simulated by generating a random number between 0 and 1 , substituting this value for $F$, and solving equation 13 for $P$. A similar process for storm duration is done with the generation of a new random number between 0 and 1 , substituting this value for $F$, and solving equation 14 for $D$. This process is best illustrated by example. A random number of 0.78687 is generated for storm depth and results in a depth of 1.33 inches using equation 13. Another random number of 0.040703 is generated for storm duration and results in a duration of 1.01 hours using equation 14. The average rainfall intensity for this storm thus is 1.33 divided by 1.01 or 1.32 inches per hour.

\section{Regional Approach by County}

\section{Example 6: Regional Estimation of Storm Occurrence}

PROBLEM: The storm interevent time for storms defined by a 40 -hour minimum interevent time in Randall County, Tex. (fig. 3A), is desired. The storm interevent time is a component of a design. The maps in this report can be used for estimation.

SOLUTION: The storm interevent time for a 40-hour minimum interevent time is not a statistic provided in this report. However, 24-hour and 48-hour minimum interevent times bracket 40 hours. At the approximate center of Randall County, the mean storm interevent time for the 24-hour minimum interevent time is about 10.5 days (table 18), and that for the 48 -hour minimum interevent time is about 12.4 days (table 18). Linear interpolation can be used to estimate the mean storm interevent time for the 40-hour minimum interevent time; the result is about 11.8 days.

\section{Example 7: Computation of the Storm-Captured Percentage}

PROBLEM: A local ordinance for a county in Texas requires that a BMP capture a 1.5-inch storm and release this storm over a 24-hour period. The county has a mean storm depth of 0.750 inch (a randomly selected value from table 19). An estimate of the percentage of storms that will be captured under the ordinance is needed.

SOLUTION: The dimensionless storm depth frequency curve using the kappa distribution (eq. 6; table 7) for a 24-hour minimum interevent time in Texas is

$$
x(F)=-0.5790+\frac{1.115}{-0.1359}\left[1-\left(\frac{1-F^{1.747}}{1.747}\right)^{-0.1359}\right],
$$

where $x(F)$ is the dimensionless multiplier (a frequency factor) for nonexceedance probability $F$. The storm depth distribution is the mean depth multiplied by the dimensionless distribution or

$$
P(F)=0.750 \times x(F),
$$

where $P(F)$ is the storm depth for nonexceedance probability $F$. The left side of the equation is set to 1.5 inches, and the storm percentage can be estimated by solving the resulting equation for $F$. The equation is

$$
\frac{1.5}{0.75}=-0.5790+\frac{1.115}{-0.1359}\left[1-\left(\frac{1-F^{1.747}}{1.747}\right)^{-0.1359}\right] .
$$

The $F$ satisfying the equality is 0.859 . Thus, under the ordinance, about 86 percent of all storms will be captured by the BMP.

\section{Example 8: Regional Estimation of the Empirical Distribution of Storm Depth}

PROBLEM: A BMP is to be built with a 36-hour drawdown time in Randall County, Tex. (fig. 3A). The empirical distribution, specifically the 50th, 75th, 90th, 98th, and 99th percentiles of storm depth, are needed as part of the design process. 
The mean storm depths for Randall County and dimensionless storm depth frequency curves are to be used.

SOLUTION: Storm depth percentiles for a 36-hour minimum interevent time are not statistics provided in this report. However, 24-hour and 48-hour minimum interevent times bracket 36 hours. The mean storm depths for the county (table 19) are 0.488 inch (24-hour minimum interevent time) and 0.597 inch (48-hour minimum interevent time). The dimensionless storm depth frequency factors for the 24-hour minimum interevent time using the kappa distribution (eq. 6; table 7) are computed from

$$
x(F)=-0.5790+\frac{1.115}{-0.1359}\left[1-\left(\frac{1-F^{1.747}}{1.747}\right)^{-0.1359}\right] .
$$

The dimensionless storm depth frequency factors for the 48-hour minimum interevent time using the kappa distribution (eq. 6; table 7) are computed from

$$
x(F)=-0.4868+\frac{1.086}{-0.1326}\left[1-\left(\frac{1-F^{1.617}}{1.617}\right)^{-0.1326}\right] .
$$

The frequency factors for the 50th, 75th, 90th, 98th, and 99th percentiles of storm depth computed from equations 18 and 19 are listed in columns 2 and 5 of table 23 (at end of report) for the 24-hour and 48-hour minimum interevent time, respectively. The storm depths are computed by multiplying the means by the frequency factors. The resulting storm depths are listed in columns 3 and 6 of table 23; and the storm depths for the 36-hour minimum interevent time (column 4 [shaded] of table 23) are computed by interpolation. The values in table 23 indicate that for a BMP in Randall County to capture, for example, 90 percent of all storms, assuming total conversion of rainfall to runoff and that the BMP is memoryless, the structure should have about 1.35 inches of storage.

\section{Example 9: Estimation of the Uncertainty of a Regional Estimate of a Storm Depth Percentile}

PROBLEM: Estimation of the uncertainty of the 99th percentile of storm depth for the 24-hour minimum interevent time for Randall County, Tex. (fig. 3A), is required as part of a sensitivity analysis of a BMP design. This storm depth percentile was computed as part of example 8 .

SOLUTION: The use of dimensionless frequency curves and the mean for a statistic provide a convenient framework to compute uncertainty. The dimensionless frequency curve is assumed to be invariant. All uncertainty is assumed to be associated with the mean storm depth. The regional analysis provides a measure of uncertainty through computation of the study-area mean storm depth and root-weighted-mean-square error (RwMSE). For the 24-hour minimum interevent time, the mean storm depth for the study area is 0.648 inch, and RwMSE is 0.043 inch (table 10). The relative error thus is $0.0664(0.043$ divided by 0.648 ). The mean storm depth for the 24-hour minimum interevent time for Randall County is 0.488 inch (table $19)$. The error for the county thus is 0.0324 inch $(0.0664$ multi- plied by 0.488$)$. The storm depth frequency factor for the 99th-percentile storm is 6.57 (column 2 of table 23). Thus, the 99th-percentile storm depth for Randall County with uncertainty is computed as $6.57 \times 0.488 \pm 6.57 \times 0.0324$ and written as $3.21 \pm 0.213$ inch.

\section{Example 10: Estimation of the Expected Storage in a BMP Using Mean Storm Interevent Time, Depth, and Duration}

$\star$ PROBLEM: An analytical solution for the expected storage of runoff in a BMP is needed for a location in Randall County, Tex. (fig. 3A). The watershed has a runoff coefficient of 0.70 and a depression storage of 0.50 inch. The BMP has a maximum storage of 0.9 inch and a controlled outflow or treatment rate of 0.0375 inch per hour. The drawdown time of the BMP thus is 24 hours ( 0.9 divided by 0.0375$)$.

SOLUTION: The mean storm interevent times for the county (table 18) are 8.21 days (8-hour minimum interevent time) and 10.5 days (24-hour minimum interevent time). The mean storm depths for the county (table 19) are 0.378 inch (8-hour minimum interevent time) and 0.488 inch (24-hour minimum interevent time). The mean storm durations for the county (table 20) are 5.36 hours (8-hour minimum interevent time) and 11.2 hours (24-hour minimum interevent time).

Adams and Papa (2000, p. 208-210) provide equations to compute the expected storage. The equations require an assumption that storm interevent time, depth, and duration are each exponentially distributed, allowing Adams and Papa (2000) to derive closed-form solutions for expected storage in a BMP. One equation (Adams and Papa, 2000, eq. 9.18a) is applicable for situations in which storm statistics are defined by a minimum interevent time less than the drawdown time of the BMP:

$$
\begin{aligned}
& E[s]=S_{A}-\frac{\Omega(\psi+\lambda)}{\lambda \psi}+\frac{\Omega}{\psi-\lambda}\left(\frac{\psi}{\lambda} e^{-(\lambda / \Omega) S_{A}}-\frac{\lambda}{\psi} e^{-(\psi / \Omega) S_{A}}\right) \\
&+\frac{e^{-\zeta S_{D}}}{\lambda / \Omega+\zeta / \phi} \\
& {\left[1-\frac{1}{\psi-\lambda}\left(\psi e^{-(\lambda / \Omega) S_{A}}-\lambda e^{-(\psi / \Omega) S_{A}}\right)\right.} \\
&\left.+\frac{\lambda / \Omega}{\psi / \Omega+\zeta / \phi}\left(1-e^{-(\psi / \Omega+\zeta / \phi) S_{A}}\right)\right],
\end{aligned}
$$

where $E[s]$ is the expected storage in the BMP at the end of a storm; $S_{A}$ is the maximum storage of the BMP in inches; $\Omega$ is the constant or controlled outflow rate from the reservoir in inches per hour; $\psi$ is the parameter for exponentially distributed interevent times in hours ${ }^{-1}(1 /$ mean interevent time); $\lambda$ is the parameter for exponentially distributed storm duration in hours $^{-1}(1 /$ mean duration $) ; \zeta$ is the parameter for exponentially distributed storm depth in inches ${ }^{-1}$ (1/mean depth); $\phi$ is a 
runoff coefficient; and $S_{D}$ is depression storage or an initial abstraction in inches.

Equation 20 is complex, but if the minimum interevent time is greater than or equal to the drawdown time, then a much simpler equation results because prior storage in the BMP is zero. The alternative equation (Adams and Papa, 2000, eq. 9.27) is

$$
E[s]=\frac{\phi}{\zeta} \times \frac{\lambda / \Omega}{\lambda / \Omega+\zeta / \phi}\left(1-e^{-(\zeta / \phi) S_{A}}\right) e^{-\zeta S_{D}},
$$

where the variables are as defined for equation 20 .

The values for the parameters when the statistics for the 8-hour minimum interevent time are used are $S_{A}=0.90 \mathrm{inch}$, $\Omega=0.0375$ inch per hour; $\psi=0.005075$ hour $^{-1}[1 /(8.21 \times 24)]$; $\lambda=0.1866$ hour $^{-1}$ (1/5.36); $\zeta=2.646$ inches $^{-1}$ (1/0.378); $\phi=0.70 ;$ and $S_{D}=0.50$ inch. Substituting these values into equation 20 produces an expected storage of 0.0748 inch.

The values for the parameters when the statistics for the 24-hour minimum interevent time are used are $S_{A}=0.90 \mathrm{inch}$; $\Omega=0.0375$ inch per hour; $\psi=0.003968$ hour $^{-1}[1 /(10.5 \times 24)]$; $\lambda=0.08929$ hour $^{-1}(1 / 11.2) ; \zeta=2.049$ inches $^{-1}(1 / 0.488)$; $\phi=0.70$; and $S_{D}=0.50$ inch. Substituting these values into equation 21 produces an expected storage of 0.0511 inch.

The two estimates of expected storage would not be assumed to be numerically equal, but should be of the same general order of magnitude. In practice, equation 21 is considerably easier to use, but it requires storm statistics derived from a minimum interevent time greater than or equal to the drawdown time of the BMP. Therefore, an important contribution of this report is that analytical solutions for BMP function are simplified by the documentation of rainfall statistics for a wide range of minimum interevent times.

\section{Summary}

The design of runoff-control structures, from simple floodwater-detention basins to sophisticated best-management practices (BMPs), such as engineered sand-filtration ponds, requires the statistical characterization of rainfall as a basis for cost-effective, risk-mitigated, hydrologic engineering design. For this report, BMPs and similar structure types are collectively referred to as BMPs. BMPs can be a substantial component of public and private drainage infrastructure, and the characteristics of rainfall for a given location strongly influence the hydrologic and hydraulic function of these structures. In 2000, the U.S. Geological Survey, in cooperation with the Texas Department of Transportation, and in collaboration with University of Houston, Lamar University, and Texas Tech University, initiated a research program of Texas rainfall characteristics to enhance hydrologic engineering design. This report presents a framework for analysis of the probabilistic, or expected, function of these structures in the context of rainfall inputs to a watershed.
Comprehensive documentation of the three statistics of storms is provided. The three statistics are (1) mean storm interevent time, (2) distribution of storm depth, and (3) distribution of storm duration. These statistics are provided for each of seven selected minimum interevent times: $6,8,12,18,24,48$, and 72 hours.

A number of studies related to statistics of rainfall and statistics of storms for BMP analysis have been done. The Texas Department of Transportation has sponsored a multifaceted research program through several research projects on rainfall characteristics in Texas from the mid-1990s through 2005. A chronological list and brief description of results of the research program through 2005 is provided.

A database of storms recorded through 2002 at National Weather Service hourly rainfall stations in eastern New Mexico, Oklahoma, and Texas was compiled. The database contains more than 155 million values of hourly rainfall (zero values included) from 774 stations. Ninety-two stations and more than 18 million values are available for eastern New Mexico; 149 stations and more than 33 million values are available for Oklahoma; and 533 stations and more than 103 million values are available for Texas. For perspective, for an 8-hour minimum interevent time, 97,491 storms in eastern New Mexico, 206,646 storms in Oklahoma, and 584,159 storms in Texas are available.

A method to define distinct storms from the hourly time series of rainfall data is needed. Distinct storms typically are defined using a minimum time interval of no rainfall; this time interval is referred to as a minimum interevent time. The approach used for this report is to distinguish long time intervals according to the drawdown, infiltration time, or treatment time of a BMP-a structural minimum interevent time. The selected structural minimum interevent times are the intervals $6,8,12$, 18, 24, 48, and 72 hours.

For each of the selected minimum interevent times, the time series of hourly rainfall for each station was parsed or separated into sequences of storms for subsequent statistical analysis. Among the assumptions made as part of the statistical analysis is an assumption that no changes in rainfall characteristics occur from year to year or from season to season.

The storm interevent time was computed as the ratio of the total duration of record (hours) divided by the number of observed storms minus the mean storm duration (hours). The storm interevent time then was converted to units of days per storm. Storm interevent time is a mean or average rate (time per storm); however for brevity, that rate is expressed as time in this report.

The Poisson distribution is suggested to model the number of storms in a given time period. The exponential distribution is suggested to model the interevent time of successive storms. The mean storm interevent time is used as a parameter for each of these distributions. Example problems using these distributions are provided.

The 1st, 2nd, 10th, 25th, 50th, 75th, 90th, 98th, and 99th percentiles of storm depth and duration were computed for each station. If the sample size in terms of number of storms was insufficient to estimate the percentile, the percentile for that 
station was recorded as a missing value. The percentiles of both depth and duration for each station are tabulated.

The L-moment statistics of distributions of both storm depth and storm duration for each station were computed and tabulated. The L-moments of storm depth and duration considered in this report are the mean, L-scale, coefficient of L-variation (L-CV), L-skew, L-kurtosis, and Tau5. L-CV is dimensionless and is defined as the ratio of $\mathrm{L}$-scale to the mean. L-skew, L-kurtosis, and Tau5 also are dimensionless. The L-moments for a given station were considered missing values unless five or more storms were observed in the data record.

The L-moments are useful because a distribution is characterized by a few numbers. Furthermore, when a suitable probability distribution is fit to the L-moments of the observed distribution, then interpolation or extrapolation to percentiles not represented in the data or conveniently tabulated is possible.

Regionwide (eastern New Mexico) or statewide (Oklahoma and Texas) record-length, weighted-average values for mean storm interevent time and storm depth and storm duration L-moments, consisting of the mean, L-CV, L-skew, L-kurtosis, and Tau 5 are tabulated. An important observation is that the regionwide or statewide mean values for the dimensionless L-moments (L-CV, L-skew, L-kurtosis, and Tau5) are all of the same general order of magnitude as minimum interevent time increases. A second important observation is that the dimensionless L-moments for eastern New Mexico, Oklahoma, and Texas are of the same general order of magnitude. Both observations indicate that distributions of storm depth and storm duration are relatively invariant with both minimum interevent time and location. Distribution similarity with minimum interevent time is graphically demonstrated. Distribution insensitivity with location also is graphically demonstrated.

Three distributions are considered for modeling the dimensionless distributions of storm depth and duration: exponential, gamma, and kappa distributions. Both the exponential and gamma distributions have precedence in analytical solutions to BMP function. In terms of accuracy, the analysis of an L-moment diagram indicates that the kappa distribution is preferable.

A dimensionless frequency curve is fit to the data using the method of L-moments by setting the mean equal to unity and L-scale equal to L-CV. The higher L-moments (L-skew, L-kurtosis, Tau5) remain unchanged. A dimensionless exponential distribution has no parameter to estimate. A dimensionless gamma distribution is fit to the mean (unity) and L-CV. A dimensionless kappa distribution is fit to the mean (unity), L-CV, L-skew, and L-kurtosis.

For rapid implementation of the results of this report, the parameters for both gamma and kappa distributions, which correspond to the L-moments of storm depth and duration (mean set to unity and L-scale set to L-CV) are tabulated.

The concept of regionalization in this report is a two-step process. First, a neighborhood smoothing of each storm statistic (mean storm interevent time, depth, and duration) for each minimum interevent time is done for each station in the study area. Second, geostatistical analysis, in the form of kriging, is done on the smoothed statistics for the stations to produce a continuously varying grid of the statistic. Kriging was done using a spherical model of the semivariogram. That semivariogram model was selected on the basis of intermediate spatial analysis as part of the kriging operation in an integrated software system.

Subsequent to regionalization of the storm statistics, grid maps of the storm statistics were evaluated through computation of selected summary and diagnostic statistics for each grid map. The evaluation documents the error of the grids to facilitate assessment of the uncertainty in regionalization of the storm statistics.

The summary and diagnostic statistics for each grid map were computed and tabulated. These statistics are weighted values based on record lengths of the rainfall stations. Summary statistics include the number of stations without missing values for each minimum interevent time, the mean of a particular storm statistic (interevent time, depth, and duration) for the study area, and the weighted standard deviation (wSD) of the particular statistic for the study area. Diagnostic statistics computed are weighted mean bias of the grid map, root-weightedmean-square error (RwMSE) of the grid map, and percentage difference between wSD and RwMSE of the grid map. The mean bias is the weighted average of the station residuals. A residual is defined as the value of the observed statistic at each station minus the statistic predicted by the grid map.

Maps depicting the magnitude and a spatial variation of mean storm interevent time, depth, and duration by county for each of the seven minimum interevent times are presented. The diagnostic statistics show that the mean biases are approximately zero. Comparison of the percentage differences between wSD and RwMSE shows that the differences are largest for mean storm depth and smallest for mean storm interevent time, which indicates that the mean storm depth maps explain relatively more variability in storm depth across the study area than the mean storm interevent time maps or mean storm duration maps. Hence, relatively less uncertainty is associated with the storm depth maps than with the maps of the other two statistics.

Mean storm interevent time, depth, and duration for each county in eastern New Mexico, Oklahoma, and Texas are tabulated. Lastly, tables of mean storm interevent time, depth, and duration are provided. Because of the large database analyzed, the regionalization of storm statistics as represented by the dimensionless kappa distribution of storm depth and duration and the tables of mean storm interevent time, depth, and duration are preferable to other methods described here for statistical characterization of storms in eastern New Mexico, Oklahoma, and Texas.

Finally, 10 example problems and solutions using sitespecific and regional approaches to possible applications of the statistics presented in this report are discussed. The example problems provide a general guide for application of this report. 


\section{References}

Adams, B.J., and Papa, F., 2000, Urban stormwater management planning with analytical probabilistic models: New York, John Wiley, 358 p.

Al-Asaadi, Rawaa, 2002, Hyetograph estimation for the State of Texas: Lubbock, Tex., Texas Tech University, M.S. thesis, $96 \mathrm{p}$.

Asquith, W.H., 1998, Depth-duration frequency of precipitation for Texas: U.S. Geological Survey Water-Resources Investigations Report 98-4044, 107 p.

Asquith, W.H., 1999, Areal-reduction factors for the precipitation of the 1-day design storm in Texas: U.S. Geological Survey Water-Resources Investigations Report 99-4267, $81 \mathrm{p}$.

Asquith, W.H., 2003, Modeling of runoff-producing rainfall hyetographs in Texas using L-moment statistics: Austin, University of Texas at Austin, Ph.D. dissertation, 386 p.

Asquith, W.H., Bumgarner, J.R., and Fahlquist, L.S., 2003, A triangular model of dimensionless runoff-producing rainfall hyetographs in Texas: Journal American Water Resources Association, v. 39, no. 4, p. 911-921.

Asquith, W.H., and Famiglietti, J.S., 2000, Rainfall arealreduction factor estimation using an annual-maxima centered approach: Journal of Hydrology, v. 230, p. 55-69.

Asquith, W.H., and Roussel, M.C., 2003, Atlas of interoccurrence intervals for selected thresholds of daily precipitation in Texas: U.S. Geological Survey Water-Resources Investigations Report 03-4281, 204 p.

Asquith, W.H., and Roussel, M.C., 2004, Atlas of depthduration frequency of precipitation in Texas: U.S. Geological Survey Scientific Investigations Report 2004-5041, 106 p.

Asquith, W.H., and Thompson, D.B., 2003, Modeling of runoff-producing rainfall hyetographs in Texas using L-moments, in Texas Section Spring Meeting, Corpus Christi, Tex., April 2-5, 2003, Proceedings: American Society of Civil Engineers.

Asquith, W.H., Roussel, M.C., Thompson, D.B., Cleveland, T.G., Fang, Xing, 2005, Summary of dimensionless Texas hyetographs and distribution of storm depth developed for Texas Department of Transportation Research Project 0-4194: Center for Transportation Research, University of Texas at Austin, Texas Department of Transportation Research Report 0-4194-4, 68 p.

Bhat, U.N., and Miller, G.K., 2002, Elements of applied stochastic processes (3rd ed.): Hoboken, N.J., John Wiley, $461 \mathrm{p}$.

Clarke, R.T., 1998, Stochastic processes for water scientistsDevelopments and applications: New York, John Wiley, $183 \mathrm{p}$.

Dingman, S.L., 2002, Physical hydrology (2d ed.): Upper Saddle, N.J., Prentice Hall, 575 p.

Environmental Systems Research Institute, Inc. (ESRI), 2002, ArcGIS 8.2: Redlands, Calif., ESRI Geographical Information System and Mapping Software.
Evans, Merran, Hastings, N.A.J., and Peacock, J. B., 2000, Statistical distributions (3rd ed.): New York, John Wiley, $221 \mathrm{p}$.

Gilchrist, W.G., 2000, Statistical modeling with quantile functions: Boca Raton, Fla., Chapman and Hall CRC Press, 320 p.

Hosking, J.R.M., 1990, L-moments: Analysis and estimation of distributions using linear combination of order statistics: Journal Royal Statistical Society, B, v. 52, no. 1, p. 105-124.

Hosking, J.R.M., 1994, The four-parameter kappa distribution: IBM Journal of Research and Development, v. 38, no. 3, p. 251-258.

Hosking, J.R.M., 1996, Fortran routines for use with the method of L-moments-Version 3: Yorktown Heights, N.Y., IBM Research Division, Research Report RC 20525.

Hosking, J.R.M., and Wallis, J.R., 1997, Regional frequency analysis-An approach based on L-moments: Cambridge, U.K., Cambridge University Press, 224 p.

Hydrosphere, 2003, NCDC hourly precipitation-West: Boulder, Colo., Hydrosphere Data Products Inc., v. 13.2, CD-ROM.

Isaaks, E.H., and Srivastava, R.M., 1989, An introduction to applied geostatistics: New York, Oxford University Press, $561 \mathrm{p}$.

Lanning-Rush, J., Asquith, W.H., and Slade, R.M., 1998, Extreme precipitation depths for Texas, excluding the TransPecos region: U.S. Geological Survey Water-Resources Investigations Report 98-4099, 38 p.

Ross, Sheldon, 1994, A first course in probability (4th ed.): New York, MacMillan College Publishing Co., 473 p.

Schueler, T.R., 1987, Controlling urban runoff-A practical manual for planning and designing urban BMPs: Washington, D.C., Metropolitan Washington Council of Governments.

Stedinger, J.R., Vogel, R.M., and Foufoula-Georgiou, Efi, 1992, Frequency analysis of extreme events, in Maidment, D.A., ed., Handbook of applied hydrology, chap. 18: New York, McGraw-Hill, p. 18.1-18.66.

Strand [now Roussel], M.C., 2003, Depth-duration frequency of annual precipitation maxima in Texas: Austin, University of Texas at Austin, M.S. thesis, 195 p.

U.S. Environmental Protection Agency, 1986, Methodology for analysis of detention basins for control of urban runoff quality: Washington, D.C., EPA 440/S-87-001.

Vogel, R.M., and Fennessey, N.M., 1993, L-moment diagrams should replace product moment diagrams: Water Resources Research, v. 29, no. 6, p. 1,745-1,752.

Wanielista, M.P., and Yousef, Y.A., 1993, Stormwater management: New York, John Wiley, 579 p.

Wilks, D.S., 1995, Statistical methods in the atmospheric sciences: San Diego, Academic Press, 467 p.

Williams-Sether, Tara, Asquith, W.H., Thompson, D.B., Cleveland, T.G., and Fang, Xing, 2004, Empirical, dimensionless, cumulative-rainfall hyetographs developed from 1959-86 storm data for selected small watersheds in Texas: U.S. Geological Survey Scientific Investigations Report 2004-5057, 125 p. 
Blank Page 
Table 1. National Weather Service hourly rainfall stations in eastern New Mexico (Hydrosphere, 2003).

[NGVD 29, National Geodetic Vertical Datum of 1929]

\begin{tabular}{|c|c|c|c|c|c|c|c|c|c|}
\hline $\begin{array}{c}\text { Station } \\
\text { no. }\end{array}$ & Station name & County & $\begin{array}{c}\text { Elevation } \\
\text { (feet above } \\
\text { NGVD 29) }\end{array}$ & $\begin{array}{c}\text { Longitude } \\
\text { (degrees/ } \\
\text { minutes) }\end{array}$ & $\begin{array}{l}\text { Latitude } \\
\text { (degrees/ } \\
\text { minutes) }\end{array}$ & $\begin{array}{c}\text { Beginning } \\
\text { month/ } \\
\text { year }\end{array}$ & $\begin{array}{c}\text { Ending } \\
\text { month/ } \\
\text { year }\end{array}$ & $\begin{array}{l}\text { Percentage } \\
\text { of record } \\
\text { with data }\end{array}$ & $\begin{array}{l}\text { Years } \\
\text { with } \\
\text { record }\end{array}$ \\
\hline 0199 & Alamogordo & Otero & 4,350 & $105^{\circ} 56^{\prime}$ & $32^{\circ} 55^{\prime}$ & $09 / 1968$ & $12 / 2002$ & 85 & 35 \\
\hline 0208 & Alamogordo Filter Plant & Otero $^{1}$ & 4,724 & $105^{\circ} 56^{\prime}$ & $32^{\circ} 58^{\prime}$ & $11 / 1958$ & $05 / 1968$ & 82 & 11 \\
\hline 0404 & Angel Fire $2 \mathrm{~S}$ & Colfax & 8,632 & $105^{\circ} 18^{\prime}$ & $36^{\circ} 22^{\prime}$ & 07/1990 & $12 / 1993$ & 76 & 4 \\
\hline 0606 & Aspen Grove Ranch & Rio Arriba & 9,708 & $106^{\circ} 11^{\prime}$ & $36^{\circ} 39^{\prime}$ & $11 / 1947$ & $01 / 1950$ & 23 & 4 \\
\hline 0646 & Aurora & Colfax $^{1}$ & 8,136 & $105^{\circ} 03^{\prime}$ & $36^{\circ} 16^{\prime}$ & $10 / 1947$ & $08 / 1960$ & 77 & 14 \\
\hline 1120 & Bonito Dam & Lincoln & 7,055 & $105^{\circ} 41^{\prime}$ & $33^{\circ} 26^{\prime}$ & $10 / 1947$ & $12 / 2002$ & 89 & 56 \\
\hline 1440 & Capitan & Lincoln & 6,480 & $105^{\circ} 35^{\prime}$ & $33^{\circ} 31^{\prime}$ & $01 / 1976$ & $12 / 2002$ & 93 & 27 \\
\hline 1446 & Caprock 4 SE & Lea & 4,272 & $103^{\circ} 38^{\prime}$ & $33^{\circ} 21^{\prime}$ & $10 / 1947$ & $12 / 1971$ & 91 & 25 \\
\hline 1469 & Carlsbad & Eddy & 3,120 & $104^{\circ} 13^{\prime}$ & $32^{\circ} 20^{\prime}$ & $10 / 1947$ & $12 / 2002$ & 92 & 56 \\
\hline 1807 & Cienega 5 SSW & Otero & 3,802 & $105^{\circ} 06^{\prime}$ & $32^{\circ} 02^{\prime}$ & $10 / 1957$ & 04/1964 & 72 & 8 \\
\hline 1840 & Circle F Ranch & Lincoln & 5,400 & $105^{\circ} 00^{\prime}$ & $33^{\circ} 54^{\prime}$ & $03 / 1980$ & $01 / 1995$ & 86 & 16 \\
\hline 1881 & Clayton 9 SSE & Union $^{1}$ & 4,905 & $103^{\circ} 06^{\prime}$ & $36^{\circ} 20^{\prime}$ & $11 / 1947$ & $11 / 1947$ & 4 & 1 \\
\hline 1887 & Clayton Municipal Airport & Union & 4,960 & $103^{\circ} 09^{\prime}$ & $36^{\circ} 26^{\prime}$ & $10 / 1947$ & $12 / 2002$ & 88 & 56 \\
\hline 1939 & Clovis & Curry & 4,290 & $103^{\circ} 12^{\prime}$ & $34^{\circ} 25^{\prime}$ & $07 / 1949$ & $12 / 2002$ & 94 & 54 \\
\hline 1950 & Clovis $8 \mathrm{NW}$ & Curry ${ }^{1}$ & 4,383 & $103^{\circ} 19^{\prime}$ & $34^{\circ} 29^{\prime}$ & $10 / 1947$ & 06/1949 & 39 & 3 \\
\hline 1956 & Clovis $3 \mathrm{~W}$ & Curry $^{1}$ & 4,272 & $103^{\circ} 14^{\prime}$ & $34^{\circ} 25^{\prime}$ & $10 / 1947$ & $06 / 1949$ & 43 & 3 \\
\hline 1961 & Clovis $6 \mathrm{SW}$ & Curry ${ }^{1}$ & 4,242 & $103^{\circ} 19^{\prime}$ & $34^{\circ} 21^{\prime}$ & $10 / 1947$ & 06/1949 & 42 & 3 \\
\hline 1963 & Clovis $13 \mathrm{~N}$ & Curry & 4,435 & $103^{\circ} 12^{\prime}$ & $34^{\circ} 35^{\prime}$ & $07 / 1949$ & $12 / 2002$ & 94 & 54 \\
\hline 1982 & Cochiti Dam & Sandoval & 5,560 & $106^{\circ} 19^{\prime}$ & $35^{\circ} 38^{\prime}$ & $04 / 1967$ & $12 / 2002$ & 92 & 36 \\
\hline 2030 & Conchas Dam & San Miguel & 4,244 & $104^{\circ} 11^{\prime}$ & $35^{\circ} 24^{\prime}$ & $10 / 1947$ & $12 / 2002$ & 94 & 56 \\
\hline 2700 & Eagle Nest & Colfax & 8,280 & $105^{\circ} 15^{\prime}$ & $36^{\circ} 33^{\prime}$ & $10 / 1947$ & $12 / 2002$ & 89 & 56 \\
\hline 3145 & Farnsworth Ranch & Lincoln & 5,400 & $105^{\circ} 00^{\prime}$ & $33^{\circ} 54^{\prime}$ & $05 / 1953$ & 05/1980 & 91 & 28 \\
\hline 3242 & Folsom & Union $^{1}$ & 6,604 & $103^{\circ} 55^{\prime}$ & $36^{\circ} 52^{\prime}$ & $10 / 1947$ & $01 / 1950$ & 17 & 4 \\
\hline 3288 & Fort Stanton & Lincoln & 6,224 & $105^{\circ} 31^{\prime}$ & $33^{\circ} 30^{\prime}$ & $05 / 1970$ & $11 / 1975$ & 58 & 6 \\
\hline 3374 & Galisteo & Santa Fe & 6,093 & $105^{\circ} 57^{\prime}$ & $35^{\circ} 24^{\prime}$ & $01 / 1958$ & $12 / 1977$ & 96 & 20 \\
\hline 4026 & Hobbs & Lea & 3,615 & $103^{\circ} 07^{\prime}$ & $32^{\circ} 43^{\prime}$ & $05 / 1948$ & $09 / 1948$ & 25 & 1 \\
\hline 4028 & Hobbs FAA Airport & Lea & 3,655 & $103^{\circ} 12^{\prime}$ & $32^{\circ} 41^{\prime}$ & $06 / 1948$ & $10 / 1958$ & 90 & 11 \\
\hline 4030 & Hobbs $13 \mathrm{~W}$ & Lea & 3,816 & $103^{\circ} 21^{\prime}$ & $32^{\circ} 42^{\prime}$ & $10 / 1996$ & $12 / 2002$ & 72 & 7 \\
\hline 4089 & Hondo $1 \mathrm{SE}$ & Lincoln & 5,270 & $105^{\circ} 15^{\prime}$ & $33^{\circ} 22^{\prime}$ & $10 / 1947$ & $12 / 2002$ & 93 & 56 \\
\hline 4112 & Hope & Eddy & 4,091 & $104^{\circ} 44^{\prime}$ & $32^{\circ} 48^{\prime}$ & $05 / 1965$ & $12 / 2002$ & 88 & 34 \\
\hline 4306 & Ione & Union $^{1}$ & 4,705 & $103^{\circ} 18^{\prime}$ & $35^{\circ} 45^{\prime}$ & $11 / 1947$ & $12 / 1961$ & 82 & 15 \\
\hline 4850 & Las Vegas $2 \mathrm{NW}$ & San Miguel & 6,604 & $105^{\circ} 16^{\prime}$ & $35^{\circ} 37^{\prime}$ & $11 / 1954$ & $06 / 1983$ & 84 & 29 \\
\hline 4856 & Las Vegas FAA Airport & San Miguel & 6,866 & $105^{\circ} 08^{\prime}$ & $35^{\circ} 39^{\prime}$ & $11 / 1947$ & $11 / 1954$ & 80 & 8 \\
\hline 4860 & Las Vegas $4 \mathrm{NW}$ & San Miguel & 6,706 & $105^{\circ} 16^{\prime}$ & $35^{\circ} 38^{\prime}$ & $05 / 1967$ & $06 / 1969$ & 68 & 3 \\
\hline 4862 & Las Vegas Sewage Plant & San Miguel & 6,349 & $105^{\circ} 12^{\prime}$ & $35^{\circ} 34^{\prime}$ & $06 / 1983$ & $12 / 2002$ & 91 & 20 \\
\hline 5370 & Maljamar & Lea & 4,122 & $103^{\circ} 45^{\prime}$ & $32^{\circ} 51^{\prime}$ & $02 / 1948$ & $12 / 2002$ & 91 & 55 \\
\hline 5651 & Mesa Service Station & Chaves $^{1}$ & 5,003 & $104^{\circ} 41^{\prime}$ & $33^{\circ} 59^{\prime}$ & $11 / 1947$ & 03/1953 & 74 & 7 \\
\hline
\end{tabular}


50 Statistical Characteristics of Storm Interevent Time, Depth, and Duration for Eastern New Mexico, Oklahoma, and Texas

Table 1. National Weather Service hourly rainfall stations in eastern New Mexico—Continued.

\begin{tabular}{|c|c|c|c|c|c|c|c|c|c|}
\hline $\begin{array}{c}\text { Station } \\
\text { no. }\end{array}$ & Station name & County & $\begin{array}{c}\text { Elevation } \\
\text { (feet above } \\
\text { NGVD 29) }\end{array}$ & $\begin{array}{c}\text { Longitude } \\
\text { (degrees/ } \\
\text { minutes) }\end{array}$ & $\begin{array}{l}\text { Latitude } \\
\text { (degrees/ } \\
\text { minutes) }\end{array}$ & $\begin{array}{c}\text { Beginning } \\
\text { month/ } \\
\text { year }\end{array}$ & $\begin{array}{l}\text { Ending } \\
\text { month/ } \\
\text { year }\end{array}$ & $\begin{array}{l}\text { Percentage } \\
\text { of record } \\
\text { with data }\end{array}$ & $\begin{array}{l}\text { Years } \\
\text { with } \\
\text { record }\end{array}$ \\
\hline 5866 & Montezuma $8 \mathrm{NW}$ & San Miguel & 7,244 & $105^{\circ} 23^{\prime}$ & $35^{\circ} 41^{\prime}$ & $08 / 1971$ & $10 / 1973$ & 55 & 3 \\
\hline 6138 & Nogal Lake & Lincoln & 7,116 & $105^{\circ} 41^{\prime}$ & $33^{\circ} 31^{\prime}$ & $11 / 1947$ & $05 / 1970$ & 80 & 24 \\
\hline 6275 & Ocate $2 \mathrm{NW}$ & Mora & 7,655 & $105^{\circ} 03^{\prime}$ & $36^{\circ} 11^{\prime}$ & $08 / 1960$ & $12 / 2002$ & 94 & 43 \\
\hline 6435 & Orogrande & Otero & 4,182 & $106^{\circ} 05^{\prime}$ & $32^{\circ} 22^{\prime}$ & $11 / 1947$ & $12 / 2002$ & 89 & 56 \\
\hline 6492 & Otto FAA Airport & Santa $\mathrm{Fe}^{1}$ & 6,234 & $106^{\circ} 01^{\prime}$ & $35^{\circ} 05^{\prime}$ & $11 / 1947$ & $07 / 1954$ & 70 & 8 \\
\hline 6619 & Pasamonte & Union & 5,650 & $103^{\circ} 44^{\prime}$ & $36^{\circ} 17^{\prime}$ & $11 / 1947$ & $03 / 1965$ & 77 & 19 \\
\hline 6659 & Pearl & Lea & 3,800 & $103^{\circ} 23^{\prime}$ & $32^{\circ} 39^{\prime}$ & $11 / 1947$ & 08/1996 & 93 & 50 \\
\hline 6797 & Philmont Ranch & Colfax $^{1}$ & 7,605 & $105^{\circ} 03^{\prime}$ & $36^{\circ} 37^{\prime}$ & $11 / 1947$ & $06 / 1957$ & 75 & 11 \\
\hline 6951 & Plaza Larga & Quay $^{1}$ & 4,072 & $103^{\circ} 36^{\prime}$ & $35^{\circ} 05^{\prime}$ & $11 / 1947$ & $12 / 1953$ & 85 & 7 \\
\hline 7094 & Progresso & Torrance & 6,297 & $105^{\circ} 53^{\prime}$ & $34^{\circ} 25^{\prime}$ & $11 / 1947$ & $12 / 2002$ & 94 & 56 \\
\hline 7254 & Ramon $8 \mathrm{SW}$ & Lincoln & 5,327 & $105^{\circ} 00^{\prime}$ & $34^{\circ} 09^{\prime}$ & $06 / 1969$ & $12 / 2002$ & 92 & 34 \\
\hline 7277 & Raton & Colfax $^{1}$ & 6,683 & $104^{\circ} 26^{\prime}$ & $36^{\circ} 54^{\prime}$ & $04 / 1948$ & $07 / 1948$ & 24 & 1 \\
\hline 7279 & Raton Filter Plant & Colfax & 6,932 & $104^{\circ} 25^{\prime}$ & $36^{\circ} 55^{\prime}$ & $09 / 1953$ & $12 / 2002$ & 91 & 39 \\
\hline 7283 & Raton Weather Bureau Airport & Colfax & 6,385 & $104^{\circ} 30^{\prime}$ & $36^{\circ} 45^{\prime}$ & $11 / 1947$ & $11 / 1968$ & 92 & 20 \\
\hline 7604 & Roswell & Chaves $^{1}$ & 3,573 & $104^{\circ} 26^{\prime}$ & $33^{\circ} 19^{\prime}$ & $11 / 1947$ & $11 / 1947$ & 4 & 1 \\
\hline 7605 & Roswell Climat & Chaves & 3,605 & $104^{\circ} 33^{\prime}$ & $33^{\circ} 24^{\prime}$ & 09/1995 & $12 / 1996$ & 45 & 2 \\
\hline 7609 & Roswell Municipal Airport & Chaves & 3,629 & $104^{\circ} 32^{\prime}$ & $33^{\circ} 24^{\prime}$ & $12 / 1947$ & $12 / 1972$ & 96 & 26 \\
\hline 7610 & Roswell Industrial Airport & Chaves & 3,649 & $104^{\circ} 30^{\prime}$ & $33^{\circ} 18^{\prime}$ & $01 / 1973$ & $12 / 2002$ & 97 & 29 \\
\hline 7638 & Roy & Harding & 5,878 & $104^{\circ} 11^{\prime}$ & $35^{\circ} 56^{\prime}$ & $11 / 1947$ & $12 / 2002$ & 92 & 56 \\
\hline 7649 & Ruidoso & Lincoln & 6,935 & $105^{\circ} 39^{\prime}$ & $33^{\circ} 21^{\prime}$ & $11 / 1947$ & $12 / 2002$ & 88 & 51 \\
\hline 7651 & Ruidoso 2 & Lincoln & 6,937 & $105^{\circ} 40^{\prime}$ & $33^{\circ} 21^{\prime}$ & 07/1987 & $06 / 1993$ & 82 & 7 \\
\hline 7735 & Sacramento & Otero & 7,316 & $105^{\circ} 34^{\prime}$ & $32^{\circ} 48^{\prime}$ & $11 / 1947$ & $11 / 1974$ & 93 & 28 \\
\hline 7736 & Sacramento no. 2 & Otero & 7,409 & $105^{\circ} 33^{\prime}$ & $32^{\circ} 47^{\prime}$ & $11 / 1974$ & $12 / 2002$ & 88 & 29 \\
\hline 7738 & Sacramento Canyon & Otero $^{1}$ & 7,405 & $105^{\circ} 43^{\prime}$ & $32^{\circ} 41^{\prime}$ & $02 / 1950$ & $03 / 1954$ & 70 & 5 \\
\hline 8072 & Santa Fe & Santa Fe & 7,205 & $105^{\circ} 54^{\prime}$ & $35^{\circ} 41^{\prime}$ & $11 / 1947$ & $03 / 1972$ & 90 & 26 \\
\hline 8078 & Santa Fe CAA Airport & Santa Fe & 6,348 & $106^{\circ} 05^{\prime}$ & $35^{\circ} 37^{\prime}$ & $11 / 1947$ & $05 / 1956$ & 83 & 10 \\
\hline 8084 & Santa Fe Caja D R & Santa $\mathrm{Fe}^{1}$ & 6,404 & $106^{\circ} 06^{\prime}$ & $35^{\circ} 43^{\prime}$ & $01 / 1948$ & $01 / 1950$ & 46 & 3 \\
\hline 8085 & Santa Fe 2 & Santa Fe & 6,756 & $105^{\circ} 58^{\prime}$ & $35^{\circ} 37^{\prime}$ & $03 / 1972$ & $12 / 2002$ & 91 & 31 \\
\hline 8187 & Sedan 7 NW & Union $^{1}$ & 4,774 & $103^{\circ} 13^{\prime}$ & $36^{\circ} 12^{\prime}$ & $11 / 1947$ & $04 / 1960$ & 72 & 14 \\
\hline 8358 & Skipworth Ranch & DeBaca & 4,183 & $104^{\circ} 29^{\prime}$ & $34^{\circ} 11^{\prime}$ & $01 / 1962$ & $01 / 1969$ & 62 & 4 \\
\hline 8501 & Springer & Colfax & 5,922 & $104^{\circ} 35^{\prime}$ & $36^{\circ} 21^{\prime}$ & $11 / 1947$ & $12 / 2002$ & 92 & 56 \\
\hline 8518 & Stanley 2 NNE & Santa Fe & 6,375 & $105^{\circ} 56^{\prime}$ & $35^{\circ} 10^{\prime}$ & $12 / 1954$ & $12 / 2002$ & 88 & 49 \\
\hline 8596 & Sumner Lake & DeBaca & 4,306 & $104^{\circ} 22^{\prime}$ & $34^{\circ} 36^{\prime}$ & $01 / 1975$ & $12 / 2002$ & 88 & 28 \\
\hline 8788 & Tererro & San Miguel $^{1}$ & 7,507 & $105^{\circ} 40^{\prime}$ & $35^{\circ} 46^{\prime}$ & $11 / 1947$ & $08 / 1961$ & 86 & 15 \\
\hline 8816 & Thomas $3 \mathrm{E}$ & Union $^{1}$ & 4,892 & $103^{\circ} 15^{\prime}$ & $36^{\circ} 15^{\prime}$ & $01 / 1955$ & $05 / 1955$ & 8 & 1 \\
\hline 9148 & Tucumcari & Quay & 4,042 & $103^{\circ} 42^{\prime}$ & $35^{\circ} 10^{\prime}$ & $01 / 1948$ & $01 / 1949$ & 50 & 2 \\
\hline 9153 & Tucumcari FAA Airport & Quay & 4,051 & $103^{\circ} 36^{\prime}$ & $35^{\circ} 11^{\prime}$ & $06 / 1948$ & $09 / 1951$ & 49 & 4 \\
\hline 9156 & Tucumcari 4 NE & Quay & 4,086 & $103^{\circ} 41^{\prime}$ & $35^{\circ} 12^{\prime}$ & $11 / 1947$ & $12 / 2002$ & 92 & 56 \\
\hline 9193 & Turquoise Bonanza Creek & Santa Fe & 6,124 & $106^{\circ} 06^{\prime}$ & $35^{\circ} 33^{\prime}$ & $02 / 1978$ & 03/1996 & 88 & 19 \\
\hline 9265 & Two Rivers Reservoir & Chaves & 4,056 & $104^{\circ} 37^{\prime}$ & $33^{\circ} 17^{\prime}$ & $07 / 1963$ & $08 / 1982$ & 89 & 20 \\
\hline 9569 & Waste Isolation Pilot Plant & Eddy & 3,418 & $103^{\circ} 47^{\prime}$ & $32^{\circ} 22^{\prime}$ & $09 / 1986$ & $12 / 2002$ & 91 & 17 \\
\hline 9686 & White Sands National Mon. & Otero & 3,995 & $106^{\circ} 10^{\prime}$ & $32^{\circ} 46^{\prime}$ & $11 / 1947$ & $12 / 2002$ & 93 & 56 \\
\hline
\end{tabular}

${ }^{1}$ County name or elevation not available in Hydrosphere (2003); supplied by authors. 
Table 251

Table 2. National Weather Service hourly rainfall stations in Oklahoma (Hydrosphere, 2003).

[NGVD 29, National Geodetic Vertical Datum of 1929]

\begin{tabular}{|c|c|c|c|c|c|c|c|c|c|}
\hline $\begin{array}{c}\text { Station } \\
\text { no. }\end{array}$ & Station name & County & $\begin{array}{c}\text { Elevation } \\
\text { (feet above } \\
\text { NGVD 29) }\end{array}$ & $\begin{array}{c}\text { Longitude } \\
\text { (degrees/ } \\
\text { minutes) }\end{array}$ & $\begin{array}{c}\text { Latitude } \\
\text { (degrees/ } \\
\text { minutes) }\end{array}$ & $\begin{array}{c}\text { Beginning } \\
\text { month/ } \\
\text { year }\end{array}$ & $\begin{array}{c}\text { Ending } \\
\text { month/ } \\
\text { year }\end{array}$ & $\begin{array}{c}\text { Percentage } \\
\text { of record } \\
\text { with data }\end{array}$ & $\begin{array}{c}\text { Years } \\
\text { with } \\
\text { record }\end{array}$ \\
\hline 0017 & Ada & Pontotoc & 1,015 & $96^{\circ} 41^{\prime}$ & $34^{\circ} 47^{\prime}$ & $04 / 1957$ & $06 / 1957$ & 22 & 1 \\
\hline 0179 & Altus Irrigation Research Station & Jackson & 1,380 & $99^{\circ} 20^{\prime}$ & $34^{\circ} 35^{\prime}$ & $01 / 1948$ & $12 / 2002$ & 83 & 35 \\
\hline 0188 & Altus $7 \mathrm{NE}$ & Jackson & 1,440 & $99^{\circ} 16^{\prime}$ & $34^{\circ} 43^{\prime}$ & $10 / 1947$ & 08/1970 & 78 & 24 \\
\hline 0215 & Ames & Major & 1,213 & $98^{\circ} 11^{\prime}$ & $36^{\circ} 14^{\prime}$ & $10 / 1947$ & $12 / 2002$ & 83 & 56 \\
\hline 0256 & Antlers & Pushmataha & 470 & $95^{\circ} 36^{\prime}$ & $34^{\circ} 13^{\prime}$ & $10 / 1947$ & $03 / 2001$ & 89 & 55 \\
\hline 0260 & Apache & Caddo & 1,280 & $98^{\circ} 21^{\prime}$ & $34^{\circ} 53^{\prime}$ & $11 / 1947$ & $06 / 1957$ & 12 & 2 \\
\hline 0292 & Ardmore & Carter & 880 & $97^{\circ} 08^{\prime}$ & $34^{\circ} 10^{\prime}$ & $04 / 1957$ & $12 / 2002$ & 71 & 21 \\
\hline 0293 & Ardmore no. 2 & Carter & 850 & $97^{\circ} 09^{\prime}$ & $34^{\circ} 09^{\prime}$ & $08 / 1960$ & 08/1994 & 86 & 26 \\
\hline 0296 & Ardmore FAA Airport & Carter & 725 & $97^{\circ} 01^{\prime}$ & $34^{\circ} 18^{\prime}$ & $10 / 1947$ & $12 / 1958$ & 90 & 12 \\
\hline 0535 & Barnsdall & Osage & 770 & $96^{\circ} 09^{\prime}$ & $36^{\circ} 33^{\prime}$ & 05/1979 & $11 / 2002$ & 80 & 19 \\
\hline 0908 & Boise City 2 E & Cimarron & 4,145 & $102^{\circ} 28^{\prime}$ & $36^{\circ} 43^{\prime}$ & $10 / 1947$ & $12 / 2002$ & 75 & 39 \\
\hline 0912 & Boise City 2 & Cimarron & 4,163 & $102^{\circ} 30^{\prime}$ & $36^{\circ} 44^{\prime}$ & $07 / 1965$ & $07 / 1983$ & 83 & 19 \\
\hline 1148 & Britton $2 \mathrm{E}$ & Oklahoma $^{1}$ & 1,171 & $97^{\circ} 30^{\prime}$ & $35^{\circ} 34^{\prime}$ & $04 / 1957$ & $06 / 1957$ & 22 & 1 \\
\hline 1168 & Broken Bow Dam & McCurtain & 443 & $94^{\circ} 42^{\prime}$ & $34^{\circ} 08^{\prime}$ & 08/1964 & 07/1997 & 88 & 34 \\
\hline 1391 & Calvin & Hughes & 800 & $96^{\circ} 15^{\prime}$ & $34^{\circ} 58^{\prime}$ & $04 / 1957$ & $06 / 1957$ & 24 & 1 \\
\hline 1436 & Caney 1 NNE & Atoka & 531 & $96^{\circ} 13^{\prime}$ & $34^{\circ} 14^{\prime}$ & $10 / 1947$ & $04 / 1978$ & 89 & 32 \\
\hline 1437 & Caney & Atoka & 560 & $96^{\circ} 12^{\prime}$ & $34^{\circ} 13^{\prime}$ & 05/1978 & $12 / 2002$ & 78 & 25 \\
\hline 1544 & Carter Tower & McCurtain & 1,300 & $94^{\circ} 46^{\prime}$ & $34^{\circ} 15^{\prime}$ & $11 / 1947$ & $12 / 2002$ & 92 & 56 \\
\hline 1684 & Chandler & Lincoln & 958 & $96^{\circ} 52^{\prime}$ & $35^{\circ} 42^{\prime}$ & $06 / 1953$ & $12 / 2002$ & 89 & 50 \\
\hline 1688 & Chandler 2 & Lincoln $^{1}$ & 860 & $96^{\circ} 52^{\prime}$ & $35^{\circ} 43^{\prime}$ & $10 / 1947$ & $06 / 1953$ & 54 & 7 \\
\hline 1750 & Chickasha Experiment Station & Grady & 1,085 & $97^{\circ} 54^{\prime}$ & $35^{\circ} 02^{\prime}$ & 03/1958 & $12 / 2002$ & 90 & 45 \\
\hline 2242 & Crescent & Logan & 1,135 & $97^{\circ} 35^{\prime}$ & $35^{\circ} 57^{\prime}$ & $04 / 1957$ & $06 / 1957$ & 24 & 1 \\
\hline 2309 & Cumberland Oil Field & Marshall $^{1}$ & 712 & $96^{\circ} 34^{\prime}$ & $34^{\circ} 05^{\prime}$ & $01 / 1948$ & $10 / 1951$ & 94 & 4 \\
\hline 2334 & Custer City $3 \mathrm{SE}$ & Custer & 1,755 & $98^{\circ} 49^{\prime}$ & $35^{\circ} 38^{\prime}$ & 08/1973 & $12 / 2002$ & 89 & 30 \\
\hline 2500 & Dibble & McClain ${ }^{1}$ & ${ }^{1} 1,170$ & $97^{\circ} 37^{\prime}$ & $35^{\circ} 02^{\prime}$ & $04 / 1957$ & $06 / 1957$ & 16 & 1 \\
\hline 2654 & Duncan Airport & Stephens & 1,105 & $97^{\circ} 57^{\prime}$ & $34^{\circ} 29^{\prime}$ & 09/1979 & $12 / 2002$ & 84 & 24 \\
\hline 2665 & Duncan $1 \mathrm{SSW}$ & Stephens & 1,132 & $97^{\circ} 58^{\prime}$ & $34^{\circ} 29^{\prime}$ & $10 / 1947$ & 08/1979 & 86 & 33 \\
\hline 2678 & Durant & Bryan & 600 & $96^{\circ} 22^{\prime}$ & $34^{\circ} 00^{\prime}$ & $04 / 1957$ & $06 / 1957$ & 16 & 1 \\
\hline 2849 & Elk City & Beckham & 1,970 & $99^{\circ} 24^{\prime}$ & $35^{\circ} 22^{\prime}$ & $10 / 1947$ & $12 / 2002$ & 89 & 52 \\
\hline 2852 & Elk City 2 & Beckham & 2,001 & $99^{\circ} 26^{\prime}$ & $35^{\circ} 25^{\prime}$ & 03/1976 & $11 / 1981$ & 90 & 6 \\
\hline 2994 & Eufaula Dam & Muskogee & 541 & $95^{\circ} 20^{\prime}$ & $35^{\circ} 18^{\prime}$ & 03/1957 & $05 / 1965$ & 87 & 9 \\
\hline 2997 & Eufaula Reservoir & Haskell & 732 & $95^{\circ} 22^{\prime}$ & $35^{\circ} 18^{\prime}$ & 05/1965 & 08/1970 & 88 & 6 \\
\hline 3002 & Eva & Texas & 3,574 & $101^{\circ} 54^{\prime}$ & $36^{\circ} 47^{\prime}$ & $10 / 1947$ & $12 / 2002$ & 89 & 56 \\
\hline 3281 & Fort Cobb & Caddo & 1,285 & $98^{\circ} 26^{\prime}$ & $35^{\circ} 06^{\prime}$ & $12 / 1952$ & $12 / 2002$ & 94 & 51 \\
\hline 3286 & Fort Gibson Dam & Cherokee & 531 & $95^{\circ} 14^{\prime}$ & $35^{\circ} 52^{\prime}$ & 05/1949 & $04 / 2001$ & 83 & 53 \\
\hline 3304 & Fort Supply 3 SE & Woodward & 2,030 & $99^{\circ} 32^{\prime}$ & $36^{\circ} 32^{\prime}$ & $10 / 1947$ & $12 / 2002$ & 90 & 56 \\
\hline 3353 & Frederick & Tillman & 1,285 & $99^{\circ} 00^{\prime}$ & $34^{\circ} 23^{\prime}$ & $04 / 1957$ & $06 / 1957$ & 21 & 1 \\
\hline 3407 & Gage Airport & Ellis & 2,191 & $99^{\circ} 46^{\prime}$ & $36^{\circ} 17^{\prime}$ & 05/1948 & $12 / 2002$ & 62 & 10 \\
\hline
\end{tabular}

Footnote at end of table. 
Table 2. National Weather Service hourly rainfall stations in Oklahoma-Continued.

\begin{tabular}{|c|c|c|c|c|c|c|c|c|c|}
\hline $\begin{array}{c}\text { Station } \\
\text { no. }\end{array}$ & Station name & County & $\begin{array}{c}\text { Elevation } \\
\text { (feet above } \\
\text { NGVD 29) }\end{array}$ & $\begin{array}{c}\text { Longitude } \\
\text { (degrees/ } \\
\text { minutes) }\end{array}$ & $\begin{array}{l}\text { Latitude } \\
\text { (degrees/ } \\
\text { minutes) }\end{array}$ & $\begin{array}{c}\text { Beginning } \\
\text { month/ } \\
\text { year }\end{array}$ & $\begin{array}{c}\text { Ending } \\
\text { month/ } \\
\text { year }\end{array}$ & $\begin{array}{c}\text { Percentage } \\
\text { of record } \\
\text { with data }\end{array}$ & $\begin{array}{l}\text { Years } \\
\text { with } \\
\text { record }\end{array}$ \\
\hline 3497 & Geary & Blaine & 1,595 & $98^{\circ} 19^{\prime}$ & $35^{\circ} 37^{\prime}$ & $10 / 1947$ & $12 / 2002$ & 94 & 56 \\
\hline 3628 & Goodwell Research Station & Texas & 3,310 & $101^{\circ} 37^{\prime}$ & $36^{\circ} 35^{\prime}$ & $10 / 1947$ & $12 / 2002$ & 89 & 56 \\
\hline 3700 & Grand River Dam & Mayes & 771 & $95^{\circ} 03^{\prime}$ & $36^{\circ} 28^{\prime}$ & $10 / 1947$ & $03 / 1980$ & 78 & 33 \\
\hline 3740 & Great Salt Plains Dam & Alfalfa & 1,200 & $98^{\circ} 07^{\prime}$ & $36^{\circ} 44^{\prime}$ & $10 / 1947$ & $12 / 2002$ & 89 & 56 \\
\hline 3830 & Guthrie SCS & $\operatorname{Logan}^{1}$ & 981 & $97^{\circ} 25^{\prime}$ & $35^{\circ} 48^{\prime}$ & $11 / 1947$ & $09 / 1957$ & 81 & 11 \\
\hline 3835 & Guymon & Texas & 3,070 & $101^{\circ} 28^{\prime}$ & $36^{\circ} 42^{\prime}$ & $05 / 1948$ & $09 / 1951$ & 63 & 4 \\
\hline 4001 & Healdton & Carter & 734 & $97^{\circ} 28^{\prime}$ & $34^{\circ} 13^{\prime}$ & $05 / 1957$ & $06 / 1957$ & 10 & 1 \\
\hline 4008 & Heavener $2 \mathrm{~N}$ & Le Flore & 592 & $94^{\circ} 35^{\prime}$ & $34^{\circ} 54^{\prime}$ & $11 / 1951$ & $06 / 1952$ & 31 & 2 \\
\hline 4010 & Heavener Experiment & Le Flore $^{1}$ & 561 & $94^{\circ} 36^{\prime}$ & $34^{\circ} 55^{\prime}$ & $10 / 1947$ & $07 / 1967$ & 89 & 21 \\
\hline 4051 & Hennepin & Garvin & 942 & $97^{\circ} 21^{\prime}$ & $34^{\circ} 31^{\prime}$ & $03 / 1948$ & $05 / 1974$ & 96 & 27 \\
\hline 4052 & Hennepin $5 \mathrm{~N}$ & Garvin & 970 & $97^{\circ} 20^{\prime}$ & $34^{\circ} 34^{\prime}$ & $05 / 1974$ & $12 / 2002$ & 90 & 29 \\
\hline 4098 & Heyburn Dam & Creek & 831 & $96^{\circ} 17^{\prime}$ & $35^{\circ} 57^{\prime}$ & $06 / 1949$ & $07 / 1997$ & 93 & 49 \\
\hline 4202 & Hobart & Kiowa & 1,547 & $99^{\circ} 05^{\prime}$ & $35^{\circ} 01^{\prime}$ & $03 / 1952$ & $12 / 2002$ & 90 & 51 \\
\hline 4204 & Hobart Municipal Airport & Kiowa & 1,556 & $99^{\circ} 03^{\prime}$ & $34^{\circ} 59^{\prime}$ & $10 / 1947$ & $11 / 1952$ & 79 & 6 \\
\hline 4384 & Hugo & Choctaw & 520 & $95^{\circ} 32^{\prime}$ & $34^{\circ} 01^{\prime}$ & $10 / 1947$ & 09/1999 & 93 & 53 \\
\hline 4386 & Hugo Dam & Choctaw & 466 & $95^{\circ} 24^{\prime}$ & $34^{\circ} 00^{\prime}$ & $01 / 1969$ & $05 / 1997$ & 88 & 29 \\
\hline 4388 & Hulah & Osage $^{1}$ & 722 & $96^{\circ} 02^{\prime}$ & $36^{\circ} 56^{\prime}$ & $10 / 1947$ & $07 / 1949$ & 54 & 3 \\
\hline 4393 & Hulah Dam & Osage & 744 & $96^{\circ} 06^{\prime}$ & $36^{\circ} 55^{\prime}$ & $12 / 1947$ & 05/1997 & 88 & 50 \\
\hline 4506 & Inola $6 \mathrm{SSW}$ & Wagoner & 545 & $95^{\circ} 33^{\prime}$ & $36^{\circ} 04^{\prime}$ & 03/1968 & $01 / 1999$ & 83 & 32 \\
\hline 4692 & Kaw Dam & Kay & 1,079 & $96^{\circ} 56^{\prime}$ & $36^{\circ} 42^{\prime}$ & $08 / 1968$ & 09/1980 & 89 & 13 \\
\hline 4812 & Keystone Dam & Tulsa & 705 & $96^{\circ} 15^{\prime}$ & $36^{\circ} 09^{\prime}$ & $08 / 1957$ & 06/1997 & 88 & 41 \\
\hline 4865 & Kingston 4 SSE & Marshall & 712 & $96^{\circ} 41^{\prime}$ & $33^{\circ} 56^{\prime}$ & $10 / 1947$ & $12 / 2002$ & 93 & 56 \\
\hline 4969 & Lake C Blackwell & Payne $^{1}$ & 951 & $97^{\circ} 11^{\prime}$ & $36^{\circ} 09^{\prime}$ & $10 / 1947$ & $12 / 1952$ & 74 & 6 \\
\hline 4975 & Lake Eufaula & Haskell & 850 & $95^{\circ} 25^{\prime}$ & $35^{\circ} 17^{\prime}$ & 09/1970 & $12 / 2002$ & 86 & 33 \\
\hline 4978 & Lake Overholser & Oklahoma & 1,270 & $97^{\circ} 39^{\prime}$ & $35^{\circ} 29^{\prime}$ & $01 / 1952$ & $12 / 2002$ & 90 & 51 \\
\hline 5063 & Lawton & Comanche & 1,150 & $98^{\circ} 27^{\prime}$ & $34^{\circ} 36^{\prime}$ & $10 / 1947$ & $12 / 1947$ & 25 & 1 \\
\hline 5068 & Lawton $2 \mathrm{~N}$ & Comanche $^{1}$ & 1,122 & $98^{\circ} 24^{\prime}$ & $34^{\circ} 39^{\prime}$ & $01 / 1948$ & $10 / 1949$ & 88 & 2 \\
\hline 5108 & Lehigh 4 SW & Coal & 695 & $96^{\circ} 16^{\prime}$ & $34^{\circ} 26^{\prime}$ & $10 / 1947$ & $04 / 2002$ & 90 & 56 \\
\hline 5329 & Lookeba $1 \mathrm{~N}$ & Caddo & 1,442 & $98^{\circ} 22^{\prime}$ & $35^{\circ} 22^{\prime}$ & $04 / 1957$ & $06 / 1957$ & 19 & 1 \\
\hline 5463 & Mackie 4 NNW & Roger Mills & 2,150 & $99^{\circ} 49^{\prime}$ & $35^{\circ} 44^{\prime}$ & $01 / 1970$ & $12 / 2002$ & 83 & 33 \\
\hline 5581 & Marlow 1 WSW & Stephens & 1,250 & $97^{\circ} 58^{\prime}$ & $34^{\circ} 38^{\prime}$ & $04 / 1957$ & $06 / 1957$ & 23 & 1 \\
\hline 5582 & Marlow 8 WSW & Stephens ${ }^{1}$ & 1,142 & $98^{\circ} 05^{\prime}$ & $34^{\circ} 37^{\prime}$ & $03 / 1958$ & $06 / 1964$ & 82 & 7 \\
\hline 5589 & Marshall & Logan & 1,045 & $97^{\circ} 37^{\prime}$ & $36^{\circ} 09^{\prime}$ & $10 / 1975$ & $12 / 2002$ & 91 & 28 \\
\hline 5648 & Mayfield & Beckham & 2,005 & $99^{\circ} 52^{\prime}$ & $35^{\circ} 20^{\prime}$ & $10 / 1947$ & $12 / 2002$ & 92 & 56 \\
\hline 5662 & McAlester $4 \mathrm{~W}$ & Pittsburg & 670 & $95^{\circ} 50^{\prime}$ & $34^{\circ} 57^{\prime}$ & $10 / 1947$ & $08 / 1957$ & 88 & 11 \\
\hline 5664 & McAlester Regional Airport & Pittsburg & 770 & $95^{\circ} 46^{\prime}$ & $34^{\circ} 52^{\prime}$ & $05 / 1980$ & $12 / 2002$ & 74 & 23 \\
\hline 6130 & Muskogee & Muskogee & 518 & $95^{\circ} 20^{\prime}$ & $35^{\circ} 46^{\prime}$ & $10 / 1947$ & $12 / 2002$ & 83 & 56 \\
\hline 6328 & Ninnekah & Grady ${ }^{1}$ & 1,161 & $97^{\circ} 56^{\prime}$ & $34^{\circ} 57^{\prime}$ & $10 / 1947$ & $12 / 1966$ & 76 & 20 \\
\hline 6391 & Norman University of Oklahoma & Cleveland $^{1}$ & 1,171 & $97^{\circ} 26^{\prime}$ & $35^{\circ} 13^{\prime}$ & $05 / 1951$ & $10 / 1951$ & 42 & 1 \\
\hline 6485 & Nowata & Nowata & 710 & $95^{\circ} 38^{\prime}$ & $36^{\circ} 41^{\prime}$ & $07 / 1949$ & $12 / 2002$ & 88 & 53 \\
\hline 6612 & Oilton & Creek & 880 & $96^{\circ} 35^{\prime}$ & $36^{\circ} 05^{\prime}$ & $07 / 1995$ & $11 / 1997$ & 8 & 3 \\
\hline 6616 & Oilton 2 SE & Creek & 880 & $96^{\circ} 34^{\prime}$ & $36^{\circ} 04^{\prime}$ & $11 / 1947$ & $12 / 2002$ & 78 & 56 \\
\hline 6620 & Okarche & Kingfisher & 1,245 & $97^{\circ} 58^{\prime}$ & $35^{\circ} 43^{\prime}$ & $06 / 1981$ & $12 / 2002$ & 89 & 22 \\
\hline 6627 & Okay 3 W Lock 17 & Wagoner & 520 & $95^{\circ} 22^{\prime}$ & $35^{\circ} 51^{\prime}$ & $06 / 1972$ & $01 / 1999$ & 76 & 28 \\
\hline 6638 & Okemah & Okfuskee & 935 & $96^{\circ} 18^{\prime}$ & $35^{\circ} 25^{\prime}$ & $10 / 1947$ & $12 / 2002$ & 86 & 54 \\
\hline 6643 & Okemah 2 & Okfuskee $^{1}$ & 801 & $96^{\circ} 18^{\prime}$ & $35^{\circ} 27^{\prime}$ & $11 / 1947$ & $10 / 1950$ & 67 & 4 \\
\hline 6656 & Oklahoma City Weather Bureau & Oklahoma & 1,263 & $97^{\circ} 32^{\prime}$ & $35^{\circ} 29^{\prime}$ & $10 / 1947$ & $03 / 1954$ & 80 & 8 \\
\hline 6661 & Oklahoma City Airport & Oklahoma & 1,304 & $97^{\circ} 36^{\prime}$ & $35^{\circ} 23^{\prime}$ & $10 / 1947$ & $12 / 2002$ & 97 & 56 \\
\hline 6729 & Oologah Dam & Rogers & 683 & $95^{\circ} 41^{\prime}$ & $36^{\circ} 26^{\prime}$ & $08 / 1956$ & $01 / 1999$ & 87 & 44 \\
\hline 6740 & Optima Lake & Texas & 2,834 & $101^{\circ} 08^{\prime}$ & $36^{\circ} 39^{\prime}$ & $10 / 1973$ & $12 / 1994$ & 90 & 22 \\
\hline 6760 & Orlando $1 \mathrm{NNE}$ & Noble & 1,089 & $97^{\circ} 22^{\prime}$ & $36^{\circ} 10^{\prime}$ & $10 / 1947$ & $10 / 1975$ & 84 & 29 \\
\hline 6859 & Paoli 2 W & Garvin & 931 & $97^{\circ} 17^{\prime}$ & $34^{\circ} 49^{\prime}$ & $10 / 1947$ & $12 / 2002$ & 93 & 56 \\
\hline
\end{tabular}


Table 253

Table 2. National Weather Service hourly rainfall stations in Oklahoma-Continued.

\begin{tabular}{|c|c|c|c|c|c|c|c|c|c|}
\hline $\begin{array}{c}\text { Station } \\
\text { no. }\end{array}$ & Station name & County & $\begin{array}{c}\text { Elevation } \\
\text { (feet above } \\
\text { NGVD 29) }\end{array}$ & $\begin{array}{c}\text { Longitude } \\
\text { (degrees/ } \\
\text { minutes) }\end{array}$ & $\begin{array}{l}\text { Latitude } \\
\text { (degrees/ } \\
\text { minutes) }\end{array}$ & $\begin{array}{c}\text { Beginning } \\
\text { month/ } \\
\text { year }\end{array}$ & $\begin{array}{l}\text { Ending } \\
\text { month/ } \\
\text { year }\end{array}$ & $\begin{array}{c}\text { Percentage } \\
\text { of record } \\
\text { with data }\end{array}$ & $\begin{array}{l}\text { Years } \\
\text { with } \\
\text { record }\end{array}$ \\
\hline 6926 & Pauls Valley 4 WSW & Garvin & 940 & $97^{\circ} 16^{\prime}$ & $34^{\circ} 43^{\prime}$ & $04 / 1957$ & $06 / 1957$ & 15 & 1 \\
\hline 6935 & Pawhuska & Osage & 835 & $96^{\circ} 20^{\prime}$ & $36^{\circ} 40^{\prime}$ & $02 / 1950$ & $12 / 2002$ & 94 & 53 \\
\hline 6940 & Pawnee & Pawnee & 835 & $96^{\circ} 48^{\prime}$ & $36^{\circ} 21^{\prime}$ & $12 / 1949$ & $12 / 1949$ & 0 & 1 \\
\hline 6944 & Pawnee $5 \mathrm{~N}$ & Pawnee & 1,000 & $96^{\circ} 48^{\prime}$ & $36^{\circ} 24^{\prime}$ & $10 / 1947$ & $12 / 2002$ & 92 & 56 \\
\hline 7080 & Pine Creek Dam & McCurtain & 490 & $95^{\circ} 05^{\prime}$ & $34^{\circ} 07^{\prime}$ & $11 / 1965$ & 06/1997 & 84 & 33 \\
\hline 7196 & Ponca City & Kay & 1,005 & $97^{\circ} 05^{\prime}$ & $36^{\circ} 43^{\prime}$ & $03 / 1952$ & $12 / 2002$ & 86 & 51 \\
\hline 7201 & Ponca City Municipal Airport & Kay & 1,000 & $97^{\circ} 06^{\prime}$ & $36^{\circ} 44^{\prime}$ & $10 / 1947$ & $02 / 1952$ & 70 & 6 \\
\hline 7309 & Pryor & Mayes & 625 & $95^{\circ} 19^{\prime}$ & $36^{\circ} 18^{\prime}$ & $02 / 1973$ & $12 / 2002$ & 79 & 30 \\
\hline 7358 & Quapaw & Ottawa & 850 & $94^{\circ} 47^{\prime}$ & $36^{\circ} 58^{\prime}$ & $12 / 1947$ & $03 / 1965$ & 82 & 19 \\
\hline 7372 & Quinton & Pittsburg & 654 & $95^{\circ} 22^{\prime}$ & $35^{\circ} 08^{\prime}$ & $04 / 1957$ & $06 / 1957$ & 24 & 1 \\
\hline 7412 & Range & Texas & 2,710 & $101^{\circ} 05^{\prime}$ & $36^{\circ} 32^{\prime}$ & $10 / 1947$ & $12 / 2002$ & 88 & 56 \\
\hline 7556 & Renfrow & Grant & 1,214 & $97^{\circ} 39^{\prime}$ & $36^{\circ} 55^{\prime}$ & 08/1992 & $12 / 2002$ & 72 & 11 \\
\hline 7588 & Reydon 7 NNE & Roger Mills ${ }^{1}$ & 2,172 & $99^{\circ} 52^{\prime}$ & $35^{\circ} 45^{\prime}$ & $10 / 1947$ & $10 / 1965$ & 82 & 19 \\
\hline 7660 & Riverside $4 \mathrm{~W}$ & Beaver & 2,450 & $100^{\circ} 25^{\prime}$ & $36^{\circ} 47^{\prime}$ & $10 / 1947$ & $12 / 2002$ & 88 & 56 \\
\hline 7675 & Robert S. Kerr Dam & Sequoyah & 493 & $94^{\circ} 46^{\prime}$ & $35^{\circ} 20^{\prime}$ & $06 / 1966$ & $10 / 2001$ & 73 & 36 \\
\hline 7705 & Roff 2 WNW & Pontotoc & 1,255 & $96^{\circ} 52^{\prime}$ & $34^{\circ} 38^{\prime}$ & $10 / 1947$ & $12 / 2002$ & 87 & 56 \\
\hline 7714 & Roll & Roger Mills & 2,303 & $99^{\circ} 43^{\prime}$ & $35^{\circ} 47^{\prime}$ & $11 / 1947$ & $01 / 1970$ & 73 & 24 \\
\hline 7732 & Rose & Mayes & 1,001 & $95^{\circ} 02^{\prime}$ & $36^{\circ} 13^{\prime}$ & $02 / 1951$ & $01 / 1974$ & 84 & 24 \\
\hline 7739 & Rose Tower & Mayes & 1,250 & $95^{\circ} 01^{\prime}$ & $36^{\circ} 10^{\prime}$ & $01 / 1974$ & $12 / 2002$ & 83 & 29 \\
\hline 8029 & Seiling $3 \mathrm{~N}$ & Major & 1,675 & $98^{\circ} 55^{\prime}$ & $36^{\circ} 11^{\prime}$ & $10 / 1947$ & $07 / 1970$ & 77 & 24 \\
\hline 8092 & Shattuck & Ellis $^{1}$ & 2,241 & $99^{\circ} 53^{\prime}$ & $36^{\circ} 16^{\prime}$ & $11 / 1947$ & $12 / 1947$ & 16 & 1 \\
\hline 8101 & Shattuck $1 \mathrm{NW}$ & Ellis & 2,195 & $99^{\circ} 53^{\prime}$ & $36^{\circ} 17^{\prime}$ & $10 / 1947$ & $12 / 2002$ & 84 & 56 \\
\hline 8290 & Snomac & Seminole & 679 & $96^{\circ} 40^{\prime}$ & $35^{\circ} 05^{\prime}$ & $10 / 1947$ & $10 / 1980$ & 90 & 34 \\
\hline 8420 & Spiro 7 NE Lock and Dam & Le Flore & 420 & $94^{\circ} 33^{\prime}$ & $35^{\circ} 19^{\prime}$ & $07 / 1972$ & 03/1989 & 65 & 18 \\
\hline 8470 & Steeley & Deleware $^{1}$ & 1,001 & $94^{\circ} 53^{\prime}$ & $36^{\circ} 18^{\prime}$ & $10 / 1947$ & $01 / 1951$ & 19 & 4 \\
\hline 8497 & Stigler $1 \mathrm{SE}$ & Haskell & 570 & $95^{\circ} 06^{\prime}$ & $35^{\circ} 14^{\prime}$ & $10 / 1947$ & $12 / 2002$ & 90 & 56 \\
\hline 8501 & Stillwater $2 \mathrm{~W}$ & Payne & 895 & $97^{\circ} 05^{\prime}$ & $36^{\circ} 07^{\prime}$ & $03 / 1948$ & $12 / 2002$ & 93 & 52 \\
\hline 8504 & Stillwater $4 \mathrm{~N}$ & Payne & 930 & $97^{\circ} 04^{\prime}$ & $36^{\circ} 10^{\prime}$ & $07 / 1980$ & $11 / 1986$ & 67 & 7 \\
\hline 8708 & Taloga & Dewey & 1,705 & $98^{\circ} 57^{\prime}$ & $36^{\circ} 02^{\prime}$ & $04 / 1957$ & $12 / 2002$ & 86 & 34 \\
\hline 8769 & Tenkiller Ferry Dam & Sequoyah & 770 & $95^{\circ} 03^{\prime}$ & $35^{\circ} 36^{\prime}$ & $04 / 1949$ & $01 / 1999$ & 82 & 51 \\
\hline 8879 & Tipton $4 \mathrm{~S}$ & Tillman & 1,362 & $99^{\circ} 08^{\prime}$ & $34^{\circ} 26^{\prime}$ & $01 / 1948$ & $08 / 1955$ & 84 & 8 \\
\hline 8884 & Tishomingo Natl. Wildlife Refuge & Johnston & 642 & $96^{\circ} 38^{\prime}$ & $34^{\circ} 11^{\prime}$ & $02 / 1948$ & $02 / 1948$ & 8 & 1 \\
\hline 8992 & Tulsa International Airport & Tulsa & 650 & $95^{\circ} 53^{\prime}$ & $36^{\circ} 11^{\prime}$ & $01 / 1948$ & $12 / 2002$ & 99 & 55 \\
\hline 9014 & Turpin & Beaver $^{1}$ & 2,743 & $100^{\circ} 52^{\prime}$ & $36^{\circ} 52^{\prime}$ & $01 / 1948$ & $10 / 1949$ & 59 & 2 \\
\hline 9023 & Tuskahoma & Pushmataha & 600 & $95^{\circ} 16^{\prime}$ & $34^{\circ} 36^{\prime}$ & $01 / 1948$ & $12 / 2002$ & 92 & 55 \\
\hline 9247 & Wagoner & Wagoner & 590 & $95^{\circ} 22^{\prime}$ & $35^{\circ} 58^{\prime}$ & $04 / 1957$ & $06 / 1957$ & 23 & 1 \\
\hline 9278 & Walters & Cotton & 1,005 & $98^{\circ} 18^{\prime}$ & $34^{\circ} 21^{\prime}$ & $04 / 1957$ & $06 / 1957$ & 21 & 1 \\
\hline 9300 & Wann 2 & Nowata $^{1}$ & 869 & $95^{\circ} 48^{\prime}$ & $36^{\circ} 55^{\prime}$ & $04 / 1957$ & $06 / 1957$ & 25 & 1 \\
\hline 9364 & Watonga & Blaine & 1,550 & $98^{\circ} 24^{\prime}$ & $35^{\circ} 51^{\prime}$ & $04 / 1957$ & $06 / 1957$ & 24 & 1 \\
\hline 9404 & Waynoka & Woods & 1,508 & $98^{\circ} 52^{\prime}$ & $36^{\circ} 34^{\prime}$ & $01 / 1948$ & $12 / 2002$ & 89 & 55 \\
\hline 9450 & Webbers Falls Dam & Muskogee & 520 & $95^{\circ} 10^{\prime}$ & $35^{\circ} 35^{\prime}$ & $06 / 1966$ & $10 / 2001$ & 79 & 36 \\
\hline 9503 & West Branch & Pawnee & 902 & $96^{\circ} 39^{\prime}$ & $36^{\circ} 15^{\prime}$ & $01 / 1948$ & $06 / 1957$ & 94 & 10 \\
\hline 9629 & Wichita Mnts. Natl. Wildlife Ref. & Comanche & 1,665 & $98^{\circ} 42^{\prime}$ & $34^{\circ} 43^{\prime}$ & $01 / 1948$ & $12 / 2002$ & 90 & 55 \\
\hline 9719 & Wister $3 \mathrm{NE}$ & Le Flore & 499 & $94^{\circ} 41^{\prime}$ & $35^{\circ} 00^{\prime}$ & $07 / 1967$ & 03/1989 & 74 & 23 \\
\hline 9724 & Wister $3 \mathrm{~S}$ & Le Flore & 525 & $94^{\circ} 42^{\prime}$ & $34^{\circ} 56^{\prime}$ & $08 / 1989$ & $12 / 2002$ & 83 & 14 \\
\hline 9748 & Wolf $4 \mathrm{~N}$ & Seminole & 900 & $96^{\circ} 40^{\prime}$ & $35^{\circ} 08^{\prime}$ & $08 / 1980$ & $12 / 2002$ & 87 & 23 \\
\hline 9762 & Woodward Field Station & Woodward & 1,991 & $99^{\circ} 24^{\prime}$ & $36^{\circ} 25^{\prime}$ & $01 / 1949$ & 06/1979 & 96 & 31 \\
\hline
\end{tabular}

${ }^{1}$ County name or elevation not available in Hydrosphere (2003); supplied by authors. 
Table 3. National Weather Service hourly rainfall stations in Texas (Hydrosphere, 2003).

[NGVD 29, National Geodetic Vertical Datum of 1929]

\begin{tabular}{|c|c|c|c|c|c|c|c|c|c|}
\hline $\begin{array}{c}\text { Station } \\
\text { no. }\end{array}$ & Station name & County & $\begin{array}{c}\text { Elevation } \\
\text { (feet above } \\
\text { NGVD 29) }\end{array}$ & $\begin{array}{c}\text { Longitude } \\
\text { (degrees/ } \\
\text { minutes) }\end{array}$ & $\begin{array}{c}\text { Latitude } \\
\text { (degrees/ } \\
\text { minutes) }\end{array}$ & $\begin{array}{c}\text { Beginning } \\
\text { month/ } \\
\text { year }\end{array}$ & $\begin{array}{c}\text { Ending } \\
\text { month/ } \\
\text { year }\end{array}$ & $\begin{array}{l}\text { Percentage } \\
\text { of record } \\
\text { with data }\end{array}$ & $\begin{array}{l}\text { Years } \\
\text { with } \\
\text { record }\end{array}$ \\
\hline 0015 & Abilene 3 & Taylor & 1,780 & $99^{\circ} 42^{\prime}$ & $32^{\circ} 26^{\prime}$ & $02 / 1948$ & $02 / 1948$ & 7 & 1 \\
\hline 0050 & Adamsville & Lampasas & 1,030 & $98^{\circ} 09^{\prime}$ & $31^{\circ} 17^{\prime}$ & 05/1963 & $10 / 1985$ & 90 & 23 \\
\hline 0054 & Addicks & Harris & 102 & $95^{\circ} 39^{\prime}$ & $29^{\circ} 46^{\prime}$ & $10 / 1947$ & 05/1948 & 32 & 2 \\
\hline 0120 & Albany & Shackelford & 1,400 & $99^{\circ} 18^{\prime}$ & $32^{\circ} 43^{\prime}$ & $04 / 1957$ & $06 / 1957$ & 19 & 1 \\
\hline 0174 & Alpine & Brewster & 4,438 & $103^{\circ} 39^{\prime}$ & $30^{\circ} 22^{\prime}$ & 08/1971 & $12 / 2002$ & 96 & 32 \\
\hline 0178 & Alpine $11 \mathrm{NW}$ & Jeff Davis & 4,544 & $103^{\circ} 46^{\prime}$ & $30^{\circ} 28^{\prime}$ & 07/1971 & 09/1971 & 23 & 1 \\
\hline 0179 & Alpine $10 \mathrm{SW}$ & Brewster & 5,033 & $103^{\circ} 47^{\prime}$ & $30^{\circ} 16^{\prime}$ & $10 / 1971$ & $04 / 1979$ & 70 & 9 \\
\hline 0202 & Alvarado 2 NNW & Johnson & 835 & $97^{\circ} 13^{\prime}$ & $32^{\circ} 26^{\prime}$ & 07/1977 & $02 / 1987$ & 64 & 8 \\
\hline 0206 & Alvord $3 \mathrm{NE}$ & Wise & 1,010 & $97^{\circ} 39^{\prime}$ & $33^{\circ} 23^{\prime}$ & $04 / 1942$ & $12 / 2002$ & 83 & 61 \\
\hline 0262 & Anna & Collin & 680 & $96^{\circ} 31^{\prime}$ & $33^{\circ} 21^{\prime}$ & 08/1946 & $10 / 1995$ & 88 & 50 \\
\hline 0271 & Antelope & Jack & 1,040 & $98^{\circ} 22^{\prime}$ & $33^{\circ} 26^{\prime}$ & $04 / 1957$ & $06 / 1957$ & 20 & 1 \\
\hline 0380 & Asherton & Dimmit $^{1}$ & 551 & $99^{\circ} 45^{\prime}$ & $28^{\circ} 26^{\prime}$ & $04 / 1941$ & $12 / 1959$ & 71 & 19 \\
\hline 0394 & Aspermont & Stonewall & 1,670 & $100^{\circ} 14^{\prime}$ & $33^{\circ} 09^{\prime}$ & $04 / 1957$ & $06 / 1957$ & 16 & 1 \\
\hline 0408 & Atlanta & Cass & 315 & $94^{\circ} 09^{\prime}$ & $33^{\circ} 07^{\prime}$ & $04 / 1957$ & $06 / 1957$ & 22 & 1 \\
\hline 0427 & Austin Water Treatment Plant & Travis & 500 & $97^{\circ} 39^{\prime}$ & $30^{\circ} 16^{\prime}$ & 09/1996 & $12 / 1997$ & 39 & 2 \\
\hline 0428 & Austin Camp Mabry & Travis & 658 & $97^{\circ} 45^{\prime}$ & $30^{\circ} 19^{\prime}$ & $08 / 1942$ & $12 / 2002$ & 97 & 61 \\
\hline 0429 & Austin-Bergstrom International & Travis & 480 & $97^{\circ} 40^{\prime}$ & $30^{\circ} 10^{\prime}$ & $01 / 1940$ & $07 / 1942$ & 85 & 3 \\
\hline 0463 & Bade Ranch & Sterling ${ }^{1}$ & $1_{2,491}$ & $101^{\circ} 10^{\prime}$ & $31^{\circ} 50^{\prime}$ & 05/1943 & $04 / 1949$ & 55 & 7 \\
\hline 0493 & Ballinger $2 \mathrm{NW}$ & Runnels & 1,755 & $99^{\circ} 58^{\prime}$ & $31^{\circ} 44^{\prime}$ & $04 / 1957$ & $06 / 1957$ & 18 & 1 \\
\hline 0495 & Balmorhea & Reeves $^{1}$ & 3,192 & $103^{\circ} 44^{\prime}$ & $30^{\circ} 59^{\prime}$ & $07 / 1942$ & 03/1960 & 69 & 17 \\
\hline 0576 & Bayview & Cameron $^{1}$ & 20 & $97^{\circ} 24^{\prime}$ & $26^{\circ} 07^{\prime}$ & $10 / 1947$ & $05 / 1950$ & 65 & 4 \\
\hline 0580 & Baylor Ranch & La Salle $^{1}$ & 400 & $98^{\circ} 59^{\prime}$ & $28^{\circ} 18^{\prime}$ & $06 / 1940$ & $04 / 1953$ & 72 & 14 \\
\hline 0587 & Baytown 2 & Harris & 30 & $95^{\circ} 01^{\prime}$ & $29^{\circ} 45^{\prime}$ & $11 / 1947$ & $08 / 1969$ & 87 & 15 \\
\hline 0605 & Beasley & Fort Bend $^{1}$ & 102 & $95^{\circ} 52^{\prime}$ & $29^{\circ} 30^{\prime}$ & $10 / 1947$ & $11 / 1950$ & 53 & 4 \\
\hline 0639 & Beeville $5 \mathrm{NE}$ & Bee & 255 & $97^{\circ} 42^{\prime}$ & $28^{\circ} 27^{\prime}$ & $08 / 1953$ & $12 / 2002$ & 90 & 50 \\
\hline 0655 & Bellville $6 \mathrm{NNE}$ & Austin & 280 & $96^{\circ} 13^{\prime}$ & $30^{\circ} 01^{\prime}$ & 09/1986 & 09/1986 & 0 & 1 \\
\hline 0665 & Belton Dam & Bell & 664 & $97^{\circ} 29^{\prime}$ & $31^{\circ} 06^{\prime}$ & 09/1951 & $12 / 1992$ & 92 & 42 \\
\hline 0689 & Benavides & Duval & 381 & $98^{\circ} 25^{\prime}$ & $27^{\circ} 36^{\prime}$ & $03 / 1940$ & $12 / 1984$ & 91 & 44 \\
\hline 0690 & Benavides 2 & Duval & 380 & $98^{\circ} 24^{\prime}$ & $27^{\circ} 35^{\prime}$ & 07/1982 & $12 / 2002$ & 77 & 21 \\
\hline 0691 & Benbrook Dam & Tarrant & 790 & $97^{\circ} 26^{\prime}$ & $32^{\circ} 38^{\prime}$ & $06 / 1949$ & $12 / 2002$ & 92 & 54 \\
\hline 0708 & Benjamin $15 \mathrm{~W}$ & King & 1,650 & $100^{\circ} 02^{\prime}$ & $33^{\circ} 35^{\prime}$ & $02 / 1989$ & $10 / 1992$ & 56 & 4 \\
\hline 0738 & Bertram 3 ENE & Burnet & 1,139 & $98^{\circ} 00^{\prime}$ & $30^{\circ} 45^{\prime}$ & $01 / 1968$ & $12 / 2002$ & 95 & 35 \\
\hline 0776 & Big Lake LCRA 140 & Reagan & 2,690 & $101^{\circ} 28^{\prime}$ & $31^{\circ} 12^{\prime}$ & $05 / 1940$ & $07 / 1990$ & 90 & 51 \\
\hline 0779 & Big Lake 2 & Reagan & 2,703 & $101^{\circ} 28^{\prime}$ & $31^{\circ} 11^{\prime}$ & $08 / 1990$ & $12 / 2002$ & 90 & 13 \\
\hline 0784 & Big Spring Field Station & Howard & 2,509 & $101^{\circ} 29^{\prime}$ & $32^{\circ} 16^{\prime}$ & $12 / 1953$ & $12 / 2002$ & 93 & 50 \\
\hline 0786 & Big Spring & Howard & 2,510 & $101^{\circ} 27^{\prime}$ & $32^{\circ} 14^{\prime}$ & $05 / 1940$ & $10 / 1953$ & 90 & 14 \\
\hline 0917 & Bon Wier & Newton & 89 & $93^{\circ} 39^{\prime}$ & $30^{\circ} 44^{\prime}$ & $05 / 1940$ & 09/1974 & 89 & 35 \\
\hline 0923 & Bonham 3 NNE & Fannin & 600 & $96^{\circ} 10^{\prime}$ & $33^{\circ} 38^{\prime}$ & $04 / 1957$ & $06 / 1957$ & 22 & 1 \\
\hline
\end{tabular}


Table 355

Table 3. National Weather Service hourly rainfall stations in Texas-Continued.

\begin{tabular}{|c|c|c|c|c|c|c|c|c|c|}
\hline $\begin{array}{c}\text { Station } \\
\text { no. }\end{array}$ & Station name & County & $\begin{array}{c}\text { Elevation } \\
\text { (feet above } \\
\text { NGVD 29) }\end{array}$ & $\begin{array}{c}\text { Longitude } \\
\text { (degrees/ } \\
\text { minutes) }\end{array}$ & $\begin{array}{l}\text { Latitude } \\
\text { (degrees/ } \\
\text { minutes) }\end{array}$ & $\begin{array}{c}\text { Beginning } \\
\text { month/ } \\
\text { year }\end{array}$ & $\begin{array}{c}\text { Ending } \\
\text { month/ } \\
\text { year }\end{array}$ & $\begin{array}{l}\text { Percentage } \\
\text { of record } \\
\text { with data }\end{array}$ & $\begin{array}{l}\text { Years } \\
\text { with } \\
\text { record }\end{array}$ \\
\hline 0926 & Bonita 4 NW & Montague & 985 & $97^{\circ} 39^{\prime}$ & $33^{\circ} 50^{\prime}$ & $02 / 1940$ & $12 / 2002$ & 93 & 63 \\
\hline 0996 & Boyd & Wise & 730 & $97^{\circ} 33^{\prime}$ & $33^{\circ} 04^{\prime}$ & $04 / 1957$ & $06 / 1957$ & 21 & 1 \\
\hline 1013 & Brackettville $22 \mathrm{~N}$ & Kinney & 1,675 & $100^{\circ} 28^{\prime}$ & $29^{\circ} 36^{\prime}$ & $10 / 1995$ & $12 / 2002$ & 67 & 8 \\
\hline 1017 & Brady & McCulloch & 1,720 & $99^{\circ} 20^{\prime}$ & $31^{\circ} 07^{\prime}$ & $05 / 1940$ & $12 / 2002$ & 93 & 63 \\
\hline 1053 & Brewers Store $5 \mathrm{SW}$ & Kimble $^{1}$ & 1,762 & $99^{\circ} 33^{\prime}$ & $30^{\circ} 41^{\prime}$ & $05 / 1940$ & $11 / 1956$ & 92 & 17 \\
\hline 1057 & Brice $2 \mathrm{~S}$ & Hall & 2,228 & $100^{\circ} 54^{\prime}$ & $34^{\circ} 41^{\prime}$ & $04 / 1941$ & 09/1982 & 93 & 42 \\
\hline 1063 & Bridgeport & Wise & 745 & $97^{\circ} 46^{\prime}$ & $33^{\circ} 12^{\prime}$ & $04 / 1957$ & 06/1957 & 22 & 1 \\
\hline 1068 & Briggs & Burnet & 1,090 & $97^{\circ} 56^{\prime}$ & $30^{\circ} 53^{\prime}$ & $02 / 1940$ & 07/1998 & 91 & 59 \\
\hline 1080 & Brite Ranch & Presidio $^{1}$ & 4,623 & $104^{\circ} 32^{\prime}$ & $30^{\circ} 20^{\prime}$ & 02/1942 & $01 / 1951$ & 58 & 8 \\
\hline 1139 & Brownwood near SCS no. 3 & Brown $^{1}$ & 1,342 & $98^{\circ} 59^{\prime}$ & $31^{\circ} 42^{\prime}$ & $01 / 1940$ & 06/1941 & 74 & 2 \\
\hline 1154 & Bryan CAA Airport & Brazos $^{1}$ & 266 & $96^{\circ} 28^{\prime}$ & $30^{\circ} 38^{\prime}$ & $07 / 1948$ & $07 / 1951$ & 59 & 4 \\
\hline 1165 & Buchanan Dam & Llano $^{1}$ & 1,020 & $98^{\circ} 25^{\prime}$ & $30^{\circ} 45^{\prime}$ & 05/1946 & 03/1964 & 77 & 18 \\
\hline 1185 & Buenavista 2 NNW & $\operatorname{Pecos}^{1}$ & 2,513 & $102^{\circ} 40^{\prime}$ & $31^{\circ} 15^{\prime}$ & 08/1942 & 09/1963 & 81 & 22 \\
\hline 1186 & Buescher Lake gage 2 & Bastrop $^{1}$ & ${ }^{1} 463$ & $97^{\circ} 09^{\prime}$ & $30^{\circ} 03^{\prime}$ & 03/1941 & $05 / 1943$ & 61 & 3 \\
\hline 1188 & Buffalo & Leon & 358 & $96^{\circ} 03^{\prime}$ & $31^{\circ} 28^{\prime}$ & $07 / 1947$ & 09/1947 & 21 & 1 \\
\hline 1245 & Burleson 2 SSW & Johnson & 771 & $97^{\circ} 20^{\prime}$ & $32^{\circ} 31^{\prime}$ & $04 / 1957$ & $06 / 1957$ & 22 & 1 \\
\hline 1246 & Burleson & Johnson & 730 & $97^{\circ} 19^{\prime}$ & $32^{\circ} 33^{\prime}$ & $12 / 1982$ & $12 / 2002$ & 92 & 21 \\
\hline 1267 & Bushland 1 WSW & Potter & 3,819 & $102^{\circ} 04^{\prime}$ & $35^{\circ} 11^{\prime}$ & $02 / 1940$ & $05 / 1953$ & 76 & 14 \\
\hline 1304 & Cadiz & $\mathrm{Bee}^{1}$ & 351 & $97^{\circ} 57^{\prime}$ & $28^{\circ} 26^{\prime}$ & 03/1940 & 08/1953 & 81 & 14 \\
\hline 1325 & Calhoun & Colorado $^{1}$ & 161 & $96^{\circ} 20^{\prime}$ & $29^{\circ} 32^{\prime}$ & 03/1940 & $03 / 1965$ & 92 & 26 \\
\hline 1438 & Canyon Dam no. 7 & Comal & 978 & $98^{\circ} 13^{\prime}$ & $29^{\circ} 55^{\prime}$ & $02 / 1961$ & 06/1993 & 89 & 33 \\
\hline 1462 & Carlsbad & Tom Green ${ }^{1}$ & 1,923 & $100^{\circ} 35^{\prime}$ & $31^{\circ} 35^{\prime}$ & $10 / 1947$ & $04 / 1949$ & 0 & 2 \\
\hline 1492 & Carta Valley & Edwards & 1,851 & $100^{\circ} 40^{\prime}$ & $29^{\circ} 47^{\prime}$ & 07/1963 & 06/1995 & 89 & 33 \\
\hline 1500 & Carthage & Panola & 340 & $94^{\circ} 21^{\prime}$ & $32^{\circ} 08^{\prime}$ & 04/1957 & $06 / 1957$ & 23 & 1 \\
\hline 1528 & Catarina & Dimmit & 560 & $99^{\circ} 37^{\prime}$ & $28^{\circ} 20^{\prime}$ & $01 / 1960$ & $10 / 2001$ & 87 & 42 \\
\hline 1541 & Cedar Creek 4 SE & Bastrop & 470 & $97^{\circ} 27^{\prime}$ & $30^{\circ} 02^{\prime}$ & $10 / 2001$ & $12 / 2002$ & 48 & 2 \\
\hline 1569 & Cego near SCS Temple 4 & Falls $^{1}$ & ${ }^{1} 500$ & $97^{\circ} 10^{\prime}$ & $31^{\circ} 15^{\prime}$ & $01 / 1940$ & 09/1943 & 78 & 4 \\
\hline 1632 & Chambers Hill Guard Station & Sabine $^{1}$ & 351 & $93^{\circ} 50^{\prime}$ & $31^{\circ} 28^{\prime}$ & 07/1947 & 08/1947 & 13 & 1 \\
\hline 1641 & Chancellor & $P e \cos ^{1}$ & 3,402 & $103^{\circ} 11^{\prime}$ & $30^{\circ} 42^{\prime}$ & $08 / 1942$ & $02 / 1955$ & 57 & 14 \\
\hline 1646 & Channing & Hartley & 3,800 & $102^{\circ} 20^{\prime}$ & $35^{\circ} 41^{\prime}$ & $01 / 1941$ & $12 / 2002$ & 90 & 62 \\
\hline 1663 & Charlotte $5 \mathrm{NNW}$ & Atascosa & 441 & $98^{\circ} 44^{\prime}$ & $28^{\circ} 55^{\prime}$ & 09/2001 & $12 / 2002$ & 32 & 2 \\
\hline 1671 & Cheapside & Gonzales & 310 & $97^{\circ} 20^{\prime}$ & $29^{\circ} 18^{\prime}$ & $05 / 1940$ & $12 / 2002$ & 91 & 63 \\
\hline 1680 & Cherokee & San Saba & 1,490 & $98^{\circ} 43^{\prime}$ & $30^{\circ} 59^{\prime}$ & $05 / 1941$ & 03/1972 & 90 & 31 \\
\hline 1694 & Childress 2 & Childress & 1,940 & $100^{\circ} 12^{\prime}$ & $34^{\circ} 25^{\prime}$ & $11 / 1997$ & $12 / 2002$ & 54 & 6 \\
\hline 1696 & Childress 3 WSW & Childress & 1,972 & $100^{\circ} 15^{\prime}$ & $34^{\circ} 26^{\prime}$ & $02 / 1940$ & 08/1975 & 86 & 31 \\
\hline 1697 & Childress $13 \mathrm{NE}$ & Childress & 1,713 & $100^{\circ} 20^{\prime}$ & $34^{\circ} 34^{\prime}$ & $02 / 1975$ & 06/1978 & 53 & 4 \\
\hline 1698 & Childress Municipal Airport & Childress & 1,951 & $100^{\circ} 16^{\prime}$ & $34^{\circ} 25^{\prime}$ & $10 / 1947$ & $12 / 2002$ & 83 & 34 \\
\hline 1720 & Choke Canyon Dam & Live Oak & 230 & $98^{\circ} 15^{\prime}$ & $28^{\circ} 28^{\prime}$ & 09/1997 & $12 / 2002$ & 79 & 6 \\
\hline 1761 & Clarendon & Donley & 2,700 & $100^{\circ} 53^{\prime}$ & $34^{\circ} 55^{\prime}$ & $07 / 1948$ & $06 / 1950$ & 45 & 3 \\
\hline 1773 & Clarksville $1 \mathrm{~W}$ & Red River & 426 & $95^{\circ} 01^{\prime}$ & $33^{\circ} 36^{\prime}$ & $09 / 1940$ & $12 / 2002$ & 93 & 58 \\
\hline
\end{tabular}

Footnote at end of table. 
Table 3. National Weather Service hourly rainfall stations in Texas-Continued.

\begin{tabular}{|c|c|c|c|c|c|c|c|c|c|}
\hline $\begin{array}{c}\text { Station } \\
\text { no. }\end{array}$ & Station name & County & $\begin{array}{c}\text { Elevation } \\
\text { (feet above } \\
\text { NGVD 29) }\end{array}$ & $\begin{array}{l}\text { Longitude } \\
\text { (degrees/ } \\
\text { minutes) }\end{array}$ & $\begin{array}{l}\text { Latitude } \\
\text { (degrees/ } \\
\text { minutes) }\end{array}$ & $\begin{array}{l}\text { Beginning } \\
\text { month/ } \\
\text { year }\end{array}$ & $\begin{array}{c}\text { Ending } \\
\text { month/ } \\
\text { year }\end{array}$ & $\begin{array}{l}\text { Percentage } \\
\text { of record } \\
\text { with data }\end{array}$ & $\begin{array}{l}\text { Years } \\
\text { with } \\
\text { record }\end{array}$ \\
\hline 1810 & Cleveland & Liberty & 196 & $95^{\circ} 05^{\prime}$ & $30^{\circ} 21^{\prime}$ & $04 / 1957$ & $06 / 1957$ & 22 & 1 \\
\hline 1823 & Clifton $9 \mathrm{E}$ & Bosque & 669 & $97^{\circ} 26^{\prime}$ & $31^{\circ} 48^{\prime}$ & $04 / 1957$ & 06/1957 & 21 & 1 \\
\hline 1870 & Coldspring 5 SSW & San Jacinto & 355 & $95^{\circ} 09^{\prime}$ & $30^{\circ} 32^{\prime}$ & $03 / 1965$ & $07 / 1970$ & 82 & 6 \\
\hline 1875 & Coleman & Coleman & 1,727 & $99^{\circ} 25^{\prime}$ & $31^{\circ} 49^{\prime}$ & $04 / 1957$ & $06 / 1957$ & 19 & 1 \\
\hline 1876 & Coleman near SCS & Coleman $^{1}$ & 2,001 & $99^{\circ} 38^{\prime}$ & $31^{\circ} 52^{\prime}$ & $04 / 1942$ & $07 / 1943$ & 62 & 2 \\
\hline 1889 & College Station Easterwood & Brazos & 305 & $96^{\circ} 21^{\prime}$ & $30^{\circ} 35^{\prime}$ & $08 / 1951$ & $12 / 2002$ & 74 & 5 \\
\hline 1903 & Colorado City & Mitchell & 2,105 & $100^{\circ} 51^{\prime}$ & $32^{\circ} 23^{\prime}$ & $06 / 1993$ & $12 / 2002$ & 89 & 10 \\
\hline 1914 & Comanche & Comanche & 1,345 & $98^{\circ} 35^{\prime}$ & $31^{\circ} 54^{\prime}$ & $04 / 1957$ & $06 / 1957$ & 18 & 1 \\
\hline 1920 & Comfort 2 & Kendall & 1,435 & $98^{\circ} 53^{\prime}$ & $29^{\circ} 57^{\prime}$ & $07 / 1990$ & $12 / 2002$ & 71 & 13 \\
\hline 1921 & Commerce $4 \mathrm{SW}$ & Hunt & 550 & $95^{\circ} 56^{\prime}$ & $33^{\circ} 12^{\prime}$ & $08 / 1948$ & $12 / 2002$ & 92 & 55 \\
\hline 1937 & Concord $1 \mathrm{~N}$ & Rusk & 541 & $94^{\circ} 35^{\prime}$ & $31^{\circ} 55^{\prime}$ & $08 / 1962$ & $10 / 1983$ & 89 & 22 \\
\hline 1956 & Conroe & Montgomery & 245 & $95^{\circ} 28^{\prime}$ & $30^{\circ} 19^{\prime}$ & $07 / 1947$ & $12 / 2002$ & 85 & 56 \\
\hline 1970 & Cooper & Delta & 480 & $95^{\circ} 41^{\prime}$ & $33^{\circ} 22^{\prime}$ & $04 / 1957$ & 06/1957 & 21 & 1 \\
\hline 2014 & Corpus Christi & Nueces & 10 & $97^{\circ} 24^{\prime}$ & $27^{\circ} 48^{\prime}$ & $01 / 1940$ & $12 / 1948$ & 100 & 8 \\
\hline 2015 & Corpus Christi WSFO Airport & Nueces & 41 & $97^{\circ} 30^{\prime}$ & $27^{\circ} 46^{\prime}$ & $10 / 1947$ & $12 / 2002$ & 98 & 56 \\
\hline 2019 & Corsicana & Navarro & 413 & $96^{\circ} 28^{\prime}$ & $32^{\circ} 06^{\prime}$ & $04 / 1957$ & $06 / 1957$ & 20 & 1 \\
\hline 2024 & Coryell City & Coryell & 971 & $97^{\circ} 37^{\prime}$ & $31^{\circ} 33^{\prime}$ & $07 / 1944$ & 03/1989 & 86 & 46 \\
\hline 2042 & Cottonwood Dam no. 1 & El Paso ${ }^{1}$ & 3,852 & $106^{\circ} 05^{\prime}$ & $31^{\circ} 33^{\prime}$ & $10 / 1947$ & $07 / 1949$ & 41 & 3 \\
\hline 2043 & Cottonwood Dam no. 2 & El Paso & $1_{385}$ & $106^{\circ} 04^{\prime}$ & $31^{\circ} 32^{\prime}$ & 08/1943 & $11 / 1948$ & 72 & 5 \\
\hline 2048 & Cotulla La Salle County Airport & La Salle & 476 & $99^{\circ} 13^{\prime}$ & $28^{\circ} 27^{\prime}$ & $04 / 1956$ & $12 / 2002$ & 89 & 47 \\
\hline 2050 & Cotulla FAA Airport & La Salle & 463 & $99^{\circ} 13^{\prime}$ & $28^{\circ} 27^{\prime}$ & $10 / 1949$ & $09 / 1951$ & 53 & 3 \\
\hline 2051 & Cotulla Hillje Ranch & La Salle & 331 & $99^{\circ} 04^{\prime}$ & $28^{\circ} 14^{\prime}$ & $04 / 1953$ & $03 / 1956$ & 63 & 4 \\
\hline 2053 & County Line & Hudspeth $^{1}$ & 3,553 & $105^{\circ} 59^{\prime}$ & $31^{\circ} 23^{\prime}$ & $03 / 1942$ & $07 / 1942$ & 41 & 1 \\
\hline 2073 & Crabb 2 W & Fort Bend ${ }^{1}$ & 112 & $95^{\circ} 45^{\prime}$ & $29^{\circ} 32^{\prime}$ & $01 / 1948$ & 09/1964 & 92 & 17 \\
\hline 2082 & Crane $2 \mathrm{E}$ & Crane & 2,602 & $102^{\circ} 18^{\prime}$ & $31^{\circ} 24^{\prime}$ & $08 / 1943$ & $12 / 2002$ & 93 & 60 \\
\hline 2086 & Cranfills Gap & Bosque & 975 & $97^{\circ} 50^{\prime}$ & $31^{\circ} 46^{\prime}$ & $05 / 1940$ & $12 / 2002$ & 81 & 63 \\
\hline 2088 & Crawford & McLennan & 705 & $97^{\circ} 27^{\prime}$ & $31^{\circ} 32^{\prime}$ & $11 / 2001$ & $12 / 2002$ & 45 & 2 \\
\hline 2090 & Crawford 4 WSW & McLennan & 842 & $97^{\circ} 31^{\prime}$ & $31^{\circ} 31^{\prime}$ & $11 / 1988$ & $06 / 1995$ & 72 & 8 \\
\hline 2096 & Cresson & Hood & 1,050 & $97^{\circ} 37^{\prime}$ & $32^{\circ} 32^{\prime}$ & $09 / 1946$ & $12 / 2002$ & 91 & 57 \\
\hline 2128 & Cross Plains 1 & Callahan & 1,742 & $99^{\circ} 10^{\prime}$ & $32^{\circ} 07^{\prime}$ & $01 / 1940$ & $10 / 1947$ & 87 & 8 \\
\hline 2131 & Cross Plains 2 & Callahan & 1,790 & $99^{\circ} 09^{\prime}$ & $32^{\circ} 07^{\prime}$ & $11 / 1947$ & $12 / 2002$ & 89 & 56 \\
\hline 2142 & Crowell & Foard & 1,455 & $99^{\circ} 43^{\prime}$ & $33^{\circ} 59^{\prime}$ & $04 / 1957$ & $06 / 1957$ & 21 & 1 \\
\hline 2160 & Crystal City & Zavala & 580 & $99^{\circ} 49^{\prime}$ & $28^{\circ} 40^{\prime}$ & $07 / 1948$ & $02 / 1949$ & 22 & 2 \\
\hline 2206 & Cypress & Harris & 150 & $95^{\circ} 41^{\prime}$ & $29^{\circ} 57^{\prime}$ & $01 / 1991$ & $12 / 2002$ & 94 & 12 \\
\hline 2238 & Dalhart & Dallam $^{1}$ & 4,003 & $102^{\circ} 29^{\prime}$ & $36^{\circ} 05^{\prime}$ & $01 / 1941$ & $12 / 1946$ & 86 & 6 \\
\hline 2240 & Dalhart FAA Airport & Hartley & 3,990 & $102^{\circ} 32^{\prime}$ & $36^{\circ} 01^{\prime}$ & $04 / 1950$ & 09/1951 & 58 & 2 \\
\hline 2242 & DFW International Airport & Tarrant & 560 & $97^{\circ} 01^{\prime}$ & $32^{\circ} 53^{\prime}$ & $02 / 1974$ & $12 / 2002$ & 97 & 29 \\
\hline 2244 & Dallas Love Field & Dallas & 440 & $96^{\circ} 51^{\prime}$ & $32^{\circ} 51^{\prime}$ & $11 / 1940$ & $12 / 2002$ & 90 & 62 \\
\hline 2247 & Dallas WFAA & Dallas $^{1}$ & 479 & $96^{\circ} 47^{\prime}$ & $32^{\circ} 46^{\prime}$ & $01 / 1940$ & $10 / 1940$ & 80 & 1 \\
\hline 2309 & Dawson & Navarro $^{1}$ & 479 & $96^{\circ} 43^{\prime}$ & $31^{\circ} 54^{\prime}$ & $12 / 1943$ & $06 / 1962$ & 77 & 19 \\
\hline 2312 & Deberry & Panola & 361 & $94^{\circ} 10^{\prime}$ & $32^{\circ} 18^{\prime}$ & $08 / 1973$ & $02 / 1984$ & 81 & 12 \\
\hline 2334 & Decatur & Wise & 1,025 & $97^{\circ} 35^{\prime}$ & $33^{\circ} 13^{\prime}$ & $08 / 1945$ & $02 / 1948$ & 57 & 4 \\
\hline 2336 & Decatur $7 \mathrm{~N}$ & Wise $^{1}$ & 902 & $97^{\circ} 35^{\prime}$ & $33^{\circ} 20^{\prime}$ & $10 / 1947$ & $10 / 1953$ & 73 & 7 \\
\hline 2354 & Dell City 5 SSW & Hudspeth & 3,770 & $105^{\circ} 14^{\prime}$ & $31^{\circ} 52^{\prime}$ & $05 / 1955$ & $09 / 1957$ & 39 & 3 \\
\hline 2355 & Delmita & Hidalgo $^{1}$ & 102 & $98^{\circ} 07^{\prime}$ & $26^{\circ} 39^{\prime}$ & $03 / 1940$ & $06 / 1941$ & 51 & 2 \\
\hline 2357 & Del Rio Weather Bureau City & Val Verde $^{1}$ & 961 & $100^{\circ} 54^{\prime}$ & $29^{\circ} 22^{\prime}$ & $02 / 1940$ & $04 / 1951$ & 87 & 12 \\
\hline 2360 & Del Rio Airport & Val Verde & 999 & $100^{\circ} 55^{\prime}$ & $29^{\circ} 22^{\prime}$ & $05 / 1951$ & $12 / 2002$ & 84 & 46 \\
\hline 2361 & Del Rio 2 NW & Val Verde & 1,080 & $100^{\circ} 54^{\prime}$ & $29^{\circ} 25^{\prime}$ & 08/1996 & $12 / 2002$ & 66 & 7 \\
\hline 2394 & Denison Dam & Grayson & 613 & $96^{\circ} 34^{\prime}$ & $33^{\circ} 49^{\prime}$ & $01 / 1940$ & $07 / 1997$ & 91 & 58 \\
\hline 2404 & Denton $2 \mathrm{SE}$ & Denton & 630 & $97^{\circ} 06^{\prime}$ & $33^{\circ} 11^{\prime}$ & $08 / 1946$ & $12 / 2002$ & 90 & 57 \\
\hline 2415 & Deport 4 NW & Lamar & 436 & $95^{\circ} 22^{\prime}$ & $33^{\circ} 33^{\prime}$ & $02 / 1944$ & $04 / 2001$ & 84 & 58 \\
\hline 2462 & Dime Box & Lee & 335 & $96^{\circ} 50^{\prime}$ & $30^{\circ} 21^{\prime}$ & 07/1981 & $12 / 2002$ & 80 & 22 \\
\hline 2528 & Dora near SCS no. 6 & Taylor $^{1}$ & 2,303 & $100^{\circ} 06^{\prime}$ & $32^{\circ} 16^{\prime}$ & $10 / 1940$ & $08 / 1943$ & 64 & 4 \\
\hline 2617 & Dumas & Moore & 3,655 & $101^{\circ} 58^{\prime}$ & $35^{\circ} 52^{\prime}$ & $01 / 1941$ & $07 / 1948$ & 83 & 7 \\
\hline
\end{tabular}


Table 357

Table 3. National Weather Service hourly rainfall stations in Texas-Continued.

\begin{tabular}{|c|c|c|c|c|c|c|c|c|c|}
\hline $\begin{array}{c}\text { Station } \\
\text { no. }\end{array}$ & Station name & County & $\begin{array}{c}\text { Elevation } \\
\text { (feet above } \\
\text { NGVD 29) }\end{array}$ & $\begin{array}{c}\text { Longitude } \\
\text { (degrees/ } \\
\text { minutes) }\end{array}$ & $\begin{array}{l}\text { Latitude } \\
\text { (degrees/ } \\
\text { minutes) }\end{array}$ & $\begin{array}{c}\text { Beginning } \\
\text { month/ } \\
\text { year }\end{array}$ & $\begin{array}{c}\text { Ending } \\
\text { month/ } \\
\text { year }\end{array}$ & $\begin{array}{l}\text { Percentage } \\
\text { of record } \\
\text { with data }\end{array}$ & $\begin{array}{l}\text { Years } \\
\text { with } \\
\text { record }\end{array}$ \\
\hline 2619 & Dumas $8 \mathrm{NE}$ & Moore & 3,553 & $101^{\circ} 53^{\prime}$ & $35^{\circ} 57^{\prime}$ & $10 / 1947$ & $02 / 1955$ & 67 & 9 \\
\hline 2675 & Eagle Lake & Colorado & 177 & $96^{\circ} 20^{\prime}$ & $29^{\circ} 36^{\prime}$ & $10 / 1965$ & $02 / 1986$ & 81 & 22 \\
\hline 2676 & Eagle Lake Research Center & Colorado & 176 & $96^{\circ} 21^{\prime}$ & $29^{\circ} 37^{\prime}$ & $04 / 1986$ & $12 / 2002$ & 84 & 17 \\
\hline 2679 & Eagle Pass & Maverick & 808 & $100^{\circ} 28^{\prime}$ & $28^{\circ} 42^{\prime}$ & $10 / 1941$ & $12 / 2002$ & 91 & 62 \\
\hline 2758 & Edinburg & Hidalgo & 96 & $98^{\circ} 09^{\prime}$ & $26^{\circ} 17^{\prime}$ & $10 / 1947$ & $05 / 1950$ & 63 & 4 \\
\hline 2794 & El Paso 32 ENE & Hudspeth & 5,240 & $105^{\circ} 57^{\prime}$ & $31^{\circ} 50^{\prime}$ & 07/1947 & 09/1947 & 22 & 1 \\
\hline 2797 & El Paso Airport & El Paso & 3,918 & $106^{\circ} 22^{\prime}$ & $31^{\circ} 48^{\prime}$ & 08/1942 & $12 / 2002$ & 97 & 61 \\
\hline 2811 & Eldorado $1 \mathrm{~N}$ & Schleicher & 2,419 & $100^{\circ} 36^{\prime}$ & $30^{\circ} 53^{\prime}$ & $03 / 1940$ & $12 / 1995$ & 92 & 50 \\
\hline 2813 & Eldorado $11 \mathrm{SW}$ & Schleicher ${ }^{1}$ & 2,431 & $100^{\circ} 46^{\prime}$ & $30^{\circ} 47^{\prime}$ & 03/1949 & $10 / 1952$ & 19 & 4 \\
\hline 3005 & Evant $1 \mathrm{SSW}$ & Coryell & 1,245 & $98^{\circ} 10^{\prime}$ & $31^{\circ} 28^{\prime}$ & $05 / 1943$ & $12 / 2002$ & 87 & 60 \\
\hline 3033 & Fabens 1 & El Paso & 3,612 & $106^{\circ} 09^{\prime}$ & $31^{\circ} 30^{\prime}$ & $02 / 1953$ & 09/1977 & 95 & 25 \\
\hline 3034 & Fabens 2 & El Paso ${ }^{1}$ & 3,652 & $106^{\circ} 09^{\prime}$ & $31^{\circ} 31^{\prime}$ & 07/1949 & $04 / 1951$ & 2 & 2 \\
\hline 3047 & Fairfield $3 \mathrm{~W}$ & Freestone & 432 & $96^{\circ} 12^{\prime}$ & $31^{\circ} 43^{\prime}$ & 04/1957 & 06/1957 & 22 & 1 \\
\hline 3103 & Fawcett Ranch & Val Verde ${ }^{1}$ & 1,503 & $100^{\circ} 54^{\prime}$ & $29^{\circ} 52^{\prime}$ & $04 / 1946$ & 08/1949 & 44 & 4 \\
\hline 3133 & Ferris & Ellis & 470 & $96^{\circ} 40^{\prime}$ & $32^{\circ} 31^{\prime}$ & 07/1946 & $12 / 2002$ & 89 & 56 \\
\hline 3156 & Fischers Store & Comal & 1,160 & $98^{\circ} 15^{\prime}$ & $29^{\circ} 58^{\prime}$ & $10 / 1995$ & $12 / 2002$ & 70 & 8 \\
\hline 3171 & Flat & Coryell & 835 & $97^{\circ} 38^{\prime}$ & $31^{\circ} 19^{\prime}$ & $02 / 1950$ & $12 / 2002$ & 91 & 53 \\
\hline 3189 & Fletcher Ranch & Presido $^{1}$ & 4,852 & $104^{\circ} 12^{\prime}$ & $30^{\circ} 10^{\prime}$ & 02/1942 & $02 / 1954$ & 72 & 12 \\
\hline 3260 & Fort Clark & Kinney $^{1}$ & 1,102 & $100^{\circ} 27^{\prime}$ & $29^{\circ} 18^{\prime}$ & 03/1941 & 03/1946 & 71 & 6 \\
\hline 3267 & Fort McIntosh & Webb $^{1}$ & 459 & $99^{\circ} 31^{\prime}$ & $27^{\circ} 30^{\prime}$ & $12 / 1940$ & $07 / 1943$ & 60 & 4 \\
\hline 3285 & Fort Worth WSFO & Tarrant & 644 & $97^{\circ} 18^{\prime}$ & $32^{\circ} 50^{\prime}$ & $05 / 1948$ & $12 / 2002$ & 93 & 44 \\
\hline 3329 & Fredericksburg & Gillespie & 1,685 & $98^{\circ} 54^{\prime}$ & $30^{\circ} 14^{\prime}$ & $04 / 1940$ & 03/1975 & 90 & 36 \\
\hline 3335 & Freeman Ranch & Harris ${ }^{1}$ & ${ }^{1} 151$ & $95^{\circ} 47^{\prime}$ & $29^{\circ} 53^{\prime}$ & $01 / 1943$ & $11 / 1946$ & 89 & 4 \\
\hline 3370 & Frisco & Collin & 740 & $96^{\circ} 49^{\prime}$ & $33^{\circ} 09^{\prime}$ & $10 / 1966$ & $12 / 2002$ & 91 & 37 \\
\hline 3410 & Gageby 3 WNW & Hemphill & 2,800 & $100^{\circ} 23^{\prime}$ & $35^{\circ} 37^{\prime}$ & $04 / 1941$ & $12 / 2002$ & 91 & 59 \\
\hline 3415 & Gainesville & Cooke & 780 & $97^{\circ} 08^{\prime}$ & $33^{\circ} 38^{\prime}$ & 09/1941 & $12 / 2002$ & 82 & 62 \\
\hline 3430 & Galveston & Galveston & 10 & $94^{\circ} 46^{\prime}$ & $29^{\circ} 20^{\prime}$ & $01 / 1940$ & $07 / 2000$ & 93 & 61 \\
\hline 3431 & Galveston Weather Bureau Ap. & Galveston & 5 & $94^{\circ} 52^{\prime}$ & $29^{\circ} 16^{\prime}$ & 01/1948 & 09/1951 & 72 & 4 \\
\hline 3441 & Garcia Lake & Deaf Smith ${ }^{1}$ & 4,203 & $103^{\circ} 01^{\prime}$ & $34^{\circ} 53^{\prime}$ & $10 / 1947$ & 08/1953 & 23 & 6 \\
\hline 3442 & Garcia Lake 12 ENE & Deaf Smith & 4,134 & $102^{\circ} 44^{\prime}$ & $34^{\circ} 55^{\prime}$ & $11 / 1943$ & 05/1971 & 66 & 21 \\
\hline 3446 & Garden City $16 \mathrm{E}$ & Sterling & 2,461 & $101^{\circ} 12^{\prime}$ & $31^{\circ} 50^{\prime}$ & $04 / 1949$ & 07/1973 & 87 & 25 \\
\hline 3460 & Garland near SCS no. 10 & Dallas $^{1}$ & 610 & $96^{\circ} 41^{\prime}$ & $32^{\circ} 57^{\prime}$ & $01 / 1940$ & $10 / 1940$ & 79 & 1 \\
\hline 3462 & Garlington Ranch & Sterling ${ }^{1}$ & $1_{2,622}$ & $100^{\circ} 53^{\prime}$ & $31^{\circ} 55^{\prime}$ & 08/1943 & $04 / 1949$ & 56 & 7 \\
\hline 3463 & Garner State Park & Uvalde $^{1}$ & 1,401 & $99^{\circ} 44^{\prime}$ & $29^{\circ} 35^{\prime}$ & 07/1948 & $12 / 1951$ & 23 & 4 \\
\hline 3476 & Lewisville Dam & Denton & 561 & $97^{\circ} 01^{\prime}$ & $33^{\circ} 04^{\prime}$ & $07 / 1949$ & $01 / 1964$ & 88 & 16 \\
\hline 3485 & Gatesville 4 SSE & Coryell & 760 & $97^{\circ} 43^{\prime}$ & $31^{\circ} 23^{\prime}$ & 04/1957 & 06/1957 & 22 & 1 \\
\hline 3507 & Georgetown Lake & Williamson & 840 & $97^{\circ} 43^{\prime}$ & $30^{\circ} 41^{\prime}$ & 07/1981 & $12 / 2002$ & 80 & 22 \\
\hline 3546 & Gilmer 4 WNW & Upshur & 390 & $95^{\circ} 02^{\prime}$ & $32^{\circ} 44^{\prime}$ & $07 / 1941$ & $12 / 2002$ & 86 & 56 \\
\hline 3547 & Gilmer & Upshur ${ }^{1}$ & 371 & $94^{\circ} 57^{\prime}$ & $32^{\circ} 44^{\prime}$ & $12 / 1950$ & 09/1956 & 65 & 7 \\
\hline 3579 & Glen Cove 2 NE & Coleman & 2,090 & $99^{\circ} 37^{\prime}$ & $31^{\circ} 52^{\prime}$ & $01 / 1940$ & $04 / 1942$ & 45 & 3 \\
\hline
\end{tabular}

Footnote at end of table. 
Table 3. National Weather Service hourly rainfall stations in Texas-Continued.

\begin{tabular}{|c|c|c|c|c|c|c|c|c|c|}
\hline $\begin{array}{c}\text { Station } \\
\text { no. }\end{array}$ & Station name & County & $\begin{array}{c}\text { Elevation } \\
\text { (feet above } \\
\text { NGVD 29) }\end{array}$ & $\begin{array}{l}\text { Longitude } \\
\text { (degrees/ } \\
\text { minutes) }\end{array}$ & $\begin{array}{l}\text { Latitude } \\
\text { (degrees/ } \\
\text { minutes) }\end{array}$ & $\begin{array}{l}\text { Beginning } \\
\text { month/ } \\
\text { year }\end{array}$ & $\begin{array}{c}\text { Ending } \\
\text { month/ } \\
\text { year }\end{array}$ & $\begin{array}{l}\text { Percentage } \\
\text { of record } \\
\text { with data }\end{array}$ & $\begin{array}{l}\text { Years } \\
\text { with } \\
\text { record }\end{array}$ \\
\hline 3642 & Gordonville & Grayson & 755 & $96^{\circ} 51^{\prime}$ & $33^{\circ} 47^{\prime}$ & $01 / 1942$ & $12 / 2002$ & 91 & 61 \\
\hline 3646 & Gorman 2 NNE & Eastland & 1,380 & $98^{\circ} 39^{\prime}$ & $32^{\circ} 14^{\prime}$ & $09 / 1951$ & 07/1999 & 93 & 49 \\
\hline 3668 & Graham & Young & 1,050 & $98^{\circ} 35^{\prime}$ & $33^{\circ} 06^{\prime}$ & $04 / 1957$ & $06 / 1957$ & 22 & 1 \\
\hline 3673 & Granbury 2 ENE & Hood & 722 & $97^{\circ} 45^{\prime}$ & $32^{\circ} 27^{\prime}$ & $04 / 1957$ & $06 / 1957$ & 23 & 1 \\
\hline 3686 & Granger Dam & Williamson & 565 & $97^{\circ} 20^{\prime}$ & $30^{\circ} 42^{\prime}$ & $05 / 1980$ & $12 / 2002$ & 88 & 23 \\
\hline 3691 & Grapevine Dam & Tarrant & 585 & $97^{\circ} 03^{\prime}$ & $32^{\circ} 57^{\prime}$ & $06 / 1949$ & $12 / 2002$ & 93 & 54 \\
\hline 3734 & Greenville KGVL Radio & Hunt & 545 & $96^{\circ} 06^{\prime}$ & $33^{\circ} 10^{\prime}$ & $04 / 1957$ & $06 / 1957$ & 23 & 1 \\
\hline 3771 & Groesbeck 2 & Limestone & 465 & $96^{\circ} 31^{\prime}$ & $31^{\circ} 31^{\prime}$ & $11 / 1977$ & $12 / 2002$ & 86 & 26 \\
\hline 3789 & Guadalupe Pass CAA Airport & Culberson & 5,452 & $104^{\circ} 48^{\prime}$ & $31^{\circ} 50^{\prime}$ & $07 / 1948$ & $08 / 1950$ & 58 & 3 \\
\hline 3826 & Gustine 2 SE & Comanche & 1,220 & $98^{\circ} 23^{\prime}$ & $31^{\circ} 49^{\prime}$ & $02 / 1984$ & 03/1989 & 80 & 6 \\
\hline 3831 & Guyer & Wilbarger & 1,161 & $98^{\circ} 56^{\prime}$ & $34^{\circ} 08^{\prime}$ & $01 / 1940$ & $07 / 1952$ & 66 & 13 \\
\hline 3841 & Hackberry & Cottle & 1,670 & $100^{\circ} 08^{\prime}$ & $33^{\circ} 56^{\prime}$ & 04/1971 & $01 / 1980$ & 55 & 10 \\
\hline 3871 & Hall Ranch & Kerr & 2,280 & $99^{\circ} 36^{\prime}$ & $30^{\circ} 08^{\prime}$ & $04 / 1940$ & $06 / 1976$ & 90 & 37 \\
\hline 3884 & Hamilton $2 \mathrm{E}$ & Hamilton & 1,260 & $98^{\circ} 05^{\prime}$ & $31^{\circ} 42^{\prime}$ & $04 / 1957$ & $06 / 1957$ & 21 & 1 \\
\hline 3941 & Harleton & Harrison & 345 & $94^{\circ} 34^{\prime}$ & $32^{\circ} 41^{\prime}$ & $09 / 1940$ & $07 / 1941$ & 40 & 2 \\
\hline 3963 & Harris Lake & Brazoria & $1_{41}$ & $95^{\circ} 33^{\prime}$ & $29^{\circ} 15^{\prime}$ & $12 / 1948$ & $01 / 1949$ & 4 & 2 \\
\hline 4040 & Hazeldell & Comanche & 1,130 & $98^{\circ} 18^{\prime}$ & $31^{\circ} 53^{\prime}$ & $07 / 1973$ & $01 / 1984$ & 82 & 12 \\
\hline 4058 & Hebbronville & Jim Hogg & 580 & $98^{\circ} 41^{\prime}$ & $27^{\circ} 19^{\prime}$ & $04 / 1957$ & $06 / 1957$ & 23 & 1 \\
\hline 4098 & Hereford & Deaf Smith & 3,820 & $102^{\circ} 24^{\prime}$ & $34^{\circ} 49^{\prime}$ & $05 / 1955$ & $12 / 2002$ & 87 & 48 \\
\hline 4100 & Hereford $1 \mathrm{SE}$ & Deaf Smith ${ }^{1}$ & 3,822 & $102^{\circ} 24^{\prime}$ & $34^{\circ} 49^{\prime}$ & $07 / 1941$ & $05 / 1955$ & 62 & 15 \\
\hline 4137 & Hico & Hamilton & 1,025 & $98^{\circ} 01^{\prime}$ & $31^{\circ} 59^{\prime}$ & $10 / 1977$ & $12 / 2002$ & 83 & 26 \\
\hline 4191 & Hindes & Atascosa & 360 & $98^{\circ} 48^{\prime}$ & $28^{\circ} 43^{\prime}$ & $02 / 1940$ & 05/1999 & 94 & 60 \\
\hline 4256 & Hondo Municipal Airport & Medina & 920 & $99^{\circ} 10^{\prime}$ & $29^{\circ} 21^{\prime}$ & $07 / 1996$ & $12 / 2002$ & 42 & 3 \\
\hline 4257 & Honey Grove & Fannin & 680 & $95^{\circ} 53^{\prime}$ & $33^{\circ} 35^{\prime}$ & $02 / 1944$ & $12 / 2002$ & 89 & 57 \\
\hline 4258 & Honey Grove 2 & Fannin & 659 & $95^{\circ} 54^{\prime}$ & $33^{\circ} 35^{\prime}$ & 03/1972 & $01 / 1975$ & 56 & 4 \\
\hline 4278 & Hords Creek Dam & Coleman & 1,942 & $99^{\circ} 33^{\prime}$ & $31^{\circ} 50^{\prime}$ & $04 / 1956$ & $12 / 2002$ & 76 & 47 \\
\hline 4299 & Hot Springs & Brewster $^{1}$ & 2,201 & $103^{\circ} 00^{\prime}$ & $29^{\circ} 11^{\prime}$ & $07 / 1942$ & $06 / 1952$ & 61 & 11 \\
\hline 4300 & Houston Bush International Ap. & Harris & 95 & $95^{\circ} 21^{\prime}$ & $29^{\circ} 59^{\prime}$ & $01 / 1970$ & $12 / 2002$ & 99 & 33 \\
\hline 4305 & Houston Weather Bureau City & Harris $^{1}$ & 43 & $95^{\circ} 22^{\prime}$ & $29^{\circ} 46^{\prime}$ & $01 / 1940$ & $05 / 1970$ & 95 & 31 \\
\hline 4307 & Houston Hobby Airport & Harris & 44 & $95^{\circ} 16^{\prime}$ & $29^{\circ} 38^{\prime}$ & $01 / 1948$ & $12 / 2002$ & 75 & 11 \\
\hline 4309 & Houston Addicks & Harris & 91 & $95^{\circ} 38^{\prime}$ & $29^{\circ} 46^{\prime}$ & $01 / 1943$ & $12 / 2002$ & 90 & 59 \\
\hline 4311 & Houston Alief & Harris & 71 & $95^{\circ} 35^{\prime}$ & $29^{\circ} 42^{\prime}$ & $01 / 1940$ & $12 / 2002$ & 90 & 62 \\
\hline 4313 & Houston Barker & Harris & 127 & $95^{\circ} 44^{\prime}$ & $29^{\circ} 49^{\prime}$ & $01 / 1943$ & $06 / 1948$ & 80 & 5 \\
\hline 4319 & Houston Golf Crest & Harris $^{1}$ & 49 & $95^{\circ} 17^{\prime}$ & $29^{\circ} 41^{\prime}$ & $06 / 1948$ & $09 / 1951$ & 35 & 4 \\
\hline 4329 & Houston Satsuma & Harris & 122 & $95^{\circ} 38^{\prime}$ & $29^{\circ} 56^{\prime}$ & $01 / 1940$ & $12 / 1990$ & 89 & 50 \\
\hline 4331 & Houston Spring Branch & Harris & 92 & $95^{\circ} 30^{\prime}$ & $29^{\circ} 48^{\prime}$ & $10 / 1950$ & $04 / 1952$ & 0 & 2 \\
\hline 4375 & Hunt $10 \mathrm{~W}$ & Kerr & 2,095 & $99^{\circ} 31^{\prime}$ & $30^{\circ} 02^{\prime}$ & 07/1976 & $12 / 2002$ & 88 & 27 \\
\hline 4392 & Hurt & Hunt $^{1}$ & 679 & $95^{\circ} 58^{\prime}$ & $33^{\circ} 13^{\prime}$ & $05 / 1940$ & $08 / 1948$ & 82 & 9 \\
\hline 4425 & Imperial & Pecos & 2,400 & $102^{\circ} 42^{\prime}$ & $31^{\circ} 16^{\prime}$ & $09 / 1963$ & $10 / 1993$ & 91 & 31 \\
\hline 4440 & Indian Gap & Hamilton & 1,575 & $98^{\circ} 25^{\prime}$ & $31^{\circ} 40^{\prime}$ & 09/1951 & $12 / 1983$ & 97 & 33 \\
\hline 4476 & Iredell & Bosque & 902 & $97^{\circ} 52^{\prime}$ & $31^{\circ} 59^{\prime}$ & $09 / 1963$ & $12 / 2002$ & 90 & 40 \\
\hline 4498 & Island Stn & $\mathrm{El} \mathrm{Paso}^{1}$ & 3,632 & $106^{\circ} 14^{\prime}$ & $31^{\circ} 32^{\prime}$ & $01 / 1942$ & $07 / 1942$ & 56 & 1 \\
\hline 4517 & Jacksboro & Jack & 1,100 & $98^{\circ} 09^{\prime}$ & $33^{\circ} 14^{\prime}$ & $05 / 1940$ & $10 / 1977$ & 92 & 38 \\
\hline 4520 & Jacksboro $1 \mathrm{NNE}$ & Jack & 1,020 & $98^{\circ} 08^{\prime}$ & $33^{\circ} 14^{\prime}$ & $11 / 1977$ & $12 / 2002$ & 82 & 26 \\
\hline 4525 & Jacksonville & Cherokee & 560 & $95^{\circ} 16^{\prime}$ & $31^{\circ} 57^{\prime}$ & $04 / 1957$ & $06 / 1957$ & 22 & 1 \\
\hline 4563 & Jasper & Jasper & 290 & $94^{\circ} 00^{\prime}$ & $30^{\circ} 54^{\prime}$ & $04 / 1957$ & $06 / 1957$ & 24 & 1 \\
\hline 4570 & Jayton & Kent & 2,010 & $100^{\circ} 34^{\prime}$ & $33^{\circ} 15^{\prime}$ & $05 / 1940$ & $12 / 2002$ & 87 & 63 \\
\hline 4577 & Jefferson & Marion & 199 & $94^{\circ} 20^{\prime}$ & $32^{\circ} 46^{\prime}$ & $02 / 1944$ & $12 / 1978$ & 91 & 35 \\
\hline 4591 & Jewett & Leon & 510 & $96^{\circ} 09^{\prime}$ & $31^{\circ} 21^{\prime}$ & $12 / 1941$ & $02 / 1991$ & 90 & 51 \\
\hline 4670 & Junction 4 SSW & Kimble & 1,747 & $99^{\circ} 48^{\prime}$ & $30^{\circ} 26^{\prime}$ & $03 / 1940$ & $12 / 2002$ & 90 & 62 \\
\hline 4671 & Junction FAA Airport & Kimble & ${ }^{1} 1,660$ & $99^{\circ} 46^{\prime}$ & $30^{\circ} 30^{\prime}$ & $10 / 1948$ & $09 / 1951$ & 62 & 4 \\
\hline 4679 & Justin & Denton & 640 & $97^{\circ} 17^{\prime}$ & $33^{\circ} 04^{\prime}$ & $01 / 1954$ & $12 / 2002$ & 93 & 49 \\
\hline 4696 & Karnes City 2 N & Karnes & 450 & $97^{\circ} 52^{\prime}$ & $28^{\circ} 54^{\prime}$ & $07 / 1947$ & $09 / 1947$ & 22 & 1 \\
\hline 4703 & Katy & Harris & 142 & $95^{\circ} 49^{\prime}$ & $29^{\circ} 47^{\prime}$ & $10 / 1947$ & $04 / 1951$ & 45 & 5 \\
\hline 4704 & Katy City & Harris & 153 & $95^{\circ} 49^{\prime}$ & $29^{\circ} 48^{\prime}$ & $01 / 1940$ & $12 / 1946$ & 94 & 7 \\
\hline
\end{tabular}

Footnote at end of table. 
Table 359

Table 3. National Weather Service hourly rainfall stations in Texas-Continued.

\begin{tabular}{|c|c|c|c|c|c|c|c|c|c|}
\hline $\begin{array}{c}\text { Station } \\
\text { no. }\end{array}$ & Station name & County & $\begin{array}{c}\text { Elevation } \\
\text { (feet above } \\
\text { NGVD 29) }\end{array}$ & $\begin{array}{c}\text { Longitude } \\
\text { (degrees/ } \\
\text { minutes) }\end{array}$ & $\begin{array}{l}\text { Latitude } \\
\text { (degrees/ } \\
\text { minutes) }\end{array}$ & $\begin{array}{c}\text { Beginning } \\
\text { month/ } \\
\text { year }\end{array}$ & $\begin{array}{c}\text { Ending } \\
\text { month/ } \\
\text { year }\end{array}$ & $\begin{array}{l}\text { Percentage } \\
\text { of record } \\
\text { with data }\end{array}$ & $\begin{array}{l}\text { Years } \\
\text { with } \\
\text { record }\end{array}$ \\
\hline 4731 & Kelly Field & Bexar $^{1}$ & 682 & $98^{\circ} 34^{\prime}$ & $29^{\circ} 23^{\prime}$ & $01 / 1941$ & $12 / 1942$ & 89 & 2 \\
\hline 4819 & Kirbyville & Jasper & 200 & $93^{\circ} 55^{\prime}$ & $30^{\circ} 37^{\prime}$ & $07 / 1974$ & $12 / 1978$ & 80 & 5 \\
\hline 4852 & Knox City & Knox & 1,532 & $99^{\circ} 49^{\prime}$ & $33^{\circ} 25^{\prime}$ & $04 / 1957$ & $06 / 1957$ & 19 & 1 \\
\hline 4866 & Kopperl 5 NNE & Hill & 620 & $97^{\circ} 28^{\prime}$ & $32^{\circ} 08^{\prime}$ & $05 / 1940$ & $12 / 2002$ & 91 & 63 \\
\hline 4880 & Kress & Swisher & 3,480 & $101^{\circ} 44^{\prime}$ & $34^{\circ} 22^{\prime}$ & $02 / 1940$ & $12 / 2002$ & 95 & 63 \\
\hline 4920 & La Pryor & Zavala & 759 & $99^{\circ} 52^{\prime}$ & $28^{\circ} 59^{\prime}$ & $02 / 1940$ & $12 / 2002$ & 88 & 60 \\
\hline 4934 & La Vernia & Wilson $^{1}$ & 479 & $98^{\circ} 06^{\prime}$ & $29^{\circ} 22^{\prime}$ & 07/1947 & 09/1947 & 17 & 1 \\
\hline 4972 & Lake Bridgeport Dam & Wise & 870 & $97^{\circ} 49^{\prime}$ & $33^{\circ} 13^{\prime}$ & 08/1946 & $12 / 2002$ & 90 & 57 \\
\hline 4973 & Lake Coffee Mill & Fannin $^{1}$ & 502 & $96^{\circ} 00^{\prime}$ & $33^{\circ} 44^{\prime}$ & $01 / 1946$ & 08/1959 & 66 & 14 \\
\hline 4982 & Lake Kemp & Baylor & 1,167 & $99^{\circ} 08^{\prime}$ & $33^{\circ} 45^{\prime}$ & 08/1974 & $12 / 2002$ & 79 & 29 \\
\hline 5018 & Lampasas & Lampasas & 1,032 & $98^{\circ} 11^{\prime}$ & $31^{\circ} 04^{\prime}$ & $04 / 1957$ & $07 / 2000$ & 87 & 16 \\
\hline 5048 & Langtry & Val Verde & 1,290 & $101^{\circ} 33^{\prime}$ & $29^{\circ} 47^{\prime}$ & $07 / 1942$ & $12 / 2002$ & 93 & 58 \\
\hline 5049 & Langtry 2 & Val Verde & 1,342 & $101^{\circ} 34^{\prime}$ & $29^{\circ} 48^{\prime}$ & $10 / 1965$ & 08/1969 & 76 & 5 \\
\hline 5056 & Laredo & Webb & 397 & $99^{\circ} 30^{\prime}$ & $27^{\circ} 30^{\prime}$ & 03/1948 & $04 / 1949$ & 5 & 2 \\
\hline 5057 & Laredo Weather Bureau Airport & Webb & 500 & $99^{\circ} 28^{\prime}$ & $27^{\circ} 32^{\prime}$ & $03 / 1944$ & $10 / 1965$ & 94 & 22 \\
\hline 5060 & Laredo 2 & Webb & 430 & $99^{\circ} 29^{\prime}$ & $27^{\circ} 34^{\prime}$ & $10 / 1965$ & $07 / 1972$ & 82 & 8 \\
\hline 5081 & Latex & Panola $^{1}$ & 302 & $94^{\circ} 06^{\prime}$ & $32^{\circ} 21^{\prime}$ & $12 / 1942$ & $07 / 1963$ & 89 & 22 \\
\hline 5094 & Lavon Dam & Collin & 510 & $96^{\circ} 29^{\prime}$ & $33^{\circ} 02^{\prime}$ & 07/1949 & $12 / 2002$ & 88 & 54 \\
\hline 5113 & Leakey & Real & 1,622 & $99^{\circ} 45^{\prime}$ & $29^{\circ} 44^{\prime}$ & $04 / 1940$ & $12 / 2002$ & 85 & 60 \\
\hline 5114 & Leakey 2 & Real & 1,601 & $99^{\circ} 50^{\prime}$ & $29^{\circ} 42^{\prime}$ & 07/1947 & 08/1947 & 0 & 1 \\
\hline 5123 & Lee Ranch & Concho $^{1}$ & 2,011 & $99^{\circ} 52^{\prime}$ & $31^{\circ} 08^{\prime}$ & $04 / 1940$ & 08/1940 & 28 & 1 \\
\hline 5303 & Loma Alta & Val Verde ${ }^{1}$ & 1,923 & $100^{\circ} 46^{\prime}$ & $29^{\circ} 55^{\prime}$ & $11 / 1942$ & 05/1963 & 89 & 22 \\
\hline 5312 & London $3 \mathrm{~N}$ & Menard & 1,800 & $99^{\circ} 34^{\prime}$ & $30^{\circ} 42^{\prime}$ & $11 / 1956$ & $12 / 2002$ & 83 & 47 \\
\hline 5341 & Longview & Gregg & 330 & $94^{\circ} 44^{\prime}$ & $32^{\circ} 27^{\prime}$ & 04/1957 & 06/1957 & 23 & 1 \\
\hline 5342 & Longview East-Tex. Rgnl. Ap. & Gregg & 365 & $94^{\circ} 42^{\prime}$ & $32^{\circ} 23^{\prime}$ & $07 / 2002$ & $12 / 2002$ & 0 & 1 \\
\hline 5348 & Longview $11 \mathrm{SE}$ & Rusk & 407 & $94^{\circ} 39^{\prime}$ & $32^{\circ} 20^{\prime}$ & $08 / 1975$ & $12 / 2002$ & 88 & 28 \\
\hline 5358 & Loraine & Mitchell & 2,270 & $100^{\circ} 43^{\prime}$ & $32^{\circ} 25^{\prime}$ & $01 / 1940$ & $02 / 1984$ & 79 & 45 \\
\hline 5398 & Lovelady & Houston & 302 & $95^{\circ} 27^{\prime}$ & $31^{\circ} 08^{\prime}$ & $01 / 1940$ & $11 / 1986$ & 93 & 47 \\
\hline 5410 & Lubbock $9 \mathrm{~N}$ & Lubbock & 3,245 & $101^{\circ} 49^{\prime}$ & $33^{\circ} 41^{\prime}$ & 09/1942 & $12 / 2002$ & 92 & 61 \\
\hline 5411 & Lubbock International Airport & Lubbock & 3,254 & $101^{\circ} 49^{\prime}$ & $33^{\circ} 39^{\prime}$ & $04 / 1940$ & $12 / 2002$ & 97 & 53 \\
\hline 5424 & Lufkin Angelina County Ap. & Angelina & 288 & $94^{\circ} 45^{\prime}$ & $31^{\circ} 14^{\prime}$ & 08/1948 & $12 / 2002$ & 59 & 7 \\
\hline 5429 & Luling & Caldwell & 400 & $97^{\circ} 39^{\prime}$ & $29^{\circ} 40^{\prime}$ & 09/1943 & $09 / 1965$ & 81 & 23 \\
\hline 5431 & Luling 5 ENE & Caldwell $^{1}$ & 390 & $97^{\circ} 34^{\prime}$ & $29^{\circ} 42^{\prime}$ & 09/1965 & 03/1967 & 16 & 3 \\
\hline 5461 & Mabank 4 SW & Henderson & 341 & $96^{\circ} 09^{\prime}$ & $32^{\circ} 20^{\prime}$ & $04 / 1940$ & $04 / 1977$ & 90 & 38 \\
\hline 5463 & Mabank 4 SW & Kaufman & 360 & $96^{\circ} 07^{\prime}$ & $32^{\circ} 21^{\prime}$ & 05/1977 & $12 / 2002$ & 79 & 26 \\
\hline 5471 & Madden Arroyo & Hudspeth $^{1}$ & 3,504 & $105^{\circ} 46^{\prime}$ & $31^{\circ} 13^{\prime}$ & $02 / 1942$ & $07 / 1942$ & 40 & 1 \\
\hline 5477 & Madisonville & Madison & 252 & $95^{\circ} 54^{\prime}$ & $30^{\circ} 56^{\prime}$ & $04 / 1957$ & $06 / 1957$ & 18 & 1 \\
\hline 5528 & Malone 3 ENE & Hill & 485 & $96^{\circ} 50^{\prime}$ & $31^{\circ} 56^{\prime}$ & $01 / 1973$ & $12 / 2002$ & 80 & 30 \\
\hline 5579 & Marathon & Brewster & 4,066 & $103^{\circ} 14^{\prime}$ & $30^{\circ} 12^{\prime}$ & $04 / 1948$ & $04 / 1948$ & 7 & 1 \\
\hline 5580 & Marble Falls & Burnet $^{1}$ & 771 & $98^{\circ} 17^{\prime}$ & $30^{\circ} 34^{\prime}$ & $04 / 1957$ & $06 / 1957$ & 22 & 1 \\
\hline 5589 & Marfa CAA Airport & Presidio $^{1}$ & 4,859 & $103^{\circ} 53^{\prime}$ & $30^{\circ} 15^{\prime}$ & $10 / 1947$ & $01 / 1952$ & 70 & 6 \\
\hline
\end{tabular}

Footnote at end of table. 
60 Statistical Characteristics of Storm Interevent Time, Depth, and Duration for Eastern New Mexico, Oklahoma, and Texas

Table 3. National Weather Service hourly rainfall stations in Texas-Continued.

\begin{tabular}{|c|c|c|c|c|c|c|c|c|c|}
\hline $\begin{array}{c}\text { Station } \\
\text { no. }\end{array}$ & Station name & County & $\begin{array}{c}\text { Elevation } \\
\text { (feet above } \\
\text { NGVD 29) }\end{array}$ & $\begin{array}{l}\text { Longitude } \\
\text { (degrees/ } \\
\text { minutes) }\end{array}$ & $\begin{array}{l}\text { Latitude } \\
\text { (degrees/ } \\
\text { minutes) }\end{array}$ & $\begin{array}{l}\text { Beginning } \\
\text { month/ } \\
\text { year }\end{array}$ & $\begin{array}{c}\text { Ending } \\
\text { month/ } \\
\text { year }\end{array}$ & $\begin{array}{l}\text { Percentage } \\
\text { of record } \\
\text { with data }\end{array}$ & $\begin{array}{l}\text { Years } \\
\text { with } \\
\text { record }\end{array}$ \\
\hline 5590 & Marfa Airport & Presidio $^{1}$ & 4,833 & $104^{\circ} 01^{\prime}$ & $30^{\circ} 22^{\prime}$ & $07 / 1942$ & $12 / 1946$ & 88 & 5 \\
\hline 5591 & Marfa Charco M R & Jeff Davis ${ }^{1}$ & 5,305 & $104^{\circ} 07^{\prime}$ & $30^{\circ} 29^{\prime}$ & $03 / 1949$ & 07/1968 & 94 & 20 \\
\hline 5592 & Marfa $9 \mathrm{~W}$ & Presidio $^{1}$ & 4,754 & $104^{\circ} 10^{\prime}$ & $30^{\circ} 18^{\prime}$ & $02 / 1952$ & 05/1969 & 87 & 18 \\
\hline 5594 & Marfa Ryan & Presidio $^{1}$ & 4,705 & $104^{\circ} 19^{\prime}$ & $30^{\circ} 22^{\prime}$ & $05 / 1951$ & 03/1959 & 62 & 9 \\
\hline 5595 & Marfa 25 WSW & Presidio $^{1}$ & 5,023 & $104^{\circ} 24^{\prime}$ & $30^{\circ} 12^{\prime}$ & $04 / 1954$ & $02 / 1955$ & 5 & 2 \\
\hline 5596 & Marfa 2 & Presidio & 4,730 & $104^{\circ} 00^{\prime}$ & $30^{\circ} 18^{\prime}$ & $07 / 1968$ & $12 / 2002$ & 92 & 34 \\
\hline 5600 & Marfa 16 SSE & Presidio & 4,662 & $103^{\circ} 53^{\prime}$ & $30^{\circ} 08^{\prime}$ & $06 / 1969$ & 06/1981 & 88 & 13 \\
\hline 5618 & Marshall & Harrison & 352 & $94^{\circ} 21^{\prime}$ & $32^{\circ} 32^{\prime}$ & $04 / 1957$ & $06 / 1957$ & 23 & 1 \\
\hline 5650 & Mason & Mason & 1,430 & $99^{\circ} 14^{\prime}$ & $30^{\circ} 44^{\prime}$ & $04 / 1957$ & $06 / 1957$ & 21 & 1 \\
\hline 5656 & Matador 2 & Motley & 2,390 & $100^{\circ} 49^{\prime}$ & $34^{\circ} 00^{\prime}$ & $10 / 1965$ & $12 / 2002$ & 91 & 38 \\
\hline 5658 & Matador & Motley & 2,290 & $100^{\circ} 49^{\prime}$ & $34^{\circ} 01^{\prime}$ & $12 / 1941$ & $10 / 1965$ & 86 & 25 \\
\hline 5661 & Mathis 4 SSW & Jim Wells & 138 & $97^{\circ} 52^{\prime}$ & $28^{\circ} 02^{\prime}$ & $09 / 1997$ & $12 / 2002$ & 79 & 6 \\
\hline 5666 & Matthews & Colorado $^{1}$ & 151 & $96^{\circ} 20^{\prime}$ & $29^{\circ} 31^{\prime}$ & $03 / 1965$ & $10 / 1965$ & 54 & 1 \\
\hline 5695 & Maypearl & Ellis & 530 & $97^{\circ} 01^{\prime}$ & $32^{\circ} 19^{\prime}$ & $12 / 1943$ & $02 / 1995$ & 85 & 53 \\
\hline 5742 & Medina & Bandera & 1,705 & $99^{\circ} 15^{\prime}$ & $29^{\circ} 48^{\prime}$ & $10 / 1947$ & $05 / 1948$ & 26 & 2 \\
\hline 5766 & McKinney $3 \mathrm{~S}$ & Collin & 595 & $96^{\circ} 37^{\prime}$ & $33^{\circ} 10^{\prime}$ & $04 / 1957$ & $06 / 1957$ & 22 & 1 \\
\hline 5770 & McLean & Gray & 2,860 & $100^{\circ} 36^{\prime}$ & $35^{\circ} 14^{\prime}$ & $10 / 1940$ & $12 / 2002$ & 91 & 63 \\
\hline 5775 & McClellan Creek Dam & Gray $^{1}$ & 3,002 & $100^{\circ} 52^{\prime}$ & $35^{\circ} 14^{\prime}$ & $02 / 1940$ & $09 / 1940$ & 34 & 1 \\
\hline 5779 & McMahan & Caldwell $^{1}$ & 4,554 & $97^{\circ} 31^{\prime}$ & $29^{\circ} 51^{\prime}$ & $06 / 1969$ & $01 / 1970$ & 26 & 2 \\
\hline 5840 & Mercury & McCulloch & 1,440 & $99^{\circ} 10^{\prime}$ & $31^{\circ} 25^{\prime}$ & $02 / 1965$ & $01 / 1975$ & 83 & 10 \\
\hline 5890 & Midland International Airport & Midland & 2,862 & $102^{\circ} 11^{\prime}$ & $31^{\circ} 56^{\prime}$ & $02 / 1941$ & $12 / 2002$ & 95 & 59 \\
\hline 5891 & Midland 4 ENE & Midland & 2,776 & $102^{\circ} 01^{\prime}$ & $32^{\circ} 01^{\prime}$ & $10 / 1947$ & $10 / 1953$ & 78 & 7 \\
\hline 5897 & Midlothian 2 & Ellis & 750 & $96^{\circ} 59^{\prime}$ & $32^{\circ} 29^{\prime}$ & $01 / 1974$ & $12 / 2002$ & 87 & 28 \\
\hline 5957 & Mineral Wells 1 SSW & Palo Pinto & 845 & $98^{\circ} 07^{\prime}$ & $32^{\circ} 47^{\prime}$ & $03 / 1952$ & $12 / 2002$ & 88 & 51 \\
\hline 5958 & Mineral Wells FCWOS Ap. & Palo Pinto & 930 & $98^{\circ} 03^{\prime}$ & $32^{\circ} 46^{\prime}$ & $03 / 1948$ & $11 / 1952$ & 90 & 5 \\
\hline 5973 & Mission Pumping Station & Hidalgo 1 & 131 & $98^{\circ} 19^{\prime}$ & $26^{\circ} 12^{\prime}$ & $10 / 1947$ & $05 / 1950$ & 63 & 4 \\
\hline 5996 & Moline & Mills & 1,385 & $98^{\circ} 19^{\prime}$ & $31^{\circ} 24^{\prime}$ & $05 / 1940$ & $12 / 2002$ & 93 & 63 \\
\hline 6017 & Monte Alto & Willacy $^{1}$ & 39 & $97^{\circ} 58^{\prime}$ & $26^{\circ} 33^{\prime}$ & $10 / 1947$ & $05 / 1950$ & 64 & 4 \\
\hline 6024 & Montgomery & Montgomery & 320 & $95^{\circ} 41^{\prime}$ & $30^{\circ} 23^{\prime}$ & $03 / 1940$ & $06 / 1948$ & 47 & 9 \\
\hline 6050 & Monkstown & Fannin & 480 & $95^{\circ} 56^{\prime}$ & $33^{\circ} 48^{\prime}$ & $12 / 1972$ & $08 / 1973$ & 16 & 2 \\
\hline 6104 & Mount Locke & Jeff Davis & 6,790 & $104^{\circ} 01^{\prime}$ & $30^{\circ} 42^{\prime}$ & $06 / 1948$ & $12 / 2002$ & 92 & 55 \\
\hline 6108 & Mount Pleasant & Titus & 425 & $95^{\circ} 00^{\prime}$ & $33^{\circ} 10^{\prime}$ & $02 / 1940$ & $12 / 2002$ & 84 & 62 \\
\hline 6136 & Muleshoe 2 & Bailey & 3,800 & $102^{\circ} 44^{\prime}$ & $34^{\circ} 13^{\prime}$ & $06 / 1941$ & $12 / 2002$ & 86 & 62 \\
\hline 6166 & Myers Ranch & Val Verde $^{1}$ & ${ }^{1} 1,826$ & $100^{\circ} 49^{\prime}$ & $29^{\circ} 48^{\prime}$ & $04 / 1940$ & $12 / 1941$ & 79 & 2 \\
\hline 6176 & Nacogdoches & Nacogdoches & 312 & $94^{\circ} 39^{\prime}$ & $31^{\circ} 36^{\prime}$ & $12 / 1947$ & $10 / 1961$ & 78 & 9 \\
\hline 6177 & Nacogdoches & Nacogdoches & 435 & $94^{\circ} 38^{\prime}$ & $31^{\circ} 37^{\prime}$ & $10 / 1947$ & $12 / 2002$ & 82 & 51 \\
\hline 6210 & Navarro Mills Dam & Navarro & 454 & $96^{\circ} 42^{\prime}$ & $31^{\circ} 57^{\prime}$ & $08 / 1962$ & $12 / 2002$ & 94 & 41 \\
\hline 6211 & Navasota & Grimes $^{1}$ & 220 & $96^{\circ} 07^{\prime}$ & $30^{\circ} 23^{\prime}$ & $11 / 1941$ & $06 / 1952$ & 57 & 12 \\
\hline 6270 & New Boston & Bowie & 345 & $94^{\circ} 24^{\prime}$ & $33^{\circ} 27^{\prime}$ & $10 / 1973$ & $12 / 2002$ & 89 & 30 \\
\hline 6275 & New Braunfels Airport & Guadalupe & 645 & $98^{\circ} 02^{\prime}$ & $29^{\circ} 42^{\prime}$ & $09 / 2002$ & $12 / 2002$ & 0 & 1 \\
\hline 6276 & New Braunfels & Comal & 710 & $98^{\circ} 07^{\prime}$ & $29^{\circ} 44^{\prime}$ & $04 / 1957$ & $06 / 1957$ & 22 & 1 \\
\hline 6335 & New Summerfield $2 \mathrm{~W}$ & Cherokee & 380 & $95^{\circ} 18^{\prime}$ & $31^{\circ} 58^{\prime}$ & $08 / 1962$ & $12 / 2002$ & 88 & 41 \\
\hline 6434 & North Fork Dam & Williamson & 883 & $97^{\circ} 43^{\prime}$ & $30^{\circ} 41^{\prime}$ & 08/1980 & $06 / 1981$ & 27 & 2 \\
\hline 6504 & O’Donnell & Lynn & 3,046 & $101^{\circ} 49^{\prime}$ & $32^{\circ} 58^{\prime}$ & $05 / 1940$ & $12 / 2002$ & 92 & 63 \\
\hline 6558 & Oklaunion & Wilbarger ${ }^{1}$ & ${ }^{1} 1,245$ & $99^{\circ} 05^{\prime}$ & $34^{\circ} 08^{\prime}$ & $02 / 1940$ & $09 / 1940$ & 64 & 1 \\
\hline 6615 & Old 8 Camp 6666 & King & 1,790 & $100^{\circ} 11^{\prime}$ & $33^{\circ} 33^{\prime}$ & $09 / 1973$ & $10 / 2000$ & 67 & 23 \\
\hline 6660 & Oplin near SCS no. 174 & Callahan $^{1}$ & 2,001 & $99^{\circ} 31^{\prime}$ & $32^{\circ} 10^{\prime}$ & $02 / 1940$ & $11 / 1943$ & 70 & 4 \\
\hline 6663 & Orange Dupont & Orange $^{1}$ & 10 & $93^{\circ} 45^{\prime}$ & $30^{\circ} 04^{\prime}$ & $11 / 1948$ & $12 / 1952$ & 25 & 5 \\
\hline 6734 & Ozona 1 SSW & Crockett & 2,340 & $101^{\circ} 12^{\prime}$ & $30^{\circ} 41^{\prime}$ & $04 / 1940$ & $12 / 2002$ & 91 & 12 \\
\hline 6736 & Ozona 8 WSW & Crockett & 2,550 & $101^{\circ} 20^{\prime}$ & $30^{\circ} 40^{\prime}$ & $02 / 1951$ & $10 / 2002$ & 94 & 52 \\
\hline 6740 & Paducah & Cottle & 1,900 & $100^{\circ} 18^{\prime}$ & $34^{\circ} 00^{\prime}$ & $04 / 1957$ & $06 / 1957$ & 21 & 1 \\
\hline 6750 & Palacios Municipal Airport & Matagorda & 12 & $96^{\circ} 15^{\prime}$ & $28^{\circ} 43^{\prime}$ & $08 / 1940$ & $12 / 2002$ & 55 & 5 \\
\hline 6757 & Palestine $2 \mathrm{NE}$ & Anderson & 465 & $95^{\circ} 36^{\prime}$ & $31^{\circ} 46^{\prime}$ & $02 / 1940$ & $12 / 2002$ & 91 & 63 \\
\hline 6775 & Pampa Weather Bureau Airport & Gray & 3,232 & $100^{\circ} 58^{\prime}$ & $35^{\circ} 32^{\prime}$ & $01 / 1941$ & $09 / 1953$ & 86 & 13 \\
\hline 6776 & Pampa 2 & Gray & 3,150 & $100^{\circ} 58^{\prime}$ & $35^{\circ} 33^{\prime}$ & $10 / 1953$ & $12 / 2002$ & 91 & 50 \\
\hline
\end{tabular}

Footnote at end of table. 
Table 361

Table 3. National Weather Service hourly rainfall stations in Texas-Continued.

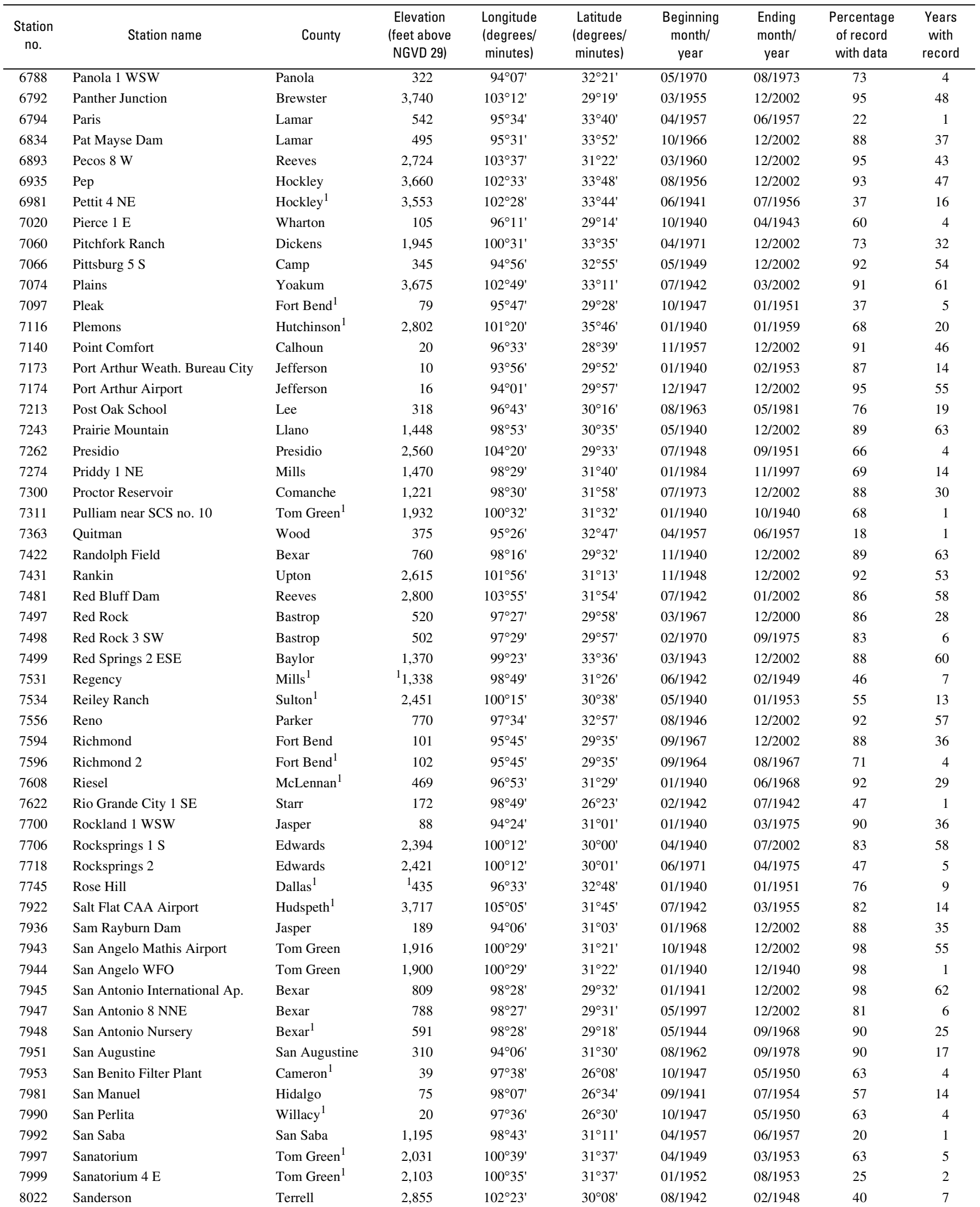

Footnote at end of table. 
Table 3. National Weather Service hourly rainfall stations in Texas-Continued.

\begin{tabular}{|c|c|c|c|c|c|c|c|c|c|}
\hline $\begin{array}{c}\text { Station } \\
\text { no. }\end{array}$ & Station name & County & $\begin{array}{c}\text { Elevation } \\
\text { (feet above } \\
\text { NGVD 29) }\end{array}$ & $\begin{array}{l}\text { Longitude } \\
\text { (degrees/ } \\
\text { minutes) }\end{array}$ & $\begin{array}{l}\text { Latitude } \\
\text { (degrees/ } \\
\text { minutes) }\end{array}$ & $\begin{array}{l}\text { Beginning } \\
\text { month/ } \\
\text { year }\end{array}$ & $\begin{array}{l}\text { Ending } \\
\text { month/ } \\
\text { year }\end{array}$ & $\begin{array}{l}\text { Percentage } \\
\text { of record } \\
\text { with data }\end{array}$ & $\begin{array}{l}\text { Years } \\
\text { with } \\
\text { record }\end{array}$ \\
\hline 8023 & Sanderson 5 NNW & Terrell & 3,080 & $102^{\circ} 25^{\prime}$ & $30^{\circ} 12^{\prime}$ & $10 / 1947$ & $12 / 2002$ & 94 & 56 \\
\hline 8047 & Santa Anna & Coleman & 1,745 & $99^{\circ} 18^{\prime}$ & $31^{\circ} 44^{\prime}$ & $05 / 1940$ & $12 / 2002$ & 86 & 63 \\
\hline 8060 & Santa Rosa & Cameron $^{1}$ & 49 & $97^{\circ} 50^{\prime}$ & $26^{\circ} 15^{\prime}$ & $10 / 1947$ & $05 / 1950$ & 65 & 4 \\
\hline 8062 & Santo & Palo Pinto ${ }^{1}$ & 833 & $98^{\circ} 14^{\prime}$ & $32^{\circ} 37^{\prime}$ & $10 / 1941$ & $07 / 1942$ & 37 & 2 \\
\hline 8068 & Saragosa & Reeves $^{1}$ & 2,992 & $103^{\circ} 39^{\prime}$ & $31^{\circ} 04^{\prime}$ & $07 / 1942$ & $12 / 1945$ & 39 & 3 \\
\hline 8081 & Sarita $7 \mathrm{E}$ & Kenedy & 38 & $97^{\circ} 41^{\prime}$ & $27^{\circ} 13^{\prime}$ & $09 / 1941$ & $12 / 2002$ & 91 & 62 \\
\hline 8089 & Satsuma & Harris & 112 & $95^{\circ} 37^{\prime}$ & $29^{\circ} 54^{\prime}$ & $10 / 1947$ & $05 / 1948$ & 32 & 2 \\
\hline 8221 & Seymour & Baylor & 1,287 & $99^{\circ} 16^{\prime}$ & $33^{\circ} 35^{\prime}$ & $04 / 1957$ & $06 / 1957$ & 21 & 1 \\
\hline 8252 & Sheffield & Pecos & 2,170 & $101^{\circ} 49^{\prime}$ & $30^{\circ} 41^{\prime}$ & $07 / 1942$ & $12 / 2002$ & 93 & 61 \\
\hline 8265 & Sheperd 2 SW & San Jacinto ${ }^{1}$ & 180 & $95^{\circ} 00^{\prime}$ & $30^{\circ} 29^{\prime}$ & $04 / 1940$ & $01 / 1965$ & 90 & 26 \\
\hline 8289 & Shinnery Store & Collingsworth ${ }^{1}$ & 1,942 & $100^{\circ} 00^{\prime}$ & $34^{\circ} 54^{\prime}$ & $08 / 1944$ & 09/1949 & 21 & 5 \\
\hline 8305 & Sierra Blanca 2 E & Hudspeth & 4,590 & $105^{\circ} 21^{\prime}$ & $31^{\circ} 10^{\prime}$ & $07 / 1942$ & $12 / 2002$ & 82 & 59 \\
\hline 8335 & Simms 4 WNW & Bowie & 322 & $94^{\circ} 34^{\prime}$ & $33^{\circ} 22^{\prime}$ & 03/1944 & $10 / 1973$ & 90 & 30 \\
\hline 8400 & Smith Brothers Ranch & Jeff Davis ${ }^{1}$ & ${ }^{1} 5,351$ & $104^{\circ} 06^{\prime}$ & $30^{\circ} 29^{\prime}$ & $01 / 1942$ & $01 / 1949$ & 77 & 8 \\
\hline 8445 & Somerville & Burleson $^{1}$ & 249 & $96^{\circ} 31^{\prime}$ & $30^{\circ} 21^{\prime}$ & $03 / 1940$ & $10 / 1963$ & 91 & 24 \\
\hline 8446 & Somerville Dam & Burleson & 263 & $96^{\circ} 32^{\prime}$ & $30^{\circ} 20^{\prime}$ & $10 / 1963$ & 03/1994 & 91 & 32 \\
\hline 8451 & Sonora Valiant Ranch & Sutton $^{1}$ & 2,441 & $100^{\circ} 12^{\prime}$ & $30^{\circ} 39^{\prime}$ & $02 / 1953$ & 03/1961 & 73 & 9 \\
\hline 8531 & Spicewood & Burnet & 850 & $98^{\circ} 09^{\prime}$ & $30^{\circ} 28^{\prime}$ & 03/1968 & $12 / 2002$ & 89 & 35 \\
\hline 8541 & Spring Branch 3 SSW & Comal & 1,190 & $98^{\circ} 26^{\prime}$ & $29^{\circ} 50^{\prime}$ & $01 / 1995$ & $07 / 1997$ & 74 & 3 \\
\hline 8544 & Spring Branch $2 \mathrm{SE}$ & Comal & 1,119 & $98^{\circ} 22^{\prime}$ & $29^{\circ} 51^{\prime}$ & $05 / 1988$ & $12 / 2002$ & 76 & 13 \\
\hline 8545 & Spring Creek & San Saba ${ }^{1}$ & ${ }^{1} 1,310$ & $98^{\circ} 48^{\prime}$ & $31^{\circ} 20^{\prime}$ & $09 / 1941$ & $03 / 1942$ & 24 & 2 \\
\hline 8563 & Springtown $4 \mathrm{~S}$ & Parker & 1,053 & $97^{\circ} 40^{\prime}$ & $32^{\circ} 54^{\prime}$ & $11 / 1977$ & $12 / 2002$ & 91 & 26 \\
\hline 8566 & Spur & Dickens & 2,297 & $100^{\circ} 52^{\prime}$ & $33^{\circ} 28^{\prime}$ & $11 / 1947$ & 03/1964 & 76 & 18 \\
\hline 8583 & Stamford 1 & Jones & 1,640 & $99^{\circ} 48^{\prime}$ & $32^{\circ} 56^{\prime}$ & $10 / 1947$ & $12 / 2002$ & 75 & 24 \\
\hline 8584 & Stamford 2 & Jones & 1,601 & $99^{\circ} 48^{\prime}$ & $32^{\circ} 57^{\prime}$ & $01 / 1940$ & $04 / 1980$ & 87 & 40 \\
\hline 8623 & Stephenville $1 \mathrm{~N}$ & Erath & 1,309 & $98^{\circ} 11^{\prime}$ & $32^{\circ} 14^{\prime}$ & $07 / 1940$ & $12 / 2002$ & 90 & 37 \\
\hline 8625 & Stephenville $6 \mathrm{SW}$ & Erath & 1,450 & $98^{\circ} 19^{\prime}$ & $32^{\circ} 10^{\prime}$ & $10 / 1947$ & $07 / 1975$ & 94 & 29 \\
\hline 8630 & Sterling City & Sterling & 2,265 & $100^{\circ} 58^{\prime}$ & $31^{\circ} 50^{\prime}$ & $02 / 1977$ & $12 / 2002$ & 84 & 26 \\
\hline 8631 & Sterling City 8 NE & Sterling & 2,710 & $100^{\circ} 52^{\prime}$ & $31^{\circ} 55^{\prime}$ & 05/1949 & 02/1977 & 83 & 29 \\
\hline 8646 & Stillhouse Hollow Dam & Bell & 706 & $97^{\circ} 31^{\prime}$ & $31^{\circ} 02^{\prime}$ & $05 / 1964$ & $12 / 2002$ & 89 & 39 \\
\hline 8647 & Stinnett & Hutchinson & 3,130 & $101^{\circ} 27^{\prime}$ & $35^{\circ} 50^{\prime}$ & $01 / 1959$ & 04/1992 & 86 & 33 \\
\hline 8677 & Stony & Denton $^{1}$ & 702 & $97^{\circ} 20^{\prime}$ & $33^{\circ} 14^{\prime}$ & $09 / 1946$ & $01 / 1954$ & 50 & 9 \\
\hline 8696 & Strawn 8 NNE & Palo Pinto & 1,180 & $98^{\circ} 28^{\prime}$ & $32^{\circ} 39^{\prime}$ & $04 / 1957$ & $06 / 1957$ & 22 & 1 \\
\hline 8743 & Sulphur Springs & Hopkins & 495 & $95^{\circ} 38^{\prime}$ & $33^{\circ} 09^{\prime}$ & $10 / 1941$ & $12 / 2002$ & 92 & 61 \\
\hline 8761 & Sunray 4 SW & Moore & 3,543 & $101^{\circ} 52^{\prime}$ & $35^{\circ} 58^{\prime}$ & $05 / 1955$ & $08 / 1984$ & 83 & 30 \\
\hline 8778 & Swan 4 NW & Smith & 450 & $95^{\circ} 25^{\prime}$ & $32^{\circ} 27^{\prime}$ & $08 / 1957$ & $12 / 2002$ & 88 & 46 \\
\hline 8845 & Tarpley & Bandera & 1,404 & $99^{\circ} 17^{\prime}$ & $29^{\circ} 40^{\prime}$ & $03 / 1940$ & $12 / 2002$ & 91 & 63 \\
\hline 8859 & Tatum & Rusk & 269 & $94^{\circ} 31^{\prime}$ & $32^{\circ} 18^{\prime}$ & $01 / 1940$ & $08 / 1975$ & 90 & 36 \\
\hline 8898 & Telephone & Fannin & 541 & $96^{\circ} 01^{\prime}$ & $33^{\circ} 47^{\prime}$ & $09 / 1959$ & $12 / 1972$ & 89 & 14 \\
\hline 8908 & Temple SCS 7R & Bell $^{1}$ & ${ }^{1} 595$ & $97^{\circ} 21^{\prime}$ & $31^{\circ} 06^{\prime}$ & $01 / 1940$ & $09 / 1940$ & 67 & 1 \\
\hline 8910 & Temple & Bell & 635 & $97^{\circ} 19^{\prime}$ & $31^{\circ} 05^{\prime}$ & $04 / 1957$ & $06 / 1957$ & 22 & 1 \\
\hline 8911 & Temple $3 \mathrm{SE}$ & Bell $^{1}$ & 650 & $97^{\circ} 21^{\prime}$ & $31^{\circ} 03^{\prime}$ & $10 / 1947$ & $06 / 1968$ & 74 & 20 \\
\hline 8924 & Terlingua & Brewster $^{1}$ & 2,592 & $103^{\circ} 33^{\prime}$ & $29^{\circ} 18^{\prime}$ & $07 / 1942$ & $02 / 1963$ & 81 & 22 \\
\hline 8929 & Terrell & Kaufman & 515 & $96^{\circ} 17^{\prime}$ & $32^{\circ} 46^{\prime}$ & $04 / 1957$ & $06 / 1957$ & 21 & 1 \\
\hline 8942 & Texarkana & Bowie & 390 & $94^{\circ} 05^{\prime}$ & $33^{\circ} 25^{\prime}$ & $09 / 1968$ & $12 / 2002$ & 89 & 35 \\
\hline 8944 & Texarkana Dam & Cass & 282 & $94^{\circ} 10^{\prime}$ & $33^{\circ} 18^{\prime}$ & $11 / 1955$ & $05 / 1972$ & 90 & 18 \\
\hline 8996 & Thompsons 3 WSW & Fort Bend & 70 & $95^{\circ} 37^{\prime}$ & $29^{\circ} 28^{\prime}$ & $05 / 1957$ & $12 / 2002$ & 88 & 46 \\
\hline 9014 & Throckmorton & Throckmorton & 1,370 & $99^{\circ} 11^{\prime}$ & $33^{\circ} 10^{\prime}$ & $04 / 1957$ & $06 / 1957$ & 19 & 1 \\
\hline 9037 & Tinnin Ranch & Reeves $^{1}$ & 3,232 & $103^{\circ} 59^{\prime}$ & $31^{\circ} 19^{\prime}$ & $07 / 1942$ & $12 / 1969$ & 92 & 28 \\
\hline 9106 & Toyah & Reeves & 2,812 & $103^{\circ} 48^{\prime}$ & $31^{\circ} 18^{\prime}$ & $12 / 1969$ & 05/1977 & 73 & 9 \\
\hline 9107 & Toyahvale & Reeves $^{1}$ & 3,343 & $103^{\circ} 46^{\prime}$ & $30^{\circ} 56^{\prime}$ & $01 / 1946$ & 02/1949 & 33 & 4 \\
\hline 9129 & Tri City Airport & Hidalgo $^{1}$ & 200 & $98^{\circ} 21^{\prime}$ & $26^{\circ} 24^{\prime}$ & $10 / 1947$ & $05 / 1950$ & 65 & 4 \\
\hline 9163 & Truscott $3 \mathrm{~W}$ & Knox & 1,571 & $99^{\circ} 51^{\prime}$ & $33^{\circ} 45^{\prime}$ & $02 / 1940$ & $12 / 2002$ & 85 & 63 \\
\hline 9213 & Tyler Pounds Field & Smith & 544 & $95^{\circ} 24^{\prime}$ & $32^{\circ} 21^{\prime}$ & 08/1948 & $12 / 2002$ & 46 & 5 \\
\hline
\end{tabular}


Table 363

Table 3. National Weather Service hourly rainfall stations in Texas-Continued.

\begin{tabular}{|c|c|c|c|c|c|c|c|c|c|}
\hline $\begin{array}{c}\text { Station } \\
\text { no. }\end{array}$ & Station name & County & $\begin{array}{c}\text { Elevation } \\
\text { (feet above } \\
\text { NGVD 29) }\end{array}$ & $\begin{array}{c}\text { Longitude } \\
\text { (degrees/ } \\
\text { minutes) }\end{array}$ & $\begin{array}{l}\text { Latitude } \\
\text { (degrees/ } \\
\text { minutes) }\end{array}$ & $\begin{array}{c}\text { Beginning } \\
\text { month/ } \\
\text { year }\end{array}$ & $\begin{array}{c}\text { Ending } \\
\text { month/ } \\
\text { year }\end{array}$ & $\begin{array}{c}\text { Percentage } \\
\text { of record } \\
\text { with data }\end{array}$ & $\begin{array}{l}\text { Years } \\
\text { with } \\
\text { record }\end{array}$ \\
\hline 9214 & Tyler 5 NE & Smith & 489 & $95^{\circ} 16^{\prime}$ & $32^{\circ} 24^{\prime}$ & $04 / 1957$ & $06 / 1957$ & 22 & 1 \\
\hline 9222 & Uhland near SCS Lockhart & Caldwell $^{1}$ & ${ }^{1} 615$ & $97^{\circ} 47^{\prime}$ & $29^{\circ} 57^{\prime}$ & $02 / 1940$ & $09 / 1943$ & 89 & 4 \\
\hline 9248 & Upland & Upton $^{1}$ & 2,602 & $102^{\circ} 00^{\prime}$ & $31^{\circ} 23^{\prime}$ & $08 / 1942$ & $07 / 1948$ & 67 & 7 \\
\hline 9266 & Uvalde near SCS no. 1 & Maverick $^{1}$ & 850 & $100^{\circ} 08^{\prime}$ & $29^{\circ} 04^{\prime}$ & $01 / 1940$ & $12 / 1941$ & 95 & 2 \\
\hline 9270 & Valentine & Jeff Davis & 4,449 & $104^{\circ} 29^{\prime}$ & $30^{\circ} 35^{\prime}$ & $05 / 1959$ & $12 / 2002$ & 94 & 44 \\
\hline 9295 & Van Horn & Culberson & 4,065 & $104^{\circ} 50^{\prime}$ & $31^{\circ} 02^{\prime}$ & $07 / 1948$ & $09 / 1951$ & 60 & 4 \\
\hline 9304 & Vancourt & Tom Green & 1,903 & $100^{\circ} 11^{\prime}$ & $31^{\circ} 21^{\prime}$ & $12 / 1947$ & $12 / 1947$ & 3 & 1 \\
\hline 9307 & Vancourt $5 \mathrm{SW}$ & Tom Green ${ }^{1}$ & 1,903 & $100^{\circ} 14^{\prime}$ & $31^{\circ} 21^{\prime}$ & $01 / 1940$ & $01 / 1949$ & 78 & 10 \\
\hline 9328 & Vega near SCS 4R & Oldham $^{1}$ & 3,934 & $102^{\circ} 25^{\prime}$ & $35^{\circ} 19^{\prime}$ & $03 / 1940$ & $02 / 1944$ & 78 & 5 \\
\hline 9329 & Vega SCS 101-2-46 & Oldham $^{1}$ & 4,022 & $102^{\circ} 26^{\prime}$ & $35^{\circ} 15^{\prime}$ & $01 / 1941$ & $05 / 1941$ & 36 & 1 \\
\hline 9345 & Veribest near SCS no. 8 & Tom Green ${ }^{1}$ & 1,821 & $100^{\circ} 15^{\prime}$ & $31^{\circ} 27^{\prime}$ & $01 / 1940$ & $03 / 1940$ & 11 & 1 \\
\hline 9363 & Victoria Weather Bureau Ap. & Victoria $^{1}$ & 115 & $97^{\circ} 05^{\prime}$ & $28^{\circ} 47^{\prime}$ & $01 / 1946$ & $06 / 1961$ & 91 & 16 \\
\hline 9364 & Victoria ASOS & Victoria & 115 & $96^{\circ} 55^{\prime}$ & $28^{\circ} 51^{\prime}$ & $03 / 1940$ & $12 / 2002$ & 98 & 48 \\
\hline 9365 & Victoria CP\&L & Victoria & 61 & $97^{\circ} 00^{\prime}$ & $28^{\circ} 47^{\prime}$ & $06 / 1948$ & $02 / 1949$ & 32 & 2 \\
\hline 9371 & View near SCS Abilene & Haskell $^{1}$ & 1,752 & $99^{\circ} 52^{\prime}$ & $33^{\circ} 22^{\prime}$ & $01 / 1940$ & $08 / 1943$ & 83 & 4 \\
\hline 9417 & Waco Dam & McLennan & 495 & $97^{\circ} 13^{\prime}$ & $31^{\circ} 36^{\prime}$ & $02 / 1965$ & $12 / 2002$ & 91 & 38 \\
\hline 9419 & Waco Regional Airport & McLennan & 500 & $97^{\circ} 13^{\prime}$ & $31^{\circ} 36^{\prime}$ & $02 / 1941$ & $12 / 2002$ & 98 & 62 \\
\hline 9435 & Walcott & Deaf Smith & 4,114 & $102^{\circ} 59^{\prime}$ & $34^{\circ} 56^{\prime}$ & $05 / 1971$ & $02 / 1975$ & 35 & 5 \\
\hline 9491 & Washington State Park & Washington & 215 & $96^{\circ} 09^{\prime}$ & $30^{\circ} 19^{\prime}$ & $06 / 1952$ & $12 / 2002$ & 87 & 51 \\
\hline 9499 & Water Valley & Tom Green & 2,120 & $100^{\circ} 43^{\prime}$ & $31^{\circ} 40^{\prime}$ & $10 / 1953$ & $12 / 2002$ & 92 & 50 \\
\hline 9522 & Waxahachie & Ellis & 630 & $96^{\circ} 51^{\prime}$ & $32^{\circ} 25^{\prime}$ & $04 / 1957$ & $06 / 1957$ & 19 & 1 \\
\hline 9527 & Wayside & Armstrong & 3,400 & $101^{\circ} 32^{\prime}$ & $34^{\circ} 47^{\prime}$ & $06 / 1941$ & $12 / 2002$ & 81 & 62 \\
\hline 9532 & Weatherford & Parker & 955 & $97^{\circ} 46^{\prime}$ & $32^{\circ} 44^{\prime}$ & $10 / 1947$ & $12 / 2002$ & 94 & 56 \\
\hline 9544 & Webster Ranch & Schleicher $^{1}$ & 2,192 & $100^{\circ} 11^{\prime}$ & $30^{\circ} 53^{\prime}$ & $09 / 1961$ & $12 / 1961$ & 13 & 1 \\
\hline 9565 & Wellington & Collingsworth & 2,040 & $100^{\circ} 12^{\prime}$ & $34^{\circ} 50^{\prime}$ & $10 / 1949$ & $12 / 2002$ & 88 & 41 \\
\hline 9570 & Wellington 2 & Collingsworth & 2,031 & $100^{\circ} 13^{\prime}$ & $34^{\circ} 51^{\prime}$ & $08 / 1971$ & $01 / 1983$ & 78 & 13 \\
\hline 9574 & Wellington $11 \mathrm{SE}$ & Collingsworth & 1,910 & $100^{\circ} 02^{\prime}$ & $34^{\circ} 48^{\prime}$ & $02 / 1983$ & $05 / 1983$ & 33 & 1 \\
\hline 9588 & Weslaco $2 \mathrm{E}$ & Hidalgo & 75 & $97^{\circ} 58^{\prime}$ & $26^{\circ} 09^{\prime}$ & $10 / 1947$ & $12 / 2002$ & 86 & 53 \\
\hline 9665 & Wheelock & Robertson & 420 & $96^{\circ} 23^{\prime}$ & $30^{\circ} 54^{\prime}$ & $06 / 1940$ & $12 / 2002$ & 91 & 63 \\
\hline 9715 & Whitney Dam & Bosque & 574 & $97^{\circ} 22^{\prime}$ & $31^{\circ} 51^{\prime}$ & $06 / 1952$ & $12 / 2002$ & 96 & 51 \\
\hline 9729 & Wichita Falls Municipal Ap. & Wichita & 1,017 & $98^{\circ} 29^{\prime}$ & $33^{\circ} 58^{\prime}$ & $05 / 1940$ & $12 / 2002$ & 98 & 63 \\
\hline 9772 & William Harris Reservoir & Brazoria & 39 & $95^{\circ} 33^{\prime}$ & $29^{\circ} 15^{\prime}$ & $03 / 1948$ & $03 / 1964$ & 86 & 17 \\
\hline 9814 & Wimberley 2 & Hays & 1,112 & $98^{\circ} 04^{\prime}$ & $29^{\circ} 58^{\prime}$ & $01 / 1940$ & $06 / 1984$ & 36 & 2 \\
\hline 9815 & Wimberley $1 \mathrm{NW}$ & Hays & 830 & $98^{\circ} 03^{\prime}$ & $30^{\circ} 00^{\prime}$ & $02 / 1989$ & $12 / 2002$ & 92 & 14 \\
\hline 9816 & Winchell 1 WNW & Brown & 1,381 & $99^{\circ} 11^{\prime}$ & $31^{\circ} 29^{\prime}$ & $02 / 1949$ & $03 / 1953$ & 44 & 5 \\
\hline 9817 & Winchell & Brown & 1,460 & $99^{\circ} 10^{\prime}$ & $31^{\circ} 27^{\prime}$ & $04 / 1953$ & $12 / 2002$ & 81 & 41 \\
\hline 9829 & Wink & Winkler & 2,790 & $103^{\circ} 09^{\prime}$ & $31^{\circ} 46^{\prime}$ & $07 / 1942$ & $04 / 1997$ & 93 & 51 \\
\hline 9830 & Wink FAA Airport & Winkler & 2,807 & $103^{\circ} 12^{\prime}$ & $31^{\circ} 46^{\prime}$ & $10 / 1947$ & $11 / 1952$ & 81 & 6 \\
\hline 9858 & Wolf Creek Dam & Ochiltree & 2,703 & $100^{\circ} 40^{\prime}$ & $36^{\circ} 14^{\prime}$ & $05 / 1941$ & $10 / 1974$ & 93 & 34 \\
\hline 9893 & Woodson & Throckmorton & 1,263 & $99^{\circ} 03^{\prime}$ & $33^{\circ} 01^{\prime}$ & $08 / 1941$ & $12 / 2002$ & 90 & 62 \\
\hline 9916 & Wright Patman Lock and Dam & Cass & 282 & $94^{\circ} 10^{\prime}$ & $33^{\circ} 18^{\prime}$ & $11 / 1981$ & $12 / 2002$ & 74 & 22 \\
\hline 9976 & Zapata 3 SW & Zapata & 320 & $99^{\circ} 15^{\prime}$ & $26^{\circ} 52^{\prime}$ & 03/1940 & $12 / 2002$ & 90 & 63 \\
\hline
\end{tabular}

${ }^{1}$ County name or elevation not available in Hydrosphere (2003); supplied by authors. 
64 Statistical Characteristics of Storm Interevent Time, Depth, and Duration for Eastern New Mexico, Oklahoma, and Texas

Table 4. Selected storm statistics for station 4570 Jayton, Texas, illustrating influence of minimum interevent time on statistics.

\begin{tabular}{lccccccc}
\hline \multirow{2}{*}{\multicolumn{1}{c}{ Statistic (units) }} & \multicolumn{7}{c}{ Minimum interevent time } \\
\cline { 2 - 7 } & 6 hours & 8 hours & 12 hours & 18 hours & 24 hours & 48 hours & 72 hours \\
\hline Mean storm interevent time (days) & 8.50 & 9.04 & 9.81 & 10.70 & 11.48 & 13.34 & 14.96 \\
Mean storm depth (inches) & .445 & .474 & .516 & .566 & .611 & .724 & .831 \\
90th-percentile storm depth (inches) & 1.10 & 1.20 & 1.30 & 1.40 & 1.50 & 1.85 & 2.10 \\
50th-percentile storm duration (hours) & 1 & 2 & 3 & 3 & 4 & 6 & 10 \\
\hline
\end{tabular}

Table 5. Regionwide or statewide record-length, weighted-average storm depth L-moments.

[L-CV, coefficient of L-variation (L-scale/mean); --, dimensionless]

\begin{tabular}{|c|c|c|c|c|c|c|}
\hline \multirow{2}{*}{$\begin{array}{c}\text { Region or } \\
\text { State and } \\
\text { minimum } \\
\text { interevent } \\
\text { time } \\
\text { (hours) }\end{array}$} & \multirow{2}{*}{$\begin{array}{l}\text { Record } \\
\text { length } \\
\text { (hours) }\end{array}$} & \multicolumn{5}{|c|}{ Storm depth L-moments } \\
\hline & & $\begin{array}{l}\text { Mean } \\
\text { (inches) }\end{array}$ & $\begin{array}{c}\text { L-CV } \\
(--)\end{array}$ & $\begin{array}{c}\text { L-skew } \\
(--)\end{array}$ & $\begin{array}{c}\text { L-kurtosis } \\
(--)\end{array}$ & $\begin{array}{c}\text { Tau5 } \\
(--)\end{array}$ \\
\hline \multicolumn{7}{|c|}{ Eastern New Mexico (92 stations) } \\
\hline 6 & $18,755,163$ & 0.276 & 0.554 & 0.530 & 0.305 & 0.178 \\
\hline 8 & $18,755,163$ & .291 & .555 & .515 & .298 & .177 \\
\hline 12 & $18,755,163$ & .315 & .558 & .508 & .291 & .177 \\
\hline 18 & $18,754,611$ & .346 & .563 & .502 & .284 & .175 \\
\hline 24 & $18,754,611$ & .386 & .567 & .495 & .276 & .172 \\
\hline 48 & $18,745,803$ & .474 & .574 & .484 & .263 & .164 \\
\hline 72 & $18,745,803$ & .556 & .582 & .484 & 261 & .162 \\
\hline \multicolumn{7}{|c|}{ Oklahoma (149 stations) } \\
\hline 6 & $33,226,434$ & .523 & .582 & .485 & .249 & .154 \\
\hline 8 & $33,226,434$ & .555 & .579 & .477 & .244 & .153 \\
\hline 12 & $33,223,650$ & .602 & .576 & .469 & .239 & .153 \\
\hline 18 & $33,223,650$ & .660 & .574 & .458 & .233 & .150 \\
\hline 24 & $33,223,650$ & .712 & .572 & .452 & .230 & .149 \\
\hline 48 & $33,221,682$ & .874 & .571 & .442 & .226 & .145 \\
\hline 72 & $33,221,682$ & 1.05 & .568 & .432 & .220 & .140 \\
\hline \multicolumn{7}{|c|}{ Texas (533 stations) } \\
\hline 6 & $103,788,249$ & .489 & .601 & .506 & .272 & .168 \\
\hline 8 & $103,788,249$ & .518 & .598 & .500 & .267 & .168 \\
\hline 12 & $103,788,249$ & .563 & .595 & .492 & .263 & .167 \\
\hline 18 & $103,785,321$ & .619 & .593 & .484 & .258 & .167 \\
\hline 24 & $103,785,321$ & .675 & .590 & .477 & .254 & .166 \\
\hline 48 & $103,773,105$ & .821 & .586 & .463 & .246 & .162 \\
\hline 72 & $103,771,089$ & .964 & .581 & .452 & .238 & .156 \\
\hline
\end{tabular}


Table 6. Regionwide or statewide record-length, weighted-average mean storm interevent time and storm duration L-moments.

[L-CV, coefficient of L-variation (L-scale/mean); --, dimensionless]

\begin{tabular}{|c|c|c|c|c|c|c|c|}
\hline \multirow{2}{*}{$\begin{array}{l}\text { Region or } \\
\text { State and } \\
\text { minimum } \\
\text { interevent } \\
\text { time } \\
\text { (hours) }\end{array}$} & \multirow{2}{*}{$\begin{array}{l}\text { Record } \\
\text { length } \\
\text { (hours) }\end{array}$} & \multirow{2}{*}{$\begin{array}{c}\text { Mean } \\
\text { interevent } \\
\text { time } \\
\text { (days) }\end{array}$} & \multicolumn{5}{|c|}{ Storm duration L-moments } \\
\hline & & & $\begin{array}{c}\text { Mean } \\
\text { (hours) }\end{array}$ & $\begin{array}{c}\mathrm{L-CV} \\
(--)\end{array}$ & $\begin{array}{c}\text { L-skew } \\
(--)\end{array}$ & $\begin{array}{c}\text { L-kurtosis } \\
(--)\end{array}$ & $\begin{array}{c}\text { Tau5 } \\
(--)\end{array}$ \\
\hline \multicolumn{8}{|c|}{ Eastern New Mexico (92 stations) } \\
\hline 6 & $18,755,115$ & 7.90 & 4.12 & 0.522 & 0.511 & 0.244 & 0.152 \\
\hline 8 & $18,754,611$ & 8.31 & 4.71 & .546 & .516 & .249 & .153 \\
\hline 12 & $18,754,611$ & 8.94 & 5.87 & .584 & .529 & .258 & .153 \\
\hline 18 & $18,754,611$ & 9.75 & 7.90 & .618 & .525 & .241 & .128 \\
\hline 24 & $18,754,611$ & 10.71 & 11.17 & 632 & .499 & .211 & .111 \\
\hline 48 & $18,745,803$ & 12.68 & 21.71 & 659 & .494 & .209 & .114 \\
\hline 72 & $18,745,803$ & 14.36 & 35.79 & .674 & .500 & .218 & .122 \\
\hline \multicolumn{8}{|c|}{ Oklahoma (149 stations) } \\
\hline 6 & $33,223,650$ & 6.34 & 5.02 & .516 & .445 & .196 & .131 \\
\hline 8 & $33,223,650$ & 6.70 & 5.70 & .532 & .447 & .197 & .128 \\
\hline 12 & $33,223,650$ & 7.22 & 6.99 & .558 & .454 & .201 & .125 \\
\hline 18 & $33,223,650$ & 7.85 & 9.02 & .582 & .456 & .195 & .114 \\
\hline 24 & $33,223,650$ & 8.39 & 11.33 & .598 & .457 & .193 & .111 \\
\hline 48 & $33,221,682$ & 9.96 & 21.77 & .638 & .473 & .200 & .107 \\
\hline 72 & $33,221,682$ & 11.41 & 37.79 & .649 & .463 & .187 & .103 \\
\hline \multicolumn{8}{|c|}{ Texas (533 stations) } \\
\hline 6 & $103,782,513$ & 7.39 & 4.94 & .525 & .477 & .231 & .155 \\
\hline 8 & $103,782,513$ & 7.80 & 5.62 & .545 & .484 & .236 & .154 \\
\hline 12 & $103,782,513$ & 8.41 & 6.91 & .576 & .495 & .239 & .147 \\
\hline 18 & $103,782,513$ & 9.17 & 9.02 & .604 & .496 & .228 & .129 \\
\hline 24 & $103,782,513$ & 9.89 & 11.68 & .617 & .484 & .211 & .117 \\
\hline 48 & $103,771,785$ & 11.62 & 21.57 & .646 & .482 & .203 & .109 \\
\hline 72 & $103,769,769$ & 13.08 & 35.39 & .657 & .475 & .195 & .107 \\
\hline
\end{tabular}


Table 7. Dimensionless gamma and kappa distributions fit to record-length, weighted-average storm depth L-moments.

[--, dimensionless $]$

\begin{tabular}{|c|c|c|c|c|c|c|}
\hline \multirow{2}{*}{$\begin{array}{l}\text { Region or } \\
\text { State and } \\
\text { minimum } \\
\text { interevent } \\
\text { time } \\
\text { (hours) }\end{array}$} & \multicolumn{2}{|c|}{ Gamma distribution parameters $^{1}$} & \multicolumn{4}{|c|}{ Kappa distribution parameters $^{1}$} \\
\hline & $\begin{array}{c}\theta \\
(--)\end{array}$ & $\begin{array}{c}\beta \\
(--)\end{array}$ & $\begin{array}{c}\xi \\
(--)\end{array}$ & $\begin{array}{c}\alpha \\
(--)\end{array}$ & $\begin{array}{c}\kappa \\
(--)\end{array}$ & $\begin{array}{c}h \\
(--)\end{array}$ \\
\hline \multicolumn{7}{|c|}{ Eastern New Mexico (92 stations) } \\
\hline 6 & 0.7592 & 1.317 & -0.4607 & 0.8958 & -0.2272 & 1.930 \\
\hline 8 & .7554 & 1.324 & -.3352 & .8498 & -.2355 & 1.709 \\
\hline 12 & .7441 & 1.344 & -.3481 & .8744 & -.2231 & 1.690 \\
\hline 18 & .7255 & 1.378 & -.3824 & .9116 & -.2079 & 1.696 \\
\hline 24 & .7111 & 1.406 & -.4173 & .9526 & -.1903 & 1.701 \\
\hline 48 & .6864 & 1.457 & -.4865 & 1.030 & -.1596 & 1.720 \\
\hline 72 & .6593 & 1.517 & -.5445 & 1.071 & -.1506 & 1.759 \\
\hline \multicolumn{7}{|c|}{ Oklahoma (149 stations) } \\
\hline 6 & .6593 & 1.517 & -.8242 & 1.275 & -.08913 & 2.023 \\
\hline 8 & .6694 & 1.494 & -.7607 & 1.253 & -.08716 & 1.945 \\
\hline 12 & .6795 & 1.472 & -.7030 & 1.234 & -.08439 & 1.871 \\
\hline 18 & .6864 & 1.457 & -.6196 & 1.203 & -.08368 & 1.761 \\
\hline 24 & .6934 & 1.442 & -.5706 & 1.183 & -.08398 & 1.701 \\
\hline 48 & 6969 & 1.435 & -.4840 & 1.143 & -.08812 & 1.588 \\
\hline 72 & .7075 & 1.413 & -.4357 & 1.133 & -.08178 & 1.518 \\
\hline \multicolumn{7}{|c|}{ Texas (533 stations) } \\
\hline 6 & .5991 & 1.669 & -.7991 & 1.186 & -.1422 & 2.041 \\
\hline 8 & .6083 & 1.644 & -.7746 & 1.188 & -.1354 & 2.001 \\
\hline 12 & .6175 & 1.619 & -.6883 & 1.151 & -.1389 & 1.896 \\
\hline 18 & .6238 & 1.603 & -.6336 & 1.135 & -.1367 & 1.818 \\
\hline 24 & .6333 & 1.579 & -.5790 & 1.115 & -.1359 & 1.747 \\
\hline 48 & .6462 & 1.548 & -.4868 & 1.086 & -.1326 & 1.617 \\
\hline 72 & .6627 & 1.509 & -.4479 & 1.087 & -.1210 & 1.556 \\
\hline
\end{tabular}

\footnotetext{
${ }^{1}$ Parameters defined in "Glossary" at end of report.
} 
Table 8. Dimensionless gamma and kappa distributions fit to record-length, weighted-average storm duration L-moments.

[--, dimensionless $]$

\begin{tabular}{|c|c|c|c|c|c|c|}
\hline \multirow{2}{*}{$\begin{array}{l}\text { Region or } \\
\text { State and } \\
\text { minimum } \\
\text { interevent } \\
\text { time } \\
\text { (hours) }\end{array}$} & \multicolumn{2}{|c|}{ Gamma distribution parameters $^{1}$} & \multicolumn{4}{|c|}{ Kappa distribution parameters ${ }^{1}$} \\
\hline & $\begin{array}{c}\theta \\
(--)\end{array}$ & $\begin{array}{c}\beta \\
(--)\end{array}$ & $\begin{array}{c}\xi \\
(--)\end{array}$ & $\begin{array}{c}\alpha \\
(--)\end{array}$ & $\begin{array}{c}\kappa \\
(--)\end{array}$ & $\begin{array}{c}h \\
(--)\end{array}$ \\
\hline \multicolumn{7}{|c|}{ Eastern New Mexico (92 stations) } \\
\hline 6 & 0.8931 & 1.120 & -1.586 & 1.793 & 0.04985 & 2.847 \\
\hline 8 & .7906 & 1.265 & -1.702 & 1.848 & .03993 & 2.877 \\
\hline 12 & .6527 & 1.532 & -2.115 & 2.087 & .04684 & 3.075 \\
\hline 18 & .5497 & 1.819 & -3.292 & 3.070 & .1684 & 3.431 \\
\hline 24 & .5119 & 1.953 & -3.796 & 3.742 & .2616 & 3.355 \\
\hline 48 & .4455 & 2.245 & -3.694 & 3.655 & .2430 & 3.237 \\
\hline 72 & .4118 & 2.428 & -3.485 & 3.389 & .1999 & 3.189 \\
\hline \multicolumn{7}{|c|}{ Oklahoma (149 stations) } \\
\hline 6 & .9209 & 1.086 & -1.081 & 1.650 & .08616 & 2.213 \\
\hline 8 & .8487 & 1.178 & -1.169 & 1.714 & .08709 & 2.237 \\
\hline 12 & .7441 & 1.344 & -1.349 & 1.832 & .08765 & 2.311 \\
\hline 18 & .6593 & 1.517 & -1.778 & 2.198 & .1388 & 2.488 \\
\hline 24 & .6083 & 1.644 & -1.999 & 2.385 & .1587 & 2.557 \\
\hline 48 & .4965 & 2.014 & -2.656 & 2.865 & .1869 & 2.820 \\
\hline 72 & .4692 & 2.131 & -3.009 & 3.267 & .2413 & 2.857 \\
\hline \multicolumn{7}{|c|}{ Texas (533 stations) } \\
\hline 6 & .8795 & 1.137 & -0.9128 & 1.384 & -.01524 & 2.214 \\
\hline 8 & .7946 & 1.259 & -1.027 & 1.444 & -.01941 & 2.277 \\
\hline 12 & 6795 & 1.472 & -1.395 & 1.683 & .006370 & 2.496 \\
\hline 18 & .5901 & 1.695 & -2.073 & 2.224 & .08957 & 2.794 \\
\hline 24 & .5525 & 1.810 & -2.483 & 2.648 & .1583 & 2.865 \\
\hline 48 & .4765 & 2.099 & -3.108 & 3.215 & .2159 & 3.010 \\
\hline 72 & .4501 & 2.222 & -3.306 & 3.449 & .2439 & 3.005 \\
\hline
\end{tabular}

${ }^{1}$ Parameters defined in "Glossary" at end of report.

Table 9. Summary statistics and diagnostic statistics of mean storm interevent time maps for each minimum interevent time.

[Record length used as weight factors. Bias computed as observed value for station minus predicted value from map. wSD, weighted standard deviation; RwMSE, root-weighted-mean-square error]

\begin{tabular}{cccc|ccc}
\hline $\begin{array}{c}\text { Minimum } \\
\text { interevent } \\
\text { time } \\
\text { (hours) }\end{array}$ & $\begin{array}{c}\text { No. of } \\
\text { stations }\end{array}$ & $\begin{array}{c}\text { Weighted-mean } \\
\text { storm interevent } \\
\text { time for } \\
\text { study area } \\
\text { (days) }\end{array}$ & $\begin{array}{c}\text { wSD of } \\
\text { storm } \\
\text { interevent } \\
\text { time for } \\
\text { study area } \\
\text { (days) }\end{array}$ & $\begin{array}{c}\text { Weighted-mean } \\
\text { bias of storm } \\
\text { interevent } \\
\text { time map } \\
\text { (days) }\end{array}$ & $\begin{array}{c}\text { RwMSE } \\
\text { of storm } \\
\text { interevent } \\
\text { time map } \\
\text { (days) }\end{array}$ & $\begin{array}{c}\text { Difference } \\
\text { between } \\
\text { wSD and } \\
\text { RwMSE } \\
\text { (percent) }\end{array}$ \\
\hline 6 & 766 & 7.22 & 2.59 & -0.042 & 1.76 & -32.0 \\
8 & 766 & 7.62 & 2.69 & -.046 & 1.84 & -31.6 \\
12 & 766 & 8.22 & 2.84 & -.045 & 1.93 & -32.0 \\
18 & 766 & 8.95 & 3.09 & -.045 & 2.15 & -30.4 \\
24 & 765 & 9.67 & 3.30 & -.053 & 2.31 & -30.0 \\
48 & 765 & 11.38 & 3.74 & -.069 & 2.63 & -29.7 \\
72 & 765 & 12.87 & 4.12 & -.087 & 3.01 & -26.9 \\
\hline
\end{tabular}


Table 10. Summary statistics and diagnostic statistics of mean storm depth maps for each minimum interevent time.

[Record length used as weight factors. Bias computed as observed value for station minus predicted value from map. wSD, weighted standard deviation; RwMSE, root-weighted-mean-square error]

\begin{tabular}{cccc|ccc}
\hline $\begin{array}{c}\text { Minimum } \\
\text { interevent } \\
\text { time } \\
\text { (hours) }\end{array}$ & $\begin{array}{c}\text { No. of } \\
\text { stations }\end{array}$ & $\begin{array}{c}\text { Weighted- } \\
\text { mean storm } \\
\text { depth for } \\
\text { study area } \\
\text { (inches) }\end{array}$ & $\begin{array}{c}\text { wSD of } \\
\text { storm depth } \\
\text { for study area } \\
\text { (inches) }\end{array}$ & $\begin{array}{c}\text { Weighted- } \\
\text { mean bias of } \\
\text { storm depth } \\
\text { map } \\
\text { (inches) }\end{array}$ & $\begin{array}{c}\text { RwMSE of } \\
\text { storm depth } \\
\text { map } \\
\text { (inches) }\end{array}$ & $\begin{array}{c}\text { Difference } \\
\text { between } \\
\text { wSD and } \\
\text { RwMSE } \\
\text { (percent) }\end{array}$ \\
\hline 6 & 755 & 0.470 & 0.114 & $-5.19 \mathrm{e}-5$ & 0.032 & -71.9 \\
8 & 754 & .498 & .124 & $-6.56 \mathrm{e}-5$ & .033 & -73.4 \\
12 & 753 & .541 & .136 & $-2.99 \mathrm{e}-5$ & .036 & -73.5 \\
18 & 751 & .595 & .151 & $4.14 \mathrm{e}-5$ & .039 & -74.2 \\
24 & 751 & .648 & .163 & $1.03 \mathrm{e}-4$ & .043 & -73.6 \\
48 & 745 & .790 & .207 & $8.95 \mathrm{e}-5$ & .059 & -71.5 \\
72 & 744 & .933 & .261 & $8.48 \mathrm{e}-5$ & .076 & -70.9 \\
\hline
\end{tabular}

Table 11. Summary statistics and diagnostic statistics of mean storm duration maps for each minimum interevent time.

[Record length used as weight factors. Bias computed as observed value for station minus value predicted from map. wSD, weighted standard deviation; RwMSE, root-weighted-mean-square error]

\begin{tabular}{cccc|ccc}
\hline $\begin{array}{c}\text { Minimum } \\
\text { interevent } \\
\text { time } \\
\text { (hours) }\end{array}$ & $\begin{array}{c}\text { No. of } \\
\text { stations }\end{array}$ & $\begin{array}{c}\text { Weighted- } \\
\text { mean storm } \\
\text { duration for } \\
\text { study area } \\
\text { (hours) }\end{array}$ & $\begin{array}{c}\text { wSD of } \\
\text { storm } \\
\text { duration for } \\
\text { study area } \\
\text { (hours) }\end{array}$ & $\begin{array}{c}\text { Weighted- } \\
\text { mean bias of } \\
\text { storm duration } \\
\text { map } \\
\text { (hours) }\end{array}$ & $\begin{array}{c}\text { RwMSE } \\
\text { of storm } \\
\text { duration } \\
\text { map } \\
\text { (hours) }\end{array}$ & $\begin{array}{c}\text { Difference } \\
\text { between }\end{array}$ \\
\hline 6 & 753 & 4.86 & 0.887 & 0.006 & 0.600 & $\begin{array}{c}\text { wSD and } \\
\text { RwMSE } \\
\text { (percent) }\end{array}$ \\
\hline 8 & 752 & 5.53 & .975 & .004 & .636 & -32.4 \\
12 & 751 & 6.80 & 1.12 & .004 & .695 & -37.9 \\
18 & 751 & 8.88 & 1.39 & .008 & .816 & -41.3 \\
24 & 751 & 11.5 & 1.76 & .008 & 1.03 & -41.5 \\
48 & 745 & 21.6 & 3.65 & .003 & 2.01 & -44.9 \\
72 & 744 & 35.9 & 7.00 & -.004 & 3.63 & -48.1 \\
\hline
\end{tabular}

Table 12. Mean storm interevent time by county for eastern New Mexico.

\begin{tabular}{|c|c|c|c|c|c|c|c|}
\hline \multirow{2}{*}{ County } & \multicolumn{7}{|c|}{ Mean storm interevent time (days) for each minimum interevent time } \\
\hline & 6 hours & 8 hours & 12 hours & 18 hours & 24 hours & 48 hours & 72 hours \\
\hline Chaves & 9.27 & 9.81 & 10.5 & 11.4 & 12.3 & 14.3 & 15.9 \\
\hline Colfax & 6.48 & 6.79 & 7.30 & 8.02 & 9.04 & 11.1 & 12.8 \\
\hline DeBaca & 9.39 & 9.92 & 10.5 & 11.2 & 12.0 & 13.9 & 15.6 \\
\hline Eddy & 10.6 & 11.1 & 11.9 & 12.8 & 13.7 & 15.7 & 17.5 \\
\hline Guadalupe & 8.20 & 8.63 & 9.23 & 10.0 & 10.9 & 13.0 & 14.7 \\
\hline Lincoln & 7.36 & 7.79 & 8.44 & 9.20 & 10.2 & 12.2 & 13.9 \\
\hline Los Alamos & 6.47 & 6.86 & 7.47 & 8.23 & 9.10 & 11.0 & 12.6 \\
\hline Mora & 6.35 & 6.70 & 7.21 & 7.95 & 9.00 & 11.1 & 12.8 \\
\hline Otero & 9.40 & 9.96 & 10.6 & 11.5 & 12.7 & 14.9 & 16.9 \\
\hline Quay & 9.70 & 10.1 & 10.7 & 11.6 & 12.8 & 15.3 & 17.5 \\
\hline Roosevelt & 8.79 & 9.32 & 9.91 & 10.7 & 11.6 & 13.5 & 15.2 \\
\hline San Miguel & 7.15 & 7.51 & 8.04 & 8.81 & 9.85 & 11.9 & 13.8 \\
\hline
\end{tabular}


Table 1369

Table 13. Mean storm depth by county for eastern New Mexico.

\begin{tabular}{|c|c|c|c|c|c|c|c|}
\hline \multirow{2}{*}{ County } & \multicolumn{7}{|c|}{ Mean storm depth (inches) for each minimum interevent time } \\
\hline & 6 hours & 8 hours & 12 hours & 18 hours & 24 hours & 48 hours & 72 hours \\
\hline Chaves & 0.294 & 0.311 & 0.336 & 0.366 & 0.401 & 0.476 & 0.543 \\
\hline Curry & .333 & .350 & .377 & .412 & .451 & .543 & .634 \\
\hline DeBaca & .280 & .294 & .315 & .339 & .367 & .434 & .498 \\
\hline Eddy & .308 & .322 & .347 & .375 & .405 & .470 & .532 \\
\hline Harding & .280 & .293 & .312 & .339 & .383 & .473 & .553 \\
\hline Lea & .349 & .367 & .394 & .427 & .461 & .541 & .609 \\
\hline Lincoln & .269 & .285 & .312 & .344 & .388 & .487 & .580 \\
\hline Los Alamos & .222 & .236 & .259 & .288 & .322 & .402 & .480 \\
\hline Mora & .252 & .266 & .288 & .321 & .369 & .471 & .568 \\
\hline Santa Fe & .221 & .235 & .256 & .285 & .319 & .395 & .471 \\
\hline Taos & .220 & .234 & .255 & .284 & .328 & .427 & .521 \\
\hline Torrance & .239 & .252 & .274 & .303 & .336 & .413 & .486 \\
\hline Union & .272 & .285 & .303 & .330 & .376 & .467 & .551 \\
\hline
\end{tabular}

Table 14. Mean storm duration by county for eastern New Mexico.

\begin{tabular}{|c|c|c|c|c|c|c|c|}
\hline \multirow{2}{*}{ County } & \multicolumn{7}{|c|}{ Mean storm duration (hours) for each minimum interevent time } \\
\hline & 6 hours & 8 hours & 12 hours & 18 hours & 24 hours & 48 hours & 72 hours \\
\hline Chaves & 4.06 & 4.65 & 5.78 & 7.62 & 10.3 & 18.7 & 29.7 \\
\hline Curry & 4.13 & 4.67 & 5.75 & 7.63 & 10.3 & 19.3 & 32.5 \\
\hline DeBaca & 4.20 & 4.74 & 5.73 & 7.25 & 9.43 & 17.4 & 28.8 \\
\hline Harding & 4.12 & 4.59 & 5.50 & 7.29 & 10.9 & 21.7 & 35.6 \\
\hline Lea & 3.90 & 4.43 & 5.47 & 7.10 & 9.31 & 16.9 & 26.5 \\
\hline Lincoln & 4.31 & 4.99 & 6.29 & 8.44 & 12.0 & 23.9 & 39.5 \\
\hline Los Alamos & 3.82 & 4.47 & 5.79 & 8.04 & 11.5 & 23.0 & 39.0 \\
\hline Mora & 4.13 & 4.74 & 5.91 & 8.26 & 12.6 & 25.8 & 43.3 \\
\hline San Miguel & 3.96 & 4.49 & 5.59 & 7.71 & 11.5 & 22.8 & 38.4 \\
\hline Santa Fe & 4.22 & 4.90 & 6.17 & 8.48 & 11.9 & 23.0 & 38.7 \\
\hline Taos & 3.97 & 4.60 & 5.85 & 8.22 & 12.6 & 27.0 & 45.7 \\
\hline Torrance & 4.19 & 4.80 & 6.00 & 8.15 & 11.3 & 21.8 & 36.2 \\
\hline Union & 4.22 & 4.73 & 5.62 & 7.42 & 11.4 & 23.0 & 38.1 \\
\hline
\end{tabular}


70 Statistical Characteristics of Storm Interevent Time, Depth, and Duration for Eastern New Mexico, Oklahoma, and Texas

Table 15. Mean storm interevent time by county for Oklahoma.

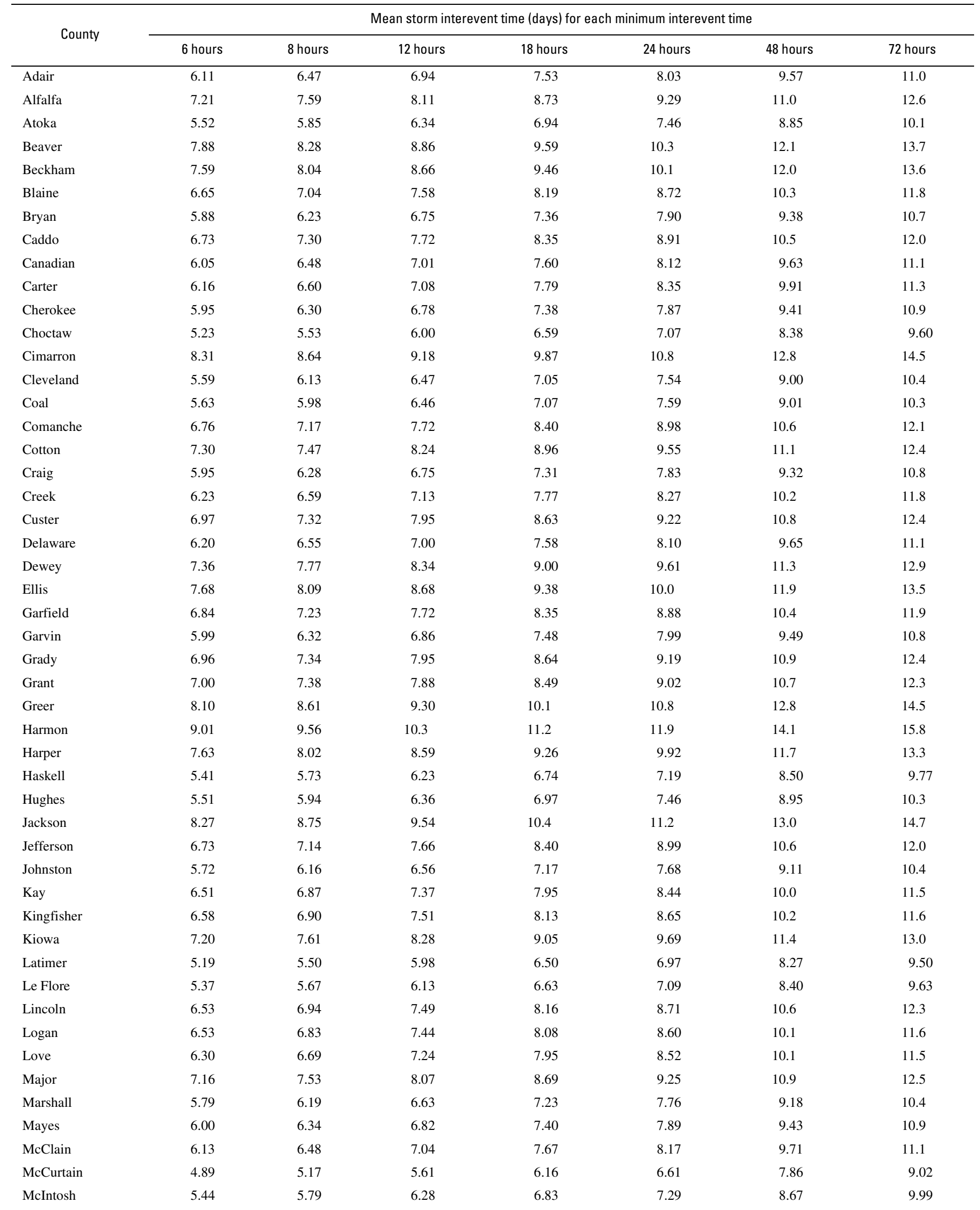


Table 1571

Table 15. Mean storm interevent time by county for Oklahoma—Continued.

\begin{tabular}{|c|c|c|c|c|c|c|c|}
\hline \multirow{2}{*}{ County } & \multicolumn{7}{|c|}{ Mean storm interevent time (days) for each minimum interevent time } \\
\hline & 6 hours & 8 hours & 12 hours & 18 hours & 24 hours & 48 hours & 72 hours \\
\hline Murray & 5.95 & 6.31 & 6.83 & 7.48 & 8.00 & 9.50 & 10.8 \\
\hline Muskogee & 5.56 & 5.90 & 6.38 & 6.96 & 7.41 & 8.84 & 10.2 \\
\hline Noble & 6.42 & 6.80 & 7.29 & 7.90 & 8.41 & 9.91 & 11.3 \\
\hline Nowata & 5.57 & 5.88 & 6.36 & 6.89 & 7.39 & 8.83 & 10.2 \\
\hline Okfuskee & 5.82 & 6.18 & 6.72 & 7.33 & 7.85 & 9.48 & 10.9 \\
\hline Oklahoma & 5.77 & 6.20 & 6.68 & 7.27 & 7.76 & 9.27 & 10.7 \\
\hline Okmulgee & 5.68 & 6.02 & 6.53 & 7.11 & 7.59 & 9.14 & 10.6 \\
\hline Osage & 5.62 & 5.96 & 6.45 & 6.99 & 7.44 & 8.90 & 10.3 \\
\hline Ottawa & 6.01 & 6.36 & 6.81 & 7.37 & 7.91 & 9.41 & 10.9 \\
\hline Pawnee & 5.88 & 6.24 & 6.77 & 7.36 & 7.85 & 9.53 & 11.0 \\
\hline Payne & 6.54 & 6.91 & 7.43 & 8.06 & 8.58 & 10.3 & 11.8 \\
\hline Pittsburg & 5.41 & 5.77 & 6.27 & 6.85 & 7.34 & 8.71 & 10.0 \\
\hline Pontotoc & 5.74 & 6.10 & 6.60 & 7.21 & 7.71 & 9.21 & 10.5 \\
\hline Pottawatomie & 5.81 & 6.24 & 6.71 & 7.31 & 7.83 & 9.43 & 10.9 \\
\hline Pushmataha & 5.01 & 5.32 & 5.77 & 6.33 & 6.81 & 8.10 & 9.29 \\
\hline Roger Mills & 7.68 & 8.10 & 8.73 & 9.48 & 10.2 & 12.0 & 13.6 \\
\hline Rogers & 5.38 & 5.69 & 6.16 & 6.69 & 7.16 & 8.56 & 9.93 \\
\hline Seminole & 5.75 & 6.11 & 6.64 & 7.24 & 7.77 & 9.36 & 10.8 \\
\hline Sequoyah & 5.97 & 6.32 & 6.82 & 7.39 & 7.88 & 9.35 & 10.8 \\
\hline Stephens & 6.80 & 7.17 & 7.68 & 8.41 & 8.98 & 10.5 & 11.9 \\
\hline Texas & 8.24 & 8.61 & 9.20 & 9.91 & 10.7 & 12.5 & 14.2 \\
\hline Tillman & 8.12 & 8.50 & 9.33 & 10.2 & 11.0 & 12.8 & 14.4 \\
\hline Tulsa & 5.35 & 5.65 & 6.12 & 6.64 & 7.06 & 8.45 & 9.79 \\
\hline Wagoner & 5.41 & 5.71 & 6.20 & 6.77 & 7.20 & 8.62 & 10.0 \\
\hline Washington & 5.41 & 5.71 & 6.22 & 6.76 & 7.21 & 8.65 & 9.99 \\
\hline Washita & 7.02 & 7.36 & 8.03 & 8.77 & 9.36 & 11.0 & 12.6 \\
\hline Woods & 7.41 & 7.79 & 8.33 & 8.97 & 9.58 & 11.3 & 12.9 \\
\hline Woodward & 7.55 & 7.93 & 8.48 & 9.13 & 9.78 & 11.6 & 13.2 \\
\hline
\end{tabular}




\section{Statistical Characteristics of Storm Interevent Time, Depth, and Duration for Eastern New Mexico, Oklahoma, and Texas}

Table 16. Mean storm depth by county for Oklahoma.

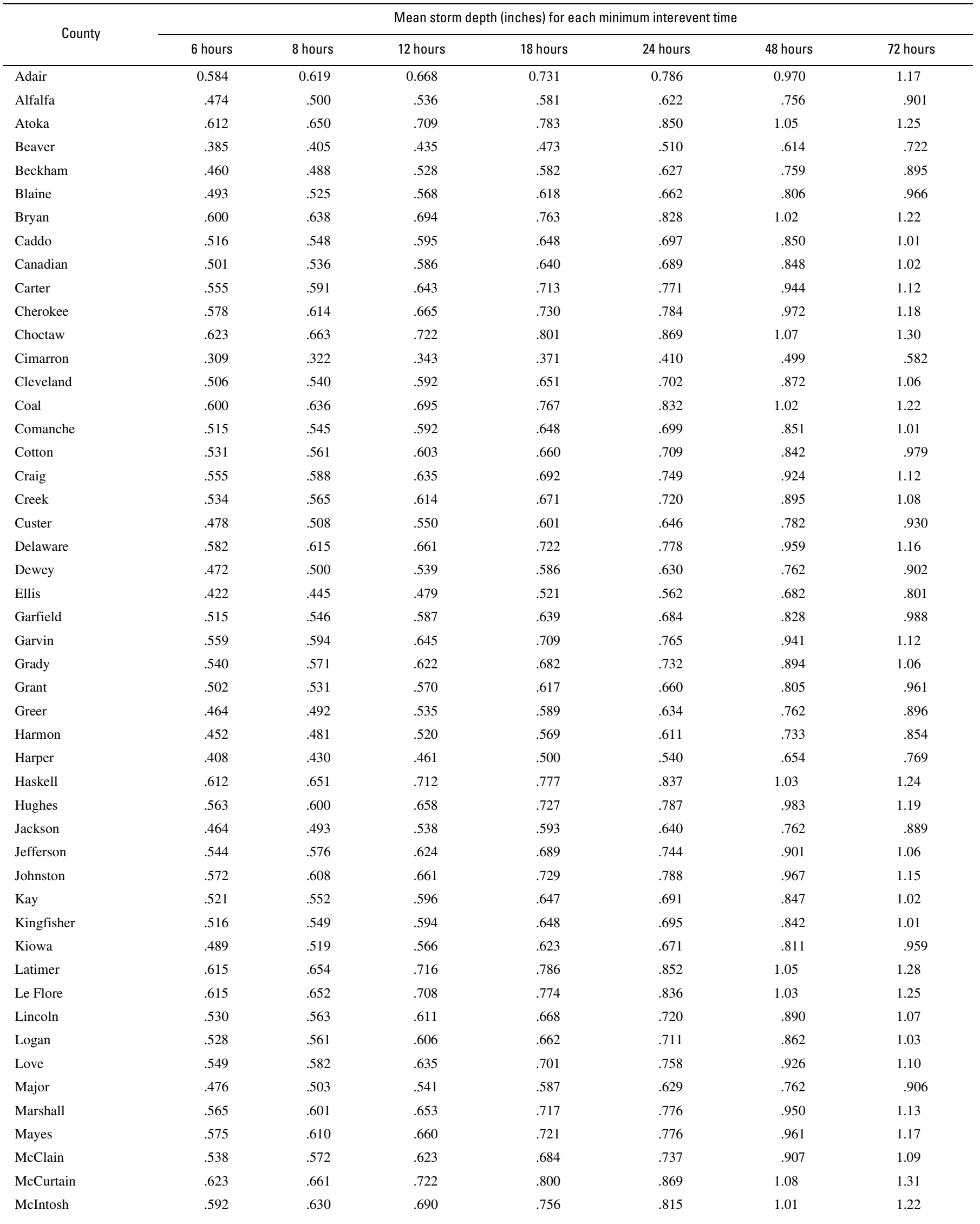


Table $16 \quad 73$

Table 16. Mean storm depth by county for Oklahoma-Continued.

\begin{tabular}{|c|c|c|c|c|c|c|c|}
\hline \multirow{2}{*}{ County } & \multicolumn{7}{|c|}{ Mean storm depth (inches) for each minimum interevent time } \\
\hline & 6 hours & 8 hours & 12 hours & 18 hours & 24 hours & 48 hours & 72 hours \\
\hline Murray & 0.554 & 0.589 & 0.641 & 0.708 & 0.763 & 0.939 & 1.12 \\
\hline Muskogee & .577 & .614 & .668 & .734 & .789 & .976 & 1.19 \\
\hline Noble & .529 & .561 & .606 & .662 & .710 & .864 & 1.03 \\
\hline Nowata & .534 & .567 & .616 & .673 & .728 & .904 & 1.10 \\
\hline Okfuskee & .555 & .590 & .646 & .710 & .768 & .958 & 1.16 \\
\hline Oklahoma & .497 & .530 & .581 & .637 & .686 & .850 & 1.03 \\
\hline Okmulgee & .555 & .589 & .643 & .706 & .760 & .945 & 1.15 \\
\hline Osage & .526 & .559 & .610 & .667 & .714 & .887 & 1.08 \\
\hline Ottawa & .559 & .591 & .637 & .695 & .752 & .926 & 1.12 \\
\hline Pawnee & .532 & .565 & .616 & .674 & .723 & .899 & 1.09 \\
\hline Payne & .533 & .565 & .609 & .665 & .713 & .872 & 1.04 \\
\hline Pittsburg & .587 & .626 & .687 & .757 & .820 & 1.01 & 1.22 \\
\hline Pontotoc & .565 & .599 & .655 & .723 & .781 & .967 & 1.16 \\
\hline Pottawatomie & .535 & .570 & .623 & .685 & .741 & .922 & 1.11 \\
\hline Pushmataha & .617 & .657 & .718 & .795 & .865 & 1.07 & 1.30 \\
\hline Roger Mills & .453 & .478 & .517 & .566 & .611 & .739 & .867 \\
\hline Rogers & .535 & .568 & .618 & .678 & .732 & .913 & 1.12 \\
\hline Seminole & .553 & .589 & .645 & .711 & .770 & .963 & 1.16 \\
\hline Sequoyah & .584 & .620 & .672 & .734 & .789 & .969 & 1.17 \\
\hline Stephens & .556 & .588 & .633 & .700 & .753 & .909 & 1.07 \\
\hline Texas & .352 & .368 & .395 & .427 & .463 & .555 & .652 \\
\hline Tillman & .502 & .532 & .580 & .639 & .693 & .825 & .956 \\
\hline Tulsa & .527 & .559 & .607 & .664 & .713 & .886 & 1.09 \\
\hline Wagoner & .545 & .579 & .630 & .694 & .744 & .928 & 1.14 \\
\hline Washington & .525 & .559 & .609 & .668 & .718 & .897 & 1.09 \\
\hline Washita & .485 & .515 & .558 & .614 & .660 & .798 & .947 \\
\hline Woods & .468 & .502 & .544 & .584 & .709 & .837 & .468 \\
\hline Woodward & .453 & .486 & .526 & .567 & .688 & .809 & .453 \\
\hline
\end{tabular}




\section{Statistical Characteristics of Storm Interevent Time, Depth, and Duration for Eastern New Mexico, Oklahoma, and Texas}

Table 17. Mean storm duration by county for Oklahoma.

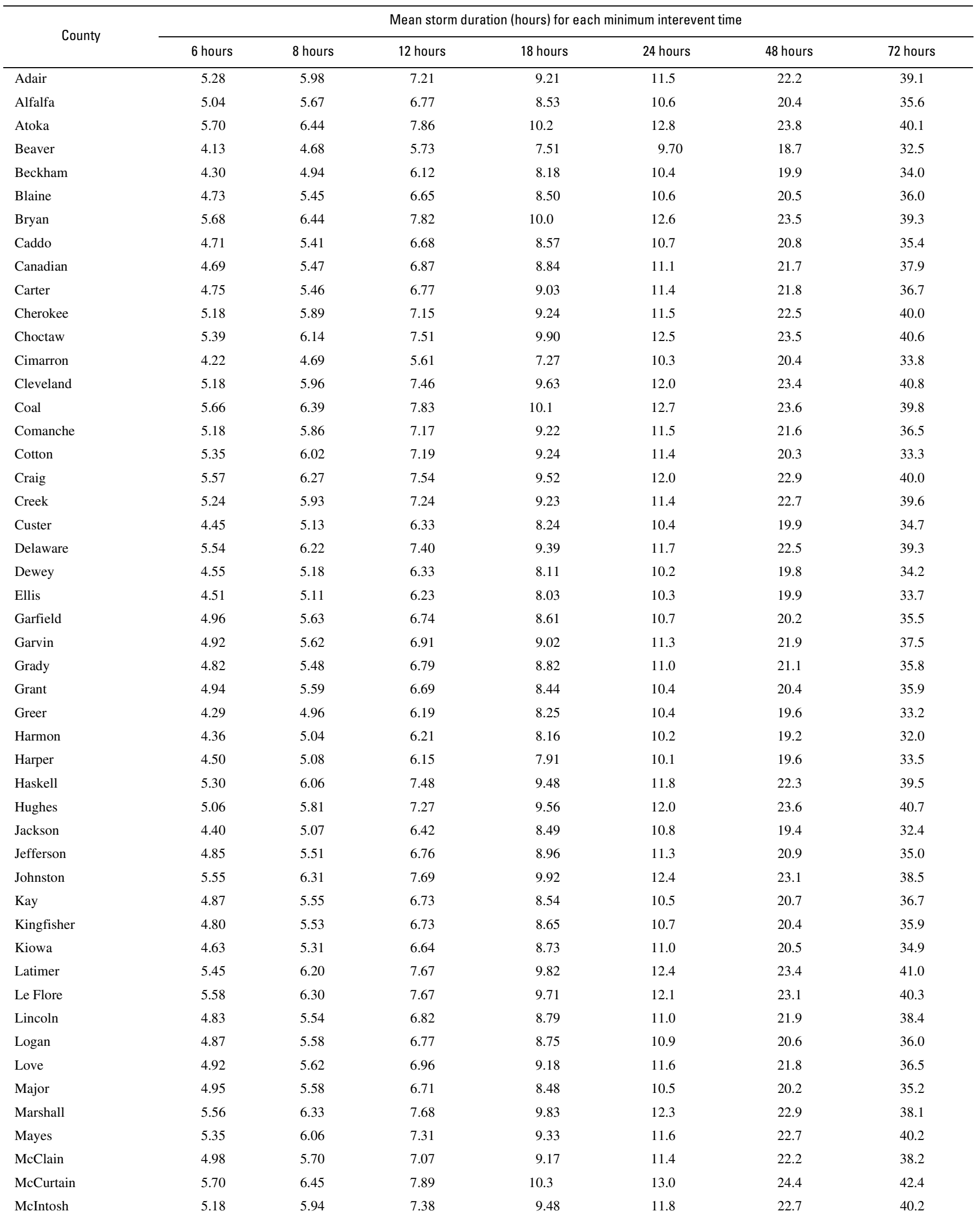


Table $17 \quad 75$

Table 17. Mean storm duration by county for Oklahoma - Continued.

\begin{tabular}{|c|c|c|c|c|c|c|c|}
\hline \multirow{2}{*}{ County } & \multicolumn{7}{|c|}{ Mean storm duration (hours) for each minimum interevent time } \\
\hline & 6 hours & 8 hours & 12 hours & 18 hours & 24 hours & 48 hours & 72 hours \\
\hline Murray & 4.93 & 5.65 & 6.98 & 9.16 & 11.5 & 22.1 & 37.5 \\
\hline Muskogee & 5.27 & 6.01 & 7.37 & 9.51 & 11.8 & 22.8 & 40.7 \\
\hline Noble & 4.80 & 5.49 & 6.68 & 8.61 & 10.7 & 20.5 & 36.2 \\
\hline Nowata & 5.39 & 6.11 & 7.45 & 9.46 & 11.9 & 23.1 & 40.7 \\
\hline Okfuskee & 4.97 & 5.70 & 7.12 & 9.24 & 11.7 & 23.2 & 40.1 \\
\hline Oklahoma & 5.15 & 5.93 & 7.41 & 9.54 & 11.9 & 23.0 & 40.4 \\
\hline Okmulgee & 5.22 & 5.94 & 7.34 & 9.45 & 11.7 & 23.1 & 40.6 \\
\hline Osage & 5.03 & 5.75 & 7.11 & 9.12 & 11.2 & 22.3 & 39.7 \\
\hline Ottawa & 5.67 & 6.38 & 7.62 & 9.61 & 12.1 & 22.9 & 39.9 \\
\hline Pawnee & 5.25 & 5.96 & 7.37 & 9.42 & 11.6 & 22.7 & 39.8 \\
\hline Payne & 4.96 & 5.64 & 6.81 & 8.74 & 10.9 & 21.0 & 36.7 \\
\hline Pittsburg & 5.23 & 6.01 & 7.48 & 9.72 & 12.2 & 23.2 & 40.4 \\
\hline Pontotoc & 5.02 & 5.72 & 7.14 & 9.34 & 11.7 & 22.8 & 38.9 \\
\hline Pottawatomie & 4.98 & 5.72 & 7.15 & 9.27 & 11.7 & 23.1 & 40.0 \\
\hline Pushmataha & 5.40 & 6.15 & 7.60 & 9.96 & 12.6 & 24.0 & 41.6 \\
\hline Roger Mills & 4.42 & 5.04 & 6.22 & 8.14 & 10.4 & 19.9 & 33.5 \\
\hline Rogers & 5.51 & 6.26 & 7.64 & 9.77 & 12.2 & 23.7 & 42.3 \\
\hline Seminole & 4.91 & 5.64 & 7.08 & 9.24 & 11.7 & 23.4 & 40.3 \\
\hline Sequoyah & 5.23 & 5.95 & 7.25 & 9.23 & 11.4 & 22.0 & 38.8 \\
\hline Stephens & 4.95 & 5.60 & 6.74 & 8.94 & 11.2 & 20.6 & 34.7 \\
\hline Texas & 4.06 & 4.55 & 5.57 & 7.23 & 9.55 & 18.4 & 31.9 \\
\hline Tillman & 4.81 & 5.46 & 6.84 & 8.98 & 11.4 & 20.2 & 32.7 \\
\hline Tulsa & 5.57 & 6.30 & 7.67 & 9.74 & 12.0 & 23.3 & 41.8 \\
\hline Wagoner & 5.44 & 6.19 & 7.58 & 9.80 & 12.0 & 23.5 & 42.6 \\
\hline Washington & 5.16 & 5.90 & 7.28 & 9.34 & 11.6 & 23.0 & 40.9 \\
\hline Washita & 4.48 & 5.15 & 6.37 & 8.43 & 10.6 & 20.1 & 34.7 \\
\hline Woods & 4.84 & 5.44 & 6.53 & 8.26 & 10.4 & 20.1 & 34.4 \\
\hline Woodward & 4.71 & 5.30 & 6.39 & 8.10 & 10.3 & 19.9 & 33.9 \\
\hline
\end{tabular}


76 Statistical Characteristics of Storm Interevent Time, Depth, and Duration for Eastern New Mexico, Oklahoma, and Texas

Table 18. Mean storm interevent time by county for Texas.

\begin{tabular}{|c|c|c|c|c|c|c|c|}
\hline \multirow{2}{*}{ County } & \multicolumn{7}{|c|}{ Mean storm interevent time (days) for each minimum interevent time } \\
\hline & 6 hours & 8 hours & 12 hours & 18 hours & 24 hours & 48 hours & 72 hours \\
\hline Anderson & 5.34 & 5.68 & 6.12 & 6.67 & 7.21 & 8.52 & 9.76 \\
\hline Andrews & 9.66 & 10.2 & 10.9 & 11.7 & 12.4 & 14.2 & 15.8 \\
\hline Angelina & 5.04 & 5.31 & 5.77 & 6.29 & 6.88 & 8.24 & 9.56 \\
\hline Aransas & 5.56 & 5.88 & 6.42 & 7.11 & 7.80 & 9.39 & 10.8 \\
\hline Archer & 7.02 & 7.30 & 8.05 & 8.74 & 9.38 & 11.0 & 12.4 \\
\hline Armstrong & 7.46 & 7.91 & 8.54 & 9.28 & 10.0 & 12.0 & 13.6 \\
\hline Atascosa & 7.28 & 7.67 & 8.35 & 9.13 & 9.94 & 11.7 & 13.1 \\
\hline Austin & 5.70 & 6.10 & 6.52 & 7.16 & 7.85 & 9.35 & 10.7 \\
\hline Bailey & 8.52 & 8.93 & 9.61 & 10.4 & 11.3 & 13.3 & 15.0 \\
\hline Bandera & 6.85 & 7.36 & 8.02 & 8.87 & 9.57 & 11.3 & 12.7 \\
\hline Bastrop & 5.75 & 6.16 & 6.73 & 7.42 & 8.04 & 9.67 & 11.0 \\
\hline Baylor & 8.14 & 8.52 & 9.28 & 10.1 & 10.8 & 12.7 & 14.3 \\
\hline Bee & 6.58 & 6.93 & 7.52 & 8.25 & 9.09 & 10.8 & 12.3 \\
\hline Bell & 6.42 & 6.77 & 7.24 & 7.85 & 8.43 & 9.94 & 11.3 \\
\hline Bexar & 6.11 & 6.40 & 7.07 & 7.82 & 8.53 & 10.1 & 11.4 \\
\hline Blanco & 6.53 & 6.85 & 7.50 & 8.20 & 8.79 & 10.3 & 11.5 \\
\hline Borden & 8.71 & 9.18 & 9.91 & 10.8 & 11.6 & 13.5 & 15.0 \\
\hline Bosque & 6.52 & 7.06 & 7.50 & 8.18 & 8.81 & 10.4 & 11.8 \\
\hline Bowie & 5.09 & 5.39 & 5.84 & 6.40 & 6.89 & 8.24 & 9.44 \\
\hline Brazoria & 5.05 & 5.33 & 5.77 & 6.34 & 7.02 & 8.50 & 9.89 \\
\hline Brazos & 5.58 & 5.91 & 6.41 & 7.06 & 7.74 & 9.22 & 10.5 \\
\hline Brewster & 11.3 & 12.2 & 12.4 & 13.3 & 14.5 & 16.9 & 18.9 \\
\hline Briscoe & 8.28 & 8.71 & 9.45 & 10.3 & 11.0 & 13.0 & 14.7 \\
\hline Brooks & 10.3 & 11.2 & 11.7 & 12.9 & 14.2 & 16.9 & 18.5 \\
\hline Brown & 8.66 & 8.82 & 9.83 & 10.7 & 11.4 & 13.4 & 14.9 \\
\hline Burleson & 5.84 & 6.14 & 6.63 & 7.30 & 7.91 & 9.39 & 10.7 \\
\hline Burnet & 6.67 & 7.00 & 7.64 & 8.28 & 8.85 & 10.4 & 11.7 \\
\hline Caldwell & 6.71 & 7.25 & 7.85 & 8.66 & 9.31 & 11.0 & 12.6 \\
\hline Calhoun & 5.05 & 5.35 & 5.83 & 6.43 & 7.10 & 8.60 & 9.88 \\
\hline Callahan & 6.65 & 7.11 & 7.68 & 8.43 & 9.14 & 10.7 & 12.0 \\
\hline Cameron & 5.81 & 6.24 & 6.84 & 7.63 & 8.57 & 10.3 & 11.8 \\
\hline Camp & 5.54 & 5.88 & 6.38 & 6.95 & 7.47 & 8.87 & 10.1 \\
\hline Carson & 7.52 & 7.98 & 8.56 & 9.30 & 10.0 & 12.0 & 13.6 \\
\hline Cass & 5.14 & 5.42 & 5.87 & 6.39 & 6.92 & 8.26 & 9.48 \\
\hline Castro & 9.13 & 9.67 & 10.4 & 11.2 & 12.1 & 14.2 & 16.0 \\
\hline Chambers & 4.28 & 4.55 & 5.01 & 5.59 & 6.27 & 7.69 & 9.16 \\
\hline Cherokee & 5.27 & 5.59 & 6.05 & 6.59 & 7.14 & 8.45 & 9.73 \\
\hline Childress & 8.85 & 9.43 & 10.1 & 11.0 & 11.7 & 13.7 & 15.4 \\
\hline Clay & 6.82 & 7.12 & 7.84 & 8.56 & 9.19 & 10.8 & 12.2 \\
\hline Cochran & 9.20 & 9.65 & 10.4 & 11.2 & 12.1 & 14.2 & 16.0 \\
\hline Coke & 9.39 & 9.45 & 10.6 & 11.6 & 12.4 & 14.4 & 15.8 \\
\hline Coleman & 8.55 & 8.99 & 9.71 & 10.5 & 11.3 & 13.3 & 14.8 \\
\hline Collin & 6.08 & 6.45 & 6.94 & 7.53 & 8.05 & 9.46 & 10.8 \\
\hline Collingsworth & 8.80 & 9.37 & 10.0 & 10.9 & 11.6 & 13.8 & 15.5 \\
\hline Colorado & 5.56 & 5.93 & 6.39 & 7.01 & 7.67 & 9.10 & 10.4 \\
\hline Comal & 6.55 & 6.87 & 7.51 & 8.25 & 8.85 & 10.3 & 11.6 \\
\hline Comanche & 7.68 & 7.82 & 8.59 & 9.21 & 9.83 & 11.3 & 12.6 \\
\hline Concho & 8.40 & 8.83 & 9.58 & 10.3 & 11.0 & 12.9 & 14.4 \\
\hline Cooke & 6.56 & 6.91 & 7.51 & 8.21 & 8.80 & 10.4 & 11.8 \\
\hline
\end{tabular}


Table 1877

Table 18. Mean storm interevent time by county for Texas-Continued.

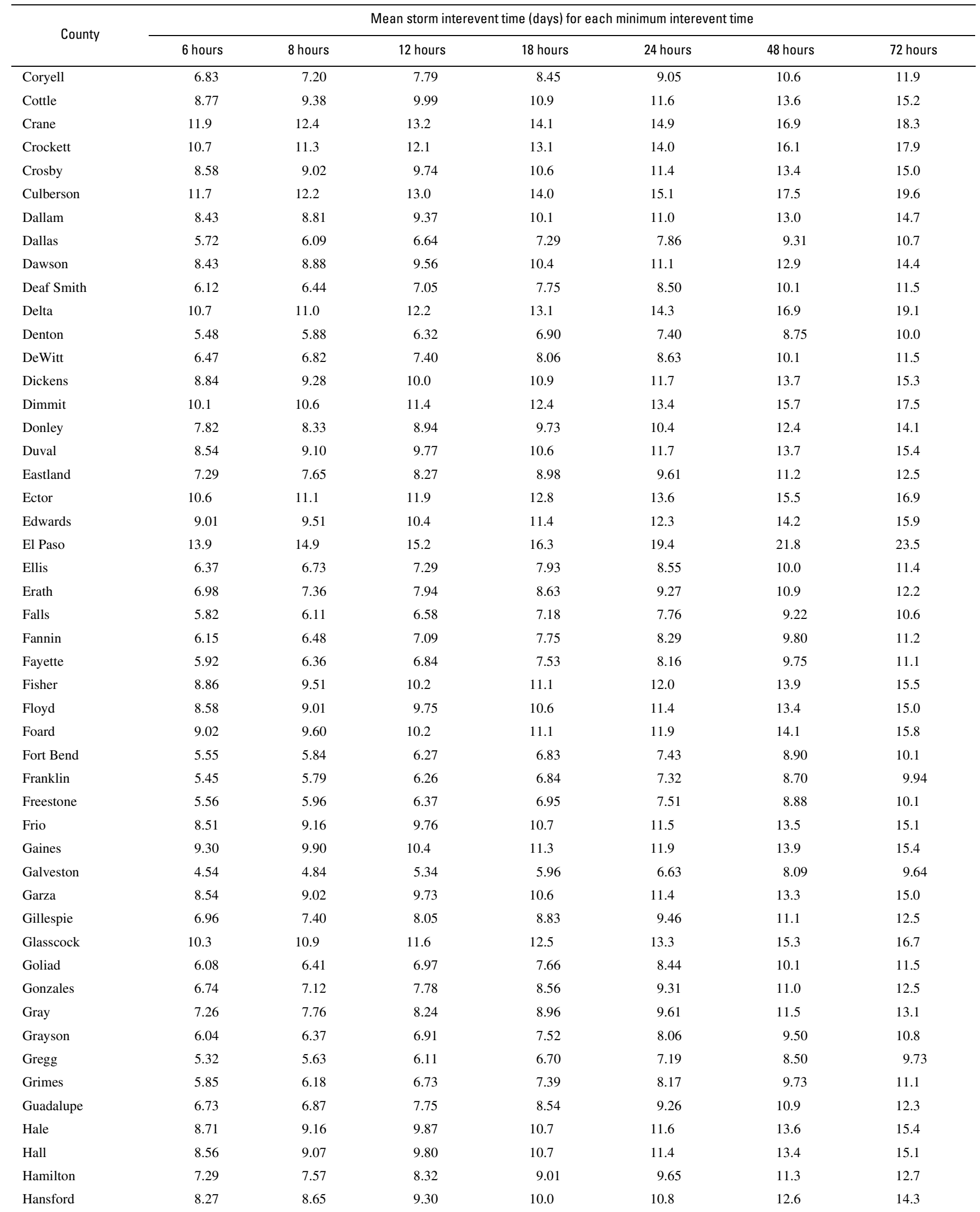


78 Statistical Characteristics of Storm Interevent Time, Depth, and Duration for Eastern New Mexico, Oklahoma, and Texas

Table 18. Mean storm interevent time by county for Texas-Continued.

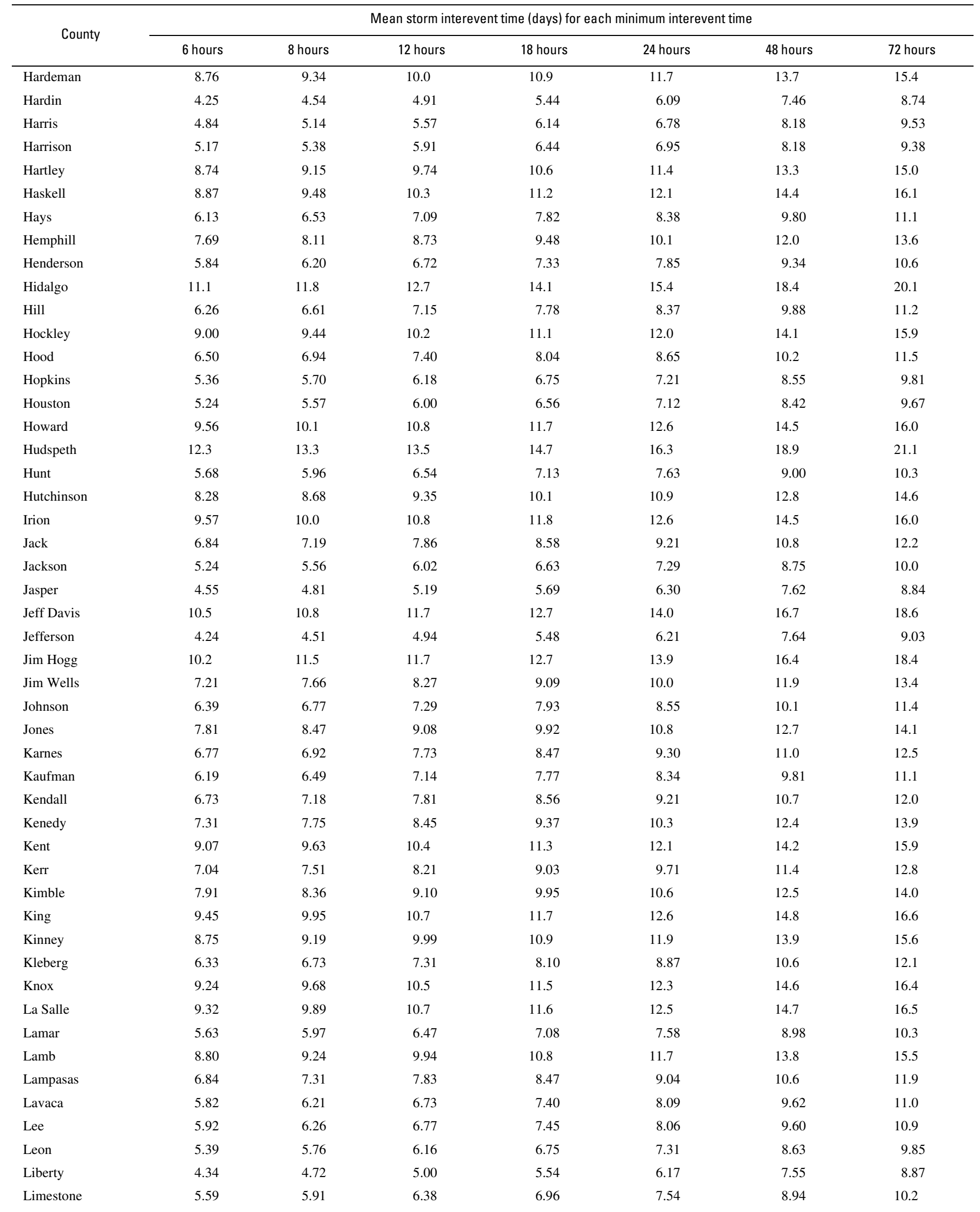


Table $18 \quad 79$

Table 18. Mean storm interevent time by county for Texas-Continued.

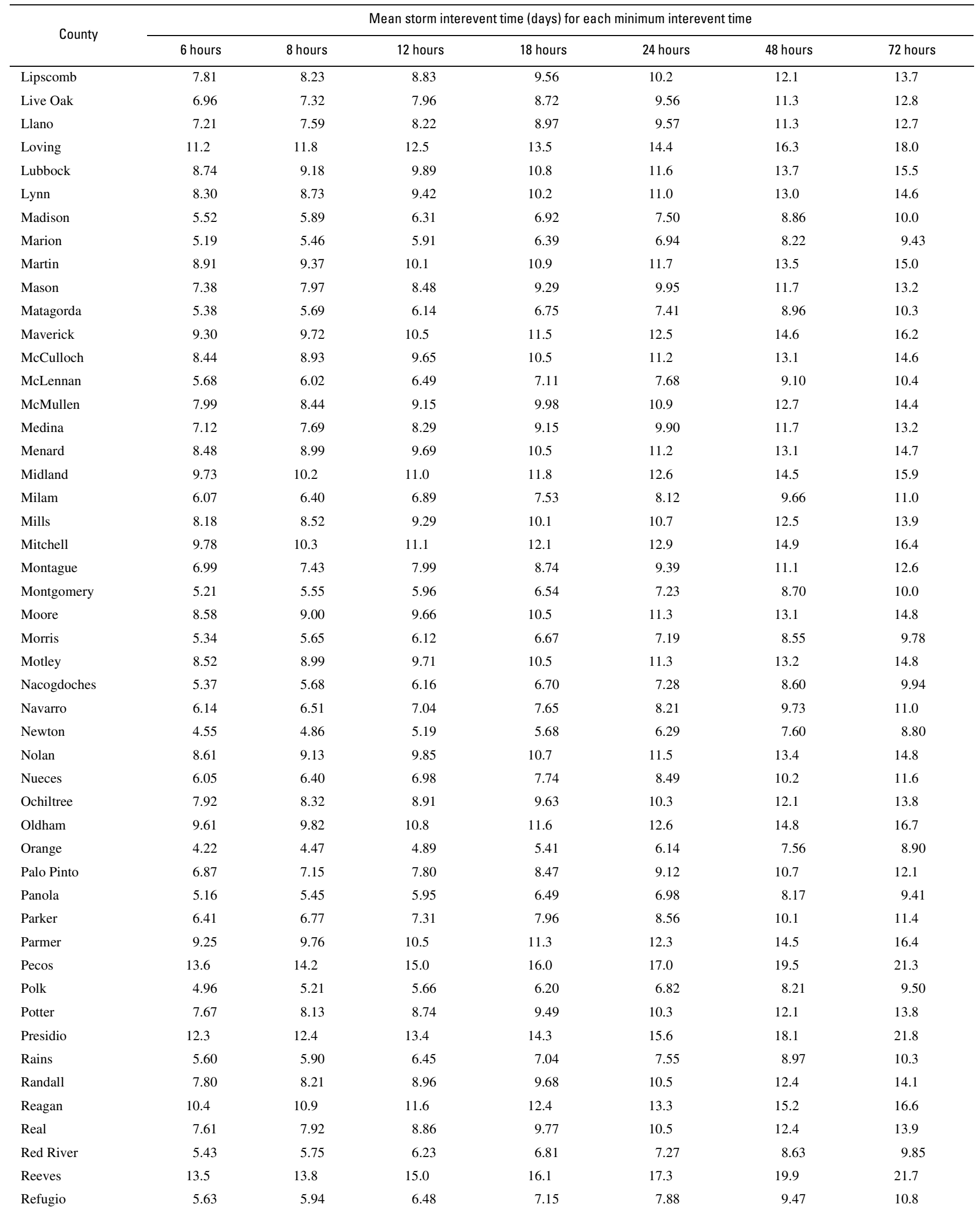


80 Statistical Characteristics of Storm Interevent Time, Depth, and Duration for Eastern New Mexico, Oklahoma, and Texas

Table 18. Mean storm interevent time by county for Texas-Continued.

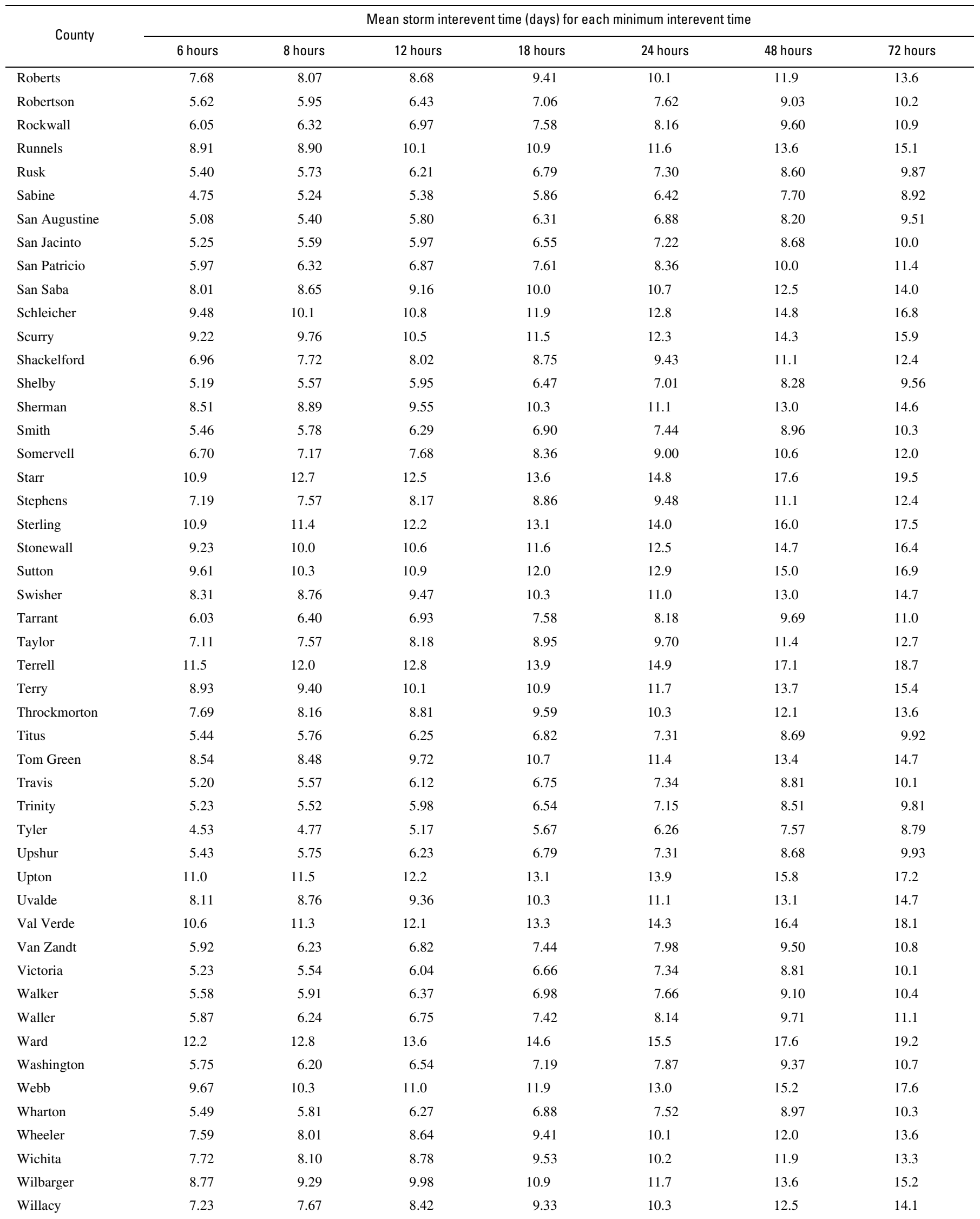


Table $18 \quad 81$

Table 18. Mean storm interevent time by county for Texas-Continued.

\begin{tabular}{|c|c|c|c|c|c|c|c|}
\hline \multirow{2}{*}{ County } & \multicolumn{7}{|c|}{ Mean storm interevent time (days) for each minimum interevent time } \\
\hline & 6 hours & 8 hours & 12 hours & 18 hours & 24 hours & 48 hours & 72 hours \\
\hline Williamson & 6.15 & 6.54 & 7.09 & 7.73 & 8.33 & 9.92 & 11.2 \\
\hline Winkler & 11.3 & 11.9 & 12.7 & 13.6 & 14.4 & 16.4 & 18.0 \\
\hline Wise & 7.12 & 7.64 & 8.13 & 8.87 & 9.52 & 11.2 & 12.7 \\
\hline Young & 7.09 & 7.34 & 8.13 & 8.84 & 9.47 & 11.1 & 12.5 \\
\hline Zapata & 10.3 & 11.2 & 11.7 & 12.5 & 13.6 & 16.0 & 18.9 \\
\hline Zavala & 9.68 & 10.2 & 11.0 & 12.0 & 12.9 & 15.1 & 16.7 \\
\hline
\end{tabular}


82 Statistical Characteristics of Storm Interevent Time, Depth, and Duration for Eastern New Mexico, Oklahoma, and Texas

Table 19. Mean storm depth by county for Texas.

\begin{tabular}{|c|c|c|c|c|c|c|c|}
\hline \multirow{2}{*}{ County } & \multicolumn{7}{|c|}{ Mean storm depth (inches) for each minimum interevent time } \\
\hline & 6 hours & 8 hours & 12 hours & 18 hours & 24 hours & 48 hours & 72 hours \\
\hline Anderson & 0.575 & 0.611 & 0.664 & 0.731 & 0.799 & 0.982 & 1.19 \\
\hline Andrews & .334 & .351 & .378 & .409 & .440 & .516 & .584 \\
\hline Angelina & .612 & .648 & .707 & .779 & .864 & 1.08 & 1.33 \\
\hline Aransas & .482 & .513 & .561 & .627 & .698 & .877 & 1.05 \\
\hline Archer & .473 & .502 & .548 & .599 & .648 & .781 & .919 \\
\hline Armstrong & .372 & .393 & .428 & .468 & .508 & .623 & .729 \\
\hline Atascosa & .494 & .524 & .571 & .629 & .691 & .834 & .967 \\
\hline Austin & .566 & .599 & .654 & .724 & .803 & .988 & 1.18 \\
\hline Bailey & .359 & .377 & .407 & .444 & .485 & .582 & .673 \\
\hline Bandera & .509 & .542 & .602 & .671 & .730 & .884 & 1.03 \\
\hline Bastrop & .513 & .550 & .605 & .674 & .738 & .921 & 1.10 \\
\hline Baylor & .491 & .518 & .564 & .616 & .664 & .801 & .926 \\
\hline Bee & .494 & .523 & .568 & .628 & .699 & .860 & 1.01 \\
\hline Bell & .533 & .563 & .606 & .661 & .715 & .870 & 1.02 \\
\hline Bexar & .479 & .510 & .560 & .626 & .689 & .846 & .987 \\
\hline Blanco & .532 & .567 & .616 & .678 & .734 & .882 & 1.02 \\
\hline Borden & .394 & .416 & .450 & .492 & .532 & .633 & .722 \\
\hline Bosque & .524 & .557 & .607 & .667 & .725 & .880 & 1.04 \\
\hline Bowie & .628 & .666 & .726 & .804 & .876 & 1.10 & 1.32 \\
\hline Brazoria & .537 & .569 & .619 & .688 & .773 & .979 & 1.21 \\
\hline Brazos & .546 & .579 & .632 & .703 & .779 & .963 & 1.15 \\
\hline Brewster & .337 & .351 & .372 & .404 & .447 & .536 & .613 \\
\hline Briscoe & .389 & .411 & .447 & .487 & .527 & .637 & .739 \\
\hline Brooks & .454 & .480 & .524 & .579 & .639 & .778 & .883 \\
\hline Brown & .516 & .543 & .589 & .645 & .695 & .831 & .952 \\
\hline Burleson & .552 & .583 & .632 & .701 & .768 & .945 & 1.12 \\
\hline Burnet & .530 & .563 & .612 & .668 & .720 & .872 & 1.01 \\
\hline Caldwell & .524 & .564 & .620 & .689 & .754 & .927 & 1.10 \\
\hline Calhoun & .509 & .541 & .593 & .659 & .739 & .938 & 1.14 \\
\hline Callahan & .446 & .476 & .519 & .574 & .626 & .753 & .875 \\
\hline Cameron & .381 & .410 & .453 & .511 & .583 & .730 & .866 \\
\hline Camp & .632 & .672 & .734 & .807 & .877 & 1.08 & 1.30 \\
\hline Carson & .376 & .397 & .431 & .471 & .512 & .628 & .738 \\
\hline Cass & .622 & .659 & .717 & .789 & .864 & 1.08 & 1.31 \\
\hline Castro & .369 & .389 & .421 & .458 & .498 & .596 & .689 \\
\hline Chambers & .516 & .552 & .613 & .694 & .790 & 1.03 & 1.31 \\
\hline Cherokee & .582 & .618 & .673 & .740 & .812 & 1.00 & 1.22 \\
\hline Childress & .436 & .465 & .502 & .550 & .588 & .699 & .809 \\
\hline Clay & .501 & .532 & .580 & .639 & .691 & .835 & .982 \\
\hline Cochran & .369 & .388 & .418 & .457 & .497 & .595 & .683 \\
\hline Coke & .450 & .475 & .513 & .562 & .601 & .712 & .801 \\
\hline Coleman & .522 & .551 & .596 & .649 & .701 & .844 & .966 \\
\hline Collin & .584 & .618 & .672 & .734 & .792 & .961 & 1.14 \\
\hline Collingsworth & .441 & .468 & .505 & .552 & .592 & .716 & .835 \\
\hline Colorado & .548 & .583 & .635 & .704 & .779 & .957 & 1.14 \\
\hline Comal & .548 & .583 & .633 & .701 & .758 & .911 & 1.05 \\
\hline Comanche & .524 & .549 & .589 & .636 & .683 & .807 & .922 \\
\hline Concho & .460 & .485 & .527 & .570 & .614 & .732 & .837 \\
\hline Cooke & .547 & .579 & .630 & .694 & .750 & .912 & 1.08 \\
\hline
\end{tabular}


Table $19 \quad 83$

Table 19. Mean storm depth by county for Texas-Continued.

\begin{tabular}{|c|c|c|c|c|c|c|c|}
\hline \multirow{2}{*}{ County } & \multicolumn{7}{|c|}{ Mean storm depth (inches) for each minimum interevent time } \\
\hline & 6 hours & 8 hours & 12 hours & 18 hours & 24 hours & 48 hours & 72 hours \\
\hline Coryell & 0.544 & 0.577 & 0.625 & 0.683 & 0.737 & 0.883 & 1.03 \\
\hline Crane & .363 & .380 & .405 & .435 & .463 & .532 & .586 \\
\hline Crockett & .414 & .435 & .468 & .507 & .547 & .638 & .718 \\
\hline Dallam & .333 & .349 & .373 & .406 & .446 & .538 & .627 \\
\hline Dallas & .533 & .569 & .625 & .691 & .754 & .924 & 1.11 \\
\hline Dawson & .363 & .383 & .415 & .452 & .489 & .584 & .669 \\
\hline Deaf Smith & .505 & .538 & .587 & .651 & .724 & .891 & 1.06 \\
\hline Delta & .371 & .392 & .423 & .458 & .500 & .599 & .692 \\
\hline Dimmit & .488 & .517 & .557 & .608 & .662 & .788 & .895 \\
\hline Donley & .403 & .427 & .463 & .507 & .546 & .668 & .781 \\
\hline Duval & .480 & .508 & .553 & .607 & .672 & .805 & .927 \\
\hline Eastland & .519 & .548 & .592 & .647 & .696 & .834 & .957 \\
\hline Ector & .340 & .356 & .383 & .413 & .442 & .512 & .571 \\
\hline Edwards & .473 & .501 & .548 & .606 & .657 & .773 & .891 \\
\hline El Paso & .213 & .223 & .237 & .253 & .275 & .326 & .365 \\
\hline Ellis & .575 & .611 & .663 & .727 & .791 & .959 & 1.13 \\
\hline Erath & .523 & .553 & .598 & .654 & .709 & .855 & .992 \\
\hline Falls & .519 & .548 & .591 & .651 & .710 & .874 & 1.05 \\
\hline Freestone & .569 & .606 & .658 & .724 & .791 & .970 & 1.16 \\
\hline Frio & .497 & .528 & .576 & .633 & .691 & .827 & .953 \\
\hline Gaines & .353 & .371 & .399 & .433 & .468 & .555 & .631 \\
\hline Galveston & .499 & .535 & .595 & .673 & .760 & .977 & 1.24 \\
\hline Garza & .405 & .428 & .465 & .508 & .550 & .658 & .756 \\
\hline Gillespie & .503 & .535 & .586 & .646 & .698 & .842 & .975 \\
\hline Glasscock & .402 & .423 & .453 & .489 & .526 & .612 & .683 \\
\hline Goliad & .502 & .533 & .580 & .642 & .716 & .887 & 1.06 \\
\hline Gonzales & .512 & .546 & .597 & .660 & .730 & .894 & 1.05 \\
\hline Gray & .405 & .428 & .463 & .506 & .547 & .671 & .789 \\
\hline Grayson & .575 & .610 & .662 & .726 & .785 & .957 & 1.14 \\
\hline Gregg & .620 & .659 & .719 & .797 & .863 & 1.06 & 1.28 \\
\hline Grimes & .567 & .600 & .657 & .727 & .813 & 1.00 & 1.20 \\
\hline Guadalupe & .512 & .545 & .595 & .661 & .725 & .883 & 1.03 \\
\hline Hale & .386 & .406 & .439 & .479 & .520 & .625 & .722 \\
\hline Hall & .412 & .437 & .475 & .519 & .558 & .669 & .774 \\
\hline Hamilton & .521 & .552 & .598 & .652 & .703 & .843 & .983 \\
\hline Hansford & .369 & .386 & .417 & .452 & .490 & .585 & .684 \\
\hline
\end{tabular}


84 Statistical Characteristics of Storm Interevent Time, Depth, and Duration for Eastern New Mexico, Oklahoma, and Texas

Table 19. Mean storm depth by county for Texas-Continued.

\begin{tabular}{|c|c|c|c|c|c|c|c|}
\hline \multirow{2}{*}{ County } & \multicolumn{7}{|c|}{ Mean storm depth (inches) for each minimum interevent time } \\
\hline & 6 hours & 8 hours & 12 hours & 18 hours & 24 hours & 48 hours & 72 hours \\
\hline Hardeman & 0.469 & 0.497 & 0.540 & 0.592 & 0.638 & 0.763 & 0.883 \\
\hline Harris & .554 & .590 & .646 & .720 & .810 & 1.03 & 1.27 \\
\hline Harrison & .618 & .656 & .712 & .783 & .855 & 1.05 & 1.27 \\
\hline Hays & .527 & .564 & .615 & .685 & .743 & .897 & 1.05 \\
\hline Hemphill & .419 & .443 & .479 & .523 & .563 & .684 & .800 \\
\hline Henderson & .595 & .633 & .690 & .759 & .821 & 1.01 & 1.20 \\
\hline Hidalgo & .433 & .459 & .502 & .557 & .618 & .751 & .841 \\
\hline Hill & .552 & .586 & .635 & .696 & .756 & .923 & 1.08 \\
\hline Houston & .578 & .614 & .668 & .736 & .811 & .998 & 1.21 \\
\hline Howard & .397 & .419 & .452 & .490 & .530 & .625 & .703 \\
\hline Hudspeth & .250 & .260 & .278 & .302 & .329 & .392 & .448 \\
\hline Hunt & .608 & .647 & .707 & .777 & .838 & 1.03 & 1.23 \\
\hline Hutchinson & .384 & .404 & .437 & .475 & .517 & .621 & .726 \\
\hline Irion & .411 & .434 & .468 & .511 & .549 & .646 & .728 \\
\hline Jack & .509 & .540 & .590 & .649 & .702 & .846 & .993 \\
\hline Jackson & .540 & .574 & .627 & .696 & .776 & .972 & 1.17 \\
\hline Jasper & .625 & .662 & .721 & .799 & .899 & 1.14 & 1.41 \\
\hline Jeff Davis & .310 & .324 & .346 & .379 & .426 & .524 & .601 \\
\hline Kendall & .533 & .569 & .623 & .687 & .745 & .892 & 1.03 \\
\hline Kenedy & .431 & .459 & .504 & .564 & .627 & .781 & .920 \\
\hline Kent & .438 & .464 & .503 & .550 & .595 & .709 & .811 \\
\hline Kerr & .495 & .527 & .582 & .645 & .699 & .843 & .976 \\
\hline Kimble & .457 & .483 & .529 & .581 & .626 & .754 & .863 \\
\hline King & .461 & .485 & .526 & .576 & .622 & .745 & .859 \\
\hline Kinney & .445 & .468 & .511 & .562 & .614 & .735 & .845 \\
\hline Kleberg & .453 & .482 & .529 & .592 & .656 & .812 & .963 \\
\hline Knox & .489 & .515 & .558 & .612 & .661 & .801 & .927 \\
\hline La Salle & .478 & .508 & .550 & .600 & .655 & .782 & .899 \\
\hline Lamar & .639 & .679 & .740 & .818 & .884 & 1.09 & 1.31 \\
\hline Lamb & .373 & .392 & .423 & .462 & .504 & .605 & .700 \\
\hline Lampasas & .517 & .549 & .596 & .649 & .697 & .840 & .976 \\
\hline Lavaca & .524 & .559 & .611 & .678 & .751 & .926 & 1.10 \\
\hline Lee & .540 & .571 & .623 & .691 & .755 & .931 & 1.10 \\
\hline Leon & .562 & .598 & .648 & .716 & .785 & .962 & 1.15 \\
\hline Liberty & .552 & .588 & .644 & .723 & .820 & 1.06 & 1.34 \\
\hline Limestone & .542 & .575 & .623 & .686 & .751 & .924 & 1.11 \\
\hline
\end{tabular}


Table $19 \quad 85$

Table 19. Mean storm depth by county for Texas-Continued.

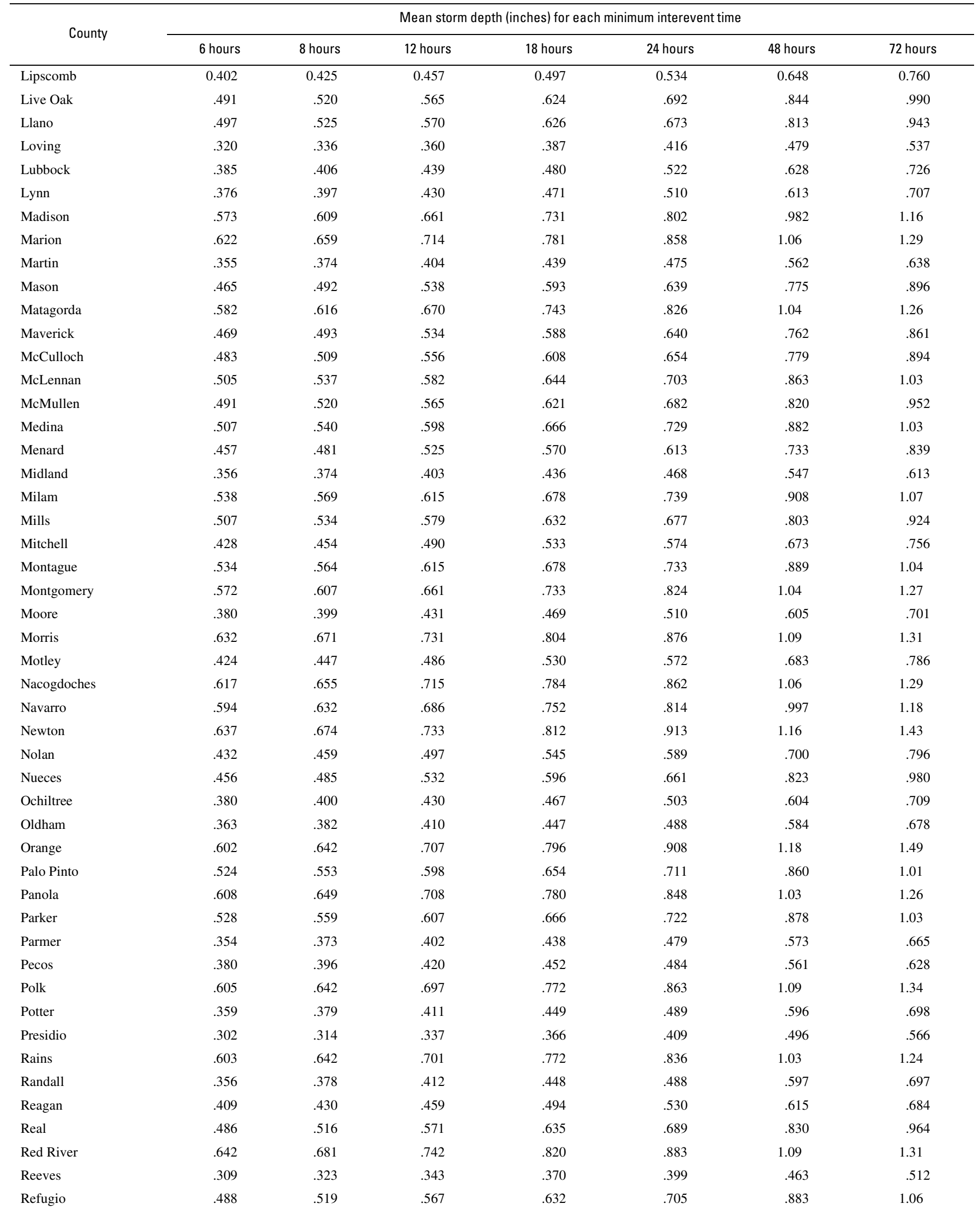


86 Statistical Characteristics of Storm Interevent Time, Depth, and Duration for Eastern New Mexico, Oklahoma, and Texas

Table 19. Mean storm depth by county for Texas-Continued.

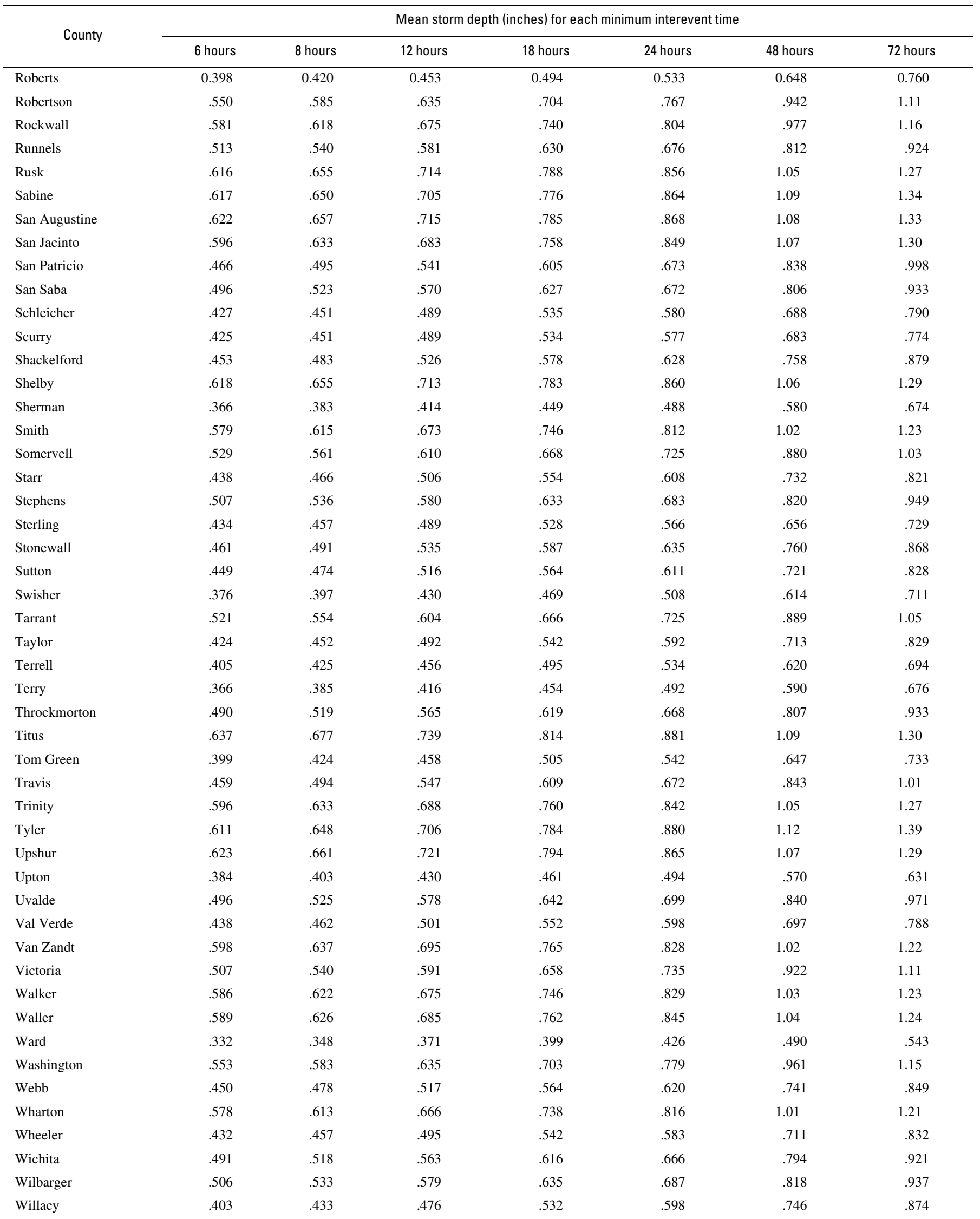


Table $19 \quad 87$

Table 19. Mean storm depth by county for Texas-Continued.

\begin{tabular}{|c|c|c|c|c|c|c|c|}
\hline \multirow{2}{*}{ County } & \multicolumn{7}{|c|}{ Mean storm depth (inches) for each minimum interevent time } \\
\hline & 6 hours & 8 hours & 12 hours & 18 hours & 24 hours & 48 hours & 72 hours \\
\hline Williamson & 0.521 & 0.556 & 0.606 & 0.666 & 0.724 & 0.892 & 1.05 \\
\hline Wilson & .499 & .530 & .577 & .639 & .706 & .862 & 1.00 \\
\hline Winkler & .331 & .347 & .372 & .401 & .429 & .496 & .553 \\
\hline Wise & .539 & .570 & .621 & .682 & .738 & .894 & 1.04 \\
\hline Wood & .604 & .641 & .701 & .774 & .844 & 1.05 & 1.27 \\
\hline Yoakum & .363 & .381 & .410 & .446 & .483 & .576 & .654 \\
\hline Young & .498 & .528 & .575 & .631 & .681 & .820 & .958 \\
\hline Zapata & .440 & .470 & .508 & .552 & .607 & .728 & .826 \\
\hline Zavala & .498 & .525 & .567 & .624 & .678 & .806 & .912 \\
\hline
\end{tabular}


88 Statistical Characteristics of Storm Interevent Time, Depth, and Duration for Eastern New Mexico, Oklahoma, and Texas

Table 20. Mean storm duration by county for Texas.

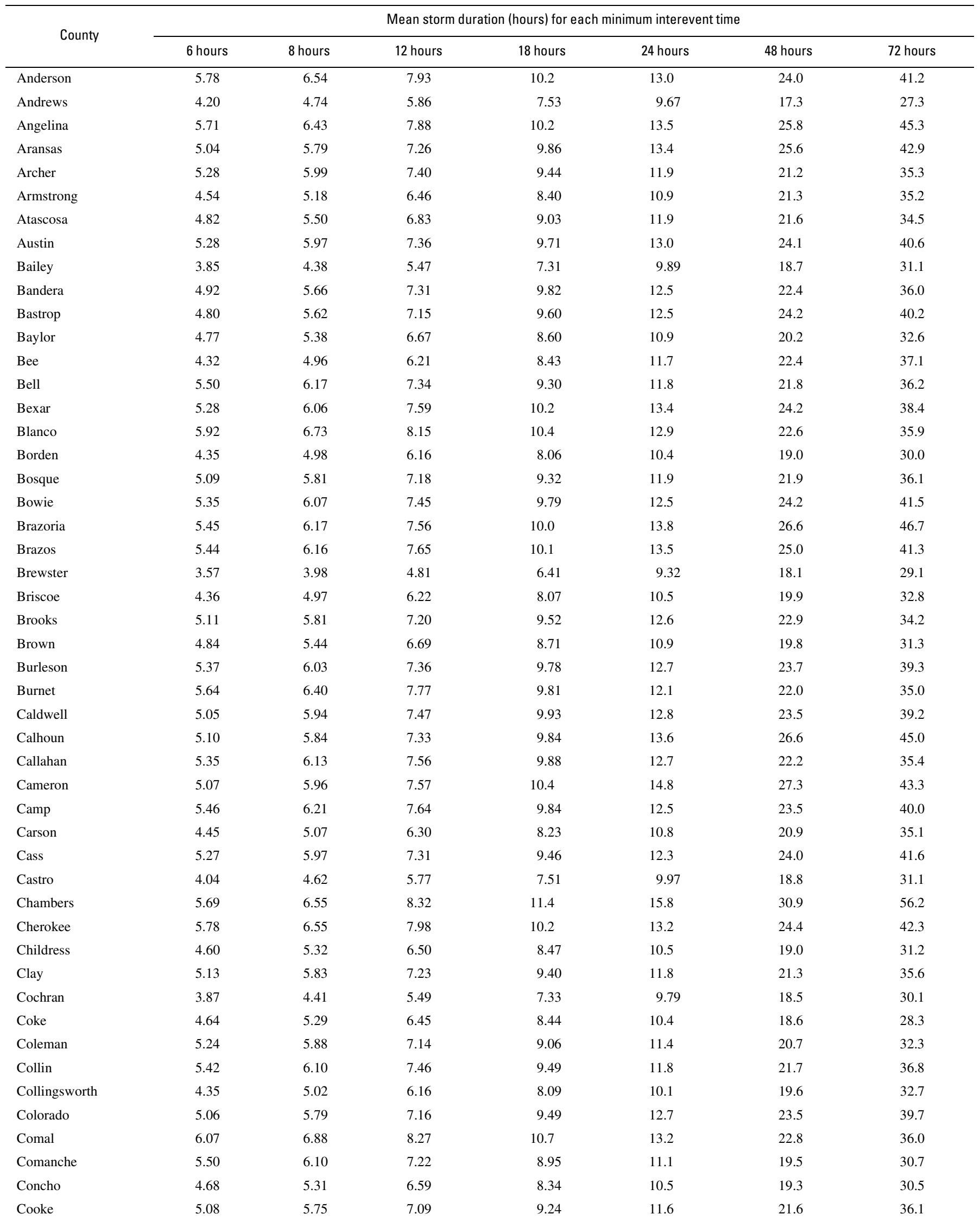


Table $20 \quad 89$

Table 20. Mean storm duration by county for Texas-Continued.

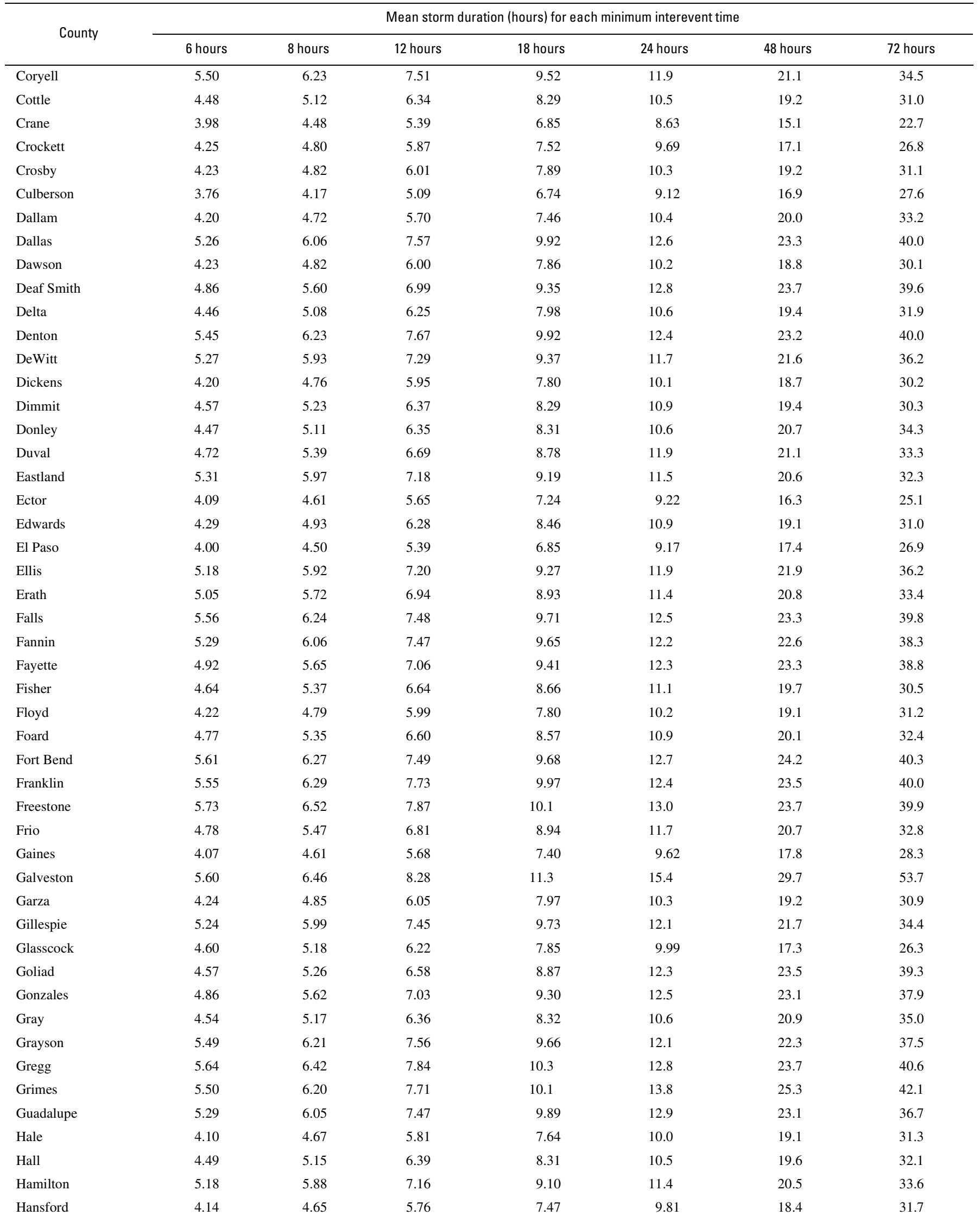


90 Statistical Characteristics of Storm Interevent Time, Depth, and Duration for Eastern New Mexico, Oklahoma, and Texas

Table 20. Mean storm duration by county for Texas—Continued.

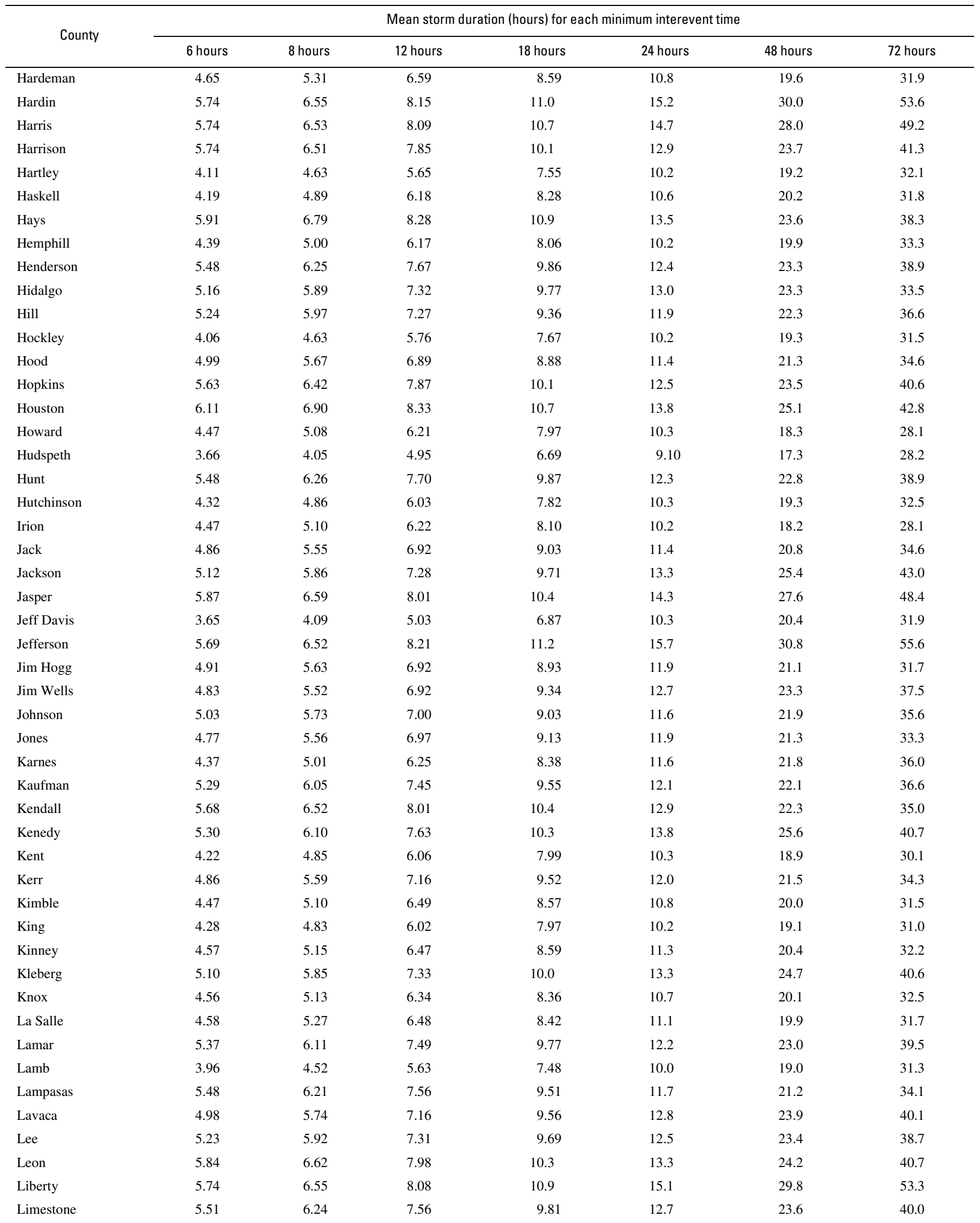


Table 2091

Table 20. Mean storm duration by county for Texas-Continued.

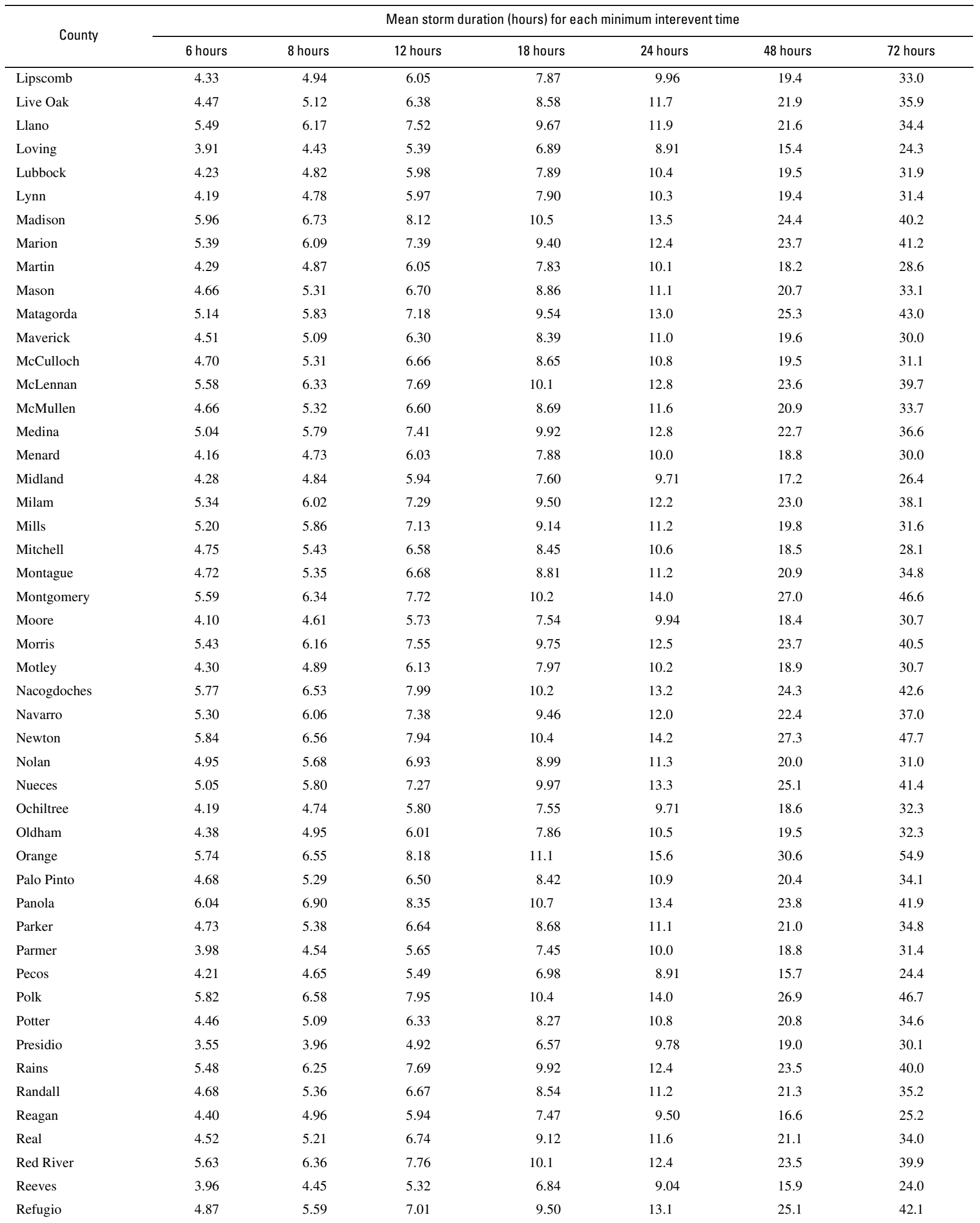


92 Statistical Characteristics of Storm Interevent Time, Depth, and Duration for Eastern New Mexico, Oklahoma, and Texas

Table 20. Mean storm duration by county for Texas—Continued.

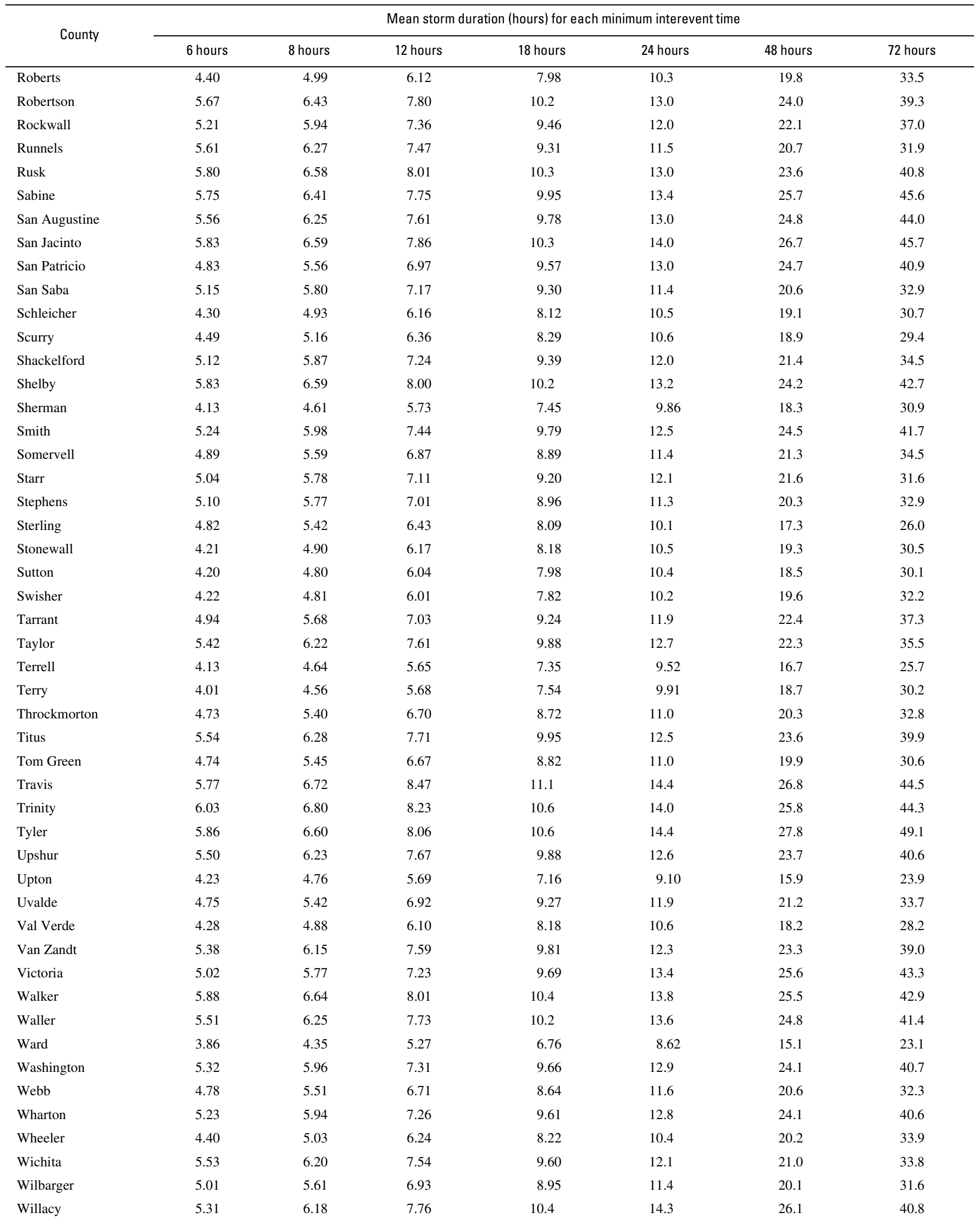


Table $20 \quad 93$

Table 20. Mean storm duration by county for Texas-Continued.

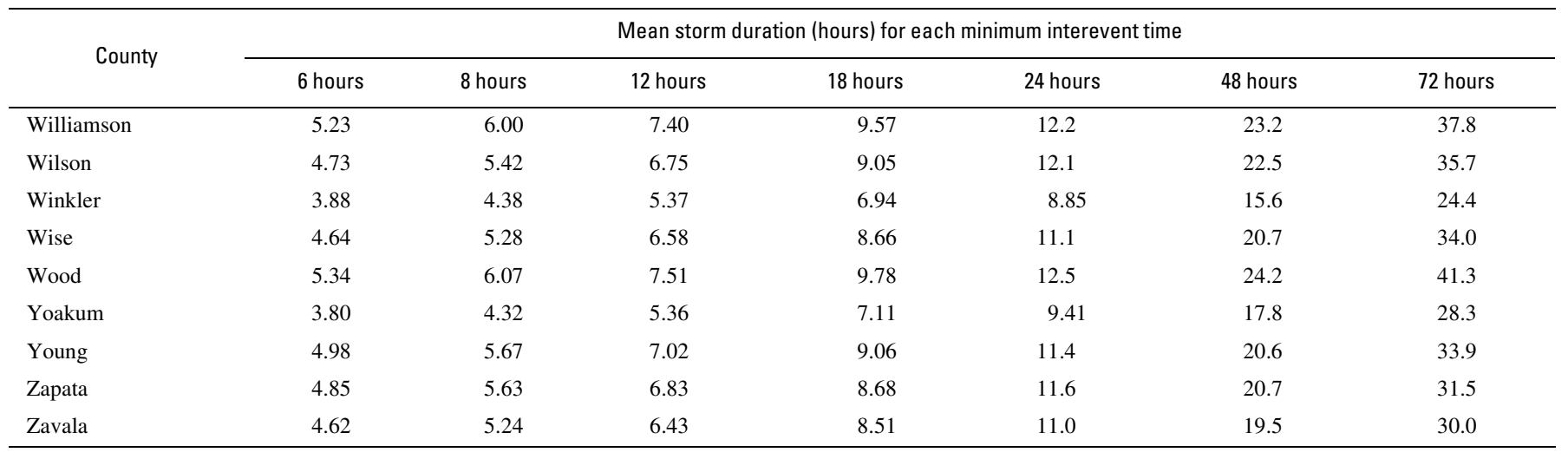




\section{Statistical Characteristics of Storm Interevent Time, Depth, and Duration for Eastern New Mexico, Oklahoma, and Texas}

Table 21. Example simulations of storm interevent time from example 2 using 24-hour minimum interevent time for station 0211 Amarillo Weather Service Office Airport, Texas.

[Nonexceedance probability is random number between 0 and 1 . Interevent time is computed as $1-(7.60-1) \times \ln (1-\mathrm{F})$, where $\mathrm{F}$ is nonexceedance probability, 7.60 days is mean interevent time, and 1 is day equivalent of 24-hour minimum interevent time. --, dimensionless]

\begin{tabular}{|c|c|}
\hline $\begin{array}{c}\text { Nonexceedance } \\
\text { probability } \\
\text { (random number) } \\
(--)\end{array}$ & $\begin{array}{l}\text { Simulated interevent } \\
\text { time-Interval between } \\
\text { successive storms } \\
\text { (days) }\end{array}$ \\
\hline 0.86910 & 14.42 \\
\hline .15608 & 2.12 \\
\hline .56156 & 6.44 \\
\hline .31575 & 3.50 \\
\hline \multirow{3}{*}{ Mean } & 9.46 \\
\hline & 35.94 \\
\hline & 7.19 \\
\hline
\end{tabular}

Table 22. Distribution of storm depth for examples 3 and 4 based on site-specific statistics for station 4311 Houston Alief, Texas.

[Empirical estimate of storm depth from appendix 4-4.5, station 4311. Continuous distribution estimate of storm depth from fitted four-parameter kappa distribution to L-moments from appendix 4-2.5, station 4311. -- dimensionless]

\begin{tabular}{ccc}
\hline $\begin{array}{c}\text { Nonexceedance } \\
\text { probability } \\
(--)\end{array}$ & $\begin{array}{c}\text { Empirical estimate } \\
\text { of storm depth } \\
\text { (inches) }\end{array}$ & $\begin{array}{c}\text { Continuous distribution } \\
\text { estimate of storm depth } \\
\text { (inches) }\end{array}$ \\
\hline 0.01 & 0.02 & 0.03 \\
.02 & .02 & .03 \\
.10 & .06 & .05 \\
.25 & .15 & .15 \\
.50 & .44 & .46 \\
.75 & 1.18 & 1.15 \\
.90 & 2.20 & 2.24 \\
.98 & 4.55 & 4.55 \\
.99 & 5.71 & 5.70 \\
\hline
\end{tabular}

Table 23. Regional values for selected storm depth percentiles in Randall County, Texas.

[Linear interpolation used to estimate storm depths for 36-hour minimum interevent time. --, dimensionless]

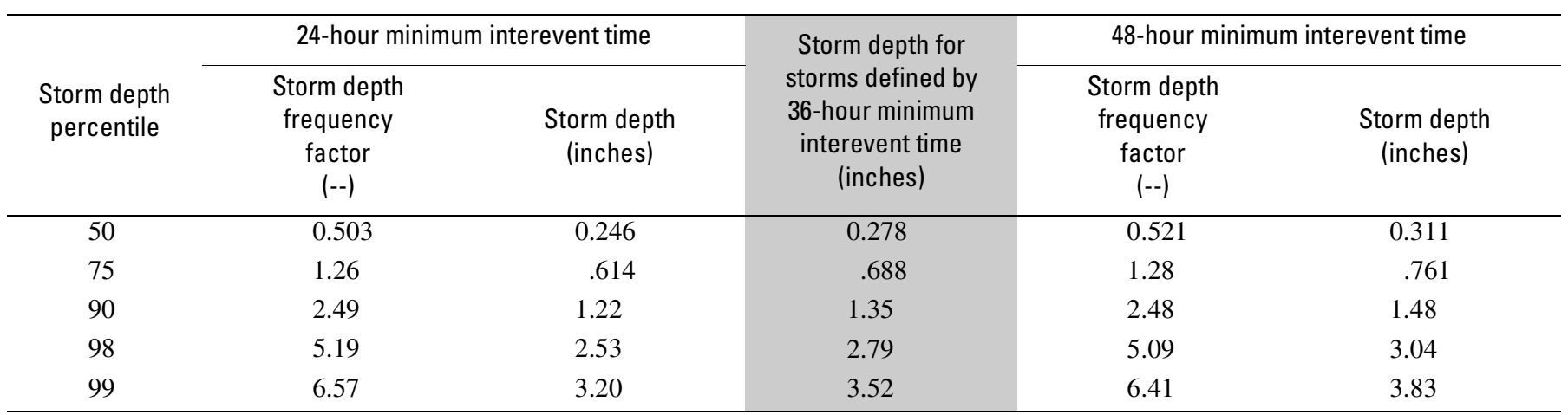


Appendix 1-

L-Moments and Sample L-Moments 
Blank Page 


\section{L-moments}

This discussion is derived mostly from Hosking (1990), Hosking and Wallis (1997), and references therein. Other references are provided as needed for specifics of L-moment theory development.

The theoretical L-moments of a random variable $X$ with a quantile function $X(F)$ for nonexceedance probability $F$ are defined from the expectations of order statistics. The order statistics for $X$ of a sample of size $n$ are formed by the ascending order $X_{1: n} \leq X_{2: n} \leq \ldots \leq X_{n: n}$. The theoretical L-moments are defined as

$\lambda_{r} \equiv \frac{1}{r} \sum_{k=0}^{r-1}(-1)^{k}\left(\begin{array}{c}r-1 \\ k\end{array}\right) E\left[X_{r-k: r}\right]$

where $r \geq 1$ is the order of the L-moment, and $E\left[X_{r-k: r}\right]$ is the expectation of the $r-k$ order statistic of a sample of size $r$. The expectation of an order statistic is

$E\left[X_{j: r}\right]=\frac{r !}{(j-1) !(r-j) !} \int_{0}^{1} X(F) \times F^{j-1}(1-F)^{r-j} \mathrm{~d} F$.

The equation for the binomial coefficients is

$\left(\begin{array}{l}a \\ b\end{array}\right)=\frac{a !}{b !(a-b) !}$.

The first four theoretical L-moments are

$$
\begin{aligned}
\lambda_{1}= & \int_{0}^{1} X(F) \mathrm{d} F, \\
\lambda_{2}= & \int_{0}^{1} X(F) \times(2 F-1) \mathrm{d} F, \\
\lambda_{3}= & \int_{0}^{1} X(F) \times\left(6 F^{2}-6 F+1\right) \mathrm{d} F, \text { and } \\
\lambda_{4}= & \int_{0} X(F) \times\left(20 F^{3}-30 F^{2}+12 F+1\right) \mathrm{d} F .
\end{aligned}
$$

The L-moment ratios are the dimensionless quantities

$\mathrm{L}-\mathrm{CV}=\frac{\lambda_{2}}{\lambda_{1}}=$ coefficient of L-variation,

$\tau_{3}=\frac{\lambda_{3}}{\lambda_{2}}=$ L-skew,

$\tau_{4}=\frac{\lambda_{4}}{\lambda_{2}}=$ L-kurtosis, and

$\tau_{5}=\frac{\lambda_{5}}{\lambda_{2}}=$ not named 


\section{Sample L-moments}

The sample L-moments are computed from the sample order statistics $x_{1: n} \leq x_{2: n} \leq \ldots \leq x_{n: n}$. In the most compact notation, the sample L-moments are

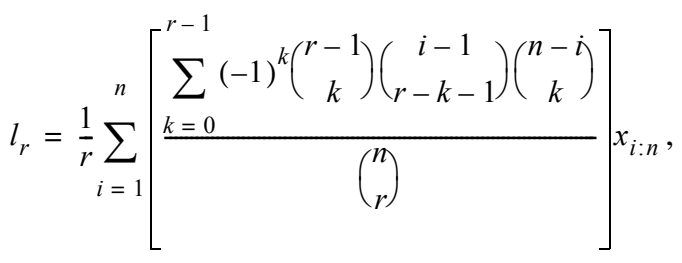

where $r$ represents the order of the L-moments and $n$ represents the sample size.

The sample L-moment ratios are

$\mathrm{L}-\mathrm{CV}=\frac{l_{2}}{l_{1}}=$ coefficient of L-variation,

$t_{3}=\frac{l_{3}}{l_{2}}=$ L-skew,

$t_{4}=\frac{l_{4}}{l_{2}}=$ L-kurtosis, and

$t_{5}=\frac{l_{5}}{l_{2}}=$ not named. 
Appendix 2-

Storm Statistics for Hourly Rainfall

Stations in Eastern New Mexico 
Blank Page 
Appendix 2-1.1. Number of storms, total duration, and mean storm interevent time defined by 6-hour minimum interevent time for hourly rainfall stations in eastern New Mexico.

\begin{tabular}{|c|c|c|c|c|c|c|c|c|c|c|c|c|c|c|c|}
\hline $\begin{array}{c}\text { Station } \\
\text { no. }\end{array}$ & $\begin{array}{c}\text { No. } \\
\text { of } \\
\text { storms }\end{array}$ & $\begin{array}{c}\text { Total } \\
\text { duration } \\
\text { (hours) }\end{array}$ & $\begin{array}{c}\text { Mean } \\
\text { storm } \\
\text { interevent } \\
\text { time } \\
\text { (days) }\end{array}$ & $\begin{array}{c}\text { Station } \\
\text { no. }\end{array}$ & $\begin{array}{c}\text { No. } \\
\text { of } \\
\text { storms }\end{array}$ & $\begin{array}{c}\text { Total } \\
\text { duration } \\
\text { (hours) }\end{array}$ & $\begin{array}{c}\text { Mean } \\
\text { storm } \\
\text { interevent } \\
\text { time } \\
\text { (days) }\end{array}$ & $\begin{array}{c}\text { Station } \\
\text { no. }\end{array}$ & $\begin{array}{c}\text { No. } \\
\text { of } \\
\text { storms }\end{array}$ & $\begin{array}{c}\text { Total } \\
\text { duration } \\
\text { (hours) }\end{array}$ & $\begin{array}{c}\text { Mean } \\
\text { storm } \\
\text { interevent } \\
\text { time } \\
\text { (days) }\end{array}$ & $\begin{array}{c}\text { Station } \\
\text { no. }\end{array}$ & $\begin{array}{c}\text { No. } \\
\text { of } \\
\text { storms }\end{array}$ & $\begin{array}{c}\text { Total } \\
\text { duration } \\
\text { (hours) }\end{array}$ & $\begin{array}{c}\text { Mean } \\
\text { storm } \\
\text { interevent } \\
\text { time } \\
\text { (days) }\end{array}$ \\
\hline 0199 & 1,367 & 300,612 & 8.99 & 1963 & 2,598 & 468,726 & 7.36 & 4860 & 141 & 19,008 & 5.42 & 7649 & 3,462 & 439,618 & 5.09 \\
\hline 0205 & 1,020 & 239,640 & 9.60 & 1982 & 1,744 & 312,660 & 7.36 & 4862 & 1,160 & 171,413 & 6.04 & 7651 & 4,53 & 52,600 & 4.69 \\
\hline 0208 & 383 & 84,000 & 8.96 & 2030 & 2,511 & 484,051 & 7.89 & 5370 & 1,906 & 481,217 & 10.36 & 7735 & 1,648 & 237,408 & 5.75 \\
\hline 0600 & 1,990 & 484,110 & 9.98 & 2207 & 486 & 140,709 & 11.90 & 6138 & 1,172 & 197,952 & 6.81 & 8072 & 1,539 & 213,312 & 5.56 \\
\hline 0606 & 82 & 19,752 & 9.88 & 2510 & 2,557 & 484,087 & 7.72 & 6275 & 2,636 & 371,738 & 5.68 & 8078 & 411 & 75,240 & 7.43 \\
\hline 0646 & 541 & 113,256 & 8.52 & 2625 & 518 & 124,200 & 9.78 & 6435 & 1,857 & 483,422 & 10.68 & 8084 & 66 & 18,288 & 11.38 \\
\hline 1120 & 3,914 & 484,063 & 4.96 & 2665 & 2,838 & 484,030 & 6.90 & 6492 & 223 & 59,160 & 10.88 & 8085 & 1,812 & 269,810 & 6.04 \\
\hline 1440 & 1,639 & 236,375 & 5.87 & 2694 & 200 & 18,288 & 3.55 & 6619 & 561 & 152,664 & 11.17 & 8187 & 500 & 109,560 & 8.96 \\
\hline 1446 & 746 & 212,592 & 11.68 & 2700 & 3,706 & 484,034 & 5.30 & 6659 & 1,797 & 428,041 & 9.74 & 8358 & 73 & 27,048 & 15.25 \\
\hline 1807 & 158 & 57,696 & 15.02 & 4026 & 1 & 2,256 & 94.00 & 7277 & 2 & 2,160 & 45.00 & 8816 & 1 & 3,624 & 151.00 \\
\hline 1840 & 635 & 130,730 & 8.46 & 4028 & 360 & 91,032 & 10.38 & 7279 & 2,311 & 335,939 & 5.92 & 9148 & 8 & 8,808 & 45.53 \\
\hline 1881 & 5 & 504 & 4.03 & 4030 & 187 & 54,485 & 12.03 & 7283 & 1,437 & 167,256 & 4.67 & 9153 & 132 & 28,248 & 8.75 \\
\hline 1887 & 3,083 & 483,991 & 6.35 & 4089 & 2,624 & 484,016 & 7.51 & 7604 & 5 & 552 & 4.32 & 9156 & 2,432 & 483,287 & 8.12 \\
\hline 1939 & 2,526 & 468,627 & 7.55 & 4112 & 1,244 & 294,907 & 9.74 & 7605 & 31 & 11,208 & 14.96 & 9193 & 981 & 159,171 & 6.64 \\
\hline 1950 & 32 & 15,336 & 19.74 & 4306 & 619 & 124,200 & 8.16 & 7609 & 1,191 & 219,864 & 7.49 & 9265 & 643 & 168,048 & 10.77 \\
\hline 1956 & 48 & 15,336 & 13.10 & 4850 & 1,510 & 242,472 & 6.52 & 7610 & 1,234 & 254,143 & 8.41 & 9569 & 594 & 142,953 & 9.91 \\
\hline 1961 & 43 & 15,336 & 14.61 & 4856 & 340 & 62,088 & 7.41 & 7638 & 2,825 & 483,293 & 6.96 & 9686 & 1,964 & 483,331 & 10.09 \\
\hline
\end{tabular}

Appendix 2-1.2. Number of storms, total duration, and mean storm interevent time defined by 8-hour minimum interevent time for hourly rainfall stations in eastern New Mexico.

\begin{tabular}{|c|c|c|c|c|c|c|c|c|c|c|c|c|c|c|c|}
\hline $\begin{array}{c}\text { Station } \\
\text { no. }\end{array}$ & $\begin{array}{c}\text { No. } \\
\text { of } \\
\text { storms }\end{array}$ & $\begin{array}{l}\text { Total } \\
\text { duration } \\
\text { (hours) }\end{array}$ & $\begin{array}{c}\text { Mean } \\
\text { storm } \\
\text { interevent } \\
\text { time } \\
\text { (days) }\end{array}$ & $\begin{array}{c}\text { Station } \\
\text { no. }\end{array}$ & $\begin{array}{c}\text { No. } \\
\text { of } \\
\text { storms }\end{array}$ & $\begin{array}{c}\text { Total } \\
\text { duration } \\
\text { (hours) }\end{array}$ & $\begin{array}{c}\text { Mean } \\
\text { storm } \\
\text { interevent } \\
\text { time } \\
\text { (days) }\end{array}$ & $\begin{array}{c}\text { Station } \\
\text { no. }\end{array}$ & $\begin{array}{l}\text { No. } \\
\text { of } \\
\text { storms }\end{array}$ & $\begin{array}{l}\text { Total } \\
\text { duration } \\
\text { (hours) }\end{array}$ & $\begin{array}{c}\text { Mean } \\
\text { storm } \\
\text { interevent } \\
\text { time } \\
\text { (days) }\end{array}$ & $\begin{array}{c}\text { Station } \\
\text { no. }\end{array}$ & $\begin{array}{c}\text { No. } \\
\text { of } \\
\text { storms }\end{array}$ & $\begin{array}{l}\text { Total } \\
\text { duration } \\
\text { (hours) }\end{array}$ & $\begin{array}{c}\text { Mean } \\
\text { storm } \\
\text { interevent } \\
\text { time } \\
\text { (days) }\end{array}$ \\
\hline 0199 & 1,274 & 300,612 & 9.62 & 1963 & 2,459 & 468,726 & 7.76 & 4860 & 137 & 19,008 & 5.57 & 7649 & 3,209 & 439,618 & 5.47 \\
\hline 0205 & 978 & 239,640 & 10.00 & 1982 & 1,656 & 312,660 & 7.74 & 4862 & 1,102 & 171,413 & 6.34 & 7651 & 417 & 52,600 & 5.07 \\
\hline 0208 & 367 & 84,000 & 9.33 & 2030 & 2,415 & 484,051 & 8.19 & 5370 & 1,807 & 481,217 & 10.91 & 7735 & 1,556 & 237,408 & 6.07 \\
\hline 0600 & 1,908 & 484,110 & 10.40 & 2207 & 466 & 140,709 & 12.40 & 6138 & 1,094 & 197,952 & 7.27 & 8072 & 1,449 & 213,312 & 5.89 \\
\hline 0606 & 80 & 19,752 & 10.12 & 2510 & 2,419 & 484,087 & 8.14 & 6275 & 2,476 & 371,738 & 6.03 & 8078 & 392 & 75,240 & 7.78 \\
\hline 0646 & 507 & 113,256 & 9.07 & 2625 & 491 & 124,200 & 10.31 & 6435 & 1,768 & 483,422 & 11.20 & 8084 & 63 & 18,288 & 11.91 \\
\hline 1120 & 3,635 & 484,063 & 5.32 & 2665 & 2,693 & 484,030 & 7.25 & 6492 & 208 & 59,160 & 11.65 & 8085 & 1,687 & 269,810 & 6.46 \\
\hline 1440 & 1,547 & 236,375 & 6.21 & 2694 & 174 & 18,288 & 4.03 & 6619 & 543 & 152,664 & 11.53 & 8187 & 477 & 109,560 & 9.38 \\
\hline 1446 & 716 & 212,592 & 12.16 & 2700 & 3,522 & 484,034 & 5.56 & 6659 & 1,705 & 428,041 & 10.25 & 8358 & 70 & 27,048 & 15.89 \\
\hline 1807 & 156 & 57,696 & 15.21 & 4026 & 1 & 2,256 & 94.00 & 7277 & 2 & 2,160 & 45.00 & 8816 & 1 & 3,624 & 151.00 \\
\hline 1840 & 601 & 130,730 & 8.92 & 4028 & 348 & 91,032 & 10.73 & 7279 & 2,186 & 335,939 & 6.25 & 9148 & 7 & 8,808 & 51.99 \\
\hline 1881 & 4 & 504 & 5.25 & 4030 & 179 & 54,485 & 12.56 & 7283 & 1,364 & 167,256 & 4.90 & 9153 & 131 & 28,248 & 8.81 \\
\hline 1887 & 2,935 & 483,991 & 6.66 & 4089 & 2,446 & 484,016 & 8.04 & 7604 & 5 & 552 & 4.32 & 9156 & 2,332 & 483,287 & 8.46 \\
\hline 1939 & 2,404 & 468,627 & 7.92 & 4112 & 1,184 & 294,907 & 10.22 & 7605 & 30 & 11,208 & 15.45 & 9193 & 918 & 159,171 & 7.08 \\
\hline 1950 & 30 & 15,336 & 21.04 & 4306 & 601 & 124,200 & 8.40 & 7609 & 1,130 & 219,864 & 7.88 & 9265 & 607 & 168,048 & 11.39 \\
\hline 1956 & 45 & 15,336 & 13.95 & 4850 & 1,435 & 242,472 & 6.85 & 7610 & 1,159 & 254,143 & 8.94 & 9569 & 569 & 142,953 & 10.34 \\
\hline 1961 & 41 & 15,336 & 15.31 & 4856 & 324 & 62,088 & 7.76 & 7638 & 2,679 & 483,293 & 7.33 & 9686 & 1,876 & 483,331 & 10.55 \\
\hline
\end{tabular}


Appendix 2-1.3. Number of storms, total duration, and mean storm interevent time defined by 12-hour minimum interevent time for hourly rainfall stations in eastern New Mexico.

\begin{tabular}{|c|c|c|c|c|c|c|c|c|c|c|c|c|c|c|c|}
\hline $\begin{array}{c}\text { Station } \\
\text { no. }\end{array}$ & $\begin{array}{c}\text { No. } \\
\text { of } \\
\text { storms }\end{array}$ & $\begin{array}{l}\text { Total } \\
\text { duration } \\
\text { (hours) }\end{array}$ & $\begin{array}{c}\text { Mean } \\
\text { storm } \\
\text { interevent } \\
\text { time } \\
\text { (days) }\end{array}$ & $\begin{array}{c}\text { Station } \\
\text { no. }\end{array}$ & $\begin{array}{c}\text { No. } \\
\text { of } \\
\text { storms }\end{array}$ & $\begin{array}{c}\text { Total } \\
\text { duration } \\
\text { (hours) }\end{array}$ & $\begin{array}{c}\text { Mean } \\
\text { storm } \\
\text { interevent } \\
\text { time } \\
\text { (days) }\end{array}$ & $\begin{array}{c}\text { Station } \\
\text { no. }\end{array}$ & $\begin{array}{c}\text { No. } \\
\text { of } \\
\text { storms }\end{array}$ & $\begin{array}{c}\text { Total } \\
\text { duration } \\
\text { (hours) }\end{array}$ & $\begin{array}{c}\text { Mean } \\
\text { storm } \\
\text { interevent } \\
\text { time } \\
\text { (days) }\end{array}$ & $\begin{array}{c}\text { Station } \\
\text { no. }\end{array}$ & $\begin{array}{c}\text { No. } \\
\text { of } \\
\text { storms }\end{array}$ & $\begin{array}{c}\text { Total } \\
\text { duration } \\
\text { (hours) }\end{array}$ & $\begin{array}{c}\text { Mean } \\
\text { storm } \\
\text { interevent } \\
\text { time } \\
\text { (days) }\end{array}$ \\
\hline 0199 & 1,174 & 300,612 & 10.41 & 1963 & 2,281 & 468,726 & 8.34 & 4860 & 128 & 19,008 & 5.94 & 7649 & 2,920 & 439,618 & 5.97 \\
\hline 0205 & 924 & 239,640 & 10.56 & 1982 & 1,511 & 312,660 & 8.44 & 4862 & 1,013 & 171,413 & 6.86 & 7651 & 376 & 52,600 & 5.58 \\
\hline 0208 & 337 & 84,000 & 10.13 & 2030 & 2,274 & 484,051 & 8.68 & 5370 & 1,664 & 481,217 & 11.82 & 7735 & 1,414 & 237,408 & 6.64 \\
\hline 0404 & 229 & 30,486 & 5.28 & 2139 & 106 & 32,112 & 12.30 & 5651 & 157 & 47,472 & 12.35 & 7736 & 1,691 & 246,584 & 5.79 \\
\hline 0600 & 1,764 & 484,110 & 11.22 & 2207 & 431 & 140,709 & 13.37 & 6138 & 995 & 197,952 & 7.96 & 8072 & 1,316 & 213,312 & 6.45 \\
\hline 0606 & 73 & 19,752 & 11.06 & 2510 & 2,232 & 484,087 & 8.79 & 6275 & 2,274 & 371,738 & 6.53 & 8078 & 364 & 75,240 & 8.35 \\
\hline 0646 & 481 & 113,256 & 9.54 & 2625 & 460 & 124,200 & 10.97 & 6435 & 1,627 & 483,422 & 12.14 & 8084 & 63 & 18,288 & 11.91 \\
\hline 1120 & 3,253 & 484,063 & 5.90 & 2665 & 2,487 & 484,030 & 7.82 & 6492 & 192 & 59,160 & 12.59 & 8085 & 1,533 & 269,810 & 7.07 \\
\hline 1440 & 1,414 & 236,375 & 6.76 & 2694 & 155 & 18,288 & 4.48 & 6619 & 522 & 152,664 & 11.98 & 8187 & 453 & 109,560 & 9.85 \\
\hline 1446 & 664 & 212,592 & 13.08 & 2700 & 3,245 & 484,034 & 6.00 & 6659 & 1,599 & 428,041 & 10.91 & 8358 & 67 & 27,048 & 16.58 \\
\hline 1807 & 150 & 57,696 & 15.81 & 4026 & 1 & 2,256 & 94.00 & 7277 & 2 & 2,160 & 45.00 & 8816 & 1 & 3,624 & 151.00 \\
\hline 1840 & 557 & 130,730 & 9.60 & 4028 & 332 & 91,032 & 11.22 & 7279 & 2,028 & 335,939 & 6.70 & 9148 & 7 & 8,808 & 51.99 \\
\hline 1881 & 3 & 504 & 7.00 & 4030 & 169 & 54,485 & 13.28 & 7283 & 1,256 & 167,256 & 5.29 & 9153 & 118 & 28,248 & 9.73 \\
\hline 1887 & 2,755 & 483,991 & 7.07 & 4089 & 2,231 & 484,016 & 8.78 & 7604 & 5 & 552 & 4.32 & 9156 & 2,186 & 483,287 & 9.00 \\
\hline 1939 & 2,225 & 468,627 & 8.53 & 4112 & 1,085 & 294,907 & 11.11 & 7605 & 26 & 11,208 & 17.76 & 9193 & 837 & 159,171 & 7.72 \\
\hline 1950 & 27 & 15,336 & 23.34 & 4306 & 566 & 124,200 & 8.89 & 7609 & 1,051 & 219,864 & 8.45 & 9265 & 568 & 168,048 & 12.15 \\
\hline 1956 & 44 & 15,336 & 14.26 & 4850 & 1,314 & 242,472 & 7.44 & 7610 & 1,068 & 254,143 & 9.66 & 9569 & 534 & 142,953 & 10.99 \\
\hline 1961 & 39 & 15,336 & 16.07 & 4856 & 302 & 62,088 & 8.29 & 7638 & 2,490 & 483,293 & 7.86 & 9686 & 1,729 & 483,331 & 11.42 \\
\hline
\end{tabular}

Appendix 2-1.4. Number of storms, total duration, and mean storm interevent time defined by 18-hour minimum interevent time for hourly rainfall stations in eastern New Mexico.

\begin{tabular}{|c|c|c|c|c|c|c|c|c|c|c|c|c|c|c|c|}
\hline $\begin{array}{c}\text { Station } \\
\text { no. }\end{array}$ & $\begin{array}{c}\text { No. } \\
\text { of } \\
\text { storms }\end{array}$ & $\begin{array}{c}\text { Total } \\
\text { duration } \\
\text { (hours) }\end{array}$ & $\begin{array}{c}\text { Mean } \\
\text { storm } \\
\text { interevent } \\
\text { time } \\
\text { (days) }\end{array}$ & $\begin{array}{c}\text { Station } \\
\text { no. }\end{array}$ & $\begin{array}{c}\text { No. } \\
\text { of } \\
\text { storms }\end{array}$ & $\begin{array}{c}\text { Total } \\
\text { duration } \\
\text { (hours) }\end{array}$ & $\begin{array}{c}\text { Mean } \\
\text { storm } \\
\text { interevent } \\
\text { time } \\
\text { (days) }\end{array}$ & $\begin{array}{c}\text { Station } \\
\text { no. }\end{array}$ & $\begin{array}{l}\text { No. } \\
\text { of } \\
\text { storms }\end{array}$ & $\begin{array}{c}\text { Total } \\
\text { duration } \\
\text { (hours) }\end{array}$ & $\begin{array}{c}\text { Mean } \\
\text { storm } \\
\text { interevent } \\
\text { time } \\
\text { (days) }\end{array}$ & $\begin{array}{c}\text { Station } \\
\text { no. }\end{array}$ & $\begin{array}{c}\text { No. } \\
\text { of } \\
\text { storms }\end{array}$ & $\begin{array}{c}\text { Total } \\
\text { duration } \\
\text { (hours) }\end{array}$ & $\begin{array}{c}\text { Mean } \\
\text { storm } \\
\text { interevent } \\
\text { time } \\
\text { (days) }\end{array}$ \\
\hline 0199 & 1,075 & 300,612 & 11.31 & 1963 & 2,061 & 468,726 & 9.16 & 4860 & 113 & 19,008 & 6.64 & 7649 & 2,594 & 439,618 & 6.65 \\
\hline 0205 & 869 & 239,640 & 11.19 & 1982 & 1,362 & 312,660 & 9.30 & 4862 & 891 & 171,413 & 7.72 & 7651 & 324 & 52,600 & 6.38 \\
\hline 0208 & 315 & 84,000 & 10.80 & 2030 & 2,096 & 484,051 & 9.36 & 5370 & 1,544 & 481,217 & 12.69 & 7735 & 1,243 & 237,408 & 7.47 \\
\hline 0600 & 1,630 & 484,110 & 12.09 & 2207 & 386 & 140,709 & 14.85 & 6138 & 900 & 197,952 & 8.73 & 8072 & 1,185 & 213,312 & 7.09 \\
\hline 0606 & 71 & 19,752 & 11.35 & 2510 & 2,010 & 484,087 & 9.69 & 6275 & 1,999 & 371,738 & 7.35 & 8078 & 328 & 75,240 & 9.20 \\
\hline 0646 & 439 & 113,256 & 10.39 & 2625 & 436 & 124,200 & 11.54 & 6435 & 1,496 & 483,422 & 13.15 & 8084 & 57 & 18,288 & 13.10 \\
\hline 1120 & 2,850 & 484,063 & 6.64 & 2665 & 2,256 & 484,030 & 8.56 & 6492 & 177 & 59,160 & 13.60 & 8085 & 1,378 & 269,810 & 7.80 \\
\hline 1440 & 1,266 & 236,375 & 7.48 & 2694 & 137 & 18,288 & 4.99 & 6619 & 488 & 152,664 & 12.77 & 8187 & 429 & 109,560 & 10.37 \\
\hline 1446 & 622 & 212,592 & 13.92 & 2700 & 2,931 & 484,034 & 6.58 & 6659 & 1,476 & 428,041 & 11.77 & 8358 & 62 & 27,048 & 17.87 \\
\hline 1807 & 140 & 57,696 & 16.89 & 4026 & 1 & 2,256 & 94.00 & 7277 & 2 & 2,160 & 45.00 & 8816 & 1 & 3,624 & 151.00 \\
\hline 1840 & 524 & 130,730 & 10.16 & 4028 & 319 & 91,032 & 11.66 & 7279 & 1,844 & 335,939 & 7.31 & 9148 & 6 & 8,808 & 60.57 \\
\hline 1881 & 3 & 504 & 7.00 & 4030 & 152 & 54,485 & 14.70 & 7283 & 1,121 & 167,256 & 5.86 & 9153 & 107 & 28,248 & 10.66 \\
\hline 1887 & 2,535 & 483,991 & 7.63 & 4089 & 2,026 & 484,016 & 9.60 & 7604 & 4 & 552 & 5.75 & 9156 & 2,015 & 483,287 & 9.71 \\
\hline 1939 & 2,052 & 468,627 & 9.20 & 4112 & 994 & 294,907 & 12.07 & 7605 & 23 & 11,208 & 19.99 & 9193 & 759 & 159,171 & 8.46 \\
\hline 1950 & 27 & 15,336 & 23.34 & 4306 & 517 & 124,200 & 9.68 & 7609 & 961 & 219,864 & 9.18 & 9265 & 516 & 168,048 & 13.31 \\
\hline 1956 & 40 & 15,336 & 15.62 & 4850 & 1,193 & 242,472 & 8.13 & 7610 & 968 & 254,143 & 10.60 & 9569 & 499 & 142,953 & 11.72 \\
\hline 1961 & 35 & 15,336 & 17.84 & 4856 & 273 & 62,088 & 9.11 & 7638 & 2,249 & 483,293 & 8.63 & 9686 & 1,610 & 483,331 & 12.22 \\
\hline
\end{tabular}


Appendix 2-1.5. Number of storms, total duration, and mean storm interevent time defined by 24-hour minimum interevent time for hourly rainfall stations in eastern New Mexico.

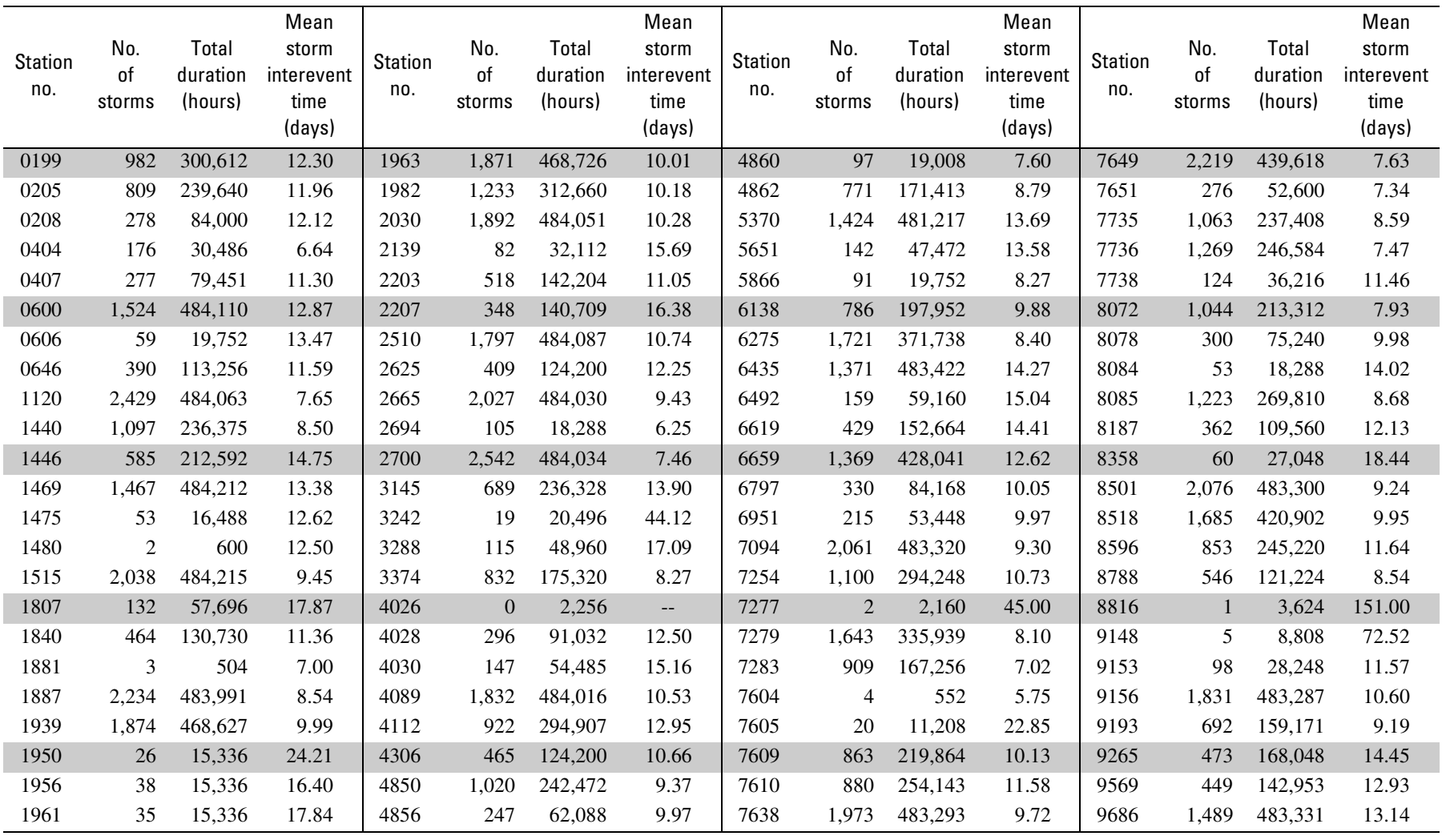

Appendix 2-1.6. Number of storms, total duration, and mean storm interevent time defined by 48-hour minimum interevent time for hourly rainfall stations in eastern New Mexico.

\begin{tabular}{|c|c|c|c|c|c|c|c|c|c|c|c|c|c|c|c|}
\hline $\begin{array}{c}\text { Station } \\
\text { no. }\end{array}$ & $\begin{array}{l}\text { No. } \\
\text { of } \\
\text { storms }\end{array}$ & $\begin{array}{c}\text { Total } \\
\text { duration } \\
\text { (hours) }\end{array}$ & $\begin{array}{c}\text { Mean } \\
\text { storm } \\
\text { interevent } \\
\text { time } \\
\text { (days) }\end{array}$ & $\begin{array}{c}\text { Station } \\
\text { no. }\end{array}$ & $\begin{array}{l}\text { No. } \\
\text { of } \\
\text { storms }\end{array}$ & $\begin{array}{c}\text { Total } \\
\text { duration } \\
\text { (hours) }\end{array}$ & $\begin{array}{c}\text { Mean } \\
\text { storm } \\
\text { interevent } \\
\text { time } \\
\text { (days) }\end{array}$ & $\begin{array}{c}\text { Station } \\
\text { no. }\end{array}$ & $\begin{array}{c}\text { No. } \\
\text { of } \\
\text { storms }\end{array}$ & $\begin{array}{c}\text { Total } \\
\text { duration } \\
\text { (hours) }\end{array}$ & $\begin{array}{c}\text { Mean } \\
\text { storm } \\
\text { interevent } \\
\text { time } \\
\text { (days) }\end{array}$ & $\begin{array}{c}\text { Station } \\
\text { no. }\end{array}$ & $\begin{array}{c}\text { No. } \\
\text { of } \\
\text { storms }\end{array}$ & $\begin{array}{c}\text { Total } \\
\text { duration } \\
\text { (hours) }\end{array}$ & $\begin{array}{c}\text { Mean } \\
\text { storm } \\
\text { interevent } \\
\text { time } \\
\text { (days) }\end{array}$ \\
\hline 0199 & 825 & 300,612 & 14.37 & 1963 & 1,543 & 468,726 & 11.83 & 4860 & 77 & 19,008 & 9.21 & 7649 & 1,640 & 439,618 & 9.81 \\
\hline 0205 & 682 & 239,640 & 13.91 & 1982 & 996 & 312,660 & 12.27 & 4862 & 593 & 171,413 & 11.00 & 7651 & 203 & 52,600 & 9.46 \\
\hline 0208 & 236 & 84,000 & 14.01 & 2030 & 1,549 & 484,051 & 12.24 & 5370 & 1,225 & 481,217 & 15.67 & 7735 & 842 & 237,408 & 10.47 \\
\hline 0600 & 1,317 & 484,110 & 14.67 & 2207 & 288 & 140,709 & 19.50 & 6138 & 608 & 197,952 & 12.34 & 8072 & 836 & 213,312 & 9.55 \\
\hline 0606 & 45 & 19,752 & 17.25 & 2510 & 1,467 & 484,087 & 12.83 & 6275 & 1,319 & 371,738 & 10.51 & 8078 & 254 & 75,240 & 11.52 \\
\hline 0646 & 329 & 113,256 & 13.46 & 2625 & 350 & 124,200 & 14.07 & 6435 & 1,168 & 483,422 & 16.50 & 8084 & 48 & 18,288 & 15.37 \\
\hline 1120 & 1,783 & 484,063 & 9.90 & 2665 & 1,646 & 484,030 & 11.27 & 6492 & 135 & 59,160 & 17.45 & 8085 & 986 & 269,810 & 10.42 \\
\hline 1440 & 867 & 236,375 & 10.37 & 2694 & 73 & 18,288 & 8.39 & 6619 & 360 & 152,664 & 16.89 & 8187 & 293 & 109,560 & 14.61 \\
\hline 1446 & 512 & 212,592 & 16.64 & 2700 & 1,927 & 484,034 & 9.37 & 6659 & 1,164 & 428,041 & 14.59 & 8358 & 53 & 27,048 & 20.67 \\
\hline 1807 & 119 & 57,696 & 19.66 & 4026 & 0 & 2,256 & -- & 7277 & 2 & 2,160 & 45.00 & 8816 & 1 & 3,624 & 151.00 \\
\hline 1840 & 379 & 130,730 & 13.59 & 4028 & 255 & 91,032 & 14.26 & 7279 & 1,303 & 335,939 & 9.83 & 9148 & 3 & 8,808 & 122.33 \\
\hline 1881 & 1 & 504 & 21.00 & 4030 & 116 & 54,485 & 18.85 & 7283 & 681 & 167,256 & 8.88 & 9153 & 72 & 28,248 & 15.18 \\
\hline 1887 & 1,754 & 483,991 & 10.48 & 4089 & 1,514 & 484,016 & 12.44 & 7604 & 3 & 552 & 7.67 & 9156 & 1,542 & 483,287 & 12.31 \\
\hline 1939 & 1,568 & 468,627 & 11.66 & 4112 & 779 & 294,907 & 15.06 & 7605 & 18 & 11,208 & 25.23 & 9193 & 556 & 159,171 & 11.09 \\
\hline 1950 & 23 & 15,336 & 27.16 & 4306 & 387 & 124,200 & 12.52 & 7609 & 714 & 219,864 & 11.94 & 9265 & 402 & 168,048 & 16.75 \\
\hline 1956 & 31 & 15,336 & 19.72 & 4850 & 802 & 242,472 & 11.53 & 7610 & 748 & 254,143 & 13.37 & 9569 & 387 & 142,953 & 14.78 \\
\hline 1961 & 30 & 15,336 & 20.57 & 4856 & 206 & 62,088 & 11.67 & 7638 & 1,594 & 483,293 & 11.69 & 9686 & 1,256 & 483,331 & 15.31 \\
\hline
\end{tabular}


104 Statistical Characteristics of Storm Interevent Time, Depth, and Duration for Eastern New Mexico, Oklahoma, and Texas

Appendix 2-1.7. Number of storms, total duration, and mean storm interevent time defined by 72-hour minimum interevent time for hourly rainfall stations in eastern New Mexico.

\begin{tabular}{|c|c|c|c|c|c|c|c|c|c|c|c|c|c|c|c|}
\hline $\begin{array}{c}\text { Station } \\
\text { no. }\end{array}$ & $\begin{array}{c}\text { No. } \\
\text { of } \\
\text { storms }\end{array}$ & $\begin{array}{c}\text { Total } \\
\text { duration } \\
\text { (hours) }\end{array}$ & $\begin{array}{c}\text { Mean } \\
\text { storm } \\
\text { interevent } \\
\text { time } \\
\text { (days) }\end{array}$ & $\begin{array}{c}\text { Station } \\
\text { no. }\end{array}$ & $\begin{array}{c}\text { No. } \\
\text { of } \\
\text { storms }\end{array}$ & $\begin{array}{c}\text { Total } \\
\text { duration } \\
\text { (hours) }\end{array}$ & $\begin{array}{c}\text { Mean } \\
\text { storm } \\
\text { interevent } \\
\text { time } \\
\text { (days) }\end{array}$ & $\begin{array}{c}\text { Station } \\
\text { no. }\end{array}$ & $\begin{array}{c}\text { No. } \\
\text { of } \\
\text { storms }\end{array}$ & $\begin{array}{c}\text { Total } \\
\text { duration } \\
\text { (hours) }\end{array}$ & $\begin{array}{c}\text { Mean } \\
\text { storm } \\
\text { interevent } \\
\text { time } \\
\text { (days) }\end{array}$ & $\begin{array}{c}\text { Station } \\
\text { no. }\end{array}$ & $\begin{array}{c}\text { No. } \\
\text { of } \\
\text { storms }\end{array}$ & $\begin{array}{c}\text { Total } \\
\text { duration } \\
\text { (hours) }\end{array}$ & $\begin{array}{c}\text { Mean } \\
\text { storm } \\
\text { interevent } \\
\text { time } \\
\text { (days) }\end{array}$ \\
\hline 0199 & 708 & 300,612 & 16.34 & 1963 & 1,305 & 468,726 & 13.54 & 4860 & 62 & 19,008 & 10.83 & 7649 & 1,337 & 439,618 & 11.47 \\
\hline 0205 & 599 & 239,640 & 15.49 & 1982 & 852 & 312,660 & 13.92 & 4862 & 489 & 171,413 & 12.83 & 7651 & 166 & 52,600 & 10.99 \\
\hline 0208 & 205 & 84,000 & 15.76 & 2030 & 1,329 & 484,051 & 13.86 & 5370 & 1,095 & 481,217 & 17.24 & 7735 & 706 & 237,408 & 12.02 \\
\hline 0404 & 96 & 30,486 & 10.57 & 2139 & 53 & 32,112 & 23.35 & 5651 & 117 & 47,472 & 16.03 & 7736 & 766 & 246,584 & 11.20 \\
\hline 0407 & 162 & 79,451 & 18.05 & 2203 & 385 & 142,204 & 14.26 & 5866 & 46 & 19,752 & 14.49 & 7738 & 91 & 36,216 & 14.89 \\
\hline 0600 & 1,157 & 484,110 & 16.36 & 2207 & 250 & 140,709 & 22.09 & 6138 & 498 & 197,952 & 14.51 & 8072 & 684 & 213,312 & 11.11 \\
\hline 0606 & 37 & 19,752 & 20.53 & 2510 & 1,281 & 484,087 & 14.33 & 6275 & 1,116 & 371,738 & 11.97 & 8078 & 214 & 75,240 & 13.21 \\
\hline 0646 & 276 & 113,256 & 15.57 & 2625 & 298 & 124,200 & 16.08 & 6435 & 1,024 & 483,422 & 18.48 & 8084 & 41 & 18,288 & 17.55 \\
\hline 1120 & 1,416 & 484,063 & 11.83 & 2665 & 1,394 & 484,030 & 12.87 & 6492 & 120 & 59,160 & 19.32 & 8085 & 832 & 269,810 & 11.89 \\
\hline 1440 & 737 & 236,375 & 11.76 & 2694 & 59 & 18,288 & 9.76 & 6619 & 312 & 152,664 & 19.11 & 8187 & 261 & 109,560 & 16.09 \\
\hline 1446 & 454 & 212,592 & 18.45 & 2700 & 1,579 & 484,034 & 10.90 & 6659 & 1,034 & 428,041 & 16.12 & 8358 & 49 & 27,048 & 22.15 \\
\hline 1469 & 1,136 & 484,212 & 16.75 & 3145 & 550 & 236,328 & 16.95 & 6797 & 218 & 84,168 & 14.27 & 8501 & 1,393 & 483,300 & 12.87 \\
\hline 1475 & 41 & 16,488 & 15.65 & 3242 & 15 & 20,496 & 55.34 & 6951 & 152 & 53,448 & 13.29 & 8518 & 1,169 & 420,902 & 13.51 \\
\hline 1480 & 1 & 600 & 25.00 & 3288 & 74 & 48,960 & 25.49 & 7094 & 1,402 & 483,320 & 12.80 & 8596 & 612 & 245,220 & 15.50 \\
\hline 1515 & 1,380 & 484,215 & 13.07 & 3374 & 573 & 175,320 & 11.19 & 7254 & 796 & 294,248 & 14.12 & 8788 & 354 & 121,224 & 12.19 \\
\hline 1807 & 108 & 57,696 & 21.40 & 4026 & 0 & 2,256 & -- & 7277 & 2 & 2,160 & 45.00 & 8816 & 1 & 3,624 & 151.00 \\
\hline 1840 & 331 & 130,730 & 15.20 & 4028 & 222 & 91,032 & 16.01 & 7279 & 1,072 & 335,939 & 11.42 & 9148 & 2 & 8,808 & 183.50 \\
\hline 1881 & 1 & 504 & 21.00 & 4030 & 104 & 54,485 & 20.75 & 7283 & 543 & 167,256 & 10.49 & 9153 & 63 & 28,248 & 16.96 \\
\hline 1887 & 1,467 & 483,991 & 12.05 & 4089 & 1,299 & 484,016 & 14.10 & 7604 & 2 & 552 & 11.50 & 9156 & 1,334 & 483,287 & 13.84 \\
\hline 1939 & 1,346 & 468,627 & 13.18 & 4112 & 691 & 294,907 & 16.66 & 7605 & 15 & 11,208 & 29.80 & 9193 & 465 & 159,171 & 12.78 \\
\hline 1950 & 21 & 15,336 & 29.51 & 4306 & 326 & 124,200 & 14.39 & 7609 & 618 & 219,864 & 13.41 & 9265 & 363 & 168,048 & 18.30 \\
\hline 1956 & 26 & 15,336 & 23.04 & 4850 & 662 & 242,472 & 13.44 & 7610 & 661 & 254,143 & 14.80 & 9569 & 330 & 142,953 & 16.91 \\
\hline 1961 & 24 & 15,336 & 25.10 & 4856 & 169 & 62,088 & 13.68 & 7638 & 1,357 & 483,293 & 13.29 & 9686 & 1,103 & 483,331 & 17.09 \\
\hline
\end{tabular}


Appendix 2-2.1. L-moments of storm depth defined by 6-hour minimum interevent time for hourly rainfall stations in eastern New Mexico.

[--, not available]

\begin{tabular}{|c|c|c|c|c|c|c|c|c|c|c|c|c|c|}
\hline $\begin{array}{c}\text { Station } \\
\text { no. }\end{array}$ & $\begin{array}{c}\text { Depth } \\
\text { mean } \\
\text { (inches) }\end{array}$ & $\begin{array}{c}\text { Depth } \\
\text { L-scale } \\
\text { (inches) }\end{array}$ & $\begin{array}{c}\text { Depth } \\
\text { L-CV } \\
\text { (dimen- } \\
\text { sionless) }\end{array}$ & $\begin{array}{l}\text { Depth } \\
\text { L-skew } \\
\text { (dimen- } \\
\text { sionless) }\end{array}$ & $\begin{array}{c}\text { Depth } \\
\text { L-kurtosis } \\
\text { (dimen- } \\
\text { sionless) }\end{array}$ & $\begin{array}{c}\text { Depth } \\
\text { Tau5 } \\
\text { (dimen- } \\
\text { sionless) }\end{array}$ & $\begin{array}{c}\text { Station } \\
\text { no. }\end{array}$ & $\begin{array}{l}\text { Depth } \\
\text { mean } \\
\text { (inches) }\end{array}$ & $\begin{array}{l}\text { Depth } \\
\text { L-scale } \\
\text { (inches) }\end{array}$ & $\begin{array}{c}\text { Depth } \\
\text { L-CV } \\
\text { (dimen- } \\
\text { sionless) }\end{array}$ & $\begin{array}{c}\text { Depth } \\
\text { L-skew } \\
\text { (dimen- } \\
\text { sionless) }\end{array}$ & $\begin{array}{l}\text { Depth } \\
\text { L-kurtosis } \\
\text { (dimen- } \\
\text { sionless) }\end{array}$ & $\begin{array}{c}\text { Depth } \\
\text { Tau5 } \\
\text { (dimen- } \\
\text { sionless) }\end{array}$ \\
\hline 0205 & .26399 & .16236 & .61503 & .53188 & .32677 & .22765 & 4862 & .28971 & .14096 & .48657 & .55900 & .27132 & .15749 \\
\hline 0208 & .21238 & .11126 & .52386 & .42549 & .20768 & .12692 & 5370 & .34465 & .19869 & .57650 & .55189 & .34540 & .22082 \\
\hline 0404 & .29155 & .14655 & .50266 & .61466 & .36450 & .26924 & 5651 & .26045 & .14685 & .56381 & .48115 & .26032 & .14918 \\
\hline 0600 & .28481 & .16297 & .57219 & .55504 & .35913 & .21296 & 6138 & .24529 & .14477 & .59021 & .50546 & .29449 & .19753 \\
\hline 0606 & .26878 & .12729 & .47357 & .36271 & .20509 & .10423 & 6275 & .25631 & .13912 & .54279 & .50064 & .29651 & .15614 \\
\hline 0646 & .29168 & .16125 & .55283 & .47300 & .28024 & .18503 & 6435 & .25634 & .14156 & .55224 & .50252 & .30459 & .17476 \\
\hline 1120 & .30475 & .15987 & .52457 & .47994 & .26385 & .14000 & 6492 & .17969 & .11034 & 61409 & .53948 & .33866 & .23680 \\
\hline 1440 & .26650 & .12826 & .48130 & .53185 & .27897 & .14270 & 6619 & .32843 & .18725 & .57013 & .48386 & .27723 & .17620 \\
\hline 1480 & -- & -- & -- & -- & -- & -- & 7094 & .23486 & .12162 & .51784 & .49660 & .30121 & .15688 \\
\hline 1515 & .22585 & .12007 & .53165 & .46540 & .26710 & .13799 & 7254 & .26117 & .14355 & .54963 & .54496 & .35284 & .19951 \\
\hline 1807 & .24778 & .13295 & .53657 & .39702 & .18541 & .11432 & 7277 & -- & -- & -- & -- & -- & -- \\
\hline 1840 & .32161 & .16667 & .51824 & .59455 & .34606 & .24809 & 7279 & .26822 & .13226 & .49310 & .54595 & .30895 & .17066 \\
\hline 1881 & -- & -- & -- & -- & -- & -- & 7283 & .19001 & .12664 & 66648 & .56644 & .33423 & .22419 \\
\hline 1887 & .23973 & .15717 & .65559 & .55225 & .32973 & .22439 & 7604 & .18200 & .11400 & .62637 & .24561 & -.31579 & -.24561 \\
\hline 1939 & .34381 & .19793 & .57568 & .51153 & .29776 & .17417 & 7605 & .27419 & .12774 & .46588 & .51306 & .16567 & .03286 \\
\hline 1950 & .42063 & .24183 & .57494 & .44714 & .23209 & .17596 & 7609 & .21892 & .14779 & .67508 & .56849 & .33348 & .22676 \\
\hline 1956 & .26333 & .16124 & .61231 & .55667 & .39536 & .32022 & 7610 & .29001 & .18276 & 63018 & .56101 & .36482 & .23056 \\
\hline 1961 & .31977 & .19870 & .62140 & .47974 & .20962 & .08397 & 7638 & .29020 & .15546 & .53569 & .51253 & .29315 & .15175 \\
\hline 2625 & .27842 & .17757 & .63778 & .59243 & .39438 & .29346 & 8084 & .21333 & .10845 & .50837 & .46517 & .30696 & .20637 \\
\hline 2665 & .25781 & .13857 & .53748 & .48062 & .27194 & .14346 & 8085 & .21802 & .11056 & .50711 & .48920 & .30298 & .14099 \\
\hline 2694 & .24075 & .14615 & .60705 & .49702 & .28883 & .22302 & 8187 & .30280 & .18026 & .59530 & .51431 & .30556 & .20610 \\
\hline 2700 & .19541 & .08872 & .45401 & .52657 & .34724 & .14818 & 8358 & .25534 & .14681 & .57496 & .52922 & .34283 & .23250 \\
\hline 3145 & .25823 & .15435 & .59774 & .53447 & .31759 & .19529 & 8501 & .27210 & .15543 & .57124 & .50632 & .30191 & .17852 \\
\hline 3242 & .39036 & .18967 & .48589 & .39728 & .14091 & .00033 & 8518 & .21598 & .11964 & .55396 & .50423 & .31043 & .18066 \\
\hline 3288 & .27219 & .14698 & .53999 & .41514 & .19806 & .10369 & 8596 & .32249 & .17025 & .52793 & .58071 & .31507 & .18989 \\
\hline 3374 & .19020 & .11337 & .59608 & .52474 & .30234 & .18589 & 8788 & .28450 & .15507 & .54508 & .43866 & .23460 & .14198 \\
\hline 4026 & -- & -- & -- & -- & -- & -- & 8816 & -- & -- & -- & -- & -- & -- \\
\hline 4028 & .30486 & .19552 & .64135 & .53391 & .29031 & .17074 & 9148 & .10250 & .03929 & .38328 & -.10909 & .04545 & .52727 \\
\hline 4030 & .33743 & .17557 & .52030 & .55494 & .26825 & .17243 & 9153 & .31371 & .19687 & .62753 & .51197 & .29828 & .20171 \\
\hline 4089 & .27467 & .15447 & .56240 & .52146 & .31147 & .16898 & 9156 & .32439 & .18045 & .55627 & .51085 & .28897 & .15352 \\
\hline 4112 & .32510 & .17852 & .54911 & .59447 & .36332 & .23386 & 9193 & .25800 & .12618 & .48905 & .61358 & .35423 & .21048 \\
\hline 4306 & .23532 & .13796 & .58626 & .48563 & .25978 & .16316 & 9265 & .32373 & .18156 & .56083 & .58822 & .37256 & .23291 \\
\hline 4850 & .24952 & .14030 & .56225 & .51771 & .33236 & .20256 & 9569 & .32879 & .16792 & .51072 & .54357 & .24645 & .13747 \\
\hline 4856 & .24503 & .15078 & .61534 & .51869 & .29042 & .18335 & 9686 & .21967 & .11781 & .53628 & .48531 & .29173 & .15428 \\
\hline
\end{tabular}


Appendix 2-2.2. L-moments of storm depth defined by 8-hour minimum interevent time for hourly rainfall stations in eastern New Mexico.

$[--$, not available]

\begin{tabular}{|c|c|c|c|c|c|c|c|c|c|c|c|c|c|}
\hline $\begin{array}{c}\text { Station } \\
\text { no. }\end{array}$ & $\begin{array}{l}\text { Depth } \\
\text { mean } \\
\text { (inches) }\end{array}$ & $\begin{array}{l}\text { Depth } \\
\text { L-scale } \\
\text { (inches) }\end{array}$ & $\begin{array}{c}\text { Depth } \\
\text { L-CV } \\
\text { (dimen- } \\
\text { sionless) }\end{array}$ & $\begin{array}{c}\text { Depth } \\
\text { L-skew } \\
\text { (dimen- } \\
\text { sionless) }\end{array}$ & $\begin{array}{c}\text { Depth } \\
\text { L-kurtosis } \\
\text { (dimen- } \\
\text { sionless) }\end{array}$ & $\begin{array}{c}\text { Depth } \\
\text { Tau5 } \\
\text { (dimen- } \\
\text { sionless) }\end{array}$ & $\begin{array}{c}\text { Station } \\
\text { no. }\end{array}$ & $\begin{array}{l}\text { Depth } \\
\text { mean } \\
\text { (inches) }\end{array}$ & $\begin{array}{l}\text { Depth } \\
\text { L-scale } \\
\text { (inches) }\end{array}$ & $\begin{array}{c}\text { Depth } \\
\text { L-CV } \\
\text { (dimen- } \\
\text { sionless) }\end{array}$ & $\begin{array}{c}\text { Depth } \\
\text { L-skew } \\
\text { (dimen- } \\
\text { sionless) }\end{array}$ & $\begin{array}{c}\text { Depth } \\
\text { L-kurtosis } \\
\text { (dimen- } \\
\text { sionless) }\end{array}$ & $\begin{array}{c}\text { Depth } \\
\text { Tau5 } \\
\text { (dimen- } \\
\text { sionless) }\end{array}$ \\
\hline 0205 & .27533 & .16810 & .61055 & .52681 & .32465 & .22687 & 4862 & .30495 & .14977 & .49113 & .54291 & .25922 & .15348 \\
\hline 0208 & .22163 & .11480 & .51795 & .42046 & .21228 & .13170 & 5370 & .36353 & .20898 & .57486 & .54487 & .33561 & .21645 \\
\hline 0404 & .32218 & .16468 & .51115 & .58422 & .33698 & .24685 & 5651 & .27440 & .15433 & .56241 & .47719 & .25254 & .14402 \\
\hline 0600 & .29705 & .17066 & .57453 & .55093 & .35020 & .20829 & 6138 & .26278 & .15389 & .58561 & .49537 & .28606 & 19223 \\
\hline 0606 & .27550 & .12820 & .46533 & .35619 & .20050 & .10239 & 6275 & .27287 & .14841 & .54388 & .49662 & .28759 & .15392 \\
\hline 0646 & .31124 & .16909 & .54328 & .46823 & .27543 & .17722 & 6435 & .26924 & .14903 & .55351 & .50374 & .30737 & .18515 \\
\hline 1120 & .32815 & .17239 & .52536 & .47198 & .25522 & .14207 & 6492 & .19264 & .11732 & .60900 & .53433 & .32983 & .22864 \\
\hline 1440 & .28235 & .13694 & .48499 & .51347 & .26167 & .14049 & 6619 & .33932 & .19282 & .56827 & .48167 & .27190 & .16859 \\
\hline 1480 & -- & -- & -- & -- & -- & -- & 7094 & .24919 & .12945 & .51948 & .48860 & .28481 & .14847 \\
\hline 1515 & .23719 & .12633 & .53259 & .46049 & .25804 & .13339 & 7254 & .27267 & .14991 & .54978 & .53985 & .34399 & 19635 \\
\hline 1807 & .25096 & .13469 & .53671 & .39714 & .18401 & .11184 & 7277 & -- & -- & -- & -- & -- & -- \\
\hline 1840 & .33980 & .17974 & .52896 & .59058 & .33973 & .23897 & 7279 & .28356 & .14166 & .49960 & .53659 & .30115 & .17444 \\
\hline 1881 & -- & -- & -- & -- & -- & -- & 7283 & .20018 & .13360 & .66738 & .56771 & .33685 & .22541 \\
\hline 1887 & .25182 & .16446 & .65309 & .54828 & .32737 & .22433 & 7604 & .18200 & .11400 & .62637 & .24561 & -.31579 & -.24561 \\
\hline 1939 & .36125 & .20803 & .57586 & .50887 & .29474 & .17609 & 7605 & .28333 & .13207 & .46613 & .48887 & .13845 & .01985 \\
\hline 1950 & .44867 & .25200 & .56166 & .42651 & .22969 & .17747 & 7609 & .23073 & .15532 & .67314 & .56716 & .33401 & .22767 \\
\hline 1956 & .28089 & .16943 & .60321 & .55362 & .38825 & .29932 & 7610 & .30877 & .19438 & .62951 & .55611 & .35487 & .22188 \\
\hline 1961 & .33537 & .20704 & .61735 & .45304 & .17762 & .07806 & 7638 & .30601 & .16476 & .53842 & .50790 & .28630 & .15162 \\
\hline 2625 & .29373 & .18612 & .63366 & .58513 & .38717 & .28665 & 8084 & .22349 & .11349 & .50779 & .45960 & .29575 & .20181 \\
\hline 2665 & .27169 & .14489 & .53330 & .47310 & .26313 & .14182 & 8085 & .23417 & .11930 & .50945 & .48308 & .28598 & .13371 \\
\hline 2694 & .27672 & .16252 & .58731 & .47906 & .28216 & .22114 & 8187 & .31740 & .18736 & .59028 & .50759 & .30017 & .20109 \\
\hline 2700 & .20562 & .09471 & .46063 & .52372 & .33258 & .14560 & 8358 & .26629 & .15082 & .56638 & .51527 & .33036 & .23403 \\
\hline 3145 & .27511 & .16326 & .59344 & .52948 & .31449 & .19924 & 8501 & .28866 & .16419 & .56880 & .49946 & .29352 & .17499 \\
\hline 3242 & .40481 & .19359 & .47822 & .37458 & .12443 & -.00483 & 8518 & .22718 & .12559 & .55280 & .50145 & .30489 & .17779 \\
\hline 3288 & .29114 & .15750 & .54098 & .42355 & .21526 & .12505 & 8596 & .33876 & .18018 & .53189 & .57446 & .30833 & .19137 \\
\hline 3374 & .20173 & .11940 & .59188 & .51461 & .29282 & .18307 & 8788 & .30346 & .16239 & .53511 & .42499 & .22235 & .13313 \\
\hline 4026 & -- & -- & -- & -- & -- & -- & 8816 & -- & -- & -- & -- & -- & -- \\
\hline 4028 & .31537 & .20321 & .64434 & .53679 & .29348 & .17488 & 9148 & .11714 & .05857 & .50000 & .15122 & -.02439 & .21951 \\
\hline 4030 & .35251 & .18470 & .52396 & .54117 & .25165 & .15808 & 9153 & .31611 & .19757 & .62500 & .51065 & .29771 & .20115 \\
\hline 4089 & .29466 & .16591 & .56307 & .51272 & .29625 & .16184 & 9156 & .33830 & .18872 & .55784 & .50543 & .27832 & .14734 \\
\hline 4112 & .34158 & .18892 & .55308 & .58589 & .35541 & .23305 & 9193 & .27571 & .13673 & .49593 & .59023 & .33202 & .20464 \\
\hline 4306 & .24236 & .14207 & .58617 & .48542 & .25905 & .16046 & 9265 & .34293 & .19541 & .56983 & .58779 & .37434 & .24073 \\
\hline 4850 & .26256 & .14826 & .56465 & .52172 & .33468 & .20640 & 9569 & .34323 & .17651 & .51427 & .53346 & .24117 & .14015 \\
\hline 4856 & .25713 & .15636 & .60810 & .50847 & .28098 & .17874 & 9686 & .22998 & .12351 & .53704 & .48226 & .28493 & .15195 \\
\hline
\end{tabular}


Appendix 2-2.3. L-moments of storm depth defined by 12-hour minimum interevent time for hourly rainfall stations in eastern New Mexico.

[--, not available]

\begin{tabular}{|c|c|c|c|c|c|c|c|c|c|c|c|c|c|}
\hline $\begin{array}{c}\text { Station } \\
\text { no. }\end{array}$ & $\begin{array}{l}\text { Depth } \\
\text { mean } \\
\text { (inches) }\end{array}$ & $\begin{array}{l}\text { Depth } \\
\text { L-scale } \\
\text { (inches) }\end{array}$ & $\begin{array}{c}\text { Depth } \\
\text { L-CV } \\
\text { (dimen- } \\
\text { sionless) }\end{array}$ & $\begin{array}{c}\text { Depth } \\
\text { L-skew } \\
\text { (dimen- } \\
\text { sionless) }\end{array}$ & $\begin{array}{c}\text { Depth } \\
\text { L-kurtosis } \\
\text { (dimen- } \\
\text { sionless) }\end{array}$ & $\begin{array}{c}\text { Depth } \\
\text { Tau5 } \\
\text { (dimen- } \\
\text { sionless) }\end{array}$ & $\begin{array}{c}\text { Station } \\
\text { no. }\end{array}$ & $\begin{array}{l}\text { Depth } \\
\text { mean } \\
\text { (inches) }\end{array}$ & $\begin{array}{l}\text { Depth } \\
\text { L-scale } \\
\text { (inches) }\end{array}$ & $\begin{array}{c}\text { Depth } \\
\text { L-CV } \\
\text { (dimen- } \\
\text { sionless) }\end{array}$ & $\begin{array}{c}\text { Depth } \\
\text { L-skew } \\
\text { (dimen- } \\
\text { sionless) }\end{array}$ & $\begin{array}{c}\text { Depth } \\
\text { L-kurtosis } \\
\text { (dimen- } \\
\text { sionless) }\end{array}$ & $\begin{array}{c}\text { Depth } \\
\text { Tau5 } \\
\text { (dimen- } \\
\text { sionless) }\end{array}$ \\
\hline 0205 & .29142 & .17889 & .61385 & .52881 & .32902 & .23406 & 4862 & .33175 & .16574 & .49961 & .52842 & .25754 & .15972 \\
\hline 0208 & .24136 & .12395 & .51354 & .43013 & .23789 & .16119 & 5370 & .39477 & .22843 & .57863 & .54037 & .33454 & .22361 \\
\hline 0404 & .36157 & .18549 & .51302 & .55644 & .33981 & .27492 & 5651 & .29363 & .17020 & .57965 & .50487 & .28995 & 18977 \\
\hline 0600 & .32130 & .18548 & .57729 & .54312 & .34005 & .21158 & 6138 & .28892 & .16914 & .58541 & .50032 & .29667 & .20141 \\
\hline 0606 & .30192 & .14094 & .46680 & .36235 & .20388 & .09707 & 6275 & .29711 & .16147 & .54346 & .49132 & .28049 & .15851 \\
\hline 0646 & .32807 & .17712 & .53988 & .46457 & .27436 & .17372 & 6435 & .29258 & .16193 & .55347 & .49927 & .29769 & .17998 \\
\hline 1120 & .36668 & .19387 & .52873 & .46520 & .24614 & .14198 & 6492 & .20870 & .12412 & .59473 & .51313 & .31512 & .22653 \\
\hline 1440 & .30890 & .15275 & .49451 & .49704 & .25348 & .14520 & 6619 & .35297 & .19861 & .56268 & .48059 & .27004 & .16158 \\
\hline 1480 & -- & -- & -- & -- & -- & -- & 7094 & .27167 & .14204 & .52284 & .47989 & .27114 & .14604 \\
\hline 1515 & .25631 & .13677 & .53360 & .45371 & .24790 & .13177 & 7254 & .29537 & .16327 & .55275 & .53361 & .32573 & .18275 \\
\hline 1807 & .26100 & .14313 & .54840 & .40621 & .18682 & .11869 & 7277 & -- & -- & -- & -- & -- & -- \\
\hline 1840 & .36664 & .19338 & .52743 & .56321 & .32213 & .23022 & 7279 & .30565 & .15477 & .50636 & .52140 & .28395 & .16851 \\
\hline 1881 & -- & -- & -- & -- & -- & -- & 7283 & .21740 & .14438 & .66415 & .56142 & .33143 & .22144 \\
\hline 1887 & .26828 & .17516 & .65291 & .54811 & .32753 & .22234 & 7604 & .18200 & .11400 & .62637 & .24561 & -.31579 & -.24561 \\
\hline 1939 & .39031 & .22555 & .57788 & .50332 & .29084 & .17897 & 7605 & .32692 & .15246 & .46635 & .42230 & .08492 & -.03457 \\
\hline 1950 & .49852 & .26091 & .52337 & .40582 & .24066 & .16482 & 7609 & .24808 & .16655 & .67136 & .56373 & .33336 & .22923 \\
\hline 1956 & .28727 & .17439 & .60704 & .54683 & .37020 & .28306 & 7610 & .33508 & .20843 & .62204 & .54213 & .33944 & .21432 \\
\hline 1961 & .35256 & .21089 & .59816 & .44118 & .16822 & .07771 & 7638 & .32924 & .17871 & .54279 & .49925 & .27544 & .14853 \\
\hline 2625 & .31352 & .19825 & .63233 & .57523 & .37406 & .27549 & 8084 & .22349 & .11349 & .50779 & .45960 & .29575 & .20181 \\
\hline 2665 & .29420 & .15709 & .53397 & .46587 & .25401 & .13998 & 8085 & .25770 & .13282 & .51541 & .47303 & .26526 & .12811 \\
\hline 2694 & .31065 & .17649 & .56813 & .45340 & .26397 & .21160 & 8187 & .33422 & .19811 & .59276 & .50444 & .29405 & .19532 \\
\hline 2700 & .22317 & .10493 & .47017 & .51445 & .31232 & .14829 & 8358 & .27821 & .15938 & .57288 & .51966 & .32782 & .21957 \\
\hline 3145 & .29284 & .17434 & .59535 & .53355 & .32109 & .20681 & 8501 & .30642 & .17439 & .56912 & .49298 & .28308 & .16787 \\
\hline 3242 & .40481 & .19359 & .47822 & .37458 & .12443 & -.00483 & 8518 & .24493 & .13698 & .55926 & .49749 & .29145 & .16652 \\
\hline 3288 & .32394 & .18124 & .55949 & .45121 & .24203 & .14905 & 8596 & .36596 & .19629 & .53637 & .55493 & .29385 & .18928 \\
\hline 3374 & .21601 & .12797 & .59241 & .51077 & .28780 & .17852 & 8788 & .32514 & .17253 & .53063 & .41485 & .21145 & .12255 \\
\hline 4026 & -- & -- & -- & -- & -- & -- & 8816 & -- & -- & -- & -- & -- & -- \\
\hline 4028 & .33057 & .21445 & .64874 & .54124 & .29621 & .17453 & 9148 & .11714 & .05857 & .50000 & .15122 & -.02439 & .21951 \\
\hline 4030 & .37337 & .19601 & .52498 & .51851 & .22949 & .14538 & 9153 & .35093 & .21969 & .62603 & .52353 & .30705 & .19986 \\
\hline 4089 & .32306 & .18271 & .56556 & .51358 & .29509 & .16833 & 9156 & .36090 & .20289 & .56218 & .50738 & .27912 & .15407 \\
\hline 4112 & .37275 & .20757 & .55686 & .57128 & .34092 & .23206 & 9193 & .30239 & .15287 & .50555 & .57216 & .31272 & .20123 \\
\hline 4306 & .25735 & .15211 & .59107 & .48594 & .25634 & .15938 & 9265 & .36648 & .21227 & .57921 & .59357 & .38970 & .26807 \\
\hline 4850 & .28674 & .16154 & .56338 & .50884 & .31755 & .19957 & 9569 & .36573 & .18997 & .51943 & .52019 & .23399 & .14064 \\
\hline 4856 & .27586 & .16662 & .60401 & .50316 & .27154 & .16455 & 9686 & .24953 & .13457 & .53930 & .47115 & .26744 & .14595 \\
\hline
\end{tabular}


Appendix 2-2.4. L-moments of storm depth defined by 18-hour minimum interevent time for hourly rainfall stations in eastern New Mexico.

$[--$, not available $]$

\begin{tabular}{|c|c|c|c|c|c|c|c|c|c|c|c|c|c|}
\hline $\begin{array}{c}\text { Station } \\
\text { no. }\end{array}$ & $\begin{array}{l}\text { Depth } \\
\text { mean } \\
\text { (inches) }\end{array}$ & $\begin{array}{l}\text { Depth } \\
\text { L-scale } \\
\text { (inches) }\end{array}$ & $\begin{array}{c}\text { Depth } \\
\text { L-CV } \\
\text { (dimen- } \\
\text { sionless) }\end{array}$ & $\begin{array}{c}\text { Depth } \\
\text { L-skew } \\
\text { (dimen- } \\
\text { sionless) }\end{array}$ & $\begin{array}{c}\text { Depth } \\
\text { L-kurtosis } \\
\text { (dimen- } \\
\text { sionless) }\end{array}$ & $\begin{array}{c}\text { Depth } \\
\text { Tau5 } \\
\text { (dimen- } \\
\text { sionless) }\end{array}$ & $\begin{array}{c}\text { Station } \\
\text { no. }\end{array}$ & $\begin{array}{l}\text { Depth } \\
\text { mean } \\
\text { (inches) }\end{array}$ & $\begin{array}{l}\text { Depth } \\
\text { L-scale } \\
\text { (inches) }\end{array}$ & $\begin{array}{c}\text { Depth } \\
\text { L-CV } \\
\text { (dimen- } \\
\text { sionless) }\end{array}$ & $\begin{array}{l}\text { Depth } \\
\text { L-skew } \\
\text { (dimen- } \\
\text { sionless) }\end{array}$ & $\begin{array}{c}\text { Depth } \\
\text { L-kurtosis } \\
\text { (dimen- } \\
\text { sionless) }\end{array}$ & $\begin{array}{c}\text { Depth } \\
\text { Tau5 } \\
\text { (dimen- } \\
\text { sionless) }\end{array}$ \\
\hline 0199 & 0.34008 & 0.17819 & 0.52396 & 0.45506 & 0.25162 & 0.14773 & 4860 & 0.31566 & 0.19630 & 0.62186 & 0.54826 & 0.30678 & 0.16471 \\
\hline 0205 & .30986 & .19166 & .61853 & .53395 & .33277 & .23496 & 4862 & .37717 & .19620 & .52019 & .52227 & .25736 & .16179 \\
\hline 0208 & .25822 & .13263 & .51363 & .42524 & .23160 & .15539 & 5370 & .42545 & .24702 & .58061 & .53747 & .33442 & .23043 \\
\hline 0404 & .40990 & .20784 & .50704 & .51795 & .31960 & .25546 & 5651 & .31361 & .17955 & .57253 & .47990 & .26249 & .17805 \\
\hline 0407 & .33802 & .16648 & .49250 & .50816 & .23847 & .15176 & 5866 & .43053 & .25490 & .59207 & .44893 & .20163 & .10686 \\
\hline 0600 & .34771 & .20286 & .58340 & .53978 & .33470 & .21440 & 6138 & .31942 & .18417 & .57658 & .48380 & .28352 & .19088 \\
\hline 0606 & .31042 & .14321 & .46134 & .34696 & .19489 & .09463 & 6275 & .33798 & .18612 & .55068 & .48235 & .26956 & .16031 \\
\hline 0646 & .35945 & .19296 & .53680 & .45260 & .25836 & .16205 & 6435 & .31820 & .17749 & .55781 & .50048 & .29706 & .18279 \\
\hline 1120 & .41853 & .22197 & .53036 & .45467 & .23550 & .14278 & 6492 & .22638 & .13532 & .59774 & .52541 & .32926 & .23829 \\
\hline 1440 & .34502 & .17326 & .50219 & .47816 & .24217 & .14161 & 6619 & .37756 & .21175 & .56083 & .47437 & .26562 & .15758 \\
\hline 1446 & .42084 & .24598 & .58451 & .48062 & .26720 & .17928 & 6659 & .44101 & .27033 & .61298 & .54924 & .33686 & .22325 \\
\hline 1469 & .39911 & .23876 & .59823 & .53728 & .32953 & .21681 & 6797 & .26354 & .15335 & .58186 & .51720 & .33417 & .23646 \\
\hline 1475 & .35945 & .25608 & .71241 & .61799 & .35927 & .21129 & 6951 & .32397 & .19220 & .59326 & .46861 & .22429 & .12856 \\
\hline 1480 & -- & -- & -- & -- & -- & -- & 7094 & .30269 & .15974 & .52774 & .46914 & .25810 & .14698 \\
\hline 1515 & .28296 & .15107 & .53390 & .44442 & .23830 & .13376 & 7254 & .32327 & .17945 & .55512 & .52286 & .30885 & .17475 \\
\hline 1807 & .27964 & .15614 & .55834 & .42449 & .21092 & .14083 & 7277 & -- & -- & -- & -- & -- & -- \\
\hline 1840 & .38973 & .20784 & .53328 & .55430 & .31417 & .22067 & 7279 & .33615 & .17547 & .52201 & .51632 & .27753 & .16261 \\
\hline 1881 & -- & -- & -- & -- & -- & -- & 7283 & .24358 & .16210 & .66548 & .55409 & .32090 & .21484 \\
\hline 1887 & .29156 & .19028 & .65262 & .54543 & .32432 & .21712 & 7604 & -- & -- & -- & -- & -- & -- \\
\hline 1939 & .42322 & .24703 & .58370 & .50436 & .29285 & .18390 & 7605 & .36957 & .17866 & .48342 & .41783 & .10177 & .00609 \\
\hline 1950 & .49852 & .26091 & .52337 & .40582 & .24066 & .16482 & 7609 & .27131 & .18091 & .66680 & .55016 & .31401 & .21006 \\
\hline 1956 & .31600 & .19133 & .60549 & .52158 & .33050 & .25089 & 7610 & .36945 & .22995 & .62241 & .54091 & .33315 & .20764 \\
\hline 1961 & .39286 & .22484 & .57232 & .39124 & .14340 & .07728 & 7638 & .36452 & .20089 & .55110 & .49461 & .26476 & .14324 \\
\hline 1963 & .40813 & .24086 & .59015 & .52834 & .30529 & .18515 & 7649 & .36261 & .20006 & .55171 & .44543 & .24024 & .14619 \\
\hline 1982 & .29640 & .14248 & .48070 & .53567 & .24990 & .15630 & 7651 & .43025 & .21610 & .50226 & .44750 & .19217 & .12473 \\
\hline 2030 & .34824 & .19746 & .56702 & .51576 & .29928 & .16995 & 7735 & .40451 & .22630 & .55945 & .46123 & .25667 & .16448 \\
\hline 2139 & .31484 & .17252 & .54797 & .43158 & .22642 & .15039 & 7736 & .46605 & .24928 & .53488 & .48217 & .25598 & .17393 \\
\hline 2203 & .46254 & .25977 & .56161 & .56393 & .33022 & .23711 & 7738 & .46120 & .26240 & .56895 & .43733 & .20635 & .09799 \\
\hline 2207 & .42153 & .22863 & .54239 & .48447 & .25615 & .16253 & 8072 & .26713 & .15316 & .57336 & .47429 & .26397 & .16320 \\
\hline 2510 & .34711 & .19007 & .54759 & .49819 & .28041 & .17296 & 8078 & .22665 & .13252 & .58468 & .50116 & .31046 & .20983 \\
\hline 2625 & .33078 & .21061 & .63669 & .57956 & .37632 & .27253 & 8084 & .24702 & .13319 & .53919 & .50511 & .32570 & .20466 \\
\hline 2665 & .32432 & .17432 & .53749 & .46352 & .25284 & .14648 & 8085 & .28668 & .15082 & .52608 & .46744 & .25562 & .13204 \\
\hline 2694 & .35146 & .19340 & .55027 & .41544 & .23997 & .20394 & 8187 & .35291 & .20918 & .59273 & .50208 & .28834 & .18715 \\
\hline 2700 & .24708 & .11993 & .48539 & .50738 & .29604 & .14913 & 8358 & .30065 & .17520 & .58274 & .54722 & .36089 & .25080 \\
\hline 3145 & .31129 & .18578 & .59681 & .52612 & .30899 & .19590 & 8501 & .33474 & .19076 & .56989 & .48081 & .26678 & .15979 \\
\hline 3242 & .54650 & .28034 & .51298 & .41563 & .16115 & .07700 & 8518 & .27136 & .15326 & .56480 & .49123 & .27978 & .16288 \\
\hline 3288 & .35385 & .19852 & .56104 & .44586 & .22059 & .12267 & 8596 & .39833 & .21495 & .53963 & .53435 & .27666 & .17978 \\
\hline 3374 & .24061 & .14362 & .59690 & .50463 & .27824 & .16896 & 8788 & .37334 & .19892 & .53282 & .41907 & .21613 & .12655 \\
\hline 4026 & -- & -- & -- & -- & -- & -- & 8816 & -- & -- & -- & -- & -- & -- \\
\hline 4028 & .34404 & .22218 & .64578 & .53230 & .28245 & .16220 & 9148 & .13667 & .05867 & .42927 & .09659 & .11932 & .25568 \\
\hline 4030 & .41513 & .21698 & .52268 & .48803 & .22090 & .15060 & 9153 & .38701 & .23561 & .60881 & .50242 & .28882 & .18615 \\
\hline 4089 & .35575 & .20297 & .57056 & .50688 & .28469 & .16570 & 9156 & .39152 & .22021 & .56245 & .49692 & .27070 & .15497 \\
\hline 4112 & .40687 & .22814 & .56072 & .55242 & .31997 & .21887 & 9193 & .33347 & .17065 & .51175 & .54443 & .29270 & .19692 \\
\hline 4306 & .28174 & .17287 & .61358 & .51387 & .28110 & .17126 & 9265 & .40341 & .23750 & .58874 & .58975 & .37823 & .25602 \\
\hline 4850 & .31583 & .18076 & .57236 & .50848 & .30913 & .19418 & 9569 & .39138 & .20421 & .52176 & .50502 & .22720 & .13793 \\
\hline 4856 & .30516 & .18565 & .60835 & .51106 & .28127 & .17496 & 9686 & .26798 & .14627 & .54582 & .47308 & .26610 & .15073 \\
\hline
\end{tabular}


Appendix 2-2.5. L-moments of storm depth defined by 24-hour minimum interevent time for hourly rainfall stations in eastern New Mexico.

[--, not available]

\begin{tabular}{|c|c|c|c|c|c|c|c|c|c|c|c|c|c|}
\hline $\begin{array}{c}\text { Station } \\
\text { no. }\end{array}$ & $\begin{array}{l}\text { Depth } \\
\text { mean } \\
\text { (inches) }\end{array}$ & $\begin{array}{c}\text { Depth } \\
\text { L-scale } \\
\text { (inches) }\end{array}$ & $\begin{array}{c}\text { Depth } \\
\text { L-CV } \\
\text { (dimen- } \\
\text { sionless) }\end{array}$ & $\begin{array}{l}\text { Depth } \\
\text { L-skew } \\
\text { (dimen- } \\
\text { sionless) }\end{array}$ & $\begin{array}{c}\text { Depth } \\
\text { L-kurtosis } \\
\text { (dimen- } \\
\text { sionless) }\end{array}$ & $\begin{array}{c}\text { Depth } \\
\text { Tau5 } \\
\text { (dimen- } \\
\text { sionless) }\end{array}$ & $\begin{array}{c}\text { Station } \\
\text { no. }\end{array}$ & $\begin{array}{l}\text { Depth } \\
\text { mean } \\
\text { (inches) }\end{array}$ & $\begin{array}{l}\text { Depth } \\
\text { L-scale } \\
\text { (inches) }\end{array}$ & $\begin{array}{c}\text { Depth } \\
\text { L-CV } \\
\text { (dimen- } \\
\text { sionless) }\end{array}$ & $\begin{array}{c}\text { Depth } \\
\text { L-skew } \\
\text { (dimen- } \\
\text { sionless) }\end{array}$ & $\begin{array}{c}\text { Depth } \\
\text { L-kurtosis } \\
\text { (dimen- } \\
\text { sionless) }\end{array}$ & $\begin{array}{c}\text { Depth } \\
\text { Tau5 } \\
\text { (dimen- } \\
\text { sionless) }\end{array}$ \\
\hline 0205 & .33284 & .20409 & .61317 & .52686 & .32967 & .23472 & 4862 & .43588 & .22969 & .52697 & .49115 & .22825 & .14001 \\
\hline 0208 & .29259 & .15247 & .52110 & .42942 & .23339 & .16100 & 5370 & .46131 & .27021 & .58575 & .53502 & .33380 & .23355 \\
\hline 0404 & .47045 & .24305 & .51662 & .50015 & .29959 & .22591 & 5651 & .32465 & .18324 & .56443 & .46816 & .26174 & .18087 \\
\hline 0600 & .37190 & .21990 & .59130 & .54036 & .33098 & .21459 & 6138 & .36575 & .21215 & .58005 & .49057 & .29632 & .20501 \\
\hline 0606 & .37356 & .17397 & .46570 & .33601 & .14878 & .06974 & 6275 & .39257 & .22249 & .56675 & .49328 & .27808 & .16990 \\
\hline 0646 & .40462 & .21905 & .54137 & .43447 & .23003 & .13863 & 6435 & .34721 & .19421 & .55935 & .49360 & .28846 & .18079 \\
\hline 1120 & .49107 & .26570 & .54105 & .45186 & .23323 & .14583 & 6492 & .25201 & .14764 & .58585 & .50515 & .31578 & .22952 \\
\hline 1440 & .39817 & .20505 & .51497 & .46670 & .23336 & .13838 & 6619 & .42949 & .23937 & .55734 & .45916 & .25380 & .15897 \\
\hline 1480 & -- & -- & -- & -- & -- & -- & 7094 & .33310 & .17810 & .53469 & .46548 & .25252 & .14683 \\
\hline 1515 & .31517 & .16845 & .53448 & .43386 & .22529 & .12948 & 7254 & .35472 & .19846 & .55949 & .51666 & .29826 & .17097 \\
\hline 1807 & .29659 & .16528 & .55726 & .39916 & .17923 & .13043 & 7277 & -- & -- & -- & -- & -- & -- \\
\hline 1840 & .44013 & .23817 & .54114 & .53242 & .29265 & .20132 & 7279 & .37727 & .19930 & .52826 & .49779 & .25637 & .15252 \\
\hline 1881 & -- & -- & -- & -- & -- & -- & 7283 & .30039 & .20036 & .66701 & .54513 & .30516 & .19473 \\
\hline 1887 & .33084 & .21584 & .65240 & .54073 & .31634 & .20759 & 7604 & -- & -- & -- & -- & -- & -- \\
\hline 1939 & .46342 & .27252 & .58807 & .50503 & .29242 & .18494 & 7605 & .42500 & .20079 & .47245 & .34906 & .07049 & .02229 \\
\hline 1950 & .51769 & .28092 & .54264 & .42185 & .22592 & .12627 & 7609 & .30212 & .20174 & .66774 & .55263 & .32166 & .21706 \\
\hline 1956 & .33263 & .19815 & .59571 & .51182 & .32426 & .24571 & 7610 & .40640 & .25462 & .62653 & .53399 & .31520 & .19046 \\
\hline 1961 & .39286 & .22484 & .57232 & .39124 & .14340 & .07728 & 7638 & .41551 & .22780 & .54824 & .47321 & .23767 & .12832 \\
\hline 2625 & .35262 & .22386 & .63487 & .57546 & .36998 & .26169 & 8084 & .26566 & .14021 & .52778 & .47113 & .30011 & .20016 \\
\hline 2665 & .36096 & .19453 & .53892 & .45265 & .23930 & .13788 & 8085 & .32302 & .16941 & .52447 & .45221 & .24361 & .13291 \\
\hline 2694 & .45857 & .24319 & .53033 & .40707 & .27255 & .23043 & 8187 & .41823 & .24999 & .59773 & .49251 & .27097 & .16319 \\
\hline 2700 & .28489 & .14183 & .49784 & .49463 & .27729 & .15310 & 8358 & .31067 & .17830 & .57393 & .54495 & .35700 & .24552 \\
\hline 3145 & .32975 & .19750 & .59893 & .52340 & .30532 & .19318 & 8501 & .37682 & .21675 & .57522 & .47893 & .26237 & .15810 \\
\hline 3242 & .57526 & .29216 & .50788 & .37767 & .12880 & .08182 & 8518 & .30147 & .17117 & .56779 & .49018 & .27863 & .16481 \\
\hline 3288 & .40000 & .22818 & .57045 & .46470 & .24806 & .14679 & 8596 & .43275 & .23668 & .54692 & .52410 & .26203 & .16052 \\
\hline 3374 & .26404 & .15813 & .59890 & .49792 & .26892 & .16005 & 8788 & .43352 & .22722 & .52414 & .40411 & .20854 & .12535 \\
\hline 4026 & -- & -- & -- & -- & -- & -- & 8816 & -- & -- & -- & -- & -- & -- \\
\hline 4028 & .37051 & .23875 & .64440 & .53210 & .28235 & .15413 & 9148 & .16400 & .09500 & .57927 & .38947 & .21053 & .46316 \\
\hline 4030 & .42925 & .22461 & .52326 & .48009 & .21665 & .15110 & 9153 & .41163 & .26041 & .63262 & .53488 & .31825 & .20658 \\
\hline 4089 & .39342 & .22927 & .58275 & .51713 & .29487 & .17766 & 9156 & .43087 & .24311 & .56424 & .48913 & .26124 & .15066 \\
\hline 4112 & .43864 & .25041 & .57087 & .55200 & .32185 & .22102 & 9193 & .36575 & .19468 & .53228 & .55919 & .31402 & .22111 \\
\hline 4306 & .31325 & .19189 & .61260 & .49693 & .25890 & .15317 & 9265 & .44008 & .25907 & .58868 & .56941 & .35867 & .24357 \\
\hline 4850 & .36939 & .21426 & .58002 & .50720 & .30067 & .18842 & 9569 & .43497 & .22962 & .52790 & .48949 & .22256 & .13742 \\
\hline 4856 & .33729 & .20870 & .61875 & .51148 & .27560 & .16193 & 9686 & .28975 & .15921 & .54949 & .47307 & .26427 & .15283 \\
\hline
\end{tabular}


Appendix 2-2.6. L-moments of storm depth defined by 48-hour minimum interevent time for hourly rainfall stations in eastern New Mexico.

$[--$, not available]

\begin{tabular}{|c|c|c|c|c|c|c|c|c|c|c|c|c|c|}
\hline $\begin{array}{c}\text { Station } \\
\text { no. }\end{array}$ & $\begin{array}{l}\text { Depth } \\
\text { mean } \\
\text { (inches) }\end{array}$ & $\begin{array}{l}\text { Depth } \\
\text { L-scale } \\
\text { (inches) }\end{array}$ & $\begin{array}{c}\text { Depth } \\
\text { L-CV } \\
\text { (dimen- } \\
\text { sionless) }\end{array}$ & $\begin{array}{c}\text { Depth } \\
\text { L-skew } \\
\text { (dimen- } \\
\text { sionless) }\end{array}$ & $\begin{array}{c}\text { Depth } \\
\text { L-kurtosis } \\
\text { (dimen- } \\
\text { sionless) }\end{array}$ & $\begin{array}{c}\text { Depth } \\
\text { Tau5 } \\
\text { (dimen- } \\
\text { sionless) }\end{array}$ & $\begin{array}{c}\text { Station } \\
\text { no. }\end{array}$ & $\begin{array}{l}\text { Depth } \\
\text { mean } \\
\text { (inches) }\end{array}$ & $\begin{array}{l}\text { Depth } \\
\text { L-scale } \\
\text { (inches) }\end{array}$ & $\begin{array}{c}\text { Depth } \\
\text { L-CV } \\
\text { (dimen- } \\
\text { sionless) }\end{array}$ & $\begin{array}{l}\text { Depth } \\
\text { L-skew } \\
\text { (dimen- } \\
\text { sionless) }\end{array}$ & $\begin{array}{c}\text { Depth } \\
\text { L-kurtosis } \\
\text { (dimen- } \\
\text { sionless) }\end{array}$ & $\begin{array}{c}\text { Depth } \\
\text { Tau5 } \\
\text { (dimen- } \\
\text { sionless) }\end{array}$ \\
\hline 0205 & .39482 & .24557 & .62198 & .52508 & .32378 & .22555 & 4862 & .56671 & .30494 & .53809 & .46078 & .20759 & .11053 \\
\hline 0208 & .34466 & .18408 & .53409 & .43951 & .23990 & .16358 & 5370 & .53562 & .31515 & .58838 & .51914 & .31405 & .21789 \\
\hline 0404 & .61791 & .32679 & .52886 & .46225 & .24830 & .15927 & 5651 & .34924 & .20351 & .58272 & .49606 & .29323 & .19902 \\
\hline 0600 & .43035 & .25335 & .58870 & .52180 & .30953 & .20406 & 6138 & .47283 & .27779 & .58751 & .48327 & .27778 & .18458 \\
\hline 0606 & .48978 & .23901 & .48800 & .34094 & .08962 & -.01920 & 6275 & .51222 & .29972 & .58514 & .49748 & .27850 & .17093 \\
\hline 0646 & .47964 & .25911 & .54023 & .40943 & .20125 & .11835 & 6435 & .40755 & .23222 & .56979 & .49029 & .27686 & .17366 \\
\hline 1120 & 66899 & .36800 & .55008 & .44528 & .23597 & .15485 & 6492 & .29681 & .17472 & .58863 & .51978 & .35692 & .28487 \\
\hline 1440 & .50379 & .26841 & .53279 & .46235 & .23559 & .14078 & 6619 & .51181 & .28461 & .55609 & .44130 & .23404 & .14586 \\
\hline 1480 & -- & -- & -- & -- & -- & -- & 7094 & .41406 & .22599 & .54578 & .45353 & .23690 & .14009 \\
\hline 1515 & .39166 & .21332 & .54466 & .44239 & .23341 & .14032 & 7254 & .42228 & .24144 & .57176 & .51216 & .28901 & .17225 \\
\hline 1807 & .32899 & .18348 & .55771 & .39866 & .17827 & .11984 & 7277 & -- & -- & -- & -- & -- & -- \\
\hline 1840 & .53884 & .30081 & .55826 & .51468 & .27254 & .18350 & 7279 & .47572 & .25837 & .54311 & .47745 & .24146 & .15008 \\
\hline 1881 & -- & -- & -- & -- & -- & -- & 7283 & .40095 & .26583 & .66298 & .52922 & .28690 & .17991 \\
\hline 1887 & .42138 & .27321 & 64838 & .52303 & .28854 & .17643 & 7604 & -- & -- & -- & -- & -- & -- \\
\hline 1939 & .55367 & .32683 & .59030 & .48895 & .26710 & .16364 & 7605 & .47222 & .22974 & .48651 & .34637 & .05903 & -.00356 \\
\hline 1950 & .58522 & .30688 & .52438 & .40031 & .18967 & .04248 & 7609 & .36433 & .24054 & .66023 & .53880 & .30701 & .19827 \\
\hline 1956 & .40774 & .24290 & .59573 & .49495 & .29175 & .20059 & 7610 & .47811 & .29965 & .62673 & .52809 & .30710 & .19154 \\
\hline 1961 & .45833 & .24730 & .53956 & .33110 & .09250 & .04765 & 7638 & .51431 & .28580 & .55570 & .45590 & .22239 & .12545 \\
\hline 2625 & .41206 & .26195 & .63572 & .55758 & .34173 & .23449 & 8084 & .29333 & .15184 & .51762 & .45492 & .27665 & .18238 \\
\hline 2665 & .44451 & .24225 & .54497 & .44006 & .22346 & .13149 & 8085 & .40066 & .21390 & .53387 & .44642 & .23305 & .12840 \\
\hline 2694 & 65959 & .34420 & .52184 & .38832 & .23440 & .17143 & 8187 & .51672 & .30935 & .59868 & .48275 & .26131 & .14714 \\
\hline 2700 & .37581 & .19742 & .52532 & .48478 & .26306 & .15510 & 8358 & .35170 & .20065 & .57053 & .53319 & .34814 & .24518 \\
\hline 3145 & .37930 & .22937 & .60471 & .51484 & .29679 & .19504 & 8501 & .47125 & .27380 & .58101 & .46840 & .24973 & .15208 \\
\hline 3242 & .64294 & .32993 & .51315 & .32953 & .08405 & .10828 & 8518 & .36704 & .20989 & .57185 & .47628 & .26067 & .15780 \\
\hline 3288 & .50000 & .28396 & .56791 & .45616 & .23539 & .11904 & 8596 & .52360 & .28923 & .55239 & .49565 & .24427 & .14910 \\
\hline 3374 & .32690 & .19388 & .59306 & .47695 & .25128 & .15328 & 8788 & .56223 & .29439 & .52362 & .39314 & .19556 & .11616 \\
\hline 4026 & -- & -- & -- & -- & -- & -- & 8816 & -- & -- & -- & -- & -- & -- \\
\hline 4028 & .43008 & .27946 & .64978 & .53619 & .28044 & .14087 & 9148 & -- & -- & -- & -- & -- & -- \\
\hline 4030 & .54397 & .28920 & .53165 & .43447 & .17391 & .11106 & 9153 & .56028 & .35937 & .64141 & .52118 & .27485 & .15967 \\
\hline 4089 & .47605 & .28699 & .60286 & .52459 & .29856 & .18589 & 9156 & .51162 & .29162 & .56999 & .47743 & .24832 & .14560 \\
\hline 4112 & .51917 & .29569 & .56955 & .52118 & .30413 & .21430 & 9193 & .45522 & .24460 & .53733 & .51526 & .29144 & .20901 \\
\hline 4306 & .37638 & .22735 & .60405 & .46542 & .22312 & .12676 & 9265 & .51781 & .31603 & 61032 & .57846 & .36834 & .25132 \\
\hline 4850 & .46980 & .27567 & .58678 & .49027 & .27494 & .17080 & 9569 & .50233 & .27231 & .54209 & .49174 & .24225 & .15421 \\
\hline 4856 & .40442 & .24594 & .60813 & .47902 & .23782 & .13150 & 9686 & .34350 & .19198 & .55890 & .46098 & .25310 & .15445 \\
\hline
\end{tabular}


Appendix 2-2.7. L-moments of storm depth defined by 72-hour minimum interevent time for hourly rainfall stations in eastern New Mexico.

[--, not available]

\begin{tabular}{|c|c|c|c|c|c|c|c|c|c|c|c|c|c|}
\hline $\begin{array}{c}\text { Station } \\
\text { no. }\end{array}$ & $\begin{array}{l}\text { Depth } \\
\text { mean } \\
\text { (inches) }\end{array}$ & $\begin{array}{l}\text { Depth } \\
\text { L-scale } \\
\text { (inches) }\end{array}$ & $\begin{array}{c}\text { Depth } \\
\text { L-CV } \\
\text { (dimen- } \\
\text { sionless) }\end{array}$ & $\begin{array}{c}\text { Depth } \\
\text { L-skew } \\
\text { (dimen- } \\
\text { sionless) }\end{array}$ & $\begin{array}{c}\text { Depth } \\
\text { L-kurtosis } \\
\text { (dimen- } \\
\text { sionless) }\end{array}$ & $\begin{array}{c}\text { Depth } \\
\text { Tau5 } \\
\text { (dimen- } \\
\text { sionless) }\end{array}$ & $\begin{array}{c}\text { Station } \\
\text { no. }\end{array}$ & $\begin{array}{l}\text { Depth } \\
\text { mean } \\
\text { (inches) }\end{array}$ & $\begin{array}{l}\text { Depth } \\
\text { L-scale } \\
\text { (inches) }\end{array}$ & $\begin{array}{c}\text { Depth } \\
\text { L-CV } \\
\text { (dimen- } \\
\text { sionless) }\end{array}$ & $\begin{array}{c}\text { Depth } \\
\text { L-skew } \\
\text { (dimen- } \\
\text { sionless) }\end{array}$ & $\begin{array}{c}\text { Depth } \\
\text { L-kurtosis } \\
\text { (dimen- } \\
\text { sionless) }\end{array}$ & $\begin{array}{c}\text { Depth } \\
\text { Tau5 } \\
\text { (dimen- } \\
\text { sionless) }\end{array}$ \\
\hline 0205 & .44953 & .28095 & .62498 & .52130 & .31441 & .21191 & 4862 & .68724 & .37837 & .55057 & .44603 & .18716 & .09068 \\
\hline 0208 & .39678 & .22120 & .55748 & .45949 & .24309 & .15425 & 5370 & .59921 & .35505 & .59253 & .51479 & .31030 & .21606 \\
\hline 0404 & .86250 & .44822 & .51968 & .41095 & .22231 & .15319 & 5651 & .39402 & .23217 & .58925 & .50025 & .29932 & .20410 \\
\hline 0600 & .48986 & .28995 & .59190 & .51429 & .30077 & .20187 & 6138 & .57727 & .35046 & .60709 & .49970 & .28494 & .18418 \\
\hline 0606 & .59081 & .27009 & .45715 & .23216 & .02114 & -.00019 & 6275 & 60539 & .36048 & .59545 & .49961 & .27930 & .17618 \\
\hline 0646 & .57174 & .34049 & .59554 & .48757 & .26764 & .16815 & 6435 & .46486 & .26940 & .57952 & .48808 & .27491 & .17519 \\
\hline 1120 & .84238 & .48314 & .57354 & .46943 & .26129 & .16714 & 6492 & .33392 & .19407 & .58118 & .51530 & .35274 & .27281 \\
\hline 1440 & .59266 & .31911 & .53844 & .44152 & .20678 & .11473 & 6619 & .59054 & .32489 & .55015 & .41644 & 19577 & 10946 \\
\hline 1480 & -- & -- & -- & -- & -- & -- & 7094 & .48966 & .26956 & .55050 & .44557 & .23056 & .13414 \\
\hline 1515 & .46545 & .25970 & .55796 & .45505 & .24302 & .14757 & 7254 & 49019 & .28271 & .57674 & .49733 & .26936 & . 15715 \\
\hline 1807 & .36250 & .19708 & .54367 & .37553 & .16691 & .12475 & 7277 & -- & -- & -- & -- & -- & -- \\
\hline 1840 & .61698 & .34575 & .56039 & .50160 & .27606 & .19763 & 7279 & .57823 & .32349 & .55945 & .47321 & .23874 & .14771 \\
\hline 1881 & -- & -- & -- & -- & -- & -- & 7283 & .50285 & .33411 & .66442 & .53204 & .29415 & 19021 \\
\hline 1887 & .50382 & .32725 & .64955 & .51495 & .27139 & .15492 & 7604 & -- & -- & -- & -- & -- & -- \\
\hline 1939 & .64438 & .38527 & .59789 & .48841 & .26204 & .15778 & 7605 & .56667 & .31524 & .55630 & .45340 & .14594 & .02852 \\
\hline 1950 & .64095 & .36924 & .57608 & .52204 & .40162 & .33736 & 7609 & .42092 & .27910 & .66307 & .53665 & .30234 & 19375 \\
\hline 1956 & .48615 & .31003 & .63772 & .58312 & .43076 & .36472 & 7610 & .54104 & .34061 & .62954 & .52401 & .29781 & .18082 \\
\hline 1961 & .57292 & .32534 & .56787 & .39676 & .21267 & .20126 & 7638 & .60413 & .34302 & .56779 & .45796 & .21937 & .12100 \\
\hline 2625 & .48396 & .31016 & .64088 & .55874 & .34713 & .24222 & 8084 & .34341 & .18043 & .52539 & .43417 & .22752 & .13915 \\
\hline 2665 & .52487 & .29184 & .55602 & .44819 & .23329 & .14011 & 8085 & .47482 & .25763 & .54259 & .45320 & .24533 & .14447 \\
\hline 2694 & .81610 & .44192 & .54150 & .40314 & .21426 & .11926 & 8187 & .57996 & .34161 & .58902 & .45896 & .22882 & .11136 \\
\hline 2700 & .45863 & .24860 & .54205 & .48351 & .26337 & .15592 & 8358 & .38041 & .22723 & .59733 & .55389 & .35556 & .24436 \\
\hline 3145 & .41309 & .25071 & .60691 & .51960 & .30587 & .20117 & 8501 & .56158 & .33022 & .58803 & .46317 & .23943 & .14905 \\
\hline 3242 & .72867 & .38324 & .52594 & .31526 & .00252 & -.03547 & 8518 & .43454 & .25022 & .57581 & .47677 & .25959 & .15569 \\
\hline 3288 & .62162 & .36344 & .58467 & .49039 & .27637 & .16418 & 8596 & .60317 & .33585 & .55681 & .47910 & .23592 & .14611 \\
\hline 3374 & .38339 & .23180 & .60462 & .48989 & .26875 & .17168 & 8788 & .66864 & .35841 & .53602 & .43481 & .25321 & . 17396 \\
\hline 4026 & -- & -- & -- & -- & -- & -- & 8816 & -- & -- & -- & -- & -- & -- \\
\hline 4028 & .49401 & .32300 & .65384 & .53854 & .28209 & .14095 & 9148 & -- & -- & -- & -- & -- & -- \\
\hline 4030 & .60673 & .32688 & .53875 & .43532 & .18761 & .12702 & 9153 & .64032 & .42868 & .66948 & .56296 & .30385 & .14870 \\
\hline 4089 & .55484 & .34279 & .61782 & .53520 & .30719 & .19241 & 9156 & .59139 & .34198 & .57825 & .47032 & .23434 & .12814 \\
\hline 4112 & .58528 & .33627 & .57453 & .50951 & .29120 & .19779 & 9193 & .54430 & .29229 & .53700 & .48540 & .27553 & .20594 \\
\hline 4306 & .44681 & .26796 & .59971 & .43374 & .18294 & .10128 & 9265 & .57344 & .35086 & .61184 & .55979 & .34030 & .22823 \\
\hline 4850 & .56915 & .33824 & .59429 & .49191 & .27688 & .17317 & 9569 & .58848 & .32331 & .54939 & .47264 & .22364 & .13170 \\
\hline 4856 & .49296 & .31609 & .64121 & .51813 & .25998 & .12013 & 9686 & .39115 & .22053 & .56379 & .46047 & .25330 & .15918 \\
\hline
\end{tabular}


Appendix 2-3.1. L-moments of storm duration defined by 6-hour minimum interevent time for hourly rainfall stations in eastern New Mexico.

$[--$, not available]

\begin{tabular}{|c|c|c|c|c|c|c|c|c|c|c|c|c|c|}
\hline $\begin{array}{c}\text { Station } \\
\text { no. }\end{array}$ & $\begin{array}{c}\text { Duration } \\
\text { mean } \\
\text { (hours) }\end{array}$ & $\begin{array}{l}\text { Duration } \\
\text { L-scale } \\
\text { (hours) }\end{array}$ & $\begin{array}{c}\text { Duration } \\
\text { L-CV } \\
\text { (dimen- } \\
\text { sionless) }\end{array}$ & $\begin{array}{l}\text { Duration } \\
\text { L-skew } \\
\text { (dimen- } \\
\text { sionless) }\end{array}$ & $\begin{array}{c}\text { Duration } \\
\text { L-kurtosis } \\
\text { (dimen- } \\
\text { sionless) }\end{array}$ & $\begin{array}{l}\text { Duration } \\
\text { Tau5 } \\
\text { (dimen- } \\
\text { sionless) }\end{array}$ & $\begin{array}{c}\text { Station } \\
\text { no. }\end{array}$ & $\begin{array}{c}\text { Duration } \\
\text { mean } \\
\text { (hours) }\end{array}$ & $\begin{array}{l}\text { Duration } \\
\text { L-scale } \\
\text { (hours) }\end{array}$ & $\begin{array}{c}\text { Duration } \\
\text { L-CV } \\
\text { (dimen- } \\
\text { sionless) }\end{array}$ & $\begin{array}{l}\text { Duration } \\
\text { L-skew } \\
\text { (dimen- } \\
\text { sionless) }\end{array}$ & $\begin{array}{l}\text { Duration } \\
\text { L-kurtosis } \\
\text { (dimen- } \\
\text { sionless) }\end{array}$ & $\begin{array}{l}\text { Duration } \\
\text { Tau5 } \\
\text { (dimen- } \\
\text { sionless) }\end{array}$ \\
\hline 0205 & 4.5853 & 2.2825 & .49778 & .45864 & .23441 & .15459 & 4862 & 2.8888 & 1.4677 & .50807 & .60066 & .28832 & .14136 \\
\hline 0208 & 4.3734 & 1.9912 & .45530 & .45909 & .27928 & .18008 & 5370 & 3.7980 & 1.9821 & .52187 & .52613 & .26512 & .18638 \\
\hline 0404 & 3.6690 & 2.0990 & .57209 & .62373 & .32958 & .18539 & 5651 & 4.4463 & 2.2420 & .50425 & .44673 & .19110 & .11704 \\
\hline 0600 & 3.6693 & 1.8897 & .51499 & .52201 & .25557 & .17516 & 6138 & 5.5700 & 2.7858 & .50015 & .43206 & .21449 & .12776 \\
\hline 0606 & 3.6585 & 2.1108 & .57695 & .60757 & .25200 & .04354 & 6275 & 4.6605 & 2.4745 & .53096 & .48351 & .22586 & .14554 \\
\hline 0646 & 4.9242 & 2.6175 & .53155 & .45758 & .18942 & .12167 & 6435 & 3.9833 & 2.0510 & .51490 & .49064 & .22412 & .14862 \\
\hline 1120 & 4.6842 & 2.5570 & .54588 & .50455 & .24230 & .16372 & 6492 & 4.1570 & 2.2512 & .54154 & .50980 & .20953 & .11105 \\
\hline 1440 & 3.2251 & 1.7060 & .52897 & .58951 & .28617 & .15462 & 6619 & 4.0891 & 2.1235 & .51931 & .50402 & .25144 & .17005 \\
\hline 1480 & -- & -- & -- & -- & -- & -- & 7094 & 4.1245 & 2.1486 & .52092 & .48604 & .21140 & .13116 \\
\hline 1515 & 3.8710 & 1.9247 & .49722 & .46925 & .20945 & .13716 & 7254 & 3.8447 & 2.0818 & .54147 & .54737 & .25714 & .14283 \\
\hline 1807 & 4.5696 & 2.0791 & .45498 & .36991 & .14760 & .08129 & 7277 & -- & -- & -- & -- & -- & -- \\
\hline 1840 & 2.8252 & 1.4395 & .50953 & .62789 & .33603 & .19324 & 7279 & 3.1973 & 1.6792 & .52520 & .58889 & .29185 & .16693 \\
\hline 1881 & 4.2000 & 2.1000 & .50000 & .42857 & .28571 & .09524 & 7283 & 4.3633 & 2.2659 & .51932 & .48824 & .23802 & .15464 \\
\hline 1887 & 4.6101 & 2.3444 & .50854 & .46122 & .22591 & .15223 & 7604 & -- & -- & -- & -- & -- & -- \\
\hline 1939 & 4.2518 & 2.2040 & .51837 & .48653 & .23378 & .16206 & 7605 & 2.5484 & 1.1204 & .43966 & .48269 & .11959 & .02272 \\
\hline 1950 & 5.4375 & 2.7923 & .51353 & .48592 & .23974 & .06775 & 7609 & 4.7263 & 2.6254 & .55550 & .54511 & .30268 & .20166 \\
\hline 1956 & 5.1875 & 2.6219 & .50543 & .42672 & .20619 & .15434 & 7610 & 4.1491 & 2.3300 & .56157 & .56654 & .28947 & .18092 \\
\hline 1961 & 6.0698 & 2.7220 & .44846 & .25845 & .10435 & .13440 & 7638 & 3.9430 & 2.0895 & .52993 & .51798 & .23938 & .15148 \\
\hline 2625 & 5.0019 & 2.5699 & .51378 & .49348 & .28123 & .18010 & 8084 & 4.0152 & 1.9942 & .49666 & .45979 & .20822 & .13839 \\
\hline 2665 & 5.0194 & 2.7133 & .54057 & .48564 & .23393 & .15785 & 8085 & 4.0204 & 2.1259 & .52876 & .49138 & .18754 & .09686 \\
\hline 2694 & 6.3500 & 3.3308 & .52454 & .42588 & .19256 & .10847 & 8187 & 4.0880 & 2.0461 & .50052 & .47290 & .23240 & .16175 \\
\hline 2700 & 3.4665 & 1.8646 & .53788 & .57080 & .26343 & .13937 & 8358 & 4.6164 & 2.3721 & .51385 & .49369 & .29992 & .24758 \\
\hline 3145 & 4.7568 & 2.4819 & .52176 & .49952 & .27916 & .19032 & 8501 & 4.1955 & 2.1809 & .51982 & .48746 & .22392 & .14415 \\
\hline 3242 & 9.0000 & 3.7011 & .41123 & .20702 & .10669 & .04432 & 8518 & 4.0859 & 2.1111 & .51668 & .48417 & .21709 & .14490 \\
\hline 3288 & 6.4911 & 3.2997 & .50835 & .41754 & .18326 & .10329 & 8596 & 2.8507 & 1.4434 & .50635 & .60713 & .30018 & .15717 \\
\hline 3374 & 5.0788 & 2.4228 & .47705 & .41337 & .20088 & .12119 & 8788 & 6.4243 & 3.4036 & .52981 & .44174 & .21841 & .14426 \\
\hline 4026 & -- & -- & -- & -- & -- & -- & 8816 & -- & -- & -- & -- & -- & -- \\
\hline 4028 & 3.7889 & 1.8240 & .48140 & .43690 & .17879 & .12742 & 9148 & 8.2500 & 3.3929 & .41126 & .04211 & .08421 & .18947 \\
\hline 4030 & 2.5829 & 1.2559 & .48623 & .62619 & .31508 & .14869 & 9153 & 4.0909 & 2.3421 & .57252 & .55869 & .23327 & .12498 \\
\hline 4089 & 4.1688 & 2.2414 & .53766 & .51998 & .24985 & .16349 & 9156 & 3.8306 & 2.0346 & .53114 & .52950 & .24907 & .15999 \\
\hline 4112 & 3.4043 & 1.8476 & .54273 & .59447 & .29486 & .16323 & 9193 & 2.8889 & 1.4827 & .51324 & 60949 & .29151 & .13440 \\
\hline 4306 & 4.8110 & 2.2994 & .47795 & .41622 & .20487 & .13591 & 9265 & 2.8834 & 1.4752 & .51163 & .61338 & .30745 & .15743 \\
\hline 4850 & 4.1325 & 2.1808 & .52771 & .50920 & .24569 & .16078 & 9569 & 2.7189 & 1.3311 & .48960 & .59491 & .27883 & .13093 \\
\hline 4856 & 4.8824 & 2.5671 & .52580 & .43423 & .14798 & .08075 & 9686 & 3.8992 & 2.0326 & .52129 & .50402 & .22124 & .12718 \\
\hline
\end{tabular}


Appendix 2-3.2. L-moments of storm duration defined by 8-hour minimum interevent time for hourly rainfall stations in eastern New Mexico.

$[--$, not available]

\begin{tabular}{|c|c|c|c|c|c|c|c|c|c|c|c|c|c|}
\hline $\begin{array}{c}\text { Station } \\
\text { no. }\end{array}$ & $\begin{array}{c}\text { Duration } \\
\text { mean } \\
\text { (hours) }\end{array}$ & $\begin{array}{l}\text { Duration } \\
\text { L-scale } \\
\text { (hours) }\end{array}$ & $\begin{array}{l}\text { Duration } \\
\text { L-CV } \\
\text { (dimen- } \\
\text { sionless) }\end{array}$ & $\begin{array}{l}\text { Duration } \\
\text { L-skew } \\
\text { (dimen- } \\
\text { sionless) }\end{array}$ & $\begin{array}{l}\text { Duration } \\
\text { L-kurtosis } \\
\text { (dimen- } \\
\text { sionless) }\end{array}$ & $\begin{array}{l}\text { Duration } \\
\text { Tau5 } \\
\text { (dimen- } \\
\text { sionless) }\end{array}$ & $\begin{array}{c}\text { Station } \\
\text { no. }\end{array}$ & $\begin{array}{c}\text { Duration } \\
\text { mean } \\
\text { (hours) }\end{array}$ & $\begin{array}{l}\text { Duration } \\
\text { L-scale } \\
\text { (hours) }\end{array}$ & $\begin{array}{l}\text { Duration } \\
\text { L-CV } \\
\text { (dimen- } \\
\text { sionless) }\end{array}$ & $\begin{array}{l}\text { Duration } \\
\text { L-skew } \\
\text { (dimen- } \\
\text { sionless) }\end{array}$ & $\begin{array}{l}\text { Duration } \\
\text { L-kurtosis } \\
\text { (dimen- } \\
\text { sionless) }\end{array}$ & $\begin{array}{l}\text { Duration } \\
\text { Tau5 } \\
\text { (dimen- } \\
\text { sionless) }\end{array}$ \\
\hline 0205 & 5.0593 & 2.6321 & .52026 & .47074 & .23615 & .14992 & 4862 & 3.3820 & 1.8321 & .54172 & .58722 & .27069 & .12525 \\
\hline 0208 & 4.8447 & 2.2871 & .47208 & .45498 & .26281 & .16319 & 5370 & 4.3592 & 2.3898 & .54822 & .53302 & .27161 & .18455 \\
\hline 0404 & 4.7315 & 2.8855 & .60984 & .60457 & .30969 & .17464 & 5651 & 5.0357 & 2.7801 & .55207 & .51746 & .26719 & .16776 \\
\hline 0600 & 4.1059 & 2.2128 & .53893 & .53092 & .26404 & .17541 & 6138 & 6.4241 & 3.3477 & .52111 & .44356 & .21864 & .12831 \\
\hline 0606 & 3.9000 & 2.2968 & .58893 & .59845 & .22830 & .00782 & 6275 & 5.3776 & 2.9650 & .55136 & .48833 & .23219 & .15039 \\
\hline 0646 & 5.6824 & 3.1042 & .54629 & .44959 & .18273 & .12286 & 6435 & 4.5102 & 2.4380 & .54056 & .50215 & .23334 & .15092 \\
\hline 1120 & 5.5381 & 3.1409 & .56715 & .50187 & .23809 & .15800 & 6492 & 4.9038 & 2.7799 & .56688 & .51230 & .21839 & .12319 \\
\hline 1440 & 3.8009 & 2.1250 & .55907 & .57551 & .26742 & .13732 & 6619 & 4.4346 & 2.4012 & .54147 & .52123 & .26246 & .16543 \\
\hline 1480 & -- & -- & -- & -- & -- & -- & 7094 & 4.7721 & 2.5899 & .54272 & .48714 & .21305 & .12821 \\
\hline 1515 & 4.3907 & 2.3158 & .52743 & .49061 & .22769 & .14372 & 7254 & 4.2984 & 2.4048 & .55947 & .54356 & .25185 & .13536 \\
\hline 1807 & 4.7115 & 2.1717 & .46093 & .38309 & .16342 & .09105 & 7277 & -- & -- & -- & -- & -- & -- \\
\hline 1840 & 3.3461 & 1.8707 & .55907 & .64305 & .35489 & .20279 & 7279 & 3.7470 & 2.0873 & .55706 & .58305 & .28568 & .16030 \\
\hline 1881 & -- & -- & -- & -- & -- & -- & 7283 & 4.9413 & 2.6700 & .54035 & .49842 & .25207 & .16812 \\
\hline 1887 & 5.1704 & 2.7414 & .53021 & .47445 & .23534 & .15463 & 7604 & -- & -- & -- & -- & -- & -- \\
\hline 1939 & 4.7941 & 2.5970 & .54170 & .49914 & .24588 & .16719 & 7605 & 2.8333 & 1.3644 & .48154 & .53713 & .21223 & .11390 \\
\hline 1950 & 6.2000 & 3.2230 & .51984 & .49307 & .22939 & .04957 & 7609 & 5.3336 & 3.0409 & .57014 & .54138 & .29700 & .19492 \\
\hline 1956 & 5.9333 & 3.1394 & .52911 & .46979 & .30173 & .29566 & 7610 & 4.8326 & 2.8078 & .58100 & .55683 & .28037 & .17478 \\
\hline 1961 & 6.6585 & 3.4695 & .52106 & .43420 & .28154 & .26281 & 7638 & 4.5121 & 2.5037 & .55488 & .52547 & .24894 & .15597 \\
\hline 2625 & 5.6314 & 3.0492 & .54146 & .50927 & .28119 & .16781 & 8084 & 4.5238 & 2.3262 & .51420 & .44351 & .16852 & .08321 \\
\hline 2665 & 5.6342 & 3.1068 & .55141 & .47926 & .22809 & .15427 & 8085 & 4.7961 & 2.6438 & .55124 & .48176 & .18147 & .09653 \\
\hline 2694 & 8.2644 & 4.4857 & .54277 & .42676 & .20552 & .13177 & 8187 & 4.5954 & 2.4604 & .53540 & .50019 & .24388 & .15166 \\
\hline 2700 & 3.9864 & 2.2547 & .56559 & .57108 & .26596 & .14172 & 8358 & 5.1000 & 2.7389 & .53704 & .50303 & .29038 & .22438 \\
\hline 3145 & 5.4952 & 3.0134 & .54837 & .50979 & .28162 & .18869 & 8501 & 4.8417 & 2.6224 & .54163 & .49072 & .22726 & .14157 \\
\hline 3242 & 9.5556 & 3.9601 & .41443 & .17266 & .05863 & .02664 & 8518 & 4.6351 & 2.4902 & .53726 & .48434 & .21218 & .13379 \\
\hline 3288 & 7.3924 & 3.7247 & .50385 & .38518 & .15714 & .09637 & 8596 & 3.3220 & 1.8252 & .54942 & .62199 & .32645 & .18471 \\
\hline 3374 & 5.7796 & 2.9032 & .50231 & .43081 & .21067 & .12652 & 8788 & 7.2833 & 3.9967 & .54874 & .44969 & .21770 & .13959 \\
\hline 4026 & -- & -- & -- & -- & -- & -- & 8816 & -- & -- & -- & -- & -- & -- \\
\hline 4028 & 4.1437 & 2.1090 & .50898 & .47122 & .21893 & .15997 & 9148 & 10.429 & 5.3333 & .51142 & .26786 & .21429 & .26786 \\
\hline 4030 & 2.9832 & 1.5442 & .51763 & .59299 & .25665 & .08163 & 9153 & 4.2137 & 2.4120 & .57241 & .54655 & .22051 & .11942 \\
\hline 4089 & 4.9419 & 2.7925 & .56505 & .52102 & .24658 & .15331 & 9156 & 4.2697 & 2.3548 & .55152 & .53305 & .25321 & .16084 \\
\hline 4112 & 3.9062 & 2.2357 & .57233 & .59634 & .29728 & .16400 & 9193 & 3.5261 & 1.9379 & .54957 & .57959 & .25574 & .11144 \\
\hline 4306 & 5.1448 & 2.5251 & .49081 & .42481 & .21114 & .14119 & 9265 & 3.4316 & 1.9186 & .55909 & .62594 & .32722 & .17444 \\
\hline 4850 & 4.6843 & 2.5878 & .55244 & .52001 & .25375 & .15825 & 9569 & 3.1265 & 1.6314 & .52179 & .58385 & .26689 & .12301 \\
\hline 4856 & 5.4383 & 2.9302 & .53882 & .43355 & .14810 & .08126 & 9686 & 4.3881 & 2.3872 & .54402 & .51106 & .22813 & .12931 \\
\hline
\end{tabular}


114 Statistical Characteristics of Storm Interevent Time, Depth, and Duration for Eastern New Mexico, Oklahoma, and Texas

Appendix 2-3.3. L-moments of storm duration defined by 12-hour minimum interevent time for hourly rainfall stations in eastern New Mexico.

$[--$, not available $]$

\begin{tabular}{|c|c|c|c|c|c|c|c|c|c|c|c|c|c|}
\hline $\begin{array}{c}\text { Station } \\
\text { no. }\end{array}$ & $\begin{array}{l}\text { Duration } \\
\text { mean } \\
\text { (hours) }\end{array}$ & $\begin{array}{l}\text { Duration } \\
\text { L-scale } \\
\text { (hours) }\end{array}$ & $\begin{array}{l}\text { Duration } \\
\text { L-CV } \\
\text { (dimen- } \\
\text { sionless) }\end{array}$ & $\begin{array}{l}\text { Duration } \\
\text { L-skew } \\
\text { (dimen- } \\
\text { sionless) }\end{array}$ & $\begin{array}{l}\text { Duration } \\
\text { L-kurtosis } \\
\text { (dimen- } \\
\text { sionless) }\end{array}$ & $\begin{array}{l}\text { Duration } \\
\text { Tau5 } \\
\text { (dimen- } \\
\text { sionless) }\end{array}$ & $\begin{array}{c}\text { Station } \\
\text { no. }\end{array}$ & $\begin{array}{c}\text { Duration } \\
\text { mean } \\
\text { (hours) }\end{array}$ & $\begin{array}{l}\text { Duration } \\
\text { L-scale } \\
\text { (hours) }\end{array}$ & $\begin{array}{l}\text { Duration } \\
\text { L-CV } \\
\text { (dimen- } \\
\text { sionless) }\end{array}$ & $\begin{array}{l}\text { Duration } \\
\text { L-skew } \\
\text { (dimen- } \\
\text { sionless) }\end{array}$ & $\begin{array}{l}\text { Duration } \\
\text { L-kurtosis } \\
\text { (dimen- } \\
\text { sionless) }\end{array}$ & $\begin{array}{l}\text { Duration } \\
\text { Tau5 } \\
\text { (dimen- } \\
\text { sionless) }\end{array}$ \\
\hline 0199 & 6.2453 & 3.7041 & 0.59310 & 0.51815 & 0.24551 & 0.15786 & 4860 & 6.0547 & 3.5402 & 0.58470 & 0.55459 & 0.35335 & 0.29728 \\
\hline 0205 & 5.9004 & 3.2773 & .55544 & .50016 & .25789 & .16005 & 4862 & 4.4965 & 2.6627 & .59216 & .58039 & .27267 & .13920 \\
\hline 0208 & 6.1217 & 3.2079 & .52402 & .48244 & .25858 & .14144 & 5370 & 5.5541 & 3.2810 & .59073 & .55292 & .29549 & .19740 \\
\hline 0404 & 6.4803 & 3.9630 & .61155 & .51752 & .20935 & .11728 & 5651 & 6.0255 & 3.5116 & .58279 & .54116 & .30062 & .20097 \\
\hline 0407 & 5.6156 & 3.5245 & .62762 & .57815 & .25957 & .11498 & 5866 & 8.7742 & 4.8505 & .55282 & .44387 & .22626 & .16006 \\
\hline 0600 & 5.2092 & 3.0191 & .57956 & .53793 & .26527 & .16424 & 6138 & 7.9759 & 4.5159 & .56619 & .48559 & .25371 & .15796 \\
\hline 0606 & 5.2329 & 3.5567 & .67968 & .69735 & .39452 & .18028 & 6275 & 6.6988 & 3.8770 & .57877 & .49820 & .24102 & .14723 \\
\hline 0646 & 6.4865 & 3.7808 & .58287 & .49519 & .22815 & .14937 & 6435 & 5.7210 & 3.3261 & .58138 & .51602 & .23927 & .14224 \\
\hline 1120 & 7.2939 & 4.3787 & .60032 & .50921 & .24077 & .14664 & 6492 & 6.0781 & 3.6155 & .59484 & .50008 & .18440 & .07841 \\
\hline 1440 & 5.0410 & 3.0334 & .60175 & .56603 & .26226 & .13981 & 6619 & 4.9847 & 2.8104 & .56380 & .53047 & .27014 & .17063 \\
\hline 1446 & 6.2500 & 3.5986 & .57577 & .54600 & .32035 & .20663 & 6659 & 5.9350 & 3.4583 & .58269 & .53423 & .28817 & .19590 \\
\hline 1469 & 5.3089 & 3.1689 & .59690 & .56722 & .29929 & .19233 & 6797 & 7.5537 & 4.1918 & .55494 & .46464 & .23617 & .14596 \\
\hline 1475 & 5.0968 & 3.2057 & .62897 & .62026 & .34597 & .25664 & 6951 & 6.1189 & 3.3886 & .55379 & .46378 & .21351 & .14500 \\
\hline 1480 & -- & -- & -- & -- & -- & -- & 7094 & 6.0412 & 3.4748 & .57519 & .49437 & .21966 & .12789 \\
\hline 1515 & 5.4976 & 3.1401 & .57117 & .51455 & .24348 & .13869 & 7254 & 5.4406 & 3.2389 & .59532 & .54256 & .24663 & .12764 \\
\hline 1807 & 5.2733 & 2.6595 & .50432 & .43810 & .19835 & .09929 & 7277 & -- & -- & -- & -- & -- & -- \\
\hline 1840 & 4.3662 & 2.6121 & .59825 & .60278 & .29308 & .13779 & 7279 & 4.7771 & 2.8750 & .60183 & .58561 & .28540 & .14842 \\
\hline 1881 & -- & -- & -- & -- & -- & -- & 7283 & 6.1752 & 3.5771 & .57927 & .52521 & .28194 & .18952 \\
\hline 1887 & 6.1074 & 3.4224 & .56036 & .49367 & .24575 & .14993 & 7604 & -- & -- & -- & -- & -- & -- \\
\hline 1939 & 5.9380 & 3.4228 & .57642 & .51906 & .26802 & .17744 & 7605 & 4.8462 & 2.8738 & .59302 & .55969 & .24710 & .09428 \\
\hline 1950 & 7.9259 & 4.5812 & .57800 & .51592 & .23296 & .06932 & 7609 & 6.4282 & 3.8487 & .59873 & .54648 & .29082 & .17979 \\
\hline 1956 & 6.3182 & 3.5328 & .55914 & .51385 & .33245 & .29484 & 7610 & 6.0459 & 3.6513 & .60392 & .54693 & .27381 & .17177 \\
\hline 1961 & 7.5641 & 4.0256 & .53220 & .44969 & .28656 & .21835 & 7638 & 5.5610 & 3.2740 & .58874 & .53530 & .25687 & .15172 \\
\hline 1963 & 5.4270 & 3.1838 & .58665 & .53875 & .26798 & .17614 & 7649 & 7.1740 & 4.2285 & .58942 & .50289 & .23993 & .14023 \\
\hline 1982 & 4.3554 & 2.6651 & .61190 & .62506 & .30784 & .13407 & 7651 & 5.9495 & 3.6780 & .61820 & .56008 & .25956 & .13078 \\
\hline 2030 & 4.6447 & 2.7017 & .58167 & .56215 & .27297 & .15570 & 7735 & 8.4873 & 4.6904 & .55264 & .45710 & .21793 & .11226 \\
\hline 2139 & 7.7264 & 4.0817 & .52827 & .38630 & .14149 & .06821 & 7736 & 6.8587 & 4.1872 & .61049 & .51965 & .23026 & .13523 \\
\hline 2203 & 4.9855 & 3.0223 & .60621 & .58668 & .29930 & .18007 & 7738 & 8.3452 & 4.8283 & .57857 & .49777 & .26719 & .17774 \\
\hline 2207 & 5.5963 & 3.3546 & .59944 & .57238 & .32213 & .21931 & 8072 & 7.3761 & 4.0775 & .55279 & .44983 & .20584 & .12450 \\
\hline 2510 & 5.9440 & 3.5224 & .59260 & .52534 & .24653 & .14727 & 8078 & 6.3242 & 3.4932 & .55236 & .45314 & .18433 & .09406 \\
\hline 2625 & 6.6391 & 3.8341 & .57750 & .53020 & .28226 & .15416 & 8084 & 4.5238 & 2.3262 & .51420 & .44351 & .16852 & .08321 \\
\hline 2665 & 6.8693 & 3.9984 & .58207 & .49327 & .23040 & .13876 & 8085 & 6.2198 & 3.6329 & .58409 & .48779 & .19719 & .11545 \\
\hline 2694 & 10.413 & 5.8287 & .55975 & .41475 & .17025 & .09022 & 8187 & 5.3355 & 3.1598 & .59221 & .57552 & .32493 & .20652 \\
\hline 2700 & 5.1310 & 3.0950 & .60321 & .56472 & .26253 & .14097 & 8358 & 5.7761 & 3.2845 & .56863 & .51667 & .27177 & .17328 \\
\hline 3145 & 6.4755 & 3.7821 & .58406 & .53705 & .29909 & .19232 & 8501 & 5.7039 & 3.2506 & .56990 & .50340 & .23405 & .13656 \\
\hline 3242 & 9.5556 & 3.9601 & .41443 & .17266 & .05863 & .02664 & 8518 & 5.7319 & 3.2883 & .57369 & .50406 & .23323 & .14428 \\
\hline 3288 & 9.3028 & 5.3468 & .57475 & .48313 & .24046 & .13723 & 8596 & 4.3201 & 2.5924 & 60009 & .61536 & .31902 & .17442 \\
\hline 3374 & 6.8387 & 3.6649 & .53590 & .45677 & .22430 & .12990 & 8788 & 8.4918 & 4.8225 & .56791 & .45049 & .19973 & .10949 \\
\hline 4026 & -- & -- & -- & -- & -- & -- & 8816 & -- & -- & -- & -- & -- & -- \\
\hline 4028 & 4.8102 & 2.6874 & .55868 & .52765 & .26696 & .17078 & 9148 & 10.429 & 5.3333 & .51142 & .26786 & .21429 & .26786 \\
\hline 4030 & 3.6982 & 2.0988 & .56750 & .59230 & .25574 & .07819 & 9153 & 5.8898 & 3.6899 & .62649 & .57565 & .29274 & .20836 \\
\hline 4089 & 6.3156 & 3.8479 & .60928 & .54843 & .27700 & .17455 & 9156 & 5.1798 & 3.0511 & .58904 & .54984 & .26884 & .16234 \\
\hline 4112 & 5.1060 & 3.1219 & .61143 & .58443 & .28756 & .16214 & 9193 & 4.7718 & 2.8595 & .59926 & .56798 & .24700 & .10863 \\
\hline 4306 & 6.0583 & 3.2246 & .53226 & .46777 & .24085 & .14941 & 9265 & 4.3187 & 2.6182 & .60624 & .62803 & .33338 & .18311 \\
\hline 4850 & 5.9779 & 3.4697 & .58041 & .51308 & .24168 & .13702 & 9569 & 3.9644 & 2.2645 & .57121 & .57876 & .26112 & .11926 \\
\hline 4856 & 6.5132 & 3.6926 & .56693 & .45590 & .17428 & .09565 & 9686 & 5.5547 & 3.2069 & .57733 & .51393 & .23276 & .13085 \\
\hline
\end{tabular}


Appendix 2-3.4. L-moments of storm duration defined by 18-hour minimum interevent time for hourly rainfall stations in eastern New Mexico.

[--, not available]

\begin{tabular}{|c|c|c|c|c|c|c|c|c|c|c|c|c|c|}
\hline $\begin{array}{c}\text { Station } \\
\text { no. }\end{array}$ & $\begin{array}{c}\text { Duration } \\
\text { mean } \\
\text { (hours) }\end{array}$ & $\begin{array}{l}\text { Duration } \\
\text { L-scale } \\
\text { (hours) }\end{array}$ & $\begin{array}{l}\text { Duration } \\
\text { L-CV } \\
\text { (dimen- } \\
\text { sionless) }\end{array}$ & $\begin{array}{l}\text { Duration } \\
\text { L-skew } \\
\text { (dimen- } \\
\text { sionless) }\end{array}$ & $\begin{array}{l}\text { Duration } \\
\text { L-kurtosis } \\
\text { (dimen- } \\
\text { sionless) }\end{array}$ & $\begin{array}{l}\text { Duration } \\
\text { Tau5 } \\
\text { (dimen- } \\
\text { sionless) }\end{array}$ & $\begin{array}{c}\text { Station } \\
\text { no. }\end{array}$ & $\begin{array}{l}\text { Duration } \\
\text { mean } \\
\text { (hours) }\end{array}$ & $\begin{array}{l}\text { Duration } \\
\text { L-scale } \\
\text { (hours) }\end{array}$ & $\begin{array}{c}\text { Duration } \\
\text { L-CV } \\
\text { (dimen- } \\
\text { sionless) }\end{array}$ & $\begin{array}{l}\text { Duration } \\
\text { L-skew } \\
\text { (dimen- } \\
\text { sionless) }\end{array}$ & $\begin{array}{l}\text { Duration } \\
\text { L-kurtosis } \\
\text { (dimen- } \\
\text { sionless) }\end{array}$ & $\begin{array}{l}\text { Duration } \\
\text { Tau5 } \\
\text { (dimen- } \\
\text { sionless) }\end{array}$ \\
\hline 0205 & 7.1991 & 4.2781 & .59426 & .52434 & .26640 & .15153 & 4862 & 7.1100 & 4.6658 & .65623 & .58008 & .26169 & .11115 \\
\hline 0208 & 7.5619 & 4.2547 & .56265 & .49418 & .24090 & .10991 & 5370 & 7.1075 & 4.4205 & 62194 & .55218 & .28150 & .17047 \\
\hline 0404 & 9.2921 & 5.6325 & .60616 & .44727 & .14829 & .09466 & 5651 & 7.4218 & 4.5366 & .61125 & .54084 & .28167 & .17305 \\
\hline 0600 & 6.8227 & 4.1878 & .61381 & .53145 & .23990 & .12545 & 6138 & 10.329 & 6.0967 & .59026 & .47299 & .21297 & . 11346 \\
\hline 0606 & 5.8169 & 3.9320 & .67596 & .65135 & .32178 & .11534 & 6275 & 9.6143 & 5.7777 & 60095 & .47294 & .19839 & .10748 \\
\hline 0646 & 8.5148 & 5.2872 & .62094 & .51197 & .22642 & .11821 & 6435 & 7.5020 & 4.6013 & .61335 & .51559 & .22650 & .11921 \\
\hline 1120 & 10.372 & 6.4649 & .62333 & .49093 & .20863 & .11705 & 6492 & 7.8814 & 4.8962 & .62124 & .49273 & .16227 & .04210 \\
\hline 1440 & 7.3033 & 4.6306 & .63405 & .54091 & .23586 & .12430 & 6619 & 6.3443 & 3.9251 & .61868 & .58153 & .32705 & .20864 \\
\hline 1480 & -- & -- & -- & -- & -- & -- & 7094 & 8.3765 & 5.0342 & 60099 & .47516 & .18634 & .09421 \\
\hline 1515 & 7.5555 & 4.6223 & .61177 & .52283 & .24433 & .13262 & 7254 & 7.2850 & 4.5332 & .62227 & .52788 & .22860 & .11598 \\
\hline 1807 & 6.6500 & 3.6697 & .55184 & .45938 & .19307 & .08341 & 7277 & -- & -- & -- & -- & -- & -- \\
\hline 1840 & 5.5382 & 3.5022 & .63237 & .59403 & .28017 & .12842 & 7279 & 6.7007 & 4.2955 & .64105 & .56883 & .25621 & .11370 \\
\hline 1881 & -- & -- & -- & -- & -- & -- & 7283 & 8.6512 & 5.3767 & .62149 & .52535 & .25022 & .13606 \\
\hline 1887 & 7.9065 & 4.7235 & .59741 & .50435 & .23729 & .12844 & 7604 & -- & -- & -- & -- & -- & -- \\
\hline 1939 & 7.6745 & 4.6710 & .60864 & .52425 & .25921 & .15337 & 7605 & 7.6087 & 4.9209 & .64675 & .54378 & .21136 & .06569 \\
\hline 1950 & 7.9259 & 4.5812 & .57800 & .51592 & .23296 & .06932 & 7609 & 8.3777 & 5.2061 & .62142 & .52602 & .24454 & .12401 \\
\hline 1956 & 8.4500 & 4.9590 & .58686 & .50312 & .27304 & .17318 & 7610 & 8.1054 & 5.1239 & .63216 & .53918 & .25819 & .15340 \\
\hline 1961 & 10.114 & 5.5361 & .54736 & .39813 & .15732 & .04779 & 7638 & 7.7208 & 4.8624 & 62979 & .53699 & .24309 & .12273 \\
\hline 2625 & 7.7936 & 4.6466 & .59621 & .52500 & .26295 & .13698 & 8084 & 6.5439 & 4.1648 & .63644 & .61285 & .36895 & 23466 \\
\hline 2665 & 9.0656 & 5.4390 & .59996 & .47596 & .20174 & .11056 & 8085 & 8.5755 & 5.2129 & 60789 & .47064 & .17056 & .08584 \\
\hline 2694 & 13.745 & 7.6895 & .55946 & .36507 & .10492 & .05551 & 8187 & 6.4662 & 4.0809 & .63112 & .59618 & .33502 & 19939 \\
\hline 2700 & 7.2402 & 4.6562 & .64311 & .55777 & .24898 & .12173 & 8358 & 7.4032 & 4.4588 & .60227 & .50977 & .21722 & .07257 \\
\hline 3145 & 7.7836 & 4.7648 & .61217 & .54425 & .28763 & .16564 & 8501 & 7.5760 & 4.5990 & .60705 & .51065 & .22735 & .11575 \\
\hline 3242 & 18.000 & 9.6263 & .53480 & .39512 & .12981 & .01554 & 8518 & 7.9167 & 4.8533 & .61305 & .51159 & .22871 & .12292 \\
\hline 3288 & 11.531 & 6.8884 & .59740 & .46652 & .18346 & .06401 & 8596 & 5.9633 & 3.7756 & .63314 & .57863 & .26688 & .12435 \\
\hline 3374 & 9.2432 & 5.2879 & .57209 & .45102 & .18488 & .08376 & 8788 & 11.915 & 7.1276 & .59821 & .43921 & .15669 & .06947 \\
\hline 4026 & -- & -- & -- & -- & -- & -- & 8816 & -- & -- & -- & -- & -- & -- \\
\hline 4028 & 5.6144 & 3.3255 & .59232 & .55242 & .28749 & .17058 & 9148 & 14.333 & 7.6000 & .53023 & .47807 & .58333 & . 10965 \\
\hline 4030 & 5.7566 & 3.5953 & .62455 & .56068 & .23026 & .08526 & 9153 & 8.2430 & 5.3643 & .65077 & .55033 & .25138 & .14362 \\
\hline 4089 & 8.4131 & 5.3761 & .63901 & .54513 & .26096 & .14678 & 9156 & 6.8531 & 4.2674 & .62270 & .54453 & .25191 & .13259 \\
\hline 4112 & 6.8883 & 4.4611 & .64763 & .57827 & .27515 & .14288 & 9193 & 6.7246 & 4.1927 & .62348 & .52447 & .19808 & .07935 \\
\hline 4306 & 8.0155 & 4.8524 & .60537 & .53681 & .28581 & .16400 & 9265 & 6.1628 & 4.0050 & 64987 & .60512 & .30293 & .15936 \\
\hline 4850 & 8.0746 & 5.0086 & .62030 & .51974 & .23024 & .11314 & 9569 & 5.2585 & 3.2419 & .61650 & .57858 & .26673 & .12767 \\
\hline 4856 & 8.8315 & 5.4417 & .61617 & .50330 & .23024 & .14547 & 9686 & 7.0410 & 4.3030 & .61114 & .52418 & .23683 & .12642 \\
\hline
\end{tabular}


Appendix 2-3.5. L-moments of storm duration defined by 24-hour minimum interevent time for hourly rainfall stations in eastern New Mexico.

$[--$, not available]

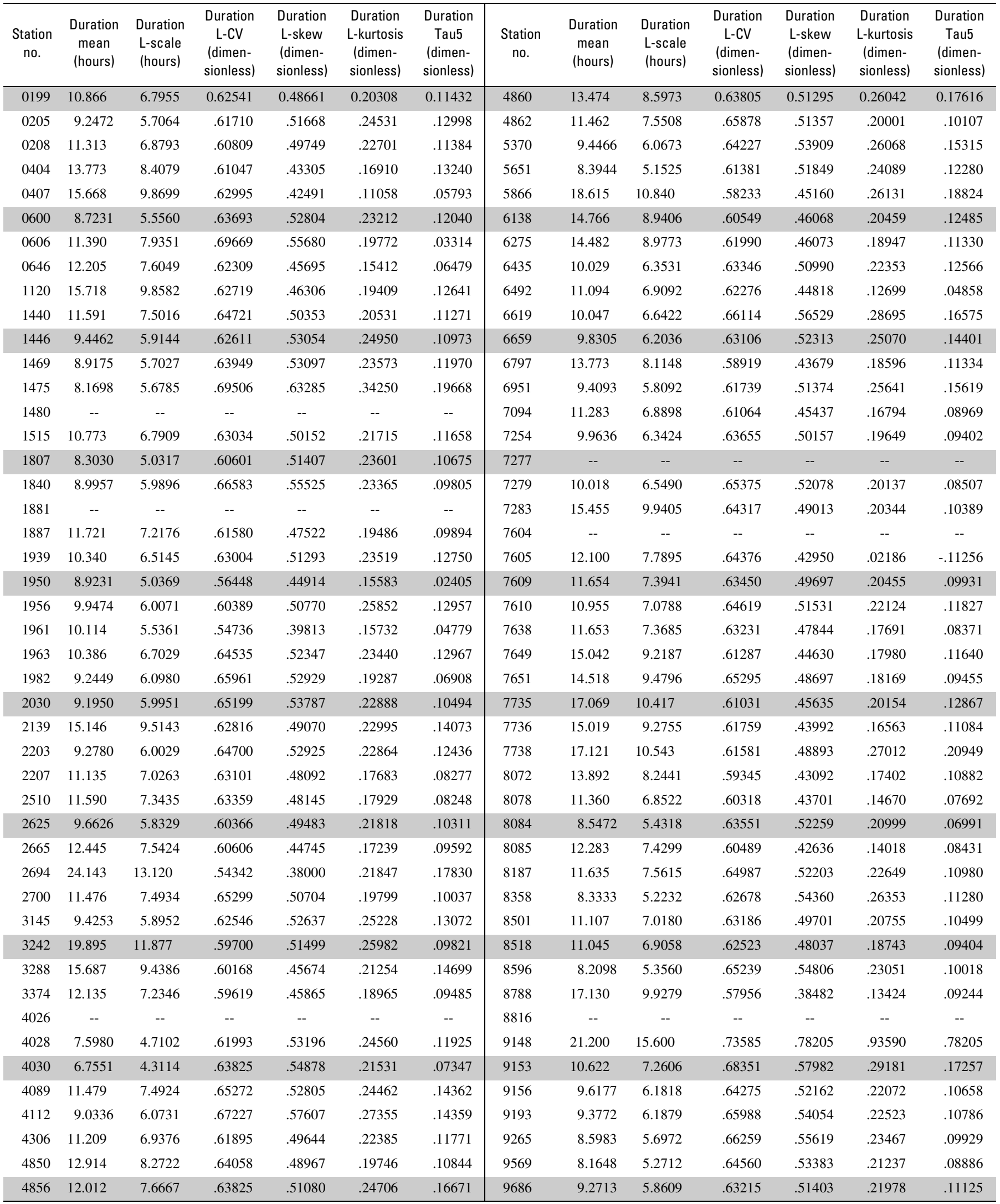


Appendix 2-3.6. L-moments of storm duration defined by 48-hour minimum interevent time for hourly rainfall stations in eastern New Mexico.

[--, not available]

\begin{tabular}{|c|c|c|c|c|c|c|c|c|c|c|c|c|c|}
\hline $\begin{array}{c}\text { Station } \\
\text { no. }\end{array}$ & $\begin{array}{c}\text { Duration } \\
\text { mean } \\
\text { (hours) }\end{array}$ & $\begin{array}{l}\text { Duration } \\
\text { L-scale } \\
\text { (hours) }\end{array}$ & $\begin{array}{l}\text { Duration } \\
\text { L-CV } \\
\text { (dimen- } \\
\text { sionless) }\end{array}$ & $\begin{array}{l}\text { Duration } \\
\text { L-skew } \\
\text { (dimen- } \\
\text { sionless) }\end{array}$ & $\begin{array}{l}\text { Duration } \\
\text { L-kurtosis } \\
\text { (dimen- } \\
\text { sionless) }\end{array}$ & $\begin{array}{l}\text { Duration } \\
\text { Tau5 } \\
\text { (dimen- } \\
\text { sionless) }\end{array}$ & $\begin{array}{c}\text { Station } \\
\text { no. }\end{array}$ & $\begin{array}{c}\text { Duration } \\
\text { mean } \\
\text { (hours) }\end{array}$ & $\begin{array}{l}\text { Duration } \\
\text { L-scale } \\
\text { (hours) }\end{array}$ & $\begin{array}{l}\text { Duration } \\
\text { L-CV } \\
\text { (dimen- } \\
\text { sionless) }\end{array}$ & $\begin{array}{l}\text { Duration } \\
\text { L-skew } \\
\text { (dimen- } \\
\text { sionless) }\end{array}$ & $\begin{array}{l}\text { Duration } \\
\text { L-kurtosis } \\
\text { (dimen- } \\
\text { sionless) }\end{array}$ & $\begin{array}{c}\text { Duration } \\
\text { Tau5 } \\
\text { (dimen- } \\
\text { sionless) }\end{array}$ \\
\hline 0205 & 17.497 & 11.605 & .66324 & .51651 & .22261 & .10076 & 4862 & 24.970 & 16.385 & .65621 & .46969 & .18666 & .11911 \\
\hline 0208 & 19.593 & 12.639 & .64506 & .50900 & .24265 & .14265 & 5370 & 16.649 & 11.176 & .67128 & .51985 & .21933 & .10249 \\
\hline 0404 & 29.060 & 19.082 & .65665 & .48867 & .22790 & .13938 & 5651 & 11.561 & 7.6099 & .65826 & .54707 & .25947 & .12598 \\
\hline 0600 & 15.463 & 10.239 & .66215 & .50944 & .21081 & .10255 & 6138 & 29.516 & 18.660 & .63218 & .45686 & .19105 & .11221 \\
\hline 0606 & 24.933 & 17.198 & 68976 & .49746 & .17167 & .07424 & 6275 & 29.592 & 19.173 & .64791 & .48645 & .23789 & .16303 \\
\hline 0646 & 21.125 & 13.877 & .65690 & .48370 & .19406 & .10593 & 6435 & 17.774 & 11.944 & .67199 & .52839 & .24604 & .13745 \\
\hline 1120 & 33.872 & 21.832 & .64455 & .46502 & .20385 & .12837 & 6492 & 19.459 & 12.478 & .64126 & .45974 & .17867 & .11119 \\
\hline 1440 & 23.776 & 16.122 & .67806 & .51892 & .24085 & .14508 & 6619 & 18.628 & 12.776 & .68588 & .53088 & .22248 & .09811 \\
\hline 1480 & -- & -- & -- & -- & -- & -- & 7094 & 22.576 & 14.402 & .63795 & .45934 & .18278 & .10316 \\
\hline 1515 & 21.791 & 14.458 & .66349 & .50641 & .23231 & .14403 & 7254 & 18.145 & 12.049 & .66406 & .49771 & .19751 & .09700 \\
\hline 1807 & 13.076 & 8.5022 & .65023 & .52324 & .22758 & .09265 & 7277 & -- & -- & -- & -- & -- & -- \\
\hline 1840 & 18.831 & 12.935 & .68688 & .51636 & .19152 & .07095 & 7279 & 21.798 & 14.705 & .67461 & .50064 & .19847 & .10010 \\
\hline 1881 & -- & -- & -- & -- & -- & -- & 7283 & 32.511 & 20.871 & .64198 & .45046 & .17487 & .10703 \\
\hline 1887 & 24.446 & 15.752 & .64433 & .47476 & .19901 & .11058 & 7604 & -- & -- & -- & -- & -- & -- \\
\hline 1939 & 19.001 & 12.564 & .66122 & .50579 & .21693 & .10903 & 7605 & 17.056 & 11.631 & .68193 & .49536 & .14217 & .02501 \\
\hline 1950 & 15.043 & 8.7984 & .58487 & .36863 & .04634 & -.03994 & 7609 & 21.311 & 13.917 & .65304 & .47965 & .18642 & .09611 \\
\hline 1956 & 21.452 & 14.716 & .68602 & .56380 & .31365 & .20805 & 7610 & 18.896 & 12.375 & 65490 & .49275 & .21414 & .13311 \\
\hline 1961 & 17.467 & 10.455 & .59858 & .40211 & .11980 & .04073 & 7638 & 22.726 & 14.638 & .64412 & .46092 & .17948 & .10654 \\
\hline 2625 & 17.283 & 11.384 & .65869 & .52158 & .23358 & .11064 & 8084 & 12.146 & 7.3551 & .60556 & .40528 & .07513 & -.00858 \\
\hline 2665 & 23.491 & 14.991 & .63818 & .46760 & .19843 & .11702 & 8085 & 23.628 & 14.932 & .63195 & .44413 & .17109 & .10106 \\
\hline 2694 & 49.137 & 27.528 & .56023 & .36373 & .16985 & .13587 & 8187 & 23.287 & 16.088 & .69088 & .53839 & .24313 & .12365 \\
\hline 2700 & 26.190 & 17.421 & .66516 & .48589 & .20230 & .12352 & 8358 & 14.283 & 9.9129 & .69404 & .57386 & .26837 & .10230 \\
\hline 3145 & 16.187 & 11.188 & .69117 & .57503 & .29359 & .15365 & 8501 & 22.448 & 14.948 & .66589 & .50615 & .22805 & .13631 \\
\hline 3242 & 26.529 & 16.316 & .61502 & .43488 & .07867 & -.09686 & 8518 & 21.046 & 13.672 & .64962 & .47882 & .19724 & .11298 \\
\hline 3288 & 28.413 & 18.992 & .66842 & .53459 & .28414 & .17495 & 8596 & 17.217 & 11.738 & .68175 & .51903 & .20421 & .09215 \\
\hline 3374 & 23.183 & 14.630 & .63107 & .46287 & .19088 & .10693 & 8788 & 32.458 & 19.624 & .60457 & .40544 & .16112 & .10874 \\
\hline 4026 & -- & -- & -- & -- & -- & -- & 8816 & -- & -- & -- & -- & -- & -- \\
\hline 4028 & 14.682 & 10.230 & .69678 & .57988 & .28736 & .14054 & 9148 & -- & -- & -- & -- & -- & -- \\
\hline 4030 & 17.267 & 11.721 & .67879 & .50021 & .16261 & .04352 & 9153 & 28.097 & 18.939 & .67406 & .46623 & .12520 & .00891 \\
\hline 4089 & 21.083 & 14.465 & 68612 & .53973 & .25817 & .14919 & 9156 & 17.973 & 12.014 & .66845 & .50499 & .20293 & .09856 \\
\hline 4112 & 17.187 & 11.712 & .68145 & .53155 & .23640 & .13024 & 9193 & 20.068 & 13.224 & .65896 & .47533 & .17457 & .09745 \\
\hline 4306 & 20.455 & 13.415 & .65584 & .50557 & .22344 & .11279 & 9265 & 16.104 & 11.517 & .71517 & .59030 & .29580 & .16617 \\
\hline 4850 & 25.706 & 17.027 & .66239 & .49113 & .21397 & .13219 & 9569 & 14.765 & 10.134 & .68637 & .54205 & .23286 & .11382 \\
\hline 4856 & 21.354 & 13.762 & .64444 & .47635 & .21051 & .13814 & 9686 & 17.386 & 11.446 & .65831 & .49464 & .19412 & .09004 \\
\hline
\end{tabular}


Appendix 2-3.7. L-moments of storm duration defined by 72-hour minimum interevent time for hourly rainfall stations in eastern New Mexico.

$[--$, not available]

\begin{tabular}{|c|c|c|c|c|c|c|c|c|c|c|c|c|c|}
\hline $\begin{array}{c}\text { Station } \\
\text { no. }\end{array}$ & $\begin{array}{c}\text { Duration } \\
\text { mean } \\
\text { (hours) }\end{array}$ & $\begin{array}{l}\text { Duration } \\
\text { L-scale } \\
\text { (hours) }\end{array}$ & $\begin{array}{l}\text { Duration } \\
\text { L-CV } \\
\text { (dimen- } \\
\text { sionless) }\end{array}$ & $\begin{array}{l}\text { Duration } \\
\text { L-skew } \\
\text { (dimen- } \\
\text { sionless) }\end{array}$ & $\begin{array}{l}\text { Duration } \\
\text { L-kurtosis } \\
\text { (dimen- } \\
\text { sionless) }\end{array}$ & $\begin{array}{l}\text { Duration } \\
\text { Tau5 } \\
\text { (dimen- } \\
\text { sionless) }\end{array}$ & $\begin{array}{c}\text { Station } \\
\text { no. }\end{array}$ & $\begin{array}{c}\text { Duration } \\
\text { mean } \\
\text { (hours) }\end{array}$ & $\begin{array}{l}\text { Duration } \\
\text { L-scale } \\
\text { (hours) }\end{array}$ & $\begin{array}{c}\text { Duration } \\
\text { L-CV } \\
\text { (dimen- } \\
\text { sionless) }\end{array}$ & $\begin{array}{l}\text { Duration } \\
\text { L-skew } \\
\text { (dimen- } \\
\text { sionless) }\end{array}$ & $\begin{array}{c}\text { Duration } \\
\text { L-kurtosis } \\
\text { (dimen- } \\
\text { sionless) }\end{array}$ & $\begin{array}{c}\text { Duration } \\
\text { Tau5 } \\
\text { (dimen- } \\
\text { sionless) }\end{array}$ \\
\hline 0205 & 28.210 & 19.128 & .67804 & .50732 & .20803 & .09692 & 4862 & 42.685 & 28.011 & .65623 & .45241 & .16620 & . 09241. \\
\hline 0208 & 31.629 & 21.870 & .69144 & .54954 & .26993 & .14073 & 5370 & 25.691 & 17.826 & .69384 & .53813 & .24718 & .13050 \\
\hline 0404 & 63.896 & 40.529 & .63429 & .43269 & .18369 & .12727 & 5651 & 20.949 & 15.014 & .71669 & .59445 & .30935 & . 16027 \\
\hline 0600 & 25.809 & 17.671 & .68468 & .52145 & .22844 & 11737 & 6138 & 49.339 & 32.669 & .66213 & .50392 & .25427 & . 16843 \\
\hline 0606 & 41.216 & 27.862 & .67599 & .43211 & .06567 & -.03338 & 6275 & 45.834 & 30.381 & .66284 & .49548 & .23853 & .15149 \\
\hline 0646 & 36.764 & 26.625 & .72421 & .57396 & .28842 & .16367 & 6435 & 28.586 & 19.775 & 69176 & .53118 & .24008 & .12400 \\
\hline 1120 & 57.970 & 38.281 & .66035 & .48599 & .23318 & .15494 & 6492 & 29.292 & 18.880 & .64456 & .44134 & .15590 & .09657 \\
\hline 1440 & 38.460 & 25.938 & .67441 & .49160 & .20519 & .10915 & 6619 & 30.763 & 21.515 & .69938 & .52720 & .21918 & .09899. \\
\hline 1480 & -- & -- & -- & -- & -- & -- & 7094 & 37.635 & 24.242 & .64413 & .45486 & .18453 & .10934 \\
\hline 1515 & 37.102 & 25.148 & .67781 & .50944 & .23580 & .14298 & 7254 & 30.887 & 21.112 & .68352 & .51055 & .21883 & .11461 \\
\hline 1807 & 20.546 & 14.198 & .69103 & .56072 & .27381 & .13416 & 7277 & -- & -- & -- & -- & -- & -- \\
\hline 1840 & 30.227 & 20.628 & .68244 & .49275 & .18225 & .08320 & 7279 & 39.330 & 26.697 & .67880 & .49299 & .20603 & .12156 \\
\hline 1881 & -- & -- & -- & -- & -- & -- & 7283 & 56.175 & 35.808 & .63744 & .43673 & .17381 & .11453 \\
\hline 1887 & 40.669 & 26.824 & .65957 & .48040 & .20616 & .12282 & 7604 & -- & -- & -- & -- & -- & -- \\
\hline 1939 & 31.846 & 21.345 & .67025 & .49113 & .19780 & .09624 & 7605 & 32.067 & 22.762 & .70983 & .51883 & .17605 & .02928 \\
\hline 1950 & 22.143 & 13.724 & .61978 & .39435 & .07983 & .02846 & 7609 & 33.892 & 23.150 & .68307 & .51958 & .24339 & .15029 \\
\hline 1956 & 36.923 & 26.231 & .71042 & .57164 & .32423 & .24560 & 7610 & 29.180 & 19.518 & .66887 & .49746 & .22167 & .13749 \\
\hline 1961 & 36.708 & 23.752 & .64704 & .44543 & .13462 & .03035 & 7638 & 37.208 & 25.301 & .67998 & .51115 & .23704 & (14159 \\
\hline 2625 & 30.909 & 21.212 & .68628 & .52476 & .22589 & .09896 & 8084 & 24.732 & 16.760 & .67766 & .49703 & .19843 & .11754 \\
\hline 2665 & 38.391 & 25.409 & .66185 & .49369 & .22988 & .14305 & 8085 & 38.819 & 25.049 & .64527 & .46318 & .20671 & .13697 \\
\hline 2694 & 75.763 & 46.231 & .61021 & .46937 & .23856 & .11124 & 8187 & 33.513 & 22.523 & .67204 & .47585 & .16199 & .06345 \\
\hline 2700 & 45.047 & 30.242 & .67133 & .48904 & .21886 & .14241 & 8358 & 20.449 & 15.125 & .73965 & .63115 & .35323 & .19625 \\
\hline 3145 & 22.918 & 16.010 & .69856 & .55981 & .27224 & .14135 & 8501 & 38.183 & 25.960 & .67989 & .50030 & .20947 & .11116 \\
\hline 3242 & 38.133 & 25.705 & .67408 & .52239 & .17206 & -.03688 & 8518 & 35.879 & 23.869 & .66525 & .49212 & .22407 & . 14377 \\
\hline 3288 & 49.757 & 33.461 & .67249 & .52901 & .29699 & .21334 & 8596 & 28.725 & 19.390 & .67501 & .47985 & .16869 & 08115 \\
\hline 3374 & 37.487 & 24.787 & .66122 & .49713 & .23494 & .14714 & 8788 & 49.932 & 32.785 & .65659 & .48374 & .23335 & .15881 \\
\hline 4026 & -- & -- & -- & -- & -- & -- & 8816 & -- & -- & -- & -- & -- & -- \\
\hline 4028 & 25.793 & 18.393 & .71311 & .56526 & .26775 & .13450 & 9148 & -- & -- & -- & -- & -- & -- \\
\hline 4030 & 25.962 & 17.735 & .68312 & .48821 & .15729 & .04478 & 9153 & 41.397 & 28.845 & 69679 & .50025 & . 17729 & .06637. \\
\hline 4089 & 34.269 & 24.184 & .70570 & .55141 & .27195 & .15996 & 9156 & 30.023 & 20.462 & .68152 & .50066 & .19740 & .09303 \\
\hline 4112 & 26.918 & 18.469 & .68615 & .51101 & .20494 & .09122 & 9193 & 35.596 & 22.662 & .63666 & .40845 & 10996 & .05901 \\
\hline 4306 & 35.613 & 23.699 & .66545 & .48246 & .18636 & .08362 & 9265 & 23.857 & 16.972 & .71139 & .55508 & .25073 & .12835 \\
\hline 4850 & 43.721 & 29.339 & .67105 & .49217 & .22332 & .14775 & 9569 & 27.355 & 18.960 & .69313 & .52140 & .22110 & .11595 \\
\hline 4856 & 39.083 & 26.856 & .68716 & .53162 & .26104 & .14857 & 9686 & 28.026 & 19.203 & .68519 & .51841 & .22445 & .11453 \\
\hline
\end{tabular}


Appendix 2-4.1. Empirical distribution of storm depth defined by 6-hour minimum interevent time for hourly rainfall stations in eastern New Mexico.

[--, not available]




Appendix 2-4.2. Empirical distribution of storm depth defined by 8-hour minimum interevent time for hourly rainfall stations in eastern New Mexico.

$[--$, not available]

$$
\text { Depth }
$$

(inches)

\begin{tabular}{|c|c|c|c|c|c|c|c|c|c|c|c|c|c|c|c|c|c|c|c|}
\hline $\begin{array}{l}\text { Sta- } \\
\text { tion } \\
\text { no. }\end{array}$ & $\begin{array}{c}1 \text { st } \\
\text { per- } \\
\text { centile }\end{array}$ & $\begin{array}{c}\text { 2nd } \\
\text { per- } \\
\text { centile }\end{array}$ & $\begin{array}{c}\text { 10th } \\
\text { per- } \\
\text { centile }\end{array}$ & $\begin{array}{c}25 \text { th } \\
\text { per- } \\
\text { centile }\end{array}$ & $\begin{array}{c}\text { 50th } \\
\text { per- } \\
\text { centile } \\
\text { (median) }\end{array}$ & $\begin{array}{c}\text { 75th } \\
\text { per- } \\
\text { centile }\end{array}$ & $\begin{array}{c}\text { 90th } \\
\text { per- } \\
\text { centile }\end{array}$ & $\begin{array}{c}\text { 98th } \\
\text { per- } \\
\text { centile }\end{array}$ & $\begin{array}{c}\text { 99th } \\
\text { per- } \\
\text { centile }\end{array}$ & $\begin{array}{l}\text { Sta- } \\
\text { tion } \\
\text { no. }\end{array}$ & $\begin{array}{c}1 \text { st } \\
\text { per- } \\
\text { centile }\end{array}$ & $\begin{array}{c}\text { 2nd } \\
\text { per- } \\
\text { centile }\end{array}$ & $\begin{array}{c}10 \text { th } \\
\text { per- } \\
\text { centile }\end{array}$ & $\begin{array}{c}25 \text { th } \\
\text { per- } \\
\text { centile }\end{array}$ & $\begin{array}{c}\text { 50th } \\
\text { per- } \\
\text { centile } \\
\text { (median) }\end{array}$ & $\begin{array}{c}\text { 75th } \\
\text { per- } \\
\text { centile }\end{array}$ & $\begin{array}{c}\text { 90th } \\
\text { per- } \\
\text { centile }\end{array}$ & $\begin{array}{c}\text { 98th } \\
\text { per- } \\
\text { centile }\end{array}$ & $\begin{array}{c}\text { 99th } \\
\text { per- } \\
\text { centile }\end{array}$ \\
\hline 0199 & 0.02 & 0.02 & 0.05 & 0.10 & 0.18 & 0.36 & 0.70 & 1.34 & 1.62 & 4860 & 0.01 & 0.01 & 0.03 & 0.05 & 0.10 & 0.27 & 0.79 & 1.89 & 2.31 \\
\hline 0205 & .01 & .01 & .03 & .05 & .13 & .30 & .64 & 1.47 & 2.27 & 4862 & .10 & .10 & .10 & .10 & .20 & .40 & .70 & 1.49 & 1.80 \\
\hline 0208 & .02 & .02 & .03 & .06 & .13 & .29 & .53 & .85 & 1.25 & 5370 & .02 & .03 & .06 & .10 & .20 & .40 & .88 & 1.81 & 2.62 \\
\hline 0404 & .10 & .10 & .10 & .10 & .20 & .35 & .72 & 1.40 & 2.88 & 5651 & .02 & .02 & .05 & .06 & .15 & .32 & .71 & 1.40 & 1.84 \\
\hline 0407 & .10 & .10 & .10 & .10 & .10 & .30 & .50 & 1.20 & 1.50 & 5866 & .02 & .02 & .04 & .06 & .17 & .47 & .88 & 1.81 & 2.52 \\
\hline 0600 & .02 & .02 & .04 & .10 & .11 & .30 & .70 & 1.70 & 2.37 & 6138 & .02 & .02 & .03 & .05 & .14 & .32 & .63 & 1.45 & 1.99 \\
\hline 0606 & -- & .03 & .04 & .10 & .20 & .35 & .62 & 1.18 & -- & 6275 & .02 & .02 & .04 & .10 & .13 & .30 & .66 & 1.35 & 1.71 \\
\hline 0646 & .02 & .02 & .04 & .09 & .19 & .37 & .72 & 1.59 & 2.02 & 6435 & .01 & .02 & .04 & .09 & .12 & .30 & .61 & 1.45 & 1.99 \\
\hline 1120 & .02 & .02 & .07 & .10 & .20 & .40 & .80 & 1.54 & 1.87 & 6492 & .01 & .01 & .02 & .04 & .09 & .22 & .44 & 1.09 & 2.28 \\
\hline 1440 & .03 & .04 & .10 & .10 & .17 & .30 & .61 & 1.30 & 1.60 & 6619 & .02 & .02 & .04 & .08 & .18 & .41 & .85 & 1.95 & 2.30 \\
\hline 1446 & .02 & .02 & .04 & .07 & .18 & .47 & .89 & 1.84 & 2.32 & 6659 & .02 & .02 & .05 & .10 & .18 & .41 & .90 & 2.15 & 3.29 \\
\hline 1469 & .02 & .02 & .05 & .10 & .15 & .40 & .80 & 2.02 & 2.57 & 6797 & .01 & .01 & .03 & .05 & .12 & .24 & .47 & 1.27 & 1.83 \\
\hline 1475 & -- & .01 & .02 & .04 & .09 & .31 & .89 & 2.44 & -- & 6951 & .01 & .01 & .03 & .05 & .12 & .40 & .72 & 1.48 & 2.24 \\
\hline 1480 & -- & -- & -- & -- & .32 & -- & -- & -- & -- & 7094 & .02 & .02 & .05 & .10 & .12 & .30 & .60 & 1.19 & 1.41 \\
\hline 1515 & .01 & .02 & .03 & .08 & .10 & .30 & .60 & 1.12 & 1.39 & 7254 & .02 & .02 & .05 & .10 & .11 & .30 & .67 & 1.44 & 2.00 \\
\hline 1807 & .02 & .02 & .03 & .05 & .15 & .33 & .61 & 1.13 & 1.40 & 7277 & -- & -- & -- & -- & .23 & -- & -- & -- & -- \\
\hline 1840 & .10 & .10 & .10 & .10 & .20 & .40 & .80 & 1.80 & 3.19 & 7279 & .03 & .04 & .10 & .10 & .14 & .30 & .60 & 1.30 & 1.76 \\
\hline 1881 & -- & -- & -- & .04 & .08 & .21 & -- & -- & -- & 7283 & .01 & .01 & .01 & .02 & .07 & .22 & .52 & 1.29 & 1.74 \\
\hline 1887 & .01 & .01 & .01 & .03 & .10 & .28 & .63 & 1.64 & 2.17 & 7604 & -- & -- & -- & .01 & .13 & .38 & -- & -- & -- \\
\hline 1939 & .01 & .02 & .05 & .10 & .20 & .41 & .90 & 1.84 & 2.49 & 7605 & -- & -- & .10 & .10 & .15 & .40 & .79 & -- & -- \\
\hline 1950 & -- & -- & .04 & .08 & .27 & .63 & .99 & -- & -- & 7609 & .01 & .01 & .01 & .03 & .08 & .26 & .59 & 1.42 & 1.98 \\
\hline 1956 & -- & -- & .03 & .06 & .15 & .30 & .64 & -- & -- & 7610 & .01 & .01 & .02 & .07 & .10 & .33 & .76 & 1.93 & 2.64 \\
\hline 1961 & -- & -- & .01 & .05 & .10 & .51 & 1.06 & -- & -- & 7638 & .02 & .03 & .06 & .10 & .16 & .37 & .75 & 1.53 & 1.93 \\
\hline 1963 & .02 & .02 & .05 & .10 & .15 & .40 & .80 & 1.86 & 2.41 & 7649 & .01 & .02 & .04 & .10 & .15 & .39 & .70 & 1.40 & 1.70 \\
\hline 1982 & .10 & .10 & .10 & .10 & .10 & .30 & .50 & 1.10 & 1.40 & 7651 & .10 & .10 & .10 & .10 & .20 & .40 & .80 & 1.50 & 1.96 \\
\hline 2030 & .01 & .02 & .05 & .10 & .14 & .36 & .70 & 1.60 & 2.00 & 7735 & .02 & .02 & .04 & .08 & .18 & .40 & .78 & 1.61 & 1.88 \\
\hline 2139 & .01 & .02 & .03 & .06 & .13 & .33 & .61 & 1.40 & 1.98 & 7736 & .03 & .03 & .10 & .10 & .20 & .41 & .90 & 1.76 & 2.10 \\
\hline 2203 & .10 & .10 & .10 & .10 & .20 & .40 & .80 & 2.50 & 2.84 & 7738 & .01 & .01 & .03 & .07 & .19 & .47 & .95 & 1.66 & 2.37 \\
\hline 2207 & .02 & .03 & .09 & .10 & .20 & .42 & .80 & 1.83 & 2.15 & 8072 & .01 & .01 & .03 & .05 & .11 & .26 & .53 & 1.22 & 1.57 \\
\hline 2510 & .02 & .03 & .05 & .10 & .14 & .36 & .70 & 1.50 & 1.81 & 8078 & .01 & .01 & .02 & .05 & .10 & .22 & .45 & 1.02 & 1.32 \\
\hline 2625 & .02 & .02 & .03 & .05 & .12 & .30 & .70 & 1.63 & 2.23 & 8084 & -- & .01 & .04 & .08 & .15 & .28 & .44 & 1.36 & -- \\
\hline 2665 & .02 & .02 & .05 & .10 & .14 & .32 & .65 & 1.30 & 1.66 & 8085 & .02 & .02 & .05 & .10 & .10 & .30 & .59 & 1.10 & 1.46 \\
\hline 2694 & .01 & .01 & .03 & .05 & .15 & .35 & .64 & 1.44 & 2.41 & 8187 & .02 & .02 & .04 & .06 & .15 & .36 & .78 & 1.92 & 2.30 \\
\hline 2700 & .02 & .03 & .06 & .10 & .10 & .20 & .45 & .90 & 1.20 & 8358 & -- & .02 & .04 & .06 & .14 & .32 & .65 & 1.88 & -- \\
\hline 3145 & .02 & .02 & .03 & .06 & .12 & .30 & .70 & 1.54 & 2.04 & 8501 & .01 & .02 & .04 & .08 & .14 & .34 & .70 & 1.50 & 1.92 \\
\hline 3242 & -- & -- & .10 & .15 & .20 & .55 & 1.01 & -- & -- & 8518 & .01 & .02 & .03 & .06 & .10 & .27 & .55 & 1.21 & 1.42 \\
\hline 3288 & .02 & .02 & .04 & .07 & .18 & .36 & .75 & 1.25 & 1.86 & 8596 & .07 & .10 & .10 & .10 & .20 & .40 & .80 & 2.00 & 2.30 \\
\hline 3374 & .01 & .02 & .02 & .04 & .10 & .23 & .52 & 1.19 & 1.44 & 8788 & .01 & .02 & .04 & .08 & .20 & .40 & .73 & 1.32 & 1.76 \\
\hline 4026 & -- & -- & -- & -- & .07 & -- & -- & -- & -- & 8816 & -- & -- & -- & -- & .04 & -- & -- & -- & -- \\
\hline 4028 & .01 & .01 & .02 & .05 & .13 & .37 & .80 & 2.10 & 2.38 & 9148 & -- & -- & -- & .02 & .13 & .20 & -- & -- & -- \\
\hline 4030 & .10 & .10 & .10 & .10 & .20 & .40 & .90 & 1.64 & 2.48 & 9153 & .01 & .01 & .02 & .04 & .15 & .39 & .86 & 1.89 & 3.26 \\
\hline 4089 & .02 & .02 & .05 & .10 & .12 & .34 & .70 & 1.52 & 1.82 & 9156 & .01 & .02 & .05 & .10 & .18 & .40 & .80 & 1.62 & 2.17 \\
\hline 4112 & .03 & .04 & .10 & .10 & .19 & .40 & .80 & 1.80 & 2.72 & 9193 & .04 & .08 & .10 & .10 & .10 & .30 & .60 & 1.30 & 1.78 \\
\hline 4306 & .01 & .02 & .03 & .05 & .12 & .31 & .60 & 1.28 & 1.60 & 9265 & .01 & .02 & .10 & .10 & .16 & .40 & .80 & 1.90 & 3.00 \\
\hline 4850 & .01 & .02 & .03 & .09 & .11 & .30 & .60 & 1.51 & 2.08 & 9569 & .10 & .10 & .10 & .10 & .20 & .40 & .80 & 1.56 & 2.10 \\
\hline 4856 & .01 & .01 & .02 & .05 & .11 & .31 & .64 & 1.60 & 2.11 & 9686 & .01 & .02 & .03 & .08 & .10 & .30 & .52 & 1.18 & 1.38 \\
\hline
\end{tabular}


Appendix 2-4.3. Empirical distribution of storm depth defined by 12-hour minimum interevent time for hourly rainfall stations in eastern New Mexico.

[--, not available]

\begin{tabular}{|c|c|c|c|c|c|c|c|c|c|c|c|c|c|c|c|c|c|c|c|}
\hline \multicolumn{20}{|c|}{$\begin{array}{c}\text { Depth } \\
\text { (inches) }\end{array}$} \\
\hline $\begin{array}{l}\text { Sta- } \\
\text { tion } \\
\text { no. }\end{array}$ & $\begin{array}{c}\text { 1st } \\
\text { per- } \\
\text { centile }\end{array}$ & $\begin{array}{c}\text { 2nd } \\
\text { per- } \\
\text { centile }\end{array}$ & $\begin{array}{c}\text { 10th } \\
\text { per- } \\
\text { centile }\end{array}$ & $\begin{array}{c}\text { 25th } \\
\text { per- } \\
\text { centile }\end{array}$ & $\begin{array}{c}\text { 50th } \\
\text { per- } \\
\text { centile } \\
\text { (median) }\end{array}$ & $\begin{array}{c}\text { 75th } \\
\text { per- } \\
\text { centile }\end{array}$ & $\begin{array}{l}\text { 90th } \\
\text { per- } \\
\text { centile }\end{array}$ & $\begin{array}{c}\text { 98th } \\
\text { per- } \\
\text { centile }\end{array}$ & $\begin{array}{c}\text { 99th } \\
\text { per- } \\
\text { centile }\end{array}$ & $\begin{array}{l}\text { Sta- } \\
\text { tion } \\
\text { no. }\end{array}$ & $\begin{array}{c}1 \text { st } \\
\text { per- } \\
\text { centile }\end{array}$ & $\begin{array}{c}\text { 2nd } \\
\text { per- } \\
\text { centile }\end{array}$ & $\begin{array}{c}\text { 10th } \\
\text { per- } \\
\text { centile }\end{array}$ & $\begin{array}{c}\text { 25th } \\
\text { per- } \\
\text { centile }\end{array}$ & $\begin{array}{c}\text { 50th } \\
\text { per- } \\
\text { centile } \\
\text { (median) }\end{array}$ & $\begin{array}{c}\text { 75th } \\
\text { per- } \\
\text { centile }\end{array}$ & $\begin{array}{c}\text { 90th } \\
\text { per- } \\
\text { centile }\end{array}$ & $\begin{array}{c}\text { 98th } \\
\text { per- } \\
\text { centile }\end{array}$ & $\begin{array}{c}\text { 99th } \\
\text { per- } \\
\text { centile }\end{array}$ \\
\hline 0199 & 0.02 & 0.02 & 0.05 & 0.10 & 0.20 & 0.40 & 0.70 & 1.50 & 1.73 & 4860 & 0.01 & 0.01 & 0.03 & 0.05 & 0.11 & 0.29 & 0.82 & 2.03 & 2.53 \\
\hline 0205 & .01 & .01 & .03 & .05 & .14 & .33 & .69 & 1.56 & 2.24 & 4862 & .10 & .10 & .10 & .10 & .20 & .40 & .80 & 1.67 & 1.90 \\
\hline 0208 & .02 & .03 & .04 & .07 & .15 & .32 & .56 & .86 & 1.57 & 5370 & .02 & .03 & .06 & .10 & .20 & .45 & .92 & 1.94 & 3.33 \\
\hline 0404 & .10 & .10 & .10 & .10 & .20 & .40 & .80 & 1.90 & 3.47 & 5651 & .02 & .02 & .05 & .06 & .15 & .37 & .72 & 1.82 & 2.46 \\
\hline 0407 & .10 & .10 & .10 & .10 & .20 & .30 & .70 & 1.40 & 1.70 & 5866 & .02 & .02 & .04 & .07 & .23 & .50 & 1.04 & 1.82 & 2.65 \\
\hline 0600 & .02 & .02 & .05 & .10 & .14 & .36 & .78 & 1.76 & 2.70 & 6138 & .02 & .02 & .03 & .06 & .15 & .35 & .69 & 1.55 & 2.20 \\
\hline 0606 & -- & .02 & .05 & .12 & .20 & .38 & .66 & 1.29 & -- & 6275 & .02 & .02 & .05 & .10 & .15 & .35 & .70 & 1.40 & 1.88 \\
\hline 0646 & .02 & .03 & .05 & .10 & .20 & .40 & .75 & 1.71 & 2.19 & 6435 & .01 & .02 & .04 & .10 & .15 & .35 & .69 & 1.60 & 2.00 \\
\hline 1120 & .02 & .03 & .08 & .10 & .20 & .48 & .90 & 1.70 & 2.10 & 6492 & .01 & .01 & .02 & .05 & .10 & .25 & .45 & 1.17 & 2.37 \\
\hline 1440 & .03 & .04 & .10 & .10 & .20 & .40 & .70 & 1.49 & 1.88 & 6619 & .02 & .02 & .05 & .09 & .20 & .42 & .88 & 1.95 & 2.30 \\
\hline 1446 & .02 & .02 & .05 & .07 & .20 & .50 & .94 & 1.93 & 2.61 & 6659 & .02 & .02 & .05 & .10 & .20 & .49 & .99 & 2.20 & 3.42 \\
\hline 1469 & .02 & .02 & .05 & .10 & .20 & .40 & .90 & 2.24 & 2.89 & 6797 & .01 & .01 & .03 & .06 & .13 & .26 & .54 & 1.51 & 1.84 \\
\hline 1475 & -- & .01 & .02 & .04 & .09 & .32 & .94 & 2.95 & -- & 6951 & .01 & .02 & .03 & .05 & .12 & .40 & .82 & 1.49 & 2.31 \\
\hline 1480 & -- & -- & -- & -- & .32 & -- & -- & -- & -- & 7094 & .02 & .03 & .05 & .10 & .15 & .31 & .63 & 1.25 & 1.50 \\
\hline 1515 & .01 & .02 & .04 & .09 & .12 & .31 & .60 & 1.20 & 1.50 & 7254 & .02 & .02 & .05 & .10 & .14 & .30 & .70 & 1.60 & 2.20 \\
\hline 1807 & .02 & .02 & .03 & .05 & .15 & .35 & .64 & 1.20 & 1.53 & 7277 & -- & -- & -- & -- & .23 & -- & -- & -- & -- \\
\hline 1840 & .10 & .10 & .10 & .10 & .20 & .40 & .90 & 1.88 & 3.28 & 7279 & .03 & .04 & .10 & .10 & .20 & .40 & .70 & 1.40 & 1.79 \\
\hline 1881 & -- & -- & -- & -- & .08 & -- & -- & -- & -- & 7283 & .01 & .01 & .01 & .03 & .08 & .25 & .55 & 1.38 & 1.84 \\
\hline 1887 & .01 & .01 & .01 & .03 & .11 & .30 & .66 & 1.83 & 2.26 & 7604 & -- & -- & -- & .01 & .13 & .38 & -- & -- & -- \\
\hline 1939 & .02 & .02 & .05 & .10 & .20 & .48 & .92 & 2.05 & 2.84 & 7605 & -- & -- & .10 & .10 & .20 & .45 & .90 & -- & -- \\
\hline 1950 & -- & -- & .07 & .15 & .30 & .66 & 1.19 & -- & -- & 7609 & .01 & .01 & .01 & .03 & .09 & .28 & .65 & 1.61 & 2.23 \\
\hline 1956 & -- & -- & .03 & .05 & .15 & .33 & .66 & -- & -- & 7610 & .01 & .01 & .02 & .08 & .13 & .40 & .80 & 2.09 & 2.84 \\
\hline 1961 & -- & -- & .04 & .05 & .10 & .52 & 1.10 & -- & -- & 7638 & .02 & .03 & .06 & .10 & .19 & .40 & .83 & 1.70 & 2.05 \\
\hline 1963 & .02 & .02 & .05 & .10 & .18 & .40 & .90 & 1.94 & 2.61 & 7649 & .01 & .02 & .04 & .10 & .19 & .40 & .80 & 1.59 & 1.90 \\
\hline 1982 & .10 & .10 & .10 & .10 & .10 & .30 & .60 & 1.28 & 1.60 & 7651 & .10 & .10 & .10 & .10 & .20 & .50 & .83 & 1.70 & 2.07 \\
\hline 2030 & .01 & .02 & .05 & .10 & .17 & .40 & .80 & 1.70 & 2.18 & 7735 & .02 & .02 & .05 & .09 & .20 & .45 & .85 & 1.76 & 2.23 \\
\hline 2139 & .01 & .02 & .04 & .07 & .15 & .36 & .62 & 1.43 & 2.05 & 7736 & .03 & .05 & .10 & .10 & .20 & .50 & .95 & 1.91 & 2.51 \\
\hline 2203 & .10 & .10 & .10 & .10 & .20 & .50 & .90 & 2.60 & 3.10 & 7738 & .01 & .01 & .03 & .07 & .20 & .49 & 1.05 & 2.04 & 2.40 \\
\hline 2207 & .02 & .03 & .09 & .10 & .20 & .50 & .86 & 1.90 & 2.66 & 8072 & .01 & .01 & .03 & .05 & .12 & .29 & .61 & 1.33 & 1.60 \\
\hline 2510 & .02 & .03 & .06 & .10 & .16 & .40 & .72 & 1.57 & 2.05 & 8078 & .01 & .01 & .02 & .05 & .11 & .24 & .48 & 1.16 & 1.42 \\
\hline 2625 & .02 & .02 & .03 & .05 & .14 & .34 & .80 & 1.66 & 2.36 & 8084 & -- & .01 & .04 & .08 & .15 & .28 & .44 & 1.36 & -- \\
\hline 2665 & .02 & .02 & .05 & .10 & .16 & .37 & .70 & 1.42 & 1.72 & 8085 & .02 & .02 & .05 & .10 & .12 & .30 & .60 & 1.24 & 1.53 \\
\hline 2694 & .02 & .02 & .03 & .05 & .19 & .43 & .72 & 1.45 & 2.89 & 8187 & .02 & .02 & .04 & .06 & .17 & .39 & .85 & 2.05 & 2.35 \\
\hline 2700 & .02 & .03 & .07 & .10 & .10 & .27 & .50 & 1.00 & 1.28 & 8358 & -- & .02 & .04 & .06 & .15 & .33 & .72 & 1.93 & -- \\
\hline 3145 & .02 & .02 & .04 & .06 & .14 & .32 & .71 & 1.80 & 2.08 & 8501 & .02 & .02 & .04 & .09 & .15 & .38 & .75 & 1.60 & 1.98 \\
\hline 3242 & -- & -- & .10 & .15 & .20 & .55 & 1.01 & -- & -- & 8518 & .02 & .02 & .03 & .07 & .10 & .30 & .60 & 1.30 & 1.56 \\
\hline 3288 & .01 & .02 & .04 & .07 & .20 & .43 & .81 & 1.65 & 2.35 & 8596 & .07 & .10 & .10 & .10 & .20 & .40 & .80 & 2.00 & 2.29 \\
\hline 3374 & .01 & .02 & .03 & .05 & .10 & .25 & .55 & 1.23 & 1.56 & 8788 & .01 & .02 & .04 & .09 & .20 & .42 & .77 & 1.38 & 1.77 \\
\hline 4026 & -- & -- & -- & -- & .07 & -- & -- & -- & -- & 8816 & -- & -- & -- & -- & .04 & -- & -- & -- & -- \\
\hline 4028 & .01 & .01 & .02 & .05 & .13 & .38 & .84 & 2.20 & 2.55 & 9148 & -- & -- & -- & .02 & .13 & .20 & -- & -- & -- \\
\hline 4030 & .10 & .10 & .10 & .10 & .20 & .50 & 1.00 & 1.66 & 2.69 & 9153 & .01 & .02 & .02 & .06 & .15 & .41 & .94 & 2.03 & 3.49 \\
\hline 4089 & .02 & .02 & .05 & .10 & .15 & .40 & .79 & 1.64 & 2.27 & 9156 & .01 & .02 & .06 & .10 & .20 & .42 & .90 & 1.80 & 2.40 \\
\hline 4112 & .03 & .05 & .10 & .10 & .20 & .40 & .80 & 1.90 & 2.90 & 9193 & .05 & .10 & .10 & .10 & .20 & .30 & .70 & 1.52 & 1.86 \\
\hline 4306 & .01 & .02 & .03 & .05 & .12 & .32 & .64 & 1.36 & 1.60 & 9265 & .01 & .02 & .10 & .10 & .20 & .40 & .80 & 2.39 & 3.20 \\
\hline 4850 & .01 & .02 & .04 & .10 & .14 & .32 & .69 & 1.65 & 2.11 & 9569 & .10 & .10 & .10 & .10 & .20 & .40 & .90 & 1.83 & 2.23 \\
\hline 4856 & .01 & .02 & .03 & .05 & .12 & .32 & .71 & 1.70 & 2.21 & 9686 & .02 & .02 & .04 & .08 & .11 & .30 & .60 & 1.20 & 1.44 \\
\hline
\end{tabular}


Appendix 2-4.4. Empirical distribution of storm depth defined by 18-hour minimum interevent time for hourly rainfall stations in eastern New Mexico.

$[--$, not available]

\begin{tabular}{|c|c|c|c|c|c|c|c|c|c|c|c|c|c|c|c|c|c|c|c|}
\hline \multicolumn{20}{|c|}{$\begin{array}{l}\text { Depth } \\
\text { (inches) }\end{array}$} \\
\hline $\begin{array}{l}\text { Sta- } \\
\text { tion } \\
\text { no. }\end{array}$ & $\begin{array}{c}1 \text { st } \\
\text { per- } \\
\text { centile }\end{array}$ & $\begin{array}{l}\text { 2nd } \\
\text { per- } \\
\text { centile }\end{array}$ & $\begin{array}{c}\text { 10th } \\
\text { per- } \\
\text { centile }\end{array}$ & $\begin{array}{l}25 \text { th } \\
\text { per- } \\
\text { centile }\end{array}$ & $\begin{array}{c}\text { 50th } \\
\text { per- } \\
\text { centile } \\
\text { (median) }\end{array}$ & $\begin{array}{c}75 \text { th } \\
\text { per- } \\
\text { centile }\end{array}$ & $\begin{array}{c}\text { 90th } \\
\text { per- } \\
\text { centile }\end{array}$ & $\begin{array}{c}\text { 98th } \\
\text { per- } \\
\text { centile }\end{array}$ & $\begin{array}{c}\text { 99th } \\
\text { per- } \\
\text { centile }\end{array}$ & $\begin{array}{l}\text { Sta- } \\
\text { tion } \\
\text { no. }\end{array}$ & $\begin{array}{c}\text { 1st } \\
\text { per- } \\
\text { centile }\end{array}$ & $\begin{array}{c}\text { 2nd } \\
\text { per- } \\
\text { centile }\end{array}$ & $\begin{array}{c}\text { 10th } \\
\text { per- } \\
\text { centile }\end{array}$ & $\begin{array}{c}\text { 25th } \\
\text { per- } \\
\text { centile }\end{array}$ & $\begin{array}{c}\text { 50th } \\
\text { per- } \\
\text { centile } \\
\text { (median) }\end{array}$ & $\begin{array}{c}\text { 75th } \\
\text { per- } \\
\text { centile }\end{array}$ & $\begin{array}{c}\text { 90th } \\
\text { per- } \\
\text { centile }\end{array}$ & $\begin{array}{c}\text { 98th } \\
\text { per- } \\
\text { centile }\end{array}$ & $\begin{array}{c}\text { 99th } \\
\text { per- } \\
\text { centile }\end{array}$ \\
\hline 0199 & 0.02 & 0.03 & 0.06 & 0.10 & 0.20 & 0.40 & 0.80 & 1.67 & 1.97 & 4860 & 0.01 & 0.02 & 0.04 & 0.06 & 0.12 & 0.34 & 0.93 & 2.21 & 2.57 \\
\hline 0205 & .01 & .01 & .03 & .05 & .15 & .35 & .74 & 1.87 & 2.43 & 4862 & .10 & .10 & .10 & .10 & .20 & .40 & .90 & 1.90 & 2.31 \\
\hline 0208 & .02 & .03 & .04 & .08 & .16 & .34 & .60 & 1.00 & 1.81 & 5370 & .02 & .03 & .07 & .10 & .20 & .50 & 1.00 & 2.20 & 3.99 \\
\hline 0404 & .10 & .10 & .10 & .10 & .20 & .50 & .90 & 2.44 & 3.50 & 5651 & .01 & .02 & .05 & .06 & .18 & .40 & .72 & 1.83 & 2.54 \\
\hline 0407 & .10 & .10 & .10 & .10 & .20 & .40 & .80 & 1.53 & 2.09 & 5866 & .02 & .02 & .03 & .07 & .24 & .54 & 1.28 & 1.97 & 2.79 \\
\hline 0600 & .02 & .02 & .05 & .10 & .17 & .40 & .80 & 2.00 & 2.86 & 6138 & .02 & .02 & .03 & .07 & .17 & .39 & .79 & 1.64 & 2.25 \\
\hline 0606 & -- & .02 & .05 & .12 & .20 & .40 & .67 & 1.30 & -- & 6275 & .02 & .03 & .05 & .10 & .20 & .40 & .80 & 1.60 & 2.10 \\
\hline 0646 & .02 & .03 & .05 & .10 & .20 & .45 & .80 & 1.83 & 2.27 & 6435 & .02 & .02 & .05 & .10 & .17 & .40 & .70 & 1.76 & 2.20 \\
\hline 1120 & .03 & .03 & .09 & .10 & .22 & .50 & 1.00 & 1.90 & 2.39 & 6492 & .01 & .01 & .02 & .05 & .12 & .28 & .51 & 1.65 & 2.42 \\
\hline 1440 & .03 & .04 & .10 & .10 & .20 & .40 & .80 & 1.70 & 2.00 & 6619 & .02 & .02 & .05 & .10 & .20 & .44 & .93 & 1.96 & 2.30 \\
\hline 1446 & .02 & .02 & .05 & .09 & .21 & .54 & .98 & 2.05 & 2.93 & 6659 & .02 & .02 & .05 & .10 & .20 & .50 & 1.05 & 2.65 & 3.58 \\
\hline 1469 & .02 & .02 & .05 & .10 & .20 & .47 & .95 & 2.30 & 3.00 & 6797 & .01 & .01 & .03 & .06 & .15 & .30 & .60 & 1.62 & 1.91 \\
\hline 1475 & -- & .01 & .02 & .04 & .08 & .33 & 1.04 & 3.10 & -- & 6951 & .01 & .02 & .03 & .05 & .15 & .43 & .85 & 1.53 & 2.40 \\
\hline 1480 & -- & -- & -- & -- & .32 & -- & -- & -- & -- & 7094 & .02 & .03 & .05 & .10 & .18 & .40 & .70 & 1.40 & 1.70 \\
\hline 1515 & .02 & .02 & .04 & .10 & .15 & .39 & .67 & 1.30 & 1.64 & 7254 & .02 & .03 & .06 & .10 & .18 & .37 & .80 & 1.70 & 2.40 \\
\hline 1807 & .02 & .02 & .03 & .05 & .15 & .36 & .69 & 1.46 & 1.69 & 7277 & -- & -- & -- & -- & .23 & -- & -- & -- & -- \\
\hline 1840 & .10 & .10 & .10 & .10 & .20 & .40 & .90 & 1.95 & 3.35 & 7279 & .03 & .04 & .10 & .10 & .20 & .40 & .80 & 1.60 & 1.96 \\
\hline 1881 & -- & -- & -- & -- & .08 & -- & -- & -- & -- & 7283 & .01 & .01 & .01 & .03 & .09 & .28 & .62 & 1.50 & 1.88 \\
\hline 1887 & .01 & .01 & .02 & .04 & .12 & .33 & .72 & 1.89 & 2.44 & 7604 & -- & -- & -- & .01 & .23 & .45 & -- & -- & -- \\
\hline 1939 & .02 & .02 & .05 & .10 & .20 & .50 & 1.02 & 2.15 & 2.94 & 7605 & -- & -- & .10 & .10 & .20 & .60 & .90 & -- & -- \\
\hline 1950 & -- & -- & .07 & .15 & .30 & .66 & 1.19 & -- & -- & 7609 & .01 & .01 & .01 & .03 & .10 & .31 & .72 & 1.76 & 2.62 \\
\hline 1956 & -- & -- & .03 & .06 & .15 & .42 & .73 & -- & -- & 7610 & .01 & .01 & .02 & .10 & .16 & .40 & .90 & 2.30 & 2.97 \\
\hline 1961 & -- & -- & .04 & .07 & .20 & .54 & 1.16 & -- & -- & 7638 & .02 & .03 & .06 & .10 & .20 & .45 & .90 & 1.90 & 2.25 \\
\hline 1963 & .02 & .02 & .05 & .10 & .20 & .50 & 1.00 & 2.24 & 2.84 & 7649 & .01 & .02 & .04 & .10 & .20 & .48 & .90 & 1.71 & 2.18 \\
\hline 1982 & .10 & .10 & .10 & .10 & .20 & .40 & .70 & 1.40 & 1.84 & 7651 & .10 & .10 & .10 & .10 & .20 & .60 & 1.05 & 1.95 & 2.38 \\
\hline 2030 & .01 & .02 & .05 & .10 & .20 & .40 & .80 & 1.80 & 2.50 & 7735 & .02 & .02 & .05 & .10 & .22 & .51 & .99 & 1.88 & 2.45 \\
\hline 2139 & -- & .02 & .04 & .07 & .17 & .42 & .71 & 1.73 & -- & 7736 & .03 & .06 & .10 & .10 & .30 & .60 & 1.10 & 2.17 & 2.80 \\
\hline 2203 & .10 & .10 & .10 & .10 & .20 & .50 & 1.00 & 2.68 & 3.45 & 7738 & .01 & .02 & .05 & .10 & .24 & .59 & 1.28 & 2.35 & 2.46 \\
\hline 2207 & .03 & .04 & .10 & .10 & .20 & .50 & 1.02 & 1.93 & 2.89 & 8072 & .01 & .01 & .03 & .06 & .14 & .32 & .68 & 1.41 & 1.63 \\
\hline 2510 & .02 & .03 & .06 & .10 & .20 & .41 & .80 & 1.80 & 2.32 & 8078 & .01 & .01 & .02 & .05 & .12 & .25 & .51 & 1.35 & 2.02 \\
\hline 2625 & .01 & .02 & .03 & .06 & .14 & .35 & .80 & 1.79 & 2.76 & 8084 & -- & .01 & .05 & .08 & .15 & .29 & .60 & 1.55 & -- \\
\hline 2665 & .02 & .03 & .05 & .10 & .20 & .40 & .80 & 1.60 & 1.90 & 8085 & .02 & .02 & .05 & .10 & .16 & .35 & .69 & 1.46 & 1.69 \\
\hline 2694 & .02 & .02 & .03 & .07 & .20 & .49 & .85 & 1.53 & 3.33 & 8187 & .02 & .02 & .04 & .07 & .18 & .40 & .93 & 2.14 & 2.42 \\
\hline 2700 & .02 & .03 & .07 & .10 & .10 & .30 & .56 & 1.17 & 1.41 & 8358 & -- & .02 & .04 & .08 & .15 & .34 & .78 & 2.37 & -- \\
\hline 3145 & .02 & .02 & .04 & .06 & .15 & .35 & .78 & 1.80 & 2.24 & 8501 & .02 & .02 & .04 & .10 & .18 & .40 & .81 & 1.70 & 2.07 \\
\hline 3242 & -- & -- & .10 & .18 & .31 & .84 & 1.58 & -- & -- & 8518 & .02 & .02 & .04 & .07 & .12 & .32 & .65 & 1.41 & 1.74 \\
\hline 3288 & .01 & .02 & .04 & .08 & .20 & .46 & .92 & 1.78 & 2.40 & 8596 & .07 & .10 & .10 & .10 & .20 & .50 & .90 & 2.10 & 2.67 \\
\hline 3374 & .01 & .02 & .03 & .05 & .11 & .28 & .63 & 1.29 & 1.65 & 8788 & .02 & .02 & .05 & .10 & .22 & .50 & .93 & 1.59 & 1.92 \\
\hline 4026 & -- & -- & -- & -- & .07 & -- & -- & -- & -- & 8816 & -- & -- & -- & -- & .04 & -- & -- & -- & -- \\
\hline 4028 & .01 & .01 & .03 & .05 & .14 & .41 & .90 & 2.23 & 2.59 & 9148 & -- & -- & -- & .04 & .14 & .22 & -- & -- & -- \\
\hline 4030 & .10 & .10 & .10 & .10 & .20 & .50 & 1.10 & 1.69 & 2.91 & 9153 & .02 & .02 & .03 & .09 & .16 & .46 & 1.01 & 2.04 & 3.69 \\
\hline 4089 & .02 & .02 & .05 & .10 & .20 & .42 & .86 & 1.80 & 2.39 & 9156 & .01 & .02 & .07 & .10 & .20 & .50 & .99 & 1.94 & 2.50 \\
\hline 4112 & .03 & .05 & .10 & .10 & .20 & .50 & .94 & 2.05 & 3.06 & 9193 & .06 & .10 & .10 & .10 & .20 & .40 & .70 & 1.60 & 2.00 \\
\hline 4306 & .01 & .02 & .03 & .05 & .12 & .33 & .69 & 1.72 & 1.97 & 9265 & .02 & .02 & .10 & .10 & .20 & .40 & .90 & 2.80 & 3.68 \\
\hline 4850 & .01 & .02 & .04 & .10 & .16 & .39 & .78 & 1.82 & 2.30 & 9569 & .10 & .10 & .10 & .10 & .20 & .50 & 1.00 & 2.00 & 2.40 \\
\hline 4856 & .01 & .02 & .03 & .05 & .13 & .40 & .75 & 1.92 & 2.40 & 9686 & .02 & .02 & .04 & .09 & .13 & .35 & .61 & 1.30 & 1.60 \\
\hline
\end{tabular}


Appendix 2-4.5. Empirical distribution of storm depth defined by 24-hour minimum interevent time for hourly rainfall stations in eastern New Mexico.

$[--$, not available $]$

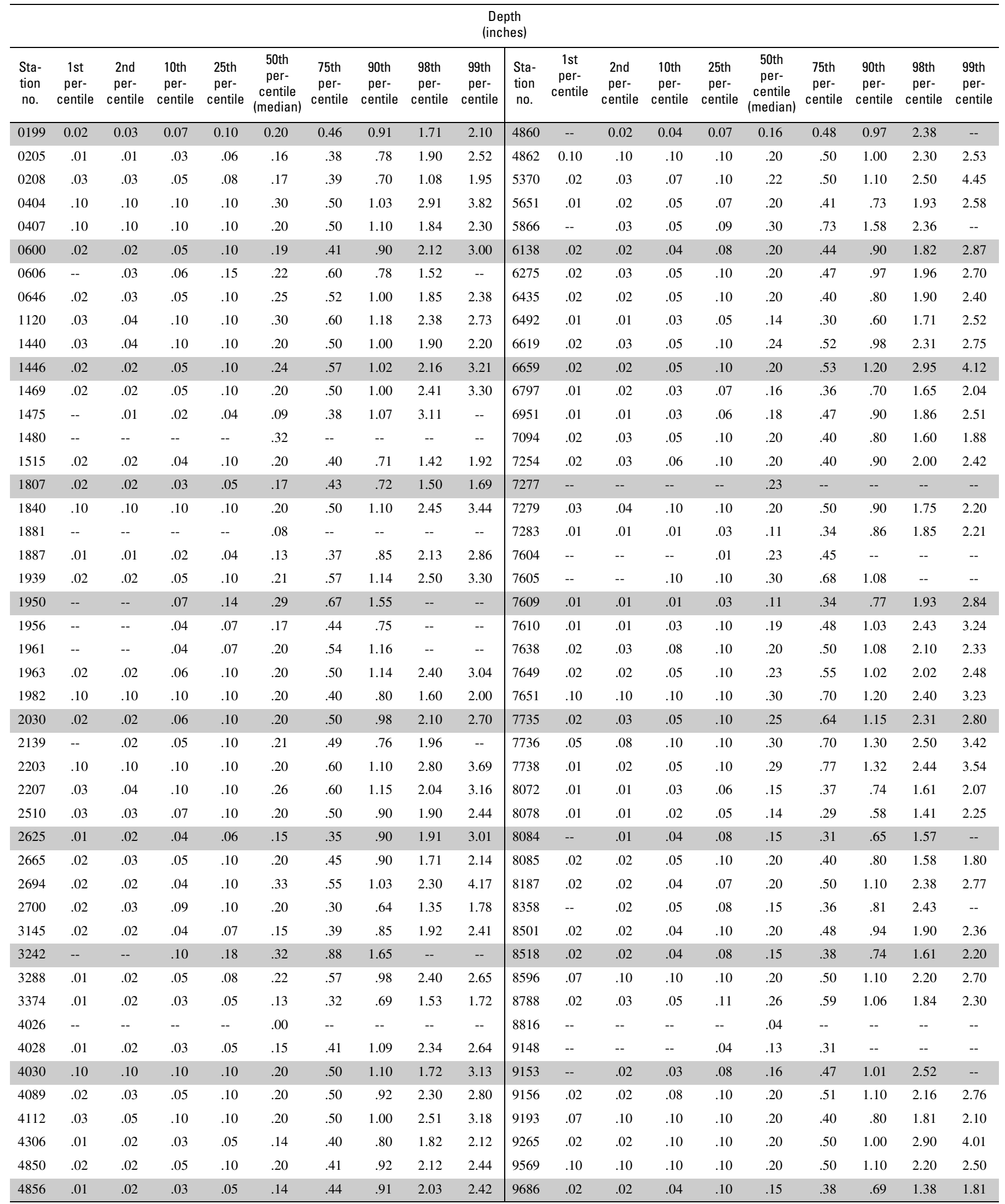


Appendix 2-4.6. Empirical distribution of storm depth defined by 48-hour minimum interevent time for hourly rainfall stations in eastern New Mexico.

$[--$, not available]

\begin{tabular}{|c|c|c|c|c|c|c|c|c|c|c|c|c|c|c|c|c|c|c|c|}
\hline \multicolumn{20}{|c|}{$\begin{array}{l}\text { Depth } \\
\text { (inches) }\end{array}$} \\
\hline $\begin{array}{l}\text { Sta- } \\
\text { tion } \\
\text { no. }\end{array}$ & $\begin{array}{c}1 \text { st } \\
\text { per- } \\
\text { centile }\end{array}$ & $\begin{array}{c}\text { 2nd } \\
\text { per- } \\
\text { centile }\end{array}$ & $\begin{array}{c}\text { 10th } \\
\text { per- } \\
\text { centile }\end{array}$ & $\begin{array}{c}25 \text { th } \\
\text { per- } \\
\text { centile }\end{array}$ & $\begin{array}{c}\text { 50th } \\
\text { per- } \\
\text { centile } \\
\text { (median) }\end{array}$ & $\begin{array}{c}\text { 75th } \\
\text { per- } \\
\text { centile }\end{array}$ & $\begin{array}{c}\text { 90th } \\
\text { per- } \\
\text { centile }\end{array}$ & $\begin{array}{c}\text { 98th } \\
\text { per- } \\
\text { centile }\end{array}$ & $\begin{array}{c}\text { 99th } \\
\text { per- } \\
\text { centile }\end{array}$ & $\begin{array}{l}\text { Sta- } \\
\text { tion } \\
\text { no. }\end{array}$ & $\begin{array}{c}\text { 1st } \\
\text { per- } \\
\text { centile }\end{array}$ & $\begin{array}{c}\text { 2nd } \\
\text { per- } \\
\text { centile }\end{array}$ & $\begin{array}{c}10 \text { th } \\
\text { per- } \\
\text { centile }\end{array}$ & $\begin{array}{c}25 \text { th } \\
\text { per- } \\
\text { centile }\end{array}$ & $\begin{array}{c}\text { 50th } \\
\text { per- } \\
\text { centile } \\
\text { (median) }\end{array}$ & $\begin{array}{c}\text { 75th } \\
\text { per- } \\
\text { centile }\end{array}$ & $\begin{array}{c}\text { 90th } \\
\text { per- } \\
\text { centile }\end{array}$ & $\begin{array}{c}\text { 98th } \\
\text { per- } \\
\text { centile }\end{array}$ & $\begin{array}{c}\text { 99th } \\
\text { per- } \\
\text { centile }\end{array}$ \\
\hline 0199 & 0.02 & 0.03 & 0.07 & 0.10 & 0.26 & 0.52 & 1.10 & 2.10 & 2.48 & 4860 & -- & 0.02 & 0.04 & 0.07 & 0.21 & 0.56 & 1.29 & 3.99 & -- \\
\hline 0205 & .01 & .01 & .03 & .07 & .19 & .46 & .98 & 2.25 & 3.09 & 4862 & 0.10 & .10 & .10 & .10 & .30 & .70 & 1.50 & 2.50 & 3.01 \\
\hline 0208 & .02 & .03 & .05 & .09 & .20 & .44 & .78 & 1.89 & 2.10 & 5370 & .02 & .03 & .08 & .10 & .29 & .60 & 1.20 & 3.27 & 4.88 \\
\hline 0404 & .10 & .10 & .10 & .20 & .35 & .72 & 1.55 & 3.43 & 4.41 & 5651 & .01 & .02 & .05 & .07 & .20 & .41 & .87 & 2.28 & 2.79 \\
\hline 0407 & .10 & .10 & .10 & .10 & .40 & .80 & 1.41 & 2.31 & 3.10 & 5866 & -- & .03 & .07 & .15 & .43 & 1.01 & 2.12 & 3.41 & -- \\
\hline 0600 & .02 & .02 & .06 & .10 & .20 & .50 & 1.00 & 2.37 & 3.40 & 6138 & .02 & .02 & .05 & .10 & .25 & .56 & 1.18 & 2.41 & 3.42 \\
\hline 0606 & -- & -- & .08 & .16 & .30 & .68 & 1.25 & -- & -- & 6275 & .02 & .03 & .06 & .10 & .26 & .60 & 1.24 & 2.90 & 3.51 \\
\hline 0646 & .02 & .03 & .05 & .10 & .30 & .64 & 1.20 & 2.18 & 2.61 & 6435 & .02 & .02 & .05 & .10 & .20 & .50 & .97 & 2.13 & 2.92 \\
\hline 1120 & .03 & .05 & .10 & .16 & .40 & .88 & 1.60 & 3.20 & 4.02 & 6492 & .01 & .01 & .03 & .06 & .16 & .35 & .63 & 1.91 & 3.64 \\
\hline 1440 & .04 & .05 & .10 & .10 & .30 & .60 & 1.30 & 2.40 & 3.02 & 6619 & .02 & .03 & .05 & .11 & .30 & .63 & 1.23 & 2.46 & 3.29 \\
\hline 1446 & .02 & .02 & .05 & .10 & .26 & .65 & 1.37 & 2.62 & 3.83 & 6659 & .02 & .02 & .05 & .10 & .25 & .65 & 1.39 & 3.39 & 5.07 \\
\hline 1469 & .02 & .02 & .05 & .10 & .24 & .60 & 1.20 & 2.86 & 3.62 & 6797 & .01 & .02 & .05 & .09 & .20 & .44 & 1.07 & 1.79 & 2.59 \\
\hline 1475 & -- & -- & .02 & .04 & .09 & .38 & 1.12 & -- & -- & 6951 & .01 & .02 & .03 & .07 & .20 & .52 & 1.11 & 2.33 & 2.94 \\
\hline 1480 & -- & -- & -- & -- & .32 & -- & -- & -- & -- & 7094 & .02 & .03 & .06 & .10 & .20 & .50 & 1.02 & 1.90 & 2.33 \\
\hline 1515 & .02 & .02 & .05 & .10 & .20 & .50 & .91 & 1.91 & 2.25 & 7254 & .02 & .03 & .07 & .10 & .20 & .50 & 1.08 & 2.40 & 3.00 \\
\hline 1807 & .02 & .02 & .03 & .05 & .18 & .47 & .83 & 1.60 & 1.70 & 7277 & -- & -- & -- & -- & .23 & -- & -- & -- & -- \\
\hline 1840 & .10 & .10 & .10 & .10 & .30 & .60 & 1.30 & 3.04 & 3.98 & 7279 & .03 & .05 & .10 & .10 & .28 & .60 & 1.10 & 2.30 & 2.90 \\
\hline 1881 & -- & -- & -- & -- & .44 & -- & -- & -- & -- & 7283 & .01 & .01 & .02 & .04 & .15 & .49 & 1.13 & 2.45 & 2.97 \\
\hline 1887 & .01 & .01 & .02 & .05 & .17 & .50 & 1.15 & 2.69 & 3.31 & 7604 & -- & -- & -- & -- & .43 & -- & -- & -- & -- \\
\hline 1939 & .02 & .02 & .06 & .10 & .28 & .70 & 1.40 & 3.01 & 3.79 & 7605 & -- & -- & .10 & .10 & .35 & .75 & 1.22 & -- & -- \\
\hline 1950 & -- & -- & .06 & .17 & .30 & .90 & 1.72 & -- & -- & 7609 & .01 & .01 & .02 & .04 & .15 & .41 & .99 & 2.26 & 2.95 \\
\hline 1956 & -- & -- & .04 & .07 & .24 & .46 & 1.21 & -- & -- & 7610 & .01 & .01 & .03 & .10 & .20 & .58 & 1.20 & 2.80 & 3.82 \\
\hline 1961 & -- & -- & .05 & .09 & .29 & .70 & 1.24 & -- & -- & 7638 & .03 & .04 & .09 & .10 & .30 & .66 & 1.30 & 2.40 & 2.90 \\
\hline 1963 & .02 & .02 & .07 & .10 & .27 & .62 & 1.40 & 2.81 & 3.76 & 7649 & .02 & .02 & .05 & .10 & .30 & .78 & 1.40 & 2.71 & 3.38 \\
\hline 1982 & .10 & .10 & .10 & .10 & .20 & .50 & .90 & 2.00 & 2.50 & 7651 & .10 & .10 & .10 & .10 & .40 & .90 & 1.70 & 3.98 & 4.20 \\
\hline 2030 & .02 & .02 & .07 & .10 & .20 & .60 & 1.20 & 2.60 & 3.02 & 7735 & .02 & .03 & .06 & .12 & .30 & .75 & 1.57 & 2.94 & 3.65 \\
\hline 2139 & -- & .02 & .05 & .13 & .27 & .62 & 1.45 & 2.47 & -- & 7736 & .05 & .08 & .10 & .20 & .40 & .90 & 1.86 & 3.74 & 4.42 \\
\hline 2203 & .10 & .10 & .10 & .10 & .30 & .70 & 1.49 & 3.20 & 4.24 & 7738 & .01 & .02 & .05 & .11 & .36 & .82 & 1.67 & 2.49 & 3.76 \\
\hline 2207 & .04 & .05 & .10 & .10 & .30 & .70 & 1.41 & 2.91 & 3.53 & 8072 & .01 & .02 & .04 & .08 & .21 & .49 & .93 & 1.86 & 2.43 \\
\hline 2510 & .03 & .03 & .08 & .10 & .24 & .60 & 1.10 & 2.36 & 3.07 & 8078 & .01 & .01 & .03 & .06 & .16 & .35 & .66 & 1.98 & 2.57 \\
\hline 2625 & .02 & .02 & .04 & .07 & .18 & .47 & 1.00 & 2.19 & 3.46 & 8084 & -- & -- & .05 & .09 & .20 & .39 & .72 & -- & -- \\
\hline 2665 & .02 & .03 & .06 & .10 & .25 & .57 & 1.10 & 2.16 & 2.60 & 8085 & .02 & .02 & .07 & .10 & .21 & .50 & 1.00 & 1.81 & 2.11 \\
\hline 2694 & -- & .03 & .06 & .17 & .46 & .89 & 1.61 & 3.64 & -- & 8187 & .02 & .02 & .04 & .10 & .26 & .58 & 1.45 & 2.95 & 3.20 \\
\hline 2700 & .03 & .04 & .10 & .10 & .20 & .49 & .90 & 1.90 & 2.27 & 8358 & -- & .03 & .05 & .10 & .19 & .38 & .92 & 2.84 & -- \\
\hline 3145 & .02 & .02 & .04 & .07 & .19 & .43 & 1.00 & 2.40 & 2.70 & 8501 & .02 & .02 & .05 & .10 & .24 & .60 & 1.19 & 2.30 & 2.97 \\
\hline 3242 & -- & -- & .10 & .17 & .35 & .93 & 1.97 & -- & -- & 8518 & .02 & .02 & .04 & .10 & .20 & .45 & .90 & 2.01 & 2.57 \\
\hline 3288 & -- & .02 & .05 & .11 & .25 & .60 & 1.35 & 2.55 & -- & 8596 & .07 & .10 & .10 & .10 & .30 & .65 & 1.30 & 2.69 & 3.19 \\
\hline 3374 & .01 & .02 & .03 & .06 & .16 & .42 & .82 & 1.72 & 2.06 & 8788 & .02 & .03 & .08 & .15 & .35 & .75 & 1.35 & 2.30 & 2.83 \\
\hline 4026 & -- & -- & -- & -- & .00 & -- & -- & -- & -- & 8816 & -- & -- & -- & -- & .04 & -- & -- & -- & -- \\
\hline 4028 & .01 & .01 & .03 & .05 & .17 & .46 & 1.36 & 2.64 & 2.92 & 9148 & -- & -- & -- & -- & .13 & -- & -- & -- & -- \\
\hline 4030 & .10 & .10 & .10 & .10 & .30 & .70 & 1.43 & 2.20 & 3.71 & 9153 & -- & .02 & .03 & .09 & .19 & .73 & 1.76 & 3.72 & -- \\
\hline 4089 & .02 & .03 & .05 & .10 & .20 & .57 & 1.20 & 2.79 & 3.70 & 9156 & .02 & .02 & .09 & .10 & .26 & .63 & 1.30 & 2.60 & 3.13 \\
\hline 4112 & .04 & .05 & .10 & .10 & .30 & .60 & 1.20 & 3.01 & 3.77 & 9193 & .07 & .10 & .10 & .10 & .26 & .50 & 1.10 & 2.09 & 2.62 \\
\hline 4306 & .01 & .02 & .03 & .05 & .18 & .50 & .97 & 1.91 & 2.52 & 9265 & .02 & .03 & .10 & .10 & .20 & .60 & 1.20 & 3.19 & 5.77 \\
\hline 4850 & .02 & .02 & .05 & .10 & .24 & .51 & 1.16 & 2.38 & 2.66 & 9569 & .10 & .10 & .10 & .10 & .30 & .60 & 1.22 & 2.50 & 2.75 \\
\hline 4856 & .01 & .02 & .03 & .06 & .19 & .47 & 1.08 & 2.34 & 2.47 & 9686 & .02 & .02 & .04 & .10 & .20 & .43 & .84 & 1.70 & 2.10 \\
\hline
\end{tabular}


Appendix 2-4.7. Empirical distribution of storm depth defined by 72-hour minimum interevent time for hourly rainfall stations in eastern New Mexico.

$[--$, not available $]$

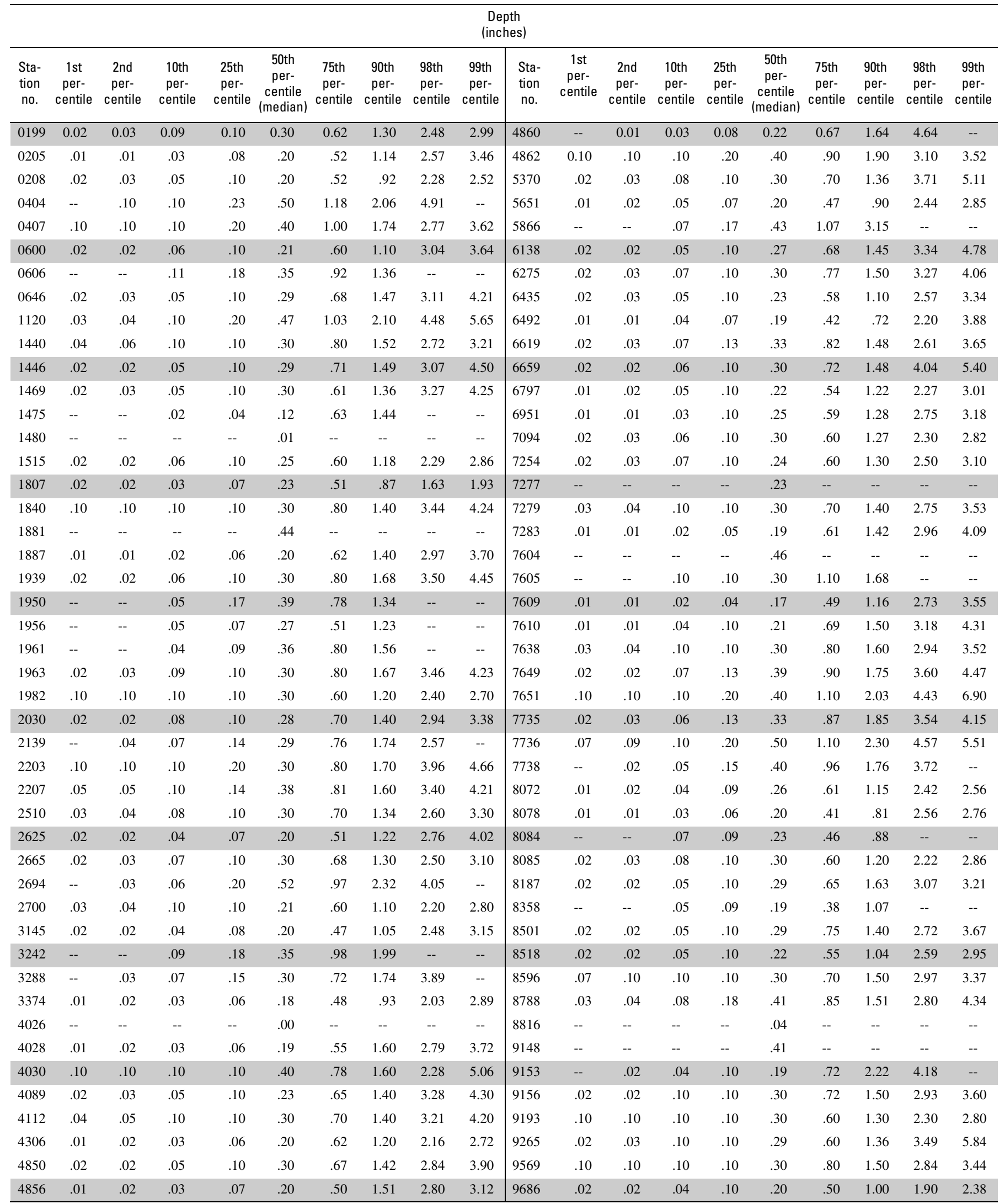


Appendix 2-5.1. Empirical distribution of storm duration defined by 6-hour minimum interevent time for hourly rainfall stations in eastern New Mexico.

$[--$, not available $]$

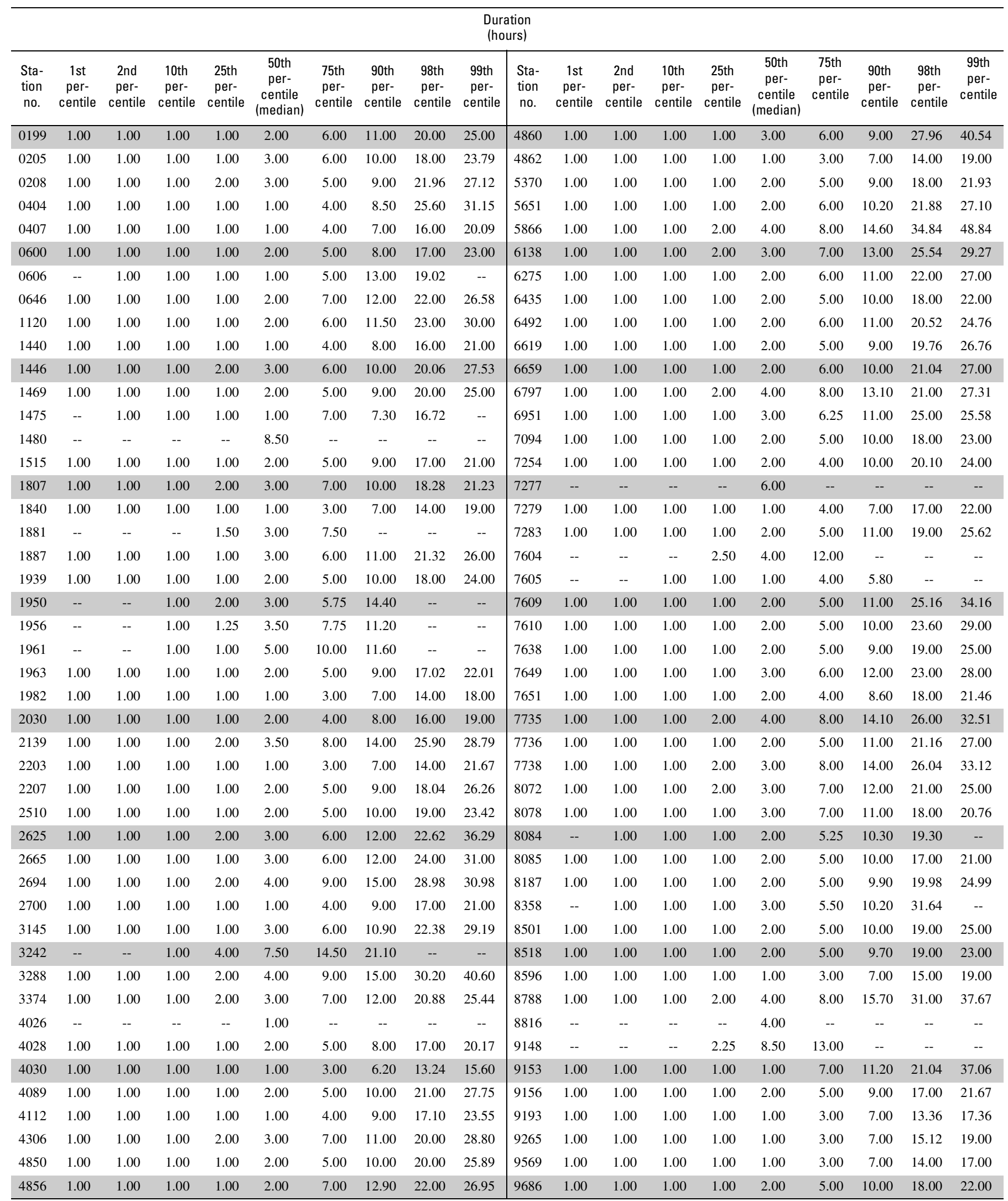


Appendix 2-5.2. Empirical distribution of storm duration defined by 8-hour minimum interevent time for hourly rainfall stations in eastern New Mexico.

[--, not available]

\begin{tabular}{|c|c|c|c|c|c|c|c|c|c|c|c|c|c|c|c|c|c|c|c|}
\hline \multicolumn{20}{|c|}{$\begin{array}{c}\begin{array}{c}\text { Duration } \\
\text { (hours) }\end{array} \\
\end{array}$} \\
\hline $\begin{array}{l}\text { Sta- } \\
\text { tion } \\
\text { no. }\end{array}$ & $\begin{array}{c}1 \text { st } \\
\text { per- } \\
\text { centile }\end{array}$ & $\begin{array}{c}\text { 2nd } \\
\text { per- } \\
\text { centile }\end{array}$ & $\begin{array}{c}10 \text { th } \\
\text { per- } \\
\text { centile }\end{array}$ & $\begin{array}{c}25 \text { th } \\
\text { per- } \\
\text { centile }\end{array}$ & $\begin{array}{c}\text { 50th } \\
\text { per- } \\
\text { centile } \\
\text { (median) }\end{array}$ & $\begin{array}{c}\text { 75th } \\
\text { per- } \\
\text { centile }\end{array}$ & $\begin{array}{c}\text { 90th } \\
\text { per- } \\
\text { centile }\end{array}$ & $\begin{array}{c}\text { 98th } \\
\text { per- } \\
\text { centile }\end{array}$ & $\begin{array}{c}\text { 99th } \\
\text { per- } \\
\text { centile }\end{array}$ & $\begin{array}{l}\text { Sta- } \\
\text { tion } \\
\text { no. }\end{array}$ & $\begin{array}{c}1 \text { st } \\
\text { per- } \\
\text { centile }\end{array}$ & $\begin{array}{c}\text { 2nd } \\
\text { per- } \\
\text { centile }\end{array}$ & $\begin{array}{c}\text { 10th } \\
\text { per- } \\
\text { centile }\end{array}$ & $\begin{array}{c}\text { 25th } \\
\text { per- } \\
\text { centile }\end{array}$ & $\begin{array}{c}\text { 50th } \\
\text { per- } \\
\text { centile } \\
\text { (median) }\end{array}$ & $\begin{array}{c}\text { 75th } \\
\text { per- } \\
\text { centile }\end{array}$ & $\begin{array}{c}90 \text { th } \\
\text { per- } \\
\text { centile }\end{array}$ & $\begin{array}{c}\text { 98th } \\
\text { per- } \\
\text { centile }\end{array}$ & $\begin{array}{r}\text { 99th } \\
\text { per- } \\
\text { centile }\end{array}$ \\
\hline 0199 & 1.00 & 1.00 & 1.00 & 1.00 & 2.00 & 7.00 & 12.00 & 26.00 & 31.00 & 4860 & 1.00 & 1.00 & 1.00 & 1.00 & 3.00 & 6.00 & 10.20 & 32.64 & 44.10 \\
\hline 0205 & 1.00 & 1.00 & 1.00 & 1.00 & 3.00 & 6.00 & 12.00 & 23.00 & 28.21 & 4862 & 1.00 & 1.00 & 1.00 & 1.00 & 1.00 & 4.00 & 9.00 & 18.00 & 21.00 \\
\hline 0407 & 1.00 & 1.00 & 1.00 & 1.00 & 1.00 & 4.00 & 10.00 & 22.94 & 29.88 & 5866 & 1.00 & 1.00 & 1.00 & 2.00 & 4.00 & 9.00 & 16.00 & 42.86 & 58.56 \\
\hline 0600 & 1.00 & 1.00 & 1.00 & 1.00 & 2.00 & 5.00 & 10.00 & 21.00 & 26.91 & 6138 & 1.00 & 1.00 & 1.00 & 2.00 & 4.00 & 8.25 & 15.00 & 28.10 & 36.00 \\
\hline 0606 & -- & 1.00 & 1.00 & 1.00 & 1.00 & 5.00 & 13.90 & 19.14 & -- & 6275 & 1.00 & 1.00 & 1.00 & 1.00 & 3.00 & 7.00 & 13.00 & 26.00 & 33.00 \\
\hline 0646 & 1.00 & 1.00 & 1.00 & 1.00 & 3.00 & 8.00 & 14.00 & 26.84 & 32.92 & 6435 & 1.00 & 1.00 & 1.00 & 1.00 & 2.00 & 6.00 & 11.00 & 20.00 & 29.00 \\
\hline 1120 & 1.00 & 1.00 & 1.00 & 1.00 & 3.00 & 7.00 & 14.00 & 29.00 & 37.00 & 6492 & 1.00 & 1.00 & 1.00 & 1.00 & 2.00 & 7.00 & 13.00 & 26.64 & 32.00 \\
\hline 1475 & -- & 1.00 & 1.00 & 1.00 & 1.00 & 7.00 & 7.30 & 16.72 & -- & 6951 & 1.00 & 1.00 & 1.00 & 1.00 & 3.00 & 7.00 & 12.30 & 26.72 & 35.01 \\
\hline 1480 & -- & -- & -- & -- & 12.00 & -- & -- & -- & -- & 7094 & 1.00 & 1.00 & 1.00 & 1.00 & 2.00 & 6.00 & 12.00 & 23.00 & 27.00 \\
\hline 1515 & 1.00 & 1.00 & 1.00 & 1.00 & 2.00 & 6.00 & 11.00 & 21.00 & 26.00 & 7254 & 1.00 & 1.00 & 1.00 & 1.00 & 2.00 & 5.00 & 11.00 & 23.00 & 27.00 \\
\hline 1807 & 1.00 & 1.00 & 1.00 & 2.00 & 3.00 & 7.00 & 10.30 & 19.86 & 23.00 & 7277 & -- & -- & -- & -- & 6.00 & -- & -- & -- & -- \\
\hline 1840 & 1.00 & 1.00 & 1.00 & 1.00 & 1.00 & 3.00 & 9.00 & 19.96 & 26.92 & 7279 & 1.00 & 1.00 & 1.00 & 1.00 & 1.00 & 4.00 & 9.00 & 21.00 & 27.00 \\
\hline 1881 & -- & -- & -- & 3.50 & 7.00 & 9.75 & -- & -- & -- & 7283 & 1.00 & 1.00 & 1.00 & 1.00 & 2.00 & 6.00 & 12.00 & 26.00 & 32.35 \\
\hline 1887 & 1.00 & 1.00 & 1.00 & 1.00 & 3.00 & & 12.00 & 24.28 & 30.64 & 7604 & -- & -- & -- & & & 12.00 & -- & -- & -- \\
\hline 1939 & 1.00 & 1.00 & 1.00 & 1.00 & 2.00 & 6.00 & 11.00 & 22.00 & 30.00 & 7605 & -- & -- & 1.00 & 1.00 & 1.00 & 4.25 & 6.80 & -- & -- \\
\hline 1950 & -- & -- & 1.10 & 2.00 & 3.00 & 8.50 & 18.40 & -- & -- & 7609 & 1.00 & 1.00 & 1.00 & 1.00 & 2.00 & 6.00 & 13.00 & 29.38 & 40.00 \\
\hline 1956 & -- & -- & 1.00 & 1.50 & 4.00 & 8.00 & 11.00 & -- & -- & 7610 & 1.00 & 1.00 & 1.00 & 1.00 & 2.00 & 6.00 & 12.00 & 26.00 & 33.00 \\
\hline 2510 & 1.00 & 1.00 & 1.00 & 1.00 & 2.00 & 6.00 & 12.00 & 24.00 & 33.00 & 8078 & 1.00 & 1.00 & 1.00 & 1.00 & 3.00 & 7.00 & 13.00 & 21.14 & 24.28 \\
\hline 2625 & 1.00 & 1.00 & 1.00 & 2.00 & 3.00 & 6.00 & 14.00 & 29.16 & 40.16 & 8084 & -- & 1.00 & 1.00 & 1.00 & 3.00 & 6.00 & 13.80 & 19.60 & -- \\
\hline 2665 & 1.00 & 1.00 & 1.00 & 1.00 & 3.00 & 7.00 & 14.00 & 28.12 & 36.06 & 8085 & 1.00 & 1.00 & 1.00 & 1.00 & 2.00 & 7.00 & 13.00 & 23.00 & 29.00 \\
\hline 2694 & 1.00 & 1.00 & 1.00 & 2.00 & 5.00 & 11.00 & 20.00 & 34.50 & 57.25 & 8187 & 1.00 & 1.00 & 1.00 & 1.00 & 2.00 & 5.00 & 12.00 & 22.88 & 27.44 \\
\hline 2700 & 1.00 & 1.00 & 1.00 & 1.00 & 1.00 & 5.00 & 10.00 & 21.00 & 27.00 & 8358 & -- & 1.00 & 1.00 & 1.00 & 3.00 & 6.00 & 12.90 & 35.58 & -- \\
\hline 3145 & 1.00 & 1.00 & 1.00 & 1.00 & 3.00 & 7.00 & 13.00 & 27.92 & 39.73 & 8501 & 1.00 & 1.00 & 1.00 & 1.00 & 2.00 & 6.00 & 12.00 & 23.00 & 29.00 \\
\hline 3242 & -- & -- & 1.00 & 4.00 & 8.00 & 15.00 & 21.20 & -- & -- & 8518 & 1.00 & 1.00 & 1.00 & 1.00 & 2.00 & 6.00 & 11.30 & 22.00 & 27.63 \\
\hline 3288 & 1.00 & 1.00 & 1.00 & 2.00 & 4.50 & 10.25 & 17.10 & 31.92 & 40.82 & 8596 & 1.00 & 1.00 & 1.00 & 1.00 & 1.00 & 4.00 & 8.00 & 19.00 & 26.27 \\
\hline 3374 & 1.00 & 1.00 & 1.00 & 2.00 & 3.00 & 8.00 & 13.00 & 25.00 & 29.10 & 8788 & 1.00 & 1.00 & 1.00 & 2.00 & 4.00 & 9.00 & 18.00 & 36.38 & 40.00 \\
\hline 4026 & -- & -- & -- & -- & 1.00 & -- & -- & -- & -- & 8816 & -- & -- & -- & -- & 4.00 & -- & -- & -- & -- \\
\hline 4028 & 1.00 & 1.00 & 1.00 & 1.00 & 2.00 & 6.00 & 9.00 & 19.04 & 22.51 & 9148 & -- & -- & -- & 1.00 & 9.00 & 17.00 & -- & -- & -- \\
\hline 4030 & 1.00 & 1.00 & 1.00 & 1.00 & 1.00 & 3.00 & 8.00 & 14.40 & 16.00 & 9153 & 1.00 & 1.00 & 1.00 & 1.00 & 1.00 & 7.00 & 13.00 & 21.16 & 37.24 \\
\hline 4089 & 1.00 & 1.00 & 1.00 & 1.00 & 2.00 & 6.00 & 12.00 & 27.00 & 31.00 & 9156 & 1.00 & 1.00 & 1.00 & 1.00 & 2.00 & 5.00 & 11.00 & 21.00 & 26.67 \\
\hline 4112 & 1.00 & 1.00 & 1.00 & 1.00 & 1.00 & 5.00 & 10.00 & 22.00 & 28.00 & 9193 & 1.00 & 1.00 & 1.00 & 1.00 & 1.00 & 4.00 & 9.00 & 19.00 & 22.00 \\
\hline 4306 & 1.00 & 1.00 & 1.00 & 2.00 & 3.00 & 7.00 & 11.00 & 24.96 & 30.96 & 9265 & 1.00 & 1.00 & 1.00 & 1.00 & 1.00 & 4.00 & 9.00 & 20.84 & 25.84 \\
\hline 4850 & 1.00 & 1.00 & 1.00 & 1.00 & 2.00 & 6.00 & 12.00 & 24.00 & 31.64 & 9569 & 1.00 & 1.00 & 1.00 & 1.00 & 1.00 & 4.00 & 8.00 & 17.00 & 19.30 \\
\hline 4856 & 1.00 & 1.00 & 1.00 & 1.00 & 3.00 & 8.00 & 13.00 & 24.00 & 29.75 & 9686 & 1.00 & 1.00 & 1.00 & 1.00 & 2.00 & 6.00 & 11.00 & 21.00 & 26.00 \\
\hline
\end{tabular}


Appendix 2-5.3. Empirical distribution of storm duration defined by 12-hour minimum interevent time for hourly rainfall stations in eastern New Mexico.

$[--$, not available $]$

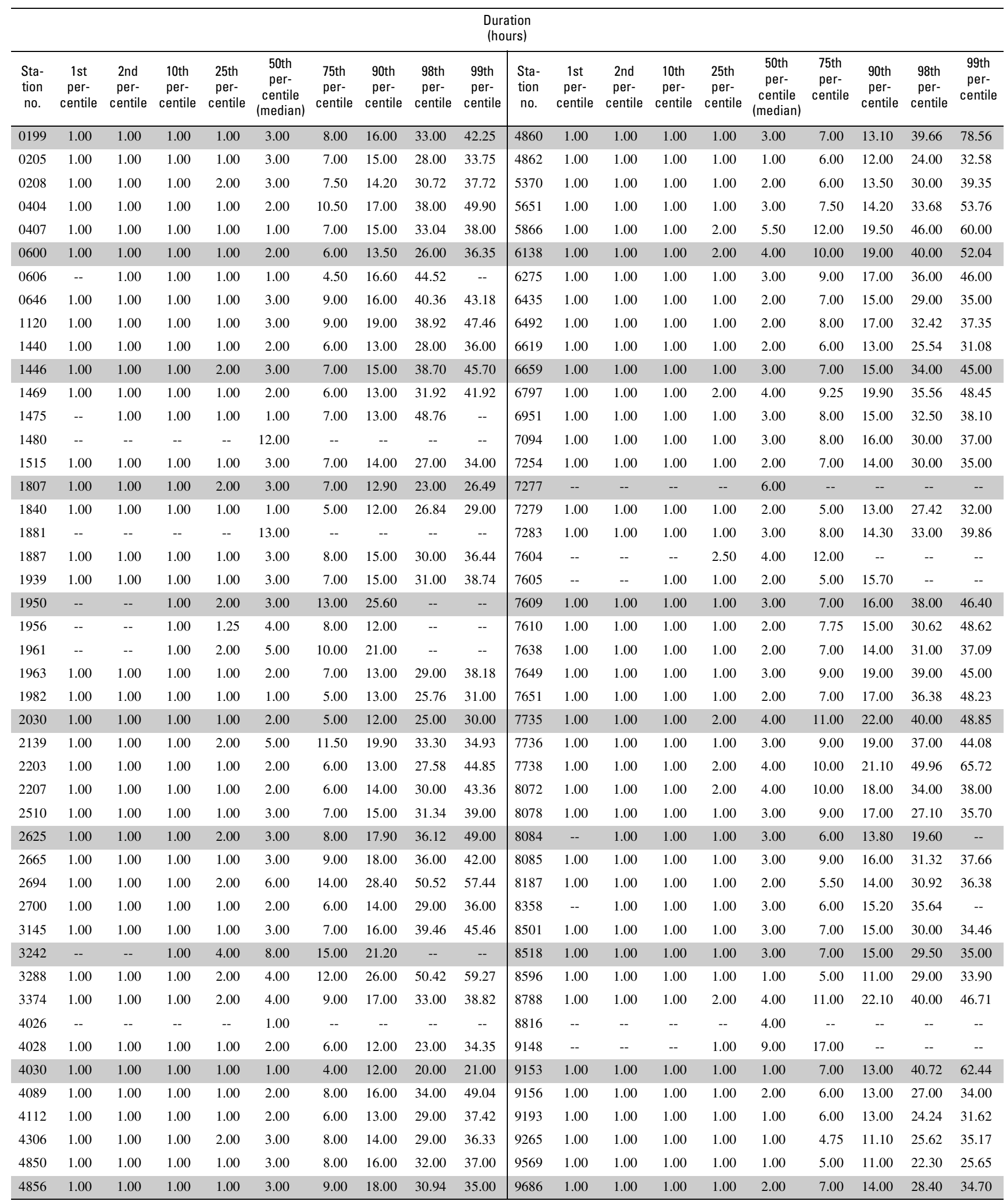


Appendix 2-5.4. Empirical distribution of storm duration defined by 18-hour minimum interevent time for hourly rainfall stations in eastern New Mexico.

[--, not available]

\begin{tabular}{|c|c|c|c|c|c|c|c|c|c|c|c|c|c|c|c|c|c|c|c|}
\hline \\
\hline $\begin{array}{l}\text { Sta- } \\
\text { tion } \\
\text { no. }\end{array}$ & $\begin{array}{c}1 \text { st } \\
\text { per- } \\
\text { centile }\end{array}$ & $\begin{array}{c}\text { 2nd } \\
\text { per- } \\
\text { centile }\end{array}$ & $\begin{array}{c}10 \text { th } \\
\text { per- } \\
\text { centile }\end{array}$ & $\begin{array}{c}25 \text { th } \\
\text { per- } \\
\text { centile }\end{array}$ & $\begin{array}{c}\text { 50th } \\
\text { per- } \\
\text { centile } \\
\text { (median) }\end{array}$ & $\begin{array}{c}75 \text { th } \\
\text { per- } \\
\text { centile }\end{array}$ & $\begin{array}{c}\text { 90th } \\
\text { per- } \\
\text { centile }\end{array}$ & $\begin{array}{c}\text { 98th } \\
\text { per- } \\
\text { centile }\end{array}$ & $\begin{array}{c}\text { 99th } \\
\text { per- } \\
\text { centile }\end{array}$ & $\begin{array}{l}\text { Sta- } \\
\text { tion } \\
\text { no. }\end{array}$ & $\begin{array}{c}1 \text { st } \\
\text { per- } \\
\text { centile }\end{array}$ & $\begin{array}{c}\text { 2nd } \\
\text { per- } \\
\text { centile }\end{array}$ & $\begin{array}{c}10 \text { th } \\
\text { per- } \\
\text { centile }\end{array}$ & $\begin{array}{l}\text { 25th } \\
\text { per- } \\
\text { centile }\end{array}$ & $\begin{array}{c}\text { 50th } \\
\text { per- } \\
\text { centile } \\
\text { (median) }\end{array}$ & $\begin{array}{c}\text { 75th } \\
\text { per- } \\
\text { centile }\end{array}$ & $\begin{array}{c}90 \text { th } \\
\text { per- } \\
\text { centile }\end{array}$ & $\begin{array}{c}\text { 98th } \\
\text { per- } \\
\text { centile }\end{array}$ & $\begin{array}{c}\text { 99th } \\
\text { per- } \\
\text { centile }\end{array}$ \\
\hline 0199 & 1.00 & 1.00 & 1.00 & 1.00 & 3.00 & 11.00 & 22.00 & 45.00 & 59.72 & 4860 & 1.00 & 1.00 & 1.00 & 1.00 & 4.00 & 9.00 & 23.60 & 65.60 & 85.92 \\
\hline 0205 & 1.00 & 1.00 & 1.00 & 1.00 & 3.00 & 9.00 & 19.00 & 39.20 & 50.30 & 4862 & 1.00 & 1.00 & 1.00 & 1.00 & 2.00 & 9.00 & 21.80 & 42.00 & 49.16 \\
\hline 0407 & 1.00 & 1.00 & 1.00 & 1.00 & 2.00 & 15.00 & 28.50 & 49.90 & 62.80 & 5866 & 1.00 & 1.00 & 1.00 & 2.00 & 5.00 & 13.50 & 26.20 & 81.56 & 91.44 \\
\hline 0600 & 1.00 & 1.00 & 1.00 & 1.00 & 2.00 & 9.00 & 19.00 & 36.00 & 42.00 & 6138 & 1.00 & 1.00 & 1.00 & 2.00 & 4.00 & 14.00 & 27.00 & 52.98 & 59.97 \\
\hline 0606 & -- & 1.00 & 1.00 & 1.00 & 1.00 & 7.00 & 19.40 & 44.56 & -- & 6275 & 1.00 & 1.00 & 1.00 & 2.00 & 4.00 & 14.00 & 26.00 & 50.00 & 58.00 \\
\hline 0646 & 1.00 & 1.00 & 1.00 & 1.00 & 3.00 & 10.00 & 26.00 & 49.40 & 54.60 & 6435 & 1.00 & 1.00 & 1.00 & 1.00 & 3.00 & 10.00 & 20.00 & 37.00 & 47.06 \\
\hline 1120 & 1.00 & 1.00 & 1.00 & 1.00 & 4.00 & 15.00 & 28.00 & 54.98 & 68.00 & 6492 & 1.00 & 1.00 & 1.00 & 1.00 & 2.00 & 12.00 & 25.00 & 37.44 & 42.66 \\
\hline 1475 & -- & 1.00 & 1.00 & 1.00 & 1.00 & 7.00 & 21.40 & 73.96 & -- & 6951 & 1.00 & 1.00 & 1.00 & 1.00 & 4.00 & 10.00 & 20.00 & 45.40 & 56.00 \\
\hline 1480 & -- & -- & -- & -- & 12.00 & -- & -- & -- & -- & 7094 & 1.00 & 1.00 & 1.00 & 1.00 & 3.00 & 12.00 & 23.00 & 43.00 & 50.31 \\
\hline 1515 & 1.00 & 1.00 & 1.00 & 1.00 & 3.00 & 10.00 & 20.00 & 42.00 & 52.87 & 7254 & 1.00 & 1.00 & 1.00 & 1.00 & 2.00 & 10.00 & 20.00 & 39.00 & 46.76 \\
\hline 1807 & 1.00 & 1.00 & 1.00 & 2.00 & 3.00 & 8.75 & 19.00 & 27.00 & 41.75 & 7277 & -- & -- & -- & -- & 6.00 & -- & -- & -- & -- \\
\hline 1840 & 1.00 & 1.00 & 1.00 & 1.00 & 1.00 & 6.00 & 17.00 & 31.50 & 42.75 & 7279 & 1.00 & 1.00 & 1.00 & 1.00 & 2.00 & 8.00 & 20.00 & 37.00 & 48.00 \\
\hline 1881 & -- & -- & -- & -- & 13.00 & -- & -- & -- & -- & 7283 & 1.00 & 1.00 & 1.00 & 1.00 & 3.00 & 11.00 & 24.00 & 49.56 & 59.34 \\
\hline 1887 & 1.00 & 1.00 & 1.00 & 1.00 & & 10.00 & & & 50.00 & 7604 & -- & -- & -- & 4.00 & 16.00 & 2 & -- & -- & -- \\
\hline 1939 & 1.00 & 1.00 & 1.00 & 1.00 & 3.00 & 9.00 & 21.00 & 41.00 & 54.47 & 7605 & -- & -- & 1.00 & 1.00 & 3.00 & 13.00 & 25.00 & -- & -- \\
\hline 1950 & -- & -- & 1.00 & 2.00 & 3.00 & 13.00 & 25.60 & -- & -- & 7609 & 1.00 & 1.00 & 1.00 & 1.00 & 3.00 & 10.50 & 24.00 & 46.00 & 55.00 \\
\hline 1956 & -- & -- & 1.00 & 2.00 & 4.00 & 9.00 & 27.40 & -- & -- & 7610 & 1.00 & 1.00 & 1.00 & 1.00 & 3.00 & 10.00 & 22.00 & 44.62 & 61.55 \\
\hline 2510 & 1.00 & 1.00 & 1.00 & 1.00 & 3.00 & 10.00 & 23.00 & 45.00 & 54.89 & 8078 & 1.00 & 1.00 & 1.00 & 1.00 & 3.00 & 12.75 & 23.10 & 42.84 & 61.39 \\
\hline 2625 & 1.00 & 1.00 & 1.00 & 2.00 & 3.00 & 10.00 & 20.30 & 41.26 & 63.15 & 8084 & -- & 1.00 & 1.00 & 1.00 & 3.00 & 6.00 & 17.60 & 47.72 & -- \\
\hline 2665 & 1.00 & 1.00 & 1.00 & 1.00 & 4.00 & 13.00 & 24.00 & 44.86 & 56.00 & 8085 & 1.00 & 1.00 & 1.00 & 1.00 & 3.00 & 13.00 & 24.00 & 41.00 & 50.42 \\
\hline 2694 & 1.00 & 1.00 & 1.00 & 2.50 & 7.00 & 22.50 & 36.00 & 57.24 & 69.16 & 8187 & 1.00 & 1.00 & 1.00 & 1.00 & 2.00 & 6.00 & 19.00 & 38.80 & 51.40 \\
\hline 2700 & 1.00 & 1.00 & 1.00 & 1.00 & 2.00 & 9.00 & 21.00 & 43.36 & 51.00 & 8358 & -- & 1.00 & 1.00 & 1.00 & 3.00 & 8.25 & 25.40 & 35.74 & -- \\
\hline 3145 & 1.00 & 1.00 & 1.00 & 1.00 & 3.00 & 9.00 & 21.00 & 45.00 & 56.28 & 8501 & 1.00 & 1.00 & 1.00 & 1.00 & 3.00 & 9.00 & 21.00 & 40.00 & 48.00 \\
\hline 3242 & -- & -- & 2.10 & 4.50 & 9.50 & 26.50 & 44.90 & -- & -- & 8518 & 1.00 & 1.00 & 1.00 & 1.00 & 3.00 & 10.00 & 22.00 & 40.00 & 52.27 \\
\hline 3288 & 1.00 & 1.00 & 1.00 & 2.00 & 5.00 & 14.50 & 33.00 & 57.28 & 63.07 & 8596 & 1.00 & 1.00 & 1.00 & 1.00 & 2.00 & 7.00 & 17.00 & 35.00 & 44.00 \\
\hline 3374 & 1.00 & 1.00 & 1.00 & 2.00 & 4.00 & 13.00 & 25.00 & 43.72 & 50.00 & 8788 & 1.00 & 1.00 & 1.00 & 2.00 & 5.00 & 18.00 & 33.00 & 55.00 & 67.95 \\
\hline 4026 & -- & -- & -- & -- & 1.00 & -- & -- & -- & -- & 8816 & -- & -- & -- & -- & 4.00 & -- & -- & -- & -- \\
\hline 4028 & 1.00 & 1.00 & 1.00 & 1.00 & 3.00 & 7.00 & 17.00 & 34.00 & 40.20 & 9148 & -- & -- & -- & 6.25 & 9.50 & 23.00 & -- & -- & -- \\
\hline 4030 & 1.00 & 1.00 & 1.00 & 1.00 & 2.00 & 8.00 & 18.70 & 41.98 & 44.47 & 9153 & 1.00 & 1.00 & 1.00 & 1.00 & 1.00 & 13.00 & 25.00 & 48.04 & 65.56 \\
\hline 4089 & 1.00 & 1.00 & 1.00 & 1.00 & 3.00 & 11.00 & 24.00 & 47.00 & 64.00 & 9156 & 1.00 & 1.00 & 1.00 & 1.00 & 2.00 & 8.00 & 19.00 & 37.68 & 44.00 \\
\hline 4112 & 1.00 & 1.00 & 1.00 & 1.00 & 2.00 & 9.00 & 19.50 & 40.10 & 54.15 & 9193 & 1.00 & 1.00 & 1.00 & 1.00 & 2.00 & 9.00 & 20.00 & 35.40 & 46.40 \\
\hline 4306 & 1.00 & 1.00 & 1.00 & 2.00 & 3.00 & 9.00 & 23.00 & 46.64 & 56.46 & 9265 & 1.00 & 1.00 & 1.00 & 1.00 & 1.00 & 7.00 & 17.00 & 40.00 & 44.83 \\
\hline 4850 & 1.00 & 1.00 & 1.00 & 1.00 & 3.00 & 10.00 & 24.00 & 40.00 & 50.18 & 9569 & 1.00 & 1.00 & 1.00 & 1.00 & 1.00 & 7.00 & 16.00 & 28.00 & 38.00 \\
\hline 4856 & 1.00 & 1.00 & 1.00 & 1.00 & 3.00 & 12.00 & 23.00 & 44.52 & 78.56 & 9686 & 1.00 & 1.00 & 1.00 & 1.00 & 3.00 & 9.00 & 19.00 & 38.78 & 45.89 \\
\hline
\end{tabular}


Appendix 2-5.5. Empirical distribution of storm duration defined by 24-hour minimum interevent time for hourly rainfall stations in eastern New Mexico.

$[--$, not available]

\begin{tabular}{|c|c|c|c|c|c|c|c|c|c|c|c|c|c|c|c|c|c|c|c|}
\hline \multicolumn{20}{|c|}{$\begin{array}{c}\text { Duration } \\
\text { (hours) }\end{array}$} \\
\hline $\begin{array}{l}\text { Sta- } \\
\text { tion } \\
\text { no. }\end{array}$ & $\begin{array}{c}1 \text { st } \\
\text { per- } \\
\text { centile }\end{array}$ & $\begin{array}{c}\text { 2nd } \\
\text { per- } \\
\text { centile }\end{array}$ & $\begin{array}{c}10 \text { th } \\
\text { per- } \\
\text { centile }\end{array}$ & $\begin{array}{c}25 \text { th } \\
\text { per- } \\
\text { centile }\end{array}$ & $\begin{array}{c}\text { 50th } \\
\text { per- } \\
\text { centile } \\
\text { (median) }\end{array}$ & $\begin{array}{c}\text { 75th } \\
\text { per- } \\
\text { centile }\end{array}$ & $\begin{array}{c}\text { 90th } \\
\text { per- } \\
\text { centile }\end{array}$ & $\begin{array}{c}\text { 98th } \\
\text { per- } \\
\text { centile }\end{array}$ & $\begin{array}{c}\text { 99th } \\
\text { per- } \\
\text { centile }\end{array}$ & $\begin{array}{l}\text { Sta- } \\
\text { tion } \\
\text { no. }\end{array}$ & $\begin{array}{c}1 \text { st } \\
\text { per- } \\
\text { centile }\end{array}$ & $\begin{array}{c}\text { 2nd } \\
\text { per- } \\
\text { centile }\end{array}$ & $\begin{array}{c}10 \text { th } \\
\text { per- } \\
\text { centile }\end{array}$ & $\begin{array}{c}25 \text { th } \\
\text { per- } \\
\text { centile }\end{array}$ & $\begin{array}{c}\text { 50th } \\
\text { per- } \\
\text { centile } \\
\text { (median) }\end{array}$ & $\begin{array}{c}75 \text { th } \\
\text { per- } \\
\text { centile }\end{array}$ & $\begin{array}{c}\text { 90th } \\
\text { per- } \\
\text { centile }\end{array}$ & $\begin{array}{c}\text { 98th } \\
\text { per- } \\
\text { centile }\end{array}$ & $\begin{array}{c}\text { 99th } \\
\text { per- } \\
\text { centile }\end{array}$ \\
\hline 0199 & 1.00 & 1.00 & 1.00 & 1.00 & 4.00 & 15.00 & 29.00 & 54.02 & 70.68 & 4860 & -- & 1.00 & 1.00 & 2.00 & 6.00 & 20.00 & 1.40 & 90.68 & -- \\
\hline 0205 & 1.00 & 1.00 & 1.00 & 2.00 & 3.00 & 12.00 & 25.00 & 51.00 & 59.70 & 4862 & 1.00 & 1.00 & 1.00 & 1.00 & 3.00 & 18.00 & 32.00 & 66.00 & 89.00 \\
\hline 0407 & 1.00 & 1.00 & 1.00 & 1.00 & 5.00 & 25.00 & 45.20 & 76.32 & 84.44 & 5866 & -- & 1.00 & 1.00 & 3.00 & 10.00 & 25.00 & 43.20 & 105.84 & -- \\
\hline 0600 & 1.00 & 1.00 & 1.00 & 1.00 & 3.00 & 12.00 & 24.00 & 46.50 & 56.00 & 6138 & 1.00 & 1.00 & 1.00 & 2.00 & 6.00 & 20.00 & 39.00 & 76.26 & 100.13 \\
\hline 0606 & -- & 1.00 & 1.00 & 1.00 & 1.00 & 17.00 & 44.00 & 73.80 & -- & 6275 & 1.00 & 1.00 & 1.00 & 2.00 & 6.00 & 1.00 & 39.00 & 75.00 & 92.56 \\
\hline 0646 & 1.00 & 1.00 & 1.00 & 1.00 & 5.00 & 18.25 & 33.90 & 58.36 & 72.00 & 6435 & 1.00 & 1.00 & 1.00 & 1.00 & 3.00 & 14.00 & 26.80 & 57.00 & 68.56 \\
\hline 1120 & 1.00 & 1.00 & 1.00 & 1.00 & 6.00 & 23.00 & 43.00 & 80.00 & 100.40 & 6492 & 1.00 & 1.00 & 1.00 & 1.00 & 3.00 & 17.00 & 32.00 & 61.80 & 71.40 \\
\hline 1475 & -- & 1.00 & 1.00 & 1.00 & 1.00 & 7.00 & 28.60 & 75.64 & -- & 6951 & 1.00 & 1.00 & 1.00 & 1.00 & 4.00 & 11.00 & 26.40 & 58.72 & 63.52 \\
\hline 1480 & -- & -- & -- & -- & 12.00 & -- & -- & -- & -- & 7094 & 1.00 & 1.00 & 1.00 & 1.00 & 4.00 & 17.00 & 29.00 & 53.00 & 65.00 \\
\hline 1515 & 1.00 & 1.00 & 1.00 & 1.00 & 4.00 & 15.00 & 28.00 & 58.22 & 71.00 & 7254 & 1.00 & 1.00 & 1.00 & 1.00 & 3.00 & 14.00 & 28.00 & 53.98 & 63.99 \\
\hline 1807 & 1.00 & 1.00 & 1.00 & 2.00 & 3.00 & 10.00 & 23.00 & 49.36 & 53.34 & \begin{tabular}{|l|}
7277 \\
\end{tabular} & -- & -- & -- & -- & 6.00 & -- & -- & -- & -- \\
\hline 1840 & 1.00 & 1.00 & 1.00 & 1.00 & 2.00 & 12.00 & 26.00 & 52.70 & 64.80 & 7279 & 1.00 & 1.00 & 1.00 & 1.00 & 3.00 & 15.00 & 28.00 & 52.12 & 64.68 \\
\hline 1881 & -- & -- & -- & -- & 13.00 & -- & -- & -- & -- & 7283 & 1.00 & 1.00 & 1.00 & 2.00 & 5.00 & 23.00 & 47.00 & 86.80 & 98.90 \\
\hline 1887 & 1.00 & 1.00 & 1.00 & 2.00 & 5.00 & 17.00 & 31.00 & 60.00 & 72.65 & 7604 & -- & -- & -- & 4.00 & 16.00 & 27.25 & -- & -- & -- \\
\hline 1939 & 1.00 & 1.00 & 1.00 & 1.00 & 4.00 & 13.00 & 28.00 & 56.00 & 72.25 & 7605 & -- & -- & 1.00 & 1.00 & 3.00 & 25.75 & 38.90 & -- & -- \\
\hline 1950 & -- & -- & 1.00 & 2.00 & 4.00 & 13.25 & 25.90 & -- & -- & 7609 & 1.00 & 1.00 & 1.00 & 1.00 & 4.00 & 16.00 & 33.00 & 64.88 & 76.08 \\
\hline 1956 & -- & -- & 1.00 & 2.00 & 4.50 & 10.25 & 28.80 & -- & -- & 7610 & 1.00 & 1.00 & 1.00 & 1.00 & 3.00 & 15.00 & 30.00 & 61.14 & 76.33 \\
\hline 2510 & 1.00 & 1.00 & 1.00 & 1.00 & 4.00 & 17.50 & 33.00 & 58.00 & 71.00 & 8078 & 1.00 & 1.00 & 1.00 & 1.00 & 4.00 & 18.00 & 29.00 & 54.98 & 66.97 \\
\hline 2625 & 1.00 & 1.00 & 1.00 & 2.00 & 4.00 & 14.00 & 26.00 & 59.40 & 65.90 & 8084 & -- & 1.00 & 1.00 & 1.00 & 3.00 & 9.50 & 27.20 & 48.36 & -- \\
\hline 2665 & 1.00 & 1.00 & 1.00 & 2.00 & 5.00 & 19.00 & 33.00 & 61.44 & 73.00 & 8085 & 1.00 & 1.00 & 1.00 & 1.00 & 5.00 & 19.00 & 32.00 & 60.52 & 68.00 \\
\hline 2694 & 1.00 & 1.00 & 1.00 & 4.00 & 17.00 & 30.00 & 52.80 & 134.04 & 157.74 & 8187 & 1.00 & 1.00 & 1.00 & 1.00 & 4.00 & 17.25 & 30.00 & 62.74 & 82.25 \\
\hline 2700 & 1.00 & 1.00 & 1.00 & 1.00 & 3.00 & 17.00 & 32.00 & 62.00 & 73.00 & 8358 & -- & 1.00 & 1.00 & 1.00 & 3.00 & 8.75 & 27.80 & 46.78 & -- \\
\hline 3145 & 1.00 & 1.00 & 1.00 & 1.50 & 3.00 & 11.50 & 26.00 & 53.40 & 67.20 & 8501 & 1.00 & 1.00 & 1.00 & 1.00 & 4.00 & 16.00 & 30.00 & 58.92 & 69.00 \\
\hline 3242 & -- & -- & 2.00 & 4.00 & 9.00 & 25.00 & 67.00 & -- & -- & 8518 & 1.00 & 1.00 & 1.00 & 1.00 & 4.00 & 16.00 & 30.00 & 56.00 & 66.28 \\
\hline 3288 & 1.00 & 1.00 & 1.00 & 2.00 & 8.00 & 25.00 & 38.20 & 92.44 & 139.12 & 8596 & 1.00 & 1.00 & 1.00 & 1.00 & 2.00 & 10.50 & 24.00 & 50.00 & 60.00 \\
\hline 3374 & 1.00 & 1.00 & 1.00 & 2.00 & 5.00 & 18.00 & 31.00 & 58.68 & 71.67 & 8788 & 1.00 & 1.00 & 1.00 & 2.00 & 9.00 & 26.00 & 45.00 & 77.06 & 89.12 \\
\hline 4026 & -- & -- & -- & -- & .00 & -- & -- & -- & -- & 8816 & -- & -- & -- & -- & 4.00 & -- & -- & -- & -- \\
\hline 4028 & 1.00 & 1.00 & 1.00 & 1.00 & 3.00 & 8.00 & 24.00 & 39.12 & 49.51 & 9148 & -- & -- & -- & 4.50 & 9.00 & 44.00 & -- & -- & -- \\
\hline 4030 & 1.00 & 1.00 & 1.00 & 1.00 & 2.00 & 9.00 & 20.00 & 43.04 & 44.52 & 9153 & -- & 1.00 & 1.00 & 1.00 & 1.00 & 13.00 & 31.60 & 79.36 & -- \\
\hline 4089 & 1.00 & 1.00 & 1.00 & 1.00 & 4.00 & 15.75 & 30.00 & 72.00 & 89.00 & 9156 & 1.00 & 1.00 & 1.00 & 1.00 & 3.00 & 13.00 & 27.00 & 52.00 & 70.04 \\
\hline 4112 & 1.00 & 1.00 & 1.00 & 1.00 & 2.00 & 12.00 & 25.00 & 59.08 & 74.08 & 9193 & 1.00 & 1.00 & 1.00 & 1.00 & 2.00 & 13.75 & 26.70 & 54.28 & 68.07 \\
\hline 4306 & 1.00 & 1.00 & 1.00 & 2.00 & 4.00 & 14.00 & 29.00 & 58.36 & 70.00 & 9265 & 1.00 & 1.00 & 1.00 & 1.00 & 2.00 & 12.00 & 25.00 & 47.04 & 61.30 \\
\hline 4850 & 1.00 & 1.00 & 1.00 & 1.00 & 4.00 & 20.00 & 34.00 & 70.74 & 85.58 & 9569 & 1.00 & 1.00 & 1.00 & 1.00 & 2.00 & 11.00 & 24.00 & 49.00 & 54.00 \\
\hline 4856 & 1.00 & 1.00 & 1.00 & 1.00 & 4.00 & 16.00 & 31.00 & 69.56 & 92.72 & 9686 & 1.00 & 1.00 & 1.00 & 1.00 & 3.00 & 13.00 & 26.00 & 50.00 & 58.00 \\
\hline
\end{tabular}


Appendix 2-5.6. Empirical distribution of storm duration defined by 48-hour minimum interevent time for hourly rainfall stations in eastern New Mexico.

[--, not available]

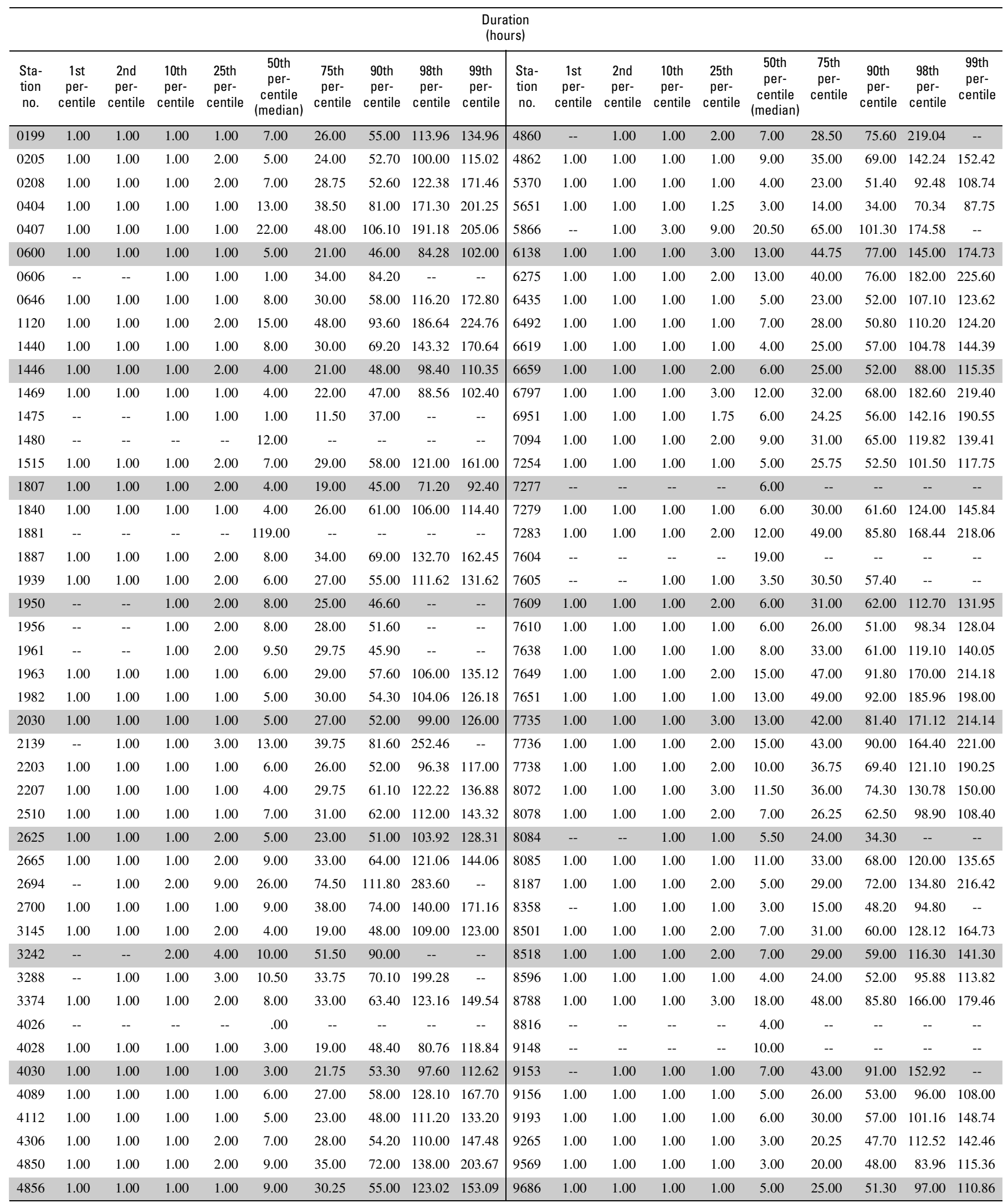


Appendix 2-5.7. Empirical distribution of storm duration defined by 72-hour minimum interevent time for hourly rainfall stations in eastern New Mexico.

$[--$, not available]

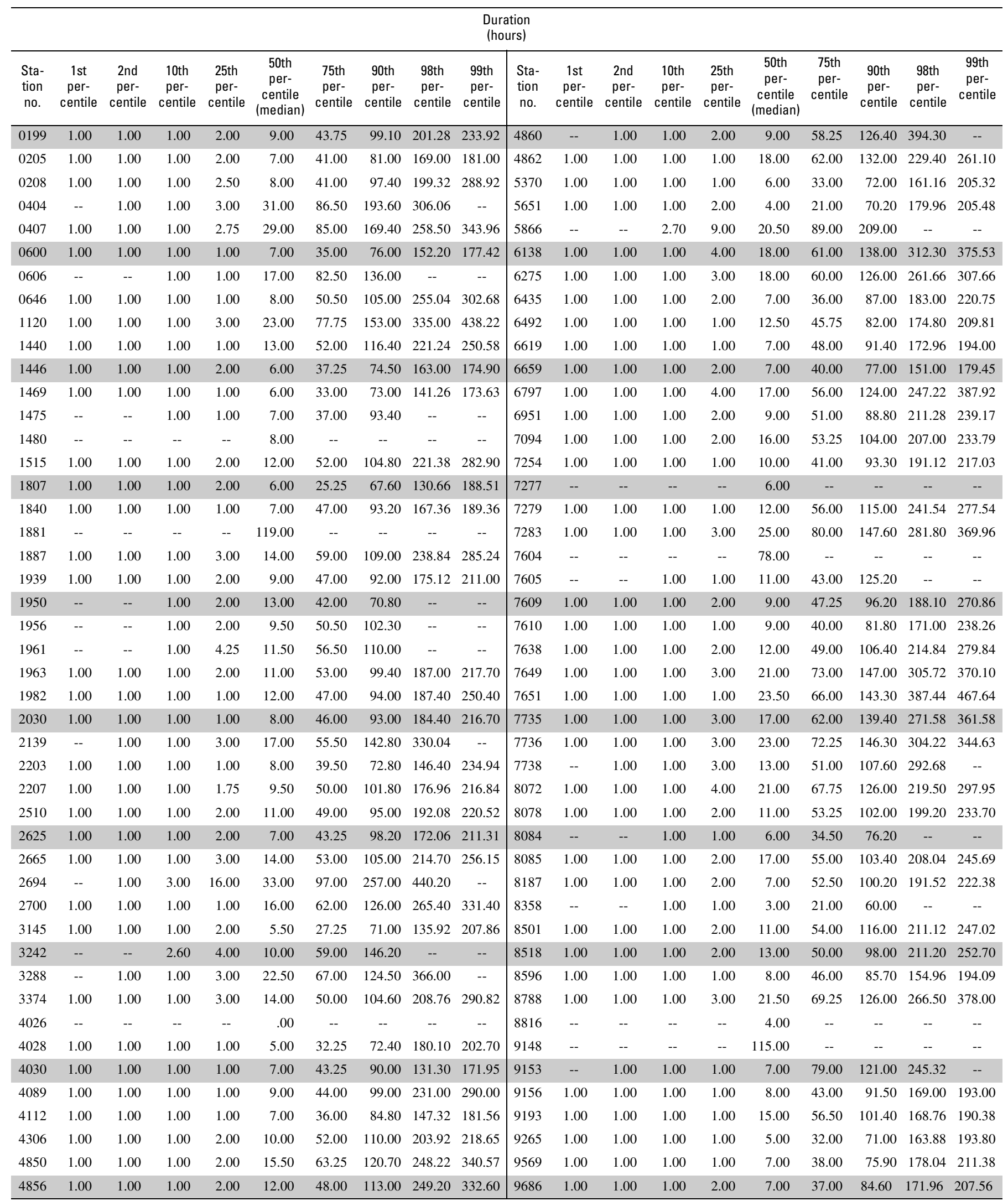


Appendix 3-

Storm Statistics for Hourly

Rainfall Stations in Oklahoma 
Blank Page 
Appendix 3-1.1. Number of storms, total duration, and mean storm interevent time defined by 6-hour minimum interevent time for hourly rainfall stations in Oklahoma.

[--, not available]

\begin{tabular}{|c|c|c|c|c|c|c|c|c|c|c|c|c|c|c|c|}
\hline $\begin{array}{c}\text { Station } \\
\text { no. }\end{array}$ & $\begin{array}{c}\text { No. } \\
\text { of } \\
\text { storms }\end{array}$ & $\begin{array}{c}\text { Total } \\
\text { duration } \\
\text { (hours) }\end{array}$ & $\begin{array}{c}\text { Mean } \\
\text { storm } \\
\text { interevent } \\
\text { time } \\
\text { (days) }\end{array}$ & $\begin{array}{c}\text { Station } \\
\text { no. }\end{array}$ & $\begin{array}{c}\text { No. } \\
\text { of } \\
\text { storms }\end{array}$ & $\begin{array}{c}\text { Total } \\
\text { duration } \\
\text { (hours) }\end{array}$ & $\begin{array}{c}\text { Mean } \\
\text { storm } \\
\text { interevent } \\
\text { time } \\
\text { (days) }\end{array}$ & $\begin{array}{c}\text { Station } \\
\text { no. }\end{array}$ & $\begin{array}{c}\text { No. } \\
\text { of } \\
\text { storms }\end{array}$ & $\begin{array}{c}\text { Total } \\
\text { duration } \\
\text { (hours) }\end{array}$ & $\begin{array}{c}\text { Mean } \\
\text { storm } \\
\text { interevent } \\
\text { time } \\
\text { (days) }\end{array}$ & $\begin{array}{c}\text { Station } \\
\text { no. }\end{array}$ & $\begin{array}{l}\text { No. } \\
\text { of } \\
\text { storms }\end{array}$ & $\begin{array}{c}\text { Total } \\
\text { duration } \\
\text { (hours) }\end{array}$ & $\begin{array}{c}\text { Mean } \\
\text { storm } \\
\text { interevent } \\
\text { time } \\
\text { (days) }\end{array}$ \\
\hline 0017 & 37 & 2,016 & 1.98 & 2665 & 1,504 & 279,696 & 7.52 & 5063 & 14 & 2,208 & 6.22 & 7412 & 2,444 & 484,014 & 8.09 \\
\hline 0026 & 1,243 & 189,192 & 6.09 & 2678 & 22 & 2,016 & 3.56 & 5068 & 133 & 16,080 & 4.82 & 7556 & 550 & 91,044 & 6.73 \\
\hline 0179 & 1,583 & 306,537 & 7.92 & 2849 & 2,567 & 448,934 & 7.10 & 5108 & 3,467 & 478,177 & 5.49 & 7588 & 677 & 158,232 & 9.51 \\
\hline 0215 & 2,625 & 483,917 & 7.44 & 2994 & 585 & 72,336 & 4.92 & 5463 & 1,615 & 288,975 & 7.30 & 7675 & 1,959 & 309,543 & 6.41 \\
\hline 0242 & 1,188 & 227,208 & 7.74 & 2997 & 343 & 46,728 & 5.41 & 5581 & 32 & 2,016 & 2.25 & 7705 & 3,260 & 484,086 & 5.99 \\
\hline 0256 & 3,676 & 468,853 & 5.11 & 3002 & 2,245 & 484,178 & 8.82 & 5582 & 343 & 55,368 & 6.47 & 7714 & 788 & 195,072 & 10.09 \\
\hline 0260 & 32 & 5,808 & 7.22 & 3281 & 2,723 & 438,744 & 6.51 & 5589 & 1,621 & 238,629 & 5.97 & 7732 & 1,268 & 201,624 & 6.37 \\
\hline 0535 & 1,168 & 162,830 & 5.64 & 3407 & 461 & 84,292 & 7.41 & 6130 & 3,432 & 484,134 & 5.64 & 8101 & 2,435 & 484,151 & 8.12 \\
\hline 0537 & 368 & 46,007 & 5.05 & 3497 & 3,194 & 484,026 & 6.12 & 6328 & 682 & 168,768 & 10.07 & 8290 & 1,972 & 290,040 & 5.92 \\
\hline 0670 & 4,021 & 484,231 & 4.81 & 3628 & 2,457 & 484,135 & 8.04 & 6391 & 41 & 4,416 & 4.33 & 8420 & 839 & 146,696 & 7.04 \\
\hline 0814 & 120 & 34,320 & 11.84 & 3700 & 1,753 & 276,120 & 6.32 & 6485 & 3,402 & 459,723 & 5.41 & 8470 & 61 & 20,496 & 13.77 \\
\hline 0908 & 1,336 & 335,099 & 10.29 & 3740 & 2,873 & 483,978 & 6.81 & 6612 & 25 & 21,154 & 35.08 & 8497 & 3,659 & 484,182 & 5.30 \\
\hline 0912 & 839 & 158,520 & 7.68 & 3830 & 502 & 86,928 & 6.97 & 6616 & 2,926 & 483,347 & 6.69 & 8501 & 3,020 & 453,753 & 6.06 \\
\hline 1148 & 31 & 2,088 & 2.47 & 3835 & 227 & 29,640 & 5.23 & 6620 & 1,286 & 188,946 & 5.95 & 8504 & 318 & 56,224 & 7.23 \\
\hline 1168 & 2,373 & 288,946 & 4.81 & 4001 & 10 & 1,296 & 5.27 & 6627 & 1,652 & 233,650 & 5.69 & 8708 & 1,660 & 295,443 & 7.26 \\
\hline 1688 & 196 & 50,400 & 10.48 & 4202 & 2,591 & 445,343 & 7.00 & 6740 & 992 & 186,220 & 7.66 & 9023 & 3,912 & 481,954 & 4.86 \\
\hline 1750 & 2,443 & 392,817 & 6.53 & 4204 & 274 & 45,192 & 6.67 & 6760 & 1,284 & 246,192 & 7.76 & 9247 & 34 & 2,016 & 2.14 \\
\hline 1855 & 43 & 2,016 & 1.69 & 4384 & 3,680 & 455,726 & 4.92 & 6859 & 3,339 & 484,210 & 5.83 & 9278 & 28 & 1,896 & 2.51 \\
\hline 1891 & 5 & 2,784 & 23.03 & 4386 & 2,052 & 248,882 & 4.80 & 6926 & 23 & 2,184 & 3.70 & 9300 & 41 & 2,184 & 1.90 \\
\hline 1900 & 2,218 & 308,928 & 5.55 & 4388 & 139 & 15,624 & 4.47 & 6935 & 3,554 & 463,634 & 5.24 & 9364 & 33 & 2,184 & 2.49 \\
\hline 1902 & 1,088 & 149,600 & 5.55 & 4393 & 2,990 & 425,017 & 5.70 & 6940 & 0 & 24 & -- & 9404 & 2,739 & 481,280 & 7.11 \\
\hline 1909 & 30 & 2,016 & 2.47 & 4506 & 1,972 & 270,888 & 5.49 & 6944 & 3,521 & 484,090 & 5.53 & 9450 & 2,100 & 309,586 & 5.96 \\
\hline 1954 & 39 & 2,016 & 1.88 & 4692 & 739 & 106,656 & 5.76 & 7080 & 2,334 & 277,331 & 4.71 & 9503 & 598 & 83,256 & 5.52 \\
\hline 2145 & 267 & 55,368 & 8.41 & 4812 & 2,552 & 349,689 & 5.48 & 7196 & 2,613 & 445,356 & 6.89 & 9629 & 2,958 & 481,788 & 6.57 \\
\hline 2242 & 31 & 2,088 & 2.44 & 4865 & 3,482 & 484,184 & 5.55 & 7201 & 314 & 38,616 & 4.89 & 9719 & 1,157 & 190,528 & 6.61 \\
\hline 2309 & 242 & 33,600 & 5.56 & 4969 & 296 & 46,056 & 6.27 & 7309 & 1,864 & 262,049 & 5.68 & 9724 & 988 & 117,472 & 4.77 \\
\hline 2334 & 1,615 & 257,534 & 6.48 & 4975 & 2,136 & 283,256 & 5.31 & 7358 & 1,124 & 151,944 & 5.37 & 9748 & 1,578 & 196,347 & 5.01 \\
\hline 2500 & 22 & 2,184 & 3.79 & 4978 & 2,842 & 446,857 & 6.36 & 7372 & 37 & 2,112 & 2.05 & 9762 & 1,527 & 267,312 & 7.07 \\
\hline 2654 & 1,318 & 204,388 & 6.29 & & & & & & & & & & & & \\
\hline
\end{tabular}


Appendix 3-1.2. Number of storms, total duration, and mean storm interevent time defined by 8-hour minimum interevent time for hourly rainfall stations in Oklahoma.

[--, not available]

\begin{tabular}{|c|c|c|c|c|c|c|c|c|c|c|c|c|c|c|c|}
\hline $\begin{array}{c}\text { Station } \\
\text { no. }\end{array}$ & $\begin{array}{c}\text { No. } \\
\text { of } \\
\text { storms }\end{array}$ & $\begin{array}{c}\text { Total } \\
\text { duration } \\
\text { (hours) }\end{array}$ & $\begin{array}{c}\text { Mean } \\
\text { storm } \\
\text { interevent } \\
\text { time } \\
\text { (days) }\end{array}$ & $\begin{array}{c}\text { Station } \\
\text { no. }\end{array}$ & $\begin{array}{c}\text { No. } \\
\text { of } \\
\text { storms }\end{array}$ & $\begin{array}{c}\text { Total } \\
\text { duration } \\
\text { (hours) }\end{array}$ & $\begin{array}{c}\text { Mean } \\
\text { storm } \\
\text { interevent } \\
\text { time } \\
\text { (days) }\end{array}$ & $\begin{array}{c}\text { Station } \\
\text { no. }\end{array}$ & $\begin{array}{c}\text { No. } \\
\text { of } \\
\text { storms }\end{array}$ & $\begin{array}{c}\text { Total } \\
\text { duration } \\
\text { (hours) }\end{array}$ & $\begin{array}{c}\text { Mean } \\
\text { storm } \\
\text { interevent } \\
\text { time } \\
\text { (days) }\end{array}$ & $\begin{array}{c}\text { Station } \\
\text { no. }\end{array}$ & $\begin{array}{c}\text { No. } \\
\text { of } \\
\text { storms }\end{array}$ & $\begin{array}{c}\text { Total } \\
\text { duration } \\
\text { (hours) }\end{array}$ & $\begin{array}{c}\text { Mean } \\
\text { storm } \\
\text { interevent } \\
\text { time } \\
\text { (days) }\end{array}$ \\
\hline 0017 & 34 & 2,016 & 2.13 & 2665 & 1,427 & 279,696 & 7.91 & 5063 & 14 & 2,208 & 6.22 & 7412 & 2,322 & 484,014 & 8.50 \\
\hline 0026 & 1,179 & 189,192 & 6.40 & 2678 & 21 & 2,016 & 3.72 & 5068 & 127 & 16,080 & 5.03 & 7556 & 509 & 91,044 & 7.25 \\
\hline 0179 & 1,483 & 306,537 & 8.43 & 2849 & 2,417 & 448,934 & 7.53 & 5108 & 3,266 & 478,177 & 5.81 & 7588 & 648 & 158,232 & 9.92 \\
\hline 0188 & 857 & 200,904 & 9.53 & 2852 & 279 & 49,728 & 7.24 & 5329 & 24 & 2,016 & 3.12 & 7660 & 2,323 & 483,990 & 8.49 \\
\hline 0215 & 2,499 & 483,917 & 7.80 & 2994 & 556 & 72,336 & 5.16 & 5463 & 1,524 & 288,975 & 7.72 & 7675 & 1,832 & 309,543 & 6.83 \\
\hline 0242 & 1,143 & 227,208 & 8.04 & 2997 & 331 & 46,728 & 5.60 & 5581 & 31 & 2,016 & 2.31 & 7705 & 3,088 & 484,086 & 6.31 \\
\hline 0256 & 3,449 & 468,853 & 5.43 & 3002 & 2,156 & 484,178 & 9.17 & 5582 & 325 & 55,368 & 6.82 & 7714 & 749 & 195,072 & 10.60 \\
\hline 0260 & 30 & 5,808 & 7.68 & 3281 & 2,559 & 438,744 & 6.91 & 5589 & 1,507 & 238,629 & 6.40 & 7732 & 1,215 & 201,624 & 6.63 \\
\hline 0292 & 959 & 181,645 & 7.68 & 3286 & 3,029 & 455,654 & 6.02 & 5648 & 2,605 & 484,213 & 7.55 & 7739 & 1,763 & 254,039 & 5.79 \\
\hline 0293 & 1,360 & 219,878 & 6.54 & 3304 & 2,569 & 483,966 & 7.62 & 5662 & 668 & 86,616 & 5.09 & 8029 & 843 & 200,160 & 9.67 \\
\hline 0296 & 686 & 98,640 & 5.70 & 3353 & 22 & 1,896 & 3.01 & 5664 & 1,248 & 198,470 & 6.41 & 8092 & 4 & 1,464 & 15.25 \\
\hline 0535 & 1,081 & 162,830 & 6.08 & 3407 & 423 & 84,292 & 8.05 & 6130 & 3,235 & 484,134 & 5.96 & 8101 & 2,303 & 484,151 & 8.57 \\
\hline 0537 & 345 & 46,007 & 5.36 & 3497 & 2,967 & 484,026 & 6.57 & 6328 & 653 & 168,768 & 10.50 & 8290 & 1,870 & 290,040 & 6.22 \\
\hline 0670 & 3,782 & 484,231 & 5.10 & 3628 & 2,341 & 484,135 & 8.42 & 6391 & 39 & 4,416 & 4.53 & 8420 & 805 & 146,696 & 7.32 \\
\hline 0814 & 118 & 34,320 & 12.04 & 3700 & 1,663 & 276,120 & 6.65 & 6485 & 3,193 & 459,723 & 5.75 & 8470 & 55 & 20,496 & 15.25 \\
\hline 0908 & 1,291 & 335,099 & 10.64 & 3740 & 2,723 & 483,978 & 7.17 & 6612 & 23 & 21,154 & 38.11 & 8497 & 3,425 & 484,182 & 5.64 \\
\hline 0912 & 805 & 158,520 & 7.99 & 3830 & 472 & 86,928 & 7.40 & 6616 & 2,752 & 483,347 & 7.10 & 8501 & 2,848 & 453,753 & 6.41 \\
\hline 1148 & 31 & 2,088 & 2.47 & 3835 & 219 & 29,640 & 5.41 & 6620 & 1,204 & 188,946 & 6.34 & 8504 & 302 & 56,224 & 7.60 \\
\hline 1168 & 2,242 & 288,946 & 5.07 & 4001 & 9 & 1,296 & 5.83 & 6627 & 1,551 & 233,650 & 6.04 & 8708 & 1,558 & 295,443 & 7.71 \\
\hline 1391 & 35 & 2,136 & 2.19 & 4008 & 45 & 5,808 & 4.96 & 6638 & 2,996 & 466,694 & 6.25 & 8769 & 2,875 & 435,941 & 6.05 \\
\hline 1436 & 1,786 & 268,080 & 5.95 & 4010 & 1,252 & 173,856 & 5.47 & 6643 & 203 & 26,304 & 5.13 & 8879 & 317 & 67,200 & 8.61 \\
\hline 1437 & 1,357 & 216,039 & 6.42 & 4051 & 1,492 & 229,608 & 6.17 & 6656 & 451 & 56,808 & 4.98 & 8884 & 10 & 672 & 2.43 \\
\hline 1544 & 4,016 & 483,436 & 4.76 & 4052 & 1,658 & 251,204 & 6.10 & 6661 & 3,807 & 484,166 & 5.01 & 8992 & 4,180 & 482,102 & 4.51 \\
\hline 1684 & 2,783 & 434,246 & 6.28 & 4098 & 2,913 & 422,152 & 5.77 & 6729 & 2,658 & 372,427 & 5.58 & 9014 & 67 & 16,080 & 9.81 \\
\hline 1688 & 182 & 50,400 & 11.26 & 4202 & 2,437 & 445,343 & 7.42 & 6740 & 956 & 186,220 & 7.93 & 9023 & 3,698 & 481,954 & 5.13 \\
\hline 1750 & 2,305 & 392,817 & 6.91 & 4204 & 253 & 45,192 & 7.20 & 6760 & 1,220 & 246,192 & 8.15 & 9247 & 31 & 2,016 & 2.32 \\
\hline 1855 & 39 & 2,016 & 1.83 & 4384 & 3,459 & 455,726 & 5.22 & 6859 & 3,140 & 484,210 & 6.18 & 9278 & 25 & 1,896 & 2.78 \\
\hline 1891 & 5 & 2,784 & 22.98 & 4386 & 1,937 & 248,882 & 5.07 & 6926 & 21 & 2,184 & 4.03 & 9300 & 37 & 2,184 & 2.07 \\
\hline 1900 & 2,112 & 308,928 & 5.81 & 4388 & 130 & 15,624 & 4.76 & 6935 & 3,314 & 463,634 & 5.60 & 9364 & 31 & 2,184 & 2.63 \\
\hline 1902 & 1,022 & 149,600 & 5.89 & 4393 & 2,837 & 425,017 & 6.00 & 6940 & 0 & 24 & -- & 9404 & 2,589 & 48,1280 & 7.51 \\
\hline 1909 & 28 & 2,016 & 2.62 & 4506 & 1,871 & 270,888 & 5.77 & 6944 & 3,289 & 484,090 & 5.90 & 9450 & 1,973 & 309,586 & 6.33 \\
\hline 1954 & 36 & 2,016 & 2.02 & 4692 & 706 & 106,656 & 6.01 & 7080 & 2,215 & 277,331 & 4.95 & 9503 & 564 & 83,256 & 5.84 \\
\hline 2145 & 254 & 55,368 & 8.83 & 4812 & 2,421 & 349,689 & 5.76 & 7196 & 2,467 & 445,356 & 7.29 & 9629 & 2,792 & 481,788 & 6.94 \\
\hline 2242 & 29 & 2,088 & 2.59 & 4865 & 3,276 & 484,184 & 5.88 & 7201 & 299 & 38,616 & 5.13 & 9719 & 1,101 & 190,528 & 6.94 \\
\hline 2309 & 226 & 33,600 & 5.93 & 4969 & 284 & 46,056 & 6.52 & 7309 & 1,732 & 262,049 & 6.09 & 9724 & 916 & 117,472 & 5.12 \\
\hline 2334 & 1,508 & 257,534 & 6.92 & 4975 & 2,004 & 283,256 & 5.65 & 7358 & 1,071 & 151,944 & 5.62 & 9748 & 1,461 & 196,347 & 5.39 \\
\hline 2500 & 21 & 2,184 & 3.95 & 4978 & 2,669 & 446,857 & 6.76 & 7372 & 32 & 2,112 & 2.32 & 9762 & 1,465 & 267,312 & 7.36 \\
\hline 2654 & 1,247 & 204,388 & 6.64 & & & & & & & & & & & & \\
\hline
\end{tabular}


Appendix 3-1.3. Number of storms, total duration, and mean storm interevent time defined by 12-hour minimum interevent time for hourly rainfall stations in Oklahoma.

[--, not available]

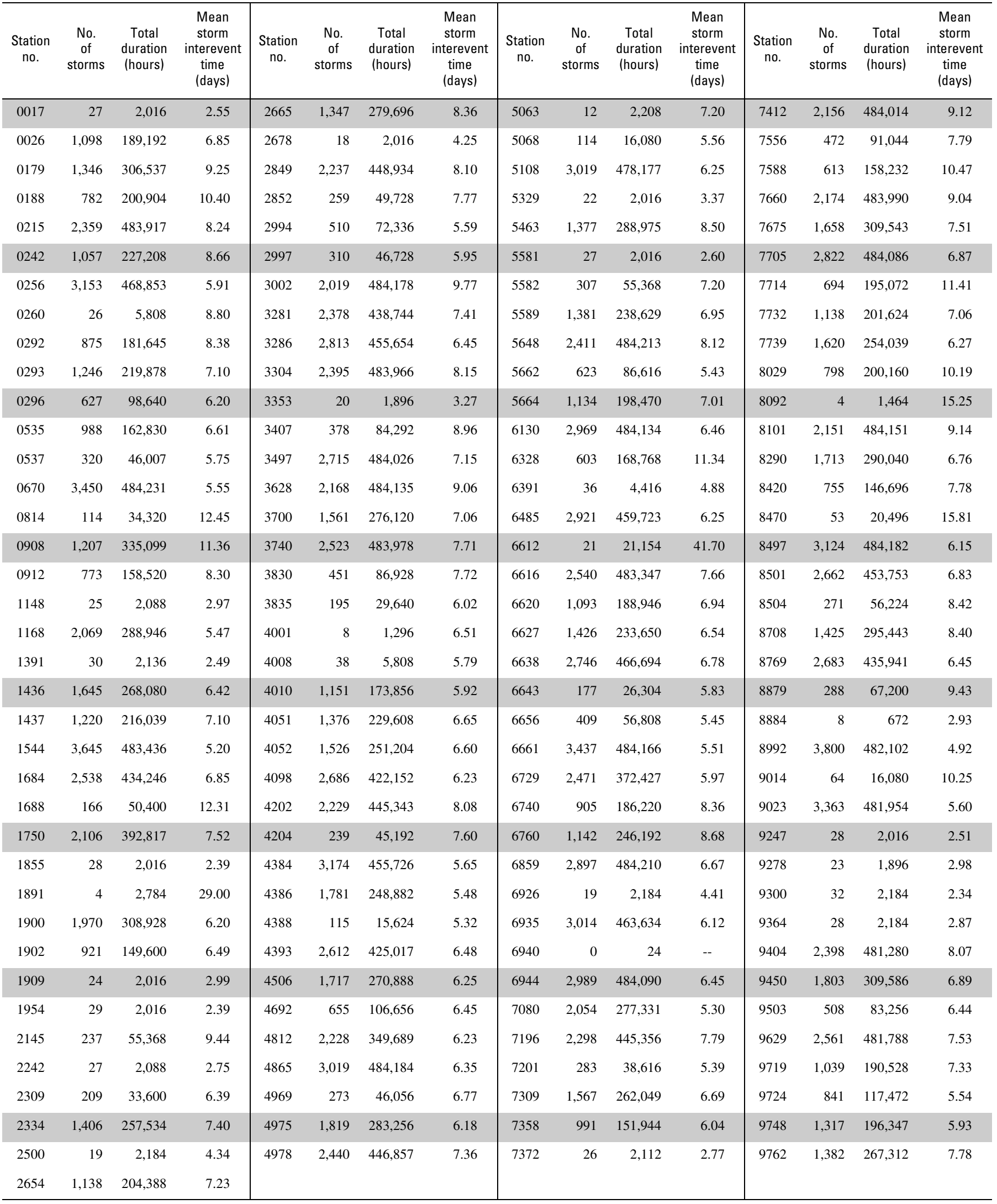


Appendix 3-1.4. Number of storms, total duration, and mean storm interevent time defined by 18-hour minimum interevent time for hourly rainfall stations in Oklahoma.

$[--$, not available $]$

\begin{tabular}{|c|c|c|c|c|c|c|c|c|c|c|c|c|c|c|c|}
\hline $\begin{array}{c}\text { Station } \\
\text { no. }\end{array}$ & $\begin{array}{c}\text { No. } \\
\text { of } \\
\text { storms }\end{array}$ & $\begin{array}{c}\text { Total } \\
\text { duration } \\
\text { (hours) }\end{array}$ & $\begin{array}{c}\text { Mean } \\
\text { storm } \\
\text { interevent } \\
\text { time } \\
\text { (days) }\end{array}$ & $\begin{array}{c}\text { Station } \\
\text { no. }\end{array}$ & $\begin{array}{c}\text { No. } \\
\text { of } \\
\text { storms }\end{array}$ & $\begin{array}{c}\text { Total } \\
\text { duration } \\
\text { (hours) }\end{array}$ & $\begin{array}{c}\text { Mean } \\
\text { storm } \\
\text { interevent } \\
\text { time } \\
\text { (days) }\end{array}$ & $\begin{array}{c}\text { Station } \\
\text { no. }\end{array}$ & $\begin{array}{c}\text { No. } \\
\text { of } \\
\text { storms }\end{array}$ & $\begin{array}{c}\text { Total } \\
\text { duration } \\
\text { (hours) }\end{array}$ & $\begin{array}{c}\text { Mean } \\
\text { storm } \\
\text { interevent } \\
\text { time } \\
\text { (days) }\end{array}$ & $\begin{array}{c}\text { Station } \\
\text { no. }\end{array}$ & $\begin{array}{c}\text { No. } \\
\text { of } \\
\text { storms }\end{array}$ & $\begin{array}{c}\text { Total } \\
\text { duration } \\
\text { (hours) }\end{array}$ & $\begin{array}{c}\text { Mean } \\
\text { storm } \\
\text { interevent } \\
\text { time } \\
\text { (days) }\end{array}$ \\
\hline 0017 & 23 & 2,016 & 2.88 & 2665 & 1,238 & 279,696 & 9.04 & 5063 & 11 & 2,208 & 7.80 & 7412 & 1,988 & 484,014 & 9.84 \\
\hline 0026 & 994 & 189,192 & 7.50 & 2678 & 13 & 2,016 & 5.68 & 5068 & 103 & 16,080 & 6.09 & 7556 & 427 & 91,044 & 8.54 \\
\hline 0179 & 1,211 & 306,537 & 10.22 & 2849 & 2,015 & 448,934 & 8.93 & 5108 & 2,753 & 478,177 & 6.80 & 7588 & 559 & 158,232 & 11.42 \\
\hline 0188 & 715 & 200,904 & 11.32 & 2852 & 236 & 49,728 & 8.47 & 5329 & 19 & 2,016 & 3.77 & 7660 & 1,992 & 483,990 & 9.81 \\
\hline 0215 & 2,171 & 483,917 & 8.90 & 2994 & 460 & 72,336 & 6.14 & 5463 & 1,268 & 288,975 & 9.18 & 7675 & 1,507 & 309,543 & 8.20 \\
\hline 0242 & 978 & 227,208 & 9.31 & 2997 & 288 & 46,728 & 6.36 & 5581 & 21 & 2,016 & 3.14 & 7705 & 2,572 & 484,086 & 7.48 \\
\hline 0256 & 2,836 & 468,853 & 6.50 & 3002 & 1,871 & 484,178 & 10.49 & 5582 & 281 & 55,368 & 7.81 & 7714 & 651 & 195,072 & 12.12 \\
\hline 0260 & 24 & 5,808 & 9.49 & 3281 & 2,200 & 438,744 & 7.96 & 5589 & 1,238 & 238,629 & 7.68 & 7732 & 1,061 & 201,624 & 7.53 \\
\hline 0292 & 769 & 181,645 & 9.45 & 3286 & 2,559 & 455,654 & 7.03 & 5648 & 2,186 & 484,213 & 8.90 & 7739 & 1,453 & 254,039 & 6.92 \\
\hline 0293 & 1,136 & 219,878 & 7.73 & 3304 & 2,202 & 483,966 & 8.81 & 5662 & 559 & 86,616 & 5.98 & 8029 & 746 & 200,160 & 10.86 \\
\hline 0296 & 564 & 98,640 & 6.83 & 3353 & 18 & 1,896 & 3.54 & 5664 & 1,025 & 198,470 & 7.70 & 8092 & 4 & 1,464 & 15.25 \\
\hline 0535 & 887 & 162,830 & 7.30 & 3407 & 341 & 84,292 & 9.86 & 6130 & 2,687 & 484,134 & 7.08 & 8101 & 1,986 & 484,151 & 9.85 \\
\hline 0537 & 291 & 46,007 & 6.26 & 3497 & 2,497 & 484,026 & 7.72 & 6328 & 554 & 168,768 & 12.29 & 8290 & 1,553 & 290,040 & 7.39 \\
\hline 0670 & 3,137 & 484,231 & 6.04 & 3628 & 2,000 & 484,135 & 9.78 & 6391 & 33 & 4,416 & 5.25 & 8420 & 703 & 146,696 & 8.31 \\
\hline 0814 & 108 & 34,320 & 13.10 & 3700 & 1,452 & 276,120 & 7.54 & 6485 & 2,668 & 459,723 & 6.78 & 8470 & 48 & 20,496 & 17.39 \\
\hline 0908 & 1,116 & 335,099 & 12.23 & 3740 & 2,352 & 483,978 & 8.23 & 6612 & 18 & 21,154 & 48.56 & 8497 & 2,855 & 484,182 & 6.67 \\
\hline 0912 & 723 & 158,520 & 8.84 & 3830 & 412 & 86,928 & 8.40 & 6616 & 2,322 & 483,347 & 8.32 & 8501 & 2,440 & 453,753 & 7.40 \\
\hline 1148 & 23 & 2,088 & 3.17 & 3835 & 175 & 29,640 & 6.63 & 6620 & 1,005 & 188,946 & 7.49 & 8504 & 242 & 56,224 & 9.36 \\
\hline 1168 & 1,881 & 288,946 & 5.95 & 4001 & 6 & 1,296 & 8.51 & 6627 & 1,297 & 233,650 & 7.13 & 8708 & 1,307 & 295,443 & 9.10 \\
\hline 1391 & 26 & 2,136 & 2.78 & 4008 & 33 & 5,808 & 6.59 & 6638 & 2,520 & 466,694 & 7.33 & 8769 & 2,467 & 435,941 & 6.97 \\
\hline 1436 & 1,512 & 268,080 & 6.94 & 4010 & 1,061 & 173,856 & 6.37 & 6643 & 158 & 26,304 & 6.46 & 8879 & 266 & 67,200 & 10.16 \\
\hline 1437 & 1,081 & 216,039 & 7.93 & 4051 & 1,256 & 229,608 & 7.23 & 6656 & 370 & 56,808 & 5.96 & 8884 & 7 & 672 & 3.15 \\
\hline 1544 & 3,242 & 483,436 & 5.78 & 4052 & 1,375 & 251,204 & 7.26 & 6661 & 3,110 & 484,166 & 6.03 & 8992 & 3,419 & 482,102 & 5.41 \\
\hline 1684 & 2,320 & 434,246 & 7.44 & 4098 & 2,472 & 422,152 & 6.71 & 6729 & 2,287 & 372,427 & 6.40 & 9014 & 62 & 16,080 & 10.56 \\
\hline 1688 & 158 & 50,400 & 12.90 & 4202 & 2,005 & 445,343 & 8.91 & 6740 & 839 & 186,220 & 8.97 & 9023 & 3,059 & 481,954 & 6.10 \\
\hline 1750 & 1,922 & 392,817 & 8.18 & 4204 & 218 & 45,192 & 8.28 & 6760 & 1,063 & 246,192 & 9.28 & 9247 & 27 & 2,016 & 2.59 \\
\hline 1855 & 23 & 2,016 & 2.78 & 4384 & 2,860 & 455,726 & 6.21 & 6859 & 2,641 & 484,210 & 7.25 & 9278 & 19 & 1,896 & 3.46 \\
\hline 1891 & 4 & 2,784 & 29.00 & 4386 & 1,602 & 248,882 & 6.03 & 6926 & 16 & 2,184 & 5.12 & 9300 & 28 & 2,184 & 2.57 \\
\hline 1900 & 1,815 & 308,928 & 6.68 & 4388 & 109 & 15,624 & 5.58 & 6935 & 2,745 & 463,634 & 6.66 & 9364 & 24 & 2,184 & 3.25 \\
\hline 1902 & 843 & 149,600 & 7.04 & 4393 & 2,400 & 425,017 & 7.00 & 6940 & 0 & 24 & -- & 9404 & 2,203 & 481,280 & 8.74 \\
\hline 1909 & 22 & 2,016 & 3.18 & 4506 & 1,560 & 270,888 & 6.82 & 6944 & 2,723 & 484,090 & 7.03 & 9450 & 1,629 & 309,586 & 7.56 \\
\hline 1954 & 22 & 2,016 & 2.97 & 4692 & 621 & 106,656 & 6.77 & 7080 & 1,843 & 277,331 & 5.84 & 9503 & 455 & 83,256 & 7.12 \\
\hline 2145 & 219 & 55,368 & 10.16 & 4812 & 2,052 & 349,689 & 6.71 & 7196 & 2,117 & 445,356 & 8.41 & 9629 & 2,342 & 481,788 & 8.18 \\
\hline 2242 & 26 & 2,088 & 2.81 & 4865 & 2,756 & 484,184 & 6.90 & 7201 & 254 & 38,616 & 5.94 & 9719 & 979 & 190,528 & 7.74 \\
\hline 2309 & 192 & 33,600 & 6.90 & 4969 & 249 & 46,056 & 7.37 & 7309 & 1,419 & 262,049 & 7.33 & 9724 & 755 & 117,472 & 6.10 \\
\hline 2334 & 1,283 & 257,534 & 8.05 & 4975 & 1,672 & 283,256 & 6.67 & 7358 & 911 & 151,944 & 6.52 & 9748 & 1,174 & 196,347 & 6.58 \\
\hline 2500 & 17 & 2,184 & 4.77 & 4978 & 2,233 & 446,857 & 7.98 & 7372 & 24 & 2,112 & 2.95 & 9762 & 1,301 & 267,312 & 8.23 \\
\hline 2654 & 1,002 & 204,388 & 8.13 & & & & & & & & & & & & \\
\hline
\end{tabular}


Appendix 3-1.5. Number of storms, total duration, and mean storm interevent time defined by 24-hour minimum interevent time for hourly rainfall stations in Oklahoma.

$[--$, not available $]$

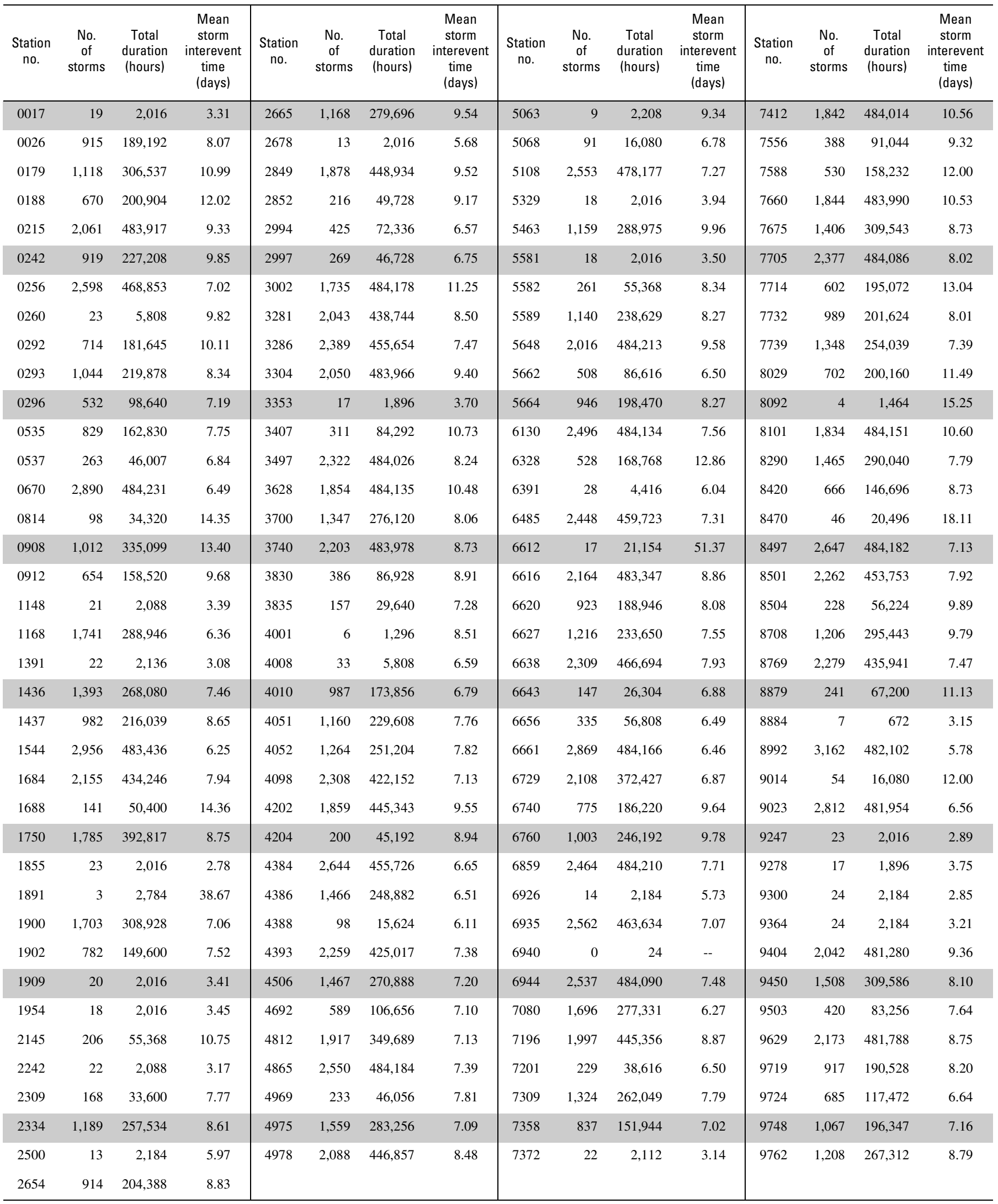


Appendix 3-1.6. Number of storms, total duration, and mean storm interevent time defined by 48-hour minimum interevent time for hourly rainfall stations in Oklahoma.

[--, not available]

\begin{tabular}{|c|c|c|c|c|c|c|c|c|c|c|c|c|c|c|c|}
\hline $\begin{array}{c}\text { Station } \\
\text { no. }\end{array}$ & $\begin{array}{c}\text { No. } \\
\text { of } \\
\text { storms }\end{array}$ & $\begin{array}{c}\text { Total } \\
\text { duration } \\
\text { (hours) }\end{array}$ & $\begin{array}{c}\text { Mean } \\
\text { storm } \\
\text { interevent } \\
\text { time } \\
\text { (days) }\end{array}$ & $\begin{array}{c}\text { Station } \\
\text { no. }\end{array}$ & $\begin{array}{c}\text { No. } \\
\text { of } \\
\text { storms }\end{array}$ & $\begin{array}{c}\text { Total } \\
\text { duration } \\
\text { (hours) }\end{array}$ & $\begin{array}{c}\text { Mean } \\
\text { storm } \\
\text { interevent } \\
\text { time } \\
\text { (days) }\end{array}$ & $\begin{array}{c}\text { Station } \\
\text { no. }\end{array}$ & $\begin{array}{c}\text { No. } \\
\text { of } \\
\text { storms }\end{array}$ & $\begin{array}{c}\text { Total } \\
\text { duration } \\
\text { (hours) }\end{array}$ & $\begin{array}{c}\text { Mean } \\
\text { storm } \\
\text { interevent } \\
\text { time } \\
\text { (days) }\end{array}$ & $\begin{array}{c}\text { Station } \\
\text { no. }\end{array}$ & $\begin{array}{c}\text { No. } \\
\text { of } \\
\text { storms }\end{array}$ & $\begin{array}{c}\text { Total } \\
\text { duration } \\
\text { (hours) }\end{array}$ & $\begin{array}{c}\text { Mean } \\
\text { storm } \\
\text { interevent } \\
\text { time } \\
\text { (days) }\end{array}$ \\
\hline 0017 & 15 & 2,016 & 3.98 & 2665 & 1,002 & 279,696 & 10.87 & 5063 & 9 & 2,208 & 9.34 & 7412 & 1,544 & 484,014 & 12.32 \\
\hline 0026 & 748 & 189,192 & 9.55 & 2678 & 8 & 2,016 & 8.77 & 5068 & 71 & 16,080 & 8.27 & 7556 & 309 & 91,044 & 11.33 \\
\hline 0179 & 943 & 306,537 & 12.77 & 2849 & 1,537 & 448,934 & 11.32 & 5108 & 2,123 & 478,177 & 8.44 & 7588 & 440 & 158,232 & 14.15 \\
\hline 0188 & 570 & 200,904 & 13.88 & 2852 & 188 & 49,728 & 10.34 & 5329 & 10 & 2,016 & 5.97 & 7660 & 1,556 & 483,990 & 12.22 \\
\hline 0215 & 1,712 & 483,917 & 10.93 & 2994 & 344 & 72,336 & 7.77 & 5463 & 930 & 288,975 & 12.06 & 7675 & 1,147 & 309,543 & 10.38 \\
\hline 0242 & 777 & 227,208 & 11.38 & 2997 & 220 & 46,728 & 7.92 & 5581 & 12 & 2,016 & 4.74 & 7705 & 1,932 & 484,086 & 9.54 \\
\hline 0256 & 2,085 & 468,853 & 8.39 & 3002 & 1,479 & 484,178 & 12.94 & 5582 & 213 & 55,368 & 9.89 & 7714 & 500 & 195,072 & 15.41 \\
\hline 0260 & 16 & 5,808 & 13.55 & 3281 & 1,687 & 438,744 & 9.99 & 5589 & 937 & 238,629 & 9.75 & 7732 & 820 & 201,624 & 9.36 \\
\hline 0292 & 575 & 181,645 & 12.21 & 3286 & 1,921 & 455,654 & 8.95 & 5648 & 1,673 & 484,213 & 11.24 & 7739 & 1,057 & 254,039 & 9.03 \\
\hline 0293 & 866 & 219,878 & 9.75 & 3304 & 1,703 & 483,966 & 11.02 & 5662 & 405 & 86,616 & 7.78 & 8029 & 578 & 200,160 & 13.64 \\
\hline 0296 & 422 & 98,640 & 8.68 & 3353 & 12 & 1,896 & 4.67 & 5664 & 774 & 198,470 & 9.78 & 8092 & 3 & 1,464 & 20.33 \\
\hline 0535 & 640 & 162,830 & 9.63 & 3407 & 250 & 84,292 & 13.00 & 6130 & 2,010 & 484,134 & 9.03 & 8101 & 1,489 & 484,151 & 12.72 \\
\hline 0537 & 205 & 46,007 & 8.35 & 3497 & 1,873 & 484,026 & 9.86 & 6328 & 443 & 168,768 & 15.04 & 8290 & 1,187 & 290,040 & 9.27 \\
\hline 0670 & 2,301 & 484,231 & 7.78 & 3628 & 1,546 & 484,135 & 12.28 & 6391 & 22 & 4,416 & 7.24 & 8420 & 554 & 146,696 & 10.20 \\
\hline 0814 & 77 & 34,320 & 17.88 & 3700 & 1,115 & 276,120 & 9.43 & 6485 & 1,960 & 459,723 & 8.78 & 8470 & 35 & 20,496 & 23.37 \\
\hline 0908 & 826 & 335,099 & 16.10 & 3740 & 1,802 & 483,978 & 10.34 & 6612 & 10 & 21,154 & 86.21 & 8497 & 2,149 & 484,182 & 8.45 \\
\hline 0912 & 551 & 158,520 & 11.21 & 3830 & 320 & 86,928 & 10.46 & 6616 & 1,746 & 483,347 & 10.64 & 8501 & 1,845 & 453,753 & 9.38 \\
\hline 1148 & 12 & 2,088 & 5.15 & 3835 & 113 & 29,640 & 9.50 & 6620 & 769 & 188,946 & 9.41 & 8504 & 188 & 56,224 & 11.70 \\
\hline 1168 & 1,428 & 288,946 & 7.44 & 4001 & 4 & 1,296 & 13.50 & 6627 & 987 & 233,650 & 8.96 & 8708 & 983 & 295,443 & 11.68 \\
\hline 1391 & 17 & 2,136 & 3.74 & 4008 & 30 & 5,808 & 7.09 & 6638 & 1,848 & 466,694 & 9.54 & 8769 & 1,855 & 435,941 & 8.85 \\
\hline 1436 & 1,138 & 268,080 & 8.79 & 4010 & 793 & 173,856 & 8.10 & 6643 & 123 & 26,304 & 7.94 & 8879 & 201 & 67,200 & 13.04 \\
\hline 1437 & 770 & 216,039 & 10.63 & 4051 & 955 & 229,608 & 9.11 & 6656 & 269 & 56,808 & 7.73 & 8884 & 4 & 672 & 7.00 \\
\hline 1544 & 2,342 & 483,436 & 7.52 & 4052 & 1,012 & 251,204 & 9.40 & 6661 & 2,266 & 484,166 & 7.79 & 8992 & 2,496 & 482,102 & 6.93 \\
\hline 1684 & 1,753 & 434,246 & 9.43 & 4098 & 1,889 & 422,152 & 8.39 & 6729 & 1,701 & 372,427 & 8.17 & 9014 & 44 & 16,080 & 14.40 \\
\hline 1688 & 121 & 50,400 & 16.50 & 4202 & 1,546 & 445,343 & 11.19 & 6740 & 647 & 186,220 & 11.27 & 9023 & 2,276 & 481,954 & 7.76 \\
\hline 1750 & 1,454 & 392,817 & 10.41 & 4204 & 169 & 45,192 & 10.30 & 6760 & 854 & 246,192 & 11.24 & 9247 & 12 & 2,016 & 4.45 \\
\hline 1855 & 13 & 2,016 & 4.19 & 4384 & 2,141 & 455,726 & 7.87 & 6859 & 2,003 & 484,210 & 9.16 & 9278 & 11 & 1,896 & 5.22 \\
\hline 1891 & 2 & 2,784 & 58.00 & 4386 & 1,194 & 248,882 & 7.66 & 6926 & 9 & 2,184 & 8.39 & 9300 & 15 & 2,184 & 3.95 \\
\hline 1900 & 1,378 & 308,928 & 8.39 & 4388 & 80 & 15,624 & 7.18 & 6935 & 2,045 & 463,634 & 8.50 & 9364 & 16 & 2,184 & 4.27 \\
\hline 1902 & 614 & 149,600 & 9.19 & 4393 & 1,849 & 425,017 & 8.70 & 6940 & 0 & 24 & -- & 9404 & 1,682 & 481,280 & 11.05 \\
\hline 1909 & 15 & 2,016 & 4.01 & 4506 & 1,179 & 270,888 & 8.60 & 6944 & 2,055 & 484,090 & 8.90 & 9450 & 1,212 & 309,586 & 9.73 \\
\hline 1954 & 14 & 2,016 & 4.07 & 4692 & 476 & 106,656 & 8.42 & 7080 & 1,363 & 277,331 & 7.46 & 9503 & 342 & 83,256 & 9.04 \\
\hline 2145 & 176 & 55,368 & 12.34 & 4812 & 1,556 & 349,689 & 8.44 & 7196 & 1,651 & 445,356 & 10.42 & 9629 & 1,773 & 481,788 & 10.40 \\
\hline 2242 & 14 & 2,088 & 4.27 & 4865 & 2,096 & 484,184 & 8.67 & 7201 & 183 & 38,616 & 7.76 & 9719 & 776 & 190,528 & 9.43 \\
\hline 2309 & 132 & 33,600 & 9.47 & 4969 & 196 & 46,056 & 9.02 & 7309 & 1,043 & 262,049 & 9.50 & 9724 & 534 & 117,472 & 8.12 \\
\hline 2334 & 986 & 257,534 & 10.09 & 4975 & 1,281 & 283,256 & 8.32 & 7358 & 682 & 151,944 & 8.29 & 9748 & 830 & 196,347 & 8.79 \\
\hline 2500 & 9 & 2,184 & 8.24 & 4978 & 1,714 & 446,857 & 10.01 & 7372 & 15 & 2,112 & 4.16 & 9762 & 1,010 & 267,312 & 10.23 \\
\hline 2654 & 741 & 204,388 & 10.58 & & & & & & & & & & & & \\
\hline
\end{tabular}


Appendix 3-1.7. Number of storms, total duration, and mean storm interevent time defined by 72-hour minimum interevent time for hourly rainfall stations in Oklahoma.

[--, not available]

\begin{tabular}{|c|c|c|c|c|c|c|c|c|c|c|c|c|c|c|c|}
\hline $\begin{array}{c}\text { Station } \\
\text { no. }\end{array}$ & $\begin{array}{c}\text { No. } \\
\text { of } \\
\text { storms }\end{array}$ & $\begin{array}{c}\text { Total } \\
\text { duration } \\
\text { (hours) }\end{array}$ & $\begin{array}{c}\text { Mean } \\
\text { storm } \\
\text { interevent } \\
\text { time } \\
\text { (days) }\end{array}$ & $\begin{array}{c}\text { Station } \\
\text { no. }\end{array}$ & $\begin{array}{c}\text { No. } \\
\text { of } \\
\text { storms }\end{array}$ & $\begin{array}{c}\text { Total } \\
\text { duration } \\
\text { (hours) }\end{array}$ & $\begin{array}{c}\text { Mean } \\
\text { storm } \\
\text { interevent } \\
\text { time } \\
\text { (days) }\end{array}$ & $\begin{array}{c}\text { Station } \\
\text { no. }\end{array}$ & $\begin{array}{c}\text { No. } \\
\text { of } \\
\text { storms }\end{array}$ & $\begin{array}{c}\text { Total } \\
\text { duration } \\
\text { (hours) }\end{array}$ & $\begin{array}{c}\text { Mean } \\
\text { storm } \\
\text { interevent } \\
\text { time } \\
\text { (days) }\end{array}$ & $\begin{array}{c}\text { Station } \\
\text { no. }\end{array}$ & $\begin{array}{c}\text { No. } \\
\text { of } \\
\text { storms }\end{array}$ & $\begin{array}{c}\text { Total } \\
\text { duration } \\
\text { (hours) }\end{array}$ & $\begin{array}{c}\text { Mean } \\
\text { storm } \\
\text { interevent } \\
\text { time } \\
\text { (days) }\end{array}$ \\
\hline 0017 & 11 & 2,016 & 4.50 & 2665 & 872 & 279,696 & 12.12 & 5063 & 7 & 2,208 & 11.28 & 7412 & 1,319 & 484,014 & 13.99 \\
\hline 0026 & 632 & 189,192 & 10.86 & 2678 & 7 & 2,016 & 9.64 & 5068 & 58 & 16,080 & 9.61 & 7556 & 264 & 91,044 & 12.84 \\
\hline 0179 & 803 & 306,537 & 14.58 & 2849 & 1,290 & 448,934 & 13.01 & 5108 & 1,794 & 478,177 & 9.55 & 7588 & 387 & 158,232 & 15.75 \\
\hline 0188 & 499 & 200,904 & 15.50 & 2852 & 165 & 49,728 & 11.46 & 5329 & 8 & 2,016 & 6.77 & 7660 & 1,325 & 483,990 & 13.92 \\
\hline 0215 & 1,437 & 483,917 & 12.55 & 2994 & 283 & 72,336 & 8.92 & 5463 & 787 & 288,975 & 13.81 & 7675 & 954 & 309,543 & 11.98 \\
\hline 0242 & 676 & 227,208 & 12.71 & 2997 & 195 & 46,728 & 8.62 & 5581 & 8 & 2,016 & 5.83 & 7705 & 1,633 & 484,086 & 10.84 \\
\hline 0256 & 1,734 & 468,853 & 9.59 & 3002 & 1,296 & 484,178 & 14.41 & 5582 & 182 & 55,368 & 11.14 & 7714 & 447 & 195,072 & 16.94 \\
\hline 0260 & 11 & 5,808 & 18.61 & 3281 & 1,435 & 438,744 & 11.32 & 5589 & 778 & 238,629 & 11.24 & 7732 & 690 & 201,624 & 10.67 \\
\hline 0292 & 481 & 181,645 & 14.13 & 3286 & 1,579 & 455,654 & 10.35 & 5648 & 1,410 & 484,213 & 12.88 & 7739 & 861 & 254,039 & 10.54 \\
\hline 0293 & 735 & 219,878 & 11.05 & 3304 & 1,454 & 483,966 & 12.49 & 5662 & 340 & 86,616 & 8.80 & 8029 & 512 & 200,160 & 15.07 \\
\hline 0296 & 352 & 98,640 & 9.92 & 3353 & 8 & 1,896 & 6.17 & 5664 & 640 & 198,470 & 11.32 & 8092 & 3 & 1,464 & 20.33 \\
\hline 0535 & 513 & 162,830 & 11.41 & 3407 & 201 & 84,292 & 15.56 & 6130 & 1,656 & 484,134 & 10.43 & 8101 & 1,271 & 484,151 & 14.48 \\
\hline 0537 & 169 & 46,007 & 9.61 & 3497 & 1,583 & 484,026 & 11.23 & 6328 & 387 & 168,768 & 16.86 & 8290 & 989 & 290,040 & 10.63 \\
\hline 0670 & 1,891 & 484,231 & 8.94 & 3628 & 1,311 & 484,135 & 14.03 & 6391 & 13 & 4,416 & 10.52 & 8420 & 460 & 146,696 & 11.78 \\
\hline 0814 & 67 & 34,320 & 20.16 & 3700 & 913 & 276,120 & 10.98 & 6485 & 1,625 & 459,723 & 10.08 & 8470 & 30 & 20,496 & 26.91 \\
\hline 0908 & 712 & 335,099 & 18.29 & 3740 & 1,494 & 483,978 & 11.97 & 6612 & 8 & 21,154 & 107.20 & 8497 & 1,763 & 484,182 & 9.76 \\
\hline 0912 & 479 & 158,520 & 12.52 & 3830 & 266 & 86,928 & 12.09 & 6616 & 1,453 & 483,347 & 12.29 & 8501 & 1,532 & 453,753 & 10.80 \\
\hline 1148 & 8 & 2,088 & 6.51 & 3835 & 85 & 29,640 & 11.78 & 6620 & 630 & 188,946 & 10.94 & 8504 & 159 & 56,224 & 13.37 \\
\hline 1168 & 1,170 & 288,946 & 8.54 & 4001 & 4 & 1,296 & 13.50 & 6627 & 794 & 233,650 & 10.54 & 8708 & 820 & 295,443 & 13.52 \\
\hline 1391 & 11 & 2,136 & 4.44 & 4008 & 27 & 5,808 & 7.62 & 6638 & 1,536 & 466,694 & 10.98 & 8769 & 1,544 & 435,941 & 10.14 \\
\hline 1436 & 954 & 268,080 & 10.01 & 4010 & 666 & 173,856 & 9.17 & 6643 & 101 & 26,304 & 9.12 & 8879 & 174 & 67,200 & 14.68 \\
\hline 1437 & 633 & 216,039 & 12.41 & 4051 & 818 & 229,608 & 10.23 & 6656 & 220 & 56,808 & 8.91 & 8884 & 2 & 672 & 14.00 \\
\hline 1544 & 1,909 & 483,436 & 8.66 & 4052 & 844 & 251,204 & 10.79 & 6661 & 1,833 & 484,166 & 9.05 & 8992 & 1,974 & 482,102 & 8.11 \\
\hline 1684 & 1,445 & 434,246 & 10.92 & 4098 & 1,572 & 422,152 & 9.59 & 6729 & 1,385 & 372,427 & 9.48 & 9014 & 36 & 16,080 & 17.03 \\
\hline 1688 & 103 & 50,400 & 18.96 & 4202 & 1,304 & 445,343 & 12.81 & 6740 & 537 & 186,220 & 13.07 & 9023 & 1,870 & 481,954 & 8.91 \\
\hline 1750 & 1,234 & 392,817 & 11.83 & 4204 & 138 & 45,192 & 12.06 & 6760 & 747 & 246,192 & 12.50 & 9247 & 9 & 2,016 & 5.12 \\
\hline 1855 & 10 & 2,016 & 4.65 & 4384 & 1,778 & 455,726 & 8.97 & 6859 & 1,667 & 484,210 & 10.50 & 9278 & 8 & 1,896 & 6.31 \\
\hline 1891 & 2 & 2,784 & 58.00 & 4386 & 972 & 248,882 & 8.86 & 6926 & 5 & 2,184 & 13.10 & 9300 & 10 & 2,184 & 4.72 \\
\hline 1900 & 1,154 & 308,928 & 9.54 & 4388 & 62 & 15,624 & 8.46 & 6935 & 1,686 & 463,634 & 9.79 & 9364 & 11 & 2,184 & 5.04 \\
\hline 1902 & 495 & 149,600 & 10.82 & 4393 & 1,524 & 425,017 & 10.03 & 6940 & 0 & 24 & -- & 9404 & 1,414 & 481,280 & 12.68 \\
\hline 1909 & 11 & 2,016 & 4.79 & 4506 & 955 & 270,888 & 10.06 & 6944 & 1,693 & 484,090 & 10.28 & 9450 & 992 & 309,586 & 11.35 \\
\hline 1954 & 10 & 2,016 & 5.02 & 4692 & 395 & 106,656 & 9.66 & 7080 & 1,102 & 277,331 & 8.65 & 9503 & 284 & 83,256 & 10.40 \\
\hline 2145 & 159 & 55,368 & 13.39 & 4812 & 1,272 & 349,689 & 9.78 & 7196 & 1,390 & 445,356 & 11.91 & 9629 & 1,491 & 481,788 & 11.90 \\
\hline 2242 & 8 & 2,088 & 5.58 & 4865 & 1,771 & 484,184 & 9.81 & 7201 & 144 & 38,616 & 9.18 & 9719 & 660 & 190,528 & 10.65 \\
\hline 2309 & 114 & 33,600 & 10.57 & 4969 & 155 & 46,056 & 10.75 & 7309 & 845 & 262,049 & 11.16 & 9724 & 426 & 117,472 & 9.54 \\
\hline 2334 & 802 & 257,534 & 11.84 & 4975 & 1,054 & 283,256 & 9.58 & 7358 & 548 & 151,944 & 9.72 & 9748 & 682 & 196,347 & 10.17 \\
\hline 2500 & 7 & 2,184 & 9.91 & 4978 & 1,439 & 446,857 & 11.46 & 7372 & 11 & 2,112 & 4.87 & 9762 & 859 & 267,312 & 11.59 \\
\hline 2654 & 611 & 204,388 & 12.30 & & & & & & & & & & & & \\
\hline
\end{tabular}


142 Statistical Characteristics of Storm Interevent Time, Depth, and Duration for Eastern New Mexico, Oklahoma, and Texas

Appendix 3-2.1. L-moments of storm depth defined by 6-hour minimum interevent time for hourly rainfall stations in Oklahoma.

[--, not available]

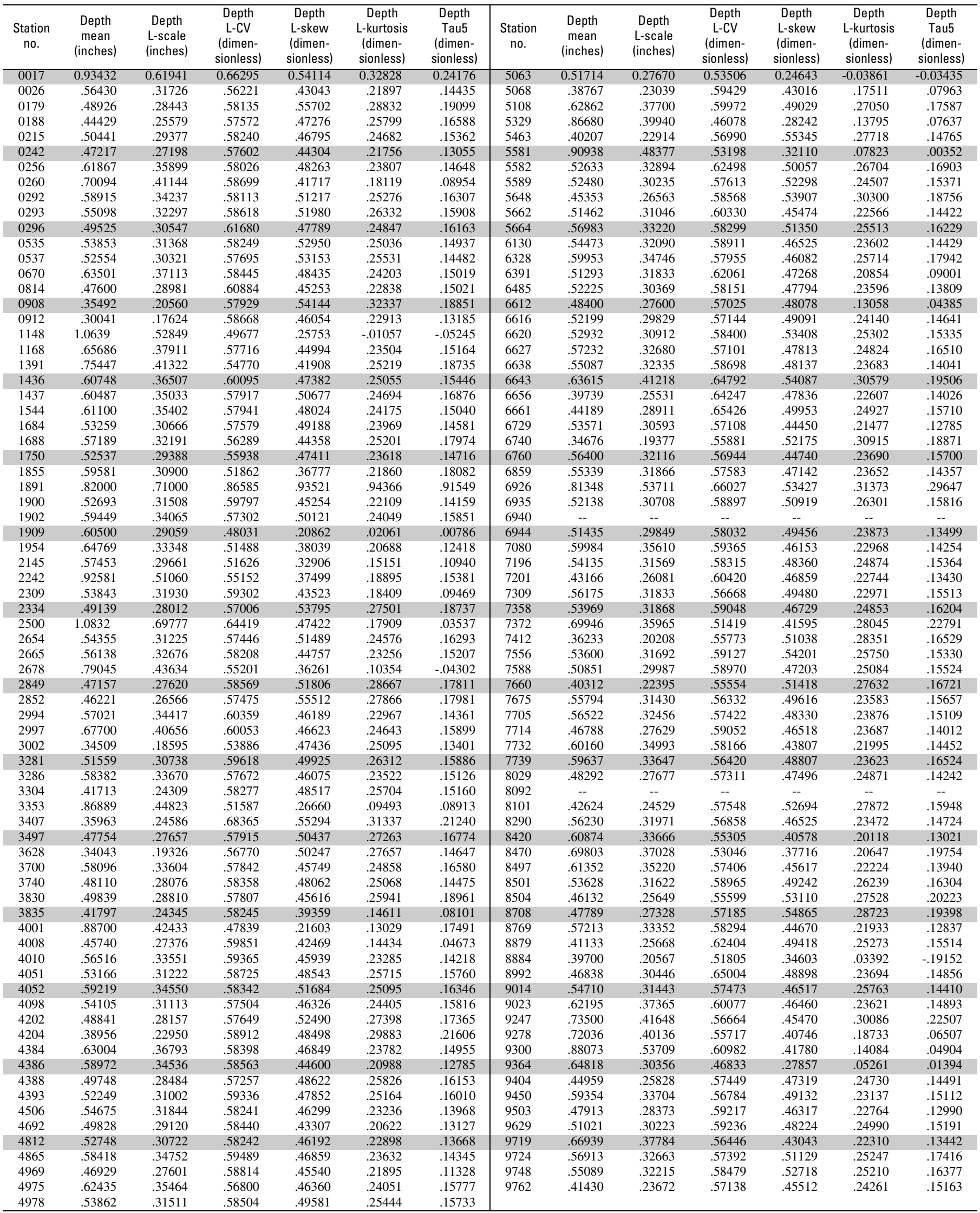


Appendix 3-2.2. L-moments of storm depth defined by 8-hour minimum interevent time for hourly rainfall stations in Oklahoma.

[--, not available]

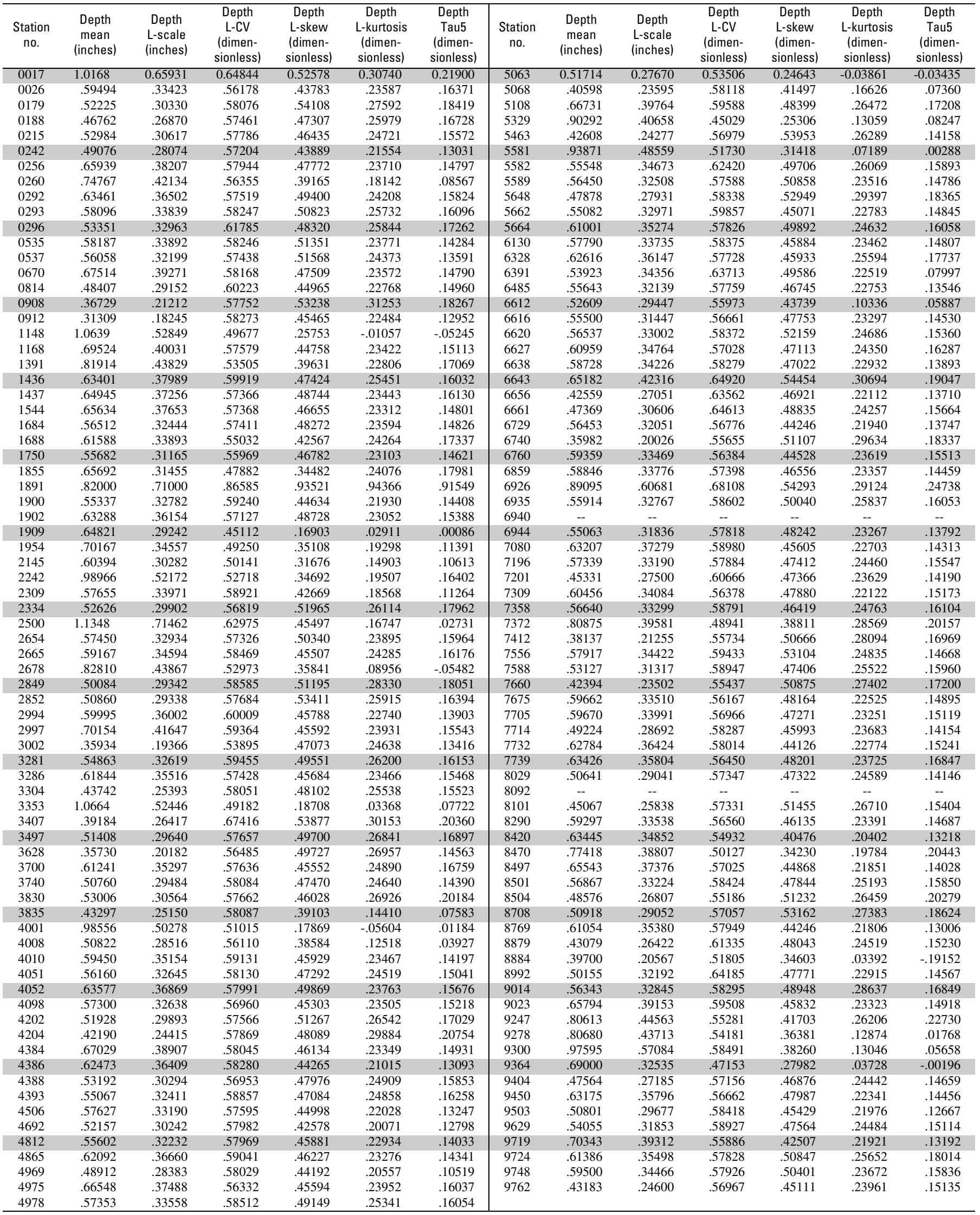


144 Statistical Characteristics of Storm Interevent Time, Depth, and Duration for Eastern New Mexico, Oklahoma, and Texas

Appendix 3-2.3. L-moments of storm depth defined by 12-hour minimum interevent time for hourly rainfall stations in Oklahoma.

[--, not available]

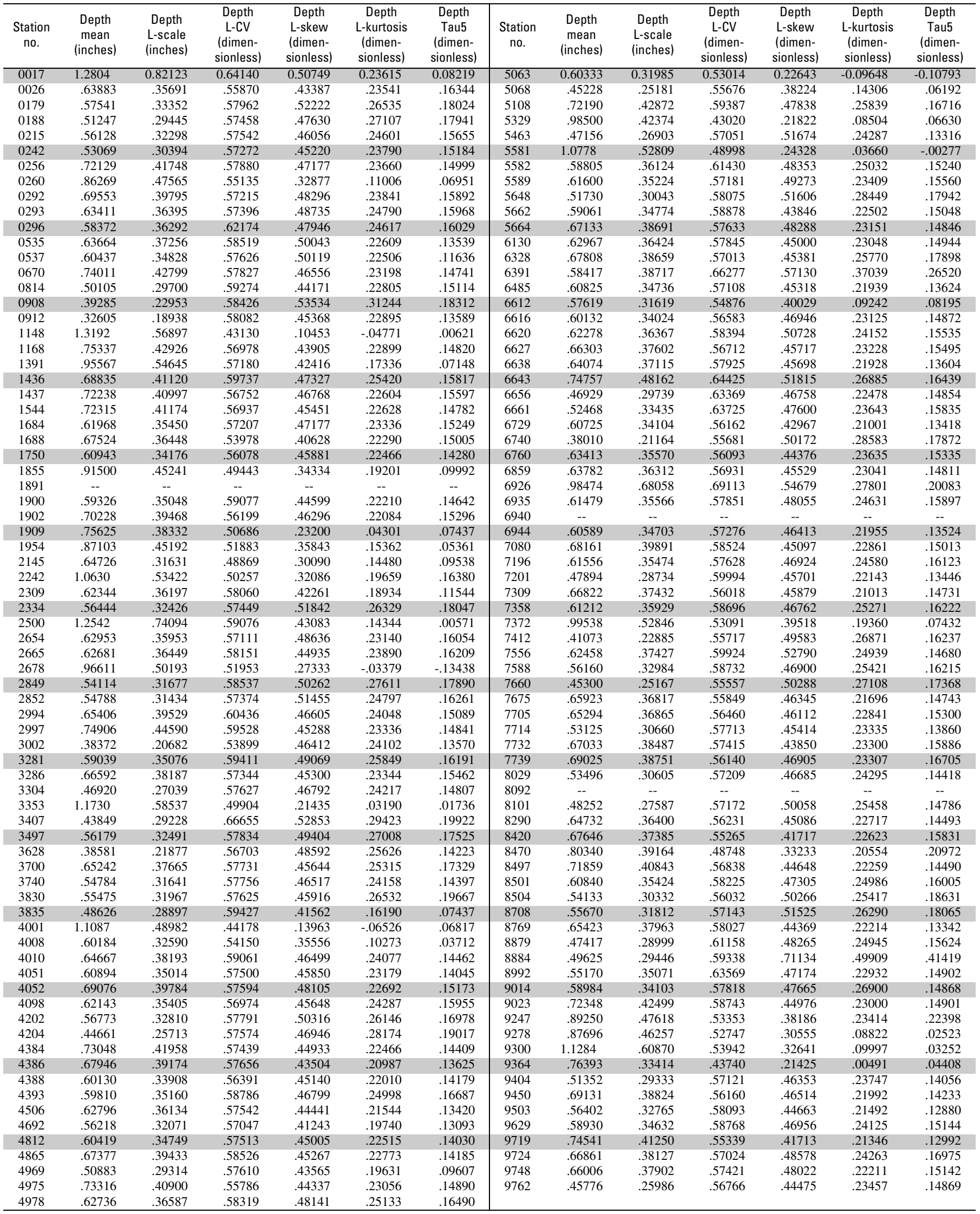


Appendix 3-2.4. L-moments of storm depth defined by 18-hour minimum interevent time for hourly rainfall stations in Oklahoma.

[--, not available]

\begin{tabular}{|c|c|c|c|c|c|c|c|c|c|c|c|c|c|}
\hline $\begin{array}{c}\text { Station } \\
\text { no. }\end{array}$ & $\begin{array}{c}\text { Depth } \\
\text { mean } \\
\text { (inches) }\end{array}$ & $\begin{array}{l}\text { Depth } \\
\text { L-scale } \\
\text { (inches) }\end{array}$ & $\begin{array}{c}\text { Depth } \\
\text { L-CV } \\
\text { (dimen- } \\
\text { sionless) }\end{array}$ & $\begin{array}{c}\text { Depth } \\
\text { L-skew } \\
\text { (dimen- } \\
\text { sionless) }\end{array}$ & $\begin{array}{c}\text { Depth } \\
\text { L-kurtosis } \\
\text { (dimen- } \\
\text { sionless) }\end{array}$ & $\begin{array}{c}\text { Depth } \\
\text { Tau5 } \\
\text { (dimen- } \\
\text { sionless) }\end{array}$ & $\begin{array}{c}\text { Station } \\
\text { no. }\end{array}$ & $\begin{array}{l}\text { Depth } \\
\text { mean } \\
\text { (inches) }\end{array}$ & $\begin{array}{l}\text { Depth } \\
\text { L-scale } \\
\text { (inches) }\end{array}$ & $\begin{array}{c}\text { Depth } \\
\text { L-CV } \\
\text { (dimen- } \\
\text { sionless) }\end{array}$ & $\begin{array}{c}\text { Depth } \\
\text { L-skew } \\
\text { (dimen- } \\
\text { sionless) }\end{array}$ & $\begin{array}{c}\text { Depth } \\
\text { L-kurtosis } \\
\text { (dimen- } \\
\text { sionless) }\end{array}$ & $\begin{array}{c}\text { Depth } \\
\text { Tau5 } \\
\text { (dimen- } \\
\text { sionless) }\end{array}$ \\
\hline 0017 & 1.5030 & 0.96372 & 0.64118 & 0.51620 & 0.24697 & 0.06539 & 5063 & 0.65818 & 0.32309 & 0.49088 & 0.16151 & -0.10204 & -0.09848 \\
\hline 0026 & .70566 & .39718 & .56285 & .43825 & .24453 & .17249 & 5068 & .50058 & .27198 & .54333 & .33874 & .10209 & .05207 \\
\hline 0179 & .63955 & .37122 & .58044 & .50779 & .25613 & .17091 & 5108 & .79166 & .46444 & .58667 & .46330 & .24617 & .15984 \\
\hline 0188 & .56049 & .32312 & .57650 & .47330 & .26528 & .17410 & 5329 & 1.1405 & .47047 & .41250 & .13120 & .05048 & .11910 \\
\hline 0215 & 60989 & .34776 & .57021 & .45205 & .24292 & .15774 & 5463 & .51210 & .29249 & .57117 & .50067 & .23134 & .12648 \\
\hline 0242 & .57356 & .32682 & .56981 & .44287 & .22969 & .14621 & 5581 & 1.3857 & .66910 & .48285 & .27683 & .12293 & .07773 \\
\hline 0256 & .80191 & .46077 & .57459 & .45824 & .22673 & .14328 & 5582 & .64246 & .39527 & .61524 & .47676 & .23870 & .14527 \\
\hline 0260 & .93458 & .56451 & 60402 & .40997 & .14489 & .04058 & 5589 & .68716 & .38806 & .56474 & .46747 & .21936 & .14741 \\
\hline 0292 & .79140 & .45081 & .56964 & .46567 & .22116 & .13464 & 5648 & .57055 & .33062 & .57948 & .50283 & .27319 & .17484 \\
\hline 0293 & .69551 & .40046 & .57577 & .48150 & .24411 & .15404 & 5662 & .65823 & .38739 & .58854 & .43651 & .22234 & .14442 \\
\hline 0296 & .64892 & .40761 & .62814 & .49112 & .26125 & .17256 & 5664 & .74272 & .42347 & .57016 & .46298 & .22122 & .14184 \\
\hline 0535 & .70913 & .41165 & .58050 & .47723 & .20979 & .12694 & 6130 & .69576 & .39814 & .57224 & .43335 & .21645 & .13979 \\
\hline 0537 & .66460 & .37809 & .56889 & .46957 & .19408 & .09494 & 6328 & .73805 & .41823 & .56666 & .43915 & .24273 & .16704 \\
\hline 0670 & .81395 & .46634 & .57294 & .45177 & .22383 & .14268 & 6391 & .63727 & .40657 & 63799 & .54947 & .36819 & .26534 \\
\hline 0814 & .52889 & .30509 & .57684 & .42191 & .21909 & .15340 & 6485 & .66593 & .37937 & .56968 & .44784 & .22207 & .14168 \\
\hline 0908 & .42488 & .24989 & .58814 & .52850 & .30448 & .18150 & 6612 & .67222 & .42255 & .62859 & .54505 & .24575 & .14018 \\
\hline 0912 & .34860 & .20334 & .58329 & .45968 & .23962 & .14591 & 6616 & .65777 & .37121 & .56434 & .46129 & .22996 & .15273 \\
\hline 1148 & 1.4339 & .62751 & .43762 & .09358 & -.06265 & -.00532 & 6620 & .67731 & .39289 & .58007 & .48839 & .22980 & .15195 \\
\hline 1168 & .82867 & .47158 & .56908 & .43669 & .22847 & .14613 & 6627 & .72897 & .40821 & .55998 & .43962 & .22033 & .14968 \\
\hline 1391 & 1.1027 & 65392 & .59302 & .47285 & .24162 & .14543 & 6638 & .69821 & .40445 & .57928 & .45122 & .21572 & .13501 \\
\hline 1436 & .74890 & .44873 & .59919 & .47118 & .24745 & .14951 & 6643 & .83747 & .53489 & .63870 & .50835 & .26398 & .16229 \\
\hline 1437 & .81526 & .45289 & .55552 & .43984 & .21219 & .14654 & 6656 & .51876 & .32077 & .61835 & .44726 & .21400 & .14796 \\
\hline 1544 & .81304 & .45867 & .56414 & .43884 & .21700 & .14331 & 6661 & .57985 & .36444 & .62851 & .46544 & .23166 & .15694 \\
\hline 1684 & .67791 & .38360 & .56586 & .45292 & .22047 & .14360 & 6729 & .65610 & .37067 & .56495 & .43137 & .21337 & .13745 \\
\hline 1688 & .70943 & .38535 & .54318 & .41496 & .23615 & .16148 & 6740 & .41000 & .22782 & .55565 & .49141 & .28006 & .18026 \\
\hline 1750 & .66778 & .37421 & .56038 & .44882 & .21835 & .14165 & 6760 & .68126 & .37709 & .55352 & .43137 & .22465 & .14475 \\
\hline 1855 & 1.1139 & .54838 & .49230 & .33141 & .14158 & .03419 & 6859 & .69965 & .39597 & .56596 & .44293 & .21608 & .13665 \\
\hline 1891 & -- & -- & -- & - & - & -- & 6926 & 1.1694 & .76079 & .65060 & .48425 & .24807 & .22481 \\
\hline 1900 & .64392 & .37851 & .58781 & .43898 & .21802 & .14687 & 6935 & .67504 & .38739 & .57388 & .46516 & .23539 & .15359 \\
\hline 1902 & .76726 & .42650 & .55587 & .44750 & .21349 & .14645 & 6940 & -- & -- & -- & -- & -- & -- \\
\hline 1909 & .82500 & .41275 & .50030 & .23558 & .08475 & .13814 & 6944 & .66508 & .37882 & .56958 & .44923 & .20908 & .13184 \\
\hline 1954 & 1.1482 & .59528 & .51846 & .26682 & .03399 & .01083 & 7080 & .75965 & .44402 & .58451 & .44682 & .22444 & .14591 \\
\hline 2145 & .70046 & .34707 & .49548 & .31329 & .15415 & .09773 & 7196 & .66819 & .38091 & .57007 & .45335 & .23355 & .15626 \\
\hline 2242 & 1.1038 & .56692 & .51359 & .31228 & .15354 & .12589 & 7201 & .53362 & .31902 & .59784 & .46470 & .25071 & .16937 \\
\hline 2309 & .67865 & .39456 & .58139 & .41427 & .17388 & .10475 & 7309 & .73791 & .40972 & .55524 & .44400 & .20916 & .15017 \\
\hline 2334 & .61855 & .35456 & .57321 & .49925 & .24819 & .17133 & 7358 & .66587 & .38809 & .58283 & .45591 & .24170 & .15502 \\
\hline 2500 & 1.4018 & .84537 & .60307 & .44208 & .14732 & -.00329 & 7372 & 1.0783 & .61772 & .57284 & .43764 & .18492 & .02284 \\
\hline 2654 & .71497 & .40211 & .56241 & .45751 & .21767 & .15813 & 7412 & .44544 & .24848 & .55784 & .48458 & .25899 & .15624 \\
\hline 2665 & .68200 & .39371 & .57729 & .44565 & .23830 & .16184 & 7556 & .69040 & .41228 & .59716 & .50792 & .22977 & .12644 \\
\hline 2678 & 1.3377 & .73500 & .54945 & .26521 & -.02559 & -.01580 & 7588 & .61585 & .36166 & .58725 & .47006 & .25367 & .15363 \\
\hline 2849 & .60075 & .35464 & .59032 & .50212 & .27680 & .18049 & 7660 & .49439 & .27345 & .55310 & .48762 & .25693 & .16455 \\
\hline 2852 & .60127 & .34199 & .56877 & .49028 & .23463 & .16018 & 7675 & .72528 & .40407 & .55713 & .45219 & .21362 & .14474 \\
\hline 2994 & .72515 & .43804 & .60406 & .47128 & .25127 & .15966 & 7705 & .71641 & .40270 & .56210 & .44975 & .22363 & .15196 \\
\hline 2997 & .80628 & .47494 & .58905 & .44278 & .22528 & .13839 & 7714 & .56634 & .32828 & .57965 & .45366 & .22839 & .12881 \\
\hline 3002 & .41407 & .22501 & .54342 & .46037 & .23817 & .13493 & 7732 & .71897 & .40863 & .56836 & .42835 & .22434 & .15124 \\
\hline 3281 & .63816 & .37825 & .59273 & .48355 & .25320 & .15971 & 7739 & .76958 & .42982 & .55851 & .45631 & .22961 & .16366 \\
\hline 3286 & .73202 & .41608 & .56840 & .44170 & .22554 & .14829 & 8029 & .57225 & .32639 & .57036 & .46396 & .24458 & .15101 \\
\hline 3304 & .51033 & .29289 & .57392 & .45767 & .23286 & .14336 & 8092 & -- & -- & -- & -- & -- & -- \\
\hline 3353 & 1.3033 & 63379 & .48628 & .19970 & .00761 & -.01681 & 8101 & .52261 & .29874 & .57163 & .48681 & .24103 & 13999 \\
\hline 3407 & .48607 & .31275 & .64342 & .50112 & .27649 & .18949 & 8290 & .71401 & .40236 & .56352 & .44716 & .22620 & .14531 \\
\hline 3497 & .61084 & .35291 & .57775 & .48697 & .26874 & .17846 & 8420 & .72650 & .39732 & .54690 & .40661 & .21990 & .15607 \\
\hline 3628 & .41822 & .23893 & .57129 & .48503 & .25734 & .14901 & 8470 & .88708 & .41922 & .47258 & .31240 & .20356 & 19986 \\
\hline 3700 & .70140 & .40333 & .57504 & .45730 & .25798 & .17778 & 8497 & .78629 & .44123 & .56115 & .43029 & .21107 & .13761 \\
\hline 3740 & .58767 & .33819 & .57549 & .45729 & .23460 & .14046 & 8501 & .66376 & .38358 & .57789 & .46184 & .24078 & .15538 \\
\hline 3830 & .60726 & .34937 & .57532 & .46086 & .27983 & .22037 & 8504 & .60620 & .33420 & .55131 & .47325 & .23510 & .16992 \\
\hline 3835 & .54183 & .31920 & .58912 & .39116 & .13188 & .05774 & 8708 & .60696 & .34548 & .56919 & .50146 & .25861 & .18151 \\
\hline 4001 & 1.4783 & .69233 & .46832 & .09581 & -.03996 & .12277 & 8769 & .71101 & .41035 & .57714 & .43583 & .21596 & .13010 \\
\hline 4008 & .69303 & .38049 & .54903 & .32939 & .05576 & .01736 & 8879 & .51338 & .31072 & .60524 & .47395 & .24723 & .15976 \\
\hline 4010 & .70153 & .41169 & .58685 & .45740 & .23648 & .14860 & 8884 & .56714 & .33286 & .58690 & .62747 & .40773 & .43920 \\
\hline 4051 & .66712 & .38384 & .57536 & .45253 & .22587 & .13511 & 8992 & .61318 & .38409 & .62640 & .45909 & .22295 & .14849 \\
\hline 4052 & .76662 & .44021 & .57422 & .46868 & .22392 & .15272 & 9014 & .60887 & .34726 & .57034 & .46884 & .26375 & .14037 \\
\hline 4098 & .67522 & .38429 & .56913 & .45187 & .24075 & .15776 & 9023 & .79537 & .46103 & .57963 & .43724 & .22205 & .14408 \\
\hline 4202 & .63116 & .36549 & .57908 & .49188 & .25274 & .16361 & 9247 & .92556 & .47769 & .51611 & .38100 & .24216 & .22797 \\
\hline 4204 & .48963 & .27866 & .56912 & .45325 & .26573 & .17889 & 9278 & 1.0616 & .49257 & .46400 & .18946 & .05420 & .04935 \\
\hline 4384 & .81067 & .46475 & .57328 & .44561 & .22631 & .14709 & 9300 & 1.2896 & .74142 & .57490 & .41827 & .22374 & .16967 \\
\hline 4386 & .75537 & .43448 & .57519 & .43304 & .21345 & .13975 & 9364 & .89125 & .42172 & .47318 & .27725 & .10850 & .18686 \\
\hline 4388 & .63440 & .35763 & .56372 & .43812 & .19763 & .11859 & 9404 & .55897 & .31837 & .56957 & .45198 & .22696 & .13461 \\
\hline 4393 & .65093 & .37858 & .58160 & .45523 & .24158 & .16285 & 9450 & .76516 & .42583 & .55653 & .44647 & .20756 & .13228 \\
\hline 4506 & .69115 & .39289 & .56846 & .43133 & .20974 & .13446 & 9503 & .62971 & .36802 & .58442 & .45440 & .22605 & .13975 \\
\hline 4692 & .59296 & .33634 & .56723 & .41136 & .20088 & .13646 & 9629 & .64441 & .37880 & .58782 & .46237 & .23244 & .14349 \\
\hline 4812 & .65601 & .37515 & .57187 & .44465 & .22383 & .14275 & 9719 & .79109 & .43395 & .54854 & .40982 & .20793 & .12603 \\
\hline 4865 & .73807 & .42989 & .58245 & .44502 & .22102 & .13878 & 9724 & .74477 & .42544 & .57124 & .47689 & .23975 & .16644 \\
\hline 4969 & .55787 & .32337 & .57964 & .44394 & .21060 & .11999 & 9748 & .74046 & .42335 & .57174 & .46369 & .21216 & .14022 \\
\hline 4975 & .79762 & .44102 & .55292 & .42934 & .22140 & .14163 & 9762 & .48626 & .27690 & .56944 & .44043 & .22934 & .14445 \\
\hline 4978 & .68551 & .39636 & .57820 & .46775 & .24295 & .16272 & & & & & & & \\
\hline
\end{tabular}


146 Statistical Characteristics of Storm Interevent Time, Depth, and Duration for Eastern New Mexico, Oklahoma, and Texas

Appendix 3-2.5. L-moments of storm depth defined by 24-hour minimum interevent time for hourly rainfall stations in Oklahoma.

[--, not available]

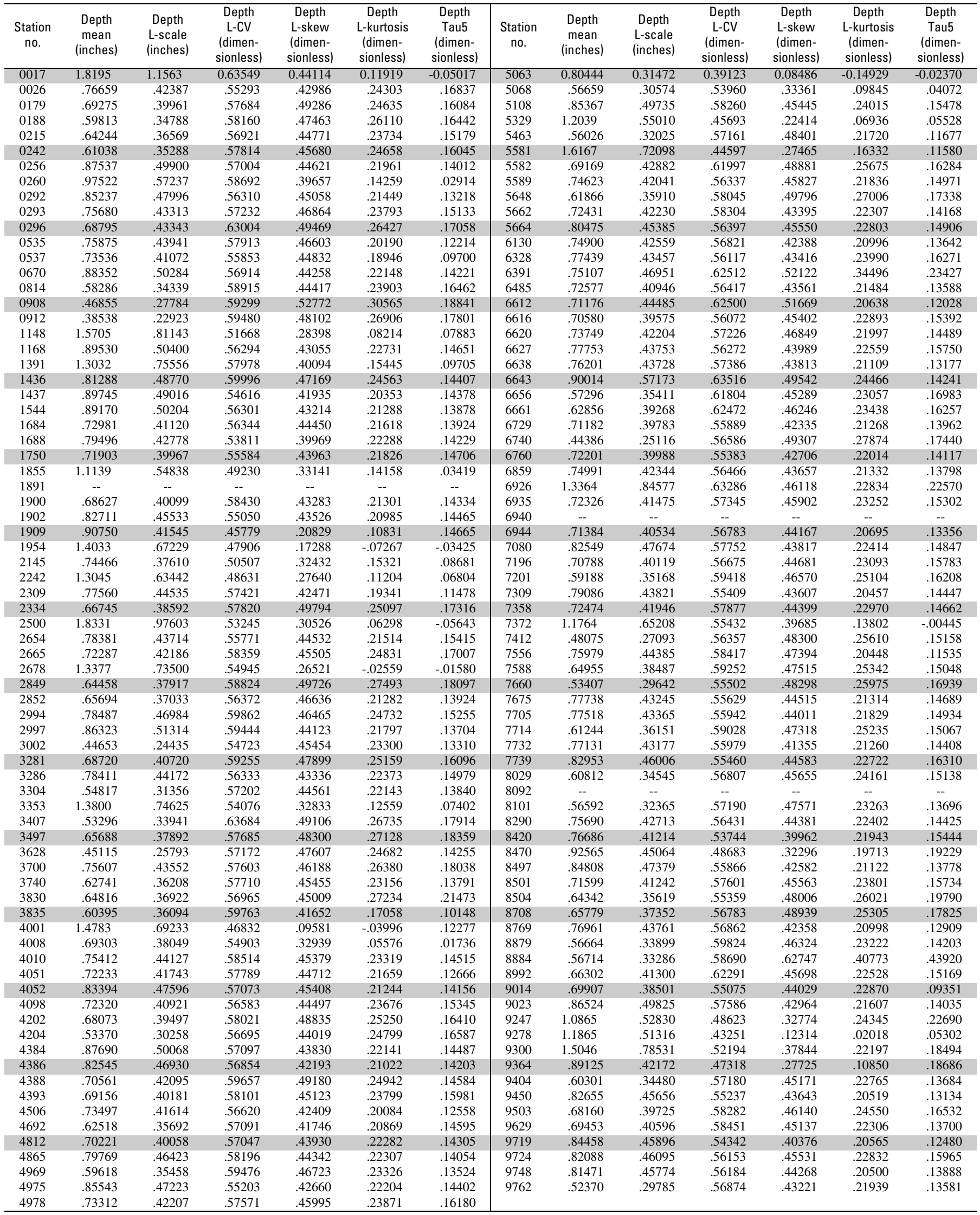


Appendix 3-2.6. L-moments of storm depth defined by 48-hour minimum interevent time for hourly rainfall stations in Oklahoma.

[--, not available]

\begin{tabular}{|c|c|c|c|c|c|c|c|c|c|c|c|c|c|}
\hline $\begin{array}{c}\text { Station } \\
\text { no. }\end{array}$ & $\begin{array}{c}\text { Depth } \\
\text { mean } \\
\text { (inches) }\end{array}$ & $\begin{array}{c}\text { Depth } \\
\text { L-scale } \\
\text { (inches) }\end{array}$ & $\begin{array}{c}\text { Depth } \\
\text { L-CV } \\
\text { (dimen- } \\
\text { sionless) }\end{array}$ & $\begin{array}{l}\text { Depth } \\
\text { L-skew } \\
\text { (dimen- } \\
\text { sionless) }\end{array}$ & $\begin{array}{c}\text { Depth } \\
\text { L-kurtosis } \\
\text { (dimen- } \\
\text { sionless) }\end{array}$ & $\begin{array}{c}\text { Depth } \\
\text { Tau5 } \\
\text { (dimen- } \\
\text { sionless) }\end{array}$ & $\begin{array}{c}\text { Station } \\
\text { no. }\end{array}$ & $\begin{array}{l}\text { Depth } \\
\text { mean } \\
\text { (inches) }\end{array}$ & $\begin{array}{l}\text { Depth } \\
\text { L-scale } \\
\text { (inches) }\end{array}$ & $\begin{array}{c}\text { Depth } \\
\text { L-CV } \\
\text { (dimen- } \\
\text { sionless) }\end{array}$ & $\begin{array}{c}\text { Depth } \\
\text { L-skew } \\
\text { (dimen- } \\
\text { sionless) }\end{array}$ & $\begin{array}{c}\text { Depth } \\
\text { L-kurtosis } \\
\text { (dimen- } \\
\text { sionless) }\end{array}$ & $\begin{array}{c}\text { Depth } \\
\text { Tau5 } \\
\text { (dimen- } \\
\text { sionless) }\end{array}$ \\
\hline 0017 & 2.2520 & 1.3622 & 0.60488 & 0.32654 & -0.04123 & -0.13760 & 5063 & 0.80444 & 0.31472 & 0.39123 & 0.08486 & -0.14929 & -0.02370 \\
\hline 0026 & .93774 & .51961 & .55411 & .43864 & .25898 & .17946 & 5068 & .72620 & .41313 & .56889 & .38291 & .14031 & .07589 \\
\hline 0179 & .82131 & .47028 & .57259 & .47023 & .23363 & .15088 & 5108 & 1.0266 & .59445 & .57906 & .44864 & .23942 & .15289 \\
\hline 0188 & .70307 & .40990 & .58302 & .46645 & .24308 & .13887 & 5329 & 2.1670 & 1.0092 & .46572 & .30139 & .10355 & -.12105 \\
\hline 0215 & .77341 & .44166 & .57106 & .44626 & .24209 & .15916 & 5463 & .69822 & .40106 & .57440 & .46702 & .21777 & .12350 \\
\hline 0242 & .72193 & .42540 & .58925 & .46337 & .24761 & .15437 & 5581 & 2.4008 & 1.0699 & .44565 & .12339 & -.07536 & -.06915 \\
\hline 0256 & 1.0908 & .61257 & .56160 & .42660 & .20863 & .13049 & 5582 & .84756 & .52090 & .61459 & .47669 & .24369 & .14760 \\
\hline 0260 & 1.4019 & .82929 & .59156 & .35067 & .00522 & -.16696 & 5589 & .90790 & .50441 & .55558 & .43712 & .21602 & .14738 \\
\hline 0292 & 1.0584 & .58691 & .55452 & .43974 & .23348 & .15894 & 5648 & .74550 & .43252 & .58018 & .48584 & .26277 & .16703 \\
\hline 0293 & .91236 & .52271 & .57292 & .45887 & .23791 & .15767 & 5662 & .90852 & .53267 & .58631 & .43543 & .22145 & .13586 \\
\hline 0296 & .86727 & .54199 & .62493 & .48338 & .25736 & .16823 & 5664 & .98358 & .55255 & .56177 & .44433 & .22751 & .14551 \\
\hline 0535 & .97875 & .56175 & .57394 & .43286 & .17641 & .09944 & 6130 & .93010 & .52716 & .56678 & .42140 & .21581 & 14067 \\
\hline 0537 & .94341 & .52324 & .55462 & .41058 & .16520 & .09399 & 6328 & .92298 & .53208 & .57648 & .45287 & .25233 & .16357 \\
\hline 0670 & 1.1097 & .62420 & .56250 & .42879 & .21866 & .13870 & 6391 & .95591 & .63405 & .66329 & .59205 & .42529 & .29577 \\
\hline 0814 & .74182 & .47063 & .63443 & .49799 & .24901 & .11913 & 6485 & .90633 & .50893 & .56153 & .42215 & .20812 & .13162 \\
\hline 0908 & .57406 & .34322 & .59789 & .51702 & .29301 & .17752 & 6612 & 1.2100 & .75444 & .62351 & .37113 & -.04040 & -.07511 \\
\hline 0912 & .45742 & .27764 & 60697 & .48341 & .26220 & .16105 & 6616 & .87477 & .49035 & .56055 & .44350 & .22565 & .14517 \\
\hline 1148 & 2.6325 & 1.2223 & .46433 & .07024 & -.15910 & .02764 & 6620 & .88518 & .51044 & .57666 & .46259 & .22630 & .15224 \\
\hline 1168 & 1.0915 & .61157 & .56028 & .42547 & .22993 & .15222 & 6627 & .95793 & .53992 & .56363 & .43414 & .22707 & .15271 \\
\hline 1391 & 1.6165 & .94007 & .58156 & .35743 & .06720 & -.02475 & 6638 & .95210 & .54202 & .56929 & .42403 & .20605 & .13106 \\
\hline 1436 & .99503 & .59172 & .59468 & .45896 & .23390 & .13526 & 6643 & 1.0758 & .71046 & .66042 & .55213 & .33027 & .22951 \\
\hline 1437 & 1.1419 & .61756 & .54080 & .41505 & .22200 & .15247 & 6656 & .71353 & .44417 & .62249 & .45995 & .23966 & .17715 \\
\hline 1544 & 1.1255 & .62529 & .55558 & .41798 & .21094 & .13522 & 6661 & .79583 & .48848 & .61380 & .45187 & .23286 & .16138 \\
\hline 1684 & .89717 & .50764 & .56582 & .44143 & .22526 & .14613 & 6729 & .88213 & .49120 & .55683 & .42265 & .21993 & .14154 \\
\hline 1688 & .92636 & .52831 & .57031 & .43706 & .24007 & .14322 & 6740 & .53167 & .30423 & .57221 & .47948 & .25176 & .14822 \\
\hline 1750 & .88272 & .48691 & .55160 & .42412 & .21740 & .14927 & 6760 & .84799 & .47526 & .56045 & .42448 & .21008 & .13032 \\
\hline 1855 & 1.7408 & 1.1133 & .63956 & .57567 & .43623 & .32691 & 6859 & .92250 & .52293 & .56686 & .43298 & .21585 & .13876 \\
\hline 1891 & -- & -- & -- & -- & -- & -- & 6926 & 1.8878 & 1.1942 & .63258 & .42492 & .14947 & -.03170 \\
\hline 1900 & .84813 & .49156 & .57958 & .42789 & .21530 & .14419 & 6935 & .90611 & .51721 & .57081 & .44260 & .22279 & .14389 \\
\hline 1902 & 1.0534 & .58121 & .55173 & .42518 & .20827 & .13414 & 6940 & -- & -- & -- & -- & -- & -- \\
\hline 1909 & 1.2100 & .72686 & .60071 & .41116 & .13290 & -.01326 & 6944 & .88127 & .49808 & .56518 & .43013 & .20426 & .12914 \\
\hline 1954 & 1.8043 & .93286 & .51702 & .22688 & -.01383 & -.00101 & 7080 & 1.0272 & .58658 & .57106 & .42364 & .21890 & .14624 \\
\hline 2145 & .87159 & .43334 & .49718 & .35255 & .19821 & .11173 & 7196 & .85623 & .48447 & .56582 & .44203 & .23508 & .15943 \\
\hline 2242 & 1.9871 & .85835 & .43195 & .09954 & .03915 & -.00662 & 7201 & .74066 & .44089 & .59527 & .45793 & .22931 & .12850 \\
\hline 2309 & .98712 & .54869 & .55585 & .36670 & .14578 & .09507 & 7309 & 1.0039 & .54614 & .54400 & .41078 & .19788 & .13627 \\
\hline 2334 & .80487 & .46670 & .57984 & .48462 & .25285 & .17857 & 7358 & .88946 & .51239 & .57608 & .43818 & .23058 & .15433 \\
\hline 2500 & 2.5222 & 1.2967 & .51410 & .20939 & -.08887 & -.17322 & 7372 & 1.4500 & .83743 & .57754 & .49936 & .31226 & .11855 \\
\hline 2654 & .96680 & .54681 & .56559 & .45600 & .23977 & .16314 & 7412 & .57354 & .32748 & .57098 & .47122 & .23961 & .14081 \\
\hline 2665 & .84262 & .49046 & .58206 & .44750 & .24055 & .16099 & 7556 & .95405 & .55302 & .57966 & .44250 & .17850 & .09405 \\
\hline 2678 & 1.9125 & 1.4089 & .73669 & .62510 & .37161 & .17085 & 7588 & .78241 & .46039 & .58843 & .45703 & .23242 & .13340 \\
\hline 2849 & .78759 & .46346 & .58845 & .48592 & .27062 & .18118 & 7660 & .63292 & .35338 & .55833 & .46889 & .24363 & .15195 \\
\hline 2852 & .75479 & .43205 & .57241 & .45795 & .19923 & .12087 & 7675 & .95292 & .52783 & .55390 & .43027 & .21195 & .14368 \\
\hline 2994 & .96968 & .57091 & .58876 & .44658 & .23618 & .14881 & 7705 & .95373 & .52916 & .55483 & .42619 & .22055 & .15401 \\
\hline 2997 & 1.0555 & .61150 & .57935 & .40006 & .17624 & .11285 & 7714 & .73738 & .43486 & .58974 & .45844 & .23258 & .13276 \\
\hline 3002 & .52382 & .29162 & .55672 & .44630 & .22174 & .12627 & 7732 & .93028 & .52136 & .56043 & .41983 & .22853 & .16211 \\
\hline 3281 & .83222 & .48778 & .58612 & .45861 & .23879 & .15568 & 7739 & 1.0579 & .57966 & .54793 & .41897 & .21361 & .15334 \\
\hline 3286 & .97514 & .54739 & .56135 & .42246 & .21753 & .14543 & 8029 & .73858 & .42371 & .57368 & .44558 & .22095 & .12867 \\
\hline 3304 & .65986 & .37974 & .57548 & .44299 & .22270 & .14376 & 8092 & -- & -- & -- & -- & -- & -- \\
\hline 3353 & 1.9550 & .89955 & .46013 & .17837 & .11426 & .05791 & 8101 & .69705 & .39966 & .57336 & .44947 & .20505 & .11592 \\
\hline 3407 & .66264 & .41940 & 63292 & .48236 & .25668 & .17398 & 8290 & .93417 & .53221 & .56972 & .44153 & .22566 & .14877 \\
\hline 3497 & .81435 & .47098 & .57835 & .47166 & .26182 & .17286 & 8420 & .92190 & .49333 & .53512 & .38846 & .20492 & .14106 \\
\hline 3628 & .54103 & .31033 & .57359 & .45395 & .22761 & .13863 & 8470 & 1.2166 & .62217 & .51141 & .37056 & .19596 & .11169 \\
\hline 3700 & .91339 & .53077 & .58110 & .46915 & .27420 & .18754 & 8497 & 1.0446 & .57725 & .55260 & .41223 & .20327 & .12696 \\
\hline 3740 & .76703 & .44464 & .57968 & .44850 & .22278 & .12704 & 8501 & .87782 & .51081 & .58191 & .45252 & .23470 & .15320 \\
\hline 3830 & .78184 & .45387 & .58051 & .46626 & .28379 & .21220 & 8504 & .78032 & .43157 & .55307 & .47526 & .28297 & .21895 \\
\hline 3835 & .83752 & .47222 & .56383 & .36970 & .15252 & .10306 & 8708 & .80502 & .46326 & .57547 & .48289 & .25115 & .16459 \\
\hline 4001 & -- & -- & -- & -- & -- & -- & 8769 & .94553 & .53994 & .57105 & .42358 & .21143 & .13347 \\
\hline 4008 & .76233 & .42941 & .56329 & .35723 & .10367 & .07743 & 8879 & .67940 & .40826 & .60091 & .46931 & .24070 & .14943 \\
\hline 4010 & .93861 & .54303 & .57855 & .43425 & .21384 & .12945 & 8884 & -- & -- & -- & -- & -- & -- \\
\hline 4051 & .87738 & .50931 & .58048 & .44299 & .21485 & .13202 & 8992 & .83993 & .51333 & .61115 & .44104 & .21684 & .14574 \\
\hline 4052 & 1.0416 & .58924 & .56571 & .44172 & .22033 & .14740 & 9014 & .85795 & .44618 & .52005 & .36441 & .13629 & .05570 \\
\hline 4098 & .88362 & .50076 & .56672 & .43701 & .23022 & .14333 & 9023 & 1.0690 & 60701 & .56782 & .42225 & .21681 & .13928 \\
\hline 4202 & .81855 & .47486 & .58012 & .47349 & .24355 & .15450 & 9247 & 1.8550 & 1.0733 & .57862 & .42730 & .21429 & .01442 \\
\hline 4204 & .63160 & 36675 & .58067 & 49549 & .31677 & .20829 & 9278 & 1.8109 & .88145 & .48675 & .27764 & .06284 & -.10864 \\
\hline 4384 & 1.0829 & .61528 & .56817 & .42727 & .21998 & .14759 & 9300 & 2.2093 & 1.2710 & .57527 & .37566 & .16448 & .09455 \\
\hline 4386 & 1.0135 & .56509 & .55757 & .40832 & .20823 & .13958 & 9364 & 1.2788 & .73442 & .57432 & .37678 & .04869 & .01869 \\
\hline 4388 & .86438 & .49038 & .56732 & .44212 & .20090 & .10999 & 9404 & .73207 & .41733 & .57007 & .43935 & .21618 & .12656 \\
\hline 4393 & .84491 & .48943 & .57927 & .44253 & .23302 & .15620 & 9450 & 1.0284 & .56809 & .55239 & .42573 & .20457 & .13015 \\
\hline 4506 & .91450 & .50543 & .55269 & .39880 & .19057 & .12143 & 9503 & .83705 & .49433 & .59057 & .48041 & .27407 & .18394 \\
\hline 4692 & .77359 & .44394 & .57386 & .41568 & .20730 & .14076 & 9629 & .85081 & .49587 & .58282 & .44243 & .21561 & .13040 \\
\hline 4812 & .86512 & .48645 & .56229 & .41997 & .21158 & .13957 & 9719 & .99804 & .53982 & .54088 & .39514 & .19981 & .11997 \\
\hline 4865 & .97048 & .55789 & .57487 & .42562 & .20872 & 13092 & 9724 & 1.0530 & .58176 & .55248 & .43537 & .22467 & .15155 \\
\hline 4969 & .70872 & .43307 & .61106 & .49105 & .26004 & .15486 & 9748 & 1.0473 & .57070 & .54490 & .41510 & .20407 & .13252 \\
\hline 4975 & 1.0400 & .58103 & .55870 & .42235 & .21074 & .12705 & 9762 & .62637 & .35943 & .57384 & .43053 & .21269 & .12737 \\
\hline 4978 & .89309 & .51365 & .57515 & .45273 & .23978 & .16103 & & & & & & & \\
\hline
\end{tabular}


148 Statistical Characteristics of Storm Interevent Time, Depth, and Duration for Eastern New Mexico, Oklahoma, and Texas

Appendix 3-2.7. L-moments of storm depth defined by 72-hour minimum interevent time for hourly rainfall stations in Oklahoma.

[--, not available]

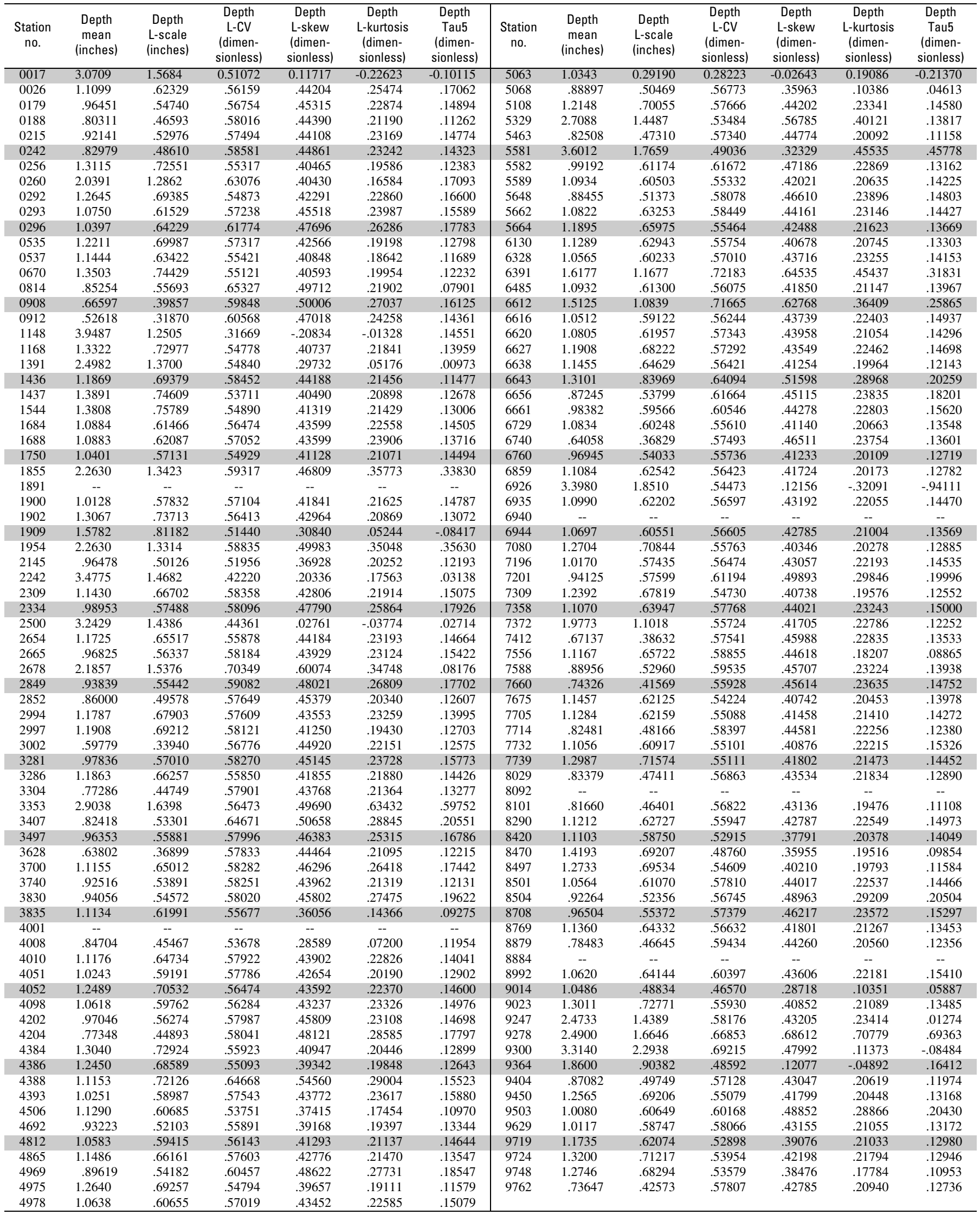


Appendix 3-3.1. L-moments of storm duration defined by 6-hour minimum interevent time for hourly rainfall stations in Oklahoma.

[--, not available]

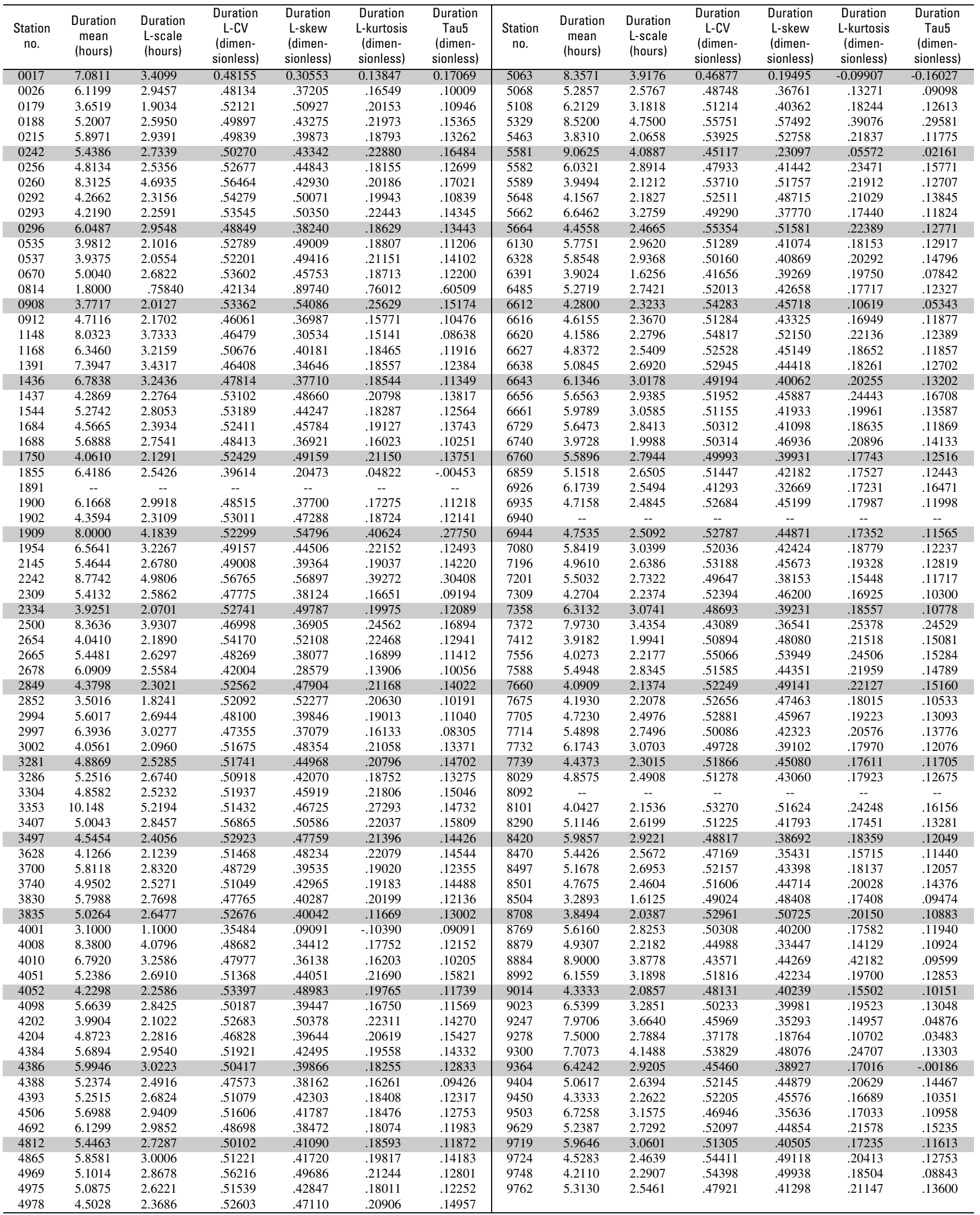


150 Statistical Characteristics of Storm Interevent Time, Depth, and Duration for Eastern New Mexico, Oklahoma, and Texas

Appendix 3-3.2. L-moments of storm duration defined by 8-hour minimum interevent time for hourly rainfall stations in Oklahoma.

[--, not available]

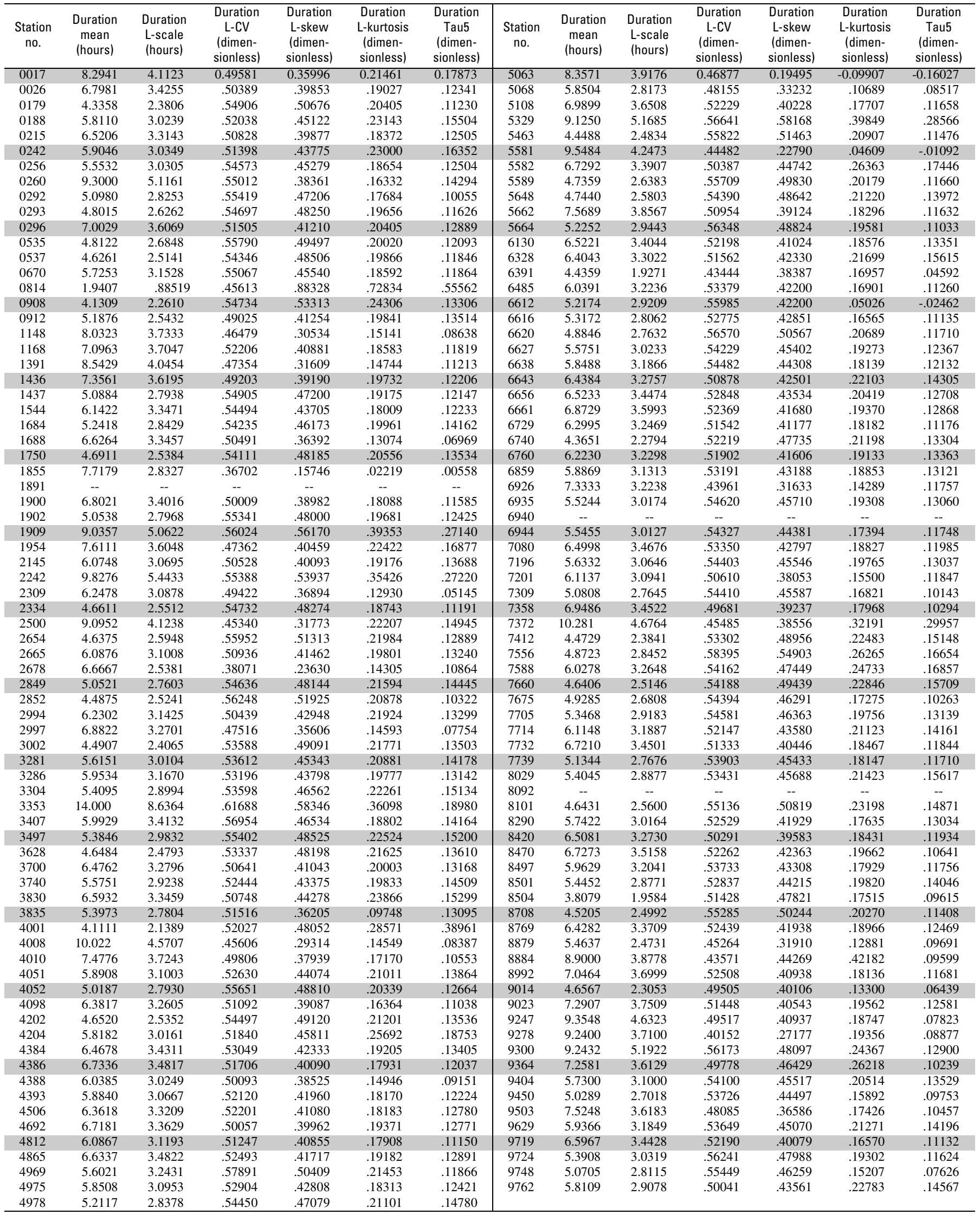


Appendix 3-3.3. L-moments of storm duration defined by 12-hour minimum interevent time for hourly rainfall stations in Oklahoma.

[--, not available]

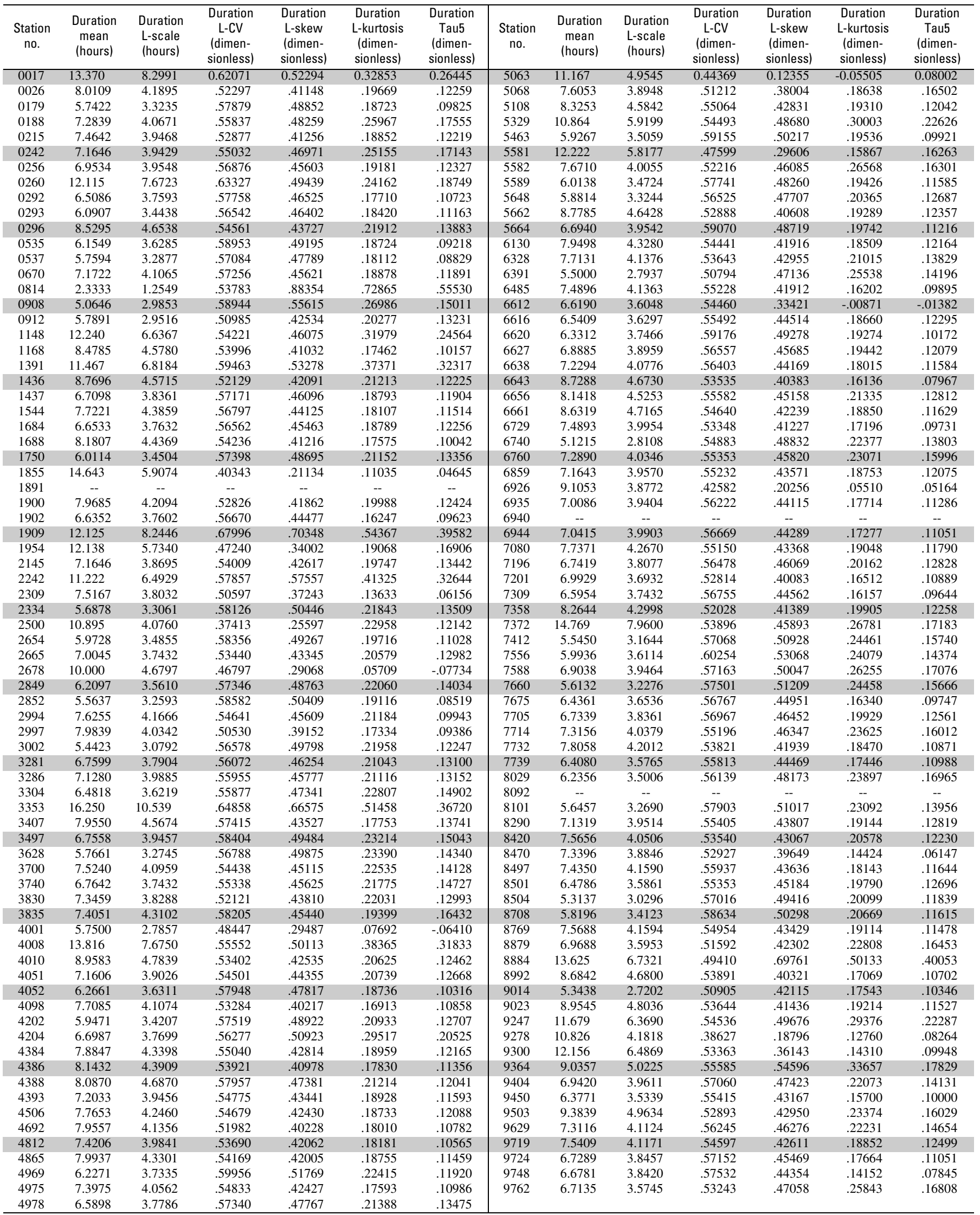


152 Statistical Characteristics of Storm Interevent Time, Depth, and Duration for Eastern New Mexico, Oklahoma, and Texas

Appendix 3-3.4. L-moments of storm duration defined by 18-hour minimum interevent time for hourly rainfall stations in Oklahoma.

[--, not available]

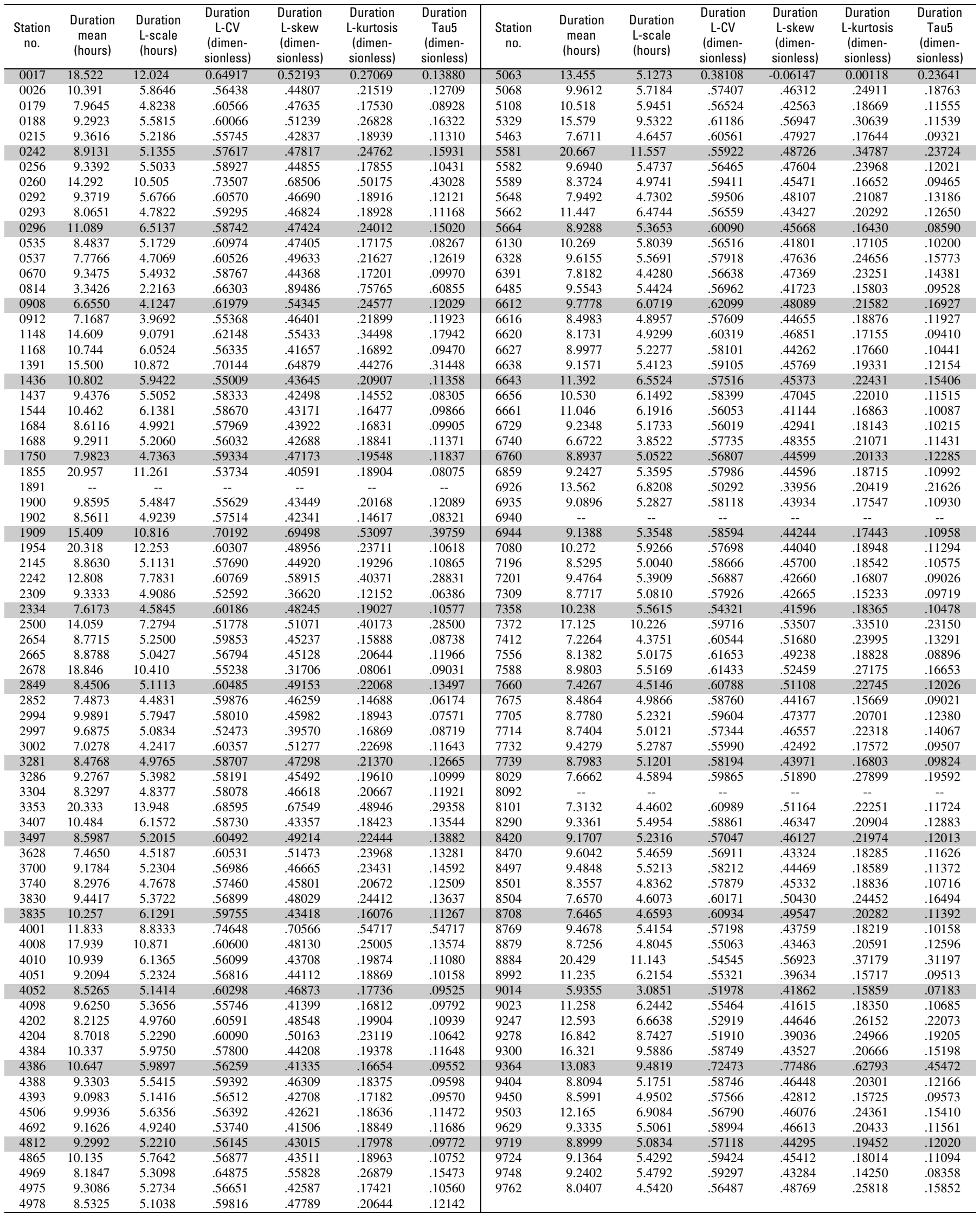


Appendix 3-3.5. L-moments of storm duration defined by 24-hour minimum interevent time for hourly rainfall stations in Oklahoma.

$[--$, not available $]$

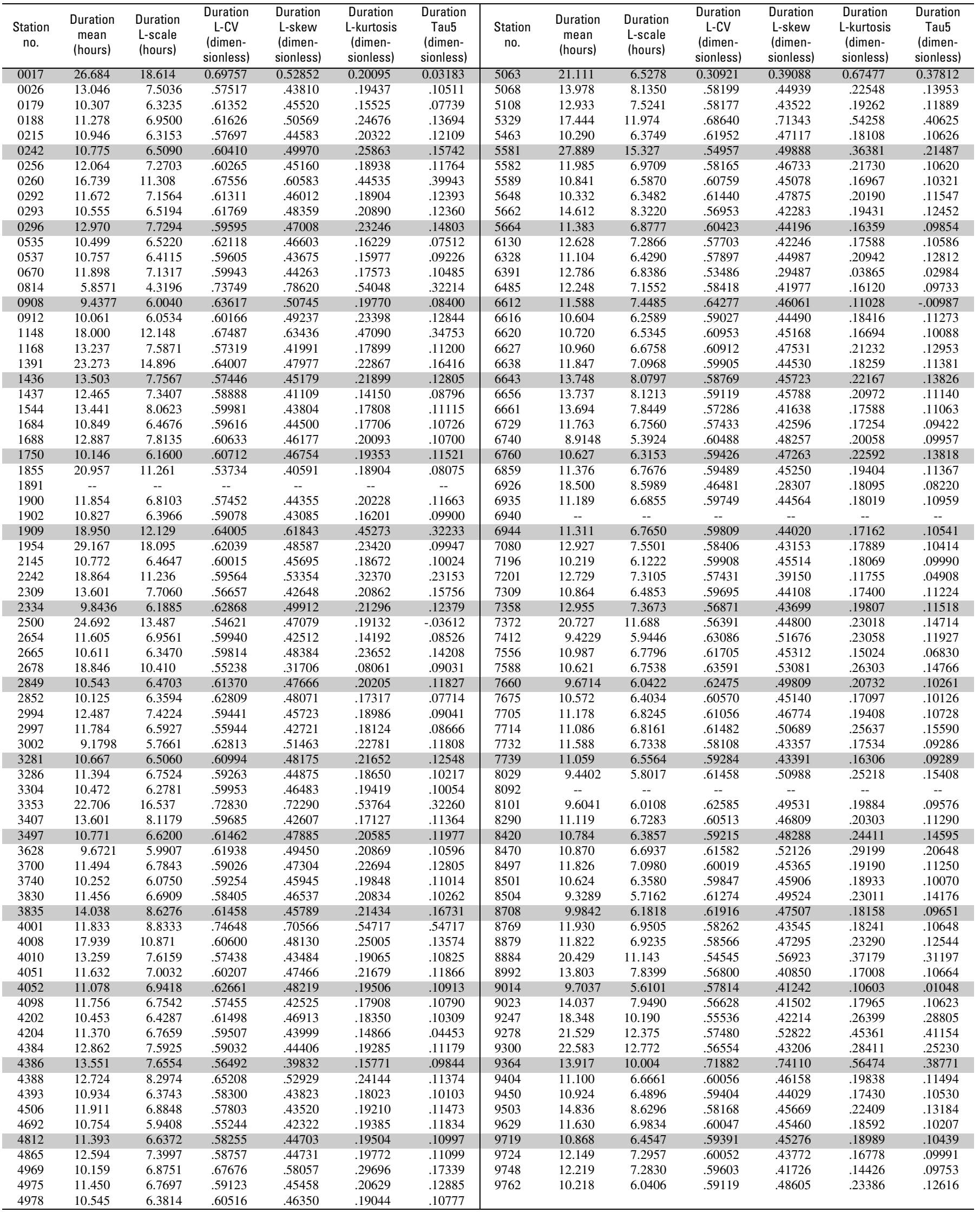


154 Statistical Characteristics of Storm Interevent Time, Depth, and Duration for Eastern New Mexico, Oklahoma, and Texas

Appendix 3-3.6. L-moments of storm duration defined by 48-hour minimum interevent time for hourly rainfall stations in Oklahoma.

[--, not available]

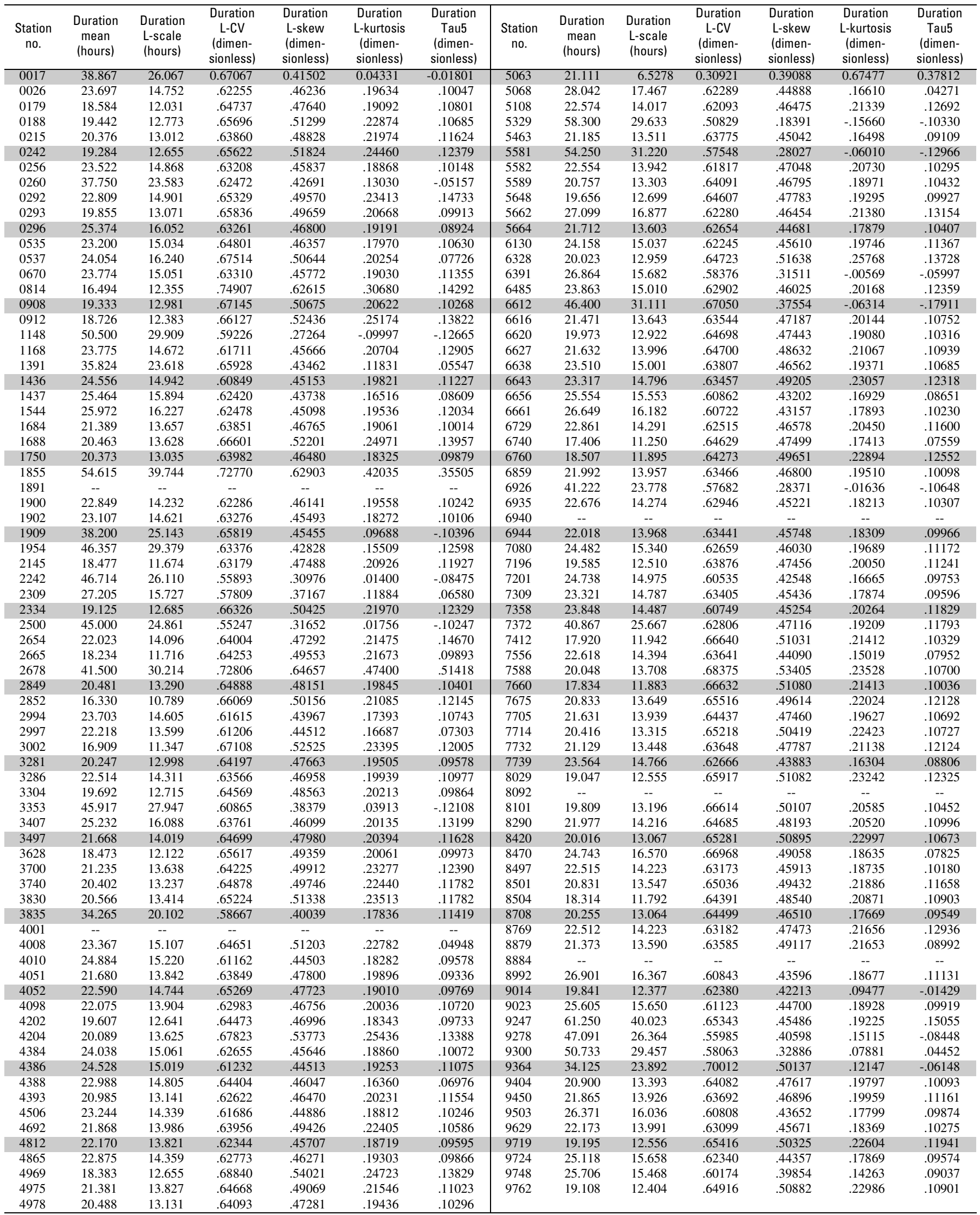


Appendix 3-3.7. L-moments of storm duration defined by 72-hour minimum interevent time for hourly rainfall stations in Oklahoma.

[--, not available]

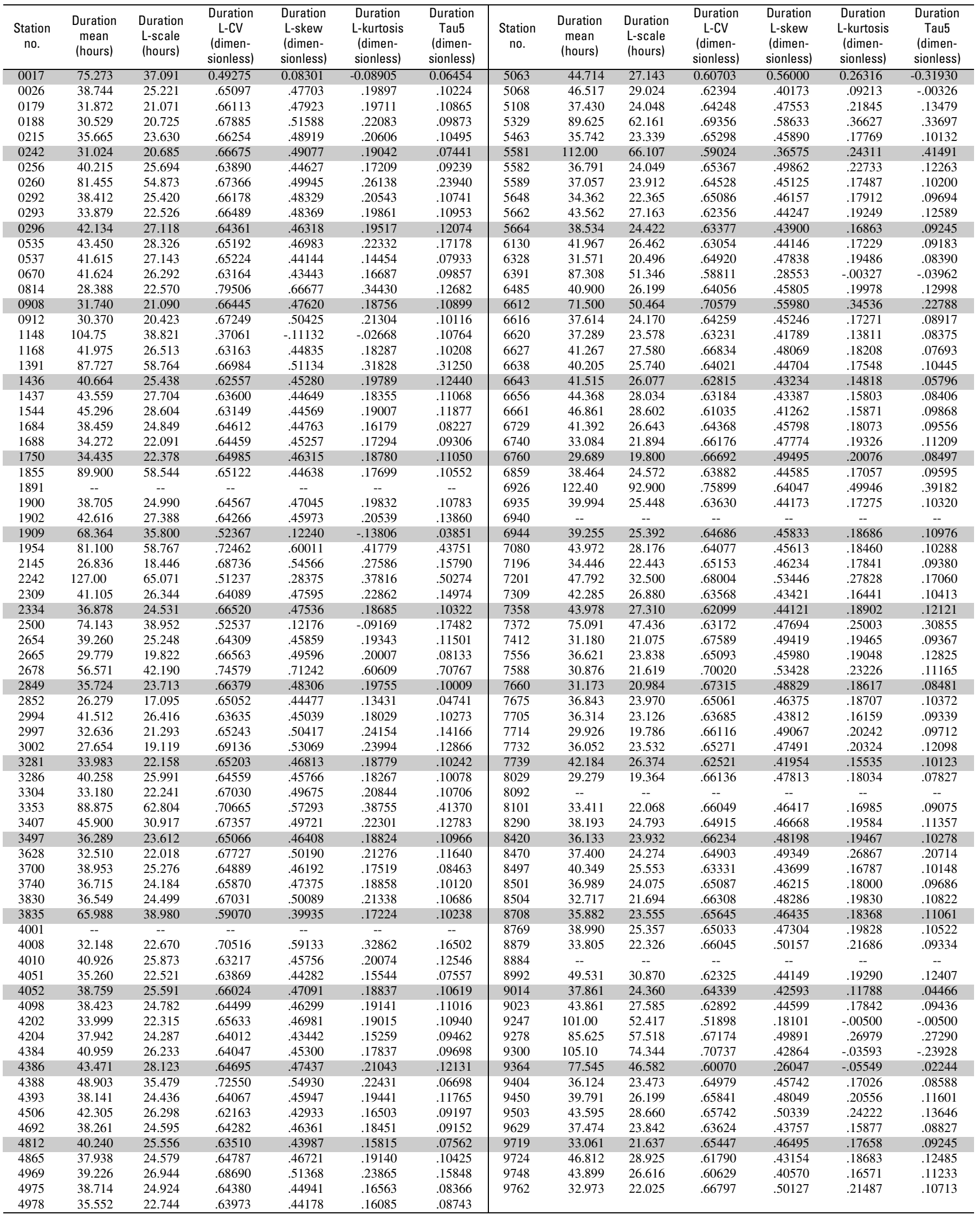


156 Statistical Characteristics of Storm Interevent Time, Depth, and Duration for Eastern New Mexico, Oklahoma, and Texas

Appendix 3-4.1. Empirical distribution of storm depth defined by 6-hour minimum interevent time for hourly rainfall stations in Oklahoma.

[--, not available]

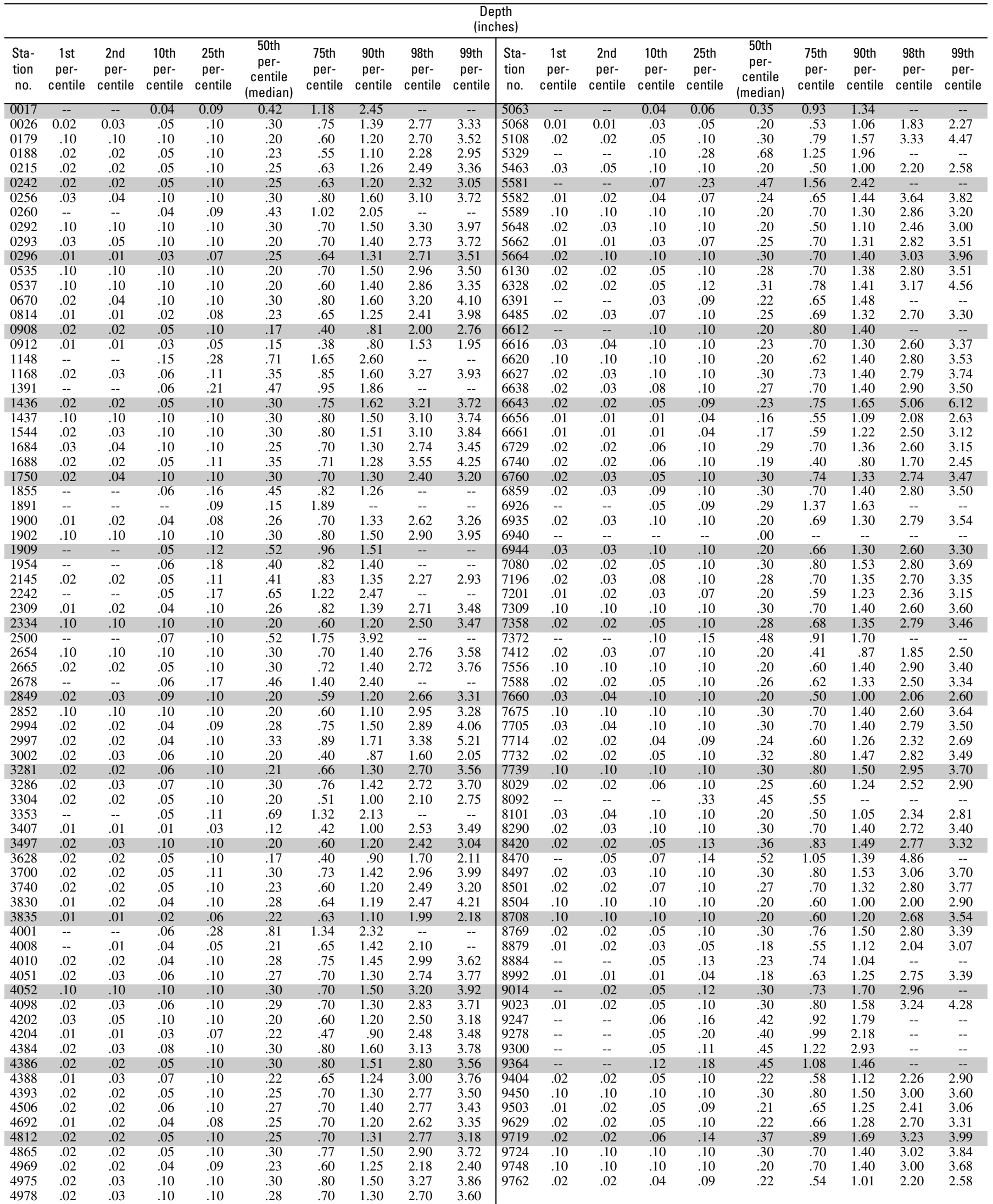


Appendix 3-4.2. Empirical distribution of storm depth defined by 8-hour minimum interevent time for hourly rainfall stations in Oklahoma.

[--, not available]

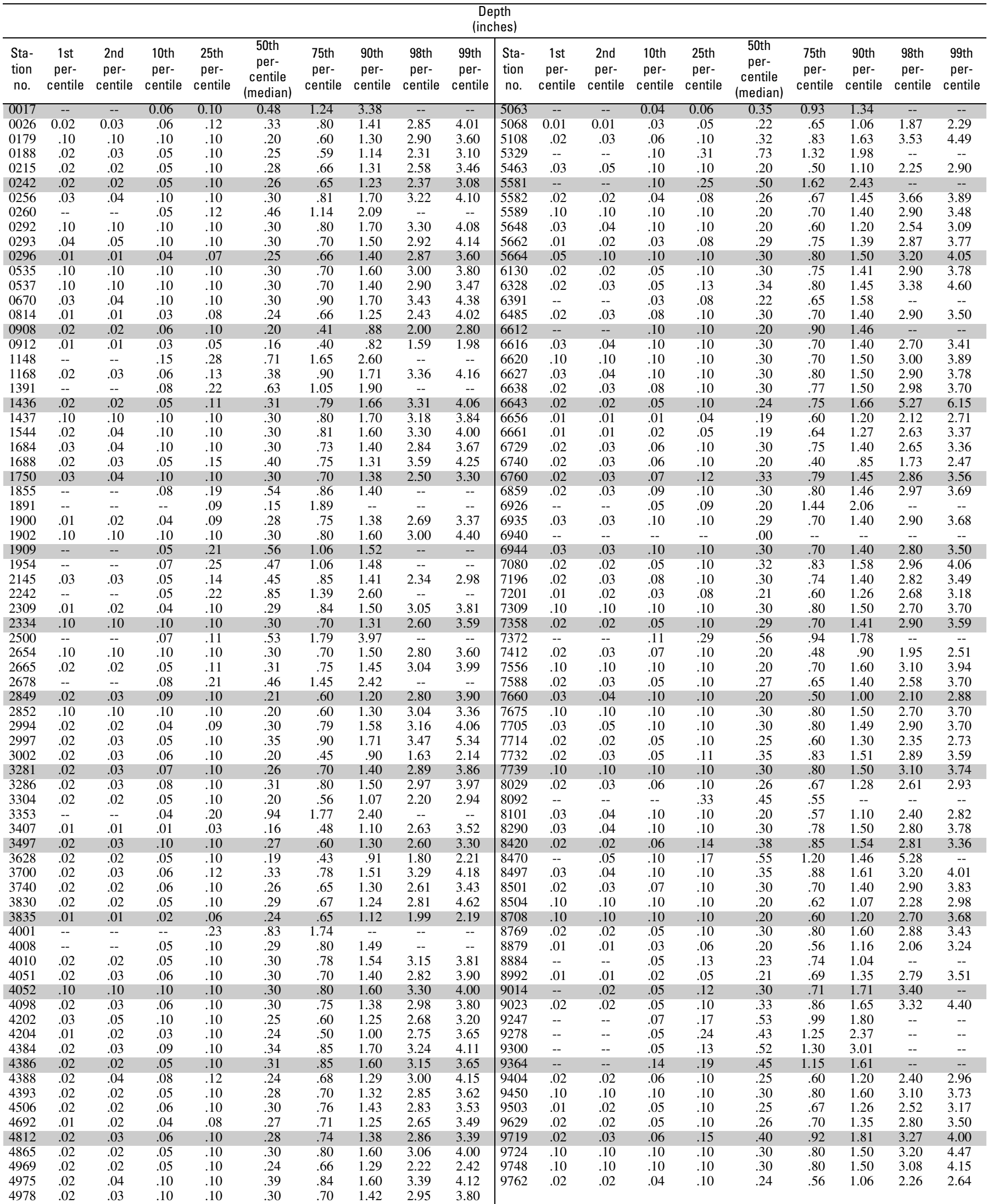


158 Statistical Characteristics of Storm Interevent Time, Depth, and Duration for Eastern New Mexico, Oklahoma, and Texas

Appendix 3-4.3. Empirical distribution of storm depth defined by 12-hour minimum interevent time for hourly rainfall stations in Oklahoma.

[--, not available]

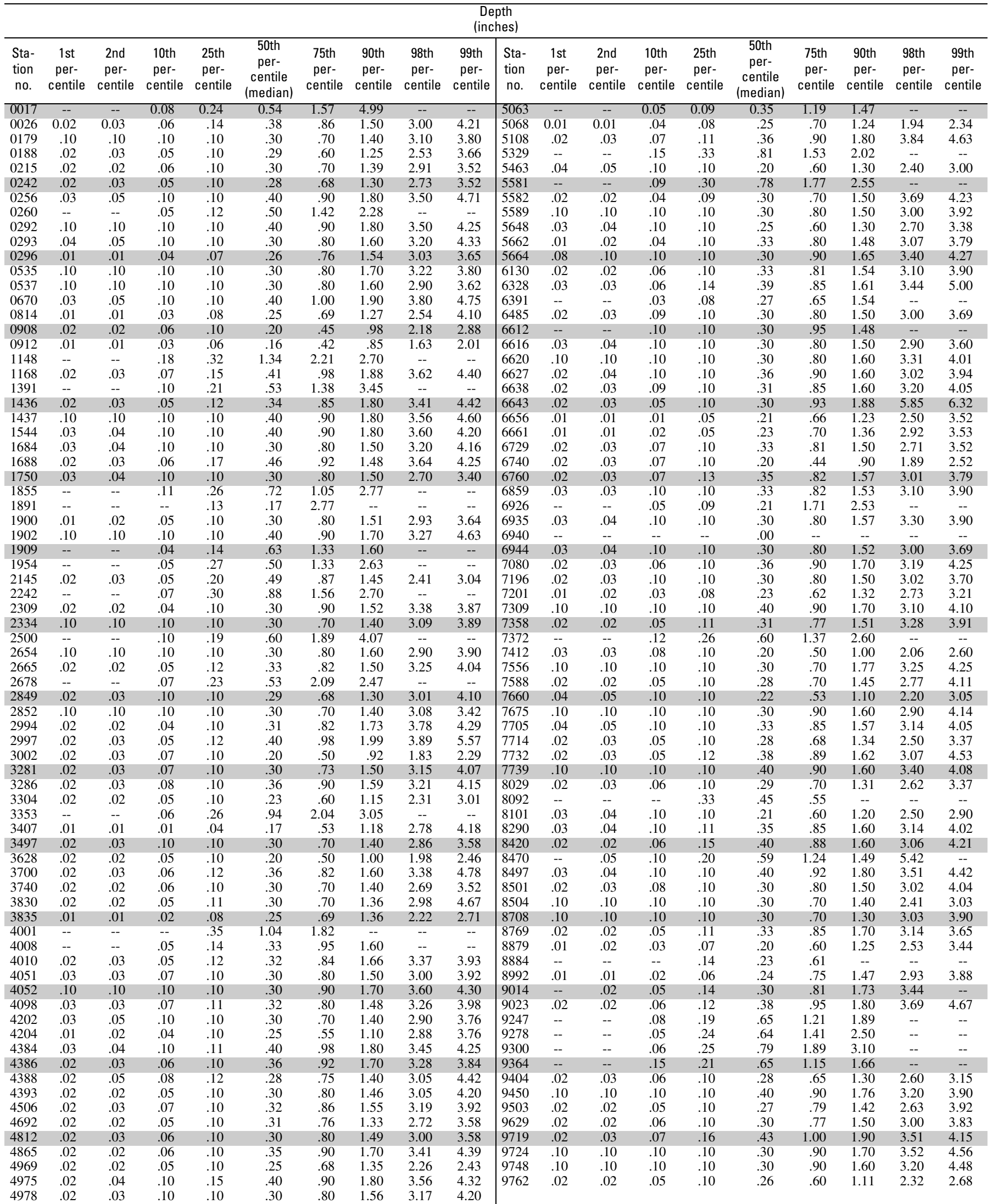


Appendix 3-4.4. Empirical distribution of storm depth defined by 18-hour minimum interevent time for hourly rainfall stations in Oklahoma.

[--, not available]

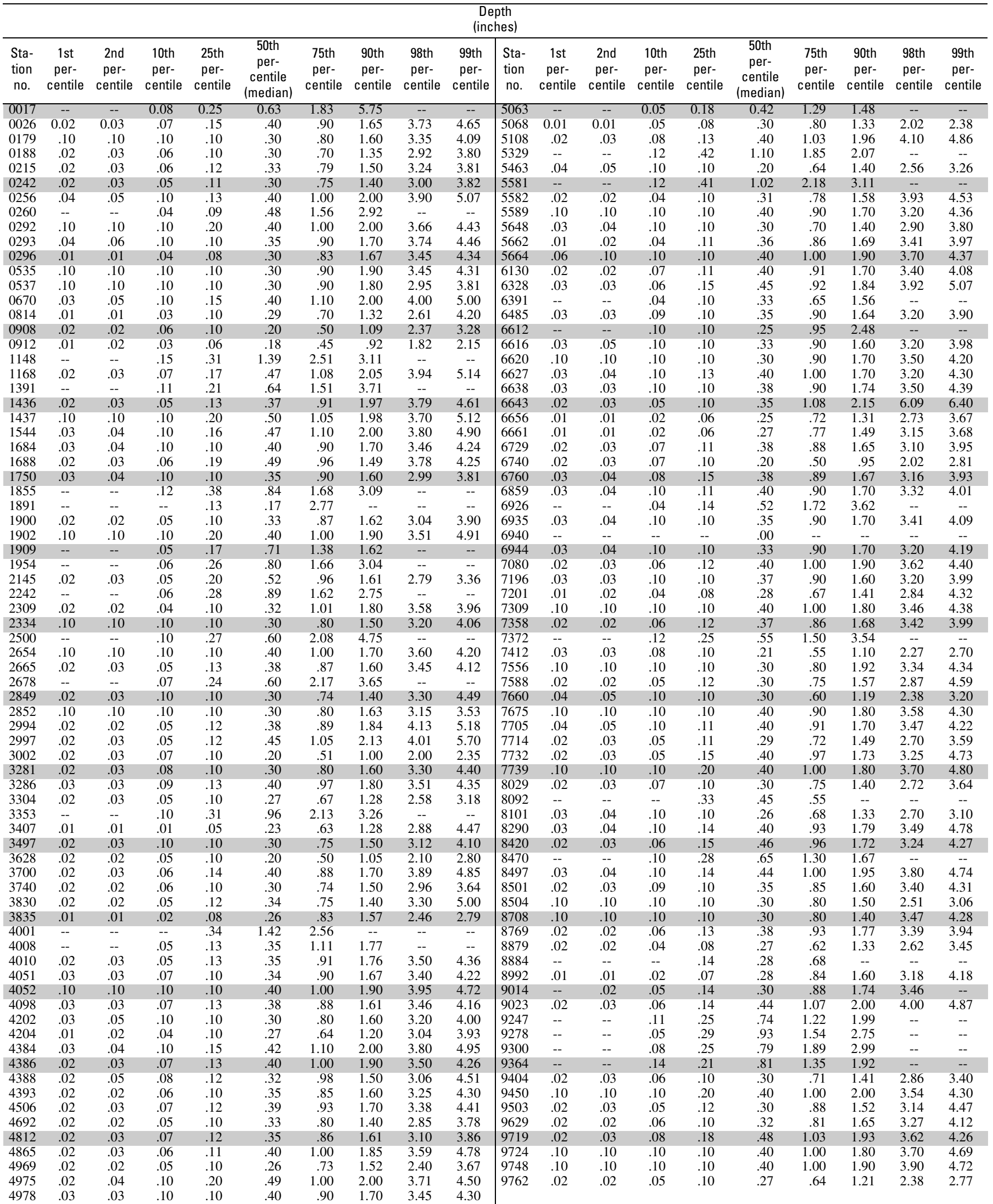


160 Statistical Characteristics of Storm Interevent Time, Depth, and Duration for Eastern New Mexico, Oklahoma, and Texas

Appendix 3-4.5. Empirical distribution of storm depth defined by 24-hour minimum interevent time for hourly rainfall stations in Oklahoma.

[--, not available]

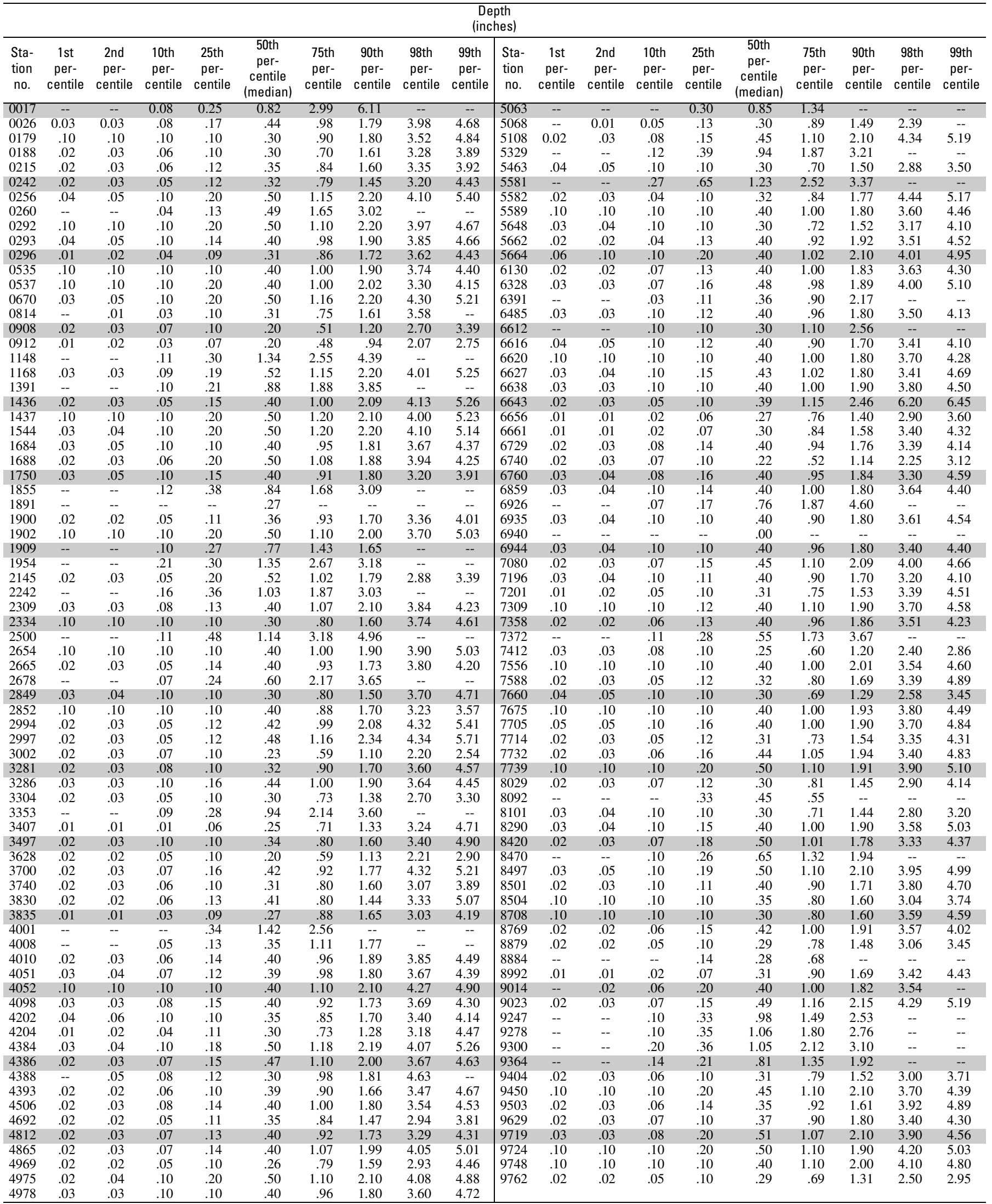


Appendix 3-4.6. Empirical distribution of storm depth defined by 48-hour minimum interevent time for hourly rainfall stations in Oklahoma.

[--, not available]

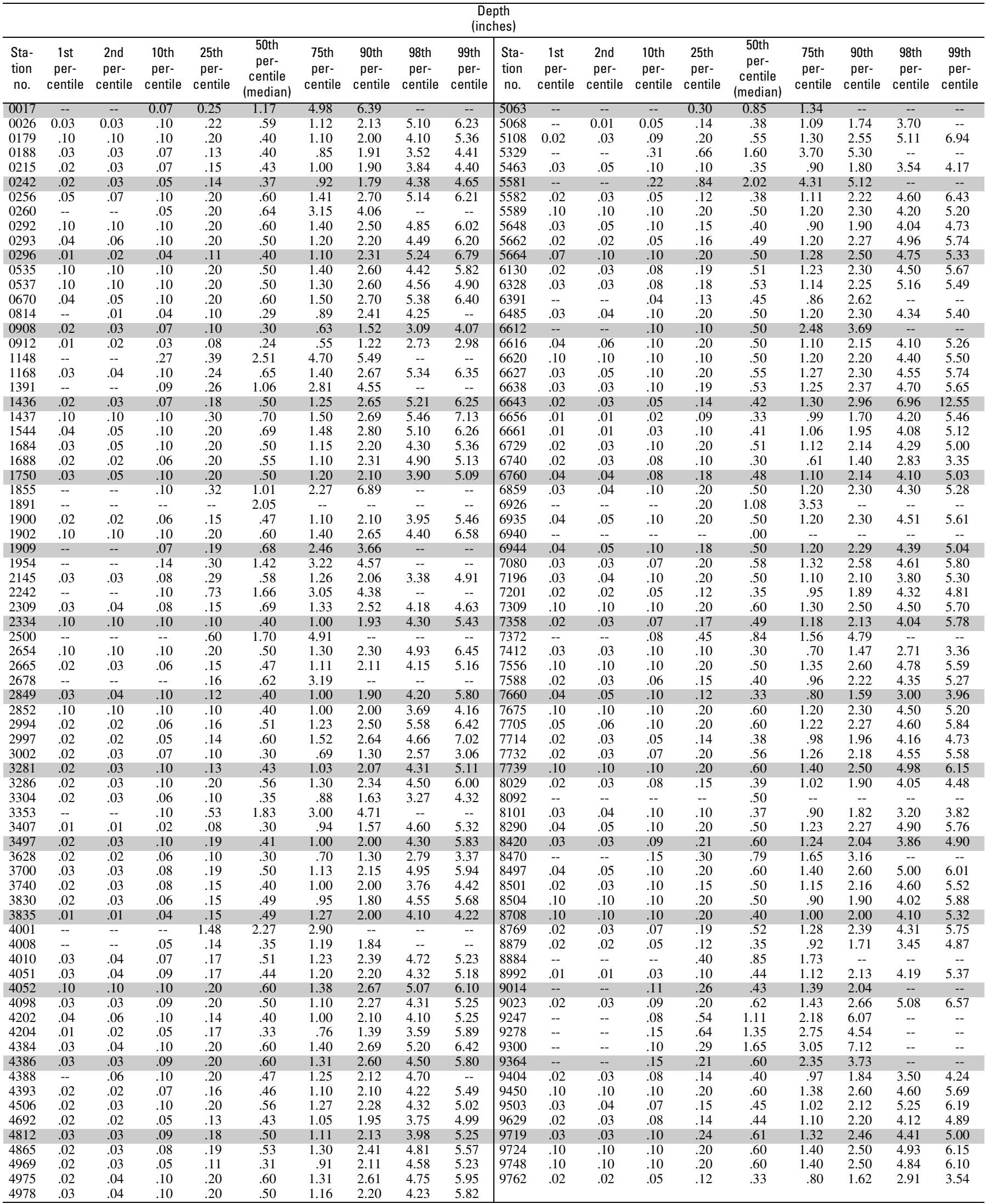


162 Statistical Characteristics of Storm Interevent Time, Depth, and Duration for Eastern New Mexico, Oklahoma, and Texas

Appendix 3-4.7. Empirical distribution of storm depth defined by 72-hour minimum interevent time for hourly rainfall stations in Oklahoma.

[--, not available]

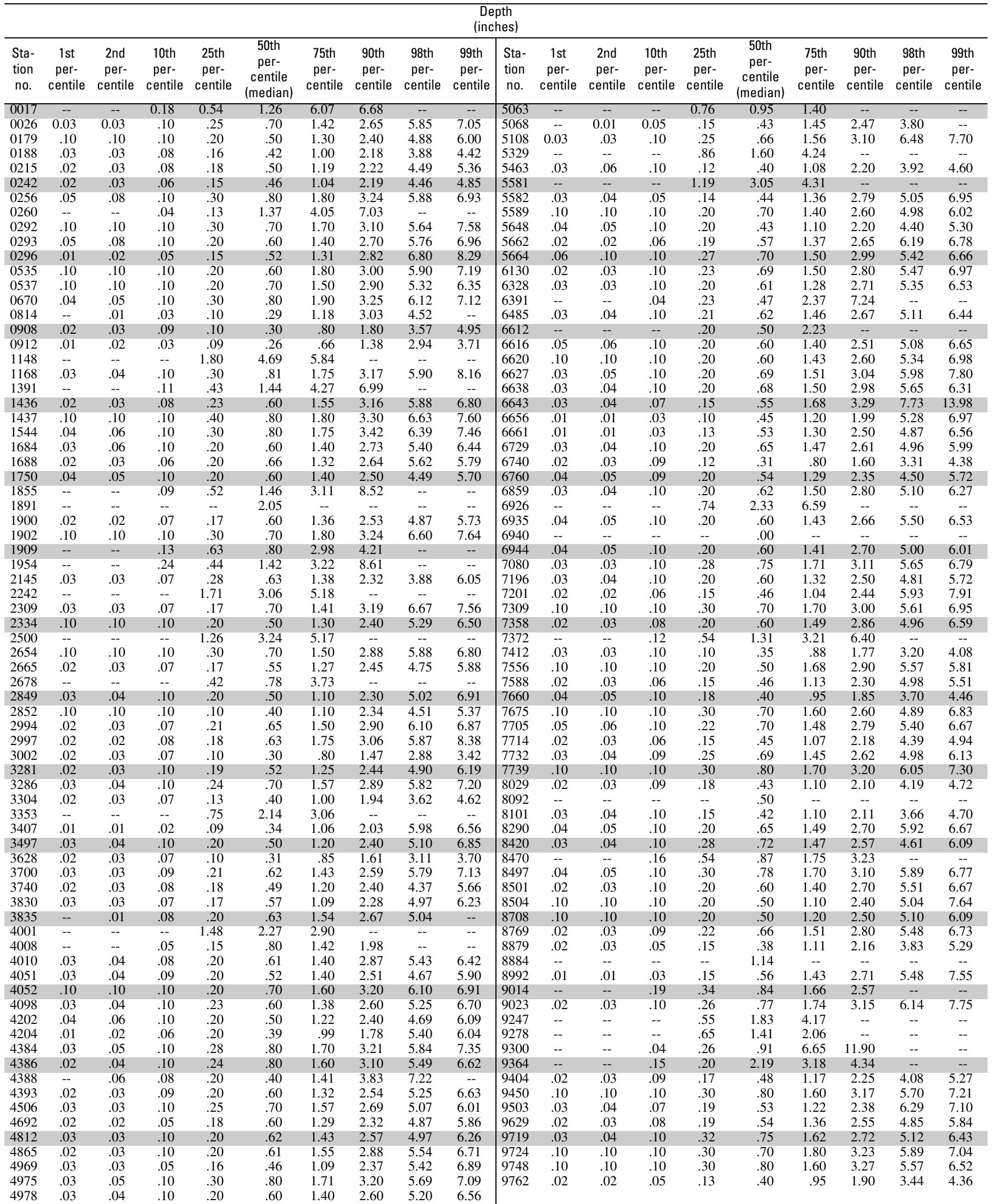


Appendix 3-5.1. Empirical distribution of storm duration defined by 6-hour minimum interevent time for hourly rainfall stations in Oklahoma.

$[--$, not available]

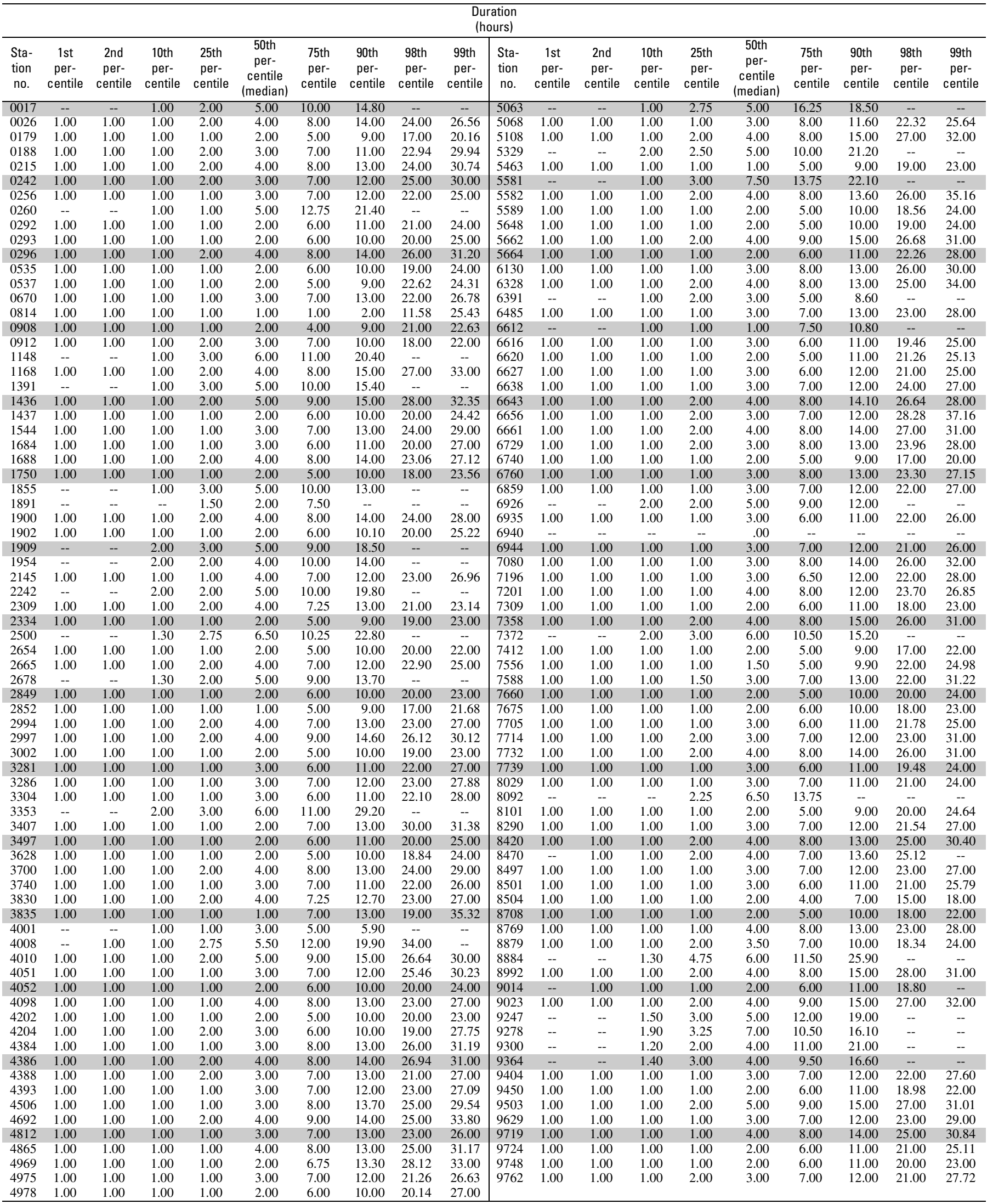


164 Statistical Characteristics of Storm Interevent Time, Depth, and Duration for Eastern New Mexico, Oklahoma, and Texas

Appendix 3-5.2. Empirical distribution of storm duration defined by 8-hour minimum interevent time for hourly rainfall stations in Oklahoma.

[--, not available]

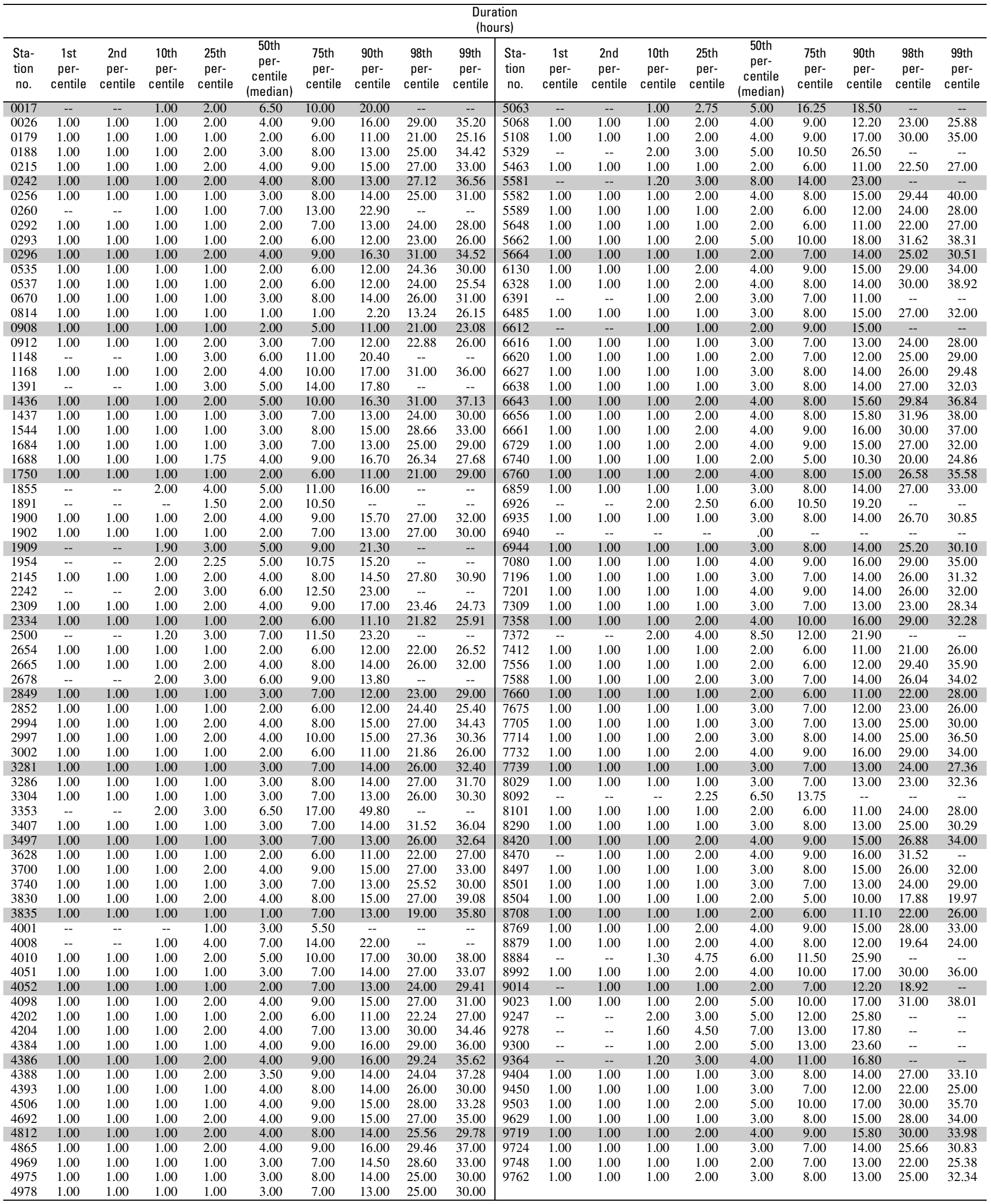


Appendix 3-5.3. Empirical distribution of storm duration defined by 12-hour minimum interevent time for hourly rainfall stations in Oklahoma.

$[--$, not available]

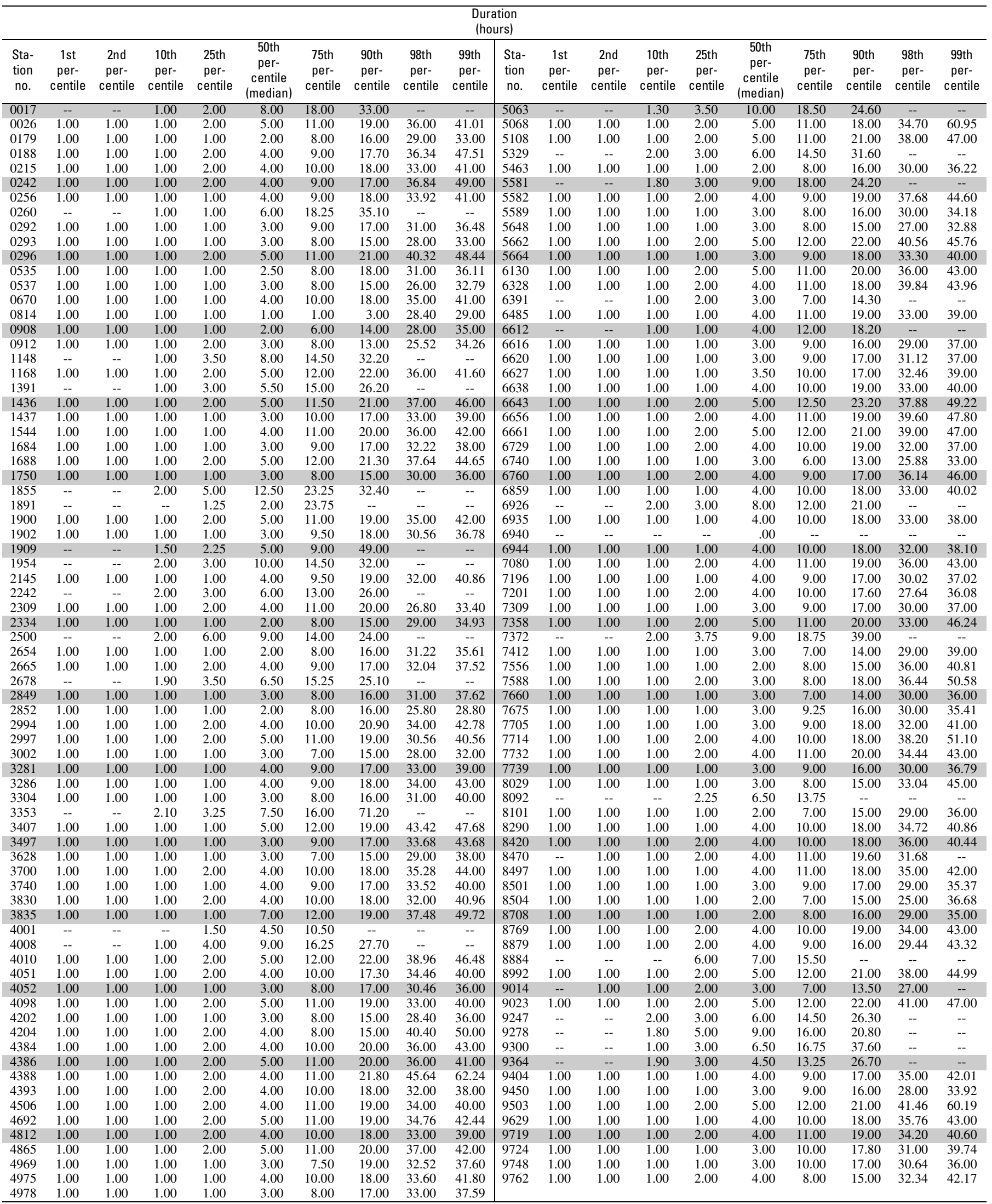


166 Statistical Characteristics of Storm Interevent Time, Depth, and Duration for Eastern New Mexico, Oklahoma, and Texas

Appendix 3-5.4. Empirical distribution of storm duration defined by 18-hour minimum interevent time for hourly rainfall stations in Oklahoma.

[--, not available]

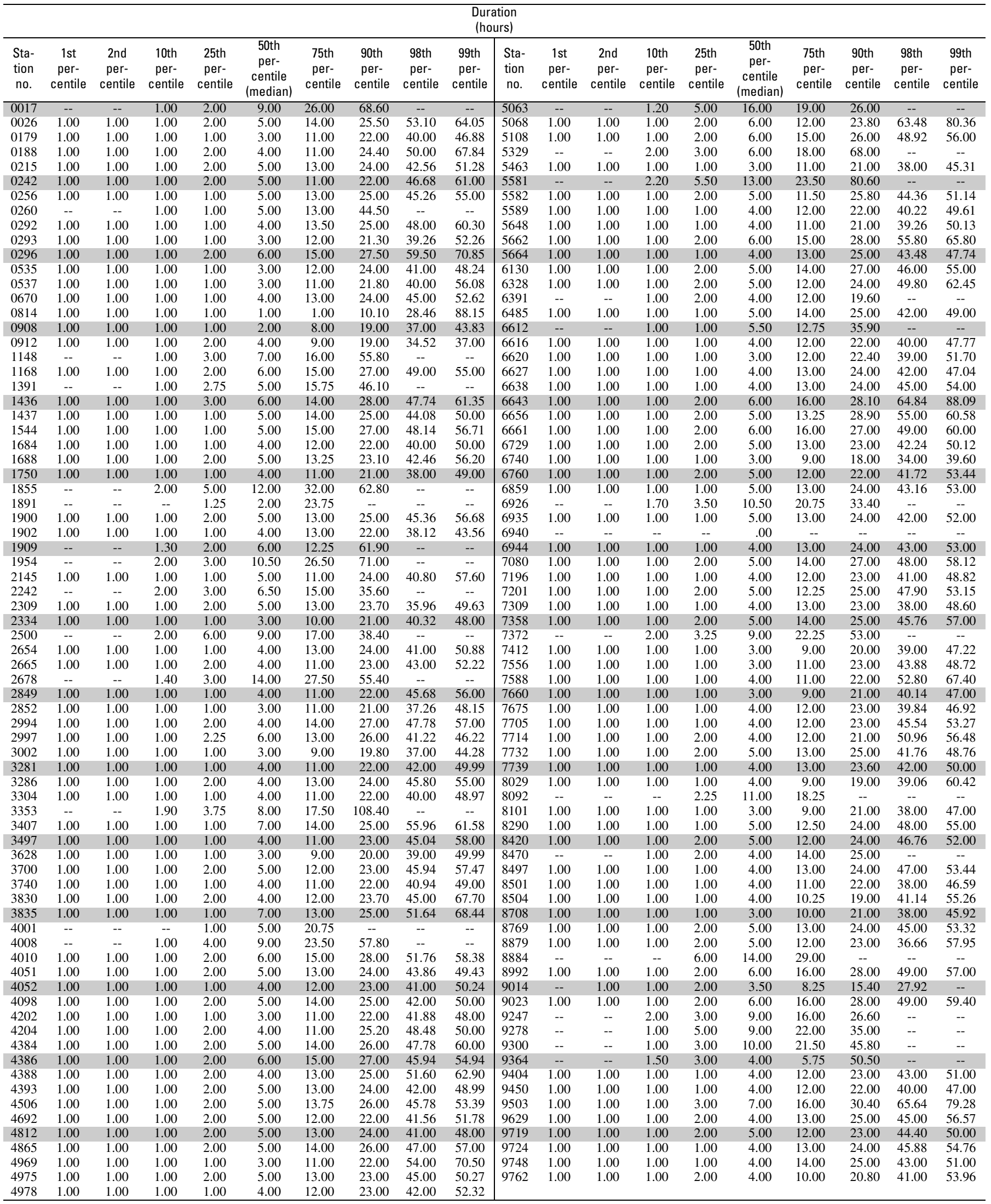


Appendix 3-5.5. Empirical distribution of storm duration defined by 24-hour minimum interevent time for hourly rainfall stations in Oklahoma.

$[--$, not available]

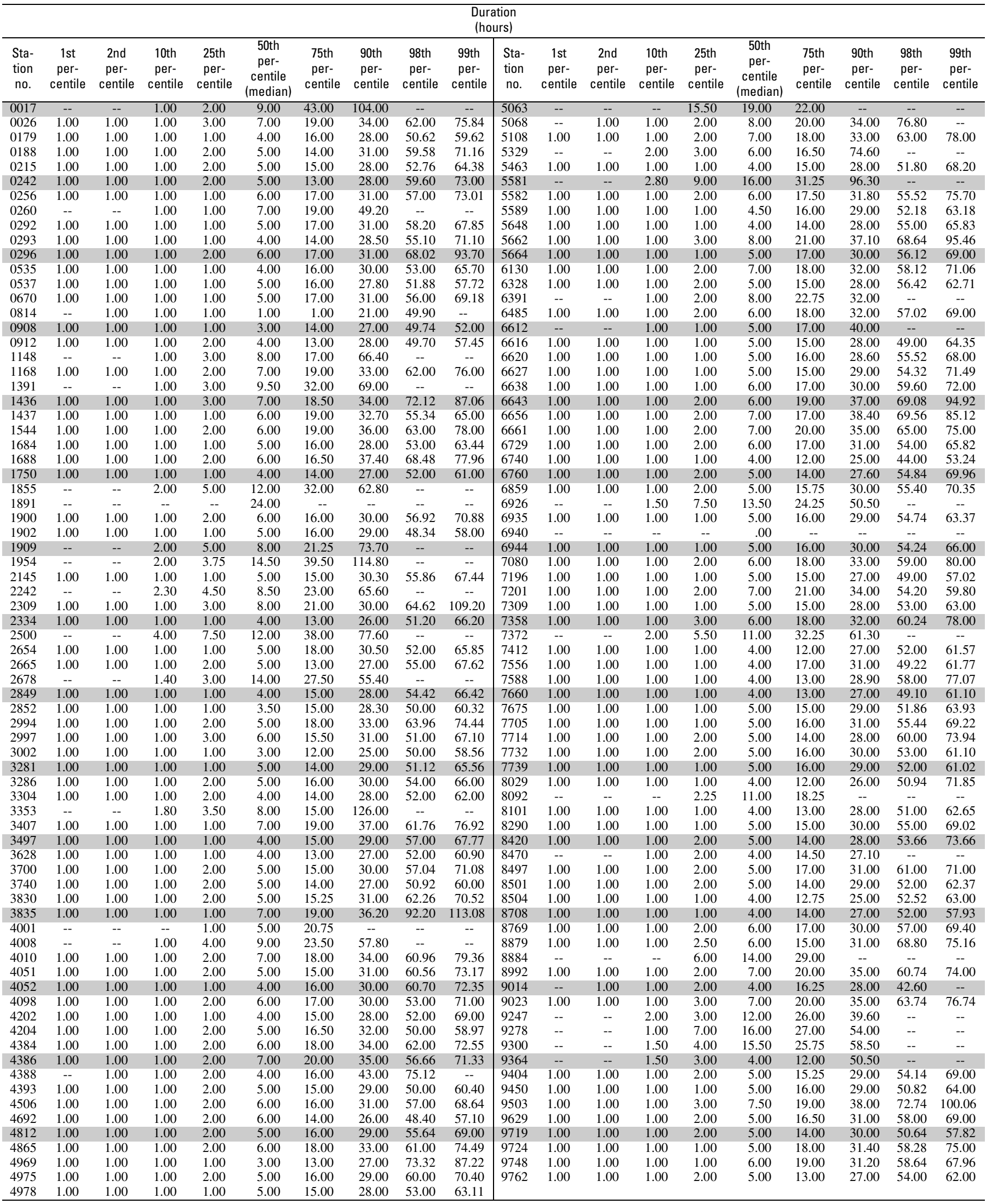


168 Statistical Characteristics of Storm Interevent Time, Depth, and Duration for Eastern New Mexico, Oklahoma, and Texas

Appendix 3-5.6. Empirical distribution of storm duration defined by 48-hour minimum interevent time for hourly rainfall stations in Oklahoma.

[--, not available]

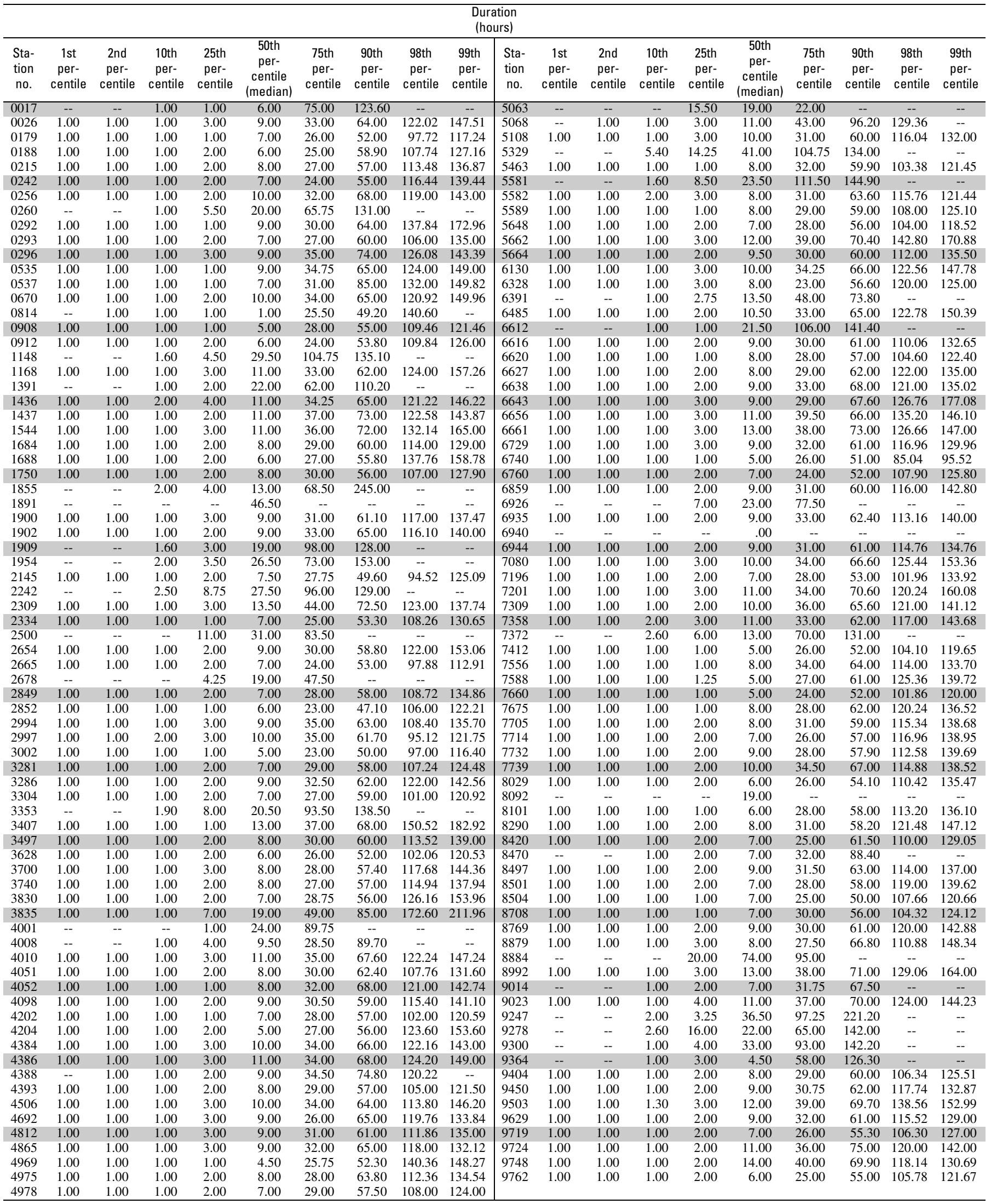


Appendix 3-5.7. Empirical distribution of storm duration defined by 72-hour minimum interevent time for hourly rainfall stations in Oklahoma.

$[--$, not available]

\begin{tabular}{|c|c|c|c|c|c|c|c|c|c|c|c|c|c|c|c|c|c|c|c|}
\hline \multicolumn{20}{|c|}{$\begin{array}{c}\begin{array}{c}\text { Duration } \\
\text { (hours) }\end{array}\end{array}$} \\
\hline $\begin{array}{l}\text { Sta- } \\
\text { tion } \\
\text { no. }\end{array}$ & $\begin{array}{c}1 \text { st } \\
\text { per- } \\
\text { centile }\end{array}$ & $\begin{array}{c}\text { 2nd } \\
\text { per- } \\
\text { centile }\end{array}$ & $\begin{array}{c}\text { 10th } \\
\text { per- } \\
\text { centile }\end{array}$ & $\begin{array}{c}25 \text { th } \\
\text { per- } \\
\text { centile }\end{array}$ & $\begin{array}{c}50 \text { th } \\
\text { per- } \\
\text { centile } \\
\text { (median) }\end{array}$ & $\begin{array}{c}75 \text { th } \\
\text { per- } \\
\text { centile }\end{array}$ & $\begin{array}{c}\text { 90th } \\
\text { per- } \\
\text { centile }\end{array}$ & $\begin{array}{c}\text { 98th } \\
\text { per- } \\
\text { centile }\end{array}$ & $\begin{array}{c}\text { 99th } \\
\text { per- } \\
\text { centile }\end{array}$ & $\begin{array}{l}\text { Sta- } \\
\text { tion } \\
\text { no. }\end{array}$ & $\begin{array}{c}1 \text { st } \\
\text { per- } \\
\text { centile }\end{array}$ & $\begin{array}{c}\text { 2nd } \\
\text { per- } \\
\text { centile }\end{array}$ & $\begin{array}{c}\text { 10th } \\
\text { per- } \\
\text { centile }\end{array}$ & $\begin{array}{c}25 \text { th } \\
\text { per- } \\
\text { centile }\end{array}$ & $\begin{array}{c}50 \text { th } \\
\text { per- } \\
\text { centile } \\
\text { (median) }\end{array}$ & $\begin{array}{c}\text { 75th } \\
\text { per- } \\
\text { centile }\end{array}$ & $\begin{array}{c}\text { 90th } \\
\text { per- } \\
\text { centile }\end{array}$ & $\begin{array}{c}\text { 98th } \\
\text { per- } \\
\text { centile }\end{array}$ & $\begin{array}{c}\text { 99th } \\
\text { per- } \\
\text { centile }\end{array}$ \\
\hline 0017 & -- & & 1.60 & 6.00 & 75.00 & 134.00 & 168.20 & -- & -- & 5063 & -- & & - & 15.00 & 19.00 & 95.00 & -- & & -- \\
\hline 0026 & 1.00 & 1.00 & 1.00 & 3.25 & 13.00 & 56.75 & 108.70 & 224.68 & 248.67 & 5068 & -- & 1.00 & 1.00 & 4.75 & 19.50 & 81.00 & 153.10 & 198.92 & -- \\
\hline 0179 & 1.00 & 1.00 & 1.00 & 2.00 & 11.00 & 45.00 & 92.60 & 180.92 & 210.96 & 5108 & 1.00 & 1.00 & 1.00 & 4.00 & 15.00 & 53.00 & 101.00 & 198.00 & 247.45 \\
\hline 0188 & 1.00 & 1.00 & 1.00 & 2.00 & 8.00 & 40.00 & 93.00 & 182.00 & 201.00 & 5329 & -- & -- & -- & 11.25 & 41.00 & 125.00 & -- & & -- \\
\hline 0215 & 1.00 & 1.00 & 1.00 & 3.00 & 12.00 & 53.00 & 103.00 & 198.72 & 236.62 & 5463 & 1.00 & 1.00 & 1.00 & 1.00 & 14.00 & 51.00 & 102.00 & 183.68 & 240.32 \\
\hline 0260 & -- & -- & 1.00 & 12.00 & 24.00 & 131.00 & 324.80 & -- & -- & 5589 & 1.00 & 1.00 & 1.00 & 2.00 & 14.00 & 57.00 & 105.10 & 171.36 & 207.30 \\
\hline 0292 & 1.00 & 1.00 & 1.00 & 2.00 & 14.00 & 52.50 & 112.00 & 224.16 & 240.54 & 5648 & 1.00 & 1.00 & 1.00 & 2.00 & 12.00 & 53.00 & 97.00 & 180.00 & 226.23 \\
\hline 0293 & 1.00 & 1.00 & 1.00 & 2.00 & 11.00 & 51.00 & 93.40 & 174.12 & 214.28 & 5662 & 1.00 & 1.00 & 2.00 & 4.00 & 20.50 & 62.00 & 115.40 & 257.08 & 285.85 \\
\hline 0296 & 1.00 & 1.00 & 1.00 & 4.00 & 17.00 & 62.00 & 111.70 & 251.60 & 330.44 & 5664 & 1.00 & 1.00 & 1.00 & 3.00 & 16.00 & 59.00 & 110.90 & 196.72 & 241.03 \\
\hline 0535 & 1.00 & 1.00 & 1.00 & 2.00 & 21.00 & 61.50 & 110.00 & 277.04 & 324.86 & 6130 & 1.00 & 1.00 & 1.00 & 4.00 & 17.00 & 63.00 & 118.00 & 212.00 & 239.00 \\
\hline 0537 & 1.00 & 1.00 & 1.00 & 2.00 & 12.00 & 73.00 & 108.00 & 216.00 & 312.60 & 6328 & 1.00 & 1.00 & 1.00 & 3.00 & 11.00 & 44.00 & 95.20 & 173.48 & 194.48 \\
\hline 0670 & 1.00 & 1.00 & 1.00 & 3.00 & 18.00 & 62.00 & 116.00 & 206.00 & 260.24 & 6391 & -- & -- & 1.00 & 5.00 & 67.00 & 162.50 & 244.60 & -- & -- \\
\hline 0814 & -- & 1.00 & 1.00 & 1.00 & 1.00 & 33.00 & 113.80 & 229.28 & -- & 6485 & 1.00 & 1.00 & 1.00 & 3.00 & 17.00 & 60.00 & 109.00 & 222.00 & 265.70 \\
\hline 1437 & 1.00 & 1.00 & 1.00 & 4.00 & 19.00 & 62.50 & 122.00 & 228.32 & 270.86 & 6656 & 1.00 & 1.00 & 1.00 & 4.00 & 17.00 & 64.25 & 128.80 & 228.12 & 272.34 \\
\hline 1544 & 1.00 & 1.00 & 1.00 & 4.00 & 20.00 & 66.50 & 121.00 & 229.00 & 272.90 & 6661 & 1.00 & 1.00 & 2.00 & 5.00 & 22.00 & 70.00 & 126.00 & 209.00 & 267.30 \\
\hline 1684 & 1.00 & 1.00 & 1.00 & 3.00 & 14.00 & 59.00 & 112.40 & 191.00 & 228.02 & 6729 & 1.00 & 1.00 & 1.00 & 3.00 & 16.00 & 61.00 & 113.00 & 215.28 & 260.28 \\
\hline 1688 & 1.00 & 1.00 & 1.00 & 3.00 & 15.00 & 54.00 & 96.60 & 198.76 & 219.24 & 6740 & 1.00 & 1.00 & 1.00 & 2.00 & 11.00 & 51.00 & 89.00 & 180.48 & 225.48 \\
\hline 1750 & 1.00 & 1.00 & 1.00 & 2.00 & 13.00 & 51.00 & 98.00 & 188.20 & 232.60 & 6760 & 1.00 & 1.00 & 1.00 & 2.00 & 9.00 & 42.00 & 91.00 & 153.28 & 179.60 \\
\hline 1855 & -- & -- & 2.20 & 8.50 & 58.50 & 145.50 & 318.60 & -- & -- & 6859 & 1.00 & 1.00 & 1.00 & 3.00 & 15.00 & 58.00 & 106.00 & 191.64 & 253.28 \\
\hline 1891 & -- & -- & -- & -- & 46.50 & -- & -- & -- & -- & 6926 & -- & -- & -- & 12.00 & 53.00 & 267.50 & -- & -- & -- \\
\hline 1900 & 1.00 & 1.00 & 1.00 & 4.00 & 14.00 & 56.25 & 110.00 & 209.20 & 253.45 & 6935 & 1.00 & 1.00 & 1.00 & 3.00 & 16.00 & 60.25 & 110.00 & 206.52 & 248.65 \\
\hline 1902 & 1.00 & 1.00 & 1.00 & 3.00 & 18.00 & 63.00 & 108.00 & 238.24 & 278.60 & 6940 & -- & -- & -- & -- & .00 & -- & -- & -- & -- \\
\hline 1909 & -- & -- & 1.40 & 9.00 & 63.00 & 120.00 & 160.00 & -- & -- & 6944 & 1.00 & 1.00 & 1.00 & 2.00 & 15.00 & 59.00 & 110.00 & 210.24 & 245.06 \\
\hline 1954 & -- & -- & 2.00 & 3.50 & 40.00 & 114.75 & 389.70 & -- & -- & 7080 & 1.00 & 1.00 & 1.00 & 4.00 & 17.00 & 65.00 & 122.00 & 226.82 & 267.88 \\
\hline 2145 & 1.00 & 1.00 & 1.00 & 2.00 & 9.00 & 32.00 & 84.00 & 162.00 & 260.00 & 7196 & 1.00 & 1.00 & 1.00 & 2.00 & 12.00 & 50.25 & 98.00 & 182.72 & 230.36 \\
\hline 2242 & -- & -- & -- & 26.00 & 120.00 & 163.75 & -- & -- & -- & 7201 & 1.00 & 1.00 & 2.00 & 3.00 & 18.00 & 59.75 & 141.00 & 297.00 & 456.55 \\
\hline 2309 & 1.00 & 1.00 & 1.50 & 3.75 & 19.50 & 56.50 & 121.50 & 262.50 & 301.00 & 7309 & 1.00 & 1.00 & 1.00 & 3.00 & 17.00 & 64.00 & 114.40 & 222.08 & 257.96 \\
\hline 2334 & 1.00 & 1.00 & 1.00 & 2.00 & 11.50 & 56.00 & 104.00 & 194.76 & 245.37 & 7358 & 1.00 & 1.00 & 2.00 & 4.00 & 21.00 & 64.75 & 112.10 & 234.00 & 281.73 \\
\hline 2500 & -- & -- & -- & 10.00 & 68.00 & 123.00 & -- & -- & -- & 7372 & -- & -- & 6.00 & 7.00 & 36.00 & 115.00 & 284.60 & -- & -- \\
\hline 3304 & 1.00 & 1.00 & 1.00 & 2.00 & & 49.00 & 93.50 & 198.70 & 231.90 & 8092 & -- & -- & -- & -- & 19.00 & -- & -- & -- & -- \\
\hline 3353 & -- & -- & -- & 9.25 & 44.50 & 115.50 & -- & -- & -- & 8101 & 1.00 & 1.00 & 1.00 & 2.00 & 10.00 & 52.00 & 95.00 & 180.56 & 203.28 \\
\hline 3407 & 1.00 & 1.00 & 1.00 & 2.00 & 16.00 & 61.50 & 132.20 & 345.16 & 360.92 & 8290 & 1.00 & 1.00 & 1.00 & 3.0 & 14.00 & 54.00 & 103.00 & 202.80 & 234.20 \\
\hline 3497 & 1.00 & 1.00 & 1.00 & 2.00 & 13. & 5 & 99.00 & 192.32 & 237.48 & 8420 & 1.00 & 1.00 & 1.00 & 3.0 & 11 & 58.00 & 97.90 & 206.46 & 228.07 \\
\hline 3628 & 1.00 & 1.00 & 1.00 & 2.00 & 9.0 & 48.00 & 91.00 & 192.52 & 233.56 & 8470 & -- & 100 & 1.00 & 3.0 & 19. & 50.50 & 133.20 & 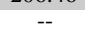 & . \\
\hline 3700 & 1.00 & 1.00 & 1.00 & & & 5900 & 112.00 & 196.72 & 239.88 & 84 & 1.0 & 1.0 & 1. & 3. & & 62.00 & 106.00 & 205.00 & 248.72 \\
\hline 3740 & 1.00 & 1.00 & 1.00 & 3.00 & & 57.00 & 107.00 & 197.00 & 234.05 & 8501 & 1.00 & 1.00 & 1.0 & 3.0 & 13.00 & 56.00 & 106.70 & 180.04 & 239.67 \\
\hline 3830 & 1.00 & 1.00 & 1.00 & 3.00 & 10 & 52.25 & 113.20 & 215.30 & 245.62 & 8504 & 1.00 & 1.00 & 1.00 & 2.0 & 11.00 & 50.00 & 92.00 & 197.00 & 278.00 \\
\hline 3835 & -- & 1.00 & 1.00 & 9. & & 88.00 & 165.40 & 319.00 & -- & 8708 & 1.00 & 1. & 1.0 & 1.2 & & 53.75 & 99.90 & 183.32 & 224.37 \\
\hline 4001 & -- & -- & -- & 1.00 & & 89.75 & 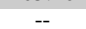 & -- & -- & 8769 & 1.0 & 1.0 & 1.0 & 3.0 & 15 & 56.00 & 114.50 & 215.30 & 247.00 \\
\hline 4008 & -- & -- & 1.00 & & & & 103.6 & -- & -- & 88 & 1.00 & 1.00 & 1.00 & 3.00 & & 45.25 & 98.00 & 211.50 & 247.00 \\
\hline 4010 & 1.00 & 1.00 & 2.00 & 4.0 & & 58.25 & 103.30 & 223.66 & 272.65 & & -- & -- & -- & -- & & -- & -- & -- & - \\
\hline 4051 & 1.00 & 1.00 & 1.00 & 3.00 & & 55.00 & 99.00 & 175.24 & 215.10 & 8992 & 1.00 & 1.00 & 2.00 & 5.00 & 22.50 & 71.00 & 128.50 & 244.50 & 313.75 \\
\hline 4052 & 1.00 & 1. & 1. & 2. & & 58.75 & 111.50 & 218.20 & 269.20 & 9014 & - & - & 1.00 & 2. & 15 & 69.75 & 111.80 & -- & - \\
\hline 4098 & 1.00 & 1.00 & 1.00 & 3.0 & 14.00 & 57.00 & 104.40 & 203.54 & 226.62 & 9023 & 1.00 & 1.00 & 2.00 & 5.0 & 18.00 & 64.00 & 122.00 & 228.00 & 264.58 \\
\hline 4202 & 1.00 & & & & & 510 & 93.00 & 191.10 & 218.95 & 9247 & - & -- & - & 23.5 & 74.00 & 178.50 & -- & -- & -- \\
\hline 4204 & 1.00 & 1.0 & 1. & 2. & & 63.25 & 106.20 & 208.08 & 264.71 & 9278 & -- & -- & -- & 11.0 & & 5.00 & -- & -- & -- \\
\hline 4384 & 1.00 & 1.0 & 1.00 & 3. & & 62.00 & 113.10 & 208.68 & 254.63 & 9300 & -- & -- & 1.00 & 3.2 & 2 & 227.00 & 338.90 & -- & -- \\
\hline 4386 & 1.00 & 1. & 1. & 4. & 17. & 65.00 & 118.70 & 231.54 & 265.08 & 936 & -- & -- & 1.0 & 2.0 & 63.00 & 141.00 & 216.40 & -- & -- \\
\hline 4388 & -- & 1.0 & & 2. & & 56.75 & 193.40 & 310.80 & -- & 94 & 1.00 & 1.00 & 1. & 2. & 12 & 55.00 & 103.50 & 187.70 & 216.85 \\
\hline 439 & 1.00 & & & 3. & & 57.00 & 99.50 & 214.00 & 246.00 & 94 & 1.00 & 1.00 & 1.0 & 3. & 14. & 58.00 & 112.00 & 211.84 & 252.12 \\
\hline 4506 & 1.00 & 1.00 & 1.00 & 4.00 & 19 & 62.00 & 115.00 & 212.64 & 249.44 & 9503 & 1.00 & 1.00 & 2.0 & 4. & 16. & 54.50 & 132.50 & 273.50 & 358.95 \\
\hline 4692 & 1.00 & 1.00 & 1.00 & 4.0 & 13 & 58.00 & 109.00 & 227.40 & 241.04 & 9629 & 1.00 & 1.00 & 1.00 & 3.00 & 15.00 & 58.00 & 103.80 & 184.16 & 224.40 \\
\hline 4812 & 1.00 & 1.00 & 1.00 & 3. & & 62.00 & 113.70 & 191.16 & 239.70 & 9719 & 1.00 & 1.0 & $1 .($ & 2.0 & 11.00 & 51.00 & 93.90 & 182.78 & 199.17 \\
\hline 4865 & 1.00 & 1.00 & 1.00 & 3.00 & 13 & 56.00 & 106.00 & 196.56 & 236.12 & 97 & 1.00 & 1.0 & 1.0 & 4.7 & 23.00 & 71.00 & 120.00 & 233.76 & 328.26 \\
\hline 4969 & 1. & & & 1. & & 54. & 112 & 235.44 & 410.04 & 97 & 1.0 & 1.0 & 1.0 & 4.0 & 23 & 66.00 & 116.00 & 219.68 & 264.17 \\
\hline 4975 & 1.00 & 1.00 & 1.00 & 3.00 & 14.00 & 61.00 & 108.50 & 198.50 & 234.45 & 9762 & 1.00 & 1.00 & 1.00 & 3.00 & 10.00 & 48.00 & 95.00 & 192.00 & 219.20 \\
\hline 4978 & 1.00 & 1.00 & 1.00 & 2.00 & 14.00 & 56.00 & 100.00 & 177.80 & 217.60 & & & & & & & & & & \\
\hline
\end{tabular}


Blank Page 
Appendix 4-

Storm Statistics for Hourly

Rainfall Stations in Texas 
Blank Page 
Appendix 4-1.1. Number of storms, total duration, and mean storm interevent time defined by 6-hour minimum interevent time for hourly rainfall stations in Texas.

[--, not available $]$

\begin{tabular}{|c|c|c|c|c|c|c|c|c|c|c|c|c|c|c|c|}
\hline $\begin{array}{c}\text { Station } \\
\text { no. }\end{array}$ & $\begin{array}{c}\text { No. } \\
\text { of } \\
\text { storms }\end{array}$ & $\begin{array}{c}\text { Total } \\
\text { duration } \\
\text { (hours) }\end{array}$ & $\begin{array}{c}\text { Mean } \\
\text { storm } \\
\text { interevent } \\
\text { time } \\
\text { (days) }\end{array}$ & $\begin{array}{c}\text { Station } \\
\text { no. }\end{array}$ & $\begin{array}{c}\text { No. } \\
\text { of } \\
\text { storms }\end{array}$ & $\begin{array}{c}\text { Total } \\
\text { duration } \\
\text { (hours) }\end{array}$ & $\begin{array}{c}\text { Mean } \\
\text { storm } \\
\text { interevent } \\
\text { time } \\
\text { (days) }\end{array}$ & $\begin{array}{c}\text { Station } \\
\text { no. }\end{array}$ & $\begin{array}{c}\text { No. } \\
\text { of } \\
\text { storms }\end{array}$ & $\begin{array}{c}\text { Total } \\
\text { duration } \\
\text { (hours) }\end{array}$ & $\begin{array}{c}\text { Mean } \\
\text { storm } \\
\text { interevent } \\
\text { time } \\
\text { (days) }\end{array}$ & $\begin{array}{c}\text { Station } \\
\text { no. }\end{array}$ & $\begin{array}{c}\text { No. } \\
\text { of } \\
\text { storms }\end{array}$ & $\begin{array}{c}\text { Total } \\
\text { duration } \\
\text { (hours) }\end{array}$ & $\begin{array}{c}\text { Mean } \\
\text { storm } \\
\text { interevent } \\
\text { time } \\
\text { (days) }\end{array}$ \\
\hline 0015 & 11 & 624 & 2.14 & 1154 & 264 & 26,928 & 4.03 & 2160 & 28 & 4,752 & 6.70 & 3463 & 54 & 30,096 & 22.95 \\
\hline 0016 & 3,737 & 543,665 & 5.83 & 1165 & 701 & 147,768 & 8.55 & 2206 & 864 & 105,121 & 4.84 & 3476 & 798 & 127,848 & 6.44 \\
\hline 0050 & 1,109 & 197,240 & 7.14 & 1185 & 407 & 185,232 & 18.79 & 2238 & 292 & 51,864 & 7.20 & 3485 & 25 & 1,968 & 3.04 \\
\hline 0054 & 60 & 5,688 & 3.77 & 1186 & 135 & 19,128 & 5.67 & 2240 & 104 & 12,864 & 4.98 & 3507 & 1,268 & 188,267 & 6.01 \\
\hline 0120 & 33 & 1,896 & 2.11 & 1188 & 11 & 1,872 & 6.87 & 2242 & 2,075 & 253,435 & 4.85 & 3546 & 3,726 & 485,758 & 5.20 \\
\hline 0145 & 204 & 28,392 & 5.60 & 1245 & 41 & 1,992 & 1.78 & 2244 & 3,957 & 535,994 & 5.42 & 3547 & 231 & 51,144 & 9.01 \\
\hline 0146 & 52 & 5,664 & 4.28 & 1246 & 1,352 & 176,017 & 5.26 & 2247 & 64 & 7,056 & 4.37 & 3579 & 89 & 19,944 & 9.06 \\
\hline 0174 & 1,542 & 275,194 & 7.30 & 1267 & 558 & 116,832 & 8.52 & 2309 & 777 & 153,600 & 7.98 & 3642 & 3,609 & 534,563 & 5.95 \\
\hline 0178 & 25 & 2,040 & 3.24 & 1304 & 659 & 117,840 & 7.22 & 2312 & 802 & 92,757 & 4.63 & 3646 & 2,345 & 419,874 & 7.19 \\
\hline 0179 & 348 & 66,456 & 7.80 & 1325 & 1,614 & 219,432 & 5.42 & 2334 & 66 & 22,344 & 13.85 & 3668 & 26 & 2,016 & 2.89 \\
\hline 0202 & 299 & 58,398 & 8.01 & 1429 & 1,423 & 213,741 & 6.04 & 2336 & 249 & 53,256 & 8.71 & 3673 & 38 & 2,016 & 1.98 \\
\hline 0206 & 2,881 & 532,111 & 7.52 & 1431 & 2,048 & 356,239 & 6.98 & 2354 & 32 & 20,976 & 27.11 & 3686 & 1,324 & 198,532 & 6.08 \\
\hline 0208 & 4 & 384 & 4.00 & 1432 & 1,469 & 246,787 & 6.73 & 2355 & 64 & 10,536 & 6.59 & 3691 & 3,195 & 469,594 & 5.94 \\
\hline 0211 & 3,890 & 542,494 & 5.60 & 1433 & 2,147 & 367,055 & 6.86 & 2357 & 588 & 98,496 & 6.75 & 3734 & 36 & 2,016 & 2.12 \\
\hline 0244 & 96 & 11,568 & 4.70 & 1434 & 2,179 & 358,448 & 6.60 & 2360 & 1,968 & 399,684 & 8.26 & 3771 & 1,629 & 220,455 & 5.48 \\
\hline 0248 & 2,159 & 529,764 & 10.06 & 1435 & 1,289 & 230,732 & 7.20 & 2361 & 212 & 56,004 & 10.85 & 3789 & 113 & 18,888 & 6.80 \\
\hline 0262 & 2,872 & 431,066 & 6.02 & 1436 & 2,453 & 367,281 & 5.96 & 2394 & 3,547 & 504,483 & 5.68 & 3826 & 240 & 45,240 & 7.61 \\
\hline 0271 & 29 & 2,016 & 2.64 & 1437 & 28 & 4,728 & 6.80 & 2404 & 3,225 & 493,624 & 6.15 & 3831 & 456 & 110,112 & 9.82 \\
\hline 0380 & 447 & 163,872 & 15.03 & 1438 & 1,745 & 284,045 & 6.52 & 2415 & 3,306 & 501,413 & 6.06 & 3841 & 169 & 77,472 & 18.90 \\
\hline 0394 & 11 & 1,992 & 7.20 & 1462 & 0 & 4,416 & -- & 2462 & 1,103 & 188,205 & 6.87 & 3871 & 1,614 & 317,328 & 7.95 \\
\hline 0408 & 34 & 2,112 & 2.40 & 1492 & 1,318 & 280,441 & 8.70 & 2528 & 129 & 24,840 & 7.74 & 3884 & 23 & 1,896 & 3.21 \\
\hline 0427 & 73 & 11,646 & 6.47 & 1500 & 33 & 2,112 & 2.41 & 2617 & 276 & 56,688 & 8.29 & 3941 & 79 & 7,608 & 3.70 \\
\hline 0428 & 4,766 & 529,122 & 4.38 & 1528 & 1,519 & 366,583 & 9.88 & 2619 & 255 & 64,992 & 10.39 & 3963 & 7 & 1,488 & 8.86 \\
\hline 0429 & 221 & 22,416 & 3.94 & 1541 & 70 & 10,953 & 6.30 & 2621 & 1,359 & 277,864 & 8.32 & 4040 & 500 & 92,806 & 7.51 \\
\hline 0463 & 127 & 51,936 & 16.81 & 1569 & 227 & 32,160 & 5.67 & 2675 & 1,267 & 178,918 & 5.68 & 4058 & 30 & 2,016 & 2.63 \\
\hline 0493 & 19 & 1,704 & 3.45 & 1632 & 7 & 1,320 & 7.86 & 2676 & 1,115 & 146,737 & 5.33 & 4098 & 1,932 & 417,420 & 8.87 \\
\hline 0495 & 373 & 138,072 & 15.24 & 1641 & 236 & 110,280 & 19.25 & 2679 & 2,486 & 536,229 & 8.81 & 4100 & 399 & 121,992 & 12.54 \\
\hline 0496 & 27 & 10,872 & 16.72 & 1646 & 2,656 & 543,287 & 8.38 & 2715 & 1,954 & 354,131 & 7.32 & 4137 & 1,336 & 221,233 & 6.74 \\
\hline 0498 & 13 & 7,920 & 25.33 & 1663 & 53 & 11,460 & 8.80 & 2744 & 1,980 & 413,376 & 8.50 & 4191 & 2,701 & 519,452 & 7.81 \\
\hline 0509 & 3,412 & 549,223 & 6.49 & 1671 & 3,674 & 548,538 & 6.02 & 2758 & 135 & 23,376 & 6.96 & 4256 & 0 & 21,906 & -- \\
\hline 0518 & 2,353 & 330,777 & 5.67 & 1680 & 1,320 & 261,792 & 8.00 & 2794 & 11 & 2,064 & 7.57 & 4257 & 3,572 & 498,651 & 5.61 \\
\hline 0521 & 40 & 5,760 & 5.77 & 1694 & 177 & 45,172 & 10.45 & 2797 & 2,663 & 529,606 & 8.11 & 4258 & 221 & 25,608 & 4.66 \\
\hline 0556 & 117 & 21,936 & 7.54 & 1696 & 1,227 & 268,080 & 8.87 & 2811 & 2,196 & 435,389 & 8.08 & 4278 & 1,750 & 409,467 & 9.51 \\
\hline 0569 & 2,893 & 399,353 & 5.55 & 1697 & 95 & 29,496 & 12.71 & 2813 & 30 & 31,728 & 43.90 & 4299 & 135 & 86,976 & 26.68 \\
\hline 0572 & 1,152 & 158,448 & 5.50 & 1698 & 1,513 & 291,219 & 7.85 & 2814 & 19 & 12,336 & 26.88 & 4300 & 3,272 & 289,264 & 3.45 \\
\hline 0576 & 160 & 23,352 & 5.84 & 1720 & 247 & 46,709 & 7.73 & 2815 & 283 & 58,942 & 8.54 & 4305 & 2,904 & 266,616 & 3.59 \\
\hline 0580 & 389 & 112,800 & 11.85 & 1761 & 139 & 16,680 & 4.80 & 2818 & 209 & 45,312 & 8.81 & 4307 & 770 & 96,430 & 4.98 \\
\hline 0587 & 1,009 & 121,272 & 4.74 & 1773 & 3,920 & 502,398 & 5.12 & 2986 & 322 & 54,240 & 6.74 & 4309 & 4,042 & 517,057 & 5.10 \\
\hline 0605 & 137 & 27,000 & 7.99 & 1810 & 26 & 2,112 & 3.19 & 3005 & 2,981 & 522,908 & 7.11 & 4311 & 4,116 & 543,389 & 5.26 \\
\hline 0639 & 2,686 & 433,077 & 6.56 & 1823 & 25 & 1,968 & 3.11 & 3033 & 700 & 216,192 & 12.70 & 4313 & 316 & 39,432 & 4.96 \\
\hline 0655 & 0 & 718 & -- & 1870 & 381 & 47,496 & 4.88 & 3034 & 2 & 6,888 & 143.50 & 4319 & 108 & 29,208 & 11.01 \\
\hline 0665 & 2,338 & 361,772 & 6.20 & 1875 & 20 & 1,752 & 3.46 & 3047 & 36 & 2,016 & 2.10 & 4329 & 3,165 & 438,183 & 5.52 \\
\hline 0689 & 1,825 & 381,486 & 8.48 & 1876 & 53 & 10,920 & 8.26 & 3103 & 37 & 29,448 & 33.01 & 4331 & 0 & 5,112 & -- \\
\hline 0690 & 759 & 179,395 & 9.72 & 1889 & 368 & 38,734 & 4.16 & 3133 & 2,976 & 486,379 & 6.57 & 4375 & 1,392 & 232,138 & 6.80 \\
\hline 0691 & 3,002 & 469,381 & 6.28 & 1903 & 398 & 83,753 & 8.64 & 3156 & 337 & 63,435 & 7.63 & 4392 & 565 & 72,624 & 5.06 \\
\hline 0708 & 155 & 32,809 & 8.67 & 1914 & 23 & 1,752 & 2.92 & 3171 & 2,700 & 463,053 & 6.90 & 4425 & 981 & 264,416 & 11.08 \\
\hline 0738 & 1,987 & 306,512 & 6.17 & 1920 & 516 & 109,392 & 8.61 & 3189 & 398 & 97,104 & 10.00 & 4440 & 1,794 & 283,440 & 6.33 \\
\hline 0776 & 1,884 & 439,998 & 9.54 & 1921 & 3,468 & 476,617 & 5.50 & 3260 & 188 & 44,448 & 9.63 & 4476 & 2,109 & 344,677 & 6.63 \\
\hline 0779 & 490 & 108,787 & 9.11 & 1937 & 1,384 & 186,288 & 5.33 & 3267 & 119 & 22,512 & 7.64 & 4498 & 14 & 4,944 & 14.62 \\
\hline 0784 & 2,097 & 429,972 & 8.39 & 1956 & 3,740 & 486,210 & 5.20 & 3270 & 1,780 & 365,659 & 8.42 & 4517 & 2,004 & 328,752 & 6.60 \\
\hline 0786 & 616 & 117,888 & 7.76 & 1970 & 31 & 1,992 & 2.45 & 3272 & 25 & 3,840 & 6.30 & 4520 & 1,352 & 220,430 & 6.64 \\
\hline 0917 & 2,556 & 300,936 & 4.65 & 2014 & 574 & 69,312 & 4.82 & 3277 & 16 & 4,176 & 10.65 & 4525 & 35 & 1,992 & 2.09 \\
\hline 0923 & 32 & 2,016 & 2.31 & 2015 & 4,052 & 484,318 & 4.76 & 3278 & 1,101 & 253,450 & 9.42 & 4563 & 31 & 2,112 & 2.59 \\
\hline 0926 & 3,560 & 551,357 & 6.23 & 2019 & 30 & 2,016 & 2.60 & 3280 & 171 & 41,832 & 10.01 & 4570 & 2,633 & 548,973 & 8.50 \\
\hline 0950 & 47 & 18,936 & 16.67 & 2024 & 2,243 & 391,758 & 7.05 & 3281 & 48 & 26,304 & 22.70 & 4577 & 2,335 & 305,664 & 5.23 \\
\hline 0996 & 32 & 1,992 & 2.38 & 2042 & 14 & 16,080 & 47.76 & 3283 & 2,000 & 247,224 & 4.89 & 4591 & 3,122 & 431,406 & 5.48 \\
\hline 1013 & 280 & 63,492 & 9.32 & 2043 & 102 & 36,960 & 14.98 & 3284 & 3,329 & 499,445 & 6.05 & 4670 & 2,858 & 541,246 & 7.71 \\
\hline 1017 & 3,239 & 549,062 & 6.87 & 2048 & 1,951 & 409,659 & 8.57 & 3285 & 2,627 & 382,496 & 5.90 & 4671 & 175 & 25,848 & 5.97 \\
\hline 1042 & 27 & 1,488 & 1.96 & 2050 & 83 & 17,448 & 8.57 & 3329 & 1,869 & 306,792 & 6.61 & 4679 & 2,957 & 429,394 & 5.86 \\
\hline 1048 & 27 & 1,728 & 2.49 & 2051 & 70 & 26,304 & 15.46 & 3335 & 247 & 33,792 & 5.44 & 4696 & 15 & 1,992 & 5.37 \\
\hline 1053 & 734 & 145,392 & 8.02 & 2053 & 11 & 3,552 & 13.31 & 3370 & 2,135 & 317,621 & 5.95 & 4703 & 134 & 31,392 & 9.57 \\
\hline 1057 & 1,881 & 363,120 & 7.81 & 2073 & 1,041 & 146,832 & 5.65 & 3410 & 2,711 & 514,264 & 7.72 & 4704 & 473 & 61,368 & 5.15 \\
\hline 1063 & 29 & 1,968 & 2.58 & 2082 & 2,000 & 519,707 & 10.67 & 3415 & 3,347 & 537,022 & 6.50 & 4731 & 146 & 17,160 & 4.66 \\
\hline 1068 & 3,066 & 512,393 & 6.74 & 2086 & 2,797 & 548,518 & 7.96 & 3430 & 5,047 & 530,963 & 4.16 & 4792 & 1,402 & 213,141 & 6.18 \\
\hline 1080 & 215 & 61,344 & 11.76 & 2088 & 63 & 10,190 & 6.54 & 3431 & 313 & 32,784 & 4.14 & 4819 & 343 & 39,480 & 4.61 \\
\hline 1081 & 1,407 & 241,368 & 6.88 & 2090 & 398 & 58,356 & 5.95 & 3441 & 45 & 42,816 & 39.43 & 4852 & 17 & 2,016 & 4.82 \\
\hline 1133 & 19 & 2,784 & 5.81 & 2096 & 3,028 & 493,125 & 6.57 & 3442 & 564 & 171,216 & 12.42 & 4866 & 3,233 & 548,790 & 6.84 \\
\hline 1136 & 4,368 & 532,054 & 4.87 & 2128 & 385 & 68,160 & 7.11 & 3446 & 721 & 213,312 & 12.14 & 4876 & 155 & 29,184 & 7.66 \\
\hline 1138 & 23 & 1,320 & 2.21 & 2131 & 2,616 & 483,317 & 7.52 & 3460 & 47 & 7,008 & 6.01 & 4878 & 3,157 & 350,328 & 4.37 \\
\hline 1139 & 104 & 13,056 & 4.92 & 2142 & 22 & 2,016 & 3.50 & 3462 & 163 & 49,920 & 12.53 & 4880 & 2,923 & 551,284 & 7.66 \\
\hline
\end{tabular}


Appendix 4-1.1. Number of storms, total duration, and mean storm interevent time defined by 6 -hour minimum interevent time for hourly rainfall stations in Texas-Continued.

\begin{tabular}{|c|c|c|c|c|c|c|c|c|c|c|c|c|c|c|c|}
\hline $\begin{array}{c}\text { Station } \\
\text { no. }\end{array}$ & $\begin{array}{c}\text { No. } \\
\text { of } \\
\text { storms }\end{array}$ & $\begin{array}{c}\text { Total } \\
\text { duration } \\
\text { (hours) }\end{array}$ & $\begin{array}{c}\text { Mean } \\
\text { storm } \\
\text { interevent } \\
\text { time } \\
\text { (days) }\end{array}$ & $\begin{array}{c}\text { Station } \\
\text { no. }\end{array}$ & $\begin{array}{c}\text { No. } \\
\text { of } \\
\text { storms }\end{array}$ & $\begin{array}{c}\text { Total } \\
\text { duration } \\
\text { (hours) }\end{array}$ & $\begin{array}{c}\text { Mean } \\
\text { storm } \\
\text { interevent } \\
\text { time } \\
\text { (days) }\end{array}$ & $\begin{array}{c}\text { Station } \\
\text { no. }\end{array}$ & $\begin{array}{c}\text { No. } \\
\text { of } \\
\text { storms }\end{array}$ & $\begin{array}{c}\text { Total } \\
\text { duration } \\
\text { (hours) }\end{array}$ & $\begin{array}{c}\text { Mean } \\
\text { storm } \\
\text { interevent } \\
\text { time } \\
\text { (days) }\end{array}$ & $\begin{array}{c}\text { Station } \\
\text { no. }\end{array}$ & $\begin{array}{c}\text { No. } \\
\text { of } \\
\text { storms }\end{array}$ & $\begin{array}{c}\text { Total } \\
\text { duration } \\
\text { (hours) }\end{array}$ & $\begin{array}{c}\text { Mean } \\
\text { storm } \\
\text { interevent } \\
\text { time } \\
\text { (days) }\end{array}$ \\
\hline 4920 & 2,278 & 524,353 & 9.41 & 5775 & 14 & 5,112 & 15.00 & 7481 & 1,622 & 495,877 & 12.57 & 8743 & 4,043 & 527,995 & 5.20 \\
\hline 4934 & 9 & 1,992 & 9.08 & 5779 & 27 & 5,880 & 8.82 & 7497 & 1,678 & 243,992 & 5.89 & 8761 & 1,118 & 256,990 & 9.42 \\
\hline 4972 & 2,943 & 494,452 & 6.79 & 5840 & 414 & 78,888 & 7.66 & 7498 & 342 & 49,632 & 5.88 & 8778 & 2,983 & 398,041 & 5.36 \\
\hline 4973 & 513 & 119,208 & 9.43 & 5890 & 2,724 & 515,614 & 7.69 & 7499 & 2,724 & 523,925 & 7.84 & 8845 & 3,555 & 550,655 & 6.25 \\
\hline 4974 & 1,491 & 340,313 & 9.32 & 5891 & 181 & 53,304 & 12.09 & 7531 & 165 & 49,968 & 12.35 & 8859 & 2,280 & 312,504 & 5.44 \\
\hline 4975 & 1,976 & 257,716 & 5.25 & 5897 & 1,707 & 245,324 & 5.82 & 7534 & 362 & 103,032 & 11.64 & 8898 & 770 & 116,904 & 6.05 \\
\hline 4978 & 169 & 47,496 & 11.53 & 5957 & 2,566 & 445,446 & 7.05 & 7556 & 3,129 & 493,793 & 6.39 & 8908 & 46 & 5,904 & 5.13 \\
\hline 4979 & 24 & 1,896 & 2.89 & 5958 & 232 & 41,136 & 7.17 & 7594 & 2,370 & 309,414 & 5.20 & 8910 & 23 & 1,968 & 3.39 \\
\hline 4982 & 1,174 & 248,714 & 8.60 & 5973 & 125 & 23,376 & 7.53 & 7596 & 177 & 26,136 & 5.90 & 8911 & 1,004 & 164,256 & 6.62 \\
\hline 5018 & 813 & 134,338 & 6.65 & 5996 & 3,420 & 549,087 & 6.47 & 7608 & 1,865 & 249,648 & 5.36 & 8924 & 477 & 181,152 & 15.67 \\
\hline 5048 & 1,834 & 503,770 & 11.29 & 6017 & 151 & 23,280 & 6.23 & 7622 & 23 & 4,152 & 7.36 & 8929 & 29 & 2,016 & 2.66 \\
\hline 5049 & 109 & 34,272 & 12.98 & 6024 & 296 & 72,480 & 9.92 & 7700 & 2,663 & 308,448 & 4.56 & 8942 & 2,480 & 300,835 & 4.85 \\
\hline 5056 & 5 & 10,104 & 83.94 & 6050 & 23 & 6,576 & 11.65 & 7706 & 2,427 & 502,063 & 8.44 & 8944 & 1,373 & 144,888 & 4.17 \\
\hline 5057 & 1,099 & 189,960 & 6.98 & 6104 & 2,892 & 478,193 & 6.74 & 7718 & 118 & 34,320 & 11.87 & 8996 & 3,294 & 399,638 & 4.82 \\
\hline 5060 & 328 & 59,904 & 7.36 & 6108 & 3,605 & 542,529 & 6.06 & 7745 & 480 & 70,872 & 5.85 & 9014 & 25 & 1,896 & 2.86 \\
\hline 5081 & 1,491 & 180,720 & 4.76 & 6136 & 2,496 & 539,302 & 8.86 & 7922 & 348 & 111,768 & 13.22 & 9037 & 776 & 240,720 & 12.73 \\
\hline 5094 & 3,129 & 468,823 & 6.05 & 6166 & 106 & 14,520 & 5.51 & 7936 & 2,603 & 306,666 & 4.70 & 9106 & 229 & 65,736 & 11.76 \\
\hline 5113 & 3,023 & 523,501 & 7.02 & 6176 & 448 & 69,264 & 6.18 & 7943 & 3,046 & 474,742 & 6.29 & 9107 & 40 & 26,784 & 27.76 \\
\hline 5114 & 0 & 1,488 & -- & 6177 & 3,106 & 440,385 & 5.69 & 7944 & 90 & 8,592 & 3.74 & 9129 & 129 & 23,376 & 7.35 \\
\hline 5123 & 17 & 3,072 & 7.34 & 6210 & 2,506 & 354,217 & 5.69 & 7945 & 4,789 & 543,166 & 4.48 & 9163 & 2,614 & 550,742 & 8.57 \\
\hline 5192 & 1,973 & 332,072 & 6.79 & 6211 & 564 & 93,000 & 6.67 & 7947 & 296 & 49,618 & 6.79 & 9213 & 292 & 38,700 & 5.30 \\
\hline 5193 & 3,814 & 550,050 & 5.79 & 6270 & 2,103 & 256,135 & 4.88 & 7948 & 1,214 & 213,840 & 7.13 & 9214 & 35 & 2,016 & 2.16 \\
\hline 5224 & 415 & 52,608 & 5.05 & 6275 & 0 & 2,920 & -- & 7951 & 1,151 & 141,648 & 4.87 & 9222 & 265 & 31,416 & 4.68 \\
\hline 5228 & 468 & 79,632 & 6.87 & 6276 & 29 & 2,016 & 2.60 & 7953 & 141 & 23,352 & 6.65 & 9248 & 143 & 52,272 & 14.98 \\
\hline 5235 & 35 & 4,392 & 4.94 & 6335 & 2,621 & 354,128 & 5.38 & 7981 & 260 & 112,728 & 17.84 & 9266 & 86 & 17,160 & 8.06 \\
\hline 5247 & 2,952 & 552,074 & 7.62 & 6434 & 23 & 8,016 & 14.25 & 7990 & 156 & 23,304 & 6.00 & 9270 & 1,914 & 382,084 & 8.18 \\
\hline 5258 & 1,010 & 177,528 & 7.07 & 6504 & 2,650 & 548,140 & 8.46 & 7992 & 28 & 1,896 & 2.59 & 9295 & 116 & 27,672 & 9.77 \\
\hline 5303 & 749 & 179,592 & 9.76 & 6558 & 34 & 5,688 & 6.72 & 7997 & 131 & 34,656 & 10.83 & 9304 & 4 & 336 & 3.50 \\
\hline 5312 & 2,113 & 404,451 & 7.81 & 6615 & 753 & 194,104 & 10.60 & 7999 & 21 & 13,992 & 27.58 & 9307 & 292 & 79,272 & 11.09 \\
\hline 5341 & 30 & 2,016 & 2.52 & 6660 & 132 & 32,880 & 10.12 & 8022 & 78 & 48,504 & 25.69 & 9328 & 198 & 34,344 & 6.99 \\
\hline 5342 & 0 & 4,404 & -- & 6663 & 119 & 36,168 & 12.51 & 8023 & 1,804 & 484,077 & 11.01 & 9329 & 16 & 3,240 & 8.10 \\
\hline 5348 & 1,876 & 240,206 & 5.14 & 6734 & 437 & 102,262 & 9.53 & 8047 & 2,778 & 548,776 & 8.03 & 9345 & 3 & 960 & 13.33 \\
\hline 5358 & 1,657 & 387,072 & 9.53 & 6736 & 2,000 & 452,975 & 9.27 & 8060 & 154 & 23,376 & 6.09 & 9363 & 1,229 & 135,816 & 4.40 \\
\hline 5398 & 3,164 & 411,020 & 5.15 & 6740 & 21 & 2,016 & 3.65 & 8062 & 47 & 6,504 & 5.47 & 9364 & 4,148 & 418,894 & 3.99 \\
\hline 5410 & 2,582 & 528,181 & 8.36 & 6750 & 298 & 37,990 & 5.09 & 8068 & 41 & 21,192 & 21.36 & 9365 & 40 & 5,664 & 5.71 \\
\hline 5411 & 3,085 & 462,238 & 6.03 & 6757 & 4,304 & 551,361 & 5.10 & 8081 & 2,981 & 537,081 & 7.30 & 9371 & 172 & 31,416 & 7.37 \\
\hline 5424 & 543 & 56,254 & 4.05 & 6775 & 639 & 110,784 & 6.99 & 8089 & 46 & 5,760 & 5.00 & 9417 & 2,278 & 331,982 & 5.83 \\
\hline 5429 & 1,172 & 193,392 & 6.66 & 6776 & 2,441 & 431,190 & 7.19 & 8221 & 24 & 1,896 & 2.93 & 9419 & 4,521 & 542,606 & 4.76 \\
\hline 5431 & 18 & 13,848 & 31.61 & 6788 & 202 & 29,256 & 5.81 & 8252 & 2,025 & 529,928 & 10.72 & 9435 & 85 & 33,600 & 16.25 \\
\hline 5461 & 2,076 & 324,672 & 6.24 & 6792 & 1,812 & 419,078 & 9.51 & 8265 & 1,862 & 217,176 & 4.58 & 9491 & 3,127 & 443,170 & 5.69 \\
\hline 5463 & 1,375 & 224,790 & 6.64 & 6794 & 30 & 2,016 & 2.43 & 8289 & 63 & 36,528 & 23.94 & 9499 & 2,056 & 431,095 & 8.57 \\
\hline 5471 & 28 & 3,480 & 5.10 & 6834 & 2,445 & 317,594 & 5.23 & 8305 & 1,882 & 512,439 & 11.21 & 9522 & 19 & 2,016 & 4.14 \\
\hline 5477 & 26 & 1,992 & 2.97 & 6893 & 1,414 & 374,777 & 10.91 & 8335 & 1,909 & 259,728 & 5.38 & 9527 & 2,498 & 539,410 & 8.85 \\
\hline 5528 & 1,384 & 262,609 & 7.67 & 6935 & 2,045 & 406,635 & 8.14 & 8400 & 258 & 62,112 & 9.88 & 9532 & 3,180 & 484,263 & 6.15 \\
\hline 5579 & 2 & 696 & 14.50 & 6981 & 216 & 132,768 & 25.38 & 8445 & 1,491 & 206,856 & 5.52 & 9544 & 5 & 2,928 & 24.27 \\
\hline 5580 & 29 & 1,944 & 2.59 & 7020 & 159 & 21,432 & 5.32 & 8446 & 2,086 & 268,785 & 5.18 & 9565 & 1,682 & 352,743 & 8.56 \\
\hline 5589 & 189 & 37,872 & 8.21 & 7060 & 1,093 & 277,917 & 10.43 & 8451 & 302 & 71,544 & 9.65 & 9570 & 580 & 100,848 & 7.10 \\
\hline 5590 & 194 & 39,024 & 8.17 & 7066 & 3,535 & 470,310 & 5.31 & 8531 & 1,798 & 305,116 & 6.82 & 9574 & 27 & 2,856 & 4.30 \\
\hline 5591 & 723 & 170,232 & 9.65 & 7074 & 2,335 & 523,667 & 9.19 & 8541 & 122 & 22,615 & 7.49 & 9588 & 2,377 & 457,802 & 7.83 \\
\hline 5592 & 612 & 151,920 & 10.18 & 7097 & 131 & 29,232 & 9.07 & 8544 & 599 & 110,871 & 7.48 & 9665 & 3,744 & 548,295 & 5.89 \\
\hline 5594 & 186 & 68,448 & 15.19 & 7116 & 679 & 167,304 & 10.05 & 8545 & 23 & 4,656 & 8.15 & 9715 & 3,018 & 443,326 & 5.91 \\
\hline 5595 & 2 & 8,016 & 167.00 & 7140 & 2,918 & 395,594 & 5.43 & 8563 & 1,460 & 220,484 & 6.12 & 9729 & 3,990 & 549,310 & 5.50 \\
\hline 5596 & 1,447 & 292,949 & 8.32 & 7173 & 1,291 & 115,200 & 3.48 & 8566 & 612 & 143,616 & 9.60 & 9772 & 1,149 & 140,832 & 4.88 \\
\hline 5600 & 467 & 105,888 & 9.26 & 7174 & 5,394 & 474,094 & 3.43 & 8583 & 1,021 & 203,370 & 8.16 & 9814 & 41 & 13,030 & 12.96 \\
\hline 5618 & 37 & 2,112 & 2.14 & 7213 & 876 & 156,336 & 7.19 & 8584 & 1,560 & 344,280 & 9.01 & 9815 & 848 & 121,848 & 5.75 \\
\hline 5650 & 22 & 1896 & 3.39 & 7243 & 3,239 & 549,034 & 6.88 & 8623 & 1,868 & 319,544 & 6.94 & 9816 & 83 & 35,472 & 17.60 \\
\hline 5656 & 1,863 & 326,279 & 7.16 & 7262 & 106 & 27,360 & 10.63 & 8625 & 1,488 & 243,840 & 6.57 & 9817 & 1,621 & 356,901 & 9.00 \\
\hline 5658 & 995 & 209,400 & 8.53 & 7274 & 471 & 121,725 & 10.56 & 8630 & 861 & 226,383 & 10.76 & 9829 & 1,684 & 436,603 & 10.63 \\
\hline 5661 & 253 & 46,672 & 7.53 & 7300 & 1,354 & 258,332 & 7.73 & 8631 & 992 & 243,264 & 9.99 & 9830 & 175 & 45,216 & 10.60 \\
\hline 5666 & 38 & 5,880 & 6.22 & 7311 & 32 & 6,600 & 8.41 & 8646 & 2,083 & 338,752 & 6.53 & 9858 & 1,514 & 293,448 & 7.86 \\
\hline 5695 & 2,606 & 448,602 & 6.95 & 7363 & 28 & 2,016 & 2.74 & 8647 & 1,482 & 282,860 & 7.79 & 9893 & 2,956 & 537,872 & 7.37 \\
\hline 5742 & 23 & 5,784 & 10.27 & 7422 & 3,350 & 544,386 & 6.57 & 8677 & 197 & 64,752 & 13.45 & 9916 & 1,245 & 185,230 & 5.98 \\
\hline 5766 & 37 & 2,016 & 2.05 & 7431 & 1,638 & 456,709 & 11.43 & 8696 & 30 & 2,016 & 2.61 & 9976 & 2,335 & 549,337 & 9.60 \\
\hline 5770 & 3,219 & 545,182 & 6.87 & & & & & & & & & & & & \\
\hline
\end{tabular}


Appendix 4-1.2. Number of storms, total duration, and mean storm interevent time defined by 8-hour minimum interevent time for hourly rainfall stations in Texas.

[--, not available]

\begin{tabular}{|c|c|c|c|c|c|c|c|c|c|c|c|c|c|c|c|}
\hline $\begin{array}{c}\text { Station } \\
\text { no. }\end{array}$ & $\begin{array}{c}\text { No. } \\
\text { of } \\
\text { storms }\end{array}$ & $\begin{array}{c}\text { Total } \\
\text { duration } \\
\text { (hours) }\end{array}$ & $\begin{array}{c}\text { Mean } \\
\text { storm } \\
\text { interevent } \\
\text { time } \\
\text { (days) }\end{array}$ & $\begin{array}{c}\text { Station } \\
\text { no. }\end{array}$ & $\begin{array}{c}\text { No. } \\
\text { of } \\
\text { storms }\end{array}$ & $\begin{array}{c}\text { Total } \\
\text { duration } \\
\text { (hours) }\end{array}$ & $\begin{array}{c}\text { Mean } \\
\text { storm } \\
\text { interevent } \\
\text { time } \\
\text { (days) }\end{array}$ & $\begin{array}{c}\text { Station } \\
\text { no. }\end{array}$ & $\begin{array}{c}\text { No. } \\
\text { of } \\
\text { storms }\end{array}$ & $\begin{array}{c}\text { Total } \\
\text { duration } \\
\text { (hours) }\end{array}$ & $\begin{array}{c}\text { Mean } \\
\text { storm } \\
\text { interevent } \\
\text { time } \\
\text { (days) }\end{array}$ & $\begin{array}{c}\text { Station } \\
\text { no. }\end{array}$ & $\begin{array}{c}\text { No. } \\
\text { of } \\
\text { storms }\end{array}$ & $\begin{array}{c}\text { Total } \\
\text { duration } \\
\text { (hours) }\end{array}$ & $\begin{array}{c}\text { Mean } \\
\text { storm } \\
\text { interevent } \\
\text { time } \\
\text { (days) }\end{array}$ \\
\hline 0015 & 10 & 624 & 2.32 & 1154 & 242 & 26,928 & 4.38 & 2160 & 28 & 4,752 & 6.70 & 3463 & 49 & 30,096 & 25.26 \\
\hline 0016 & 3,492 & 543,665 & 6.23 & 1165 & 665 & 147,768 & 9.00 & 2206 & 813 & 105,121 & 5.13 & 3476 & 761 & 127,848 & 6.74 \\
\hline 0050 & 1,042 & 197,240 & 7.59 & 1185 & 397 & 185,232 & 19.25 & 2238 & 273 & 51864 & 7.68 & 3485 & 25 & 1,968 & 3.04 \\
\hline 0054 & 58 & 5,688 & 3.89 & 1186 & 125 & 19,128 & 6.11 & 2240 & 104 & 12864 & 4.98 & 3507 & 1,178 & 188,267 & 6.45 \\
\hline 0120 & 29 & 1,896 & 2.36 & 1188 & 10 & 1,872 & 7.53 & 2242 & 1,936 & 253435 & 5.18 & 3546 & 3,504 & 485,758 & 5.51 \\
\hline 0145 & 191 & 28,392 & 5.97 & 1245 & 36 & 1,992 & 1.99 & 2244 & 3,689 & 535994 & 5.80 & 3547 & 219 & 51,144 & 9.49 \\
\hline 0146 & 49 & 5,664 & 4.52 & 1246 & 1,264 & 176,017 & 5.61 & 2247 & 62 & 7056 & 4.51 & 3579 & 79 & 19,944 & 10.17 \\
\hline 0174 & 1,467 & 275,194 & 7.66 & 1267 & 524 & 116,832 & 9.05 & 2309 & 721 & 153600 & 8.58 & 3642 & 3,374 & 534,563 & 6.34 \\
\hline 0178 & 24 & 2,040 & 3.37 & 1304 & 616 & 117,840 & 7.71 & 2312 & 746 & 92757 & 4.96 & 3646 & 2,231 & 419,874 & 7.55 \\
\hline 0179 & 336 & 66,456 & 8.07 & 1325 & 1,519 & 219,432 & 5.74 & 2334 & 65 & 22344 & 14.06 & 3668 & 24 & 2,016 & 3.10 \\
\hline 0202 & 288 & 58,398 & 8.31 & 1429 & 1,351 & 213,741 & 6.35 & 2336 & 236 & 53256 & 9.17 & 3673 & 31 & 2,016 & 2.37 \\
\hline 0206 & 2,730 & 532,111 & 7.92 & 1431 & 1,922 & 356,239 & 7.42 & 2354 & 31 & 20976 & 27.97 & 3686 & 1,236 & 198,532 & 6.49 \\
\hline 0208 & 4 & 384 & 4.00 & 1432 & 1,376 & 246,787 & 7.17 & 2355 & 59 & 10536 & 7.12 & 3691 & 3,000 & 469,594 & 6.31 \\
\hline 0211 & 3,654 & 542,494 & 5.94 & 1433 & 2,015 & 367,055 & 7.29 & 2357 & 556 & 98496 & 7.12 & 3734 & 33 & 2,016 & 2.29 \\
\hline 0244 & 92 & 11,568 & 4.89 & 1434 & 2,058 & 358,448 & 6.97 & 2360 & 1,857 & 399684 & 8.74 & 3771 & 1,528 & 220,455 & 5.82 \\
\hline 0248 & 2,060 & 529,764 & 10.53 & 1435 & 1,211 & 230,732 & 7.65 & 2361 & 203 & 56004 & 11.32 & 3789 & 113 & 18,888 & 6.79 \\
\hline 0262 & 2,719 & 431,066 & 6.34 & 1436 & 2,279 & 367,281 & 6.40 & 2394 & 3,343 & 504483 & 6.01 & 3826 & 232 & 45,240 & 7.86 \\
\hline 0271 & 25 & 2,016 & 3.02 & 1437 & 27 & 4,728 & 7.03 & 2404 & 3,066 & 493624 & 6.45 & 3831 & 436 & 110,112 & 10.26 \\
\hline 0380 & 422 & 163,872 & 15.91 & 1438 & 1,643 & 284,045 & 6.91 & 2415 & 3,126 & 501413 & 6.39 & 3841 & 165 & 77,472 & 19.36 \\
\hline 0394 & 11 & 1,992 & 7.20 & 1462 & 0 & 4,416 & -- & 2462 & 1,050 & 188205 & 7.21 & 3871 & 1,515 & 317,328 & 8.45 \\
\hline 0408 & 32 & 2,112 & 2.53 & 1492 & 1,244 & 280,441 & 9.20 & 2528 & 125 & 24840 & 7.98 & 3884 & 23 & 1,896 & 3.21 \\
\hline 0427 & 67 & 11,646 & 7.03 & 1500 & 28 & 2,112 & 2.78 & 2617 & 264 & 56688 & 8.66 & 3941 & 74 & 7,608 & 3.93 \\
\hline 0428 & 4,404 & 529,122 & 4.72 & 1528 & 1,433 & 366,583 & 10.46 & 2619 & 242 & 64992 & 10.93 & 3963 & 7 & 1,488 & 8.86 \\
\hline 0429 & 195 & 22,416 & 4.43 & 1541 & 66 & 10,953 & 6.67 & 2621 & 1,298 & 277864 & 8.70 & 4040 & 471 & 92,806 & 7.95 \\
\hline 0463 & 117 & 51,936 & 18.23 & 1569 & 221 & 32,160 & 5.81 & 2675 & 1,194 & 178918 & 6.01 & 4058 & 30 & 2,016 & 2.63 \\
\hline 0493 & 18 & 1,704 & 3.63 & 1632 & 7 & 1,320 & 7.86 & 2676 & 1,042 & 146737 & 5.69 & 4098 & 1,826 & 417,420 & 9.37 \\
\hline 0495 & 357 & 138,072 & 15.91 & 1641 & 227 & 110,280 & 20.01 & 2679 & 2,350 & 536229 & 9.30 & 4100 & 371 & 121,992 & 13.46 \\
\hline 0496 & 27 & 10,872 & 16.72 & 1646 & 2,525 & 543,287 & 8.80 & 2715 & 1,854 & 354131 & 7.70 & 4137 & 1,269 & 221,233 & 7.09 \\
\hline 0498 & 12 & 7,920 & 27.44 & 1663 & 48 & 11,460 & 9.69 & 2744 & 1,887 & 413376 & 8.91 & 4191 & 2,551 & 519,452 & 8.25 \\
\hline 0509 & 3,173 & 549,223 & 6.96 & 1671 & 3,440 & 548,538 & 6.41 & 2758 & 123 & 23376 & 7.61 & 4256 & 0 & 21,906 & -- \\
\hline 0518 & 2,211 & 330,777 & 6.02 & 1680 & 1,259 & 261,792 & 8.37 & 2794 & 11 & 2064 & 7.57 & 4257 & 3,359 & 498,651 & 5.95 \\
\hline 0521 & 35 & 5,760 & 6.55 & 1694 & 165 & 45,172 & 11.19 & 2797 & 2,548 & 529606 & 8.46 & 4258 & 204 & 25,608 & 5.02 \\
\hline 0556 & 115 & 21,936 & 7.67 & 1696 & 1,149 & 268,080 & 9.45 & 2811 & 2,074 & 435389 & 8.54 & 4278 & 1,671 & 409,467 & 9.94 \\
\hline 0569 & 2,724 & 399,353 & 5.88 & 1697 & 90 & 29,496 & 13.40 & 2813 & 30 & 31728 & 43.90 & 4299 & 129 & 86,976 & 27.91 \\
\hline 0572 & 1,096 & 158,448 & 5.77 & 1698 & 1,411 & 291,219 & 8.40 & 2814 & 17 & 12336 & 30.02 & 4300 & 3,055 & 289,264 & 3.67 \\
\hline 0576 & 151 & 23,352 & 6.17 & 1720 & 237 & 46,709 & 8.04 & 2815 & 264 & 58942 & 9.14 & 4305 & 2,708 & 266,616 & 3.83 \\
\hline 0580 & 364 & 112,800 & 12.65 & 1761 & 123 & 16,680 & 5.40 & 2818 & 198 & 45312 & 9.28 & 4307 & 695 & 96,430 & 5.49 \\
\hline 0587 & 959 & 121,272 & 4.98 & 1773 & 3,716 & 502,398 & 5.39 & 2986 & 292 & 54240 & 7.40 & 4309 & 3,843 & 517,057 & 5.35 \\
\hline 0605 & 131 & 27,000 & 8.34 & 1810 & 24 & 2,112 & 3.44 & 3005 & 2,815 & 522,908 & 7.51 & 4311 & 3,900 & 543,389 & 5.54 \\
\hline 0639 & 2,542 & 433,077 & 6.91 & 1823 & 25 & 1,968 & 3.11 & 3033 & 672 & 216,192 & 13.22 & 4313 & 300 & 39,432 & 5.21 \\
\hline 0655 & 0 & 718 & -- & 1870 & 357 & 47,496 & 5.19 & 3034 & 2 & 6,888 & 143.50 & 4319 & 105 & 29,208 & 11.32 \\
\hline 0665 & 2,217 & 361,772 & 6.52 & 1875 & 20 & 1,752 & 3.46 & 3047 & 35 & 2,016 & 2.15 & 4329 & 3,007 & 438,183 & 5.80 \\
\hline 0689 & 1,723 & 381,486 & 8.97 & 1876 & 52 & 10,920 & 8.42 & 3103 & 34 & 29,448 & 35.90 & 4331 & 0 & 5,112 & -- \\
\hline 0690 & 726 & 179,395 & 10.15 & 1889 & 334 & 38,734 & 4.55 & 3133 & 2,797 & 486,379 & 6.97 & 4375 & 1,307 & 232,138 & 7.23 \\
\hline 0691 & 2,839 & 469,381 & 6.63 & 1903 & 378 & 83,753 & 9.08 & 3156 & 317 & 63,435 & 8.09 & 4392 & 508 & 72,624 & 5.60 \\
\hline 0708 & 148 & 32,809 & 9.07 & 1914 & 22 & 1,752 & 3.04 & 3171 & 2,548 & 463,053 & 7.29 & 4425 & 926 & 264,416 & 11.73 \\
\hline 0738 & 1,859 & 306,512 & 6.58 & 1920 & 491 & 109,392 & 9.03 & 3189 & 378 & 97,104 & 10.51 & 4440 & 1,679 & 283,440 & 6.74 \\
\hline 0776 & 1,792 & 439,998 & 10.02 & 1921 & 3,260 & 476,617 & 5.83 & 3260 & 183 & 44,448 & 9.89 & 4476 & 1,972 & 344,677 & 7.07 \\
\hline 0779 & 463 & 108,787 & 9.63 & 1937 & 1,300 & 186,288 & 5.65 & 3267 & 112 & 22,512 & 8.10 & 4498 & 13 & 4,944 & 15.72 \\
\hline 0784 & 1,995 & 429,972 & 8.80 & 1956 & 3,516 & 486,210 & 5.52 & 3270 & 1,691 & 365,659 & 8.85 & 4517 & 1,893 & 328,752 & 6.97 \\
\hline 0786 & 576 & 117,888 & 8.28 & 1970 & 29 & 1,992 & 2.60 & 3272 & 24 & 3,840 & 6.55 & 4520 & 1,267 & 220,430 & 7.07 \\
\hline 0917 & 2,426 & 300,936 & 4.88 & 2014 & 527 & 69,312 & 5.23 & 3277 & 16 & 4,176 & 10.65 & 4525 & 35 & 1,992 & 2.09 \\
\hline 0923 & 27 & 2,016 & 2.69 & 2015 & 3,791 & 484,318 & 5.07 & 3278 & 1,061 & 253,450 & 9.77 & 4563 & 30 & 2,112 & 2.66 \\
\hline 0926 & 3,374 & 551,357 & 6.56 & 2019 & 29 & 2,016 & 2.68 & 3280 & 166 & 41,832 & 10.31 & 4570 & 2,471 & 548,973 & 9.04 \\
\hline 0950 & 46 & 18,936 & 17.03 & 2024 & 2,114 & 391,758 & 7.46 & 3281 & 46 & 26,304 & 23.68 & 4577 & 2,209 & 305,664 & 5.52 \\
\hline 0996 & 30 & 1,992 & 2.53 & 2042 & 13 & 16,080 & 51.41 & 3283 & 1,851 & 247,224 & 5.26 & 4591 & 2,938 & 431,406 & 5.81 \\
\hline 1013 & 266 & 63,492 & 9.79 & 2043 & 94 & 36,960 & 16.23 & 3284 & 3,115 & 499,445 & 6.45 & 4670 & 2,704 & 541,246 & 8.14 \\
\hline 1017 & 3,047 & 549,062 & 7.28 & 2048 & 1,832 & 409,659 & 9.11 & 3285 & 2,479 & 382,496 & 6.24 & 4671 & 169 & 25,848 & 6.18 \\
\hline 1042 & 26 & 1,488 & 2.03 & 2050 & 80 & 17,448 & 8.88 & 3329 & 1,740 & 306,792 & 7.08 & 4679 & 2,769 & 429,394 & 6.24 \\
\hline 1048 & 27 & 1,728 & 2.49 & 2051 & 67 & 26,304 & 16.14 & 3335 & 232 & 33,792 & 5.78 & 4696 & 15 & 1,992 & 5.37 \\
\hline 1053 & 697 & 145,392 & 8.43 & 2053 & 10 & 3,552 & 14.62 & 3370 & 2,028 & 317,621 & 6.25 & 4703 & 126 & 31,392 & 10.16 \\
\hline 1057 & 1,770 & 363,120 & 8.28 & 2073 & 995 & 146,832 & 5.90 & 3410 & 2,563 & 514,264 & 8.15 & 4704 & 434 & 61,368 & 5.59 \\
\hline 1063 & 26 & 1,968 & 2.85 & 2082 & 1,903 & 519,707 & 11.20 & 3415 & 3,170 & 537,022 & 6.84 & 4731 & 137 & 17,160 & 4.95 \\
\hline 1068 & 2,882 & 512,393 & 7.15 & 2086 & 2,626 & 548,518 & 8.46 & 3430 & 4,690 & 530,963 & 4.46 & 4792 & 1,310 & 213,141 & 6.59 \\
\hline 1080 & 209 & 61,344 & 12.09 & 2088 & 57 & 10,190 & 7.20 & 3431 & 292 & 32,784 & 4.42 & 4819 & 315 & 39,480 & 4.99 \\
\hline 1081 & 1,326 & 241,368 & 7.29 & 2090 & 375 & 58,356 & 6.29 & 3441 & 44 & 42,816 & 40.32 & 4852 & 17 & 2,016 & 4.82 \\
\hline 1133 & 17 & 2,784 & 6.46 & 2096 & 2,837 & 493,125 & 6.99 & 3442 & 539 & 171,216 & 12.98 & 4866 & 3,037 & 548,790 & 7.26 \\
\hline 1136 & 4,047 & 532,054 & 5.23 & 2128 & 360 & 68,160 & 7.59 & 3446 & 698 & 213,312 & 12.53 & 4876 & 146 & 29,184 & 8.11 \\
\hline 1138 & 21 & 1,320 & 2.40 & 2131 & 2,462 & 483,317 & 7.98 & 3460 & 44 & 7,008 & 6.40 & 4878 & 2,969 & 350,328 & 4.63 \\
\hline 1139 & 101 & 13,056 & 5.06 & 2142 & 20 & 2,016 & 3.83 & 3462 & 152 & 49,920 & 13.42 & 4880 & 2,788 & 551,284 & 8.02 \\
\hline
\end{tabular}


176 Statistical Characteristics of Storm Interevent Time, Depth, and Duration for Eastern New Mexico, Oklahoma, and Texas

Appendix 4-1.2. Number of storms, total duration, and mean storm interevent time defined by 8-hour minimum interevent time for hourly rainfall stations in Texas-Continued.

\begin{tabular}{|c|c|c|c|c|c|c|c|c|c|c|c|c|c|c|c|}
\hline $\begin{array}{c}\text { Station } \\
\text { no. }\end{array}$ & $\begin{array}{c}\text { No. } \\
\text { of } \\
\text { storms }\end{array}$ & $\begin{array}{c}\text { Total } \\
\text { duration } \\
\text { (hours) }\end{array}$ & $\begin{array}{c}\text { Mean } \\
\text { storm } \\
\text { interevent } \\
\text { time } \\
\text { (days) }\end{array}$ & $\begin{array}{c}\text { Station } \\
\text { no. }\end{array}$ & $\begin{array}{c}\text { No. } \\
\text { of } \\
\text { storms }\end{array}$ & $\begin{array}{c}\text { Total } \\
\text { duration } \\
\text { (hours) }\end{array}$ & $\begin{array}{c}\text { Mean } \\
\text { storm } \\
\text { interevent } \\
\text { time } \\
\text { (days) }\end{array}$ & $\begin{array}{c}\text { Station } \\
\text { no. }\end{array}$ & $\begin{array}{c}\text { No. } \\
\text { of } \\
\text { storms }\end{array}$ & $\begin{array}{c}\text { Total } \\
\text { duration } \\
\text { (hours) }\end{array}$ & $\begin{array}{c}\text { Mean } \\
\text { storm } \\
\text { interevent } \\
\text { time } \\
\text { (days) }\end{array}$ & $\begin{array}{c}\text { Station } \\
\text { no. }\end{array}$ & $\begin{array}{c}\text { No. } \\
\text { of } \\
\text { storms }\end{array}$ & $\begin{array}{c}\text { Total } \\
\text { duration } \\
\text { (hours) }\end{array}$ & $\begin{array}{c}\text { Mean } \\
\text { storm } \\
\text { interevent } \\
\text { time } \\
\text { (days) }\end{array}$ \\
\hline 4920 & 2,172 & 524,353 & 9.85 & 5775 & 14 & 5112 & 15.00 & 7481 & 1,548 & 495,877 & 13.16 & 8743 & 3,795 & 527995 & 5.52 \\
\hline 4934 & 8 & 1,992 & 10.18 & 5779 & 22 & 5880 & 10.77 & 7497 & 1,557 & 243,992 & 6.33 & 8761 & 1,076 & 256,990 & 9.78 \\
\hline 4972 & 2,772 & 494,452 & 7.20 & 5840 & 394 & 78888 & 8.04 & 7498 & 318 & 49,632 & 6.30 & 8778 & 2,815 & 398,041 & 5.66 \\
\hline 4973 & 486 & 119,208 & 9.93 & 5890 & 2,582 & 515614 & 8.09 & 7499 & 2,576 & 523,925 & 8.27 & 8845 & 3,339 & 550,655 & 6.63 \\
\hline 4974 & 1,397 & 340,313 & 9.93 & 5891 & 170 & 53304 & 12.85 & 7531 & 150 & 49,968 & 13.56 & 8859 & 2,143 & 312,504 & 5.77 \\
\hline 4975 & 1,830 & 257,716 & 5.65 & 5897 & 1,600 & 245324 & 6.20 & 7534 & 348 & 103,032 & 12.10 & 8898 & 733 & 116,904 & 6.34 \\
\hline 4978 & 157 & 47,496 & 12.39 & 5957 & 2,439 & 445446 & 7.40 & 7556 & 2,961 & 493,793 & 6.73 & 8908 & 43 & 5,904 & 5.46 \\
\hline 4979 & 22 & 1,896 & 3.13 & 5958 & 219 & 41136 & 7.58 & 7594 & 2,254 & 309,414 & 5.45 & 8910 & 22 & 1,968 & 3.53 \\
\hline 4982 & 1,126 & 248,714 & 8.95 & 5973 & 118 & 23376 & 7.96 & 7596 & 174 & 26,136 & 6.00 & 8911 & 960 & 164,256 & 6.91 \\
\hline 5018 & 763 & 134,338 & 7.06 & 5996 & 3,219 & 549087 & 6.86 & 7608 & 1,782 & 249,648 & 5.60 & 8924 & 465 & 181,152 & 16.07 \\
\hline 5048 & 1,742 & 503,770 & 11.87 & 6017 & 140 & 23280 & 6.70 & 7622 & 22 & 4,152 & 7.69 & 8929 & 26 & 2,016 & 2.93 \\
\hline 5049 & 103 & 34,272 & 13.72 & 6024 & 281 & 72480 & 10.44 & 7700 & 2,532 & 308,448 & 4.78 & 8942 & 2,335 & 300,835 & 5.14 \\
\hline 5056 & 5 & 10,104 & 83.94 & 6050 & 20 & 6,576 & 13.36 & 7706 & 2,288 & 502,063 & 8.94 & 8944 & 1,309 & 144,888 & 4.36 \\
\hline 5057 & 1,019 & 189,960 & 7.51 & 6104 & 2,774 & 478,193 & 7.01 & 7718 & 113 & 34,320 & 12.38 & 8996 & 3,129 & 399,638 & 5.06 \\
\hline 5060 & 313 & 59,904 & 7.70 & 6108 & 3,360 & 542,529 & 6.48 & 7745 & 440 & 70,872 & 6.35 & 9014 & 23 & 1,896 & 3.08 \\
\hline 5081 & 1,383 & 180,720 & 5.12 & 6136 & 2,386 & 539,302 & 9.26 & 7922 & 339 & 111,768 & 13.56 & 9037 & 733 & 240,720 & 13.46 \\
\hline 5094 & 2,953 & 468,823 & 6.40 & 6166 & 98 & 14,520 & 5.94 & 7936 & 2,461 & 306,666 & 4.95 & 9106 & 217 & 65,736 & 12.40 \\
\hline 5113 & 2,848 & 523,501 & 7.43 & 6176 & 420 & 69,264 & 6.57 & 7943 & 2,840 & 474,742 & 6.72 & 9107 & 40 & 26,784 & 27.76 \\
\hline 5114 & 0 & 1,488 & -- & 6177 & 2,930 & 440,385 & 6.02 & 7944 & 84 & 8,592 & 3.99 & 9129 & 121 & 23,376 & 7.82 \\
\hline 5123 & 16 & 3,072 & 7.78 & 6210 & 2,368 & 354,217 & 6.01 & 7945 & 4,454 & 543,166 & 4.80 & 9163 & 2,487 & 550,742 & 8.99 \\
\hline 5192 & 1,879 & 332,072 & 7.12 & 6211 & 535 & 93,000 & 7.02 & 7947 & 270 & 49,618 & 7.42 & 9213 & 258 & 38,700 & 5.96 \\
\hline 5193 & 3,590 & 550,050 & 6.13 & 6270 & 1,969 & 256,135 & 5.20 & 7948 & 1,146 & 213,840 & 7.54 & 9214 & 33 & 2,016 & 2.27 \\
\hline 5224 & 389 & 52,608 & 5.37 & 6275 & 0 & 2,920 & -- & 7951 & 1,101 & 141,648 & 5.08 & 9222 & 243 & 31,416 & 5.08 \\
\hline 5228 & 447 & 79,632 & 7.18 & 6276 & 28 & 2,016 & 2.67 & 7953 & 133 & 23,352 & 7.04 & 9248 & 137 & 52,272 & 15.63 \\
\hline 5235 & 33 & 4,392 & 5.23 & 6335 & 2,485 & 354,128 & 5.66 & 7981 & 250 & 112,728 & 18.55 & 9266 & 83 & 17,160 & 8.34 \\
\hline 5247 & 2,779 & 552,074 & 8.08 & 6434 & 23 & 8,016 & 14.25 & 7990 & 143 & 23,304 & 6.52 & 9270 & 1,830 & 382,084 & 8.55 \\
\hline 5258 & 963 & 177,528 & 7.40 & 6504 & 2,525 & 548,140 & 8.86 & 7992 & 28 & 1,896 & 2.59 & 9295 & 115 & 27,672 & 9.86 \\
\hline 5303 & 710 & 179,592 & 10.28 & 6558 & 32 & 5,688 & 7.12 & 7997 & 125 & 34,656 & 11.34 & 9304 & 4 & 336 & 3.50 \\
\hline 5312 & 1,997 & 404,451 & 8.25 & 6615 & 706 & 194,104 & 11.29 & 7999 & 19 & 13,992 & 30.45 & 9307 & 275 & 79272 & 11.75 \\
\hline 5341 & 29 & 2,016 & 2.59 & 6660 & 123 & 32,880 & 10.84 & 8022 & 74 & 48,504 & 27.07 & 9328 & 187 & 34344 & 7.38 \\
\hline 5342 & 0 & 4,404 & -- & 6663 & 113 & 36,168 & 13.16 & 8023 & 1,715 & 484,077 & 11.56 & 9329 & 16 & 3240 & 8.10 \\
\hline 5348 & 1,756 & 240,206 & 5.47 & 6734 & 410 & 102,262 & 10.14 & 8047 & 2,624 & 548,776 & 8.49 & 9345 & 3 & 960 & 13.33 \\
\hline 5358 & 1,560 & 387,072 & 10.11 & 6736 & 1,898 & 452,975 & 9.76 & 8060 & 140 & 23,376 & 6.68 & 9363 & 1,140 & 135816 & 4.72 \\
\hline 5398 & 2,986 & 411,020 & 5.44 & 6740 & 17 & 2,016 & 4.44 & 8062 & 45 & 6,504 & 5.70 & 9364 & 3,882 & 418894 & 4.25 \\
\hline 5410 & 2,460 & 528,181 & 8.76 & 6750 & 281 & 37,990 & 5.38 & 8068 & 39 & 21,192 & 22.44 & 9365 & 38 & 5664 & 6.00 \\
\hline 5411 & 2,881 & 462,238 & 6.44 & 6757 & 4,044 & 551,361 & 5.42 & 8081 & 2,814 & 537,081 & 7.71 & 9371 & 160 & 31416 & 7.90 \\
\hline 5424 & 486 & 56,254 & 4.49 & 6775 & 604 & 110,784 & 7.38 & 8089 & 43 & 5,760 & 5.33 & 9417 & 2,163 & 331982 & 6.13 \\
\hline 5429 & 1,098 & 193,392 & 7.09 & 6776 & 2,315 & 431,190 & 7.56 & 8221 & 21 & 1,896 & 3.32 & 9419 & 4,206 & 542606 & 5.09 \\
\hline 5431 & 18 & 13,848 & 31.61 & 6788 & 191 & 29,256 & 6.13 & 8252 & 1,940 & 529,928 & 11.18 & 9435 & 83 & 33600 & 16.63 \\
\hline 5461 & 1,952 & 324,672 & 6.62 & 6792 & 1,742 & 419,078 & 9.88 & 8265 & 1,763 & 217,176 & 4.82 & 9491 & 2,967 & 443170 & 5.98 \\
\hline 5463 & 1,289 & 224790 & 7.06 & 6794 & 29 & 2,016 & 2.51 & 8289 & 55 & 36,528 & 27.38 & 9499 & 1,947 & 431095 & 9.04 \\
\hline 5471 & 27 & 3480 & 5.28 & 6834 & 2,295 & 317,594 & 5.55 & 8305 & 1,804 & 512,439 & 11.68 & 9522 & 17 & 2016 & 4.59 \\
\hline 5477 & 25 & 1992 & 3.08 & 6893 & 1,347 & 374,777 & 11.43 & 8335 & 1,806 & 259,728 & 5.67 & 9527 & 2,371 & 539410 & 9.31 \\
\hline 5528 & 1,317 & 262609 & 8.05 & 6935 & 1,938 & 406,635 & 8.58 & 8400 & 239 & 62,112 & 10.65 & 9532 & 3,024 & 484263 & 6.45 \\
\hline 5579 & 1 & 696 & 29.00 & 6981 & 207 & 132,768 & 26.47 & 8445 & 1,409 & 206,856 & 5.83 & 9544 & 5 & 2928 & 24.27 \\
\hline 5580 & 29 & 1944 & 2.59 & 7020 & 146 & 21,432 & 5.77 & 8446 & 1,987 & 268,785 & 5.42 & 9565 & 1,591 & 352743 & 9.03 \\
\hline 5589 & 181 & 37872 & 8.56 & 7060 & 1,048 & 277,917 & 10.87 & 8451 & 282 & 71,544 & 10.32 & 9570 & 550 & 100848 & 7.48 \\
\hline 5590 & 185 & 39024 & 8.56 & 7066 & 3,348 & 470,310 & 5.59 & 8531 & 1,717 & 305,116 & 7.13 & 9574 & 25 & 2856 & 4.61 \\
\hline 5591 & 693 & 170232 & 10.05 & 7074 & 2,225 & 523,667 & 9.63 & 8541 & 111 & 22,615 & 8.21 & 9588 & 2,218 & 457802 & 8.37 \\
\hline 5592 & 591 & 151920 & 10.53 & 7097 & 121 & 29,232 & 9.80 & 8544 & 563 & 110,871 & 7.94 & 9665 & 3,518 & 548295 & 6.25 \\
\hline 5594 & 177 & 68448 & 15.95 & 7116 & 646 & 167,304 & 10.55 & 8545 & 22 & 4,656 & 8.51 & 9715 & 2,863 & 443326 & 6.21 \\
\hline 5595 & 2 & 8016 & 167.00 & 7140 & 2,760 & 395,594 & 5.73 & 8563 & 1,375 & 220,484 & 6.48 & 9729 & 3,741 & 549310 & 5.85 \\
\hline 5596 & 1,384 & 292949 & 8.69 & 7173 & 1,210 & 115,200 & 3.70 & 8566 & 583 & 143,616 & 10.07 & 9772 & 1,093 & 140832 & 5.12 \\
\hline 5600 & 452 & 105888 & 9.55 & 7174 & 5,042 & 474,094 & 3.65 & 8583 & 948 & 203,370 & 8.76 & 9814 & 38 & 13030 & 13.96 \\
\hline 5618 & 37 & 2112 & 2.13 & 7213 & 838 & 156,336 & 7.50 & 8584 & 1,457 & 344,280 & 9.62 & 9815 & 785 & 121848 & 6.19 \\
\hline 5650 & 20 & 1896 & 3.69 & 7243 & 3,063 & 549,034 & 7.26 & 8623 & 1,753 & 319,544 & 7.38 & 9816 & 80 & 35472 & 18.24 \\
\hline 5656 & 1,753 & 326279 & 7.59 & 7262 & 105 & 27,360 & 10.73 & 8625 & 1,398 & 243,840 & 6.97 & 9817 & 1,548 & 356901 & 9.41 \\
\hline 5658 & 945 & 209400 & 8.97 & 7274 & 459 & 121,725 & 10.82 & 8630 & 821 & 226,383 & 11.27 & 9829 & 1,609 & 436603 & 11.12 \\
\hline 5661 & 240 & 46672 & 7.92 & 7300 & 1,302 & 258,332 & 8.03 & 8631 & 939 & 243,264 & 10.54 & 9830 & 165 & 45216 & 11.23 \\
\hline 5666 & 36 & 5880 & 6.55 & 7311 & 32 & 6,600 & 8.41 & 8646 & 1,979 & 338,752 & 6.86 & 9858 & 1,450 & 293448 & 8.19 \\
\hline 5695 & 2,452 & 448602 & 7.36 & 7363 & 28 & 2,016 & 2.74 & 8647 & 1,413 & 282860 & 8.16 & 9893 & 2,790 & 537872 & 7.79 \\
\hline 5742 & 21 & 5784 & 11.22 & 7422 & 3,169 & 544,386 & 6.93 & 8677 & 183 & 64,752 & 14.46 & 9916 & 1,166 & 185230 & 6.36 \\
\hline 5766 & 34 & 2016 & 2.21 & 7431 & 1,562 & 456,709 & 11.97 & 8696 & 29 & 2,016 & 2.69 & 9976 & 2,181 & 549337 & 10.26 \\
\hline 5770 & 3,038 & 545182 & 7.26 & & & & & & & & & & & & \\
\hline
\end{tabular}


Appendix 4-1.3. Number of storms, total duration, and mean storm interevent time defined by 12-hour minimum interevent time for hourly rainfall stations in Texas.

[--, not available]

\begin{tabular}{|c|c|c|c|c|c|c|c|c|c|c|c|c|c|c|c|}
\hline $\begin{array}{c}\text { Station } \\
\text { no. }\end{array}$ & $\begin{array}{c}\text { No. } \\
\text { of } \\
\text { storms }\end{array}$ & $\begin{array}{c}\text { Total } \\
\text { duration } \\
\text { (hours) }\end{array}$ & $\begin{array}{c}\text { Mean } \\
\text { storm } \\
\text { interevent } \\
\text { time } \\
\text { (days) }\end{array}$ & $\begin{array}{c}\text { Station } \\
\text { no. }\end{array}$ & $\begin{array}{c}\text { No. } \\
\text { of } \\
\text { storms }\end{array}$ & $\begin{array}{c}\text { Total } \\
\text { duration } \\
\text { (hours) }\end{array}$ & $\begin{array}{c}\text { Mean } \\
\text { storm } \\
\text { interevent } \\
\text { time } \\
\text { (days) }\end{array}$ & $\begin{array}{c}\text { Station } \\
\text { no. }\end{array}$ & $\begin{array}{c}\text { No. } \\
\text { of } \\
\text { storms }\end{array}$ & $\begin{array}{c}\text { Total } \\
\text { duration } \\
\text { (hours) }\end{array}$ & $\begin{array}{c}\text { Mean } \\
\text { storm } \\
\text { interevent } \\
\text { time } \\
\text { (days) }\end{array}$ & $\begin{array}{c}\text { Station } \\
\text { no. }\end{array}$ & $\begin{array}{c}\text { No. } \\
\text { of } \\
\text { storms }\end{array}$ & $\begin{array}{l}\text { Total } \\
\text { duration } \\
\text { (hours) }\end{array}$ & $\begin{array}{c}\text { Mean } \\
\text { storm } \\
\text { interevent } \\
\text { time } \\
\text { (days) }\end{array}$ \\
\hline 0015 & 7 & 624 & 3.14 & 1154 & 215 & 26,928 & 4.87 & 2160 & 23 & 4,752 & 8.04 & 3463 & 48 & 30,096 & 25.78 \\
\hline 0016 & 3,189 & 543,665 & 6.78 & 1165 & 612 & 147,768 & 9.74 & 2206 & 759 & 105,121 & 5.47 & 3476 & 709 & 127,848 & 7.21 \\
\hline 0050 & 965 & 197,240 & 8.16 & 1185 & 379 & 185,232 & 20.15 & 2238 & 252 & 51,864 & 8.29 & 3485 & 22 & 1,968 & 3.38 \\
\hline 0054 & 53 & 5,688 & 4.22 & 1186 & 117 & 19,128 & 6.50 & 2240 & 94 & 12,864 & 5.46 & 3507 & 1,078 & 188,267 & 7.02 \\
\hline 0120 & 22 & 1,896 & 2.99 & 1188 & 10 & 1,872 & 7.53 & 2242 & 1,745 & 253,435 & 5.70 & 3546 & 3,210 & 485,758 & 5.98 \\
\hline 0145 & 179 & 28,392 & 6.33 & 1245 & 27 & 1,992 & 2.50 & 2244 & 3,345 & 535,994 & 6.35 & 3547 & 202 & 51,144 & 10.25 \\
\hline 0146 & 44 & 5,664 & 4.99 & 1246 & 1,140 & 176,017 & 6.18 & 2247 & 57 & 7,056 & 4.87 & 3579 & 77 & 19,944 & 10.42 \\
\hline 0174 & 1,356 & 275,194 & 8.26 & 1267 & 484 & 116,832 & 9.77 & 2309 & 662 & 153,600 & 9.31 & 3642 & 3,115 & 534,563 & 6.84 \\
\hline 0178 & 22 & 2,040 & 3.62 & 1304 & 561 & 117,840 & 8.42 & 2312 & 679 & 92,757 & 5.41 & 3646 & 2,106 & 419,874 & 7.97 \\
\hline 0179 & 321 & 66,456 & 8.43 & 1325 & 1,392 & 219,432 & 6.23 & 2334 & 60 & 22,344 & 15.19 & 3668 & 20 & 2,016 & 3.65 \\
\hline 0202 & 269 & 58,398 & 8.87 & 1429 & 1,254 & 213,741 & 6.81 & 2336 & 226 & 53,256 & 9.56 & 3673 & 25 & 2,016 & 2.83 \\
\hline 0206 & 2,505 & 532,111 & 8.59 & 1431 & 1,766 & 356,239 & 8.04 & 2354 & 27 & 20,976 & 32.06 & 3686 & 1,122 & 198,532 & 7.11 \\
\hline 0208 & 3 & 384 & 5.33 & 1432 & 1,283 & 246,787 & 7.66 & 2355 & 54 & 10,536 & 7.75 & 3691 & 2,764 & 469,594 & 6.81 \\
\hline 0211 & 3,334 & 542,494 & 6.47 & 1433 & 1,849 & 367,055 & 7.91 & 2357 & 512 & 98,496 & 7.70 & 3734 & 28 & 2,016 & 2.61 \\
\hline 0244 & 80 & 11,568 & 5.56 & 1434 & 1,907 & 358,448 & 7.49 & 2360 & 1,690 & 399,684 & 9.56 & 3771 & 1,418 & 220,455 & 6.24 \\
\hline 0248 & 1,915 & 529,764 & 11.30 & 1435 & 1,127 & 230,732 & 8.19 & 2361 & 186 & 56,004 & 12.32 & 3789 & 105 & 18,888 & 7.28 \\
\hline 0262 & 2,499 & 431,066 & 6.87 & 1436 & 2,106 & 367,281 & 6.89 & 2394 & 3,092 & 504,483 & 6.46 & 3826 & 212 & 45,240 & 8.57 \\
\hline 0271 & 22 & 2,016 & 3.36 & 1437 & 23 & 4,728 & 8.19 & 2404 & 2,821 & 493,624 & 6.98 & 3831 & 402 & 110,112 & 11.10 \\
\hline 0380 & 402 & 163,872 & 16.68 & 1438 & 1,509 & 284,045 & 7.48 & 2415 & 2,881 & 501,413 & 6.90 & 3841 & 152 & 77,472 & 20.98 \\
\hline 0394 & 9 & 1,992 & 8.71 & 1462 & 0 & 4,416 & -- & 2462 & 979 & 188,205 & 7.70 & 3871 & 1,377 & 317,328 & 9.26 \\
\hline 0408 & 27 & 2,112 & 2.91 & 1492 & 1,132 & 280,441 & 10.08 & 2528 & 116 & 24,840 & 8.57 & 3884 & 21 & 1,896 & 3.48 \\
\hline 0427 & 57 & 11,646 & 8.20 & 1500 & 27 & 2,112 & 2.87 & 2617 & 244 & 56,688 & 9.34 & 3941 & 64 & 7,608 & 4.49 \\
\hline 0428 & 3,936 & 529,122 & 5.23 & 1528 & 1,323 & 366,583 & 11.29 & 2619 & 220 & 64,992 & 11.98 & 3963 & 7 & 1,488 & 8.86 \\
\hline 0429 & 174 & 22,416 & 4.92 & 1541 & 62 & 10,953 & 7.08 & 2621 & 1,212 & 277,864 & 9.28 & 4040 & 448 & 92,806 & 8.34 \\
\hline 0463 & 115 & 51,936 & 18.54 & 1569 & 200 & 32,160 & 6.38 & 2675 & 1,109 & 178,918 & 6.44 & 4058 & 26 & 2,016 & 2.97 \\
\hline 0493 & 17 & 1,704 & 3.82 & 1632 & 7 & 1,320 & 7.86 & 2676 & 959 & 146,737 & 6.15 & 4098 & 1,683 & 417,420 & 10.13 \\
\hline 0495 & 341 & 138,072 & 16.64 & 1641 & 214 & 110,280 & 21.20 & 2679 & 2,134 & 536,229 & 10.20 & 4100 & 344 & 121,992 & 14.49 \\
\hline 0496 & 27 & 10,872 & 16.72 & 1646 & 2,356 & 543,287 & 9.40 & 2715 & 1,717 & 354,131 & 8.28 & 4137 & 1,149 & 221,233 & 7.78 \\
\hline 0498 & 12 & 7,920 & 27.44 & 1663 & 42 & 11,460 & 11.02 & 2744 & 1,724 & 413,376 & 9.71 & 4191 & 2,343 & 519,452 & 8.95 \\
\hline 0509 & 2,856 & 549,223 & 7.69 & 1671 & 3,150 & 548,538 & 6.96 & 2758 & 110 & 23,376 & 8.47 & 4256 & 0 & 21,906 & -- \\
\hline 0518 & 2,037 & 330,777 & 6.50 & 1680 & 1,169 & 261,792 & 8.99 & 2794 & 11 & 2,064 & 7.54 & 4257 & 3,066 & 498,651 & 6.48 \\
\hline 0521 & 33 & 5,760 & 6.92 & 1694 & 146 & 45,172 & 12.59 & 2797 & 2,370 & 529,606 & 9.07 & 4258 & 182 & 25,608 & 5.58 \\
\hline 0556 & 111 & 21,936 & 7.93 & 1696 & 1,080 & 268,080 & 10.03 & 2811 & 1,910 & 435,389 & 9.24 & 4278 & 1,555 & 409,467 & 10.66 \\
\hline 0569 & 2,517 & 399,353 & 6.33 & 1697 & 85 & 29,496 & 14.17 & 2813 & 27 & 31,728 & 48.74 & 4299 & 125 & 86,976 & 28.79 \\
\hline 0572 & 1,017 & 158,448 & 6.19 & 1698 & 1,293 & 291,219 & 9.13 & 2814 & 17 & 12,336 & 30.02 & 4300 & 2,768 & 289,264 & 4.01 \\
\hline 0576 & 131 & 23,352 & 7.05 & 1720 & 216 & 46,709 & 8.78 & 2815 & 247 & 58,942 & 9.74 & 4305 & 2,438 & 266,616 & 4.21 \\
\hline 0580 & 338 & 112,800 & 13.59 & 1761 & 111 & 16,680 & 5.93 & 2818 & 184 & 45,312 & 9.96 & 4307 & 609 & 96,430 & 6.21 \\
\hline 0587 & 872 & 121,272 & 5.43 & 1773 & 3,387 & 502,398 & 5.88 & 2986 & 267 & 54,240 & 8.06 & 4309 & 3,564 & 517,057 & 5.74 \\
\hline 0605 & 120 & 27,000 & 9.07 & 1810 & 24 & 2,112 & 3.42 & 3005 & 2,623 & 522,908 & 8.03 & 4311 & 3,587 & 543,389 & 5.99 \\
\hline 0639 & 2,358 & 433,077 & 7.42 & 1823 & 23 & 1,968 & 3.33 & 3033 & 646 & 216,192 & 13.73 & 4313 & 266 & 39,432 & 5.82 \\
\hline 0655 & 0 & 718 & -- & 1870 & 340 & 47,496 & 5.43 & 3034 & 2 & 6,888 & 143.50 & 4319 & 98 & 29,208 & 12.10 \\
\hline 0665 & 2,070 & 361,772 & 6.95 & 1875 & 17 & 1,752 & 4.01 & 3047 & 34 & 2,016 & 2.20 & 4329 & 2,770 & 438,183 & 6.26 \\
\hline 0689 & 1,576 & 381,486 & 9.77 & 1876 & 49 & 10,920 & 8.91 & 3103 & 33 & 29,448 & 36.97 & 4331 & 0 & 5,112 & -- \\
\hline 0690 & 671 & 179,395 & 10.95 & 1889 & 268 & 38,734 & 5.58 & 3133 & 2,583 & 486,379 & 7.51 & 4375 & 1,187 & 232,138 & 7.92 \\
\hline 0691 & 2,667 & 469,381 & 7.03 & 1903 & 349 & 83,753 & 9.81 & 3156 & 285 & 63,435 & 8.95 & 4392 & 458 & 72,624 & 6.16 \\
\hline 0708 & 135 & 32,809 & 9.90 & 1914 & 20 & 1,752 & 3.29 & 3171 & 2,369 & 463,053 & 7.82 & 4425 & 856 & 264,416 & 12.65 \\
\hline 0738 & 1,716 & 306,512 & 7.09 & 1920 & 456 & 109,392 & 9.69 & 3189 & 345 & 97,104 & 11.48 & 4440 & 1,544 & 283,440 & 7.30 \\
\hline 0776 & 1,669 & 439,998 & 10.73 & 1921 & 3,003 & 476,617 & 6.30 & 3260 & 169 & 44,448 & 10.68 & 4476 & 1,805 & 344,677 & 7.69 \\
\hline 0779 & 441 & 108,787 & 10.09 & 1937 & 1,187 & 186,288 & 6.16 & 3267 & 107 & 22,512 & 8.46 & 4498 & 11 & 4,944 & 18.51 \\
\hline 0784 & 1,846 & 429,972 & 9.48 & 1956 & 3,262 & 486,210 & 5.91 & 3270 & 1,552 & 365,659 & 9.61 & 4517 & 1,735 & 328,752 & 7.57 \\
\hline 0786 & 524 & 117,888 & 9.06 & 1970 & 24 & 1,992 & 3.04 & 3272 & 23 & 3,840 & 6.82 & 4520 & 1,144 & 220,430 & 7.79 \\
\hline 0917 & 2,251 & 300,936 & 5.23 & 2014 & 479 & 69,312 & 5.72 & 3277 & 15 & 4,176 & 11.32 & 4525 & 30 & 1,992 & 2.36 \\
\hline 0923 & 27 & 2,016 & 2.67 & 2015 & 3,428 & 484,318 & 5.57 & 3278 & 1,001 & 253,450 & 10.33 & 4563 & 28 & 2,112 & 2.82 \\
\hline 0926 & 3,112 & 551,357 & 7.08 & 2019 & 25 & 2,016 & 3.03 & 3280 & 158 & 41,832 & 10.81 & 4570 & 2,269 & 548,973 & 9.81 \\
\hline 0950 & 45 & 18,936 & 17.40 & 2024 & 1,944 & 391,758 & 8.08 & 3281 & 45 & 26,304 & 24.20 & 4577 & 2,048 & 305,664 & 5.92 \\
\hline 0996 & 24 & 1,992 & 3.04 & 2042 & 12 & 16,080 & 55.66 & 3283 & 1,671 & 247,224 & 5.79 & 4591 & 2,707 & 431,406 & 6.27 \\
\hline 1013 & 245 & 63,492 & 10.60 & 2043 & 86 & 36,960 & 17.70 & 3284 & 2,856 & 499,445 & 7.00 & 4670 & 2,484 & 541,246 & 8.82 \\
\hline 1017 & 2,775 & 549,062 & 7.96 & 2048 & 1,690 & 409,659 & 9.84 & 3285 & 2,243 & 382,496 & 6.85 & 4671 & 148 & 25,848 & 6.99 \\
\hline 1042 & 20 & 1,488 & 2.52 & 2050 & 70 & 17,448 & 10.08 & 3329 & 1,595 & 306,792 & 7.69 & 4679 & 2,505 & 429,394 & 6.86 \\
\hline 1048 & 27 & 1,728 & 2.49 & 2051 & 65 & 26,304 & 16.62 & 3335 & 208 & 33,792 & 6.40 & 4696 & 14 & 1,992 & 5.76 \\
\hline 1053 & 633 & 145,392 & 9.24 & 2053 & 10 & 3,552 & 14.62 & 3370 & 1,897 & 317,621 & 6.65 & 4703 & 121 & 31,392 & 10.56 \\
\hline 1057 & 1,643 & 363,120 & 8.89 & 2073 & 919 & 146,832 & 6.36 & 3410 & 2,356 & 514,264 & 8.84 & 4704 & 397 & 61,368 & 6.07 \\
\hline 1063 & 22 & 1,968 & 3.28 & 2082 & 1,789 & 519,707 & 11.89 & 3415 & 2,884 & 537,022 & 7.48 & 4731 & 124 & 17,160 & 5.42 \\
\hline 1068 & 2,649 & 512,393 & 7.75 & 2086 & 2,406 & 548,518 & 9.20 & 3430 & 4,192 & 530,963 & 4.94 & 4792 & 1,195 & 213,141 & 7.19 \\
\hline 1080 & 193 & 61,344 & 13.06 & 2088 & 49 & 10,190 & 8.31 & 3431 & 246 & 32,784 & 5.16 & 4819 & 288 & 39,480 & 5.42 \\
\hline 1081 & 1,229 & 241,368 & 7.83 & 2090 & 349 & 58,356 & 6.73 & 3441 & 41 & 42,816 & 43.24 & 4852 & 16 & 2,016 & 5.10 \\
\hline 1133 & 16 & 2,784 & 6.84 & 2096 & 2,614 & 493,125 & 7.55 & 3442 & 499 & 171,216 & 13.99 & 4866 & 2,803 & 548,790 & 7.84 \\
\hline 1136 & 3,650 & 532,054 & 5.76 & 2128 & 323 & 68,160 & 8.41 & 3446 & 654 & 213,312 & 13.34 & 4876 & 137 & 29,184 & 8.62 \\
\hline 1138 & 19 & 1,320 & 2.61 & 2131 & 2,250 & 483,317 & 8.69 & 3460 & 41 & 7,008 & 6.84 & 4878 & 2,716 & 350,328 & 5.02 \\
\hline 1139 & 89 & 13,056 & 5.69 & 2142 & 19 & 2,016 & 4.01 & 3462 & 144 & 49,920 & 14.14 & 4880 & 2,595 & 551,284 & 8.59 \\
\hline
\end{tabular}


178 Statistical Characteristics of Storm Interevent Time, Depth, and Duration for Eastern New Mexico, Oklahoma, and Texas

Appendix 4-1.3. Number of storms, total duration, and mean storm interevent time defined by 12-hour minimum interevent time for hourly rainfall stations in Texas-Continued.

\begin{tabular}{|c|c|c|c|c|c|c|c|c|c|c|c|c|c|c|c|}
\hline $\begin{array}{c}\text { Station } \\
\text { no. }\end{array}$ & $\begin{array}{c}\text { No. } \\
\text { of } \\
\text { storms }\end{array}$ & $\begin{array}{l}\text { Total } \\
\text { duration } \\
\text { (hours) }\end{array}$ & $\begin{array}{c}\text { Mean } \\
\text { storm } \\
\text { interevent } \\
\text { time } \\
\text { (days) }\end{array}$ & $\begin{array}{c}\text { Station } \\
\text { no. }\end{array}$ & $\begin{array}{c}\text { No. } \\
\text { of } \\
\text { storms }\end{array}$ & $\begin{array}{c}\text { Total } \\
\text { duration } \\
\text { (hours) }\end{array}$ & $\begin{array}{c}\text { Mean } \\
\text { storm } \\
\text { interevent } \\
\text { time } \\
\text { (days) }\end{array}$ & $\begin{array}{c}\text { Station } \\
\text { no. }\end{array}$ & $\begin{array}{c}\text { No. } \\
\text { of } \\
\text { storms }\end{array}$ & $\begin{array}{c}\text { Total } \\
\text { duration } \\
\text { (hours) }\end{array}$ & $\begin{array}{c}\text { Mean } \\
\text { storm } \\
\text { interevent } \\
\text { time } \\
\text { (days) }\end{array}$ & $\begin{array}{c}\text { Station } \\
\text { no. }\end{array}$ & $\begin{array}{c}\text { No. } \\
\text { of } \\
\text { storms }\end{array}$ & $\begin{array}{c}\text { Total } \\
\text { duration } \\
\text { (hours) }\end{array}$ & $\begin{array}{c}\text { Mean } \\
\text { storm } \\
\text { interevent } \\
\text { time } \\
\text { (days) }\end{array}$ \\
\hline 4920 & 2,036 & 524,353 & 10.48 & 5775 & 13 & 5,112 & 16.12 & 7481 & 1,448 & 495,877 & 14.04 & 8743 & 3,466 & 527,995 & 6.01 \\
\hline 4934 & 7 & 1,992 & 11.53 & 5779 & 19 & 5,880 & 12.39 & 7497 & 1,406 & 243,992 & 6.97 & 8761 & 1,007 & 256,990 & 10.42 \\
\hline 4972 & 2,552 & 494,452 & 7.78 & 5840 & 355 & 78,888 & 8.88 & 7498 & 295 & 49,632 & 6.76 & 8778 & 2,557 & 398,041 & 6.19 \\
\hline 4973 & 446 & 119,208 & 10.79 & 5890 & 2,354 & 515,614 & 8.84 & 7499 & 2,349 & 523,925 & 9.03 & 8845 & 2,985 & 550,655 & 7.37 \\
\hline 4974 & 1,289 & 340,313 & 10.73 & 5891 & 159 & 53,304 & 13.71 & 7531 & 139 & 49,968 & 14.60 & 8859 & 1,987 & 312,504 & 6.19 \\
\hline 4975 & 1,674 & 257,716 & 6.14 & 5897 & 1,455 & 245,324 & 6.77 & 7534 & 320 & 103,032 & 13.12 & 8898 & 674 & 116,904 & 6.86 \\
\hline 4978 & 143 & 47,496 & 13.56 & 5957 & 2,261 & 445,446 & 7.95 & 7556 & 2,729 & 493,793 & 7.27 & 8908 & 36 & 5,904 & 6.47 \\
\hline 4979 & 21 & 1,896 & 3.26 & 5958 & 199 & 41,136 & 8.30 & 7594 & 2,115 & 309,414 & 5.79 & 8910 & 20 & 1,968 & 3.85 \\
\hline 4982 & 1,052 & 248,714 & 9.56 & 5973 & 106 & 23,376 & 8.81 & 7596 & 167 & 26,136 & 6.23 & 8911 & 904 & 164,256 & 7.31 \\
\hline 5018 & 709 & 134,338 & 7.57 & 5996 & 2,931 & 549,087 & 7.49 & 7608 & 1,644 & 249,648 & 6.03 & 8924 & 439 & 181,152 & 17.00 \\
\hline 5048 & 1,614 & 503,770 & 12.78 & 6017 & 131 & 23,280 & 7.14 & 7622 & 22 & 4,152 & 7.69 & 8929 & 22 & 2,016 & 3.38 \\
\hline 5049 & 99 & 34,272 & 14.26 & 6024 & 258 & 72,480 & 11.33 & 7700 & 2,311 & 308,448 & 5.20 & 8942 & 2,131 & 300,835 & 5.59 \\
\hline 5056 & 5 & 10,104 & 83.94 & 6050 & 20 & 6,576 & 13.36 & 7706 & 2,086 & 502,063 & 9.77 & 8944 & 1,205 & 144,888 & 4.70 \\
\hline 5057 & 941 & 189,960 & 8.10 & 6104 & 2,586 & 478,193 & 7.49 & 7718 & 102 & 34,320 & 13.68 & 8996 & 2,924 & 399,638 & 5.39 \\
\hline 5060 & 294 & 59,904 & 8.17 & 6108 & 3,086 & 542,529 & 7.02 & 7745 & 395 & 70,872 & 7.04 & 9014 & 23 & 1,896 & 3.08 \\
\hline 5081 & 1,270 & 180,720 & 5.54 & 6136 & 2,211 & 539,302 & 9.96 & 7922 & 319 & 111,768 & 14.39 & 9037 & 693 & 240,720 & 14.21 \\
\hline 5094 & 2,691 & 468,823 & 6.98 & 6166 & 92 & 14,520 & 6.30 & 7936 & 2,277 & 306,666 & 5.32 & 9106 & 201 & 65,736 & 13.35 \\
\hline 5113 & 2,559 & 523,501 & 8.23 & 6176 & 390 & 69,264 & 7.04 & 7943 & 2,606 & 474,742 & 7.29 & 9107 & 35 & 26,784 & 31.67 \\
\hline 5114 & 0 & 1,488 & -- & 6177 & 2,669 & 440,385 & 6.57 & 7944 & 76 & 8,592 & 4.37 & 9129 & 109 & 23,376 & 8.63 \\
\hline 5123 & 15 & 3,072 & 8.27 & 6210 & 2,184 & 354,217 & 6.48 & 7945 & 3,982 & 543,166 & 5.32 & 9163 & 2,301 & 550,742 & 9.69 \\
\hline 5192 & 1,724 & 332,072 & 7.72 & 6211 & 483 & 93,000 & 7.73 & 7947 & 249 & 49,618 & 8.01 & 9213 & 224 & 38,700 & 6.80 \\
\hline 5193 & 3,265 & 550,050 & 6.71 & 6270 & 1,804 & 256,135 & 5.64 & 7948 & 1,060 & 213,840 & 8.12 & 9214 & 30 & 2,016 & 2.46 \\
\hline 5224 & 348 & 52,608 & 5.96 & 6275 & 0 & 2,920 & -- & 7951 & 1,028 & 141,648 & 5.41 & 9222 & 210 & 31,416 & 5.83 \\
\hline 5228 & 433 & 79,632 & 7.40 & 6276 & 24 & 2,016 & 3.05 & 7953 & 121 & 23,352 & 7.70 & 9248 & 128 & 52,272 & 16.70 \\
\hline 5235 & 30 & 4,392 & 5.71 & 6335 & 2,302 & 354,128 & 6.08 & 7981 & 230 & 112,728 & 20.13 & 9266 & 74 & 17,160 & 9.31 \\
\hline 5247 & 2,556 & 552,074 & 8.75 & 6434 & 19 & 8,016 & 17.16 & 7990 & 130 & 23,304 & 7.13 & 9270 & 1,710 & 382,084 & 9.12 \\
\hline 5258 & 883 & 177,528 & 8.03 & 6504 & 2,317 & 548,140 & 9.62 & 7992 & 23 & 1,896 & 3.08 & 9295 & 105 & 27,672 & 10.75 \\
\hline 5303 & 651 & 179,592 & 11.17 & 6558 & 30 & 5,688 & 7.57 & 7997 & 120 & 34,656 & 11.79 & 9304 & 4 & 336 & 3.50 \\
\hline 5312 & 1,826 & 404,451 & 8.99 & 6615 & 648 & 194,104 & 12.27 & 7999 & 19 & 13,992 & 30.45 & 9307 & 254 & 79,272 & 12.69 \\
\hline 5341 & 27 & 2,016 & 2.76 & 6660 & 112 & 32,880 & 11.86 & 8022 & 68 & 48,504 & 29.42 & 9328 & 178 & 34,344 & 7.73 \\
\hline 5342 & 0 & 4,404 & -- & 6663 & 103 & 36,168 & 14.41 & 8023 & 1,592 & 484,077 & 12.43 & 9329 & 15 & 3,240 & 8.62 \\
\hline 5348 & 1,595 & 240,206 & 5.98 & 6734 & 374 & 102,262 & 11.08 & 8047 & 2,416 & 548,776 & 9.19 & 9345 & 2 & 960 & 20.00 \\
\hline 5358 & 1,456 & 387,072 & 10.80 & 6736 & 1,764 & 452,975 & 10.47 & 8060 & 128 & 23,376 & 7.27 & 9363 & 1,024 & 135,816 & 5.21 \\
\hline 5398 & 2,765 & 411,020 & 5.85 & 6740 & 15 & 2,016 & 4.98 & 8062 & 41 & 6,504 & 6.23 & 9364 & 3,509 & 418,894 & 4.66 \\
\hline 5410 & 2,293 & 528,181 & 9.37 & 6750 & 245 & 37,990 & 6.10 & 8068 & 36 & 21,192 & 24.28 & 9365 & 34 & 5,664 & 6.66 \\
\hline 5411 & 2,636 & 462,238 & 7.00 & 6757 & 3,712 & 551,361 & 5.86 & 8081 & 2,584 & 537,081 & 8.36 & 9371 & 147 & 31,416 & 8.56 \\
\hline 5424 & 426 & 56,254 & 5.07 & 6775 & 554 & 110,784 & 8.02 & 8089 & 40 & 5,760 & 5.71 & 9417 & 2,009 & 331,982 & 6.57 \\
\hline 5429 & 1,015 & 193,392 & 7.64 & 6776 & 2,149 & 431,190 & 8.12 & 8221 & 19 & 1,896 & 3.63 & 9419 & 3,820 & 542,606 & 5.57 \\
\hline 5431 & 16 & 13,848 & 35.51 & 6788 & 173 & 29,256 & 6.73 & 8252 & 1,799 & 529,928 & 12.03 & 9435 & 77 & 33,600 & 17.90 \\
\hline 5461 & 1,787 & 324,672 & 7.20 & 6792 & 1,650 & 419,078 & 10.41 & 8265 & 1,637 & 217,176 & 5.16 & 9491 & 2,716 & 443,170 & 6.49 \\
\hline 5463 & 1,191 & 224,790 & 7.61 & 6794 & 25 & 2,016 & 2.82 & 8289 & 53 & 36,528 & 28.40 & 9499 & 1,784 & 431,095 & 9.83 \\
\hline 5471 & 25 & 3,480 & 5.66 & 6834 & 2,122 & 317,594 & 5.97 & 8305 & 1,697 & 512,439 & 12.39 & 9522 & 15 & 2,016 & 5.15 \\
\hline 5477 & 24 & 1,992 & 3.18 & 6893 & 1,268 & 374,777 & 12.12 & 8335 & 1,664 & 259,728 & 6.12 & 9527 & 2,156 & 539,410 & 10.20 \\
\hline 5528 & 1,228 & 262,609 & 8.60 & 6935 & 1,801 & 406,635 & 9.20 & 8400 & 216 & 62,112 & 11.74 & 9532 & 2,788 & 484,263 & 6.97 \\
\hline 5579 & 1 & 696 & 29.00 & 6981 & 192 & 132,768 & 28.51 & 8445 & 1,314 & 206,856 & 6.22 & 9544 & 5 & 2,928 & 24.27 \\
\hline 5580 & 27 & 1,944 & 2.73 & 7020 & 127 & 21,432 & 6.57 & 8446 & 1,826 & 268,785 & 5.87 & 9565 & 1,473 & 352,743 & 9.72 \\
\hline 5589 & 172 & 37,872 & 8.99 & 7060 & 958 & 277,917 & 11.86 & 8451 & 259 & 71,544 & 11.20 & 9570 & 512 & 100,848 & 8.00 \\
\hline 5590 & 170 & 39,024 & 9.27 & 7066 & 3,052 & 470,310 & 6.09 & 8531 & 1,597 & 305,116 & 7.63 & 9574 & 22 & 2,856 & 5.18 \\
\hline 5591 & 656 & 170,232 & 10.60 & 7074 & 2,077 & 523,667 & 10.28 & 8541 & 102 & 22,615 & 8.90 & 9588 & 2,030 & 457,802 & 9.11 \\
\hline 5592 & 545 & 151,920 & 11.38 & 7097 & 111 & 29,232 & 10.65 & 8544 & 524 & 110,871 & 8.50 & 9665 & 3,246 & 548,295 & 6.74 \\
\hline 5594 & 171 & 68,448 & 16.50 & 7116 & 608 & 167,304 & 11.19 & 8545 & 20 & 4,656 & 9.31 & 9715 & 2,640 & 443,326 & 6.70 \\
\hline 5595 & 2 & 8,016 & 167.00 & 7140 & 2,542 & 395,594 & 6.19 & 8563 & 1,245 & 220,484 & 7.12 & 9729 & 3,406 & 549,310 & 6.38 \\
\hline 5596 & 1,282 & 292,949 & 9.35 & 7173 & 1,101 & 115,200 & 4.02 & 8566 & 559 & 143,616 & 10.48 & 9772 & 1,016 & 140,832 & 5.48 \\
\hline 5600 & 424 & 105,888 & 10.16 & 7174 & 4,518 & 474,094 & 4.02 & 8583 & 856 & 203,370 & 9.66 & 9814 & 35 & 13,030 & 15.13 \\
\hline 5618 & 33 & 2,112 & 2.34 & 7213 & 776 & 156,336 & 8.07 & 8584 & 1,335 & 344,280 & 10.47 & 9815 & 728 & 121,848 & 6.64 \\
\hline 5650 & 19 & 1,896 & 3.86 & 7243 & 2,800 & 549,034 & 7.90 & 8623 & 1,605 & 319,544 & 8.02 & 9816 & 74 & 35,472 & 19.69 \\
\hline 5656 & 1,591 & 326,279 & 8.33 & 7262 & 102 & 27,360 & 11.03 & 8625 & 1,298 & 243,840 & 7.48 & 9817 & 1,424 & 356,901 & 10.20 \\
\hline 5658 & 876 & 209,400 & 9.64 & 7274 & 431 & 121,725 & 11.50 & 8630 & 782 & 226,383 & 11.81 & 9829 & 1,490 & 436,603 & 11.97 \\
\hline 5661 & 221 & 46,672 & 8.57 & 7300 & 1,221 & 258,332 & 8.54 & 8631 & 860 & 243,264 & 11.48 & 9830 & 156 & 45,216 & 11.85 \\
\hline 5666 & 35 & 5,880 & 6.72 & 7311 & 30 & 6,600 & 8.94 & 8646 & 1,854 & 338,752 & 7.30 & 9858 & 1,373 & 293,448 & 8.63 \\
\hline 5695 & 2,279 & 448,602 & 7.89 & 7363 & 27 & 2,016 & 2.82 & 8647 & 1,285 & 282,860 & 8.94 & 9893 & 2,580 & 537,872 & 8.40 \\
\hline 5742 & 19 & 5,784 & 12.37 & 7422 & 2,899 & 544,386 & 7.54 & 8677 & 167 & 64,752 & 15.81 & 9916 & 1,079 & 185,230 & 6.84 \\
\hline 5766 & 30 & 2,016 & 2.44 & 7431 & 1,475 & 456,709 & 12.65 & 8696 & 26 & 2,016 & 2.94 & 9976 & 2,015 & 549,337 & 11.08 \\
\hline 5770 & 2,812 & 545,182 & 7.81 & & & & & & & & & & & & \\
\hline
\end{tabular}


Appendix 4-1.4. Number of storms, total duration, and mean storm interevent time defined by 18-hour minimum interevent time for hourly rainfall stations in Texas.

$[--$, not available $]$

\begin{tabular}{|c|c|c|c|c|c|c|c|c|c|c|c|c|c|c|c|}
\hline $\begin{array}{c}\text { Station } \\
\text { no. }\end{array}$ & $\begin{array}{c}\text { No. } \\
\text { of } \\
\text { storms }\end{array}$ & $\begin{array}{c}\text { Total } \\
\text { duration } \\
\text { (hours) }\end{array}$ & $\begin{array}{c}\text { Mean } \\
\text { storm } \\
\text { interevent } \\
\text { time } \\
\text { (days) }\end{array}$ & $\begin{array}{c}\text { Station } \\
\text { no. }\end{array}$ & $\begin{array}{c}\text { No. } \\
\text { of } \\
\text { storms }\end{array}$ & $\begin{array}{c}\text { Total } \\
\text { duration } \\
\text { (hours) }\end{array}$ & $\begin{array}{c}\text { Mean } \\
\text { storm } \\
\text { interevent } \\
\text { time } \\
\text { (days) }\end{array}$ & $\begin{array}{c}\text { Station } \\
\text { no. }\end{array}$ & $\begin{array}{c}\text { No. } \\
\text { of } \\
\text { storms }\end{array}$ & $\begin{array}{c}\text { Total } \\
\text { duration } \\
\text { (hours) }\end{array}$ & $\begin{array}{c}\text { Mean } \\
\text { storm } \\
\text { interevent } \\
\text { time } \\
\text { (days) }\end{array}$ & $\begin{array}{c}\text { Station } \\
\text { no. }\end{array}$ & $\begin{array}{c}\text { No. } \\
\text { of } \\
\text { storms }\end{array}$ & $\begin{array}{c}\text { Total } \\
\text { duration } \\
\text { (hours) }\end{array}$ & $\begin{array}{c}\text { Mean } \\
\text { storm } \\
\text { interevent } \\
\text { time } \\
\text { (days) }\end{array}$ \\
\hline 0015 & 7 & 624 & 3.14 & 1154 & 185 & 26,928 & 5.55 & 2160 & 22 & 4,752 & 8.40 & 3463 & 44 & 30,096 & 28.07 \\
\hline 0016 & 2,882 & 543,665 & 7.44 & 1165 & 566 & 147,768 & 10.49 & 2206 & 697 & 105,121 & 5.90 & 3476 & 643 & 127,848 & 7.88 \\
\hline 0050 & 900 & 197,240 & 8.71 & 1185 & 361 & 185,232 & 21.13 & 2238 & 219 & 51,864 & 9.45 & 3485 & 22 & 1,968 & 3.38 \\
\hline 0054 & 48 & 5,688 & 4.59 & 1186 & 100 & 19,128 & 7.50 & 2240 & 81 & 12,864 & 6.24 & 3507 & 975 & 188,267 & 7.69 \\
\hline 0120 & 17 & 1,896 & 3.69 & 1188 & 8 & 1,872 & 9.16 & 2242 & 1,582 & 253,435 & 6.23 & 3546 & 2,921 & 485,758 & 6.52 \\
\hline 0145 & 155 & 28,392 & 7.21 & 1245 & 22 & 1,992 & 3.00 & 2244 & 2,991 & 535,994 & 7.03 & 3547 & 192 & 51,144 & 10.75 \\
\hline 0146 & 41 & 5,664 & 5.31 & 1246 & 1,021 & 176,017 & 6.83 & 2247 & 48 & 7,056 & 5.65 & 3579 & 71 & 19,944 & 11.26 \\
\hline 0174 & 1,217 & 275,194 & 9.13 & 1267 & 448 & 116,832 & 10.50 & 2309 & 596 & 153,600 & 10.28 & 3642 & 2,837 & 534,563 & 7.45 \\
\hline 0178 & 18 & 2,040 & 4.34 & 1304 & 502 & 117,840 & 9.34 & 2312 & 607 & 92,757 & 5.98 & 3646 & 1,931 & 419,874 & 8.64 \\
\hline 0179 & 292 & 66,456 & 9.20 & 1325 & 1,249 & 219,432 & 6.87 & 2334 & 55 & 22,344 & 16.52 & 3668 & 18 & 2,016 & 3.99 \\
\hline 0202 & 247 & 58,398 & 9.60 & 1429 & 1,119 & 213,741 & 7.56 & 2336 & 208 & 53,256 & 10.34 & 3673 & 22 & 2,016 & 3.14 \\
\hline 0206 & 2,277 & 532,111 & 9.40 & 1431 & 1,598 & 356,239 & 8.82 & 2354 & 25 & 20,976 & 34.58 & 3686 & 1,012 & 198,532 & 7.82 \\
\hline 0208 & 3 & 384 & 5.33 & 1432 & 1,162 & 246,787 & 8.39 & 2355 & 46 & 10,536 & 9.01 & 3691 & 2,509 & 469,594 & 7.44 \\
\hline 0211 & 3,059 & 542,494 & 7.00 & 1433 & 1,688 & 367,055 & 8.60 & 2357 & 466 & 98,496 & 8.40 & 3734 & 23 & 2,016 & 3.05 \\
\hline 0244 & 69 & 11,568 & 6.35 & 1434 & 1,724 & 358,448 & 8.23 & 2360 & 1,490 & 399,684 & 10.77 & 3771 & 1,297 & 220,455 & 6.77 \\
\hline 0248 & 1,775 & 529,764 & 12.15 & 1435 & 1,024 & 230,732 & 8.95 & 2361 & 170 & 56,004 & 13.41 & 3789 & 96 & 18,888 & 7.89 \\
\hline 0262 & 2,268 & 431,066 & 7.50 & 1436 & 1,915 & 367,281 & 7.52 & 2394 & 2,835 & 504,483 & 7.00 & 3826 & 203 & 45,240 & 8.92 \\
\hline 0271 & 17 & 2,016 & 4.20 & 1437 & 22 & 4,728 & 8.53 & 2404 & 2,587 & 493,624 & 7.56 & 3831 & 360 & 110,112 & 12.32 \\
\hline 0380 & 372 & 163,872 & 17.98 & 1438 & 1,375 & 284,045 & 8.16 & 2415 & 2,629 & 501,413 & 7.51 & 3841 & 141 & 77,472 & 22.57 \\
\hline 0394 & 8 & 1,992 & 9.73 & 1462 & 0 & 4,416 & -- & 2462 & 888 & 188,205 & 8.43 & 3871 & 1,258 & 317,328 & 10.08 \\
\hline 0408 & 24 & 2,112 & 3.20 & 1492 & 1,023 & 280,441 & 11.09 & 2528 & 106 & 24,840 & 9.33 & 3884 & 20 & 1,896 & 3.63 \\
\hline 0427 & 49 & 11,646 & 9.44 & 1500 & 24 & 2,112 & 3.15 & 2617 & 227 & 56,688 & 9.99 & 3941 & 56 & 7,608 & 5.04 \\
\hline 0428 & 3,500 & 529,122 & 5.81 & 1528 & 1,218 & 366,583 & 12.22 & 2619 & 207 & 64,992 & 12.70 & 3963 & 6 & 1,488 & 10.19 \\
\hline 0429 & 151 & 22,416 & 5.58 & 1541 & 57 & 10,953 & 7.64 & 2621 & 1,115 & 277,864 & 10.04 & 4040 & 416 & 92,806 & 8.94 \\
\hline 0463 & 106 & 51,936 & 20.06 & 1569 & 168 & 32,160 & 7.48 & 2675 & 1,008 & 178,918 & 7.03 & 4058 & 20 & 2,016 & 3.67 \\
\hline 0493 & 16 & 1,704 & 3.98 & 1632 & 7 & 1,320 & 7.73 & 2676 & 871 & 146,737 & 6.71 & 4098 & 1,546 & 417,420 & 10.98 \\
\hline 0495 & 314 & 138,072 & 18.02 & 1641 & 195 & 110,280 & 23.21 & 2679 & 1,938 & 536,229 & 11.18 & 4100 & 324 & 121,992 & 15.35 \\
\hline 0496 & 27 & 10,872 & 16.72 & 1646 & 2,145 & 543,287 & 10.27 & 2715 & 1,590 & 354,131 & 8.90 & 4137 & 1,046 & 221,233 & 8.49 \\
\hline 0498 & 11 & 7,920 & 29.87 & 1663 & 37 & 11,460 & 12.42 & 2744 & 1,604 & 413,376 & 10.39 & 4191 & 2,134 & 519,452 & 9.77 \\
\hline 0509 & 2,593 & 549,223 & 8.40 & 1671 & 2,833 & 548,538 & 7.68 & 2758 & 101 & 23,376 & 9.17 & 4256 & 0 & 21,906 & -- \\
\hline 0518 & 1,863 & 330,777 & 7.05 & 1680 & 1,070 & 261,792 & 9.77 & 2794 & 11 & 2,064 & 7.54 & 4257 & 2,773 & 498,651 & 7.11 \\
\hline 0521 & 32 & 5,760 & 7.12 & 1694 & 137 & 45,172 & 13.38 & 2797 & 2,170 & 529,606 & 9.85 & 4258 & 162 & 25,608 & 6.20 \\
\hline 0556 & 100 & 21,936 & 8.74 & 1696 & 992 & 268,080 & 10.87 & 2811 & 1,740 & 435,389 & 10.08 & 4278 & 1,446 & 409,467 & 11.41 \\
\hline 0569 & 2,268 & 399,353 & 6.96 & 1697 & 83 & 29,496 & 14.49 & 2813 & 22 & 31,728 & 59.73 & 4299 & 118 & 86,976 & 30.46 \\
\hline 0572 & 914 & 158,448 & 6.82 & 1698 & 1,162 & 291,219 & 10.09 & 2814 & 16 & 12,336 & 31.81 & 4300 & 2,423 & 289,264 & 4.50 \\
\hline 0576 & 119 & 23,352 & 7.70 & 1720 & 199 & 46,709 & 9.48 & 2815 & 230 & 58,942 & 10.41 & 4305 & 2,148 & 266,616 & 4.70 \\
\hline 0580 & 312 & 112,800 & 14.67 & 1761 & 97 & 16,680 & 6.68 & 2818 & 172 & 45,312 & 10.61 & 4307 & 529 & 96,430 & 7.05 \\
\hline 0587 & 789 & 121,272 & 5.94 & 1773 & 3,059 & 502,398 & 6.44 & 2986 & 244 & 54,240 & 8.77 & 4309 & 3,263 & 517,057 & 6.21 \\
\hline 0605 & 110 & 27,000 & 9.84 & 1810 & 22 & 2,112 & 3.68 & 3005 & 2,396 & 522,908 & 8.73 & 4311 & 3,272 & 543,389 & 6.51 \\
\hline 0639 & 2,146 & 433,077 & 8.09 & 1823 & 21 & 1,968 & 3.58 & 3033 & 621 & 216,192 & 14.26 & 4313 & 236 & 39,432 & 6.49 \\
\hline 0655 & 0 & 718 & -- & 1870 & 307 & 47,496 & 5.95 & 3034 & 2 & 6,888 & 143.50 & 4319 & 85 & 29,208 & 13.86 \\
\hline 0665 & 1,900 & 361,772 & 7.52 & 1875 & 16 & 1,752 & 4.18 & 3047 & 23 & 2,016 & 2.99 & 4329 & 2,533 & 438,183 & 6.79 \\
\hline 0689 & 1,443 & 381,486 & 10.61 & 1876 & 43 & 10,920 & 10.09 & 3103 & 28 & 29,448 & 43.46 & 4331 & 0 & 5,112 & -- \\
\hline 0690 & 615 & 179,395 & 11.89 & 1889 & 229 & 38,734 & 6.43 & 3133 & 2,368 & 486,379 & 8.14 & 4375 & 1,061 & 232,138 & 8.79 \\
\hline 0691 & 2,464 & 469,381 & 7.56 & 1903 & 322 & 83,753 & 10.57 & 3156 & 252 & 63,435 & 10.05 & 4392 & 417 & 72,624 & 6.71 \\
\hline 0708 & 125 & 32,809 & 10.64 & 1914 & 19 & 1,752 & 3.44 & 3171 & 2,169 & 463,053 & 8.48 & 4425 & 780 & 264,416 & 13.83 \\
\hline 0738 & 1,578 & 306,512 & 7.66 & 1920 & 416 & 109,392 & 10.57 & 3189 & 319 & 97,104 & 12.37 & 4440 & 1,415 & 283,440 & 7.91 \\
\hline 0776 & 1,564 & 439,998 & 11.41 & 1921 & 2,732 & 476,617 & 6.86 & 3260 & 155 & 44,448 & 11.59 & 4476 & 1,645 & 344,677 & 8.37 \\
\hline 0779 & 395 & 108,787 & 11.20 & 1937 & 1,101 & 186,288 & 6.59 & 3267 & 100 & 22,512 & 9.01 & 4498 & 11 & 4,944 & 18.51 \\
\hline 0784 & 1,696 & 429,972 & 10.26 & 1956 & 2,963 & 486,210 & 6.45 & 3270 & 1,431 & 365,659 & 10.37 & 4517 & 1,575 & 328,752 & 8.28 \\
\hline 0786 & 481 & 117,888 & 9.82 & 1970 & 22 & 1,992 & 3.26 & 3272 & 19 & 3,840 & 8.09 & 4520 & 1,035 & 220,430 & 8.54 \\
\hline 0917 & 2,045 & 300,936 & 5.70 & 2014 & 420 & 69,312 & 6.43 & 3277 & 14 & 4,176 & 12.14 & 4525 & 21 & 1,992 & 3.13 \\
\hline 0923 & 23 & 2,016 & 3.03 & 2015 & 3,007 & 484,318 & 6.26 & 3278 & 931 & 253,450 & 11.06 & 4563 & 26 & 2,112 & 2.99 \\
\hline 0926 & 2,834 & 551,357 & 7.71 & 2019 & 20 & 2,016 & 3.63 & 3280 & 150 & 41,832 & 11.35 & 4570 & 2,070 & 548,973 & 10.70 \\
\hline 0950 & 43 & 18,936 & 18.17 & 2024 & 1,779 & 391,758 & 8.77 & 3281 & 43 & 26,304 & 25.30 & 4577 & 1,880 & 305,664 & 6.40 \\
\hline 0996 & 20 & 1,992 & 3.53 & 2042 & 10 & 16,080 & 66.67 & 3283 & 1,487 & 247,224 & 6.43 & 4591 & 2,445 & 431,406 & 6.88 \\
\hline 1013 & 224 & 63,492 & 11.54 & 2043 & 80 & 36,960 & 18.99 & 3284 & 2,555 & 499,445 & 7.76 & 4670 & 2,255 & 541,246 & 9.66 \\
\hline 1017 & 2,524 & 549,062 & 8.69 & 2048 & 1,545 & 409,659 & 10.71 & 3285 & 2,025 & 382,496 & 7.53 & 4671 & 134 & 25,848 & 7.64 \\
\hline 1042 & 18 & 1,488 & 2.70 & 2050 & 60 & 17,448 & 11.65 & 3329 & 1,431 & 306,792 & 8.50 & 4679 & 2,262 & 429,394 & 7.53 \\
\hline 1048 & 24 & 1,728 & 2.70 & 2051 & 61 & 26,304 & 17.67 & 3335 & 187 & 33,792 & 7.05 & 4696 & 12 & 1,992 & 6.58 \\
\hline 1053 & 567 & 145,392 & 10.25 & 2053 & 10 & 3,552 & 14.62 & 3370 & 1,769 & 317,621 & 7.09 & 4703 & 109 & 31,392 & 11.65 \\
\hline 1057 & 1,523 & 363,120 & 9.55 & 2073 & 837 & 146,832 & 6.92 & 3410 & 2,151 & 514,264 & 9.62 & 4704 & 350 & 61,368 & 6.80 \\
\hline 1063 & 19 & 1,968 & 3.71 & 2082 & 1,673 & 519,707 & 12.67 & 3415 & 2,596 & 537,022 & 8.25 & 4731 & 109 & 17,160 & 6.09 \\
\hline 1068 & 2,425 & 512,393 & 8.41 & 2086 & 2,193 & 548,518 & 10.04 & 3430 & 3,704 & 530,963 & 5.51 & 4792 & 1,076 & 213,141 & 7.92 \\
\hline 1080 & 171 & 61,344 & 14.65 & 2088 & 47 & 10,190 & 8.65 & 3431 & 211 & 32,784 & 5.90 & 4819 & 263 & 39,480 & 5.88 \\
\hline 1081 & 1,132 & 241,368 & 8.45 & 2090 & 312 & 58,356 & 7.46 & 3441 & 37 & 42,816 & 47.84 & 4852 & 16 & 2,016 & 5.10 \\
\hline 1133 & 14 & 2,784 & 7.71 & 2096 & 2,372 & 493,125 & 8.27 & 3442 & 463 & 171,216 & 15.03 & 4866 & 2,583 & 548,790 & 8.45 \\
\hline 1136 & 3,178 & 532,054 & 6.52 & 2128 & 288 & 68,160 & 9.36 & 3446 & 623 & 213,312 & 13.98 & 4876 & 122 & 29,184 & 9.61 \\
\hline 1138 & 17 & 1,320 & 2.85 & 2131 & 2,028 & 483,317 & 9.58 & 3460 & 36 & 7,008 & 7.71 & 4878 & 2,433 & 350,328 & 5.54 \\
\hline 1139 & 77 & 13,056 & 6.48 & 2142 & 16 & 2,016 & 4.65 & 3462 & 128 & 49,920 & 15.84 & 4880 & 2,391 & 551,284 & 9.27 \\
\hline
\end{tabular}


Appendix 4-1.4. Number of storms, total duration, and mean storm interevent time defined by 18-hour minimum interevent time for hourly rainfall stations in Texas-Continued.

\begin{tabular}{|c|c|c|c|c|c|c|c|c|c|c|c|c|c|c|c|}
\hline $\begin{array}{c}\text { Station } \\
\text { no. }\end{array}$ & $\begin{array}{c}\text { No. } \\
\text { of } \\
\text { storms }\end{array}$ & $\begin{array}{c}\text { Total } \\
\text { duration } \\
\text { (hours) }\end{array}$ & $\begin{array}{c}\text { Mean } \\
\text { storm } \\
\text { interevent } \\
\text { time } \\
\text { (days) }\end{array}$ & $\begin{array}{c}\text { Station } \\
\text { no. }\end{array}$ & $\begin{array}{c}\text { No. } \\
\text { of } \\
\text { storms }\end{array}$ & $\begin{array}{c}\text { Total } \\
\text { duration } \\
\text { (hours) }\end{array}$ & $\begin{array}{c}\text { Mean } \\
\text { storm } \\
\text { interevent } \\
\text { time } \\
\text { (days) }\end{array}$ & $\begin{array}{c}\text { Station } \\
\text { no. }\end{array}$ & $\begin{array}{c}\text { No. } \\
\text { of } \\
\text { storms }\end{array}$ & $\begin{array}{c}\text { Total } \\
\text { duration } \\
\text { (hours) }\end{array}$ & $\begin{array}{c}\text { Mean } \\
\text { storm } \\
\text { interevent } \\
\text { time } \\
\text { (days) }\end{array}$ & $\begin{array}{c}\text { Station } \\
\text { no. }\end{array}$ & $\begin{array}{c}\text { No. } \\
\text { of } \\
\text { storms }\end{array}$ & $\begin{array}{c}\text { Total } \\
\text { duration } \\
\text { (hours) }\end{array}$ & $\begin{array}{c}\text { Mean } \\
\text { storm } \\
\text { interevent } \\
\text { time } \\
\text { (days) }\end{array}$ \\
\hline 4920 & 1,835 & 524,353 & 11.57 & 5775 & 12 & 5,112 & 17.54 & 7481 & 1,351 & 495,877 & 15.00 & 8743 & 3,146 & 527,995 & 6.56 \\
\hline 4934 & 7 & 1,992 & 11.53 & 5779 & 19 & 5,880 & 12.39 & 7497 & 1,259 & 243,992 & 7.71 & 8761 & 924 & 256,990 & 11.31 \\
\hline 4972 & 2,328 & 494,452 & 8.47 & 5840 & 332 & 78,888 & 9.45 & 7498 & 267 & 49,632 & 7.41 & 8778 & 2,297 & 398,041 & 6.83 \\
\hline 4973 & 401 & 119,208 & 11.93 & 5890 & 2,146 & 515,614 & 9.64 & 7499 & 2,133 & 523,925 & 9.89 & 8845 & 2,671 & 550,655 & 8.17 \\
\hline 4974 & 1,175 & 340,313 & 11.71 & 5891 & 152 & 53,304 & 14.32 & 7531 & 125 & 49,968 & 16.17 & 8859 & 1,789 & 312,504 & 6.81 \\
\hline 4975 & 1,523 & 257,716 & 6.69 & 5897 & 1,304 & 245,324 & 7.49 & 7534 & 294 & 103,032 & 14.23 & 8898 & 621 & 116,904 & 7.39 \\
\hline 4978 & 133 & 47,496 & 14.54 & 5957 & 2,077 & 445,446 & 8.61 & 7556 & 2,500 & 493,793 & 7.89 & 8908 & 31 & 5,904 & 7.42 \\
\hline 4979 & 18 & 1,896 & 3.70 & 5958 & 176 & 41,136 & 9.31 & 7594 & 1,935 & 309,414 & 6.27 & 8910 & 20 & 1,968 & 3.82 \\
\hline 4982 & 979 & 248,714 & 10.23 & 5973 & 90 & 23,376 & 10.27 & 7596 & 157 & 26,136 & 6.59 & 8911 & 825 & 164,256 & 7.96 \\
\hline 5018 & 666 & 134,338 & 8.02 & 5996 & 2,680 & 549,087 & 8.14 & 7608 & 1,503 & 249,648 & 6.54 & 8924 & 418 & 181,152 & 17.82 \\
\hline 5048 & 1,474 & 503,770 & 13.93 & 6017 & 120 & 23,280 & 7.74 & 7622 & 21 & 4,152 & 7.99 & 8929 & 18 & 2,016 & 3.99 \\
\hline 5049 & 91 & 34,272 & 15.47 & 6024 & 234 & 72,480 & 12.43 & 7700 & 2,103 & 308,448 & 5.65 & 8942 & 1,911 & 300,835 & 6.16 \\
\hline 5056 & 5 & 10,104 & 83.94 & 6050 & 19 & 6,576 & 14.03 & 7706 & 1,889 & 502,063 & 10.72 & 8944 & 1,098 & 144,888 & 5.10 \\
\hline 5057 & 843 & 189,960 & 8.97 & 6104 & 2,343 & 478,193 & 8.21 & 7718 & 82 & 34,320 & 16.86 & 8996 & 2,653 & 399,638 & 5.88 \\
\hline 5060 & 270 & 59,904 & 8.85 & 6108 & 2,809 & 542,529 & 7.65 & 7745 & 356 & 70,872 & 7.74 & 9014 & 20 & 1,896 & 3.45 \\
\hline 5081 & 1,154 & 180,720 & 6.03 & 6136 & 2,008 & 539,302 & 10.91 & 7922 & 303 & 111,768 & 15.11 & 9037 & 651 & 240,720 & 15.09 \\
\hline 5094 & 2,451 & 468,823 & 7.61 & 6166 & 85 & 14,520 & 6.76 & 7936 & 2,056 & 306,666 & 5.83 & 9106 & 181 & 65,736 & 14.76 \\
\hline 5113 & 2,295 & 523,501 & 9.10 & 6176 & 358 & 69,264 & 7.62 & 7943 & 2,354 & 474,742 & 8.01 & 9107 & 31 & 26,784 & 35.67 \\
\hline 5114 & 0 & 1,488 & -- & 6177 & 2,433 & 440,385 & 7.15 & 7944 & 67 & 8,592 & 4.87 & 9129 & 97 & 23,376 & 9.62 \\
\hline 5123 & 15 & 3,072 & 8.27 & 6210 & 1,982 & 354,217 & 7.08 & 7945 & 3,486 & 543,166 & 6.00 & 9163 & 2,107 & 550,742 & 10.53 \\
\hline 5192 & 1,592 & 332,072 & 8.31 & 6211 & 440 & 93,000 & 8.43 & 7947 & 217 & 49,618 & 9.11 & 9213 & 204 & 38,700 & 7.40 \\
\hline 5193 & 2,945 & 550,050 & 7.37 & 6270 & 1,611 & 256,135 & 6.24 & 7948 & 973 & 213,840 & 8.79 & 9214 & 28 & 2,016 & 2.60 \\
\hline 5224 & 317 & 52,608 & 6.48 & 6275 & 0 & 2,920 & -- & 7951 & 948 & 141,648 & 5.82 & 9222 & 187 & 31,416 & 6.47 \\
\hline 5228 & 393 & 79,632 & 8.09 & 6276 & 21 & 2,016 & 3.40 & 7953 & 110 & 23,352 & 8.40 & 9248 & 116 & 52,272 & 18.36 \\
\hline 5235 & 27 & 4,392 & 6.27 & 6335 & 2,128 & 354,128 & 6.52 & 7981 & 206 & 112,728 & 22.40 & 9266 & 69 & 17,160 & 9.92 \\
\hline 5247 & 2,325 & 552,074 & 9.56 & 6434 & 17 & 8,016 & 19.11 & 7990 & 108 & 23,304 & 8.46 & 9270 & 1,538 & 382,084 & 10.07 \\
\hline 5258 & 803 & 177,528 & 8.77 & 6504 & 2,117 & 548,140 & 10.47 & 7992 & 20 & 1,896 & 3.46 & 9295 & 97 & 27,672 & 11.58 \\
\hline 5303 & 596 & 179,592 & 12.15 & 6558 & 29 & 5,688 & 7.78 & 7997 & 103 & 34,656 & 13.64 & 9304 & 4 & 336 & 3.50 \\
\hline 5312 & 1,669 & 404,451 & 9.78 & 6615 & 582 & 194,104 & 13.59 & 7999 & 17 & 13,992 & 33.97 & 9307 & 242 & 79,272 & 13.29 \\
\hline 5341 & 22 & 2,016 & 3.25 & 6660 & 97 & 32,880 & 13.61 & 8022 & 60 & 48,504 & 33.27 & 9328 & 158 & 34,344 & 8.63 \\
\hline 5342 & 0 & 4,404 & -- & 6663 & 95 & 36,168 & 15.57 & 8023 & 1,461 & 484,077 & 13.49 & 9329 & 12 & 3,240 & 10.58 \\
\hline 5348 & 1,433 & 240,206 & 6.59 & 6734 & 347 & 102,262 & 11.90 & 8047 & 2,201 & 548,776 & 10.03 & 9345 & 2 & 960 & 20.00 \\
\hline 5358 & 1,336 & 387,072 & 11.72 & 6736 & 1,641 & 452,975 & 11.21 & 8060 & 118 & 23,376 & 7.83 & 9363 & 887 & 135,816 & 5.92 \\
\hline 5398 & 2,529 & 411,020 & 6.34 & 6740 & 13 & 2,016 & 5.65 & 8062 & 40 & 6,504 & 6.37 & 9364 & 3,140 & 418,894 & 5.14 \\
\hline 5410 & 2,105 & 528,181 & 10.15 & 6750 & 217 & 37,990 & 6.81 & 8068 & 34 & 21,192 & 25.67 & 9365 & 31 & 5,664 & 7.25 \\
\hline 5411 & 2,378 & 462,238 & 7.70 & 6757 & 3,346 & 551,361 & 6.44 & 8081 & 2,335 & 537,081 & 9.19 & 9371 & 132 & 31,416 & 9.47 \\
\hline 5424 & 365 & 56,254 & 5.80 & 6775 & 507 & 110,784 & 8.70 & 8089 & 38 & 5,760 & 5.98 & 9417 & 1,837 & 331,982 & 7.13 \\
\hline 5429 & 934 & 193,392 & 8.25 & 6776 & 1,973 & 431,190 & 8.79 & 8221 & 19 & 1,896 & 3.63 & 9419 & 3,380 & 542,606 & 6.21 \\
\hline 5431 & 14 & 13,848 & 40.49 & 6788 & 162 & 29,256 & 7.14 & 8252 & 1,664 & 529,928 & 12.96 & 9435 & 73 & 33,600 & 18.84 \\
\hline 5461 & 1,645 & 324,672 & 7.77 & 6792 & 1,530 & 419,078 & 11.17 & 8265 & 1,455 & 217,176 & 5.73 & 9491 & 2,453 & 443,170 & 7.13 \\
\hline 5463 & 1,079 & 224,790 & 8.34 & 6794 & 19 & 2,016 & 3.53 & 8289 & 50 & 36,528 & 30.07 & 9499 & 1,630 & 431,095 & 10.70 \\
\hline 5471 & 20 & 3,480 & 6.95 & 6834 & 1,903 & 317,594 & 6.59 & 8305 & 1,540 & 512,439 & 13.59 & 9522 & 11 & 2,016 & 6.78 \\
\hline 5477 & 23 & 1,992 & 3.30 & 6893 & 1,178 & 374,777 & 13.00 & 8335 & 1,515 & 259,728 & 6.67 & 9527 & 1,956 & 539,410 & 11.18 \\
\hline 5528 & 1,152 & 262,609 & 9.13 & 6935 & 1,658 & 406,635 & 9.94 & 8400 & 206 & 62,112 & 12.28 & 9532 & 2,555 & 484,263 & 7.55 \\
\hline 5579 & 1 & 696 & 29.00 & 6981 & 176 & 132,768 & 31.04 & 8445 & 1,191 & 206,856 & 6.80 & 9544 & 4 & 2,928 & 30.50 \\
\hline 5580 & 24 & 1,944 & 2.99 & 7020 & 115 & 21,432 & 7.20 & 8446 & 1,636 & 268,785 & 6.48 & 9565 & 1,339 & 352,743 & 10.64 \\
\hline 5589 & 156 & 37,872 & 9.85 & 7060 & 876 & 277,917 & 12.91 & 8451 & 235 & 71,544 & 12.28 & 9570 & 467 & 100,848 & 8.72 \\
\hline 5590 & 157 & 39,024 & 10.00 & 7066 & 2,765 & 470,310 & 6.66 & 8531 & 1,462 & 305,116 & 8.28 & 9574 & 22 & 2,856 & 5.18 \\
\hline 5591 & 606 & 170,232 & 11.42 & 7074 & 1,919 & 523,667 & 11.08 & 8541 & 94 & 22,615 & 9.60 & 9588 & 1,824 & 457,802 & 10.07 \\
\hline 5592 & 513 & 151,920 & 12.06 & 7097 & 102 & 29,232 & 11.54 & 8544 & 467 & 110,871 & 9.46 & 9665 & 2,916 & 548,295 & 7.43 \\
\hline 5594 & 162 & 68,448 & 17.39 & 7116 & 560 & 167,304 & 12.09 & 8545 & 16 & 4,656 & 11.49 & 9715 & 2,416 & 443,326 & 7.27 \\
\hline 5595 & 2 & 8,016 & 167.00 & 7140 & 2,313 & 395,594 & 6.74 & 8563 & 1,123 & 220,484 & 7.83 & 9729 & 3,094 & 549,310 & 6.97 \\
\hline 5596 & 1,162 & 292,949 & 10.25 & 7173 & 956 & 115,200 & 4.54 & 8566 & 521 & 143,616 & 11.20 & 9772 & 925 & 140,832 & 5.95 \\
\hline 5600 & 388 & 105,888 & 11.04 & 7174 & 3,971 & 474,094 & 4.50 & 8583 & 768 & 203,370 & 10.70 & 9814 & 32 & 13,030 & 16.49 \\
\hline 5618 & 28 & 2,112 & 2.65 & 7213 & 700 & 156,336 & 8.88 & 8584 & 1,230 & 344,280 & 11.31 & 9815 & 645 & 121,848 & 7.42 \\
\hline 5650 & 19 & 1,896 & 3.86 & 7243 & 2,518 & 549,034 & 8.72 & 8623 & 1,444 & 319,544 & 8.85 & 9816 & 71 & 35,472 & 20.50 \\
\hline 5656 & 1,442 & 326,279 & 9.13 & 7262 & 89 & 27,360 & 12.54 & 8625 & 1,192 & 243,840 & 8.09 & 9817 & 1,285 & 356,901 & 11.23 \\
\hline 5658 & 825 & 209,400 & 10.20 & 7274 & 405 & 121,725 & 12.20 & 8630 & 729 & 226,383 & 12.63 & 9829 & 1,373 & 436,603 & 12.94 \\
\hline 5661 & 196 & 46,672 & 9.58 & 7300 & 1,141 & 258,332 & 9.09 & 8631 & 791 & 243,264 & 12.43 & 9830 & 146 & 45,216 & 12.62 \\
\hline 5666 & 32 & 5,880 & 7.29 & 7311 & 26 & 6,600 & 10.23 & 8646 & 1,721 & 338,752 & 7.81 & 9858 & 1,288 & 293,448 & 9.16 \\
\hline 5695 & 2,085 & 448,602 & 8.57 & 7363 & 20 & 2,016 & 3.60 & 8647 & 1,180 & 282,860 & 9.68 & 9893 & 2,365 & 537,872 & 9.11 \\
\hline 5742 & 16 & 5,784 & 14.56 & 7422 & 2,597 & 544,386 & 8.35 & 8677 & 145 & 64,752 & 18.12 & 9916 & 982 & 185,230 & 7.46 \\
\hline 5766 & 23 & 2,016 & 3.00 & 7431 & 1,382 & 456,709 & 13.46 & 8696 & 23 & 2,016 & 3.25 & 9976 & 1,856 & 549,337 & 11.97 \\
\hline 5770 & 2,558 & 545,182 & 8.53 & & & & & & & & & & & & \\
\hline
\end{tabular}


Appendix 4-1.5. Number of storms, total duration, and mean storm interevent time defined by 24-hour minimum interevent time for hourly rainfall stations in Texas.

[--, not available]

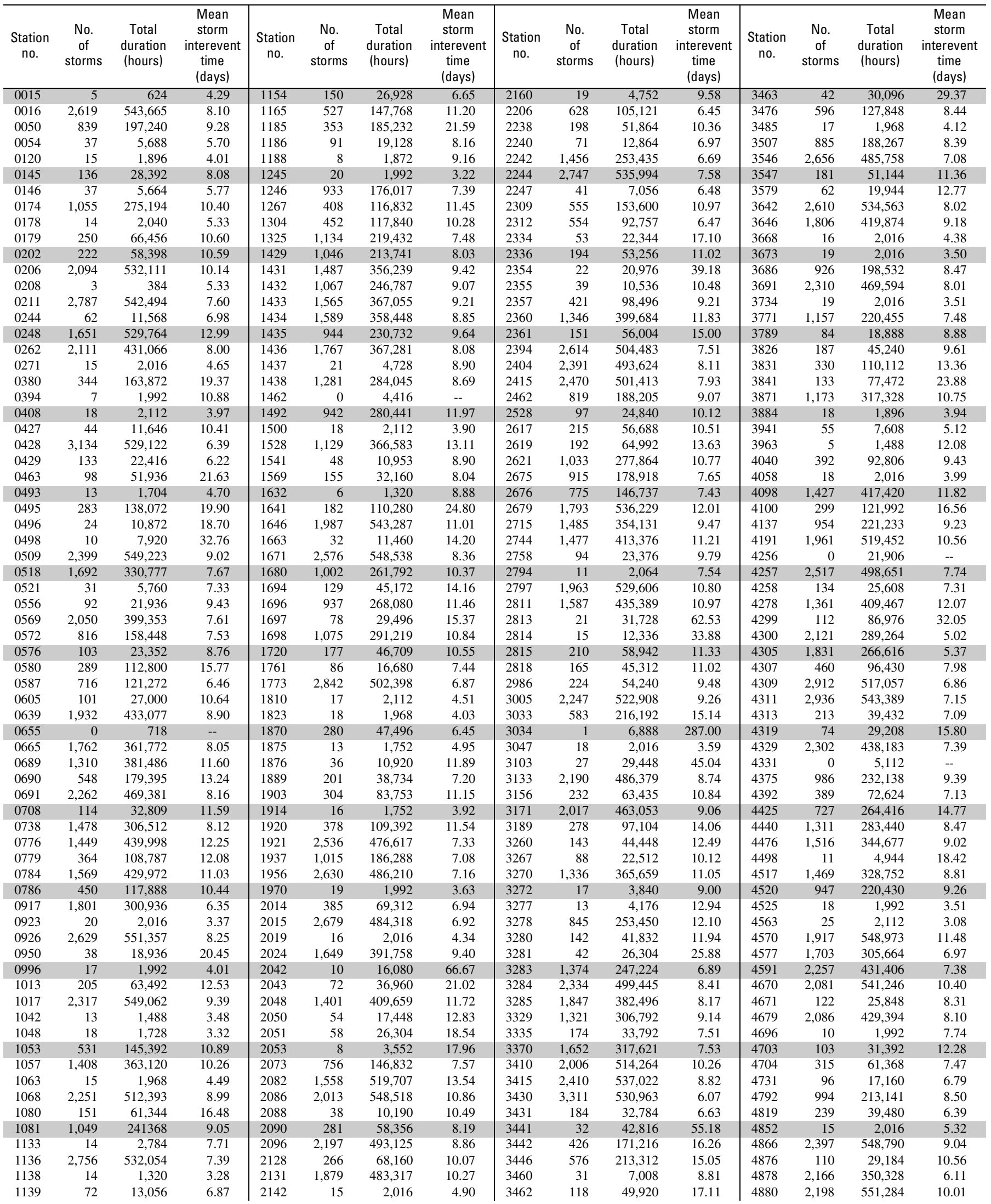


Appendix 4-1.5. Number of storms, total duration, and mean storm interevent time defined by 24-hour minimum interevent time for hourly rainfall stations in Texas-Continued.

\begin{tabular}{|c|c|c|c|c|c|c|c|c|c|c|c|c|c|c|c|}
\hline $\begin{array}{c}\text { Station } \\
\text { no. }\end{array}$ & $\begin{array}{c}\text { No. } \\
\text { of } \\
\text { storms }\end{array}$ & $\begin{array}{l}\text { Total } \\
\text { duration } \\
\text { (hours) }\end{array}$ & $\begin{array}{c}\text { Mean } \\
\text { storm } \\
\text { interevent } \\
\text { time } \\
\text { (days) }\end{array}$ & $\begin{array}{c}\text { Station } \\
\text { no. }\end{array}$ & $\begin{array}{c}\text { No. } \\
\text { of } \\
\text { storms }\end{array}$ & $\begin{array}{c}\text { Total } \\
\text { duration } \\
\text { (hours) }\end{array}$ & $\begin{array}{c}\text { Mean } \\
\text { storm } \\
\text { interevent } \\
\text { time } \\
\text { (days) }\end{array}$ & $\begin{array}{c}\text { Station } \\
\text { no. }\end{array}$ & $\begin{array}{c}\text { No. } \\
\text { of } \\
\text { storms }\end{array}$ & $\begin{array}{c}\text { Total } \\
\text { duration } \\
\text { (hours) }\end{array}$ & $\begin{array}{c}\text { Mean } \\
\text { storm } \\
\text { interevent } \\
\text { time } \\
\text { (days) }\end{array}$ & $\begin{array}{c}\text { Station } \\
\text { no. }\end{array}$ & $\begin{array}{c}\text { No. } \\
\text { of } \\
\text { storms }\end{array}$ & $\begin{array}{c}\text { Total } \\
\text { duration } \\
\text { (hours) }\end{array}$ & $\begin{array}{c}\text { Mean } \\
\text { storm } \\
\text { interevent } \\
\text { time } \\
\text { (days) }\end{array}$ \\
\hline 4920 & 1,678 & 524,353 & 12.57 & 5775 & 12 & 5,112 & 17.54 & 7481 & 1,265 & 495,877 & 15.96 & 8743 & 2,934 & 527,995 & 6.97 \\
\hline 4934 & 6 & 1,992 & 13.47 & 5779 & 18 & 5,880 & 13.04 & 7497 & 1,158 & 243,992 & 8.31 & 8761 & 843 & 256,990 & 12.31 \\
\hline 4972 & 2,158 & 494,452 & 9.07 & 5840 & 315 & 78,888 & 9.92 & 7498 & 245 & 49,632 & 8.00 & 8778 & 2,122 & 398,041 & 7.32 \\
\hline 4973 & 380 & 119,208 & 12.54 & 5890 & 1,985 & 515,614 & 10.35 & 7499 & 1,980 & 523,925 & 10.59 & 8845 & 2,435 & 550,655 & 8.88 \\
\hline 4974 & 1,076 & 340,313 & 12.71 & 5891 & 143 & 53,304 & 15.17 & 7531 & 116 & 49,968 & 17.36 & 8859 & 1,668 & 312,504 & 7.24 \\
\hline 4975 & 1,397 & 257,716 & 7.22 & 5897 & 1,183 & 245,324 & 8.17 & 7534 & 274 & 103,032 & 15.21 & 8898 & 580 & 116,904 & 7.86 \\
\hline 4978 & 120 & 47,496 & 16.02 & 5957 & 1,910 & 445,446 & 9.29 & 7556 & 2,319 & 493,793 & 8.43 & 8908 & 30 & 5,904 & 7.64 \\
\hline 4979 & 16 & 1,896 & 4.05 & 5958 & 161 & 41,136 & 10.09 & 7594 & 1,781 & 309,414 & 6.74 & 8910 & 17 & 1,968 & 4.34 \\
\hline 4982 & 909 & 248,714 & 10.95 & 5973 & 85 & 23,376 & 10.83 & 7596 & 148 & 26,136 & 6.94 & 8911 & 746 & 164,256 & 8.71 \\
\hline 5018 & 610 & 134,338 & 8.68 & 5996 & 2,493 & 549,087 & 8.69 & 7608 & 1,366 & 249,648 & 7.11 & 8924 & 384 & 181,152 & 19.33 \\
\hline 5048 & 1,374 & 503,770 & 14.89 & 6017 & 112 & 23,280 & 8.23 & 7622 & 19 & 4,152 & 8.73 & 8929 & 14 & 2,016 & 4.88 \\
\hline 5049 & 88 & 34,272 & 15.96 & 6024 & 212 & 72,480 & 13.63 & 7700 & 1,875 & 308,448 & 6.23 & 8942 & 1,744 & 300,835 & 6.67 \\
\hline 5056 & 5 & 10,104 & 83.78 & 6050 & 19 & 6,576 & 14.03 & 7706 & 1,726 & 502,063 & 11.65 & 8944 & 988 & 144,888 & 5.57 \\
\hline 5057 & 749 & 189,960 & 9.99 & 6104 & 1,973 & 478,193 & 9.58 & 7718 & 79 & 34,320 & 17.47 & 8996 & 2,351 & 399,638 & 6.53 \\
\hline 5060 & 230 & 59,904 & 10.24 & 6108 & 2,594 & 542,529 & 8.22 & 7745 & 321 & 70,872 & 8.49 & 9014 & 17 & 1,896 & 3.88 \\
\hline 5081 & 1,049 & 180,720 & 6.55 & 6136 & 1,826 & 539,302 & 11.91 & 7922 & 281 & 111,768 & 16.23 & 9037 & 606 & 240,720 & 16.15 \\
\hline 5094 & 2,259 & 468,823 & 8.18 & 6166 & 77 & 14,520 & 7.38 & 7936 & 1,847 & 306,666 & 6.40 & 9106 & 166 & 65,736 & 16.02 \\
\hline 5113 & 2,122 & 523,501 & 9.77 & 6176 & 326 & 69,264 & 8.28 & 7943 & 2,196 & 474,742 & 8.52 & 9107 & 31 & 26,784 & 35.67 \\
\hline 5114 & 0 & 1,488 & -- & 6177 & 2,212 & 440,385 & 7.78 & 7944 & 57 & 8,592 & 5.57 & 9129 & 85 & 23,376 & 10.87 \\
\hline 5123 & 14 & 3,072 & 8.79 & 6210 & 1,832 & 354,217 & 7.59 & 7945 & 3,119 & 543,166 & 6.60 & 9163 & 1,942 & 550,742 & 11.35 \\
\hline 5192 & 1,480 & 332,072 & 8.88 & 6211 & 364 & 93,000 & 10.00 & 7947 & 195 & 49,618 & 10.04 & 9213 & 185 & 38,700 & 8.06 \\
\hline 5193 & 2,692 & 550,050 & 7.98 & 6270 & 1,461 & 256,135 & 6.80 & 7948 & 890 & 213,840 & 9.53 & 9214 & 22 & 2,016 & 3.07 \\
\hline 5224 & 287 & 52,608 & 7.07 & 6275 & 0 & 2,920 & -- & 7951 & 865 & 141,648 & 6.30 & 9222 & 165 & 31,416 & 7.23 \\
\hline 5228 & 354 & 79,632 & 8.89 & 6276 & 20 & 2,016 & 3.52 & 7953 & 96 & 23,352 & 9.50 & 9248 & 107 & 52,272 & 19.84 \\
\hline 5235 & 23 & 4,392 & 7.23 & 6335 & 1,957 & 354,128 & 7.02 & 7981 & 188 & 112,728 & 24.47 & 9266 & 67 & 17,160 & 10.19 \\
\hline 5247 & 2,177 & 552,074 & 10.15 & 6434 & 17 & 8,016 & 19.11 & 7990 & 94 & 23,304 & 9.59 & 9270 & 1,376 & 382,084 & 11.16 \\
\hline 5258 & 752 & 177,528 & 9.31 & 6504 & 1,945 & 548,140 & 11.33 & 7992 & 17 & 1,896 & 3.91 & 9295 & 90 & 27,672 & 12.43 \\
\hline 5303 & 548 & 179,592 & 13.14 & 6558 & 23 & 5,688 & 9.69 & 7997 & 92 & 34,656 & 15.22 & 9304 & 3 & 336 & 4.67 \\
\hline 5312 & 1,555 & 404,451 & 10.43 & 6615 & 544 & 194,104 & 14.48 & 7999 & 16 & 13,992 & 36.04 & 9307 & 223 & 79,272 & 14.35 \\
\hline 5341 & 19 & 2,016 & 3.62 & 6660 & 85 & 32,880 & 15.43 & 8022 & 54 & 48,504 & 36.87 & 9328 & 147 & 34,344 & 9.22 \\
\hline 5342 & 0 & 4,404 & -- & 6663 & 69 & 36,168 & 21.09 & 8023 & 1,368 & 484,077 & 14.35 & 9329 & 10 & 3,240 & 12.53 \\
\hline 5348 & 1,319 & 240,206 & 7.09 & 6734 & 321 & 102,262 & 12.79 & 8047 & 2,019 & 548,776 & 10.85 & 9345 & 2 & 960 & 20.00 \\
\hline 5358 & 1,252 & 387,072 & 12.45 & 6736 & 1,524 & 452,975 & 12.00 & 8060 & 106 & 23,376 & 8.62 & 9363 & 768 & 135,816 & 6.71 \\
\hline 5398 & 2,273 & 411,020 & 6.95 & 6740 & 12 & 2,016 & 5.98 & 8062 & 33 & 6,504 & 7.53 & 9364 & 2,731 & 418,894 & 5.78 \\
\hline 5410 & 1,966 & 528,181 & 10.81 & 6750 & 193 & 37,990 & 7.54 & 8068 & 30 & 21,192 & 28.97 & 9365 & 23 & 5,664 & 9.55 \\
\hline 5411 & 2,167 & 462,238 & 8.37 & 6757 & 3,039 & 551,361 & 7.00 & 8081 & 2,138 & 537,081 & 9.96 & 9371 & 121 & 31,416 & 10.25 \\
\hline 5424 & 317 & 56,254 & 6.54 & 6775 & 466 & 110,784 & 9.39 & 8089 & 37 & 5,760 & 6.09 & 9417 & 1,688 & 331,982 & 7.68 \\
\hline 5429 & 827 & 193,392 & 9.20 & 6776 & 1,810 & 431,190 & 9.50 & 8221 & 17 & 1,896 & 3.90 & 9419 & 3,086 & 542,606 & 6.73 \\
\hline 5431 & 14 & 13,848 & 40.49 & 6788 & 151 & 29,256 & 7.60 & 8252 & 1,535 & 529,928 & 13.97 & 9435 & 67 & 33,600 & 20.46 \\
\hline 5461 & 1,554 & 324,672 & 8.17 & 6792 & 1,389 & 419,078 & 12.22 & 8265 & 1,298 & 217,176 & 6.32 & 9491 & 2,210 & 443,170 & 7.82 \\
\hline 5463 & 1,000 & 224,790 & 8.93 & 6794 & 16 & 2,016 & 4.02 & 8289 & 48 & 36,528 & 31.29 & 9499 & 1,534 & 431,095 & 11.31 \\
\hline 5471 & 16 & 3,480 & 8.48 & 6834 & 1,764 & 317,594 & 7.05 & 8305 & 1,420 & 512,439 & 14.67 & 9522 & 8 & 2,016 & 8.99 \\
\hline 5477 & 17 & 1,992 & 4.16 & 6893 & 1,105 & 374,777 & 13.80 & 8335 & 1,415 & 259,728 & 7.08 & 9527 & 1,803 & 539,410 & 12.06 \\
\hline 5528 & 1,074 & 262,609 & 9.73 & 6935 & 1,515 & 406,635 & 10.80 & 8400 & 183 & 62,112 & 13.72 & 9532 & 2,347 & 484,263 & 8.14 \\
\hline 5579 & 1 & 696 & 29.00 & 6981 & 158 & 132,768 & 34.48 & 8445 & 1,077 & 206,856 & 7.43 & 9544 & 4 & 2,928 & 30.50 \\
\hline 5580 & 20 & 1,944 & 3.41 & 7020 & 107 & 21,432 & 7.68 & 8446 & 1,492 & 268,785 & 7.02 & 9565 & 1,260 & 352,743 & 11.25 \\
\hline 5589 & 141 & 37,872 & 10.81 & 7060 & 815 & 277,917 & 13.81 & 8451 & 222 & 71,544 & 12.95 & 9570 & 422 & 100,848 & 9.55 \\
\hline 5590 & 141 & 39,024 & 11.05 & 7066 & 2,556 & 470,310 & 7.14 & 8531 & 1,352 & 305,116 & 8.89 & 9574 & 19 & 2,856 & 5.87 \\
\hline 5591 & 545 & 170,232 & 12.60 & 7074 & 1,775 & 523,667 & 11.91 & 8541 & 91 & 22,615 & 9.89 & 9588 & 1,619 & 457,802 & 11.24 \\
\hline 5592 & 456 & 151,920 & 13.46 & 7097 & 92 & 29,232 & 12.70 & 8544 & 434 & 110,871 & 10.12 & 9665 & 2,687 & 548,295 & 7.99 \\
\hline 5594 & 151 & 68,448 & 18.59 & 7116 & 513 & 167,304 & 13.12 & 8545 & 15 & 4,656 & 12.21 & 9715 & 2,236 & 443,326 & 7.79 \\
\hline 5595 & 2 & 8,016 & 167.00 & 7140 & 2,094 & 395,594 & 7.35 & 8563 & 1,048 & 220,484 & 8.33 & 9729 & 2,854 & 549,310 & 7.48 \\
\hline 5596 & 1,025 & 292,949 & 11.51 & 7173 & 823 & 115,200 & 5.14 & 8566 & 469 & 143,616 & 12.34 & 9772 & 820 & 140,832 & 6.61 \\
\hline 5600 & 347 & 105,888 & 12.25 & 7174 & 3,474 & 474,094 & 5.02 & 8583 & 706 & 203,370 & 11.56 & 9814 & 32 & 13,030 & 16.49 \\
\hline 5618 & 24 & 2,112 & 2.94 & 7213 & 647 & 156,336 & 9.54 & 8584 & 1,125 & 344,280 & 12.29 & 9815 & 593 & 121,848 & 7.99 \\
\hline 5650 & 17 & 1,896 & 4.22 & 7243 & 2,340 & 549,034 & 9.32 & 8623 & 1,339 & 319,544 & 9.48 & 9816 & 65 & 35,472 & 22.33 \\
\hline 5656 & 1,323 & 326,279 & 9.87 & 7262 & 80 & 27,360 & 13.85 & 8625 & 1,094 & 243,840 & 8.74 & 9817 & 1,206 & 356,901 & 11.91 \\
\hline 5658 & 768 & 209,400 & 10.90 & 7274 & 383 & 121,725 & 12.85 & 8630 & 687 & 226,383 & 13.35 & 9829 & 1,274 & 436,603 & 13.88 \\
\hline 5661 & 174 & 46,672 & 10.69 & 7300 & 1,061 & 258,332 & 9.71 & 8631 & 740 & 243,264 & 13.22 & 9830 & 135 & 45,216 & 13.58 \\
\hline 5666 & 28 & 5,880 & 8.21 & 7311 & 25 & 6,600 & 10.60 & 8646 & 1,595 & 338,752 & 8.36 & 9858 & 1,222 & 293,448 & 9.61 \\
\hline 5695 & 1,925 & 448,602 & 9.22 & 7363 & 17 & 2,016 & 4.07 & 8647 & 1,078 & 282,860 & 10.51 & 9893 & 2,199 & 537,872 & 9.73 \\
\hline 5742 & 14 & 5,784 & 16.51 & 7422 & 2,361 & 544,386 & 9.10 & 8677 & 135 & 64,752 & 19.40 & 9916 & 917 & 185,230 & 7.93 \\
\hline 5766 & 23 & 2,016 & 3.00 & 7431 & 1,302 & 456,709 & 14.24 & 8696 & 19 & 2,016 & 3.76 & 9976 & 1,692 & 549,337 & 13.05 \\
\hline 5770 & 2,383 & 545,182 & 9.09 & & & & & & & & & & & & \\
\hline
\end{tabular}


Appendix 4-1.6. Number of storms, total duration, and mean storm interevent time defined by 48-hour minimum interevent time for hourly rainfall stations in Texas.

[--, not available]

\begin{tabular}{|c|c|c|c|c|c|c|c|c|c|c|c|c|c|c|c|}
\hline $\begin{array}{c}\text { Station } \\
\text { no. }\end{array}$ & $\begin{array}{c}\text { No. } \\
\text { of } \\
\text { storms }\end{array}$ & $\begin{array}{c}\text { Total } \\
\text { duration } \\
\text { (hours) }\end{array}$ & $\begin{array}{c}\text { Mean } \\
\text { storm } \\
\text { interevent } \\
\text { time } \\
\text { (days) }\end{array}$ & $\begin{array}{c}\text { Station } \\
\text { no. }\end{array}$ & $\begin{array}{c}\text { No. } \\
\text { of } \\
\text { storms }\end{array}$ & $\begin{array}{c}\text { Total } \\
\text { duration } \\
\text { (hours) }\end{array}$ & $\begin{array}{c}\text { Mean } \\
\text { storm } \\
\text { interevent } \\
\text { time } \\
\text { (days) }\end{array}$ & $\begin{array}{c}\text { Station } \\
\text { no. }\end{array}$ & $\begin{array}{c}\text { No. } \\
\text { of } \\
\text { storms }\end{array}$ & $\begin{array}{c}\text { Total } \\
\text { duration } \\
\text { (hours) }\end{array}$ & $\begin{array}{c}\text { Mean } \\
\text { storm } \\
\text { interevent } \\
\text { time } \\
\text { (days) }\end{array}$ & $\begin{array}{c}\text { Station } \\
\text { no. }\end{array}$ & $\begin{array}{c}\text { No. } \\
\text { of } \\
\text { storms }\end{array}$ & $\begin{array}{c}\text { Total } \\
\text { duration } \\
\text { (hours) }\end{array}$ & $\begin{array}{c}\text { Mean } \\
\text { storm } \\
\text { interevent } \\
\text { time } \\
\text { (days) }\end{array}$ \\
\hline 0015 & 4 & 624 & 6.50 & 1154 & 108 & 26,928 & 8.62 & 2160 & 15 & 4,752 & 11.76 & 3463 & 35 & 30,096 & 34.93 \\
\hline 0016 & 2,147 & 543,665 & 9.57 & 1165 & 434 & 147,768 & 13.28 & 2206 & 505 & 105,121 & 7.68 & 3476 & 492 & 127,848 & 9.91 \\
\hline 0050 & 703 & 197,240 & 10.79 & 1185 & 316 & 185,232 & 23.97 & 2238 & 165 & 51,864 & 12.13 & 3485 & 14 & 1,968 & 4.76 \\
\hline 0054 & 31 & 5,688 & 6.46 & 1186 & 65 & 19,128 & 10.87 & 2240 & 53 & 12,864 & 8.88 & 3507 & 699 & 188,267 & 10.25 \\
\hline 0120 & 11 & 1,896 & 4.98 & 1188 & 6 & 1,872 & 11.69 & 2242 & 1,172 & 253,435 & 7.96 & 3546 & 2,135 & 485,758 & 8.46 \\
\hline 0145 & 110 & 28,392 & 9.62 & 1245 & 12 & 1,992 & 4.63 & 2244 & 2,223 & 535,994 & 9.03 & 3547 & 153 & 51,144 & 13.16 \\
\hline 0146 & 27 & 5,664 & 7.31 & 1246 & 728 & 176,017 & 9.08 & 2247 & 31 & 7,056 & 8.14 & 3579 & 51 & 19,944 & 15.20 \\
\hline 0174 & 847 & 275,194 & 12.61 & 1267 & 334 & 116,832 & 13.67 & 2309 & 454 & 153,600 & 13.09 & 3642 & 2,150 & 534,563 & 9.43 \\
\hline 0178 & 10 & 2,040 & 6.90 & 1304 & 380 & 117,840 & 11.95 & 2312 & 448 & 92,757 & 7.66 & 3646 & 1,516 & 419,874 & 10.66 \\
\hline 0179 & 196 & 66,456 & 13.13 & 1325 & 955 & 219,432 & 8.62 & 2334 & 44 & 22,344 & 20.31 & 3668 & 8 & 2,016 & 7.33 \\
\hline 0202 & 179 & 58,398 & 12.79 & 1429 & 861 & 213,741 & 9.45 & 2336 & 162 & 53,256 & 12.89 & 3673 & 10 & 2,016 & 5.69 \\
\hline 0206 & 1,731 & 532,111 & 11.97 & 1431 & 1,230 & 356,239 & 11.09 & 2354 & 18 & 20,976 & 47.60 & 3686 & 739 & 198,532 & 10.26 \\
\hline 0208 & 3 & 384 & 5.33 & 1432 & 902 & 246,787 & 10.46 & 2355 & 33 & 10,536 & 12.13 & 3691 & 1,893 & 469,594 & 9.46 \\
\hline 0211 & 2,229 & 542,494 & 9.14 & 1433 & 1,320 & 367,055 & 10.65 & 2357 & 334 & 98,496 & 11.24 & 3734 & 10 & 2,016 & 5.57 \\
\hline 0244 & 50 & 11,568 & 8.32 & 1434 & 1,331 & 358,448 & 10.30 & 2360 & 1,124 & 399,684 & 13.88 & 3771 & 923 & 220,455 & 9.02 \\
\hline 0248 & 1,423 & 529,764 & 14.85 & 1435 & 816 & 230,732 & 10.93 & 2361 & 128 & 56,004 & 17.44 & 3789 & 67 & 18,888 & 10.77 \\
\hline 0262 & 1,745 & 431,066 & 9.37 & 1436 & 1,478 & 367,281 & 9.38 & 2394 & 2,118 & 504,483 & 8.94 & 3826 & 159 & 45,240 & 11.06 \\
\hline 0271 & 10 & 2,016 & 6.45 & 1437 & 19 & 4,728 & 9.69 & 2404 & 1,980 & 493,624 & 9.49 & 3831 & 279 & 110,112 & 15.55 \\
\hline 0380 & 290 & 163,872 & 22.72 & 1438 & 1,085 & 284,045 & 10.01 & 2415 & 2,022 & 501,413 & 9.37 & 3841 & 109 & 77,472 & 28.81 \\
\hline 0394 & 6 & 1,992 & 12.39 & 1462 & 0 & 4,416 & -- & 2462 & 671 & 188,205 & 10.75 & 3871 & 997 & 317,328 & 12.39 \\
\hline 0408 & 10 & 2,112 & 6.07 & 1492 & 818 & 280,441 & 13.56 & 2528 & 83 & 24,840 & 11.59 & 3884 & 13 & 1,896 & 4.87 \\
\hline 0427 & 33 & 11,646 & 13.31 & 1500 & 13 & 2,112 & 4.83 & 2617 & 178 & 56,688 & 12.40 & 3941 & 47 & 7,608 & 5.72 \\
\hline 0428 & 2,437 & 529,122 & 7.80 & 1528 & 955 & 366,583 & 15.24 & 2619 & 168 & 64,992 & 15.36 & 3963 & 4 & 1,488 & 15.50 \\
\hline 0429 & 107 & 22,416 & 7.38 & 1541 & 40 & 10,953 & 10.42 & 2621 & 880 & 277,864 & 12.39 & 4040 & 327 & 92,806 & 11.02 \\
\hline 0463 & 86 & 51,936 & 24.43 & 1569 & 121 & 32,160 & 9.90 & 2675 & 747 & 178,918 & 9.05 & 4058 & 12 & 2,016 & 5.42 \\
\hline 0493 & 10 & 1,704 & 5.62 & 1632 & 5 & 1,320 & 10.38 & 2676 & 598 & 146,737 & 9.21 & 4098 & 1,201 & 417,420 & 13.78 \\
\hline 0495 & 245 & 138,072 & 22.76 & 1641 & 155 & 110,280 & 28.89 & 2679 & 1,491 & 536,229 & 14.16 & 4100 & 247 & 121,992 & 19.75 \\
\hline 0496 & 22 & 10,872 & 20.30 & 1646 & 1,675 & 543,287 & 12.80 & 2715 & 1,246 & 354,131 & 11.00 & 4137 & 769 & 221,233 & 11.10 \\
\hline 0498 & 9 & 7,920 & 36.19 & 1663 & 27 & 11,460 & 16.60 & 2744 & 1,263 & 413,376 & 12.87 & 4191 & 1,646 & 519,452 & 12.30 \\
\hline 0509 & 1,984 & 549,223 & 10.61 & 1671 & 2,125 & 548,538 & 9.83 & 2758 & 72 & 23,376 & 12.33 & 4256 & 0 & 21,906 & -- \\
\hline 0518 & 1,369 & 330,777 & 9.14 & 1680 & 849 & 261,792 & 11.99 & 2794 & 10 & 2,064 & 8.13 & 4257 & 2,057 & 498,651 & 9.15 \\
\hline 0521 & 27 & 5,760 & 8.18 & 1694 & 110 & 45,172 & 16.36 & 2797 & 1,622 & 529,606 & 12.76 & 4258 & 105 & 25,608 & 8.94 \\
\hline 0556 & 80 & 21,936 & 10.62 & 1696 & 804 & 268,080 & 13.12 & 2811 & 1,345 & 435,389 & 12.69 & 4278 & 1,130 & 409,467 & 14.26 \\
\hline 0569 & 1,612 & 399,353 & 9.29 & 1697 & 74 & 29,496 & 16.13 & 2813 & 19 & 31,728 & 68.97 & 4299 & 100 & 86,976 & 35.71 \\
\hline 0572 & 671 & 158,448 & 8.85 & 1698 & 893 & 291,219 & 12.76 & 2814 & 10 & 12,336 & 50.25 & 4300 & 1,580 & 289,264 & 6.24 \\
\hline 0576 & 83 & 23,352 & 10.57 & 1720 & 145 & 46,709 & 12.56 & 2815 & 176 & 58,942 & 13.23 & 4305 & 1,382 & 266,616 & 6.64 \\
\hline 0580 & 242 & 112,800 & 18.56 & 1761 & 60 & 16,680 & 10.09 & 2818 & 142 & 45,312 & 12.59 & 4307 & 361 & 96,430 & 9.77 \\
\hline 0587 & 572 & 121,272 & 7.72 & 1773 & 2,278 & 502,398 & 8.21 & 2986 & 181 & 54,240 & 11.42 & 4309 & 2,327 & 517,057 & 8.22 \\
\hline 0605 & 82 & 27,000 & 12.77 & 1810 & 12 & 2,112 & 5.68 & 3005 & 1,853 & 522,908 & 10.92 & 4311 & 2,373 & 543,389 & 8.50 \\
\hline 0639 & 1,572 & 433,077 & 10.60 & 1823 & 11 & 1,968 & 5.75 & 3033 & 494 & 216,192 & 17.60 & 4313 & 171 & 39,432 & 8.49 \\
\hline 0655 & 0 & 718 & -- & 1870 & 226 & 47,496 & 7.65 & 3034 & 1 & 6,888 & 287.00 & 4319 & 60 & 29,208 & 19.17 \\
\hline 0665 & 1,468 & 361,772 & 9.37 & 1875 & 11 & 1,752 & 5.51 & 3047 & 11 & 2,016 & 5.04 & 4329 & 1,877 & 438,183 & 8.73 \\
\hline 0689 & 1,113 & 381,486 & 13.41 & 1876 & 32 & 10,920 & 13.17 & 3103 & 25 & 29,448 & 48.52 & 4331 & 0 & 5,112 & -- \\
\hline 0690 & 459 & 179,395 & 15.53 & 1889 & 153 & 38,734 & 9.01 & 3133 & 1,841 & 486,379 & 10.11 & 4375 & 806 & 232,138 & 11.16 \\
\hline 0691 & 1,861 & 469,381 & 9.60 & 1903 & 255 & 83,753 & 13.02 & 3156 & 197 & 63,435 & 12.52 & 4392 & 310 & 72,624 & 8.58 \\
\hline 0708 & 82 & 32,809 & 15.59 & 1914 & 10 & 1,752 & 5.28 & 3171 & 1,688 & 463,053 & 10.54 & 4425 & 630 & 264,416 & 16.84 \\
\hline 0738 & 1,222 & 306,512 & 9.52 & 1920 & 326 & 109,392 & 13.16 & 3189 & 229 & 97,104 & 16.78 & 4440 & 1,102 & 283,440 & 9.81 \\
\hline 0776 & 1,257 & 439,998 & 13.90 & 1921 & 2,080 & 476,617 & 8.62 & 3260 & 120 & 44,448 & 14.60 & 4476 & 1,248 & 344,677 & 10.64 \\
\hline 0779 & 305 & 108,787 & 14.15 & 1937 & 847 & 186,288 & 8.19 & 3267 & 69 & 22,512 & 12.55 & 4498 & 9 & 4,944 & 22.35 \\
\hline 0784 & 1,312 & 429,972 & 12.91 & 1956 & 2,071 & 486,210 & 8.70 & 3270 & 1,110 & 365,659 & 13.01 & 4517 & 1,224 & 328,752 & 10.29 \\
\hline 0786 & 378 & 117,888 & 12.16 & 1970 & 10 & 1,992 & 5.58 & 3272 & 12 & 3,840 & 11.99 & 4520 & 775 & 220,430 & 10.99 \\
\hline 0917 & 1,437 & 300,936 & 7.59 & 2014 & 292 & 69,312 & 8.70 & 3277 & 12 & 4,176 & 13.92 & 4525 & 12 & 1,992 & 4.58 \\
\hline 0923 & 10 & 2,016 & 5.40 & 2015 & 2,090 & 484,318 & 8.47 & 3278 & 710 & 253,450 & 14.14 & 4563 & 15 & 2,112 & 4.29 \\
\hline 0926 & 2,174 & 551,357 & 9.67 & 2019 & 10 & 2,016 & 6.24 & 3280 & 124 & 41,832 & 13.46 & 4570 & 1,618 & 548,973 & 13.34 \\
\hline 0950 & 27 & 18,936 & 28.13 & 2024 & 1,396 & 391,758 & 10.84 & 3281 & 38 & 26,304 & 28.45 & 4577 & 1,379 & 305,664 & 8.27 \\
\hline 0996 & 8 & 1,992 & 7.13 & 2042 & 9 & 16,080 & 73.88 & 3283 & 1,090 & 247,224 & 8.31 & 4591 & 1,869 & 431,406 & 8.61 \\
\hline 1013 & 169 & 63,492 & 14.90 & 2043 & 62 & 36,960 & 24.23 & 3284 & 1,911 & 499,445 & 9.95 & 4670 & 1,728 & 541,246 & 12.23 \\
\hline 1017 & 1,945 & 549,062 & 10.91 & 2048 & 1,160 & 409,659 & 13.86 & 3285 & 1,512 & 382,496 & 9.66 & 4671 & 99 & 25,848 & 9.87 \\
\hline 1042 & 7 & 1,488 & 5.72 & 2050 & 52 & 17,448 & 13.27 & 3329 & 1,101 & 306,792 & 10.68 & 4679 & 1,697 & 429,394 & 9.62 \\
\hline 1048 & 11 & 1,728 & 4.58 & 2051 & 51 & 26,304 & 20.89 & 3335 & 150 & 33,792 & 8.50 & 4696 & 7 & 1,992 & 10.46 \\
\hline 1053 & 449 & 145,392 & 12.61 & 2053 & 6 & 3,552 & 23.51 & 3370 & 1,380 & 317,621 & 8.74 & 4703 & 79 & 31,392 & 15.56 \\
\hline 1057 & 1,179 & 363,120 & 11.97 & 2073 & 622 & 146,832 & 8.89 & 3410 & 1,674 & 514,264 & 12.01 & 4704 & 261 & 61,368 & 8.70 \\
\hline 1063 & 10 & 1,968 & 5.91 & 2082 & 1,354 & 519,707 & 15.36 & 3415 & 1,958 & 537,022 & 10.52 & 4731 & 78 & 17,160 & 8.03 \\
\hline 1068 & 1,873 & 512,393 & 10.52 & 2086 & 1,677 & 548,518 & 12.75 & 3430 & 2,576 & 530,963 & 7.39 & 4792 & 797 & 213,141 & 10.25 \\
\hline 1080 & 122 & 61,344 & 20.06 & 2088 & 32 & 10,190 & 12.20 & 3431 & 134 & 32,784 & 8.52 & 4819 & 184 & 39,480 & 7.88 \\
\hline 1081 & 873 & 241,368 & 10.58 & 2090 & 232 & 58,356 & 9.61 & 3441 & 25 & 42,816 & 70.21 & 4852 & 11 & 2,016 & 6.79 \\
\hline 1133 & 11 & 2,784 & 9.41 & 2096 & 1,811 & 493,125 & 10.43 & 3442 & 368 & 171,216 & 18.60 & 4866 & 1,994 & 548,790 & 10.57 \\
\hline 1136 & 2,147 & 532,054 & 9.08 & 2128 & 230 & 68,160 & 11.41 & 3446 & 502 & 213,312 & 17.06 & 4876 & 89 & 29,184 & 12.76 \\
\hline 1138 & 9 & 1,320 & 4.36 & 2131 & 1,553 & 483,317 & 12.13 & 3460 & 27 & 7,008 & 9.92 & 4878 & 1,708 & 350,328 & 7.37 \\
\hline 1139 & 55 & 13,056 & 8.62 & 2142 & 8 & 2,016 & 8.22 & 3462 & 101 & 49,920 & 19.71 & 4880 & 1,835 & 551,284 & 11.71 \\
\hline
\end{tabular}


Appendix 4-1.6. Number of storms, total duration, and mean storm interevent time defined by 48-hour minimum interevent time for hourly rainfall stations in Texas-Continued.

\begin{tabular}{|c|c|c|c|c|c|c|c|c|c|c|c|c|c|c|c|}
\hline $\begin{array}{c}\text { Station } \\
\text { no. }\end{array}$ & $\begin{array}{c}\text { No. } \\
\text { of } \\
\text { storms }\end{array}$ & $\begin{array}{l}\text { Total } \\
\text { duration } \\
\text { (hours) }\end{array}$ & $\begin{array}{c}\text { Mean } \\
\text { storm } \\
\text { interevent } \\
\text { time } \\
\text { (days) }\end{array}$ & $\begin{array}{c}\text { Station } \\
\text { no. }\end{array}$ & $\begin{array}{c}\text { No. } \\
\text { of } \\
\text { storms }\end{array}$ & $\begin{array}{c}\text { Total } \\
\text { duration } \\
\text { (hours) }\end{array}$ & $\begin{array}{c}\text { Mean } \\
\text { storm } \\
\text { interevent } \\
\text { time } \\
\text { (days) }\end{array}$ & $\begin{array}{c}\text { Station } \\
\text { no. }\end{array}$ & $\begin{array}{c}\text { No. } \\
\text { of } \\
\text { storms }\end{array}$ & $\begin{array}{c}\text { Total } \\
\text { duration } \\
\text { (hours) }\end{array}$ & $\begin{array}{c}\text { Mean } \\
\text { storm } \\
\text { interevent } \\
\text { time } \\
\text { (days) }\end{array}$ & $\begin{array}{c}\text { Station } \\
\text { no. }\end{array}$ & $\begin{array}{c}\text { No. } \\
\text { of } \\
\text { storms }\end{array}$ & $\begin{array}{c}\text { Total } \\
\text { duration } \\
\text { (hours) }\end{array}$ & $\begin{array}{c}\text { Mean } \\
\text { storm } \\
\text { interevent } \\
\text { time } \\
\text { (days) }\end{array}$ \\
\hline 4920 & 1,430 & 524,353 & 14.50 & 5775 & 8 & 5112 & 25.62 & 7481 & 1,103 & 495,877 & 18.10 & 8743 & 2,383 & 527,995 & 8.25 \\
\hline 4934 & 5 & 1,992 & 15.92 & 5779 & 18 & 5880 & 13.04 & 7497 & 921 & 243,992 & 10.08 & 8761 & 722 & 256,990 & 14.13 \\
\hline 4972 & 1,810 & 494,452 & 10.54 & 5840 & 271 & 78888 & 11.30 & 7498 & 197 & 49,632 & 9.60 & 8778 & 1,678 & 398,041 & 8.88 \\
\hline 4973 & 314 & 119,208 & 14.88 & 5890 & 1,665 & 515614 & 12.07 & 7499 & 1,622 & 523,925 & 12.61 & 8845 & 2,013 & 550,655 & 10.44 \\
\hline 4974 & 912 & 340,313 & 14.73 & 5891 & 124 & 53304 & 17.28 & 7531 & 96 & 49,968 & 20.68 & 8859 & 1,385 & 312,504 & 8.43 \\
\hline 4975 & 1,129 & 257,716 & 8.59 & 5897 & 967 & 245324 & 9.67 & 7534 & 233 & 103,032 & 17.62 & 8898 & 488 & 116,904 & 9.07 \\
\hline 4978 & 104 & 47,496 & 18.26 & 5957 & 1,582 & 445446 & 10.91 & 7556 & 1,909 & 493,793 & 9.94 & 8908 & 24 & 5,904 & 9.19 \\
\hline 4979 & 8 & 1,896 & 6.65 & 5958 & 136 & 41136 & 11.67 & 7594 & 1,444 & 309,414 & 7.97 & 8910 & 13 & 1,968 & 5.24 \\
\hline 4982 & 772 & 248,714 & 12.64 & 5973 & 70 & 23376 & 12.87 & 7596 & 122 & 26,136 & 8.12 & 8911 & 590 & 164,256 & 10.64 \\
\hline 5018 & 514 & 134,338 & 10.03 & 5996 & 2,051 & 549087 & 10.25 & 7608 & 1,095 & 249,648 & 8.52 & 8924 & 327 & 181,152 & 22.45 \\
\hline 5048 & 1,190 & 503,770 & 16.97 & 6017 & 84 & 23280 & 10.50 & 7622 & 17 & 4,152 & 9.61 & 8929 & 11 & 2,016 & 5.89 \\
\hline 5049 & 72 & 34,272 & 19.20 & 6024 & 183 & 72480 & 15.57 & 7700 & 1,480 & 308,448 & 7.51 & 8942 & 1,378 & 300,835 & 8.07 \\
\hline 5056 & 4 & 10,104 & 105.25 & 6050 & 17 & 6,576 & 15.51 & 7706 & 1,454 & 502,063 & 13.56 & 8944 & 775 & 144,888 & 6.71 \\
\hline 5057 & 613 & 189,960 & 11.89 & 6104 & 1,519 & 478,193 & 12.02 & 7718 & 71 & 34,320 & 19.27 & 8996 & 1,850 & 399,638 & 7.91 \\
\hline 5060 & 194 & 59,904 & 11.88 & 6108 & 2,116 & 542,529 & 9.75 & 7745 & 269 & 70,872 & 9.86 & 9014 & 10 & 1,896 & 5.48 \\
\hline 5081 & 854 & 180,720 & 7.72 & 6136 & 1,530 & 539,302 & 13.93 & 7922 & 243 & 111,768 & 18.55 & 9037 & 538 & 240,720 & 18.01 \\
\hline 5094 & 1,844 & 468,823 & 9.70 & 6166 & 63 & 14,520 & 8.68 & 7936 & 1,447 & 306,666 & 7.77 & 9106 & 147 & 65,736 & 17.90 \\
\hline 5113 & 1,740 & 523,501 & 11.61 & 6176 & 265 & 69,264 & 9.86 & 7943 & 1,801 & 474,742 & 10.08 & 9107 & 26 & 26,784 & 42.24 \\
\hline 5114 & 0 & 1,488 & -- & 6177 & 1,808 & 440,385 & 9.19 & 7944 & 43 & 8,592 & 6.97 & 9129 & 69 & 23,376 & 13.04 \\
\hline 5123 & 12 & 3,072 & 10.08 & 6210 & 1,484 & 354,217 & 9.03 & 7945 & 2,460 & 543,166 & 7.99 & 9163 & 1,625 & 550,742 & 13.29 \\
\hline 5192 & 1,226 & 332,072 & 10.42 & 6211 & 291 & 93,000 & 12.11 & 7947 & 152 & 49,618 & 12.47 & 9213 & 141 & 38,700 & 10.09 \\
\hline 5193 & 2,181 & 550,050 & 9.52 & 6270 & 1,182 & 256,135 & 8.06 & 7948 & 737 & 213,840 & 11.21 & 9214 & 12 & 2,016 & 4.53 \\
\hline 5224 & 221 & 52,608 & 8.75 & 6275 & 0 & 2,920 & -- & 7951 & 718 & 141,648 & 7.28 & 9222 & 139 & 31,416 & 8.32 \\
\hline 5228 & 289 & 79,632 & 10.56 & 6276 & 10 & 2,016 & 5.67 & 7953 & 81 & 23,352 & 11.01 & 9248 & 92 & 52,272 & 22.80 \\
\hline 5235 & 20 & 4,392 & 8.05 & 6335 & 1,619 & 354,128 & 8.18 & 7981 & 156 & 112,728 & 29.20 & 9266 & 56 & 17,160 & 11.93 \\
\hline 5247 & 1,783 & 552,074 & 12.08 & 6434 & 15 & 8,016 & 21.48 & 7990 & 76 & 23,304 & 11.53 & 9270 & 1,089 & 382,084 & 13.71 \\
\hline 5258 & 630 & 177,528 & 10.83 & 6504 & 1,632 & 548,140 & 13.22 & 7992 & 12 & 1,896 & 4.85 & 9295 & 78 & 27,672 & 14.09 \\
\hline 5303 & 467 & 179,592 & 15.18 & 6558 & 22 & 5,688 & 10.09 & 7997 & 80 & 34,656 & 17.34 & 9304 & 3 & 336 & 4.67 \\
\hline 5312 & 1,258 & 404,451 & 12.56 & 6615 & 453 & 194,104 & 17.10 & 7999 & 13 & 13,992 & 44.06 & 9307 & 192 & 79,272 & 16.45 \\
\hline 5341 & 10 & 2,016 & 5.65 & 6660 & 76 & 32,880 & 17.08 & 8022 & 49 & 48,504 & 40.54 & 9328 & 120 & 34,344 & 10.99 \\
\hline 5342 & 0 & 4,404 & -- & 6663 & 51 & 36,168 & 28.03 & 8023 & 1,172 & 484,077 & 16.51 & 9329 & 9 & 3,240 & 13.81 \\
\hline 5348 & 1,066 & 240,206 & 8.43 & 6734 & 261 & 102,262 & 15.39 & 8047 & 1,666 & 548,776 & 12.85 & 9345 & 2 & 960 & 20.00 \\
\hline 5358 & 1,056 & 387,072 & 14.49 & 6736 & 1,305 & 452,975 & 13.78 & 8060 & 83 & 23,376 & 10.63 & 9363 & 595 & 135,816 & 8.24 \\
\hline 5398 & 1,852 & 411,020 & 8.21 & 6740 & 11 & 2,016 & 6.35 & 8062 & 30 & 6,504 & 8.15 & 9364 & 2,095 & 418,894 & 7.09 \\
\hline 5410 & 1,641 & 528,181 & 12.67 & 6750 & 151 & 37,990 & 9.19 & 8068 & 22 & 21,192 & 39.01 & 9365 & 21 & 5,664 & 10.34 \\
\hline 5411 & 1,750 & 462,238 & 10.01 & 6757 & 2,454 & 551,361 & 8.33 & 8081 & 1,778 & 537,081 & 11.69 & 9371 & 97 & 31,416 & 12.44 \\
\hline 5424 & 230 & 56,254 & 8.44 & 6775 & 383 & 110,784 & 11.12 & 8089 & 31 & 5,760 & 6.99 & 9417 & 1,376 & 331,982 & 9.10 \\
\hline 5429 & 677 & 193,392 & 10.93 & 6776 & 1,480 & 431,190 & 11.30 & 8221 & 10 & 1,896 & 5.53 & 9419 & 2,470 & 542,606 & 8.05 \\
\hline 5431 & 12 & 13,848 & 47.03 & 6788 & 131 & 29,256 & 8.54 & 8252 & 1,325 & 529,928 & 15.97 & 9435 & 57 & 33,600 & 23.79 \\
\hline 5461 & 1,299 & 324,672 & 9.50 & 6792 & 1,164 & 419,078 & 14.30 & 8265 & 1,029 & 217,176 & 7.60 & 9491 & 1,784 & 443,170 & 9.34 \\
\hline 5463 & 812 & 224,790 & 10.67 & 6794 & 9 & 2,016 & 6.28 & 8289 & 37 & 36,528 & 40.16 & 9499 & 1,285 & 431,095 & 13.23 \\
\hline 5471 & 13 & 3,480 & 10.11 & 6834 & 1,428 & 317,594 & 8.36 & 8305 & 1,189 & 512,439 & 17.25 & 9522 & 5 & 2,016 & 13.54 \\
\hline 5477 & 12 & 1,992 & 5.38 & 6893 & 951 & 374,777 & 15.81 & 8335 & 1,149 & 259,728 & 8.38 & 9527 & 1,467 & 539,410 & 14.49 \\
\hline 5528 & 900 & 262,609 & 11.34 & 6935 & 1,262 & 406,635 & 12.68 & 8400 & 145 & 62,112 & 16.94 & 9532 & 1,931 & 484,263 & 9.59 \\
\hline 5579 & 1 & 696 & 29.00 & 6981 & 132 & 132,768 & 41.02 & 8445 & 874 & 206,856 & 8.82 & 9544 & 4 & 2,928 & 30.50 \\
\hline 5580 & 15 & 1,944 & 4.11 & 7020 & 92 & 21,432 & 8.69 & 8446 & 1,216 & 268,785 & 8.28 & 9565 & 1,046 & 352,743 & 13.26 \\
\hline 5589 & 111 & 37,872 & 13.35 & 7060 & 688 & 277,917 & 16.09 & 8451 & 190 & 71,544 & 14.90 & 9570 & 347 & 100,848 & 11.31 \\
\hline 5590 & 108 & 39,024 & 13.98 & 7066 & 2,091 & 470,310 & 8.40 & 8531 & 1,122 & 305,116 & 10.42 & 9574 & 15 & 2,856 & 7.01 \\
\hline 5591 & 463 & 170,232 & 14.57 & 7074 & 1,497 & 523,667 & 13.86 & 8541 & 77 & 22,615 & 11.41 & 9588 & 1,350 & 457,802 & 13.19 \\
\hline 5592 & 386 & 151,920 & 15.65 & 7097 & 66 & 29,232 & 17.16 & 8544 & 351 & 110,871 & 12.17 & 9665 & 2,187 & 548,295 & 9.49 \\
\hline 5594 & 129 & 68,448 & 21.53 & 7116 & 429 & 167,304 & 15.42 & 8545 & 13 & 4,656 & 13.81 & 9715 & 1,837 & 443,326 & 9.17 \\
\hline 5595 & 2 & 8,016 & 167.00 & 7140 & 1,663 & 395,594 & 8.89 & 8563 & 845 & 220,484 & 9.98 & 9729 & 2,341 & 549,310 & 8.81 \\
\hline 5596 & 827 & 292,949 & 13.92 & 7173 & 631 & 115,200 & 6.26 & 8566 & 383 & 143,616 & 14.77 & 9772 & 665 & 140,832 & 7.82 \\
\hline 5600 & 296 & 105,888 & 14.10 & 7174 & 2,617 & 474,094 & 6.19 & 8583 & 566 & 203,370 & 14.08 & 9814 & 29 & 13,030 & 18.07 \\
\hline 5618 & 13 & 2,112 & 4.23 & 7213 & 535 & 156,336 & 11.24 & 8584 & 947 & 344,280 & 14.33 & 9815 & 482 & 121,848 & 9.51 \\
\hline 5650 & 12 & 1,896 & 5.45 & 7243 & 1,898 & 549,034 & 11.16 & 8623 & 1,109 & 319,544 & 11.15 & 9816 & 56 & 35,472 & 25.70 \\
\hline 5656 & 1,089 & 326,279 & 11.69 & 7262 & 66 & 27,360 & 16.47 & 8625 & 915 & 243,840 & 10.17 & 9817 & 1,014 & 356,901 & 13.89 \\
\hline 5658 & 646 & 209,400 & 12.69 & 7274 & 348 & 121,725 & 14.00 & 8630 & 592 & 226,383 & 15.26 & 9829 & 1,099 & 436,603 & 15.87 \\
\hline 5661 & 136 & 46,672 & 13.28 & 7300 & 899 & 258,332 & 11.21 & 8631 & 644 & 243,264 & 14.98 & 9830 & 119 & 45,216 & 15.20 \\
\hline 5666 & 22 & 5,880 & 10.06 & 7311 & 23 & 6,600 & 11.41 & 8646 & 1,327 & 338,752 & 9.76 & 9858 & 1,032 & 293,448 & 11.11 \\
\hline 5695 & 1,612 & 448,602 & 10.72 & 7363 & 11 & 2,016 & 5.44 & 8647 & 896 & 282,860 & 12.36 & 9893 & 1,850 & 537,872 & 11.30 \\
\hline 5742 & 13 & 5,784 & 17.63 & 7422 & 1,940 & 544,386 & 10.77 & 8677 & 113 & 64,752 & 22.89 & 9916 & 733 & 185,230 & 9.56 \\
\hline 5766 & 9 & 2,016 & 6.02 & 7431 & 1,133 & 456,709 & 16.15 & 8696 & 10 & 2,016 & 5.77 & 9976 & 1,419 & 549,337 & 15.29 \\
\hline 5770 & 1,922 & 545,182 & 10.92 & & & & & & & & & & & & \\
\hline
\end{tabular}


Appendix 4-1.7. Number of storms, total duration, and mean storm interevent time defined by 72-hour minimum interevent time for hourly rainfall stations in Texas.

$[--$, not available $]$

\begin{tabular}{|c|c|c|c|c|c|c|c|c|c|c|c|c|c|c|c|}
\hline $\begin{array}{c}\text { Station } \\
\text { no. }\end{array}$ & $\begin{array}{c}\text { No. } \\
\text { of } \\
\text { storms }\end{array}$ & $\begin{array}{c}\text { Total } \\
\text { duration } \\
\text { (hours) }\end{array}$ & $\begin{array}{c}\text { Mean } \\
\text { storm } \\
\text { interevent } \\
\text { time } \\
\text { (days) }\end{array}$ & $\begin{array}{c}\text { Station } \\
\text { no. }\end{array}$ & $\begin{array}{c}\text { No. } \\
\text { of } \\
\text { storms }\end{array}$ & $\begin{array}{c}\text { Total } \\
\text { duration } \\
\text { (hours) }\end{array}$ & $\begin{array}{c}\text { Mean } \\
\text { storm } \\
\text { interevent } \\
\text { time } \\
\text { (days) }\end{array}$ & $\begin{array}{c}\text { Station } \\
\text { no. }\end{array}$ & $\begin{array}{c}\text { No. } \\
\text { of } \\
\text { storms }\end{array}$ & $\begin{array}{c}\text { Total } \\
\text { duration } \\
\text { (hours) }\end{array}$ & $\begin{array}{c}\text { Mean } \\
\text { storm } \\
\text { interevent } \\
\text { time } \\
\text { (days) }\end{array}$ & $\begin{array}{c}\text { Station } \\
\text { no. }\end{array}$ & $\begin{array}{c}\text { No. } \\
\text { of } \\
\text { storms }\end{array}$ & $\begin{array}{c}\text { Total } \\
\text { duration } \\
\text { (hours) }\end{array}$ & $\begin{array}{c}\text { Mean } \\
\text { storm } \\
\text { interevent } \\
\text { time } \\
\text { (days) }\end{array}$ \\
\hline 0015 & 3 & 624 & 8.67 & 1154 & 89 & 26,928 & 9.94 & 2160 & 12 & 4,752 & 13.98 & 3463 & 30 & 30,096 & 40.38 \\
\hline 0016 & 1,819 & 543,665 & 10.85 & 1165 & 379 & 147,768 & 14.87 & 2206 & 425 & 105,121 & 8.68 & 3476 & 423 & 127,848 & 11.13 \\
\hline 0050 & 614 & 197,240 & 12.00 & 1185 & 292 & 185,232 & 25.74 & 2238 & 144 & 51,864 & 13.52 & 3485 & 10 & 1,968 & 6.03 \\
\hline 0054 & 21 & 5,688 & 8.32 & 1186 & 56 & 19,128 & 12.21 & 2240 & 46 & 12,864 & 9.84 & 3507 & 590 & 188,267 & 11.69 \\
\hline 0120 & 8 & 1,896 & 6.27 & 1188 & 5 & 1,872 & 13.52 & 2242 & 952 & 253,435 & 9.24 & 3546 & 1,758 & 485,758 & 9.75 \\
\hline 0145 & 92 & 28,392 & 10.99 & 1245 & 9 & 1,992 & 5.37 & 2244 & 1,805 & 535,994 & 10.55 & 3547 & 135 & 51,144 & 14.59 \\
\hline 0146 & 23 & 5,664 & 8.13 & 1246 & 622 & 176,017 & 10.20 & 2247 & 26 & 7,056 & 9.26 & 3579 & 46 & 19,944 & 16.59 \\
\hline 0174 & 712 & 275,194 & 14.53 & 1267 & 285 & 116,832 & 15.59 & 2309 & 381 & 153,600 & 15.14 & 3642 & 1,813 & 534,563 & 10.73 \\
\hline 0178 & 6 & 2,040 & 9.89 & 1304 & 335 & 117,840 & 13.25 & 2312 & 349 & 92,757 & 9.14 & 3646 & 1,328 & 419,874 & 11.82 \\
\hline 0179 & 168 & 66,456 & 14.91 & 1325 & 803 & 219,432 & 9.78 & 2334 & 38 & 22,344 & 23.15 & 3668 & 6 & 2,016 & 9.47 \\
\hline 0202 & 148 & 58,398 & 14.94 & 1429 & 743 & 213,741 & 10.56 & 2336 & 151 & 53,256 & 13.67 & 3673 & 9 & 2,016 & 6.07 \\
\hline 0206 & 1,480 & 532,111 & 13.58 & 1431 & 1,060 & 356,239 & 12.46 & 2354 & 15 & 20,976 & 56.62 & 3686 & 632 & 198,532 & 11.56 \\
\hline 0208 & 2 & 384 & 8.00 & 1432 & 774 & 246,787 & 11.78 & 2355 & 32 & 10,536 & 12.43 & 3691 & 1,609 & 469,594 & 10.69 \\
\hline 0211 & 1,870 & 542,494 & 10.42 & 1433 & 1,147 & 367,055 & 11.89 & 2357 & 285 & 98,496 & 12.75 & 3734 & 7 & 2,016 & 7.33 \\
\hline 0244 & 40 & 11,568 & 9.77 & 1434 & 1,159 & 358,448 & 11.45 & 2360 & 982 & 399,684 & 15.53 & 3771 & 765 & 220,455 & 10.38 \\
\hline 0248 & 1,261 & 529,764 & 16.45 & 1435 & 710 & 230,732 & 12.19 & 2361 & 113 & 56,004 & 19.42 & 3789 & 53 & 18,888 & 12.97 \\
\hline 0262 & 1,462 & 431,066 & 10.71 & 1436 & 1,274 & 367,281 & 10.48 & 2394 & 1,774 & 504,483 & 10.19 & 3826 & 137 & 45,240 & 12.44 \\
\hline 0271 & 8 & 2,016 & 7.37 & 1437 & 17 & 4,728 & 10.55 & 2404 & 1,667 & 493,624 & 10.81 & 3831 & 252 & 110,112 & 16.95 \\
\hline 0380 & 268 & 163,872 & 24.38 & 1438 & 939 & 284,045 & 11.17 & 2415 & 1,698 & 501,413 & 10.69 & 3841 & 98 & 77,472 & 31.75 \\
\hline 0394 & 5 & 1,992 & 14.33 & 1462 & 0 & 4,416 & -- & 2462 & 581 & 188,205 & 12.06 & 3871 & 881 & 317,328 & 13.69 \\
\hline 0408 & 8 & 2,112 & 7.27 & 1492 & 709 & 280,441 & 15.27 & 2528 & 71 & 24,840 & 13.12 & 3884 & 8 & 1,896 & 6.85 \\
\hline 0427 & 27 & 11,646 & 15.77 & 1500 & 9 & 2,112 & 6.27 & 2617 & 158 & 56,688 & 13.65 & 3941 & 39 & 7,608 & 6.39 \\
\hline 0428 & 1,982 & 529,122 & 9.03 & 1528 & 836 & 366,583 & 17.06 & 2619 & 145 & 64,992 & 17.41 & 3963 & 4 & 1,488 & 15.50 \\
\hline 0429 & 88 & 22,416 & 8.44 & 1541 & 33 & 10,953 & 12.08 & 2621 & 785 & 277,864 & 13.59 & 4040 & 291 & 92,806 & 12.08 \\
\hline 0463 & 78 & 51,936 & 26.66 & 1569 & 103 & 32,160 & 11.18 & 2675 & 621 & 178,918 & 10.38 & 4058 & 9 & 2,016 & 6.88 \\
\hline 0493 & 9 & 1,704 & 6.00 & 1632 & 5 & 1,320 & 10.38 & 2676 & 495 & 146,737 & 10.60 & 4098 & 1,034 & 417,420 & 15.61 \\
\hline 0495 & 222 & 138,072 & 24.86 & 1641 & 137 & 110,280 & 32.37 & 2679 & 1,323 & 536,229 & 15.65 & 4100 & 218 & 121,992 & 22.05 \\
\hline 0496 & 22 & 10,872 & 20.30 & 1646 & 1,430 & 543,287 & 14.57 & 2715 & 1,096 & 354,131 & 12.17 & 4137 & 642 & 221,233 & 12.81 \\
\hline 0498 & 8 & 7,920 & 40.34 & 1663 & 24 & 11,460 & 18.33 & 2744 & 1,100 & 413,376 & 14.41 & 4191 & 1,421 & 519,452 & 13.86 \\
\hline 0509 & 1,710 & 549,223 & 11.92 & 1671 & 1,799 & 548,538 & 11.17 & 2758 & 61 & 23,376 & 14.09 & 4256 & 0 & 21,906 & -- \\
\hline 0518 & 1,148 & 330,777 & 10.43 & 1680 & 736 & 261,792 & 13.46 & 2794 & 7 & 2,064 & 10.48 & 4257 & 1,721 & 498,651 & 10.45 \\
\hline 0521 & 21 & 5,760 & 9.78 & 1694 & 98 & 45,172 & 18.05 & 2797 & 1,401 & 529,606 & 14.38 & 4258 & 84 & 25,608 & 10.58 \\
\hline 0556 & 71 & 21,936 & 11.65 & 1696 & 699 & 268,080 & 14.73 & 2811 & 1,174 & 435,389 & 14.18 & 4278 & 992 & 409,467 & 15.90 \\
\hline 0569 & 1,328 & 399,353 & 10.75 & 1697 & 63 & 29,496 & 18.51 & 2813 & 16 & 31,728 & 81.46 & 4299 & 93 & 86,976 & 38.22 \\
\hline 0572 & 559 & 158,448 & 10.12 & 1698 & 770 & 291,219 & 14.41 & 2814 & 7 & 12,336 & 70.76 & 4300 & 1,214 & 289,264 & 7.38 \\
\hline 0576 & 75 & 23,352 & 11.43 & 1720 & 120 & 46,709 & 14.64 & 2815 & 157 & 58,942 & 14.53 & 4305 & 1,083 & 266,616 & 7.79 \\
\hline 0580 & 217 & 112,800 & 20.42 & 1761 & 47 & 16,680 & 12.20 & 2818 & 124 & 45,312 & 14.05 & 4307 & 270 & 96,430 & 12.21 \\
\hline 0587 & 453 & 121,272 & 9.10 & 1773 & 1,905 & 502,398 & 9.34 & 2986 & 152 & 54,240 & 13.12 & 4309 & 1,915 & 517,057 & 9.45 \\
\hline 0605 & 69 & 27,000 & 14.74 & 1810 & 9 & 2,112 & 7.02 & 3005 & 1,572 & 522,908 & 12.43 & 4311 & 1,993 & 543,389 & 9.65 \\
\hline 0639 & 1,320 & 433,077 & 12.16 & 1823 & 8 & 1,968 & 7.10 & 3033 & 454 & 216,192 & 18.93 & 4313 & 145 & 39,432 & 9.56 \\
\hline 0655 & 0 & 718 & -- & 1870 & 183 & 47,496 & 8.87 & 3034 & 1 & 6,888 & 287.00 & 4319 & 45 & 29,208 & 24.76 \\
\hline 0665 & 1,246 & 361,772 & 10.59 & 1875 & 10 & 1,752 & 5.85 & 3047 & 9 & 2,016 & 6.11 & 4329 & 1,579 & 438,183 & 9.91 \\
\hline 0689 & 972 & 381,486 & 15.00 & 1876 & 32 & 10,920 & 13.17 & 3103 & 23 & 29,448 & 52.53 & 4331 & 0 & 5,112 & -- \\
\hline 0690 & 397 & 179,395 & 17.57 & 1889 & 129 & 38,734 & 10.21 & 3133 & 1,573 & 486,379 & 11.41 & 4375 & 696 & 232,138 & 12.53 \\
\hline 0691 & 1,606 & 469,381 & 10.74 & 1903 & 227 & 83,753 & 14.33 & 3156 & 178 & 63,435 & 13.61 & 4392 & 252 & 72,624 & 10.00 \\
\hline 0708 & 68 & 32,809 & 18.27 & 1914 & 7 & 1,752 & 6.55 & 3171 & 1,459 & 463,053 & 11.81 & 4425 & 563 & 264,416 & 18.54 \\
\hline 0738 & 1,063 & 306,512 & 10.57 & 1920 & 289 & 109,392 & 14.54 & 3189 & 199 & 97,104 & 18.92 & 4440 & 953 & 283,440 & 10.96 \\
\hline 0776 & 1,112 & 439,998 & 15.40 & 1921 & 1,724 & 476,617 & 9.89 & 3260 & 112 & 44,448 & 15.48 & 4476 & 1,077 & 344,677 & 11.94 \\
\hline 0779 & 280 & 108,787 & 15.19 & 1937 & 704 & 186,288 & 9.34 & 3267 & 60 & 22,512 & 14.06 & 4498 & 9 & 4,944 & 22.35 \\
\hline 0784 & 1,155 & 429,972 & 14.32 & 1956 & 1,691 & 486,210 & 10.10 & 3270 & 973 & 365,659 & 14.49 & 4517 & 1,059 & 328,752 & 11.51 \\
\hline 0786 & 341 & 117,888 & 13.21 & 1970 & 7 & 1,992 & 7.33 & 3272 & 9 & 3,840 & 15.20 & 4520 & 645 & 220,430 & 12.72 \\
\hline 0917 & 1,171 & 300,936 & 8.76 & 2014 & 235 & 69,312 & 10.22 & 3277 & 11 & 4,176 & 14.98 & 4525 & 7 & 1,992 & 6.73 \\
\hline 0923 & 8 & 2,016 & 6.50 & 2015 & 1,727 & 484,318 & 9.73 & 3278 & 615 & 253,450 & 15.93 & 4563 & 9 & 2,112 & 6.54 \\
\hline 0926 & 1,856 & 551,357 & 10.91 & 2019 & 8 & 2,016 & 7.53 & 3280 & 113 & 41,832 & 14.53 & 4570 & 1,409 & 548,973 & 14.96 \\
\hline 0950 & 25 & 18,936 & 30.18 & 2024 & 1,192 & 391,758 & 12.27 & 3281 & 36 & 26,304 & 29.87 & 4577 & 1,143 & 305,664 & 9.46 \\
\hline 0996 & 8 & 1,992 & 7.13 & 2042 & 8 & 16,080 & 82.82 & 3283 & 895 & 247,224 & 9.58 & 4591 & 1,570 & 431,406 & 9.78 \\
\hline 1013 & 135 & 63,492 & 18.05 & 2043 & 58 & 36,960 & 25.73 & 3284 & 1,610 & 499,445 & 11.35 & 4670 & 1,518 & 541,246 & 13.58 \\
\hline 1017 & 1,674 & 549,062 & 12.28 & 2048 & 997 & 409,659 & 15.72 & 3285 & 1,259 & 382,496 & 11.11 & 4671 & 85 & 25,848 & 11.08 \\
\hline 1042 & 6 & 1,488 & 8.15 & 2050 & 47 & 17,448 & 14.41 & 3329 & 954 & 306,792 & 11.94 & 4679 & 1,434 & 429,394 & 10.93 \\
\hline 1048 & 9 & 1,728 & 4.97 & 2051 & 43 & 26,304 & 24.32 & 3335 & 126 & 33,792 & 9.64 & 4696 & 7 & 1,992 & 10.46 \\
\hline 1053 & 405 & 145,392 & 13.71 & 2053 & 6 & 3,552 & 23.51 & 3370 & 1,170 & 317,621 & 9.87 & 4703 & 66 & 31,392 & 18.13 \\
\hline 1057 & 1,004 & 363,120 & 13.62 & 2073 & 513 & 146,832 & 10.26 & 3410 & 1,447 & 514,264 & 13.51 & 4704 & 220 & 61,368 & 9.86 \\
\hline 1063 & 8 & 1,968 & 6.83 & 2082 & 1,237 & 519,707 & 16.58 & 3415 & 1,638 & 537,022 & 12.10 & 4731 & 65 & 17,160 & 9.14 \\
\hline 1068 & 1,616 & 512,393 & 11.80 & 2086 & 1,421 & 548,518 & 14.60 & 3430 & 2,062 & 530,963 & 8.62 & 4792 & 700 & 213,141 & 11.32 \\
\hline 1080 & 108 & 61,344 & 22.34 & 2088 & 28 & 10,190 & 13.59 & 3431 & 109 & 32,784 & 9.89 & 4819 & 149 & 39,480 & 9.17 \\
\hline 1081 & 748 & 241,368 & 11.93 & 2090 & 201 & 58,356 & 10.72 & 3441 & 22 & 42,816 & 79.43 & 4852 & 8 & 2,016 & 8.76 \\
\hline 1133 & 10 & 2,784 & 10.12 & 2096 & 1,563 & 493,125 & 11.71 & 3442 & 322 & 171,216 & 20.88 & 4866 & 1,752 & 548,790 & 11.69 \\
\hline 1136 & 1,772 & 532,054 & 10.48 & 2128 & 201 & 68,160 & 12.70 & 3446 & 454 & 213,312 & 18.60 & 4876 & 76 & 29,184 & 14.55 \\
\hline 1138 & 6 & 1,320 & 5.35 & 2131 & 1,349 & 483,317 & 13.59 & 3460 & 24 & 7,008 & 10.85 & 4878 & 1,400 & 350,328 & 8.45 \\
\hline 1139 & 41 & 13,056 & 10.80 & 2142 & 5 & 2,016 & 11.68 & 3462 & 91 & 49,920 & 21.60 & 4880 & 1,588 & 551,284 & 13.15 \\
\hline
\end{tabular}


Appendix 4-1.7. Number of storms, total duration, and mean storm interevent time defined by 72-hour minimum interevent time for hourly rainfall stations in Texas-Continued.

\begin{tabular}{|c|c|c|c|c|c|c|c|c|c|c|c|c|c|c|c|}
\hline $\begin{array}{c}\text { Station } \\
\text { no. }\end{array}$ & $\begin{array}{c}\text { No. } \\
\text { of } \\
\text { storms }\end{array}$ & $\begin{array}{l}\text { Total } \\
\text { duration } \\
\text { (hours) }\end{array}$ & $\begin{array}{c}\text { Mean } \\
\text { storm } \\
\text { interevent } \\
\text { time } \\
\text { (days) }\end{array}$ & $\begin{array}{c}\text { Station } \\
\text { no. }\end{array}$ & $\begin{array}{c}\text { No. } \\
\text { of } \\
\text { storms }\end{array}$ & $\begin{array}{c}\text { Total } \\
\text { duration } \\
\text { (hours) }\end{array}$ & $\begin{array}{c}\text { Mean } \\
\text { storm } \\
\text { interevent } \\
\text { time } \\
\text { (days) }\end{array}$ & $\begin{array}{c}\text { Station } \\
\text { no. }\end{array}$ & $\begin{array}{c}\text { No. } \\
\text { of } \\
\text { storms }\end{array}$ & $\begin{array}{c}\text { Total } \\
\text { duration } \\
\text { (hours) }\end{array}$ & $\begin{array}{c}\text { Mean } \\
\text { storm } \\
\text { interevent } \\
\text { time } \\
\text { (days) }\end{array}$ & $\begin{array}{c}\text { Station } \\
\text { no. }\end{array}$ & $\begin{array}{c}\text { No. } \\
\text { of } \\
\text { storms }\end{array}$ & $\begin{array}{c}\text { Total } \\
\text { duration } \\
\text { (hours) }\end{array}$ & $\begin{array}{c}\text { Mean } \\
\text { storm } \\
\text { interevent } \\
\text { time } \\
\text { (days) }\end{array}$ \\
\hline 4920 & 1,263 & 524,353 & 16.09 & 5775 & 7 & 5,112 & 28.95 & 7481 & 984 & 495,877 & 19.98 & 8743 & 1,967 & 527,995 & 9.48 \\
\hline 4934 & 5 & 1,992 & 15.92 & 5779 & 15 & 5,880 & 15.12 & 7497 & 776 & 243,992 & 11.50 & 8761 & 639 & 256,990 & 15.64 \\
\hline 4972 & 1,542 & 494,452 & 11.95 & 5840 & 239 & 78,888 & 12.49 & 7498 & 163 & 49,632 & 11.08 & 8778 & 1,409 & 398,041 & 10.10 \\
\hline 4973 & 271 & 119,208 & 16.85 & 5890 & 1,456 & 515,614 & 13.44 & 7499 & 1,412 & 523,925 & 14.11 & 8845 & 1,710 & 550,655 & 11.86 \\
\hline 4974 & 808 & 340,313 & 16.32 & 5891 & 111 & 53,304 & 19.03 & 7531 & 82 & 49,968 & 23.86 & 8859 & 1,161 & 312,504 & 9.58 \\
\hline 4975 & 934 & 257,716 & 9.87 & 5897 & 809 & 245,324 & 11.09 & 7534 & 200 & 103,032 & 20.11 & 8898 & 417 & 116,904 & 10.20 \\
\hline 4978 & 88 & 47,496 & 21.11 & 5957 & 1,346 & 445,446 & 12.39 & 7556 & 1,621 & 493,793 & 11.27 & 8908 & 17 & 5,904 & 11.93 \\
\hline 4979 & 6 & 1,896 & 8.50 & 5958 & 118 & 41,136 & 13.07 & 7594 & 1,224 & 309,414 & 8.96 & 8910 & 9 & 1,968 & 6.90 \\
\hline 4982 & 671 & 248,714 & 14.17 & 5973 & 58 & 23,376 & 15.00 & 7596 & 112 & 26,136 & 8.63 & 8911 & 479 & 164,256 & 12.53 \\
\hline 5018 & 436 & 134,338 & 11.39 & 5996 & 1,766 & 549,087 & 11.51 & 7608 & 889 & 249,648 & 9.91 & 8924 & 297 & 181,152 & 24.47 \\
\hline 5048 & 1,085 & 503,770 & 18.37 & 6017 & 70 & 23,280 & 12.10 & 7622 & 14 & 4,152 & 11.11 & 8929 & 9 & 2,016 & 7.10 \\
\hline 5049 & 63 & 34,272 & 21.60 & 6024 & 161 & 72,480 & 17.36 & 7700 & 1,189 & 308,448 & 8.74 & 8942 & 1,129 & 300,835 & 9.31 \\
\hline 5056 & 2 & 10,104 & 210.50 & 6050 & 15 & 6,576 & 17.25 & 7706 & 1,262 & 502,063 & 15.25 & 8944 & 623 & 144,888 & 7.76 \\
\hline 5057 & 521 & 189,960 & 13.54 & 6104 & 1,291 & 478,193 & 13.72 & 7718 & 63 & 34,320 & 21.39 & 8996 & 1,517 & 399,638 & 9.09 \\
\hline 5060 & 153 & 59,904 & 14.40 & 6108 & 1,778 & 542,529 & 11.13 & 7745 & 217 & 70,872 & 11.63 & 9014 & 7 & 1,896 & 7.19 \\
\hline 5081 & 709 & 180,720 & 8.80 & 6136 & 1,323 & 539,302 & 15.72 & 7922 & 210 & 111,768 & 21.08 & 9037 & 488 & 240,720 & 19.61 \\
\hline 5094 & 1,566 & 468,823 & 10.99 & 6166 & 56 & 14,520 & 9.46 & 7936 & 1,173 & 306,666 & 9.01 & 9106 & 134 & 65,736 & 19.40 \\
\hline 5113 & 1,494 & 523,501 & 13.11 & 6176 & 219 & 69,264 & 11.45 & 7943 & 1,569 & 474,742 & 11.21 & 9107 & 24 & 26,784 & 45.53 \\
\hline 5114 & 0 & 1,488 & -- & 6177 & 1,486 & 440,385 & 10.65 & 7944 & 33 & 8,592 & 8.44 & 9129 & 63 & 23,376 & 14.05 \\
\hline 5123 & 11 & 3,072 & 10.78 & 6210 & 1,259 & 354,217 & 10.20 & 7945 & 2,034 & 543,166 & 9.14 & 9163 & 1,397 & 550,742 & 15.05 \\
\hline 5192 & 1,018 & 332,072 & 12.06 & 6211 & 241 & 93,000 & 14.10 & 7947 & 129 & 49,618 & 14.23 & 9213 & 117 & 38,700 & 11.64 \\
\hline 5193 & 1,851 & 550,050 & 10.77 & 6270 & 978 & 256,135 & 9.23 & 7948 & 657 & 213,840 & 12.27 & 9214 & 6 & 2,016 & 7.35 \\
\hline 5224 & 176 & 52,608 & 10.36 & 6275 & 0 & 2,920 & -- & 7951 & 585 & 141,648 & 8.37 & 9222 & 117 & 31,416 & 9.43 \\
\hline 5228 & 239 & 79,632 & 12.26 & 6276 & 8 & 2,016 & 6.81 & 7953 & 70 & 23,352 & 12.38 & 9248 & 86 & 52,272 & 24.33 \\
\hline 5235 & 17 & 4,392 & 9.07 & 6335 & 1,354 & 354,128 & 9.30 & 7981 & 145 & 112,728 & 31.24 & 9266 & 52 & 17,160 & 12.67 \\
\hline 5247 & 1,530 & 552,074 & 13.66 & 6434 & 14 & 8,016 & 22.82 & 7990 & 58 & 23,304 & 14.33 & 9270 & 935 & 382,084 & 15.57 \\
\hline 5258 & 533 & 177,528 & 12.36 & 6504 & 1,414 & 548,140 & 14.89 & 7992 & 6 & 1,896 & 7.53 & 9295 & 61 & 27,672 & 17.29 \\
\hline 5303 & 417 & 179,592 & 16.70 & 6558 & 20 & 5,688 & 10.97 & 7997 & 74 & 34,656 & 18.58 & 9304 & 2 & 336 & 7.00 \\
\hline 5312 & 1,091 & 404,451 & 14.10 & 6615 & 398 & 194,104 & 19.13 & 7999 & 13 & 13,992 & 44.06 & 9307 & 171 & 79,272 & 18.18 \\
\hline 5341 & 7 & 2,016 & 7.51 & 6660 & 67 & 32,880 & 19.05 & 8022 & 46 & 48,504 & 43.02 & 9328 & 102 & 34,344 & 12.48 \\
\hline 5342 & 0 & 4,404 & -- & 6663 & 40 & 36,168 & 35.11 & 8023 & 1,042 & 484,077 & 18.26 & 9329 & 7 & 3,240 & 17.04 \\
\hline 5348 & 876 & 240,206 & 9.72 & 6734 & 214 & 102,262 & 18.23 & 8047 & 1,444 & 548,776 & 14.45 & 9345 & 2 & 960 & 20.00 \\
\hline 5358 & 937 & 387,072 & 16.02 & 6736 & 1,161 & 452,975 & 15.18 & 8060 & 72 & 23,376 & 11.88 & 9363 & 475 & 135,816 & 9.69 \\
\hline 5398 & 1,527 & 411,020 & 9.43 & 6740 & 10 & 2,016 & 7.02 & 8062 & 26 & 6,504 & 8.99 & 9364 & 1,671 & 418,894 & 8.27 \\
\hline 5410 & 1,424 & 528,181 & 14.22 & 6750 & 124 & 37,990 & 10.63 & 8068 & 21 & 21,192 & 40.72 & 9365 & 18 & 5,664 & 11.50 \\
\hline 5411 & 1,481 & 462,238 & 11.39 & 6757 & 2,008 & 551,361 & 9.63 & 8081 & 1,513 & 537,081 & 13.30 & 9371 & 82 & 31,416 & 14.25 \\
\hline 5424 & 180 & 56,254 & 10.10 & 6775 & 322 & 110,784 & 12.76 & 8089 & 26 & 5,760 & 7.84 & 9417 & 1,168 & 331,982 & 10.29 \\
\hline 5429 & 577 & 193,392 & 12.39 & 6776 & 1,251 & 431,190 & 12.92 & 8221 & 8 & 1,896 & 6.34 & 9419 & 2,022 & 542,606 & 9.28 \\
\hline 5431 & 11 & 13,848 & 51.11 & 6788 & 106 & 29,256 & 9.96 & 8252 & 1,194 & 529,928 & 17.45 & 9435 & 50 & 33,600 & 26.77 \\
\hline 5461 & 1,120 & 324,672 & 10.62 & 6792 & 1,017 & 419,078 & 16.01 & 8265 & 846 & 217,176 & 8.70 & 9491 & 1,484 & 443,170 & 10.73 \\
\hline 5463 & 691 & 224,790 & 12.11 & 6794 & 6 & 2,016 & 8.56 & 8289 & 35 & 36,528 & 42.30 & 9499 & 1,139 & 431,095 & 14.61 \\
\hline 5471 & 9 & 3,480 & 13.60 & 6834 & 1,174 & 317,594 & 9.65 & 8305 & 1,047 & 512,439 & 19.26 & 9522 & 4 & 2,016 & 21.00 \\
\hline 5477 & 11 & 1,992 & 5.66 & 6893 & 854 & 374,777 & 17.32 & 8335 & 983 & 259,728 & 9.39 & 9527 & 1,270 & 539,410 & 16.35 \\
\hline 5528 & 797 & 262,609 & 12.48 & 6935 & 1,102 & 406,635 & 14.16 & 8400 & 127 & 62,112 & 19.00 & 9532 & 1,637 & 484,263 & 10.87 \\
\hline 5579 & 1 & 696 & 29.00 & 6981 & 114 & 132,768 & 47.13 & 8445 & 724 & 206,856 & 10.14 & 9544 & 4 & 2,928 & 30.50 \\
\hline 5580 & 8 & 1,944 & 6.02 & 7020 & 83 & 21,432 & 9.40 & 8446 & 998 & 268,785 & 9.55 & 9565 & 905 & 352,743 & 14.94 \\
\hline 5589 & 96 & 37,872 & 15.06 & 7060 & 590 & 277,917 & 18.35 & 8451 & 168 & 71,544 & 16.53 & 9570 & 284 & 100,848 & 13.27 \\
\hline 5590 & 90 & 39,024 & 16.28 & 7066 & 1,740 & 470,310 & 9.60 & 8531 & 967 & 305,116 & 11.69 & 9574 & 11 & 2,856 & 8.58 \\
\hline 5591 & 413 & 170,232 & 16.04 & 7074 & 1,323 & 523,667 & 15.35 & 8541 & 68 & 22,615 & 12.58 & 9588 & 1,181 & 457,802 & 14.72 \\
\hline 5592 & 340 & 151,920 & 17.43 & 7097 & 59 & 29,232 & 18.89 & 8544 & 299 & 110,871 & 13.86 & 9665 & 1,892 & 548,295 & 10.59 \\
\hline 5594 & 114 & 68,448 & 24.03 & 7116 & 368 & 167,304 & 17.57 & 8545 & 12 & 4,656 & 14.72 & 9715 & 1,551 & 443,326 & 10.41 \\
\hline 5595 & 1 & 8,016 & 334.00 & 7140 & 1,404 & 395,594 & 10.07 & 8563 & 733 & 220,484 & 11.14 & 9729 & 1,953 & 549,310 & 10.08 \\
\hline 5596 & 707 & 292,949 & 15.87 & 7173 & 485 & 115,200 & 7.40 & 8566 & 342 & 143,616 & 16.24 & 9772 & 531 & 140,832 & 9.17 \\
\hline 5600 & 269 & 105,888 & 15.26 & 7174 & 1,991 & 474,094 & 7.35 & 8583 & 496 & 203,370 & 15.72 & 9814 & 22 & 13,030 & 23.01 \\
\hline 5618 & 9 & 2,112 & 5.54 & 7213 & 456 & 156,336 & 12.76 & 8584 & 845 & 344,280 & 15.76 & 9815 & 402 & 121,848 & 10.91 \\
\hline 5650 & 8 & 1,896 & 7.30 & 7243 & 1,617 & 549,034 & 12.67 & 8623 & 968 & 319,544 & 12.42 & 9816 & 52 & 35,472 & 27.48 \\
\hline 5656 & 928 & 326,279 & 13.29 & 7262 & 60 & 27,360 & 17.84 & 8625 & 789 & 243,840 & 11.40 & 9817 & 887 & 356,901 & 15.53 \\
\hline 5658 & 572 & 209,400 & 14.00 & 7274 & 318 & 121,725 & 15.09 & 8630 & 534 & 226,383 & 16.65 & 9829 & 981 & 436,603 & 17.48 \\
\hline 5661 & 122 & 46,672 & 14.53 & 7300 & 787 & 258,332 & 12.45 & 8631 & 580 & 243,264 & 16.36 & 9830 & 105 & 45,216 & 16.90 \\
\hline 5666 & 21 & 5,880 & 10.42 & 7311 & 20 & 6,600 & 12.73 & 8646 & 1,139 & 338,752 & 10.96 & 9858 & 890 & 293,448 & 12.49 \\
\hline 5695 & 1,381 & 448,602 & 12.10 & 7363 & 8 & 2,016 & 6.86 & 8647 & 758 & 282,860 & 14.16 & 9893 & 1,594 & 537,872 & 12.72 \\
\hline 5742 & 10 & 5,784 & 22.45 & 7422 & 1,685 & 544,386 & 12.02 & 8677 & 103 & 64,752 & 24.90 & 9916 & 608 & 185,230 & 11.02 \\
\hline 5766 & 7 & 2,016 & 7.05 & 7431 & 1,022 & 456,709 & 17.63 & 8696 & 8 & 2,016 & 6.93 & 9976 & 1,258 & 549,337 & 16.93 \\
\hline 5770 & 1,633 & 545,182 & 12.42 & & & & & & & & & & & & \\
\hline
\end{tabular}


Appendix 4-2.1. L-moments of storm depth defined by 6-hour minimum interevent time for hourly rainfall stations in Texas.

$[--$, not available $]$

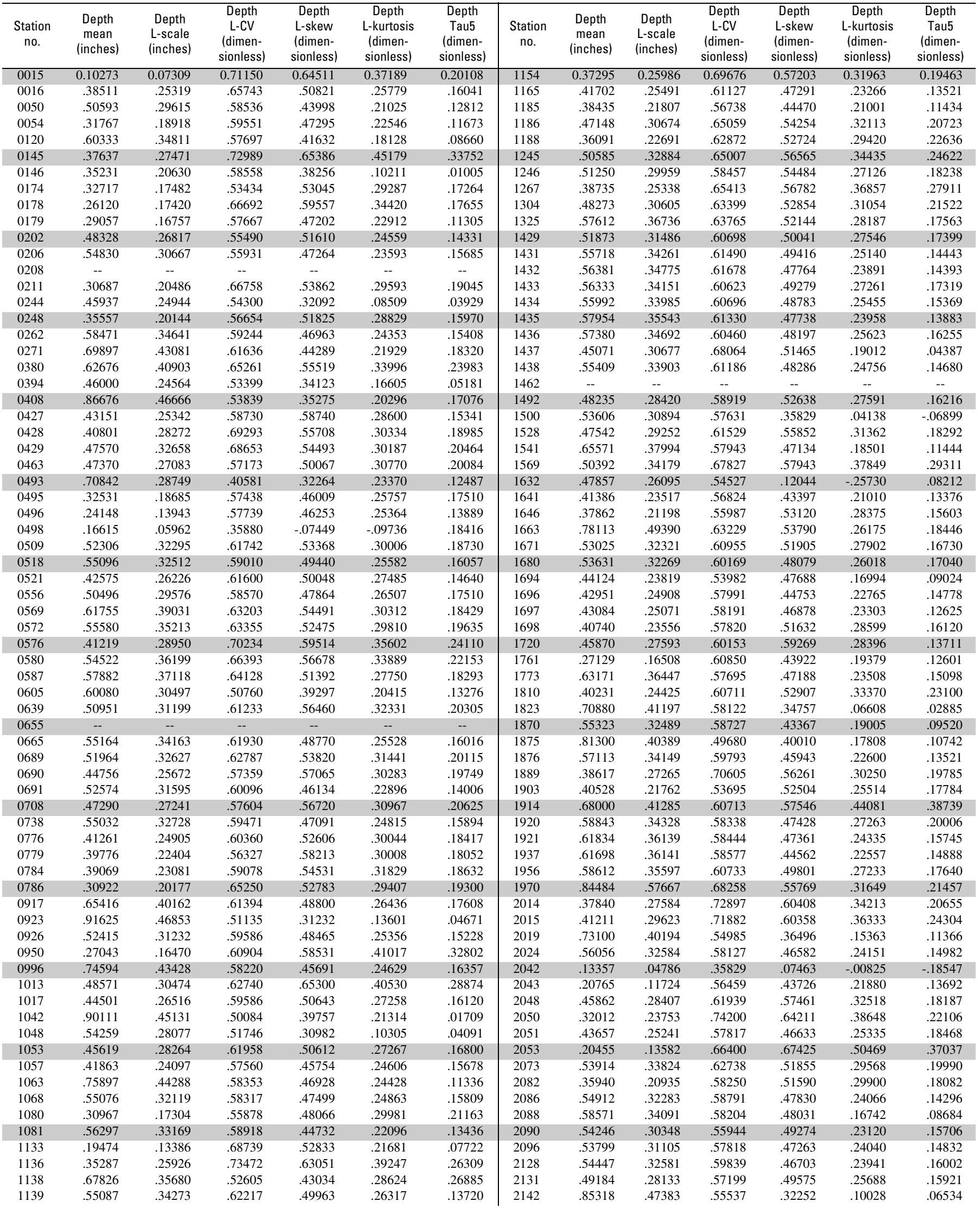


188 Statistical Characteristics of Storm Interevent Time, Depth, and Duration for Eastern New Mexico, Oklahoma, and Texas

Appendix 4-2.1. L-moments of storm depth defined by 6-hour minimum interevent time for hourly rainfall stations in Texas-Continued.

\begin{tabular}{|c|c|c|c|c|c|c|c|c|c|c|c|c|c|}
\hline $\begin{array}{c}\text { Station } \\
\text { no. }\end{array}$ & $\begin{array}{c}\text { Depth } \\
\text { mean } \\
\text { (inches) }\end{array}$ & $\begin{array}{l}\text { Depth } \\
\text { L-scale } \\
\text { (inches) }\end{array}$ & $\begin{array}{c}\text { Depth } \\
\text { L-CV } \\
\text { (dimen- } \\
\text { sionless) }\end{array}$ & $\begin{array}{c}\text { Depth } \\
\text { L-skew } \\
\text { (dimen- } \\
\text { sionless) }\end{array}$ & $\begin{array}{c}\text { Depth } \\
\text { L-kurtosis } \\
\text { (dimen- } \\
\text { sionless) }\end{array}$ & $\begin{array}{c}\text { Depth } \\
\text { Tau5 } \\
\text { (dimen- } \\
\text { sionless) }\end{array}$ & $\begin{array}{c}\text { Station } \\
\text { no. }\end{array}$ & $\begin{array}{l}\text { Depth } \\
\text { mean } \\
\text { (inches) }\end{array}$ & $\begin{array}{l}\text { Depth } \\
\text { L-scale } \\
\text { (inches) }\end{array}$ & $\begin{array}{c}\text { Depth } \\
\text { L-CV } \\
\text { (dimen- } \\
\text { sionless) }\end{array}$ & $\begin{array}{c}\text { Depth } \\
\text { L-skew } \\
\text { (dimen- } \\
\text { sionless) }\end{array}$ & $\begin{array}{c}\text { Depth } \\
\text { L-kurtosis } \\
\text { (dimen- } \\
\text { sionless) }\end{array}$ & $\begin{array}{c}\text { Depth } \\
\text { Tau5 } \\
\text { (dimen- } \\
\text { sionless) }\end{array}$ \\
\hline 2160 & 0.35393 & 0.25266 & 0.71387 & 0.63987 & 0.48189 & 0.43958 & 3463 & 0.57796 & 0.38248 & 0.66178 & 0.54666 & 0.33190 & 0.23110 \\
\hline 2206 & .63094 & .38936 & .61712 & .49922 & .27600 & .18420 & 3476 & .51896 & .31352 & .60413 & .47538 & .25510 & .16409 \\
\hline 2238 & .28596 & .18844 & .65896 & .51030 & .23599 & .11797 & 3485 & .85000 & .58343 & .68639 & .54956 & .23572 & .08493 \\
\hline 2240 & .29692 & .18344 & .61782 & .47167 & .25047 & .16892 & 3507 & .53783 & .31832 & .59187 & .53393 & .26053 & .15370 \\
\hline 2242 & .47025 & .29839 & .63454 & .46639 & .21445 & .12610 & 3546 & .62530 & .37356 & .59741 & .47935 & .24933 & .15486 \\
\hline 2244 & .51028 & .32155 & .63015 & .49969 & .26691 & .15831 & 3547 & .56264 & .31819 & .56553 & .40559 & .16975 & .08942 \\
\hline 2247 & .47141 & .31449 & .66713 & .53286 & .29139 & .18442 & 3579 & .54494 & .33286 & .61082 & .45246 & .15987 & .02009 \\
\hline 2309 & .64591 & .35534 & .55013 & .43439 & .24804 & .17470 & 3642 & .58049 & .34581 & .59573 & .49410 & .26623 & .16956 \\
\hline 2312 & .56517 & .31381 & .55525 & .45691 & .20066 & .13229 & 3646 & .54983 & .32145 & .58463 & .46968 & .25736 & .17237 \\
\hline 2334 & .86652 & .50452 & .58224 & .43889 & .22269 & .11724 & 3668 & .96923 & .47086 & .48581 & .33088 & .18698 & .06500 \\
\hline 2336 & .57361 & .30709 & .53536 & .37208 & .16869 & .11351 & 3673 & .57263 & .33303 & .58158 & .31873 & .01401 & -.01899 \\
\hline 2354 & .29313 & .16558 & .56489 & .41317 & .10682 & .01352 & 3686 & .52518 & .30744 & .58539 & .51197 & .23870 & .13232 \\
\hline 2355 & .52078 & .36299 & .69701 & .58502 & .33352 & .20328 & 3691 & .52548 & .30601 & .58234 & .49501 & .25282 & .15018 \\
\hline 2357 & .34483 & .25423 & .73727 & .62312 & .37095 & .23987 & 3734 & .95472 & .62726 & .65701 & .49419 & .23494 & .13027 \\
\hline 2360 & .34481 & .24565 & .71243 & .59687 & .34942 & .22339 & 3771 & .53689 & .31531 & .58728 & .53338 & .25017 & .15534 \\
\hline 2361 & .42939 & .28500 & .66374 & .63623 & .46414 & .37668 & 3789 & .19301 & .10453 & .54156 & .48778 & .30871 & .20100 \\
\hline 2394 & .56322 & .34067 & .60486 & .47560 & .24690 & .15569 & 3826 & .51554 & .28060 & .54427 & .41172 & .21081 & .12137 \\
\hline 2404 & .53951 & .32617 & .60456 & .49020 & .26389 & .17104 & 3831 & .59480 & .35510 & .59700 & .47435 & .25595 & .17683 \\
\hline 2415 & .66429 & .38936 & .58614 & .45605 & .23526 & .14865 & 3841 & .59947 & .34542 & .57622 & .44398 & .22626 & .14654 \\
\hline 2462 & .62580 & .36495 & .58317 & .45455 & .22228 & .13095 & 3871 & .47012 & .27812 & .59159 & .46201 & .23631 & .15154 \\
\hline 2528 & .50264 & .29585 & .58859 & .47049 & .22560 & .10947 & 3884 & .80261 & .54292 & .67645 & .55535 & .26392 & .10502 \\
\hline 2617 & .43054 & .26831 & .62320 & .51341 & .30064 & .20717 & 3941 & .65924 & .38688 & .58686 & .35337 & .08984 & .06642 \\
\hline 2619 & .42882 & .23861 & .55642 & .43716 & .22746 & .14274 & 3963 & .07143 & .03619 & .50667 & .60000 & .43421 & .25000 \\
\hline 2621 & .45582 & .26534 & .58211 & .48523 & .26519 & .16302 & 4040 & .48728 & .27829 & .57110 & .44620 & .22634 & .13323 \\
\hline 2675 & .55219 & .32929 & .59634 & .51837 & .28291 & .17897 & 4058 & .68600 & .42402 & .61811 & .48495 & .25737 & .17063 \\
\hline 2676 & .52780 & .30929 & .58600 & .53486 & .24715 & .14262 & 4098 & .34196 & .18330 & .53603 & .52525 & .27241 & .14526 \\
\hline 2679 & .44955 & .29104 & .64741 & .58786 & .35071 & .19977 & 4100 & .45659 & .28002 & .61328 & .49340 & .26101 & .17015 \\
\hline 2715 & .52991 & .30542 & .57636 & .45408 & .23233 & .14584 & 4137 & .51324 & .28721 & .55961 & .50162 & .23164 & .15078 \\
\hline 2744 & .46579 & .26988 & .57940 & .47603 & .25136 & .15519 & 4191 & .50140 & .31425 & .62675 & .54900 & .31181 & .18452 \\
\hline 2758 & .35852 & .26331 & .73444 & .65758 & .42084 & .26239 & 4256 & -- & -- & -- & -- & -- & -- \\
\hline 2794 & .43091 & .29836 & .69241 & .50599 & .16870 & -.00965 & 4257 & .63689 & .37017 & .58122 & .47965 & .24037 & .15371 \\
\hline 2797 & .18629 & .11972 & .64264 & .53117 & .29567 & .19060 & 4258 & .52534 & .30120 & .57335 & .52184 & .25993 & .19374 \\
\hline 2811 & .43527 & .25804 & .59283 & .51365 & .27793 & .15596 & 4278 & .56291 & .33703 & .59874 & .46470 & .23371 & .14364 \\
\hline 2813 & .51000 & .30064 & .58950 & .48240 & .28391 & .15941 & 4299 & .31326 & .14964 & .47770 & .32900 & .13472 & .07297 \\
\hline 2814 & .19211 & .14363 & .74764 & .68116 & .40922 & .15200 & 4300 & .48359 & .33348 & .68959 & .55473 & .30999 & .20372 \\
\hline 2815 & .44452 & .25095 & .56455 & .56912 & .31902 & .21757 & 4305 & .45608 & .31956 & .70067 & .56758 & .31853 & .20616 \\
\hline 2818 & .52617 & .31022 & .58958 & .44493 & .22154 & .14458 & 4307 & .48370 & .33012 & .68248 & .54560 & .31312 & .22645 \\
\hline 2986 & .68068 & .38730 & .56898 & .38587 & .15389 & .10248 & 4309 & .60818 & .38143 & .62716 & .49899 & .26815 & .17589 \\
\hline 3005 & .51452 & .29801 & .57920 & .47028 & .23668 & .14078 & 4311 & .63377 & .39448 & .62243 & .48941 & .25416 & .15637 \\
\hline 3033 & .24866 & .13789 & .55454 & .50079 & .31452 & .21421 & 4313 & 63927 & .42308 & .66181 & .55858 & .34205 & .23717 \\
\hline 3034 & -- & -- & -- & -- & -- & -- & 4319 & .48870 & .28273 & .57854 & .43289 & .18443 & .09245 \\
\hline 3047 & .50278 & .30476 & .60616 & .42639 & .20019 & .16062 & 4329 & .62298 & .38374 & 61597 & .49958 & .28091 & .18377 \\
\hline 3103 & .67108 & .44935 & .66960 & .60223 & .36463 & .19422 & 4331 & -- & -- & -- & -- & -- & -- \\
\hline 3133 & .59412 & .34529 & .58118 & .46084 & .24292 & .15787 & 4375 & .51151 & .30516 & .59659 & .57878 & .32265 & .22703 \\
\hline 3156 & .64430 & .40159 & .62330 & .57332 & .34627 & .24025 & 4392 & .65496 & .40825 & 62333 & .47524 & .23833 & .15113 \\
\hline 3171 & .56607 & .33668 & .59476 & .48066 & .25898 & .16383 & 4425 & .36121 & .20516 & .56798 & .54306 & .30187 & .16995 \\
\hline 3189 & .28741 & .17064 & .59371 & .50890 & .29933 & .18843 & 4440 & .46991 & .28818 & .61326 & .48837 & .25752 & .16708 \\
\hline 3260 & .48255 & .28776 & .59632 & .43926 & .20881 & .13146 & 4476 & .50312 & .28323 & .56296 & .48234 & .24016 & .14845 \\
\hline 3267 & .39286 & .25609 & .65186 & .52747 & .28768 & .19374 & 4498 & .18786 & .10291 & .54782 & .37960 & .16080 & .08062 \\
\hline 3270 & .43669 & .24825 & .56848 & .54475 & .28248 & .17959 & 4517 & .51965 & .30810 & .59291 & .45784 & .23818 & .15582 \\
\hline 3272 & .10560 & .05267 & .49874 & .43110 & .24368 & .11626 & 4520 & .48794 & .27833 & .57041 & .53128 & .25238 & .15436 \\
\hline 3277 & .15188 & .09588 & .63128 & .55113 & .36774 & .37100 & 4525 & .62200 & .41081 & 66046 & .52526 & .24375 & .12004 \\
\hline 3278 & .39856 & .22708 & .56974 & .48791 & .25743 & .14148 & 4563 & .45387 & .26224 & .57778 & .41084 & .19507 & .17368 \\
\hline 3280 & .30175 & .18747 & .62127 & .51414 & .26925 & .15074 & 4570 & .44469 & .26201 & .58919 & .52039 & .29161 & .17772 \\
\hline 3281 & .33083 & .16108 & .48690 & .32733 & .12487 & .04654 & 4577 & .62373 & .35470 & .56868 & .45106 & .22825 & .14839 \\
\hline 3283 & .45116 & .29577 & .65557 & .50396 & .25826 & .16000 & 4591 & .56113 & .34110 & 60789 & .48079 & .25468 & .16198 \\
\hline 3284 & .50865 & .30210 & .59393 & .48895 & .25155 & .14164 & 4670 & .43253 & .25721 & .59467 & .51307 & .27852 & .15891 \\
\hline 3285 & .52849 & .30566 & .57836 & .50485 & .23175 & .13108 & 4671 & .35189 & .22007 & .62539 & .47165 & .22292 & .13790 \\
\hline 3329 & .48343 & .31017 & .64161 & .54439 & .32310 & .21832 & 4679 & .52132 & .31123 & .59701 & .53061 & .28319 & .18002 \\
\hline 3335 & .70202 & .45155 & .64322 & .54276 & .34056 & .25069 & 4696 & .39667 & .20438 & .51525 & .31307 & .07735 & .01284 \\
\hline 3370 & .62761 & .36250 & .57758 & .44338 & .23055 & .14860 & 4703 & .40291 & .24623 & .61112 & .50958 & .26651 & .12813 \\
\hline 3410 & .43194 & .23879 & .55283 & .49910 & .26309 & .16569 & 4704 & .68461 & .44496 & 64995 & .53024 & .30252 & .20060 \\
\hline 3415 & .52706 & .31065 & .58940 & .49665 & .26253 & .16396 & 4731 & .36966 & .26351 & .71286 & .61285 & .40664 & .31788 \\
\hline 3430 & .47884 & .33154 & .69237 & .56414 & .32559 & .21970 & 4792 & .50592 & .28343 & .56023 & .50424 & .23038 & .14476 \\
\hline 3431 & .45700 & .33068 & .72359 & .61494 & .37996 & .26526 & 4819 & .59329 & .32851 & .55371 & .47129 & .21500 & .13559 \\
\hline 3441 & .51711 & .30534 & .59048 & .44662 & .22461 & .13401 & 4852 & .83353 & .41581 & .49885 & .48584 & .36665 & .23067 \\
\hline 3442 & .35652 & .21241 & .59578 & .50445 & .28132 & .17480 & 4866 & .57106 & .33047 & .57869 & .46234 & .23316 & .14521 \\
\hline 3446 & .42297 & .23876 & .56448 & .45768 & .23951 & .14695 & 4876 & 69032 & .41326 & .59865 & .51321 & .23876 & .13591 \\
\hline 3460 & .63043 & .40039 & .63511 & .50424 & .29996 & .26012 & 4878 & .66325 & .41218 & .62145 & .50052 & .27472 & .17835 \\
\hline 3462 & .42325 & .23472 & .55457 & .44361 & .22165 & .12042 & 4880 & .39443 & .22336 & .56629 & .48330 & .26267 & .15769 \\
\hline
\end{tabular}


Appendix 4-2.1. L-moments of storm depth defined by 6-hour minimum interevent time for hourly rainfall stations in Texas-Continued.

\begin{tabular}{|c|c|c|c|c|c|c|c|c|c|c|c|c|c|}
\hline $\begin{array}{c}\text { Station } \\
\text { no. }\end{array}$ & $\begin{array}{l}\text { Depth } \\
\text { mean } \\
\text { (inches) }\end{array}$ & $\begin{array}{c}\text { Depth } \\
\text { L-scale } \\
\text { (inches) }\end{array}$ & $\begin{array}{c}\text { Depth } \\
\text { L-CV } \\
\text { (dimen- } \\
\text { sionless) }\end{array}$ & $\begin{array}{c}\text { Depth } \\
\text { L-skew } \\
\text { (dimen- } \\
\text { sionless) }\end{array}$ & $\begin{array}{c}\text { Depth } \\
\text { L-kurtosis } \\
\text { (dimen- } \\
\text { sionless) }\end{array}$ & $\begin{array}{c}\text { Depth } \\
\text { Tau5 } \\
\text { (dimen- } \\
\text { sionless) }\end{array}$ & $\begin{array}{c}\text { Station } \\
\text { no. }\end{array}$ & $\begin{array}{l}\text { Depth } \\
\text { mean } \\
\text { (inches) }\end{array}$ & $\begin{array}{l}\text { Depth } \\
\text { L-scale } \\
\text { (inches) }\end{array}$ & $\begin{array}{c}\text { Depth } \\
\text { L-CV } \\
\text { (dimen- } \\
\text { sionless) }\end{array}$ & $\begin{array}{c}\text { Depth } \\
\text { L-skew } \\
\text { (dimen- } \\
\text { sionless) }\end{array}$ & $\begin{array}{c}\text { Depth } \\
\text { L-kurtosis } \\
\text { (dimen- } \\
\text { sionless) }\end{array}$ & $\begin{array}{c}\text { Depth } \\
\text { Tau5 } \\
\text { (dimen- } \\
\text { sionless) }\end{array}$ \\
\hline 4920 & 0.49863 & 0.30690 & 0.61549 & 0.54151 & 0.29829 & 0.17284 & 5957 & 0.53416 & 0.30789 & 0.57641 & 0.48655 & 0.24884 & 0.15379 \\
\hline 4934 & .28556 & .19306 & .67607 & .78541 & .72354 & .71778 & 5958 & .47763 & .27945 & .58508 & .42856 & .20354 & .11666 \\
\hline 4972 & .49645 & .29008 & .58431 & .49382 & .26559 & .16718 & 5973 & .36608 & .25798 & .70470 & .59201 & .32359 & .17626 \\
\hline 4973 & .68575 & .37677 & .54942 & .41330 & .21582 & .14518 & 5996 & .50025 & .29601 & .59172 & .49598 & .25873 & .15552 \\
\hline 4974 & .40036 & .23563 & .58856 & .49283 & .26989 & .16838 & 6017 & .36755 & .24659 & .67090 & .54520 & .28995 & .16378 \\
\hline 4975 & .61108 & .34574 & .56578 & .48114 & .22469 & .15456 & 6024 & .78236 & .50237 & .64212 & .51053 & .28295 & .19913 \\
\hline 4978 & .49828 & .33417 & .67063 & .56179 & .31864 & .19642 & 6050 & .58000 & .30083 & .51867 & .35199 & .10282 & -.04957 \\
\hline 4979 & .80208 & .44969 & .56066 & .36094 & .12350 & .03920 & 6104 & .31854 & .17695 & .55549 & .49559 & .28193 & .16536 \\
\hline 4982 & .47869 & .27794 & .58064 & .46671 & .25461 & .16525 & 6108 & .65047 & .37540 & .57713 & .46398 & .23069 & .14677 \\
\hline 5018 & .52827 & .30137 & .57048 & .42213 & .19617 & .12787 & 6136 & .34527 & .18852 & .54601 & .51441 & .26995 & .14320 \\
\hline 5048 & .39877 & .23926 & .60001 & .57227 & .33178 & .19581 & 6166 & .32877 & .20971 & .63787 & .52523 & .26177 & .13332 \\
\hline 5049 & .38532 & .21920 & .56887 & .63054 & .40229 & .32201 & 6176 & .63663 & .39482 & .62017 & .49362 & .27409 & .18135 \\
\hline 5056 & .43200 & .18600 & .43056 & .26882 & .67742 & .11828 & 6177 & .63509 & .37422 & .58923 & .48895 & .25746 & .16362 \\
\hline 5057 & .29699 & .21368 & .71949 & .62642 & .39415 & .26035 & 6210 & .58126 & .34346 & .59088 & .49485 & .25647 & .16117 \\
\hline 5060 & .48899 & .34538 & .70630 & .59781 & .36613 & .25487 & 6211 & .54397 & .34146 & .62772 & .48631 & .25093 & .15968 \\
\hline 5081 & .58164 & .34131 & .58680 & .43064 & .20642 & .13776 & 6270 & .63866 & .36775 & .57581 & .50005 & .25169 & .17370 \\
\hline 5094 & .55994 & .32945 & .58837 & .49523 & .25889 & .15693 & 6275 & -- & -- & -- & -- & -- & -- \\
\hline 5113 & .48934 & .30676 & .62688 & .55952 & .32155 & .19155 & 6276 & .77897 & .39672 & .50930 & .33823 & .14401 & .08090 \\
\hline 5114 & -- & -- & -- & -- & -- & -- & 6335 & .58320 & .34074 & .58426 & .44581 & .22081 & .13620 \\
\hline 5123 & .57471 & .33353 & .58035 & .45670 & .20011 & .01503 & 6434 & .49000 & .27063 & .55231 & .34073 & .07576 & .01695 \\
\hline 5192 & .58607 & .34675 & .59165 & .47710 & .24498 & .14339 & 6504 & .38259 & .21941 & .57350 & .52615 & .28819 & .16216 \\
\hline 5193 & .52918 & .32429 & .61283 & .50514 & .26370 & .15519 & 6558 & .54441 & .30739 & .56463 & .38885 & .17648 & .20086 \\
\hline 5224 & .62817 & .38067 & .60600 & .47001 & .24728 & .15401 & 6615 & .44494 & .25711 & .57786 & .57970 & .33332 & .23375 \\
\hline 5228 & .55594 & .33663 & .60551 & .44710 & .19354 & .09752 & 6660 & .53788 & .32286 & .60025 & .47097 & .24045 & .15679 \\
\hline 5235 & .47000 & .29899 & .63615 & .49552 & .25966 & .19008 & 6663 & .45025 & .27539 & .61163 & .49156 & .25127 & .11830 \\
\hline 5247 & .38880 & .21866 & .56239 & .50524 & .26729 & .14576 & 6734 & .39414 & .24293 & .61635 & .49495 & .26611 & .17075 \\
\hline 5258 & .56638 & .33364 & .58907 & .45650 & .23491 & .14717 & 6736 & .42620 & .24987 & .58627 & .53258 & .29037 & .16412 \\
\hline 5303 & .50642 & .32516 & .64207 & .54815 & .33304 & .24206 & 6740 & .93000 & .51505 & .55381 & .38093 & .15513 & .10980 \\
\hline 5312 & .47556 & .27596 & .58029 & .52741 & .28020 & .17781 & 6750 & .55872 & .38788 & .69423 & .57160 & .34587 & .24552 \\
\hline 5341 & .86367 & .52748 & .61075 & .51412 & .30136 & .20661 & 6757 & .56290 & .34684 & .61617 & .48161 & .24912 & .15411 \\
\hline 5342 & -- & -- & -- & -- & -- & -- & 6775 & .37136 & .22526 & .60657 & .47741 & .25503 & .16520 \\
\hline 5348 & .62013 & .35183 & .56735 & .45440 & .19062 & .10752 & 6776 & .39114 & .21870 & .55912 & .50299 & .27114 & .15568 \\
\hline 5358 & .45098 & .26739 & .59292 & .48140 & .25093 & .14440 & 6788 & .62396 & .36501 & .58499 & .44531 & .23817 & .15692 \\
\hline 5398 & .59630 & .35665 & .59810 & .47640 & .24998 & .15969 & 6792 & .32082 & .17576 & .54783 & .50498 & .28010 & .14612 \\
\hline 5410 & .39955 & .23024 & .57624 & .51173 & .29345 & .18298 & 6794 & 1.0297 & .59509 & .57795 & .39198 & .18884 & .12318 \\
\hline 5411 & .31104 & .20859 & .67061 & .54112 & .29608 & .18727 & 6834 & .64368 & .36638 & .56919 & .48180 & .22945 & .15791 \\
\hline 5424 & .49908 & .34754 & .69636 & .53929 & .27044 & .16384 & 6893 & .30182 & .16476 & .54589 & .56335 & .35145 & .21660 \\
\hline 5429 & .49691 & .32161 & .64722 & .53489 & .30194 & .19536 & 6935 & .38924 & .21788 & .55975 & .54001 & .30840 & .19484 \\
\hline 5431 & .80333 & .43980 & .54747 & .51438 & .31970 & .15448 & 6981 & .56023 & .32243 & .57553 & .45226 & .24891 & .17040 \\
\hline 5461 & .64000 & .38346 & .59915 & .45910 & .23461 & .15170 & 7020 & .54107 & .35142 & 64949 & .51531 & .28862 & .20169 \\
\hline 5463 & .60189 & .35114 & .58340 & .51516 & .25118 & .16342 & 7060 & .44810 & .25511 & .56931 & .50271 & .25505 & .13913 \\
\hline 5471 & .14893 & .10340 & .69429 & .67306 & .50461 & .40570 & 7066 & .62944 & .36802 & .58467 & .46636 & .23373 & .14342 \\
\hline 5477 & .65962 & .41691 & .63205 & .66113 & .52864 & .41852 & 7074 & .38024 & .22224 & .58448 & .53279 & .32049 & .20304 \\
\hline 5528 & .63973 & .36498 & .57052 & .44077 & .22803 & .14608 & 7097 & .51076 & .30671 & .60050 & .51694 & .28967 & .15890 \\
\hline 5579 & -- & -- & -- & -- & -- & -- & 7116 & .40272 & .23915 & .59384 & .47085 & .24914 & .16442 \\
\hline 5580 & .53862 & .27057 & .50233 & .29332 & .09515 & .04973 & 7140 & .60646 & .39835 & .65685 & .55720 & .32893 & .21510 \\
\hline 5589 & .26296 & .14917 & .56725 & .40687 & .18116 & .10191 & 7173 & .59258 & .42166 & .71157 & .58476 & .34628 & .23953 \\
\hline 5590 & .29835 & .17337 & .58111 & .46983 & .22987 & .11450 & 7174 & .55435 & .38313 & 69113 & .55942 & .32175 & .21939 \\
\hline 5591 & .31961 & .17015 & .53238 & .41501 & .20776 & .12335 & 7213 & .57377 & .34247 & .59688 & .47087 & .25207 & .16221 \\
\hline 5592 & .28170 & .16144 & .57311 & .47804 & .26539 & .16951 & 7243 & .46638 & .27342 & .58628 & .51499 & .27810 & .16906 \\
\hline 5594 & .29231 & .16606 & .56810 & .47587 & .26533 & .16644 & 7262 & .18358 & .10380 & .56538 & .42442 & .19268 & .09712 \\
\hline 5595 & -- & -- & -- & -- & -- & -- & 7274 & .61157 & .33376 & .54574 & .44329 & .23955 & .16212 \\
\hline 5596 & .33209 & .17088 & .51455 & .54533 & .26422 & .14906 & 7300 & .53100 & .31183 & .58725 & .45619 & .23646 & .15273 \\
\hline 5600 & .33420 & .19442 & .58176 & .49551 & .28618 & .17977 & 7311 & .59844 & .34608 & .57830 & .51767 & .28823 & .15480 \\
\hline 5618 & .54432 & .31595 & .58044 & .43414 & .17689 & .06824 & 7363 & .57857 & .29709 & .51349 & .24783 & .01853 & .03859 \\
\hline 5650 & .49273 & .23251 & .47189 & .37284 & .30940 & .21829 & 7422 & .51161 & .31706 & .61974 & .53306 & .30009 & .18724 \\
\hline 5656 & .40282 & .22611 & .56130 & .56893 & .30005 & .18897 & 7431 & .39772 & .23365 & .58748 & .51650 & .29363 & .17951 \\
\hline 5658 & .41658 & .25370 & 60901 & .51152 & .29581 & .19714 & 7481 & .31994 & .19098 & .59690 & .56542 & .36030 & .22542 \\
\hline 5661 & .51344 & .32172 & .62659 & .63399 & .37950 & .26256 & 7497 & .51385 & .29895 & .58179 & .53768 & .26544 & .16847 \\
\hline 5666 & .44421 & .26923 & .60609 & .49722 & .28664 & .18376 & 7498 & .54178 & .32197 & .59429 & .55051 & .29572 & .20617 \\
\hline 5695 & .58942 & .34099 & .57851 & .44786 & .22979 & .14917 & 7499 & .50094 & .28430 & .56753 & .50372 & .26046 & .16646 \\
\hline 5742 & .22870 & .14063 & 61493 & .51843 & .26965 & .16921 & 7531 & .51764 & .29763 & .57499 & .41165 & .18632 & .10618 \\
\hline 5766 & .65730 & .41495 & .63130 & .46933 & .17224 & .00922 & 7534 & .55019 & .34932 & .63490 & .53941 & .34191 & .26103 \\
\hline 5770 & .41628 & .24174 & .58070 & .50763 & .28028 & .16488 & 7556 & .53799 & .31735 & .58989 & .51715 & .27960 & .18000 \\
\hline 5775 & .34643 & .19049 & .54988 & .32881 & -.04007 & -.06288 & 7594 & .64424 & .38136 & .59195 & .45555 & .23144 & .14858 \\
\hline 5779 & .53556 & .35134 & .65603 & .54199 & .28944 & .13464 & 7596 & .67751 & .40225 & .59372 & .40666 & .14959 & .07738 \\
\hline 5840 & .53469 & .32008 & .59864 & .46620 & .23375 & .13867 & 7608 & .50713 & .32322 & .63736 & .49565 & .25267 & .15393 \\
\hline 5890 & .29393 & .19713 & .67066 & .54640 & .30281 & .19133 & 7622 & .21261 & .16941 & .79680 & .72202 & .48393 & .32758 \\
\hline 5891 & .34657 & .19167 & .55305 & .41911 & .18924 & .10712 & 7700 & .58010 & .36320 & .62610 & .49044 & .25497 & .16080 \\
\hline 5897 & .55009 & .32378 & .58860 & .53243 & .26189 & .16846 & 7706 & .44288 & .26719 & .60329 & .52688 & .29460 & .17626 \\
\hline
\end{tabular}


190 Statistical Characteristics of Storm Interevent Time, Depth, and Duration for Eastern New Mexico, Oklahoma, and Texas

Appendix 4-2.1. L-moments of storm depth defined by 6-hour minimum interevent time for hourly rainfall stations in Texas-Continued.

\begin{tabular}{|c|c|c|c|c|c|c|c|c|c|c|c|c|c|}
\hline $\begin{array}{c}\text { Station } \\
\text { no. }\end{array}$ & $\begin{array}{c}\text { Depth } \\
\text { mean } \\
\text { (inches) }\end{array}$ & $\begin{array}{l}\text { Depth } \\
\text { L-scale } \\
\text { (inches) }\end{array}$ & $\begin{array}{c}\text { Depth } \\
\text { L-CV } \\
\text { (dimen- } \\
\text { sionless) }\end{array}$ & $\begin{array}{c}\text { Depth } \\
\text { L-skew } \\
\text { (dimen- } \\
\text { sionless) }\end{array}$ & $\begin{array}{c}\text { Depth } \\
\text { L-kurtosis } \\
\text { (dimen- } \\
\text { sionless) }\end{array}$ & $\begin{array}{c}\text { Depth } \\
\text { Tau5 } \\
\text { (dimen- } \\
\text { sionless) }\end{array}$ & $\begin{array}{c}\text { Station } \\
\text { no. }\end{array}$ & $\begin{array}{l}\text { Depth } \\
\text { mean } \\
\text { (inches) }\end{array}$ & $\begin{array}{l}\text { Depth } \\
\text { L-scale } \\
\text { (inches) }\end{array}$ & $\begin{array}{c}\text { Depth } \\
\text { L-CV } \\
\text { (dimen- } \\
\text { sionless) }\end{array}$ & $\begin{array}{c}\text { Depth } \\
\text { L-skew } \\
\text { (dimen- } \\
\text { sionless) }\end{array}$ & $\begin{array}{c}\text { Depth } \\
\text { L-kurtosis } \\
\text { (dimen- } \\
\text { sionless) }\end{array}$ & $\begin{array}{c}\text { Depth } \\
\text { Tau5 } \\
\text { (dimen- } \\
\text { sionless) }\end{array}$ \\
\hline 7718 & 0.53907 & 0.33452 & 0.62055 & 0.51993 & 0.29646 & 0.17699 & 8910 & 0.61435 & 0.35466 & 0.57730 & 0.36970 & 0.05964 & -0.05403 \\
\hline 7745 & .60829 & .39002 & .64117 & .50490 & .26386 & .16514 & 8911 & .47805 & .30587 & 63984 & .50415 & .26152 & .15873 \\
\hline 7922 & .23187 & .13679 & .58994 & .48456 & .27291 & .17415 & 8924 & .28811 & .15657 & .54344 & .44369 & .23272 & .13064 \\
\hline 7936 & .64678 & .38090 & .58892 & .47923 & .24674 & .16225 & 8929 & .72138 & .44365 & .61500 & .39238 & .04403 & -.06798 \\
\hline 7943 & .33734 & .22581 & .66937 & .53444 & .28434 & .17510 & 8942 & .62511 & .36705 & .58718 & .50093 & .24869 & .15636 \\
\hline 7944 & .34200 & .23928 & .69964 & .56345 & .29989 & .17381 & 8944 & .54441 & .33423 & .61393 & .47101 & .24233 & .15510 \\
\hline 7945 & .38871 & .27727 & .71332 & .58929 & .34071 & .22350 & 8996 & .54426 & .34268 & 62964 & .50793 & .27698 & .17827 \\
\hline 7947 & .58074 & .36699 & .63193 & .58924 & .36316 & .25732 & 9014 & .65480 & .33463 & .51105 & .32803 & .09424 & -.00198 \\
\hline 7948 & .49748 & .32415 & .65159 & .53923 & .30131 & .18608 & 9037 & .29985 & .18082 & .60303 & .54986 & .35710 & .25723 \\
\hline 7951 & .59927 & .34281 & .57205 & .42901 & .20638 & .12094 & 9106 & .26559 & .16895 & .63611 & .60140 & .41472 & .31434 \\
\hline 7953 & .40121 & .26007 & .64823 & .53676 & .29415 & .16490 & 9107 & .32425 & .19487 & 60097 & .47751 & .23448 & .14193 \\
\hline 7981 & .48238 & .30333 & .62882 & .48235 & .22201 & .11395 & 9129 & .31465 & .22230 & .70648 & .63682 & .39804 & .24632 \\
\hline 7990 & .40237 & .28238 & .70178 & .58582 & .33630 & .21154 & 9163 & .49342 & .29080 & .58937 & .49176 & .26350 & .16008 \\
\hline 7992 & .57893 & .33430 & .57744 & .42384 & .09079 & -.04199 & 9213 & .42699 & .27517 & .64445 & .48321 & .22500 & .13458 \\
\hline 7997 & .43687 & .26495 & .60647 & .47113 & .23228 & .12542 & 9214 & .79629 & .45138 & .56685 & .46017 & .24052 & .12315 \\
\hline 7999 & .29619 & .19643 & .66318 & .55129 & .36806 & .32070 & 9222 & .44570 & .28613 & 64197 & .49355 & .24236 & .14033 \\
\hline 8022 & .37949 & .23687 & .62418 & .49773 & .21189 & .07528 & 9248 & .40545 & .23564 & .58118 & .45676 & .20234 & .08574 \\
\hline 8023 & .41615 & .24578 & .59060 & .53024 & .30316 & .18630 & 9266 & .48337 & .27289 & .56456 & .44093 & .21542 & .10330 \\
\hline 8047 & .51555 & .30293 & .58758 & .48060 & .24312 & .14296 & 9270 & .30410 & .16183 & .53216 & .54649 & .31568 & .17438 \\
\hline 8060 & .38643 & .26713 & .69127 & .58146 & .30994 & .15480 & 9295 & .19586 & .12208 & 62330 & .51621 & .28043 & .16154 \\
\hline 8062 & .60213 & .41528 & .68969 & .55220 & .29066 & .15742 & 9304 & -- & -- & -- & -- & -- & -- \\
\hline 8068 & .24585 & .16616 & .67584 & .60444 & .36846 & .23661 & 9307 & .51281 & .28213 & .55017 & .43566 & .21656 & .12305 \\
\hline 8081 & .50216 & .31566 & .62861 & .55335 & .32416 & .20727 & 9328 & .39485 & .22796 & .57735 & .43435 & .18327 & .09143 \\
\hline 8089 & .43239 & .24544 & .56764 & .38744 & .13614 & .04299 & 9329 & .45312 & .26621 & .58749 & .28422 & -.10783 & -.14229 \\
\hline 8221 & .58375 & .31103 & .53282 & .38605 & .23214 & .16789 & 9345 & -- & -- & -- & -- & -- & -- \\
\hline 8252 & .40571 & .23646 & .58282 & .51492 & .28501 & .16399 & 9363 & .37165 & .25932 & 69774 & .57971 & .33819 & .22223 \\
\hline 8265 & 61648 & .40290 & .65354 & .55182 & .32976 & .22734 & 9364 & .43703 & .30771 & .70411 & .58126 & .33758 & .22222 \\
\hline 8289 & .40000 & .19675 & .49187 & .25516 & .07714 & .06652 & 9365 & .27875 & .17612 & .63183 & 64908 & .46733 & .33785 \\
\hline 8305 & .27072 & .14830 & .54781 & .53090 & .32651 & .18432 & 9371 & .51395 & .29700 & .57787 & .43075 & .18282 & .08967 \\
\hline 8335 & .65963 & .38630 & .58564 & .44070 & .22196 & .14629 & 9417 & .53150 & .32327 & .60823 & .46554 & .22001 & .12315 \\
\hline 8400 & .32012 & .19026 & .59435 & .51963 & .32519 & .22764 & 9419 & .43258 & .28854 & .66702 & .51266 & .25228 & .14735 \\
\hline 8445 & .57037 & .36643 & .64245 & .52304 & .28759 & .18503 & 9435 & .43447 & .24233 & .55777 & .45062 & .25049 & 18377 \\
\hline 8446 & .50997 & .30253 & .59322 & .50688 & .25723 & .13813 & 9491 & .56034 & .33819 & .60355 & .49473 & .25730 & .15255 \\
\hline 8451 & .41060 & .24312 & .59211 & .45302 & .20095 & 10277 & 9499 & .43913 & .25276 & .57558 & .51896 & .26679 & .15001 \\
\hline 8531 & .55067 & .32043 & .58189 & .46328 & .24398 & .15535 & 9522 & .87263 & .55632 & .63752 & .43818 & .11445 & -.02741 \\
\hline 8541 & .51779 & .29320 & .56626 & .43641 & .23540 & .14485 & 9527 & .37282 & .20711 & .55553 & .53711 & .28422 & .15566 \\
\hline 8544 & .58289 & .35631 & .61129 & .53071 & .28807 & .17832 & 9532 & .51907 & .30483 & .58727 & .48901 & .25985 & .16453 \\
\hline 8545 & .26217 & .13368 & .50987 & .25876 & .05808 & .08900 & 9544 & 1.2500 & 1.1260 & .90080 & .96803 & .95560 & .90586 \\
\hline 8563 & .56719 & .32856 & .57928 & .52297 & .26423 & .18092 & 9565 & .46615 & .27146 & .58235 & .51046 & .28274 & .17602 \\
\hline 8566 & .43655 & .25424 & .58239 & .44185 & .20877 & .11296 & 9570 & .42572 & .23656 & .55567 & .54990 & .28769 & .18471 \\
\hline 8583 & .43061 & .24400 & .56664 & .55210 & .25525 & .13805 & 9574 & .42963 & .24644 & .57361 & .56569 & .27133 & .14594 \\
\hline 8584 & .51463 & .29625 & .57566 & .47578 & .23050 & .13514 & 9588 & .43068 & .27134 & .63001 & .57024 & .34217 & .20225 \\
\hline 8623 & .52556 & .29514 & .56157 & .47977 & .23876 & .14669 & 9665 & .58354 & .34692 & .59451 & .48418 & .25156 & .15744 \\
\hline 8625 & .51480 & .31122 & .60455 & .48111 & .25575 & .16908 & 9715 & .53752 & .31534 & .58667 & .46764 & .23174 & .13838 \\
\hline 8630 & .42914 & .24465 & .57010 & .47873 & .23769 & .12511 & 9729 & .42891 & .27916 & .65086 & .50096 & .25243 & .15598 \\
\hline 8631 & .45616 & .27439 & .60153 & .49631 & .27444 & .17201 & 9772 & .55369 & .36728 & .66333 & .56045 & .33394 & .22579 \\
\hline 8646 & .53350 & .31988 & .59959 & .47186 & .24124 & .14922 & 9814 & .52390 & .30344 & .57919 & .37729 & .11123 & .02417 \\
\hline 8647 & .37070 & .21323 & .57523 & .54861 & .31076 & .17809 & 9815 & .55328 & .33743 & 60988 & .51234 & .28771 & .18410 \\
\hline 8677 & .47401 & .27280 & .57552 & .45235 & .22893 & .12652 & 9816 & .54169 & .34963 & .64544 & .52382 & .30635 & .22371 \\
\hline 8696 & .63833 & .33226 & .52052 & .30729 & .13393 & .14698 & 9817 & .49724 & .28090 & .56493 & .49345 & .24775 & .15897 \\
\hline 8743 & .62340 & .37219 & .59704 & .47965 & .25321 & .16121 & 9829 & .32126 & .18864 & .58718 & .54975 & .33658 & .19485 \\
\hline 8761 & .37565 & .20959 & .55794 & .52619 & .30262 & .18524 & 9830 & .25463 & .15375 & 60383 & .49307 & .25526 & .14657 \\
\hline 8778 & .57635 & .33805 & .58653 & .49720 & .24964 & .15705 & 9858 & .42228 & .23902 & .56603 & .45472 & .24944 & .16272 \\
\hline 8845 & .52296 & .32588 & .62314 & .54532 & .30638 & .18413 & 9893 & .49094 & .28892 & .58851 & .48964 & .26177 & .16145 \\
\hline 8859 & .63032 & .36844 & .58453 & .44170 & .22239 & .14389 & 9916 & .64072 & .36611 & .57141 & .45420 & .21001 & .12291 \\
\hline 8898 & .61606 & .36411 & .59103 & .46179 & .24480 & .15773 & 9976 & .44450 & .27500 & .61869 & .54525 & .31184 & .18407 \\
\hline 8908 & .48543 & .30937 & .63730 & .52598 & .30246 & .22045 & & & & & & & \\
\hline
\end{tabular}


Appendix 4-2.2. L-moments of storm depth defined by 8-hour minimum interevent time for hourly rainfall stations in Texas.

$[--$, not available $]$

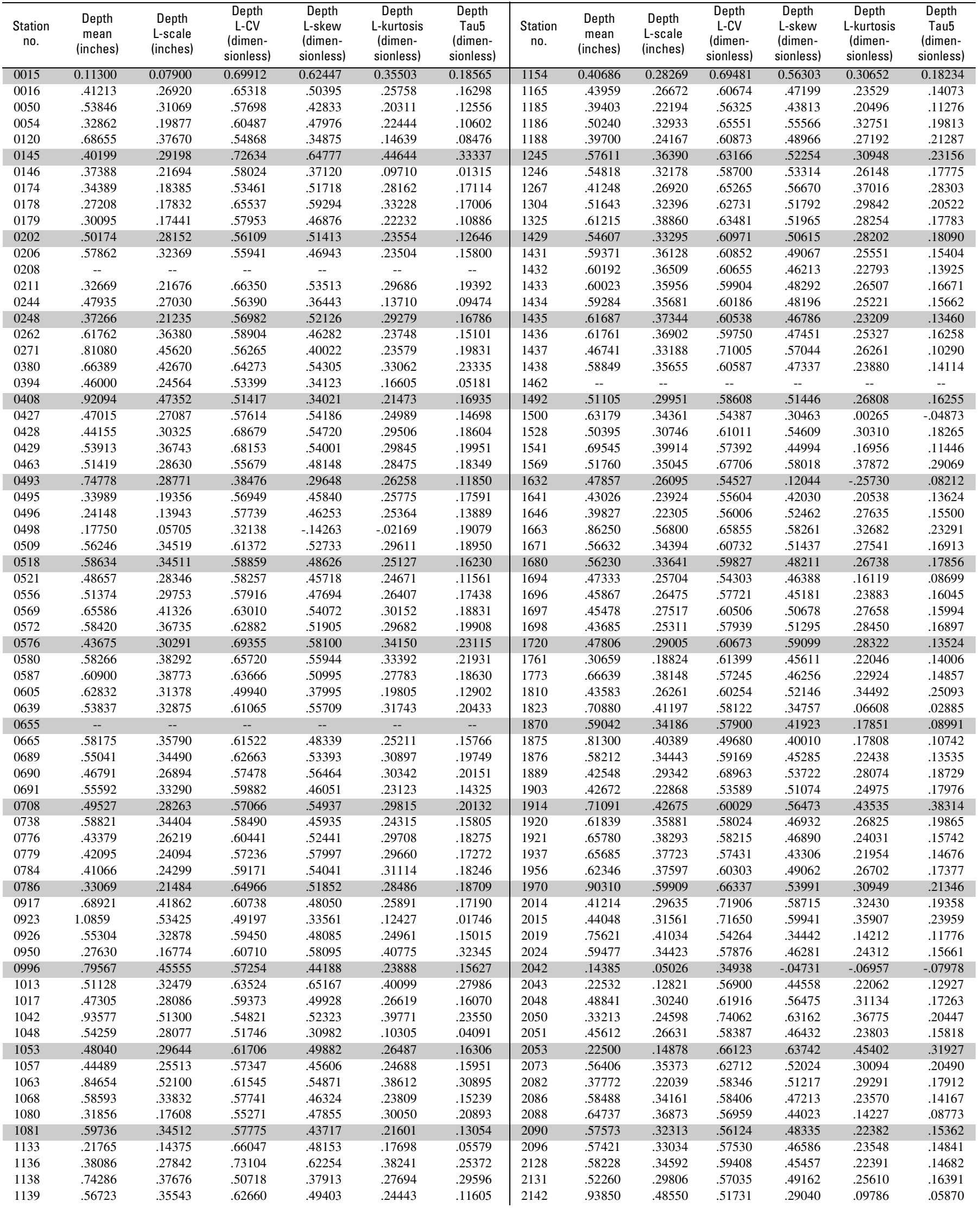


192 Statistical Characteristics of Storm Interevent Time, Depth, and Duration for Eastern New Mexico, Oklahoma, and Texas

Appendix 4-2.2. L-moments of storm depth defined by 8-hour minimum interevent time for hourly rainfall stations in Texas-Continued.

\begin{tabular}{|c|c|c|c|c|c|c|c|c|c|c|c|c|c|}
\hline $\begin{array}{c}\text { Station } \\
\text { no. }\end{array}$ & $\begin{array}{c}\text { Depth } \\
\text { mean } \\
\text { (inches) }\end{array}$ & $\begin{array}{c}\text { Depth } \\
\text { L-scale } \\
\text { (inches) }\end{array}$ & $\begin{array}{c}\text { Depth } \\
\text { L-CV } \\
\text { (dimen- } \\
\text { sionless) }\end{array}$ & $\begin{array}{c}\text { Depth } \\
\text { L-skew } \\
\text { (dimen- } \\
\text { sionless) }\end{array}$ & $\begin{array}{c}\text { Depth } \\
\text { L-kurtosis } \\
\text { (dimen- } \\
\text { sionless) }\end{array}$ & $\begin{array}{c}\text { Depth } \\
\text { Tau5 } \\
\text { (dimen- } \\
\text { sionless) }\end{array}$ & $\begin{array}{c}\text { Station } \\
\text { no. }\end{array}$ & $\begin{array}{l}\text { Depth } \\
\text { mean } \\
\text { (inches) }\end{array}$ & $\begin{array}{l}\text { Depth } \\
\text { L-scale } \\
\text { (inches) }\end{array}$ & $\begin{array}{c}\text { Depth } \\
\text { L-CV } \\
\text { (dimen- } \\
\text { sionless) }\end{array}$ & $\begin{array}{c}\text { Depth } \\
\text { L-skew } \\
\text { (dimen- } \\
\text { sionless) }\end{array}$ & $\begin{array}{c}\text { Depth } \\
\text { L-kurtosis } \\
\text { (dimen- } \\
\text { sionless) }\end{array}$ & $\begin{array}{c}\text { Depth } \\
\text { Tau5 } \\
\text { (dimen- } \\
\text { sionless) }\end{array}$ \\
\hline 2160 & 0.35393 & 0.25266 & 0.71387 & 0.63987 & 0.48189 & 0.43958 & 3463 & 0.63694 & 0.40807 & 0.64067 & 0.52378 & 0.31248 & 0.21677 \\
\hline 2206 & .67052 & .40776 & .60812 & .48819 & .26725 & .17663 & 3476 & .54419 & .32607 & .59918 & .46746 & .24691 & .15818 \\
\hline 2238 & .30586 & .20271 & .66274 & .51990 & .24987 & .12889 & 3485 & .85000 & .58343 & .68639 & .54956 & .23572 & .08493 \\
\hline 2240 & .29692 & .18344 & .61782 & .47167 & .25047 & .16892 & 3507 & .57892 & .34222 & .59113 & .52022 & .25152 & .15211 \\
\hline 2242 & .50401 & .31804 & .63103 & .46317 & .21735 & .13365 & 3546 & .66491 & .39506 & .59415 & .47416 & .24705 & .15719 \\
\hline 2244 & .54735 & .34254 & .62582 & .49067 & .25853 & .15417 & 3547 & .59347 & .33698 & .56781 & .41169 & .18000 & .10054 \\
\hline 2247 & .48661 & .32076 & .65917 & .52583 & .28850 & .18353 & 3579 & .61392 & .36583 & .59588 & .46008 & .18193 & .05975 \\
\hline 2309 & .69607 & .37980 & .54563 & .43200 & .24660 & .17323 & 3642 & .62092 & .36673 & .59063 & .48580 & .26114 & .16846 \\
\hline 2312 & .60760 & .33177 & .54604 & .43364 & .19088 & .13022 & 3646 & .57792 & .33648 & .58223 & .46675 & .25561 & .17036 \\
\hline 2334 & .87985 & .50804 & .57742 & .43652 & .22316 & .11547 & 3668 & 1.0500 & .50964 & .48537 & .34780 & .21279 & .07527 \\
\hline 2336 & .60521 & .32544 & .53773 & .36919 & .15707 & .10208 & 3673 & .70194 & .40065 & .57077 & .30108 & .01472 & .01246 \\
\hline 2354 & .30258 & .16927 & .55942 & .38996 & .08867 & .02118 & 3686 & .56257 & .32766 & .58243 & .50039 & .23238 & .13748 \\
\hline 2355 & .56492 & .39524 & .69965 & .59890 & .36209 & .23597 & 3691 & .55964 & .32514 & .58098 & .48977 & .25354 & .15674 \\
\hline 2357 & .36468 & .26695 & .73202 & .61179 & .35689 & .22902 & 3734 & 1.0415 & 67769 & .65068 & .47945 & .20202 & .08706 \\
\hline 2360 & .36542 & .25996 & .71139 & .59495 & .34818 & .22233 & 3771 & .57238 & .33697 & .58871 & .52358 & .24497 & .15476 \\
\hline 2361 & .44842 & .29744 & .66329 & .63240 & .46276 & .37847 & 3789 & .19301 & .10453 & .54156 & .48778 & .30871 & .20100 \\
\hline 2394 & .59759 & .35838 & .59970 & .46871 & .24290 & .15374 & 3826 & .53332 & .28770 & .53945 & .40767 & .20587 & .11767 \\
\hline 2404 & .56749 & .34208 & .60279 & .48348 & .25758 & .16730 & 3831 & .62209 & .36731 & .59045 & .46970 & .25300 & .17061 \\
\hline 2415 & .70254 & .40682 & .57907 & .44808 & .22992 & .14497 & 3841 & .61400 & .34873 & .56797 & .43860 & .22512 & .14524 \\
\hline 2462 & .65739 & .37800 & .57500 & .44304 & .21416 & .12826 & 3871 & .50084 & .29318 & .58538 & .45739 & .23451 & .15228 \\
\hline 2528 & .51872 & .30111 & .58048 & .45996 & .22079 & .10467 & 3884 & .80261 & .54292 & .67645 & .55535 & .26392 & .10502 \\
\hline 2617 & .45011 & .27701 & .61543 & .51070 & .29942 & .20403 & 3941 & .70378 & .40944 & .58177 & .34473 & .08411 & .06465 \\
\hline 2619 & .45186 & .25381 & .56171 & .45135 & .25426 & .17654 & 3963 & .07143 & .03619 & .50667 & .60000 & .43421 & .25000 \\
\hline 2621 & .47724 & .27630 & .57895 & .48042 & .25717 & .15645 & 4040 & .51728 & .29205 & .56458 & .43574 & .21648 & .12605 \\
\hline 2675 & .58595 & .34987 & .59709 & .51522 & .28244 & .18125 & 4058 & .68600 & .42402 & .61811 & .48495 & .25737 & .17063 \\
\hline 2676 & .56478 & .32965 & .58368 & .51872 & .23831 & .14228 & 4098 & .36181 & .19483 & .53849 & .51615 & .26615 & .14760 \\
\hline 2679 & .47557 & .30726 & .64609 & .58082 & .33960 & .19360 & 4100 & .49105 & .29841 & 60769 & .49806 & .27636 & .18814 \\
\hline 2715 & .55850 & .32149 & .57563 & .45421 & .23592 & .15136 & 4137 & .54034 & .30200 & .55892 & .49308 & .23111 & .15541 \\
\hline 2744 & .48874 & .28091 & .57476 & .47111 & .24791 & .15545 & 4191 & .53088 & .33086 & .62323 & .54384 & .30415 & .18194 \\
\hline 2758 & .39350 & .28350 & .72047 & .63667 & .39521 & .23820 & 4256 & -- & -- & -- & -- & -- & -- \\
\hline 2794 & .43091 & .29836 & .69241 & .50599 & .16870 & -.00965 & 4257 & .67727 & .39215 & .57901 & .47244 & .23590 & .15247 \\
\hline 2797 & .19470 & .12471 & .64052 & .52870 & .29453 & .18969 & 4258 & .56912 & .32602 & .57286 & .51028 & .25967 & .19879 \\
\hline 2811 & .46087 & .27294 & .59222 & .50564 & .26792 & .15050 & 4278 & .58952 & .35085 & .59514 & .46146 & .23307 & .14445 \\
\hline 2813 & .51000 & .30064 & .58950 & .48240 & .28391 & .15941 & 4299 & .32783 & .15522 & .47348 & .32593 & .13492 & .06345 \\
\hline 2814 & .21471 & .15750 & .73356 & .64893 & .35201 & .08353 & 4300 & .51794 & .35415 & .68377 & .54755 & .30565 & .20245 \\
\hline 2815 & .47652 & .26791 & .56222 & .54360 & .29305 & .19919 & 4305 & .48909 & .33942 & .69397 & .55735 & .30926 & .20103 \\
\hline 2818 & .55540 & .32604 & .58704 & .43348 & .20931 & .13802 & 4307 & .53590 & .36646 & .68382 & .54822 & .31858 & .23174 \\
\hline 2986 & .75062 & .42142 & .56143 & .38814 & .16507 & .11109 & 4309 & 63968 & .39725 & .62102 & .49294 & .26420 & .17332 \\
\hline 3005 & .54486 & .31327 & .57496 & .46119 & .22898 & .13887 & 4311 & .66887 & .41196 & .61591 & .48126 & .24902 & .15552 \\
\hline 3033 & .25902 & .14294 & .55185 & .49692 & .30895 & .20904 & 4313 & .67337 & .44337 & .65844 & .55584 & .33565 & .22608 \\
\hline 3034 & -- & -- & -- & -- & -- & -- & 4319 & .50267 & .28589 & .56874 & .42444 & .18235 & .09264 \\
\hline 3047 & .51714 & .30857 & .59669 & .41881 & 20079 & .16225 & 4329 & .65572 & .40083 & .61128 & .49394 & .27656 & .18099 \\
\hline 3103 & .73029 & .47019 & .64383 & .59465 & .34268 & .18287 & 4331 & -- & -- & -- & -- & -- & -- \\
\hline 3133 & .63214 & .36522 & .57776 & .45727 & .24378 & .16162 & 4375 & .54477 & .32636 & .59907 & .57137 & .32053 & .22899 \\
\hline 3156 & .68495 & .42144 & .61529 & .55467 & .33301 & .23480 & 4392 & .72844 & .44784 & .61479 & .47704 & .25136 & .16681 \\
\hline 3171 & .59984 & .35342 & .58918 & .47208 & .25018 & .15666 & 4425 & .38267 & .21804 & .56979 & .53389 & .28791 & .16181 \\
\hline 3189 & .30262 & .17856 & .59004 & .51479 & .30431 & .18557 & 4440 & .50210 & .30426 & .60597 & .47976 & .25163 & .16268 \\
\hline 3260 & .49530 & .29115 & .58782 & .43101 & .20742 & .13073 & 4476 & .53807 & .30288 & .56290 & .47616 & .23961 & .15194 \\
\hline 3267 & .41741 & .27327 & .65467 & .54141 & .30873 & .21286 & 4498 & .20231 & .10603 & .52408 & .35517 & .16027 & .03276 \\
\hline 3270 & .45967 & .26178 & .56950 & .53664 & .27624 & .17978 & 4517 & .55012 & .32435 & .58959 & .45242 & .23373 & .15462 \\
\hline 3272 & .11000 & .05442 & .49473 & .39693 & .21940 & .12995 & 4520 & .52068 & .29832 & .57294 & .52343 & .25111 & .15743 \\
\hline 3277 & .15188 & .09588 & .63128 & .55113 & .36774 & .37100 & 4525 & .62200 & .41081 & 66046 & .52526 & .24375 & .12004 \\
\hline 3278 & .41358 & .23674 & .57242 & .49115 & .26297 & .14839 & 4563 & .46900 & .28176 & 60076 & .44688 & .21533 & .16729 \\
\hline 3280 & .31084 & .19783 & .63642 & .54190 & .30373 & .18256 & 4570 & .47385 & .27957 & .59001 & .51570 & .28635 & .17788 \\
\hline 3281 & .34522 & .16901 & .48959 & .30841 & .10088 & .03939 & 4577 & .65931 & .37203 & .56427 & .44568 & .22846 & .15106 \\
\hline 3283 & .48748 & .31736 & .65103 & .49845 & .25598 & .15964 & 4591 & .59627 & .35752 & .59959 & .47241 & .24820 & .15581 \\
\hline 3284 & .54359 & .32073 & .59003 & .48047 & .24551 & .14092 & 4670 & .45716 & .27087 & .59251 & .50586 & .27185 & .15857 \\
\hline 3285 & .56004 & .32271 & .57623 & .49401 & .22726 & .13401 & 4671 & .36438 & .22519 & .61801 & .45778 & .21233 & .13448 \\
\hline 3329 & .51927 & .33069 & .63683 & .53518 & .31312 & .21245 & 4679 & .55672 & .33283 & .59785 & .52369 & .27861 & .17917 \\
\hline 3335 & .74741 & .47391 & .63407 & .53381 & .33551 & .24260 & 4696 & .39667 & .20438 & .51525 & .31307 & .07735 & .01284 \\
\hline 3370 & .66072 & .38093 & .57653 & .44194 & .22805 & .14480 & 4703 & .42849 & .25401 & .59279 & .49651 & .25407 & .12005 \\
\hline 3410 & .45688 & .25270 & .55310 & .49404 & .26015 & .16854 & 4704 & .74613 & .47075 & 63092 & .51600 & .29696 & .19811 \\
\hline 3415 & .55649 & .32677 & .58720 & .48813 & .25667 & .16450 & 4731 & .39394 & .27912 & .70853 & 60792 & .40458 & .31596 \\
\hline 3430 & .51529 & .35335 & .68573 & .55550 & .31884 & .21550 & 4792 & .54145 & .30381 & .56110 & .49436 & .22800 & .14682 \\
\hline 3431 & .48986 & .35425 & .72315 & .60960 & .37379 & .26379 & 4819 & .64603 & .35485 & .54928 & .45453 & .20885 & .13513 \\
\hline 3441 & .52886 & .30881 & .58391 & .43895 & .22149 & .13087 & 4852 & .83353 & .41581 & .49885 & .48584 & .36665 & .23067 \\
\hline 3442 & .37306 & .21972 & .58896 & .49958 & .27911 & .17389 & 4866 & .60792 & .34827 & .57290 & .45288 & .22617 & .14261 \\
\hline 3446 & .43691 & .24430 & .55916 & .45317 & .23986 & .14846 & 4876 & .73288 & .43123 & .58841 & .49308 & .22906 & .13270 \\
\hline 3460 & .67341 & .41207 & .61191 & .49257 & .30148 & .26878 & 4878 & .70525 & .43357 & 61477 & .49390 & .27037 & .17404 \\
\hline 3462 & .45388 & .24642 & .54291 & .42610 & .19866 & .10529 & 4880 & .41353 & .23314 & .56379 & .47612 & .25500 & .15585 \\
\hline
\end{tabular}


Appendix 4-2.2. L-moments of storm depth defined by 8-hour minimum interevent time for hourly rainfall stations in Texas-Continued.

\begin{tabular}{|c|c|c|c|c|c|c|c|c|c|c|c|c|c|}
\hline $\begin{array}{c}\text { Station } \\
\text { no. }\end{array}$ & $\begin{array}{c}\text { Depth } \\
\text { mean } \\
\text { (inches) }\end{array}$ & $\begin{array}{c}\text { Depth } \\
\text { L-scale } \\
\text { (inches) }\end{array}$ & $\begin{array}{c}\text { Depth } \\
\text { L-CV } \\
\text { (dimen- } \\
\text { sionless) }\end{array}$ & $\begin{array}{c}\text { Depth } \\
\text { L-skew } \\
\text { (dimen- } \\
\text { sionless) }\end{array}$ & $\begin{array}{c}\text { Depth } \\
\text { L-kurtosis } \\
\text { (dimen- } \\
\text { sionless) }\end{array}$ & $\begin{array}{c}\text { Depth } \\
\text { Tau5 } \\
\text { (dimen- } \\
\text { sionless) }\end{array}$ & $\begin{array}{c}\text { Station } \\
\text { no. }\end{array}$ & $\begin{array}{c}\text { Depth } \\
\text { mean } \\
\text { (inches) }\end{array}$ & $\begin{array}{c}\text { Depth } \\
\text { L-scale } \\
\text { (inches) }\end{array}$ & $\begin{array}{c}\text { Depth } \\
\text { L-CV } \\
\text { (dimen- } \\
\text { sionless) }\end{array}$ & $\begin{array}{c}\text { Depth } \\
\text { L-skew } \\
\text { (dimen- } \\
\text { sionless) }\end{array}$ & $\begin{array}{c}\text { Depth } \\
\text { L-kurtosis } \\
\text { (dimen- } \\
\text { sionless) }\end{array}$ & $\begin{array}{c}\text { Depth } \\
\text { Tau5 } \\
\text { (dimen- } \\
\text { sionless) }\end{array}$ \\
\hline 4920 & 0.52297 & 0.32105 & 0.61390 & 0.53713 & 0.29458 & 0.17553 & 5957 & 0.56197 & 0.32581 & 0.57976 & 0.48800 & 0.25358 & 0.16306 \\
\hline 4934 & .32125 & .21339 & .66426 & .77071 & .71381 & .68870 & 5958 & .50598 & .29103 & .57518 & .42228 & .20322 & .11500 \\
\hline 4972 & .52708 & .30565 & .57990 & .48431 & .25997 & .16881 & 5973 & .38780 & .27195 & .70127 & .59001 & .32269 & .17721 \\
\hline 4973 & .72385 & .39077 & .53985 & .40877 & .22112 & .15341 & 5996 & .53149 & .31358 & .59000 & .49133 & .25574 & .15734 \\
\hline 4974 & .42729 & .24980 & .58461 & .49003 & .27015 & .17085 & 6017 & .39643 & .26202 & .66096 & .53636 & .27871 & .15126 \\
\hline 4975 & .65983 & .36992 & .56062 & .46486 & .21880 & .15345 & 6024 & .82413 & .52495 & .63697 & .50463 & .27945 & .20041 \\
\hline 4978 & .53637 & .35184 & .65596 & .53980 & .29984 & .18402 & 6050 & 66700 & .31868 & .47779 & .30559 & .01394 & -.08970 \\
\hline 4979 & .87500 & .51851 & .59258 & .47256 & .28165 & .18238 & 6104 & .33209 & .18532 & .55804 & .49792 & .28605 & .17171 \\
\hline 4982 & .49909 & .29091 & .58288 & .46857 & .25487 & .16320 & 6108 & 69790 & .39949 & .57241 & .45397 & .22620 & .14622 \\
\hline 5018 & .56288 & .32073 & .56979 & .42667 & .20423 & .13428 & 6136 & .36119 & .19760 & .54708 & .50948 & .26649 & .14815 \\
\hline 5048 & .41983 & .25304 & .60273 & .56899 & .32777 & .19764 & 6166 & .35561 & .22362 & .62883 & .52295 & .26189 & .13461 \\
\hline 5049 & .40777 & .23113 & .56681 & .60860 & .38370 & .31199 & 6176 & 67907 & .41928 & .61744 & .49130 & .27197 & .17550 \\
\hline 5056 & .43200 & .18600 & .43056 & .26882 & .67742 & .11828 & 6177 & .67324 & .39419 & .58552 & .48127 & .25312 & .16287 \\
\hline 5057 & .32030 & .22969 & .71711 & .61836 & .38222 & .24840 & 6210 & .61514 & .36062 & .58624 & .48398 & .24820 & .15831 \\
\hline 5060 & .51243 & .35823 & .69908 & .58728 & .35604 & .24811 & 6211 & .57346 & .35941 & .62673 & .48355 & .24692 & .15518 \\
\hline 5081 & .62706 & .36329 & .57935 & .42741 & .20874 & .13891 & 6270 & .68212 & .39113 & .57341 & .48900 & .24719 & .17149 \\
\hline 5094 & .59331 & .34745 & .58562 & .48717 & .25294 & .15455 & 6275 & -- & -- & -- & -- & -- & -- \\
\hline 5113 & .51941 & .32530 & .62630 & .55458 & .31619 & .19143 & 6276 & .80679 & .42097 & .52178 & .33688 & .10862 & .03604 \\
\hline 5114 & -- & -- & -- & -- & -- & -- & 6335 & 61512 & .35563 & .57815 & .43862 & .21575 & .13472 \\
\hline 5123 & .61062 & .33996 & .55674 & .44464 & .18737 & -.00649 & 6434 & .49000 & .27063 & .55231 & .34073 & .07576 & .01695 \\
\hline 5192 & .61539 & .36264 & .58928 & .47198 & .24262 & .14581 & 6504 & .40153 & .23111 & .57557 & .52278 & .28409 & .16226 \\
\hline 5193 & .56219 & .34157 & .60756 & .49686 & .25674 & .15399 & 6558 & .57844 & .33983 & .58749 & .41687 & .17826 & .16473 \\
\hline 5224 & .67015 & .40388 & 60267 & .46371 & .23763 & .14351 & 6615 & .47456 & .27664 & .58294 & .57299 & .32580 & .22818 \\
\hline 5228 & .58206 & .35158 & 60404 & .44598 & .19502 & .10199 & 6660 & .57724 & .34098 & .59072 & .45819 & .23090 & .14873 \\
\hline 5235 & .49848 & .32646 & .65490 & .50470 & .23795 & .14846 & 6663 & .47416 & .28777 & .60690 & .47844 & .23534 & .10042 \\
\hline 5247 & .41301 & .23204 & .56182 & .49720 & .26074 & .14610 & 6734 & .42010 & .25470 & .60628 & .48329 & .25652 & .16222 \\
\hline 5258 & .59402 & .34778 & .58546 & .45386 & .23357 & .14490 & 6736 & .44910 & .26316 & .58597 & .52683 & .28492 & .16687 \\
\hline 5303 & .53424 & .34190 & 63998 & .54555 & .32999 & .23978 & 6740 & 1.1488 & .55500 & .48310 & .28771 & .16845 & .08528 \\
\hline 5312 & .50318 & .29315 & .58260 & .52125 & .27345 & .17484 & 6750 & .59253 & .41485 & .70013 & .58322 & .36343 & .26258 \\
\hline 5341 & .89345 & .57222 & .64046 & .56904 & .36654 & .25987 & 6757 & .59909 & .36585 & 61067 & .47372 & .24442 & .15420 \\
\hline 5342 & -- & -- & -- & -- & -- & -- & 6775 & .39288 & .23483 & .59772 & .46531 & .24514 & .15789 \\
\hline 5348 & .66251 & .37565 & .56701 & .44835 & .19282 & .11269 & 6776 & .41243 & .23091 & .55987 & .49875 & .26863 & .15914 \\
\hline 5358 & .47902 & .28169 & .58806 & .47429 & .24493 & .14262 & 6788 & 65990 & .37558 & .56915 & .42634 & .23173 & .15542 \\
\hline 5398 & .63185 & .37493 & .59339 & .46870 & .24383 & .15693 & 6792 & .33371 & .18226 & .54616 & .49644 & .26993 & .14330 \\
\hline 5410 & .41937 & .24187 & .57674 & .50907 & .29165 & .18549 & 6794 & 1.0652 & .59980 & .56310 & .38590 & .18999 & .12107 \\
\hline 5411 & .33306 & .22217 & .66704 & .53672 & .29472 & .18869 & 6834 & .68575 & .38787 & .56562 & .46835 & .22227 & .15447 \\
\hline 5424 & .55753 & .38532 & .69112 & .53200 & .26741 & .16534 & 6893 & .31683 & .17325 & .54684 & .55183 & .33683 & .21139 \\
\hline 5429 & .53040 & .34102 & .64295 & .52758 & .29334 & .18702 & 6935 & .41073 & .23011 & .56024 & .53535 & .30759 & .20128 \\
\hline 5431 & .80333 & .43980 & .54747 & .51438 & .31970 & .15448 & 6981 & .58459 & .33971 & .58111 & .46307 & .25511 & .16822 \\
\hline 5461 & .68066 & .40485 & .59480 & .45544 & .23276 & .15001 & 7020 & .58925 & .37129 & .63011 & .49212 & .27166 & .19243 \\
\hline 5463 & .64205 & .37463 & .58349 & .50670 & .24868 & .16471 & 7060 & .46734 & .26585 & .56887 & .49892 & .25532 & .14357 \\
\hline 5471 & .15444 & .10963 & .70983 & .68489 & .50322 & .38546 & 7066 & .66459 & .38652 & .58159 & .45940 & .22847 & .14064 \\
\hline 5477 & .68600 & .46167 & .67298 & .74405 & .67395 & .62374 & 7074 & .39904 & .23302 & .58395 & .52641 & .31257 & .19936 \\
\hline 5528 & .67228 & .38148 & .56744 & .43717 & .22394 & .14167 & 7097 & .55298 & .32896 & .59489 & .50205 & .27141 & .14054 \\
\hline 5579 & -- & -- & -- & -- & -- & -- & 7116 & .42330 & .24852 & .58710 & .46677 & .25214 & .17054 \\
\hline 5580 & .53862 & .27057 & .50233 & .29332 & .09515 & .04973 & 7140 & 64117 & .42178 & .65782 & .55862 & .33309 & .22234 \\
\hline 5589 & .27459 & .15539 & .56591 & .40078 & .17235 & .09713 & 7173 & .63225 & .44836 & .70915 & .58104 & .34251 & .23476 \\
\hline 5590 & .31286 & .18107 & .57874 & .46157 & .21646 & .09780 & 7174 & .59305 & .40722 & .68665 & .55402 & .31944 & .22039 \\
\hline 5591 & .33345 & .17627 & .52862 & .41195 & .20060 & .11445 & 7213 & .59979 & .35641 & .59423 & .46753 & .25002 & .16093 \\
\hline 5592 & .29171 & .16655 & .57096 & .47691 & .26371 & .16756 & 7243 & .49317 & .28824 & .58446 & .50641 & .26988 & .16753 \\
\hline 5594 & .30718 & .17362 & .56522 & .47096 & .24897 & .14330 & 7262 & .18533 & .10677 & .57609 & .44882 & .22203 & .12044 \\
\hline 5595 & -- & -- & -- & -- & -- & -- & 7274 & .62756 & .34193 & .54485 & .44555 & .24399 & .16515 \\
\hline 5596 & .34720 & .17945 & .51684 & .53912 & .26231 & .15472 & 7300 & .55221 & .32328 & .58542 & .45190 & .23152 & .14993 \\
\hline 5600 & .34529 & .20166 & .58403 & .49824 & .28974 & .18222 & 7311 & .59844 & .34608 & .57830 & .51767 & .28823 & .15480 \\
\hline 5618 & .54432 & .31595 & .58044 & .43414 & .17689 & .06824 & 7363 & .57857 & .29709 & .51349 & .24783 & .01853 & .03859 \\
\hline 5650 & .54200 & .23321 & .43028 & .35929 & .36565 & .19130 & 7422 & .54083 & .33301 & .61573 & .52348 & .29200 & .18544 \\
\hline 5656 & .42810 & .24212 & .56556 & .55827 & .29191 & .18703 & 7431 & .41707 & .24578 & .58931 & .51411 & .29043 & .18036 \\
\hline 5658 & .43862 & .26684 & 60835 & .50807 & .28848 & .18703 & 7481 & .33524 & .20130 & .60046 & .56724 & .36014 & .22793 \\
\hline 5661 & .54125 & .34256 & .63291 & .63377 & .38209 & .26445 & 7497 & .55378 & .32143 & .58043 & .52080 & .25416 & .16416 \\
\hline 5666 & .46889 & .27443 & .58527 & .49242 & .28487 & .17953 & 7498 & .58267 & .34330 & .58918 & .53049 & .28042 & .20135 \\
\hline 5695 & .62644 & .35954 & .57394 & .44551 & .23274 & .15489 & 7499 & .52972 & .29998 & .56629 & .49433 & .25295 & .16529 \\
\hline 5742 & .25048 & .15138 & .60437 & .50454 & .24380 & .14163 & 7531 & .56940 & .31973 & .56153 & .40715 & .17577 & .08519 \\
\hline 5766 & .71529 & .46009 & .64322 & .52953 & .26075 & .10359 & 7534 & .57233 & .36311 & .63445 & .53740 & .33522 & .25259 \\
\hline 5770 & .44109 & .25478 & .57762 & .50043 & .27133 & .16013 & 7556 & .56851 & .33437 & .58814 & .51186 & .27766 & .18297 \\
\hline 5775 & .34643 & .19049 & .54988 & .32881 & -.04007 & -.06288 & 7594 & 67740 & .39966 & .59000 & .45394 & .23167 & .15121 \\
\hline 5779 & .65727 & .39199 & .59639 & .48066 & .24625 & .07866 & 7596 & .68920 & .41133 & .59683 & .41483 & .16078 & .08494 \\
\hline 5840 & .56183 & .33553 & .59722 & .46412 & .22953 & .13560 & 7608 & .53075 & .33766 & .63619 & .49275 & .25231 & .15739 \\
\hline 5890 & .31010 & .20684 & .66701 & .54252 & .30110 & .19101 & 7622 & .22227 & .17790 & .80037 & .72060 & .47714 & .32193 \\
\hline 5891 & .36900 & .19846 & .53783 & .39619 & .17842 & .10733 & 7700 & 61011 & .37954 & .62208 & .48615 & .25360 & .16077 \\
\hline 5897 & .58688 & .34584 & .58928 & .52189 & .25518 & .16707 & 7706 & .46979 & .28225 & .60079 & .51872 & .28742 & .17526 \\
\hline
\end{tabular}


194 Statistical Characteristics of Storm Interevent Time, Depth, and Duration for Eastern New Mexico, Oklahoma, and Texas

Appendix 4-2.2. L-moments of storm depth defined by 8-hour minimum interevent time for hourly rainfall stations in Texas-Continued.

\begin{tabular}{|c|c|c|c|c|c|c|c|c|c|c|c|c|c|}
\hline $\begin{array}{c}\text { Station } \\
\text { no. }\end{array}$ & $\begin{array}{c}\text { Depth } \\
\text { mean } \\
\text { (inches) }\end{array}$ & $\begin{array}{c}\text { Depth } \\
\text { L-scale } \\
\text { (inches) }\end{array}$ & $\begin{array}{c}\text { Depth } \\
\text { L-CV } \\
\text { (dimen- } \\
\text { sionless) }\end{array}$ & $\begin{array}{c}\text { Depth } \\
\text { L-skew } \\
\text { (dimen- } \\
\text { sionless) }\end{array}$ & $\begin{array}{c}\text { Depth } \\
\text { L-kurtosis } \\
\text { (dimen- } \\
\text { sionless) }\end{array}$ & $\begin{array}{c}\text { Depth } \\
\text { Tau5 } \\
\text { (dimen- } \\
\text { sionless) }\end{array}$ & $\begin{array}{c}\text { Station } \\
\text { no. }\end{array}$ & $\begin{array}{l}\text { Depth } \\
\text { mean } \\
\text { (inches) }\end{array}$ & $\begin{array}{c}\text { Depth } \\
\text { L-scale } \\
\text { (inches) }\end{array}$ & $\begin{array}{c}\text { Depth } \\
\text { L-CV } \\
\text { (dimen- } \\
\text { sionless) }\end{array}$ & $\begin{array}{c}\text { Depth } \\
\text { L-skew } \\
\text { (dimen- } \\
\text { sionless) }\end{array}$ & $\begin{array}{c}\text { Depth } \\
\text { L-kurtosis } \\
\text { (dimen- } \\
\text { sionless) }\end{array}$ & $\begin{array}{c}\text { Depth } \\
\text { Tau5 } \\
\text { (dimen- } \\
\text { sionless) }\end{array}$ \\
\hline 7718 & 0.56292 & 0.35054 & 0.62271 & 0.52325 & 0.29268 & 0.17041 & 8910 & 0.64227 & 0.36236 & 0.56418 & 0.34553 & 0.04315 & -0.06205 \\
\hline 7745 & .66359 & .41582 & .62663 & .48903 & .25968 & .17343 & 8911 & .49996 & .31913 & .63831 & .50120 & .26168 & .16132 \\
\hline 7922 & .23802 & .14097 & .59224 & .48519 & .27025 & .16887 & 8924 & .29555 & .15861 & .53665 & .43813 & .22773 & .12618 \\
\hline 7936 & .68410 & .40162 & .58708 & .47591 & .25011 & .17067 & 8929 & .80462 & .46837 & .58210 & .34468 & .01082 & -.05684 \\
\hline 7943 & .36181 & .24071 & .66529 & .52896 & .28020 & .17169 & 8942 & .66393 & .38705 & .58297 & .49014 & .24373 & .15350 \\
\hline 7944 & .36643 & .25720 & .70190 & .56645 & .30363 & .17095 & 8944 & .57103 & .35054 & .61388 & .47104 & .24359 & .15613 \\
\hline 7945 & .41795 & .29588 & .70794 & .58281 & .33753 & .22427 & 8996 & .57296 & .35833 & .62541 & .50243 & .27332 & .17829 \\
\hline 7947 & .63667 & .40256 & .63230 & .57903 & .35601 & .25774 & 9014 & .71174 & .34079 & .47881 & .28837 & .08279 & .01061 \\
\hline 7948 & .52700 & .34037 & .64587 & .53037 & .29274 & .18012 & 9037 & .31744 & .19083 & .60117 & .54670 & .35125 & .24935 \\
\hline 7951 & .62649 & .35425 & .56546 & .42364 & .20625 & .12570 & 9106 & .28028 & .17904 & .63879 & .59563 & .40198 & .30038 \\
\hline 7953 & .42534 & .26916 & .63282 & .52227 & .28598 & .16155 & 9107 & .32425 & .19487 & .60097 & .47751 & .23448 & .14193 \\
\hline 7981 & .50168 & .31947 & .63680 & .49738 & .23447 & .11785 & 9129 & .33545 & .23287 & 69419 & .62100 & .38272 & .23748 \\
\hline 7990 & .43895 & .30823 & .70219 & .59780 & .36668 & .25148 & 9163 & .51821 & .30372 & .58608 & .48806 & .26296 & .16388 \\
\hline 7992 & .57893 & .33430 & .57744 & .42384 & .09079 & -.04199 & 9213 & .48326 & .30749 & .63628 & .48717 & .25569 & .17568 \\
\hline 7997 & .45784 & .27970 & .61092 & .48360 & .23637 & .11650 & 9214 & .84455 & .47250 & .55947 & .44385 & .21225 & .10906 \\
\hline 7999 & .32737 & .21971 & .67113 & .55778 & .34309 & .24567 & 9222 & .48605 & .30582 & .62920 & .47975 & .23752 & .14530 \\
\hline 8022 & .40000 & .24731 & .61828 & .48905 & .20516 & .08084 & 9248 & .42321 & .24287 & .57388 & .44221 & .18637 & .07647 \\
\hline 8023 & .43775 & .25844 & .59038 & .53039 & .30366 & .19049 & 9266 & .50084 & .28466 & .56836 & .43403 & .19826 & .08590 \\
\hline 8047 & .54581 & .31859 & .58371 & .47283 & .23744 & .14257 & 9270 & .31797 & .17015 & .53509 & .53970 & .30384 & .16925 \\
\hline 8060 & .42507 & .28908 & .68009 & .56564 & .29365 & .13878 & 9295 & .19757 & .12254 & .62024 & .51481 & .27985 & .16032 \\
\hline 8062 & .62889 & .43338 & .68913 & .54209 & .27677 & .15435 & 9304 & -- & -- & -- & -- & -- & -- \\
\hline 8068 & .25846 & .17182 & .66479 & .59214 & .35957 & .22701 & 9307 & .54451 & .29518 & .54211 & .43459 & .22759 & .13901 \\
\hline 8081 & .53196 & .33433 & .62848 & .55202 & .32469 & .21240 & 9328 & .41807 & .24207 & .57901 & .43655 & .19480 & .11087 \\
\hline 8089 & .46256 & .26012 & .56235 & .35282 & .08976 & .01938 & 9329 & .45312 & .26621 & .58749 & .28422 & -.10783 & -.14229 \\
\hline 8221 & .66714 & .31671 & .47473 & .36685 & .25215 & .17857 & 9345 & -- & -- & -- & -- & -- & -- \\
\hline 8252 & .42349 & .24612 & .58118 & .50877 & .27803 & .16081 & 9363 & .40067 & .27787 & .69353 & .57477 & .33660 & .22282 \\
\hline 8265 & .65110 & .42136 & .64714 & .54244 & .32155 & .22324 & 9364 & .46697 & .32692 & .70008 & .57804 & .33939 & .22826 \\
\hline 8289 & .45818 & .21741 & .47450 & .21799 & .03403 & .02819 & 9365 & .29342 & .18173 & .61934 & .64189 & .46226 & .33322 \\
\hline 8305 & .28242 & .15478 & .54806 & .52774 & .32159 & .18574 & 9371 & .55250 & .31683 & .57346 & .42474 & .17230 & .07776 \\
\hline 8335 & .69724 & .40366 & .57894 & .43473 & .21894 & .14295 & 9417 & .55975 & .33736 & .60269 & .45931 & .21569 & .12008 \\
\hline 8400 & .34556 & .20450 & .59178 & .51740 & .32474 & .22596 & 9419 & .46498 & .30584 & .65775 & .50046 & .24304 & .14201 \\
\hline 8445 & .60356 & .38339 & .63521 & .51592 & .28267 & .18228 & 9435 & .44494 & .24555 & .55187 & .44202 & .24529 & .18550 \\
\hline 8446 & .53538 & .31557 & .58944 & .49451 & .24512 & .13180 & 9491 & .59056 & .35327 & .59820 & .48256 & .24535 & .14618 \\
\hline 8451 & .43972 & .26464 & .60185 & .48292 & .25022 & .15473 & 9499 & .46372 & .26680 & .57535 & .50934 & .25773 & .14736 \\
\hline 8531 & .57665 & .33267 & .57691 & .45700 & .24236 & .15722 & 9522 & .97529 & .58603 & .60087 & .40115 & .08166 & -.04390 \\
\hline 8541 & .56910 & .32296 & .56749 & .42333 & .22318 & .14837 & 9527 & .39279 & .21769 & .55422 & .52337 & .27086 & .15206 \\
\hline 8544 & .62016 & .37641 & .60695 & .52476 & .28477 & .18188 & 9532 & .54585 & .31966 & .58563 & .48331 & .25707 & .16546 \\
\hline 8545 & .27409 & .13435 & .49017 & .23751 & .06235 & .10152 & 9544 & 1.2500 & 1.1260 & .90080 & .96803 & .95560 & .90586 \\
\hline 8563 & .60225 & .34704 & .57623 & .50810 & .25359 & .17376 & 9565 & .49281 & .28611 & .58058 & .49922 & .27189 & .17028 \\
\hline 8566 & .45827 & .26672 & .58201 & .44257 & .21162 & .11705 & 9570 & .44895 & .25001 & .55688 & .54415 & .28969 & .18762 \\
\hline 8583 & .46377 & .26344 & .56804 & .53155 & .23618 & .12869 & 9574 & .46400 & .27033 & .58261 & .54763 & .23461 & .10686 \\
\hline 8584 & .55102 & .31513 & .57191 & .46427 & .22208 & .13212 & 9588 & .46156 & .29091 & .63028 & .56205 & .32875 & .19226 \\
\hline 8623 & .56003 & .31251 & .55802 & .47046 & .23051 & .14147 & 9665 & .62102 & .36676 & .59057 & .47713 & .24833 & .15977 \\
\hline 8625 & .54794 & .32857 & .59965 & .47795 & .25483 & .17042 & 9715 & .56662 & .33031 & .58295 & .46316 & .23082 & .14322 \\
\hline 8630 & .45005 & .25606 & .56896 & .46880 & .22741 & .12034 & 9729 & .45746 & .29530 & .64552 & .49218 & .24455 & .15074 \\
\hline 8631 & .48191 & .28465 & .59068 & .48584 & .26488 & .16336 & 9772 & .58206 & .38459 & .66074 & .55900 & .33413 & .22609 \\
\hline 8646 & .56154 & .33324 & .59344 & .46260 & .23447 & .14701 & 9814 & .56526 & .32758 & .57952 & .40171 & .14923 & .06278 \\
\hline 8647 & .38880 & .22296 & .57346 & .53730 & .29544 & .17091 & 9815 & .59768 & .36084 & .60374 & .50487 & .28016 & .18042 \\
\hline 8677 & .51027 & .28299 & .55459 & .43434 & .21744 & .11862 & 9816 & .56200 & .35600 & .63345 & .51555 & .30519 & .22460 \\
\hline 8696 & .66034 & .36342 & .55035 & .36586 & .17278 & .14793 & 9817 & .52068 & .29341 & .56350 & .48572 & .24222 & .15782 \\
\hline 8743 & .66414 & .39294 & .59165 & .47223 & .24897 & .16194 & 9829 & .33623 & .19821 & .58949 & .54758 & .33076 & .19181 \\
\hline 8761 & .39032 & .21712 & .55628 & .51999 & .29611 & .18399 & 9830 & .27006 & .16132 & .59735 & .48159 & .23788 & .12953 \\
\hline 8778 & .61075 & .35629 & .58337 & .48709 & .24435 & .15775 & 9858 & .44050 & .24903 & .56534 & .45769 & .25360 & .16391 \\
\hline 8845 & .55679 & .34543 & .62039 & .53905 & .29900 & .18254 & 9893 & .52015 & .30502 & .58641 & .48279 & .25607 & .16028 \\
\hline 8859 & .67061 & .38656 & .57643 & .43403 & .21847 & .14410 & 9916 & .68413 & .38861 & .56804 & .44633 & .20793 & .12509 \\
\hline 8898 & .64716 & .38377 & .59300 & .47203 & .25927 & .16993 & 9976 & .47588 & .29158 & .61272 & .53327 & .29957 & .18024 \\
\hline 8908 & .51930 & .32031 & .61681 & .51075 & .29940 & .22084 & & & & & & & \\
\hline
\end{tabular}


Appendix 4-2.3. L-moments of storm depth defined by 12-hour minimum interevent time for hourly rainfall stations in Texas.

$[--$, not available $]$

\begin{tabular}{|c|c|c|c|c|c|c|c|c|c|c|c|c|c|}
\hline $\begin{array}{c}\text { Station } \\
\text { no. }\end{array}$ & $\begin{array}{l}\text { Depth } \\
\text { mean } \\
\text { (inches) }\end{array}$ & $\begin{array}{c}\text { Depth } \\
\text { L-scale } \\
\text { (inches) }\end{array}$ & $\begin{array}{c}\text { Depth } \\
\text { L-CV } \\
\text { (dimen- } \\
\text { sionless) }\end{array}$ & $\begin{array}{c}\text { Depth } \\
\text { L-skew } \\
\text { (dimen- } \\
\text { sionless) }\end{array}$ & $\begin{array}{c}\text { Depth } \\
\text { L-kurtosis } \\
\text { (dimen- } \\
\text { sionless) }\end{array}$ & $\begin{array}{c}\text { Depth } \\
\text { Tau5 } \\
\text { (dimen- } \\
\text { sionless) }\end{array}$ & $\begin{array}{c}\text { Station } \\
\text { no. }\end{array}$ & $\begin{array}{l}\text { Depth } \\
\text { mean } \\
\text { (inches) }\end{array}$ & $\begin{array}{l}\text { Depth } \\
\text { L-scale } \\
\text { (inches) }\end{array}$ & $\begin{array}{c}\text { Depth } \\
\text { L-CV } \\
\text { (dimen- } \\
\text { sionless) }\end{array}$ & $\begin{array}{l}\text { Depth } \\
\text { L-skew } \\
\text { (dimen- } \\
\text { sionless) }\end{array}$ & $\begin{array}{c}\text { Depth } \\
\text { L-kurtosis } \\
\text { (dimen- } \\
\text { sionless) }\end{array}$ & $\begin{array}{c}\text { Depth } \\
\text { Tau5 } \\
\text { (dimen- } \\
\text { sionless) }\end{array}$ \\
\hline 0015 & 0.16143 & 0.12905 & 0.79941 & 0.81255 & 0.70849 & 0.64207 & 1154 & 0.45795 & 0.31144 & 0.68006 & 0.53824 & 0.28282 & 0.16880 \\
\hline 0016 & .45129 & .29110 & .64505 & .49382 & .25124 & .15931 & 1165 & .47766 & .28672 & .60025 & .45850 & .22182 & .13145 \\
\hline 0050 & .58143 & .32808 & .56427 & .41163 & .19275 & .12250 & 1185 & .41264 & .22963 & .55650 & .43287 & .20710 & .11926 \\
\hline 0054 & .35962 & .21406 & .59523 & .44374 & .18470 & .08440 & 1186 & .53675 & .34684 & 64619 & .54719 & .32244 & .18867 \\
\hline 0120 & .90500 & .45067 & .49798 & .21676 & .02554 & -.00634 & 1188 & .39700 & .24167 & .60873 & .48966 & .27192 & .21287 \\
\hline 0145 & .42894 & .31151 & .72624 & .64928 & .45010 & .33903 & 1245 & .76815 & .45174 & .58809 & .40646 & .21405 & .21783 \\
\hline 0146 & .41636 & .22708 & .54539 & .32619 & .07886 & .01056 & 1246 & .60781 & .36015 & .59254 & .52588 & .26316 & .18460 \\
\hline 0174 & .37204 & .20304 & .54575 & .51982 & .28577 & .17755 & 1267 & .44657 & .29088 & .65137 & .56614 & .37211 & .28621 \\
\hline 0178 & .29682 & .18755 & .63188 & .57002 & .30733 & .17407 & 1304 & .56706 & .34893 & .61533 & .50300 & .28993 & .20435 \\
\hline 0179 & .31502 & .18332 & .58193 & .46825 & .21732 & .10430 & 1325 & .66800 & .41826 & .62614 & .50575 & .26896 & .16918 \\
\hline 0202 & .53717 & .30028 & .55900 & .49693 & .22461 & .12394 & 1429 & .58831 & .35667 & .60627 & .49930 & .27815 & .17917 \\
\hline 0206 & .63059 & .34887 & .55324 & .45146 & .22538 & .15401 & 1431 & .64616 & .38919 & .60231 & .47754 & .24437 & .14927 \\
\hline 0208 & -- & -- & -- & -- & -- & -- & 1432 & .64555 & .38974 & .60374 & .46162 & .23075 & .14068 \\
\hline 0211 & .35804 & .23554 & .65784 & .52636 & .28842 & .18619 & 1433 & .65412 & .38903 & .59474 & .47729 & .26321 & .16621 \\
\hline 0244 & .55125 & .30706 & .55703 & .33180 & .08285 & .04459 & 1434 & .63978 & .38112 & .59570 & .47233 & .24406 & .15227 \\
\hline 0248 & .40087 & .23004 & .57385 & .51966 & .29434 & .17576 & 1435 & .66285 & .39584 & .59718 & .45722 & .22615 & .13253 \\
\hline 0262 & .67199 & .39095 & .58178 & .45297 & .23572 & .15515 & 1436 & .66834 & .39695 & .59393 & .47264 & .25611 & .16629 \\
\hline 0271 & .92136 & .51539 & .55938 & .40585 & .20871 & .11058 & 1437 & .54870 & .40470 & .73757 & .61313 & .32704 & .16792 \\
\hline 0380 & .69692 & .44345 & .63631 & .53310 & .32043 & .22596 & 1438 & .64075 & .38446 & .60002 & .46675 & .23408 & .13862 \\
\hline 0394 & .56222 & .33278 & .59190 & .48390 & .38111 & .21846 & 1462 & -- & -- & -- & -- & -- & -- \\
\hline 0408 & 1.0915 & .57296 & .52494 & .31774 & .16430 & .12722 & 1492 & .56161 & .32915 & .58609 & .50515 & .26260 & .16568 \\
\hline 0427 & .55263 & .33947 & .61429 & .57153 & .28239 & .16999 & 1500 & .65519 & .37219 & .56807 & .35382 & .05419 & -.00250 \\
\hline 0428 & .49405 & .33577 & .67964 & .53670 & .28615 & .17995 & 1528 & .54585 & .33216 & .60851 & .53311 & .28735 & .17686 \\
\hline 0429 & .60420 & .40547 & .67109 & .52717 & .28968 & .19427 & 1541 & .74032 & .43165 & .58306 & .46682 & .19860 & .13581 \\
\hline 0463 & .52313 & .29680 & .56736 & .49974 & .30789 & .21163 & 1569 & .57195 & .38107 & .66627 & .56563 & .36439 & .27699 \\
\hline 0493 & .79176 & .31684 & .40017 & .25607 & .16762 & .07348 & 1632 & .47857 & .26095 & .54527 & .12044 & -.25730 & .08212 \\
\hline 0495 & .35584 & .20172 & .56689 & .45533 & .25707 & .17333 & 1641 & .45640 & .25184 & .55180 & .41393 & .19700 & .12920 \\
\hline 0496 & .24148 & .13943 & .57739 & .46253 & .25364 & .13889 & 1646 & .42683 & .24159 & .56600 & .52231 & .27831 & .16404 \\
\hline 0498 & .17750 & .05705 & .32138 & -.14263 & -.02169 & .19079 & 1663 & .98571 & .65017 & .65960 & .57720 & .32154 & .20063 \\
\hline 0509 & .62489 & .38060 & .60907 & .51808 & .29025 & .19091 & 1671 & .61846 & .37451 & .60556 & .50930 & .27122 & .16945 \\
\hline 0518 & .63643 & .37509 & .58936 & .48186 & .24994 & .16232 & 1680 & .60559 & .35968 & .59394 & .47650 & .26361 & .17471 \\
\hline 0521 & .51606 & .29314 & .56804 & .45243 & .24887 & .11467 & 1694 & .53493 & .29312 & .54795 & .44674 & .15656 & .09537 \\
\hline 0556 & .53225 & .30861 & .57983 & .47878 & .26197 & .16313 & 1696 & .48797 & .27932 & .57240 & .44126 & .22830 & .15306 \\
\hline 0569 & .70980 & .44806 & .63125 & .54109 & .30480 & .19473 & 1697 & .48153 & .28922 & .60063 & .50264 & .26964 & .14365 \\
\hline 0572 & .62958 & .39388 & .62562 & .51315 & .29092 & .19736 & 1698 & .47672 & .27769 & .58251 & .51092 & .28532 & .17834 \\
\hline 0576 & .50344 & .34736 & .68997 & .58793 & .36764 & .27724 & 1720 & .52454 & .32286 & .61551 & .58104 & .27160 & .12342 \\
\hline 0580 & .62749 & .40311 & .64242 & .55042 & .32855 & .21772 & 1761 & .33973 & .20388 & .60013 & .42749 & .19509 & .12248 \\
\hline 0587 & .66976 & .42027 & .62749 & .49246 & .26130 & .17778 & 1773 & .73112 & .41666 & .56989 & .45379 & .22678 & .14879 \\
\hline 0605 & .68592 & .34341 & .50066 & .38795 & .21192 & .13500 & 1810 & .43583 & .26261 & .60254 & .52146 & .34492 & .25093 \\
\hline 0639 & .58038 & .35493 & .61155 & .54921 & .30783 & .19795 & 1823 & .77043 & .44743 & .58075 & .34349 & .03860 & -.01276 \\
\hline 0655 & -- & -- & -- & -- & -- & -- & 1870 & .61994 & .35903 & .57913 & .41864 & .17374 & .07860 \\
\hline 0665 & .62306 & .38037 & .61049 & .47557 & .24635 & .15517 & 1875 & .95647 & .45897 & .47986 & .31407 & .09296 & .09516 \\
\hline 0689 & .60172 & .37554 & .62410 & .52978 & .30809 & .20182 & 1876 & .61776 & .36766 & .59516 & .44209 & .20161 & .10574 \\
\hline 0690 & .50626 & .29168 & .57614 & .54559 & .28534 & .19097 & 1889 & .53026 & .35447 & .66847 & .50743 & .25691 & .17116 \\
\hline 0691 & .59178 & .35389 & .59801 & .45546 & .22406 & .13677 & 1903 & .46218 & .24957 & .53999 & .49894 & .24544 & .18060 \\
\hline 0708 & .54296 & .31309 & .57663 & .55750 & .32969 & .23420 & 1914 & .78200 & .45795 & .58561 & .55040 & .39231 & .38574 \\
\hline 0738 & .63723 & .37040 & .58127 & .45686 & .24612 & .16416 & 1920 & .66586 & .38428 & .57713 & .46613 & .26941 & .20409 \\
\hline 0776 & .46576 & .28017 & .60154 & .51476 & .28818 & .17936 & 1921 & .71409 & .41177 & .57663 & .45614 & .23180 & .15172 \\
\hline 0779 & .44195 & .25229 & .57085 & .56257 & .28191 & .16635 & 1937 & .71938 & .40650 & .56508 & .42652 & .22590 & .15722 \\
\hline 0784 & .44381 & .26461 & .59623 & .53440 & .30092 & .17657 & 1956 & .67201 & .40360 & .60058 & .48670 & .26780 & .17886 \\
\hline 0786 & .36351 & .23707 & .65217 & .51651 & .27437 & .16832 & 1970 & 1.0913 & .74734 & .68484 & .59879 & .43039 & .36674 \\
\hline 0917 & .74279 & .44610 & .60057 & .47327 & .25477 & .16769 & 2014 & .45344 & .32836 & .72413 & .59092 & .32651 & .19473 \\
\hline 0923 & 1.0859 & .53425 & .49197 & .33561 & .12427 & .01746 & 2015 & .48713 & .34857 & .71556 & .59692 & .35767 & .23930 \\
\hline 0926 & .59960 & .35254 & .58797 & .47081 & .24168 & .14692 & 2019 & .87720 & .47200 & .53808 & .28423 & .05080 & .06433 \\
\hline 0950 & .28244 & .17189 & .60858 & .57900 & .40621 & .32166 & 2024 & .64673 & .36861 & .56995 & .45129 & .23976 & .15871 \\
\hline 0996 & .99458 & .60020 & .60347 & .48483 & .29877 & .23995 & 2042 & .15583 & .05795 & .37190 & -.03895 & -.06580 & .02876 \\
\hline 1013 & .55510 & .35892 & .64659 & .65418 & .41069 & .29594 & 2043 & .24628 & .14367 & .58337 & .45368 & .20791 & .10084 \\
\hline 1017 & .51942 & .30733 & .59167 & .49159 & .26487 & .16922 & 2048 & .52945 & .32986 & .62303 & .56520 & .31803 & .19023 \\
\hline 1042 & 1.2165 & .59082 & .48567 & .50431 & .34382 & .22325 & 2050 & .37957 & .28729 & .75689 & .66537 & .43603 & .29936 \\
\hline 1048 & .54259 & .28077 & .51746 & .30982 & .10305 & .04091 & 2051 & .47015 & .27176 & .57802 & .44790 & .22777 & .16273 \\
\hline 1053 & .52897 & .32266 & .60998 & .49211 & .26125 & .16124 & 2053 & .22500 & .14878 & .66123 & .63742 & .45402 & .31927 \\
\hline 1057 & .47928 & .27261 & .56879 & .45129 & .24397 & .15769 & 2073 & .61071 & .38210 & .62567 & .52260 & .30985 & .21775 \\
\hline 1063 & 1.0005 & .59535 & .59508 & .51198 & .36594 & .29763 & 2082 & .40179 & .23454 & .58373 & .50748 & .28743 & .17974 \\
\hline 1068 & .63746 & .36513 & .57279 & .45372 & .22985 & .14660 & 2086 & .63836 & .37021 & .57995 & .46404 & .23594 & .14967 \\
\hline 1080 & .34497 & .18448 & .53476 & .45828 & .28923 & .20703 & 2088 & .75306 & .41641 & .55296 & .37999 & .10573 & .10543 \\
\hline 1081 & .64451 & .37024 & .57446 & .43476 & .21597 & .13117 & 2090 & .61862 & .34960 & .56512 & .48258 & .23256 & .16572 \\
\hline 1133 & .23125 & .15967 & .69045 & .52699 & .22311 & .09873 & 2096 & .62319 & .35697 & .57281 & .46301 & .24188 & .16102 \\
\hline 1136 & .42228 & .30791 & .72917 & .61723 & .37628 & .24940 & 2128 & .64898 & .37648 & .58011 & .44257 & .22154 & .15064 \\
\hline 1138 & .82105 & .43509 & .52991 & .34853 & .17125 & .20881 & 2131 & .57184 & .32406 & .56670 & .47439 & .24114 & .15605 \\
\hline 1139 & .64371 & .38763 & .60218 & .44511 & .19822 & .09487 & 2142 & .98789 & .48532 & .49127 & .28339 & .09465 & .06287 \\
\hline
\end{tabular}


196 Statistical Characteristics of Storm Interevent Time, Depth, and Duration for Eastern New Mexico, Oklahoma, and Texas

Appendix 4-2.3. L-moments of storm depth defined by 12-hour minimum interevent time for hourly rainfall stations in Texas-

Continued.

\begin{tabular}{|c|c|c|c|c|c|c|c|c|c|c|c|c|c|}
\hline $\begin{array}{c}\text { Station } \\
\text { no. }\end{array}$ & $\begin{array}{c}\text { Depth } \\
\text { mean } \\
\text { (inches) }\end{array}$ & $\begin{array}{l}\text { Depth } \\
\text { L-scale } \\
\text { (inches) }\end{array}$ & $\begin{array}{c}\text { Depth } \\
\text { L-CV } \\
\text { (dimen- } \\
\text { sionless) }\end{array}$ & $\begin{array}{l}\text { Depth } \\
\text { L-skew } \\
\text { (dimen- } \\
\text { sionless) }\end{array}$ & $\begin{array}{c}\text { Depth } \\
\text { L-kurtosis } \\
\text { (dimen- } \\
\text { sionless) }\end{array}$ & $\begin{array}{c}\text { Depth } \\
\text { Tau5 } \\
\text { (dimen- } \\
\text { sionless) }\end{array}$ & $\begin{array}{c}\text { Station } \\
\text { no. }\end{array}$ & $\begin{array}{c}\text { Depth } \\
\text { mean } \\
\text { (inches) }\end{array}$ & $\begin{array}{c}\text { Depth } \\
\text { L-scale } \\
\text { (inches) }\end{array}$ & $\begin{array}{c}\text { Depth } \\
\text { L-CV } \\
\text { (dimen- } \\
\text { sionless) }\end{array}$ & $\begin{array}{c}\text { Depth } \\
\text { L-skew } \\
\text { (dimen- } \\
\text { sionless) }\end{array}$ & $\begin{array}{c}\text { Depth } \\
\text { L-kurtosis } \\
\text { (dimen- } \\
\text { sionless) }\end{array}$ & $\begin{array}{c}\text { Depth } \\
\text { Tau5 } \\
\text { (dimen- } \\
\text { sionless) }\end{array}$ \\
\hline 2160 & 0.43087 & 0.32411 & 0.75222 & 0.71941 & 0.61026 & 0.57505 & 3463 & 0.65021 & 0.41812 & 0.64305 & 0.51658 & 0.29446 & 0.20259 \\
\hline 2206 & .71822 & .43547 & .60631 & .49066 & .27650 & .18865 & 3476 & .58410 & .34957 & .59846 & .46830 & .24610 & .15357 \\
\hline 2238 & .33135 & .21630 & .65277 & .50259 & .23486 & .12016 & 3485 & .96591 & .62842 & .65060 & .50541 & .18597 & .09606 \\
\hline 2240 & .32851 & .20192 & .61467 & .48323 & .26214 & .15760 & 3507 & .63263 & .37431 & .59167 & .50526 & .23849 & .14583 \\
\hline 2242 & .55917 & .34753 & .62151 & .44882 & .20882 & .13170 & 3546 & .72581 & .42719 & .58857 & .46534 & .24410 & .15998 \\
\hline 2244 & .60364 & .37398 & .61954 & .48019 & .25069 & .15244 & 3547 & .64342 & .36110 & .56122 & .39326 & .15740 & .08370 \\
\hline 2247 & .52930 & .34553 & .65280 & .50036 & .25253 & .15667 & 3579 & .62987 & .37170 & .59012 & .44984 & .17253 & .05783 \\
\hline 2309 & .75811 & .40429 & .53329 & .41807 & .23788 & .16216 & 3642 & .67255 & .39580 & .58851 & .48389 & .26447 & .17560 \\
\hline 2312 & .66756 & .36025 & .53965 & .42275 & .19504 & .13765 & 3646 & .61222 & .35369 & .57771 & .45996 & .24973 & .16747 \\
\hline 2334 & .95317 & .52912 & .55511 & .41005 & .20774 & .10171 & 3668 & 1.2600 & .54347 & .43133 & .37830 & .15546 & .00173 \\
\hline 2336 & 63199 & .33779 & .53448 & .36028 & .14725 & .09400 & 3673 & .87040 & .48803 & .56070 & .26008 & -.02863 & -.00590 \\
\hline 2354 & .34741 & .18786 & .54076 & .37970 & .08268 & -.00345 & 3686 & .61973 & .35903 & .57933 & .48126 & .21617 & .12907 \\
\hline 2355 & .61722 & .42914 & .69528 & .59753 & .36538 & .23242 & 3691 & .60742 & .35211 & .57969 & .48066 & .24786 & .15502 \\
\hline 2357 & .39602 & .28781 & .72676 & .60722 & .35832 & .23523 & 3734 & 1.2275 & .76954 & .62691 & .43032 & .15685 & .06485 \\
\hline 2360 & .40153 & .28264 & .70393 & .58143 & .33332 & .21134 & 3771 & .61678 & .36190 & .58676 & .50819 & .23595 & .15185 \\
\hline 2361 & .48941 & .32639 & .66690 & .62773 & .45205 & .37071 & 3789 & .20771 & .11391 & .54842 & .47919 & .28933 & .18440 \\
\hline 2394 & .64610 & .38582 & .59716 & .46866 & .24726 & .15863 & 3826 & .58363 & .31391 & .53785 & .40861 & .20721 & .12027 \\
\hline 2404 & .61678 & .37039 & .60053 & .47772 & .25359 & .16578 & 3831 & .67470 & .39314 & .58268 & .46056 & .25205 & .17220 \\
\hline 2415 & .76228 & .43787 & .57442 & .44157 & .22745 & .14554 & 3841 & .66651 & .38328 & .57506 & .45726 & .24423 & .15098 \\
\hline 2462 & .70507 & .40265 & .57108 & .43926 & .21724 & .13682 & 3871 & .55103 & .32010 & .58092 & .46002 & .24614 & .16381 \\
\hline 2528 & .55897 & .32366 & .57903 & .44663 & .19628 & .08049 & 3884 & .87905 & .57290 & .65173 & .51916 & .24431 & .09439 \\
\hline 2617 & .48701 & .29634 & 60849 & .50883 & .30062 & .20745 & 3941 & .81375 & .47949 & .58923 & .38698 & .14497 & .10783 \\
\hline 2619 & .49705 & .27237 & .54798 & .44307 & .24953 & .17110 & 3963 & .07143 & .03619 & .50667 & 60000 & .43421 & .25000 \\
\hline 2621 & .51111 & .29366 & .57456 & .47034 & .24951 & .15448 & 4040 & .54384 & .30844 & .56715 & .44351 & .23179 & .14926 \\
\hline 2675 & .63087 & .37736 & .59817 & .51544 & .29102 & .19665 & 4058 & .79154 & .51197 & .64680 & .58065 & .43040 & .39313 \\
\hline 2676 & .61366 & .36056 & .58756 & .51022 & .23227 & .13740 & 4098 & .39255 & .21347 & .54379 & .50324 & .25589 & .14725 \\
\hline 2679 & .52370 & .33875 & .64685 & .57517 & .33183 & .19313 & 4100 & .52959 & .31614 & .59695 & .48804 & .27238 & .19034 \\
\hline 2715 & .60306 & .34301 & .56879 & .44327 & .23055 & .15028 & 4137 & .59677 & .33240 & .55700 & .47198 & .21853 & .15180 \\
\hline 2744 & .53495 & .30667 & .57327 & .46494 & .24168 & .15387 & 4191 & .57801 & .35784 & .61909 & .53459 & .29460 & .17790 \\
\hline 2758 & .44000 & .30874 & .70169 & .61251 & .36542 & .21129 & 4256 & -- & -- & -- & -- & -- & -- \\
\hline 2794 & .43091 & .29836 & .69241 & .50599 & .16870 & -.00965 & 4257 & .74200 & .42464 & .57230 & .45994 & .23314 & .15500 \\
\hline 2797 & .20932 & .13407 & .64048 & .52785 & .29689 & .19468 & 4258 & .63791 & .36231 & .56797 & .49072 & .25725 & .20023 \\
\hline 2811 & .50045 & .29640 & .59228 & .49900 & .26248 & .15131 & 4278 & .63350 & .37507 & .59207 & .45114 & .21889 & .13312 \\
\hline 2813 & .56667 & .31516 & .55616 & .45088 & .27980 & .13580 & 4299 & .33832 & .15895 & .46983 & .33319 & .14650 & .07384 \\
\hline 2814 & .21471 & .15750 & .73356 & 64893 & .35201 & .08353 & 4300 & .57164 & .38724 & .67741 & .54318 & .30771 & .20747 \\
\hline 2815 & .50931 & .28340 & .55644 & .52275 & .28467 & .20091 & 4305 & .54326 & .37362 & .68774 & .54955 & .30451 & .19990 \\
\hline 2818 & .59766 & .35063 & .58666 & .43966 & .22245 & .14878 & 4307 & .61158 & .41849 & .68429 & .54913 & .31912 & .22790 \\
\hline 2986 & .82090 & .45994 & .56029 & .38734 & .16333 & .10479 & 4309 & .68975 & .42747 & .61974 & .49222 & .26590 & .17588 \\
\hline 3005 & .58474 & .33214 & .56801 & .44820 & .21942 & .13795 & 4311 & .72724 & .44314 & .60934 & .47620 & .24820 & .15699 \\
\hline 3033 & .26944 & .14961 & .55527 & .49553 & .30138 & .19998 & 4313 & .75944 & .48489 & .63849 & .53904 & .31899 & .20546 \\
\hline 3034 & -- & -- & -- & -- & -- & -- & 4319 & .53857 & .30346 & .56346 & .41970 & .18417 & .10404 \\
\hline 3047 & .53235 & .31296 & .58788 & .40829 & .19968 & .16157 & 4329 & .71182 & .43107 & .60559 & .48784 & .27248 & .17655 \\
\hline 3103 & .75242 & .48805 & .64864 & .58730 & .32086 & .15765 & 4331 & -- & -- & -- & -- & -- & -- \\
\hline 3133 & .68451 & .39154 & .57200 & .44844 & .24132 & .16327 & 4375 & .59985 & .35960 & .59948 & .55183 & .30140 & .21324 \\
\hline 3156 & .76186 & .46437 & .60952 & .53678 & .31958 & .22496 & 4392 & .80797 & .48450 & .59965 & .46170 & .24445 & .16628 \\
\hline 3171 & .64516 & .37778 & .58556 & .46428 & .24207 & .15085 & 4425 & .41396 & .23723 & .57306 & .52309 & .27700 & .16172 \\
\hline 3189 & .33157 & .19393 & .58489 & .51964 & .30666 & .18460 & 4440 & .54600 & .32874 & .60210 & .47456 & .24874 & .16198 \\
\hline 3260 & .53633 & .31391 & .58530 & .42876 & .21074 & .13734 & 4476 & .58785 & .32894 & .55957 & .46025 & .22722 & .14858 \\
\hline 3267 & .43664 & .28795 & .65948 & .55226 & .32272 & .22065 & 4498 & .23909 & .11836 & .49506 & .23605 & .04506 & .00512 \\
\hline 3270 & .50084 & .28400 & .56704 & .51473 & .26043 & .17510 & 4517 & .60022 & .35072 & .58432 & .44972 & .23455 & .15241 \\
\hline 3272 & .11478 & .05858 & .51033 & .38944 & .16300 & .05834 & 4520 & .57657 & .33029 & .57284 & .50777 & .24716 & .16130 \\
\hline 3277 & .16200 & .10590 & .65373 & .56489 & .35010 & .31797 & 4525 & .72567 & .49748 & .68555 & .57199 & .31678 & .19170 \\
\hline 3278 & .43837 & .24954 & .56925 & .47917 & .25125 & .14151 & 4563 & .50250 & .29245 & .58198 & .41481 & .20251 & .17938 \\
\hline 3280 & .32658 & .20757 & .63558 & .53108 & .28899 & .16986 & 4570 & .51603 & .30267 & .58653 & .50001 & .27178 & .17020 \\
\hline 3281 & .35289 & .17029 & .48257 & .29522 & .09804 & .04112 & 4577 & .71114 & .40044 & .56309 & .44476 & .23258 & .15518 \\
\hline 3283 & .53999 & .34674 & .64213 & .49012 & .25567 & .16342 & 4591 & .64716 & .38237 & .59084 & .46401 & .24728 & .16029 \\
\hline 3284 & .59289 & .34760 & .58629 & .47361 & .24053 & .14099 & 4670 & .49760 & .29500 & .59285 & .49888 & .26816 & .16351 \\
\hline 3285 & .61897 & .35705 & .57685 & .48430 & .22769 & .14155 & 4671 & .41608 & .25513 & .61318 & .44591 & .20378 & .13226 \\
\hline 3329 & .56648 & .35985 & .63524 & .53357 & .31548 & .21734 & 4679 & 61539 & .36687 & .59615 & .51106 & .27029 & .18014 \\
\hline 3335 & .83365 & .51495 & .61770 & .51260 & .31174 & .22141 & 4696 & .34286 & .17374 & .50673 & .32321 & .12138 & .10465 \\
\hline 3370 & .70635 & .40366 & .57147 & .43447 & .22464 & .14466 & 4703 & .44620 & .26107 & .58509 & .48355 & .23768 & .11194 \\
\hline 3410 & .49702 & .27400 & .55128 & .47929 & .24837 & .16418 & 4704 & .81567 & .50671 & .62122 & .50501 & .28525 & .18575 \\
\hline 3415 & .61168 & .35651 & .58284 & .47604 & .25052 & .16318 & 4731 & .43524 & .30140 & .69250 & .59030 & .38960 & .30024 \\
\hline 3430 & .57651 & .39205 & .68004 & .54901 & .31630 & .21654 & 4792 & .59356 & .33194 & .55924 & .47381 & .21460 & .14263 \\
\hline 3431 & .58146 & .42198 & .72572 & .61565 & .38870 & .28251 & 4819 & .70660 & .38773 & .54873 & .43846 & .19369 & .12328 \\
\hline 3441 & .56756 & .34327 & .60481 & .47635 & .25388 & .14030 & 4852 & .88562 & .43246 & .48831 & .43799 & .36173 & .27439 \\
\hline 3442 & .40297 & .23379 & .58018 & .48824 & .26981 & .16738 & 4866 & .65867 & .37554 & .57015 & .44797 & .22646 & .14703 \\
\hline 3446 & .46630 & .25888 & .55519 & .44628 & .23181 & .14451 & 4876 & .78102 & .45895 & .58763 & .48930 & .23697 & .14733 \\
\hline 3460 & .72268 & .43112 & .59656 & .46500 & .29291 & .27538 & 4878 & .77095 & .46895 & .60828 & .48617 & .26503 & .17143 \\
\hline 3462 & .47910 & .25608 & .53450 & .41430 & .19217 & .10754 & 4880 & .44429 & .25197 & .56715 & .47727 & .25678 & .15928 \\
\hline
\end{tabular}


Appendix 4-2.3. L-moments of storm depth defined by 12-hour minimum interevent time for hourly rainfall stations in TexasContinued.

\begin{tabular}{|c|c|c|c|c|c|c|c|c|c|c|c|c|c|}
\hline $\begin{array}{c}\text { Station } \\
\text { no. }\end{array}$ & $\begin{array}{c}\text { Depth } \\
\text { mean } \\
\text { (inches) }\end{array}$ & $\begin{array}{c}\text { Depth } \\
\text { L-scale } \\
\text { (inches) }\end{array}$ & $\begin{array}{c}\text { Depth } \\
\text { L-CV } \\
\text { (dimen- } \\
\text { sionless) }\end{array}$ & $\begin{array}{c}\text { Depth } \\
\text { L-skew } \\
\text { (dimen- } \\
\text { sionless) }\end{array}$ & $\begin{array}{c}\text { Depth } \\
\text { L-kurtosis } \\
\text { (dimen- } \\
\text { sionless) }\end{array}$ & $\begin{array}{c}\text { Depth } \\
\text { Tau5 } \\
\text { (dimen- } \\
\text { sionless) }\end{array}$ & $\begin{array}{c}\text { Station } \\
\text { no. }\end{array}$ & $\begin{array}{c}\text { Depth } \\
\text { mean } \\
\text { (inches) }\end{array}$ & $\begin{array}{l}\text { Depth } \\
\text { L-scale } \\
\text { (inches) }\end{array}$ & $\begin{array}{c}\text { Depth } \\
\text { L-CV } \\
\text { (dimen- } \\
\text { sionless) }\end{array}$ & $\begin{array}{c}\text { Depth } \\
\text { L-skew } \\
\text { (dimen- } \\
\text { sionless) }\end{array}$ & $\begin{array}{c}\text { Depth } \\
\text { L-kurtosis } \\
\text { (dimen- } \\
\text { sionless) }\end{array}$ & $\begin{array}{c}\text { Depth } \\
\text { Tau5 } \\
\text { (dimen- } \\
\text { sionless) }\end{array}$ \\
\hline 4920 & 0.55790 & 0.34102 & 0.61126 & 0.52557 & 0.28264 & 0.16853 & 5957 & 0.60621 & 0.35072 & 0.57854 & 0.47865 & 0.24368 & 0.15759 \\
\hline 4934 & .36714 & .24857 & .67704 & .72874 & .63218 & .57854 & 5958 & .55683 & .31708 & .56943 & .39835 & .17331 & .09320 \\
\hline 4972 & .57252 & .33030 & .57692 & .47828 & .25845 & .17262 & 5973 & .43170 & .29738 & .68886 & .56476 & .29912 & .16703 \\
\hline 4973 & .78877 & .42110 & .53388 & .40540 & .22767 & .16567 & 5996 & .58372 & .34278 & .58723 & .48115 & .24694 & .15512 \\
\hline 4974 & .46310 & .27175 & .58681 & .49185 & .27505 & .18190 & 6017 & .42366 & .27389 & .64649 & .51247 & .25975 & .14360 \\
\hline 4975 & .72132 & .40183 & .55707 & .45240 & .21669 & .15223 & 6024 & .89760 & .55998 & .62386 & .48749 & .26730 & .19383 \\
\hline 4978 & .58888 & .37590 & .63833 & .51259 & .27771 & .16848 & 6050 & .66700 & .31868 & .47779 & .30559 & .01394 & -.08970 \\
\hline 4979 & .91667 & .52581 & .57361 & .46734 & .28798 & .17044 & 6104 & .35623 & .20056 & .56301 & .49583 & .28551 & .17778 \\
\hline 4982 & .53420 & .30903 & .57849 & .46029 & .24676 & .15941 & 6108 & .75986 & .43071 & .56683 & .44402 & .22375 & .14620 \\
\hline 5018 & .60575 & .33944 & .56037 & .41482 & .19654 & .12908 & 6136 & .38978 & .21386 & .54868 & .49764 & .25826 & .15094 \\
\hline 5048 & .45312 & .27348 & .60355 & .55392 & .31091 & .19020 & 6166 & .37880 & .23154 & .61123 & .50281 & .24763 & .13020 \\
\hline 5049 & .42424 & .23843 & .56200 & .59627 & .38241 & .31264 & 6176 & .73131 & .44628 & .61024 & .49030 & .27808 & .17994 \\
\hline 5056 & .43200 & .18600 & .43056 & .26882 & .67742 & .11828 & 6177 & .73907 & .43223 & .58482 & .47611 & .25207 & .16455 \\
\hline 5057 & .34685 & .24742 & .71332 & .60966 & .36965 & .23458 & 6210 & .66696 & .38885 & .58302 & .47332 & .24322 & .15781 \\
\hline 5060 & .54554 & .38362 & .70320 & .59089 & .35732 & .24606 & 6211 & .63520 & .38851 & .61163 & .46479 & .23232 & .14700 \\
\hline 5081 & .68286 & .39070 & .57216 & .41996 & .20647 & .13833 & 6270 & .74451 & .42608 & .57229 & .47845 & .24304 & .16873 \\
\hline 5094 & .65107 & .37798 & .58054 & .47101 & .24168 & .15097 & 6275 & -- & -- & -- & -- & -- & -- \\
\hline 5113 & .57807 & .36279 & .62760 & .54808 & .31159 & .19577 & 6276 & .94125 & .48766 & .51810 & .28786 & .06129 & .03673 \\
\hline 5114 & -- & -- & -- & -- & -- & -- & 6335 & .66402 & .37982 & .57200 & .43331 & .21671 & .14067 \\
\hline 5123 & .65133 & .40114 & 61588 & .56622 & .34537 & .13957 & 6434 & .59316 & .33304 & .56147 & .38501 & .12317 & .09667 \\
\hline 5192 & .67071 & .39392 & .58732 & .46726 & .24272 & .14888 & 6504 & .43757 & .25327 & .57880 & .51380 & .27903 & .16758 \\
\hline 5193 & .61816 & .37378 & .60466 & .49072 & .25302 & .15593 & 6558 & .61700 & .36033 & .58401 & .38924 & .14039 & .14724 \\
\hline 5224 & .74911 & .45137 & .60254 & .45881 & .23770 & .15060 & 6615 & .51704 & .30625 & .59231 & .57186 & .33143 & .23739 \\
\hline 5228 & .60088 & .36349 & 60493 & .44652 & .19722 & .10760 & 6660 & .63393 & .37057 & .58456 & .43745 & .21262 & .13198 \\
\hline 5235 & .54833 & .34417 & .62767 & .46800 & .22067 & .15210 & 6663 & .51534 & .31139 & .60424 & .46915 & .22971 & .10018 \\
\hline 5247 & .44904 & .25136 & .55976 & .48411 & .25125 & .14700 & 6734 & .46053 & .27556 & .59834 & .48063 & .26275 & .16918 \\
\hline 5258 & .64784 & .37852 & .58428 & .45136 & .22708 & .13542 & 6736 & .48322 & .28436 & .58847 & .52295 & .28205 & .16979 \\
\hline 5303 & .58266 & .37072 & .63626 & .53840 & .31839 & .23175 & 6740 & 1.3020 & 69067 & .53047 & .28977 & .04037 & -.06832 \\
\hline 5312 & .55030 & .32100 & .58333 & .51106 & .26515 & .17449 & 6750 & .67959 & .47296 & .69594 & .58005 & .36184 & .25795 \\
\hline 5341 & .95963 & .59707 & .62218 & .55221 & .36238 & .25745 & 6757 & .65268 & .39363 & .60310 & .46396 & .23803 & .15273 \\
\hline 5342 & -- & -- & -- & -- & -- & -- & 6775 & .42834 & .25665 & .59917 & .46809 & .24944 & .16117 \\
\hline 5348 & .72938 & .40953 & .56148 & .43579 & .19252 & .12008 & 6776 & .44429 & .24821 & .55866 & .48733 & .25994 & .15658 \\
\hline 5358 & .51323 & .30160 & .58764 & .47250 & .24164 & .13929 & 6788 & .72855 & .40662 & .55811 & .42184 & .23631 & .15500 \\
\hline 5398 & .68235 & .40207 & .58925 & .46208 & .23924 & .15408 & 6792 & .35232 & .19322 & .54843 & .48636 & .25989 & .14026 \\
\hline 5410 & .44991 & .25953 & .57684 & .50405 & .28742 & .18705 & 6794 & 1.2356 & .65647 & .53129 & .37400 & .14830 & .04740 \\
\hline 5411 & .36402 & .24008 & .65952 & .52489 & .28528 & .18429 & 6834 & .74166 & .41820 & .56387 & .45812 & .21891 & .15278 \\
\hline 5424 & .63606 & .42780 & .67258 & .51357 & .26322 & .17084 & 6893 & .33657 & .18676 & .55489 & .55179 & .33434 & .21392 \\
\hline 5429 & .57377 & .36646 & .63868 & .51650 & .27962 & .17690 & 6935 & .44197 & .24779 & .56065 & .52618 & .30362 & .20257 \\
\hline 5431 & .90375 & .47725 & .52808 & .45918 & .26568 & .11987 & 6981 & .63026 & .36232 & .57487 & .45840 & .25344 & .16763 \\
\hline 5461 & .74350 & .43723 & .58807 & .45232 & .23483 & .15204 & 7020 & .67740 & .40866 & .60328 & .48639 & .29543 & .22130 \\
\hline 5463 & .69488 & .40260 & .57938 & .48912 & .23822 & .16119 & 7060 & .51124 & .28890 & .56509 & .48536 & .24602 & .14582 \\
\hline 5471 & .16680 & .11500 & .68945 & .67924 & .50136 & .37977 & 7066 & .72905 & .41858 & .57414 & .44452 & .21826 & .13573 \\
\hline 5477 & .71458 & .48868 & .68386 & .74244 & .65843 & .59902 & 7074 & .42747 & .24958 & .58386 & .51795 & .30507 & .19955 \\
\hline 5528 & .72100 & .40587 & .56293 & .43276 & .22321 & .14237 & 7097 & .60279 & .35793 & .59379 & .50503 & .28137 & .15921 \\
\hline 5579 & -- & -- & -- & -- & -- & -- & 7116 & .44975 & .26128 & .58094 & .45724 & .24088 & .16142 \\
\hline 5580 & .57852 & .29476 & .50950 & .29608 & .10343 & .06746 & 7140 & .69616 & .45537 & .65411 & .55134 & .32681 & .21929 \\
\hline 5589 & .28895 & .16246 & .56223 & .39308 & .16693 & .09547 & 7173 & .69484 & .48964 & .70468 & .57491 & .33452 & .22413 \\
\hline 5590 & .34047 & .20189 & .59297 & .50349 & .28067 & .16859 & 7174 & .66183 & .44715 & .67563 & .53947 & .30819 & .21320 \\
\hline 5591 & .35226 & .18544 & .52643 & .41346 & .21164 & .12931 & 7213 & .64771 & .38005 & .58677 & .45846 & .24293 & .15435 \\
\hline 5592 & .31633 & .17982 & .56845 & .46962 & .25758 & .16783 & 7243 & .53950 & .31347 & .58105 & .49496 & .26346 & .17258 \\
\hline 5594 & .31795 & .18192 & .57217 & .47122 & .24571 & .14634 & 7262 & .19078 & .10951 & .57400 & .43768 & .21000 & .11324 \\
\hline 5595 & -- & -- & -- & -- & -- & -- & 7274 & .66833 & .36328 & .54357 & .44510 & .25046 & .16861 \\
\hline 5596 & .37483 & .19452 & .51897 & .52049 & .25102 & .15500 & 7300 & .58885 & .34342 & .58320 & .44781 & .22846 & .14830 \\
\hline 5600 & .36809 & .21407 & .58156 & .50132 & .29722 & .19172 & 7311 & .63833 & .35967 & .56345 & .51850 & .27762 & .15910 \\
\hline 5618 & .61030 & .34371 & .56318 & .41042 & .14197 & .04799 & 7363 & .60000 & .30211 & .50351 & .21687 & .00838 & .06075 \\
\hline 5650 & .57053 & .25713 & .45070 & .35194 & .29942 & .11496 & 7422 & .59120 & .36132 & .61117 & .51462 & .28644 & .18749 \\
\hline 5656 & .47169 & .26812 & .56842 & .54015 & .28278 & .18709 & 7431 & .44167 & .25867 & .58566 & .50374 & .27909 & .17483 \\
\hline 5658 & .47317 & .28667 & .60585 & .50500 & .28685 & .18966 & 7481 & .35839 & .21637 & .60372 & .56428 & .35652 & .23074 \\
\hline 5661 & .58778 & .38030 & .64702 & .64901 & .41615 & .30700 & 7497 & .61326 & .35805 & .58386 & .51478 & .25776 & .17275 \\
\hline 5666 & .48229 & .28176 & .58423 & .47543 & .26354 & .17689 & 7498 & .62810 & .36697 & .58425 & .50959 & .26564 & .19588 \\
\hline 5695 & .67400 & .38381 & .56945 & .44036 & .23170 & .15618 & 7499 & .58092 & .32815 & .56489 & .48349 & .24727 & .16679 \\
\hline 5742 & .27684 & .15772 & .56971 & .47916 & .22896 & .15492 & 7531 & .61446 & .34354 & .55909 & .40298 & .17424 & .08837 \\
\hline 5766 & .81067 & .50441 & .62222 & .48576 & .20481 & .05951 & 7534 & .62241 & .38714 & .62201 & .52607 & .32238 & .24118 \\
\hline 5770 & .47654 & .27552 & .57817 & .49627 & .26966 & .16382 & 7556 & .61674 & .36224 & .58735 & .50038 & .26629 & .17541 \\
\hline 5775 & .37308 & .20038 & .53711 & .25982 & -.10434 & -.01392 & 7594 & .72191 & .42456 & .58810 & .45674 & .23869 & .15682 \\
\hline 5779 & .76105 & .42322 & .55609 & .44407 & .19698 & .03313 & 7596 & .71808 & .42760 & .59547 & .40817 & .15424 & .08522 \\
\hline 5840 & .62355 & .36974 & .59296 & .45394 & .21902 & .12773 & 7608 & .57530 & .36168 & .62868 & .48192 & .24613 & .15629 \\
\hline 5890 & .34013 & .22639 & .66561 & .54185 & .30379 & .19499 & 7622 & .22227 & .17790 & .80037 & .72060 & .47714 & .32193 \\
\hline 5891 & .39453 & .20974 & .53163 & .38432 & .16298 & .09964 & 7700 & .66846 & .41115 & .61507 & .47594 & .24481 & .15360 \\
\hline 5897 & .64537 & .37772 & .58527 & .50166 & .24486 & .16814 & 7706 & .51528 & .30817 & .59807 & .50919 & .27933 & .17442 \\
\hline
\end{tabular}


198 Statistical Characteristics of Storm Interevent Time, Depth, and Duration for Eastern New Mexico, Oklahoma, and Texas

Appendix 4-2.3. L-moments of storm depth defined by 12-hour minimum interevent time for hourly rainfall stations in TexasContinued.

\begin{tabular}{|c|c|c|c|c|c|c|c|c|c|c|c|c|c|}
\hline $\begin{array}{c}\text { Station } \\
\text { no. }\end{array}$ & $\begin{array}{c}\text { Depth } \\
\text { mean } \\
\text { (inches) }\end{array}$ & $\begin{array}{c}\text { Depth } \\
\text { L-scale } \\
\text { (inches) }\end{array}$ & $\begin{array}{c}\text { Depth } \\
\text { L-CV } \\
\text { (dimen- } \\
\text { sionless) }\end{array}$ & $\begin{array}{l}\text { Depth } \\
\text { L-skew } \\
\text { (dimen- } \\
\text { sionless) }\end{array}$ & $\begin{array}{c}\text { Depth } \\
\text { L-kurtosis } \\
\text { (dimen- } \\
\text { sionless) }\end{array}$ & $\begin{array}{c}\text { Depth } \\
\text { Tau5 } \\
\text { (dimen- } \\
\text { sionless) }\end{array}$ & $\begin{array}{c}\text { Station } \\
\text { no. }\end{array}$ & $\begin{array}{c}\text { Depth } \\
\text { mean } \\
\text { (inches) }\end{array}$ & $\begin{array}{l}\text { Depth } \\
\text { L-scale } \\
\text { (inches) }\end{array}$ & $\begin{array}{c}\text { Depth } \\
\text { L-CV } \\
\text { (dimen- } \\
\text { sionless) }\end{array}$ & $\begin{array}{l}\text { Depth } \\
\text { L-skew } \\
\text { (dimen- } \\
\text { sionless) }\end{array}$ & $\begin{array}{c}\text { Depth } \\
\text { L-kurtosis } \\
\text { (dimen- } \\
\text { sionless) }\end{array}$ & $\begin{array}{c}\text { Depth } \\
\text { Tau5 } \\
\text { (dimen- } \\
\text { sionless) }\end{array}$ \\
\hline 7718 & 0.62363 & 0.37990 & 0.60918 & 0.50647 & 0.27617 & 0.15690 & 8910 & 0.70650 & 0.38166 & 0.54021 & 0.29665 & 0.03253 & -0.04321 \\
\hline 7745 & .73919 & .44685 & .60452 & .46432 & .24655 & .17008 & 8911 & .53093 & .33805 & .63671 & .49564 & .25746 & .16095 \\
\hline 7922 & .25295 & .14749 & .58308 & .47230 & .26279 & .16360 & 8924 & .31305 & .16579 & .52959 & .42882 & .21894 & .11763 \\
\hline 7936 & .73929 & .43138 & .58351 & .46889 & .24760 & .17162 & 8929 & .95091 & .53580 & .56346 & .31309 & .01221 & -.01702 \\
\hline 7943 & .39430 & .26215 & .66484 & .52733 & .28011 & .17310 & 8942 & .72748 & .42560 & .58503 & .48291 & .24070 & .15344 \\
\hline 7944 & .40500 & .27489 & .67875 & .53795 & .28089 & .15345 & 8944 & .62032 & .38178 & .61546 & .47001 & .24150 & .15312 \\
\hline 7945 & 46749 & .32725 & .70001 & .57110 & .32855 & .22116 & 8996 & .61313 & .38117 & .62167 & .49774 & .27170 & .17886 \\
\hline 7947 & 69036 & .43513 & .63029 & .57133 & .35031 & .25518 & 9014 & .71174 & .34079 & .47881 & .28837 & .08279 & .01061 \\
\hline 7948 & .56975 & .36369 & .63832 & .51699 & .27926 & .16905 & 9037 & .33576 & .20162 & .60050 & .54318 & .34737 & .24545 \\
\hline 7951 & 67097 & .37469 & .55842 & .41662 & .20176 & .12160 & 9106 & .30259 & .19196 & .63439 & .58547 & .38556 & .28438 \\
\hline 7953 & .46752 & .29252 & .62569 & .51620 & .28361 & .16461 & 9107 & .37057 & .22343 & .60293 & .44933 & .18851 & .12487 \\
\hline 7981 & .54530 & .33930 & .62223 & .47475 & .21045 & .09804 & 9129 & .37239 & .25312 & .67973 & .59657 & .34988 & .21559 \\
\hline 7990 & .48285 & .33224 & .68808 & .58336 & .35628 & .23804 & 9163 & .56010 & .32661 & .58312 & .47972 & .25668 & .16205 \\
\hline 7992 & .70478 & .39233 & .55667 & .38940 & .07829 & -.04344 & 9213 & .55661 & .34733 & .62401 & .46529 & .22988 & .14650 \\
\hline 7997 & .47692 & .29346 & .61534 & .49299 & .25297 & .13565 & 9214 & .92900 & .53861 & .57977 & .48620 & .26373 & .14435 \\
\hline 7999 & .32737 & .21971 & .67113 & .55778 & .34309 & .24567 & 9222 & .55910 & .35131 & .62835 & .47734 & .22977 & .13098 \\
\hline 8022 & .43529 & .26711 & .61363 & .46970 & .18391 & .06443 & 9248 & .45297 & .25756 & .56860 & .44349 & .19634 & .09899 \\
\hline 8023 & .47157 & .27763 & .58874 & .52191 & .29585 & .18908 & 9266 & .56176 & .31679 & .56393 & .41736 & .16439 & .05934 \\
\hline 8047 & .59280 & .34175 & .57650 & .45761 & .22568 & .13831 & 9270 & .34029 & .18393 & .54051 & .53295 & .29670 & .17036 \\
\hline 8060 & .46492 & .31292 & .67305 & .55543 & .28779 & .14083 & 9295 & .21638 & .13544 & .62595 & .53388 & .32075 & .21735 \\
\hline 8062 & .68512 & .50527 & .73749 & .61684 & .35777 & .20157 & 9304 & -- & -- & -- & -- & -- & -- \\
\hline 8068 & .28000 & .18357 & .65561 & .55825 & .32289 & .21352 & 9307 & .58953 & .32006 & .54291 & .43908 & .24104 & .15580 \\
\hline 8081 & .57931 & .36511 & .63025 & .55060 & .32392 & .21493 & 9328 & .43921 & .24796 & .56456 & .42114 & .18629 & .10713 \\
\hline 8089 & .49725 & .28806 & .57930 & .38787 & .13470 & .05739 & 9329 & .48333 & .26990 & .55842 & .24971 & -.14014 & -.12035 \\
\hline 8221 & .73737 & .33076 & .44857 & .32655 & .24033 & .14816 & 9345 & -- & -- & -- & -- & -- & -- \\
\hline 8252 & .45668 & .26402 & .57813 & .49875 & .26929 & .16201 & 9363 & .44605 & .30707 & .68841 & .56564 & .32752 & .21621 \\
\hline 8265 & .70119 & 44917 & .64059 & .53230 & .31432 & .21967 & 9364 & .51661 & .36071 & .69822 & .57597 & .33941 & .22995 \\
\hline 8289 & .47547 & .22080 & .46438 & .20473 & .03851 & .02900 & 9365 & .32794 & .20937 & .63843 & .64646 & .45802 & .32395 \\
\hline 8305 & .30023 & .16541 & .55093 & .52146 & .31434 & .18674 & 9371 & .60136 & .34870 & .57986 & .43093 & .17020 & .07561 \\
\hline 8335 & .75674 & .43293 & .57211 & .42973 & .22039 & .14638 & 9417 & .60266 & .36076 & .59861 & .45291 & .21196 & .12015 \\
\hline 8400 & .38236 & .22631 & .59187 & .51518 & .32541 & .23243 & 9419 & .51197 & .33254 & .64953 & .49057 & .23927 & .14476 \\
\hline 8445 & .64720 & .40780 & .63010 & .50930 & .27871 & .18153 & 9435 & .47961 & .27509 & .57356 & .48933 & .31105 & .25907 \\
\hline 8446 & .58258 & .34374 & .59003 & .49069 & .24638 & .13880 & 9491 & .64514 & .38378 & .59489 & .47535 & .24107 & .14633 \\
\hline 8451 & .47876 & .28661 & .59865 & .48632 & .25751 & .15968 & 9499 & .50609 & .29050 & .57401 & .49036 & .23643 & .13460 \\
\hline 8531 & 61998 & .35486 & .57237 & .44780 & .23555 & .15275 & 9522 & 1.1053 & .65467 & .59228 & .33090 & -.02320 & -.10442 \\
\hline 8541 & .61931 & .36195 & .58443 & .44061 & .22410 & .14133 & 9527 & .43156 & .24134 & .55921 & .51677 & .26947 & .15991 \\
\hline 8544 & .66632 & .40398 & .60629 & .51507 & .27068 & .16957 & 9532 & .59205 & .34592 & .58427 & .47496 & .25004 & .16280 \\
\hline 8545 & .30150 & .14513 & .48137 & .18102 & .02057 & .10041 & 9544 & 1.2500 & 1.1260 & .90080 & .96803 & .95560 & .90586 \\
\hline 8563 & .66514 & .37909 & .56993 & .48410 & .23956 & .16875 & 9565 & .53229 & .31120 & .58464 & .50053 & .27578 & .17350 \\
\hline 8566 & .47794 & .28321 & .59257 & .45594 & .22346 & .12987 & 9570 & .48227 & .26989 & .55962 & .52858 & .27245 & .17182 \\
\hline 8583 & .51361 & .29314 & .57075 & .51189 & .22206 & .12326 & 9574 & .52727 & .29654 & .56240 & .47124 & .17610 & .10626 \\
\hline 8584 & 60137 & .34518 & .57398 & .46131 & .22504 & .14164 & 9588 & .50430 & .31869 & .63195 & .55598 & .32065 & .18919 \\
\hline 8623 & .61168 & .33934 & .55476 & .45519 & .21949 & .13639 & 9665 & .67306 & .39277 & .58356 & .46734 & .24250 & .15947 \\
\hline 8625 & .59015 & .35337 & .59878 & .47824 & .25855 & .17632 & 9715 & .61448 & .35738 & .58159 & .45758 & .22814 & .14599 \\
\hline 8630 & .47249 & .26728 & .56568 & .46251 & .22513 & .12361 & 9729 & .50245 & .31989 & .63667 & .48142 & .24067 & .15324 \\
\hline 8631 & .52617 & .31059 & .59028 & .48636 & .26775 & .16861 & 9772 & .62617 & .40819 & .65188 & .54281 & .31857 & .21594 \\
\hline 8646 & .59940 & .35331 & .58945 & .45769 & .23245 & .14706 & 9814 & .61371 & .33995 & .55392 & .36670 & .13471 & .06075 \\
\hline 8647 & .42753 & .24558 & .57442 & .52599 & .28579 & .17707 & 9815 & .64448 & .38754 & .60132 & .50556 & .28781 & .19201 \\
\hline 8677 & .55856 & .30735 & .55026 & .43553 & .23015 & .14235 & 9816 & .60757 & .39459 & .64947 & .53566 & .31004 & .20258 \\
\hline 8696 & .73654 & .39352 & .53429 & .33496 & .14206 & .10838 & 9817 & .56603 & .31881 & .56324 & .47962 & .24190 & .16055 \\
\hline 8743 & .72718 & .42783 & .58835 & .46408 & .24177 & .15671 & 9829 & .36309 & .21560 & .59379 & .54755 & .32740 & .19436 \\
\hline 8761 & .41706 & .23354 & .55997 & .51529 & .29004 & .18452 & 9830 & .28564 & .17306 & .60586 & .49262 & .24280 & .12822 \\
\hline 8778 & .67237 & .38818 & .57732 & .47228 & .23906 & .15974 & 9858 & .46521 & .26192 & .56302 & .45329 & .24480 & .15337 \\
\hline 8845 & .62282 & .38368 & .61603 & .52576 & .28691 & .18012 & 9893 & .56249 & .32820 & .58347 & .47486 & .25342 & .16364 \\
\hline 8859 & .72326 & .41024 & .56721 & .42451 & .21292 & .14054 & 9916 & .73930 & .41484 & .56114 & .42949 & .19162 & .11593 \\
\hline 8898 & .70381 & .41479 & .58935 & .47114 & .25962 & .16793 & 9976 & .51503 & .31647 & .61447 & .53111 & .29758 & .18359 \\
\hline 8908 & .61639 & .39106 & .63443 & .52859 & .31249 & .23155 & & & & & & & \\
\hline
\end{tabular}


Appendix 4-2.4. L-moments of storm depth defined by 18-hour minimum interevent time for hourly rainfall stations in Texas.

[--, not available]

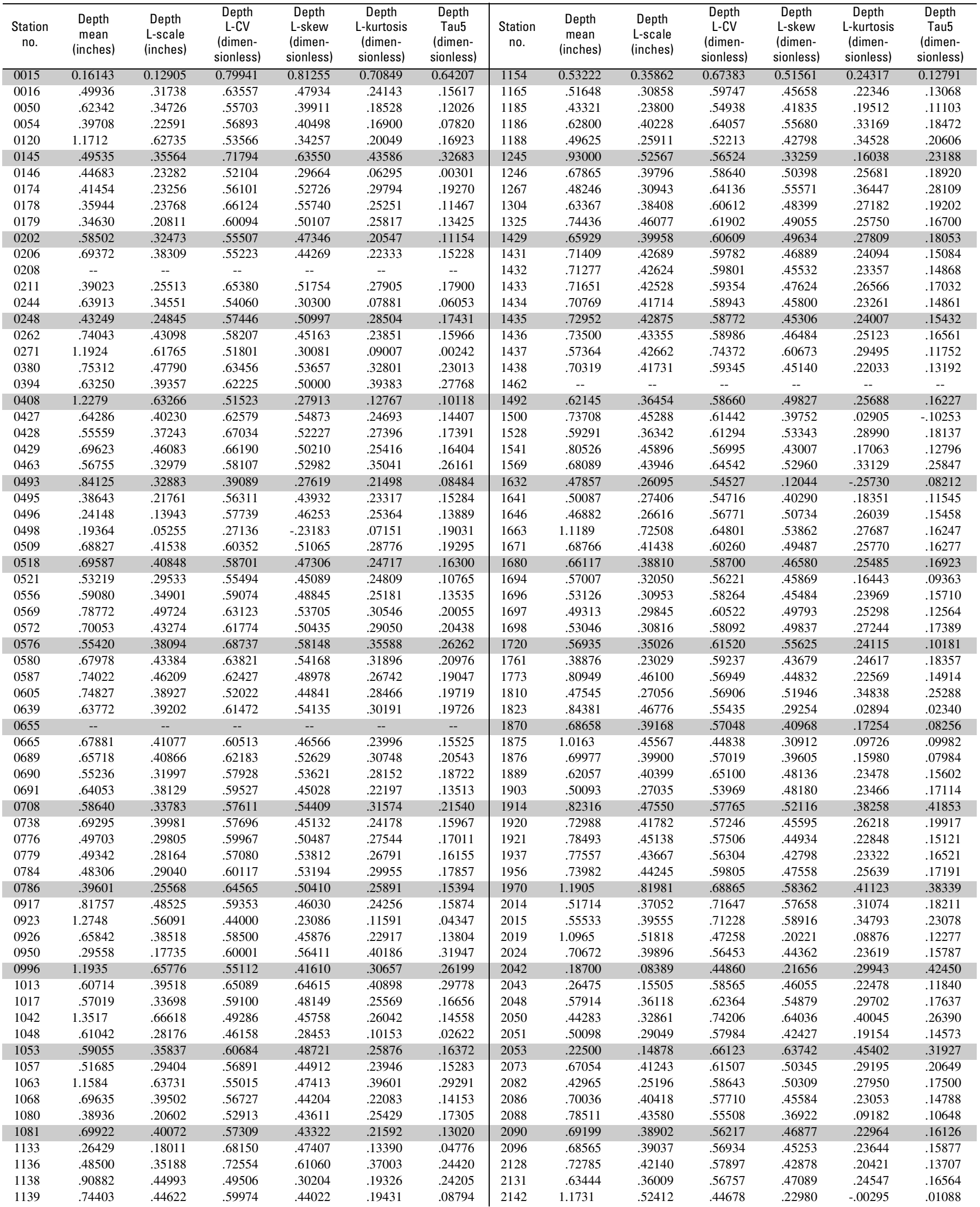


200 Statistical Characteristics of Storm Interevent Time, Depth, and Duration for Eastern New Mexico, Oklahoma, and Texas

Appendix 4-2.4. L-moments of storm depth defined by 18-hour minimum interevent time for hourly rainfall stations in Texas-

Continued.

\begin{tabular}{|c|c|c|c|c|c|c|c|c|c|c|c|c|c|}
\hline $\begin{array}{c}\text { Station } \\
\text { no. }\end{array}$ & $\begin{array}{c}\text { Depth } \\
\text { mean } \\
\text { (inches) }\end{array}$ & $\begin{array}{l}\text { Depth } \\
\text { L-scale } \\
\text { (inches) }\end{array}$ & $\begin{array}{c}\text { Depth } \\
\text { L-CV } \\
\text { (dimen- } \\
\text { sionless) }\end{array}$ & $\begin{array}{c}\text { Depth } \\
\text { L-skew } \\
\text { (dimen- } \\
\text { sionless) }\end{array}$ & $\begin{array}{c}\text { Depth } \\
\text { L-kurtosis } \\
\text { (dimen- } \\
\text { sionless) }\end{array}$ & $\begin{array}{c}\text { Depth } \\
\text { Tau5 } \\
\text { (dimen- } \\
\text { sionless) }\end{array}$ & $\begin{array}{c}\text { Station } \\
\text { no. }\end{array}$ & $\begin{array}{c}\text { Depth } \\
\text { mean } \\
\text { (inches) }\end{array}$ & $\begin{array}{l}\text { Depth } \\
\text { L-scale } \\
\text { (inches) }\end{array}$ & $\begin{array}{c}\text { Depth } \\
\text { L-CV } \\
\text { (dimen- } \\
\text { sionless) }\end{array}$ & $\begin{array}{c}\text { Depth } \\
\text { L-skew } \\
\text { (dimen- } \\
\text { sionless) }\end{array}$ & $\begin{array}{c}\text { Depth } \\
\text { L-kurtosis } \\
\text { (dimen- } \\
\text { sionless) }\end{array}$ & $\begin{array}{c}\text { Depth } \\
\text { Tau5 } \\
\text { (dimen- } \\
\text { sionless) }\end{array}$ \\
\hline 2160 & 0.45000 & 0.33403 & 0.74228 & 0.71862 & 0.61626 & 0.57945 & 3463 & 0.70932 & 0.44053 & 0.62107 & 0.48674 & 0.28150 & 0.20110 \\
\hline 2206 & .78211 & .47190 & 60336 & .47707 & .25683 & .17075 & 3476 & .64406 & .38368 & .59573 & .46722 & .25131 & .16317 \\
\hline 2238 & .38128 & .25625 & 67209 & .53505 & .27025 & .14546 & 3485 & .96591 & .62842 & .65060 & .50541 & .18597 & .09606 \\
\hline 2240 & .38012 & .23150 & 60900 & .44642 & .21203 & .12661 & 3507 & 69946 & .41581 & .59447 & .49665 & .23231 & .13915 \\
\hline 2242 & .61679 & .37931 & 61497 & .44294 & .21028 & .13638 & 3546 & .79762 & .46582 & .58401 & .46036 & .24634 & .16488 \\
\hline 2244 & .67507 & .41315 & .61202 & .46866 & .24250 & .15029 & 3547 & .67693 & .38206 & .56440 & .39620 & .16046 & .08682 \\
\hline 2247 & .62854 & .40377 & .64240 & .47446 & .22532 & .14616 & 3579 & .68310 & .40151 & .58778 & .44269 & .15630 & .04555 \\
\hline 2309 & .84109 & .44106 & .52439 & .40951 & .24009 & .16688 & 3642 & .73845 & .43271 & .58598 & .47724 & .26150 & .17541 \\
\hline 2312 & .74674 & .39975 & .53532 & .40971 & .19314 & .13703 & 3646 & .66771 & .38609 & .57824 & .46124 & .25302 & .17111 \\
\hline 2334 & 1.0398 & .56753 & .54580 & .41381 & .21173 & .08727 & 3668 & 1.4000 & .57549 & .41106 & .35379 & .17968 & .01222 \\
\hline 2336 & .68668 & .36071 & .52529 & .35760 & .15383 & .10097 & 3673 & .98909 & .51169 & .51733 & .18109 & -.05047 & .01439 \\
\hline 2354 & .37520 & .20797 & .55428 & .38997 & .09673 & .02118 & 3686 & .68709 & .39509 & .57501 & .46857 & .21903 & .14286 \\
\hline 2355 & .66283 & .46298 & 69849 & .58296 & .34559 & .23706 & 3691 & .66916 & .38669 & .57788 & .47038 & .24314 & .15613 \\
\hline 2357 & .43511 & .31545 & .72499 & 60050 & .34917 & .22557 & 3734 & 1.4943 & .90830 & .60782 & .37302 & .08651 & .01332 \\
\hline 2360 & .45542 & .31834 & .69899 & .57211 & .32578 & .21090 & 3771 & .67425 & .39771 & .58986 & .49873 & .22948 & .14800 \\
\hline 2361 & .53547 & .36255 & .67706 & 63701 & .45893 & .37479 & 3789 & .22719 & .12464 & .54864 & .45432 & .26205 & .17180 \\
\hline 2394 & .70467 & .41786 & .59298 & .46105 & .24162 & .15455 & 3826 & 60951 & .32871 & .53931 & .40733 & .21289 & .13222 \\
\hline 2404 & .67257 & .40006 & .59482 & .46938 & .25131 & .17004 & 3831 & .75342 & .43918 & .58291 & .45707 & .24406 & .15719 \\
\hline 2415 & .83535 & .47673 & .57069 & .43892 & .22972 & .14992 & 3841 & .71851 & .41517 & .57782 & .45463 & .23935 & .14953 \\
\hline 2462 & .77732 & .44052 & .56671 & .43238 & .21361 & .13743 & 3871 & .60316 & .34783 & .57669 & .45466 & .24441 & .16255 \\
\hline 2528 & .61170 & .36264 & .59284 & .43863 & .15297 & .02319 & 3884 & .92300 & .59568 & .64538 & .49019 & .21285 & .09746 \\
\hline 2617 & .52348 & .31498 & .60170 & .49581 & .28887 & .20041 & 3941 & .93000 & .52354 & .56295 & .33359 & .10509 & .10336 \\
\hline 2619 & .52826 & .28710 & .54348 & .43062 & .23946 & .16865 & 3963 & .08333 & .04933 & .59200 & .67568 & .45946 & .20270 \\
\hline 2621 & .55557 & .32101 & .57780 & .47244 & .25299 & .15771 & 4040 & .58567 & .32732 & .55887 & .43053 & .23065 & .15448 \\
\hline 2675 & .69408 & .41730 & .60123 & .51555 & .29343 & .19967 & 4058 & 1.0290 & 66947 & .65061 & .55144 & .36672 & .32576 \\
\hline 2676 & .67566 & .39808 & .58917 & .49756 & .22343 & .13291 & 4098 & .42734 & .23436 & .54842 & .49701 & .25507 & .15154 \\
\hline 2679 & .57667 & .37298 & 64679 & .56676 & .32160 & .18887 & 4100 & .56228 & .33635 & .59819 & .48480 & .26867 & .18739 \\
\hline 2715 & .65123 & .36842 & .56573 & .44133 & .23229 & .15170 & 4137 & .65554 & .36465 & .55626 & .45884 & .21301 & .14954 \\
\hline 2744 & .57498 & .32846 & .57126 & .46214 & .24252 & .15781 & 4191 & .63462 & .39054 & .61540 & .52430 & .28703 & .17814 \\
\hline 2758 & .47921 & .33658 & .70237 & 60195 & .34458 & .19364 & 4256 & -- & -- & -- & -- & -- & -- \\
\hline 2794 & .43091 & .29836 & .69241 & .50599 & .16870 & -.00965 & 4257 & .81988 & .46685 & .56942 & .45167 & .23273 & .15859 \\
\hline 2797 & .22862 & .14582 & .63783 & .51977 & .28974 & .18998 & 4258 & .71667 & .40355 & .56309 & .46890 & .24383 & .18773 \\
\hline 2811 & .54934 & .32608 & .59359 & .49280 & .25243 & .14238 & 4278 & .68125 & .40173 & .58970 & .44649 & .21777 & .13434 \\
\hline 2813 & .67636 & .35740 & .52842 & .42645 & .23315 & .04773 & 4299 & .35839 & .16674 & .46525 & .32118 & .13418 & .05702 \\
\hline 2814 & .22812 & .16854 & .73881 & .63221 & .30029 & .00876 & 4300 & .65304 & .43804 & .67077 & .53337 & .29874 & .20031 \\
\hline 2815 & .54696 & .30647 & .56031 & .52706 & .30743 & .22793 & 4305 & .61660 & .41609 & .67480 & .53035 & .28782 & .19048 \\
\hline 2818 & .63936 & .38009 & .59449 & .47190 & .26974 & .19451 & 4307 & .70406 & .47685 & .67729 & .54754 & .32780 & .23877 \\
\hline 2986 & .89262 & .48355 & .54171 & .36050 & .14796 & .10114 & 4309 & .75338 & .46392 & .61579 & .48459 & .26090 & .17512 \\
\hline 3005 & .64014 & .35986 & .56216 & .43699 & .21362 & .13788 & 4311 & .79725 & .48002 & .60210 & .46620 & .24397 & .15784 \\
\hline 3033 & .28029 & .15726 & .56106 & .49773 & .30060 & .19845 & 4313 & .85597 & .52550 & .61393 & .51355 & .30063 & .18937 \\
\hline 3034 & -- & -- & -- & -- & -- & -- & 4319 & .62094 & .33363 & .53730 & .38501 & .16515 & .11567 \\
\hline 3047 & .78696 & .46569 & .59176 & .40781 & .19185 & .15451 & 4329 & .77842 & .46540 & .59787 & .48089 & .27026 & .17703 \\
\hline 3103 & .88679 & .57583 & .64935 & .61785 & .35803 & .18334 & 4331 & -- & -- & -- & -- & -- & -- \\
\hline 3133 & .74666 & .42515 & .56940 & .44254 & .23620 & .15787 & 4375 & 67108 & .40568 & .60452 & .54854 & .30711 & .21638 \\
\hline 3156 & .86163 & .52691 & .61153 & .52566 & .31055 & .21762 & 4392 & .88741 & .53326 & .60092 & .46677 & .25484 & .17477 \\
\hline 3171 & .70465 & .40578 & .57586 & .45082 & .23508 & .15061 & 4425 & .45429 & .26017 & .57268 & .50597 & .26142 & .15499 \\
\hline 3189 & .35859 & .21350 & .59540 & .53160 & .31713 & .19776 & 4440 & .59577 & .35828 & .60138 & .47382 & .25039 & .16404 \\
\hline 3260 & .58477 & .34530 & .59049 & .43507 & .20952 & .12309 & 4476 & .64503 & .35722 & .55380 & .44913 & .22706 & .15027 \\
\hline 3267 & .46720 & .31534 & 67496 & .58663 & .36630 & .25808 & 4498 & .23909 & .11836 & .49506 & .23605 & .04506 & .00512 \\
\hline 3270 & .54319 & .30812 & .56724 & .50127 & .25379 & .17468 & 4517 & .66119 & .38216 & .57799 & .44131 & .22887 & .14611 \\
\hline 3272 & .13895 & .06930 & .49874 & .30037 & .10983 & .08910 & 4520 & .63729 & .35985 & .56466 & .47610 & .22449 & .15296 \\
\hline 3277 & .17143 & .11077 & .64615 & .54464 & .34479 & .33883 & 4525 & 1.0367 & .75510 & .72839 & .57986 & .24763 & .03538 \\
\hline 3278 & .47122 & .27029 & .57359 & .47433 & .24339 & .13676 & 4563 & .54115 & .33848 & .62547 & .48798 & .26894 & .22470 \\
\hline 3280 & .34400 & .21494 & .62484 & .51284 & .27829 & .16666 & 4570 & .56564 & .33244 & .58772 & .49540 & .26837 & .16886 \\
\hline 3281 & .36930 & .18315 & .49592 & .31909 & .12656 & .06467 & 4577 & .77445 & .43387 & .56023 & .44124 & .23625 & .15862 \\
\hline 3283 & .60677 & .38624 & .63656 & .48583 & .25432 & .16031 & 4591 & .71650 & .41887 & .58460 & .45481 & .24188 & .15796 \\
\hline 3284 & .66272 & .38494 & .58085 & .46257 & .23502 & .14238 & 4670 & .54813 & .32179 & .58707 & .48204 & .25485 & .16058 \\
\hline 3285 & .68486 & .39031 & .56992 & .46384 & .21720 & .13720 & 4671 & .45955 & .27756 & .60398 & .45062 & .22523 & .15382 \\
\hline 3329 & .63140 & .39842 & .63101 & .52534 & .30728 & .21171 & 4679 & .68150 & .40607 & .59584 & .50276 & .26770 & .18040 \\
\hline 3335 & .92727 & .56280 & 60694 & .50673 & .30054 & .20514 & 4696 & .40000 & .20667 & .51667 & .32859 & .08431 & .01931 \\
\hline 3370 & .75746 & .43283 & .57143 & .43486 & .22809 & .14934 & 4703 & .49532 & .29708 & .59977 & .49889 & .26114 & .14038 \\
\hline 3410 & .54435 & .29994 & .55100 & .46699 & .24002 & .16045 & 4704 & .92520 & .57402 & .62043 & .50577 & .28216 & .17682 \\
\hline 3415 & .67954 & .39469 & .58082 & .46690 & .24287 & .15868 & 4731 & .49514 & .34456 & .69588 & .59474 & .39171 & .29277 \\
\hline 3430 & .65246 & .44072 & .67548 & .54475 & .31666 & .21991 & 4792 & .65920 & .36839 & .55884 & .46210 & .21147 & .13801 \\
\hline 3431 & .67791 & .48426 & .71433 & 60175 & .38060 & .28389 & 4819 & .77376 & .42723 & .55215 & .43824 & .20009 & .12495 \\
\hline 3441 & .62892 & .38764 & .61636 & .47986 & .22709 & .10206 & 4852 & .88562 & .43246 & .48831 & .43799 & .36173 & .27439 \\
\hline 3442 & .43430 & .25521 & .58764 & .49030 & .26330 & .15346 & 4866 & .71477 & .40453 & .56596 & .44240 & .22828 & .15192 \\
\hline 3446 & .48950 & .26770 & .54689 & .43062 & .21978 & .14144 & 4876 & .87705 & .52707 & .60096 & .49683 & .24699 & .15686 \\
\hline 3460 & .82306 & .47780 & .58052 & .41448 & .24731 & .27373 & 4878 & .86062 & .51609 & .59968 & .47696 & .25841 & .16412 \\
\hline 3462 & .53836 & .28626 & .53172 & .40883 & .20521 & .14376 & 4880 & .48219 & .27580 & .57198 & .47903 & .25967 & .16405 \\
\hline
\end{tabular}


Appendix 4-2.4. L-moments of storm depth defined by 18-hour minimum interevent time for hourly rainfall stations in TexasContinued.

\begin{tabular}{|c|c|c|c|c|c|c|c|c|c|c|c|c|c|}
\hline $\begin{array}{c}\text { Station } \\
\text { no. }\end{array}$ & $\begin{array}{c}\text { Depth } \\
\text { mean } \\
\text { (inches) }\end{array}$ & $\begin{array}{c}\text { Depth } \\
\text { L-scale } \\
\text { (inches) }\end{array}$ & $\begin{array}{c}\text { Depth } \\
\text { L-CV } \\
\text { (dimen- } \\
\text { sionless) }\end{array}$ & $\begin{array}{c}\text { Depth } \\
\text { L-skew } \\
\text { (dimen- } \\
\text { sionless) }\end{array}$ & $\begin{array}{c}\text { Depth } \\
\text { L-kurtosis } \\
\text { (dimen- } \\
\text { sionless) }\end{array}$ & $\begin{array}{c}\text { Depth } \\
\text { Tau5 } \\
\text { (dimen- } \\
\text { sionless) }\end{array}$ & $\begin{array}{c}\text { Station } \\
\text { no. }\end{array}$ & $\begin{array}{l}\text { Depth } \\
\text { mean } \\
\text { (inches) }\end{array}$ & $\begin{array}{l}\text { Depth } \\
\text { L-scale } \\
\text { (inches) }\end{array}$ & $\begin{array}{c}\text { Depth } \\
\text { L-CV } \\
\text { (dimen- } \\
\text { sionless) }\end{array}$ & $\begin{array}{c}\text { Depth } \\
\text { L-skew } \\
\text { (dimen- } \\
\text { sionless) }\end{array}$ & $\begin{array}{c}\text { Depth } \\
\text { L-kurtosis } \\
\text { (dimen- } \\
\text { sionless) }\end{array}$ & $\begin{array}{c}\text { Depth } \\
\text { Tau5 } \\
\text { (dimen- } \\
\text { sionless) }\end{array}$ \\
\hline 4920 & 0.61901 & 0.37646 & 0.60816 & 0.51356 & 0.27495 & .17153 & 5957 & 0.65992 & 0.38244 & 0.57953 & 0.47745 & 0.25027 & 0.16997 \\
\hline 4934 & .36714 & .24857 & .67704 & .72874 & .63218 & .57854 & 5958 & .62960 & .35753 & .56787 & .39598 & .16570 & .07877 \\
\hline 4972 & .62760 & .36140 & .57584 & .47209 & .25529 & .17158 & 5973 & .50844 & .34589 & .68029 & .55140 & .28194 & .15061 \\
\hline 4973 & .87728 & .45613 & .51993 & .39080 & .22433 & .16178 & 5996 & .63813 & .37140 & .58201 & .46674 & .23468 & .14927 \\
\hline 4974 & .50803 & .29915 & .58884 & .49044 & .27702 & .18489 & 6017 & .46250 & .29108 & .62936 & .48690 & .24036 & .13188 \\
\hline 4975 & .79284 & .44264 & .55830 & .44867 & .22094 & .15574 & 6024 & .98966 & .61403 & .62044 & .48539 & .26714 & .19472 \\
\hline 4978 & .63316 & .39251 & .61992 & .48915 & .26285 & .16336 & 6050 & .70211 & .32620 & .46460 & .30137 & .01414 & -.07950 \\
\hline 4979 & 1.0694 & .57657 & .53913 & .41508 & .24564 & .13222 & 6104 & .39300 & .22526 & .57317 & .50773 & .30081 & .19724 \\
\hline 4982 & .57403 & .32903 & .57318 & .44911 & .23688 & .15293 & 6108 & .83479 & .46949 & .56241 & .43411 & .22017 & .14567 \\
\hline 5018 & .64486 & .35513 & .55071 & .40141 & .18643 & .12275 & 6136 & .42902 & .23693 & .55226 & .49109 & .25965 & .16105 \\
\hline 5048 & .49602 & .29880 & .60240 & .53931 & .29750 & .18714 & 6166 & .41000 & .24689 & .60216 & .49059 & .24107 & .13171 \\
\hline 5049 & .46154 & .26190 & .56746 & .59235 & .38789 & .31075 & 6176 & .79668 & .47776 & .59969 & .47646 & .26638 & .16988 \\
\hline 5056 & .43200 & .18600 & .43056 & .26882 & .67742 & .11828 & 6177 & .81076 & .47062 & .58046 & .46561 & .24520 & .16020 \\
\hline 5057 & .38718 & .27383 & .70724 & .59968 & .35899 & .22649 & 6210 & .73493 & .42767 & .58192 & .46306 & .23471 & .15465 \\
\hline 5060 & .59404 & .41986 & .70679 & .59393 & .36070 & .24798 & 6211 & .69357 & .42624 & .61456 & .46810 & .23399 & .14556 \\
\hline 5081 & .75150 & .42556 & .56629 & .41242 & .20787 & .14472 & 6270 & .83371 & .47109 & .56505 & .45851 & .23408 & .16455 \\
\hline 5094 & .71483 & .41334 & .57824 & .46440 & .24131 & .15322 & 6275 & -- & -- & -- & -- & -- & -- \\
\hline 5113 & .64457 & .40307 & .62534 & .54122 & .30967 & .20013 & 6276 & 1.0757 & .52052 & .48389 & .18528 & .01742 & .07976 \\
\hline 5114 & -- & -- & -- & -- & -- & -- & 6335 & .71831 & .40945 & .57001 & .43096 & .21804 & .14211 \\
\hline 5123 & .65133 & .40114 & .61588 & .56622 & .34537 & .13957 & 6434 & .66294 & .34434 & .51941 & .33649 & .12141 & .12260 \\
\hline 5192 & .72633 & .42521 & .58542 & .46507 & .24244 & .14718 & 6504 & .47891 & .27849 & .58150 & .50663 & .27158 & .16574 \\
\hline 5193 & .68532 & .41128 & .60012 & .47840 & .24316 & .15238 & 6558 & .63828 & .38589 & .60458 & .41319 & .12958 & .09096 \\
\hline 5224 & .82237 & .48997 & .59581 & .45172 & .24408 & .16603 & 6615 & .57567 & .34289 & .59564 & .55662 & .32194 & .23456 \\
\hline 5228 & .66204 & .40013 & .60439 & .44264 & .20057 & .12101 & 6660 & .73196 & .43753 & .59775 & .47701 & .26184 & .16425 \\
\hline 5235 & .60926 & .36516 & .59935 & .42014 & .20256 & .15941 & 6663 & .55874 & .34009 & .60868 & .48883 & .26599 & .15270 \\
\hline 5247 & .49366 & .27794 & .56302 & .47671 & .24720 & .15035 & 6734 & .49637 & .29437 & .59305 & .46933 & .25525 & .16522 \\
\hline 5258 & .71238 & .41360 & .58060 & .45752 & .24315 & .14820 & 6736 & .51944 & .30631 & .58970 & .51609 & .27772 & .17140 \\
\hline 5303 & .63643 & .40443 & .63548 & .53561 & .31651 & .22802 & 6740 & 1.5023 & .78744 & .52415 & .23161 & -.09360 & -.22178 \\
\hline 5312 & .60207 & .35230 & .58515 & .50469 & .26071 & .17417 & 6750 & .76705 & .53511 & .69762 & .57230 & .34225 & .23197 \\
\hline 5341 & 1.1777 & .73305 & .62243 & .54997 & .30889 & .14983 & 6757 & .72407 & .43389 & .59923 & .46257 & .24222 & .15923 \\
\hline 5342 & -- & -- & -- & -- & -- & -- & 6775 & .46805 & .27613 & .58996 & .45477 & .24035 & .15926 \\
\hline 5348 & .81184 & .45400 & .55922 & .42539 & .18941 & .11639 & 6776 & .48392 & .27097 & .55994 & .47836 & .25392 & .15751 \\
\hline 5358 & .55933 & .32781 & .58608 & .46835 & .24386 & .14838 & 6788 & .77802 & .42242 & .54293 & .39965 & .22370 & .14854 \\
\hline 5398 & .74601 & .43661 & .58526 & .45732 & .23900 & .15587 & 6792 & .37995 & .21169 & .55716 & .48949 & .26220 & .14771 \\
\hline 5410 & .49009 & .28424 & .57997 & .50294 & .29132 & .19680 & 6794 & 1.6258 & .74778 & .45995 & .18235 & .08183 & .11124 \\
\hline 5411 & .40351 & .26636 & .66011 & .52597 & .28809 & .18678 & 6834 & .82701 & .46653 & .56411 & .45550 & .22863 & .16089 \\
\hline 5424 & .74236 & .48182 & .64904 & .48032 & .23841 & .15818 & 6893 & .36228 & .20436 & .56410 & .54910 & .33452 & .21923 \\
\hline 5429 & .62351 & .39462 & .63291 & .50596 & .26877 & .16904 & 6935 & .48008 & .27193 & .56642 & .52087 & .29883 & .20303 \\
\hline 5431 & 1.0329 & .52538 & .50867 & .38590 & .18418 & .09813 & 6981 & .68756 & .40210 & .58482 & .48412 & .28389 & .19270 \\
\hline 5461 & .80768 & .46947 & .58125 & .44862 & .23919 & .15794 & 7020 & .74739 & .44205 & .59146 & .46755 & .27647 & .21154 \\
\hline 5463 & .76701 & .44202 & .57629 & .47605 & .23593 & .16363 & 7060 & .55910 & .31734 & .56759 & .47425 & .23255 & .13496 \\
\hline 5471 & .20350 & .13603 & .66843 & .65873 & .46059 & .31783 & 7066 & .80472 & .45942 & .57090 & .43858 & .22088 & .14267 \\
\hline 5477 & .74565 & .51308 & .68810 & .73104 & 63699 & .58232 & 7074 & .46267 & .27302 & .59010 & .51805 & .30519 & .20288 \\
\hline 5528 & .76857 & .42711 & .55572 & .42028 & .21258 & .13490 & 7097 & .65598 & .37937 & .57833 & .48014 & .25849 & .14185 \\
\hline 5579 & -- & -- & -- & -- & -- & -- & 7116 & .48830 & .28295 & .57946 & .46174 & .25196 & .17397 \\
\hline 5580 & .65083 & .32051 & .49246 & .29207 & .10480 & .02007 & 7140 & .76508 & .50271 & .65706 & .55295 & .32872 & .22116 \\
\hline 5589 & .31782 & .17908 & .56347 & .39757 & .17707 & .10539 & 7173 & .79990 & .55218 & .69032 & .55268 & .31352 & .20992 \\
\hline 5590 & .36783 & .21465 & .58354 & .47915 & .25699 & .15848 & 7174 & .75300 & .50422 & .66961 & .53059 & .30105 & .20868 \\
\hline 5591 & .38132 & .20100 & .52712 & .41781 & .22367 & .14440 & 7213 & .71803 & .41676 & .58042 & .44740 & .23228 & .14277 \\
\hline 5592 & .33606 & .19196 & .57122 & .47387 & .26205 & .16866 & 7243 & .59964 & .34782 & .58004 & .48576 & .25676 & .17113 \\
\hline 5594 & .33309 & .19101 & .57347 & .47845 & .25867 & .15746 & 7262 & .21865 & .12727 & .58204 & .44325 & .22794 & .14702 \\
\hline 5595 & -- & -- & -- & -- & -- & -- & 7274 & .71123 & .38731 & .54456 & .44609 & .24908 & .16309 \\
\hline 5596 & .41354 & .22057 & .53338 & .52455 & .26768 & .17679 & 7300 & .63013 & .36594 & .58074 & .44562 & .23120 & .15124 \\
\hline 5600 & .40224 & .23765 & .59080 & .50341 & .29505 & .19258 & 7311 & .73654 & .42863 & .58195 & .52154 & .26491 & .15414 \\
\hline 5618 & .71929 & .40201 & .55890 & .40210 & .14848 & .05476 & 7363 & .81000 & .38805 & .47908 & .21434 & .05225 & .07345 \\
\hline 5650 & .57053 & .25713 & .45070 & .35194 & .29942 & .11496 & 7422 & .65995 & .40447 & .61288 & .50754 & .28065 & .18555 \\
\hline 5656 & .52043 & .29834 & .57326 & .52683 & .27021 & .17979 & 7431 & .47139 & .27449 & .58231 & .49780 & .27337 & .17173 \\
\hline 5658 & .50242 & .30646 & 60997 & .51640 & .30477 & .20442 & 7481 & .38409 & .23316 & .60704 & .56268 & .35525 & .23454 \\
\hline 5661 & .66276 & .44000 & .66389 & .65635 & .43175 & .32816 & 7497 & .68486 & .39728 & .58009 & .49502 & .24833 & .17318 \\
\hline 5666 & .52750 & .30792 & .58374 & .45234 & .21988 & .12890 & 7498 & .69397 & .40601 & .58505 & .49487 & .25015 & .18203 \\
\hline 5695 & .73630 & .41616 & .56520 & .44069 & .23980 & .16363 & 7499 & .63974 & .36349 & .56818 & .47999 & .24529 & .16580 \\
\hline 5742 & .32875 & .22033 & .67022 & 68289 & .55196 & .52503 & 7531 & .68328 & .38739 & .56696 & .41141 & .17847 & .09471 \\
\hline 5766 & 1.0574 & .57229 & .54123 & .38271 & .13802 & .04368 & 7534 & .67745 & .41872 & .61808 & .51717 & .31473 & .23396 \\
\hline 5770 & .52385 & .30377 & .57988 & .48624 & .25979 & .15989 & 7556 & .67323 & .39512 & .58690 & .49702 & .26915 & .18151 \\
\hline 5775 & .36417 & .20523 & .56355 & .30690 & -.11308 & -.08810 & 7594 & .78907 & .46040 & .58348 & .45461 & .24382 & .16384 \\
\hline 5779 & .76105 & .42322 & .55609 & .44407 & .19698 & .03313 & 7596 & .76382 & .44808 & .58663 & .38508 & .13391 & .08046 \\
\hline 5840 & .66675 & .39327 & .58983 & .45308 & .22831 & .14135 & 7608 & .62927 & .39579 & .62897 & .48157 & .24935 & .16277 \\
\hline 5890 & .37310 & .24537 & .65766 & .53099 & .29610 & .18987 & 7622 & .23286 & .18471 & .79325 & .70806 & .46303 & .31352 \\
\hline 5891 & .41270 & .21912 & .53094 & .39030 & .16851 & .10120 & 7700 & .73457 & .44522 & .60609 & .46516 & .24028 & .15273 \\
\hline 5897 & .72010 & .41756 & .57986 & .47993 & .23189 & .16083 & 7706 & .56902 & .33834 & .59460 & .49913 & .27489 & .17743 \\
\hline
\end{tabular}


Appendix 4-2.4. L-moments of storm depth defined by 18-hour minimum interevent time for hourly rainfall stations in TexasContinued.

\begin{tabular}{|c|c|c|c|c|c|c|c|c|c|c|c|c|c|}
\hline $\begin{array}{c}\text { Station } \\
\text { no. }\end{array}$ & $\begin{array}{l}\text { Depth } \\
\text { mean } \\
\text { (inches) }\end{array}$ & $\begin{array}{l}\text { Depth } \\
\text { L-scale } \\
\text { (inches) }\end{array}$ & $\begin{array}{c}\text { Depth } \\
\text { L-CV } \\
\text { (dimen- } \\
\text { sionless) }\end{array}$ & $\begin{array}{c}\text { Depth } \\
\text { L-skew } \\
\text { (dimen- } \\
\text { sionless) }\end{array}$ & $\begin{array}{c}\text { Depth } \\
\text { L-kurtosis } \\
\text { (dimen- } \\
\text { sionless) }\end{array}$ & $\begin{array}{c}\text { Depth } \\
\text { Tau5 } \\
\text { (dimen- } \\
\text { sionless) }\end{array}$ & $\begin{array}{c}\text { Station } \\
\text { no. }\end{array}$ & $\begin{array}{l}\text { Depth } \\
\text { mean } \\
\text { (inches) }\end{array}$ & $\begin{array}{l}\text { Depth } \\
\text { L-scale } \\
\text { (inches) }\end{array}$ & $\begin{array}{c}\text { Depth } \\
\text { L-CV } \\
\text { (dimen- } \\
\text { sionless) }\end{array}$ & $\begin{array}{l}\text { Depth } \\
\text { L-skew } \\
\text { (dimen- } \\
\text { sionless) }\end{array}$ & $\begin{array}{l}\text { Depth } \\
\text { L-kurtosis } \\
\text { (dimen- } \\
\text { sionless) }\end{array}$ & $\begin{array}{c}\text { Depth } \\
\text { Tau5 } \\
\text { (dimen- } \\
\text { sionless) }\end{array}$ \\
\hline 7718 & 0.77573 & 0.46945 & 0.60518 & 0.50376 & 0.28494 & 0.18276 & 8910 & 0.70650 & 0.38166 & 0.54021 & 0.29665 & 0.03253 & -0.04321 \\
\hline 7745 & .82017 & .49608 & .60485 & .48104 & .27541 & .19435 & 9107 & .41839 & .24834 & .59357 & .45102 & .18256 & .12444 \\
\hline 7922 & .26630 & .15459 & .58050 & .46894 & .26293 & .16173 & 9129 & .41845 & .28372 & .67803 & .59593 & .35031 & .20736 \\
\hline 7936 & .81875 & .47233 & .57689 & .45634 & .24222 & .17198 & 9163 & .61168 & .35554 & .58126 & .47348 & .25237 & .15988 \\
\hline 7943 & .43647 & .28695 & .65743 & .51436 & .26741 & .16327 & 9213 & .61118 & .38457 & .62923 & .47015 & .23452 & .15065 \\
\hline 7944 & .45940 & .30176 & .65686 & .49201 & .23370 & .12928 & 9214 & .99536 & .61597 & .61884 & .55350 & .35015 & .22789 \\
\hline 7945 & .53387 & .37077 & .69450 & .56183 & .31995 & .21424 & 8911 & .58177 & .36296 & .62388 & .47991 & .24773 & .15859 \\
\hline 7947 & .79217 & .50929 & .64291 & .57165 & .34512 & .24771 & 8924 & .32878 & .17302 & .52626 & .42163 & .20753 & .10316 \\
\hline 7948 & .62070 & .39175 & .63114 & .50525 & .26679 & .15905 & 8929 & 1.1622 & .58320 & .50180 & .16099 & -.03597 & .08066 \\
\hline 7951 & .72759 & .40287 & .55370 & .40966 & .19613 & .11762 & 8942 & .81123 & .46831 & .57729 & .46524 & .23610 & .15458 \\
\hline 7953 & .51427 & .31500 & .61252 & .49751 & .26678 & .15275 & 8944 & .68064 & .41452 & .60901 & .46271 & .24316 & .16065 \\
\hline 7981 & .60845 & .37493 & .61621 & .45341 & .18327 & .07566 & 8996 & .67574 & .41806 & .61868 & .49012 & .26668 & .17974 \\
\hline 7990 & .58093 & .40700 & .70061 & .60280 & .36774 & .21859 & 9014 & .81850 & .40418 & .49381 & .37557 & .21132 & .13798 \\
\hline 7992 & .81050 & .42582 & .52537 & .31706 & .03105 & -.07188 & 9037 & .35739 & .21513 & 60196 & .53987 & .34378 & .24293 \\
\hline 7997 & .55563 & .33975 & .61146 & .50289 & .28314 & .16188 & 9106 & .33602 & .22196 & .66055 & .62114 & .43201 & .33414 \\
\hline 7999 & .36588 & .23294 & .63666 & .53144 & .34091 & .24009 & 9222 & .62775 & .39025 & .62166 & .48239 & .24588 & .14777 \\
\hline 8022 & .49300 & .30321 & .61504 & .47131 & .17096 & .04555 & 9248 & .49983 & .28623 & .57266 & .44237 & .20172 & 10947 \\
\hline 8023 & .51385 & .30144 & .58662 & .50901 & .28311 & .18289 & 9266 & .60246 & .33669 & .55885 & .40366 & .15275 & .06020 \\
\hline 8047 & .65070 & .37518 & .57658 & .44999 & .22018 & .13863 & 9270 & .37834 & .20776 & .54913 & .52735 & .30036 & .18851 \\
\hline 8060 & .50432 & .33707 & .66836 & .54892 & .27827 & .13387 & 9295 & .23423 & .14770 & .63058 & .54716 & .34384 & .23353 \\
\hline 8062 & .70225 & .51866 & .73857 & .61124 & .34326 & .18541 & 9304 & -- & -- & -- & -- & -- & -- \\
\hline 8068 & .29647 & .19576 & .66029 & .55922 & .31093 & .18190 & 9307 & .61876 & .33168 & .53604 & .42617 & .22693 & .14951 \\
\hline 8081 & .64109 & .40384 & .62993 & .54296 & .31692 & .21438 & 9328 & .49481 & .28363 & .57321 & .44729 & .23066 & .14963 \\
\hline 8089 & .52342 & .29703 & .56749 & .36118 & .11296 & .05501 & 9329 & .60417 & .37068 & .61354 & .36611 & .04122 & .10975 \\
\hline 8221 & .73737 & .33076 & .44857 & .32655 & .24033 & .14816 & 9345 & -- & -- & -- & -- & -- & -- \\
\hline 8252 & .49373 & .28491 & .57705 & .48661 & .25704 & .15723 & 9363 & .51495 & .35061 & .68087 & .55125 & .31093 & .19953 \\
\hline 8265 & .78889 & .49643 & .62927 & .51484 & .29926 & .21007 & 9364 & .57724 & .40068 & .69414 & .56964 & .33382 & .22652 \\
\hline 8289 & .50400 & .22962 & .45560 & .17924 & .01368 & .01921 & 9365 & .35968 & .23467 & .65244 & .67607 & .49292 & .34953 \\
\hline 8305 & .33082 & .18563 & .56111 & .52719 & .32209 & .20424 & 9371 & .66970 & .38792 & .57925 & .40881 & .13551 & .04705 \\
\hline 8335 & .83116 & .47000 & .56548 & .42239 & .22076 & .15122 & 9417 & .65909 & .39150 & .59399 & .44303 & .20534 & .11990 \\
\hline 8400 & .40092 & .23429 & .58437 & .51020 & .32294 & .22671 & 9419 & .57861 & .36831 & .63653 & .47244 & .22632 & .13806 \\
\hline 8445 & .71404 & .44178 & .61870 & .49296 & .26559 & .17342 & 9435 & .50589 & .28280 & .55901 & .48065 & .31627 & .26791 \\
\hline 8446 & .65024 & .37925 & .58324 & .46819 & .23036 & .13319 & 9491 & .71430 & .42196 & .59073 & .46397 & .23411 & .14676 \\
\hline 8451 & .52766 & .32087 & .60810 & .50246 & .27657 & .17705 & 9499 & .55390 & .31980 & .57735 & .47886 & .22409 & .12725 \\
\hline 8531 & .67723 & .38431 & .56747 & .43685 & .22879 & .15056 & 9522 & 1.5073 & .95164 & .63136 & .46567 & .14740 & .05397 \\
\hline 8541 & .67202 & .38462 & .57234 & .41986 & .20944 & .13006 & 9527 & .47569 & .26701 & .56132 & .50479 & .26318 & .16278 \\
\hline 8544 & .74764 & .46064 & .61612 & .52642 & .29214 & .20237 & 9532 & .64604 & .37745 & .58425 & .47150 & .25239 & .16956 \\
\hline 8545 & .37688 & .18454 & .48966 & .23427 & .08570 & .15104 & 9544 & -- & -- & -- & -- & -- & -- \\
\hline 8563 & .73731 & .42019 & .56989 & .47858 & .24713 & .17834 & 9565 & .58556 & .34270 & .58526 & .49020 & .26612 & .16726 \\
\hline 8566 & .51280 & .30342 & .59170 & .44997 & .21745 & .12864 & 9570 & .52874 & .29659 & .56094 & .50762 & .24604 & .14592 \\
\hline 8583 & .57246 & .32930 & .57524 & .50310 & .22774 & .14046 & 9574 & .52727 & .29654 & .56240 & .47124 & .17610 & .10626 \\
\hline 8584 & .65271 & .37487 & .57433 & .45934 & .22595 & .14409 & 9588 & .56126 & .35573 & .63381 & .55059 & .31233 & .18694 \\
\hline 8623 & .67983 & .37491 & .55147 & .44403 & .21365 & .13513 & 9665 & .74923 & .43378 & .57897 & .45844 & .23845 & .16002 \\
\hline 8625 & .64263 & .37982 & .59104 & .46440 & .25027 & .17474 & 9715 & .67145 & .38665 & .57584 & .44726 & .22367 & .14306 \\
\hline 8630 & .50684 & .28634 & .56495 & .44825 & .21258 & .12029 & 9729 & .55312 & .34931 & .63153 & .47234 & .23366 & .15085 \\
\hline 8631 & .57207 & .33487 & .58537 & .47471 & .25317 & .15234 & 9772 & .68777 & .45422 & .66042 & .55852 & .33886 & .23313 \\
\hline 8646 & .64572 & .38001 & .58851 & .45401 & .23003 & .14678 & 9814 & .67125 & .40808 & .60795 & .45551 & .21103 & .11327 \\
\hline 8647 & .46557 & .26838 & .57645 & .51397 & .27696 & .17736 & 9815 & .72741 & .43832 & .60258 & .49489 & .27143 & .17718 \\
\hline 8677 & .64331 & .35303 & .54878 & .43064 & .20961 & .11931 & 9816 & .63324 & .41274 & .65179 & .53088 & .28850 & .17300 \\
\hline 8696 & .83261 & .47202 & .56691 & .36519 & .14446 & .09684 & 9817 & .62725 & .35467 & .56544 & .47427 & .24544 & .17015 \\
\hline 8743 & .80100 & .46732 & .58342 & .45501 & .23804 & .15632 & 9829 & .39403 & .23443 & .59495 & .53791 & .31620 & .19156 \\
\hline 8761 & .45452 & .25684 & .56508 & .50873 & .27696 & .16912 & 9830 & .30521 & .18397 & .60279 & .48490 & .22995 & .11117 \\
\hline 8778 & .74848 & .42878 & .57287 & .46075 & .23549 & .15779 & 9858 & .49591 & .27981 & .56424 & .45285 & .24221 & .14947 \\
\hline 8845 & .69604 & .42815 & .61512 & .51881 & .28355 & .18003 & 9893 & .61362 & .35707 & .58191 & .46693 & .25138 & .16888 \\
\hline 8859 & .80328 & .44917 & .55916 & .41406 & .21216 & .14493 & 9916 & .81232 & .45376 & .55859 & .42479 & .19670 & .12334 \\
\hline 8898 & .76388 & .44408 & .58135 & .46194 & .25203 & .15934 & 9976 & .55915 & .34352 & .61435 & .52334 & .28943 & .18040 \\
\hline 8908 & .71581 & .42953 & .60006 & .47792 & .28751 & .23412 & & & & & & & \\
\hline
\end{tabular}


Appendix 4-2.5. L-moments of storm depth defined by 24-hour minimum interevent time for hourly rainfall stations in Texas.

$[--$, not available $]$

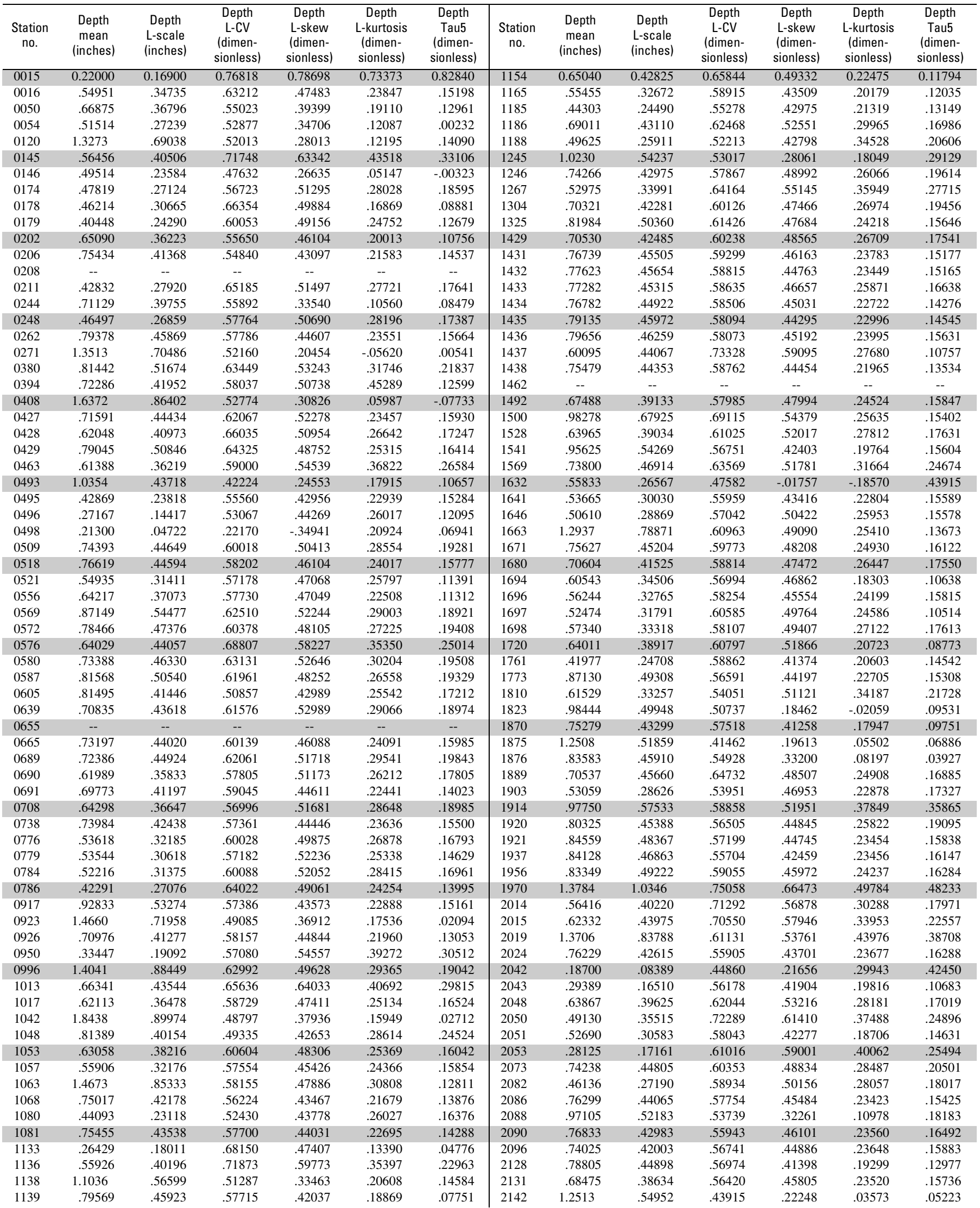


204 Statistical Characteristics of Storm Interevent Time, Depth, and Duration for Eastern New Mexico, Oklahoma, and Texas

Appendix 4-2.5. L-moments of storm depth defined by 24-hour minimum interevent time for hourly rainfall stations in Texas-

Continued.

\begin{tabular}{|c|c|c|c|c|c|c|c|c|c|c|c|c|c|}
\hline $\begin{array}{c}\text { Station } \\
\text { no. }\end{array}$ & $\begin{array}{c}\text { Depth } \\
\text { mean } \\
\text { (inches) }\end{array}$ & $\begin{array}{l}\text { Depth } \\
\text { L-scale } \\
\text { (inches) }\end{array}$ & $\begin{array}{c}\text { Depth } \\
\text { L-CV } \\
\text { (dimen- } \\
\text { sionless) }\end{array}$ & $\begin{array}{c}\text { Depth } \\
\text { L-skew } \\
\text { (dimen- } \\
\text { sionless) }\end{array}$ & $\begin{array}{c}\text { Depth } \\
\text { L-kurtosis } \\
\text { (dimen- } \\
\text { sionless) }\end{array}$ & $\begin{array}{c}\text { Depth } \\
\text { Tau5 } \\
\text { (dimen- } \\
\text { sionless) }\end{array}$ & $\begin{array}{c}\text { Station } \\
\text { no. }\end{array}$ & $\begin{array}{c}\text { Depth } \\
\text { mean } \\
\text { (inches) }\end{array}$ & $\begin{array}{l}\text { Depth } \\
\text { L-scale } \\
\text { (inches) }\end{array}$ & $\begin{array}{c}\text { Depth } \\
\text { L-CV } \\
\text { (dimen- } \\
\text { sionless) }\end{array}$ & $\begin{array}{c}\text { Depth } \\
\text { L-skew } \\
\text { (dimen- } \\
\text { sionless) }\end{array}$ & $\begin{array}{c}\text { Depth } \\
\text { L-kurtosis } \\
\text { (dimen- } \\
\text { sionless) }\end{array}$ & $\begin{array}{c}\text { Depth } \\
\text { Tau5 } \\
\text { (dimen- } \\
\text { sionless) }\end{array}$ \\
\hline 2160 & 0.52105 & 0.38877 & 0.74613 & 0.70567 & 0.58835 & 0.53667 & 3463 & 0.74310 & 0.45685 & 0.61479 & 0.47271 & 0.26103 & 0.18851 \\
\hline 2206 & .86804 & .51373 & .59183 & .45358 & .23881 & .16553 & 3476 & .69485 & .40923 & .58895 & .45956 & .24594 & .15735 \\
\hline 2238 & .42172 & .27791 & .65900 & .51285 & .24790 & .12914 & 3485 & 1.2500 & .89338 & .71471 & .62281 & .36325 & .23155 \\
\hline 2240 & .43366 & .26965 & .62179 & .46348 & .22366 & .11291 & 3507 & .77059 & .45395 & .58909 & .47652 & .22073 & .13912 \\
\hline 2242 & .67016 & .40682 & 60704 & .43297 & .20579 & .13482 & 3546 & .87720 & .50875 & .57997 & .45570 & .24677 & .16689 \\
\hline 2244 & .73503 & .44714 & .60833 & .46417 & .24144 & .15117 & 3547 & .71807 & .39989 & .55690 & .38582 & .15017 & .07884 \\
\hline 2247 & .73585 & .47359 & .64359 & .46587 & .21665 & .14670 & 3579 & .78226 & .44011 & .56262 & .40516 & .12369 & .04776 \\
\hline 2309 & .90323 & .47924 & .53059 & .42749 & .26291 & .19034 & 3642 & .80267 & .46599 & .58054 & .46689 & .25450 & .17267 \\
\hline 2312 & .81818 & .43864 & .53612 & .41406 & .20449 & .13696 & 3646 & .71392 & .41245 & .57772 & .45574 & .24553 & .16354 \\
\hline 2334 & 1.0791 & .61169 & .56688 & .45044 & .25622 & .14298 & 3668 & 1.5750 & .65950 & .41873 & .31961 & .19599 & .08839 \\
\hline 2336 & .73624 & .38629 & .52468 & .34465 & .13574 & .08838 & 3673 & 1.1453 & .66117 & .57731 & .26610 & -.04417 & -.02303 \\
\hline 2354 & .42636 & .23623 & .55407 & .36630 & .05198 & -.04824 & 3686 & .75091 & .42191 & .56187 & .44204 & .20621 & .14053 \\
\hline 2355 & .78179 & .51950 & .66450 & .54139 & .31739 & .22297 & 3691 & .72680 & .41697 & .57370 & .45663 & .22964 & .14622 \\
\hline 2357 & .48162 & .34578 & .71796 & .58573 & .32879 & .20465 & 3734 & 1.8089 & 1.0539 & .58258 & .30965 & .02178 & -.01449 \\
\hline 2360 & .50410 & .34718 & .68871 & .55845 & .31551 & .20807 & 3771 & .75583 & .43714 & .57836 & .46691 & .21036 & .13987 \\
\hline 2361 & .60285 & .40528 & .67227 & .62022 & .44180 & .36298 & 3789 & .25964 & .14035 & .54057 & .42739 & .23087 & .14470 \\
\hline 2394 & .76425 & .45075 & .58979 & .45601 & .23927 & .15397 & 3826 & 66166 & .35856 & .54191 & .40667 & .21210 & .13902 \\
\hline 2404 & .72770 & .43144 & .59289 & .46583 & .25254 & .17630 & 3831 & .82191 & .47857 & .58226 & .45187 & .23562 & .14693 \\
\hline 2415 & .88912 & .50430 & .56719 & .43722 & .23237 & .15350 & 3841 & .76173 & .43852 & .57569 & .45636 & .25316 & .17255 \\
\hline 2462 & .84281 & .47299 & .56121 & .42428 & .20742 & .12972 & 3871 & .64686 & .37333 & .57715 & .45515 & .24570 & .16303 \\
\hline 2528 & .66330 & .38740 & .58405 & .42111 & .13067 & .00247 & 3884 & 1.0256 & .65621 & .63986 & .46504 & .15371 & .01235 \\
\hline 2617 & .55223 & .33112 & .59960 & .48729 & .27394 & .18239 & 3941 & .94691 & .54249 & .57291 & .35202 & .12310 & .12253 \\
\hline 2619 & .56953 & .30731 & .53959 & .41455 & .22492 & .15892 & 3963 & .10000 & .05700 & .57000 & .50877 & .38596 & .87719 \\
\hline 2621 & .59967 & .34548 & .57612 & .46859 & .24817 & .15052 & 4040 & .62153 & .34531 & .55558 & .43046 & .23130 & .15362 \\
\hline 2675 & .76462 & .45225 & .59147 & .49803 & .27992 & .19377 & 4058 & 1.1433 & .75974 & .66449 & .57253 & .35731 & .24771 \\
\hline 2676 & .75935 & .44999 & .59259 & .49662 & .24052 & .15945 & 4098 & .46297 & .25751 & .55620 & .49701 & .25757 & .15394 \\
\hline 2679 & .62330 & .40131 & .64385 & .55197 & .30410 & .17898 & 4100 & 60930 & .35977 & .59047 & .47340 & .25902 & .18050 \\
\hline 2715 & .69727 & .39020 & .55961 & .43362 & .23065 & .15227 & 4137 & .71875 & .39981 & .55626 & .45495 & .22424 & .16473 \\
\hline 2744 & .62410 & .35700 & .57202 & .45960 & .24137 & .15716 & 4191 & .69061 & .42267 & .61203 & .51202 & .27558 & .17087 \\
\hline 2758 & .51489 & .35618 & .69175 & .58282 & .31922 & .17105 & 4256 & -- & -- & -- & -- & -- & -- \\
\hline 2794 & .43091 & .29836 & .69241 & .50599 & .16870 & -.00965 & 4257 & .90327 & .51106 & .56579 & .44503 & .23629 & .16425 \\
\hline 2797 & .25273 & .16057 & .63535 & .51471 & .28694 & .18877 & 4258 & .86642 & .47325 & .54622 & .43339 & .23521 & .18243 \\
\hline 2811 & .60230 & .35751 & .59357 & .48958 & .25162 & .14757 & 4278 & .72380 & .42644 & .58917 & .44842 & .22342 & .13865 \\
\hline 2813 & .70857 & .38471 & .54294 & .42252 & .19943 & .00717 & 4299 & .37759 & .17457 & .46234 & .30420 & .10798 & .04137 \\
\hline 2814 & .24333 & .17695 & .72720 & 60296 & .26534 & -.01688 & 4300 & .74602 & .49273 & .66048 & .51956 & .28853 & .19536 \\
\hline 2815 & .59905 & .33570 & .56039 & .51141 & .28983 & .20097 & 4305 & .72335 & .47518 & .65691 & .50657 & .27087 & .18167 \\
\hline 2818 & .66648 & .39732 & .59614 & .47249 & .26966 & .19266 & 4307 & .80967 & .53684 & .66303 & .53532 & .32425 & .23648 \\
\hline 2986 & .97232 & .52981 & .54489 & .38853 & .20184 & .16051 & 4309 & .84419 & .51176 & .60621 & .47220 & .25463 & .17545 \\
\hline 3005 & .68259 & .38463 & .56350 & .44073 & .22322 & .15000 & 4311 & .88849 & .52954 & .59600 & .45778 & .23879 & .15473 \\
\hline 3033 & .29856 & .17059 & .57137 & .50032 & .29419 & .18818 & 4313 & .94840 & .56651 & .59733 & .50020 & .28986 & .18350 \\
\hline 3034 & -- & -- & -- & -- & -- & -- & 4319 & .71324 & .39950 & .56012 & .45062 & .23395 & .13605 \\
\hline 3047 & 1.0056 & .57680 & .57361 & .38225 & .12078 & .03092 & 4329 & .85653 & .50666 & .59153 & .46988 & .25965 & .17057 \\
\hline 3103 & .91963 & .59718 & .64937 & 60613 & .34128 & .16851 & 4331 & -- & -- & -- & -- & -- & -- \\
\hline 3133 & .80735 & .45973 & .56943 & .43883 & .23646 & .16073 & 4375 & .72213 & .43366 & .60054 & .53379 & .29664 & .21037 \\
\hline 3156 & .93591 & .57141 & .61055 & .52389 & .31592 & .22973 & 4392 & .95129 & .56965 & .59882 & .46630 & .26292 & .18855 \\
\hline 3171 & .75775 & .43457 & .57349 & .44726 & .23475 & .15185 & 4425 & .48741 & .28223 & .57903 & .50538 & .26517 & .15934 \\
\hline 3189 & .41147 & .23977 & .58270 & .51822 & .30228 & .19282 & 4440 & .64304 & .38306 & .59571 & .46580 & .24885 & .16660 \\
\hline 3260 & .63385 & .37413 & .59026 & .43944 & .21051 & .11436 & 4476 & .69991 & .38651 & .55222 & .44273 & .22497 & .14948 \\
\hline 3267 & .53091 & .36473 & .68700 & 60419 & .39605 & .29077 & 4498 & .23909 & .11836 & .49506 & .23605 & .04506 & .00512 \\
\hline 3270 & .58181 & .33000 & .56720 & .49316 & .25379 & .17730 & 4517 & .70890 & .40762 & .57501 & .43385 & .22126 & .14054 \\
\hline 3272 & .15118 & .07257 & .48006 & .24174 & .10103 & .11831 & 4520 & .69652 & .39417 & .56592 & .46953 & .22579 & .15523 \\
\hline 3277 & .18462 & .11423 & 61875 & .53719 & .35262 & .36554 & 4525 & 1.2094 & .92108 & .76157 & .65530 & .38131 & .17264 \\
\hline 3278 & .51918 & .30202 & .58171 & .48255 & .25409 & .14974 & 4563 & .56280 & .35317 & .62752 & .48782 & .27605 & .24136 \\
\hline 3280 & .36338 & .22921 & .63078 & .50938 & .25946 & .13886 & 4570 & .61079 & .35969 & .58889 & .49491 & .27051 & .17147 \\
\hline 3281 & .37810 & .18433 & .48753 & .30871 & .12545 & .06205 & 4577 & .85494 & .47531 & .55595 & .43914 & .24513 & .17104 \\
\hline 3283 & .65667 & .41926 & .63847 & .49103 & .26018 & .16137 & 4591 & .77619 & .45091 & .58093 & .44911 & .24016 & .15972 \\
\hline 3284 & .72548 & .41755 & .57556 & .45105 & .22653 & .13662 & 4670 & .59396 & .34703 & .58427 & .47748 & .25339 & .16038 \\
\hline 3285 & .75086 & .42482 & .56578 & .45078 & .21261 & .13545 & 4671 & .49934 & .30429 & .60939 & .44824 & .21102 & .13341 \\
\hline 3329 & .68397 & .42859 & .62662 & .51937 & .30230 & .20960 & 4679 & .73900 & .43878 & .59374 & .49510 & .26543 & .18010 \\
\hline 3335 & .99655 & .59219 & .59424 & .48830 & .28370 & .19673 & 4696 & .48000 & .23956 & .49907 & .16419 & -.08468 & .09508 \\
\hline 3370 & .81111 & .46203 & .56962 & .43311 & .22872 & .14840 & 4703 & .52417 & .30662 & .58496 & .48338 & .24730 & .13210 \\
\hline 3410 & .58299 & .32358 & .55504 & .46624 & .23998 & .15785 & 4704 & 1.0280 & 62862 & .61150 & .48494 & .26302 & .16214 \\
\hline 3415 & .73198 & .42297 & .57785 & .46236 & .24459 & .16117 & 4731 & .56219 & .38996 & .69365 & .59075 & .38837 & .29284 \\
\hline 3430 & .72991 & .48774 & .66822 & .53536 & .30855 & .21244 & 4792 & .71358 & .39722 & .55666 & .45278 & .21205 & .14111 \\
\hline 3431 & .77739 & .55309 & .71147 & 60705 & .39771 & .30245 & 4819 & .85146 & .46078 & .54116 & .40420 & .17521 & .11734 \\
\hline 3441 & .72719 & .41652 & .57279 & .43761 & .20340 & .07215 & 4852 & .94467 & .45876 & .48563 & .43655 & .37026 & .24860 \\
\hline 3442 & .47202 & .27542 & .58349 & .47263 & .24113 & .13203 & 4866 & .77023 & .43493 & .56468 & .44017 & .22878 & .15104 \\
\hline 3446 & .52944 & .29290 & .55322 & .44191 & .23498 & .15479 & 4876 & .97273 & .58332 & .59967 & .48253 & .23117 & .14066 \\
\hline 3460 & .95581 & .53611 & .56090 & .36116 & .21793 & .28168 & 4878 & .96671 & .56816 & .58772 & .46002 & .24664 & .15574 \\
\hline 3462 & .58398 & .29925 & .51243 & .37995 & .18940 & .13936 & 4880 & .52453 & .30361 & .57883 & .48197 & .26191 & .16523 \\
\hline
\end{tabular}


Appendix 4-2.5. L-moments of storm depth defined by 24-hour minimum interevent time for hourly rainfall stations in TexasContinued.

\begin{tabular}{|c|c|c|c|c|c|c|c|c|c|c|c|c|c|}
\hline $\begin{array}{c}\text { Station } \\
\text { no. }\end{array}$ & $\begin{array}{c}\text { Depth } \\
\text { mean } \\
\text { (inches) }\end{array}$ & $\begin{array}{c}\text { Depth } \\
\text { L-scale } \\
\text { (inches) }\end{array}$ & $\begin{array}{c}\text { Depth } \\
\text { L-CV } \\
\text { (dimen- } \\
\text { sionless) }\end{array}$ & $\begin{array}{c}\text { Depth } \\
\text { L-skew } \\
\text { (dimen- } \\
\text { sionless) }\end{array}$ & $\begin{array}{c}\text { Depth } \\
\text { L-kurtosis } \\
\text { (dimen- } \\
\text { sionless) }\end{array}$ & $\begin{array}{c}\text { Depth } \\
\text { Tau5 } \\
\text { (dimen- } \\
\text { sionless) }\end{array}$ & $\begin{array}{c}\text { Station } \\
\text { no. }\end{array}$ & $\begin{array}{l}\text { Depth } \\
\text { mean } \\
\text { (inches) }\end{array}$ & $\begin{array}{l}\text { Depth } \\
\text { L-scale } \\
\text { (inches) }\end{array}$ & $\begin{array}{c}\text { Depth } \\
\text { L-CV } \\
\text { (dimen- } \\
\text { sionless) }\end{array}$ & $\begin{array}{c}\text { Depth } \\
\text { L-skew } \\
\text { (dimen- } \\
\text { sionless) }\end{array}$ & $\begin{array}{c}\text { Depth } \\
\text { L-kurtosis } \\
\text { (dimen- } \\
\text { sionless) }\end{array}$ & $\begin{array}{c}\text { Depth } \\
\text { Tau5 } \\
\text { (dimen- } \\
\text { sionless) }\end{array}$ \\
\hline 4920 & 0.67692 & 0.40980 & 0.60539 & 0.50113 & 0.26021 & 0.15934 & 5957 & 0.71762 & 0.41360 & 0.57635 & 0.46541 & 0.24261 & 0.16642 \\
\hline 4934 & .38667 & .28067 & .72586 & .77553 & .64964 & .49287 & 5958 & .68826 & .38488 & .55921 & .36675 & .12812 & .05699 \\
\hline 4972 & .67704 & .38799 & .57306 & .46339 & .24889 & .16752 & 5973 & .53835 & .37332 & .69345 & .57763 & .31929 & .18701 \\
\hline 4973 & .92576 & .48206 & .52071 & .39853 & .22885 & .15621 & 5996 & .68600 & .39672 & .57830 & .46171 & .23702 & .15678 \\
\hline 4974 & .55477 & .32952 & .59398 & .49534 & .28158 & .18719 & 6017 & .49554 & .31281 & .63125 & .48811 & .23557 & .11939 \\
\hline 4975 & .86435 & .47730 & .55221 & .43284 & .21337 & .15229 & 6024 & 1.0924 & .65139 & .59632 & .46350 & .26694 & .19990 \\
\hline 4978 & .70175 & .43875 & .62522 & .48441 & .23993 & .13004 & 6050 & .70211 & .32620 & .46460 & .30137 & .01414 & -.07950 \\
\hline 4979 & 1.2031 & .65038 & .54057 & .39683 & .15406 & -.00484 & 6104 & .46666 & .27175 & .58233 & .50284 & .29621 & .20032 \\
\hline 4982 & .61824 & .35513 & .57442 & .44658 & .23248 & .14884 & 6108 & .90398 & .50539 & .55907 & .42812 & .21856 & .14686 \\
\hline 5018 & .70407 & .38544 & .54744 & .39807 & .19255 & .13071 & 6136 & .47179 & .26396 & .55950 & .48903 & .25762 & .15963 \\
\hline 5048 & .53212 & .32066 & .60261 & .53072 & .28892 & .18174 & 6166 & .45260 & .26496 & .58542 & .47617 & .23519 & .11590 \\
\hline 5049 & .47727 & .26946 & .56459 & .57982 & .37978 & .30932 & 6176 & .87488 & .52144 & .59602 & .47705 & .27584 & .17534 \\
\hline 5056 & .43200 & .18600 & .43056 & .26882 & .67742 & .11828 & 6177 & .89177 & .51257 & .57477 & .45901 & .24913 & .16913 \\
\hline 5057 & .43577 & .30252 & .69422 & .57934 & .33681 & .20708 & 6210 & .79511 & .45704 & .57481 & .44728 & .22245 & .14674 \\
\hline 5060 & .69735 & .48457 & .69487 & .57589 & .34468 & .23270 & 6211 & .83838 & .50445 & 60169 & .44147 & .21186 & .13135 \\
\hline 5081 & .82672 & .45735 & .55321 & .39835 & .20264 & .13918 & 6270 & .91930 & .51102 & .55588 & .44367 & .23125 & .16125 \\
\hline 5094 & .77558 & .44574 & .57471 & .45739 & .23907 & .15145 & 6275 & -- & -- & -- & -- & -- & -- \\
\hline 5113 & .69712 & .43429 & .62298 & .53367 & .30373 & .19817 & 6276 & 1.1295 & .55355 & .49009 & .16073 & -.03778 & .05689 \\
\hline 5114 & -- & -- & -- & -- & -- & -- & 6335 & .78108 & .44065 & .56415 & .42528 & .21860 & .14430 \\
\hline 5123 & .69786 & .44467 & .63719 & .55715 & .27423 & .01679 & 6434 & .66294 & .34434 & .51941 & .33649 & .12141 & .12260 \\
\hline 5192 & .78129 & .45286 & .57963 & .45611 & .23584 & .14391 & 6504 & .52124 & .30334 & .58196 & .49437 & .25828 & .15800 \\
\hline 5193 & .74973 & .44479 & .59326 & .46553 & .23608 & .15359 & 6558 & .77043 & .48431 & .62862 & .48936 & .29121 & .30356 \\
\hline 5224 & .90833 & .55357 & .60944 & .48052 & .28886 & .21457 & 6615 & .61588 & .36725 & .59629 & .54857 & .32135 & .24023 \\
\hline 5228 & .73497 & .43723 & .59490 & .42663 & .18924 & .11776 & 6660 & .82188 & .48536 & .59055 & .47269 & .24660 & .13113 \\
\hline 5235 & .71522 & .40850 & .57115 & .35656 & .15116 & .14486 & 6663 & .76928 & .43042 & .55951 & .41920 & .20026 & .12580 \\
\hline 5247 & .52722 & .29605 & .56154 & .46527 & .23624 & .14326 & 6734 & .53657 & .31887 & .59426 & .46813 & .25065 & .15937 \\
\hline 5258 & .76069 & .44219 & .58130 & .46378 & .25075 & .15170 & 6736 & .55928 & .32911 & .58845 & .50962 & .27754 & .17584 \\
\hline 5303 & .69217 & .43481 & .62818 & .52222 & .30341 & .21948 & 6740 & 1.6275 & .88659 & .54476 & .24768 & -.07294 & -.12604 \\
\hline 5312 & .64621 & .37892 & .58638 & .49937 & .25655 & .17012 & 6750 & .86244 & 60239 & .69848 & .56187 & .32126 & .21266 \\
\hline 5341 & 1.3637 & .90199 & .66143 & .56701 & .27846 & .05322 & 6757 & .79721 & .47281 & .59308 & .45472 & .24015 & .15978 \\
\hline 5342 & -- & -- & -- & -- & -- & -- & 6775 & .50923 & .29965 & .58843 & .44784 & .22955 & .14551 \\
\hline 5348 & .88200 & .49081 & .55648 & .41990 & .19259 & .11933 & 6776 & .52750 & .29731 & .56362 & .47461 & .25188 & .15809 \\
\hline 5358 & .59686 & .34840 & .58372 & .46173 & .23626 & .14269 & 6788 & .83470 & .46005 & .55116 & .42849 & .25469 & .15663 \\
\hline 5398 & .83003 & .47765 & .57546 & .44903 & .24058 & .15968 & 6792 & .41852 & .23535 & .56233 & .47974 & .25049 & .14342 \\
\hline 5410 & .52474 & .30523 & .58169 & .49937 & .28590 & .19397 & 6794 & 1.9306 & .94588 & .48993 & .22585 & .02468 & .00395 \\
\hline 5411 & .44280 & .29048 & .65601 & .51978 & .28442 & .18659 & 6834 & .89218 & .49978 & .56018 & .44713 & .22891 & .16292 \\
\hline 5424 & .85476 & .55196 & .64575 & .47844 & .24001 & .15920 & 6893 & .38622 & .21964 & .56870 & .54310 & .32620 & .21335 \\
\hline 5429 & .70418 & .43429 & .61672 & .48389 & .25279 & .16305 & 6935 & .52500 & .30135 & .57399 & .51720 & .29385 & .19556 \\
\hline 5431 & 1.0329 & .52538 & .50867 & .38590 & .18418 & .09813 & 6981 & .76589 & .43687 & .57041 & .45621 & .26117 & .17825 \\
\hline 5461 & .85498 & .49680 & .58106 & .45177 & .24693 & .16673 & 7020 & .80327 & .48871 & .60840 & .48915 & .28807 & .20920 \\
\hline 5463 & .82760 & .47062 & .56865 & .45927 & .22773 & .15865 & 7060 & .60094 & .34131 & .56795 & .46947 & .22843 & .13310 \\
\hline 5471 & .25438 & .16246 & .63866 & .61587 & .39869 & .26261 & 7066 & .87052 & .49151 & .56462 & .43088 & .22140 & .14602 \\
\hline 5477 & 1.0088 & .76993 & .76319 & .81721 & .75943 & .72088 & 7074 & .49984 & .29785 & .59590 & .52037 & .31064 & .21245 \\
\hline 5528 & .82439 & .45246 & .54885 & .40823 & .20639 & .13020 & 7097 & .72728 & .41042 & .56432 & .45268 & .23485 & .12546 \\
\hline 5579 & -- & -- & -- & -- & -- & -- & 7116 & .53304 & .30429 & .57085 & .44876 & .24418 & .16935 \\
\hline 5580 & .78100 & .36916 & .47267 & .22360 & -.00245 & -.08559 & 7140 & .84510 & .55251 & .65378 & .54284 & .31652 & .20978 \\
\hline 5589 & .35163 & .19896 & .56583 & .39408 & .16219 & .07913 & 7173 & .92916 & 62289 & .67038 & .52713 & .29574 & .20103 \\
\hline 5590 & .40745 & .24458 & .60027 & .49044 & .25354 & .14390 & 7174 & .86073 & .56731 & 65910 & .51869 & .29415 & .20456 \\
\hline 5591 & .42400 & .22155 & .52252 & .41335 & .22723 & .14944 & 7213 & .77685 & .44625 & .57444 & .43736 & .22511 & .13990 \\
\hline 5592 & .37807 & .22123 & .58515 & .48687 & .27443 & .17838 & 7243 & .64525 & .37008 & .57354 & .46889 & .24006 & .16095 \\
\hline 5594 & .35735 & .19959 & .55852 & .45803 & .24218 & .14685 & 7262 & .24138 & .14282 & .59171 & .45121 & .24129 & .16926 \\
\hline 5595 & -- & -- & -- & -- & -- & -- & 7274 & .75209 & .41470 & .55140 & .45780 & .25903 & .16652 \\
\hline 5596 & .46881 & .25755 & .54936 & .52813 & .28290 & .18946 & 7300 & .67764 & .39285 & .57973 & .44327 & .23125 & .15181 \\
\hline 5600 & .44977 & .26968 & .59959 & .51574 & .31174 & .21361 & 7311 & .76600 & .44497 & .58090 & .50375 & .24424 & .14364 \\
\hline 5618 & .83917 & .51033 & .60813 & .49131 & .26664 & .17940 & 7363 & .95294 & .49699 & .52153 & .21947 & -.03585 & .00592 \\
\hline 5650 & .63765 & .29647 & .46494 & .47550 & .38917 & .20704 & 7422 & .72591 & .44218 & .60914 & .49507 & .26821 & .17906 \\
\hline 5656 & .56724 & .32609 & .57487 & .51331 & .25919 & .16928 & 7431 & .50035 & .29382 & .58722 & .50413 & .27898 & .17650 \\
\hline 5658 & .53971 & .33034 & .61206 & .51643 & .30699 & .20861 & 7481 & .41020 & .25009 & 60968 & .55859 & .35133 & .23500 \\
\hline 5661 & .74655 & .49846 & .66768 & .64313 & .41855 & .31631 & 7497 & .74459 & .42953 & .57687 & .48335 & .24538 & .17615 \\
\hline 5666 & .60286 & .38437 & .63757 & .53641 & .32195 & .24495 & 7498 & .75629 & .44192 & .58434 & .48175 & .24148 & .18407 \\
\hline 5695 & .79739 & .44821 & .56210 & .44108 & .24705 & .17078 & 7499 & .68918 & .39149 & .56805 & .47218 & .23995 & .15839 \\
\hline 5742 & .37571 & .24604 & .65487 & .67329 & .57093 & .52720 & 7531 & .73629 & .41234 & .56003 & .40489 & .18392 & .10829 \\
\hline 5766 & 1.0574 & .57229 & .54123 & .38271 & .13802 & .04368 & 7534 & .72690 & .44824 & .61665 & .51067 & .30941 & .23174 \\
\hline 5770 & .56232 & .32582 & .57942 & .47809 & .24978 & .15235 & 7556 & .72577 & .42615 & .58716 & .49085 & .26343 & .17439 \\
\hline 5775 & .36417 & .20523 & .56355 & .30690 & -.11308 & -.08810 & 7594 & .85730 & .49421 & .57647 & .44474 & .23915 & .16156 \\
\hline 5779 & .80333 & .43974 & .54739 & .40638 & .16808 & .03413 & 7596 & .81027 & .47105 & .58135 & .37687 & .12897 & .07379 \\
\hline 5840 & .70273 & .41506 & .59064 & .45251 & .22161 & .13244 & 7608 & .69238 & .43142 & 62309 & .47802 & .25304 & .16886 \\
\hline 5890 & .40336 & .26395 & .65438 & .52422 & .28943 & .18494 & 7622 & .25737 & .19942 & .77482 & 68197 & .43872 & .29883 \\
\hline 5891 & .43867 & .23059 & .52565 & .38539 & .17140 & .10276 & 7700 & .82390 & .48729 & .59145 & .44398 & .22614 & .14592 \\
\hline 5897 & .79375 & .45762 & .57653 & .46774 & .22971 & .16109 & 7706 & .62276 & .37027 & .59456 & .49740 & .27551 & .17739 \\
\hline
\end{tabular}


Appendix 4-2.5. L-moments of storm depth defined by 24-hour minimum interevent time for hourly rainfall stations in TexasContinued.

\begin{tabular}{|c|c|c|c|c|c|c|c|c|c|c|c|c|c|}
\hline $\begin{array}{c}\text { Station } \\
\text { no. }\end{array}$ & $\begin{array}{l}\text { Depth } \\
\text { mean } \\
\text { (inches) }\end{array}$ & $\begin{array}{l}\text { Depth } \\
\text { L-scale } \\
\text { (inches) }\end{array}$ & $\begin{array}{c}\text { Depth } \\
\text { L-CV } \\
\text { (dimen- } \\
\text { sionless) }\end{array}$ & $\begin{array}{c}\text { Depth } \\
\text { L-skew } \\
\text { (dimen- } \\
\text { sionless) }\end{array}$ & $\begin{array}{c}\text { Depth } \\
\text { L-kurtosis } \\
\text { (dimen- } \\
\text { sionless) }\end{array}$ & $\begin{array}{c}\text { Depth } \\
\text { Tau5 } \\
\text { (dimen- } \\
\text { sionless) }\end{array}$ & $\begin{array}{c}\text { Station } \\
\text { no. }\end{array}$ & $\begin{array}{l}\text { Depth } \\
\text { mean } \\
\text { (inches) }\end{array}$ & $\begin{array}{l}\text { Depth } \\
\text { L-scale } \\
\text { (inches) }\end{array}$ & $\begin{array}{c}\text { Depth } \\
\text { L-CV } \\
\text { (dimen- } \\
\text { sionless) }\end{array}$ & $\begin{array}{l}\text { Depth } \\
\text { L-skew } \\
\text { (dimen- } \\
\text { sionless) }\end{array}$ & $\begin{array}{l}\text { Depth } \\
\text { L-kurtosis } \\
\text { (dimen- } \\
\text { sionless) }\end{array}$ & $\begin{array}{c}\text { Depth } \\
\text { Tau5 } \\
\text { (dimen- } \\
\text { sionless) }\end{array}$ \\
\hline 7718 & 0.80519 & 0.47914 & 0.59507 & 0.49209 & 0.28075 & 0.17880 & 8910 & 0.83118 & 0.49765 & 0.59873 & 0.40290 & 0.14408 & 0.05599 \\
\hline 7745 & .90960 & .54698 & .60135 & .47467 & .26671 & .18474 & 8911 & .64338 & .39337 & .61141 & .46169 & .23603 & .15528 \\
\hline 7922 & .28676 & .16478 & .57463 & .45934 & .25232 & .15425 & 8924 & .35789 & .18774 & .52457 & .41736 & .20718 & .10536 \\
\hline 7936 & .91140 & .52016 & .57072 & .44433 & .23577 & .16830 & 8929 & 1.4943 & 1.0552 & .70613 & .61669 & .48725 & .54717 \\
\hline 7943 & .46788 & .30671 & .65554 & .51221 & .26682 & .16364 & 8942 & .88892 & .50457 & .56762 & .44669 & .22652 & .14968 \\
\hline 7944 & .54000 & .33991 & .62947 & .46177 & .21511 & .10481 & 8944 & .75642 & .45684 & .60395 & .45876 & .24459 & .16499 \\
\hline 7945 & .59669 & .40840 & .68445 & .54801 & .30882 & .20814 & 8996 & .76254 & .46362 & .60800 & .47264 & .25112 & .16960 \\
\hline 7947 & .88154 & .55325 & .62759 & .54978 & .33266 & .24756 & 9014 & .96294 & .43875 & .45564 & .32375 & .20106 & .08604 \\
\hline 7948 & .67858 & .42708 & .62937 & .50804 & .27622 & .16996 & 9037 & .38393 & .22880 & .59593 & .53313 & .33941 & .23816 \\
\hline 7951 & .79741 & .43670 & .54765 & .40449 & .20261 & .13151 & 9106 & .36639 & .23932 & .65319 & .59900 & .40979 & .32544 \\
\hline 7953 & .58927 & .37587 & .63785 & .53327 & .29740 & .16221 & 9107 & .41839 & .24834 & .59357 & .45102 & .18256 & .12444 \\
\hline 7981 & .66670 & .41324 & .61982 & .47141 & .21171 & .09803 & 9129 & .47753 & .32564 & .68194 & .59215 & .34924 & .21514 \\
\hline 7990 & .66745 & .45804 & .68626 & .57759 & .33232 & .18069 & 9163 & .66365 & .38621 & .58195 & .47032 & .24905 & .15492 \\
\hline 7992 & .95353 & .51515 & .54025 & .27057 & -.02989 & -.01579 & 9213 & .67395 & .41278 & .61248 & .44820 & .22462 & .14873 \\
\hline 7997 & .59000 & .36630 & .62085 & .51374 & .30800 & .21858 & 9214 & 1.2668 & .83232 & .65701 & .63027 & .44189 & .31677 \\
\hline 7999 & .38875 & .25650 & .65981 & .54080 & .30815 & .17772 & 9222 & .71012 & .43297 & .60971 & .46956 & .23358 & .13957 \\
\hline 8022 & .54778 & .33040 & .60316 & .43354 & .12207 & .02274 & 9248 & .54187 & .30510 & .56305 & .41993 & .17647 & .09918 \\
\hline 8023 & .54879 & .32108 & .58507 & .50289 & .27994 & .18594 & 9266 & .62045 & .34338 & .55344 & .39468 & .14700 & .05624 \\
\hline 8047 & .70935 & .40472 & .57055 & .44086 & .21862 & .14116 & 9270 & .42289 & .23402 & .55340 & .51261 & .28741 & .18523 \\
\hline 8060 & .56142 & .37343 & .66517 & .53070 & .25473 & .11794 & 9295 & .25133 & .15822 & .62954 & .53667 & .32729 & .21395 \\
\hline 8062 & .85121 & .60100 & .70606 & .55291 & .27905 & .13495 & 9304 & -- & -- & -- & -- & -- & -- \\
\hline 8068 & .33600 & .21322 & .63458 & .52096 & .28187 & .15252 & 9307 & .67148 & .36017 & .53638 & .42088 & .21390 & .12496 \\
\hline 8081 & .70016 & .44024 & .62877 & .53983 & .31787 & .22081 & 9328 & .53184 & .30781 & .57876 & .45057 & .23452 & .15322 \\
\hline 8089 & .53757 & .29983 & .55776 & .34847 & .11078 & .05739 & 9329 & .72500 & .39144 & .53992 & .25802 & .05377 & .26398 \\
\hline 8221 & .82412 & .38779 & .47056 & .29905 & .14697 & .03923 & 9345 & -- & -- & -- & -- & -- & -- \\
\hline 8252 & .53522 & .30810 & .57564 & .47827 & .25128 & .15581 & 9363 & .59474 & .40431 & .67981 & .54954 & .31295 & .20840 \\
\hline 8265 & .88431 & .54395 & .61511 & .49350 & .28046 & .19632 & 9364 & .66368 & .45427 & .68447 & .55106 & .31338 & .21186 \\
\hline 8289 & .52500 & .23628 & .45005 & .15446 & -.01247 & .01306 & 9365 & .48000 & .29542 & .61545 & .60492 & .42249 & .31311 \\
\hline 8305 & .35878 & .20347 & .56713 & .52154 & .31388 & .20236 & 9371 & .73058 & .41978 & .57459 & .39914 & .12219 & .03001 \\
\hline 8335 & .88990 & .49747 & .55902 & .41536 & .22032 & .15404 & 9417 & .71727 & .41806 & .58284 & .42652 & .19689 & .11882 \\
\hline 8400 & .45131 & .26436 & .58577 & .50540 & .31887 & .22371 & 9419 & .63370 & .39782 & .62778 & .46542 & .22937 & .14837 \\
\hline 8445 & .78962 & .48022 & .60816 & .47554 & .25339 & .16892 & 9435 & .55119 & .30136 & .54674 & .45594 & .30909 & .27169 \\
\hline 8446 & .71300 & .41021 & .57533 & .45061 & .22055 & .13418 & 9491 & .79285 & .46217 & .58293 & .44915 & .22487 & .14361 \\
\hline 8451 & .55856 & .33887 & .60669 & .49437 & .26936 & .17256 & 9499 & .58857 & .33988 & .57748 & .47109 & .21471 & .11941 \\
\hline 8531 & .73233 & .41406 & .56540 & .43353 & .22531 & .14534 & 9522 & 2.0725 & 1.4675 & .70808 & .56072 & .26040 & .15697 \\
\hline 8541 & .69418 & .39790 & .57320 & .42843 & .21701 & .12740 & 9527 & .51606 & .29223 & .56628 & .50062 & .26224 & .16171 \\
\hline 8544 & .80449 & .49505 & .61536 & .51917 & .28285 & .19444 & 9532 & .70330 & .40924 & .58189 & .46554 & .25032 & .16904 \\
\hline 8545 & .40200 & .18771 & .46695 & .17855 & .10178 & .21255 & 9544 & -- & -- & -- & -- & -- & -- \\
\hline 8563 & .79008 & .45108 & .57093 & .47398 & .24495 & .17366 & 9565 & .62227 & .36633 & .58870 & .49157 & .26943 & .16906 \\
\hline 8566 & .56966 & .33511 & .58827 & .44550 & .21001 & .12022 & 9570 & .58512 & .32587 & .55694 & .48326 & .22261 & .12514 \\
\hline 8583 & .62273 & .35548 & .57083 & .48139 & .21262 & .13392 & 9574 & .61053 & .34854 & .57088 & .45302 & .15700 & .08150 \\
\hline 8584 & .71361 & .41033 & .57500 & .45394 & .21928 & .13783 & 9588 & .63232 & .40165 & 63519 & .54076 & .29757 & .17699 \\
\hline 8623 & .73314 & .40647 & .55443 & .44579 & .22291 & .14690 & 9665 & .81309 & .46685 & .57417 & .45425 & .24270 & .16692 \\
\hline 8625 & .70020 & .40933 & .58459 & .45881 & .24854 & .17099 & 9715 & .72551 & .41665 & .57429 & .44680 & .23263 & .15578 \\
\hline 8630 & .53783 & .30364 & .56456 & .44117 & .20562 & .11417 & 9729 & .59963 & .37506 & .62549 & .46558 & .23186 & .15284 \\
\hline 8631 & .61150 & .35707 & .58393 & .47111 & .25050 & .15084 & 9772 & .77584 & .50483 & .65069 & .54090 & .32107 & .21966 \\
\hline 8646 & .69673 & .40432 & .58032 & .44170 & .22188 & .14409 & 9814 & .67125 & .40808 & 60795 & .45551 & .21103 & .11327 \\
\hline 8647 & .50962 & .29415 & .57719 & .50555 & .27042 & .17192 & 9815 & .79120 & .47593 & .60153 & .49215 & .27032 & .18093 \\
\hline 8677 & 69096 & .38826 & .56190 & .46237 & .24744 & .16000 & 9816 & .69154 & .43881 & .63455 & .52466 & .27427 & .14765 \\
\hline 8696 & 1.0079 & .60047 & .59576 & .44235 & .21963 & .10008 & 9817 & .66834 & .37643 & .56323 & .46507 & .24141 & .16806 \\
\hline 8743 & .85888 & .49955 & .58163 & .45218 & .23918 & .15886 & 9829 & .42445 & .25268 & .59530 & .52872 & .30568 & .18797 \\
\hline 8761 & .49820 & .28459 & .57124 & .50437 & .26819 & .16125 & 9830 & .33007 & .20039 & .60712 & .49342 & .23717 & .11141 \\
\hline 8778 & .81021 & .45790 & .56517 & .44889 & .23150 & .15533 & 9858 & .52269 & .29731 & .56880 & .45955 & .24515 & .14706 \\
\hline 8845 & .76350 & .46697 & .61162 & .50564 & .27016 & .17261 & 9893 & .65987 & .38521 & .58376 & .46109 & .24154 & .15849 \\
\hline 8859 & .86130 & .47837 & .55540 & .41421 & .21800 & .14925 & 9916 & .86990 & .47861 & .55019 & .40638 & .18260 & .11491 \\
\hline 8898 & .81788 & .47064 & .57543 & .45234 & .24125 & .14801 & 9976 & .61335 & .37339 & .60877 & .50948 & .27611 & .17630 \\
\hline 8908 & .73967 & .43557 & .58888 & .46861 & .29014 & .23783 & & & & & & & \\
\hline
\end{tabular}


Appendix 4-2.6. L-moments of storm depth defined by 48-hour minimum interevent time for hourly rainfall stations in Texas.

$[--$, not available $]$

\begin{tabular}{|c|c|c|c|c|c|c|c|c|c|c|c|c|c|}
\hline $\begin{array}{c}\text { Station } \\
\text { no. }\end{array}$ & $\begin{array}{l}\text { Depth } \\
\text { mean } \\
\text { (inches) }\end{array}$ & $\begin{array}{c}\text { Depth } \\
\text { L-scale } \\
\text { (inches) }\end{array}$ & $\begin{array}{l}\text { Depth } \\
\text { L-CV } \\
\text { (dimen- } \\
\text { sionless) }\end{array}$ & $\begin{array}{c}\text { Depth } \\
\text { L-skew } \\
\text { (dimen- } \\
\text { sionless) }\end{array}$ & $\begin{array}{c}\text { Depth } \\
\text { L-kurtosis } \\
\text { (dimen- } \\
\text { sionless) }\end{array}$ & $\begin{array}{l}\text { Depth } \\
\text { Tau5 } \\
\text { (dimen- } \\
\text { sionless) }\end{array}$ & $\begin{array}{c}\text { Station } \\
\text { no. }\end{array}$ & $\begin{array}{c}\text { Depth } \\
\text { mean } \\
\text { (inches) }\end{array}$ & $\begin{array}{c}\text { Depth } \\
\text { L-scale } \\
\text { (inches) }\end{array}$ & $\begin{array}{c}\text { Depth } \\
\text { L-CV } \\
\text { (dimen- } \\
\text { sionless) }\end{array}$ & $\begin{array}{c}\text { Depth } \\
\text { L-skew } \\
\text { (dimen- } \\
\text { sionless) }\end{array}$ & $\begin{array}{c}\text { Depth } \\
\text { L-kurtosis } \\
\text { (dimen- } \\
\text { sionless) }\end{array}$ & $\begin{array}{c}\text { Depth } \\
\text { Tau5 } \\
\text { (dimen- } \\
\text { sionless) }\end{array}$ \\
\hline 0015 & -- & -- & -- & -- & -- & -- & 1154 & 0.90333 & 0.55334 & 0.61255 & 0.43539 & 0.19295 & 0.11139 \\
\hline 0054 & .61484 & .36430 & .59251 & .57910 & .45730 & .33613 & 1186 & .96615 & .61283 & .63430 & .50245 & .27190 & .15359 \\
\hline 0120 & 1.8100 & 1.1549 & .63807 & .43976 & .23869 & .31158 & 1188 & .66167 & .28300 & .42771 & .31684 & .28151 & .87750 \\
\hline 0145 & .69564 & .48582 & .69838 & .59541 & .38842 & .29582 & 1245 & 1.7025 & 1.2922 & .75900 & .67528 & .50077 & .40718 \\
\hline 0179 & .51592 & .32372 & .62746 & .53243 & .31367 & .20839 & 1325 & .97351 & .58664 & . 60260 & .46251 & .23547 & .15343 \\
\hline 0202 & .80726 & .43945 & .54436 & .42280 & .18498 & .11030 & 1429 & .85684 & .51207 & .59763 & .47914 & .26661 & .18072 \\
\hline 0206 & .91253 & .49834 & .54611 & .41418 & .20118 & .12549 & 1431 & .92773 & .54197 & .58418 & .44416 & .23106 & .15560 \\
\hline 0208 & -- & -- & -- & -- & -- & -- & 1432 & .91823 & .53591 & .58364 & .43849 & .23331 & .15808 \\
\hline 0211 & .53554 & .34480 & .64384 & .50036 & .26423 & .16807 & 1433 & .91627 & .53312 & .58184 & .45788 & .25402 & .16307 \\
\hline 0244 & .88200 & .48150 & .54592 & .32550 & .09877 & .07126 & 1434 & .91665 & .53370 & .58223 & .43956 & .22229 & .14374 \\
\hline 0248 & .53947 & .31208 & .57850 & .49237 & .26742 & .16794 & 1435 & .91548 & .52642 & .57502 & .41569 & .19974 & .13081 \\
\hline 0408 & 2.9470 & 1.9992 & .67839 & .50203 & .14844 & -.14600 & 1492 & .77719 & .44967 & .57859 & .46670 & .24056 & .16288 \\
\hline 0427 & .95455 & .59072 & .61885 & .48517 & .21461 & .15358 & 1500 & 1.3608 & .96808 & .71142 & .62914 & .44822 & .42129 \\
\hline 0428 & .79794 & .51200 & .64166 & .48479 & .25132 & .16486 & 1528 & .75619 & .45800 & .60567 & .50201 & .26631 & .17398 \\
\hline 0429 & .98252 & .64013 & .65152 & .49142 & .25244 & .16263 & 1541 & 1.1475 & .68429 & .59634 & .50276 & .30670 & .22725 \\
\hline 0463 & 69953 & .42079 & .60153 & .55679 & .38119 & .26197 & 1569 & .94537 & .59664 & .63112 & .49661 & .28346 & .21993 \\
\hline 0493 & 1.3460 & .42867 & .31847 & .18040 & .21962 & .33152 & 1632 & .67000 & .25700 & .38358 & -.24514 & .08560 & -.27237 \\
\hline 0495 & .49518 & .27274 & .55078 & .42961 & .22263 & .13374 & 1641 & .63013 & .34673 & .55025 & .42383 & .22629 & .14985 \\
\hline 0496 & .29636 & .14667 & .49489 & .43211 & .27007 & .09429 & 1646 & .60037 & .34343 & .57204 & .48575 & .24602 & .15141 \\
\hline 0498 & .23667 & .07083 & .29930 & - -01176 & .36975 & .29804 & 1663 & 1.5333 & 1.0262 & .66927 & .58567 & .39145 & .32376 \\
\hline 0509 & .89954 & .52845 & .58747 & .47840 & .27171 & .19050 & 1671 & .91678 & .54068 & .58976 & .46296 & .23675 & .15276 \\
\hline 0518 & .94697 & .54250 & .57288 & .44457 & .23730 & .15847 & 1680 & .83327 & .47870 & .57448 & .45270 & .24883 & .16792 \\
\hline 0521 & .63074 & .38165 & .60509 & .53716 & .33917 & .19105 & 1694 & .71000 & .41457 & .58390 & .47358 & .19081 & .09314 \\
\hline 0556 & .73850 & .42516 & .57571 & .44472 & .19311 & .09387 & 1696 & .65460 & .37774 & .57706 & .43735 & .21976 & .13893 \\
\hline 0569 & 1.1083 & 68077 & .61425 & .49363 & .26556 & .17138 & 1697 & .55311 & .33424 & .60430 & .47710 & .22422 & .10571 \\
\hline 0572 & .95422 & .57434 & .60189 & .47608 & .26892 & .19052 & 1698 & 69026 & .39905 & .57811 & .47558 & .25798 & .16446 \\
\hline 0691 & .84808 & .49204 & .58018 & .44227 & .23932 & .15974 & 1903 & .63255 & .33595 & .53110 & .44697 & .23324 & .16786 \\
\hline 0708 & .89390 & .51140 & .57210 & .46569 & .22033 & .12268 & 1914 & 1.5640 & .90711 & .57999 & .53926 & .44167 & .46834 \\
\hline 0738 & .89483 & .50841 & .56817 & .43341 & .23168 & .15589 & 1920 & .93138 & .51368 & .55152 & .42365 & .23935 & .18331 \\
\hline 0776 & .61807 & .37260 & .60284 & .49138 & .26113 & .16431 & 1921 & 1.0310 & .58459 & .56703 & .43277 & .22415 & .14957 \\
\hline 0779 & .63902 & .37305 & .58379 & .52949 & .28233 & .16622 & 1937 & 1.0081 & .55458 & .55010 & .42309 & .24006 & .15749 \\
\hline 0784 & .62437 & .37249 & .59659 & .49771 & .26395 & 16196 & 1956 & 1.0585 & .61208 & .57827 & .43412 & .22480 & .15649 \\
\hline 0786 & .50325 & .30936 & .61472 & .45736 & .21943 & .12678 & 1970 & 2.6190 & 2.2461 & .85762 & .76658 & .53007 & .35981 \\
\hline 0917 & 1.1635 & .65608 & .56389 & .42828 & .23225 & .15035 & 2014 & .74384 & .51193 & .68823 & .52833 & .26202 & .14906 \\
\hline 0923 & 2.9320 & 1.8871 & .64363 & .44292 & .07957 & -.20193 & 2015 & .79898 & .55285 & .69194 & .55718 & .31893 & .21249 \\
\hline 0926 & .85831 & .49832 & .58058 & .44361 & .22195 & .13924 & 2019 & 2.1930 & 1.4310 & .65253 & .44840 & .13996 & -.05264 \\
\hline 0950 & .47074 & .30339 & .64450 & .58448 & .37265 & .25256 & 2024 & .90044 & .49860 & .55373 & .42831 & .23303 & .16159 \\
\hline 0996 & 2.8512 & 1.8223 & .63913 & .35228 & .01029 & .01068 & 2042 & .20778 & .09611 & .46257 & .20231 & .25681 & .28324 \\
\hline 1013 & .80473 & .52922 & .65763 & .62695 & .41366 & .31595 & 2043 & .33597 & .18768 & .55863 & .42287 & .23921 & .18008 \\
\hline 1017 & .73993 & .42976 & .58081 & .45571 & .24242 & .16319 & 2048 & .77135 & .46904 & 60807 & .49718 & .25770 & .16554 \\
\hline 1042 & 3.1971 & 1.8843 & .58937 & .49356 & .34647 & .18575 & 2050 & .51019 & .36823 & .72175 & .60470 & .35899 & .23554 \\
\hline 1048 & 1.3318 & .85709 & .64355 & .68420 & .65069 & .58054 & 2051 & .59922 & .36581 & .61048 & .46486 & .20948 & .12113 \\
\hline 1053 & .74575 & .44058 & .59079 & .44987 & .22014 & .13745 & 2053 & .37500 & .24433 & .65156 & .57299 & .32469 & .09550 \\
\hline 1057 & .66765 & .38758 & .58052 & .45256 & .23491 & .14617 & 2073 & .90232 & .53568 & .59367 & .48565 & .29687 & .21406 \\
\hline 1063 & 2.2010 & 1.2990 & .59019 & .29920 & .03699 & .12394 & 2082 & .53087 & .31320 & .58997 & .49572 & .27913 & .18342 \\
\hline 1068 & .90157 & .50440 & .55947 & .42203 & .21210 & .13845 & 2086 & .91586 & .52194 & .56990 & .44164 & .22883 & .15083 \\
\hline 1080 & .54574 & .29560 & .54165 & .44224 & .26401 & .18245 & 2088 & 1.1531 & .59204 & .51342 & .32416 & .19963 & .26294 \\
\hline 1081 & .90667 & .52308 & .57693 & .45852 & .26072 & .17235 & 2090 & .93060 & .50377 & .54134 & .42428 & .21424 & .14645 \\
\hline 1133 & .33636 & .21109 & .62757 & .35688 & .04967 & .10336 & 2096 & .89803 & .50530 & .56267 & .44351 & .24217 & .16161 \\
\hline 1136 & .71789 & .50322 & .70097 & .56753 & .32128 & .20599 & 2128 & .91139 & .52221 & .57298 & .40156 & .17066 & .11000 \\
\hline 1138 & 1.7167 & .95694 & .55744 & .44798 & .35745 & .25495 & 2131 & .82849 & .46467 & .56087 & .44201 & .22869 & .15365 \\
\hline 1139 & 1.0398 & .61227 & .58882 & .39460 & .13053 & .04507 & 2142 & 2.2450 & .63071 & .28094 & -.22537 & .13250 & -.21348 \\
\hline
\end{tabular}


208 Statistical Characteristics of Storm Interevent Time, Depth, and Duration for Eastern New Mexico, Oklahoma, and Texas

Appendix 4-2.6. L-moments of storm depth defined by 48-hour minimum interevent time for hourly rainfall stations in Texas-

Continued.

\begin{tabular}{|c|c|c|c|c|c|c|c|c|c|c|c|c|c|}
\hline $\begin{array}{c}\text { Station } \\
\text { no. }\end{array}$ & $\begin{array}{c}\text { Depth } \\
\text { mean } \\
\text { (inches) }\end{array}$ & $\begin{array}{c}\text { Depth } \\
\text { L-scale } \\
\text { (inches) }\end{array}$ & $\begin{array}{c}\text { Depth } \\
\text { L-CV } \\
\text { (dimen- } \\
\text { sionless) }\end{array}$ & $\begin{array}{c}\text { Depth } \\
\text { L-skew } \\
\text { (dimen- } \\
\text { sionless) }\end{array}$ & $\begin{array}{c}\text { Depth } \\
\text { L-kurtosis } \\
\text { (dimen- } \\
\text { sionless) }\end{array}$ & $\begin{array}{c}\text { Depth } \\
\text { Tau5 } \\
\text { (dimen- } \\
\text { sionless) }\end{array}$ & $\begin{array}{c}\text { Station } \\
\text { no. }\end{array}$ & $\begin{array}{c}\text { Depth } \\
\text { mean } \\
\text { (inches) }\end{array}$ & $\begin{array}{c}\text { Depth } \\
\text { L-scale } \\
\text { (inches) }\end{array}$ & $\begin{array}{c}\text { Depth } \\
\text { L-CV } \\
\text { (dimen- } \\
\text { sionless) }\end{array}$ & $\begin{array}{c}\text { Depth } \\
\text { L-skew } \\
\text { (dimen- } \\
\text { sionless) }\end{array}$ & $\begin{array}{c}\text { Depth } \\
\text { L-kurtosis } \\
\text { (dimen- } \\
\text { sionless) }\end{array}$ & $\begin{array}{c}\text { Depth } \\
\text { Tau5 } \\
\text { (dimen- } \\
\text { sionless) }\end{array}$ \\
\hline 2160 & 0.66000 & 0.48181 & 0.73001 & 0.66266 & 0.51556 & 0.46974 & 3463 & 0.89171 & 0.58866 & 0.66014 & 0.55719 & 0.37378 & 0.31178 \\
\hline 2206 & 1.0760 & 64438 & .59889 & .45169 & .23063 & .15443 & 3476 & .84173 & .50070 & .59484 & .46112 & .24734 & .16506 \\
\hline 2238 & .50606 & .33051 & .65311 & .50274 & .22884 & .09639 & 3485 & 1.5179 & 1.1217 & .73900 & .61274 & .27771 & .04666 \\
\hline 2240 & .57113 & .33643 & .58906 & .40021 & .16378 & .07767 & 3507 & .97564 & .55997 & .57396 & .44075 & .20560 & .12971 \\
\hline 2242 & .83256 & .49023 & .58883 & .41657 & .20626 & .13587 & 3546 & 1.0913 & .62222 & .57018 & .44072 & .23775 & .15758 \\
\hline 2244 & .90830 & .54323 & .59808 & .45212 & .23776 & .15163 & 3547 & .84948 & .47033 & .55367 & .38805 & .17023 & .11079 \\
\hline 2247 & .97323 & .58925 & .60546 & .41376 & .17180 & .09861 & 3579 & .95098 & .49108 & .51640 & .31005 & .08447 & .05900 \\
\hline 2309 & 1.1042 & .58091 & .52611 & .41904 & .25383 & .18332 & 3642 & .97441 & .55865 & .57332 & 44596 & .23684 & .15892 \\
\hline 2312 & 1.0118 & .52803 & .52190 & .39689 & .20397 & .12473 & 3646 & .85049 & .48771 & .57345 & .44355 & .23550 & .15614 \\
\hline 2334 & 1.2998 & .69257 & .53284 & .37378 & .20804 & .11767 & 3668 & 3.1500 & 1.5957 & .50658 & .48791 & .58326 & .60385 \\
\hline 2336 & .88167 & .46903 & .53198 & .35974 & .13586 & .07514 & 3673 & 2.1460 & 1.5351 & .71534 & .55588 & .33266 & .35242 \\
\hline 2354 & .52111 & .30837 & .59175 & .42534 & .14148 & .06067 & 3686 & .94092 & .51460 & .54691 & .41327 & .20535 & .14766 \\
\hline 2355 & .92394 & .58633 & .63459 & .50673 & .29102 & .17547 & 3691 & .88690 & .50392 & .56818 & .44122 & .22797 & .15188 \\
\hline 2357 & .60683 & .42582 & .70172 & .55921 & .30362 & .19125 & 3734 & 3.4370 & 2.5886 & .75314 & .60574 & .36117 & .27098 \\
\hline 2360 & .60367 & .41021 & .67953 & .54682 & .31020 & .21207 & 3771 & .94724 & .53562 & .56545 & .43107 & .19677 & .13286 \\
\hline 2361 & .71117 & .48810 & .68633 & .63180 & .45875 & .38331 & 3789 & .32552 & .18371 & .56435 & .45743 & .26123 & .17032 \\
\hline 2394 & .94322 & .54865 & .58169 & .43586 & .21921 & .13826 & 3826 & .77818 & .41065 & .52770 & .40876 & .23842 & .16704 \\
\hline 2404 & .87875 & .51573 & .58689 & .45564 & .24552 & .16750 & 3831 & .97215 & .55776 & .57373 & .41448 & .18881 & .11636 \\
\hline 2415 & 1.0861 & .61453 & .56581 & .43320 & .22983 & .14967 & 3841 & .92945 & .52721 & .56723 & .43398 & .22100 & .14284 \\
\hline 2462 & 1.0287 & .58140 & .56518 & .44014 & .23044 & .14627 & 3871 & .76105 & .43654 & .57359 & .44670 & .23861 & .15585 \\
\hline 2528 & .77518 & .43060 & .55549 & .35547 & .07078 & -.02533 & 3884 & 1.4200 & .87679 & .61746 & .35001 & -.00861 & -.06024 \\
\hline 2617 & .66702 & .39552 & .59297 & .49728 & .31530 & .23250 & 3941 & 1.1081 & .61188 & .55219 & .29057 & .07774 & .11667 \\
\hline 2619 & .65089 & .35550 & .54617 & .41534 & .21439 & .13852 & 3963 & -- & -- & -- & -- & -- & -- \\
\hline 2621 & .70393 & .40867 & .58055 & .46720 & .24840 & .15133 & 4040 & .74508 & .41249 & .55363 & .43512 & .24773 & .16515 \\
\hline 2675 & .93659 & .55401 & .59152 & .48882 & .27726 & .19224 & 4058 & 1.7150 & 1.3341 & .77790 & .75884 & .63070 & .54819 \\
\hline 2676 & .98411 & .58163 & .59102 & .47848 & .24642 & .17980 & 4098 & .55009 & .30781 & .55956 & .47660 & .24486 & .14778 \\
\hline 2679 & .74955 & .48256 & .64380 & .54103 & .29910 & .18317 & 4100 & .73688 & .44331 & .60160 & .50024 & .30364 & .21378 \\
\hline 2715 & .83102 & .46508 & .55965 & .43168 & .23637 & .16123 & 4137 & .89166 & .48773 & .54699 & .43082 & .22268 & .16415 \\
\hline 2744 & .72985 & .41688 & .57118 & .44525 & .22788 & .14912 & 4191 & .82277 & .49527 & .60196 & .48567 & .24979 & .15215 \\
\hline 2758 & 67222 & .47109 & .70080 & .59855 & .34821 & .20048 & 4256 & -- & -- & -- & -- & -- & -- \\
\hline 2794 & .47400 & .32244 & .68026 & .46468 & .11527 & -.05048 & 4257 & 1.1053 & .61877 & .55984 & .43084 & .22997 & .15647 \\
\hline 2797 & .30586 & .19426 & .63512 & .51226 & .28966 & .19671 & 4258 & 1.1057 & .61361 & .55494 & .43161 & .22318 & .15040 \\
\hline 2811 & .71054 & .42206 & .59400 & .47582 & .23756 & .14446 & 4278 & .87176 & .51368 & .58924 & .44919 & .23081 & .14770 \\
\hline 2813 & .78316 & .42778 & .54622 & .36840 & .11625 & -.04478 & 4299 & .42290 & .19374 & .45813 & .27534 & .08740 & .03753 \\
\hline 2814 & .36500 & .28767 & .78813 & .70742 & .47980 & .33121 & 4300 & 1.0013 & .63784 & .63698 & .48786 & .26310 & .17702 \\
\hline 2815 & .71477 & .41003 & .57365 & .50985 & .27860 & .16385 & 4305 & .95836 & .61578 & .64253 & .48347 & .25099 & .16751 \\
\hline 2818 & .77444 & .46549 & 60107 & .47508 & .26919 & .19007 & 4307 & 1.0317 & .68548 & .66441 & .54844 & .34514 & .25188 \\
\hline 2986 & 1.2019 & .64588 & .53737 & .37037 & .18414 & .14740 & 4309 & 1.0564 & .62661 & .59315 & .45163 & .24133 & .16488 \\
\hline 3005 & .82772 & .45944 & .55506 & .42570 & .22099 & .15345 & 4311 & 1.0993 & .64117 & .58327 & .43362 & .21724 & .13939 \\
\hline 3033 & .35235 & .20493 & .58162 & .50579 & .30755 & .21336 & 4313 & 1.1813 & .67521 & .57156 & .45690 & .24712 & .15282 \\
\hline 3034 & -- & -- & -- & -- & -- & -- & 4319 & .87967 & .48618 & .55269 & .44246 & .21955 & .11746 \\
\hline 3047 & 1.6455 & 1.1853 & .72033 & .72071 & .67403 & 62868 & 4329 & 1.0505 & 61041 & .58108 & .45191 & .24365 & .15942 \\
\hline 3103 & .99320 & 64087 & .64525 & .58301 & .31180 & .14061 & 4331 & -- & -- & -- & -- & -- & -- \\
\hline 3133 & .96033 & .54023 & .56254 & .42956 & .23564 & .15905 & 4375 & .88340 & .52381 & .59295 & .50771 & .28501 & .20053 \\
\hline 3156 & 1.1022 & 66982 & .60772 & .52089 & .32339 & .23033 & 4392 & 1.1937 & .70945 & .59432 & .45405 & .23681 & .15169 \\
\hline 3171 & .90544 & .51246 & .56598 & .43480 & .22911 & .14731 & 4425 & .56246 & .32679 & .58100 & .49108 & .25663 & .16008 \\
\hline 3189 & .49952 & .29405 & .58867 & .51453 & .29972 & .19686 & 4440 & .76499 & .44772 & .58526 & .45110 & .24657 & .17048 \\
\hline 3260 & .75533 & .44655 & .59120 & .41225 & .16356 & .07791 & 4476 & .85022 & .46114 & .54238 & .42444 & .22307 & .14829 \\
\hline 3267 & .65826 & .44718 & .67933 & .59815 & .39451 & .28190 & 4498 & .27667 & .12389 & .44779 & .14029 & .03587 & .01025 \\
\hline 3270 & .70027 & .39438 & .56319 & .47154 & .24898 & .17709 & 4517 & .85080 & .48960 & .57545 & .43196 & .21997 & .13654 \\
\hline 3272 & .21417 & .10992 & .51326 & .21406 & -.02228 & .00758 & 4520 & .85110 & .47632 & .55966 & .44328 & .21954 & .15606 \\
\hline 3277 & .20000 & .11879 & .59394 & .51276 & .36650 & .40115 & 4525 & 1.8142 & 1.4831 & .81751 & .77134 & .60484 & .45318 \\
\hline 3278 & 61790 & .36167 & .58532 & .47168 & .23862 & .14020 & 4563 & .93800 & .60162 & .64138 & .43849 & .13165 & -.00992 \\
\hline 3280 & .41613 & .25799 & 61997 & .48289 & .23267 & .11937 & 4570 & .72366 & .42284 & .58430 & .47752 & .25644 & .16154 \\
\hline 3281 & .41789 & .21622 & .51739 & .36792 & .18523 & .12351 & 4577 & 1.0558 & .58402 & .55314 & .43154 & .24442 & .16785 \\
\hline 3283 & .82776 & .51847 & .62636 & .47225 & .24586 & .15377 & 4591 & .93660 & .54456 & .58142 & .44107 & .23052 & .15222 \\
\hline 3284 & .88606 & .50781 & .57311 & .44254 & .22822 & .14358 & 4670 & .71530 & .41324 & .57772 & .45834 & .24163 & .15814 \\
\hline 3285 & .91715 & .51394 & .56036 & .43248 & .20564 & .12954 & 4671 & .61535 & .37448 & .60856 & .43505 & .18987 & .10521 \\
\hline 3329 & .82064 & .50334 & .61335 & .49224 & .27616 & .19225 & 4679 & .90840 & .53567 & .58969 & .48086 & .26391 & .18113 \\
\hline 3335 & 1.1560 & 64953 & .56188 & .45692 & .25754 & .18257 & 4696 & .68571 & .19905 & .29028 & -.06029 & .34450 & -.36842 \\
\hline 3370 & .97098 & .55038 & .56683 & .42922 & .22670 & .14066 & 4703 & .68342 & .38831 & .56818 & .44942 & .23204 & .14822 \\
\hline 3410 & .69733 & .39016 & .55951 & .45605 & .23396 & .14868 & 4704 & 1.2407 & .72823 & .58695 & .43826 & .21452 & .12627 \\
\hline 3415 & .90096 & .51773 & .57464 & .45013 & .23894 & .15729 & 4731 & .69154 & .46797 & .67670 & .56125 & .35570 & .25900 \\
\hline 3430 & .93817 & 60932 & .64948 & .50696 & .27918 & .18585 & 4792 & .88996 & .49296 & .55392 & .42230 & .18705 & .11922 \\
\hline 3431 & 1.0675 & .73398 & .68760 & .56810 & .34736 & .24937 & 4819 & 1.1060 & .55606 & .50278 & .32657 & .14297 & .10497 \\
\hline 3441 & .93080 & .53950 & .57961 & .49593 & .30349 & .19496 & 4852 & 1.2882 & .70909 & .55046 & .36803 & .11154 & -.13077 \\
\hline 3442 & .54641 & .31827 & .58247 & .46720 & .23528 & .12260 & 4866 & .92590 & .52047 & .56212 & .42777 & .22347 & .14778 \\
\hline 3446 & 60749 & .33025 & .54363 & .41957 & .22684 & .16017 & 4876 & 1.1989 & .70110 & .58480 & .46354 & .23586 & .14120 \\
\hline 3460 & 1.0974 & .56994 & .51935 & .30223 & .23792 & .32756 & 4878 & 1.2259 & .70021 & .57120 & .43122 & .22073 & .13458 \\
\hline 3462 & .68228 & .35786 & .52451 & .42880 & .27102 & .21526 & 4880 & .62829 & .36525 & .58134 & .47671 & .26181 & .16877 \\
\hline
\end{tabular}


Appendix 4-2.6. L-moments of storm depth defined by 48-hour minimum interevent time for hourly rainfall stations in TexasContinued.

\begin{tabular}{|c|c|c|c|c|c|c|c|c|c|c|c|c|c|}
\hline $\begin{array}{c}\text { Station } \\
\text { no. }\end{array}$ & $\begin{array}{c}\text { Depth } \\
\text { mean } \\
\text { (inches) }\end{array}$ & $\begin{array}{l}\text { Depth } \\
\text { L-scale } \\
\text { (inches) }\end{array}$ & $\begin{array}{c}\text { Depth } \\
\text { L-CV } \\
\text { (dimen- } \\
\text { sionless) }\end{array}$ & $\begin{array}{c}\text { Depth } \\
\text { L-skew } \\
\text { (dimen- } \\
\text { sionless) }\end{array}$ & $\begin{array}{c}\text { Depth } \\
\text { L-kurtosis } \\
\text { (dimen- } \\
\text { sionless) }\end{array}$ & $\begin{array}{c}\text { Depth } \\
\text { Tau5 } \\
\text { (dimen- } \\
\text { sionless) }\end{array}$ & $\begin{array}{c}\text { Station } \\
\text { no. }\end{array}$ & $\begin{array}{l}\text { Depth } \\
\text { mean } \\
\text { (inches) }\end{array}$ & $\begin{array}{c}\text { Depth } \\
\text { L-scale } \\
\text { (inches) }\end{array}$ & $\begin{array}{c}\text { Depth } \\
\text { L-CV } \\
\text { (dimen- } \\
\text { sionless) }\end{array}$ & $\begin{array}{c}\text { Depth } \\
\text { L-skew } \\
\text { (dimen- } \\
\text { sionless) }\end{array}$ & $\begin{array}{c}\text { Depth } \\
\text { L-kurtosis } \\
\text { (dimen- } \\
\text { sionless) }\end{array}$ & $\begin{array}{c}\text { Depth } \\
\text { Tau5 } \\
\text { (dimen- } \\
\text { sionless) }\end{array}$ \\
\hline 4920 & 0.79432 & 0.47599 & 0.59924 & 0.48459 & 0.25134 & 0.16022 & 5957 & 0.86640 & 0.49659 & 0.57316 & 0.45455 & 0.24283 & 0.16722 \\
\hline 4934 & .46400 & .35400 & .76293 & .76836 & .60452 & .49153 & 5958 & .81478 & .45039 & .55277 & .36902 & .15251 & .08490 \\
\hline 4972 & .80722 & .45931 & .56901 & .45017 & .23796 & .15386 & 5973 & .65371 & .44593 & .68214 & .55160 & .29237 & .16834 \\
\hline 4973 & 1.1204 & .61055 & .54497 & .43593 & .25314 & .16280 & 5996 & .83384 & .47572 & .57052 & .44106 & .22791 & .15641 \\
\hline 4974 & .65453 & .38797 & .59274 & .48706 & .27815 & .18358 & 6017 & .66071 & .42798 & .64775 & .51703 & .27374 & .15372 \\
\hline 4975 & 1.0695 & .57518 & .53779 & .40896 & .21140 & .14922 & 6024 & 1.2655 & .73452 & .58043 & .43246 & .23801 & .18085 \\
\hline 4978 & .80971 & .49542 & .61185 & .46045 & .21966 & .11653 & 6050 & .78471 & .38838 & .49494 & .41151 & .13079 & .02860 \\
\hline 4979 & 2.4062 & 1.4952 & .62137 & .49624 & .50460 & .61830 & 6104 & .60614 & .36582 & .60352 & .51145 & .29374 & .19235 \\
\hline 4982 & .72795 & .42102 & .57836 & .44499 & .22305 & .13340 & 6108 & 1.1082 & .61577 & .55565 & .41302 & .20793 & .13559 \\
\hline 5018 & .83556 & .46663 & .55846 & .40933 & .20354 & .13834 & 6136 & .56306 & .31745 & .56379 & .47268 & .23931 & .14379 \\
\hline 5048 & .61439 & .36822 & .59933 & .51789 & .28687 & .19297 & 6166 & .55317 & .30986 & .56014 & .41603 & .16975 & .06747 \\
\hline 5049 & .58333 & .33599 & .57599 & .54993 & .33553 & .25483 & 6176 & 1.0763 & .62442 & .58017 & .46917 & .27972 & .17070 \\
\hline 5056 & -- & -- & -- & -- & -- & -- & 6177 & 1.0910 & .62168 & .56981 & .44453 & .23977 & .16153 \\
\hline 5057 & .53245 & .36208 & .68002 & .54845 & .29746 & .17489 & 6210 & .98156 & .55842 & .56890 & .43258 & .22095 & .14843 \\
\hline 5060 & .82675 & .56524 & 68369 & .55441 & .32413 & .21798 & 6211 & 1.0487 & .61106 & .58269 & .41525 & .19660 & .12463 \\
\hline 5081 & 1.0146 & .56289 & .55478 & .40168 & .20744 & .13923 & 6270 & 1.1363 & .61750 & .54343 & .41680 & .21565 & .14496 \\
\hline 5094 & .95013 & .54083 & .56922 & .44336 & .23329 & .15025 & 6275 & -- & -- & -- & -- & -- & -- \\
\hline 5113 & .84896 & .52381 & .61700 & .51403 & .28617 & .18455 & 6276 & 2.2590 & 1.4599 & .64625 & .44341 & .19803 & .21851 \\
\hline 5114 & -- & -- & -- & -- & -- & -- & 6335 & .94414 & .52678 & .55794 & .41153 & .20816 & .13832 \\
\hline 5123 & .81417 & .49250 & 60491 & .49503 & .19192 & -.08276 & 6434 & .75133 & .34752 & .46254 & .28912 & .16763 & .12480 \\
\hline 5192 & .94316 & .53938 & .57189 & .44333 & .22803 & .13663 & 6504 & .62121 & .36440 & .58659 & .48340 & .25162 & .15830 \\
\hline 5193 & .92539 & .54037 & .58393 & .45068 & .23367 & .15675 & 6558 & .80545 & .50000 & 62077 & .46642 & .27720 & .32125 \\
\hline 5224 & 1.1796 & .68516 & .58085 & .46161 & .28618 & .20050 & 6615 & .73960 & .44459 & .60112 & .53534 & .31379 & .22472 \\
\hline 5228 & .90028 & .53310 & .59215 & .42928 & .20312 & .12971 & 6660 & .91921 & .52313 & .56911 & .43841 & .22653 & .11947 \\
\hline 5235 & .82250 & .48361 & .58797 & .44329 & .29137 & .24974 & 6663 & 1.0153 & .56373 & .55524 & .42427 & .24126 & .16296 \\
\hline 5247 & .64372 & .36189 & .56218 & .44471 & .21701 & .12562 & 6734 & .65992 & .38522 & .58373 & .44812 & .23513 & .14975 \\
\hline 5258 & .90800 & .52828 & .58180 & .46846 & .26364 & .16941 & 6736 & .65313 & .38629 & .59144 & .50652 & .28170 & .18339 \\
\hline 5303 & .81223 & .50804 & .62549 & .52032 & .30803 & .21883 & 6740 & 1.7755 & .94418 & .53180 & .15643 & -.16599 & -.04750 \\
\hline 5312 & .79877 & .46550 & .58277 & .47712 & .24764 & .16555 & 6750 & 1.1023 & .77331 & .70153 & .57108 & .33003 & .20845 \\
\hline 5341 & 2.5910 & 2.0057 & .77409 & .69727 & .43321 & .12251 & 6757 & .98643 & .57145 & .57931 & .43282 & .22608 & .15231 \\
\hline 5342 & -- & -- & -- & -- & -- & -- & 6775 & .61948 & .35900 & .57952 & .43933 & .22360 & .13916 \\
\hline 5348 & 1.0913 & .59261 & .54301 & .39426 & .18206 & .10993 & 6776 & .64512 & .36540 & .56641 & .46235 & .24426 & .15849 \\
\hline 5358 & .70764 & .41471 & .58604 & .46640 & .24634 & .15038 & 6788 & .96214 & .53494 & .55599 & .41995 & .22234 & .11625 \\
\hline 5398 & 1.0185 & .57397 & .56353 & .42508 & .22307 & .14584 & 6792 & .49942 & .28026 & .56116 & .45881 & .23255 & .13887 \\
\hline 5410 & .62867 & .37076 & .58976 & .48979 & .27225 & .18107 & 6794 & 3.4322 & 2.0500 & .59728 & .32466 & -.08420 & -.28970 \\
\hline 5411 & .54831 & .35830 & .65346 & .51368 & .27912 & .18226 & 6834 & 1.1021 & .61270 & .55593 & .43180 & .22209 & .15018 \\
\hline 5424 & 1.1781 & .73676 & .62539 & .45137 & .22439 & .15019 & 6893 & .44876 & .25621 & .57094 & .52543 & .31318 & .21032 \\
\hline 5429 & .86021 & .52264 & .60758 & .47156 & .24811 & .16766 & 6935 & .63025 & .36474 & .57872 & .50199 & .27921 & .18295 \\
\hline 5431 & 1.2050 & .61227 & .50811 & .32294 & .03770 & -.04863 & 6981 & .91674 & .51930 & .56646 & .42637 & .22535 & .15100 \\
\hline 5461 & 1.0228 & .58681 & .57374 & .44086 & .24085 & .16415 & 7020 & .93424 & .57834 & 61905 & .50028 & .29219 & .19883 \\
\hline 5463 & 1.0192 & .57513 & .56429 & .44291 & .22506 & .15156 & 7060 & .71188 & .40850 & .57384 & .47545 & .25067 & .16202 \\
\hline 5471 & .31308 & .19769 & .63145 & .51539 & .27626 & .25897 & 7066 & 1.0641 & .59727 & .56129 & .42288 & .21879 & .14165 \\
\hline 5477 & 1.4292 & 1.1490 & .80398 & .83620 & .75666 & .71198 & 7074 & .59266 & .35680 & .60203 & .50609 & .28971 & .19551 \\
\hline 5528 & .98316 & .54507 & .55441 & .42052 & .22547 & .14466 & 7097 & 1.0117 & .55796 & .55153 & .40369 & .17865 & .08601 \\
\hline 5579 & -- & -- & -- & -- & -- & -- & 7116 & .63741 & .36728 & .57620 & .45928 & .26325 & .18407 \\
\hline 5580 & 1.0413 & .47124 & .45253 & .07353 & -.09547 & .01191 & 7140 & 1.0641 & .68038 & .63938 & .51534 & .28744 & .18493 \\
\hline 5589 & .44667 & .23915 & .53541 & .30841 & .07831 & .03602 & 7173 & 1.2119 & .78362 & .64661 & .49837 & .27529 & .18230 \\
\hline 5590 & .53194 & .30638 & .57596 & .45235 & .24682 & .17309 & 7174 & 1.1426 & .73417 & .64255 & .49516 & .27292 & .18664 \\
\hline 5591 & .49909 & .26829 & .53756 & .41810 & .23183 & .16156 & 7213 & .93948 & .52957 & .56369 & .41700 & .20009 & .11489 \\
\hline 5592 & .44663 & .25979 & .58167 & .47097 & .26171 & .16920 & 7243 & .79552 & .45240 & .56868 & .45094 & .23328 & .15723 \\
\hline 5594 & .41829 & .24360 & .58237 & .51771 & .31867 & .21273 & 7262 & .27576 & .15930 & .57767 & .43081 & .23499 & .17376 \\
\hline 5595 & -- & -- & -- & -- & -- & -- & 7274 & .82773 & .45675 & .55181 & .45335 & .25962 & .16863 \\
\hline 5596 & .58105 & .32687 & .56254 & .51151 & .27719 & .18885 & 7300 & .79976 & .46076 & .57612 & .44038 & .23774 & .15835 \\
\hline 5600 & .52726 & .31650 & .60028 & .50765 & .30469 & .22187 & 7311 & .83261 & .47387 & .56914 & .46861 & .20711 & .13283 \\
\hline 5618 & 1.5492 & 1.0310 & .66551 & .60663 & .49194 & .47512 & 7363 & 1.4727 & 1.1036 & .74938 & .62779 & .35695 & .22103 \\
\hline 5650 & .90333 & .43970 & .48675 & .36030 & .13094 & -.08626 & 7422 & .88267 & .52998 & .60043 & .47866 & .26277 & .18151 \\
\hline 5656 & .68913 & .39917 & .57924 & .49638 & .25674 & .17184 & 7431 & .57499 & .33774 & .58739 & .49140 & .26851 & .17578 \\
\hline 5658 & .64164 & .38748 & 60390 & .50000 & .29350 & .19989 & 7481 & .47044 & .28696 & 60997 & .54530 & .33665 & .22676 \\
\hline 5661 & .95515 & .62775 & .65723 & .59930 & .37625 & .27477 & 7497 & .93620 & .53482 & .57127 & .45917 & .24144 & .18248 \\
\hline 5666 & .76727 & .50022 & .65194 & .54548 & .32009 & .19943 & 7498 & .94056 & .55225 & .58715 & .47948 & .26111 & .19771 \\
\hline 5695 & .95183 & .53016 & .55699 & .43318 & .24090 & .16205 & 7499 & .84129 & .47364 & .56299 & .45511 & .23651 & .15712 \\
\hline 5742 & .40462 & .27462 & .67871 & .68806 & .57805 & .54427 & 7531 & .88969 & .49695 & .55857 & .41353 & .20315 & .11536 \\
\hline 5766 & 2.5756 & 1.6425 & .63773 & .58126 & .33548 & .06393 & 7534 & .85481 & .51797 & .60595 & .49936 & .31174 & .24202 \\
\hline 5770 & .69720 & .40853 & .58596 & .47018 & .24210 & .14367 & 7556 & .88165 & .51240 & .58118 & .46945 & .24581 & .15707 \\
\hline 5775 & .54625 & .31696 & .58025 & .32282 & .00732 & .07042 & 7594 & 1.0573 & .60092 & .56834 & .42975 & .22704 & .15262 \\
\hline 5779 & .80333 & .43974 & .54739 & .40638 & .16808 & .03413 & 7596 & .98295 & .55970 & .56940 & .37104 & .14306 & .09265 \\
\hline 5840 & .81683 & .47757 & .58466 & .44139 & .22110 & .13627 & 7608 & .86374 & .52613 & 60913 & .45367 & .23354 & .16219 \\
\hline 5890 & .48088 & .31131 & .64736 & .51466 & .28432 & .18639 & 7622 & .28765 & .21882 & .76074 & .64503 & .39142 & .28283 \\
\hline 5891 & .50589 & .25641 & .50686 & .34837 & .14809 & .09284 & 7700 & 1.0438 & .59902 & .57389 & .42573 & .22009 & .14311 \\
\hline 5897 & .97105 & .54858 & .56493 & .44455 & .22533 & .15404 & 7706 & .73926 & .43821 & .59277 & .48595 & .26946 & .17978 \\
\hline
\end{tabular}


Appendix 4-2.6. L-moments of storm depth defined by 48-hour minimum interevent time for hourly rainfall stations in TexasContinued.

\begin{tabular}{|c|c|c|c|c|c|c|c|c|c|c|c|c|c|}
\hline $\begin{array}{c}\text { Station } \\
\text { no. }\end{array}$ & $\begin{array}{c}\text { Depth } \\
\text { mean } \\
\text { (inches) }\end{array}$ & $\begin{array}{l}\text { Depth } \\
\text { L-scale } \\
\text { (inches) }\end{array}$ & $\begin{array}{c}\text { Depth } \\
\text { L-CV } \\
\text { (dimen- } \\
\text { sionless) }\end{array}$ & $\begin{array}{c}\text { Depth } \\
\text { L-skew } \\
\text { (dimen- } \\
\text { sionless) }\end{array}$ & $\begin{array}{c}\text { Depth } \\
\text { L-kurtosis } \\
\text { (dimen- } \\
\text { sionless) }\end{array}$ & $\begin{array}{c}\text { Depth } \\
\text { Tau5 } \\
\text { (dimen- } \\
\text { sionless) }\end{array}$ & $\begin{array}{c}\text { Station } \\
\text { no. }\end{array}$ & $\begin{array}{l}\text { Depth } \\
\text { mean } \\
\text { (inches) }\end{array}$ & $\begin{array}{c}\text { Depth } \\
\text { L-scale } \\
\text { (inches) }\end{array}$ & $\begin{array}{c}\text { Depth } \\
\text { L-CV } \\
\text { (dimen- } \\
\text { sionless) }\end{array}$ & $\begin{array}{l}\text { Depth } \\
\text { L-skew } \\
\text { (dimen- } \\
\text { sionless) }\end{array}$ & $\begin{array}{l}\text { Depth } \\
\text { L-kurtosis } \\
\text { (dimen- } \\
\text { sionless) }\end{array}$ & $\begin{array}{c}\text { Depth } \\
\text { Tau5 } \\
\text { (dimen- } \\
\text { sionless) }\end{array}$ \\
\hline 7718 & 0.89592 & 0.53734 & 0.59976 & 0.50568 & 0.31166 & 0.21059 & 8910 & 1.0869 & 0.68923 & 0.63411 & 0.45688 & 0.16995 & -0.00035 \\
\hline 7745 & 1.0854 & .63831 & .58807 & .45236 & .24795 & .16768 & 8911 & .81349 & .48541 & .59670 & .43858 & .21877 & .14560 \\
\hline 7922 & .33160 & .19053 & .57458 & .45715 & .25183 & .15994 & 8924 & .41957 & .21776 & .51901 & .40290 & .19662 & .10312 \\
\hline 7936 & 1.1618 & .65266 & .56176 & .43011 & .23513 & .16544 & 8929 & 1.9018 & 1.4536 & .76434 & .70248 & .57380 & .62487 \\
\hline 7943 & .57049 & .37005 & .64865 & .50021 & .25589 & .15374 & 8942 & 1.1250 & .63058 & .56051 & .43425 & .22805 & .14686 \\
\hline 7944 & .71581 & .41464 & .57926 & .37223 & .12930 & .04664 & 8944 & .96431 & .58365 & .60526 & .47301 & .26965 & .18677 \\
\hline 7945 & .75653 & .50488 & .66736 & .52562 & .29443 & .20245 & 8996 & .96904 & .57582 & .59422 & .44896 & .23317 & .15466 \\
\hline 7947 & 1.1309 & .68889 & .60914 & .51509 & .30929 & .22526 & 9014 & 1.6370 & .92500 & .56506 & .44793 & .35547 & .38715 \\
\hline 7948 & .81946 & .50905 & .62121 & .49060 & .26865 & .18205 & 9037 & .43245 & .26059 & .60258 & .53372 & .33398 & .23074 \\
\hline 7951 & .96067 & .53057 & .55229 & .41200 & .20882 & .13311 & 9106 & .41374 & .27508 & .66485 & .61479 & .42746 & .33763 \\
\hline 7953 & .69840 & .45544 & .65213 & .53106 & .26216 & .09578 & 9107 & .49885 & .30451 & .61042 & .45668 & .20478 & .16321 \\
\hline 7981 & .80288 & .48759 & .60729 & .46193 & .21000 & .09964 & 9129 & .58826 & .38782 & .65927 & .54227 & .28618 & .16163 \\
\hline 7990 & .82553 & .56334 & .68240 & .55141 & .27890 & .11657 & 9163 & .79311 & .45850 & .57811 & .45575 & .23340 & .13719 \\
\hline 7992 & 1.3508 & .71583 & .52992 & .23357 & .01662 & .09059 & 9213 & .88426 & .52726 & .59628 & .42320 & .21167 & .14940 \\
\hline 7997 & .65763 & .39448 & .59985 & .49263 & .30028 & .21389 & 9214 & 2.3225 & 1.5869 & .68327 & .65500 & .48982 & .44772 \\
\hline 7999 & .47846 & .29615 & .61897 & .48477 & .24274 & .07834 & 9222 & .84295 & .50276 & .59643 & .45357 & .22442 & .12530 \\
\hline 8022 & .57898 & .34080 & .58862 & .41159 & .11456 & .02696 & 9248 & .63022 & .35236 & .55911 & .43208 & .20273 & .12674 \\
\hline 8023 & .64056 & .37530 & .58589 & .48964 & .26158 & .17087 & 9266 & .74232 & .40333 & .54334 & .36354 & .11731 & .04654 \\
\hline 8047 & .85965 & .48943 & .56933 & .43412 & .21844 & .14103 & 9270 & .53433 & .30981 & .57980 & .52647 & .30939 & .20821 \\
\hline 8060 & .71699 & .48089 & .67071 & .51973 & .22767 & .08543 & 9295 & .29000 & .18838 & .64959 & .53376 & .28862 & .16118 \\
\hline 8062 & .93633 & .64489 & .68873 & .52073 & .23865 & .10599 & 9304 & -- & -- & -- & -- & -- & -- \\
\hline 8068 & .45818 & .28576 & .62368 & .50142 & .28011 & .14453 & 9307 & .77885 & .40781 & .52361 & .40217 & .20928 & .12547 \\
\hline 8081 & .84191 & .52801 & .62716 & .52483 & .30431 & .21191 & 9328 & .65150 & .37850 & .58097 & .44184 & .22033 & .12467 \\
\hline 8089 & .64161 & .36112 & .56283 & .36365 & .15561 & .11960 & 9329 & .80556 & .39139 & .48586 & .21282 & .10828 & .35121 \\
\hline 8221 & 1.4010 & .62856 & .44865 & .33065 & .25307 & .07804 & 9345 & -- & -- & -- & -- & -- & -- \\
\hline 8252 & .61959 & .36007 & .58114 & .47750 & .25063 & .15702 & 9363 & .76766 & .50378 & .65626 & .51061 & .27092 & .17189 \\
\hline 8265 & 1.1155 & .67273 & .60308 & .47420 & .26757 & .18570 & 9364 & .86509 & .57947 & .66984 & .53174 & .29792 & .20301 \\
\hline 8289 & .68108 & .25233 & .37048 & .09400 & .02702 & .11310 & 9365 & .52571 & .32648 & .62101 & .57626 & .36416 & .24864 \\
\hline 8305 & .42849 & .24537 & .57265 & .50883 & .29968 & .19980 & 9371 & .91134 & .49208 & .53995 & .35581 & .12540 & .06905 \\
\hline 8335 & 1.0959 & .61507 & .56124 & .41695 & .22946 & .16536 & 9417 & .87991 & .50818 & .57754 & .42441 & .21147 & .13709 \\
\hline 8400 & .56931 & .32516 & .57114 & .47755 & .28600 & .19161 & 9419 & .79174 & .48587 & .61367 & .44862 & .22427 & .14959 \\
\hline 8445 & .97302 & .59285 & 60929 & .47652 & .25522 & .16839 & 9435 & 64789 & .35227 & .54372 & .43849 & .29564 & .25098 \\
\hline 8446 & .87484 & .49965 & .57113 & .43281 & .20964 & .12880 & 9491 & .98217 & .56091 & .57109 & .43155 & .22295 & .14851 \\
\hline 8451 & .65263 & .39193 & .60054 & .47946 & .25288 & .15558 & 9499 & .70261 & .40591 & .57772 & .45465 & .20278 & .11118 \\
\hline 8531 & .88242 & .48907 & .55424 & .41462 & .21602 & .14332 & 9522 & 3.3160 & 2.8510 & .85977 & .83655 & .63346 & .40196 \\
\hline 8541 & .82039 & .47511 & .57912 & .45968 & .24446 & .12412 & 9527 & .63425 & .36336 & .57290 & .48531 & .24729 & .14886 \\
\hline 8544 & .99473 & .60203 & .60522 & .50930 & .29770 & .21274 & 9532 & .85481 & .49359 & .57742 & .45862 & .25379 & .17272 \\
\hline 8545 & .46385 & .18744 & .40409 & .14924 & .22311 & .24383 & 9544 & -- & -- & -- & -- & -- & -- \\
\hline 8563 & .97976 & .55585 & .56733 & .45672 & .24021 & .16490 & 9565 & .74958 & .44451 & .59302 & .47749 & .24507 & .14530 \\
\hline 8566 & .69757 & .40694 & .58336 & .44351 & .21994 & .13843 & 9570 & .71159 & .39609 & .55663 & .46506 & .22651 & .14142 \\
\hline 8583 & .77659 & .44193 & .56906 & .45530 & .21157 & .15245 & 9574 & .77333 & .42381 & .54803 & .38462 & .13829 & .09454 \\
\hline 8584 & .84774 & .48261 & .56929 & .43638 & .21352 & .14114 & 9588 & .75832 & .48334 & .63738 & .53001 & .28434 & .16747 \\
\hline 8623 & .88519 & .48659 & .54970 & .42490 & .20729 & .13208 & 9665 & .99898 & .56598 & .56656 & .43887 & .23834 & .16602 \\
\hline 8625 & .83718 & .48249 & .57633 & .44819 & .24234 & .16105 & 9715 & .88309 & .50073 & .56702 & .43009 & .22449 & .15261 \\
\hline 8630 & .62414 & .35512 & .56898 & .43725 & .20355 & .11700 & 9729 & .73103 & .45053 & .61629 & .45364 & .22504 & .14695 \\
\hline 8631 & .70266 & .40619 & .57807 & .46310 & .24939 & .15496 & 9772 & .95668 & .60932 & .63692 & .51864 & .30112 & .20257 \\
\hline 8646 & .83744 & .48340 & .57723 & .42823 & .20975 & .13570 & 9814 & .74069 & .44276 & .59777 & .40680 & .15577 & .09759 \\
\hline 8647 & .61314 & .35675 & .58185 & .49348 & .26348 & .16498 & 9815 & .97305 & .57792 & .59393 & .47343 & .26002 & .18299 \\
\hline 8677 & .82549 & .45985 & .55707 & .46363 & .25018 & .14968 & 9816 & .80268 & .50430 & .62827 & .51413 & .26740 & .13202 \\
\hline 8696 & 1.9150 & 1.1621 & .60685 & .30892 & -.00795 & -.00574 & 9817 & .79489 & .44603 & .56112 & .44524 & .22734 & .15388 \\
\hline 8743 & 1.0574 & .61265 & .57938 & .44468 & .23771 & .15760 & 9829 & .49204 & .29559 & .60075 & .52148 & .29859 & .18747 \\
\hline 8761 & .58169 & .33772 & .58058 & .49367 & .25341 & .14734 & 9830 & .37445 & .23084 & .61646 & .50414 & .25651 & .14911 \\
\hline 8778 & 1.0246 & .56426 & .55072 & .42054 & .21681 & .14549 & 9858 & .61892 & .35757 & .57773 & .45947 & .24189 & .14697 \\
\hline 8845 & .92356 & .55839 & .60461 & .48487 & .25428 & .16530 & 9893 & .78436 & .45575 & .58105 & .45584 & .24192 & .15691 \\
\hline 8859 & 1.0373 & .57127 & .55074 & .40887 & .21388 & .14263 & 9916 & 1.0883 & .58216 & .53494 & .38797 & .18933 & .12307 \\
\hline 8898 & .97207 & .56196 & .57811 & .44873 & .23710 & .15280 & 9976 & .73135 & .44325 & .60607 & .49006 & .25798 & .16465 \\
\hline 8908 & .92458 & .55679 & .60221 & .47594 & .27052 & .20928 & & & & & & & \\
\hline
\end{tabular}


Appendix 4-2.7. L-moments of storm depth defined by 72-hour minimum interevent time for hourly rainfall stations in Texas.

[--, not available]

\begin{tabular}{|c|c|c|c|c|c|c|c|c|c|c|c|c|c|}
\hline $\begin{array}{c}\text { Station } \\
\text { no. }\end{array}$ & $\begin{array}{c}\text { Depth } \\
\text { mean } \\
\text { (inches) }\end{array}$ & $\begin{array}{c}\text { Depth } \\
\text { L-scale } \\
\text { (inches) }\end{array}$ & $\begin{array}{l}\text { Depth } \\
\text { L-CV } \\
\text { (dimen- } \\
\text { sionless) }\end{array}$ & $\begin{array}{l}\text { Depth } \\
\text { L-skew } \\
\text { (dimen- } \\
\text { sionless) }\end{array}$ & $\begin{array}{l}\text { Depth } \\
\text { L-kurtosis } \\
\text { (dimen- } \\
\text { sionless) }\end{array}$ & $\begin{array}{l}\text { Depth } \\
\text { Tau5 } \\
\text { (dimen- } \\
\text { sionless) }\end{array}$ & $\begin{array}{c}\text { Station } \\
\text { no. }\end{array}$ & $\begin{array}{c}\text { Depth } \\
\text { mean } \\
\text { (inches) }\end{array}$ & $\begin{array}{c}\text { Depth } \\
\text { L-scale } \\
\text { (inches) }\end{array}$ & $\begin{array}{l}\text { Depth } \\
\text { L-CV } \\
\text { (dimen- } \\
\text { sionless) }\end{array}$ & $\begin{array}{l}\text { Depth } \\
\text { L-skew } \\
\text { (dimen- } \\
\text { sionless) }\end{array}$ & $\begin{array}{c}\text { Depth } \\
\text { L-kurtosis } \\
\text { (dimen- } \\
\text { sionless) }\end{array}$ & $\begin{array}{c}\text { Depth } \\
\text { Tau5 } \\
\text { (dimen- } \\
\text { sionless) }\end{array}$ \\
\hline 0015 & -- & -- & -- & -- & -- & -- & 1154 & 1.0909 & 0.66687 & 0.61131 & 0.43595 & 0.18909 & 0.09968 \\
\hline 0054 & .90762 & .52595 & .57949 & .56502 & .40463 & .23852 & 1186 & 1.1214 & .74634 & .66552 & .55270 & .31849 & .17137 \\
\hline 0120 & 2.4300 & 1.4289 & .58804 & .37391 & .36266 & .63109 & 1188 & .79400 & .24600 & .30982 & .47967 & .63415 & .99187 \\
\hline 0145 & .83174 & .57865 & .69571 & .58620 & .38347 & .30496 & 1245 & 2.2700 & 1.7197 & .75759 & .65134 & .46016 & .41373 \\
\hline 0179 & .60190 & .37682 & .62604 & .52035 & .30920 & .22080 & 1325 & 1.1578 & .68676 & .59317 & .44367 & .22283 & .14941 \\
\hline 0202 & .97635 & .51893 & .53150 & .38752 & .16198 & .10048 & 1429 & .99292 & .58535 & .58953 & .45574 & .23809 & .15667 \\
\hline 0206 & 1.0673 & .58115 & .54451 & .41195 & .21280 & .14154 & 1431 & 1.0765 & .61988 & .57582 & .42620 & .21087 & .13393 \\
\hline 0208 & -- & -- & -- & -- & -- & -- & 1432 & 1.0701 & .62223 & .58148 & .42818 & .22400 & .15736 \\
\hline 0211 & .63835 & .41033 & .64280 & .49210 & .25216 & .15683 & 1433 & 1.0545 & .60584 & .57455 & .44194 & .23834 & .15050 \\
\hline 0244 & 1.1025 & .54528 & .49459 & .23652 & .10489 & .12652 & 1434 & 1.0527 & .60463 & .57437 & .42003 & .20579 & .13405 \\
\hline 0248 & .60878 & .35502 & .58317 & .48636 & .26086 & .16575 & 1435 & 1.0522 & .59787 & .56823 & .40049 & .18665 & .11997 \\
\hline 0427 & 1.1667 & .71225 & .61050 & .45072 & .17768 & .10352 & 1500 & 1.8967 & 1.2144 & .64030 & .56914 & .47785 & .63181 \\
\hline 0428 & .98112 & .60688 & .61856 & .45177 & .22666 & .15033 & 1528 & .86383 & .52256 & .60494 & .49085 & .25732 & .16870 \\
\hline 0429 & 1.1947 & .78517 & .65723 & .48742 & .24042 & .15088 & 1541 & 1.3909 & .81288 & .58442 & .46655 & .24108 & .13002 \\
\hline 0463 & .77128 & .46379 & .60133 & .56587 & .40493 & .29001 & 1569 & 1.1106 & .68428 & .61614 & .47772 & .26246 & .19976 \\
\hline 0493 & 1.4956 & .48250 & .32262 & .14352 & .19936 & .12106 & 1632 & .67000 & .25700 & .38358 & -.24514 & .08560 & -.27237 \\
\hline 0495 & .54649 & .30591 & .55978 & .44245 & .23957 & .16123 & 1641 & .71292 & .41009 & .57523 & .46961 & .27395 & .17998 \\
\hline 0496 & .29636 & .14667 & .49489 & .43211 & .27007 & .09429 & 1646 & .70323 & .40609 & .57746 & .47575 & .23813 & .15119 \\
\hline 0498 & .26625 & .06446 & .24212 & .16898 & .58449 & .20776 & 1663 & 1.7250 & 1.1112 & .64419 & .55230 & .38097 & .32276 \\
\hline 0509 & 1.0437 & 60566 & .58032 & .46235 & .25955 & .17930 & 1671 & 1.0829 & .63784 & .58901 & .45374 & .23000 & .14949 \\
\hline 0518 & 1.1293 & .63612 & .56330 & .43341 & .23941 & .16061 & 1680 & .96121 & .55307 & .57539 & .45051 & .24985 & .17037 \\
\hline 0521 & .81095 & .54033 & .66629 & .62740 & .50018 & .42490 & 1694 & .79694 & .47935 & .60149 & .50094 & .23462 & .13019 \\
\hline 0556 & .83211 & .48167 & .57886 & .42870 & .18454 & .10576 & 1696 & .75290 & .43341 & .57565 & .42977 & .21464 & .13450 \\
\hline 0569 & 1.3453 & .80993 & .60205 & .46631 & .24134 & .15595 & 1697 & .64968 & .39436 & .60701 & .45516 & .18473 & .05616 \\
\hline 0572 & 1.1454 & .68515 & .59818 & .47119 & .26786 & .19326 & 1698 & .80052 & .46827 & .58495 & .47588 & .25361 & .15199 \\
\hline 0708 & 1.0779 & .61016 & .56604 & .44213 & .20307 & .10620 & 1914 & 2.2343 & 1.2276 & .54945 & .56788 & .56905 & .57060 \\
\hline 0738 & 1.0287 & .58265 & .56641 & .42152 & .21814 & .14129 & 1920 & 1.0506 & .58081 & .55283 & .41368 & .22298 & .16755 \\
\hline 0776 & .69867 & .42102 & .60260 & .48993 & .26280 & .16362 & 1921 & 1.2439 & 69067 & .55526 & .41578 & .21888 & .14815 \\
\hline 0779 & .69607 & .40727 & .58510 & .51621 & .26866 & .15559 & 1937 & 1.2129 & .66569 & .54883 & .43006 & .24581 & .14739 \\
\hline 0784 & .70924 & .43021 & .60658 & .50237 & .26837 & .16769 & 1956 & 1.2962 & .73470 & .56682 & .41981 & .22219 & .15417 \\
\hline 0786 & .55786 & .34260 & .61414 & .45314 & .21790 & .12955 & 1970 & 3.7243 & 2.9829 & .80092 & .67088 & .42529 & .42369 \\
\hline 0917 & 1.4278 & .79056 & .55370 & .40865 & .21743 & .13807 & 2014 & .92426 & .62712 & .67851 & .51695 & .25274 & .13838 \\
\hline 0923 & 3.5463 & 2.2045 & .62163 & .28732 & -.14346 & -.17197 & 2015 & .96692 & .66145 & .68408 & .54696 & .31035 & .20470 \\
\hline 0926 & 1.0054 & .58122 & .57812 & .43894 & .22464 & .14507 & 2019 & 2.7075 & 1.6361 & .60427 & .30474 & .00742 & -.00546 \\
\hline 0950 & .50840 & .32403 & .63736 & .56394 & .35266 & .23907 & 2024 & 1.0545 & .57578 & .54600 & .41448 & .22618 & .15661 \\
\hline 0996 & 2.8512 & 1.8223 & .63913 & .35228 & .01029 & .01068 & 2042 & .23375 & .13018 & .55691 & .37174 & .32785 & .41015 \\
\hline 1013 & 1.0074 & .65289 & .64809 & .60110 & .39147 & .28267 & 2043 & .35914 & .20285 & .56483 & .45210 & .27549 & .20093 \\
\hline 1017 & .85971 & .50063 & .58233 & .45387 & .24460 & .16806 & 2048 & .89746 & .54090 & .60270 & .48216 & .24959 & .16192 \\
\hline 1042 & 2.0200 & .99000 & .49010 & .31313 & .28620 & .36027 & 2050 & .56447 & .41071 & .72761 & .62131 & .39053 & .26800 \\
\hline 1048 & 1.6278 & 1.0550 & .64812 & .70902 & .57158 & .37463 & 2051 & .71070 & .43702 & .61492 & .47060 & .21080 & .08968 \\
\hline 1053 & .82677 & .48839 & .59072 & .44647 & .21478 & .13014 & 2053 & .37500 & .24433 & .65156 & .57299 & .32469 & .09550 \\
\hline 1057 & .78104 & .45016 & .57636 & .43790 & .22123 & .13553 & 2073 & 1.0940 & .65085 & .59491 & .49159 & .30641 & .22039 \\
\hline 1063 & 2.7513 & 1.7945 & .65224 & .43835 & .21564 & .27396 & 2082 & .58108 & .34407 & .59212 & .49558 & .28320 & .19104 \\
\hline 1068 & 1.0438 & .57917 & .55487 & .40932 & .20380 & .13055 & 2086 & 1.0809 & .60415 & .55896 & .42575 & .22404 & .15051 \\
\hline 1080 & .61648 & .35371 & .57376 & .48540 & .29689 & .19908 & 2088 & 1.3179 & .68135 & .51701 & .31392 & .15801 & .18846 \\
\hline 1081 & 1.0582 & .59656 & .56376 & .43789 & .24123 & .15056 & 2090 & 1.0741 & .56080 & .52209 & .39543 & .20278 & .13656 \\
\hline 1133 & .37000 & .22889 & .61862 & .30291 & -.02913 & .09466 & 2096 & 1.0405 & .57597 & .55355 & .42372 & .22907 & .15334 \\
\hline 1136 & .86982 & .59838 & .68793 & .54554 & .29890 & .19131 & 2128 & 1.0429 & .59864 & .57402 & .40648 & .18482 & .11932 \\
\hline 1138 & 2.5750 & 1.3510 & .52466 & .55070 & .33506 & .26400 & 2131 & .95378 & .53544 & .56139 & .43677 & .22556 & .14653 \\
\hline 1139 & 1.3827 & .76254 & .55149 & .32694 & .09372 & .04128 & 2142 & 3.5920 & 1.3060 & .36359 & .82542 & .73201 & .51455 \\
\hline
\end{tabular}


212 Statistical Characteristics of Storm Interevent Time, Depth, and Duration for Eastern New Mexico, Oklahoma, and Texas

Appendix 4-2.7. L-moments of storm depth defined by 72-hour minimum interevent time for hourly rainfall stations in Texas-

Continued.

\begin{tabular}{|c|c|c|c|c|c|c|c|c|c|c|c|c|c|}
\hline $\begin{array}{c}\text { Station } \\
\text { no. }\end{array}$ & $\begin{array}{c}\text { Depth } \\
\text { mean } \\
\text { (inches) }\end{array}$ & $\begin{array}{c}\text { Depth } \\
\text { L-scale } \\
\text { (inches) }\end{array}$ & $\begin{array}{c}\text { Depth } \\
\text { L-CV } \\
\text { (dimen- } \\
\text { sionless) }\end{array}$ & $\begin{array}{c}\text { Depth } \\
\text { L-skew } \\
\text { (dimen- } \\
\text { sionless) }\end{array}$ & $\begin{array}{c}\text { Depth } \\
\text { L-kurtosis } \\
\text { (dimen- } \\
\text { sionless) }\end{array}$ & $\begin{array}{c}\text { Depth } \\
\text { Tau5 } \\
\text { (dimen- } \\
\text { sionless) }\end{array}$ & $\begin{array}{c}\text { Station } \\
\text { no. }\end{array}$ & $\begin{array}{c}\text { Depth } \\
\text { mean } \\
\text { (inches) }\end{array}$ & $\begin{array}{c}\text { Depth } \\
\text { L-scale } \\
\text { (inches) }\end{array}$ & $\begin{array}{c}\text { Depth } \\
\text { L-CV } \\
\text { (dimen- } \\
\text { sionless) }\end{array}$ & $\begin{array}{c}\text { Depth } \\
\text { L-skew } \\
\text { (dimen- } \\
\text { sionless) }\end{array}$ & $\begin{array}{c}\text { Depth } \\
\text { L-kurtosis } \\
\text { (dimen- } \\
\text { sionless) }\end{array}$ & $\begin{array}{c}\text { Depth } \\
\text { Tau5 } \\
\text { (dimen- } \\
\text { sionless) }\end{array}$ \\
\hline 2160 & 0.82500 & 0.58864 & 0.71350 & 0.65266 & 0.53951 & 0.53651 & 3463 & 1.0403 & 0.65431 & 0.62894 & 0.51530 & 0.35613 & 0.31701 \\
\hline 2206 & 1.2713 & .74245 & .58401 & .42026 & .20389 & .14070 & 3476 & .97903 & .57434 & .58664 & .44434 & .23460 & .15661 \\
\hline 2238 & .57986 & .38564 & .66506 & .51003 & .22162 & .07723 & 3485 & 2.1130 & 1.6557 & .78356 & .65694 & .30081 & -.06228 \\
\hline 2240 & .65804 & .37064 & .56325 & .37140 & .14437 & .03942 & 3507 & 1.1559 & .64724 & .55995 & .41628 & .19018 & .11094 \\
\hline 2242 & 1.0250 & .58643 & .57215 & .40015 & .20529 & .13749 & 3546 & 1.3253 & .74774 & .56421 & .42173 & .21926 & .14621 \\
\hline 2244 & 1.1186 & .65267 & .58345 & .43708 & .23338 & .15304 & 3547 & .96274 & .53176 & .55234 & .38937 & .17600 & .11119 \\
\hline 2247 & 1.1604 & .69248 & .59676 & .41623 & .20228 & .14246 & 3579 & 1.0543 & .55502 & .52641 & .31526 & .08094 & .05798 \\
\hline 2309 & 1.3157 & 69119 & .52533 & .40834 & .24496 & .17643 & 3642 & 1.1555 & .66005 & .57121 & .43896 & .23081 & .15195 \\
\hline 2312 & 1.2988 & .66545 & .51237 & .36292 & .15940 & .07553 & 3646 & .97089 & .55240 & .56896 & .43439 & .23350 & .15690 \\
\hline 2334 & 1.5050 & .88961 & .59110 & .45316 & .28639 & .23022 & 3668 & 4.1383 & 2.2997 & .55570 & .68677 & .74417 & .64212 \\
\hline 2336 & .93695 & .50613 & .54018 & .37015 & .14984 & .08586 & 3673 & 2.3844 & 1.6978 & .71202 & .51716 & .25790 & .32671 \\
\hline 2354 & .62533 & .33695 & .53884 & .31795 & .09890 & .10927 & 3686 & 1.1002 & .59388 & .53978 & .39978 & .19875 & .13971 \\
\hline 2355 & .95281 & 60916 & .63933 & .49704 & .26204 & .14857 & 3691 & 1.0434 & .59373 & .56901 & .43689 & .23047 & .15653 \\
\hline 2357 & .71116 & .48235 & .67826 & .52971 & .28323 & .18323 & 3734 & 4.6500 & 3.3062 & .71101 & .51289 & .26401 & .22858 \\
\hline 2360 & 69096 & .46563 & .67389 & .54164 & .30982 & .21459 & 3771 & 1.1429 & .63312 & .55397 & .41264 & .19686 & .13063 \\
\hline 2361 & .80558 & .55414 & .68788 & .63354 & .46741 & .39479 & 3789 & .41019 & .22529 & .54924 & .45088 & .26870 & .17120 \\
\hline 2394 & 1.1261 & .64343 & .57137 & .42316 & .21183 & .12924 & 3826 & .90314 & .47855 & .52988 & .40488 & .22280 & .14262 \\
\hline 2404 & 1.0437 & .59861 & .57352 & .43430 & .22912 & .15683 & 3831 & 1.0763 & .61741 & .57364 & .41256 & .18748 & .11501 \\
\hline 2415 & 1.2934 & .72785 & .56276 & .42340 & .22281 & .14346 & 3841 & 1.0338 & .58460 & .56550 & .41867 & .18615 & .10625 \\
\hline 2462 & 1.1772 & .65845 & .55934 & .41783 & .21296 & .13944 & 3871 & .86126 & .49898 & .57937 & .45008 & .24153 & .15849 \\
\hline 2528 & .90620 & .48570 & .53598 & .33422 & .07565 & -.00060 & 3884 & 2.2788 & 1.4730 & .64642 & .42951 & .17590 & .25470 \\
\hline 2617 & .75146 & .44764 & .59569 & .50352 & .31061 & .20753 & 3941 & 1.3354 & .70310 & .52652 & .27443 & .10014 & .12388 \\
\hline 2619 & .75414 & .42836 & .56802 & .42021 & .18516 & .08677 & 3963 & -- & -- & -- & -- & -- & -- \\
\hline 2621 & .78912 & .46273 & .58638 & .47071 & .25100 & .15311 & 4040 & .83725 & .47540 & .56781 & .43863 & .23659 & .15914 \\
\hline 2675 & 1.1266 & .66007 & .58589 & .47534 & .27171 & .18529 & 4058 & 2.2567 & 1.6508 & .73154 & .73808 & .64172 & .48653 \\
\hline 2676 & 1.1889 & .69405 & .58378 & .45052 & .22236 & .16334 & 4098 & .63894 & .35975 & .56304 & .46540 & .23325 & .13723 \\
\hline 2679 & .84473 & .54555 & .64583 & .53505 & .29175 & .17874 & 4100 & .83069 & .50515 & .60811 & .50264 & .29678 & .19822 \\
\hline 2715 & .94475 & .53114 & .56219 & .42908 & .23282 & .15599 & 4137 & 1.0681 & .57281 & .53632 & .41075 & .21738 & .15719 \\
\hline 2744 & .83800 & .47776 & .57012 & .43672 & .21763 & .14096 & 4191 & .95305 & .57382 & .60209 & .47543 & .23974 & .14461 \\
\hline 2758 & .79344 & .54719 & .68964 & .54997 & .27617 & .15318 & 4256 & -- & -- & -- & -- & -- & -- \\
\hline 2794 & .67714 & .39238 & .57947 & .22136 & -.06917 & -.08617 & 4257 & 1.3210 & .73172 & .55389 & .41924 & .22532 & .15058 \\
\hline 2797 & .35410 & .22377 & 63193 & .50352 & .28354 & .19455 & 4258 & 1.3821 & .79144 & .57262 & .46156 & .25693 & .15719 \\
\hline 2811 & .81403 & .47566 & .58432 & .45676 & .22549 & .13939 & 4278 & .99303 & .57465 & .57868 & .43252 & .21911 & .14061 \\
\hline 2813 & .93000 & .52933 & .56918 & .36164 & .08296 & -.05198 & 4299 & .45473 & .20817 & .45778 & .27675 & .09087 & .03916 \\
\hline 2814 & .52143 & .40333 & .77352 & .62834 & .28689 & -.07910 & 4300 & 1.3025 & .80665 & .61929 & .46918 & .25033 & .16445 \\
\hline 2815 & .80127 & .47513 & .59296 & .52248 & .28065 & .14984 & 4305 & 1.2230 & .76055 & .62189 & .45524 & .23324 & .15857 \\
\hline 2818 & .88685 & .53174 & .59958 & .47265 & .28191 & .22563 & 4307 & 1.3794 & .88637 & .64256 & .51246 & .31258 & .23373 \\
\hline 2986 & 1.4312 & .76291 & .53304 & .37313 & .20973 & .16289 & 4309 & 1.2837 & .74405 & .57962 & .43128 & .23117 & .15842 \\
\hline 3005 & .97568 & .53194 & .54520 & .40673 & .21267 & .14574 & 4311 & 1.3089 & .75646 & .57794 & .42347 & .21053 & .13511 \\
\hline 3033 & .38339 & .22573 & .58877 & .51265 & .31691 & .22520 & 4313 & 1.3932 & .82335 & .59099 & .47388 & .26498 & .17148 \\
\hline 3034 & -- & -- & -- & -- & -- & -- & 4319 & 1.1729 & .63557 & .54188 & .41794 & .22498 & .12915 \\
\hline 3047 & 1.9244 & 1.4814 & .76977 & .71429 & .56886 & .48646 & 4329 & 1.2487 & .72386 & .57968 & .44590 & .23948 & .15739 \\
\hline 3103 & 1.0796 & 69186 & .64087 & .54066 & .25357 & .11512 & 4331 & -- & -- & -- & -- & -- & -- \\
\hline 3133 & 1.1239 & .62421 & .55538 & .41931 & .22763 & .14842 & 4375 & 1.0230 & .60258 & .58902 & .49552 & .27978 & .19213 \\
\hline 3156 & 1.2198 & .73808 & .60507 & .51042 & .30688 & .21106 & 4392 & 1.4685 & .83684 & .56988 & .41637 & .21348 & .14053 \\
\hline 3171 & 1.0476 & .58776 & .56107 & .42031 & .21354 & .13521 & 4425 & .62940 & .36242 & .57582 & .47616 & .25087 & .16213 \\
\hline 3189 & .57482 & .33083 & .57554 & .48405 & .27196 & .17659 & 4440 & .88460 & .51253 & .57939 & 44190 & .24365 & .16939 \\
\hline 3260 & .80929 & .47501 & .58695 & .39550 & .14518 & .07185 & 4476 & .98521 & .52997 & .53792 & .41733 & .22621 & .14732 \\
\hline 3267 & .75700 & .49631 & .65562 & .56382 & .36083 & .25731 & 4498 & .27667 & .12389 & .44779 & .14029 & .03587 & .01025 \\
\hline 3270 & .79887 & .44959 & .56279 & .46265 & .24656 & .17167 & 4517 & .98336 & .56413 & .57367 & .43035 & .22106 & .13958 \\
\hline 3272 & .28556 & .14472 & .50681 & .19879 & .10200 & .26213 & 4520 & 1.0225 & .57722 & .56453 & .45726 & .25463 & .18314 \\
\hline 3277 & .21818 & .13709 & .62833 & .51370 & .26393 & .21662 & 4525 & 3.0943 & 2.4119 & .77947 & .70259 & .48746 & .15420 \\
\hline 3278 & .71335 & .42204 & .59163 & .46749 & .23020 & .13595 & 4563 & 1.4111 & .86417 & .61240 & .29320 & -.08853 & -.12949 \\
\hline 3280 & .45664 & .27795 & 60869 & .46871 & .22304 & .10890 & 4570 & .83100 & .48651 & .58546 & .47340 & .25394 & .16215 \\
\hline 3281 & .44111 & .22002 & .49878 & .35404 & .18003 & .11633 & 4577 & 1.2695 & .70618 & .55627 & .42568 & .23499 & .16366 \\
\hline 3283 & 1.0081 & .62395 & .61892 & .46408 & .24480 & .15910 & 4591 & 1.1150 & .64264 & .57638 & .42762 & .21820 & .14245 \\
\hline 3284 & 1.0517 & .59378 & .56459 & .42851 & .22019 & .13829 & 4670 & .81425 & .47124 & .57875 & .45530 & .24080 & .15911 \\
\hline 3285 & 1.1015 & .61635 & .55958 & .42155 & .19985 & .12635 & 4671 & .71671 & .43887 & .61235 & .43100 & .16836 & .07366 \\
\hline 3329 & .94709 & .57544 & 60759 & .47595 & .25857 & .17773 & 4679 & 1.0750 & .62647 & .58276 & .47145 & .26782 & .18764 \\
\hline 3335 & 1.3762 & .75940 & .55181 & .43057 & .23413 & .16113 & 4696 & .68571 & .19905 & .29028 & -.06029 & .34450 & -.36842 \\
\hline 3370 & 1.1453 & .64043 & .55920 & .41770 & .22077 & .13887 & 4703 & .81803 & .48206 & .58930 & .51030 & .29724 & .18335 \\
\hline 3410 & .80672 & .45241 & .56080 & .44227 & .22289 & .14260 & 4704 & 1.4719 & .85911 & .58367 & .42922 & .21250 & .12760 \\
\hline 3415 & 1.0770 & 60919 & .56565 & .43306 & .22936 & .15255 & 4731 & .82985 & .53534 & .64511 & .52603 & .31681 & .22198 \\
\hline 3430 & 1.1720 & .74226 & .63332 & .48161 & .25742 & .17175 & 4792 & 1.0133 & .55855 & .55123 & .41342 & .19216 & .12452 \\
\hline 3431 & 1.3123 & .86695 & .66064 & .53666 & .32241 & .22398 & 4819 & 1.3658 & 69718 & .51047 & .34903 & .16249 & .09221 \\
\hline 3441 & 1.0577 & .59474 & .56228 & .44819 & .28781 & .20935 & 4852 & 1.7250 & .84429 & .48944 & .07910 & -.06557 & .02876 \\
\hline 3442 & .62447 & .36414 & .58311 & .45431 & .21508 & .10745 & 4866 & 1.0538 & .58671 & .55676 & .41699 & .21664 & .14217 \\
\hline 3446 & .67172 & .36508 & .54350 & .41785 & .22688 & .15611 & 4876 & 1.3737 & .81586 & .59392 & .48876 & .28336 & .19690 \\
\hline 3460 & 1.2346 & .64563 & .52296 & .30795 & .23564 & .29042 & 4878 & 1.4956 & .84445 & .56464 & .41697 & .20615 & .12165 \\
\hline 3462 & .75725 & .40448 & .53414 & .44609 & .29047 & .22953 & 4880 & .72602 & .42394 & .58392 & .46856 & .24864 & .15571 \\
\hline
\end{tabular}


Appendix 4-2.7. L-moments of storm depth defined by 72-hour minimum interevent time for hourly rainfall stations in TexasContinued.

\begin{tabular}{|c|c|c|c|c|c|c|c|c|c|c|c|c|c|}
\hline $\begin{array}{c}\text { Station } \\
\text { no. }\end{array}$ & $\begin{array}{c}\text { Depth } \\
\text { mean } \\
\text { (inches) }\end{array}$ & $\begin{array}{l}\text { Depth } \\
\text { L-scale } \\
\text { (inches) }\end{array}$ & $\begin{array}{c}\text { Depth } \\
\text { L-CV } \\
\text { (dimen- } \\
\text { sionless) }\end{array}$ & $\begin{array}{c}\text { Depth } \\
\text { L-skew } \\
\text { (dimen- } \\
\text { sionless) }\end{array}$ & $\begin{array}{c}\text { Depth } \\
\text { L-kurtosis } \\
\text { (dimen- } \\
\text { sionless) }\end{array}$ & $\begin{array}{c}\text { Depth } \\
\text { Tau5 } \\
\text { (dimen- } \\
\text { sionless) }\end{array}$ & $\begin{array}{c}\text { Station } \\
\text { no. }\end{array}$ & $\begin{array}{l}\text { Depth } \\
\text { mean } \\
\text { (inches) }\end{array}$ & $\begin{array}{c}\text { Depth } \\
\text { L-scale } \\
\text { (inches) }\end{array}$ & $\begin{array}{c}\text { Depth } \\
\text { L-CV } \\
\text { (dimen- } \\
\text { sionless) }\end{array}$ & $\begin{array}{c}\text { Depth } \\
\text { L-skew } \\
\text { (dimen- } \\
\text { sionless) }\end{array}$ & $\begin{array}{c}\text { Depth } \\
\text { L-kurtosis } \\
\text { (dimen- } \\
\text { sionless) }\end{array}$ & $\begin{array}{c}\text { Depth } \\
\text { Tau5 } \\
\text { (dimen- } \\
\text { sionless) }\end{array}$ \\
\hline 4920 & 0.89935 & 0.53758 & 0.59774 & 0.47074 & 0.23347 & 0.14203 & 5957 & 1.0183 & 0.57759 & 0.56721 & 0.44702 & 0.25286 & 0.18039 \\
\hline 4934 & .46400 & .35400 & .76293 & .76836 & .60452 & .49153 & 5958 & .93907 & .50850 & .54149 & .35565 & .16257 & .10845 \\
\hline 4972 & .94751 & .53252 & .56202 & .43756 & .23303 & .14802 & 5973 & .78897 & .52264 & .66243 & .50302 & .22472 & .11502 \\
\hline 4973 & 1.2981 & .70794 & .54536 & .45077 & .27121 & .17072 & 5996 & .96840 & .54760 & .56547 & .42599 & .21778 & .14710 \\
\hline 4974 & .73877 & .44616 & 60392 & .49743 & .28523 & .18636 & 6017 & .79286 & .50908 & .64209 & .49539 & .24280 & .12039 \\
\hline 4975 & 1.2928 & .68955 & .53337 & .38850 & .19564 & .13487 & 6024 & 1.4384 & .81249 & .56486 & .40960 & .21750 & .16109 \\
\hline 4978 & .95693 & .61371 & .64133 & .51981 & .30124 & .20659 & 6050 & .88933 & .42171 & .47419 & .37850 & .16015 & .07220 \\
\hline 4979 & 3.1833 & 2.1500 & .67539 & .62341 & .68295 & .63643 & 6104 & .71319 & .43525 & .61028 & .51367 & .29372 & .18858 \\
\hline 4982 & .83753 & .48394 & .57782 & .44678 & .23012 & .13916 & 6108 & 1.3189 & .72156 & .54711 & .39083 & .18873 & .12210 \\
\hline 5018 & .98498 & .54985 & .55824 & .41188 & .21717 & .15804 & 6136 & .65116 & .36812 & .56533 & .46488 & .23502 & .14050 \\
\hline 5048 & .67385 & .40468 & .60054 & .51427 & .28754 & .19614 & 6166 & .62232 & .32769 & .52656 & .36444 & .14106 & .05696 \\
\hline 5049 & .66667 & .37952 & .56928 & .51566 & .30558 & .23615 & 6176 & 1.2772 & .73995 & .57936 & .46400 & .25339 & .12899 \\
\hline 5056 & -- & -- & -- & -- & -- & -- & 6177 & 1.3274 & .74042 & .55778 & .42210 & .22700 & .15306 \\
\hline 5057 & .62647 & .41490 & .66228 & .52034 & .27074 & .15895 & 6210 & 1.1570 & .64460 & .55714 & .41457 & .21599 & .14221 \\
\hline 5060 & 1.0483 & .71938 & .68624 & .53881 & .29008 & .17640 & 6211 & 1.2663 & .69713 & .55054 & .36540 & .16314 & .10340 \\
\hline 5081 & 1.2221 & .67082 & .54889 & .39060 & .20222 & .13278 & 6270 & 1.3733 & .73585 & .53582 & .39872 & .20710 & .13845 \\
\hline 5094 & 1.1188 & .63271 & .56553 & .43298 & .22725 & .14771 & 6275 & -- & -- & -- & -- & -- & -- \\
\hline 5113 & .98875 & .60776 & .61468 & .50287 & .27754 & .17953 & 6276 & 2.4562 & 1.6534 & .67314 & .48007 & .22130 & .16859 \\
\hline 5114 & -- & -- & -- & -- & -- & -- & 6335 & 1.1289 & .61998 & .54918 & .39294 & .19427 & .12591 \\
\hline 5123 & .88818 & .52673 & .59304 & .42607 & .12266 & -.06674 & 6434 & .80500 & .34236 & .42530 & .28743 & .19800 & .09850 \\
\hline 5192 & 1.1351 & .64133 & .56498 & .43132 & .22438 & .13880 & 6504 & .71620 & .42332 & .59107 & .48210 & .25636 & .16422 \\
\hline 5193 & 1.0904 & .62673 & .57479 & .43034 & .21818 & .14646 & 6558 & .88350 & .51871 & .58711 & .44231 & .30327 & .35004 \\
\hline 5224 & 1.4812 & .85849 & .57959 & .42747 & .22475 & .14433 & 6615 & .84181 & .49825 & .59187 & .51467 & .30115 & .21314 \\
\hline 5228 & 1.0886 & .63217 & .58071 & .40849 & .19291 & .13174 & 6660 & 1.0427 & .56792 & .54467 & .40135 & .19493 & .09346 \\
\hline 5235 & .96765 & .62397 & .64483 & .55522 & .39177 & .32372 & 6663 & 1.2908 & .75561 & .58540 & .45011 & .22834 & .12724 \\
\hline 5247 & .75016 & .42445 & .56581 & .43712 & .20772 & .11743 & 6734 & .80486 & .46307 & .57534 & .43060 & .21539 & .13641 \\
\hline 5258 & 1.0732 & .61468 & .57273 & .45387 & .25253 & .16150 & 6736 & .73414 & .43506 & .59261 & .49773 & .27517 & .17977 \\
\hline 5303 & .90962 & .56758 & .62398 & .51316 & .30003 & .21259 & 6740 & 1.9330 & .95744 & .49532 & .07677 & -.17032 & .02721 \\
\hline 5312 & .92104 & .53570 & .58162 & .47170 & .25174 & .16993 & 6750 & 1.3423 & .92679 & .69043 & .54993 & .29709 & .17115 \\
\hline 5341 & 3.6243 & 2.6076 & .71948 & .61359 & .22188 & -.20581 & 6757 & 1.2054 & .68744 & .57028 & .41664 & .20980 & .13534 \\
\hline 5342 & -- & -- & -- & -- & -- & -- & 6775 & .73683 & .43219 & .58655 & .43846 & .21651 & .14071 \\
\hline 5348 & 1.3280 & .70177 & .52843 & .35836 & .14743 & .07480 & 6776 & .76321 & .43658 & .57203 & .44861 & .22393 & .14284 \\
\hline 5358 & .79751 & .46406 & .58189 & .45698 & .24469 & .15806 & 6788 & 1.1891 & 64449 & .54202 & .36828 & .16059 & .08090 \\
\hline 5398 & 1.2353 & .68325 & .55311 & .41400 & .22201 & .14290 & 6792 & .57161 & .32469 & .56803 & .46967 & .24583 & .15532 \\
\hline 5410 & .72447 & .43251 & .59700 & .48836 & .26792 & .17545 & 6794 & 4.9417 & 2.1763 & .44040 & .04809 & -.36238 & .18533 \\
\hline 5411 & .64791 & .42092 & .64965 & .50549 & .26774 & .16622 & 6834 & 1.3405 & .72800 & .54306 & .40009 & .19709 & .12721 \\
\hline 5424 & 1.5053 & .91260 & .60624 & .44580 & .25178 & .18410 & 6893 & .49973 & .28783 & .57598 & .52275 & .30834 & .20782 \\
\hline 5429 & 1.0093 & .60244 & .59689 & .45089 & .23651 & .16621 & 6935 & .72176 & .42464 & .58834 & .50618 & .28313 & .18253 \\
\hline 5431 & 1.3145 & .65945 & .50166 & .24198 & -.04885 & -.02734 & 6981 & 1.0607 & .59302 & .55909 & .42719 & .23917 & .15942 \\
\hline 5461 & 1.1862 & .67188 & .56639 & .42463 & .22378 & .15268 & 7020 & 1.0190 & 63981 & .62786 & .51553 & .32082 & .23455 \\
\hline 5463 & 1.1977 & .66034 & .55135 & .40741 & .19323 & .12741 & 7060 & .83012 & .47904 & .57707 & .46918 & .24736 & .15629 \\
\hline 5471 & .45222 & .25528 & .56450 & .39717 & .20954 & .36126 & 7066 & 1.2788 & .70943 & .55477 & .40591 & .20321 & .12728 \\
\hline 5477 & 1.5591 & 1.2664 & .81224 & .82101 & .71106 & .65674 & 7074 & .67060 & .40696 & .60686 & .50784 & .29331 & .19903 \\
\hline 5528 & 1.1102 & .60918 & .54870 & .41136 & .22072 & .14363 & 7097 & 1.1317 & .63037 & .55702 & .40448 & .19293 & .11122 \\
\hline 5579 & -- & -- & -- & -- & -- & -- & 7116 & .74307 & .43224 & .58169 & .44855 & .23597 & .15653 \\
\hline 5580 & 1.9425 & .95607 & .49219 & .23048 & .27531 & .37430 & 7140 & 1.2604 & .79502 & .63075 & .50092 & .27609 & .18096 \\
\hline 5589 & .51542 & .27458 & .53273 & .30103 & .07941 & .03718 & 7173 & 1.5767 & .99367 & .63022 & .46398 & .23156 & .14124 \\
\hline 5590 & .63833 & .36997 & .57959 & .43220 & .21165 & .13755 & 7174 & 1.5018 & .92635 & .61681 & .45231 & .23256 & .15597 \\
\hline 5591 & .55952 & .30907 & .55239 & .43453 & .24015 & .15713 & 7213 & 1.1022 & .60593 & .54973 & .40116 & .19823 & .11820 \\
\hline 5592 & .50706 & .30060 & .59284 & .49373 & .29688 & .20640 & 7243 & .93376 & .52725 & .56466 & .43560 & .22487 & .15204 \\
\hline 5594 & .47333 & .27605 & .58321 & .50278 & .29368 & .19221 & 7262 & .30333 & .17742 & .58491 & .44062 & .23872 & .17630 \\
\hline 5595 & -- & -- & -- & -- & -- & -- & 7274 & .90582 & .49714 & .54883 & .44069 & .24397 & .15857 \\
\hline 5596 & .67967 & .38442 & .56559 & .49429 & .26351 & .17390 & 7300 & .91357 & .51938 & .56852 & .42419 & .22003 & .13985 \\
\hline 5600 & .58019 & .34580 & .59602 & .50649 & .31707 & .23766 & 7311 & .95750 & .53124 & .55482 & .38697 & .13182 & .13438 \\
\hline 5618 & 2.1167 & 1.4736 & 69619 & .60275 & .48606 & .52980 & 7363 & 1.9075 & 1.3704 & .71840 & .56346 & .25541 & .16940 \\
\hline 5650 & 1.3313 & .72268 & .54286 & .56808 & .26365 & .05263 & 7422 & 1.0162 & .60577 & .59608 & .46753 & .25397 & .17147 \\
\hline 5656 & .80869 & .46542 & .57553 & .47633 & .24561 & .16275 & 7431 & .63744 & .37585 & .58963 & .48706 & .26696 & .17660 \\
\hline 5658 & .72465 & .43679 & .60276 & .49508 & .28522 & .18761 & 7481 & .52734 & .32024 & .60728 & .52806 & .31485 & .21073 \\
\hline 5661 & 1.0648 & .69035 & .64836 & .57884 & .35508 & .24863 & 7497 & 1.1111 & .62200 & .55979 & .44172 & .24161 & .17805 \\
\hline 5666 & .80381 & .52333 & .65107 & .52134 & .28187 & .18725 & 7498 & 1.1367 & .65415 & .57545 & .45385 & .26204 & .21881 \\
\hline 5695 & 1.1110 & .60848 & .54767 & .42528 & .24125 & .15871 & 7499 & .96641 & .54234 & .56119 & .44587 & .23087 & .15163 \\
\hline 5742 & .50500 & .36767 & .72805 & .76745 & 66099 & .59459 & 7531 & 1.0388 & .57991 & .55826 & .41747 & .19884 & .08734 \\
\hline 5766 & 3.3114 & 2.0076 & .60627 & .43776 & .09749 & -.14635 & 7534 & .99585 & .59669 & .59917 & .49013 & .30651 & .24197 \\
\hline 5770 & .82059 & .47834 & .58293 & .45721 & .23039 & .13845 & 7556 & 1.0383 & .59749 & .57545 & .45632 & .24255 & .15766 \\
\hline 5775 & .62429 & .33381 & .53471 & .20285 & -.00000 & .25678 & 7594 & 1.2473 & .70354 & .56406 & .41537 & .21389 & .14264 \\
\hline 5779 & .96400 & .48257 & .50059 & .31603 & .16693 & .01304 & 7596 & 1.0707 & .60955 & .56929 & .34974 & .10494 & .06042 \\
\hline 5840 & .92619 & .54709 & .59069 & .46131 & .25286 & .16573 & 7608 & 1.0632 & .63156 & .59402 & .43102 & .22050 & .16114 \\
\hline 5890 & .54991 & .35160 & 63937 & .49860 & .26696 & .17258 & 7622 & .34929 & .25610 & .73321 & .57670 & .30711 & .26705 \\
\hline 5891 & .56514 & .29388 & .52002 & .36398 & .16586 & .10677 & 7700 & 1.2993 & .72921 & .56125 & .40881 & .21218 & .13753 \\
\hline 5897 & 1.1607 & .64785 & .55815 & .42246 & .20797 & .14000 & 7706 & .85173 & .50137 & .58866 & .47536 & .26505 & .18276 \\
\hline
\end{tabular}


Appendix 4-2.7. L-moments of storm depth defined by 72-hour minimum interevent time for hourly rainfall stations in TexasContinued.

\begin{tabular}{|c|c|c|c|c|c|c|c|c|c|c|c|c|c|}
\hline $\begin{array}{c}\text { Station } \\
\text { no. }\end{array}$ & $\begin{array}{l}\text { Depth } \\
\text { mean } \\
\text { (inches) }\end{array}$ & $\begin{array}{l}\text { Depth } \\
\text { L-scale } \\
\text { (inches) }\end{array}$ & $\begin{array}{c}\text { Depth } \\
\text { L-CV } \\
\text { (dimen- } \\
\text { sionless) }\end{array}$ & $\begin{array}{c}\text { Depth } \\
\text { L-skew } \\
\text { (dimen- } \\
\text { sionless) }\end{array}$ & $\begin{array}{c}\text { Depth } \\
\text { L-kurtosis } \\
\text { (dimen- } \\
\text { sionless) }\end{array}$ & $\begin{array}{c}\text { Depth } \\
\text { Tau5 } \\
\text { (dimen- } \\
\text { sionless) }\end{array}$ & $\begin{array}{c}\text { Station } \\
\text { no. }\end{array}$ & $\begin{array}{l}\text { Depth } \\
\text { mean } \\
\text { (inches) }\end{array}$ & $\begin{array}{c}\text { Depth } \\
\text { L-scale } \\
\text { (inches) }\end{array}$ & $\begin{array}{c}\text { Depth } \\
\text { L-CV } \\
\text { (dimen- } \\
\text { sionless) }\end{array}$ & $\begin{array}{l}\text { Depth } \\
\text { L-skew } \\
\text { (dimen- } \\
\text { sionless) }\end{array}$ & $\begin{array}{l}\text { Depth } \\
\text { L-kurtosis } \\
\text { (dimen- } \\
\text { sionless) }\end{array}$ & $\begin{array}{c}\text { Depth } \\
\text { Tau5 } \\
\text { (dimen- } \\
\text { sionless) }\end{array}$ \\
\hline 7718 & 1.0097 & 0.60460 & 0.59881 & 0.51459 & 0.33265 & 0.22120 & 8910 & 1.5478 & 1.1989 & 0.77459 & 0.67337 & 0.44525 & 0.29525 \\
\hline 7745 & 1.3455 & .77678 & .57730 & .43481 & .24109 & .15584 & 8911 & 1.0020 & .58671 & .58553 & .42188 & .20653 & .13227 \\
\hline 7922 & .38371 & .22096 & .57583 & .46245 & .26956 & .18826 & 8924 & .46195 & .23841 & .51609 & .38871 & .18168 & .09363 \\
\hline 7936 & 1.4332 & .79395 & .55398 & .41090 & .21540 & .14577 & 8929 & 2.1478 & 1.6961 & .78971 & .72655 & .59057 & .62501 \\
\hline 7943 & .65485 & .41901 & .63985 & .48634 & .24385 & .14789 & 8942 & 1.3731 & .75625 & .55075 & .41265 & .20713 & .12097 \\
\hline 7944 & .92455 & .55737 & .60286 & .43377 & .19936 & .07272 & 8944 & 1.1996 & .72148 & .60145 & .46268 & .25835 & .17519 \\
\hline 7945 & .91498 & .60023 & .65601 & .50612 & .27545 & .18600 & 8996 & 1.1818 & 68998 & .58386 & .42559 & .21488 & .14669 \\
\hline 7947 & 1.3326 & .78129 & .58631 & .47766 & .28226 & .19452 & 9014 & 2.3257 & 1.3090 & .56286 & .55591 & .58931 & .56966 \\
\hline 7948 & .91924 & .56547 & .61515 & .47291 & .24810 & .16769 & 9037 & .47676 & .28629 & 60049 & .52188 & .31487 & .20696 \\
\hline 7951 & 1.1791 & .65040 & .55162 & .40710 & .20832 & .13596 & 9106 & .45388 & .30236 & .66617 & .60865 & .41774 & .32865 \\
\hline 7953 & .80814 & .52622 & .65115 & .53758 & .28384 & .12189 & 9107 & .54042 & .32183 & .59552 & .42762 & .19200 & .16833 \\
\hline 7981 & .86379 & .52742 & .61059 & .47307 & .22579 & .11376 & 9129 & .64429 & .41896 & .65027 & .51864 & .25813 & .13370 \\
\hline 7990 & 1.0817 & .72172 & .66719 & .52187 & .26121 & .12501 & 9163 & .92255 & .53376 & .57857 & .45074 & .23242 & .14015 \\
\hline 7992 & 2.6850 & 1.3563 & .50515 & .43352 & .33030 & -.15483 & 9213 & 1.0656 & .64656 & .60674 & .44515 & .24268 & .18400 \\
\hline 7997 & .70473 & .41923 & .59488 & .47757 & .27897 & .19053 & 9214 & 4.5550 & 2.9223 & .64157 & .62861 & .57910 & .33478 \\
\hline 7999 & .47846 & .29615 & .61897 & .48477 & .24274 & .07834 & 9222 & 1.0015 & .59822 & .59735 & .47004 & .25441 & .14469 \\
\hline 8022 & .61674 & .36461 & .59120 & .40068 & .09474 & .01150 & 9248 & .64070 & .36584 & .57100 & .44324 & .21175 & .14661 \\
\hline 8023 & .72048 & .42235 & .58621 & .48441 & .26225 & .17278 & 9266 & .79942 & .42268 & .52873 & .34665 & .10081 & .03584 \\
\hline 8047 & 99049 & .55668 & .56203 & .42184 & .21538 & .13867 & 9270 & .62234 & .36298 & .58325 & .50635 & .28005 & .17994 \\
\hline 8060 & .82653 & .53934 & .65254 & .48414 & .19553 & .06751 & 9295 & .37082 & .23423 & .63165 & .48632 & .23629 & .12458 \\
\hline 8062 & 1.0804 & .71269 & .65967 & .47419 & .19614 & .07044 & 9304 & -- & -- & -- & -- & -- & -- \\
\hline 8068 & .48000 & .29071 & .60565 & .49619 & .27932 & .12876 & 9307 & .87450 & .43879 & .50176 & .37938 & .19631 & .10401 \\
\hline 8081 & .98937 & .61811 & .62475 & .51481 & .29384 & .20104 & 9328 & .76647 & .46926 & .61224 & .48914 & .25077 & .12639 \\
\hline 8089 & .76500 & .42048 & .54964 & .33837 & .15960 & .16389 & 9329 & 1.0357 & .66667 & .64368 & .43114 & .02143 & -.16286 \\
\hline 8221 & 1.7512 & .91232 & .52095 & .41202 & .39049 & .34351 & 9345 & -- & -- & -- & -- & -- & -- \\
\hline 8252 & .68757 & .39549 & .57520 & .46039 & .23701 & .15425 & 9363 & .96160 & .61226 & .63671 & .47719 & .24214 & .15553 \\
\hline 8265 & 1.3568 & .80830 & .59575 & .45770 & .24881 & .16716 & 9364 & 1.0846 & .71318 & .65755 & .51496 & .28430 & .19287 \\
\hline 8289 & .72000 & .27971 & .38849 & .15861 & .08209 & .12576 & 9365 & .61333 & .35686 & .58184 & .55041 & .35650 & .21561 \\
\hline 8305 & .48660 & .28095 & .57738 & .51122 & .30406 & .20261 & 9371 & 1.0780 & .56712 & .52606 & .29490 & .07650 & .05468 \\
\hline 8335 & 1.2790 & .70995 & .55507 & .40324 & .21748 & .15104 & 9417 & 1.0366 & .59355 & .57259 & .41229 & .20403 & .13694 \\
\hline 8400 & .65000 & .36503 & .56159 & .46621 & .28829 & .19718 & 9419 & .96716 & .58003 & .59972 & .43255 & .21635 & .14698 \\
\hline 8445 & 1.1742 & .70334 & .59901 & .45393 & .22944 & .14520 & 9435 & .73860 & .38080 & .51556 & .41226 & .28046 & .23816 \\
\hline 8446 & 1.0659 & .59526 & .55844 & .41243 & .20118 & .12501 & 9491 & 1.1807 & .66603 & .56409 & .41634 & .20874 & .13116 \\
\hline 8451 & .73810 & .44444 & .60215 & .47805 & .25079 & .15183 & 9499 & .78971 & .45858 & .58070 & .45894 & .21779 & .12808 \\
\hline 8531 & 1.0203 & .55840 & .54727 & .39146 & .19235 & .12280 & 9522 & -- & -- & -- & -- & -- & -- \\
\hline 8541 & .92897 & .54408 & .58568 & .47689 & .26875 & .15828 & 9527 & .73209 & .42411 & .57931 & .47633 & .23242 & .13331 \\
\hline 8544 & 1.1677 & .69460 & .59483 & .49084 & .28650 & .19919 & 9532 & 1.0083 & .57661 & .57185 & .44537 & .24616 & .16888 \\
\hline 8545 & .50250 & .18977 & .37766 & .08886 & .26121 & .26081 & 9544 & -- & -- & -- & -- & -- & -- \\
\hline 8563 & 1.1295 & .63352 & .56091 & .44934 & .24442 & .16718 & 9565 & .86636 & .51662 & .59630 & .47751 & .24568 & .14428 \\
\hline 8566 & .78120 & .45406 & .58124 & .43580 & .20916 & .12199 & 9570 & .86944 & .48233 & .55476 & .44793 & .22245 & .14513 \\
\hline 8583 & .88619 & .49627 & .56001 & .43714 & .21259 & .15695 & 9574 & 1.0545 & .45818 & .43448 & .25397 & .10053 & .05952 \\
\hline 8584 & .95007 & .53782 & .56608 & .42491 & .20732 & .13716 & 9588 & .86683 & .54189 & .62513 & .50146 & .25789 & .15171 \\
\hline 8623 & 1.0141 & .54729 & .53967 & .40536 & .19719 & .12505 & 9665 & 1.1534 & 64377 & .55813 & .42456 & .22765 & .15556 \\
\hline 8625 & .97087 & .54981 & .56631 & .42845 & .22948 & .15700 & 9715 & 1.0459 & .58735 & .56156 & .42328 & .22479 & .14974 \\
\hline 8630 & .69193 & .39335 & .56848 & .43733 & .21138 & .12605 & 9729 & .87627 & .53244 & .60762 & .44149 & .21604 & .13949 \\
\hline 8631 & .78019 & .45272 & .58027 & .45631 & .23907 & .14614 & 9772 & 1.1981 & .76263 & .63654 & .50043 & .27253 & .17627 \\
\hline 8646 & .97566 & .55519 & .56904 & .41300 & .19879 & .12810 & 9814 & .97636 & .53909 & .55214 & .33074 & .12439 & .08799 \\
\hline 8647 & .72476 & .42131 & .58131 & .47208 & .24239 & .14902 & 9815 & 1.1667 & .67830 & .58139 & .45746 & .25480 & .18041 \\
\hline 8677 & .90563 & .50007 & .55218 & .44516 & .22499 & .12983 & 9816 & .86442 & .54757 & .63345 & .50880 & .25338 & .11632 \\
\hline 8696 & 2.3825 & 1.5907 & .66767 & .45914 & .18186 & .11495 & 9817 & .90870 & .50843 & .55951 & .44124 & .23505 & .15991 \\
\hline 8743 & 1.2804 & .72482 & .56608 & .42398 & .22232 & .14107 & 9829 & .55122 & .33090 & .60030 & .50927 & .28592 & .17871 \\
\hline 8761 & .65725 & .38213 & .58141 & .47340 & .23010 & .13149 & 9830 & .42371 & .25971 & .61294 & .46650 & .20689 & .12010 \\
\hline 8778 & 1.2202 & .65552 & .53722 & .39030 & .19416 & .12786 & 9858 & .71767 & .41827 & .58281 & .45311 & .23234 & .13999 \\
\hline 8845 & 1.0872 & .65234 & .60001 & .47165 & .24403 & .15768 & 9893 & .91033 & .52488 & .57658 & .44897 & .23717 & .14750 \\
\hline 8859 & 1.2374 & .67213 & .54317 & .38887 & .19626 & .12912 & 9916 & 1.3120 & .68342 & .52090 & .36420 & .17204 & .09856 \\
\hline 8898 & 1.1376 & .64160 & .56401 & .41929 & .21443 & .14111 & 9976 & .82495 & .49912 & .60502 & .48446 & .25709 & .16516 \\
\hline 8908 & 1.3053 & .74051 & .56732 & .35081 & .16670 & .21231 & & & & & & & \\
\hline
\end{tabular}


Appendix 4-3.1. L-moments of storm duration defined by 6-hour minimum interevent time for hourly rainfall stations in Texas.

$[--$, not available $]$

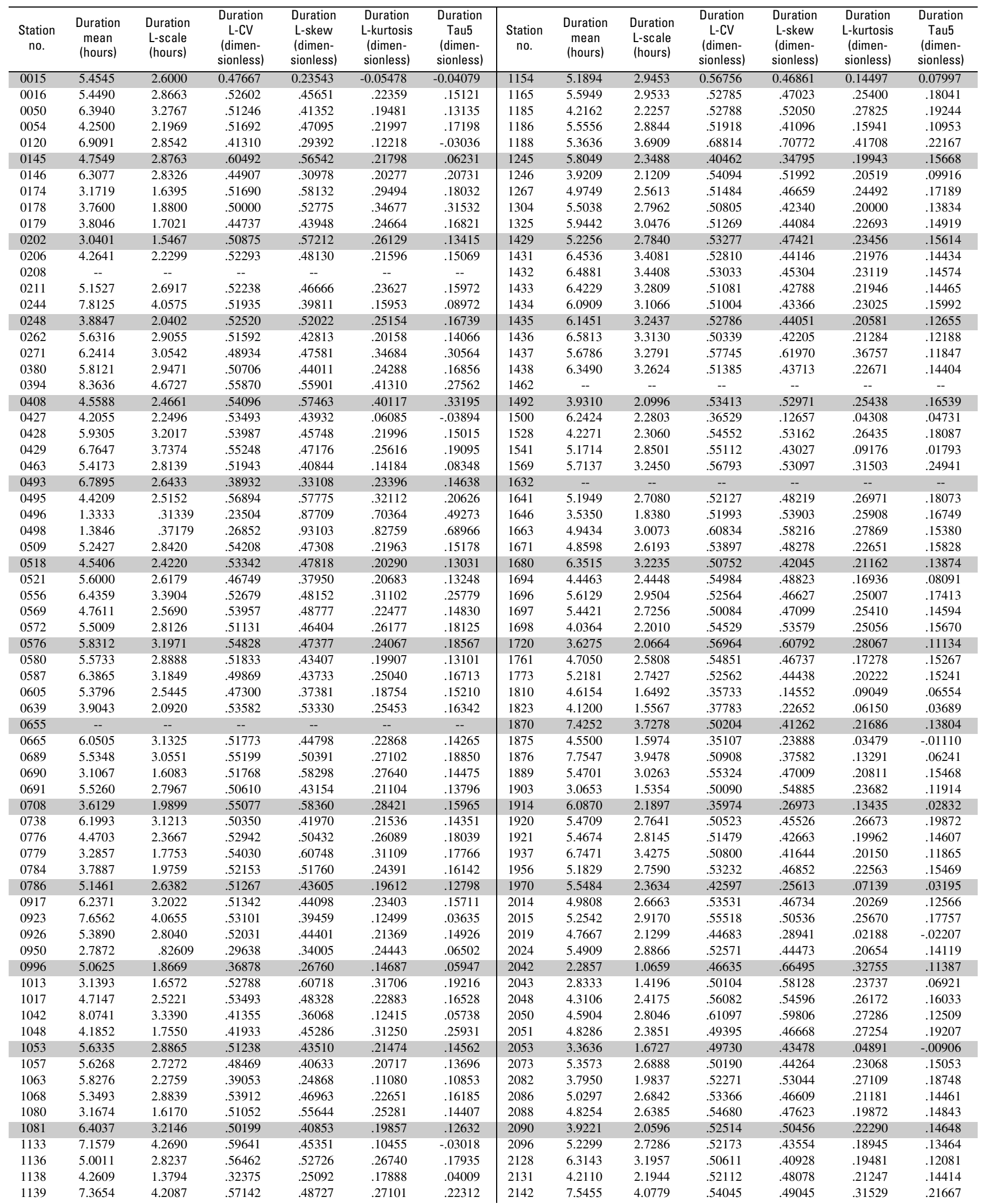


216 Statistical Characteristics of Storm Interevent Time, Depth, and Duration for Eastern New Mexico, Oklahoma, and Texas

Appendix 4-3.1. L-moments of storm duration defined by 6-hour minimum interevent time for hourly rainfall stations in TexasContinued.

\begin{tabular}{|c|c|c|c|c|c|c|c|c|c|c|c|c|c|}
\hline $\begin{array}{c}\text { Station } \\
\text { no. }\end{array}$ & $\begin{array}{l}\text { Duration } \\
\text { mean } \\
\text { (hours) }\end{array}$ & $\begin{array}{l}\text { Duration } \\
\text { L-scale } \\
\text { (hours) }\end{array}$ & $\begin{array}{l}\text { Duration } \\
\text { L-CV } \\
\text { (dimen- } \\
\text { sionless) }\end{array}$ & $\begin{array}{l}\text { Duration } \\
\text { L-skew } \\
\text { (dimen- } \\
\text { sionless) }\end{array}$ & $\begin{array}{l}\text { Duration } \\
\text { L-kurtosis } \\
\text { (dimen- } \\
\text { sionless) }\end{array}$ & $\begin{array}{c}\text { Duration } \\
\text { Tau5 } \\
\text { (dimen- } \\
\text { sionless) }\end{array}$ & $\begin{array}{c}\text { Station } \\
\text { no. }\end{array}$ & $\begin{array}{c}\text { Duration } \\
\text { mean } \\
\text { (hours) }\end{array}$ & $\begin{array}{l}\text { Duration } \\
\text { L-scale } \\
\text { (hours) }\end{array}$ & $\begin{array}{c}\text { Duration } \\
\text { L-CV } \\
\text { (dimen- } \\
\text { sionless) }\end{array}$ & $\begin{array}{c}\text { Duration } \\
\text { L-skew } \\
\text { (dimen- } \\
\text { sionless) }\end{array}$ & $\begin{array}{l}\text { Duration } \\
\text { L-kurtosis } \\
\text { (dimen- } \\
\text { sionless) }\end{array}$ & $\begin{array}{c}\text { Duration } \\
\text { Tau5 } \\
\text { (dimen- } \\
\text { sionless) }\end{array}$ \\
\hline 2160 & 8.9286 & 5.1190 & 0.57333 & 0.38032 & 0.08422 & 0.05073 & 3463 & 6.5741 & 3.6509 & 0.55535 & 0.46361 & 0.20852 & 0.10057 \\
\hline 2206 & 5.4583 & 2.7878 & .51074 & .46261 & .25360 & .17050 & 3476 & 5.6278 & 2.8338 & .50353 & .42530 & .21254 & .14765 \\
\hline 2238 & 4.8390 & 2.6196 & .54135 & .48119 & .21470 & .14395 & 3485 & 5.6400 & 2.1967 & .38948 & .24761 & .15428 & .19748 \\
\hline 2240 & 4.2308 & 2.3447 & .55419 & .48956 & .13002 & .02404 & 3507 & 4.1293 & 2.2848 & .55330 & .53818 & .24388 & .14480 \\
\hline 2242 & 5.7961 & 2.9876 & .51544 & .42974 & .20691 & .14135 & 3546 & 5.5397 & 2.9783 & .53763 & .45339 & .20415 & .14113 \\
\hline 2244 & 5.3341 & 2.8919 & .54216 & .46893 & .21637 & .14996 & 3547 & 5.2251 & 2.7490 & .52611 & .45273 & .21502 & .15711 \\
\hline 2247 & 5.2656 & 2.2413 & .42565 & .29347 & .15315 & .12333 & 3579 & 6.6517 & 3.6507 & .54883 & .48834 & .27575 & .19346 \\
\hline 2309 & 6.1892 & 2.9930 & .48358 & .36688 & .16346 & .11066 & 3642 & 5.3696 & 2.7633 & .51462 & .41906 & .17776 & .12244 \\
\hline 2312 & 4.4377 & 2.3195 & .52269 & .45587 & .17338 & .10644 & 3646 & 6.3727 & 3.1094 & .48792 & .40058 & .20732 & .13873 \\
\hline 2334 & 6.2273 & 3.3154 & .53240 & .39713 & .12792 & .07429 & 3668 & 8.2692 & 3.3000 & .39907 & .28811 & .14675 & .06126 \\
\hline 2336 & 4.8434 & 2.4347 & .50268 & .43758 & .21328 & .15709 & 3673 & 5.4211 & 2.6060 & .48071 & .36108 & .14687 & .09958 \\
\hline 2354 & 4.9375 & 2.6250 & .53165 & .47143 & .20160 & .08234 & 3686 & 4.0831 & 2.1446 & .52523 & .47635 & .17731 & .10726 \\
\hline 2355 & 6.5000 & 3.3581 & .51664 & .44756 & .20670 & .07656 & 3691 & 4.4426 & 2.3328 & .52510 & .47096 & .20349 & .13950 \\
\hline 2357 & 5.5289 & 3.1155 & .56349 & .50985 & .25097 & .14784 & 3734 & 5.1389 & 2.3944 & .46595 & .32871 & .11507 & .11800 \\
\hline 2360 & 4.8440 & 2.5981 & .53635 & .49364 & .24761 & .16788 & 3771 & 3.9061 & 2.1072 & .53948 & .52012 & .21025 & .11127 \\
\hline 2361 & 3.8208 & 1.9113 & .50024 & .48973 & .24291 & .17055 & 3789 & 4.0265 & 2.3894 & .59341 & .61199 & .29021 & .13982 \\
\hline 2394 & 5.9780 & 3.0324 & .50725 & .41132 & .19410 & .13204 & 3826 & 5.9458 & 2.8054 & .47183 & .34506 & .11743 & .05690 \\
\hline 2404 & 5.4735 & 2.8574 & .52204 & .43864 & .20392 & .14207 & 3831 & 5.7061 & 2.9145 & .51077 & .44262 & .23152 & .15908 \\
\hline 2415 & 6.2995 & 3.1980 & .50766 & .41277 & .20422 & .14181 & 3841 & 4.7337 & 2.3209 & .49028 & .43351 & .22292 & .16589 \\
\hline 2462 & 5.6573 & 2.9201 & .51616 & .41534 & .17354 & .11017 & 3871 & 5.7776 & 2.8783 & .49818 & .42194 & .21532 & .14083 \\
\hline 2528 & 6.7132 & 3.7484 & .55837 & .49644 & .30164 & .24813 & 3884 & 5.4348 & 1.8142 & .33382 & .15157 & .14410 & .10639 \\
\hline 2617 & 6.3659 & 3.3506 & .52633 & .45666 & .24001 & .15352 & 3941 & 7.4810 & 3.9046 & .52193 & .35812 & .12390 & .09295 \\
\hline 2619 & 5.5882 & 2.8680 & .51321 & .41657 & .19264 & .14584 & 3963 & -- & -- & -- & -- & -- & -- \\
\hline 2621 & 4.8536 & 2.5638 & .52824 & .49007 & .25963 & .18300 & 4040 & 5.4720 & 2.6945 & .49241 & .43403 & .23983 & .16207 \\
\hline 2675 & 4.9116 & 2.5664 & .52251 & .45846 & .21247 & .14245 & 4058 & 4.0667 & 1.6943 & .41662 & .30481 & .10733 & .02303 \\
\hline 2676 & 3.5713 & 1.9031 & .53288 & .54893 & .24809 & .14372 & 4098 & 3.1874 & 1.5987 & .50158 & .53878 & .24170 & .13606 \\
\hline 2679 & 4.3174 & 2.3238 & .53824 & .50692 & .23513 & .15642 & 4100 & 4.8521 & 2.4386 & .50258 & .43555 & .20331 & .13592 \\
\hline 2715 & 5.5461 & 2.8085 & .50639 & .42888 & .20937 & .13530 & 4137 & 3.7373 & 1.9372 & .51835 & .50412 & .21487 & .13598 \\
\hline 2744 & 4.7500 & 2.5333 & .53332 & .49381 & .25196 & .17611 & 4191 & 4.8867 & 2.6351 & .53925 & .49153 & .24268 & .16848 \\
\hline 2758 & 6.1037 & 3.4695 & .56843 & .53618 & .32186 & .22432 & 4256 & -- & -- & -- & -- & -- & -- \\
\hline 2794 & 5.9091 & 3.7636 & .63692 & .61997 & .39614 & .33816 & 4257 & 4.8707 & 2.6309 & .54015 & .47628 & .21219 & .14865 \\
\hline 2797 & 4.2753 & 2.2462 & .52540 & .50296 & .25224 & .17278 & 4258 & 4.1176 & 2.2424 & .54458 & .50333 & .18253 & .08674 \\
\hline 2811 & 4.3388 & 2.3396 & .53922 & .51898 & .26069 & .17786 & 4278 & 5.8269 & 2.9419 & .50488 & .44208 & .23798 & .15787 \\
\hline 2813 & 4.0333 & 2.0402 & .50584 & .51638 & .32175 & .25954 & 4299 & 3.8741 & 1.9059 & .49197 & .46013 & .20491 & .12914 \\
\hline 2814 & 4.0526 & 2.5848 & .63781 & .68592 & .32060 & -.00439 & 4300 & 5.7042 & 3.0749 & .53906 & .46817 & .23230 & .15901 \\
\hline 2815 & 3.2580 & 1.6486 & .50602 & .53491 & .23520 & .13209 & 4305 & 5.5844 & 3.0474 & .54571 & .47780 & .23446 & .15973 \\
\hline 2818 & 5.4785 & 2.9531 & .53904 & .46915 & .21999 & .13946 & 4307 & 5.6052 & 3.1538 & .56266 & .47449 & .20289 & .14976 \\
\hline 2986 & 6.7081 & 3.4165 & .50931 & .37304 & .14050 & .09237 & 4309 & 5.5549 & 2.8710 & .51684 & .46116 & .24165 & .15517 \\
\hline 3005 & 4.8641 & 2.5966 & .53383 & .47070 & .21271 & .14815 & 4311 & 5.7529 & 2.9650 & .51540 & .44369 & .23029 & .16017 \\
\hline 3033 & 4.0600 & 1.8523 & .45624 & .42991 & .23133 & .15350 & 4313 & 5.8323 & 3.1041 & .53223 & .45658 & .23077 & .15541 \\
\hline 3034 & -- & -- & -- & -- & -- & -- & 4319 & 6.1574 & 2.8969 & .47048 & .38833 & .22662 & .15358 \\
\hline 3047 & 5.6944 & 2.8024 & .49213 & .39804 & .19750 & .15304 & 4329 & 5.8998 & 3.0987 & .52522 & .45014 & .22230 & .14156 \\
\hline 3103 & 3.6486 & 1.8709 & .51276 & .47122 & .12973 & .02370 & 4331 & -- & -- & -- & -- & -- & -- \\
\hline 3133 & 5.8438 & 2.9942 & .51238 & .41707 & .19766 & .14375 & 4375 & 3.5474 & 1.8619 & .52486 & .53389 & .23325 & .13801 \\
\hline 3156 & 5.2226 & 2.9800 & .57061 & .51793 & .24506 & .14874 & 4392 & 7.1646 & 3.6286 & .50646 & .39825 & .19753 & .13521 \\
\hline 3171 & 5.9478 & 3.0697 & .51610 & .44041 & .22788 & .15602 & 4425 & 3.5087 & 1.8879 & .53807 & .57570 & .28470 & .16719 \\
\hline 3189 & 4.0553 & 2.1650 & .53387 & .53202 & .27148 & .17860 & 4440 & 6.1104 & 3.0496 & .49909 & .43639 & .23324 & .14439 \\
\hline 3260 & 5.2128 & 2.6514 & .50863 & .40962 & .15807 & .09790 & 4476 & 4.4087 & 2.3269 & .52779 & .48393 & .22177 & .15437 \\
\hline 3267 & 5.8992 & 3.1458 & .53327 & .47698 & .25416 & .15017 & 4498 & 2.2857 & .74725 & .32692 & .25735 & .01404 & .05147 \\
\hline 3270 & 3.3337 & 1.7467 & .52395 & .56599 & .27300 & .16799 & 4517 & 5.7410 & 2.9164 & .50799 & .41799 & .20442 & .15116 \\
\hline 3272 & 2.4000 & 1.0700 & .44583 & .59529 & .32234 & .23309 & 4520 & 3.6509 & 1.9397 & .53129 & .53351 & .22971 & .13170 \\
\hline 3277 & 5.5000 & 3.6000 & .65455 & .62500 & .30632 & .17628 & 4525 & 6.7714 & 3.2840 & .48498 & .42161 & .19812 & .06646 \\
\hline 3278 & 4.0182 & 2.0771 & .51693 & .52249 & .29600 & .22531 & 4563 & 6.0645 & 3.1355 & .51702 & .43366 & .25196 & .22529 \\
\hline 3280 & 4.2807 & 2.1564 & .50374 & .50355 & .30681 & .23612 & 4570 & 4.5116 & 2.4424 & .54136 & .51232 & .25557 & .17420 \\
\hline 3281 & 3.1250 & 1.6046 & .51348 & .59308 & .32111 & .19519 & 4577 & 5.3135 & 2.8794 & .54190 & .46686 & .21406 & .15370 \\
\hline 3283 & 6.2380 & 3.3153 & .53148 & .44090 & .21259 & .14658 & 4591 & 6.6336 & 3.3457 & .50437 & .40869 & .19893 & .12482 \\
\hline 3284 & 4.7474 & 2.5335 & .53366 & .47614 & .21768 & .15325 & 4670 & 4.2456 & 2.2352 & .52647 & .49300 & .22871 & .15641 \\
\hline 3285 & 4.0011 & 2.1381 & .53438 & .50650 & .20803 & .12150 & 4671 & 4.3600 & 2.4382 & .55921 & .49727 & .15358 & .05813 \\
\hline 3329 & 5.4195 & 2.8541 & .52665 & .45208 & .21623 & .14732 & 4679 & 4.4532 & 2.3935 & .53749 & .48457 & .20254 & .13406 \\
\hline 3335 & 6.1336 & 3.1726 & .51725 & .40652 & .17418 & .11925 & 4696 & 3.9333 & 1.6190 & .41162 & .19638 & -.03394 & .08552 \\
\hline 3370 & 6.0539 & 3.0488 & .50362 & .42720 & .21629 & .13581 & 4703 & 4.6866 & 2.6748 & .57073 & .54781 & .27352 & .16700 \\
\hline 3410 & 4.3364 & 2.2690 & .52325 & .47995 & .21835 & .15308 & 4704 & 6.1945 & 3.3392 & .53905 & .44201 & .19536 & .12192 \\
\hline 3415 & 4.5414 & 2.4144 & .53165 & .47331 & .20035 & .13590 & 4731 & 5.7466 & 3.3262 & .57881 & .49998 & .21202 & .11741 \\
\hline 3430 & 5.3008 & 2.8521 & .53805 & .46817 & .22047 & .15273 & 4792 & 3.7703 & 1.9628 & .52059 & .50437 & .21588 & .14254 \\
\hline 3431 & 5.4665 & 2.9163 & .53349 & .38996 & .09526 & .08788 & 4819 & 4.5656 & 2.3762 & .52046 & .43959 & .15512 & .09365 \\
\hline 3441 & 5.1778 & 2.7646 & .53394 & .42614 & .13419 & .08240 & 4852 & 2.8235 & 1.6176 & .57292 & .79273 & .57922 & .42657 \\
\hline 3442 & 5.5337 & 2.5793 & .46611 & .38216 & .20006 & .15121 & 4866 & 5.6081 & 2.9979 & .53456 & .45654 & .21850 & .15333 \\
\hline 3446 & 4.5922 & 2.2879 & .49822 & .47420 & .27180 & .19709 & 4876 & 4.4774 & 2.3910 & .53400 & .48426 & .21432 & .14937 \\
\hline 3460 & 4.8298 & 2.2831 & .47271 & .39800 & .18436 & .10767 & 4878 & 6.1562 & 3.1596 & .51324 & .43035 & .21801 & .14550 \\
\hline 3462 & 5.4847 & 2.7399 & .49956 & .39548 & .16228 & .09280 & 4880 & 4.6603 & 2.4337 & .52221 & .47634 & .23645 & .16820 \\
\hline
\end{tabular}


Appendix 4-3.1. L-moments of storm duration defined by 6-hour minimum interevent time for hourly rainfall stations in TexasContinued.

\begin{tabular}{|c|c|c|c|c|c|c|c|c|c|c|c|c|c|}
\hline $\begin{array}{c}\text { Station } \\
\text { no. }\end{array}$ & $\begin{array}{c}\text { Duration } \\
\text { mean } \\
\text { (hours) }\end{array}$ & $\begin{array}{l}\text { Duration } \\
\text { L-scale } \\
\text { (hours) }\end{array}$ & $\begin{array}{c}\text { Duration } \\
\text { L-CV } \\
\text { (dimen- } \\
\text { sionless) }\end{array}$ & $\begin{array}{l}\text { Duration } \\
\text { L-skew } \\
\text { (dimen- } \\
\text { sionless) }\end{array}$ & $\begin{array}{l}\text { Duration } \\
\text { L-kurtosis } \\
\text { (dimen- } \\
\text { sionless) }\end{array}$ & $\begin{array}{l}\text { Duration } \\
\text { Tau5 } \\
\text { (dimen- } \\
\text { sionless) }\end{array}$ & $\begin{array}{c}\text { Station } \\
\text { no. }\end{array}$ & $\begin{array}{l}\text { Duration } \\
\text { mean } \\
\text { (hours) }\end{array}$ & $\begin{array}{l}\text { Duration } \\
\text { L-scale } \\
\text { (hours) }\end{array}$ & $\begin{array}{l}\text { Duration } \\
\text { L-CV } \\
\text { (dimen- } \\
\text { sionless) }\end{array}$ & $\begin{array}{l}\text { Duration } \\
\text { L-skew } \\
\text { (dimen- } \\
\text { sionless) }\end{array}$ & $\begin{array}{l}\text { Duration } \\
\text { L-kurtosis } \\
\text { (dimen- } \\
\text { sionless) }\end{array}$ & $\begin{array}{c}\text { Duration } \\
\text { Tau5 } \\
\text { (dimen- } \\
\text { sionless) }\end{array}$ \\
\hline 4920 & 4.4104 & 2.3637 & 0.53594 & 0.49818 & 0.23082 & 0.15580 & 5957 & 4.4271 & 2.3473 & 0.53021 & 0.49295 & 0.23817 & 0.17093 \\
\hline 4934 & 3.4444 & 1.9167 & .55645 & .71014 & .61698 & .60870 & 5958 & 5.2026 & 2.4892 & .47846 & .40490 & .19489 & .09931 \\
\hline 4972 & 4.9800 & 2.6213 & .52636 & .46062 & .21761 & .15715 & 5973 & 6.3440 & 3.3564 & .52906 & .48130 & .32025 & .27682 \\
\hline 4973 & 6.1735 & 2.9411 & .47641 & .36388 & .17095 & .11616 & 5996 & 5.2485 & 2.7941 & .53236 & .45415 & .20418 & .14265 \\
\hline 4974 & 4.6177 & 2.3180 & .50199 & .44689 & .22294 & .16932 & 6017 & 4.5364 & 2.2606 & .49832 & .42750 & .17239 & .09728 \\
\hline 4975 & 4.3209 & 2.2774 & .52708 & .47528 & .19658 & .13005 & 6024 & 6.7162 & 3.7102 & .55243 & .47659 & .25757 & .18144 \\
\hline 4978 & 4.3432 & 2.2902 & .52731 & .47967 & .21276 & .15733 & 6050 & 6.3478 & 2.6561 & .41843 & .20663 & .09290 & .09330 \\
\hline 4979 & 9.5833 & 4.6957 & .48998 & .37717 & .18450 & .06250 & 6104 & 3.7082 & 1.8791 & .50676 & .52264 & .28399 & .20541 \\
\hline 4982 & 5.4651 & 2.7558 & .50426 & .44558 & .23181 & .14927 & 6108 & 5.1126 & 2.7343 & .53482 & .45890 & .20255 & .14333 \\
\hline 5018 & 5.7355 & 2.7901 & .48646 & .43479 & .24081 & .15641 & 6136 & 3.3458 & 1.7171 & .51321 & .54413 & .25438 & .15414 \\
\hline 5048 & 3.8375 & 2.0698 & .53937 & .54785 & .26697 & .16405 & 6166 & 4.6792 & 2.3258 & .49704 & .45205 & .22787 & .14792 \\
\hline 5049 & 2.8257 & 1.3593 & .48106 & .52724 & .16987 & .02304 & 6176 & 6.4040 & 3.4073 & .53205 & .43576 & .19107 & .11125 \\
\hline 5056 & 6.2000 & 3.9000 & .62903 & .48718 & .10256 & .30769 & 6177 & 5.1429 & 2.7345 & .53170 & .46296 & .21603 & .15050 \\
\hline 5057 & 5.3157 & 3.0594 & .57553 & .54024 & .28663 & .19008 & 6210 & 4.7749 & 2.5126 & .52621 & .46068 & .20482 & .14410 \\
\hline 5060 & 6.0183 & 3.4258 & .56923 & .55469 & .34926 & .25740 & 6211 & 4.8564 & 2.7252 & .56117 & .51304 & .23779 & .15749 \\
\hline 5081 & 6.8652 & 3.4246 & .49884 & .38874 & .17969 & .11498 & 6270 & 4.5701 & 2.4596 & .53820 & .48063 & .20249 & .13606 \\
\hline 5094 & 4.5654 & 2.4108 & .52806 & .47219 & .20582 & .13568 & 6275 & -- & -- & -- & -- & -- & -- \\
\hline 5113 & 4.7883 & 2.6051 & .54406 & .49672 & .23646 & .15946 & 6276 & 7.1034 & 3.0640 & .43135 & .29528 & .13238 & .03390 \\
\hline 5114 & -- & -- & -- & -- & -- & -- & 6335 & 6.0572 & 3.0448 & .50267 & .41675 & .20602 & .13020 \\
\hline 5123 & 4.6471 & 2.4926 & .53639 & .58348 & .44416 & .35444 & 6434 & 6.6087 & 3.0949 & .46830 & .34866 & .20909 & .14895 \\
\hline 5192 & 5.3452 & 2.7949 & .52289 & .44548 & .20695 & .14059 & 6504 & 3.9011 & 2.0862 & .53478 & .53150 & .25261 & .16161 \\
\hline 5193 & 5.2677 & 2.8047 & .53243 & .46173 & .21689 & .14740 & 6558 & 6.0882 & 2.9652 & .48704 & .34919 & .14094 & .11756 \\
\hline 5224 & 5.4627 & 2.7980 & .51220 & .40943 & .17621 & .13499 & 6615 & 3.3001 & 1.7240 & .52239 & .57035 & .28079 & .17310 \\
\hline 5228 & 5.2821 & 2.9175 & .55234 & .48534 & .22702 & .16114 & 6660 & 6.2803 & 3.2698 & .52064 & .43307 & .23744 & .19321 \\
\hline 5235 & 6.8857 & 3.6605 & .53161 & .39603 & .12519 & .05303 & 6663 & 3.6471 & 2.0014 & .54878 & .57386 & .26882 & .13798 \\
\hline 5247 & 4.1467 & 2.1680 & .52282 & .50140 & .24884 & .17977 & 6734 & 5.2243 & 2.6835 & .51366 & .43498 & .18925 & .11111 \\
\hline 5258 & 6.2050 & 3.1284 & .50418 & .42327 & .21985 & .14903 & 6736 & 3.9300 & 2.1119 & .53738 & .54326 & .27883 & .19285 \\
\hline 5303 & 5.6075 & 2.7955 & .49852 & .44670 & .23710 & .14434 & 6740 & 8.3810 & 3.7667 & .44943 & .28259 & .14732 & .10687 \\
\hline 5312 & 3.9087 & 2.1000 & .53726 & .53735 & .26285 & .17633 & 6750 & 5.4329 & 3.2093 & .59071 & .52320 & .23410 & .16989 \\
\hline 5341 & 6.6000 & 3.4414 & .52142 & .47781 & .26127 & .09842 & 6757 & 5.5980 & 2.9682 & .53023 & .44878 & .20837 & .14016 \\
\hline 5342 & -- & -- & -- & -- & -- & -- & 6775 & 5.4977 & 2.9211 & .53134 & .47199 & .24693 & .17124 \\
\hline 5348 & 4.7127 & 2.5095 & .53249 & .46337 & .18747 & .11784 & 6776 & 4.1680 & 2.2021 & .52833 & .50678 & .24740 & .17264 \\
\hline 5358 & 4.8238 & 2.3401 & .48512 & .42708 & .22729 & .16822 & 6788 & 5.4208 & 2.4464 & .45130 & .34682 & .16162 & .09334 \\
\hline 5398 & 6.2724 & 3.2215 & .51360 & .42706 & .20995 & .13240 & 6792 & 3.1115 & 1.5447 & .49644 & .55763 & .28938 & .19299 \\
\hline 5410 & 4.0205 & 2.1314 & .53013 & .52508 & .26637 & .18367 & 6794 & 8.8333 & 4.2885 & .48549 & .38362 & .24587 & .15249 \\
\hline 5411 & 5.0318 & 2.6641 & .52947 & .47431 & .23694 & .16734 & 6834 & 4.4294 & 2.3503 & .53061 & .47504 & .19535 & .12617 \\
\hline 5424 & 6.4604 & 3.7915 & .58688 & .48819 & .21851 & .16877 & 6893 & 3.3267 & 1.7389 & .52269 & .57418 & .29505 & .18882 \\
\hline 5429 & 5.1894 & 2.7292 & .52592 & .47188 & .24340 & .16592 & 6935 & 3.4034 & 1.7336 & .50936 & .53523 & .25888 & .17140 \\
\hline 5431 & 10.667 & 4.4248 & .41483 & .17725 & .13331 & .08752 & 6981 & 5.5787 & 2.8536 & .51151 & .46323 & .25284 & .15384 \\
\hline 5461 & 6.5848 & 3.3878 & .51448 & .41924 & .20381 & .13394 & 7020 & 7.1069 & 3.7565 & .52857 & .41617 & .18165 & .10407 \\
\hline 5463 & 4.1447 & 2.2191 & .53540 & .50280 & .21400 & .12981 & 7060 & 3.8307 & 2.0423 & .53313 & .53994 & .26678 & .17246 \\
\hline 5471 & 1.8214 & .73942 & .40595 & .81120 & .59868 & .41800 & 7066 & 5.6549 & 2.9548 & .52252 & .43517 & .20410 & .14367 \\
\hline 5477 & 5.2692 & 3.0508 & .57898 & .51160 & .18651 & .01701 & 7074 & 3.8261 & 1.9886 & .51974 & .51546 & .24928 & .16935 \\
\hline 5528 & 5.6077 & 2.8236 & .50353 & .42539 & .20123 & .12324 & 7097 & 5.3511 & 2.6661 & .49823 & .40493 & .17186 & .10785 \\
\hline 5579 & -- & -- & -- & -- & -- & -- & 7116 & 5.1753 & 2.5984 & .50208 & .44431 & .23487 & .16620 \\
\hline 5580 & 4.9310 & 2.0640 & .41858 & .37311 & .18895 & .03944 & 7140 & 5.1988 & 2.7661 & .53206 & .47491 & .23349 & .15241 \\
\hline 5589 & 3.3704 & 1.6009 & .47498 & .45574 & .17039 & .09909 & 7173 & 5.6429 & 3.1316 & .55497 & .48598 & .22819 & .14221 \\
\hline 5590 & 5.0567 & 2.8062 & .55495 & .54876 & .32370 & .21263 & 7174 & 5.6600 & 3.0886 & .54569 & .47297 & .22882 & .15846 \\
\hline 5591 & 3.9198 & 1.8306 & .46703 & .46846 & .27268 & .18904 & 7213 & 5.8870 & 3.0944 & .52563 & .45276 & .22074 & .13595 \\
\hline 5592 & 3.9690 & 1.8331 & .46187 & .46580 & .27562 & .18510 & 7243 & 4.4776 & 2.4650 & .55052 & .52620 & .26310 & .18148 \\
\hline 5594 & 3.3441 & 1.5232 & .45550 & .49608 & .28656 & .17974 & 7262 & 3.0189 & 1.7247 & .57131 & .72151 & .43090 & .21332 \\
\hline 5595 & -- & -- & -- & -- & -- & -- & 7274 & 5.1040 & 2.5551 & .50061 & .40222 & .17466 & .14526 \\
\hline 5596 & 2.6586 & 1.2845 & .48314 & .60102 & .29781 & .16046 & 7300 & 5.2179 & 2.6166 & .50148 & .43296 & .21501 & .14164 \\
\hline 5600 & 4.6188 & 2.3410 & .50684 & .50702 & .30101 & .19694 & 7311 & 4.4688 & 2.1220 & .47485 & .32960 & .07275 & .10899 \\
\hline 5618 & 5.8108 & 2.3799 & .40956 & .30235 & .07647 & .01617 & 7363 & 6.3571 & 3.0159 & .47441 & .35061 & .12652 & .03542 \\
\hline 5650 & 4.7727 & 2.1017 & .44036 & .43254 & .22505 & .12445 & 7422 & 4.8137 & 2.6099 & .54218 & .49368 & .23535 & .15878 \\
\hline 5656 & 3.2652 & 1.7299 & .52980 & .58837 & .29298 & .17221 & 7431 & 4.5720 & 2.4496 & .53577 & .51425 & .27571 & .19279 \\
\hline 5658 & 5.7548 & 2.9470 & .51210 & .45510 & .25337 & .17957 & 7481 & 4.0974 & 2.1558 & .52613 & .51238 & .25106 & .16038 \\
\hline 5661 & 3.7984 & 2.1563 & .56768 & .59465 & .29001 & .15528 & 7497 & 4.0167 & 2.1786 & .54239 & .52518 & .23157 & .14076 \\
\hline 5666 & 5.5526 & 2.8997 & .52222 & .44166 & .21556 & .17521 & 7498 & 4.0965 & 2.2841 & .55758 & .55531 & .26987 & .16914 \\
\hline 5695 & 5.4555 & 2.8748 & .52696 & .43745 & .18877 & .12710 & 7499 & 4.2357 & 2.2421 & .52934 & .48310 & .19571 & .11888 \\
\hline 5742 & 4.9130 & 2.2332 & .45455 & .26321 & .05537 & .11137 & 7531 & 6.4121 & 3.4712 & .54136 & .44779 & .20174 & .12349 \\
\hline 5766 & 5.2432 & 2.4625 & .46964 & .33972 & .07898 & -.00101 & 7534 & 5.2044 & 2.8429 & .54624 & .49321 & .23965 & .15005 \\
\hline 5770 & 4.5704 & 2.4447 & .53490 & .49401 & .23461 & .15588 & 7556 & 4.5174 & 2.4027 & .53188 & .48124 & .21451 & .14610 \\
\hline 5775 & 5.2143 & 2.9176 & .55954 & .44821 & .11060 & -.00479 & 7594 & 5.7734 & 2.9661 & .51375 & .43877 & .22031 & .14622 \\
\hline 5779 & 6.1111 & 3.0513 & .49930 & .32751 & .12979 & .22218 & 7596 & 6.0621 & 3.3514 & .55284 & .51183 & .30305 & .21921 \\
\hline 5840 & 6.6014 & 3.3545 & .50815 & .43644 & .24139 & .15925 & 7608 & 5.2622 & 2.7965 & .53143 & .44978 & .19736 & .13713 \\
\hline 5890 & 4.8451 & 2.5799 & .53247 & .49649 & .26341 & .18498 & 7622 & 3.8261 & 2.1818 & .57025 & .61180 & .32428 & .15824 \\
\hline 5891 & 4.4420 & 2.2249 & .50088 & .47127 & .24224 & .15067 & 7700 & 6.5062 & 3.3098 & .50872 & .41755 & .21011 & .14037 \\
\hline 5897 & 3.9414 & 2.1008 & .53302 & .50894 & .20904 & .11862 & 7706 & 4.2287 & 2.2557 & .53343 & .50563 & .23411 & .15088 \\
\hline
\end{tabular}


Appendix 4-3.1. L-moments of storm duration defined by 6-hour minimum interevent time for hourly rainfall stations in TexasContinued.

\begin{tabular}{|c|c|c|c|c|c|c|c|c|c|c|c|c|c|}
\hline $\begin{array}{c}\text { Station } \\
\text { no. }\end{array}$ & $\begin{array}{l}\text { Duration } \\
\text { mean } \\
\text { (hours) }\end{array}$ & $\begin{array}{l}\text { Duration } \\
\text { L-scale } \\
\text { (hours) }\end{array}$ & $\begin{array}{l}\text { Duration } \\
\text { L-CV } \\
\text { (dimen- } \\
\text { sionless) }\end{array}$ & $\begin{array}{l}\text { Duration } \\
\text { L-skew } \\
\text { (dimen- } \\
\text { sionless) }\end{array}$ & $\begin{array}{c}\text { Duration } \\
\text { L-kurtosis } \\
\text { (dimen- } \\
\text { sionless) }\end{array}$ & $\begin{array}{c}\text { Duration } \\
\text { Tau5 } \\
\text { (dimen- } \\
\text { sionless) }\end{array}$ & $\begin{array}{c}\text { Station } \\
\text { no. }\end{array}$ & $\begin{array}{l}\text { Duration } \\
\text { mean } \\
\text { (hours) }\end{array}$ & $\begin{array}{l}\text { Duration } \\
\text { L-scale } \\
\text { (hours) }\end{array}$ & $\begin{array}{c}\text { Duration } \\
\text { L-CV } \\
\text { (dimen- } \\
\text { sionless) }\end{array}$ & $\begin{array}{l}\text { Duration } \\
\text { L-skew } \\
\text { (dimen- } \\
\text { sionless) }\end{array}$ & $\begin{array}{c}\text { Duration } \\
\text { L-kurtosis } \\
\text { (dimen- } \\
\text { sionless) }\end{array}$ & $\begin{array}{c}\text { Duration } \\
\text { Tau5 } \\
\text { (dimen- } \\
\text { sionless) }\end{array}$ \\
\hline 7718 & 5.9746 & 3.0696 & 0.51378 & 0.41418 & 0.20496 & 0.17032 & 9037 & 4.7178 & 2.4534 & 0.52003 & 0.49003 & 0.26133 & 0.17705 \\
\hline 7745 & 7.3208 & 3.8513 & .52608 & .42246 & .21938 & .16461 & 9106 & 4.7729 & 2.4827 & .52016 & .50907 & .31472 & .24133 \\
\hline 7922 & 3.9569 & 2.1161 & .53479 & .52001 & .22440 & .11559 & 9107 & 3.3250 & 1.8994 & .57124 & .67096 & .38475 & .21740 \\
\hline 7936 & 5.0292 & 2.6337 & .52369 & .45518 & .21186 & .14747 & 9129 & 4.8527 & 2.5993 & .53564 & .49780 & .26916 & .20003 \\
\hline 7943 & 4.9783 & 2.5804 & .51833 & .47194 & .24787 & .17430 & 9163 & 5.0057 & 2.6661 & .53262 & .47668 & .23010 & .15160 \\
\hline 7944 & 5.6444 & 3.0934 & .54804 & .45153 & .19071 & .15062 & 9213 & 5.4178 & 3.0752 & .56761 & .46964 & .17307 & .13378 \\
\hline 7945 & 5.8048 & 3.1758 & .54711 & .47307 & .23159 & .16054 & 9214 & 5.7429 & 2.5815 & .44952 & .41477 & .18964 & -.00372 \\
\hline 7947 & 4.6318 & 2.6052 & .56247 & .54266 & .28996 & .22091 & 8910 & 4.1739 & 1.4783 & .35417 & .28953 & .15852 & .07127 \\
\hline 7948 & 5.0527 & 2.7266 & .53962 & .48763 & .24266 & .16786 & 8911 & 4.7629 & 2.5400 & .53329 & .48809 & .24197 & .16640 \\
\hline 7951 & 6.1503 & 3.0586 & .49731 & .41638 & .20990 & .12727 & 8924 & 3.6310 & 1.7279 & .47588 & .49491 & .28012 & .19039 \\
\hline 7953 & 5.9007 & 3.2072 & .54353 & .50369 & .29655 & .21258 & 8929 & 5.7586 & 3.1700 & .55047 & .45852 & .18498 & .09462 \\
\hline 7981 & 5.3269 & 2.8984 & .54411 & .47128 & .20713 & .12360 & 8942 & 4.8802 & 2.6576 & .54455 & .47206 & .18922 & .11980 \\
\hline 7990 & 5.3974 & 2.9035 & .53794 & .46225 & .21735 & .15857 & 8944 & 5.4902 & 2.9480 & .53696 & .46232 & .22148 & .15744 \\
\hline 7992 & 5.4643 & 1.7976 & .32898 & .18560 & .10762 & .01848 & 8996 & 5.5662 & 2.8709 & .51578 & .46504 & .24944 & .15985 \\
\hline 7997 & 4.5954 & 2.1461 & .46701 & .39440 & .19055 & .13431 & 9014 & 7.2400 & 2.9367 & .40562 & .28530 & .23481 & .20264 \\
\hline 7999 & 4.4286 & 2.4429 & .55161 & .47122 & .12332 & .03555 & 9222 & 6.1132 & 3.2772 & .53609 & .42887 & .17637 & .11079 \\
\hline 8022 & 5.2051 & 2.8258 & .54290 & .51554 & .29940 & .21865 & 9248 & 5.9930 & 3.3662 & .56169 & .48156 & .20714 & .09496 \\
\hline 8023 & 4.1757 & 2.2495 & .53870 & .53899 & .28908 & .19793 & 9266 & 6.1047 & 3.0617 & .50154 & .43120 & .22203 & .13487 \\
\hline 8047 & 4.7217 & 2.5204 & .53378 & .48539 & .23699 & .17487 & 9270 & 3.2283 & 1.6144 & .50008 & .54038 & .26453 & .17658 \\
\hline 8060 & 5.5519 & 2.9977 & .53993 & .47264 & .22866 & .14887 & 9295 & 3.9828 & 2.2111 & .55517 & .52745 & .19593 & .10733 \\
\hline 8062 & 7.0638 & 3.9130 & .55395 & .48514 & .27511 & .21406 & 9304 & - & -- & -- & -- & -- & -- \\
\hline 8068 & 4.2683 & 2.3305 & .54600 & .51125 & .22987 & .16859 & 9307 & 5.4144 & 2.8092 & .51885 & .45235 & .23671 & .17362 \\
\hline 8081 & 5.0657 & 2.7607 & .54497 & .49488 & .25077 & .17950 & 9328 & 5.7475 & 3.3016 & .57444 & .54003 & .31095 & .22074 \\
\hline 8089 & 5.1087 & 2.4700 & .48350 & .36570 & .12646 & .05880 & 9329 & 8.0625 & 4.6792 & .58036 & .45223 & .19026 & .06659 \\
\hline 8221 & 8.6250 & 4.3822 & .50809 & .43688 & .29857 & .21568 & 9345 & -- & -- & -- & -- & -- & -- \\
\hline 8252 & 4.3156 & 2.3413 & .54252 & .53697 & .28865 & .19900 & 9363 & 4.9528 & 2.6741 & .53991 & .48230 & .22359 & .14702 \\
\hline 8265 & 6.7218 & 3.4943 & .51985 & .44637 & .23224 & .14285 & 9364 & 5.1420 & 2.8271 & .54981 & .50263 & .25331 & .16854 \\
\hline 8289 & 5.3016 & 2.8582 & .53912 & .46029 & .21430 & .16644 & 9365 & 4.4500 & 2.1218 & .47681 & .40223 & .19749 & .16984 \\
\hline 8305 & 3.3098 & 1.6502 & .49858 & .53031 & .25997 & .17074 & 9371 & 5.8721 & 3.0080 & .51226 & .40707 & .16591 & .09745 \\
\hline 8335 & 6.9036 & 3.5652 & .51643 & .41998 & .21124 & .14244 & 9417 & 5.7221 & 2.9534 & .51613 & .43760 & .21748 & .14977 \\
\hline 8400 & 3.5194 & 1.8919 & .53757 & .57764 & .29709 & .19286 & 9419 & 5.8845 & 3.1320 & .53224 & .45518 & .22566 & .15384 \\
\hline 8445 & 6.2314 & 3.2319 & .51865 & .44707 & .23411 & .15120 & 9435 & 5.3765 & 3.1154 & .57945 & .57187 & .36000 & .27453 \\
\hline 8446 & 4.5695 & 2.4287 & .53150 & .48124 & .21642 & .14188 & 9491 & 5.2785 & 2.7690 & .52458 & .44982 & .20724 & .13599 \\
\hline 8451 & 5.2219 & 2.5965 & .49723 & .42656 & .19912 & .11225 & 9499 & 3.9883 & 2.1310 & .53430 & .52376 & .24917 & .16646 \\
\hline 8531 & 6.0367 & 3.1704 & .52518 & .45534 & .23569 & .15471 & 9522 & 6.7895 & 2.8947 & .42636 & .34759 & .14528 & -.00386 \\
\hline 8541 & 5.4918 & 2.3638 & .43042 & .38859 & .23043 & .11645 & 9527 & 3.5108 & 1.8390 & .52380 & .54806 & .26526 & .17032 \\
\hline 8544 & 5.6878 & 3.0077 & .52881 & .45780 & .23430 & .16243 & 9532 & 4.6909 & 2.4856 & .52987 & .47680 & .22752 & .17048 \\
\hline 8545 & 6.9130 & 3.7273 & .53917 & .40282 & .16028 & .11600 & 9544 & -- & -- & -- & -- & -- & -- \\
\hline 8563 & 4.0829 & 2.1411 & .52441 & .48016 & .19202 & .13064 & 9565 & 4.3472 & 2.2524 & .51812 & .47573 & .22324 & .15979 \\
\hline 8566 & 4.1830 & 2.1655 & .51769 & .48825 & .23582 & .16578 & 9570 & 3.3672 & 1.8028 & .53539 & .58987 & .30382 & .19186 \\
\hline 8583 & 3.4594 & 1.8083 & .52274 & .53713 & .23125 & .13312 & 9574 & 2.5926 & 1.1425 & .44066 & .54294 & .31147 & .28035 \\
\hline 8584 & 4.5641 & 2.4088 & .52777 & .47930 & .22473 & .15961 & 9588 & 4.6761 & 2.5792 & .55157 & .51152 & .24115 & .15697 \\
\hline 8623 & 4.4684 & 2.3684 & .53003 & .48397 & .22320 & .15881 & 9665 & 5.2054 & 2.7448 & .52729 & .45728 & .21517 & .14662 \\
\hline 8625 & 6.2164 & 3.0359 & .48837 & .41110 & .21624 & .14592 & 9715 & 5.1296 & 2.6952 & .52542 & .45249 & .20452 & .13445 \\
\hline 8630 & 4.6911 & 2.4562 & .52359 & .47986 & .23975 & .16548 & 9729 & 5.6754 & 2.9603 & .52160 & .44411 & .21435 & .14094 \\
\hline 8631 & 5.3962 & 2.7872 & .51652 & .46663 & .25160 & .16845 & 9772 & 5.4265 & 2.8357 & .52257 & .47188 & .24760 & .15969 \\
\hline 8646 & 5.8877 & 2.9909 & .50800 & .42097 & .20471 & .13811 & 9814 & 6.7073 & 3.2902 & .49055 & .37258 & .12966 & .01567 \\
\hline 8647 & 3.8259 & 2.0416 & .53364 & .53179 & .24555 & .15182 & 9815 & 5.7618 & 3.0108 & .52254 & .45194 & .23471 & .16657 \\
\hline 8677 & 5.9188 & 2.6962 & .45553 & .35460 & .19458 & .13997 & 9816 & 5.0723 & 2.6873 & .52981 & .48044 & .24768 & .16448 \\
\hline 8696 & 4.6000 & 1.9241 & .41829 & .25960 & .06611 & .05518 & 9817 & 4.2128 & 2.2175 & .52637 & .49152 & .22402 & .15518 \\
\hline 8743 & 5.8476 & 3.0475 & .52114 & .43325 & .20970 & .14837 & 9829 & 4.0540 & 2.2011 & .54293 & .54729 & .28581 & .19185 \\
\hline 8761 & 3.7263 & 1.9652 & .52739 & .54117 & .27430 & .18819 & 9830 & 3.8800 & 1.9524 & .50319 & .46221 & .18431 & .12271 \\
\hline 8778 & 4.8693 & 2.6424 & .54268 & .47723 & .20457 & .13361 & 9858 & 5.2774 & 2.6624 & .50448 & .45013 & .24198 & .16463 \\
\hline 8845 & 4.9601 & 2.7075 & .54586 & .48498 & .22082 & .15169 & 9893 & 5.0338 & 2.6594 & .52830 & .46706 & .23162 & .17470 \\
\hline 8859 & 6.5746 & 3.3822 & .51443 & .41502 & .20143 & .14071 & 9916 & 5.3783 & 2.8324 & .52663 & .42520 & .16606 & .11380 \\
\hline 8898 & 6.6844 & 3.2612 & .48789 & .39566 & .19975 & .12095 & 9976 & 4.7576 & 2.6578 & .55864 & .53504 & .28410 & .19798 \\
\hline 8908 & 5.3478 & 2.4251 & .45348 & .36530 & .20046 & .13462 & & & & & & & \\
\hline
\end{tabular}


Appendix 4-3.2. L-moments of storm duration defined by 8-hour minimum interevent time for hourly rainfall stations in Texas.

$[--$, not available $]$

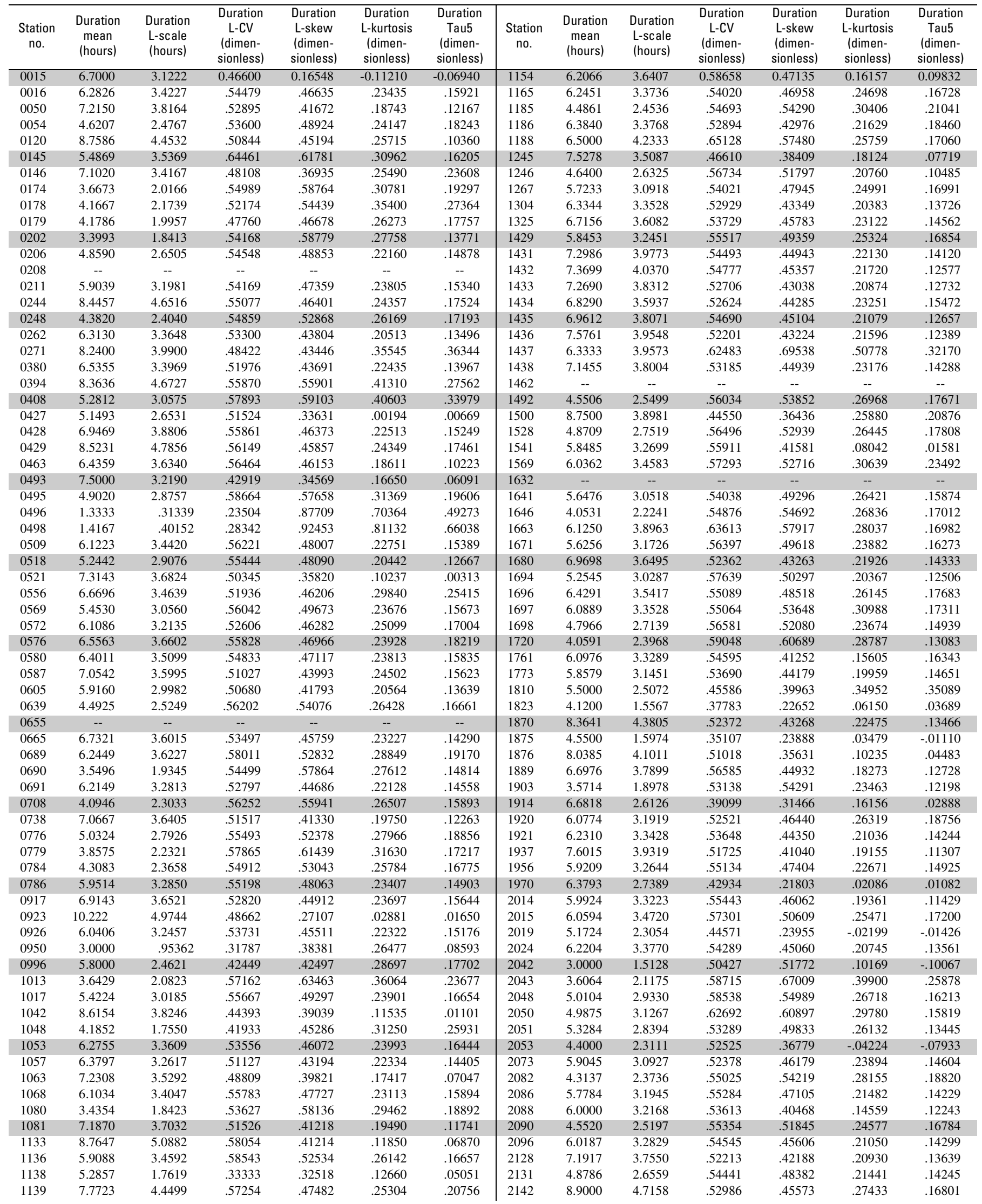


220 Statistical Characteristics of Storm Interevent Time, Depth, and Duration for Eastern New Mexico, Oklahoma, and Texas

Appendix 4-3.2. L-moments of storm duration defined by 8-hour minimum interevent time for hourly rainfall stations in TexasContinued.

\begin{tabular}{|c|c|c|c|c|c|c|c|c|c|c|c|c|c|}
\hline $\begin{array}{c}\text { Station } \\
\text { no. }\end{array}$ & $\begin{array}{l}\text { Duration } \\
\text { mean } \\
\text { (hours) }\end{array}$ & $\begin{array}{l}\text { Duration } \\
\text { L-scale } \\
\text { (hours) }\end{array}$ & $\begin{array}{l}\text { Duration } \\
\text { L-CV } \\
\text { (dimen- } \\
\text { sionless) }\end{array}$ & $\begin{array}{l}\text { Duration } \\
\text { L-skew } \\
\text { (dimen- } \\
\text { sionless) }\end{array}$ & $\begin{array}{l}\text { Duration } \\
\text { L-kurtosis } \\
\text { (dimen- } \\
\text { sionless) }\end{array}$ & $\begin{array}{c}\text { Duration } \\
\text { Tau5 } \\
\text { (dimen- } \\
\text { sionless) }\end{array}$ & $\begin{array}{c}\text { Station } \\
\text { no. }\end{array}$ & $\begin{array}{l}\text { Duration } \\
\text { mean } \\
\text { (hours) }\end{array}$ & $\begin{array}{l}\text { Duration } \\
\text { L-scale } \\
\text { (hours) }\end{array}$ & $\begin{array}{c}\text { Duration } \\
\text { L-CV } \\
\text { (dimen- } \\
\text { sionless) }\end{array}$ & $\begin{array}{l}\text { Duration } \\
\text { L-skew } \\
\text { (dimen- } \\
\text { sionless) }\end{array}$ & $\begin{array}{l}\text { Duration } \\
\text { L-kurtosis } \\
\text { (dimen- } \\
\text { sionless) }\end{array}$ & $\begin{array}{c}\text { Duration } \\
\text { Tau5 } \\
\text { (dimen- } \\
\text { sionless) }\end{array}$ \\
\hline 2160 & 8.9286 & 5.1190 & 0.57333 & 0.38032 & 0.08422 & 0.05073 & 3463 & 7.8776 & 4.1276 & 0.52396 & 0.37185 & 0.12905 & 0.06188 \\
\hline 2206 & 6.2066 & 3.2668 & .52634 & .46103 & .23857 & .14653 & 3476 & 6.2168 & 3.2156 & .51725 & .42886 & .20899 & .13900 \\
\hline 2238 & 5.6484 & 3.2583 & .57687 & .52524 & .27730 & .20050 & 3485 & 5.6400 & 2.1967 & .38948 & .24761 & .15428 & .19748 \\
\hline 2240 & 4.2308 & 2.3447 & .55419 & .48956 & .13002 & .02404 & 3507 & 4.9346 & 2.8394 & .57540 & .52531 & .23099 & .13603 \\
\hline 2242 & 6.6787 & 3.5710 & .53469 & .43779 & .20591 & .13026 & 3546 & 6.2991 & 3.4891 & .55390 & .45783 & .20548 & .13625 \\
\hline 2244 & 6.1925 & 3.4573 & .55830 & .46762 & .21224 & .13930 & 3547 & 5.8584 & 3.2798 & .55983 & .49384 & .25544 & .18372 \\
\hline 2247 & 5.6613 & 2.4223 & .42786 & .31217 & .19142 & .15602 & 3579 & 8.3291 & 4.6777 & .56161 & .48340 & .27065 & .18269 \\
\hline 2309 & 7.1678 & 3.7205 & .51905 & .41000 & .19102 & .12187 & 3642 & 6.1971 & 3.2977 & .53213 & .42780 & .18785 & .12592 \\
\hline 2312 & 5.2547 & 2.8037 & .53357 & .43307 & .15441 & .09364 & 3646 & 7.0296 & 3.5506 & .50509 & .41312 & .21110 & .13812 \\
\hline 2334 & 6.4154 & 3.4346 & .53537 & .39278 & .11724 & .05922 & 3668 & 9.5417 & 4.2917 & .44978 & .32307 & .11667 & .02575 \\
\hline 2336 & 5.4703 & 2.8816 & .52677 & .44538 & .20045 & .12908 & 3673 & 8.0968 & 4.4237 & .54635 & .52670 & .37310 & .26917 \\
\hline 2354 & 5.2903 & 2.9785 & .56301 & .51171 & .22845 & .07762 & 3686 & 4.8341 & 2.6411 & .54635 & .47193 & .18319 & .11872 \\
\hline 2355 & 7.6102 & 4.1251 & .54205 & .45137 & .19377 & .07205 & 3691 & 5.1537 & 2.8124 & .54571 & .47458 & .20981 & .14062 \\
\hline 2357 & 6.2194 & 3.6211 & .58223 & .51586 & .25331 & .15058 & 3734 & 6.1515 & 3.0398 & .49415 & .38213 & .17337 & .11480 \\
\hline 2360 & 5.5256 & 3.0883 & .55891 & .50790 & .26371 & .17840 & 3771 & 4.5955 & 2.5990 & .56555 & .52021 & .21597 & .11933 \\
\hline 2361 & 4.2709 & 2.2666 & .53070 & .52171 & .28350 & .20645 & 3789 & 4.0796 & 2.4093 & .59056 & .60031 & .27783 & .13723 \\
\hline 2394 & 6.7374 & 3.5304 & .52400 & .42177 & .19990 & .13183 & 3826 & 6.3707 & 3.1062 & .48758 & .37125 & .14634 & .08477 \\
\hline 2404 & 6.0936 & 3.2687 & .53641 & .44511 & .20590 & .13348 & 3831 & 6.2683 & 3.3084 & .52779 & .44708 & .22600 & .15453 \\
\hline 2415 & 7.0333 & 3.6413 & .51773 & .41208 & .19877 & .13346 & 3841 & 5.0000 & 2.5546 & .51092 & .45410 & .22580 & .14751 \\
\hline 2462 & 6.2695 & 3.3201 & .52957 & .42424 & .18516 & .12122 & 3871 & 6.5736 & 3.4017 & .51749 & .43368 & .21511 & .12929 \\
\hline 2528 & 7.1280 & 4.0138 & .56310 & .48943 & .28776 & .23049 & 3884 & 5.4348 & 1.8142 & .33382 & .15157 & .14410 & .10639 \\
\hline 2617 & 6.9545 & 3.6647 & .52695 & .43821 & .21732 & .13670 & 3941 & 8.4054 & 4.5268 & .53856 & .37610 & .13903 & .10358 \\
\hline 2619 & 6.2397 & 3.4117 & .54677 & .47269 & .26219 & .21142 & 3963 & -- & -- & -- & -- & -- & -- \\
\hline 2621 & 5.3883 & 2.9382 & .54530 & .49240 & .25106 & .16387 & 4040 & 6.2017 & 3.1994 & .51589 & .44350 & .23229 & .14929 \\
\hline 2675 & 5.6005 & 3.0719 & .54850 & .48015 & .23123 & .14834 & 4058 & 4.0667 & 1.6943 & .41662 & .30481 & .10733 & .02303 \\
\hline 2676 & 4.2774 & 2.3931 & .55949 & .53411 & .23231 & .12902 & 4098 & 3.7453 & 2.0009 & .53423 & .53901 & .24542 & .13842 \\
\hline 2679 & 4.9387 & 2.7924 & .56542 & .52320 & .25323 & .16650 & 4100 & 5.7035 & 2.9871 & .52374 & .44793 & .21839 & .14382 \\
\hline 2715 & 6.1920 & 3.2549 & .52567 & .44420 & .21690 & .13067 & 4137 & 4.2805 & 2.3107 & .53981 & .49907 & .20707 & .12212 \\
\hline 2744 & 5.3021 & 2.9230 & .55129 & .50126 & .25716 & .17390 & 4191 & 5.5500 & 3.1187 & .56193 & .50259 & .24900 & .16476 \\
\hline 2758 & 7.3089 & 4.2168 & .57694 & .50656 & .26351 & .15218 & 4256 & -- & -- & -- & -- & -- & -- \\
\hline 2794 & 5.9091 & 3.7636 & .63692 & .61997 & .39614 & .33816 & 4257 & 5.5918 & 3.1404 & .56161 & .48357 & .21543 & .14102 \\
\hline 2797 & 4.7618 & 2.5915 & .54423 & .50862 & .25456 & .16695 & 4258 & 4.9951 & 2.8467 & .56990 & .50211 & .19922 & .11873 \\
\hline 2811 & 4.9725 & 2.7988 & .56286 & .52751 & .26882 & .17739 & 4278 & 6.4105 & 3.3613 & .52433 & .46337 & .25738 & .17291 \\
\hline 2813 & 4.0333 & 2.0402 & .50584 & .51638 & .32175 & .25954 & 4299 & 4.3566 & 2.2955 & .52691 & .49959 & .24638 & .15664 \\
\hline 2814 & 5.2353 & 3.5956 & .68680 & .69325 & .33947 & .02548 & 4300 & 6.5673 & 3.6794 & .56027 & .47861 & .23601 & .15247 \\
\hline 2815 & 3.9773 & 2.1444 & .53916 & .51665 & .20922 & .10301 & 4305 & 6.4553 & 3.6023 & .55803 & .46790 & .21857 & .14137 \\
\hline 2818 & 6.1465 & 3.4062 & .55417 & .46253 & .19847 & .11153 & 4307 & 6.8906 & 4.0811 & .59226 & .49720 & .23424 & .16798 \\
\hline 2986 & 8.0616 & 4.3128 & .53497 & .41041 & .18844 & .13294 & 4309 & 6.1790 & 3.3206 & .53740 & .47478 & .24578 & .15055 \\
\hline 3005 & 5.5350 & 3.0513 & .55127 & .47397 & .21605 & .14797 & 4311 & 6.4303 & 3.4388 & .53478 & .45840 & .23778 & .15734 \\
\hline 3033 & 4.5030 & 2.1942 & .48729 & .46342 & .25113 & .15349 & 4313 & 6.4800 & 3.4994 & .54003 & .45203 & .22524 & .15002 \\
\hline 3034 & -- & -- & -- & -- & -- & -- & 4319 & 6.5048 & 3.0984 & .47632 & .39153 & .21680 & .13144 \\
\hline 3047 & 6.0571 & 3.0538 & .50416 & .43713 & .25946 & .21616 & 4329 & 6.5504 & 3.5670 & .54455 & .46444 & .23089 & .14464 \\
\hline 3103 & 4.5882 & 2.3761 & .51787 & .38006 & .02457 & -.03852 & 4331 & -- & -- & -- & -- & -- & -- \\
\hline 3133 & 6.6264 & 3.5066 & .52919 & .42599 & .20058 & .13745 & 4375 & 4.1951 & 2.3589 & .56230 & .55393 & .26588 & .16681 \\
\hline 3156 & 5.9621 & 3.3863 & .56797 & .47916 & .20631 & .12728 & 4392 & 8.6752 & 4.5642 & .52613 & .41446 & .20898 & .13934 \\
\hline 3171 & 6.6900 & 3.5436 & .52969 & .43953 & .21656 & .13846 & 4425 & 4.1069 & 2.3642 & .57566 & .59451 & .31359 & .19373 \\
\hline 3189 & 4.6270 & 2.5640 & .55414 & .53563 & .28108 & .18981 & 4440 & 6.9696 & 3.5949 & .51580 & .44082 & .22646 & .13408 \\
\hline 3260 & 5.4918 & 2.8352 & .51626 & .41313 & .16152 & .09938 & 4476 & 5.1648 & 2.8349 & .54889 & .48429 & .22265 & .14855 \\
\hline 3267 & 6.6786 & 3.7761 & .56540 & .52389 & .31945 & .22662 & 4498 & 2.9231 & 1.2436 & .42544 & .45829 & .28116 & .26367 \\
\hline 3270 & 3.8474 & 2.1326 & .55428 & .56961 & .27970 & .17099 & 4517 & 6.4517 & 3.4235 & .53063 & .44133 & .22286 & .15811 \\
\hline 3272 & 2.7917 & 1.3786 & .49384 & .59646 & .29706 & .15016 & 4520 & 4.3268 & 2.4220 & .55977 & .53138 & .23258 & .13634 \\
\hline 3277 & 5.5000 & 3.6000 & .65455 & 62500 & .30632 & .17628 & 4525 & 6.7714 & 3.2840 & .48498 & .42161 & .19812 & .06646 \\
\hline 3278 & 4.4156 & 2.3727 & .53735 & .53911 & .31840 & .24278 & 4563 & 6.5000 & 3.7207 & .57241 & .56123 & .42873 & .42377 \\
\hline 3280 & 4.6024 & 2.4022 & .52194 & .51663 & .30649 & .21368 & 4570 & 5.2295 & 2.9639 & .56677 & .52071 & .26023 & .17003 \\
\hline 3281 & 3.5217 & 1.9758 & .56104 & .63514 & .36601 & .22564 & 4577 & 5.9828 & 3.3179 & .55457 & .46830 & .21821 & .15470 \\
\hline 3283 & 7.2653 & 3.9944 & .54979 & .45220 & .22292 & .15010 & 4591 & 7.4523 & 3.8467 & .51617 & .41030 & .19421 & .11790 \\
\hline 3284 & 5.5159 & 3.0223 & .54793 & .46848 & .21169 & .14530 & 4670 & 4.8539 & 2.6815 & .55245 & .50696 & .24310 & .16289 \\
\hline 3285 & 4.6269 & 2.5549 & .55218 & .49612 & .20113 & .11914 & 4671 & 4.7278 & 2.6574 & .56209 & .48030 & .14779 & .07304 \\
\hline 3329 & 6.2966 & 3.4185 & .54292 & .45196 & .21165 & .13613 & 4679 & 5.1950 & 2.8950 & .55727 & .48230 & .20119 & .12828 \\
\hline 3335 & 6.9483 & 3.7229 & .53581 & .42558 & .19692 & .14103 & 4696 & 3.9333 & 1.6190 & .41162 & .19638 & -.03394 & .08552 \\
\hline 3370 & 6.7106 & 3.5114 & .52327 & .43830 & .21674 & .13259 & 4703 & 5.3889 & 3.0749 & .57059 & .50951 & .23553 & .14548 \\
\hline 3410 & 4.9602 & 2.7127 & .54688 & .49080 & .22887 & .15383 & 4704 & 7.3525 & 4.0152 & .54610 & .42530 & .17711 & .10508 \\
\hline 3415 & 5.1536 & 2.8257 & .54829 & .47314 & .20038 & .13089 & 4731 & 6.5620 & 3.7860 & .57695 & .45876 & .16128 & .08069 \\
\hline 3430 & 6.1951 & 3.4249 & .55284 & .46589 & .22073 & .15146 & 4792 & 4.4977 & 2.4801 & .55141 & .51167 & .23182 & .15765 \\
\hline 3431 & 6.2911 & 3.5691 & .56732 & .43973 & .15158 & .11199 & 4819 & 5.5460 & 2.9701 & .53554 & .42613 & .15416 & .10180 \\
\hline 3441 & 5.4318 & 2.8145 & .51815 & .39048 & .11890 & .09227 & 4852 & 2.8235 & 1.6176 & .57292 & .79273 & .57922 & .42657 \\
\hline 3442 & 6.0853 & 2.9724 & .48845 & .41104 & .22235 & .16392 & 4866 & 6.3843 & 3.5008 & .54835 & .45636 & .21500 & .14511 \\
\hline 3446 & 4.9556 & 2.5488 & .51432 & .48278 & .27395 & .19441 & 4876 & 5.1438 & 2.7903 & .54246 & .46839 & .21058 & .15590 \\
\hline 3460 & 5.5909 & 2.6797 & .47930 & .36376 & .15043 & .09520 & 4878 & 6.9545 & 3.7008 & .53214 & .44186 & .22119 & .14228 \\
\hline 3462 & 6.3553 & 3.3817 & .53210 & .42982 & .18658 & .10525 & 4880 & 5.2005 & 2.8136 & .54102 & .48465 & .24113 & .16546 \\
\hline
\end{tabular}


Appendix 4-3.2. L-moments of storm duration defined by 8-hour minimum interevent time for hourly rainfall stations in TexasContinued.

\begin{tabular}{|c|c|c|c|c|c|c|c|c|c|c|c|c|c|}
\hline $\begin{array}{c}\text { Station } \\
\text { no. }\end{array}$ & $\begin{array}{c}\text { Duration } \\
\text { mean } \\
\text { (hours) }\end{array}$ & $\begin{array}{l}\text { Duration } \\
\text { L-scale } \\
\text { (hours) }\end{array}$ & $\begin{array}{c}\text { Duration } \\
\text { L-CV } \\
\text { (dimen- } \\
\text { sionless) }\end{array}$ & $\begin{array}{l}\text { Duration } \\
\text { L-skew } \\
\text { (dimen- } \\
\text { sionless) }\end{array}$ & $\begin{array}{l}\text { Duration } \\
\text { L-kurtosis } \\
\text { (dimen- } \\
\text { sionless) }\end{array}$ & $\begin{array}{l}\text { Duration } \\
\text { Tau5 } \\
\text { (dimen- } \\
\text { sionless) }\end{array}$ & $\begin{array}{c}\text { Station } \\
\text { no. }\end{array}$ & $\begin{array}{c}\text { Duration } \\
\text { mean } \\
\text { (hours) }\end{array}$ & $\begin{array}{c}\text { Duration } \\
\text { L-scale } \\
\text { (hours) }\end{array}$ & $\begin{array}{l}\text { Duration } \\
\text { L-CV } \\
\text { (dimen- } \\
\text { sionless) }\end{array}$ & $\begin{array}{l}\text { Duration } \\
\text { L-skew } \\
\text { (dimen- } \\
\text { sionless) }\end{array}$ & $\begin{array}{l}\text { Duration } \\
\text { L-kurtosis } \\
\text { (dimen- } \\
\text { sionless) }\end{array}$ & $\begin{array}{c}\text { Duration } \\
\text { Tau5 } \\
\text { (dimen- } \\
\text { sionless) }\end{array}$ \\
\hline 4920 & 4.9443 & 2.7640 & 0.55902 & 0.51377 & 0.24880 & 0.16668 & 5957 & 4.9918 & 2.7575 & 0.55240 & 0.50481 & 0.25080 & 0.17659 \\
\hline 4934 & 4.6250 & 2.8393 & 61390 & .58491 & .18239 & -.20755 & 5958 & 5.8950 & 3.0899 & .52416 & .46865 & .25045 & .14752 \\
\hline 4972 & 5.6861 & 3.0605 & .53824 & .45451 & .21161 & .14767 & 5973 & 7.1186 & 3.8435 & .53993 & .47683 & .29810 & .24088 \\
\hline 4973 & 6.8724 & 3.3896 & .49322 & .37661 & .17039 & .10205 & 5996 & 5.9783 & 3.2633 & .54586 & .45202 & .20079 & .13561 \\
\hline 4974 & 5.3672 & 2.8340 & .52802 & .46148 & .23146 & .16530 & 6017 & 5.3857 & 2.7746 & .51518 & .41147 & .14701 & .07576 \\
\hline 4975 & 5.1825 & 2.8401 & .54802 & .47062 & .19695 & .12651 & 6024 & 7.4164 & 4.1285 & .55668 & .46358 & .23772 & .16184 \\
\hline 4978 & 5.1656 & 2.8580 & .55327 & .47120 & .18636 & .12012 & 6050 & 8.2500 & 2.7974 & .33907 & .03920 & .08657 & .05760 \\
\hline 4979 & 11.091 & 5.6234 & .50703 & .40554 & .25611 & .17016 & 6104 & 4.1435 & 2.2154 & .53467 & .54266 & .30336 & .21204 \\
\hline 4982 & 5.9751 & 3.1402 & .52555 & .46709 & .24642 & .15560 & 6108 & 5.9539 & 3.2880 & .55225 & .45812 & .20020 & .13662 \\
\hline 5018 & 6.5334 & 3.3439 & .51181 & .46294 & .26744 & .17860 & 6136 & 3.8022 & 2.0664 & .54347 & .55821 & .27631 & .17258 \\
\hline 5048 & 4.3817 & 2.4795 & .56586 & .55731 & .28089 & .17505 & 6166 & 5.5918 & 3.0065 & .53766 & .50136 & .28399 & .18953 \\
\hline 5049 & 3.3495 & 1.7070 & .50963 & .49487 & .13378 & .00533 & 6176 & 7.2929 & 3.9592 & .54289 & .42941 & .18177 & .10822 \\
\hline 5056 & 6.2000 & 3.9000 & .62903 & .48718 & .10256 & .30769 & 6177 & 5.8375 & 3.1860 & .54577 & .46284 & .21606 & .14769 \\
\hline 5057 & 6.2287 & 3.7444 & .60116 & .54934 & .28921 & .18138 & 6210 & 5.4320 & 2.9322 & .53979 & .45753 & .20217 & .13592 \\
\hline 5060 & 6.6198 & 3.8291 & .57843 & .54313 & .32447 & .22748 & 6211 & 5.4692 & 3.2054 & .58608 & .53143 & .25550 & .16302 \\
\hline 5081 & 7.9067 & 4.0640 & .51400 & .40232 & .19395 & .12602 & 6270 & 5.3220 & 2.9449 & .55335 & .47099 & .19502 & .12888 \\
\hline 5094 & 5.2221 & 2.8366 & .54318 & .46847 & .20501 & .13528 & 6275 & -- & -- & -- & -- & -- & -- \\
\hline 5113 & 5.4779 & 3.0959 & .56517 & .50647 & .24797 & .16427 & 6276 & 7.8571 & 3.5926 & .45724 & .36230 & .21813 & .12114 \\
\hline 5114 & -- & -- & -- & -- & -- & -- & 6335 & 6.7429 & 3.4694 & .51453 & .41918 & .20213 & .12280 \\
\hline 5123 & 5.3125 & 2.8042 & .52784 & .51772 & .33560 & .23815 & 6434 & 6.6087 & 3.0949 & .46830 & .34866 & .20909 & .14895 \\
\hline 5192 & 5.9351 & 3.1941 & .53818 & .44979 & .20482 & .13222 & 6504 & 4.4131 & 2.4595 & .55733 & .53544 & .25680 & .16111 \\
\hline 5193 & 5.9992 & 3.3006 & .55018 & .46894 & .22355 & .14986 & 6558 & 6.8750 & 3.8690 & .56276 & .49338 & .27498 & .19380 \\
\hline 5224 & 6.2545 & 3.3709 & .53896 & .44291 & .21252 & .15690 & 6615 & 3.9561 & 2.2204 & .56127 & .57599 & .28965 & .17738 \\
\hline 5228 & 5.8434 & 3.3512 & .57351 & .50437 & .24801 & .17689 & 6660 & 7.2195 & 3.9944 & .55328 & .45033 & .21958 & .15206 \\
\hline 5235 & 7.6667 & 4.0909 & .53360 & .34970 & .05827 & .01599 & 6663 & 4.1770 & 2.4083 & .57657 & .57762 & .27501 & .14769 \\
\hline 5247 & 4.8064 & 2.6286 & .54691 & .50700 & .25462 & .17843 & 6734 & 6.0000 & 3.2087 & .53478 & .44270 & .19298 & .11599 \\
\hline 5258 & 6.8235 & 3.5243 & .51650 & .42397 & .20917 & .13166 & 6736 & 4.4868 & 2.5280 & .56343 & .55169 & .28683 & .19160 \\
\hline 5303 & 6.2662 & 3.2837 & .52404 & .46899 & .24917 & .14775 & 6740 & 11.941 & 5.7868 & .48461 & .36722 & .24578 & .14759 \\
\hline 5312 & 4.5138 & 2.5419 & .56315 & .54212 & .27024 & .18175 & 6750 & 6.1281 & 3.7908 & .61860 & .55427 & .27278 & .19056 \\
\hline 5341 & 7.2759 & 4.1182 & .56601 & .54226 & .32575 & .15904 & 6757 & 6.3729 & 3.4726 & .54490 & .45180 & .21130 & .14208 \\
\hline 5342 & -- & -- & -- & -- & -- & -- & 6775 & 6.1821 & 3.3492 & .54175 & .46153 & .22328 & .13790 \\
\hline 5348 & 5.4772 & 3.0107 & .54969 & .46314 & .19306 & .12330 & 6776 & 4.7495 & 2.6005 & .54754 & .50584 & .24535 & .16606 \\
\hline 5358 & 5.5218 & 2.8444 & .51513 & .45217 & .23918 & .16397 & 6788 & 6.1099 & 2.8507 & .46657 & .35518 & .16428 & .10040 \\
\hline 5398 & 7.0288 & 3.7239 & .52981 & .43586 & .21265 & .13110 & 6792 & 3.4937 & 1.8257 & .52257 & .55844 & .28660 & .18200 \\
\hline 5410 & 4.5394 & 2.5208 & .55531 & .53772 & .27950 & .18884 & 6794 & 9.3793 & 4.5025 & .48004 & .38159 & .22712 & .10548 \\
\hline 5411 & 5.8417 & 3.2045 & .54856 & .47666 & .23516 & .15902 & 6834 & 5.1434 & 2.8063 & .54561 & .46460 & .18742 & .11933 \\
\hline 5424 & 7.9362 & 4.7937 & .60403 & .49329 & .23601 & .18255 & 6893 & 3.8122 & 2.1121 & .55405 & .57917 & .29622 & .18002 \\
\hline 5429 & 5.9781 & 3.3270 & .55652 & .49257 & .25244 & .16384 & 6935 & 3.9453 & 2.1489 & .54466 & .55501 & .28610 & .18947 \\
\hline 5431 & 10.667 & 4.4248 & .41483 & .17725 & .13331 & .08752 & 6981 & 6.0966 & 3.2340 & .53046 & .47497 & .25123 & .14379 \\
\hline 5461 & 7.4175 & 3.9401 & .53119 & .43262 & .21375 & .13877 & 7020 & 8.3288 & 4.4111 & .52962 & .40024 & .16913 & .10063 \\
\hline 5463 & 4.8464 & 2.6960 & .55629 & .49415 & .20097 & .11278 & 7060 & 4.2719 & 2.3344 & .54644 & .52563 & .24668 & .14975 \\
\hline 5471 & 2.1481 & 1.0427 & .48541 & .81683 & .58497 & .34389 & 7066 & 6.3339 & 3.4074 & .53796 & .44398 & .21081 & .14475 \\
\hline 5477 & 5.7200 & 3.6900 & .64510 & .65995 & .43448 & .30813 & 7074 & 4.3335 & 2.3521 & .54278 & .51999 & .25208 & .16381 \\
\hline 5528 & 6.2232 & 3.2234 & .51797 & .43105 & .20335 & .12420 & 7097 & 6.3306 & 3.4441 & .54404 & .47436 & .23875 & .14522 \\
\hline 5579 & -- & -- & -- & -- & -- & -- & 7116 & 5.7663 & 3.0142 & .52272 & .46047 & .24532 & .16811 \\
\hline 5580 & 4.9310 & 2.0640 & .41858 & .37311 & .18895 & .03944 & 7140 & 5.8659 & 3.2742 & .55817 & .49759 & .25257 & .16211 \\
\hline 5589 & 3.7901 & 1.9529 & .51526 & .51416 & .25045 & .16434 & 7173 & 6.4537 & 3.7297 & .57792 & .50368 & .24816 & .15866 \\
\hline 5590 & 5.6162 & 3.2331 & .57568 & .55160 & .30865 & .18410 & 7174 & 6.5032 & 3.6462 & .56068 & .47328 & .22808 & .15620 \\
\hline 5591 & 4.3694 & 2.1663 & .49578 & .49600 & .29313 & .19670 & 7213 & 6.4475 & 3.5332 & .54800 & .47422 & .23473 & .13849 \\
\hline 5592 & 4.3435 & 2.1371 & .49203 & .51019 & .32201 & .21993 & 7243 & 5.1103 & 2.8961 & .56672 & .52122 & .25795 & .17431 \\
\hline 5594 & 3.8249 & 1.9542 & .51091 & .56846 & .36433 & .24399 & 7262 & 3.1048 & 1.7965 & .57863 & .71950 & .42590 & .20435 \\
\hline 5595 & -- & -- & -- & -- & -- & -- & 7274 & 5.4052 & 2.7710 & .51265 & .41808 & .19428 & .16247 \\
\hline 5596 & 3.0759 & 1.6083 & .52287 & .60365 & .30334 & .16457 & 7300 & 5.6828 & 2.9496 & .51903 & .44526 & .21823 & .13671 \\
\hline 5600 & 4.9867 & 2.6585 & .53311 & .53934 & .33220 & .21776 & 7311 & 4.4688 & 2.1220 & .47485 & .32960 & .07275 & .10899 \\
\hline 5618 & 6.0000 & 2.4670 & .41116 & .27989 & .04431 & .00009 & 7363 & 6.3571 & 3.0159 & .47441 & .35061 & .12652 & .03542 \\
\hline 5650 & 6.2000 & 2.5632 & .41341 & .23956 & .09409 & .10118 & 7422 & 5.4613 & 3.0437 & .55731 & .49469 & .24095 & .16495 \\
\hline 5656 & 3.8728 & 2.1918 & .56595 & .58863 & .29314 & .16662 & 7431 & 5.1101 & 2.8498 & .55768 & .52686 & .28495 & .19176 \\
\hline 5658 & 6.4021 & 3.4181 & .53391 & .47041 & .25597 & .17080 & 7481 & 4.6008 & 2.5449 & .55314 & .53491 & .28060 & .18729 \\
\hline 5661 & 4.3833 & 2.6147 & .59651 & .60465 & .31448 & .19159 & 7497 & 4.8227 & 2.7413 & .56841 & .52285 & .23707 & .14931 \\
\hline 5666 & 6.2222 & 3.3571 & .53954 & .45393 & .21765 & .14264 & 7498 & 4.8962 & 2.8156 & .57505 & .52753 & .23335 & .13690 \\
\hline 5695 & 6.2068 & 3.3785 & .54433 & .44832 & .20262 & .13733 & 7499 & 4.8525 & 2.6760 & .55148 & .48918 & .20458 & .12383 \\
\hline 5742 & 6.0476 & 3.0619 & .50630 & .33601 & .09593 & .09148 & 7531 & 7.7067 & 4.2442 & .55072 & .45334 & .23006 & .16008 \\
\hline 5766 & 6.2647 & 3.0419 & .48556 & .36083 & .12383 & .06601 & 7534 & 5.6724 & 3.1889 & .56218 & .50828 & .25934 & .16562 \\
\hline 5770 & 5.2255 & 2.8873 & .55255 & .49348 & .23428 & .15421 & 7556 & 5.1375 & 2.8234 & .54958 & .48290 & .21659 & .14272 \\
\hline 5775 & 5.2143 & 2.9176 & .55954 & .44821 & .11060 & -.00479 & 7594 & 6.3980 & 3.4338 & .53670 & .46409 & .24152 & .15826 \\
\hline 5779 & 8.9091 & 5.0216 & .56365 & .44897 & .28870 & .29527 & 7596 & 6.2701 & 3.5090 & .55964 & .51382 & .29757 & .20812 \\
\hline 5840 & 7.2640 & 3.8299 & .52725 & .45647 & .25305 & .15991 & 7608 & 5.8098 & 3.1674 & .54518 & .45381 & .20002 & .13744 \\
\hline 5890 & 5.4628 & 3.0009 & .54934 & .49859 & .26099 & .17713 & 7622 & 4.2727 & 2.6494 & .62006 & .67843 & .42389 & .27004 \\
\hline 5891 & 5.1471 & 2.6501 & .51487 & .43878 & .18396 & .09753 & 7700 & 7.1714 & 3.7674 & .52534 & .43570 & .22520 & .14806 \\
\hline 5897 & 4.6338 & 2.5826 & .55734 & .50598 & .20862 & .11754 & 7706 & 4.8816 & 2.7241 & .55804 & .51336 & .24192 & .15087 \\
\hline
\end{tabular}


Appendix 4-3.2. L-moments of storm duration defined by 8-hour minimum interevent time for hourly rainfall stations in TexasContinued.

\begin{tabular}{|c|c|c|c|c|c|c|c|c|c|c|c|c|c|}
\hline $\begin{array}{c}\text { Station } \\
\text { no. }\end{array}$ & $\begin{array}{c}\text { Duration } \\
\text { mean } \\
\text { (hours) }\end{array}$ & $\begin{array}{l}\text { Duration } \\
\text { L-scale } \\
\text { (hours) }\end{array}$ & $\begin{array}{l}\text { Duration } \\
\text { L-CV } \\
\text { (dimen- } \\
\text { sionless) }\end{array}$ & $\begin{array}{l}\text { Duration } \\
\text { L-skew } \\
\text { (dimen- } \\
\text { sionless) }\end{array}$ & $\begin{array}{c}\text { Duration } \\
\text { L-kurtosis } \\
\text { (dimen- } \\
\text { sionless) }\end{array}$ & $\begin{array}{l}\text { Duration } \\
\text { Tau5 } \\
\text { (dimen- } \\
\text { sionless) }\end{array}$ & $\begin{array}{c}\text { Station } \\
\text { no. }\end{array}$ & $\begin{array}{c}\text { Duration } \\
\text { mean } \\
\text { (hours) }\end{array}$ & $\begin{array}{l}\text { Duration } \\
\text { L-scale } \\
\text { (hours) }\end{array}$ & $\begin{array}{c}\text { Duration } \\
\text { L-CV } \\
\text { (dimen- } \\
\text { sionless) }\end{array}$ & $\begin{array}{l}\text { Duration } \\
\text { L-skew } \\
\text { (dimen- } \\
\text { sionless) }\end{array}$ & $\begin{array}{c}\text { Duration } \\
\text { L-kurtosis } \\
\text { (dimen- } \\
\text { sionless) }\end{array}$ & $\begin{array}{c}\text { Duration } \\
\text { Tau5 } \\
\text { (dimen- } \\
\text { sionless) }\end{array}$ \\
\hline 7718 & 6.5221 & 3.4686 & 0.53181 & 0.44082 & 0.23763 & 0.19500 & 8910 & 4.6364 & 1.6450 & 0.35481 & 0.23842 & 0.11967 & 0.00166 \\
\hline 7745 & 8.5591 & 4.6737 & .54605 & .42850 & .20855 & .14111 & 8911 & 5.2781 & 2.9474 & .55841 & .50934 & .25905 & .17397 \\
\hline 7922 & 4.2360 & 2.3302 & .55009 & .53190 & .24404 & .13598 & 8924 & 3.8860 & 1.9204 & .49417 & .51179 & .29592 & .19798 \\
\hline 7936 & 5.6932 & 3.0937 & .54339 & .47164 & .23399 & .16555 & 8929 & 7.1538 & 3.9785 & .55613 & .43271 & .18743 & .12847 \\
\hline 7943 & 5.8113 & 3.1615 & .54403 & .48393 & .25255 & .16984 & 8942 & 5.5863 & 3.0808 & .55150 & .45496 & .17986 & .12032 \\
\hline 7944 & 6.5238 & 3.8069 & .58355 & .49834 & .24555 & .19514 & 8944 & 6.0688 & 3.3733 & .55585 & .47646 & .23352 & .16458 \\
\hline 7945 & 6.7265 & 3.7852 & .56273 & .47560 & .23273 & .15557 & 8996 & 6.2029 & 3.2890 & .53022 & .46692 & .24443 & .15312 \\
\hline 7947 & 5.6963 & 3.3828 & .59387 & .55129 & .30374 & .23275 & 9014 & 8.4783 & 3.6482 & .43030 & .32178 & .19161 & .09831 \\
\hline 7948 & 5.7391 & 3.1774 & .55363 & .48180 & .23196 & .15500 & 9037 & 5.3779 & 2.9509 & .54870 & .50585 & .26607 & .17302 \\
\hline 7951 & 6.7221 & 3.4128 & .50770 & .41672 & .20792 & .13144 & 9106 & 5.4147 & 2.9807 & .55048 & .53577 & .33557 & .24996 \\
\hline 7953 & 6.6541 & 3.7650 & .56581 & .51458 & .28326 & .17171 & 9107 & 3.3250 & 1.8994 & .57124 & .67096 & .38475 & .21740 \\
\hline 7981 & 5.7920 & 3.1926 & .55122 & .46298 & .19434 & .11137 & 9129 & 5.6033 & 3.0351 & .54167 & .47082 & .24022 & .17991 \\
\hline 7990 & 6.4825 & 3.7188 & .57367 & .50531 & .26957 & .19298 & 9163 & 5.5790 & 3.0441 & .54563 & .47571 & .22720 & .14664 \\
\hline 7992 & 5.4643 & 1.7976 & .32898 & .18560 & .10762 & .01848 & 9213 & 6.9225 & 4.0845 & .59003 & .47513 & .20456 & .17415 \\
\hline 7997 & 5.1360 & 2.4401 & .47510 & .39111 & .18660 & .11943 & 9214 & 6.6970 & 3.4072 & .50877 & .54243 & .37455 & .20050 \\
\hline 7999 & 5.5263 & 3.0175 & .54603 & .38235 & .02745 & -.03001 & 9222 & 7.2716 & 3.8565 & .53036 & .39187 & .15545 & .10992 \\
\hline 8022 & 5.8108 & 3.4247 & .58936 & .57614 & .36237 & .27350 & 9248 & 6.5474 & 3.7575 & .57389 & .48365 & .20400 & .09384 \\
\hline 8023 & 4.7283 & 2.6666 & .56397 & .54906 & .29463 & .19328 & 9266 & 6.5542 & 3.4331 & .52381 & .44710 & .21324 & .10258 \\
\hline 8047 & 5.3788 & 2.9689 & .55197 & .48984 & .24249 & .17539 & 9270 & 3.6667 & 1.9539 & .53288 & .55826 & .28778 & .19075 \\
\hline 8060 & 6.7643 & 3.8154 & .56405 & .47566 & .21974 & .13018 & 9295 & 4.0696 & 2.2456 & .55181 & .51166 & .18276 & .10885 \\
\hline 8062 & 7.6444 & 4.5303 & .59263 & .54725 & .34597 & .28004 & 9304 & -- & -- & -- & -- & -- & -- \\
\hline 8068 & 4.7949 & 2.7989 & .58373 & .54659 & .25300 & .15764 & 9307 & 6.1564 & 3.2633 & .53007 & .44266 & .21773 & .14972 \\
\hline 8081 & 5.7527 & 3.2604 & .56676 & .50891 & .26425 & .18336 & 9328 & 6.4973 & 3.8184 & .58768 & .53254 & .28940 & .18829 \\
\hline 8089 & 5.9302 & 2.9424 & .49617 & .33033 & .05991 & .00855 & 9329 & 8.0625 & 4.6792 & .58036 & .45223 & .19026 & .06659 \\
\hline 8221 & 10.714 & 5.2667 & .49156 & .38517 & .25780 & .18059 & 9345 & -- & -- & -- & -- & -- & -- \\
\hline 8252 & 4.7902 & 2.6796 & .55939 & .53788 & .28545 & .18917 & 9363 & 5.8474 & 3.3049 & .56520 & .48941 & .22615 & .14415 \\
\hline 8265 & 7.4594 & 3.9758 & .53298 & .44701 & .22197 & .12828 & 9364 & 5.9369 & 3.3714 & .56788 & .50509 & .25538 & .16800 \\
\hline 8289 & 7.0000 & 4.2700 & .61000 & .52793 & .25042 & .13429 & 9365 & 5.0263 & 2.2966 & .45691 & .33550 & .15850 & .13803 \\
\hline 8305 & 3.7334 & 1.9652 & .52638 & .54072 & .27346 & .17988 & 9371 & 6.8063 & 3.7466 & .55046 & .47388 & .24841 & .16993 \\
\hline 8335 & 7.6639 & 4.0715 & .53126 & .43119 & .22044 & .15041 & 9417 & 6.3694 & 3.3741 & .52974 & .43967 & .21365 & .14351 \\
\hline 8400 & 4.3264 & 2.5443 & .58809 & .60325 & .33021 & .21840 & 9419 & 6.8088 & 3.6818 & .54074 & .44313 & .21010 & .13758 \\
\hline 8445 & 6.9759 & 3.7249 & .53397 & .45526 & .24132 & .15990 & 9435 & 5.6747 & 3.4002 & .59919 & .58828 & .36169 & .25269 \\
\hline 8446 & 5.1178 & 2.8117 & .54939 & .48621 & .22004 & .14026 & 9491 & 5.9083 & 3.1851 & .53908 & .45415 & .20999 & .13473 \\
\hline 8451 & 6.0567 & 3.2178 & .53128 & .46665 & .24493 & .15611 & 9499 & 4.5722 & 2.5454 & .55671 & .52394 & .24806 & .16006 \\
\hline 8531 & 6.6267 & 3.5385 & .53398 & .44939 & .22203 & .14087 & 9522 & 8.3529 & 3.7794 & .45246 & .35486 & .16954 & .08291 \\
\hline 8541 & 6.6937 & 3.2729 & .48895 & .43689 & .23160 & .10026 & 9527 & 4.0451 & 2.2215 & .54918 & .54539 & .26355 & .16696 \\
\hline 8544 & 6.4618 & 3.5624 & .55130 & .47365 & .24281 & .16217 & 9532 & 5.2682 & 2.8782 & .54634 & .48209 & .23367 & .16906 \\
\hline 8545 & 7.5000 & 3.8939 & .51919 & .34786 & .12086 & .11502 & 9544 & -- & -- & -- & -- & -- & -- \\
\hline 8563 & 4.7375 & 2.5413 & .53643 & .46229 & .18425 & .13232 & 9565 & 4.9654 & 2.6725 & .53822 & .48327 & .23437 & .16469 \\
\hline 8566 & 4.7050 & 2.5738 & .54703 & .50970 & .24885 & .16011 & 9570 & 3.9200 & 2.1917 & .55911 & .57518 & .29102 & .18439 \\
\hline 8583 & 4.2289 & 2.3457 & .55469 & .52363 & .21563 & .11464 & 9574 & 3.6400 & 1.9000 & .52198 & .49657 & .15457 & .02140 \\
\hline 8584 & 5.3500 & 2.9546 & .55226 & .49246 & .24507 & .17570 & 9588 & 5.4739 & 3.1760 & .58022 & .52492 & .25242 & .15972 \\
\hline 8623 & 5.1860 & 2.8394 & .54752 & .47769 & .21451 & .14445 & 9665 & 5.9525 & 3.2701 & .54935 & .47254 & .22857 & .15099 \\
\hline 8625 & 7.0329 & 3.5575 & .50583 & .42431 & .22380 & .15060 & 9715 & 5.7555 & 3.0941 & .53759 & .44924 & .19956 & .12889 \\
\hline 8630 & 5.2363 & 2.8329 & .54101 & .48302 & .23688 & .15692 & 9729 & 6.4812 & 3.4826 & .53735 & .44869 & .21805 & .14337 \\
\hline 8631 & 6.0596 & 3.2439 & .53532 & .47753 & .26233 & .18003 & 9772 & 6.0393 & 3.2958 & .54571 & .49191 & .26428 & .17176 \\
\hline 8646 & 6.5376 & 3.4014 & .52028 & .42335 & .20160 & .13169 & 9814 & 7.7632 & 4.0946 & .52744 & .47780 & .28178 & .18822 \\
\hline 8647 & 4.3255 & 2.4022 & .55535 & .53297 & .24661 & .14772 & 9815 & 6.7414 & 3.6422 & .54027 & .45070 & .22381 & .15161 \\
\hline 8677 & 6.8852 & 3.1767 & .46137 & .34142 & .16279 & .09442 & 9816 & 5.5875 & 3.0239 & .54119 & .46302 & .20683 & .12101 \\
\hline 8696 & 4.9655 & 2.3621 & .47569 & .40609 & .22342 & .17852 & 9817 & 4.7196 & 2.5657 & .54363 & .49266 & .22510 & .15200 \\
\hline 8743 & 6.6530 & 3.5628 & .53552 & .43458 & .20414 & .13592 & 9829 & 4.5420 & 2.5590 & .56343 & .55184 & .29059 & .19193 \\
\hline 8761 & 4.1255 & 2.2365 & .54213 & .53190 & .26108 & .17283 & 9830 & 4.4909 & 2.3336 & .51963 & .46706 & .21522 & .15798 \\
\hline 8778 & 5.5474 & 3.0790 & .55504 & .47045 & .20075 & .13211 & 9858 & 5.7841 & 3.0374 & .52512 & .46715 & .25213 & .16834 \\
\hline 8845 & 5.6957 & 3.2332 & .56765 & .49362 & .22713 & .14907 & 9893 & 5.7176 & 3.1354 & .54838 & .47730 & .23949 & .17415 \\
\hline 8859 & 7.4069 & 3.8981 & .52628 & .42139 & .20823 & .14280 & 9916 & 6.1758 & 3.3445 & .54155 & .43104 & .17753 & .12075 \\
\hline 8898 & 7.3492 & 3.7297 & .50749 & .42127 & .22459 & .14310 & 9976 & 5.5530 & 3.2057 & .57730 & .53106 & .27632 & .18316 \\
\hline 8908 & 6.1628 & 3.0078 & .48805 & .41052 & .22120 & .11897 & & & & & & & \\
\hline
\end{tabular}


Appendix 4-3.3. L-moments of storm duration defined by 12-hour minimum interevent time for hourly rainfall stations in Texas.

$[--$, not available $]$

\begin{tabular}{|c|c|c|c|c|c|c|c|c|c|c|c|c|c|}
\hline $\begin{array}{c}\text { Station } \\
\text { n. }\end{array}$ & $\begin{array}{c}\text { Duration } \\
\text { mean } \\
\text { (hours) }\end{array}$ & $\begin{array}{l}\text { Duration } \\
\text { L-scale } \\
\text { (hours) }\end{array}$ & $\begin{array}{l}\text { Duration } \\
\text { L-CV } \\
\text { (dimen- } \\
\text { sionless) }\end{array}$ & $\begin{array}{l}\text { Duration } \\
\text { L-skew } \\
\text { (dimen- } \\
\text { sionless) }\end{array}$ & $\begin{array}{l}\text { Duration } \\
\text { L-kurtosis } \\
\text { (dimen- } \\
\text { sionless) }\end{array}$ & $\begin{array}{c}\text { Duration } \\
\text { Tau5 } \\
\text { (dimen- } \\
\text { sionless) }\end{array}$ & $\begin{array}{c}\text { Station } \\
\text { no. }\end{array}$ & $\begin{array}{c}\text { Duration } \\
\text { mean } \\
\text { (hours) }\end{array}$ & $\begin{array}{l}\text { Duration } \\
\text { L-scale } \\
\text { (hours) }\end{array}$ & $\begin{array}{l}\text { Duration } \\
\text { L-CV } \\
\text { (dimen- } \\
\text { sionless) }\end{array}$ & $\begin{array}{l}\text { Duration } \\
\text { L-skew } \\
\text { (dimen- } \\
\text { sionless) }\end{array}$ & $\begin{array}{l}\text { Duration } \\
\text { L-kurtosis } \\
\text { (dimen- } \\
\text { sionless) }\end{array}$ & $\begin{array}{l}\text { Duration } \\
\text { Tau5 } \\
\text { (dimen- } \\
\text { sionless) }\end{array}$ \\
\hline 0015 & 13.857 & 8.3333 & 0.60137 & 0.60686 & 0.63429 & 0.62286 & 1154 & 8.3535 & 5.1238 & 0.61337 & 0.47364 & 0.17159 & 0.10701 \\
\hline 0050 & 8.5492 & 4.6422 & .54300 & .41728 & .18387 & .11508 & 1185 & 5.0950 & 2.9414 & .57732 & .55946 & .30744 & .19388 \\
\hline 0054 & 5.9245 & 3.2612 & .55047 & .44376 & .17006 & .08606 & 1186 & 7.4957 & 4.3145 & .57559 & .50012 & .28830 & .22759 \\
\hline 0120 & 14.455 & 8.6926 & .60138 & .51051 & .25890 & .10077 & 1188 & 6.5000 & 4.2333 & .65128 & .57480 & .25759 & .17060 \\
\hline 0145 & 6.5810 & 4.3933 & .66758 & .62079 & .32248 & .18431 & 1245 & 13.667 & 7.5869 & .55514 & .49933 & .32242 & .23838 \\
\hline 0178 & 5.9091 & 3.4113 & .57729 & .53604 & .26109 & .07093 & 1304 & 7.8930 & 4.3749 & .55428 & .44427 & .20915 & .13191 \\
\hline 0179 & 4.8037 & 2.5573 & .53236 & .54658 & .34456 & .23539 & 1325 & 8.1968 & 4.6503 & .56733 & .46948 & .22216 & .12123 \\
\hline 0202 & 4.3271 & 2.5765 & .59542 & .60309 & .29967 & .15148 & 1429 & 7.0223 & 4.0335 & .57439 & .48726 & .23373 & .14298 \\
\hline 0206 & 6.1429 & 3.5111 & .57157 & .48084 & .20602 & .12281 & 1431 & 8.7622 & 5.0195 & .57285 & .47131 & .23099 & .13500 \\
\hline 0208 & -- & -- & -- & -- & -- & -- & 1432 & 8.5838 & 4.9277 & .57407 & .47735 & .23409 & .13421 \\
\hline 0211 & 7.3653 & 4.1957 & .56965 & .48022 & .23138 & .13560 & 1433 & 8.7518 & 4.9056 & .56052 & .46163 & .22649 & .13019 \\
\hline 0244 & 11.188 & 6.2419 & .55794 & .41975 & .18271 & .12798 & 1434 & 8.1143 & 4.4693 & .55080 & .45633 & .22856 & .13353 \\
\hline 0408 & 8.2963 & 4.7721 & .57521 & .53256 & .36466 & .31122 & 1492 & 5.9090 & 3.5590 & .60231 & .54930 & .27612 & .16978 \\
\hline 0427 & 7.5965 & 4.7425 & .62430 & .52361 & .24572 & .17932 & 1500 & 9.4444 & 4.7550 & .50347 & .49718 & .40725 & .35963 \\
\hline 0428 & 8.8882 & 5.2070 & .58583 & .47105 & .21968 & .13575 & 1528 & 6.0446 & 3.6488 & .60365 & .55061 & .28491 & .18520 \\
\hline 0429 & 10.638 & 6.3407 & .59604 & .49359 & .25593 & .14792 & 1541 & 6.8226 & 3.9569 & .57997 & .43018 & .10360 & .03805 \\
\hline 0463 & 6.7130 & 3.8552 & .57429 & .46668 & .18137 & .08663 & 1569 & 7.6500 & 4.5483 & .59455 & .52283 & .28940 & .19957 \\
\hline 0493 & 8.5882 & 3.9044 & .45462 & .30471 & .06403 & -.02405 & 1632 & -- & -- & -- & -- & -- & -- \\
\hline 0495 & 5.5777 & 3.4343 & 61572 & .59497 & .33058 & .20693 & 1641 & 6.5514 & 3.7503 & .57244 & .51372 & .26824 & . 14956. \\
\hline 0496 & 1.3333 & .31339 & .23504 & .87709 & .70364 & .49273 & 1646 & 5.0178 & 2.9481 & .58753 & .55595 & .27415 & .16290 \\
\hline 0498 & 1.4167 & .40152 & .28342 & .92453 & .81132 & .66038 & 1663 & 8.3571 & 5.3653 & .64200 & .52863 & .22127 & .11508 \\
\hline 0509 & 7.8431 & 4.6517 & .59309 & .49537 & .23914 & .15140 & 1671 & 7.0095 & 4.1740 & .59547 & .50797 & .24107 & .14829 \\
\hline 0518 & 6.4894 & 3.7803 & .58253 & .49098 & .21663 & .13243 & 1680 & 8.2258 & 4.5378 & .55166 & .45015 & .21972 & .13447 \\
\hline 0521 & 8.4242 & 4.3390 & .51506 & .39418 & .14700 & .04749 & 1694 & 7.1370 & 4.2798 & .59967 & .48320 & .18957 & .11844 \\
\hline 0556 & 7.2523 & 3.8154 & .52610 & .45542 & .28224 & .22824 & 1696 & 7.4472 & 4.2998 & .57738 & .49775 & .25559 & .15561 \\
\hline 0569 & 6.6722 & 3.9453 & .59131 & .51364 & .25188 & .15998 & 1697 & 7.0235 & 3.9213 & .55831 & .50509 & .25474 & .12283 \\
\hline 0572 & 7.3107 & 4.0847 & .55873 & .48343 & .25231 & .15502 & 1698 & 6.0874 & 3.6166 & .59412 & .51743 & .23615 & .14880 \\
\hline 0691 & 7.2118 & 4.0088 & .55586 & .46814 & .23203 & .14638 & 1903 & 4.6476 & 2.6827 & .57724 & .53742 & .22256 & .10027 \\
\hline 0708 & 5.4000 & 3.1748 & .58792 & .52735 & .23795 & .14777 & 1914 & 8.6500 & 4.2974 & .49681 & .57624 & .41825 & .31005 \\
\hline 0738 & 8.4516 & 4.5573 & .53922 & .42532 & .19169 & .10434 & 1920 & 7.2829 & 4.1254 & .56645 & .49665 & .26969 & .17057 \\
\hline 0776 & 6.0977 & 3.5467 & .58164 & .52278 & .26455 & .16043 & 1921 & 7.5644 & 4.1863 & .55341 & .43874 & .19508 & .11885 \\
\hline 0779 & 4.5170 & 2.7181 & .60176 & .60270 & .30557 & .16928 & 1937 & 9.2165 & 5.0737 & .55050 & .43887 & .20675 & .12049 \\
\hline 0784 & 5.4106 & 3.2174 & .59464 & .55952 & .29364 & .19131 & 1956 & 7.1140 & 4.0919 & .57519 & .48467 & .23289 & .14622 \\
\hline 0786 & 7.4714 & 4.4259 & .59238 & .51057 & .25476 & .15299 & 1970 & 9.9583 & 5.8098 & .58341 & .55988 & .42771 & .34065 \\
\hline 0917 & 8.1826 & 4.5340 & .55410 & .46187 & .23198 & .13944 & 2014 & 7.5344 & 4.5548 & .60453 & .51238 & .24129 & .14092 \\
\hline 0923 & 10.593 & 4.9715 & .46934 & .23521 & .03032 & .04066 & 2015 & 7.7057 & 4.6775 & .60702 & .51919 & .25541 & .15631 \\
\hline 0926 & 7.3535 & 4.1160 & .55974 & .45555 & .20894 & .12615 & 2019 & 7.8800 & 4.2500 & .53934 & .45616 & .31704 & .35701 \\
\hline 0950 & 3.2444 & 1.1859 & .36550 & .48449 & .37836 & .22613 & 2024 & 7.5689 & 4.2822 & .56576 & .45478 & .20169 & .12227 \\
\hline 0996 & 10.042 & 4.3569 & .43388 & .23863 & -.05160 & -.06203 & 2042 & 4.0833 & 2.1136 & .51763 & .39642 & .00836 & -.05854 \\
\hline 1013 & 4.7306 & 2.9439 & .62230 & .63361 & .35005 & .20856 & 2043 & 4.8488 & 3.1192 & .64328 & .66250 & .37596 & .21021 \\
\hline 1017 & 6.8796 & 4.0427 & .58763 & .50310 & .24484 & .15919 & 2048 & 6.2107 & 3.8299 & .61666 & .55753 & .27607 & .16650 \\
\hline 1042 & 13.850 & 5.9447 & .42922 & .27490 & .15363 & .28529 & 2050 & 7.2571 & 5.0265 & .69263 & .63858 & .32399 & .14664 \\
\hline 1048 & 4.1852 & 1.7550 & .41933 & .45286 & .31250 & .25931 & 2051 & 5.7692 & 3.2163 & .55750 & .51812 & .26807 & .12688 \\
\hline 1053 & 7.8610 & 4.3775 & .55687 & .45793 & .22541 & .14748 & 2053 & 4.4000 & 2.3111 & .52525 & .36779 & -.04224 & -.07933 \\
\hline 1057 & 7.6135 & 4.1111 & .53997 & .45297 & .23345 & .14649 & 2073 & 7.1632 & 4.1182 & .57490 & .51856 & .28667 & .17487 \\
\hline 1063 & 10.636 & 5.1126 & .48067 & .26647 & .05943 & .04839 & 2082 & 5.1861 & 3.0331 & .58485 & .55522 & .29090 & .18498 \\
\hline 1068 & 7.4621 & 4.3166 & .57848 & .48008 & .23006 & .14993 & 2086 & 7.1633 & 4.1198 & .57513 & .47153 & .21096 & .13095 \\
\hline 1080 & 4.5026 & 2.6448 & .58738 & .57809 & .28367 & .16194 & 2088 & 8.4082 & 4.5799 & .54470 & .34501 & .05653 & .01217 \\
\hline 1081 & 8.4898 & 4.5619 & .53734 & .41799 & .18305 & .10010 & 2090 & 5.5845 & 3.2604 & .58384 & .52842 & .26259 & .17745 \\
\hline 1133 & 9.8750 & 5.8750 & .59494 & .42675 & .14169 & .07607 & 2096 & 7.3305 & 4.1429 & .56516 & .45625 & .20533 & .13141 \\
\hline 1136 & 7.5682 & 4.6607 & .61582 & .53176 & .26264 & .15955 & 2128 & 9.0898 & 4.9651 & .54623 & .43233 & .20982 & .12895 \\
\hline 1138 & 6.8947 & 2.8772 & .41730 & .42037 & .17235 & .03916 & 2131 & 6.2111 & 3.5610 & .57332 & .48107 & .20480 & .12166 \\
\hline 1139 & 10.056 & 5.5993 & .55681 & .42751 & .22223 & .17961 & 2142 & 9.8421 & 5.2690 & .53535 & .49259 & .30926 & .17885 \\
\hline
\end{tabular}


224 Statistical Characteristics of Storm Interevent Time, Depth, and Duration for Eastern New Mexico, Oklahoma, and Texas

Appendix 4-3.3. L-moments of storm duration defined by 12-hour minimum interevent time for hourly rainfall stations in TexasContinued.

\begin{tabular}{|c|c|c|c|c|c|c|c|c|c|c|c|c|c|}
\hline $\begin{array}{c}\text { Station } \\
\text { no. }\end{array}$ & $\begin{array}{l}\text { Duration } \\
\text { mean } \\
\text { (hours) }\end{array}$ & $\begin{array}{l}\text { Duration } \\
\text { L-scale } \\
\text { (hours) }\end{array}$ & $\begin{array}{l}\text { Duration } \\
\text { L-CV } \\
\text { (dimen- } \\
\text { sionless) }\end{array}$ & $\begin{array}{l}\text { Duration } \\
\text { L-skew } \\
\text { (dimen- } \\
\text { sionless) }\end{array}$ & $\begin{array}{c}\text { Duration } \\
\text { L-kurtosis } \\
\text { (dimen- } \\
\text { sionless) }\end{array}$ & $\begin{array}{c}\text { Duration } \\
\text { Tau5 } \\
\text { (dimen- } \\
\text { sionless) }\end{array}$ & $\begin{array}{c}\text { Station } \\
\text { no. }\end{array}$ & $\begin{array}{c}\text { Duration } \\
\text { mean } \\
\text { (hours) }\end{array}$ & $\begin{array}{l}\text { Duration } \\
\text { L-scale } \\
\text { (hours) }\end{array}$ & $\begin{array}{c}\text { Duration } \\
\text { L-CV } \\
\text { (dimen- } \\
\text { sionless) }\end{array}$ & $\begin{array}{l}\text { Duration } \\
\text { L-skew } \\
\text { (dimen- } \\
\text { sionless) }\end{array}$ & $\begin{array}{l}\text { Duration } \\
\text { L-kurtosis } \\
\text { (dimen- } \\
\text { sionless) }\end{array}$ & $\begin{array}{c}\text { Duration } \\
\text { Tau5 } \\
\text { (dimen- } \\
\text { sionless) }\end{array}$ \\
\hline 2160 & 13.739 & 8.3123 & 0.60501 & 0.42137 & 0.14163 & 0.05583 & 3463 & 8.2500 & 4.4778 & 0.54277 & 0.39365 & 0.12722 & 0.03383 \\
\hline 2206 & 7.3109 & 4.1069 & .56175 & .49717 & .27037 & .17106 & 3476 & 7.3752 & 4.0631 & .55091 & .45503 & .21565 & .12530 \\
\hline 2238 & 6.8968 & 4.1585 & 60296 & .53303 & .27742 & .18112 & 3485 & 8.2727 & 3.4459 & .41654 & .26457 & .16404 & .04047 \\
\hline 2240 & 5.8511 & 3.4399 & .58792 & .46178 & .10288 & .00965 & 3507 & 6.2616 & 3.7923 & .60564 & .52175 & .22074 & .11730 \\
\hline 2242 & 8.4372 & 4.7291 & .56050 & .44106 & .19412 & .11006 & 3546 & 7.7402 & 4.4533 & .57535 & .46106 & .20279 & .12542 \\
\hline 2244 & 7.7892 & 4.5148 & .57961 & .46665 & .20576 & .12552 & 3547 & 7.1436 & 4.2194 & .59065 & .50311 & .24295 & .14783 \\
\hline 2247 & 6.9649 & 3.3421 & .47985 & .38533 & .22676 & .14308 & 3579 & 8.8182 & 5.1042 & .57883 & .49717 & .26967 & .16880 \\
\hline 2309 & 8.6375 & 4.5998 & .53254 & .40629 & .18339 & .10997 & 3642 & 7.4950 & 4.1392 & .55226 & .43367 & .18894 & .11965 \\
\hline 2312 & 6.7069 & 3.7516 & .55936 & .44503 & .18229 & .12225 & 3646 & 8.0033 & 4.2002 & .52481 & .42465 & .20951 & .12833 \\
\hline 2334 & 7.7500 & 4.3048 & .55546 & .38020 & .07890 & .01703 & 3668 & 13.250 & 5.9026 & .44548 & .25338 & .07232 & .09535 \\
\hline 2336 & 6.1062 & 3.3771 & .55307 & .45987 & .19341 & .10657 & 3673 & 12.720 & 7.1233 & .56001 & .43000 & .17530 & .05809 \\
\hline 2354 & 7.3704 & 4.3960 & .59644 & .47847 & .15275 & .00200 & 3686 & 6.2781 & 3.6394 & .57969 & .48341 & .20599 & .13528 \\
\hline 2355 & 9.0926 & 5.4504 & .59943 & .52588 & .26918 & .12858 & 3691 & 6.3969 & 3.6685 & .57349 & .48077 & .21319 & .13346 \\
\hline 2357 & 7.5684 & 4.5612 & .60266 & .51328 & .23923 & .12655 & 3734 & 9.2857 & 5.3836 & .57977 & .43977 & .16894 & .06382 \\
\hline 2360 & 6.9929 & 4.1030 & .58673 & .50288 & .24137 & .14329 & 3771 & 5.6756 & 3.3267 & .58614 & .50444 & .20363 & .11426 \\
\hline 2361 & 5.5215 & 3.2028 & .58006 & .53334 & .26912 & .16216 & 3789 & 5.2286 & 3.3505 & .64082 & .62480 & .32028 & .17627 \\
\hline 2394 & 8.0420 & 4.4093 & .54828 & .43958 & .20830 & .12758 & 3826 & 7.8302 & 4.0199 & .51338 & .38251 & .16001 & .11550 \\
\hline 2404 & 7.4350 & 4.1776 & .56189 & .45089 & .19897 & .11830 & 3831 & 7.6194 & 4.3265 & .56783 & .48227 & .24573 & .15589 \\
\hline 2415 & 8.4269 & 4.5799 & .54348 & .42855 & .19987 & .12150 & 3841 & 6.2434 & 3.5359 & .56633 & .50186 & .25920 & .16703 \\
\hline 2462 & 7.4116 & 4.0993 & .55309 & .43726 & .18795 & .11141 & 3871 & 8.1707 & 4.6264 & .56622 & .48712 & .25698 & .15336 \\
\hline 2528 & 8.4052 & 5.0711 & .60334 & .51938 & .28188 & .18256 & 3884 & 6.7143 & 2.4143 & .35957 & .15665 & .14201 & .06208 \\
\hline 2617 & 8.2582 & 4.5034 & .54533 & .45454 & .23948 & .15972 & 3941 & 11.203 & 6.3460 & .56645 & .44591 & .26270 & .21976 \\
\hline 2619 & 7.8227 & 4.4582 & .56991 & .46864 & .23976 & .17705 & 3963 & -- & -- & -- & -- & -- & -- \\
\hline 2621 & 6.4554 & 3.7075 & .57433 & .50068 & .24416 & .14514 & 4040 & 7.0045 & 3.7953 & .54184 & .45893 & .22881 & .13448 \\
\hline 2675 & 6.7466 & 3.8834 & .57561 & .49043 & .23153 & .13583 & 4058 & 6.3462 & 3.5708 & .56267 & .59306 & .42093 & .25671 \\
\hline 2676 & 5.4703 & 3.2868 & .60084 & .54658 & .24936 & .13830 & 4098 & 4.8556 & 2.7976 & .57616 & .53774 & .24776 & .14101 \\
\hline 2679 & 6.3805 & 3.8580 & 60466 & .53728 & .26250 & .15695 & 4100 & 6.8924 & 3.8243 & .55485 & .46774 & .23418 & .15580 \\
\hline 2715 & 7.4321 & 4.0571 & .54589 & .43919 & .19645 & .11130 & 4137 & 5.7058 & 3.3184 & .58158 & .50677 & .22085 & .13094 \\
\hline 2744 & 6.6885 & 3.9512 & .59075 & .51972 & .26123 & .15829 & 4191 & 6.8681 & 4.0514 & .58989 & .50484 & .23605 & .13598 \\
\hline 2758 & 9.2818 & 5.5671 & .59978 & .50747 & .26093 & .16175 & 4256 & -- & -- & -- & -- & -- & -- \\
\hline 2794 & 6.7273 & 3.9636 & .58919 & .51376 & .30810 & .29434 & 4257 & 7.0199 & 4.0602 & .57838 & .46780 & .19487 & .11723 \\
\hline 2797 & 5.8156 & 3.3881 & .58258 & .53004 & .26764 & .16212 & 4258 & 6.7143 & 4.0192 & .59860 & .48616 & .17599 & .08505 \\
\hline 2811 & 6.2099 & 3.6972 & .59537 & .53351 & .26769 & .16626 & 4278 & 7.5820 & 4.2082 & .55503 & .47872 & .25228 & .15523 \\
\hline 2813 & 5.4444 & 3.0256 & .55573 & .50102 & .26083 & .16987 & 4299 & 4.8080 & 2.6430 & .54970 & .51783 & .26535 & .16844 \\
\hline 2814 & 5.2353 & 3.5956 & .68680 & .69325 & .33947 & .02548 & 4300 & 8.2283 & 4.8213 & .58593 & .48553 & .23459 & .14353 \\
\hline 2815 & 4.8988 & 2.7048 & .55213 & .47665 & .17422 & .08687 & 4305 & 8.2067 & 4.8628 & .59254 & .49005 & .23235 & .14067 \\
\hline 2818 & 7.3207 & 4.2734 & .58374 & .49124 & .23490 & .14700 & 4307 & 9.2759 & 5.8647 & .63225 & .51659 & .23233 & .12938 \\
\hline 2986 & 9.7116 & 5.4731 & .56356 & .43467 & .19066 & .11018 & 4309 & 7.3889 & 4.2206 & .57121 & .49948 & .25876 & .15278 \\
\hline 3005 & 6.6332 & 3.7917 & .57163 & .47413 & .21159 & .13628 & 4311 & 7.8107 & 4.4382 & .56822 & .48422 & .24902 & .15019 \\
\hline 3033 & 5.0511 & 2.6293 & .52053 & .50021 & .27711 & .15908 & 4313 & 8.5188 & 4.8634 & .57090 & .46361 & .22168 & .12950 \\
\hline 3034 & -- & -- & -- & -- & -- & -- & 4319 & 7.6224 & 3.9938 & .52395 & .44697 & .23923 & .12251 \\
\hline 3047 & 6.4706 & 3.2496 & .50220 & .42046 & .22792 & .17707 & 4329 & 7.9134 & 4.5529 & .57534 & .48236 & .23535 & .13796 \\
\hline 3103 & 5.0606 & 2.8106 & .55539 & .44635 & .11277 & .04434 & 4331 & -- & -- & -- & -- & -- & -- \\
\hline 3133 & 7.9462 & 4.3594 & .54862 & .43122 & .19515 & .12163 & 4375 & 5.5687 & 3.3839 & .60767 & .56005 & .27152 & .16084 \\
\hline 3156 & 7.6772 & 4.4560 & .58043 & .45547 & .18387 & .11364 & 4392 & 10.692 & 5.9516 & .55663 & .44072 & .21048 & .12315 \\
\hline 3171 & 7.8966 & 4.3800 & .55468 & .45798 & .22797 & .14249 & 4425 & 5.2114 & 3.2217 & .61820 & .59960 & .31469 & .18551 \\
\hline 3189 & 5.9768 & 3.6105 & 60408 & .55714 & .29076 & .18431 & 4440 & 8.4048 & 4.5827 & .54525 & .45621 & .22342 & .12485 \\
\hline 3260 & 6.7160 & 3.8291 & .57015 & .48656 & .24280 & .16393 & 4476 & 6.5130 & 3.7229 & .57160 & .47184 & .19938 & .11681 \\
\hline 3267 & 7.3271 & 4.2303 & .57735 & .52566 & .32338 & .23790 & 4498 & 5.2727 & 3.4909 & .66207 & .72222 & .55295 & .48177 \\
\hline 3270 & 5.0387 & 2.9885 & .59311 & .55460 & .25867 & .14531 & 4517 & 7.8876 & 4.4238 & .56085 & .45575 & .22051 & .14305 \\
\hline 3272 & 3.2609 & 1.7154 & .52606 & .56155 & .21494 & .02105 & 4520 & 5.8086 & 3.4241 & .58948 & .50763 & .20865 & .11598 \\
\hline 3277 & 6.6000 & 4.4571 & .67532 & .61144 & .26529 & .07612 & 4525 & 9.7667 & 5.9713 & .61139 & .58856 & .38008 & .23425 \\
\hline 3278 & 5.2338 & 3.0226 & .57752 & .57312 & .34871 & .24636 & 4563 & 7.7500 & 4.3056 & .55556 & .50996 & .37923 & .36227 \\
\hline 3280 & 5.3228 & 2.9758 & .55906 & .55120 & .33390 & .21902 & 4570 & 6.5174 & 3.8571 & .59182 & .51521 & .24803 & .15495 \\
\hline 3281 & 3.7778 & 2.2111 & .58529 & .65132 & .37785 & .21967 & 4577 & 7.1870 & 4.1857 & .58240 & .48724 & .23135 & .14882 \\
\hline 3283 & 9.0575 & 5.1413 & .56763 & .44828 & .20763 & .12695 & 4591 & 8.8992 & 4.8481 & .54478 & .43332 & .20407 & .11771 \\
\hline 3284 & 6.8648 & 3.9509 & .57552 & .47867 & .21803 & .13640 & 4670 & 6.1272 & 3.5769 & .58377 & .51310 & .24884 & .16040 \\
\hline 3285 & 6.0927 & 3.5556 & .58359 & .49022 & .19858 & .11455 & 4671 & 6.9527 & 4.2268 & .60794 & .50297 & .21684 & .15797 \\
\hline 3329 & 7.7248 & 4.4674 & .57832 & .48221 & .23251 & .13872 & 4679 & 6.7325 & 3.9487 & .58650 & .48427 & .20495 & .12642 \\
\hline 3335 & 8.8558 & 4.9513 & .55910 & .42271 & .16786 & .08770 & 4696 & 4.1429 & 1.6264 & .39257 & .15541 & -.01966 & .11241 \\
\hline 3370 & 7.8266 & 4.2771 & .54648 & .44516 & .20858 & .12048 & 4703 & 6.0579 & 3.5044 & .57849 & .49561 & .22174 & .14040 \\
\hline 3410 & 6.2233 & 3.6072 & .57963 & .50156 & .23741 & .15258 & 4704 & 8.9093 & 4.9873 & .55979 & .41601 & .15637 & .08213 \\
\hline 3415 & 6.5957 & 3.8154 & .57847 & .48263 & .21458 & .13723 & 4731 & 8.2419 & 4.8505 & .58852 & .43571 & .12586 & .03832 \\
\hline 3430 & 8.0384 & 4.6390 & .57711 & .46666 & .21671 & .14014 & 4792 & 5.8184 & 3.4202 & .58782 & .51533 & .23193 & .14010 \\
\hline 3431 & 9.4919 & 5.9452 & .62634 & .48811 & .19032 & .10043 & 4819 & 6.9514 & 3.9061 & .56191 & .43510 & .16287 & .09878 \\
\hline 3441 & 6.5610 & 3.8012 & .57937 & .48363 & .20542 & .10960 & 4852 & 3.6250 & 2.3917 & .65977 & .84370 & .68794 & .58372 \\
\hline 3442 & 7.3226 & 3.8898 & .53120 & .45580 & .24675 & .16395 & 4866 & 7.7035 & 4.3841 & .56911 & .46100 & .21283 & .13570 \\
\hline 3446 & 5.8976 & 3.2972 & .55908 & .53137 & .31318 & .20722 & 4876 & 6.0949 & 3.4128 & .55995 & .46658 & .21202 & .15277 \\
\hline 3460 & 6.7561 & 3.5537 & .52599 & .41920 & .18549 & .10301 & 4878 & 8.4680 & 4.7445 & .56029 & .45896 & .22282 & .13035 \\
\hline 3462 & 7.2014 & 3.9323 & .54604 & .44751 & .22095 & .15124 & 4880 & 6.2859 & 3.6262 & .57688 & .51207 & .26346 & .16992 \\
\hline
\end{tabular}


Appendix 4-3.3. L-moments of storm duration defined by 12-hour minimum interevent time for hourly rainfall stations in TexasContinued.

\begin{tabular}{|c|c|c|c|c|c|c|c|c|c|c|c|c|c|}
\hline $\begin{array}{c}\text { Station } \\
\text { no. }\end{array}$ & $\begin{array}{c}\text { Duration } \\
\text { mean } \\
\text { (hours) }\end{array}$ & $\begin{array}{l}\text { Duration } \\
\text { L-scale } \\
\text { (hours) }\end{array}$ & $\begin{array}{c}\text { Duration } \\
\text { L-CV } \\
\text { (dimen- } \\
\text { sionless) }\end{array}$ & $\begin{array}{l}\text { Duration } \\
\text { L-skew } \\
\text { (dimen- } \\
\text { sionless) }\end{array}$ & $\begin{array}{l}\text { Duration } \\
\text { L-kurtosis } \\
\text { (dimen- } \\
\text { sionless) }\end{array}$ & $\begin{array}{c}\text { Duration } \\
\text { Tau5 } \\
\text { (dimen- } \\
\text { sionless) }\end{array}$ & $\begin{array}{c}\text { Station } \\
\text { no. }\end{array}$ & $\begin{array}{c}\text { Duration } \\
\text { mean } \\
\text { (hours) }\end{array}$ & $\begin{array}{c}\text { Duration } \\
\text { L-scale } \\
\text { (hours) }\end{array}$ & $\begin{array}{l}\text { Duration } \\
\text { L-CV } \\
\text { (dimen- } \\
\text { sionless) }\end{array}$ & $\begin{array}{l}\text { Duration } \\
\text { L-skew } \\
\text { (dimen- } \\
\text { sionless) }\end{array}$ & $\begin{array}{l}\text { Duration } \\
\text { L-kurtosis } \\
\text { (dimen- } \\
\text { sionless) }\end{array}$ & $\begin{array}{c}\text { Duration } \\
\text { Tau5 } \\
\text { (dimen- } \\
\text { sionless) }\end{array}$ \\
\hline 4920 & 5.9126 & 3.4644 & 0.58593 & 0.52148 & 0.25144 & 0.15515 & 5957 & 6.1106 & 3.5313 & 0.57791 & 0.49981 & 0.23147 & 0.14296 \\
\hline 4934 & 7.8571 & 4.8571 & .61818 & .42353 & .00000 & -.09804 & 5958 & 7.4824 & 4.0719 & .54419 & .44240 & .19252 & .09148 \\
\hline 4972 & 6.9996 & 3.9145 & .55925 & .45258 & .20148 & .12727 & 5973 & 9.0000 & 5.2417 & .58241 & .49071 & .27318 & .20176 \\
\hline 4973 & 8.3363 & 4.3984 & .52762 & .40234 & .17216 & .08948 & 5996 & 7.4927 & 4.3102 & .57525 & .46830 & .21280 & .13371 \\
\hline 4974 & 6.5912 & 3.6724 & .55717 & .47590 & .24060 & .16347 & 6017 & 6.4351 & 3.5937 & .55845 & .46078 & .18891 & .09224 \\
\hline 4975 & 6.5364 & 3.7133 & .56809 & .45897 & .18429 & .11056 & 6024 & 8.8953 & 5.0622 & .56909 & .44973 & .20970 & .13413 \\
\hline 4978 & 6.6224 & 3.8923 & .58776 & .47636 & .17954 & .09841 & 6050 & 8.2500 & 2.7974 & .33907 & .03920 & .08657 & .05760 \\
\hline 4979 & 12.000 & 5.9857 & .49881 & .37947 & .21136 & .10926 & 6104 & 5.1226 & 2.9916 & .58400 & .57405 & .32681 & .21165 \\
\hline 4982 & 7.0456 & 3.8542 & .54704 & .46346 & .22831 & .13850 & 6108 & 7.3127 & 4.2118 & .57595 & .46873 & .21235 & .14131 \\
\hline 5018 & 7.7419 & 4.1591 & .53722 & .46499 & .24150 & .13456 & 6136 & 4.8476 & 2.8262 & .58302 & .55552 & .27238 & .16280 \\
\hline 5048 & 5.4888 & 3.3206 & .60498 & .56819 & .28885 & .17042 & 6166 & 6.6087 & 3.6976 & .55950 & .51383 & .29287 & .18882 \\
\hline 5049 & 3.8384 & 2.0000 & .52105 & .46113 & .11025 & .01406 & 6176 & 8.5667 & 4.8229 & .56298 & .44726 & .21118 & .14558 \\
\hline 5056 & 6.2000 & 3.9000 & .62903 & .48718 & .10256 & .30769 & 6177 & 7.3248 & 4.2631 & .58201 & .48935 & .23650 & .15086 \\
\hline 5057 & 7.5027 & 4.6813 & .62395 & .55411 & .28940 & .17332 & 6210 & 6.6795 & 3.7528 & .56184 & .45779 & .19829 & .12296 \\
\hline 5060 & 7.6259 & 4.6364 & .60798 & .56867 & .33975 & .21961 & 6211 & 7.0704 & 4.4121 & .62403 & .54507 & .26402 & .15903 \\
\hline 5081 & 9.4417 & 5.0589 & .53580 & .41714 & .20215 & .12950 & 6270 & 6.6580 & 3.8375 & .57638 & .47137 & .20022 & .13124 \\
\hline 5094 & 6.6418 & 3.7910 & .57078 & .46908 & .20032 & .12118 & 6275 & -- & -- & -- & -- & -- & -- \\
\hline 5113 & 7.1575 & 4.3472 & .60736 & .52820 & .26182 & .16039 & 6276 & 10.833 & 5.5543 & .51271 & .35498 & .09777 & -.04596 \\
\hline 5114 & -- & -- & -- & -- & -- & -- & 6335 & 8.0261 & 4.3121 & .53726 & .43232 & .20410 & .11626 \\
\hline 5123 & 6.2667 & 3.4952 & .55775 & .47935 & .22029 & .11069 & 6434 & 10.158 & 5.2456 & .51641 & .34724 & .11593 & -.00130 \\
\hline 5192 & 7.3115 & 4.1383 & .56601 & .46434 & .21337 & .13025 & 6504 & 5.6401 & 3.3409 & .59235 & .53609 & .25490 & .14980 \\
\hline 5193 & 7.5320 & 4.3608 & .57897 & .47851 & .22249 & .13506 & 6558 & 8.0000 & 4.3839 & .54799 & .41093 & .18261 & .14588 \\
\hline 5224 & 8.1207 & 4.6809 & .57642 & .46554 & .22449 & .15562 & 6615 & 5.1636 & 3.1507 & .61017 & .58930 & .30992 & .19375 \\
\hline 5228 & 6.3441 & 3.7202 & .58641 & .51019 & .24801 & .16439 & 6660 & 8.8214 & 5.2405 & .59407 & .49082 & .24552 & .14940 \\
\hline 5235 & 9.4000 & 4.9655 & .52825 & .34127 & .10340 & .08901 & 6663 & 5.3398 & 3.3990 & .63654 & .61564 & .31374 & .16617 \\
\hline 5247 & 6.0442 & 3.5132 & .58125 & .51430 & .25150 & .15820 & 6734 & 7.5000 & 4.1906 & .55875 & .44720 & .19054 & .10676 \\
\hline 5258 & 8.2763 & 4.5238 & .54660 & .43517 & .19723 & .10779 & 6736 & 5.5278 & 3.3025 & .59744 & .56122 & .29569 & .18935 \\
\hline 5303 & 7.7035 & 4.3172 & .56042 & .49064 & .24936 & .13289 & 6740 & 14.867 & 9.3333 & .62780 & .62716 & .51962 & .42306 \\
\hline 5312 & 5.8187 & 3.4939 & .60046 & .54294 & .26220 & .15769 & 6750 & 8.5592 & 5.3722 & .62766 & .51014 & .22376 & .14005 \\
\hline 5341 & 8.5185 & 4.7236 & .55452 & .45008 & .19747 & .06931 & 6757 & 7.7885 & 4.4245 & .56807 & .45856 & .20974 & .13196 \\
\hline 5342 & -- & -- & -- & -- & -- & -- & 6775 & 7.5993 & 4.3724 & .57537 & .47929 & .22095 & .11589 \\
\hline 5348 & 6.9774 & 3.9952 & .57259 & .46301 & .19868 & .12899 & 6776 & 5.8460 & 3.3619 & .57508 & .50745 & .24362 & .15594 \\
\hline 5358 & 6.5962 & 3.6140 & .54790 & .47846 & .25700 & .16951 & 6788 & 7.6590 & 3.8384 & .50116 & .39173 & .17868 & .08808 \\
\hline 5398 & 8.3468 & 4.6387 & .55574 & .45182 & .21276 & .11887 & 6792 & 4.2158 & 2.3862 & .56602 & .57632 & .30586 & .19025 \\
\hline 5410 & 5.5608 & 3.2971 & .59292 & .55511 & .29329 & .18778 & 6794 & 12.840 & 6.0433 & .47066 & .36296 & .17625 & .02881 \\
\hline 5411 & 7.2618 & 4.1906 & .57708 & .48825 & .23899 & .14986 & 6834 & 6.3096 & 3.6049 & .57133 & .47055 & .19277 & .11548 \\
\hline 5424 & 10.481 & 6.5139 & .62148 & .49574 & .24393 & .17791 & 6893 & 4.6333 & 2.7580 & .59527 & .59284 & .31133 & .18826 \\
\hline 5429 & 7.2325 & 4.2926 & .59351 & .51997 & .26933 & .16615 & 6935 & 4.9645 & 2.8561 & .57530 & .54268 & .27069 & .16858 \\
\hline 5431 & 13.312 & 5.8375 & .43850 & .18997 & .02840 & -.03965 & 6981 & 7.3177 & 4.1658 & .56927 & .50420 & .25879 & .13207 \\
\hline 5461 & 8.9519 & 5.0151 & .56023 & .45581 & .22634 & .14088 & 7020 & 11.039 & 5.7970 & .52512 & .36661 & .14629 & .10068 \\
\hline 5463 & 6.0243 & 3.5018 & .58128 & .48929 & .19458 & .10174 & 7060 & 5.5553 & 3.2536 & .58567 & .52942 & .25261 & .15516 \\
\hline 5471 & 3.4400 & 1.9333 & .56202 & .57886 & .15619 & -.12842 & 7066 & 7.8601 & 4.3984 & .55958 & .44542 & .20136 & .12545 \\
\hline 5477 & 6.6250 & 4.2409 & .64014 & .60475 & .34577 & .21013 & 7074 & 5.3134 & 3.0773 & .57915 & .53438 & .26472 & .16382 \\
\hline 5528 & 7.3404 & 3.9719 & .54110 & .43794 & .19995 & .11918 & 7097 & 7.7568 & 4.5790 & .59033 & .52814 & .29668 & .19446 \\
\hline 5579 & -- & -- & -- & -- & -- & -- & 7116 & 6.7237 & 3.7085 & .55156 & .47648 & .23820 & .13766 \\
\hline 5580 & 6.4074 & 3.0057 & .46910 & .33846 & .07763 & -.03530 & 7140 & 7.1817 & 4.2076 & .58587 & .50659 & .25344 & .15455 \\
\hline 5589 & 4.4942 & 2.4277 & .54018 & .49893 & .21531 & .10806 & 7173 & 8.0400 & 4.8741 & .60624 & .51865 & .25992 & .15799 \\
\hline 5590 & 6.9529 & 4.1360 & .59486 & .53582 & .27434 & .14296 & 7174 & 8.3508 & 4.8803 & .58441 & .47648 & .22666 & .14761 \\
\hline 5591 & 5.1601 & 2.8226 & .54701 & .54874 & .33621 & .22110 & 7213 & 7.7126 & 4.5018 & .58370 & .50088 & .24979 & .14265 \\
\hline 5592 & 5.5211 & 3.0874 & .55920 & .56660 & .35980 & .23726 & 7243 & 6.4807 & 3.8619 & .59590 & .52171 & .25320 & .15870 \\
\hline 5594 & 4.3041 & 2.4462 & .56833 & .64352 & .44855 & .31273 & 7262 & 3.5196 & 2.1494 & .61069 & .71680 & .41659 & .18356 \\
\hline 5595 & -- & -- & -- & -- & -- & -- & 7274 & 6.3712 & 3.4795 & .54613 & .44502 & .20476 & .14810 \\
\hline 5596 & 4.0835 & 2.3869 & .58453 & .60550 & .30769 & .16430 & 7300 & 6.6798 & 3.6472 & .54600 & .46003 & .21999 & .12731 \\
\hline 5600 & 5.9528 & 3.4600 & .58124 & .58821 & .37607 & .24283 & 7311 & 5.3667 & 2.8563 & .53223 & .43932 & .21571 & .22400 \\
\hline 5618 & 7.9394 & 3.3731 & .42486 & .28094 & .12411 & .15294 & 7363 & 6.8889 & 3.5242 & .51158 & .40249 & .14737 & .01931 \\
\hline 5650 & 7.1053 & 2.8772 & .40494 & .19225 & .09344 & .09684 & 7422 & 6.8389 & 4.0145 & .58701 & .50263 & .24157 & .15179 \\
\hline 5656 & 5.2131 & 3.1374 & .60183 & .55787 & .25546 & .13675 & 7431 & 5.9573 & 3.4473 & .57867 & .52665 & .27514 & .17273 \\
\hline 5658 & 7.6450 & 4.3835 & .57338 & .50478 & .27398 & .16913 & 7481 & 5.5711 & 3.2989 & .59213 & .56117 & .30736 & .19995 \\
\hline 5661 & 5.5747 & 3.5189 & .63124 & .60068 & .31166 & .18843 & 7497 & 6.3414 & 3.8208 & .60252 & .52379 & .24057 & .14880 \\
\hline 5666 & 6.7143 & 3.7025 & .55144 & .44976 & .19266 & .09626 & 7498 & 6.0237 & 3.5766 & .59375 & .51046 & .21675 & .12947 \\
\hline 5695 & 7.3778 & 4.2104 & .57069 & .46416 & .21153 & .13695 & 7499 & 6.2286 & 3.6537 & .58661 & .49764 & .21216 & .12373 \\
\hline 5742 & 7.6316 & 3.9708 & .52031 & .35164 & .11656 & .04934 & 7531 & 9.0863 & 5.0592 & .55679 & .42156 & .17780 & .11322 \\
\hline 5766 & 8.5667 & 4.3138 & .50356 & .30311 & .04830 & .03595 & 7534 & 6.9938 & 3.9758 & .56848 & .46708 & .20504 & .12309 \\
\hline 5770 & 6.4132 & 3.7095 & .57841 & .49907 & .23934 & .15444 & 7556 & 6.3782 & 3.6805 & .57705 & .48234 & .20582 & .12157 \\
\hline 5775 & 6.3077 & 3.6538 & .57927 & .44689 & .10335 & -.04508 & 7594 & 7.4298 & 4.2194 & .56791 & .49234 & .25847 & .15913 \\
\hline 5779 & 12.000 & 7.2105 & .60088 & .47388 & .29100 & .28812 & 7596 & 6.9940 & 4.0967 & .58575 & .53418 & .30815 & .20985 \\
\hline 5840 & 9.0817 & 5.1790 & .57026 & .48884 & .26244 & .15279 & 7608 & 7.0821 & 4.0391 & .57033 & .46269 & .20293 & .12995 \\
\hline 5890 & 6.9027 & 3.9976 & .57914 & .50343 & .25554 & .16237 & 7622 & 4.2727 & 2.6494 & .62006 & .67843 & .42389 & .27004 \\
\hline 5891 & 6.1321 & 3.3855 & .55209 & .46633 & .20328 & .11236 & 7700 & 8.7572 & 4.8309 & .55164 & .44362 & .21306 & .12645 \\
\hline 5897 & 6.0254 & 3.5147 & .58331 & .48510 & .18102 & .08912 & 7706 & 6.2809 & 3.7153 & .59152 & .51752 & .24429 & .14783 \\
\hline
\end{tabular}


Appendix 4-3.3. L-moments of storm duration defined by 12-hour minimum interevent time for hourly rainfall stations in TexasContinued.

\begin{tabular}{|c|c|c|c|c|c|c|c|c|c|c|c|c|c|}
\hline $\begin{array}{c}\text { Station } \\
\text { no. }\end{array}$ & $\begin{array}{c}\text { Duration } \\
\text { mean } \\
\text { (hours) }\end{array}$ & $\begin{array}{l}\text { Duration } \\
\text { L-scale } \\
\text { (hours) }\end{array}$ & $\begin{array}{c}\text { Duration } \\
\text { L-CV } \\
\text { (dimen- } \\
\text { sionless) }\end{array}$ & $\begin{array}{l}\text { Duration } \\
\text { L-skew } \\
\text { (dimen- } \\
\text { sionless) }\end{array}$ & $\begin{array}{c}\text { Duration } \\
\text { L-kurtosis } \\
\text { (dimen- } \\
\text { sionless) }\end{array}$ & $\begin{array}{c}\text { Duration } \\
\text { Tau5 } \\
\text { (dimen- } \\
\text { sionless) }\end{array}$ & $\begin{array}{c}\text { Station } \\
\text { no. }\end{array}$ & $\begin{array}{c}\text { Duration } \\
\text { mean } \\
\text { (hours) }\end{array}$ & $\begin{array}{l}\text { Duration } \\
\text { L-scale } \\
\text { (hours) }\end{array}$ & $\begin{array}{c}\text { Duration } \\
\text { L-CV } \\
\text { (dimen- } \\
\text { sionless) }\end{array}$ & $\begin{array}{l}\text { Duration } \\
\text { L-skew } \\
\text { (dimen- } \\
\text { sionless) }\end{array}$ & $\begin{array}{c}\text { Duration } \\
\text { L-kurtosis } \\
\text { (dimen- } \\
\text { sionless) }\end{array}$ & $\begin{array}{c}\text { Duration } \\
\text { Tau5 } \\
\text { (dimen- } \\
\text { sionless) }\end{array}$ \\
\hline 7718 & 8.1667 & 4.5833 & 0.56122 & 0.43529 & 0.18363 & 0.10423 & 8910 & 6.1000 & 2.6684 & 0.43745 & 0.42538 & 0.28027 & 0.17496 \\
\hline 7745 & 10.562 & 5.8733 & .55608 & .41367 & .18262 & .11226 & 8911 & 6.1869 & 3.6167 & .58457 & .52302 & .26844 & .17100 \\
\hline 7922 & 5.1003 & 3.0145 & .59104 & .55773 & .26989 & .14482 & 8924 & 4.6788 & 2.5793 & .55126 & .56580 & .34098 & .21930 \\
\hline 7936 & 6.9025 & 3.9208 & .56803 & .47892 & .23293 & .15210 & 8929 & 10.455 & 6.5758 & 62899 & .51547 & .23825 & .09409 \\
\hline 7943 & 7.1734 & 4.1685 & .58110 & .50740 & .26137 & .15871 & 8942 & 7.0432 & 4.1224 & .58530 & .47784 & .20343 & .12572 \\
\hline 7944 & 8.1842 & 5.0007 & .61102 & .50741 & .23907 & .15554 & 8944 & 7.4091 & 4.3162 & .58255 & .49179 & .24544 & .16221 \\
\hline 7945 & 8.6236 & 5.0900 & .59024 & .48067 & .22388 & .13444 & 8996 & 7.2863 & 4.0780 & .55968 & .48613 & .25188 & .15262 \\
\hline 7947 & 6.9598 & 4.2933 & .61687 & .55580 & .31064 & .22959 & 9014 & 8.4783 & 3.6482 & .43030 & .32178 & .19161 & .09831 \\
\hline 7948 & 6.9613 & 4.0430 & .58078 & .48676 & .22317 & .13493 & 9037 & 6.2280 & 3.6398 & .58442 & .54158 & .30414 & .20531 \\
\hline 7951 & 7.8541 & 4.2370 & .53947 & .44743 & .22692 & .13915 & 9106 & 6.5970 & 3.8616 & .58535 & .54880 & .32348 & .20660 \\
\hline 7953 & 8.2645 & 5.0302 & .60865 & .55479 & .31527 & .18731 & 9107 & 5.1714 & 3.3429 & .64641 & .64587 & .35389 & .19974 \\
\hline 7981 & 7.1043 & 4.0513 & .57026 & .45565 & .17847 & .08768 & 9129 & 7.3119 & 4.1505 & .56764 & .44550 & .17204 & .08553 \\
\hline 7990 & 8.1000 & 4.7822 & .59039 & .47956 & .21106 & .11651 & 9163 & 6.7744 & 3.8654 & .57059 & .47961 & .22220 & .13504 \\
\hline 7992 & 8.6087 & 3.7945 & .44077 & .31131 & .14018 & .02655 & 9213 & 9.6205 & 5.8817 & .61137 & .47632 & .21321 & .15518 \\
\hline 7997 & 5.7417 & 2.9953 & .52168 & .46627 & .25308 & .16349 & 9214 & 8.2333 & 4.8701 & .59151 & .63509 & .48165 & .34176 \\
\hline 7999 & 5.5263 & 3.0175 & .54603 & .38235 & .02745 & -.03001 & 9222 & 9.7619 & 5.6457 & .57834 & .42641 & .15166 & .07001 \\
\hline 8022 & 7.1765 & 4.5601 & .63543 & .59827 & .35027 & .22304 & 9248 & 7.6250 & 4.5668 & .59893 & .49788 & .21351 & .10594 \\
\hline 8023 & 5.7952 & 3.4677 & .59837 & .55931 & .29710 & .18139 & 9266 & 8.5676 & 4.9678 & .57984 & .51134 & .27804 & .16729 \\
\hline 8047 & 6.6399 & 3.8327 & .57722 & .48994 & .23226 & .15117 & 9270 & 4.5895 & 2.6879 & .58566 & .58390 & .30968 & .18909 \\
\hline 8060 & 8.2188 & 4.8761 & .59329 & .48055 & .19208 & .07406 & 9295 & 5.5048 & 3.3005 & .59958 & .54747 & .28434 & .24432 \\
\hline 8062 & 9.0488 & 5.8012 & .64111 & .58626 & .33172 & .17853 & 9304 & -- & -- & -- & -- & -- & -- \\
\hline 8068 & 6.0278 & 3.4865 & .57841 & .46375 & .15148 & .07167 & 9307 & 7.4449 & 4.2287 & .56800 & .46967 & .21657 & .11877 \\
\hline 8081 & 7.1018 & 4.2741 & .60184 & .53133 & .27910 & .17893 & 9328 & 7.3146 & 4.3388 & .59317 & .51749 & .27274 & .18493 \\
\hline 8089 & 7.0250 & 3.6788 & .52368 & .37607 & .12881 & .09359 & 9329 & 9.2000 & 4.8095 & .52277 & .38340 & .17974 & .02698 \\
\hline 8221 & 12.737 & 6.6023 & .51837 & .37842 & .15197 & -.00134 & 9345 & -- & -- & -- & -- & -- & -- \\
\hline 8252 & 5.8988 & 3.5156 & .59599 & .55297 & .29198 & .17643 & 9363 & 7.5820 & 4.5456 & .59952 & .50678 & .24433 & .15477 \\
\hline 8265 & 8.7520 & 4.8704 & .55649 & .45788 & .21661 & .11531 & 9364 & 7.5571 & 4.5570 & .60301 & .52396 & .26650 & .16165 \\
\hline 8289 & 7.6604 & 4.8440 & .63234 & .55061 & .27276 & .15486 & 9365 & 6.6765 & 3.8601 & .57816 & .54930 & .37088 & .29914 \\
\hline 8305 & 4.5598 & 2.6021 & .57065 & .56262 & .29565 & .18600 & 9371 & 8.1905 & 4.7585 & .58099 & .48512 & .23477 & .13445 \\
\hline 8335 & 9.1028 & 4.9450 & .54324 & .42687 & .20913 & .13787 & 9417 & 7.5719 & 4.2333 & .55908 & .46282 & .22566 & .14185 \\
\hline 8400 & 5.8287 & 3.7561 & .64442 & .62635 & .35539 & .22367 & 9419 & 8.4340 & 4.7480 & .56295 & .44955 & .20795 & .12729 \\
\hline 8445 & 8.1476 & 4.5292 & .55589 & .46752 & .23909 & .14465 & 9435 & 6.8442 & 4.3257 & .63203 & .60970 & .37565 & .23676 \\
\hline 8446 & 6.4173 & 3.7279 & .58091 & .49973 & .23583 & .14768 & 9491 & 7.3089 & 4.1792 & .57179 & .47998 & .23190 & .14163 \\
\hline 8451 & 7.4517 & 4.2403 & .56904 & .49455 & .25418 & .14471 & 9499 & 5.8442 & 3.4778 & .59509 & .53262 & .25090 & .14605 \\
\hline 8531 & 7.8284 & 4.3693 & .55813 & .45872 & .22054 & .13586 & 9522 & 10.867 & 4.7714 & .43909 & .23875 & .05113 & .04373 \\
\hline 8541 & 8.1176 & 4.3545 & .53642 & .46031 & .20962 & .06961 & 9527 & 5.3706 & 3.1886 & .59371 & .54594 & .25925 & .14987 \\
\hline 8544 & 7.6450 & 4.3636 & .57077 & .47789 & .23418 & .13826 & 9532 & 6.5004 & 3.7362 & .57477 & .48746 & .22888 & .15014 \\
\hline 8545 & 9.2500 & 5.0658 & .54765 & .31810 & .00973 & -.01592 & 9544 & -- & -- & -- & -- & -- & -- \\
\hline 8563 & 6.2104 & 3.4784 & .56009 & .44853 & .17698 & .12302 & 9565 & 6.1066 & 3.5153 & .57565 & .51144 & .26050 & .17099 \\
\hline 8566 & 5.3220 & 3.0933 & .58123 & .54998 & .29953 & .20584 & 9570 & 4.9082 & 2.9067 & .59222 & .55908 & .26137 & .14499 \\
\hline 8583 & 5.6986 & 3.3414 & .58636 & .49671 & .18205 & .08263 & 9574 & 5.5000 & 3.1580 & .57418 & .45236 & .09372 & -.00688 \\
\hline 8584 & 6.6944 & 3.9492 & .58993 & .51029 & .24620 & .15013 & 9588 & 6.8404 & 4.2133 & .61594 & .54439 & .27019 & .16355 \\
\hline 8623 & 6.5383 & 3.7155 & .56826 & .46400 & .19216 & .11346 & 9665 & 7.2384 & 4.1371 & .57155 & .47485 & .22221 & .13470 \\
\hline 8625 & 8.2804 & 4.4568 & .53823 & .45373 & .23870 & .14874 & 9715 & 7.0303 & 3.9382 & .56017 & .45349 & .19810 & .12027 \\
\hline 8630 & 5.9680 & 3.3373 & .55920 & .48690 & .23579 & .15156 & 9729 & 8.0382 & 4.4781 & .55710 & .44688 & .20672 & .12696 \\
\hline 8631 & 7.4500 & 4.3364 & .58207 & .52799 & .30810 & .20862 & 9772 & 7.2018 & 4.1432 & .57530 & .50208 & .25406 & .14573 \\
\hline 8646 & 7.6154 & 4.1451 & .54431 & .44274 & .21478 & .13805 & 9814 & 9.2286 & 4.9210 & .53324 & .42418 & .18485 & .11329 \\
\hline 8647 & 5.6825 & 3.3551 & .59044 & .52188 & .23022 & .13012 & 9815 & 7.9986 & 4.4614 & .55777 & .45641 & .22589 & .14890 \\
\hline 8677 & 8.3832 & 4.1997 & .50096 & .39916 & .19842 & .10742 & 9816 & 6.7162 & 3.8802 & .57774 & .47723 & .20300 & .12285 \\
\hline 8696 & 7.0385 & 3.2815 & .46623 & .26770 & .07520 & .06828 & 9817 & 5.9494 & 3.4169 & .57432 & .49570 & .22474 & .13993 \\
\hline 8743 & 8.1645 & 4.6160 & .56538 & .45536 & .21385 & .13236 & 9829 & 5.6503 & 3.4136 & .60415 & .57055 & .30771 & .19811 \\
\hline 8761 & 5.0516 & 2.9207 & .57818 & .54096 & .26874 & .17283 & 9830 & 5.3269 & 3.0288 & .56859 & .53505 & .30910 & .25298 \\
\hline 8778 & 7.0458 & 4.0538 & .57535 & .46573 & .19982 & .12831 & 9858 & 6.6293 & 3.6534 & .55110 & .48305 & .25398 & .15690 \\
\hline 8845 & 7.4868 & 4.5117 & .60263 & .50630 & .23677 & .14705 & 9893 & 6.9481 & 3.9917 & .57450 & .48610 & .23800 & .15675 \\
\hline 8859 & 8.7297 & 4.8042 & .55033 & .43733 & .21217 & .13577 & 9916 & 7.4449 & 4.1646 & .55939 & .43530 & .18205 & .11886 \\
\hline 8898 & 8.8175 & 4.6921 & .53213 & .42545 & .20457 & .11513 & 9976 & 6.7787 & 4.1337 & .60981 & .54809 & .28957 & .18512 \\
\hline 8908 & 8.7500 & 5.2198 & .59655 & .57068 & .37142 & .23680 & & & & & & & \\
\hline
\end{tabular}


Appendix 4-3.4. L-moments of storm duration defined by 18-hour minimum interevent time for hourly rainfall stations in Texas.

$[--$, not available $]$

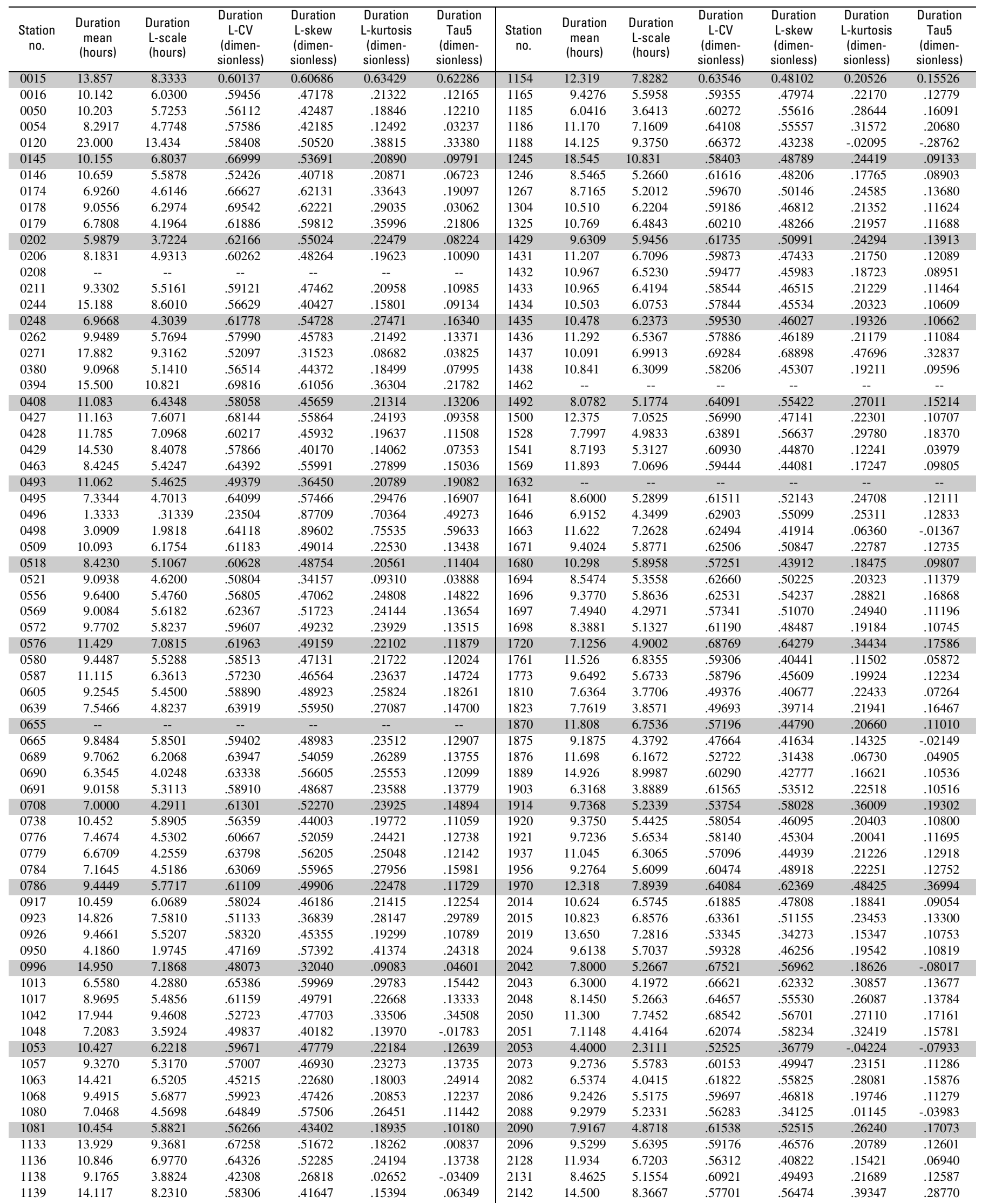


228 Statistical Characteristics of Storm Interevent Time, Depth, and Duration for Eastern New Mexico, Oklahoma, and Texas

Appendix 4-3.4. L-moments of storm duration defined by 18-hour minimum interevent time for hourly rainfall stations in TexasContinued.

\begin{tabular}{|c|c|c|c|c|c|c|c|c|c|c|c|c|c|}
\hline $\begin{array}{c}\text { Station } \\
\text { no. }\end{array}$ & $\begin{array}{l}\text { Duration } \\
\text { mean } \\
\text { (hours) }\end{array}$ & $\begin{array}{l}\text { Duration } \\
\text { L-scale } \\
\text { (hours) }\end{array}$ & $\begin{array}{l}\text { Duration } \\
\text { L-CV } \\
\text { (dimen- } \\
\text { sionless) }\end{array}$ & $\begin{array}{c}\text { Duration } \\
\text { L-skew } \\
\text { (dimen- } \\
\text { sionless) }\end{array}$ & $\begin{array}{c}\text { Duration } \\
\text { L-kurtosis } \\
\text { (dimen- } \\
\text { sionless) }\end{array}$ & $\begin{array}{c}\text { Duration } \\
\text { Tau5 } \\
\text { (dimen- } \\
\text { sionless) }\end{array}$ & $\begin{array}{c}\text { Station } \\
\text { no. }\end{array}$ & $\begin{array}{c}\text { Duration } \\
\text { mean } \\
\text { (hours) }\end{array}$ & $\begin{array}{c}\text { Duration } \\
\text { L-scale } \\
\text { (hours) }\end{array}$ & $\begin{array}{c}\text { Duration } \\
\text { L-CV } \\
\text { (dimen- } \\
\text { sionless) }\end{array}$ & $\begin{array}{l}\text { Duration } \\
\text { L-skew } \\
\text { (dimen- } \\
\text { sionless) }\end{array}$ & $\begin{array}{l}\text { Duration } \\
\text { L-kurtosis } \\
\text { (dimen- } \\
\text { sionless) }\end{array}$ & $\begin{array}{c}\text { Duration } \\
\text { Tau5 } \\
\text { (dimen- } \\
\text { sionless) }\end{array}$ \\
\hline 2160 & 14.318 & 8.4697 & 0.59153 & 0.40419 & 0.13703 & 0.04969 & 3463 & 10.432 & 5.8631 & 0.56204 & 0.37616 & 0.10832 & 0.06569 \\
\hline 2206 & 9.2238 & 5.4361 & .58936 & .49009 & .23373 & .12611 & 3476 & 9.6563 & 5.6687 & .58704 & .47048 & 20991 & .10707 \\
\hline 2238 & 10.068 & 6.4172 & .63736 & .53206 & .26486 & .16026 & 3485 & 8.2727 & 3.4459 & .41654 & .26457 & .16404 & .04047 \\
\hline 2240 & 9.1481 & 5.8056 & .63462 & .47970 & .14522 & .06835 & 3507 & 8.4287 & 5.2675 & .62495 & .50558 & .20972 & .11941 \\
\hline 2242 & 10.779 & 6.2066 & .57578 & .43418 & .17905 & .09587 & 3546 & 9.9216 & 5.8708 & .59172 & .45515 & .19245 & .11450 \\
\hline 2244 & 10.406 & 6.2056 & .59635 & .45665 & .19304 & .11725 & 3547 & 8.2604 & 5.1123 & .61890 & .53565 & .28017 & .17579 \\
\hline 2247 & 11.396 & 6.1910 & .54327 & .41106 & .17612 & .03515 & 3579 & 10.775 & 6.3400 & .58842 & .45882 & .18351 & .07087 \\
\hline 2309 & 11.069 & 6.2520 & .56483 & .42214 & .17764 & .09638 & 3642 & 9.6306 & 5.5710 & .57847 & .44840 & .19663 & .11599 \\
\hline 2312 & 9.2471 & 5.4430 & .58862 & .45223 & .18866 & .12165 & 3646 & 10.027 & 5.6219 & .56065 & .44874 & .21420 & .12474 \\
\hline 2334 & 9.7091 & 5.5657 & .57324 & .36672 & .04233 & -.02032 & 3668 & 16.222 & 8.3203 & .51289 & 40377 & .16850 & .06481 \\
\hline 2336 & 7.8990 & 4.6768 & .59207 & .48117 & .20791 & .11751 & 3673 & 16.182 & 8.3636 & .51685 & .31040 & .10154 & .06980 \\
\hline 2354 & 9.1200 & 5.9633 & .65387 & .58349 & .32878 & .22312 & 3686 & 8.5208 & 5.1837 & 60836 & .48640 & .20679 & .12335 \\
\hline 2355 & 12.717 & 8.6391 & .67932 & .59201 & .30714 & .13014 & 3691 & 8.4847 & 5.0682 & .59734 & .47463 & .20022 & .11595 \\
\hline 2357 & 9.7468 & 6.1712 & .63315 & .51770 & .22578 & .10430 & 3734 & 14.391 & 8.5929 & .59709 & .44697 & .22427 & .17551 \\
\hline 2360 & 9.8550 & 6.1648 & .62555 & .51125 & .23444 & .12964 & 3771 & 7.5220 & 4.6503 & .61823 & .50668 & .20125 & .10024 \\
\hline 2361 & 7.4765 & 4.6990 & .62851 & .54625 & .25158 & .10987 & 3789 & 7.3125 & 5.0191 & .68637 & .63947 & .35692 & .22522 \\
\hline 2394 & 10.059 & 5.7428 & .57093 & .44424 & .19940 & .11408 & 3826 & 8.7980 & 4.9026 & .55724 & .46562 & .24918 & .17970 \\
\hline 2404 & 9.4078 & 5.4959 & .58418 & .45260 & .18895 & .10232 & 3831 & 10.239 & 5.9740 & .58346 & .44440 & .18146 & .09938 \\
\hline 2415 & 10.599 & 6.0347 & .56936 & .44199 & .20238 & .11909 & 3841 & 7.8369 & 4.7877 & 61092 & .53154 & .27547 & .17432 \\
\hline 2462 & 9.6464 & 5.7577 & .59688 & .47397 & .20761 & .10839 & 3871 & 10.306 & 6.1126 & .59311 & .48391 & .22864 & .12114 \\
\hline 2528 & 10.509 & 6.7452 & .64182 & .53883 & .26939 & .13917 & 3884 & 7.6500 & 3.2658 & .42690 & .29412 & .20984 & .13856 \\
\hline 2617 & 9.9736 & 5.6674 & .56824 & .45109 & .20884 & .11324 & 3941 & 14.857 & 8.5922 & .57832 & .42361 & .18591 & .09115 \\
\hline 2619 & 9.1884 & 5.6338 & .61314 & .51061 & .25661 & .16535 & 3963 & -- & -- & -- & -- & -- & -- \\
\hline 2621 & 8.2592 & 4.9994 & .60532 & .50077 & .21972 & .10752 & 4040 & 8.6442 & 4.9252 & .56977 & .46253 & .21201 & .11646 \\
\hline 2675 & 8.8760 & 5.4281 & .61155 & .50768 & .23896 & .13325 & 4058 & 12.750 & 7.5289 & .59051 & .43283 & .10247 & -.06898 \\
\hline 2676 & 7.4948 & 4.7773 & .63742 & .54772 & .24840 & .13039 & 4098 & 6.5750 & 4.0706 & .61911 & .54663 & .25545 & .13849 \\
\hline 2679 & 8.4799 & 5.3728 & 63359 & .53355 & .24630 & .13228 & 4100 & 8.1543 & 4.7901 & .58743 & .49669 & .25368 & .15893 \\
\hline 2715 & 9.1849 & 5.2243 & .56879 & .44688 & .19512 & .10734 & 4137 & 7.6730 & 4.5995 & .59943 & .47744 & .18399 & .09856 \\
\hline 2744 & 8.2594 & 5.0711 & .61398 & .52160 & .25478 & .14680 & 4191 & 8.9728 & 5.5085 & .61390 & .50005 & .22115 & .11973 \\
\hline 2758 & 11.376 & 7.3529 & .64634 & .58059 & .35231 & .22962 & 4256 & -- & -- & -- & -- & -- & -- \\
\hline 2794 & 6.7273 & 3.9636 & .58919 & .51376 & .30810 & .29434 & 4257 & 9.2614 & 5.5241 & .59646 & .45582 & .17753 & .10139 \\
\hline 2797 & 7.6779 & 4.7201 & 61477 & .52131 & .23391 & .11373 & 4258 & 9.3210 & 5.6536 & .60654 & .44674 & .14187 & .07873 \\
\hline 2811 & 8.1994 & 5.1576 & 62902 & .53683 & .25500 & .13779 & 4278 & 9.2261 & 5.4009 & .58539 & .49406 & .25222 & .14664 \\
\hline 2813 & 8.7273 & 5.7273 & .65625 & .56871 & .27318 & .10557 & 4299 & 5.9576 & 3.6611 & .61452 & .58702 & .32912 & .19407 \\
\hline 2814 & 7.6250 & 5.6750 & .74426 & .70925 & .37455 & .09375 & 4300 & 11.459 & 6.9451 & .60606 & .46733 & 19979 & .11299 \\
\hline 2815 & 6.3435 & 3.7086 & .58463 & .47664 & .16738 & .06722 & 4305 & 11.272 & 6.9055 & .61260 & .47826 & .20980 & .12128 \\
\hline 2818 & 8.8198 & 5.5123 & 62499 & .54158 & .29456 & .20346 & 4307 & 13.015 & 8.3017 & .63785 & .48575 & .20072 & .11754 \\
\hline 2986 & 11.889 & 6.7242 & .56556 & .41447 & .16769 & .09387 & 4309 & 9.3935 & 5.6333 & .59970 & .50182 & .24308 & .13374 \\
\hline 3005 & 8.6039 & 5.1455 & .59804 & .47653 & .20406 & .11880 & 4311 & 9.9428 & 5.9602 & .59945 & .49131 & .23550 & .13128 \\
\hline 3033 & 5.8535 & 3.2795 & .56027 & .53155 & .29333 & .15897 & 4313 & 11.436 & 6.5439 & .57220 & .40399 & .12651 & .04153 \\
\hline 3034 & -- & -- & -- & -- & -- & -- & 4319 & 10.894 & 5.8118 & .53348 & .37333 & .13216 & .05762 \\
\hline 3047 & 15.957 & 9.7984 & 61407 & .48044 & .19633 & .05790 & 4329 & 10.014 & 6.0165 & .60082 & .48427 & .22089 & .12022 \\
\hline 3103 & 8.6071 & 5.3664 & .62348 & .47798 & .16065 & .08541 & 4331 & -- & -- & -- & -- & -- & -- \\
\hline 3133 & 9.9709 & 5.6821 & .56987 & .43822 & .19224 & .10959 & 4375 & 7.9340 & 5.0961 & .64231 & .55436 & .26810 & .15764 \\
\hline 3156 & 10.552 & 6.4681 & 61300 & .47116 & .19419 & .11345 & 4392 & 13.132 & 7.4714 & .56895 & .43363 & .20172 & .13396 \\
\hline 3171 & 9.9479 & 5.7206 & .57506 & .45036 & .20014 & .11227 & 4425 & 7.1269 & 4.5915 & .64424 & .56613 & .26021 & .12627 \\
\hline 3189 & 7.6176 & 4.9265 & .64673 & .58359 & .31483 & .19760 & 4440 & 10.500 & 5.9952 & .57099 & .46642 & .22424 & .12683 \\
\hline 3260 & 8.6129 & 5.0243 & .58335 & .45001 & .16932 & .07523 & 4476 & 8.5447 & 5.0464 & .59058 & .46176 & .18744 & .10844 \\
\hline 3267 & 8.9100 & 5.5219 & 61974 & .55555 & .31967 & .19475 & 4498 & 5.2727 & 3.4909 & .66207 & .72222 & .55295 & .48177 \\
\hline 3270 & 6.6681 & 4.1562 & .62330 & .54118 & .23628 & .11634 & 4517 & 10.120 & 5.9888 & .59178 & .46832 & .21234 & .12031 \\
\hline 3272 & 7.8421 & 5.0643 & .64579 & .52615 & .17071 & .00633 & 4520 & 7.9469 & 4.8581 & .61132 & .48315 & .17501 & .08158 \\
\hline 3277 & 7.0000 & 4.6813 & .66876 & .58451 & .23496 & .06466 & 4525 & 19.619 & 13.729 & .69976 & .56982 & .24789 & .04821 \\
\hline 3278 & 6.6842 & 4.1279 & 61756 & .57505 & .31898 & .18680 & 4563 & 9.4231 & 6.0723 & .64441 & .62769 & .46875 & .39819 \\
\hline 3280 & 6.3733 & 3.7797 & .59305 & .56189 & .32372 & .19262 & 4570 & 8.5193 & 5.2341 & .61439 & .50636 & .22987 & .13181 \\
\hline 3281 & 4.6279 & 2.8538 & .61665 & .62407 & .31487 & .11997 & 4577 & 9.0888 & 5.4752 & .60241 & .48017 & .21065 & .12354 \\
\hline 3283 & 11.978 & 7.0294 & .58684 & .44005 & .18261 & .10181 & 4591 & 11.403 & 6.5380 & .57336 & .44730 & .20556 & .11801 \\
\hline 3284 & 9.3558 & 5.6209 & 60079 & .47678 & .21102 & .12613 & 4670 & 8.2013 & 5.0069 & .61049 & .50419 & .22599 & .12636 \\
\hline 3285 & 8.2800 & 5.0036 & .60430 & .47744 & .18980 & .10740 & 4671 & 9.4179 & 5.8800 & .62435 & .49340 & .21528 & .14800 \\
\hline 3329 & 10.289 & 6.1921 & 60184 & .47876 & .21736 & .12313 & 4679 & 8.9991 & 5.4789 & .60882 & .47860 & .19657 & .11499 \\
\hline 3335 & 11.524 & 6.7065 & .58195 & .43000 & .15971 & .06688 & 4696 & 8.0833 & 5.3864 & .66635 & .64669 & .44866 & .31786 \\
\hline 3370 & 9.4296 & 5.3577 & .56818 & .44880 & .19861 & .11103 & 4703 & 8.3211 & 5.4117 & .65035 & .58196 & .33046 & .23390 \\
\hline 3410 & 8.1799 & 4.9973 & 61092 & .50541 & .22893 & .13034 & 4704 & 12.054 & 6.9255 & .57452 & .41769 & .17235 & .11623 \\
\hline 3415 & 8.9056 & 5.3795 & 60406 & .48050 & .20779 & .12476 & 4731 & 11.284 & 7.0267 & .62269 & .47197 & .19513 & .13565 \\
\hline 3430 & 11.004 & 6.6524 & 60454 & .47316 & .21516 & .13369 & 4792 & 8.0353 & 4.9893 & .62092 & .51759 & .23336 & .13343 \\
\hline 3431 & 13.820 & 8.4684 & 61277 & .41828 & .12280 & .07027 & 4819 & 8.9278 & 5.2855 & .59203 & .45747 & .18934 & .11934 \\
\hline 3441 & 8.9730 & 5.8228 & .64893 & .54881 & .24737 & .10367 & 4852 & 3.6250 & 2.3917 & .65977 & .84370 & .68794 & .58372 \\
\hline 3442 & 9.0043 & 5.1017 & .56659 & 47999 & .25155 & .15251 & 4866 & 9.5753 & 5.6413 & .58915 & .46504 & .20753 & .12084 \\
\hline 3446 & 6.9181 & 4.0352 & .58328 & .52752 & .28440 & .16572 & 4876 & 8.6721 & 5.2826 & .60915 & .49412 & .22825 & .15088 \\
\hline 3460 & 9.6667 & 5.1524 & .53300 & .32277 & .03686 & -.01861 & 4878 & 11.137 & 6.4710 & .58104 & .44812 & .19873 & .11642 \\
\hline 3462 & 9.8281 & 5.6620 & .57611 & .44599 & .19901 & .12344 & 4880 & 8.0498 & 4.8740 & .60548 & .51538 & .25434 & .15005 \\
\hline
\end{tabular}


Appendix 4-3.4. L-moments of storm duration defined by 18-hour minimum interevent time for hourly rainfall stations in TexasContinued.

\begin{tabular}{|c|c|c|c|c|c|c|c|c|c|c|c|c|c|}
\hline $\begin{array}{c}\text { Station } \\
\text { no. }\end{array}$ & $\begin{array}{l}\text { Duration } \\
\text { mean } \\
\text { (hours) }\end{array}$ & $\begin{array}{l}\text { Duration } \\
\text { L-scale } \\
\text { (hours) }\end{array}$ & $\begin{array}{c}\text { Duration } \\
\text { L-CV } \\
\text { (dimen- } \\
\text { sionless) }\end{array}$ & $\begin{array}{l}\text { Duration } \\
\text { L-skew } \\
\text { (dimen- } \\
\text { sionless) }\end{array}$ & $\begin{array}{c}\text { Duration } \\
\text { L-kurtosis } \\
\text { (dimen- } \\
\text { sionless) }\end{array}$ & $\begin{array}{c}\text { Duration } \\
\text { Tau5 } \\
\text { (dimen- } \\
\text { sionless) }\end{array}$ & $\begin{array}{c}\text { Station } \\
\text { no. }\end{array}$ & $\begin{array}{c}\text { Duration } \\
\text { mean } \\
\text { (hours) }\end{array}$ & $\begin{array}{l}\text { Duration } \\
\text { L-scale } \\
\text { (hours) }\end{array}$ & $\begin{array}{c}\text { Duration } \\
\text { L-CV } \\
\text { (dimen- } \\
\text { sionless) }\end{array}$ & $\begin{array}{l}\text { Duration } \\
\text { L-skew } \\
\text { (dimen- } \\
\text { sionless) }\end{array}$ & $\begin{array}{l}\text { Duration } \\
\text { L-kurtosis } \\
\text { (dimen- } \\
\text { sionless) }\end{array}$ & $\begin{array}{c}\text { Duration } \\
\text { Tau5 } \\
\text { (dimen- } \\
\text { sionless) }\end{array}$ \\
\hline 4920 & 8.1232 & 5.0815 & 0.62555 & 0.53257 & 0.25598 & 0.14840 & 5957 & 7.8970 & 4.7741 & 0.60454 & 0.49965 & 0.22443 & 0.13035 \\
\hline 4934 & 7.8571 & 4.8571 & 61818 & .42353 & .00000 & -.09804 & 5958 & 10.386 & 6.1070 & .58798 & .46022 & .19348 & .09766 \\
\hline 4972 & 9.0623 & 5.3718 & .59276 & .47231 & .20811 & .11542 & 5973 & 13.178 & 7.6137 & .57777 & .44558 & .20753 & .11219 \\
\hline 4973 & 10.923 & 5.8868 & .53895 & .38543 & .14266 & .07005 & 5996 & 9.5030 & 5.6509 & .59464 & .46723 & .20487 & .12147 \\
\hline 4974 & 8.6128 & 5.1500 & .59795 & .49792 & .24216 & .14320 & 6017 & 8.3167 & 5.1028 & .61356 & .51774 & .23344 & .10047 \\
\hline 4975 & 8.5857 & 5.0871 & .59251 & .45828 & .17741 & .09591 & 6024 & 11.321 & 6.6725 & .58942 & .44294 & .18984 & .12209 \\
\hline 4978 & 8.1278 & 4.9870 & .61357 & .48921 & .19269 & .10853 & 6050 & 9.3684 & 3.2807 & .35019 & .15445 & .16876 & .08362 \\
\hline 4979 & 16.611 & 10.062 & .60574 & .53500 & .29498 & .07576 & 6104 & 7.1652 & 4.5983 & .64175 & .59481 & .33002 & .19414 \\
\hline 4982 & 8.6323 & 4.8833 & .56571 & .44893 & .19171 & .10163 & 6108 & 9.4443 & 5.6601 & .59931 & .46919 & .20084 & .11906 \\
\hline 5018 & 9.1426 & 5.0631 & .55379 & .45720 & .21594 & .10897 & 6136 & 6.7968 & 4.2317 & .62260 & .54633 & .25406 & .13511 \\
\hline 5048 & 7.3725 & 4.6483 & .63050 & .54446 & .24896 & .12732 & 6166 & 8.5294 & 4.8333 & .56667 & .44735 & .17779 & .06780 \\
\hline 5049 & 5.4286 & 3.1629 & .58264 & .50814 & .21368 & .14215 & 6176 & 10.617 & 6.2143 & .58530 & .45870 & .21609 & .14869 \\
\hline 5056 & 6.2000 & 3.9000 & .62903 & .48718 & .10256 & .30769 & 6177 & 9.4262 & 5.6539 & .59981 & .47956 & .21744 & .13111 \\
\hline 5057 & 10.065 & 6.4883 & .64463 & .54136 & .26596 & .15061 & 6210 & 8.8315 & 5.2272 & .59188 & .46550 & .19496 & .10743 \\
\hline 5060 & 9.5593 & 6.1392 & .64223 & .57351 & .31723 & .18449 & 6211 & 9.1045 & 5.9858 & .65745 & .55312 & .25150 & .12332 \\
\hline 5081 & 11.843 & 6.6668 & .56293 & .43709 & .21685 & .14660 & 6270 & 9.1639 & 5.4732 & .59726 & .46164 & .18910 & .11463 \\
\hline 5094 & 8.7009 & 5.1724 & .59446 & .46879 & .19794 & .11793 & 6275 & -- & -- & -- & -- & -- & -- \\
\hline 5113 & 9.6619 & 6.0547 & .62666 & .51165 & .23465 & .13388 & 6276 & 14.429 & 8.3524 & .57888 & .40743 & .12671 & .02888 \\
\hline 5114 & -- & -- & -- & -- & -- & -- & 6335 & 9.8524 & 5.5557 & .56389 & .44503 & .20380 & .11376 \\
\hline 5123 & 6.2667 & 3.4952 & .55775 & .47935 & .22029 & .11069 & 6434 & 12.882 & 5.9853 & .46461 & .33071 & .10881 & -.02344 \\
\hline 5192 & 9.1131 & 5.3472 & .58677 & .46541 & .20485 & .11586 & 6504 & 7.5342 & 4.7115 & .62534 & .53609 & .25035 & .14188 \\
\hline 5193 & 9.9171 & 5.9329 & .59825 & .46722 & .19840 & .11006 & 6558 & 9.3793 & 5.4532 & .58141 & .45247 & .20772 & .12524 \\
\hline 5224 & 10.372 & 6.0702 & .58524 & .44576 & .19283 & .11750 & 6615 & 7.3780 & 4.7473 & .64344 & .56044 & .25763 & .12750 \\
\hline 5228 & 8.4249 & 5.2125 & 61869 & .51428 & .23920 & .13990 & 6660 & 12.361 & 7.5473 & .61058 & .46896 & .19386 & .08219 \\
\hline 5235 & 12.074 & 6.0940 & .50472 & .22113 & -.01150 & .04325 & 6663 & 6.9579 & 4.6611 & 66991 & .61058 & .29985 & .14215 \\
\hline 5247 & 8.0791 & 4.9744 & .61571 & .52296 & .25303 & .14898 & 6734 & 9.1960 & 5.3504 & .58182 & .45591 & .19634 & .11758 \\
\hline 5258 & 10.526 & 5.9759 & .56776 & .43118 & .18048 & .09520 & 6736 & 7.0225 & 4.4179 & 62910 & .56508 & .29092 & .17103 \\
\hline 5303 & 9.7450 & 5.7815 & .59328 & .49965 & .23535 & .10824 & 6740 & 19.385 & 12.705 & .65542 & .53252 & .26034 & .12861 \\
\hline 5312 & 7.6866 & 4.8651 & .63292 & .54488 & .25709 & .14206 & 6750 & 11.539 & 7.4569 & .64623 & .49371 & .18176 & .07915 \\
\hline 5341 & 13.545 & 7.6494 & .56472 & .35501 & .00925 & -.10319 & 6757 & 10.220 & 6.0487 & .59184 & .46405 & .21059 & .13093 \\
\hline 5342 & -- & -- & -- & -- & -- & -- & 6775 & 9.6410 & 5.6588 & .58695 & .46233 & .20022 & .10883 \\
\hline 5348 & 9.3999 & 5.6253 & .59844 & .46850 & .20453 & .13099 & 6776 & 7.6513 & 4.6532 & 60816 & .51475 & .24180 & .13974 \\
\hline 5358 & 8.4686 & 4.9808 & .58815 & .50066 & .25594 & .14685 & 6788 & 9.1605 & 4.6506 & .50768 & .36678 & .14201 & .06362 \\
\hline 5398 & 10.472 & 6.0267 & .57552 & .45393 & .20724 & .11768 & 6792 & 5.7078 & 3.5801 & .62723 & .60140 & .32229 & .18136 \\
\hline 5410 & 7.3392 & 4.5964 & .62628 & .55060 & .27060 & .14850 & 6794 & 21.474 & 11.152 & .51934 & .36506 & .26008 & .28841 \\
\hline 5411 & 9.6106 & 5.8277 & .60638 & .49453 & .23478 & .13973 & 6834 & 8.6574 & 5.1901 & .59950 & .47148 & .19181 & .10703 \\
\hline 5424 & 14.888 & 9.1493 & .61456 & .44286 & .17641 & .10841 & 6893 & 6.0866 & 3.8893 & .63900 & .60377 & .32576 & .19912 \\
\hline 5429 & 9.1146 & 5.6042 & .61486 & .50788 & .23119 & .11585 & 6935 & 6.6001 & 4.1253 & .62504 & .56563 & .28483 & .15946 \\
\hline 5431 & 17.357 & 8.3571 & .48148 & .24129 & .13095 & .19945 & 6981 & 9.3125 & 5.5951 & 60082 & .51025 & .24021 & .10019 \\
\hline 5461 & 10.970 & 6.4253 & .58570 & .46896 & .22650 & .13245 & 7020 & 13.557 & 7.3643 & .54323 & .38419 & .17055 & .12171 \\
\hline 5463 & 8.1390 & 4.9646 & 60997 & .49084 & .19994 & .10741 & 7060 & 7.4315 & 4.5841 & .61685 & .51719 & .22024 & .11005 \\
\hline 5471 & 7.2500 & 5.2132 & .71906 & .68602 & .38296 & .19516 & 7066 & 10.171 & 5.9284 & .58285 & .45261 & .20001 & .11662 \\
\hline 5477 & 7.5217 & 4.9209 & .65423 & .58830 & .29696 & .13769 & 7074 & 6.9359 & 4.2827 & 61746 & .54573 & .26986 & .15601 \\
\hline 5528 & 8.7812 & 5.0115 & .57070 & .45848 & .20567 & .11205 & 7097 & 9.6667 & 5.7758 & .59749 & .48018 & .21530 & .12079 \\
\hline 5579 & -- & -- & -- & -- & -- & -- & 7116 & 8.5196 & 5.0395 & .59151 & .49930 & .24453 & .13669 \\
\hline 5580 & 9.1250 & 4.7844 & .52432 & .42308 & .19216 & .10344 & 7140 & 9.3182 & 5.7383 & .61581 & .51235 & .24535 & .13938 \\
\hline 5589 & 6.4423 & 4.0426 & .62750 & .58247 & .31274 & .18723 & 7173 & 11.474 & 7.0900 & .61793 & .47866 & .19608 & .09516 \\
\hline 5590 & 8.6497 & 5.2020 & .60141 & .49488 & .21364 & .09916 & 7174 & 11.498 & 6.9817 & .60721 & .47006 & .20748 & .12626 \\
\hline 5591 & 6.8020 & 4.1603 & 61164 & .58806 & .35266 & .21647 & 7213 & 10.134 & 6.1139 & 60329 & .48304 & .21700 & .12047 \\
\hline 5592 & 6.7466 & 4.0443 & .59947 & .57315 & .33463 & .19632 & 7243 & 8.8106 & 5.4915 & .62329 & .51195 & .22851 & .12574 \\
\hline 5594 & 5.2407 & 3.1967 & 60998 & .64072 & .41167 & .25337 & 7262 & 6.5169 & 4.5444 & .69734 & .66795 & .35239 & .15107 \\
\hline 5595 & -- & -- & -- & -- & -- & -- & 7274 & 7.7358 & 4.4170 & .57099 & .45380 & .19588 & .11431 \\
\hline 5596 & 6.0164 & 3.9042 & .64892 & .61173 & .31153 & .15847 & 7300 & 8.1928 & 4.7668 & .58183 & .48583 & .23492 & .13544 \\
\hline 5600 & 7.8351 & 5.0060 & 63893 & 61049 & .36360 & .20421 & 7311 & 8.3846 & 4.9908 & .59523 & .47441 & .21112 & .11627 \\
\hline 5618 & 11.857 & 6.2116 & .52387 & .35313 & .07711 & .00696 & 7363 & 14.450 & 8.6395 & .59789 & .48523 & .26173 & .19441 \\
\hline 5650 & 7.1053 & 2.8772 & .40494 & .19225 & .09344 & .09684 & 7422 & 9.3188 & 5.7508 & .61711 & .50190 & .22710 & .12831 \\
\hline 5656 & 7.2205 & 4.5714 & 63311 & .53879 & .22837 & .11090 & 7431 & 7.3365 & 4.4453 & 60592 & .52782 & .26044 & .14710 \\
\hline 5658 & 8.9721 & 5.3252 & .59353 & .51454 & .27550 & .16405 & 7481 & 7.0104 & 4.3550 & .62122 & .56110 & .29593 & .17634 \\
\hline 5661 & 8.1327 & 5.5505 & .68250 & .61121 & .31424 & .17274 & 7497 & 8.7752 & 5.4236 & .61805 & .49190 & .20273 & .11991 \\
\hline 5666 & 8.7500 & 4.9415 & .56475 & .38662 & .06724 & -.03165 & 7498 & 8.1199 & 5.1364 & .63257 & .52732 & .23251 & .13069 \\
\hline 5695 & 9.3760 & 5.5165 & .58836 & .45804 & .19630 & .11661 & 7499 & 8.3197 & 5.1179 & .61515 & .49582 & .20331 & .10760 \\
\hline 5742 & 12.000 & 7.4083 & .61736 & .44111 & .16272 & .14994 & 7531 & 11.704 & 6.9653 & .59512 & .45441 & .18698 & .08906 \\
\hline 5766 & 15.652 & 5.9209 & .37828 & .09079 & .11644 & .15491 & 7534 & 8.9184 & 5.4637 & .61264 & .50310 & .22966 & .13220 \\
\hline 5770 & 8.4910 & 5.1552 & .60714 & .49768 & .22481 & .12941 & 7556 & 8.2756 & 4.9761 & 60129 & .47904 & .19428 & .10325 \\
\hline 5775 & 5.0833 & 2.8712 & .56483 & .48496 & .17678 & .04661 & 7594 & 9.4682 & 5.6651 & .59832 & .49922 & .24766 & .14189 \\
\hline 5779 & 12.000 & 7.2105 & .60088 & .47388 & .29100 & .28812 & 7596 & 8.3057 & 5.1008 & .61414 & .53468 & .27289 & .15034 \\
\hline 5840 & 10.699 & 6.3355 & .59217 & .48684 & .23940 & .13311 & 7608 & 9.0951 & 5.4351 & .59759 & .47550 & .21196 & .12890 \\
\hline 5890 & 8.9758 & 5.4465 & .60680 & .50174 & .23399 & .12827 & 7622 & 6.0000 & 3.8286 & .63810 & .56206 & .19154 & -.02204 \\
\hline 5891 & 7.0789 & 4.0973 & .57881 & .49886 & .24840 & .15993 & 7700 & 11.059 & 6.3806 & .57696 & .45306 & .20781 & .11675 \\
\hline 5897 & 8.3926 & 5.1214 & .61022 & .47902 & .18030 & .09175 & 7706 & 8.4484 & 5.2188 & .61772 & .51008 & .22901 & .12842 \\
\hline
\end{tabular}


Appendix 4-3.4. L-moments of storm duration defined by 18-hour minimum interevent time for hourly rainfall stations in TexasContinued.

\begin{tabular}{|c|c|c|c|c|c|c|c|c|c|c|c|c|c|}
\hline $\begin{array}{c}\text { Station } \\
\text { no. }\end{array}$ & $\begin{array}{l}\text { Duration } \\
\text { mean } \\
\text { (hours) }\end{array}$ & $\begin{array}{l}\text { Duration } \\
\text { L-scale } \\
\text { (hours) }\end{array}$ & $\begin{array}{l}\text { Duration } \\
\text { L-CV } \\
\text { (dimen- } \\
\text { sionless) }\end{array}$ & $\begin{array}{l}\text { Duration } \\
\text { L-skew } \\
\text { (dimen- } \\
\text { sionless) }\end{array}$ & $\begin{array}{l}\text { Duration } \\
\text { L-kurtosis } \\
\text { (dimen- } \\
\text { sionless) }\end{array}$ & $\begin{array}{c}\text { Duration } \\
\text { Tau5 } \\
\text { (dimen- } \\
\text { sionless) }\end{array}$ & $\begin{array}{c}\text { Station } \\
\text { no. }\end{array}$ & $\begin{array}{l}\text { Duration } \\
\text { mean } \\
\text { (hours) }\end{array}$ & $\begin{array}{l}\text { Duration } \\
\text { L-scale } \\
\text { (hours) }\end{array}$ & $\begin{array}{c}\text { Duration } \\
\text { L-CV } \\
\text { (dimen- } \\
\text { sionless) }\end{array}$ & $\begin{array}{l}\text { Duration } \\
\text { L-skew } \\
\text { (dimen- } \\
\text { sionless) }\end{array}$ & $\begin{array}{l}\text { Duration } \\
\text { L-kurtosis } \\
\text { (dimen- } \\
\text { sionless) }\end{array}$ & $\begin{array}{c}\text { Duration } \\
\text { Tau5 } \\
\text { (dimen- } \\
\text { sionless) }\end{array}$ \\
\hline 7718 & 13.793 & 8.2428 & 0.59762 & 0.42107 & 0.15831 & 0.11216 & 8910 & 6.7000 & 2.8895 & 0.43126 & 0.38251 & 0.22515 & 0.09757 \\
\hline 7745 & 13.298 & 7.4469 & .56001 & .38940 & .15328 & .09847 & 8911 & 8.1685 & 4.9629 & .60757 & .51030 & .24197 & .13700 \\
\hline 7922 & 6.1485 & 3.8311 & 62309 & .57421 & .28821 & .15436 & 8924 & 5.6029 & 3.3235 & .59318 & .58809 & .34105 & .18673 \\
\hline 7936 & 9.1892 & 5.4713 & .59541 & .47962 & .22009 & .12969 & 8929 & 16.167 & 10.134 & .62684 & .45735 & .19905 & .16100 \\
\hline 7943 & 9.4613 & 5.7164 & 60419 & .49421 & .22706 & .11972 & 8942 & 9.4877 & 5.6473 & .59523 & .45405 & .18307 & .11348 \\
\hline 7944 & 11.388 & 7.3157 & .64240 & .51912 & .24379 & .14850 & 8944 & 9.5264 & 5.7140 & .59981 & .47741 & .21577 & .12769 \\
\hline 7945 & 11.896 & 7.2831 & .61225 & .47342 & .20697 & .12216 & 8996 & 9.5126 & 5.6543 & .59440 & .49214 & .23634 & .13267 \\
\hline 7947 & 10.134 & 6.5660 & .64794 & .53910 & .26155 & .14911 & 9014 & 11.900 & 6.2105 & .52189 & .50028 & .40728 & .33709 \\
\hline 7948 & 8.8458 & 5.3603 & 60596 & .48297 & .19751 & .09651 & 9037 & 7.4946 & 4.6466 & 61999 & .56655 & .32058 & .20511 \\
\hline 7951 & 9.7563 & 5.5453 & .56838 & .45516 & .21623 & .12710 & 9106 & 8.9006 & 5.7625 & .64743 & .60311 & .37205 & .24397 \\
\hline 7953 & 10.600 & 6.6554 & .62787 & .53380 & .26283 & .13217 & 9107 & 7.8387 & 5.9484 & .75885 & .76363 & .52256 & .32725 \\
\hline 7981 & 9.5049 & 6.1399 & .64597 & .55981 & .29633 & .17683 & 9129 & 9.9897 & 6.1052 & .61115 & .44540 & .11783 & .01200 \\
\hline 7990 & 12.750 & 8.1957 & .64280 & .51964 & .25977 & .17386 & 9163 & 8.7466 & 5.2301 & .59796 & .47960 & .20544 & .10899 \\
\hline 7992 & 11.850 & 6.1079 & .51543 & .47810 & .24745 & .06474 & 9213 & 12.152 & 7.7627 & .63880 & .49968 & .23079 & .15351 \\
\hline 7997 & 9.0097 & 5.4875 & 60907 & .54002 & .30672 & .19013 & 9214 & 9.6786 & 6.2500 & .64576 & .67336 & .49663 & .33597 \\
\hline 7999 & 7.8235 & 4.7941 & .61278 & .48896 & .21209 & .18452 & 9222 & 12.668 & 7.3454 & .57981 & .42302 & .17931 & .11732 \\
\hline 8022 & 9.8333 & 6.4407 & 65498 & .54163 & .22810 & .08610 & 9248 & 9.9483 & 6.1666 & 61986 & .47696 & .16935 & .06549 \\
\hline 8023 & 7.6099 & 4.8182 & .63316 & .56283 & .28918 & .16382 & 9266 & 10.522 & 5.9629 & .56672 & .45689 & .21814 & .12999 \\
\hline 8047 & 8.6888 & 5.2735 & .60694 & .49400 & .22331 & .13138 & 9270 & 6.7516 & 4.3484 & 64406 & .59210 & .30807 & .16914 \\
\hline 8060 & 10.203 & 6.3041 & 61784 & .51647 & .26021 & .16097 & 9295 & 7.3608 & 4.6173 & .62728 & .52389 & .22168 & .12686 \\
\hline 8062 & 9.7000 & 6.1885 & 63799 & .55343 & .27954 & .13911 & 9304 & -- & -- & -- & -- & -- & -- \\
\hline 8068 & 7.2647 & 4.5071 & .62041 & .51397 & .21155 & .12084 & 9307 & 8.5413 & 5.0093 & .58648 & .47044 & .20106 & .09780 \\
\hline 8081 & 9.4090 & 5.9350 & 63078 & .52870 & .25649 & .14576 & 9328 & 10.152 & 6.6811 & .65812 & .57065 & .30294 & .17591 \\
\hline 8089 & 8.1316 & 4.6181 & .56792 & .42461 & .14393 & .06855 & 9329 & 16.167 & 10.061 & .62231 & .48825 & .23594 & .07957 \\
\hline 8221 & 12.737 & 6.6023 & .51837 & .37842 & .15197 & -.00134 & 9345 & -- & -- & -- & -- & -- & -- \\
\hline 8252 & 7.5228 & 4.6872 & .62307 & .54768 & .27144 & .14497 & 9363 & 11.000 & 6.8683 & .62439 & .49013 & .21108 & .11970 \\
\hline 8265 & 11.656 & 6.7816 & .58179 & .45380 & .19653 & .10131 & 9364 & 10.111 & 6.3368 & .62673 & .51109 & .23437 & .12862 \\
\hline 8289 & 8.9600 & 5.5698 & .62163 & .50918 & .22558 & .10981 & 9365 & 8.6129 & 5.4860 & .63695 & .60629 & .40709 & .28932 \\
\hline 8305 & 6.5071 & 4.0839 & .62760 & .56904 & .27892 & .14092 & 9371 & 10.765 & 6.5573 & 60912 & .47408 & .19437 & .09098 \\
\hline 8335 & 11.418 & 6.4791 & .56745 & .43538 & .20276 & .12671 & 9417 & 9.6249 & 5.6766 & .58978 & .47858 & .22944 & .13814 \\
\hline 8400 & 6.8107 & 4.4946 & .65993 & .61596 & .33569 & .19863 & 9419 & 11.386 & 6.7028 & .58868 & .45047 & .19394 & .11043 \\
\hline 8445 & 10.479 & 6.0758 & .57978 & .46373 & .21515 & .11756 & 9435 & 8.0411 & 5.2317 & .65062 & .59796 & .33846 & .18819 \\
\hline 8446 & 8.8435 & 5.3155 & 60106 & .47739 & .20076 & .11002 & 9491 & 9.6355 & 5.7288 & .59455 & .47554 & .21277 & .11568 \\
\hline 8451 & 9.6851 & 5.9367 & .61297 & .51726 & .24620 & .11206 & 9499 & 7.7442 & 4.8632 & .62798 & .53292 & .24107 & .12782 \\
\hline 8531 & 9.8673 & 5.7719 & .58495 & .46834 & .21725 & .12642 & 9522 & 20.636 & 10.491 & .50837 & .38648 & .20999 & .14298 \\
\hline 8541 & 10.128 & 5.6939 & .56221 & .45318 & .18936 & .06805 & 9527 & 7.3855 & 4.6124 & .62453 & .53061 & .23298 & .11720 \\
\hline 8544 & 10.340 & 6.2869 & 60799 & .49664 & .24418 & .14925 & 9532 & 8.4114 & 5.0621 & .60182 & .49003 & .22026 & .12903 \\
\hline 8545 & 15.125 & 7.9750 & .52727 & .24138 & .01995 & .07475 & 9544 & -- & -- & -- & -- & -- & -- \\
\hline 8563 & 8.4265 & 4.9176 & .58358 & .44757 & .18009 & .11697 & 9565 & 8.1710 & 4.9707 & .60833 & .51622 & .25584 & .15760 \\
\hline 8566 & 6.8100 & 4.2665 & .62650 & .56587 & .29267 & .17493 & 9570 & 6.7794 & 4.2716 & .63009 & .54831 & .24013 & .11429 \\
\hline 8583 & 8.0690 & 4.9977 & 61937 & .49671 & .19233 & .09936 & 9574 & 5.5000 & 3.1580 & .57418 & .45236 & .09372 & -.00688 \\
\hline 8584 & 8.4984 & 5.2073 & .61274 & .50356 & .22256 & .11549 & 9588 & 9.2440 & 6.0389 & .65328 & .55701 & .27301 & .15274 \\
\hline 8623 & 8.8539 & 5.3467 & 60389 & .48180 & .20736 & .11868 & 9665 & 9.6708 & 5.8174 & .60155 & .48324 & .21908 & .12104 \\
\hline 8625 & 10.299 & 5.7811 & .56135 & .44541 & .20700 & .11822 & 9715 & 9.0195 & 5.2457 & .58160 & .45561 & .19399 & .10835 \\
\hline 8630 & 7.4431 & 4.3885 & .58961 & .49430 & .22283 & .11628 & 9729 & 10.296 & 5.9471 & .57761 & .44302 & .18956 & .10897 \\
\hline 8631 & 9.3262 & 5.7054 & .61176 & .52856 & .28073 & .16391 & 9772 & 9.3719 & 5.7859 & .61737 & .52811 & .27263 & .16384 \\
\hline 8646 & 9.2841 & 5.3566 & .57696 & .46809 & .22545 & .13507 & 9814 & 11.438 & 6.4798 & .56654 & .44001 & .19640 & .13243 \\
\hline 8647 & 7.4653 & 4.5889 & .61471 & .50775 & .21066 & .11419 & 9815 & 10.912 & 6.4801 & .59387 & .47870 & .23620 & .14591 \\
\hline 8677 & 11.752 & 6.3624 & .54140 & .41049 & .17534 & .08731 & 9816 & 7.5493 & 4.5408 & 60149 & .48687 & .19048 & .09160 \\
\hline 8696 & 9.6957 & 4.7589 & .49083 & .30897 & .16718 & .17324 & 9817 & 8.1471 & 4.9514 & 60776 & .49740 & .21874 & .12572 \\
\hline 8743 & 10.447 & 6.0870 & .58266 & .44984 & .19759 & .11456 & 9829 & 7.3598 & 4.6614 & .63336 & .56609 & .29225 & .16879 \\
\hline 8761 & 6.7911 & 4.1995 & .61838 & .54288 & .25659 & .14182 & 9830 & 6.7397 & 4.1821 & .62052 & .57847 & .33871 & .23639 \\
\hline 8778 & 9.4667 & 5.6282 & .59452 & .45838 & .19054 & .11547 & 9858 & 8.0295 & 4.6981 & .58511 & .50430 & .25813 & .14514 \\
\hline 8845 & 10.059 & 6.2795 & .62428 & .50057 & .22646 & .13695 & 9893 & 8.8778 & 5.3215 & .59942 & .48797 & .22590 & .13254 \\
\hline 8859 & 11.269 & 6.6114 & .58667 & .45715 & .20576 & .11335 & 9916 & 9.5957 & 5.4949 & .57264 & .42063 & .15884 & .09340 \\
\hline 8898 & 10.775 & 6.0791 & .56421 & .44945 & .21415 & .12068 & 9976 & 8.5873 & 5.4181 & .63094 & .53838 & .26479 & .15287 \\
\hline 8908 & 12.452 & 7.1462 & .57392 & .40579 & .15649 & .11230 & & & & & & & \\
\hline
\end{tabular}


Appendix 4-3.5. L-moments of storm duration defined by 24-hour minimum interevent time for hourly rainfall stations in Texas.

$[--$, not available $]$

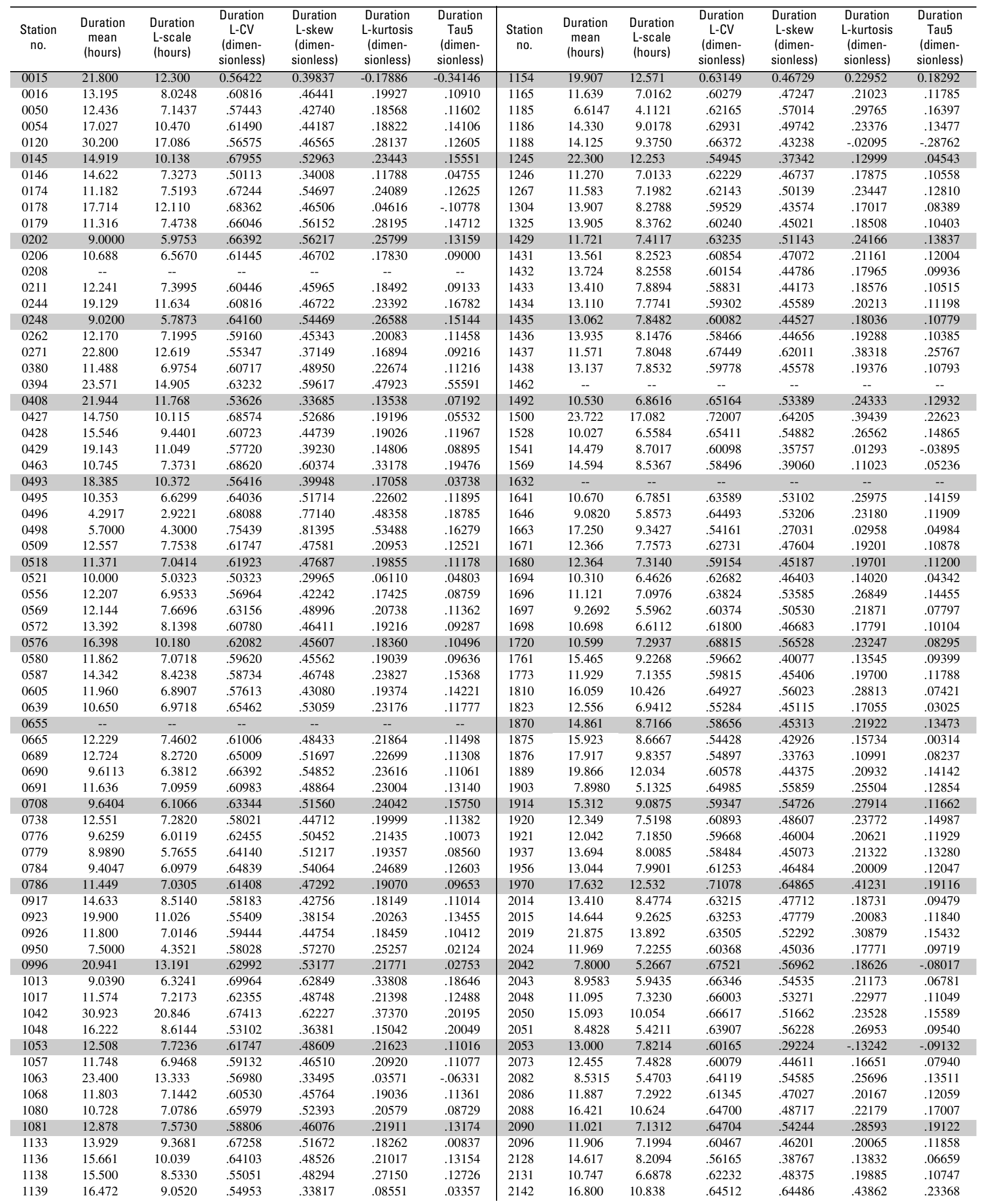


232 Statistical Characteristics of Storm Interevent Time, Depth, and Duration for Eastern New Mexico, Oklahoma, and Texas

Appendix 4-3.5. L-moments of storm duration defined by 24-hour minimum interevent time for hourly rainfall stations in TexasContinued.

\begin{tabular}{|c|c|c|c|c|c|c|c|c|c|c|c|c|c|}
\hline $\begin{array}{c}\text { Station } \\
\text { no. }\end{array}$ & $\begin{array}{l}\text { Duration } \\
\text { mean } \\
\text { (hours) }\end{array}$ & $\begin{array}{l}\text { Duration } \\
\text { L-scale } \\
\text { (hours) }\end{array}$ & $\begin{array}{l}\text { Duration } \\
\text { L-CV } \\
\text { (dimen- } \\
\text { sionless) }\end{array}$ & $\begin{array}{c}\text { Duration } \\
\text { L-skew } \\
\text { (dimen- } \\
\text { sionless) }\end{array}$ & $\begin{array}{l}\text { Duration } \\
\text { L-kurtosis } \\
\text { (dimen- } \\
\text { sionless) }\end{array}$ & $\begin{array}{c}\text { Duration } \\
\text { Tau5 } \\
\text { (dimen- } \\
\text { sionless) }\end{array}$ & $\begin{array}{c}\text { Station } \\
\text { no. }\end{array}$ & $\begin{array}{c}\text { Duration } \\
\text { mean } \\
\text { (hours) }\end{array}$ & $\begin{array}{l}\text { Duration } \\
\text { L-scale } \\
\text { (hours) }\end{array}$ & $\begin{array}{c}\text { Duration } \\
\text { L-CV } \\
\text { (dimen- } \\
\text { sionless) }\end{array}$ & $\begin{array}{l}\text { Duration } \\
\text { L-skew } \\
\text { (dimen- } \\
\text { sionless) }\end{array}$ & $\begin{array}{l}\text { Duration } \\
\text { L-kurtosis } \\
\text { (dimen- } \\
\text { sionless) }\end{array}$ & $\begin{array}{c}\text { Duration } \\
\text { Tau5 } \\
\text { (dimen- } \\
\text { sionless) }\end{array}$ \\
\hline 2160 & 20.211 & 11.620 & 0.57494 & 0.26368 & -0.09232 & -0.10933 & 3463 & 11.810 & 7.1614 & 0.60641 & 0.47665 & 0.24077 & 0.18367 \\
\hline 2206 & 12.484 & 7.5584 & 60544 & .46891 & .19846 & .10080 & 3476 & 12.050 & 7.3352 & 60871 & .48114 & .21603 & .11342 \\
\hline 2238 & 13.303 & 8.3204 & .62545 & .48337 & .21786 & .13251 & 3485 & 16.941 & 9.1471 & .53993 & .43023 & .21291 & .01224 \\
\hline 2240 & 14.000 & 8.5875 & .61339 & .41467 & .13003 & .10933 & 3507 & 11.354 & 7.2449 & .63811 & .49174 & .19673 & .11010 \\
\hline 2242 & 13.488 & 7.8273 & .58030 & .41788 & .16186 & .08996 & 3546 & 12.980 & 7.8117 & 60184 & .45474 & .20115 & .12672 \\
\hline 2244 & 13.128 & 7.9151 & .60291 & .44590 & .18307 & .11183 & 3547 & 10.022 & 6.3460 & .63320 & .52726 & .25967 & .15155 \\
\hline 2247 & 16.683 & 9.7073 & .58187 & .42211 & .17993 & .11545 & 3579 & 15.161 & 9.2041 & 60708 & .46832 & .21039 & .13063 \\
\hline 2309 & 13.393 & 8.0254 & .59923 & .46336 & .21208 & .11950 & 3642 & 12.230 & 7.2619 & .59378 & .45057 & .19786 & .11974 \\
\hline 2312 & 12.103 & 7.2858 & 60199 & .45475 & .20594 & .14912 & 3646 & 12.132 & 7.0622 & .58210 & .46175 & .21897 & .12467 \\
\hline 2334 & 11.302 & 6.1139 & .54097 & .27401 & -.03246 & -.03029 & 3668 & 21.000 & 12.550 & .59762 & .47268 & .14813 & -.00934 \\
\hline 2336 & 9.9485 & 6.1370 & .61688 & .49881 & .23316 & .14565 & 3673 & 22.211 & 11.994 & .54002 & .25322 & -.04848 & -.06399 \\
\hline 2354 & 13.227 & 8.4134 & .63607 & .49627 & .21436 & .12099 & 3686 & 11.181 & 6.9128 & .61824 & .47218 & .19231 & .11003 \\
\hline 2355 & 18.615 & 12.406 & .66645 & .49796 & .15783 & .00604 & 3691 & 10.987 & 6.7033 & 61011 & 46277 & .18334 & .10048 \\
\hline 2357 & 12.941 & 8.3516 & .64538 & .49752 & .19284 & .07851 & 3734 & 21.895 & 13.930 & .63622 & .46585 & .18275 & .04419 \\
\hline 2360 & 13.049 & 8.1451 & .62419 & .47424 & .19423 & .10992 & 3771 & 10.927 & 6.7868 & .62113 & .45993 & .15680 & .07541 \\
\hline 2361 & 10.974 & 7.2167 & .65765 & .53592 & .23881 & .12204 & 3789 & 11.643 & 8.2797 & .71114 & .61420 & .32740 & .19795 \\
\hline 2394 & 12.643 & 7.3942 & .58483 & .44223 & .19080 & .10651 & 3826 & 11.283 & 6.6858 & .59253 & .48657 & .24131 & .13846 \\
\hline 2404 & 11.853 & 7.0867 & .59789 & .44806 & .18088 & .09946 & 3831 & 12.979 & 7.8404 & 60409 & .45265 & .17720 & .08191 \\
\hline 2415 & 12.579 & 7.2598 & .57712 & .44117 & .20298 & .12361 & 3841 & 9.4812 & 5.8167 & 61350 & .49394 & .21323 & .10923 \\
\hline 2462 & 12.177 & 7.4686 & .61333 & .47615 & .20816 & .11265 & 3871 & 12.536 & 7.6051 & 60665 & .47609 & .20846 & .10478 \\
\hline 2528 & 13.134 & 8.3636 & .63679 & .48493 & .17912 & .04957 & 3884 & 10.778 & 5.4183 & .50273 & .35706 & .16466 & .06303 \\
\hline 2617 & 11.544 & 6.5881 & .57069 & .42534 & .16317 & .06374 & 3941 & 15.455 & 9.1030 & .58902 & .44492 & .21828 & .13191 \\
\hline 2619 & 11.495 & 7.3095 & 63589 & .50823 & .23549 & .14091 & 3963 & -- & -- & -- & -- & -- & -- \\
\hline 2621 & 10.531 & 6.5196 & 61906 & .48413 & .19440 & .09211 & 4040 & 10.416 & 6.2242 & .59758 & .48617 & .23562 & .14352 \\
\hline 2675 & 11.851 & 7.3243 & .61801 & .48417 & .22100 & .14014 & 4058 & 16.333 & 9.9412 & 60864 & .45537 & .13240 & -.01603 \\
\hline 2676 & 10.938 & 7.1817 & .65658 & .52898 & .23391 & .13271 & 4098 & 8.8108 & 5.6716 & .64372 & .53486 & .23271 & .11120 \\
\hline 2679 & 10.794 & 6.9301 & .64206 & .51449 & .22556 & .12325 & 4100 & 10.548 & 6.2195 & .58961 & .45168 & .19070 & .10579 \\
\hline 2715 & 11.261 & 6.5805 & .58435 & .45131 & .19758 & .11093 & 4137 & 10.396 & 6.3215 & .60806 & .45210 & .16365 & .09410 \\
\hline 2744 & 10.727 & 6.8653 & 63999 & .53026 & .25716 & .14225 & 4191 & 11.568 & 7.2114 & 62339 & .48296 & .19958 & .10546 \\
\hline 2758 & 13.809 & 9.0435 & .65492 & .53924 & .25848 & .12005 & 4256 & -- & -- & -- & -- & -- & -- \\
\hline 2794 & 6.7273 & 3.9636 & .58919 & .51376 & .30810 & .29434 & 4257 & 12.313 & 7.4677 & .60647 & .45309 & .18858 & .11971 \\
\hline 2797 & 10.632 & 6.6592 & .62633 & .49143 & .19938 & .09812 & 4258 & 15.597 & 9.6172 & 61661 & .42797 & .14674 & .09518 \\
\hline 2811 & 10.962 & 7.0960 & .64735 & .52423 & .23504 & .12492 & 4278 & 11.069 & 6.6108 & .59723 & .47952 & .22084 & .11595 \\
\hline 2813 & 10.048 & 6.9714 & .69384 & .62460 & .36439 & .22678 & 4299 & 7.3750 & 4.6705 & .63329 & .56243 & .27171 & .11505 \\
\hline 2814 & 9.4000 & 6.6000 & .70213 & .57176 & .17471 & -.01699 & 4300 & 15.988 & 9.7143 & 60761 & .44803 & .19426 & .12553 \\
\hline 2815 & 8.8667 & 5.5050 & .62087 & .49063 & .18697 & .08429 & 4305 & 16.785 & 10.023 & .59717 & .42283 & .17126 & .11385 \\
\hline 2818 & 10.061 & 6.6029 & .65631 & .57960 & .33656 & .23534 & 4307 & 18.143 & 11.332 & .62459 & .45062 & .19155 & .13690 \\
\hline 2986 & 14.723 & 8.6591 & .58813 & .43846 & .18449 & .09395 & 4309 & 13.007 & 7.8566 & .60405 & .46658 & .20166 & .10947 \\
\hline 3005 & 10.522 & 6.4608 & .61405 & .47935 & .20569 & .11963 & 4311 & 13.434 & 8.1389 & .60585 & .46763 & .20844 & .11670 \\
\hline 3033 & 7.5798 & 4.6221 & 60980 & .55363 & .28719 & .13021 & 4313 & 14.897 & 8.2952 & .55685 & .36733 & .10359 & .03650 \\
\hline 3034 & -- & -- & -- & -- & -- & -- & 4319 & 15.608 & 9.0250 & .57822 & .42461 & .17597 & .09380 \\
\hline 3047 & 25.778 & 16.523 & 64097 & .51567 & .26389 & .13761 & 4329 & 13.044 & 7.9577 & .61005 & .46795 & .20063 & .10986 \\
\hline 3103 & 9.6296 & 6.1083 & 63432 & .48541 & .16558 & .06357 & 4331 & -- & -- & -- & -- & -- & -- \\
\hline 3133 & 12.441 & 7.3883 & .59388 & .45776 & .20509 & .11452 & 4375 & 10.078 & 6.5568 & .65060 & .53405 & .24488 & .13838 \\
\hline 3156 & 13.216 & 8.2068 & 62100 & .47171 & .20817 & .13638 & 4392 & 15.501 & 8.9452 & .57706 & .42976 & .19688 & .13584 \\
\hline 3171 & 12.250 & 7.2916 & .59521 & .45991 & .20192 & .10879 & 4425 & 9.1114 & 6.0490 & .66389 & .56464 & .26590 & .14009 \\
\hline 3189 & 11.752 & 7.7108 & .65614 & .53475 & .25589 & .15680 & 4440 & 12.967 & 7.5227 & .58013 & .45541 & .21038 & .12397 \\
\hline 3260 & 11.049 & 6.6298 & 60004 & .45034 & .16876 & .07770 & 4476 & 10.992 & 6.6660 & .60644 & .45708 & .17887 & .09952 \\
\hline 3267 & 12.841 & 8.1392 & 63385 & .50193 & .22157 & .11226 & 4498 & 7.2727 & 4.7455 & .65250 & .59132 & .27842 & .01916 \\
\hline 3270 & 8.5756 & 5.5107 & .64260 & .53332 & .22991 & .11526 & 4517 & 12.320 & 7.3858 & .59950 & .45528 & .19207 & .10602 \\
\hline 3272 & 10.000 & 6.0662 & .60662 & .39055 & .01766 & -.06023 & 4520 & 10.559 & 6.5368 & .61909 & .45771 & .15303 & .07733 \\
\hline 3277 & 10.692 & 7.1538 & 66906 & .53079 & .21701 & .12708 & 4525 & 26.444 & 19.176 & .72516 & .60395 & .30138 & .09228 \\
\hline 3278 & 9.4272 & 6.1691 & .65439 & .57075 & .29475 & .15847 & 4563 & 10.520 & 7.0933 & .67427 & .66676 & .52662 & .46994 \\
\hline 3280 & 7.9225 & 5.1187 & 64610 & .61101 & .36389 & .20705 & 4570 & 10.812 & 6.8129 & .63014 & .50258 & .22402 & .12740 \\
\hline 3281 & 5.1905 & 3.4158 & 65809 & .67968 & .40834 & .23356 & 4577 & 12.201 & 7.4237 & .60843 & .46830 & .21016 & .13176 \\
\hline 3283 & 14.636 & 8.7640 & .59879 & .44452 & .19089 & .11616 & 4591 & 14.049 & 8.1827 & .58245 & .44412 & .20463 & .12513 \\
\hline 3284 & 12.170 & 7.4875 & .61524 & .47400 & .20838 & .12581 & 4670 & 10.600 & 6.5888 & .62157 & .48840 & .20756 & .11368 \\
\hline 3285 & 11.026 & 6.8068 & .61735 & .46813 & .18535 & .10677 & 4671 & 12.410 & 7.8793 & .63492 & .48432 & .20519 & .12682 \\
\hline 3329 & 12.824 & 7.7649 & 60548 & .45846 & .19559 & .11511 & 4679 & 11.457 & 7.0129 & .61209 & .45389 & .17122 & .09993 \\
\hline 3335 & 13.874 & 7.9696 & .57444 & .42355 & .18643 & .12213 & 4696 & 13.400 & 9.5333 & .71144 & .55420 & .14461 & -.14161 \\
\hline 3370 & 11.537 & 6.7435 & .58452 & .44814 & .19061 & .10387 & 4703 & 9.9417 & 6.3122 & .63492 & .51458 & .24053 & .16197 \\
\hline 3410 & 10.210 & 6.3677 & .62365 & .49376 & .21055 & .11351 & 4704 & 15.654 & 9.1707 & .58584 & .42281 & .18184 & .12011 \\
\hline 3415 & 11.189 & 6.8179 & 60933 & .46464 & .19242 & .11217 & 4731 & 15.760 & 8.8690 & .56274 & .35025 & .12797 & .14000 \\
\hline 3430 & 14.709 & 8.9739 & 61010 & .45762 & .20075 & .12436 & 4792 & 10.384 & 6.5500 & .63076 & .49942 & .21417 & .12237 \\
\hline 3431 & 19.168 & 12.059 & .62913 & .44575 & .17833 & .12640 & 4819 & 11.858 & 7.2036 & .60750 & .46095 & .20176 & .12980 \\
\hline 3441 & 13.625 & 8.9355 & .65582 & .51002 & .21830 & .11279 & 4852 & 6.6667 & 4.7524 & .71286 & .66795 & .28395 & -.05788 \\
\hline 3442 & 11.575 & 6.7861 & .58626 & .46534 & .21266 & .11047 & 4866 & 11.882 & 7.1922 & .60530 & .46688 & .20438 & .11735 \\
\hline 3446 & 9.1163 & 5.6799 & .62304 & .53743 & .27383 & .14748 & 4876 & 11.800 & 7.3656 & .62421 & .47726 & .19872 & .11614 \\
\hline 3460 & 14.516 & 8.2344 & .56726 & .37578 & .15762 & .16248 & 4878 & 15.023 & 8.8454 & .58881 & .43357 & .18526 & .11342 \\
\hline 3462 & 12.492 & 7.2269 & .57854 & .43634 & .18835 & .09883 & 4880 & 10.568 & 6.6447 & .62877 & .51329 & .23995 & .12952 \\
\hline
\end{tabular}


Appendix 4-3.5. L-moments of storm duration defined by 24-hour minimum interevent time for hourly rainfall stations in TexasContinued.

\begin{tabular}{|c|c|c|c|c|c|c|c|c|c|c|c|c|c|}
\hline $\begin{array}{c}\text { Station } \\
\text { no. }\end{array}$ & $\begin{array}{l}\text { Duration } \\
\text { mean } \\
\text { (hours) }\end{array}$ & $\begin{array}{l}\text { Duration } \\
\text { L-scale } \\
\text { (hours) }\end{array}$ & $\begin{array}{c}\text { Duration } \\
\text { L-CV } \\
\text { (dimen- } \\
\text { sionless) }\end{array}$ & $\begin{array}{l}\text { Duration } \\
\text { L-skew } \\
\text { (dimen- } \\
\text { sionless) }\end{array}$ & $\begin{array}{l}\text { Duration } \\
\text { L-kurtosis } \\
\text { (dimen- } \\
\text { sionless) }\end{array}$ & $\begin{array}{l}\text { Duration } \\
\text { Tau5 } \\
\text { (dimen- } \\
\text { sionless) }\end{array}$ & $\begin{array}{c}\text { Station } \\
\text { no. }\end{array}$ & $\begin{array}{c}\text { Duration } \\
\text { mean } \\
\text { (hours) }\end{array}$ & $\begin{array}{l}\text { Duration } \\
\text { L-scale } \\
\text { (hours) }\end{array}$ & $\begin{array}{c}\text { Duration } \\
\text { L-CV } \\
\text { (dimen- } \\
\text { sionless) }\end{array}$ & $\begin{array}{l}\text { Duration } \\
\text { L-skew } \\
\text { (dimen- } \\
\text { sionless) }\end{array}$ & $\begin{array}{l}\text { Duration } \\
\text { L-kurtosis } \\
\text { (dimen- } \\
\text { sionless) }\end{array}$ & $\begin{array}{c}\text { Duration } \\
\text { Tau5 } \\
\text { (dimen- } \\
\text { sionless) }\end{array}$ \\
\hline 4920 & 10.804 & 6.8349 & 0.63263 & 0.50270 & 0.21827 & 0.11779 & 5957 & 10.375 & 6.4798 & 0.62457 & 0.49695 & 0.21859 & 0.12394 \\
\hline 4934 & 8.6667 & 5.3333 & .61538 & .35000 & -.12500 & .00000 & 5958 & 13.248 & 7.8082 & .58936 & .43659 & .16866 & .08484 \\
\hline 4972 & 11.399 & 6.9202 & .60709 & .46930 & .20061 & .10854 & 5973 & 15.129 & 9.0669 & .59929 & .47698 & .25484 & .17173 \\
\hline 4973 & 12.682 & 7.1155 & .56109 & .42064 & .18894 & .12047 & 5996 & 11.742 & 7.1142 & .60587 & .46050 & .19235 & .10843 \\
\hline 4974 & 11.283 & 7.0003 & .62040 & .50237 & .24005 & .13697 & 6017 & 10.357 & 6.5742 & .63475 & .52523 & .24416 & .12300 \\
\hline 4975 & 11.219 & 6.8023 & .60631 & .45214 & .17048 & .08728 & 6024 & 14.651 & 8.2057 & .56008 & .37026 & .14049 & .10925 \\
\hline 4978 & 11.333 & 7.4598 & .65822 & .55337 & .30058 & .21935 & 6050 & 9.3684 & 3.2807 & .35019 & .15445 & .16876 & .08362 \\
\hline 4979 & 21.188 & 13.546 & .63933 & .58597 & .32781 & .09382 & 6104 & 12.373 & 8.2254 & .66478 & .54055 & .24961 & .13461 \\
\hline 4982 & 10.886 & 6.3800 & .58609 & .45327 & .18982 & .10078 & 6108 & 11.953 & 7.2336 & .60517 & .45861 & .19487 & .11645 \\
\hline 5018 & 11.857 & 6.8753 & .57983 & .46287 & .21034 & .10986 & 6136 & 9.5296 & 6.1579 & .64619 & .52795 & .22496 & .10634 \\
\hline 5048 & 9.3828 & 6.0250 & .64213 & .52723 & .23061 & .11810 & 6166 & 11.532 & 6.6709 & .57844 & .39874 & .10479 & .03466 \\
\hline 5049 & 6.3068 & 3.8593 & .61193 & .53462 & .24436 & .15209 & 6176 & 13.666 & 8.1366 & .59541 & .45553 & .22281 & .16775 \\
\hline 5056 & 10.000 & 5.1000 & .51000 & .09804 & -.27451 & -.58824 & 6177 & 12.395 & 7.6080 & .61379 & .47643 & .21532 & .13021 \\
\hline 5057 & 13.891 & 8.8604 & .63788 & .48774 & .20271 & .10820 & 6210 & 11.223 & 6.7907 & .60506 & .45627 & .17749 & .08783 \\
\hline 5060 & 14.774 & 9.6120 & .65061 & .53306 & .27126 & .16789 & 6211 & 15.604 & 9.7919 & .62751 & .47012 & .20255 & .12824 \\
\hline 5081 & 15.081 & 8.5740 & .56853 & .42259 & .19576 & .12337 & 6270 & 12.185 & 7.3358 & .60201 & .44513 & .18098 & .11328 \\
\hline 5094 & 11.162 & 6.7864 & .60797 & .46191 & .18968 & .11203 & 6275 & -- & -- & -- & -- & -- & -- \\
\hline 5113 & 12.114 & 7.6350 & .63028 & .49009 & .20973 & .11662 & 6276 & 16.250 & 9.8079 & .60356 & .42706 & .12420 & -.00234 \\
\hline 5114 & -- & -- & -- & -- & -- & -- & 6335 & 12.498 & 7.2461 & .57980 & .44627 & .20199 & .11606 \\
\hline 5123 & 8.3571 & 4.9945 & .59763 & .46755 & .16192 & .02790 & 6434 & 12.882 & 5.9853 & .46461 & .33071 & .10881 & -.02344 \\
\hline 5192 & 11.334 & 6.7381 & .59448 & .44652 & .17547 & .08931 & 6504 & 9.9856 & 6.3534 & .63626 & .50733 & .20975 & .10457 \\
\hline 5193 & 12.761 & 7.7790 & .60960 & .46099 & .19248 & .10973 & 6558 & 14.739 & 8.3755 & .56825 & .28551 & -.04760 & -.05733 \\
\hline 5224 & 13.571 & 8.3027 & .61178 & .47427 & .23143 & .15942 & 6615 & 9.3015 & 6.1263 & .65864 & .55013 & .24585 & .12100 \\
\hline 5228 & 11.669 & 7.5193 & .64436 & .52485 & .25427 & .15193 & 6660 & 16.388 & 9.6485 & .58874 & .39485 & .10848 & .01876 \\
\hline 5235 & 17.478 & 9.5375 & .54568 & .24670 & -.03499 & -.00619 & 6663 & 17.913 & 10.529 & .58778 & .34686 & .04890 & .02044 \\
\hline 5247 & 10.017 & 6.3020 & .62916 & .51079 & .23153 & .12854 & 6734 & 11.586 & 7.0088 & .60495 & .46869 & .20494 & .12251 \\
\hline 5258 & 12.589 & 7.3235 & .58173 & .44233 & .19572 & .11568 & 6736 & 9.1234 & 5.8650 & .64286 & .53656 & .24189 & .11758 \\
\hline 5303 & 12.372 & 7.4916 & .60552 & .48189 & .21103 & .10172 & 6740 & 24.500 & 16.348 & .66729 & .55885 & .34322 & .28962 \\
\hline 5312 & 9.7376 & 6.3418 & .65126 & .54047 & .24921 & .13412 & 6750 & 15.767 & 10.366 & .65743 & .47896 & .16423 & .08112 \\
\hline 5341 & 19.105 & 13.485 & .70585 & .63257 & .38026 & .20313 & 6757 & 13.311 & 8.0450 & .60438 & .46065 & .20691 & .12728 \\
\hline 5342 & -- & -- & -- & -- & -- & -- & 6775 & 12.266 & 7.3348 & .59797 & .44578 & .17553 & .09675 \\
\hline 5348 & 11.969 & 7.3131 & .61101 & .47085 & .21352 & .13984 & 6776 & 10.180 & 6.4484 & .63343 & .51288 & .22881 & .12137 \\
\hline 5358 & 10.387 & 6.3356 & .60993 & .50038 & .23631 & .11887 & 6788 & 11.325 & 6.0543 & .53462 & .39496 & .15745 & .07440 \\
\hline 5398 & 13.937 & 8.2359 & .59096 & .45317 & .20783 & .12594 & 6792 & 8.3521 & 5.4653 & .65436 & .56214 & .25861 & .12097 \\
\hline 5410 & 9.3143 & 5.9973 & .64388 & .53637 & .24203 & .11710 & 6794 & 29.438 & 16.387 & .55669 & .37104 & .16245 & .07747 \\
\hline 5411 & 12.536 & 7.7249 & .61620 & .47501 & .20602 & .11803 & 6834 & 10.925 & 6.6520 & .60887 & .46146 & .18429 & .10501 \\
\hline 5424 & 20.407 & 12.860 & .63018 & .46670 & .21592 & .13863 & 6893 & 7.8534 & 5.2235 & .66513 & .59639 & .30935 & .17560 \\
\hline 5429 & 13.018 & 8.0050 & .61491 & .47106 & 19899 & .10966 & 6935 & 9.1327 & 5.9193 & .64815 & .54241 & .24444 & .11806 \\
\hline 5431 & 17.357 & 8.3571 & .48148 & .24129 & .13095 & .19945 & 6981 & 12.709 & 7.7909 & .61303 & .47983 & .20836 & .11622 \\
\hline 5461 & 12.804 & 7.7440 & .60482 & .48375 & .23517 & .13510 & 7020 & 16.047 & 9.2717 & .57780 & .41544 & .16942 & .08883 \\
\hline 5463 & 10.404 & 6.4464 & .61960 & .47325 & .17741 & .08599 & 7060 & 9.5178 & 6.1032 & .64125 & .52259 & .22435 & .11212 \\
\hline 5471 & 14.000 & 9.1583 & .65417 & .41531 & -.00786 & -.10299 & 7066 & 12.667 & 7.4815 & .59064 & .44528 & .19342 & .11518 \\
\hline 5477 & 17.294 & 14.000 & .80952 & .76324 & .56242 & .44877 & 7074 & 9.1268 & 5.8966 & .64607 & .54499 & .25542 & .13044 \\
\hline 5528 & 10.907 & 6.4131 & .58799 & .45012 & .18162 & .08637 & 7097 & 12.837 & 7.6394 & .59511 & .44219 & .18765 & .12534 \\
\hline 5579 & -- & -- & -- & -- & -- & -- & 7116 & 11.158 & 6.6425 & .59532 & .45719 & .18605 & .09492 \\
\hline 5580 & 15.400 & 9.3737 & .60868 & .41475 & .05374 & -.09682 & 7140 & 12.472 & 7.8016 & .62551 & .49039 & .21551 & .11851 \\
\hline 5589 & 9.2128 & 5.8220 & .63195 & .51389 & .21786 & .10729 & 7173 & 16.616 & 10.139 & .61017 & .43198 & .15664 & .08542 \\
\hline 5590 & 11.652 & 7.2855 & .62523 & .49180 & .20053 & .09088 & 7174 & 16.091 & 9.7324 & .60482 & .44207 & .18983 & .12500 \\
\hline 5591 & 9.8899 & 6.2736 & .63435 & .53946 & .26156 & .12852 & 7213 & 12.635 & 7.8278 & .61952 & .48701 & .21860 & .12234 \\
\hline 5592 & 10.154 & 6.5552 & .64561 & .56576 & .29610 & .15840 & 7243 & 11.035 & 6.9090 & .62609 & .48494 & .19861 & .10866 \\
\hline 5594 & 7.1391 & 4.5727 & .64052 & .59981 & .31444 & .12687 & 7262 & 9.5375 & 6.8983 & .72328 & .64447 & .32687 & .14005 \\
\hline 5595 & -- & -- & -- & -- & -- & -- & 7274 & 9.3316 & 5.5965 & .59973 & .48421 & .22894 & .14029 \\
\hline 5596 & 9.5766 & 6.4120 & .66955 & .55581 & .24011 & .11202 & 7300 & 10.345 & 6.1857 & .59794 & .47529 & .21297 & .11773 \\
\hline 5600 & 11.222 & 7.6129 & .67840 & .60243 & .32779 & .16538 & 7311 & 9.5600 & 5.9000 & .61715 & .46824 & .15422 & .03007 \\
\hline 5618 & 17.458 & 10.857 & .62187 & .52578 & .31368 & .24785 & 7363 & 20.824 & 13.860 & .66561 & .55438 & .29663 & .14813 \\
\hline 5650 & 10.294 & 4.8971 & .47571 & .41141 & .36208 & .27166 & 7422 & 12.262 & 7.6803 & .62634 & .48639 & .21234 & .12427 \\
\hline 5656 & 9.6841 & 6.2338 & .64372 & .51037 & .19783 & .09179 & 7431 & 9.0284 & 5.6451 & .62525 & .52446 & .24902 & .13558 \\
\hline 5658 & 11.171 & 6.7868 & .60756 & .49534 & .23488 & .12393 & 7481 & 8.8917 & 5.7597 & .64776 & .56362 & .28645 & .15445 \\
\hline 5661 & 11.718 & 8.0225 & .68461 & .56307 & .25962 & .13942 & 7497 & 11.302 & 7.1314 & .63097 & .48811 & .20692 & .13142 \\
\hline 5666 & 12.929 & 8.2751 & .64007 & .48374 & .16948 & .05415 & 7498 & 10.686 & 6.7921 & .63562 & .49061 & .19036 & .10226 \\
\hline 5695 & 11.846 & 7.1720 & .60545 & .46384 & .20158 & .11979 & 7499 & 10.545 & 6.6032 & .62616 & .48500 & .19476 & .10668 \\
\hline 5742 & 16.929 & 10.005 & .59104 & .41571 & .25116 & .34232 & 7531 & 14.172 & 8.5810 & .60547 & .45296 & .17125 & .05605 \\
\hline 5766 & 15.652 & 5.9209 & .37828 & .09079 & .11644 & .15491 & 7534 & 11.026 & 6.9357 & .62905 & .50279 & .22619 & .12610 \\
\hline 5770 & 10.615 & 6.5445 & .61655 & .47665 & .19155 & .09914 & 7556 & 10.520 & 6.4537 & .61344 & .46503 & .17405 & .08587 \\
\hline 5775 & 5.0833 & 2.8712 & .56483 & .48496 & .17678 & .04661 & 7594 & 12.071 & 7.4299 & .61553 & .48885 & .22238 & .11767 \\
\hline 5779 & 13.667 & 8.6928 & .63606 & .49173 & .23684 & .16165 & 7596 & 10.047 & 6.1114 & .60826 & .48662 & .20881 & .10284 \\
\hline 5840 & 12.410 & 7.2758 & .58631 & .45424 & .19667 & .09726 & 7608 & 12.081 & 7.3928 & .61192 & .46622 & .19665 & .11310 \\
\hline 5890 & 11.356 & 7.0317 & .61922 & .48962 & .21576 & .11716 & 7622 & 9.0000 & 5.9591 & .66212 & .53253 & .17941 & .03005 \\
\hline 5891 & 8.7552 & 5.3529 & .61139 & .51399 & .24941 & .14694 & 7700 & 14.887 & 8.6210 & .57910 & .42683 & .18144 & .10529 \\
\hline 5897 & 11.324 & 6.9615 & .61477 & .45086 & .15783 & .08570 & 7706 & 11.165 & 7.0297 & .62962 & .49423 & .21017 & .11435 \\
\hline
\end{tabular}


Appendix 4-3.5. L-moments of storm duration defined by 24-hour minimum interevent time for hourly rainfall stations in TexasContinued.

\begin{tabular}{|c|c|c|c|c|c|c|c|c|c|c|c|c|c|}
\hline $\begin{array}{c}\text { Station } \\
\text { no. }\end{array}$ & $\begin{array}{l}\text { Duration } \\
\text { mean } \\
\text { (hours) }\end{array}$ & $\begin{array}{l}\text { Duration } \\
\text { L-scale } \\
\text { (hours) }\end{array}$ & $\begin{array}{l}\text { Duration } \\
\text { L-CV } \\
\text { (dimen- } \\
\text { sionless) }\end{array}$ & $\begin{array}{l}\text { Duration } \\
\text { L-skew } \\
\text { (dimen- } \\
\text { sionless) }\end{array}$ & $\begin{array}{l}\text { Duration } \\
\text { L-kurtosis } \\
\text { (dimen- } \\
\text { sionless) }\end{array}$ & $\begin{array}{l}\text { Duration } \\
\text { Tau5 } \\
\text { (dimen- } \\
\text { sionless) }\end{array}$ & $\begin{array}{c}\text { Station } \\
\text { no. }\end{array}$ & $\begin{array}{c}\text { Duration } \\
\text { mean } \\
\text { (hours) }\end{array}$ & $\begin{array}{l}\text { Duration } \\
\text { L-scale } \\
\text { (hours) }\end{array}$ & $\begin{array}{l}\text { Duration } \\
\text { L-CV } \\
\text { (dimen- } \\
\text { sionless) }\end{array}$ & $\begin{array}{l}\text { Duration } \\
\text { L-skew } \\
\text { (dimen- } \\
\text { sionless) }\end{array}$ & $\begin{array}{l}\text { Duration } \\
\text { L-kurtosis } \\
\text { (dimen- } \\
\text { sionless) }\end{array}$ & $\begin{array}{c}\text { Duration } \\
\text { Tau5 } \\
\text { (dimen- } \\
\text { sionless) }\end{array}$ \\
\hline 7718 & 15.051 & 9.0990 & 0.60456 & 0.41791 & 0.14201 & 0.08764 & 8910 & 11.588 & 6.8750 & 0.59327 & 0.56021 & 0.35966 & 0.21440 \\
\hline 7745 & 16.947 & 9.7098 & .57295 & .38740 & .14776 & .10142 & 8911 & 11.212 & 6.9752 & .62213 & .48568 & .20385 & .10457 \\
\hline 7922 & 8.1495 & 5.3287 & .65387 & .57373 & .28099 & .13946 & 8924 & 7.8724 & 4.9544 & .62933 & .55288 & .25712 & .09249 \\
\hline 7936 & 12.546 & 7.6069 & .60633 & .46802 & .21507 & .13674 & 8929 & 26.929 & 20.984 & .77923 & .68002 & .43226 & .29477 \\
\hline 7943 & 11.609 & 7.2055 & .62069 & .49609 & .22564 & .12138 & 8942 & 12.341 & 7.4937 & .60721 & .45560 & .19426 & .12535 \\
\hline 7944 & 17.035 & 10.305 & .60490 & .40934 & .13139 & .07348 & 8944 & 12.883 & 7.8341 & .60812 & .45880 & .19208 & .10571 \\
\hline 7945 & 15.678 & 9.5691 & .61035 & .44861 & .18940 & .12132 & 8996 & 13.363 & 8.1353 & 60880 & .47233 & .20915 & .11682 \\
\hline 7947 & 13.574 & 8.7482 & .64446 & .49820 & .21516 & .11706 & 9014 & 18.353 & 10.338 & .56330 & .41081 & .16196 & .02353 \\
\hline 7948 & 11.602 & 7.3153 & .63051 & .49711 & .21565 & .11470 & 9037 & 9.5627 & 6.0650 & .63424 & .54407 & .27752 & .15573 \\
\hline 7951 & 12.669 & 7.4117 & .58501 & .45481 & .21349 & .13005 & 9106 & 11.536 & 7.3645 & .63839 & .52643 & .25699 & .15264 \\
\hline 7953 & 15.146 & 9.9833 & .65915 & .53109 & .23259 & .09466 & 9107 & 7.8387 & 5.9484 & .75885 & .76363 & .52256 & .32725 \\
\hline 7981 & 12.394 & 8.0327 & .64813 & .51663 & .22713 & .10961 & 9129 & 14.200 & 9.5465 & .67229 & .53922 & .24310 & .11581 \\
\hline 7990 & 17.777 & 11.964 & .67301 & .54560 & .27928 & .16584 & 9163 & 11.235 & 6.9136 & .61538 & .48020 & .20649 & .11435 \\
\hline 7992 & 17.765 & 9.5956 & .54015 & .33916 & .00591 & -.12838 & 9213 & 15.708 & 10.259 & .65309 & .51590 & .26165 & .17890 \\
\hline 7997 & 11.457 & 7.0318 & .61378 & .50118 & .24340 & .12953 & 9214 & 18.045 & 13.292 & .73660 & .73845 & .52755 & .32715 \\
\hline 7999 & 9.4375 & 5.9792 & .63355 & .50443 & .21170 & .09768 & 9222 & 16.945 & 9.1370 & .53920 & .34547 & .13866 & .11214 \\
\hline 8022 & 13.370 & 8.7904 & .65745 & .51438 & .20501 & .09074 & 9248 & 12.477 & 7.7925 & .62456 & .45607 & .15670 & .08480 \\
\hline 8023 & 9.5256 & 6.1114 & .64157 & .53520 & .24832 & .13065 & 9266 & 11.478 & 6.5369 & .56953 & .43889 & .18883 & .09941 \\
\hline 8047 & 11.328 & 6.9934 & .61733 & .47871 & .20427 & .11514 & 9270 & 9.9557 & 6.5797 & .66090 & .54883 & .24776 & .12389 \\
\hline 8060 & 13.566 & 8.8365 & .65137 & .53346 & .26324 & .15199 & 9295 & 9.1333 & 5.8881 & .64469 & .52676 & .22887 & .12321 \\
\hline 8062 & 16.273 & 10.019 & .61569 & .41520 & .09774 & .04190 & 9304 & -- & -- & -- & -- & -- & -- \\
\hline 8068 & 11.167 & 6.7046 & .60041 & .41412 & .11508 & .06177 & 9307 & 11.004 & 6.6406 & .60344 & .46081 & .17252 & .05805 \\
\hline 8081 & 12.168 & 7.7215 & .63455 & .49898 & .21799 & .11861 & 9328 & 12.429 & 8.2136 & 66086 & .54600 & .27426 & .16788 \\
\hline 8089 & 9.4324 & 5.6772 & .60188 & .48552 & .21686 & .11210 & 9329 & 23.200 & 14.578 & .62835 & .41044 & .11776 & .02325 \\
\hline 8221 & 17.941 & 11.603 & .64672 & .57643 & .34148 & .11446 & 9345 & -- & -- & -- & -- & -- & -- \\
\hline 8252 & 9.8632 & 6.2475 & .63342 & .51780 & .22922 & .11232 & 9363 & 15.862 & 9.9730 & .62874 & .46986 & .20489 & .13774 \\
\hline 8265 & 15.549 & 9.0038 & .57905 & .42187 & .17269 & .10176 & 9364 & 14.681 & 9.2253 & .62838 & .47158 & .19317 & .10960 \\
\hline 8289 & 10.125 & 6.2917 & .62140 & .47697 & .16907 & .05326 & 9365 & 17.000 & 10.763 & .63311 & .47589 & .13777 & -.03606 \\
\hline 8305 & 8.7845 & 5.7445 & .65394 & .55917 & .26150 & .12629 & 9371 & 13.587 & 8.0028 & .58901 & .38755 & .09047 & .03146 \\
\hline 8335 & 13.654 & 7.9388 & .58141 & .43978 & .20163 & .12286 & 9417 & 12.285 & 7.3039 & .59454 & .45731 & .20173 & .11523 \\
\hline 8400 & 10.235 & 6.6819 & .65285 & .51484 & .19412 & .08435 & 9419 & 14.409 & 8.5726 & .59494 & .43934 & .18248 & .10639 \\
\hline 8445 & 13.729 & 8.1083 & .59060 & .44511 & .18867 & .10394 & 9435 & 10.433 & 6.8308 & .65474 & .53743 & .22737 & .07399 \\
\hline 8446 & 11.657 & 7.0966 & .60880 & .45548 & .17433 & .08946 & 9491 & 12.930 & 7.8306 & 60560 & .45977 & .19601 & .11272 \\
\hline 8451 & 11.473 & 6.9661 & .60717 & .47035 & .17588 & .05251 & 9499 & 9.5013 & 6.1025 & .64228 & .52449 & .22645 & .11268 \\
\hline 8531 & 12.315 & 7.4365 & .60385 & .46901 & .20713 & .11639 & 9522 & 36.125 & 24.518 & 67870 & .57465 & .26438 & -.04734 \\
\hline 8541 & 11.154 & 6.5871 & .59056 & .50379 & .25223 & .12803 & 9527 & 9.7404 & 6.2820 & .64494 & .52292 & .22156 & .10871 \\
\hline 8544 & 12.677 & 7.8834 & .62184 & .49802 & .24030 & .13957 & 9532 & 10.972 & 6.7231 & .61273 & .47697 & .20593 & .11665 \\
\hline 8545 & 17.400 & 10.381 & .59661 & .37177 & .10056 & .07533 & 9544 & -- & -- & -- & -- & -- & -- \\
\hline 8563 & 10.470 & 6.2365 & .59563 & .44154 & .16987 & .10136 & 9565 & 9.9437 & 6.2214 & 62566 & .51407 & .24212 & .13673 \\
\hline 8566 & 9.9701 & 6.3856 & .64047 & .53192 & .25129 & .13562 & 9570 & 9.6611 & 6.2092 & .64270 & .50224 & .17467 & .05716 \\
\hline 8583 & 10.552 & 6.5574 & .62142 & .46484 & .16461 & .08727 & 9574 & 9.4211 & 6.0000 & .63687 & .49708 & .19268 & .12053 \\
\hline 8584 & 11.169 & 7.1139 & .63694 & .50632 & .21639 & .10452 & 9588 & 13.015 & 8.6633 & .66565 & .53640 & .24914 & .14035 \\
\hline 8623 & 11.128 & 6.9530 & .62480 & .49244 & .21709 & .12320 & 9665 & 12.234 & 7.5131 & .61411 & .47732 & .21161 & .12060 \\
\hline 8625 & 13.040 & 7.5508 & .57904 & .44525 & .19797 & .11135 & 9715 & 11.386 & 6.7903 & .59635 & .45598 & .19573 & .11444 \\
\hline 8630 & 9.1485 & 5.5719 & .60905 & .49569 & .22094 & .11655 & 9729 & 12.896 & 7.5710 & .58707 & .43873 & .18718 & .11361 \\
\hline 8631 & 11.369 & 7.0896 & .62359 & .51333 & .24988 & .13550 & 9772 & 13.183 & 8.2360 & .62475 & .49124 & .22614 & .13671 \\
\hline 8646 & 11.641 & 6.8976 & .59251 & .46412 & .21048 & .11773 & 9814 & 11.438 & 6.4798 & .56654 & .44001 & .19640 & .13243 \\
\hline 8647 & 10.091 & 6.3572 & .62999 & .49478 & .20302 & .11355 & 9815 & 13.646 & 8.3474 & .61172 & .48574 & .24360 & .15802 \\
\hline 8677 & 14.119 & 8.1836 & .57964 & .45759 & .22090 & .13158 & 9816 & 9.8000 & 5.9437 & .60651 & .44662 & .13025 & .02419 \\
\hline 8696 & 15.947 & 9.1287 & .57242 & .41003 & .13427 & -.03293 & 9817 & 10.030 & 6.2557 & .62371 & .49728 & .21726 & .12438 \\
\hline 8743 & 12.671 & 7.5364 & .59475 & .45306 & .20181 & .12123 & 9829 & 9.5047 & 6.1166 & .64354 & .53440 & .24177 & .11916 \\
\hline 8761 & 9.3867 & 5.9984 & .63903 & .51678 & .21424 & .10348 & 9830 & 9.0148 & 5.5007 & 61019 & .49574 & .22221 & .11994 \\
\hline 8778 & 11.929 & 7.2153 & .60484 & .45582 & .19151 & .11588 & 9858 & 9.5671 & 5.7744 & .60357 & .50431 & .24530 & .12799 \\
\hline 8845 & 13.021 & 8.1647 & .62706 & .47903 & .20827 & .13126 & 9893 & 11.073 & 6.8743 & 62083 & .49557 & .22751 & .13043 \\
\hline 8859 & 13.540 & 8.1551 & .60229 & .46482 & .20978 & .11692 & 9916 & 11.724 & 6.7567 & .57631 & .41526 & .16612 & .10779 \\
\hline 8898 & 12.966 & 7.3651 & .56805 & .42528 & .17733 & .09607 & 9976 & 11.400 & 7.2990 & .64029 & .51094 & .22545 & .11991 \\
\hline 8908 & 13.533 & 7.5655 & .55903 & .35999 & .11772 & .10520 & & & & & & & \\
\hline
\end{tabular}


Appendix 4-3.6. L-moments of storm duration defined by 48-hour minimum interevent time for hourly rainfall stations in Texas.

$[--$, not available $]$

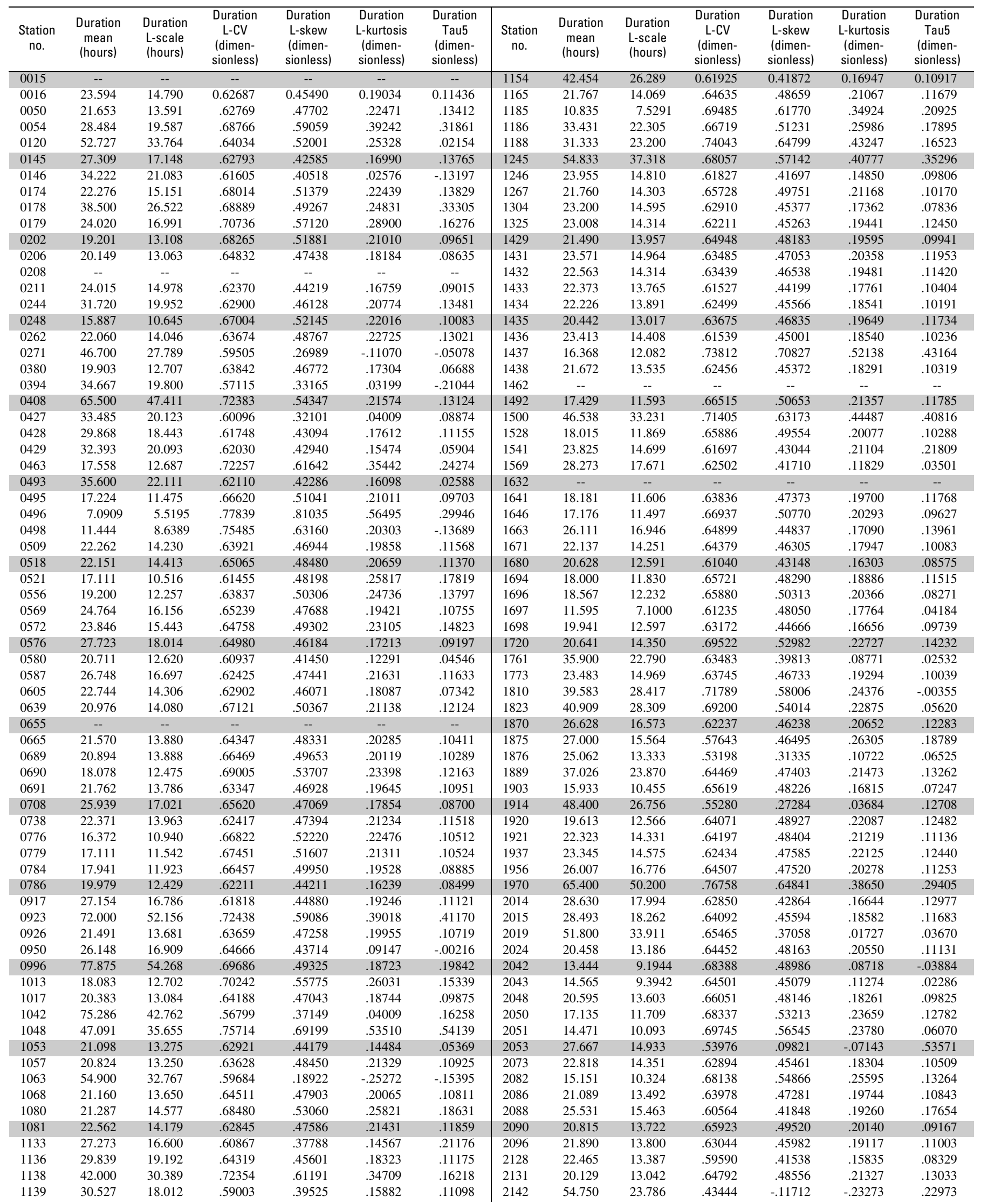


236 Statistical Characteristics of Storm Interevent Time, Depth, and Duration for Eastern New Mexico, Oklahoma, and Texas

Appendix 4-3.6. L-moments of storm duration defined by 48-hour minimum interevent time for hourly rainfall stations in TexasContinued.

\begin{tabular}{|c|c|c|c|c|c|c|c|c|c|c|c|c|c|}
\hline $\begin{array}{c}\text { Station } \\
\text { no. }\end{array}$ & $\begin{array}{c}\text { Duration } \\
\text { mean } \\
\text { (hours) }\end{array}$ & $\begin{array}{l}\text { Duration } \\
\text { L-scale } \\
\text { (hours) }\end{array}$ & $\begin{array}{c}\text { Duration } \\
\text { L-CV } \\
\text { (dimen- } \\
\text { sionless) }\end{array}$ & $\begin{array}{l}\text { Duration } \\
\text { L-skew } \\
\text { (dimen- } \\
\text { sionless) }\end{array}$ & $\begin{array}{c}\text { Duration } \\
\text { L-kurtosis } \\
\text { (dimen- } \\
\text { sionless) }\end{array}$ & $\begin{array}{c}\text { Duration } \\
\text { Tau5 } \\
\text { (dimen- } \\
\text { sionless) }\end{array}$ & $\begin{array}{c}\text { Station } \\
\text { no. }\end{array}$ & $\begin{array}{l}\text { Duration } \\
\text { mean } \\
\text { (hours) }\end{array}$ & $\begin{array}{l}\text { Duration } \\
\text { L-scale } \\
\text { (hours) }\end{array}$ & $\begin{array}{c}\text { Duration } \\
\text { L-CV } \\
\text { (dimen- } \\
\text { sionless) }\end{array}$ & $\begin{array}{l}\text { Duration } \\
\text { L-skew } \\
\text { (dimen- } \\
\text { sionless) }\end{array}$ & $\begin{array}{l}\text { Duration } \\
\text { L-kurtosis } \\
\text { (dimen- } \\
\text { sionless) }\end{array}$ & $\begin{array}{c}\text { Duration } \\
\text { Tau5 } \\
\text { (dimen- } \\
\text { sionless) }\end{array}$ \\
\hline 2160 & 34.533 & 21.629 & 0.62631 & 0.39667 & 0.12949 & 0.11788 & 3463 & 21.457 & 14.664 & 0.68340 & 0.55645 & 0.29859 & 0.18304 \\
\hline 2206 & 23.846 & 15.650 & .65630 & .50404 & .23507 & .14201 & 3476 & 21.923 & 14.475 & .66027 & .50745 & .22484 & .11768 \\
\hline 2238 & 23.152 & 14.557 & .62879 & .45106 & .18788 & .12159 & 3485 & 26.429 & 17.484 & .66154 & 61942 & .40703 & .16585 \\
\hline 2240 & 29.509 & 17.918 & .60720 & .36240 & .06917 & .02537 & 3507 & 23.303 & 15.319 & .65736 & .48624 & .20688 & .12021 \\
\hline 2242 & 25.191 & 15.535 & .61669 & .44942 & .20138 & .12924 & 3546 & 24.460 & 15.424 & .63060 & .45904 & .19344 & .11167 \\
\hline 2244 & 24.373 & 15.464 & .63449 & .45955 & .18976 & .10797 & 3547 & 18.444 & 11.816 & .64065 & .45916 & .16387 & .08279 \\
\hline 2247 & 32.194 & 20.865 & .64810 & .51078 & .25058 & .11919 & 3579 & 26.373 & 15.549 & .58959 & .37488 & .10414 & .07759 \\
\hline 2309 & 24.046 & 15.155 & .63022 & .44872 & .16036 & .06433 & 3642 & 22.204 & 14.118 & .63582 & .47494 & .20796 & .11595 \\
\hline 2312 & 23.098 & 14.286 & .61847 & .44405 & .18688 & .11187 & 3646 & 21.142 & 13.215 & .62505 & .47700 & .21605 & .11697 \\
\hline 2334 & 20.295 & 11.645 & .57379 & .32855 & .06990 & .06035 & 3668 & 76.000 & 41.179 & .54182 & .22897 & .06158 & .37467 \\
\hline 2336 & 19.494 & 13.184 & .67633 & .53069 & .24597 & .13249 & 3673 & 65.100 & 50.411 & .77436 & .69925 & .51156 & .41250 \\
\hline 2354 & 22.833 & 16.703 & .73150 & .58066 & .24638 & .08614 & 3686 & 22.524 & 14.157 & .62856 & .44660 & .17829 & .11276 \\
\hline 2355 & 28.152 & 16.379 & .58181 & .30362 & -.00896 & -.02571 & 3691 & 21.139 & 13.760 & .65091 & .49119 & .22021 & .13310 \\
\hline 2357 & 25.195 & 16.023 & .63598 & .42941 & .13325 & .06981 & 3734 & 67.900 & 52.256 & .76960 & .65448 & .43167 & .34999 \\
\hline 2360 & 22.425 & 14.554 & .64899 & .47813 & .19827 & .11406 & 3771 & 22.347 & 14.078 & .62998 & .43214 & .14739 & .08235 \\
\hline 2361 & 19.016 & 13.472 & .70845 & .58382 & .31060 & .19652 & 3789 & 23.388 & 16.358 & .69943 & .52970 & .21381 & .08946 \\
\hline 2394 & 23.714 & 14.705 & .62010 & .45384 & .19184 & .10692 & 3826 & 19.088 & 12.008 & .62909 & .50069 & .25176 & .15722 \\
\hline 2404 & 21.474 & 13.627 & .63458 & .47214 & .20743 & .12387 & 3831 & 21.487 & 13.646 & .63508 & .44207 & .13237 & .03442 \\
\hline 2415 & 23.060 & 14.392 & .62413 & .46482 & .20160 & .11001 & 3841 & 19.385 & 12.605 & .65023 & .47540 & .17470 & .07085 \\
\hline 2462 & 22.413 & 14.742 & .65774 & .51310 & .25274 & .15707 & 3871 & 20.906 & 13.141 & .62860 & .45880 & .17698 & .08656 \\
\hline 2528 & 21.217 & 12.984 & .61197 & .38396 & .06608 & -.00019 & 3884 & 29.077 & 17.744 & .61023 & .40003 & .08671 & -.01594 \\
\hline 2617 & 20.972 & 12.730 & .60698 & .42591 & .14934 & .07259 & 3941 & 24.617 & 15.492 & .62933 & .45627 & .19820 & .12795 \\
\hline 2619 & 18.155 & 11.859 & .65322 & .48863 & .19822 & .10187 & 3963 & -- & -- & -- & -- & -- & -- \\
\hline 2621 & 18.416 & 12.031 & .65327 & .48975 & .19381 & .08836 & 4040 & 19.315 & 12.425 & .64331 & .49225 & .22266 & .13188 \\
\hline 2675 & 22.380 & 14.752 & .65913 & .50579 & .23619 & .14033 & 4058 & 37.917 & 30.841 & .81339 & .75986 & .51249 & .23966 \\
\hline 2676 & 24.405 & 15.685 & .64271 & .44516 & .15766 & .09766 & 4098 & 16.833 & 11.446 & .67996 & .53283 & .23480 & .11864 \\
\hline 2679 & 19.859 & 12.974 & .65331 & .48058 & .18943 & .10263 & 4100 & 19.972 & 13.031 & .65248 & .49935 & .21570 & .09921 \\
\hline 2715 & 20.189 & 12.761 & .63208 & .48018 & .21134 & .11235 & 4137 & 21.316 & 13.772 & .64609 & .47837 & .20806 & .13029 \\
\hline 2744 & 18.443 & 12.116 & .65694 & .49796 & .20327 & .09384 & 4191 & 20.473 & 13.145 & .64209 & .46739 & .18079 & .09450 \\
\hline 2758 & 28.653 & 17.925 & .62561 & .41375 & .10429 & .01916 & 4256 & -- & -- & -- & -- & -- & -- \\
\hline 2794 & 11.200 & 7.4000 & .66071 & .53378 & .26158 & .16592 & 4257 & 22.868 & 14.747 & .64489 & .47947 & .21123 & .12512 \\
\hline 2797 & 20.241 & 13.335 & .65881 & .49988 & .21603 & .11781 & 4258 & 29.429 & 19.747 & .67101 & .50445 & .22075 & .10386 \\
\hline 2811 & 19.245 & 12.861 & .66829 & .50570 & .20809 & .10317 & 4278 & 20.180 & 13.087 & .64850 & .49523 & .21018 & .09909 \\
\hline 2813 & 14.579 & 9.9474 & .68231 & .53128 & .19299 & .01076 & 4299 & 12.610 & 8.3839 & .66486 & .54907 & .25303 & .10030 \\
\hline 2814 & 27.700 & 18.589 & .67108 & .36312 & -.13590 & -.26270 & 4300 & 33.265 & 20.844 & .62661 & .44759 & .19499 & .12572 \\
\hline 2815 & 17.330 & 11.327 & .65360 & .47916 & .16909 & .06664 & 4305 & 33.556 & 21.205 & .63193 & .45947 & .20563 & .12299 \\
\hline 2818 & 16.958 & 11.808 & .69634 & .56980 & .28237 & .14918 & 4307 & 32.704 & 20.809 & .63630 & .46210 & .21566 & .14498 \\
\hline 2986 & 25.547 & 16.083 & .62955 & .46691 & .20954 & .12812 & 4309 & 24.948 & 15.832 & .63460 & .46507 & .19074 & .10278 \\
\hline 3005 & 20.079 & 13.216 & .65820 & .49898 & .21624 & .11640 & 4311 & 24.943 & 15.834 & .63482 & .46474 & .18997 & .10015 \\
\hline 3033 & 15.348 & 10.485 & .68317 & .56669 & .26761 & .10681 & 4313 & 26.947 & 15.263 & .56642 & .37490 & .14474 & .09439 \\
\hline 3034 & -- & -- & -- & -- & -- & -- & 4319 & 26.617 & 16.525 & .62086 & .46717 & .20319 & .10207 \\
\hline 3047 & 62.364 & 43.218 & 69300 & .56920 & .41909 & .42175 & 4329 & 23.853 & 15.204 & .63740 & .47080 & .19957 & .10848 \\
\hline 3103 & 13.320 & 9.3133 & 69920 & .56572 & .25283 & .13083 & 4331 & -- & -- & -- & -- & -- & -- \\
\hline 3133 & 21.607 & 13.744 & .63609 & .48129 & .21471 & .11822 & 4375 & 20.058 & 13.367 & .66643 & .50097 & .21051 & .11734 \\
\hline 3156 & 21.579 & 13.633 & .63178 & .45498 & .18197 & .10342 & 4392 & 28.258 & 17.207 & .60892 & .42353 & .15826 & .07932 \\
\hline 3171 & 21.291 & 13.324 & .62583 & .45981 & .18786 & .09735 & 4425 & 15.667 & 10.970 & .70021 & .56799 & .26801 & .13799 \\
\hline 3189 & 21.262 & 14.411 & .67778 & .51329 & .22063 & .12781 & 4440 & 21.803 & 13.320 & .61093 & .45168 & .18928 & .10404 \\
\hline 3260 & 19.933 & 13.468 & .67565 & .53816 & .26553 & .15218 & 4476 & 20.779 & 12.959 & .62366 & .44278 & .16787 & .09166 \\
\hline 3267 & 25.087 & 15.321 & .61073 & .42415 & .15945 & .09792 & 4498 & 12.889 & 8.5000 & .65948 & .46032 & .05696 & -.08870 \\
\hline 3270 & 17.237 & 11.627 & .67455 & .52318 & .23082 & .12758 & 4517 & 21.664 & 13.868 & .64015 & .47557 & .19978 & .10648 \\
\hline 3272 & 32.167 & 22.212 & .69053 & .49332 & .19259 & .15223 & 4520 & 20.564 & 13.175 & .64068 & .46112 & .18783 & .12726 \\
\hline 3277 & 14.000 & 8.9091 & .63636 & .40612 & .04082 & -.00340 & 4525 & 56.000 & 44.591 & .79627 & .68277 & .43969 & .32909 \\
\hline 3278 & 17.706 & 12.348 & 69742 & .57216 & .28981 & .16393 & 4563 & 37.933 & 27.600 & .72759 & .57276 & .22480 & -.00589 \\
\hline 3280 & 14.363 & 10.133 & .70546 & .59435 & .28710 & .10871 & 4570 & 19.093 & 12.448 & .65197 & .48582 & .19660 & .10006 \\
\hline 3281 & 9.3684 & 7.2063 & .76921 & .75339 & .51084 & .31948 & 4577 & 23.292 & 15.254 & .65493 & .49760 & .22367 & .12121 \\
\hline 3283 & 27.442 & 17.215 & .62730 & .45024 & .18410 & .10218 & 4591 & 24.219 & 15.497 & .63985 & .49051 & .22447 & .11780 \\
\hline 3284 & 22.607 & 14.647 & .64791 & .48727 & .22074 & .13416 & 4670 & 19.712 & 12.744 & .64649 & .47174 & .17896 & .08728 \\
\hline 3285 & 21.145 & 13.744 & 64997 & .48358 & .20847 & .12411 & 4671 & 24.111 & 15.368 & .63738 & .44172 & .16339 & .10377 \\
\hline 3329 & 22.426 & 13.920 & .62073 & .43724 & .16345 & .08831 & 4679 & 22.130 & 14.318 & .64701 & .47674 & .20230 & .12097 \\
\hline 3335 & 21.207 & 12.565 & .59251 & .42733 & .19032 & .12439 & 4696 & 33.429 & 16.238 & .48575 & .14252 & .11437 & .33138 \\
\hline 3370 & 20.443 & 13.121 & .64185 & .49629 & .23233 & .13675 & 4703 & 23.848 & 15.521 & .65082 & .46518 & .17575 & .09600 \\
\hline 3410 & 18.980 & 12.420 & .65438 & .49140 & .20237 & .10288 & 4704 & 26.391 & 15.715 & .59547 & .42370 & .19445 & .13093 \\
\hline 3415 & 21.751 & 14.092 & .64789 & .48308 & .20658 & .11278 & 4731 & 27.372 & 17.535 & .64063 & .46060 & .20373 & .13682 \\
\hline 3430 & 28.710 & 17.992 & .62667 & .44794 & .18688 & .11175 & 4792 & 21.473 & 14.359 & .66872 & .50431 & .21378 & .11322 \\
\hline 3431 & 40.276 & 25.262 & .62723 & .44326 & .21351 & .16292 & 4819 & 25.467 & 16.337 & .64150 & .46466 & .18415 & .08766 \\
\hline 3441 & 27.640 & 18.613 & .67342 & .48100 & .15594 & .01696 & 4852 & 20.273 & 16.855 & .83139 & .74685 & .43995 & .13754 \\
\hline 3442 & 18.851 & 11.428 & .60627 & .44656 & .17513 & .07594 & 4866 & 21.490 & 13.667 & .63594 & .46882 & .19515 & .10691 \\
\hline 3446 & 15.422 & 10.150 & .65813 & .51641 & .21406 & .07566 & 4876 & 21.697 & 13.722 & .63246 & .46306 & .20590 & .13335 \\
\hline 3460 & 21.519 & 13.692 & .63630 & .49225 & .27231 & .21054 & 4878 & 28.254 & 17.591 & .62261 & .44466 & .17646 & .09131 \\
\hline 3462 & 21.178 & 14.045 & .66318 & .53765 & .27993 & .15810 & 4880 & 19.361 & 12.677 & .65476 & .49797 & .21344 & .11021 \\
\hline
\end{tabular}


Appendix 4-3.6. L-moments of storm duration defined by 48-hour minimum interevent time for hourly rainfall stations in TexasContinued.

\begin{tabular}{|c|c|c|c|c|c|c|c|c|c|c|c|c|c|}
\hline $\begin{array}{c}\text { Station } \\
\text { no. }\end{array}$ & $\begin{array}{l}\text { Duration } \\
\text { mean } \\
\text { (hours) }\end{array}$ & $\begin{array}{l}\text { Duration } \\
\text { L-scale } \\
\text { (hours) }\end{array}$ & $\begin{array}{c}\text { Duration } \\
\text { L-CV } \\
\text { (dimen- } \\
\text { sionless) }\end{array}$ & $\begin{array}{l}\text { Duration } \\
\text { L-skew } \\
\text { (dimen- } \\
\text { sionless) }\end{array}$ & $\begin{array}{l}\text { Duration } \\
\text { L-kurtosis } \\
\text { (dimen- } \\
\text { sionless) }\end{array}$ & $\begin{array}{c}\text { Duration } \\
\text { Tau5 } \\
\text { (dimen- } \\
\text { sionless) }\end{array}$ & $\begin{array}{c}\text { Station } \\
\text { no. }\end{array}$ & $\begin{array}{c}\text { Duration } \\
\text { mean } \\
\text { (hours) }\end{array}$ & $\begin{array}{l}\text { Duration } \\
\text { L-scale } \\
\text { (hours) }\end{array}$ & $\begin{array}{c}\text { Duration } \\
\text { L-CV } \\
\text { (dimen- } \\
\text { sionless) }\end{array}$ & $\begin{array}{c}\text { Duration } \\
\text { L-skew } \\
\text { (dimen- } \\
\text { sionless) }\end{array}$ & $\begin{array}{l}\text { Duration } \\
\text { L-kurtosis } \\
\text { (dimen- } \\
\text { sionless) }\end{array}$ & $\begin{array}{c}\text { Duration } \\
\text { Tau5 } \\
\text { (dimen- } \\
\text { sionless) }\end{array}$ \\
\hline 4920 & 18.591 & 12.140 & 0.65301 & 0.48583 & 0.19195 & 0.09434 & 5957 & 19.618 & 12.851 & 0.65506 & 0.49295 & 0.20706 & 0.10741 \\
\hline 4934 & 16.400 & 13.000 & .79268 & .64615 & .15385 & -.43077 & 5958 & 22.478 & 14.184 & .63103 & .46781 & .19623 & .10362 \\
\hline 4972 & 20.218 & 12.818 & .63401 & .46671 & .18964 & .09895 & 5973 & 25.129 & 15.749 & .62673 & .44468 & .16962 & .07464 \\
\hline 4973 & 22.548 & 14.359 & .63682 & .51997 & .28896 & .19024 & 5996 & 21.722 & 13.861 & .63811 & .46755 & .18865 & .09626 \\
\hline 4974 & 19.571 & 12.590 & .64327 & .47442 & .17955 & .07472 & 6017 & 25.202 & 16.393 & .65044 & .45880 & .15219 & .05695 \\
\hline 4975 & 22.155 & 13.866 & .62588 & 44977 & .18654 & .11680 & 6024 & 22.492 & 13.127 & .58364 & .40970 & .18650 & .11948 \\
\hline 4978 & 18.548 & 12.273 & .66168 & .50672 & .21800 & .10417 & 6050 & 14.647 & 7.5956 & .51857 & .46680 & .37782 & .25616 \\
\hline 4979 & 77.375 & 43.589 & .56335 & .24867 & .01270 & .24293 & 6104 & 26.305 & 17.965 & .68296 & .51912 & .22856 & .12220 \\
\hline 4982 & 18.865 & 11.912 & .63141 & .47724 & .20160 & .09937 & 6108 & 22.439 & 14.367 & .64026 & .47022 & .19707 & .10990 \\
\hline 5018 & 20.556 & 13.492 & .65635 & .54060 & .28670 & .17108 & 6136 & 18.132 & 12.253 & .67577 & .52111 & .22450 & .11618 \\
\hline 5048 & 16.068 & 10.526 & .65508 & .49195 & .19347 & .09902 & 6166 & 22.206 & 13.217 & .59517 & .40205 & .13148 & .07155 \\
\hline 5049 & 15.097 & 10.399 & 68879 & .53999 & .21941 & .08640 & 6176 & 24.657 & 15.283 & .61983 & .45328 & .19506 & .10483 \\
\hline 5056 & -- & -- & -- & -- & -- & -- & 6177 & 22.951 & 14.663 & .63889 & .47121 & .19866 & .10863 \\
\hline 5057 & 24.564 & 16.098 & .65533 & .47960 & .19237 & .10140 & 6210 & 22.084 & 14.213 & .64357 & .47159 & .18956 & .09905 \\
\hline 5060 & 23.670 & 15.459 & .65311 & .48251 & .19467 & .09627 & 6211 & 28.883 & 18.093 & .62641 & .43968 & .17802 & .11375 \\
\hline 5081 & 26.311 & 16.194 & .61548 & .45485 & .19666 & .10446 & 6270 & 23.233 & 14.547 & .62614 & .44616 & .17677 & .10024 \\
\hline 5094 & 21.463 & 13.806 & 64326 & .47971 & .20983 & .12283 & 6275 & -- & -- & -- & -- & -- & -- \\
\hline 5113 & 22.234 & 14.199 & .63864 & .45563 & .17173 & .09290 & 6276 & 65.500 & 53.322 & .81408 & .73390 & .55020 & .47927 \\
\hline 5114 & -- & -- & -- & -- & -- & -- & 6335 & 22.314 & 13.910 & .62338 & .46637 & .20365 & .10956 \\
\hline 5123 & 14.000 & 7.0455 & .50325 & .16000 & -.11971 & -.09642 & 6434 & 18.800 & 9.9429 & .52888 & .37666 & .15193 & .09814 \\
\hline 5192 & 20.670 & 12.973 & .62759 & .45547 & .18009 & .09478 & 6504 & 18.536 & 12.428 & .67048 & .51150 & .21494 & .10810 \\
\hline 5193 & 23.793 & 15.210 & 63924 & .47023 & .19737 & .10901 & 6558 & 16.500 & 10.223 & .61957 & .40360 & .10434 & .09212 \\
\hline 5224 & 28.000 & 17.097 & .61061 & .42244 & .16106 & .08666 & 6615 & 18.013 & 12.341 & .68513 & .53533 & .24346 & .14445 \\
\hline 5228 & 21.990 & 14.636 & .66558 & .49795 & .20596 & .10637 & 6660 & 22.750 & 13.242 & .58205 & .35572 & .08039 & .03385 \\
\hline 5235 & 26.500 & 16.026 & 60477 & .36793 & .06771 & .00452 & 6663 & 36.412 & 23.573 & .64741 & .44716 & .16895 & .10011 \\
\hline 5247 & 19.815 & 12.965 & .65432 & .49116 & .20610 & .10875 & 6734 & 22.429 & 13.796 & .61508 & .42543 & .14704 & .07244 \\
\hline 5258 & 21.862 & 13.824 & .63234 & .48192 & .21745 & .11852 & 6736 & 16.495 & 11.220 & 68019 & .53907 & .24416 & .12409 \\
\hline 5303 & 20.343 & 12.746 & .62657 & .45676 & .16564 & .07205 & 6740 & 30.909 & 20.782 & .67235 & .52931 & .23812 & .04447 \\
\hline 5312 & 20.050 & 13.359 & .66628 & .49675 & .19627 & .09508 & 6750 & 30.960 & 20.252 & .65413 & .45723 & .18357 & .13848 \\
\hline 5341 & 65.900 & 55.300 & .83915 & .76643 & .57784 & .49699 & 6757 & 24.775 & 15.756 & .63597 & .46638 & .19457 & .10292 \\
\hline 5342 & -- & -- & -- & -- & -- & -- & 6775 & 22.488 & 13.742 & .61107 & .42421 & .15577 & .08592 \\
\hline 5348 & 23.098 & 14.302 & .61921 & .43645 & .17134 & .10074 & 6776 & 20.111 & 13.260 & .65933 & .49311 & .20355 & .10989 \\
\hline 5358 & 18.741 & 12.041 & .64253 & .48522 & .19950 & .09439 & 6788 & 18.374 & 11.122 & .60532 & .46892 & .21334 & .10979 \\
\hline 5398 & 24.910 & 15.562 & .62474 & .45972 & .19327 & .10144 & 6792 & 16.874 & 11.576 & .68606 & .53823 & .23442 & .11638 \\
\hline 5410 & 17.892 & 12.091 & 67577 & .51883 & .21501 & .10214 & 6794 & 73.222 & 52.333 & .71472 & .60707 & .37671 & .25781 \\
\hline 5411 & 23.814 & 15.145 & .63598 & .46459 & .19596 & .11569 & 6834 & 21.648 & 13.907 & .64240 & .47353 & .20124 & .11959 \\
\hline 5424 & 41.935 & 26.149 & .62356 & .42048 & .15557 & .08692 & 6893 & 14.678 & 10.140 & .69084 & .55745 & .25488 & .12613 \\
\hline 5429 & 23.458 & 15.189 & .64751 & .48173 & .20181 & .10866 & 6935 & 17.889 & 12.043 & .67319 & .51287 & .20742 & .09567 \\
\hline 5431 & 25.333 & 15.606 & .61603 & .52583 & .37282 & .23948 & 6981 & 21.311 & 13.293 & .62379 & 44998 & .18851 & .12702 \\
\hline 5461 & 21.965 & 14.136 & .64360 & .48464 & .20415 & .09897 & 7020 & 24.478 & 14.528 & .59350 & .41515 & .16891 & .09055 \\
\hline 5463 & 20.826 & 13.233 & .63541 & .44250 & .14812 & .07386 & 7060 & 17.811 & 12.044 & .67619 & .52541 & .23447 & .13213 \\
\hline 5471 & 25.154 & 17.577 & 69878 & .45813 & .03143 & -.09933 & 7066 & 23.277 & 14.660 & .62980 & .46500 & .19742 & .10560 \\
\hline 5477 & 36.833 & 32.818 & .89099 & .86251 & .74177 & .67636 & 7074 & 17.262 & 11.744 & .68036 & .52921 & .22238 & .09651 \\
\hline 5528 & 19.697 & 12.680 & .64378 & .49089 & .21668 & .12051 & 7097 & 30.955 & 21.075 & .68085 & .52716 & .26246 & .17014 \\
\hline 5579 & -- & -- & -- & -- & -- & -- & 7116 & 19.956 & 12.691 & .63597 & .47721 & .20566 & .11708 \\
\hline 5580 & 31.000 & 16.429 & .52995 & .17672 & -.10457 & .00975 & 7140 & 24.604 & 15.821 & .64305 & .47027 & .19266 & .10608 \\
\hline 5589 & 20.874 & 14.248 & .68256 & .53169 & .24917 & .15369 & 7173 & 32.350 & 20.022 & .61893 & .43262 & .18556 & .13189 \\
\hline 5590 & 25.907 & 15.732 & .60726 & .39773 & .13297 & .09713 & 7174 & 32.713 & 20.544 & .62802 & .45615 & .20701 & .13222 \\
\hline 5591 & 17.877 & 12.546 & .70181 & .57908 & .28386 & .12881 & 7213 & 22.486 & 14.511 & .64533 & .47250 & .18566 & .09207 \\
\hline 5592 & 18.036 & 12.237 & .67844 & .54961 & .26693 & .14547 & 7243 & 21.403 & 13.782 & .64392 & .46707 & .18348 & .10145 \\
\hline 5594 & 13.922 & 10.099 & .72540 & .64794 & .37269 & .19452 & 7262 & 19.258 & 13.808 & .71700 & .56212 & .24470 & .13623 \\
\hline 5595 & -- & -- & -- & -- & -- & -- & 7274 & 13.833 & 9.0205 & .65208 & .53690 & .27644 & .15999 \\
\hline 5596 & 20.063 & 13.700 & .68287 & .51557 & .21121 & .11304 & 7300 & 18.355 & 11.747 & .63997 & .48947 & .21780 & .12169 \\
\hline 5600 & 19.318 & 13.470 & .69731 & .56481 & .27159 & .13831 & 7311 & 13.130 & 8.1779 & .62282 & .43451 & .09791 & -.03027 \\
\hline 5618 & 60.846 & 40.474 & .66519 & .56679 & .39393 & .36206 & 7363 & 52.818 & 42.255 & .80000 & .71142 & .49082 & .37794 \\
\hline 5650 & 27.167 & 15.758 & .58003 & .39827 & .12821 & .00497 & 7422 & 22.246 & 14.210 & .63875 & .45967 & .18137 & .10635 \\
\hline 5656 & 19.093 & 12.623 & 66116 & .48508 & .18567 & .10119 & 7431 & 15.579 & 10.644 & .68323 & .55846 & .27210 & .13975 \\
\hline 5658 & 19.690 & 12.533 & .63650 & .48222 & .20302 & .09800 & 7481 & 15.281 & 10.283 & .67295 & .52925 & .22044 & .08399 \\
\hline 5661 & 24.375 & 15.924 & .65328 & .45388 & .15826 & .10061 & 7497 & 23.005 & 14.745 & .64093 & .45883 & .18177 & .10660 \\
\hline 5666 & 25.773 & 15.439 & .59906 & .34984 & .06807 & .10150 & 7498 & 21.599 & 14.618 & .67680 & .52098 & .24153 & .14772 \\
\hline 5695 & 21.051 & 13.472 & .63995 & .47493 & .19942 & .10531 & 7499 & 20.469 & 13.276 & .64860 & .47693 & .19504 & .11173 \\
\hline 5742 & 21.769 & 15.692 & .72085 & 66399 & .54590 & .57908 & 7531 & 24.167 & 14.536 & .60150 & .38803 & .09427 & .02998 \\
\hline 5766 & 79.556 & 34.500 & .43366 & .27490 & .15689 & .11548 & 7534 & 19.339 & 12.217 & .63174 & .43267 & .12069 & .03326 \\
\hline 5770 & 21.464 & 13.879 & 64659 & .47325 & .18864 & .10017 & 7556 & 20.124 & 13.104 & .65117 & .48560 & .20468 & .11573 \\
\hline 5775 & 24.125 & 13.339 & .55292 & .11379 & -.23963 & .05756 & 7594 & 23.010 & 14.815 & .64385 & .47842 & .19751 & .09856 \\
\hline 5779 & 13.667 & 8.6928 & .63606 & .49173 & .23684 & .16165 & 7596 & 19.262 & 12.184 & .63252 & .44206 & .13611 & .04234 \\
\hline 5840 & 19.845 & 12.842 & 64710 & .50592 & .23218 & .11059 & 7608 & 23.601 & 14.971 & .63433 & .45338 & .17925 & .10511 \\
\hline 5890 & 20.094 & 13.059 & 64989 & .49446 & .21819 & .12245 & 7622 & 13.588 & 9.6838 & .71266 & .55444 & .16835 & -.02523 \\
\hline 5891 & 15.258 & 9.6857 & .63479 & .46831 & .16581 & .06248 & 7700 & 28.132 & 17.227 & .61236 & .43718 & .17314 & .08972 \\
\hline 5897 & 21.613 & 13.555 & 62716 & .43904 & .16589 & .10157 & 7706 & 19.795 & 13.036 & .65854 & .49154 & .19958 & .10396 \\
\hline
\end{tabular}


Appendix 4-3.6. L-moments of storm duration defined by 48-hour minimum interevent time for hourly rainfall stations in TexasContinued.

\begin{tabular}{|c|c|c|c|c|c|c|c|c|c|c|c|c|c|}
\hline $\begin{array}{c}\text { Station } \\
\text { no. }\end{array}$ & $\begin{array}{l}\text { Duration } \\
\text { mean } \\
\text { (hours) }\end{array}$ & $\begin{array}{l}\text { Duration } \\
\text { L-scale } \\
\text { (hours) }\end{array}$ & $\begin{array}{l}\text { Duration } \\
\text { L-CV } \\
\text { (dimen- } \\
\text { sionless) }\end{array}$ & $\begin{array}{l}\text { Duration } \\
\text { L-skew } \\
\text { (dimen- } \\
\text { sionless) }\end{array}$ & $\begin{array}{c}\text { Duration } \\
\text { L-kurtosis } \\
\text { (dimen- } \\
\text { sionless) }\end{array}$ & $\begin{array}{l}\text { Duration } \\
\text { Tau5 } \\
\text { (dimen- } \\
\text { sionless) }\end{array}$ & $\begin{array}{c}\text { Station } \\
\text { no. }\end{array}$ & $\begin{array}{l}\text { Duration } \\
\text { mean } \\
\text { (hours) }\end{array}$ & $\begin{array}{l}\text { Duration } \\
\text { L-scale } \\
\text { (hours) }\end{array}$ & $\begin{array}{l}\text { Duration } \\
\text { L-CV } \\
\text { (dimen- } \\
\text { sionless) }\end{array}$ & $\begin{array}{l}\text { Duration } \\
\text { L-skew } \\
\text { (dimen- } \\
\text { sionless) }\end{array}$ & $\begin{array}{l}\text { Duration } \\
\text { L-kurtosis } \\
\text { (dimen- } \\
\text { sionless) }\end{array}$ & $\begin{array}{c}\text { Duration } \\
\text { Tau5 } \\
\text { (dimen- } \\
\text { sionless) }\end{array}$ \\
\hline 7718 & 20.859 & 13.724 & 0.65793 & 0.51504 & 0.27790 & 0.22425 & 8910 & 25.538 & 16.577 & 0.64910 & 0.50179 & 0.20882 & 0.08395 \\
\hline 7745 & 26.770 & 16.007 & .59794 & .39418 & .13265 & .07151 & 8911 & 23.114 & 15.414 & 66689 & .50275 & .21465 & .11155 \\
\hline 7922 & 14.724 & 10.027 & 68100 & .55267 & .26002 & .13242 & 8924 & 15.196 & 10.473 & .68920 & .57411 & .29471 & .17234 \\
\hline 7936 & 25.443 & 16.298 & .64058 & .47748 & .21228 & .12289 & 8929 & 41.818 & 36.418 & .87087 & .83325 & .70752 & .66192 \\
\hline 7943 & 21.656 & 13.979 & .64547 & .48115 & .20023 & .10543 & 8942 & 24.663 & 15.706 & .63685 & .46632 & .20066 & .11832 \\
\hline 7944 & 32.558 & 17.967 & .55184 & .30808 & .06707 & .01168 & 8944 & 25.828 & 16.420 & .63575 & .45081 & .16818 & .08446 \\
\hline 7945 & 29.117 & 18.084 & 62110 & .43683 & .18323 & .12124 & 8996 & 26.268 & 16.733 & .63700 & .46890 & .20003 & .11365 \\
\hline 7947 & 27.197 & 17.514 & .64394 & .46300 & .20156 & .15203 & 9014 & 58.100 & 35.811 & .61637 & .41374 & .15485 & .16491 \\
\hline 7948 & 20.997 & 13.737 & .65421 & .48441 & .19686 & .10619 & 9037 & 15.110 & 10.195 & .67475 & .55111 & .26335 & .12651 \\
\hline 7951 & 22.538 & 14.222 & .63105 & .47862 & .21716 & .12009 & 9106 & 17.551 & 12.177 & .69381 & .57506 & .30445 & .18964 \\
\hline 7953 & 24.074 & 15.701 & .65219 & .47633 & .16617 & .06903 & 9107 & 16.423 & 13.017 & .79260 & .70778 & .40989 & .17553 \\
\hline 7981 & 21.744 & 14.369 & .66083 & .49923 & .21514 & .11885 & 9129 & 25.942 & 16.864 & .65007 & .45021 & .12660 & .02357 \\
\hline 7990 & 29.803 & 20.971 & .70366 & .55694 & .27782 & .16104 & 9163 & 20.009 & 12.864 & .64292 & .47623 & .19344 & .09949 \\
\hline 7992 & 41.500 & 28.242 & .68054 & .52532 & .25376 & .17132 & 9213 & 32.426 & 20.078 & 61920 & .40636 & .13231 & .07359 \\
\hline 7997 & 16.950 & 10.202 & .60188 & .41008 & .11402 & .03081 & 9214 & 59.250 & 40.826 & .68904 & .50492 & .19589 & .17066 \\
\hline 7999 & 18.769 & 11.269 & .60041 & .33850 & -.00745 & -.02661 & 9222 & 21.766 & 14.478 & .60229 & .46638 & .27424 & .22005 \\
\hline 8022 & 16.939 & 10.763 & 63539 & .44896 & .12924 & .04498 & 9248 & 24.974 & 15.488 & .62551 & .43329 & .15594 & .09549 \\
\hline 8023 & 16.877 & 11.456 & .67881 & .53603 & .24159 & .12345 & 9266 & 22.345 & 14.377 & .65169 & .51853 & .25358 & .13996 \\
\hline 8047 & 20.989 & 13.562 & .64615 & .47947 & .20101 & .11133 & 9270 & 19.026 & 12.299 & 69209 & .53692 & .24138 & .12854 \\
\hline 8060 & 26.614 & 18.563 & 69749 & .54921 & .25860 & .13421 & 9295 & 21.967 & 14.065 & .75315 & .68547 & .46530 & .34483 \\
\hline 8062 & 21.300 & 11.500 & .53991 & .25166 & .01762 & .10415 & 9304 & -- & -- & -- & -- & -- & -- \\
\hline 8068 & 27.136 & 15.968 & .58842 & .36438 & .14353 & .15915 & 9307 & 18.099 & 11.665 & .64453 & .50071 & .23272 & .13440 \\
\hline 8081 & 21.444 & 14.084 & .65676 & .49158 & .20784 & .11522 & 9328 & 22.558 & 14.740 & .65340 & .46574 & .15572 & .05491 \\
\hline 8089 & 18.097 & 12.032 & .66488 & .51769 & .20959 & .06303 & 9329 & 28.556 & 16.583 & .58074 & .28691 & -.06126 & -.15028 \\
\hline 8221 & 56.800 & 31.267 & .55047 & .28998 & -.11864 & -.09524 & 9345 & -- & -- & -- & -- & -- & -- \\
\hline 8252 & 16.767 & 11.283 & .67293 & .52566 & .22601 & .10511 & 9363 & 30.538 & 19.080 & 62479 & .43486 & .18152 & .12745 \\
\hline 8265 & 28.739 & 17.568 & .61131 & .43762 & .17996 & .10449 & 9364 & 29.719 & 18.782 & 63201 & .44726 & .18548 & .12083 \\
\hline 8289 & 23.459 & 13.616 & .58039 & .35454 & .11048 & .10364 & 9365 & 21.476 & 13.871 & .64590 & .44015 & .06448 & -.08103 \\
\hline 8305 & 17.061 & 11.793 & .69125 & .54977 & .24666 & .11589 & 9371 & 25.351 & 14.701 & .57990 & .33879 & .07648 & .06924 \\
\hline 8335 & 24.829 & 15.535 & 62569 & .46239 & .20012 & .10910 & 9417 & 22.811 & 14.330 & .62821 & .46492 & .19777 & .10919 \\
\hline 8400 & 21.766 & 14.478 & .66519 & .47750 & .15999 & .05799 & 9419 & 26.502 & 16.422 & .61965 & .44358 & .18402 & .10834 \\
\hline 8445 & 24.974 & 15.488 & 62018 & .44924 & .18565 & .10729 & 9435 & 18.404 & 12.860 & 69879 & .54773 & .22280 & .06463 \\
\hline 8446 & 22.345 & 14.377 & .64339 & .47037 & .18982 & .10198 & 9491 & 24.298 & 15.282 & .62894 & .45469 & .18541 & .10424 \\
\hline 8451 & 19.026 & 12.299 & .64642 & .48514 & .18758 & .07783 & 9499 & 17.955 & 11.987 & .66763 & .50624 & .20745 & .10547 \\
\hline 8531 & 21.967 & 14.065 & .64029 & .47981 & .20690 & .11232 & 9522 & 78.200 & 72.400 & .92583 & .94475 & .90331 & .88674 \\
\hline 8541 & 19.766 & 12.650 & .63996 & .52894 & .25003 & .11604 & 9527 & 19.846 & 13.356 & .67300 & .50591 & .20320 & .09795 \\
\hline 8544 & 23.832 & 14.653 & .61486 & .42622 & .14735 & .06845 & 9532 & 20.709 & 13.267 & 64061 & .47597 & .20202 & .11281 \\
\hline 8545 & 26.615 & 17.244 & .64788 & .50145 & .27658 & .14329 & 9544 & -- & -- & -- & -- & -- & -- \\
\hline 8563 & 21.405 & 13.657 & .63805 & .47017 & .20553 & .12789 & 9565 & 19.078 & 12.769 & 66929 & .51635 & .22180 & .10358 \\
\hline 8566 & 20.392 & 13.787 & .67610 & .51941 & .22134 & .10303 & 9570 & 19.222 & 12.756 & .66361 & .48091 & .16879 & .08118 \\
\hline 8583 & 21.473 & 13.877 & .64624 & .46754 & .19277 & .12636 & 9574 & 22.200 & 11.448 & .51566 & .16933 & -.05817 & .02521 \\
\hline 8584 & 19.667 & 12.772 & 64938 & .47021 & .17058 & .07978 & 9588 & 22.444 & 15.278 & .68068 & .52236 & .23457 & .13291 \\
\hline 8623 & 20.576 & 13.390 & .65074 & .48749 & .20718 & .11271 & 9665 & 23.020 & 14.847 & 64497 & .47645 & .19579 & .10065 \\
\hline 8625 & 22.398 & 13.741 & .61351 & .45334 & .19167 & .10353 & 9715 & 21.287 & 13.497 & .63406 & .46929 & .19924 & .11127 \\
\hline 8630 & 16.226 & 10.656 & .65672 & .51031 & .21980 & .10422 & 9729 & 23.173 & 14.262 & .61548 & .44519 & .18553 & .10697 \\
\hline 8631 & 18.199 & 11.970 & .65776 & .50732 & .21825 & .10305 & 9772 & 24.141 & 15.548 & .64405 & .49090 & .23302 & .14776 \\
\hline 8646 & 21.038 & 13.626 & .64768 & .50156 & .22903 & .11840 & 9814 & 15.690 & 9.9384 & .63344 & .54052 & .32163 & .22326 \\
\hline 8647 & 19.103 & 12.810 & 67059 & .51620 & .23163 & .13236 & 9815 & 24.515 & 15.465 & .63084 & .46493 & .19516 & .09960 \\
\hline 8677 & 23.549 & 14.914 & .63332 & .48863 & .22000 & .11346 & 9816 & 16.643 & 12.014 & .72189 & .63678 & .39184 & .25647 \\
\hline 8696 & 63.100 & 39.344 & .62353 & .35287 & .01349 & .06538 & 9817 & 18.541 & 12.215 & .65881 & 49990 & .21293 & .11420 \\
\hline 8743 & 23.549 & 14.797 & .62837 & .45644 & .18352 & .09446 & 9829 & 16.501 & 11.169 & .67685 & .53217 & .23360 & .11245 \\
\hline 8761 & 16.846 & 11.407 & .67714 & .52799 & .23301 & .12493 & 9830 & 15.269 & 10.407 & .68159 & .56839 & .29959 & .17956 \\
\hline 8778 & 24.161 & 15.290 & .63285 & .45726 & .18502 & .09890 & 9858 & 17.675 & 11.591 & .65576 & .51600 & .22915 & .09814 \\
\hline 8845 & 22.918 & 14.591 & .63667 & .45143 & .17049 & .09350 & 9893 & 19.508 & 12.571 & .64441 & .47951 & .19380 & .09370 \\
\hline 8859 & 23.276 & 15.047 & .64648 & .49374 & .22564 & .12656 & 9916 & 23.330 & 14.177 & 60769 & .43455 & .18500 & .11159 \\
\hline 8898 & 21.963 & 13.616 & .61997 & .46898 & .21352 & .12616 & 9976 & 20.195 & 13.098 & .64859 & .47101 & .17505 & .08370 \\
\hline 8908 & 25.542 & 17.904 & .70097 & .60579 & .38349 & .25643 & & & & & & & \\
\hline
\end{tabular}


Appendix 4-3.7. L-moments of storm duration defined by 72-hour minimum interevent time for hourly rainfall stations in Texas.

$[--$, not available $]$

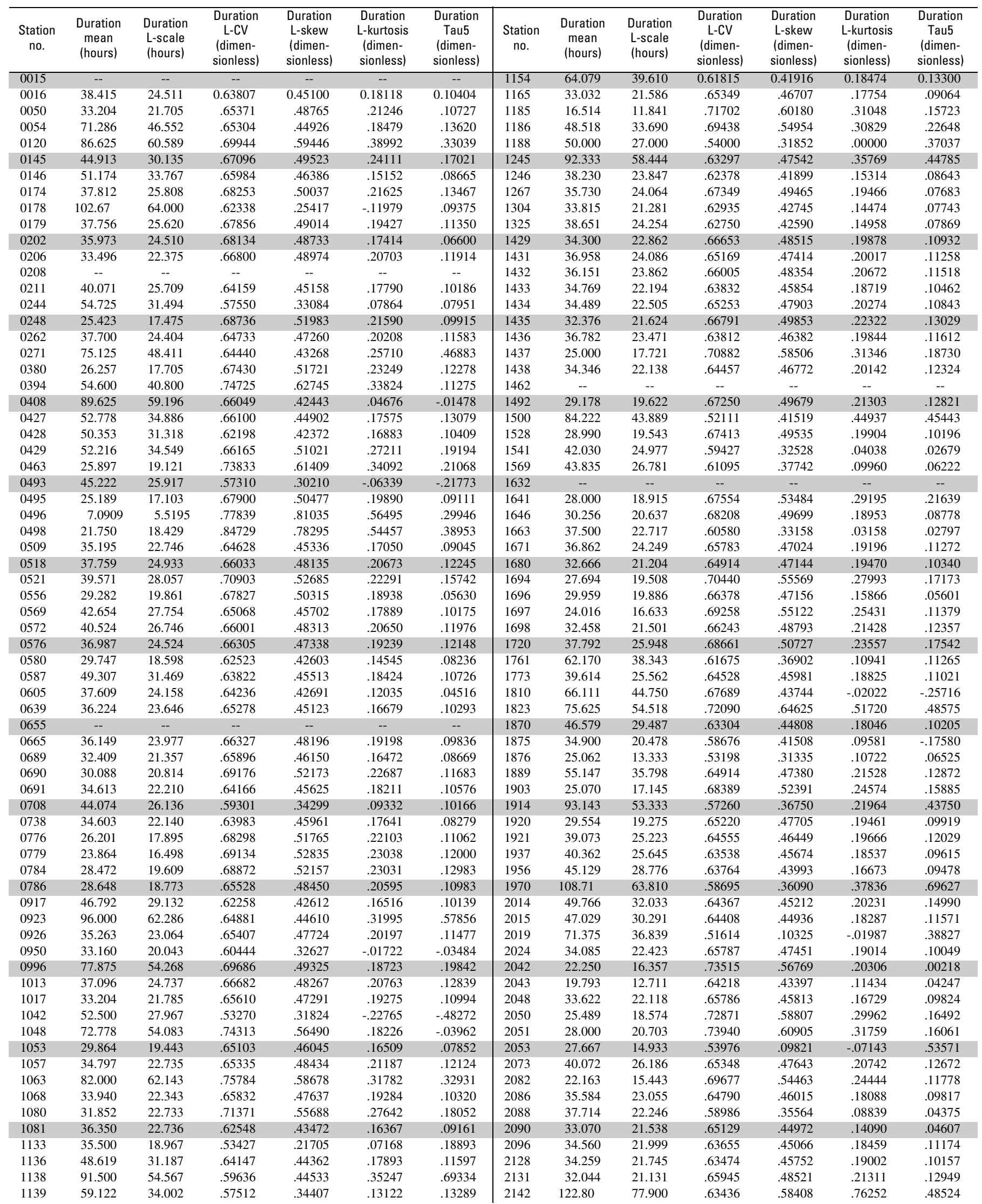


240 Statistical Characteristics of Storm Interevent Time, Depth, and Duration for Eastern New Mexico, Oklahoma, and Texas

Appendix 4-3.7. L-moments of storm duration defined by 72-hour minimum interevent time for hourly rainfall stations in TexasContinued.

\begin{tabular}{|c|c|c|c|c|c|c|c|c|c|c|c|c|c|}
\hline $\begin{array}{c}\text { Station } \\
\text { no. }\end{array}$ & $\begin{array}{l}\text { Duration } \\
\text { mean } \\
\text { (hours) }\end{array}$ & $\begin{array}{l}\text { Duration } \\
\text { L-scale } \\
\text { (hours) }\end{array}$ & $\begin{array}{l}\text { Duration } \\
\text { L-CV } \\
\text { (dimen- } \\
\text { sionless) }\end{array}$ & $\begin{array}{l}\text { Duration } \\
\text { L-skew } \\
\text { (dimen- } \\
\text { sionless) }\end{array}$ & $\begin{array}{c}\text { Duration } \\
\text { L-kurtosis } \\
\text { (dimen- } \\
\text { sionless) }\end{array}$ & $\begin{array}{c}\text { Duration } \\
\text { Tau5 } \\
\text { (dimen- } \\
\text { sionless) }\end{array}$ & $\begin{array}{c}\text { Station } \\
\text { no. }\end{array}$ & $\begin{array}{c}\text { Duration } \\
\text { mean } \\
\text { (hours) }\end{array}$ & $\begin{array}{l}\text { Duration } \\
\text { L-scale } \\
\text { (hours) }\end{array}$ & $\begin{array}{c}\text { Duration } \\
\text { L-CV } \\
\text { (dimen- } \\
\text { sionless) }\end{array}$ & $\begin{array}{l}\text { Duration } \\
\text { L-skew } \\
\text { (dimen- } \\
\text { sionless) }\end{array}$ & $\begin{array}{c}\text { Duration } \\
\text { L-kurtosis } \\
\text { (dimen- } \\
\text { sionless) }\end{array}$ & $\begin{array}{c}\text { Duration } \\
\text { Tau5 } \\
\text { (dimen- } \\
\text { sionless) }\end{array}$ \\
\hline 2160 & 60.500 & 36.773 & 0.60781 & 0.33201 & 0.05974 & 0.06840 & 3463 & 33.967 & 22.930 & 0.67507 & 0.45934 & 0.09031 & -0.07028 \\
\hline 2206 & 38.981 & 25.684 & .65889 & .47253 & .18157 & .08770 & 3476 & 35.180 & 23.026 & .65453 & .46248 & .17025 & .08774 \\
\hline 2238 & 35.569 & 23.463 & .65963 & .48833 & .22618 & .14897 & 3485 & 52.100 & 39.500 & .75816 & .65654 & .40808 & .22897 \\
\hline 2240 & 43.500 & 27.662 & .63592 & .45982 & .22965 & .17214 & 3507 & 38.580 & 24.835 & .64374 & .45253 & .18662 & .12003 \\
\hline 2242 & 44.516 & 27.979 & .62851 & .45104 & .20340 & .13000 & 3546 & 42.378 & 26.924 & .63533 & .43997 & .17089 & .10361 \\
\hline 2244 & 43.718 & 27.984 & .64010 & .44993 & .18235 & .10843 & 3547 & 28.593 & 18.924 & .66185 & .46351 & .15919 & .08363 \\
\hline 2247 & 49.192 & 33.435 & 67969 & .52908 & .27523 & .18446 & 3579 & 35.522 & 21.529 & .60607 & .39009 & .11424 & .05396 \\
\hline 2309 & 39.874 & 26.126 & .65521 & .46323 & .17684 & .09793 & 3642 & 37.259 & 23.944 & .64263 & .45877 & .18739 & .10745 \\
\hline 2312 & 46.358 & 29.471 & .63573 & .45219 & .18800 & .10273 & 3646 & 32.479 & 21.082 & 64909 & .48375 & .20859 & .10700 \\
\hline 2334 & 32.316 & 20.892 & .64649 & .43616 & .13963 & .06377 & 3668 & 108.83 & 74.033 & .68025 & .51643 & .38766 & .68663 \\
\hline 2336 & 24.629 & 16.774 & .68105 & .51796 & .22271 & .10980 & 3673 & 78.333 & 57.056 & .72837 & .60259 & .38726 & .36695 \\
\hline 2354 & 39.533 & 21.600 & .54637 & .21517 & .02184 & .22039 & 3686 & 36.597 & 23.360 & .63831 & .44763 & .17899 & .10774 \\
\hline 2355 & 30.875 & 18.359 & .59462 & .31797 & -.00285 & -.01817 & 3691 & 35.229 & 23.089 & .65540 & .46733 & .18326 & .09857 \\
\hline 2357 & 39.663 & 24.557 & 61913 & .40129 & .13769 & .09951 & 3734 & 112.14 & 71.095 & .63397 & .41112 & .24113 & .38580 \\
\hline 2360 & 34.304 & 22.741 & .66291 & .48805 & .21359 & .12649 & 3771 & 39.065 & 24.385 & .62421 & .42335 & .17126 & .12191 \\
\hline 2361 & 29.425 & 21.140 & .71845 & .57772 & .30037 & .17673 & 3789 & 45.038 & 30.340 & .67365 & .46978 & .17043 & .08653 \\
\hline 2394 & 39.790 & 25.155 & .63220 & .44791 & .18133 & .10338 & 3826 & 31.774 & 20.533 & .64624 & .49776 & .24426 & .14768 \\
\hline 2404 & 36.618 & 23.377 & .63840 & .45253 & .18385 & .10718 & 3831 & 30.254 & 19.514 & .64500 & .45752 & .17636 & .10507 \\
\hline 2415 & 38.668 & 25.171 & .65095 & .47760 & .20502 & .11388 & 3841 & 28.561 & 19.402 & .67931 & .49862 & .18231 & .05541 \\
\hline 2462 & 34.387 & 22.734 & .66112 & .48805 & .21466 & .12429 & 3871 & 31.587 & 20.600 & .65217 & .47774 & .19390 & .09801 \\
\hline 2528 & 34.972 & 19.498 & .55753 & .26356 & -.00708 & .01281 & 3884 & 72.500 & 48.786 & .67291 & .46925 & .22328 & .40264 \\
\hline 2617 & 31.247 & 20.016 & .64058 & .47082 & .20286 & .11883 & 3941 & 41.692 & 22.262 & .53395 & .22608 & .00820 & .07158 \\
\hline 2619 & 30.359 & 20.896 & .68831 & .51666 & .21982 & .10790 & 3963 & -- & -- & -- & -- & -- & -- \\
\hline 2621 & 27.794 & 18.774 & .67546 & .51003 & .22390 & .12062 & 4040 & 28.986 & 19.887 & .68607 & .53500 & .25447 & .13956 \\
\hline 2675 & 39.058 & 25.866 & .66224 & .47169 & .17679 & .08044 & 4058 & 58.778 & 41.889 & .71267 & .53467 & .19098 & .09435 \\
\hline 2676 & 41.941 & 27.033 & .64453 & .43620 & .15454 & .08897 & 4098 & 29.121 & 19.940 & .68475 & .50563 & .20286 & .10144 \\
\hline 2679 & 29.828 & 19.763 & .66256 & .48238 & .20486 & .13380 & 4100 & 30.280 & 20.467 & .67593 & .50648 & .20663 & .08433 \\
\hline 2715 & 31.114 & 20.663 & .66411 & .51344 & .24947 & .14884 & 4137 & 37.048 & 23.903 & .64518 & .46261 & .20259 & .13249 \\
\hline 2744 & 29.937 & 20.243 & .67618 & .50597 & .21905 & .12312 & 4191 & 32.913 & 21.785 & .66190 & .47974 & .19479 & .10725 \\
\hline 2758 & 45.049 & 31.581 & .70103 & .55177 & .29996 & .23765 & 4256 & -- & -- & -- & -- & -- & -- \\
\hline 2794 & 43.286 & 19.476 & .44994 & -.03374 & -.08802 & .12714 & 4257 & 38.951 & 25.507 & .65485 & .47136 & .19833 & .11868 \\
\hline 2797 & 32.857 & 22.385 & .68130 & .52218 & .24926 & .14936 & 4258 & 50.964 & 35.797 & .70240 & .53486 & .24624 & .12458 \\
\hline 2811 & 30.599 & 20.417 & .66725 & .47781 & .17536 & .08012 & 4278 & 31.246 & 20.519 & .65669 & .47394 & .17729 & .07804 \\
\hline 2813 & 27.875 & 21.575 & .77399 & .68863 & .46717 & .35482 & 4299 & 17.968 & 12.660 & .70462 & .58152 & .28609 & .12678 \\
\hline 2814 & 64.143 & 28.571 & .44543 & -.00200 & .28500 & .72833 & 4300 & 61.184 & 38.487 & .62904 & .43501 & .17692 & .10347 \\
\hline 2815 & 26.701 & 19.067 & .71409 & .57242 & .29522 & .18361 & 4305 & 59.298 & 36.133 & .60935 & .39614 & .14469 & .09666 \\
\hline 2818 & 28.113 & 19.582 & .69654 & .52667 & .21438 & .09018 & 4307 & 64.037 & 39.264 & .61315 & .40166 & .15587 & .10649 \\
\hline 2986 & 41.901 & 26.315 & .62802 & .42630 & .14773 & .06931 & 4309 & 43.147 & 27.535 & .63817 & .44642 & .17399 & .10204 \\
\hline 3005 & 34.260 & 22.951 & 66992 & .49083 & .20244 & .10584 & 4311 & 41.071 & 26.552 & .64648 & .45894 & .18142 & .10069 \\
\hline 3033 & 21.967 & 15.426 & .70224 & .56339 & .25467 & .09658 & 4313 & 42.559 & 26.554 & .62393 & .46625 & .23478 & .15042 \\
\hline 3034 & -- & -- & -- & -- & -- & -- & 4319 & 54.911 & 34.459 & .62753 & .42393 & .14918 & .08285 \\
\hline 3047 & 77.333 & 53.167 & .68750 & .47962 & .24392 & .33856 & 4329 & 39.573 & 25.635 & .64780 & .46369 & .19167 & .11201 \\
\hline 3103 & 19.739 & 14.379 & .72847 & .58439 & .25929 & .08256 & 4331 & -- & -- & -- & -- & -- & -- \\
\hline 3133 & 35.404 & 22.375 & .63201 & .43843 & .15713 & .07829 & 4375 & 32.741 & 21.701 & .66279 & .47851 & .19775 & .11946 \\
\hline 3156 & 29.848 & 19.224 & .64404 & .45141 & .16382 & .07919 & 4392 & 48.242 & 28.653 & .59394 & .38727 & .15157 & .11791 \\
\hline 3171 & 33.979 & 22.035 & .64847 & .47137 & .19665 & .11176 & 4425 & 24.703 & 17.699 & .71646 & .56693 & .26842 & .14072 \\
\hline 3189 & 33.829 & 22.107 & .65350 & .46055 & .18743 & .12952 & 4440 & 34.450 & 21.800 & .63280 & .45861 & .18683 & .10009 \\
\hline 3260 & 25.384 & 17.545 & 69117 & .54494 & .26170 & .13393 & 4476 & 33.393 & 21.611 & .64718 & .46826 & .19526 & .10815 \\
\hline 3267 & 37.650 & 23.725 & 63013 & .42643 & .13148 & .03840 & 4498 & 12.889 & 8.5000 & .65948 & .46032 & .05696 & -.08870 \\
\hline 3270 & 28.110 & 18.782 & .66817 & .47741 & .17325 & .08358 & 4517 & 34.232 & 22.223 & .64918 & .46775 & .19209 & .10727 \\
\hline 3272 & 61.889 & 50.917 & .82271 & .72239 & .50549 & .42506 & 4520 & 36.512 & 24.146 & .66133 & .48289 & .21568 & .13925 \\
\hline 3277 & 20.000 & 11.255 & .56273 & .25687 & -.04200 & -.03231 & 4525 & 123.14 & 94.667 & .76875 & .65252 & .50855 & .65744 \\
\hline 3278 & 29.776 & 21.332 & .71644 & .56268 & .26145 & .12482 & 4563 & 77.667 & 57.444 & .73963 & .47707 & -.04449 & -.37110 \\
\hline 3280 & 21.434 & 15.309 & .71427 & .57302 & .26159 & .10700 & 4570 & 30.607 & 20.274 & .66241 & .47494 & .18115 & .09207 \\
\hline 3281 & 13.694 & 10.398 & .75926 & .67192 & .36547 & .14696 & 4577 & 40.335 & 26.950 & .66815 & .49426 & .21673 & .12284 \\
\hline 3283 & 46.239 & 29.296 & .63358 & .44265 & .17955 & .11129 & 4591 & 40.171 & 26.068 & .64893 & .46824 & .18960 & .10157 \\
\hline 3284 & 37.852 & 24.673 & .65184 & .46608 & .18924 & .10650 & 4670 & 30.553 & 20.378 & .66697 & .48978 & .20364 & .11255 \\
\hline 3285 & 37.244 & 25.057 & .67280 & .50296 & .23094 & .13815 & 4671 & 38.235 & 24.843 & .64974 & .47728 & .25333 & .22797 \\
\hline 3329 & 35.066 & 22.736 & .64837 & .46329 & .18367 & .09718 & 4679 & 37.052 & 23.851 & .64370 & .45272 & .18306 & .11538 \\
\hline 3335 & 36.722 & 22.091 & 60157 & .41453 & .16234 & .09101 & 4696 & 33.429 & 16.238 & .48575 & .14252 & .11437 & .33138 \\
\hline 3370 & 34.632 & 23.083 & .66653 & .50136 & .22178 & .11613 & 4703 & 40.424 & 27.812 & .68801 & .49511 & .17771 & .06129 \\
\hline 3410 & 31.150 & 20.919 & .67155 & .49110 & .19690 & .09920 & 4704 & 42.350 & 26.106 & .61643 & .43254 & .18408 & .11285 \\
\hline 3415 & 37.336 & 24.099 & .64545 & .45720 & .18373 & .10608 & 4731 & 44.738 & 29.400 & .65716 & .49151 & .24598 & .15290 \\
\hline 3430 & 50.658 & 31.905 & 62980 & .43145 & .17016 & .10809 & 4792 & 32.750 & 22.096 & .67468 & .49686 & .20854 & .11200 \\
\hline 3431 & 63.422 & 38.068 & .60024 & .37276 & .11466 & .05234 & 4819 & 44.899 & 28.904 & .64375 & .44390 & .16429 & .09522 \\
\hline 3441 & 39.818 & 27.126 & .68124 & .46384 & .12042 & .00366 & 4852 & 41.875 & 24.018 & .57356 & .22825 & -.01859 & .25948 \\
\hline 3442 & 30.534 & 19.474 & 63779 & .46415 & .18355 & .08284 & 4866 & 32.629 & 21.332 & .65378 & .48231 & .21048 & .11990 \\
\hline 3446 & 23.405 & 15.545 & .66416 & .48139 & .16233 & .04625 & 4876 & 34.803 & 21.955 & .63083 & .46184 & .23483 & .17442 \\
\hline 3460 & 31.542 & 21.524 & .68238 & .50740 & .18554 & .01598 & 4878 & 47.546 & 29.605 & .62265 & .42112 & .15593 & .09340 \\
\hline 3462 & 30.121 & 20.171 & 66966 & .52152 & .25195 & .13987 & 4880 & 31.647 & 21.191 & .66962 & .49418 & .20446 & .10398 \\
\hline
\end{tabular}


Appendix 4-3.7. L-moments of storm duration defined by 72-hour minimum interevent time for hourly rainfall stations in TexasContinued.

\begin{tabular}{|c|c|c|c|c|c|c|c|c|c|c|c|c|c|}
\hline $\begin{array}{c}\text { Station } \\
\text { no. }\end{array}$ & $\begin{array}{l}\text { Duration } \\
\text { mean } \\
\text { (hours) }\end{array}$ & $\begin{array}{l}\text { Duration } \\
\text { L-scale } \\
\text { (hours) }\end{array}$ & $\begin{array}{c}\text { Duration } \\
\text { L-CV } \\
\text { (dimen- } \\
\text { sionless) }\end{array}$ & $\begin{array}{l}\text { Duration } \\
\text { L-skew } \\
\text { (dimen- } \\
\text { sionless) }\end{array}$ & $\begin{array}{l}\text { Duration } \\
\text { L-kurtosis } \\
\text { (dimen- } \\
\text { sionless) }\end{array}$ & $\begin{array}{c}\text { Duration } \\
\text { Tau5 } \\
\text { (dimen- } \\
\text { sionless) }\end{array}$ & $\begin{array}{c}\text { Station } \\
\text { no. }\end{array}$ & $\begin{array}{c}\text { Duration } \\
\text { mean } \\
\text { (hours) }\end{array}$ & $\begin{array}{l}\text { Duration } \\
\text { L-scale } \\
\text { (hours) }\end{array}$ & $\begin{array}{c}\text { Duration } \\
\text { L-CV } \\
\text { (dimen- } \\
\text { sionless) }\end{array}$ & $\begin{array}{l}\text { Duration } \\
\text { L-skew } \\
\text { (dimen- } \\
\text { sionless) }\end{array}$ & $\begin{array}{l}\text { Duration } \\
\text { L-kurtosis } \\
\text { (dimen- } \\
\text { sionless) }\end{array}$ & $\begin{array}{c}\text { Duration } \\
\text { Tau5 } \\
\text { (dimen- } \\
\text { sionless) }\end{array}$ \\
\hline 4920 & 28.964 & 19.333 & 0.66751 & 0.48758 & 0.19450 & 0.09878 & 5957 & 33.492 & 22.338 & 0.66695 & 0.49263 & 0.21315 & 0.12086 \\
\hline 4934 & 16.400 & 13.000 & .79268 & .64615 & .15385 & -.43077 & 5958 & 34.890 & 21.399 & .61332 & .40436 & .11661 & .03122 \\
\hline 4972 & 33.882 & 22.071 & .65141 & .47022 & .18968 & .10142 & 5973 & 42.931 & 27.405 & .63836 & .43916 & .15885 & .07633 \\
\hline 4973 & 35.472 & 23.050 & 64979 & .50115 & .25083 & .16378 & 5996 & 34.677 & 22.504 & .64896 & .46172 & .18165 & .10119 \\
\hline 4974 & 29.615 & 19.977 & .67455 & .51312 & .23228 & .13027 & 6017 & 42.186 & 26.711 & .63318 & .39529 & .08947 & .04435 \\
\hline 4975 & 39.152 & 25.085 & .64071 & .44462 & .17033 & .09677 & 6024 & 33.472 & 20.423 & .61014 & .41500 & .15525 & .07565 \\
\hline 4978 & 33.193 & 23.094 & .69574 & .56087 & .31496 & .22456 & 6050 & 24.400 & 15.638 & .64091 & .59224 & .42120 & .21801 \\
\hline 4979 & 112.00 & 74.800 & .66786 & .49332 & .32487 & .50579 & 6104 & 41.220 & 28.243 & .68518 & .50753 & .22000 & .12252 \\
\hline 4982 & 30.502 & 20.098 & .65891 & .49448 & .21089 & .10139 & 6108 & 38.085 & 24.851 & .65252 & .46898 & .19657 & .11637 \\
\hline 5018 & 34.789 & 23.803 & .68421 & .54111 & .26705 & .14866 & 6136 & 30.395 & 20.643 & .67914 & .49777 & .19844 & .10015 \\
\hline 5048 & 23.330 & 15.672 & .67175 & .50143 & .20796 & .11050 & 6166 & 32.321 & 18.583 & .57495 & .33583 & .06681 & .03121 \\
\hline 5049 & 25.556 & 17.654 & .69082 & .51124 & .19922 & .10071 & 6176 & 41.356 & 26.691 & .64539 & .47564 & .20588 & .10425 \\
\hline 5056 & -- & -- & -- & -- & -- & -- & 6177 & 40.677 & 26.308 & .64675 & .45403 & .17219 & .08953 \\
\hline 5057 & 39.568 & 25.667 & .64867 & .44287 & .15108 & .07650 & 6210 & 36.587 & 23.842 & .65166 & .46891 & .19518 & .11472 \\
\hline 5060 & 45.856 & 29.694 & .64755 & .42271 & .11460 & .05037 & 6211 & 47.593 & 29.571 & .62132 & .42746 & .18993 & .14464 \\
\hline 5081 & 43.731 & 27.374 & .62598 & .43307 & .16246 & .09122 & 6270 & 40.401 & 25.825 & .63922 & .45226 & .18973 & .11965 \\
\hline 5094 & 35.711 & 23.485 & .65763 & .47510 & .19353 & .10400 & 6275 & -- & -- & -- & -- & -- & -- \\
\hline 5113 & 35.724 & 23.311 & .65252 & .46405 & .18596 & .10822 & 6276 & 88.500 & 72.071 & .81437 & .72646 & .57384 & .59812 \\
\hline 5114 & -- & -- & -- & -- & -- & -- & 6335 & 38.277 & 24.554 & .64148 & .46194 & .18597 & .09552 \\
\hline 5123 & 20.545 & 11.200 & .54513 & .29437 & .16396 & .32738 & 6434 & 24.857 & 12.791 & .51459 & .34665 & .09579 & .07002 \\
\hline 5192 & 36.781 & 23.821 & .64764 & .46327 & .18896 & .10865 & 6504 & 30.382 & 20.931 & .68893 & .52367 & .23301 & .11996 \\
\hline 5193 & 38.583 & 24.907 & .64556 & .45830 & .18248 & .10018 & 6558 & 21.050 & 12.008 & .57045 & .32252 & .07865 & .09841 \\
\hline 5224 & 50.347 & 31.328 & .62225 & .41532 & .15661 & .10842 & 6615 & 28.661 & 19.811 & .69121 & .52181 & .22830 & .12591 \\
\hline 5228 & 38.908 & 26.076 & .67019 & .48624 & .20366 & .12025 & 6660 & 33.433 & 19.982 & .59769 & .36061 & .07191 & .02371 \\
\hline 5235 & 40.765 & 28.750 & .70527 & .59059 & .42273 & .43695 & 6663 & 61.600 & 40.045 & .65008 & .42221 & .13143 & .09333 \\
\hline 5247 & 32.897 & 22.040 & .66998 & .49373 & .20913 & .11487 & 6734 & 40.257 & 25.584 & .63551 & .43970 & .16632 & .08973 \\
\hline 5258 & 36.473 & 23.660 & .64870 & .47796 & .20837 & .12013 & 6736 & 25.885 & 17.818 & .68834 & .51873 & .21220 & .09540 \\
\hline 5303 & 29.983 & 19.471 & .64941 & .47738 & .19705 & .11349 & 6740 & 33.200 & 22.200 & .66867 & .49474 & .19294 & .03028 \\
\hline 5312 & 32.379 & 22.160 & .68441 & .51115 & .22096 & .12282 & 6750 & 51.323 & 32.639 & .63596 & .43279 & .17973 & .12171 \\
\hline 5341 & 107.71 & 79.952 & .74226 & .67933 & .66647 & .79273 & 6757 & 43.487 & 27.925 & .64214 & .45020 & .17598 & .10009 \\
\hline 5342 & -- & -- & -- & -- & -- & -- & 6775 & 37.693 & 23.802 & .63149 & .43081 & .14743 & .06895 \\
\hline 5348 & 40.989 & 25.967 & .63352 & .43794 & .16760 & .09574 & 6776 & 34.621 & 23.396 & .67578 & .49426 & .20497 & .11462 \\
\hline 5358 & 28.735 & 18.837 & .65554 & .47819 & .18775 & .08923 & 6788 & 37.057 & 22.925 & .61865 & .41087 & .11164 & .02123 \\
\hline 5398 & 42.887 & 26.820 & .62538 & .42432 & .15203 & .08753 & 6792 & 27.841 & 19.272 & .69224 & .52159 & .22203 & .11758 \\
\hline 5410 & 29.612 & 20.759 & .70102 & .53986 & .24705 & .13405 & 6794 & 130.67 & 53.000 & .40561 & .53711 & .78616 & .52830 \\
\hline 5411 & 38.843 & 25.253 & .65012 & .46415 & .18643 & .10065 & 6834 & 39.017 & 25.187 & .64553 & .44902 & .16853 & .09169 \\
\hline 5424 & 70.161 & 44.140 & .62913 & .43743 & .18943 & .11755 & 6893 & 23.105 & 16.339 & .70715 & .55211 & .24730 & .12213 \\
\hline 5429 & 37.910 & 24.999 & .65943 & .47828 & .19486 & .10384 & 6935 & 29.074 & 20.210 & .69512 & .52872 & .23103 & .11749 \\
\hline 5431 & 32.273 & 19.782 & .61296 & .40625 & .12607 & -.00689 & 6981 & 33.579 & 20.950 & .62391 & .43258 & .16549 & .08415 \\
\hline 5461 & 34.993 & 23.157 & .66176 & .48413 & .19399 & .09370 & 7020 & 32.723 & 19.996 & .61108 & .42839 & .18042 & .11388 \\
\hline 5463 & 34.741 & 22.490 & .64737 & .44924 & .16249 & .08417 & 7060 & 30.531 & 21.581 & .70686 & .55148 & .26456 & .15054 \\
\hline 5471 & 60.222 & 45.361 & .75323 & .60616 & .39332 & .44266 & 7066 & 39.813 & 25.681 & .64504 & .46254 & .18852 & .10413 \\
\hline 5477 & 45.273 & 39.618 & .87510 & .81612 & .63056 & .47575 & 7074 & 27.327 & 18.760 & .68650 & .51265 & .20677 & .09426 \\
\hline 5528 & 29.892 & 19.673 & .65813 & .48260 & .19562 & .10108 & 7097 & 42.169 & 28.989 & .68744 & .52105 & .25392 & .16834 \\
\hline 5579 & -- & -- & -- & -- & -- & -- & 7116 & 32.943 & 22.316 & .67742 & .51785 & .23396 & .11432 \\
\hline 5580 & 98.625 & 56.125 & .56907 & .22176 & -.15495 & -.33058 & 7140 & 40.108 & 26.370 & .65746 & .47622 & .20061 & .11630 \\
\hline 5589 & 33.146 & 21.465 & .64760 & .44756 & .16614 & .10970 & 7173 & 59.889 & 38.066 & .63562 & .45276 & .21147 & .14715 \\
\hline 5590 & 42.767 & 26.876 & .62842 & .40790 & .12683 & .07406 & 7174 & 61.635 & 38.758 & .62883 & .43252 & .17220 & .10053 \\
\hline 5591 & 27.119 & 19.004 & .70078 & .53559 & .21716 & .07922 & 7213 & 36.713 & 24.111 & .65676 & .47288 & .19531 & .11658 \\
\hline 5592 & 28.526 & 19.805 & .69427 & .54080 & .25020 & .13067 & 7243 & 35.351 & 23.214 & .65668 & .46889 & .19000 & .11523 \\
\hline 5594 & 23.754 & 16.996 & .71549 & .56656 & .24159 & .07665 & 7262 & 27.883 & 19.090 & .68464 & .49994 & .21393 & .17330 \\
\hline 5595 & -- & -- & -- & -- & -- & -- & 7274 & 20.632 & 13.716 & .66479 & .51618 & .22765 & .10111 \\
\hline 5596 & 33.499 & 22.272 & .66485 & .45645 & .14809 & .07400 & 7300 & 29.371 & 19.690 & .67040 & .51324 & .23630 & .12890 \\
\hline 5600 & 27.301 & 18.838 & 69000 & .52887 & .23457 & .12906 & 7311 & 24.450 & 17.545 & .71758 & .56622 & .24721 & .06869 \\
\hline 5618 & 101.78 & 77.444 & .76092 & .63292 & .38051 & .30078 & 7363 & 87.250 & 56.500 & .64756 & .44943 & .32870 & .52023 \\
\hline 5650 & 61.875 & 47.696 & .77085 & .68776 & .39049 & .09547 & 7422 & 34.607 & 22.477 & .64949 & .46248 & .18480 & .10422 \\
\hline 5656 & 32.700 & 21.900 & .66971 & .48347 & .19600 & .11376 & 7431 & 23.809 & 16.513 & .69354 & .53788 & .23691 & .10927 \\
\hline 5658 & 30.010 & 19.379 & .64575 & .46379 & .17187 & .07231 & 7481 & 24.317 & 16.363 & .67291 & .49262 & .17664 & .06296 \\
\hline 5661 & 33.795 & 21.654 & .64075 & .42524 & .14163 & .09733 & 7497 & 38.460 & 24.361 & .63341 & .43271 & .16378 & .10062 \\
\hline 5666 & 29.952 & 19.529 & .65199 & .44329 & .13994 & .09241 & 7498 & 38.485 & 26.462 & .68760 & .49922 & .18417 & .06299 \\
\hline 5695 & 34.361 & 22.145 & .64447 & .44992 & .16269 & .08022 & 7499 & 32.441 & 21.234 & .65454 & .46932 & .19044 & .10984 \\
\hline 5742 & 39.700 & 29.633 & .74643 & .71260 & .59465 & .49138 & 7531 & 36.744 & 22.731 & .61862 & .41486 & .13779 & .07471 \\
\hline 5766 & 118.71 & 47.095 & .39671 & .09767 & .07280 & -.18402 & 7534 & 32.405 & 21.370 & .65946 & .46309 & .16426 & .07928 \\
\hline 5770 & 35.688 & 23.275 & .65218 & .45848 & .17298 & .09509 & 7556 & 34.086 & 22.512 & .66045 & .47860 & .19834 & .11399 \\
\hline 5775 & 35.571 & 21.524 & .60509 & .36372 & .25885 & .51549 & 7594 & 37.702 & 24.749 & .65644 & .46956 & .18211 & .09342 \\
\hline 5779 & 29.067 & 16.933 & .58257 & .30752 & -.00826 & -.07024 & 7596 & 26.312 & 17.390 & .66089 & .48581 & .19768 & .09960 \\
\hline 5840 & 30.368 & 20.619 & .67896 & .53472 & .26331 & .14866 & 7608 & 42.937 & 27.105 & .63128 & .42154 & .14859 & .09457 \\
\hline 5890 & 31.600 & 20.961 & .66332 & .48824 & .20320 & .10710 & 7622 & 29.857 & 25.593 & .85720 & .80485 & .62928 & .54282 \\
\hline 5891 & 23.595 & 15.817 & .67035 & .52169 & .25285 & .15379 & 7700 & 49.717 & 30.464 & .61276 & .41318 & .15364 & .09510 \\
\hline 5897 & 37.099 & 23.626 & .63683 & .44060 & .16972 & .09882 & 7706 & 31.899 & 21.036 & .65946 & .47102 & .18098 & .09608 \\
\hline
\end{tabular}


Appendix 4-3.7. L-moments of storm duration defined by 72-hour minimum interevent time for hourly rainfall stations in TexasContinued.

\begin{tabular}{|c|c|c|c|c|c|c|c|c|c|c|c|c|c|}
\hline $\begin{array}{c}\text { Station } \\
\text { no. }\end{array}$ & $\begin{array}{l}\text { Duration } \\
\text { mean } \\
\text { (hours) }\end{array}$ & $\begin{array}{l}\text { Duration } \\
\text { L-scale } \\
\text { (hours) }\end{array}$ & $\begin{array}{c}\text { Duration } \\
\text { L-CV } \\
\text { (dimen- } \\
\text { sionless) }\end{array}$ & $\begin{array}{l}\text { Duration } \\
\text { L-skew } \\
\text { (dimen- } \\
\text { sionless) }\end{array}$ & $\begin{array}{l}\text { Duration } \\
\text { L-kurtosis } \\
\text { (dimen- } \\
\text { sionless) }\end{array}$ & $\begin{array}{l}\text { Duration } \\
\text { Tau5 } \\
\text { (dimen- } \\
\text { sionless) }\end{array}$ & $\begin{array}{c}\text { Station } \\
\text { no. }\end{array}$ & $\begin{array}{c}\text { Duration } \\
\text { mean } \\
\text { (hours) }\end{array}$ & $\begin{array}{l}\text { Duration } \\
\text { L-scale } \\
\text { (hours) }\end{array}$ & $\begin{array}{l}\text { Duration } \\
\text { L-CV } \\
\text { (dimen- } \\
\text { sionless) }\end{array}$ & $\begin{array}{l}\text { Duration } \\
\text { L-skew } \\
\text { (dimen- } \\
\text { sionless) }\end{array}$ & $\begin{array}{l}\text { Duration } \\
\text { L-kurtosis } \\
\text { (dimen- } \\
\text { sionless) }\end{array}$ & $\begin{array}{c}\text { Duration } \\
\text { Tau5 } \\
\text { (dimen- } \\
\text { sionless) }\end{array}$ \\
\hline 7718 & 31.333 & 21.322 & 0.68048 & 0.51344 & 0.23927 & 0.15565 & 8910 & 53.000 & 40.583 & 0.76572 & 0.59519 & 0.22216 & -0.00235 \\
\hline 7745 & 47.429 & 28.927 & 60991 & .40102 & .15541 & .11810 & 8911 & 42.284 & 28.533 & .67481 & .48640 & .19239 & .10133 \\
\hline 7922 & 26.310 & 18.630 & .70812 & .56126 & .27051 & .14243 & 8924 & 22.744 & 15.964 & .70188 & .55940 & .27136 & .15164 \\
\hline 7936 & 45.130 & 29.044 & .64358 & .46016 & .19423 & .11438 & 8929 & 53.667 & 46.694 & .87008 & .79723 & .59590 & .45755 \\
\hline 7943 & 33.631 & 21.845 & .64954 & .46493 & .18136 & .09574 & 8942 & 43.049 & 27.883 & .64771 & .46195 & .19481 & .11945 \\
\hline 7944 & 57.818 & 32.030 & .55398 & .26691 & .00723 & -.00202 & 8944 & 46.432 & 30.202 & .65045 & .45606 & .18088 & .11043 \\
\hline 7945 & 47.617 & 30.018 & .63040 & .43531 & .17628 & .10701 & 8996 & 45.176 & 28.970 & .64127 & .45057 & .18165 & .11310 \\
\hline 7947 & 43.101 & 27.888 & .64705 & .46245 & .21156 & .15599 & 9014 & 98.286 & 66.333 & .67490 & .58363 & .37688 & .44293 \\
\hline 7948 & 30.898 & 20.738 & .67117 & .49916 & .21602 & .11997 & 9037 & 22.572 & 15.369 & .68091 & .51826 & .20918 & .07929 \\
\hline 7951 & 41.260 & 27.055 & .65571 & .47952 & .20027 & .10525 & 9106 & 24.873 & 17.624 & .70855 & .56927 & .28192 & .15403 \\
\hline 7953 & 36.571 & 23.657 & .64687 & .42409 & .09855 & .03478 & 9107 & 23.167 & 18.138 & .78292 & .65369 & .31378 & .07239 \\
\hline 7981 & 27.593 & 18.054 & .65431 & .47039 & .17831 & .09286 & 9129 & 33.952 & 22.040 & .64914 & .43382 & .10984 & .02204 \\
\hline 7990 & 57.793 & 37.337 & .64605 & .43704 & .16013 & .09641 & 9163 & 33.019 & 21.895 & .66310 & .48609 & .19835 & .09738 \\
\hline 7992 & 135.17 & 98.700 & .73021 & .64404 & .47991 & .62985 & 9213 & 51.470 & 34.161 & .66371 & .47382 & .20306 & .12582 \\
\hline 7997 & 22.405 & 13.755 & .61393 & .41388 & .12952 & .06740 & 9214 & 159.50 & 89.900 & .56364 & .49722 & .70337 & .55432 \\
\hline 7999 & 18.769 & 11.269 & .60041 & .33850 & -.00745 & -.02661 & 9222 & 42.299 & 26.099 & .61701 & .44177 & .19477 & .10586 \\
\hline 8022 & 21.935 & 14.592 & .66524 & .49681 & .20087 & .11508 & 9248 & 23.884 & 15.394 & .64453 & .46673 & .18931 & .10283 \\
\hline 8023 & 26.236 & 18.065 & .68855 & .52125 & .21784 & .10144 & 9266 & 25.981 & 16.454 & .63330 & .45387 & .16521 & .06723 \\
\hline 8047 & 33.247 & 21.713 & .65307 & .46392 & .17902 & .09780 & 9270 & 34.991 & 23.873 & .68224 & .49139 & .18364 & .08532 \\
\hline 8060 & 39.667 & 27.219 & .68619 & .50728 & .20950 & .10991 & 9295 & 38.574 & 26.468 & .68616 & .47964 & .14954 & .04395 \\
\hline 8062 & 34.500 & 18.995 & .55059 & .35284 & .23126 & .24846 & 9304 & -- & -- & -- & -- & -- & -- \\
\hline 8068 & 31.810 & 17.138 & .53877 & .29902 & .11903 & .13077 & 9307 & 27.222 & 18.080 & .66415 & .50309 & .22058 & .11293 \\
\hline 8081 & 35.707 & 23.734 & .66470 & .48318 & .20339 & .12124 & 9328 & 37.265 & 25.335 & .67985 & .48878 & .18051 & .07485 \\
\hline 8089 & 33.308 & 23.446 & .70393 & .51250 & .15754 & .01000 & 9329 & 54.000 & 39.000 & .72222 & .51648 & .17949 & .12332 \\
\hline 8221 & 84.875 & 60.411 & .71176 & .63110 & .38457 & .33727 & 9345 & -- & -- & -- & -- & -- & -- \\
\hline 8252 & 25.094 & 17.064 & .68001 & .50763 & .20268 & .09421 & 9363 & 53.267 & 32.594 & .61189 & .39045 & .13236 & .08923 \\
\hline 8265 & 47.913 & 29.564 & .61704 & .41985 & .15997 & .10093 & 9364 & 52.276 & 33.044 & .63209 & .43471 & .17887 & .11760 \\
\hline 8289 & 28.343 & 16.592 & .58539 & .35168 & .09544 & .07313 & 9365 & 38.722 & 22.493 & .58089 & .26496 & -.03672 & .07057 \\
\hline 8305 & 27.309 & 18.912 & .69251 & .52317 & .22015 & .10924 & 9371 & 41.134 & 25.293 & .61489 & .38681 & .11477 & .07568 \\
\hline 8335 & 38.757 & 24.716 & .63773 & .45441 & .18687 & .11158 & 9417 & 37.336 & 24.484 & .65578 & .48153 & .20465 & .11007 \\
\hline 8400 & 33.102 & 21.727 & .65637 & .44172 & .13141 & .06352 & 9419 & 45.588 & 28.754 & .63075 & .44073 & .17779 & .10436 \\
\hline 8445 & 42.331 & 26.523 & .62655 & .43132 & .15798 & .08932 & 9435 & 29.440 & 20.263 & .68828 & .50476 & .20293 & .12340 \\
\hline 8446 & 40.141 & 25.952 & .64651 & .45033 & .16927 & .09323 & 9491 & 41.135 & 26.398 & .64175 & .45693 & .18912 & 10997 \\
\hline 8451 & 29.149 & 18.525 & .63552 & .42769 & .11951 & .03834 & 9499 & 27.826 & 18.682 & .67137 & .48851 & .18893 & .09359 \\
\hline 8531 & 34.980 & 23.308 & .66632 & .49854 & .22361 & .12590 & 9522 & -- & -- & -- & -- & -- & -- \\
\hline 8541 & 30.544 & 20.425 & .66870 & .55838 & .30338 & .18480 & 9527 & 32.221 & 22.134 & .68692 & .51016 & .21461 & .11546 \\
\hline 8544 & 38.211 & 24.026 & .62879 & .42893 & .15734 & .09456 & 9532 & 35.045 & 22.653 & .64639 & .45774 & .17875 & .09795 \\
\hline 8545 & 34.750 & 19.583 & .56355 & .39157 & .13681 & -.05777 & 9544 & -- & -- & -- & -- & -- & -- \\
\hline 8563 & 33.363 & 21.377 & .64074 & .45943 & .19723 & .12396 & 9565 & 31.312 & 21.246 & .67854 & .49443 & .18286 & .06951 \\
\hline 8566 & 30.094 & 20.532 & .68227 & .50519 & .19919 & .08334 & 9570 & 36.546 & 24.125 & .66012 & .46252 & .17897 & .11474 \\
\hline 8583 & 32.629 & 21.045 & .64496 & .44711 & .16571 & .09105 & 9574 & 53.727 & 19.255 & .35838 & .10922 & .19657 & .06217 \\
\hline 8584 & 29.208 & 19.405 & .66436 & .47698 & .18146 & .09474 & 9588 & 34.340 & 23.304 & .67864 & .49822 & .20564 & .10866 \\
\hline 8623 & 32.096 & 20.746 & .64636 & .45533 & .17061 & .08733 & 9665 & 35.701 & 23.094 & .64688 & .45827 & .17887 & .09915 \\
\hline 8625 & 35.384 & 21.972 & .62097 & .43127 & .15527 & .07445 & 9715 & 36.021 & 23.513 & .65276 & .47095 & .19207 & .10487 \\
\hline 8630 & 24.375 & 16.399 & .67279 & .51338 & .22661 & .11916 & 9729 & 39.451 & 24.933 & .63201 & .45203 & .19018 & .11029 \\
\hline 8631 & 26.750 & 18.019 & .67361 & .50330 & .20367 & .09084 & 9772 & 45.213 & 29.728 & .65752 & .47588 & .19909 & .11216 \\
\hline 8646 & 34.385 & 22.679 & .65955 & .48870 & .20651 & .10485 & 9814 & 40.136 & 26.141 & .65130 & .43034 & .06893 & -.06505 \\
\hline 8647 & 33.243 & 22.502 & .67691 & .49706 & .20676 & .11331 & 9815 & 41.351 & 26.736 & .64658 & .47074 & .20781 & .12859 \\
\hline 8677 & 31.155 & 19.891 & .63845 & .47006 & .18188 & .07660 & 9816 & 22.558 & 16.459 & .72964 & .61363 & .34286 & .20249 \\
\hline 8696 & 85.625 & 63.482 & .74140 & .59381 & .33727 & .32208 & 9817 & 29.684 & 20.088 & .67671 & .50972 & .22673 & .12818 \\
\hline 8743 & 40.906 & 25.885 & .63278 & .44360 & .17848 & .10931 & 9829 & 25.513 & 17.349 & .68002 & .50610 & .20241 & .09654 \\
\hline 8761 & 26.897 & 18.689 & .69484 & .52341 & .21216 & .08768 & 9830 & 24.914 & 18.085 & .72590 & .60128 & .31649 & .15866 \\
\hline 8778 & 40.145 & 25.666 & .63933 & .44467 & .16747 & .08911 & 9858 & 29.952 & 20.330 & .67874 & .51407 & .21757 & .09727 \\
\hline 8845 & 37.496 & 24.506 & .65355 & .46432 & .18825 & .11219 & 9893 & 32.139 & 21.026 & .65420 & .46926 & .18129 & .09105 \\
\hline 8859 & 39.225 & 25.363 & .64661 & .45773 & .17681 & .09411 & 9916 & 40.153 & 25.336 & .63099 & .45622 & .20671 & .13022 \\
\hline 8898 & 35.532 & 22.627 & .63680 & .46758 & .19594 & .09773 & 9976 & 30.413 & 20.184 & .66365 & .48121 & .19348 & .10802 \\
\hline 8908 & 60.941 & 37.838 & .62090 & .34357 & .04808 & .12377 & & & & & & & \\
\hline
\end{tabular}


Appendix 4-4.1. Empirical distribution of storm depth defined by 6-hour minimum interevent time for hourly rainfall stations in Texas.

$[--$, not available $]$

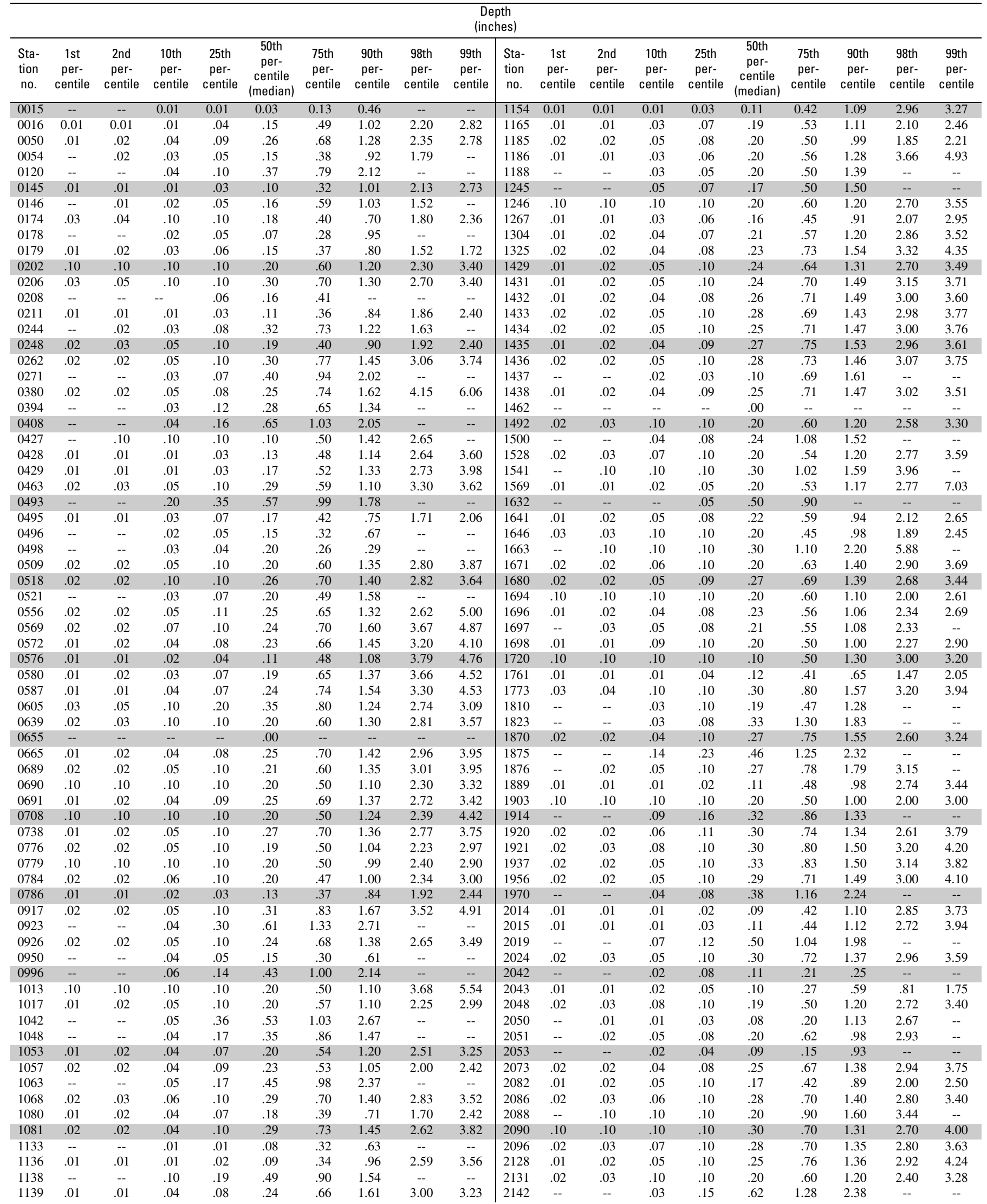


Appendix 4-4.1. Empirical distribution of storm depth defined by 6-hour minimum interevent time for hourly rainfall stations in TexasContinued.

\begin{tabular}{|c|c|c|c|c|c|c|c|c|c|c|c|c|c|c|c|c|c|c|c|}
\hline \multicolumn{20}{|c|}{$\begin{array}{c}\text { Depth } \\
\text { (inches) }\end{array}$} \\
\hline $\begin{array}{l}\text { Sta- } \\
\text { tion } \\
\text { no. }\end{array}$ & $\begin{array}{c}1 \text { st } \\
\text { per- } \\
\text { centile }\end{array}$ & $\begin{array}{c}\text { 2nd } \\
\text { per- } \\
\text { centile }\end{array}$ & $\begin{array}{c}10 \text { th } \\
\text { per- } \\
\text { centile }\end{array}$ & $\begin{array}{c}25 \text { th } \\
\text { per- } \\
\text { centile }\end{array}$ & $\begin{array}{c}\text { 50th } \\
\text { per- } \\
\text { centile } \\
\text { (median) }\end{array}$ & $\begin{array}{c}\text { 75th } \\
\text { per- } \\
\text { centile }\end{array}$ & $\begin{array}{c}\text { 90th } \\
\text { per- } \\
\text { centile }\end{array}$ & $\begin{array}{c}\text { 98th } \\
\text { per- } \\
\text { centile }\end{array}$ & $\begin{array}{c}\text { 99th } \\
\text { per- } \\
\text { centile }\end{array}$ & $\begin{array}{l}\text { Sta- } \\
\text { tion } \\
\text { no. }\end{array}$ & $\begin{array}{c}\text { 1st } \\
\text { per- } \\
\text { centile }\end{array}$ & $\begin{array}{c}\text { 2nd } \\
\text { per- } \\
\text { centile }\end{array}$ & $\begin{array}{c}10 \text { th } \\
\text { per- } \\
\text { centile }\end{array}$ & $\begin{array}{c}\text { 25th } \\
\text { per- } \\
\text { centile }\end{array}$ & $\begin{array}{c}\text { 50th } \\
\text { per- } \\
\text { centile } \\
\text { (median) }\end{array}$ & $\begin{array}{c}\text { 75th } \\
\text { per- } \\
\text { centile }\end{array}$ & $\begin{array}{c}\text { 90th } \\
\text { per- } \\
\text { centile }\end{array}$ & $\begin{array}{c}\text { 98th } \\
\text { per- } \\
\text { centile }\end{array}$ & $\begin{array}{c}\text { 99th } \\
\text { per- } \\
\text { centile }\end{array}$ \\
\hline 2160 & -- & -- & 0.01 & 0.03 & 0.12 & 0.39 & 0.64 & -- & -- & 3463 & -- & 0.01 & 0.03 & 0.06 & 0.25 & 0.63 & 1.65 & 5.14 & -- \\
\hline 2206 & 0.02 & 0.02 & .05 & .10 & .30 & .78 & 1.67 & 3.19 & 4.40 & 3476 & 0.01 & .02 & .04 & .09 & .25 & .65 & 1.30 & 2.65 & 3.54 \\
\hline 2238 & .01 & .01 & .01 & .03 & .10 & .38 & .87 & 1.75 & 2.05 & 3485 & -- & -- & .06 & .10 & .17 & 1.41 & 2.42 & -- & -- \\
\hline 2240 & .01 & .01 & .01 & .04 & .14 & .41 & .76 & 1.94 & 2.02 & 3507 & .05 & .07 & .10 & .10 & .20 & .70 & 1.40 & 2.96 & 3.53 \\
\hline 2242 & .01 & .01 & .02 & .05 & .20 & .65 & 1.31 & 2.47 & 3.25 & 3546 & .02 & .02 & .06 & .10 & .30 & .80 & 1.60 & 3.29 & 4.00 \\
\hline 2244 & .01 & .01 & .02 & .10 & .20 & .61 & 1.34 & 2.85 & 3.70 & 3547 & .02 & .02 & .05 & .11 & .30 & .77 & 1.47 & 2.59 & 2.99 \\
\hline 2247 & -- & .01 & .02 & .05 & .18 & .58 & 1.33 & 3.53 & -- & 3579 & -- & .02 & .04 & .09 & .23 & .84 & 1.65 & 2.90 & -- \\
\hline 2309 & .02 & .03 & .08 & .15 & .38 & .81 & 1.45 & 3.17 & 4.42 & 3642 & .02 & .02 & .07 & .10 & .28 & .72 & 1.45 & 3.00 & 3.79 \\
\hline 2312 & .04 & .07 & .10 & .10 & .30 & .79 & 1.37 & 2.70 & 3.46 & 3646 & .02 & .03 & .05 & .10 & .28 & .69 & 1.31 & 2.90 & 3.89 \\
\hline 2334 & -- & .02 & .06 & .14 & .55 & 1.13 & 2.39 & 4.39 & -- & 3668 & -- & -- & .10 & .33 & .68 & 1.27 & 2.54 & -- & -- \\
\hline 2336 & .02 & .03 & .05 & .15 & .35 & .85 & 1.35 & 2.25 & 3.60 & 3673 & -- & -- & .03 & .06 & .30 & 1.10 & 1.51 & -- & -- \\
\hline 2354 & -- & -- & .04 & .06 & .12 & .51 & .76 & -- & -- & 3686 & .03 & .05 & .10 & .10 & .20 & .70 & 1.40 & 2.40 & 3.10 \\
\hline 2355 & -- & .01 & .03 & .05 & .16 & .54 & 1.54 & 4.31 & -- & 3691 & .02 & .03 & .10 & .10 & .24 & .64 & 1.30 & 2.70 & 3.20 \\
\hline 2357 & .01 & .01 & .01 & .02 & .07 & .36 & .96 & 2.56 & 3.59 & 3734 & -- & -- & .03 & .09 & .38 & 1.22 & 3.24 & -- & -- \\
\hline 2360 & .01 & .01 & .01 & .03 & .09 & .36 & .96 & 2.35 & 3.09 & 3771 & .10 & .10 & .10 & .10 & .20 & .70 & 1.40 & 3.14 & 3.90 \\
\hline 2361 & .02 & .02 & .04 & .10 & .15 & .47 & .86 & 2.00 & 4.21 & 3789 & .01 & .01 & .03 & .06 & .11 & .24 & .46 & 1.02 & 2.02 \\
\hline 2394 & .02 & .02 & .04 & .10 & .27 & .71 & 1.43 & 2.90 & 3.93 & 3826 & .01 & .02 & .05 & .13 & .32 & .73 & 1.28 & 2.41 & 2.66 \\
\hline 2404 & .02 & .02 & .05 & .10 & .25 & .70 & 1.34 & 2.91 & 3.59 & 3831 & .02 & .03 & .05 & .10 & .28 & .81 & 1.39 & 2.96 & 3.74 \\
\hline 2415 & .02 & .02 & .05 & .11 & .34 & .86 & 1.66 & 3.36 & 4.08 & 3841 & .02 & .02 & .05 & .12 & .29 & .81 & 1.48 & 3.34 & 4.75 \\
\hline 2462 & .02 & .03 & .06 & .10 & .30 & .84 & 1.61 & 3.14 & 3.91 & 3871 & .01 & .02 & .04 & .08 & .23 & .63 & 1.19 & 2.27 & 3.16 \\
\hline 2528 & .03 & .03 & .05 & .09 & .24 & .64 & 1.36 & 2.55 & 2.77 & 3884 & -- & -- & .06 & .09 & .24 & .96 & 2.98 & -- & -- \\
\hline 2617 & .01 & .01 & .03 & .07 & .21 & .50 & 1.01 & 2.67 & 3.35 & 3941 & -- & .02 & .03 & .06 & .35 & 1.00 & 1.71 & 3.15 & -- \\
\hline 2619 & .02 & .02 & .05 & .10 & .23 & .54 & 1.05 & 2.16 & 2.94 & 3963 & -- & -- & -- & .03 & .05 & .10 & -- & -- & -- \\
\hline 2621 & .02 & .02 & .05 & .10 & .23 & .57 & 1.15 & 2.26 & 2.70 & 4040 & .02 & .02 & .05 & .10 & .26 & .62 & 1.23 & 2.48 & 3.13 \\
\hline 2675 & .02 & .03 & .09 & .10 & .25 & .70 & 1.37 & 3.07 & 3.83 & 4058 & -- & -- & .05 & .08 & .42 & .86 & 2.16 & -- & -- \\
\hline 2676 & .10 & .10 & .10 & .10 & .20 & .60 & 1.40 & 2.80 & 3.78 & 4098 & .03 & .04 & .10 & .10 & .20 & .40 & .80 & 1.70 & 2.10 \\
\hline 2679 & .01 & .02 & .05 & .10 & .13 & .50 & 1.20 & 2.88 & 3.98 & 4100 & .02 & .02 & .04 & .07 & .21 & .59 & 1.27 & 2.30 & 4.01 \\
\hline 2715 & .02 & .02 & .05 & .10 & .28 & .70 & 1.31 & 2.66 & 3.47 & 4137 & .10 & .10 & .10 & .10 & .20 & .70 & 1.30 & 2.43 & 3.10 \\
\hline 2744 & .02 & .02 & .05 & .10 & .21 & .60 & 1.17 & 2.30 & 3.02 & 4191 & .02 & .02 & .05 & .10 & .20 & .57 & 1.30 & 2.85 & 3.58 \\
\hline 2758 & .01 & .01 & .02 & .03 & .09 & .29 & 1.17 & 3.29 & 4.38 & 4256 & -- & -- & -- & -- & .00 & -- & -- & -- & -- \\
\hline 2794 & -- & -- & .01 & .04 & .16 & .66 & 1.61 & -- & -- & 4257 & .03 & .05 & .10 & .10 & .30 & .80 & 1.60 & 3.30 & 4.30 \\
\hline 2797 & .01 & .01 & .01 & .02 & .08 & .22 & .50 & 1.12 & 1.52 & 4258 & .10 & .10 & .10 & .10 & .20 & .70 & 1.20 & 2.56 & 4.18 \\
\hline 2811 & .01 & .02 & .05 & .10 & .20 & .50 & 1.10 & 2.30 & 2.98 & 4278 & .02 & .02 & .05 & .09 & .27 & .74 & 1.46 & 2.99 & 3.64 \\
\hline 2813 & -- & -- & .05 & .09 & .30 & .58 & 1.37 & -- & -- & 4299 & .01 & .03 & .05 & .10 & .25 & .43 & .72 & 1.17 & 1.24 \\
\hline 2814 & -- & -- & .01 & .03 & .04 & .13 & .92 & -- & -- & 4300 & .01 & .01 & .01 & .04 & .16 & .57 & 1.31 & 2.84 & 3.66 \\
\hline 2815 & .10 & .10 & .10 & .10 & .20 & .50 & 1.06 & 2.70 & 3.44 & 4305 & .01 & .01 & .01 & .03 & .14 & .52 & 1.26 & 2.94 & 3.57 \\
\hline 2818 & .02 & .02 & .04 & .09 & .30 & .68 & 1.30 & 3.03 & 3.46 & 4307 & .01 & .01 & .01 & .04 & .18 & .58 & 1.26 & 2.68 & 3.69 \\
\hline 2986 & .01 & .02 & .05 & .10 & .35 & 1.03 & 1.71 & 2.77 & 3.57 & 4309 & .01 & .02 & .04 & .08 & .26 & .77 & 1.55 & 3.26 & 4.46 \\
\hline 3005 & .02 & .02 & .06 & .10 & .26 & .69 & 1.30 & 2.56 & 3.24 & 4311 & .01 & .02 & .04 & .10 & .29 & .80 & 1.69 & 3.34 & 4.39 \\
\hline 3033 & .02 & .02 & .04 & .07 & .13 & .30 & .55 & 1.16 & 1.83 & 4313 & .01 & .01 & .04 & .08 & .25 & .74 & 1.60 & 3.91 & 5.20 \\
\hline 3034 & -- & -- & -- & -- & .43 & -- & -- & -- & -- & 4319 & .02 & .02 & .05 & .10 & .25 & .75 & 1.30 & 2.36 & 3.34 \\
\hline 3047 & -- & -- & .03 & .05 & .34 & .72 & 1.32 & -- & -- & 4329 & .02 & .02 & .05 & .10 & .29 & .75 & 1.60 & 3.48 & 4.45 \\
\hline 3103 & -- & -- & .05 & .10 & .24 & .82 & 1.93 & -- & -- & 4331 & -- & -- & -- & -- & .00 & -- & -- & -- & -- \\
\hline 3133 & .02 & .02 & .06 & .10 & .30 & .79 & 1.46 & 3.00 & 3.86 & 4375 & .10 & .10 & .10 & .10 & .20 & .60 & 1.20 & 2.90 & 4.31 \\
\hline 3156 & .04 & .05 & .10 & .10 & .30 & .70 & 1.61 & 4.37 & 5.90 & 4392 & .01 & .01 & .04 & .08 & .28 & .87 & 1.71 & 3.55 & 4.24 \\
\hline 3171 & .02 & .02 & .05 & .10 & .28 & .71 & 1.40 & 2.98 & 3.99 & 4425 & .03 & .03 & .08 & .10 & .15 & .40 & .89 & 1.90 & 2.45 \\
\hline 3189 & .01 & .01 & .03 & .06 & .13 & .35 & .70 & 1.45 & 2.35 & 4440 & .02 & .02 & .04 & .07 & .22 & .60 & 1.20 & 2.61 & 3.09 \\
\hline 3260 & .01 & .01 & .03 & .07 & .25 & .70 & 1.25 & 2.39 & 3.46 & 4476 & .03 & .04 & .10 & .10 & .26 & .65 & 1.24 & 2.64 & 3.20 \\
\hline 3267 & .01 & .01 & .02 & .05 & .15 & .55 & .97 & 2.75 & 3.69 & 4498 & -- & -- & .02 & .03 & .13 & .28 & .57 & -- & -- \\
\hline 3270 & .04 & .07 & .10 & .10 & .20 & .50 & 1.10 & 2.27 & 3.12 & 4517 & .02 & .02 & .04 & .09 & .26 & .69 & 1.30 & 2.65 & 3.34 \\
\hline 3272 & -- & -- & .02 & .04 & .06 & .12 & .32 & -- & -- & 4520 & .10 & .10 & .10 & .10 & .20 & .60 & 1.30 & 2.50 & 3.30 \\
\hline 3277 & -- & -- & .02 & .02 & .08 & .21 & .46 & -- & -- & 4525 & -- & -- & .04 & .06 & .25 & .78 & 2.44 & -- & -- \\
\hline 3278 & .02 & .02 & .05 & .10 & .20 & .50 & 1.00 & 2.07 & 2.50 & 4563 & -- & -- & .03 & .06 & .29 & .60 & 1.52 & -- & -- \\
\hline 3280 & .01 & .01 & .03 & .05 & .13 & .35 & .77 & 1.82 & 1.95 & 4570 & .02 & .02 & .06 & .10 & .20 & .50 & 1.10 & 2.34 & 2.80 \\
\hline 3281 & -- & -- & .04 & .11 & .23 & .49 & .92 & -- & -- & 4577 & .02 & .03 & .09 & .10 & .33 & .80 & 1.50 & 2.92 & 3.79 \\
\hline 3283 & .01 & .01 & .01 & .04 & .18 & .57 & 1.26 & 2.63 & 3.11 & 4591 & .01 & .02 & .04 & .10 & .27 & .70 & 1.47 & 3.09 & 3.89 \\
\hline 3284 & .01 & .01 & .06 & .10 & .22 & .65 & 1.30 & 2.61 & 3.48 & 4670 & .02 & .02 & .05 & .10 & .20 & .50 & 1.10 & 2.20 & 2.84 \\
\hline 3285 & .03 & .06 & .10 & .10 & .20 & .70 & 1.40 & 2.60 & 3.20 & 4671 & .01 & .01 & .02 & .04 & .15 & .48 & .92 & 2.04 & 2.59 \\
\hline 3329 & .01 & .02 & .04 & .08 & .20 & .57 & 1.22 & 2.70 & 3.53 & 4679 & .03 & .04 & .10 & .10 & .20 & .70 & 1.30 & 2.82 & 3.90 \\
\hline 3335 & .01 & .02 & .04 & .10 & .30 & .85 & 1.66 & 4.12 & 6.78 & 4696 & -- & -- & .05 & .10 & .27 & .66 & 1.13 & -- & -- \\
\hline 3370 & .02 & .02 & .05 & .12 & .33 & .82 & 1.55 & 3.22 & 3.85 & 4703 & .01 & .02 & .04 & .08 & .16 & .51 & 1.07 & 2.21 & 2.93 \\
\hline 3410 & .03 & .04 & .10 & .10 & .20 & .51 & 1.01 & 2.20 & 2.90 & 4704 & .02 & .02 & .04 & .08 & .29 & .84 & 1.82 & 4.04 & 4.55 \\
\hline 3415 & .02 & .02 & .08 & .10 & .24 & .67 & 1.32 & 2.60 & 3.52 & 4731 & .01 & .01 & .01 & .03 & .14 & .41 & .95 & 2.91 & 5.75 \\
\hline 3430 & .01 & .01 & .01 & .04 & .15 & .56 & 1.26 & 2.99 & 4.14 & 4792 & .10 & .10 & .10 & .10 & .20 & .70 & 1.30 & 2.59 & 3.10 \\
\hline 3431 & .01 & .01 & .01 & .04 & .12 & .50 & 1.23 & 3.26 & 4.08 & 4819 & .10 & .10 & .10 & .10 & .30 & .80 & 1.50 & 3.07 & 3.76 \\
\hline 3441 & -- & -- & .04 & .09 & .30 & .74 & 1.61 & -- & -- & 4852 & -- & -- & .17 & .35 & .50 & 1.07 & 2.38 & -- & -- \\
\hline 3442 & .02 & .02 & .04 & .07 & .17 & .43 & .94 & 1.85 & 2.37 & 4866 & .02 & .02 & .06 & .10 & .30 & .75 & 1.40 & 2.80 & 3.57 \\
\hline 3446 & .02 & .03 & .05 & .10 & .22 & .52 & 1.07 & 2.02 & 2.71 & 4876 & .10 & .10 & .10 & .10 & .30 & .80 & 1.90 & 4.15 & 4.53 \\
\hline 3460 & -- & -- & .04 & .08 & .41 & .90 & 1.45 & -- & -- & 4878 & .01 & .02 & .05 & .10 & .30 & .82 & 1.68 & 3.86 & 4.77 \\
\hline 3462 & .02 & .02 & .05 & .10 & .21 & .50 & 1.05 & 1.86 & 2.89 & 4880 & .02 & .02 & .05 & .10 & .20 & .50 & .95 & 1.92 & 2.56 \\
\hline
\end{tabular}


Appendix 4-4.1. Empirical distribution of storm depth defined by 6-hour minimum interevent time for hourly rainfall stations in TexasContinued.

\begin{tabular}{|c|c|c|c|c|c|c|c|c|c|c|c|c|c|c|c|c|c|c|c|}
\hline \multicolumn{20}{|c|}{$\begin{array}{c}\text { Depth } \\
\text { (inches) }\end{array}$} \\
\hline $\begin{array}{l}\text { Sta- } \\
\text { tion } \\
\text { no. }\end{array}$ & $\begin{array}{c}1 \text { st } \\
\text { per- } \\
\text { centile }\end{array}$ & $\begin{array}{c}\text { 2nd } \\
\text { per- } \\
\text { centile }\end{array}$ & $\begin{array}{c}\text { 10th } \\
\text { per- } \\
\text { centile }\end{array}$ & $\begin{array}{c}25 \text { th } \\
\text { per- } \\
\text { centile }\end{array}$ & $\begin{array}{c}\text { 50th } \\
\text { per- } \\
\text { centile } \\
\text { (median) }\end{array}$ & $\begin{array}{c}\text { 75th } \\
\text { per- } \\
\text { centile }\end{array}$ & $\begin{array}{c}\text { 90th } \\
\text { per- } \\
\text { centile }\end{array}$ & $\begin{array}{c}\text { 98th } \\
\text { per- } \\
\text { centile }\end{array}$ & $\begin{array}{c}\text { 99th } \\
\text { per- } \\
\text { centile }\end{array}$ & $\begin{array}{l}\text { Sta- } \\
\text { tion } \\
\text { no. }\end{array}$ & $\begin{array}{c}1 \text { st } \\
\text { per- } \\
\text { centile }\end{array}$ & $\begin{array}{c}\text { 2nd } \\
\text { per- } \\
\text { centile }\end{array}$ & $\begin{array}{c}10 \text { th } \\
\text { per- } \\
\text { centile }\end{array}$ & $\begin{array}{c}25 \text { th } \\
\text { per- } \\
\text { centile }\end{array}$ & $\begin{array}{c}\text { 50th } \\
\text { per- } \\
\text { centile } \\
\text { (median) }\end{array}$ & $\begin{array}{c}\text { 75th } \\
\text { per- } \\
\text { centile }\end{array}$ & $\begin{array}{c}\text { 90th } \\
\text { per- } \\
\text { centile }\end{array}$ & $\begin{array}{c}\text { 98th } \\
\text { per- } \\
\text { centile }\end{array}$ & $\begin{array}{c}\text { 99th } \\
\text { per- } \\
\text { centile }\end{array}$ \\
\hline 4920 & 0.02 & 0.03 & 0.06 & 0.10 & 0.20 & 0.60 & 1.30 & 2.83 & 3.73 & 5957 & 0.02 & 0.03 & 0.10 & 0.10 & 0.29 & 0.70 & 1.34 & 2.57 & 3.44 \\
\hline 4934 & -- & -- & -- & .07 & .12 & .23 & -- & -- & - & 5958 & .01 & .01 & .03 & .09 & .25 & .62 & 1.22 & 2.31 & 3.07 \\
\hline 4972 & .02 & .03 & .07 & .10 & .23 & .60 & 1.20 & 2.70 & 3.42 & 5973 & .01 & .01 & .02 & .04 & .09 & .39 & .97 & 2.93 & 3.40 \\
\hline 4973 & .02 & .03 & .07 & .15 & .40 & .91 & 1.65 & 3.00 & 3.69 & 5996 & .02 & .02 & .06 & .10 & .21 & .61 & 1.25 & 2.64 & 3.22 \\
\hline 4974 & .02 & .02 & .05 & .10 & .20 & .50 & 1.00 & 2.14 & 2.50 & 6017 & .01 & .01 & .02 & .04 & .13 & .42 & 1.04 & 2.51 & 2.80 \\
\hline 4975 & .10 & .10 & .10 & .10 & .30 & .80 & 1.50 & 2.90 & 4.20 & 6024 & .02 & .02 & .04 & .10 & .32 & 1.02 & 1.99 & 4.34 & 6.22 \\
\hline 4978 & .02 & .02 & .03 & .05 & .18 & .53 & 1.48 & 3.06 & 4.91 & 6050 & -- & -- & .06 & .18 & .35 & .95 & 1.70 & -- & -- \\
\hline 4979 & -- & -- & .06 & .11 & .50 & 1.15 & 2.51 & -- & -- & 6104 & .02 & .02 & .05 & .10 & .16 & .40 & .80 & 1.58 & 2.08 \\
\hline 4982 & .02 & .02 & .05 & .10 & .25 & .60 & 1.18 & 2.44 & 3.01 & 6108 & .02 & .03 & .10 & .10 & .30 & .83 & 1.64 & 3.20 & 4.34 \\
\hline 5018 & .02 & .02 & .05 & .10 & .29 & .72 & 1.32 & 2.22 & 2.83 & 6136 & .02 & .03 & .09 & .10 & .18 & .40 & .83 & 1.79 & 2.30 \\
\hline 5048 & .02 & .03 & .07 & .10 & .14 & .48 & 1.00 & 2.30 & 3.14 & 6166 & .01 & .01 & .03 & .05 & .12 & .38 & 1.03 & 1.77 & 2.84 \\
\hline 5049 & .10 & .10 & .10 & .10 & .20 & .40 & .80 & 3.78 & 4.93 & 6176 & .01 & .02 & .04 & .09 & .30 & .79 & 1.76 & 3.84 & 4.85 \\
\hline 5056 & -- & -- & -- & .20 & .37 & .69 & -- & -- & -- & 6177 & .02 & .03 & .10 & .10 & .30 & .80 & 1.60 & 3.38 & 4.30 \\
\hline 5057 & .01 & .01 & .01 & .03 & .08 & .27 & .79 & 2.18 & 2.90 & 6210 & .02 & .03 & .10 & .10 & .30 & .72 & 1.48 & 3.06 & 4.00 \\
\hline 5060 & .01 & .01 & .02 & .04 & .15 & .55 & 1.26 & 3.40 & 5.98 & 6211 & .01 & .01 & .03 & .07 & .24 & .70 & 1.43 & 2.91 & 4.04 \\
\hline 5081 & .01 & .02 & .04 & .09 & .30 & .78 & 1.48 & 2.90 & 3.67 & 6270 & .10 & .10 & .10 & .10 & .30 & .80 & 1.60 & 3.50 & 4.10 \\
\hline 5094 & .02 & .02 & .10 & .10 & .29 & .70 & 1.40 & 3.00 & 3.71 & 6275 & -- & -- & -- & -- & .00 & -- & -- & -- & -- \\
\hline 5113 & .02 & .02 & .05 & .10 & .20 & .55 & 1.30 & 2.90 & 3.79 & 6276 & -- & -- & .09 & .18 & .46 & 1.09 & 2.27 & -- & -- \\
\hline 5114 & -- & -- & -- & -- & .00 & -- & -- & -- & -- & 6335 & .02 & .02 & .05 & .10 & .30 & .80 & 1.48 & 2.79 & 3.37 \\
\hline 5123 & -- & -- & .06 & .14 & .25 & .80 & 1.94 & -- & -- & 6434 & -- & -- & .05 & .10 & .32 & .73 & 1.47 & -- & -- \\
\hline 5192 & .02 & .02 & .06 & .10 & .30 & .73 & 1.50 & 3.08 & 3.50 & 6504 & .02 & .03 & .07 & .10 & .20 & .47 & .97 & 1.95 & 2.85 \\
\hline 5193 & .02 & .02 & .05 & .10 & .20 & .66 & 1.40 & 2.77 & 3.63 & 6558 & -- & -- & .05 & .06 & .41 & .83 & 1.15 & -- & -- \\
\hline 5224 & .01 & .02 & .04 & .10 & .31 & .80 & 1.60 & 3.37 & 4.60 & 6615 & .08 & .10 & .10 & .10 & .20 & .50 & 1.05 & 2.30 & 3.50 \\
\hline 5228 & .01 & .02 & .04 & .08 & .24 & .77 & 1.48 & 2.85 & 3.45 & 6660 & .02 & .02 & .05 & .09 & .26 & .73 & 1.35 & 3.50 & 3.76 \\
\hline 5235 & -- & -- & .03 & .05 & .28 & .60 & 1.34 & -- & -- & 6663 & .01 & .01 & .04 & .10 & .20 & .55 & 1.35 & 2.72 & 3.08 \\
\hline 5247 & .02 & .03 & .08 & .10 & .20 & .50 & 1.00 & 1.94 & 2.40 & 6734 & .01 & .01 & .03 & .05 & .18 & .51 & 1.00 & 2.26 & 3.04 \\
\hline 5258 & .02 & .02 & .05 & .10 & .28 & .72 & 1.45 & 2.92 & 3.76 & 6736 & .02 & .03 & .08 & .10 & .20 & .50 & 1.10 & 2.37 & 3.10 \\
\hline 5303 & .01 & .02 & .04 & .07 & .20 & .60 & 1.24 & 2.68 & 4.20 & 6740 & - & -- & .10 & .18 & .58 & 1.35 & 3.05 & -- & - \\
\hline 5312 & .03 & .04 & .10 & .10 & .20 & .60 & 1.15 & 2.40 & 3.34 & 6750 & .01 & .01 & .01 & .04 & .22 & .61 & 1.34 & 3.25 & 7.14 \\
\hline 5341 & -- & -- & .07 & .20 & .36 & 1.20 & 1.70 & -- & -- & 6757 & .01 & .01 & .04 & .10 & .25 & .73 & 1.44 & 2.92 & 3.60 \\
\hline 5342 & -- & -- & -- & - & .00 & -- & -- & -- & -- & 6775 & .01 & .01 & .03 & .06 & .19 & .48 & .91 & 2.10 & 2.88 \\
\hline 5348 & .03 & .05 & .10 & .10 & .30 & .80 & 1.66 & 2.90 & 3.42 & 6776 & .02 & .03 & .08 & .10 & .20 & .50 & .96 & 2.00 & 2.70 \\
\hline 5358 & .02 & .02 & .05 & .10 & .20 & .56 & 1.16 & 2.40 & 2.80 & 6788 & .02 & .02 & .04 & .10 & .35 & .75 & 1.62 & 3.55 & 4.56 \\
\hline 5398 & .02 & .02 & .05 & .10 & .30 & .75 & 1.50 & 3.04 & 3.99 & 6792 & .02 & .02 & .05 & .10 & .17 & .40 & .80 & 1.56 & 1.96 \\
\hline 5410 & .02 & .02 & .05 & .10 & .20 & .50 & .92 & 2.21 & 3.02 & 6794 & -- & - & .02 & .20 & .55 & 1.44 & 2.84 & -- & -- \\
\hline 5411 & .01 & .01 & .01 & .03 & .11 & .36 & .85 & 1.86 & 2.56 & 6834 & .10 & .10 & .10 & .10 & .30 & .80 & 1.60 & 3.11 & 4.00 \\
\hline 5424 & .01 & .01 & .01 & .03 & .14 & .61 & 1.52 & 2.85 & 3.18 & 6893 & .02 & .03 & .07 & .10 & .12 & .30 & .70 & 1.60 & 2.35 \\
\hline 5429 & .02 & .02 & .03 & .07 & .20 & .60 & 1.30 & 2.66 & 4.21 & 6935 & .02 & .03 & .10 & .10 & .20 & .46 & .90 & 2.00 & 2.90 \\
\hline 5431 & -- & -- & .13 & .25 & .46 & .96 & 2.69 & -- & -- & 6981 & .01 & .02 & .05 & .10 & .28 & .71 & 1.39 & 2.79 & 4.56 \\
\hline 5461 & .01 & .02 & .05 & .10 & .31 & .85 & 1.59 & 3.40 & 4.10 & 7020 & .01 & .01 & .02 & .06 & .24 & .71 & 1.37 & 3.17 & 5.22 \\
\hline 5463 & .10 & .10 & .10 & .10 & .30 & .70 & 1.60 & 3.05 & 3.80 & 7060 & .02 & .03 & .10 & .10 & .20 & .53 & 1.20 & 2.30 & 2.83 \\
\hline 5471 & -- & -- & .01 & .02 & .06 & .11 & .38 & -- & -- & 7066 & .02 & .03 & .08 & .10 & .30 & .80 & 1.60 & 3.11 & 3.90 \\
\hline 5477 & -- & -- & .08 & .15 & .28 & .60 & 1.94 & -- & -- & 7074 & .02 & .02 & .05 & .10 & .20 & .43 & .90 & 2.30 & 2.83 \\
\hline 5528 & .02 & .03 & .06 & .12 & .34 & .85 & 1.56 & 3.21 & 3.96 & 7097 & .02 & .02 & .05 & .10 & .22 & .55 & 1.33 & 3.14 & 3.52 \\
\hline 5579 & - & -- & -- & - & .28 & -- & -- & - & - & 7116 & .02 & .02 & .03 & .07 & .20 & .53 & 1.03 & 2.28 & 3.03 \\
\hline 5580 & -- & -- & .05 & .11 & .42 & .84 & 1.39 & -- & -- & 7140 & .01 & .02 & .04 & .09 & .22 & .70 & 1.59 & 3.65 & 4.98 \\
\hline 5589 & .01 & .01 & .02 & .05 & .15 & .36 & .66 & 1.11 & 1.43 & 7173 & .01 & .01 & .01 & .04 & .17 & .65 & 1.51 & 4.49 & 5.78 \\
\hline 5590 & .01 & .02 & .03 & .06 & .14 & .36 & .83 & 1.41 & 1.81 & 7174 & .01 & .01 & .01 & .04 & .19 & .65 & 1.45 & 3.55 & 4.77 \\
\hline 5591 & .02 & .02 & .04 & .09 & .20 & .43 & .76 & 1.45 & 1.67 & 7213 & .02 & .02 & .05 & .10 & .29 & .73 & 1.44 & 3.21 & 4.03 \\
\hline 5592 & .01 & .02 & .03 & .06 & .15 & .35 & .64 & 1.56 & 1.89 & 7243 & .02 & .03 & .08 & .10 & .20 & .60 & 1.15 & 2.40 & 3.00 \\
\hline 5594 & .01 & .02 & .03 & .07 & .15 & .34 & .72 & 1.79 & 1.89 & 7262 & .01 & .01 & .02 & .04 & .10 & .26 & .51 & .99 & 1.10 \\
\hline 5595 & -- & -- & -- & -- & .03 & -- & -- & -- & -- & 7274 & .03 & .04 & .10 & .14 & .35 & .76 & 1.40 & 2.93 & 4.05 \\
\hline 5596 & .10 & .10 & .10 & .10 & .20 & .40 & .80 & 1.60 & 1.95 & 7300 & .02 & .02 & .04 & .10 & .27 & .70 & 1.30 & 2.65 & 3.25 \\
\hline 5600 & .01 & .02 & .04 & .07 & .16 & .40 & .76 & 1.85 & 2.26 & 7311 & -- & -- & .10 & .14 & .28 & .71 & 1.77 & -- & -- \\
\hline 5618 & -- & -- & .05 & .11 & .29 & .84 & 1.56 & -- & -- & 7363 & -- & -- & .04 & .10 & .45 & .94 & 1.46 & -- & -- \\
\hline 5650 & -- & -- & .07 & .17 & .42 & .56 & 1.41 & -- & -- & 7422 & .01 & .02 & .05 & .10 & .20 & .60 & 1.30 & 2.83 & 3.74 \\
\hline 5656 & .08 & .10 & .10 & .10 & .20 & .50 & 1.00 & 2.10 & 2.90 & 7431 & .02 & .02 & .05 & .10 & .20 & .50 & .99 & 2.09 & 2.67 \\
\hline 5658 & .02 & .02 & .04 & .07 & .20 & .51 & .99 & 2.29 & 3.36 & 7481 & .02 & .02 & .05 & .10 & .12 & .34 & .80 & 1.89 & 2.49 \\
\hline 5661 & .10 & .10 & .10 & .10 & .20 & .55 & 1.20 & 3.48 & 5.85 & 7497 & .10 & .10 & .10 & .10 & .20 & .60 & 1.30 & 2.74 & 3.32 \\
\hline 5666 & -- & -- & .04 & .06 & .25 & .56 & 1.14 & -- & -- & 7498 & .06 & .09 & .10 & .10 & .20 & .70 & 1.38 & 2.61 & 4.84 \\
\hline 5695 & .02 & .02 & .05 & .10 & .30 & .76 & 1.45 & 2.93 & 3.65 & 7499 & .03 & .05 & .10 & .10 & .24 & .60 & 1.20 & 2.48 & 3.00 \\
\hline 5742 & -- & -- & .02 & .04 & .09 & .30 & .71 & -- & -- & 7531 & .01 & .02 & .04 & .09 & .30 & .70 & 1.39 & 2.29 & 3.08 \\
\hline 5766 & -- & -- & .03 & .10 & .24 & .81 & 2.21 & -- & -- & 7534 & .01 & .02 & .04 & .07 & .25 & .63 & 1.25 & 3.22 & 3.83 \\
\hline 5770 & .02 & .02 & .05 & .10 & .20 & .50 & 1.00 & 2.20 & 3.00 & 7556 & .02 & .03 & .10 & .10 & .25 & .68 & 1.30 & 2.84 & 3.70 \\
\hline 5775 & - & -- & .05 & .06 & .18 & .70 & .92 & - & -- & 7594 & .02 & .02 & .05 & .11 & .34 & .85 & 1.61 & 3.30 & 3.93 \\
\hline 5779 & -- & -- & .03 & .07 & .23 & .50 & 1.90 & -- & -- & 7596 & .01 & .02 & .04 & .09 & .34 & 1.05 & 1.90 & 3.08 & 3.90 \\
\hline 5840 & .02 & .02 & .05 & .09 & .26 & .70 & 1.45 & 2.76 & 3.22 & 7608 & .01 & .01 & .02 & .06 & .21 & .65 & 1.36 & 2.76 & 3.39 \\
\hline 5890 & .01 & .01 & .01 & .03 & .10 & .35 & .82 & 1.90 & 2.39 & 7622 & -- & -- & .01 & .01 & .04 & .13 & .70 & -- & -- \\
\hline 5891 & .02 & .02 & .04 & .08 & .20 & .50 & .92 & 1.80 & 1.96 & 7700 & .01 & .02 & .04 & .08 & .26 & .75 & 1.52 & 3.29 & 4.15 \\
\hline 5897 & .10 & .10 & .10 & .10 & .20 & .70 & 1.40 & 3.00 & 3.70 & 7706 & .01 & .02 & .05 & .10 & .20 & .52 & 1.11 & 2.40 & 3.20 \\
\hline
\end{tabular}


Appendix 4-4.1. Empirical distribution of storm depth defined by 6-hour minimum interevent time for hourly rainfall stations in TexasContinued.

\begin{tabular}{|c|c|c|c|c|c|c|c|c|c|c|c|c|c|c|c|c|c|c|c|}
\hline \multicolumn{20}{|c|}{$\begin{array}{c}\text { Depth } \\
\text { (inches) }\end{array}$} \\
\hline $\begin{array}{l}\text { Sta- } \\
\text { tion } \\
\text { no. }\end{array}$ & $\begin{array}{c}1 \text { st } \\
\text { per- } \\
\text { centile }\end{array}$ & $\begin{array}{c}2 \text { 2nd } \\
\text { per- } \\
\text { centile }\end{array}$ & $\begin{array}{c}10 \text { th } \\
\text { per- } \\
\text { centile }\end{array}$ & $\begin{array}{c}25 \text { th } \\
\text { per- } \\
\text { centile }\end{array}$ & $\begin{array}{c}\text { 50th } \\
\text { per- } \\
\text { centile } \\
\text { (median) }\end{array}$ & $\begin{array}{c}\text { 75th } \\
\text { per- } \\
\text { centile }\end{array}$ & $\begin{array}{c}\text { 90th } \\
\text { per- } \\
\text { centile }\end{array}$ & $\begin{array}{c}\text { 98th } \\
\text { per- } \\
\text { centile }\end{array}$ & $\begin{array}{c}\text { 99th } \\
\text { per- } \\
\text { centile }\end{array}$ & $\begin{array}{l}\text { Sta- } \\
\text { tion } \\
\text { no. }\end{array}$ & $\begin{array}{c}\text { 1st } \\
\text { per- } \\
\text { centile }\end{array}$ & $\begin{array}{c}\text { 2nd } \\
\text { per- } \\
\text { centile }\end{array}$ & $\begin{array}{c}10 \text { th } \\
\text { per- } \\
\text { centile }\end{array}$ & $\begin{array}{c}25 \text { th } \\
\text { per- } \\
\text { centile }\end{array}$ & $\begin{array}{c}\text { 50th } \\
\text { per- } \\
\text { centile } \\
\text { (median) }\end{array}$ & $\begin{array}{c}\text { 75th } \\
\text { per- } \\
\text { centile }\end{array}$ & $\begin{array}{c}\text { 90th } \\
\text { per- } \\
\text { centile }\end{array}$ & $\begin{array}{c}\text { 98th } \\
\text { per- } \\
\text { centile }\end{array}$ & $\begin{array}{l}\text { 99th } \\
\text { per- } \\
\text { centile }\end{array}$ \\
\hline 7718 & 0.01 & 0.02 & 0.05 & 0.10 & 0.25 & 0.61 & 1.60 & 2.96 & 4.38 & 8910 & -- & -- & 0.05 & 0.10 & 0.39 & 1.10 & 1.87 & -- & -- \\
\hline 7745 & .01 & .01 & .03 & .08 & .25 & .75 & 1.69 & 3.71 & 4.19 & 8911 & 0.01 & 0.01 & .03 & .06 & .20 & .59 & 1.27 & 2.70 & 3.44 \\
\hline 7922 & .01 & .01 & .02 & .05 & .12 & .29 & .63 & 1.18 & 1.88 & 8924 & .02 & .02 & .04 & .07 & .17 & .34 & .75 & 1.43 & 1.76 \\
\hline 7936 & .02 & .03 & .10 & .10 & .30 & .84 & 1.60 & 3.18 & 4.29 & 8929 & -- & -- & .04 & .10 & .26 & 1.56 & 2.20 & -- & -- \\
\hline 7943 & .01 & .01 & .01 & .03 & .12 & .40 & .95 & 2.05 & 2.68 & 8942 & .03 & .06 & .10 & .10 & .30 & .80 & 1.60 & 3.40 & 4.40 \\
\hline 7944 & -- & .01 & .01 & .03 & .11 & .39 & .92 & 2.25 & -- & 8944 & .01 & .01 & .03 & .08 & .26 & .72 & 1.39 & 2.67 & 3.89 \\
\hline 7945 & .01 & .01 & .01 & .03 & .11 & .43 & 1.05 & 2.64 & 3.66 & 8996 & .01 & .02 & .04 & .08 & .24 & .66 & 1.43 & 3.02 & 3.79 \\
\hline 7947 & .03 & .04 & .10 & .10 & .20 & .62 & 1.40 & 3.53 & 4.84 & 9014 & -- & -- & .07 & .19 & .41 & .95 & 1.84 & -- & -- \\
\hline 7948 & .01 & .02 & .03 & .07 & .20 & .58 & 1.33 & 3.07 & 3.97 & 9037 & .02 & .02 & .04 & .06 & .15 & .31 & .68 & 1.60 & 2.22 \\
\hline 7951 & .02 & .02 & .05 & .11 & .32 & .80 & 1.52 & 2.74 & 3.35 & 9106 & .01 & .02 & .03 & .05 & .11 & .27 & .55 & 1.74 & 2.44 \\
\hline 7953 & .01 & .01 & .02 & .05 & .15 & .45 & 1.18 & 2.95 & 3.03 & 9107 & -- & -- & .03 & .06 & .14 & .48 & .80 & -- & -- \\
\hline 7981 & .02 & .02 & .03 & .06 & .23 & .60 & 1.53 & 2.49 & 3.16 & 9129 & .01 & .01 & .02 & .04 & .08 & .31 & .82 & 2.76 & 3.27 \\
\hline 7990 & .01 & .01 & .02 & .04 & .11 & .49 & .98 & 3.05 & 3.70 & 9163 & .02 & .02 & .05 & .10 & .23 & .60 & 1.25 & 2.63 & 3.28 \\
\hline 7992 & -- & -- & .09 & .10 & .28 & .93 & 1.79 & -- & -- & 9213 & .01 & .01 & .02 & .04 & .17 & .57 & 1.24 & 2.24 & 2.77 \\
\hline 7997 & .01 & .01 & .03 & .07 & .23 & .56 & 1.25 & 2.42 & 2.61 & 9214 & -- & -- & .08 & .21 & .35 & 1.27 & 1.88 & -- & -- \\
\hline 7999 & -- & -- & .01 & .03 & .16 & .32 & .86 & -- & -- & 9222 & .01 & .01 & .02 & .04 & .18 & .55 & 1.23 & 2.66 & 3.20 \\
\hline 8022 & -- & .01 & .05 & .05 & .14 & .59 & 1.10 & 2.08 & -- & 9248 & .01 & .02 & .05 & .09 & .20 & .58 & 1.15 & 2.09 & 2.24 \\
\hline 8023 & .02 & .03 & .06 & .10 & .20 & .50 & 1.06 & 2.16 & 3.02 & 9266 & -- & .02 & .05 & .11 & .24 & .72 & 1.29 & 2.67 & -- \\
\hline 8047 & .02 & .02 & .06 & .10 & .24 & .67 & 1.30 & 2.60 & 3.20 & 9270 & .02 & .03 & .09 & .10 & .14 & .33 & .70 & 1.50 & 2.09 \\
\hline 8060 & .01 & .01 & .02 & .04 & .12 & .37 & 1.31 & 2.71 & 3.16 & 9295 & .01 & .01 & .02 & .03 & .09 & .24 & .49 & 1.19 & 1.37 \\
\hline 8062 & -- & -- & .02 & .05 & .21 & .70 & 2.19 & -- & -- & 9304 & -- & -- & -- & .10 & .17 & .26 & -- & -- & -- \\
\hline 8068 & -- & -- & .02 & .03 & .10 & .28 & .82 & -- & -- & 9307 & .02 & .03 & .07 & .11 & .29 & .70 & 1.20 & 2.41 & 2.83 \\
\hline 8081 & .02 & .02 & .05 & .10 & .20 & .60 & 1.27 & 2.87 & 3.82 & 9328 & .02 & .02 & .04 & .08 & .18 & .54 & 1.15 & 1.69 & 2.87 \\
\hline 8089 & -- & -- & .04 & .08 & .21 & .62 & 1.17 & -- & -- & 9329 & -- & -- & .02 & .06 & .17 & .97 & 1.23 & -- & -- \\
\hline 8221 & -- & -- & .04 & .15 & .41 & .74 & 1.71 & -- & -- & 9345 & -- & -- & -- & -- & .70 & -- & -- & -- & -- \\
\hline 8252 & .02 & .02 & .05 & .10 & .20 & .50 & 1.00 & 2.16 & 2.69 & 9363 & .01 & .01 & .01 & .03 & .11 & .42 & .95 & 2.59 & 3.44 \\
\hline 8265 & .01 & .02 & .04 & .08 & .25 & .70 & 1.55 & 3.42 & 5.76 & 9364 & .01 & .01 & .01 & .03 & .13 & .50 & 1.18 & 2.85 & 3.90 \\
\hline 8289 & -- & .01 & .03 & .10 & .33 & .60 & .98 & 1.48 & -- & 9365 & -- & -- & .04 & .07 & .10 & .29 & .65 & -- & -- \\
\hline 8305 & .02 & .02 & .05 & .10 & .10 & .30 & .62 & 1.40 & 1.90 & 9371 & .02 & .02 & .05 & .09 & .25 & .71 & 1.38 & 2.58 & 2.76 \\
\hline 8335 & .02 & .02 & .05 & .10 & .35 & .86 & 1.65 & 3.32 & 4.18 & 9417 & .01 & .02 & .04 & .09 & .25 & .70 & 1.42 & 2.77 & 3.25 \\
\hline 8400 & .02 & .02 & .03 & .07 & .16 & .37 & .77 & 2.04 & 2.93 & 9419 & .01 & .01 & .01 & .04 & .15 & .55 & 1.23 & 2.60 & 3.16 \\
\hline 8445 & .01 & .02 & .04 & .07 & .24 & .69 & 1.50 & 3.10 & 4.27 & 9435 & -- & .03 & .06 & .10 & .23 & .59 & 1.02 & 2.57 & -- \\
\hline 8446 & .02 & .02 & .09 & .10 & .20 & .60 & 1.36 & 2.70 & 3.34 & 9491 & .02 & .02 & .05 & .10 & .25 & .70 & 1.46 & 2.92 & 3.50 \\
\hline 8451 & .01 & .02 & .04 & .07 & .20 & .57 & 1.08 & 2.12 & 2.30 & 9499 & .02 & .03 & .10 & .10 & .20 & .54 & 1.10 & 2.30 & 3.12 \\
\hline 8531 & .02 & .02 & .05 & .10 & .28 & .71 & 1.35 & 2.78 & 3.30 & 9522 & -- & -- & .04 & .09 & .25 & 1.27 & 2.72 & -- & -- \\
\hline 8541 & .01 & .01 & .05 & .10 & .29 & .65 & 1.28 & 2.47 & 3.68 & 9527 & .03 & .04 & .10 & .10 & .20 & .41 & .90 & 1.90 & 2.30 \\
\hline 8544 & .02 & .03 & .08 & .10 & .26 & .70 & 1.46 & 3.10 & 4.10 & 9532 & .02 & .02 & .06 & .10 & .24 & .69 & 1.26 & 2.70 & 3.52 \\
\hline 8545 & -- & -- & .02 & .06 & .21 & .41 & .61 & -- & -- & 9544 & -- & -- & -- & .11 & .13 & 2.95 & -- & -- & -- \\
\hline 8563 & .10 & .10 & .10 & .10 & .30 & .70 & 1.40 & 3.18 & 4.20 & 9565 & .02 & .03 & .07 & .10 & .20 & .57 & 1.20 & 2.49 & 3.20 \\
\hline 8566 & .01 & .01 & .04 & .08 & .22 & .58 & 1.18 & 2.18 & 2.53 & 9570 & .10 & .10 & .10 & .10 & .20 & .50 & 1.10 & 2.30 & 2.94 \\
\hline 8583 & .10 & .10 & .10 & .10 & .20 & .50 & 1.10 & 2.26 & 2.60 & 9574 & -- & -- & .10 & .10 & .20 & .50 & 1.40 & -- & -- \\
\hline 8584 & .02 & .03 & .08 & .10 & .23 & .70 & 1.25 & 2.66 & 3.06 & 9588 & .01 & .02 & .05 & .10 & .18 & .46 & 1.10 & 2.68 & 3.53 \\
\hline 8623 & .03 & .04 & .10 & .10 & .30 & .70 & 1.30 & 2.60 & 3.30 & 9665 & .02 & .02 & .07 & .10 & .30 & .76 & 1.50 & 3.08 & 4.01 \\
\hline 8625 & .01 & .02 & .04 & .09 & .24 & .69 & 1.27 & 2.76 & 3.61 & 9715 & .02 & .02 & .05 & .10 & .27 & .70 & 1.30 & 2.72 & 3.38 \\
\hline 8630 & .02 & .03 & .06 & .10 & .20 & .52 & 1.15 & 2.14 & 2.51 & 9729 & .01 & .01 & .02 & .04 & .17 & .55 & 1.17 & 2.47 & 3.00 \\
\hline 8631 & .01 & .02 & .05 & .08 & .22 & .55 & 1.21 & 2.55 & 3.52 & 9772 & .01 & .02 & .03 & .07 & .21 & .63 & 1.40 & 3.45 & 4.75 \\
\hline 8646 & .02 & .02 & .04 & .10 & .26 & .69 & 1.36 & 2.69 & 3.29 & 9814 & -- & -- & .03 & .09 & .32 & .85 & 1.62 & -- & -- \\
\hline 8647 & .02 & .03 & .08 & .10 & .16 & .42 & .90 & 2.10 & 2.70 & 9815 & .02 & .02 & .05 & .10 & .23 & .66 & 1.38 & 2.81 & 4.10 \\
\hline 8677 & .01 & .02 & .05 & .10 & .24 & .62 & 1.25 & 2.51 & 3.03 & 9816 & -- & .01 & .03 & .06 & .25 & .74 & 1.27 & 4.22 & -- \\
\hline 8696 & -- & -- & .06 & .10 & .43 & .94 & 1.39 & -- & -- & 9817 & .03 & .04 & .10 & .10 & .23 & .65 & 1.20 & 2.60 & 3.25 \\
\hline 8743 & .02 & .02 & .06 & .10 & .30 & .80 & 1.55 & 3.46 & 4.34 & 9829 & .02 & .02 & .05 & .10 & .13 & .35 & .80 & 1.80 & 2.27 \\
\hline 8761 & .02 & .03 & .10 & .10 & .20 & .41 & .87 & 1.90 & 2.72 & 9830 & .01 & .01 & .03 & .05 & .10 & .31 & .72 & 1.34 & 2.17 \\
\hline 8778 & .03 & .04 & .10 & .10 & .30 & .75 & 1.40 & 3.00 & 3.70 & 9858 & .02 & .02 & .05 & .10 & .23 & .55 & .99 & 2.10 & 2.64 \\
\hline 8845 & .02 & .02 & .06 & .10 & .20 & .60 & 1.35 & 3.10 & 3.90 & 9893 & .02 & .02 & .05 & .10 & .22 & .60 & 1.21 & 2.50 & 3.30 \\
\hline 8859 & .01 & .02 & .05 & .11 & .32 & .85 & 1.57 & 3.14 & 3.84 & 9916 & .02 & .04 & .10 & .10 & .30 & .83 & 1.60 & 3.21 & 3.80 \\
\hline 8898 & .02 & .02 & .05 & .11 & .32 & .75 & 1.56 & 3.19 & 4.02 & 9976 & .01 & .02 & .05 & .10 & .20 & .50 & 1.19 & 2.47 & 3.24 \\
\hline 8908 & -- & -- & .04 & .08 & .21 & .60 & 1.26 & -- & -- & & & & & & & & & & \\
\hline
\end{tabular}


Appendix 4-4.2. Empirical distribution of storm depth defined by 8-hour minimum interevent time for hourly rainfall stations in Texas.

$[--$, not available $]$

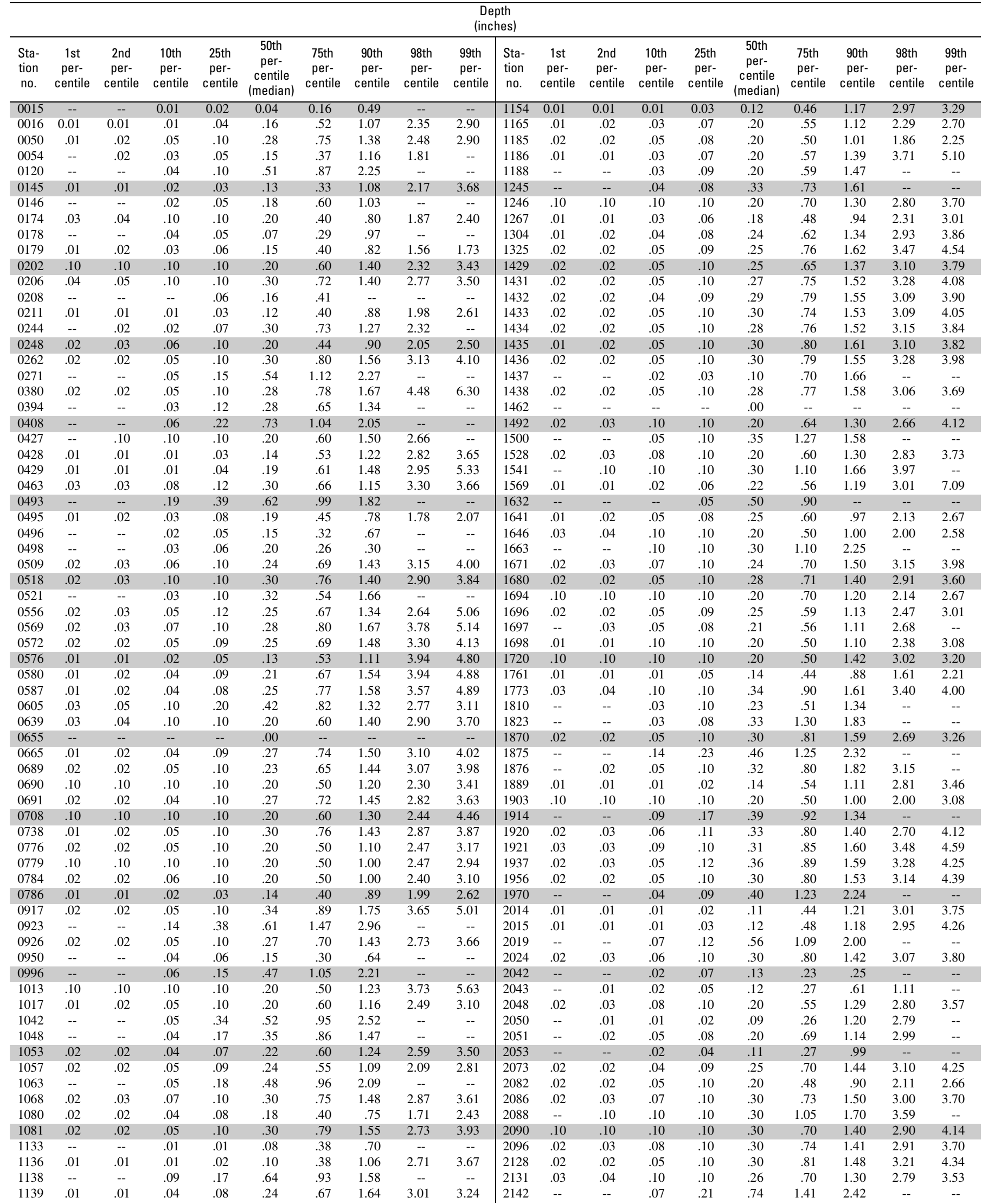


248 Statistical Characteristics of Storm Interevent Time, Depth, and Duration for Eastern New Mexico, Oklahoma, and Texas

Appendix 4-4.2. Empirical distribution of storm depth defined by 8-hour minimum interevent time for hourly rainfall stations in TexasContinued.

\begin{tabular}{|c|c|c|c|c|c|c|c|c|c|c|c|c|c|c|c|c|c|c|c|}
\hline \multicolumn{20}{|c|}{$\begin{array}{c}\text { Depth } \\
\text { (inches) }\end{array}$} \\
\hline $\begin{array}{l}\text { Sta- } \\
\text { tion } \\
\text { no. }\end{array}$ & $\begin{array}{c}\text { 1st } \\
\text { per- } \\
\text { centile }\end{array}$ & $\begin{array}{c}\text { 2nd } \\
\text { per- } \\
\text { centile }\end{array}$ & $\begin{array}{c}\text { 10th } \\
\text { per- } \\
\text { centile }\end{array}$ & $\begin{array}{c}\text { 25th } \\
\text { per- } \\
\text { centile }\end{array}$ & $\begin{array}{c}\text { 50th } \\
\text { per- } \\
\text { centile } \\
\text { (median) }\end{array}$ & $\begin{array}{c}\text { 75th } \\
\text { per- } \\
\text { centile }\end{array}$ & $\begin{array}{c}\text { 90th } \\
\text { per- } \\
\text { centile }\end{array}$ & $\begin{array}{c}\text { 98th } \\
\text { per- } \\
\text { centile }\end{array}$ & $\begin{array}{c}\text { 99th } \\
\text { per- } \\
\text { centile }\end{array}$ & $\begin{array}{l}\text { Sta- } \\
\text { tion } \\
\text { no. }\end{array}$ & $\begin{array}{c}\text { 1st } \\
\text { per- } \\
\text { centile }\end{array}$ & $\begin{array}{c}\text { 2nd } \\
\text { per- } \\
\text { centile }\end{array}$ & $\begin{array}{c}\text { 10th } \\
\text { per- } \\
\text { centile }\end{array}$ & $\begin{array}{l}\text { 25th } \\
\text { per- } \\
\text { centile }\end{array}$ & $\begin{array}{c}\text { 50th } \\
\text { per- } \\
\text { centile } \\
\text { (median) }\end{array}$ & $\begin{array}{c}\text { 75th } \\
\text { per- } \\
\text { centile }\end{array}$ & $\begin{array}{c}\text { 90th } \\
\text { per- } \\
\text { centile }\end{array}$ & $\begin{array}{c}\text { 98th } \\
\text { per- } \\
\text { centile }\end{array}$ & $\begin{array}{c}\text { 99th } \\
\text { per- } \\
\text { centile }\end{array}$ \\
\hline 2160 & -- & -- & 0.01 & 0.03 & 0.12 & 0.39 & 0.64 & -- & -- & 3463 & -- & -- & 0.03 & 0.07 & 0.28 & 0.77 & 1.79 & -- & -- \\
\hline 2206 & 0.02 & 0.03 & .05 & .10 & .32 & .83 & 1.79 & 3.22 & 4.87 & 3476 & 0.01 & 0.02 & .04 & .09 & .26 & .69 & 1.35 & 2.69 & 3.84 \\
\hline 2238 & .01 & .01 & .02 & .03 & .10 & .38 & .95 & 2.05 & 2.29 & 3485 & -- & -- & .06 & .10 & .17 & 1.41 & 2.42 & -- & -- \\
\hline 2240 & .01 & .01 & .01 & .04 & .14 & .41 & .76 & 1.94 & 2.02 & 3507 & .05 & .07 & .10 & .10 & .21 & .70 & 1.50 & 3.05 & 3.64 \\
\hline 2242 & .01 & .01 & .02 & .05 & .23 & .68 & 1.39 & 2.63 & 3.46 & 3546 & .02 & .03 & .06 & .10 & .30 & .86 & 1.65 & 3.42 & 4.21 \\
\hline 2244 & .01 & .01 & .02 & .10 & .24 & .70 & 1.40 & 3.05 & 3.90 & 3547 & .02 & .02 & .05 & .11 & .30 & .79 & 1.55 & 2.81 & 3.20 \\
\hline 2247 & -- & .01 & .02 & .05 & .18 & .60 & 1.33 & 3.59 & -- & 3579 & -- & .03 & .05 & .12 & .25 & .95 & 1.65 & 3.32 & -- \\
\hline 2309 & .03 & .03 & .08 & .17 & .42 & .91 & 1.51 & 3.33 & 4.58 & 3642 & .02 & .03 & .07 & .10 & .30 & .80 & 1.55 & 3.14 & 3.91 \\
\hline 2312 & .04 & .07 & .10 & .10 & .34 & .80 & 1.50 & 2.81 & 3.55 & 3646 & .02 & .03 & .05 & .11 & .30 & .72 & 1.39 & 2.97 & 4.12 \\
\hline 2334 & -- & .02 & .08 & .17 & .55 & 1.18 & 2.42 & 4.43 & -- & 3668 & -- & -- & .09 & .37 & .76 & 1.32 & 2.82 & -- & -- \\
\hline 2336 & .02 & .03 & .05 & .15 & .35 & .90 & 1.47 & 2.49 & 3.63 & 3673 & -- & -- & .04 & .08 & .35 & 1.23 & 1.91 & -- & -- \\
\hline 2354 & -- & -- & .04 & .06 & .12 & .52 & .76 & -- & -- & 3686 & .03 & .06 & .10 & .10 & .21 & .70 & 1.50 & 2.50 & 3.46 \\
\hline 2355 & -- & .01 & .03 & .06 & .20 & .54 & 1.76 & 4.78 & -- & 3691 & .02 & .03 & .10 & .10 & .30 & .70 & 1.40 & 2.90 & 3.40 \\
\hline 2357 & .01 & .01 & .01 & .02 & .08 & .39 & 1.03 & 2.61 & 3.72 & 3734 & -- & -- & .03 & .11 & .38 & 1.40 & 3.33 & -- & -- \\
\hline 2360 & .01 & .01 & .01 & .03 & .10 & .38 & 1.04 & 2.46 & 3.15 & 3771 & .10 & .10 & .10 & .10 & .20 & .70 & 1.50 & 3.34 & 3.90 \\
\hline 2361 & .02 & .02 & .04 & .10 & .19 & .50 & .91 & 2.28 & 4.41 & 3789 & .01 & .01 & .03 & .06 & .11 & .24 & .46 & 1.02 & 2.02 \\
\hline 2394 & .02 & .02 & .05 & .10 & .30 & .77 & 1.50 & 3.06 & 4.13 & 3826 & .01 & .02 & .06 & .13 & .33 & .76 & 1.32 & 2.45 & 2.66 \\
\hline 2404 & .02 & .02 & .05 & .10 & .26 & .73 & 1.40 & 2.99 & 3.61 & 3831 & .02 & .03 & .05 & .11 & .31 & .82 & 1.46 & 2.98 & 3.76 \\
\hline 2415 & .02 & .03 & .06 & .13 & .38 & .93 & 1.75 & 3.49 & 4.21 & 3841 & .02 & .02 & .05 & .14 & .30 & .83 & 1.52 & 3.45 & 4.81 \\
\hline 2462 & .02 & .03 & .07 & .12 & .33 & .89 & 1.72 & 3.15 & 3.93 & 3871 & .02 & .02 & .05 & .09 & .25 & .67 & 1.25 & 2.41 & 3.19 \\
\hline 2528 & .03 & .03 & .05 & .10 & .25 & .67 & 1.43 & 2.57 & 2.77 & 3884 & -- & -- & .06 & .09 & .24 & .96 & 2.98 & -- & -- \\
\hline 2617 & .01 & .01 & .04 & .07 & .23 & .52 & 1.07 & 2.75 & 3.50 & 3941 & -- & .01 & .04 & .06 & .39 & 1.11 & 1.82 & 3.30 & -- \\
\hline 2619 & .01 & .02 & .05 & .10 & .24 & .56 & 1.06 & 2.22 & 3.78 & 3963 & -- & -- & -- & .03 & .05 & .10 & -- & -- & -- \\
\hline 2621 & .02 & .03 & .05 & .10 & .24 & .60 & 1.22 & 2.35 & 2.79 & 4040 & .02 & .02 & .05 & .10 & .28 & .65 & 1.30 & 2.51 & 3.14 \\
\hline 2675 & .02 & .03 & .10 & .10 & .29 & .71 & 1.48 & 3.21 & 3.95 & 4058 & -- & -- & .05 & .08 & .42 & .86 & 2.16 & -- & -- \\
\hline 2676 & .10 & .10 & .10 & .10 & .20 & .70 & 1.50 & 2.91 & 3.91 & 4098 & .03 & .04 & .10 & .10 & .20 & .40 & .90 & 1.88 & 2.16 \\
\hline 2679 & .02 & .02 & .05 & .10 & .16 & .50 & 1.21 & 3.00 & 4.07 & 4100 & .02 & .02 & .05 & .08 & .23 & .62 & 1.31 & 2.95 & 4.26 \\
\hline 2715 & .02 & .03 & .05 & .10 & .30 & .72 & 1.39 & 2.85 & 3.52 & 4137 & .10 & .10 & .10 & .10 & .30 & .70 & 1.30 & 2.70 & 3.40 \\
\hline 2744 & .02 & .03 & .05 & .10 & .25 & .62 & 1.20 & 2.50 & 3.14 & 4191 & .02 & .03 & .05 & .10 & .20 & .61 & 1.40 & 2.99 & 3.90 \\
\hline 2758 & .01 & .01 & .02 & .04 & .11 & .35 & 1.22 & 3.65 & 4.39 & 4256 & -- & -- & -- & -- & .00 & -- & -- & -- & -- \\
\hline 2794 & -- & -- & .01 & .04 & .16 & .66 & 1.61 & -- & -- & 4257 & .03 & .05 & .10 & .10 & .35 & .90 & 1.70 & 3.41 & 4.36 \\
\hline 2797 & .01 & .01 & .01 & .03 & .08 & .23 & .52 & 1.19 & 1.61 & 4258 & .10 & .10 & .10 & .10 & .30 & .70 & 1.30 & 2.68 & 4.35 \\
\hline 2811 & .02 & .02 & .06 & .10 & .20 & .58 & 1.20 & 2.38 & 3.10 & 4278 & .02 & .02 & .05 & .10 & .30 & .76 & 1.51 & 3.01 & 3.64 \\
\hline 2813 & -- & -- & .05 & .09 & .30 & .58 & 1.37 & -- & -- & 4299 & .01 & .03 & .05 & .10 & .25 & .45 & .80 & 1.22 & 1.24 \\
\hline 2814 & -- & -- & .01 & .03 & .05 & .15 & .96 & -- & -- & 4300 & .01 & .01 & .01 & .04 & .17 & .61 & 1.39 & 3.05 & 4.18 \\
\hline 2815 & .10 & .10 & .10 & .10 & .20 & .57 & 1.20 & 2.70 & 3.62 & 4305 & .01 & .01 & .01 & .03 & .15 & .57 & 1.34 & 3.05 & 3.97 \\
\hline 2818 & .02 & .02 & .04 & .09 & .32 & .72 & 1.37 & 3.20 & 3.49 & 4307 & .01 & .01 & .01 & .04 & .20 & .62 & 1.37 & 2.82 & 4.94 \\
\hline 2986 & .02 & .02 & .06 & .13 & .42 & 1.12 & 1.83 & 3.46 & 3.86 & 4309 & .02 & .02 & .05 & .10 & .29 & .81 & 1.63 & 3.43 & 4.56 \\
\hline 3005 & .02 & .02 & .07 & .10 & .30 & .70 & 1.35 & 2.70 & 3.50 & 4311 & .01 & .02 & .05 & .10 & .31 & .86 & 1.76 & 3.41 & 4.55 \\
\hline 3033 & .02 & .02 & .04 & .07 & .14 & .30 & .60 & 1.30 & 1.85 & 4313 & .01 & .02 & .04 & .10 & .26 & .75 & 1.75 & 4.11 & 5.38 \\
\hline 3034 & -- & -- & -- & -- & .43 & -- & -- & -- & -- & 4319 & .02 & .03 & .05 & .11 & .30 & .78 & 1.31 & 2.37 & 3.38 \\
\hline 3047 & -- & -- & .03 & .05 & .34 & .73 & 1.37 & -- & -- & 4329 & .02 & .02 & .05 & .11 & .30 & .80 & 1.67 & 3.69 & 4.60 \\
\hline 3103 & -- & -- & .10 & .14 & .27 & .93 & 2.02 & -- & -- & 4331 & -- & -- & -- & -- & .00 & -- & -- & -- & -- \\
\hline 3133 & .02 & .03 & .06 & .10 & .34 & .80 & 1.52 & 3.30 & 3.95 & 4375 & .10 & .10 & .10 & .10 & .20 & .60 & 1.30 & 3.10 & 4.68 \\
\hline 3156 & .04 & .05 & .10 & .10 & .30 & .70 & 1.70 & 4.68 & 5.90 & 4392 & .01 & .02 & .05 & .10 & .35 & .97 & 1.95 & 3.83 & 5.84 \\
\hline 3171 & .02 & .03 & .05 & .10 & .30 & .77 & 1.50 & 3.07 & 4.06 & 4425 & .03 & .03 & .08 & .10 & .19 & .48 & .95 & 2.00 & 2.57 \\
\hline 3189 & .01 & .02 & .03 & .08 & .14 & .35 & .74 & 1.48 & 2.39 & 4440 & .02 & .02 & .04 & .08 & .24 & .64 & 1.25 & 2.73 & 3.29 \\
\hline 3260 & .01 & .01 & .03 & .08 & .27 & .72 & 1.28 & 2.41 & 3.51 & 4476 & .03 & .04 & .10 & .10 & .30 & .70 & 1.30 & 2.80 & 3.50 \\
\hline 3267 & .01 & .01 & .03 & .05 & .17 & .56 & 1.05 & 3.47 & 3.88 & 4498 & -- & -- & .02 & .04 & .14 & .32 & .58 & -- & -- \\
\hline 3270 & .05 & .09 & .10 & .10 & .20 & .52 & 1.10 & 2.31 & 3.30 & 4517 & .02 & .02 & .04 & .10 & .28 & .73 & 1.35 & 2.75 & 3.43 \\
\hline 3272 & -- & -- & .02 & .04 & .09 & .13 & .33 & -- & -- & 4520 & .10 & .10 & .10 & .10 & .20 & .60 & 1.30 & 2.76 & 3.33 \\
\hline 3277 & -- & -- & .02 & .02 & .08 & .21 & .46 & -- & -- & 4525 & -- & -- & .04 & .06 & .25 & .78 & 2.44 & -- & -- \\
\hline 3278 & .02 & .02 & .05 & .10 & .20 & .50 & 1.02 & 2.16 & 2.66 & 4563 & -- & -- & .03 & .06 & .26 & .61 & 1.68 & -- & -- \\
\hline 3280 & .01 & .01 & .03 & .05 & .12 & .35 & .77 & 1.90 & 2.39 & 4570 & .02 & .03 & .07 & .10 & .20 & .57 & 1.20 & 2.43 & 2.95 \\
\hline 3281 & -- & -- & .04 & .10 & .24 & .50 & .93 & -- & -- & 4577 & .02 & .03 & .10 & .10 & .39 & .88 & 1.60 & 3.22 & 4.08 \\
\hline 3283 & .01 & .01 & .01 & .05 & .20 & .60 & 1.31 & 2.81 & 3.65 & 4591 & .02 & .02 & .05 & .10 & .30 & .75 & 1.54 & 3.21 & 3.91 \\
\hline 3284 & .01 & .01 & .07 & .10 & .28 & .70 & 1.40 & 2.79 & 3.50 & 4670 & .02 & .02 & .05 & .10 & .20 & .55 & 1.19 & 2.46 & 2.93 \\
\hline 3285 & .03 & .07 & .10 & .10 & .30 & .70 & 1.50 & 2.70 & 3.32 & 4671 & .01 & .01 & .02 & .04 & .16 & .55 & .93 & 2.08 & 2.63 \\
\hline 3329 & .01 & .02 & .04 & .09 & .20 & .60 & 1.30 & 2.80 & 3.77 & 4679 & .03 & .04 & .10 & .10 & .24 & .70 & 1.40 & 3.04 & 4.03 \\
\hline 3335 & .01 & .02 & .04 & .10 & .33 & .86 & 1.75 & 4.29 & 7.40 & 4696 & -- & -- & .05 & .10 & .27 & .66 & 1.13 & -- & -- \\
\hline 3370 & .02 & .02 & .05 & .13 & .35 & .87 & 1.63 & 3.39 & 4.04 & 4703 & .02 & .02 & .05 & .10 & .17 & .53 & 1.17 & 2.32 & 2.98 \\
\hline 3410 & .04 & .05 & .10 & .10 & .21 & .59 & 1.08 & 2.30 & 3.00 & 4704 & .02 & .02 & .05 & .11 & .32 & .90 & 1.91 & 4.23 & 5.17 \\
\hline 3415 & .02 & .03 & .09 & .10 & .30 & .70 & 1.40 & 2.79 & 3.70 & 4731 & .01 & .01 & .01 & .03 & .15 & .43 & 1.03 & 3.04 & 6.19 \\
\hline 3430 & .01 & .01 & .01 & .04 & .18 & .61 & 1.34 & 3.27 & 4.31 & 4792 & .10 & .10 & .10 & .10 & .30 & .70 & 1.40 & 2.70 & 3.30 \\
\hline 3431 & .01 & .01 & .01 & .04 & .13 & .57 & 1.34 & 3.52 & 4.71 & 4819 & .10 & .10 & .10 & .10 & .30 & .90 & 1.54 & 3.60 & 3.78 \\
\hline 3441 & -- & -- & .04 & .09 & .30 & .75 & 1.63 & -- & -- & 4852 & -- & -- & .17 & .35 & .50 & 1.07 & 2.38 & -- & -- \\
\hline 3442 & .02 & .02 & .04 & .08 & .17 & .45 & .95 & 1.95 & 2.50 & 4866 & .02 & .03 & .07 & .10 & .30 & .80 & 1.50 & 2.90 & 3.68 \\
\hline 3446 & .02 & .03 & .05 & .10 & .25 & .53 & 1.10 & 2.10 & 2.77 & 4876 & .10 & .10 & .10 & .10 & .30 & 1.00 & 2.02 & 4.22 & 4.61 \\
\hline 3460 & -- & -- & .06 & .10 & .41 & .92 & 1.48 & -- & -- & 4878 & .02 & .02 & .05 & .10 & .33 & .86 & 1.76 & 4.08 & 5.10 \\
\hline 3462 & .02 & .02 & .06 & .10 & .27 & .67 & 1.13 & 1.87 & 2.98 & 4880 & .02 & .02 & .06 & .10 & .20 & .50 & 1.00 & 2.04 & 2.61 \\
\hline
\end{tabular}


Appendix 4-4.2. Empirical distribution of storm depth defined by 8-hour minimum interevent time for hourly rainfall stations in TexasContinued.

\begin{tabular}{|c|c|c|c|c|c|c|c|c|c|c|c|c|c|c|c|c|c|c|c|}
\hline \multicolumn{20}{|c|}{$\begin{array}{c}\text { Depth } \\
\text { (inches) }\end{array}$} \\
\hline $\begin{array}{l}\text { Sta- } \\
\text { tion } \\
\text { no. }\end{array}$ & $\begin{array}{c}\text { 1st } \\
\text { per- } \\
\text { centile }\end{array}$ & $\begin{array}{l}\text { 2nd } \\
\text { per- } \\
\text { centile }\end{array}$ & $\begin{array}{c}10 \text { th } \\
\text { per- } \\
\text { centile }\end{array}$ & $\begin{array}{c}25 \text { th } \\
\text { per- } \\
\text { centile }\end{array}$ & $\begin{array}{c}\text { 50th } \\
\text { per- } \\
\text { centile } \\
\text { (median) }\end{array}$ & $\begin{array}{c}\text { 75th } \\
\text { per- } \\
\text { centile }\end{array}$ & $\begin{array}{c}\text { 90th } \\
\text { per- } \\
\text { centile }\end{array}$ & $\begin{array}{c}\text { 98th } \\
\text { per- } \\
\text { centile }\end{array}$ & $\begin{array}{c}\text { 99th } \\
\text { per- } \\
\text { centile }\end{array}$ & $\begin{array}{l}\text { Sta- } \\
\text { tion } \\
\text { no. }\end{array}$ & $\begin{array}{c}\text { 1st } \\
\text { per- } \\
\text { centile }\end{array}$ & $\begin{array}{c}\text { 2nd } \\
\text { per- } \\
\text { centile }\end{array}$ & $\begin{array}{c}10 \text { th } \\
\text { per- } \\
\text { centile }\end{array}$ & $\begin{array}{c}25 \text { th } \\
\text { per- } \\
\text { centile }\end{array}$ & $\begin{array}{c}\text { 50th } \\
\text { per- } \\
\text { centile } \\
\text { (median) }\end{array}$ & $\begin{array}{c}\text { 75th } \\
\text { per- } \\
\text { centile }\end{array}$ & $\begin{array}{c}\text { 90th } \\
\text { per- } \\
\text { centile }\end{array}$ & $\begin{array}{c}\text { 98th } \\
\text { per- } \\
\text { centile }\end{array}$ & $\begin{array}{c}\text { 99th } \\
\text { per- } \\
\text { centile }\end{array}$ \\
\hline 4920 & 0.02 & 0.03 & 0.07 & 0.10 & 0.20 & 0.62 & 1.34 & 3.03 & 4.01 & 5957 & 0.02 & 0.03 & 0.10 & 0.10 & 0.30 & 0.70 & 1.40 & 2.70 & 3.50 \\
\hline 4934 & -- & -- & -- & .09 & .14 & .26 & -- & -- & -- & 5958 & .01 & .01 & .03 & .10 & .30 & .65 & 1.27 & 2.37 & 3.14 \\
\hline 4972 & .02 & .03 & .07 & .10 & .27 & .69 & 1.30 & 2.78 & 3.51 & 5973 & .01 & .01 & .02 & .04 & .10 & .41 & 1.06 & 2.97 & 3.80 \\
\hline 4973 & .02 & .04 & .08 & .16 & .45 & .95 & 1.66 & 3.32 & 3.85 & 5996 & .02 & .03 & .07 & .10 & .25 & .70 & 1.30 & 2.77 & 3.65 \\
\hline 4974 & .02 & .02 & .05 & .10 & .20 & .52 & 1.03 & 2.34 & 2.70 & 6017 & .01 & .01 & .02 & .05 & .15 & .48 & 1.21 & 2.55 & 2.83 \\
\hline 4975 & .10 & .10 & .10 & .10 & .40 & .90 & 1.60 & 3.04 & 4.20 & 6024 & .02 & .02 & .05 & .10 & .35 & 1.07 & 2.01 & 4.79 & 6.24 \\
\hline 4978 & .02 & .02 & .04 & .07 & .21 & .58 & 1.52 & 3.07 & 5.01 & 6050 & -- & -- & .10 & .23 & .38 & 1.01 & 1.72 & -- & -- \\
\hline 4979 & -- & -- & .06 & .14 & .50 & 1.09 & 2.75 & -- & -- & 6104 & .02 & .02 & .05 & .10 & .18 & .40 & .80 & 1.65 & 2.20 \\
\hline 4982 & .02 & .02 & .05 & .10 & .26 & .62 & 1.23 & 2.59 & 3.27 & 6108 & .02 & .03 & .10 & .10 & .40 & .90 & 1.76 & 3.39 & 4.60 \\
\hline 5018 & .02 & .02 & .05 & .11 & .31 & .77 & 1.39 & 2.47 & 3.17 & 6136 & .02 & .03 & .10 & .10 & .20 & .45 & .90 & 1.81 & 2.40 \\
\hline 5048 & .02 & .03 & .08 & .10 & .16 & .50 & 1.00 & 2.50 & 3.30 & 6166 & -- & .02 & .04 & .05 & .14 & .40 & 1.06 & 1.93 & -- \\
\hline 5049 & .10 & .10 & .10 & .10 & .20 & .50 & .86 & 4.09 & 4.97 & 6176 & .01 & .02 & .04 & .11 & .33 & .80 & 1.84 & 3.92 & 5.26 \\
\hline 5056 & -- & -- & -- & .20 & .37 & .69 & -- & -- & -- & 6177 & .02 & .03 & .10 & .10 & .31 & .84 & 1.70 & 3.50 & 4.44 \\
\hline 5057 & .01 & .01 & .01 & .03 & .09 & .30 & .90 & 2.24 & 3.07 & 6210 & .02 & .03 & .10 & .10 & .30 & .80 & 1.50 & 3.16 & 4.20 \\
\hline 5060 & .01 & .01 & .02 & .05 & .15 & .56 & 1.29 & 3.59 & 6.09 & 6211 & .01 & .01 & .03 & .07 & .25 & .80 & 1.48 & 3.05 & 4.18 \\
\hline 5081 & .02 & .02 & .05 & .10 & .35 & .85 & 1.59 & 2.98 & 3.91 & 6270 & .10 & .10 & .10 & .10 & .40 & .90 & 1.70 & 3.60 & 4.36 \\
\hline 5094 & .02 & .03 & .10 & .10 & .30 & .75 & 1.50 & 3.20 & 3.80 & 6275 & -- & -- & -- & -- & .00 & -- & -- & -- & -- \\
\hline 5113 & .02 & .02 & .06 & .10 & .20 & .60 & 1.36 & 3.10 & 3.90 & 6276 & -- & -- & .09 & .18 & .46 & 1.32 & 2.27 & -- & -- \\
\hline 5114 & -- & -- & -- & -- & .00 & -- & -- & -- & -- & 6335 & .02 & .02 & .05 & .10 & .32 & .84 & 1.51 & 2.90 & 3.45 \\
\hline 5123 & -- & -- & .07 & .19 & .32 & .93 & 1.97 & -- & -- & 6434 & -- & -- & .05 & .10 & .32 & .73 & 1.47 & -- & -- \\
\hline 5192 & .02 & .02 & .06 & .10 & .30 & .80 & 1.59 & 3.10 & 3.71 & 6504 & .02 & .03 & .07 & .10 & .20 & .50 & 1.00 & 2.10 & 2.90 \\
\hline 5193 & .02 & .02 & .05 & .10 & .25 & .70 & 1.45 & 3.00 & 3.83 & 6558 & -- & -- & .05 & .06 & .41 & .88 & 1.69 & -- & -- \\
\hline 5224 & .01 & .02 & .04 & .10 & .33 & .93 & 1.70 & 3.76 & 4.63 & 6615 & .09 & .10 & .10 & .10 & .20 & .50 & 1.20 & 2.50 & 3.78 \\
\hline 5228 & .01 & .02 & .04 & .09 & .26 & .78 & 1.59 & 2.92 & 3.56 & 6660 & .02 & .02 & .05 & .10 & .30 & .75 & 1.51 & 3.53 & 3.78 \\
\hline 5235 & -- & -- & .02 & .05 & .21 & .71 & 1.65 & -- & -- & 6663 & .01 & .01 & .03 & .10 & .20 & .57 & 1.35 & 2.80 & 3.09 \\
\hline 5247 & .02 & .03 & .08 & .10 & .20 & .50 & 1.02 & 2.00 & 2.60 & 6734 & .01 & .01 & .04 & .07 & .20 & .55 & 1.05 & 2.32 & 3.15 \\
\hline 5258 & .02 & .02 & .05 & .10 & .30 & .78 & 1.52 & 3.16 & 3.77 & 6736 & .02 & .03 & .09 & .10 & .20 & .53 & 1.10 & 2.40 & 3.20 \\
\hline 5303 & .01 & .02 & .05 & .08 & .22 & .62 & 1.29 & 2.93 & 4.28 & 6740 & -- & -- & .10 & .32 & .82 & 1.54 & 3.31 & -- & -- \\
\hline 5312 & .03 & .04 & .10 & .10 & .20 & .60 & 1.23 & 2.60 & 3.50 & 6750 & .01 & .01 & .01 & .04 & .23 & .62 & 1.52 & 3.36 & 8.80 \\
\hline 5341 & -- & -- & .07 & .18 & .34 & 1.21 & 1.71 & -- & -- & 6757 & .01 & .01 & .04 & .10 & .28 & .80 & 1.50 & 3.15 & 3.89 \\
\hline 5342 & -- & -- & -- & -- & .00 & -- & -- & -- & -- & 6775 & .01 & .01 & .03 & .06 & .20 & .50 & .98 & 2.10 & 2.88 \\
\hline 5348 & .03 & .05 & .10 & .10 & .30 & .90 & 1.70 & 3.30 & 3.74 & 6776 & .02 & .03 & .08 & .10 & .20 & .50 & 1.00 & 2.13 & 2.80 \\
\hline 5358 & .02 & .02 & .05 & .10 & .23 & .60 & 1.22 & 2.44 & 2.95 & 6788 & .02 & .02 & .05 & .12 & .42 & .83 & 1.67 & 3.59 & 4.59 \\
\hline 5398 & .02 & .02 & .05 & .10 & .31 & .81 & 1.60 & 3.20 & 4.11 & 6792 & .02 & .02 & .06 & .10 & .20 & .40 & .80 & 1.60 & 1.99 \\
\hline 5410 & .02 & .02 & .06 & .10 & .20 & .50 & 1.00 & 2.27 & 3.09 & 6794 & -- & -- & .06 & .21 & .55 & 1.45 & 2.84 & -- & -- \\
\hline 5411 & .01 & .01 & .01 & .03 & .13 & .39 & .88 & 2.00 & 2.78 & 6834 & .10 & .10 & .10 & .10 & .40 & .90 & 1.70 & 3.30 & 4.20 \\
\hline 5424 & .01 & .01 & .01 & .03 & .17 & .70 & 1.67 & 2.95 & 3.67 & 6893 & .02 & .03 & .08 & .10 & .15 & .40 & .70 & 1.70 & 2.40 \\
\hline 5429 & .02 & .02 & .03 & .08 & .20 & .62 & 1.39 & 2.79 & 4.31 & 6935 & .03 & .04 & .10 & .10 & .20 & .50 & .93 & 2.04 & 3.10 \\
\hline 5431 & -- & -- & .13 & .25 & .46 & .96 & 2.69 & -- & -- & 6981 & .02 & .02 & .05 & .11 & .28 & .72 & 1.46 & 3.57 & 4.63 \\
\hline 5461 & .02 & .02 & .05 & .11 & .34 & .90 & 1.70 & 3.51 & 4.24 & 7020 & .01 & .01 & .03 & .07 & .28 & .84 & 1.68 & 3.22 & 5.63 \\
\hline 5463 & .10 & .10 & .10 & .10 & .30 & .80 & 1.70 & 3.22 & 4.35 & 7060 & .02 & .03 & .10 & .10 & .20 & .53 & 1.22 & 2.30 & 2.89 \\
\hline 5471 & -- & -- & .01 & .02 & .05 & .10 & .43 & -- & -- & 7066 & .02 & .03 & .08 & .10 & .34 & .87 & 1.70 & 3.26 & 4.12 \\
\hline 5477 & -- & -- & .08 & .15 & .26 & .54 & 1.24 & -- & -- & 7074 & .02 & .02 & .05 & .10 & .20 & .49 & .90 & 2.30 & 2.89 \\
\hline 5528 & .02 & .03 & .06 & .14 & .36 & .90 & 1.61 & 3.30 & 4.00 & 7097 & .02 & .02 & .05 & .11 & .25 & .56 & 1.62 & 3.26 & 3.54 \\
\hline 5579 & -- & -- & -- & -- & .55 & -- & -- & -- & -- & 7116 & .02 & .02 & .04 & .08 & .23 & .55 & 1.05 & 2.42 & 3.38 \\
\hline 5580 & -- & -- & .05 & .11 & .42 & .84 & 1.39 & -- & -- & 7140 & .01 & .02 & .04 & .10 & .24 & .73 & 1.65 & 3.98 & 5.32 \\
\hline 5589 & .01 & .01 & .02 & .05 & .15 & .39 & .69 & 1.21 & 1.45 & 7173 & .01 & .01 & .01 & .04 & .19 & .68 & 1.66 & 4.64 & 5.86 \\
\hline 5590 & .01 & .02 & .03 & .07 & .15 & .39 & .88 & 1.43 & 1.89 & 7174 & .01 & .01 & .01 & .05 & .21 & .70 & 1.54 & 3.77 & 5.14 \\
\hline 5591 & .02 & .02 & .05 & .09 & .20 & .47 & .80 & 1.50 & 1.69 & 7213 & .02 & .02 & .05 & .10 & .30 & .78 & 1.55 & 3.30 & 4.22 \\
\hline 5592 & .02 & .02 & .04 & .06 & .15 & .36 & .66 & 1.60 & 1.90 & 7243 & .02 & .03 & .09 & .10 & .20 & .60 & 1.20 & 2.57 & 3.10 \\
\hline 5594 & .02 & .02 & .04 & .07 & .16 & .39 & .75 & 1.79 & 1.92 & 7262 & .01 & .01 & .02 & .04 & .10 & .25 & .52 & 1.03 & 1.11 \\
\hline 5595 & -- & -- & -- & -- & .03 & -- & -- & -- & -- & 7274 & .04 & .04 & .10 & .15 & .36 & .79 & 1.40 & 3.38 & 4.12 \\
\hline 5596 & .10 & .10 & .10 & .10 & .20 & .40 & .90 & 1.73 & 2.10 & 7300 & .02 & .02 & .05 & .10 & .28 & .73 & 1.35 & 2.80 & 3.34 \\
\hline 5600 & .01 & .02 & .04 & .08 & .17 & .41 & .77 & 1.91 & 2.47 & 7311 & -- & -- & .10 & .14 & .28 & .71 & 1.77 & -- & -- \\
\hline 5618 & -- & -- & .05 & .11 & .29 & .84 & 1.56 & -- & -- & 7363 & -- & -- & .04 & .10 & .45 & .94 & 1.46 & -- & -- \\
\hline 5650 & -- & -- & .09 & .23 & .45 & .61 & 1.53 & -- & -- & 7422 & .02 & .02 & .05 & .10 & .21 & .67 & 1.35 & 3.04 & 4.04 \\
\hline 5656 & .08 & .10 & .10 & .10 & .20 & .50 & 1.10 & 2.39 & 3.12 & 7431 & .02 & .02 & .05 & .10 & .20 & .50 & 1.00 & 2.19 & 2.94 \\
\hline 5658 & .02 & .02 & .04 & .08 & .20 & .54 & 1.10 & 2.33 & 3.40 & 7481 & .02 & .02 & .05 & .10 & .13 & .36 & .80 & 1.99 & 2.60 \\
\hline 5661 & .10 & .10 & .10 & .10 & .20 & .60 & 1.29 & 4.32 & 6.01 & 7497 & .10 & .10 & .10 & .10 & .28 & .70 & 1.40 & 2.90 & 3.54 \\
\hline 5666 & -- & -- & .05 & .09 & .27 & .59 & 1.27 & -- & -- & 7498 & .07 & .09 & .10 & .10 & .30 & .70 & 1.40 & 2.66 & 4.99 \\
\hline 5695 & .02 & .03 & .05 & .10 & .35 & .80 & 1.51 & 3.11 & 3.90 & 7499 & .04 & .05 & .10 & .10 & .30 & .70 & 1.30 & 2.57 & 3.52 \\
\hline 5742 & -- & -- & .03 & .05 & .09 & .34 & .75 & -- & -- & 7531 & .02 & .03 & .05 & .10 & .34 & .71 & 1.56 & 2.32 & 3.18 \\
\hline 5766 & -- & -- & .05 & .12 & .27 & .73 & 2.42 & -- & -- & 7534 & .01 & .02 & .04 & .08 & .25 & .68 & 1.29 & 3.24 & 3.88 \\
\hline 5770 & .02 & .03 & .06 & .10 & .20 & .53 & 1.10 & 2.30 & 3.02 & 7556 & .02 & .04 & .10 & .10 & .30 & .70 & 1.40 & 2.93 & 3.94 \\
\hline 5775 & -- & -- & .05 & .06 & .18 & .70 & .92 & -- & -- & 7594 & .02 & .02 & .05 & .11 & .35 & .89 & 1.72 & 3.40 & 4.20 \\
\hline 5779 & -- & -- & .04 & .13 & .35 & .72 & 2.27 & -- & -- & 7596 & .01 & .02 & .04 & .09 & .34 & 1.05 & 1.91 & 3.28 & 3.94 \\
\hline 5840 & .02 & .02 & .05 & .10 & .29 & .73 & 1.52 & 2.80 & 3.55 & 7608 & .01 & .01 & .02 & .06 & .22 & .68 & 1.42 & 2.86 & 3.55 \\
\hline 5890 & .01 & .01 & .01 & .03 & .11 & .36 & .85 & 2.02 & 2.49 & 7622 & -- & -- & .01 & .01 & .04 & .18 & .71 & -- & -- \\
\hline 5891 & .02 & .02 & .05 & .09 & .23 & .50 & .92 & 1.81 & 2.04 & 7700 & .01 & .02 & .04 & .09 & .28 & .77 & 1.56 & 3.45 & 4.21 \\
\hline 5897 & .10 & .10 & .10 & .10 & .30 & .70 & 1.50 & 3.20 & 3.90 & 7706 & .02 & .02 & .05 & .10 & .20 & 60 & 1.20 & 2.56 & 3.33 \\
\hline
\end{tabular}


Appendix 4-4.2. Empirical distribution of storm depth defined by 8-hour minimum interevent time for hourly rainfall stations in TexasContinued.

\begin{tabular}{|c|c|c|c|c|c|c|c|c|c|c|c|c|c|c|c|c|c|c|c|}
\hline \multicolumn{20}{|c|}{$\begin{array}{c}\text { Depth } \\
\text { (inches) }\end{array}$} \\
\hline $\begin{array}{l}\text { Sta- } \\
\text { tion } \\
\text { no. }\end{array}$ & $\begin{array}{c}\text { 1st } \\
\text { per- } \\
\text { centile }\end{array}$ & $\begin{array}{c}\text { 2nd } \\
\text { per- } \\
\text { centile }\end{array}$ & $\begin{array}{c}10 \text { th } \\
\text { per- } \\
\text { centile }\end{array}$ & $\begin{array}{c}25 \text { th } \\
\text { per- } \\
\text { centile }\end{array}$ & $\begin{array}{c}\text { 50th } \\
\text { per- } \\
\text { centile } \\
\text { (median) }\end{array}$ & $\begin{array}{c}\text { 75th } \\
\text { per- } \\
\text { centile }\end{array}$ & $\begin{array}{c}\text { 90th } \\
\text { per- } \\
\text { centile }\end{array}$ & $\begin{array}{c}\text { 98th } \\
\text { per- } \\
\text { centile }\end{array}$ & $\begin{array}{c}\text { 99th } \\
\text { per- } \\
\text { centile }\end{array}$ & $\begin{array}{l}\text { Sta- } \\
\text { tion } \\
\text { no. }\end{array}$ & $\begin{array}{c}\text { 1st } \\
\text { per- } \\
\text { centile }\end{array}$ & $\begin{array}{c}\text { 2nd } \\
\text { per- } \\
\text { centile }\end{array}$ & $\begin{array}{c}10 \text { th } \\
\text { per- } \\
\text { centile }\end{array}$ & $\begin{array}{c}25 \text { th } \\
\text { per- } \\
\text { centile }\end{array}$ & $\begin{array}{c}\text { 50th } \\
\text { per- } \\
\text { centile } \\
\text { (median) }\end{array}$ & $\begin{array}{c}\text { 75th } \\
\text { per- } \\
\text { centile }\end{array}$ & $\begin{array}{c}\text { 90th } \\
\text { per- } \\
\text { centile }\end{array}$ & $\begin{array}{c}\text { 98th } \\
\text { per- } \\
\text { centile }\end{array}$ & $\begin{array}{c}\text { 99th } \\
\text { per- } \\
\text { centile }\end{array}$ \\
\hline 7718 & 0.01 & 0.02 & 0.05 & 0.10 & 0.25 & 0.65 & 1.62 & 3.31 & 4.52 & 8910 & -- & -- & 0.05 & 0.12 & 0.39 & 1.13 & 1.89 & -- & -- \\
\hline 7745 & .01 & .02 & .04 & .09 & .29 & .83 & 1.74 & 3.91 & 5.14 & 8911 & 0.01 & 0.01 & .03 & .06 & .21 & .62 & 1.32 & 2.79 & 3.50 \\
\hline 7922 & .01 & .01 & .02 & .05 & .12 & .29 & .65 & 1.18 & 1.89 & 8924 & .02 & .02 & .05 & .08 & .18 & .35 & .77 & 1.43 & 1.77 \\
\hline 7936 & .03 & .03 & .10 & .10 & .32 & .90 & 1.65 & 3.43 & 4.55 & 8929 & -- & -- & .05 & .12 & .36 & 1.60 & 2.26 & -- & -- \\
\hline 7943 & .01 & .01 & .01 & .04 & .13 & .43 & 1.01 & 2.15 & 2.78 & 8942 & .03 & .06 & .10 & .10 & .30 & .80 & 1.70 & 3.43 & 4.53 \\
\hline 7944 & -- & .01 & .01 & .03 & .12 & .40 & 1.33 & 2.48 & -- & 8944 & .01 & .02 & .03 & .09 & .27 & .75 & 1.44 & 2.93 & 4.03 \\
\hline 7945 & .01 & .01 & .01 & .03 & .12 & .48 & 1.09 & 2.83 & 3.83 & 8996 & .01 & .02 & .04 & .08 & .25 & .71 & 1.48 & 3.13 & 3.89 \\
\hline 7947 & .03 & .04 & .10 & .10 & .23 & .72 & 1.50 & 4.06 & 4.94 & 9014 & -- & -- & .11 & .23 & .50 & .99 & 1.85 & -- & -- \\
\hline 7948 & .01 & .02 & .03 & .07 & .20 & .60 & 1.39 & 3.23 & 4.10 & 9037 & .02 & .02 & .04 & .07 & .15 & .35 & .76 & 1.67 & 2.30 \\
\hline 7951 & .02 & .02 & .05 & .12 & .34 & .82 & 1.57 & 2.80 & 3.37 & 9106 & .01 & .02 & .03 & .05 & .12 & .33 & .61 & 1.95 & 2.46 \\
\hline 7953 & .01 & .02 & .03 & .05 & .19 & .58 & 1.20 & 2.98 & 3.06 & 9107 & -- & -- & .03 & .06 & .14 & .48 & .80 & -- & -- \\
\hline 7981 & .02 & .02 & .03 & .06 & .23 & .60 & 1.57 & 2.94 & 3.54 & 9129 & .01 & .01 & .02 & .05 & .10 & .34 & .83 & 2.89 & 3.28 \\
\hline 7990 & .01 & .01 & .02 & .04 & .15 & .50 & .99 & 3.50 & 4.65 & 9163 & .02 & .03 & .06 & .10 & .26 & .63 & 1.30 & 2.70 & 3.31 \\
\hline 7992 & -- & -- & .09 & .10 & .28 & .93 & 1.79 & -- & -- & 9213 & .01 & .01 & .02 & .05 & .23 & .65 & 1.27 & 2.30 & 4.31 \\
\hline 7997 & .01 & .02 & .04 & .07 & .23 & .56 & 1.38 & 2.45 & 2.61 & 9214 & -- & -- & .11 & .23 & .35 & 1.41 & 1.89 & -- & -- \\
\hline 7999 & -- & -- & .02 & .03 & .16 & .32 & .93 & -- & -- & 9222 & .01 & .01 & .02 & .06 & .22 & .66 & 1.28 & 2.83 & 3.94 \\
\hline 8022 & -- & .01 & .05 & .05 & .16 & .60 & 1.12 & 2.27 & -- & 9248 & .02 & .02 & .05 & .10 & .20 & .58 & 1.17 & 2.10 & 2.25 \\
\hline 8023 & .02 & .03 & .06 & .10 & .20 & .50 & 1.10 & 2.38 & 3.09 & 9266 & -- & .02 & .04 & .11 & .25 & .77 & 1.34 & 2.68 & -- \\
\hline 8047 & .02 & .03 & .06 & .10 & .28 & .70 & 1.35 & 2.71 & 3.44 & 9270 & .03 & .03 & .09 & .10 & .15 & .38 & .78 & 1.60 & 2.10 \\
\hline 8060 & .01 & .01 & .03 & .05 & .15 & .43 & 1.35 & 2.80 & 3.19 & 9295 & .01 & .01 & .02 & .03 & .10 & .24 & .50 & 1.19 & 1.38 \\
\hline 8062 & -- & -- & .02 & .04 & .24 & .86 & 2.23 & -- & -- & 9304 & -- & -- & -- & .10 & .17 & .26 & -- & -- & -- \\
\hline 8068 & -- & -- & .02 & .04 & .10 & .31 & .90 & -- & -- & 9307 & .03 & .03 & .08 & .12 & .30 & .70 & 1.27 & 2.49 & 3.33 \\
\hline 8081 & .02 & .02 & .05 & .10 & .20 & .60 & 1.30 & 2.98 & 4.34 & 9328 & .02 & .02 & .04 & .08 & .21 & .60 & 1.18 & 1.97 & 2.89 \\
\hline 8089 & -- & -- & .03 & .08 & .20 & .80 & 1.19 & -- & -- & 9329 & -- & -- & .02 & .06 & .17 & .97 & 1.23 & -- & -- \\
\hline 8221 & -- & -- & .10 & .20 & .55 & .88 & 2.06 & -- & -- & 9345 & -- & -- & -- & -- & .70 & -- & -- & -- & -- \\
\hline 8252 & .02 & .02 & .06 & .10 & .20 & .50 & 1.07 & 2.20 & 2.87 & 9363 & .01 & .01 & .01 & .04 & .13 & .46 & 1.02 & 2.77 & 3.52 \\
\hline 8265 & .02 & .02 & .05 & .09 & .26 & .75 & 1.65 & 3.46 & 6.06 & 9364 & .01 & .01 & .01 & .04 & .15 & .53 & 1.27 & 3.00 & 4.09 \\
\hline 8289 & -- & .02 & .04 & .14 & .39 & .77 & 1.00 & 1.52 & -- & 9365 & -- & -- & .05 & .08 & .11 & .30 & .67 & -- & -- \\
\hline 8305 & .02 & .02 & .05 & .10 & .12 & .30 & .69 & 1.40 & 1.90 & 9371 & .02 & .03 & .05 & .10 & .27 & .75 & 1.45 & 2.60 & 2.81 \\
\hline 8335 & .02 & .02 & .05 & .12 & .37 & .90 & 1.73 & 3.43 & 4.30 & 9417 & .01 & .02 & .04 & .10 & .27 & .74 & 1.48 & 2.84 & 3.34 \\
\hline 8400 & .02 & .02 & .03 & .08 & .17 & .40 & .80 & 2.11 & 3.11 & 9419 & .01 & .01 & .01 & .04 & .17 & .60 & 1.30 & 2.70 & 3.32 \\
\hline 8445 & .02 & .02 & .04 & .08 & .25 & .73 & 1.58 & 3.41 & 4.36 & 9435 & -- & .03 & .06 & .10 & .25 & .60 & 1.04 & 2.64 & -- \\
\hline 8446 & .02 & .02 & .10 & .10 & .23 & .67 & 1.40 & 2.73 & 3.40 & 9491 & .02 & .02 & .06 & .10 & .30 & .77 & 1.54 & 3.00 & 3.72 \\
\hline 8451 & .01 & .02 & .04 & .08 & .20 & .58 & 1.08 & 2.34 & 3.27 & 9499 & .02 & .03 & .10 & .10 & .20 & .60 & 1.20 & 2.40 & 3.20 \\
\hline 8531 & .02 & .03 & .05 & .11 & .30 & .75 & 1.40 & 2.79 & 3.43 & 9522 & -- & -- & .05 & .20 & .40 & 1.68 & 2.92 & -- & -- \\
\hline 8541 & .01 & .01 & .05 & .12 & .34 & .73 & 1.34 & 2.98 & 3.90 & 9527 & .03 & .04 & .10 & .10 & .20 & .50 & 1.00 & 2.00 & 2.40 \\
\hline 8544 & .03 & .04 & .09 & .10 & .28 & .75 & 1.51 & 3.39 & 4.59 & 9532 & .02 & .02 & .07 & .10 & .28 & .70 & 1.31 & 2.83 & 3.93 \\
\hline 8545 & -- & -- & .02 & .07 & .23 & .42 & .61 & -- & -- & 9544 & -- & -- & -- & .11 & .13 & 2.95 & -- & -- & -- \\
\hline 8563 & .10 & .10 & .10 & .10 & .30 & .80 & 1.44 & 3.25 & 4.20 & 9565 & .02 & .03 & .08 & .10 & .25 & .60 & 1.29 & 2.54 & 3.61 \\
\hline 8566 & .01 & .02 & .04 & .09 & .23 & .61 & 1.20 & 2.38 & 2.68 & 9570 & .10 & .10 & .10 & .10 & .20 & .50 & 1.19 & 2.35 & 3.20 \\
\hline 8583 & .10 & .10 & .10 & .10 & .20 & .60 & 1.20 & 2.30 & 2.80 & 9574 & -- & -- & .10 & .10 & .20 & .60 & 1.50 & -- & -- \\
\hline 8584 & .02 & .03 & .09 & .10 & .27 & .74 & 1.39 & 2.70 & 3.20 & 9588 & .01 & .02 & .05 & .10 & .20 & .50 & 1.20 & 2.89 & 3.70 \\
\hline 8623 & .04 & .05 & .10 & .10 & .30 & .70 & 1.40 & 2.70 & 3.30 & 9665 & .02 & .03 & .07 & .10 & .30 & .80 & 1.57 & 3.20 & 4.40 \\
\hline 8625 & .02 & .02 & .05 & .10 & .26 & .72 & 1.32 & 2.85 & 3.69 & 9715 & .02 & .02 & .06 & .10 & .30 & .79 & 1.40 & 2.80 & 3.65 \\
\hline 8630 & .02 & .03 & .06 & .10 & .21 & .57 & 1.19 & 2.21 & 2.52 & 9729 & .01 & .01 & .02 & .05 & .19 & .60 & 1.25 & 2.58 & 3.20 \\
\hline 8631 & .02 & .02 & .05 & .10 & .25 & .57 & 1.30 & 2.57 & 3.53 & 9772 & .01 & .02 & .04 & .07 & .22 & .65 & 1.45 & 3.77 & 4.90 \\
\hline 8646 & .02 & .02 & .05 & .10 & .29 & .73 & 1.42 & 2.74 & 3.35 & 9814 & -- & -- & .04 & .10 & .36 & .88 & 1.67 & -- & -- \\
\hline 8647 & .02 & .03 & .09 & .10 & .20 & .50 & .95 & 2.14 & 2.70 & 9815 & .02 & .03 & .06 & .10 & .26 & .71 & 1.50 & 2.94 & 4.15 \\
\hline 8677 & .01 & .02 & .05 & .12 & .25 & .65 & 1.29 & 2.61 & 3.08 & 9816 & -- & .01 & .04 & .07 & .26 & .76 & 1.29 & 4.27 & -- \\
\hline 8696 & -- & -- & .06 & .10 & .42 & .96 & 1.44 & -- & -- & 9817 & .03 & .05 & .10 & .10 & .29 & .70 & 1.25 & 2.60 & 3.33 \\
\hline 8743 & .02 & .02 & .07 & .10 & .31 & .85 & 1.62 & 3.69 & 4.46 & 9829 & .02 & .02 & .05 & .10 & .14 & .40 & .84 & 1.90 & 2.32 \\
\hline 8761 & .02 & .03 & .10 & .10 & .20 & .49 & .90 & 1.99 & 2.90 & 9830 & .01 & .01 & .03 & .05 & .11 & .38 & .74 & 1.36 & 2.21 \\
\hline 8778 & .03 & .04 & .10 & .10 & .30 & .80 & 1.50 & 3.10 & 3.75 & 9858 & .02 & .02 & .05 & .10 & .24 & .55 & 1.04 & 2.29 & 2.71 \\
\hline 8845 & .02 & .03 & .07 & .10 & .20 & .68 & 1.42 & 3.18 & 4.15 & 9893 & .02 & .02 & .05 & .10 & .25 & .67 & 1.30 & 2.60 & 3.51 \\
\hline 8859 & .02 & .02 & .05 & .13 & .35 & .93 & 1.64 & 3.19 & 3.88 & 9916 & .02 & .04 & .10 & .10 & .39 & .90 & 1.71 & 3.47 & 4.10 \\
\hline 8898 & .02 & .03 & .05 & .12 & .34 & .78 & 1.59 & 3.71 & 4.25 & 9976 & .02 & .02 & .05 & .10 & .20 & .56 & 1.22 & 2.59 & 3.32 \\
\hline 8908 & -- & -- & .04 & .10 & .22 & .62 & 1.33 & -- & -- & & & & & & & & & & \\
\hline
\end{tabular}


Appendix 4-4.3. Empirical distribution of storm depth defined by 12-hour minimum interevent time for hourly rainfall stations in Texas.

$[--$, not available $]$

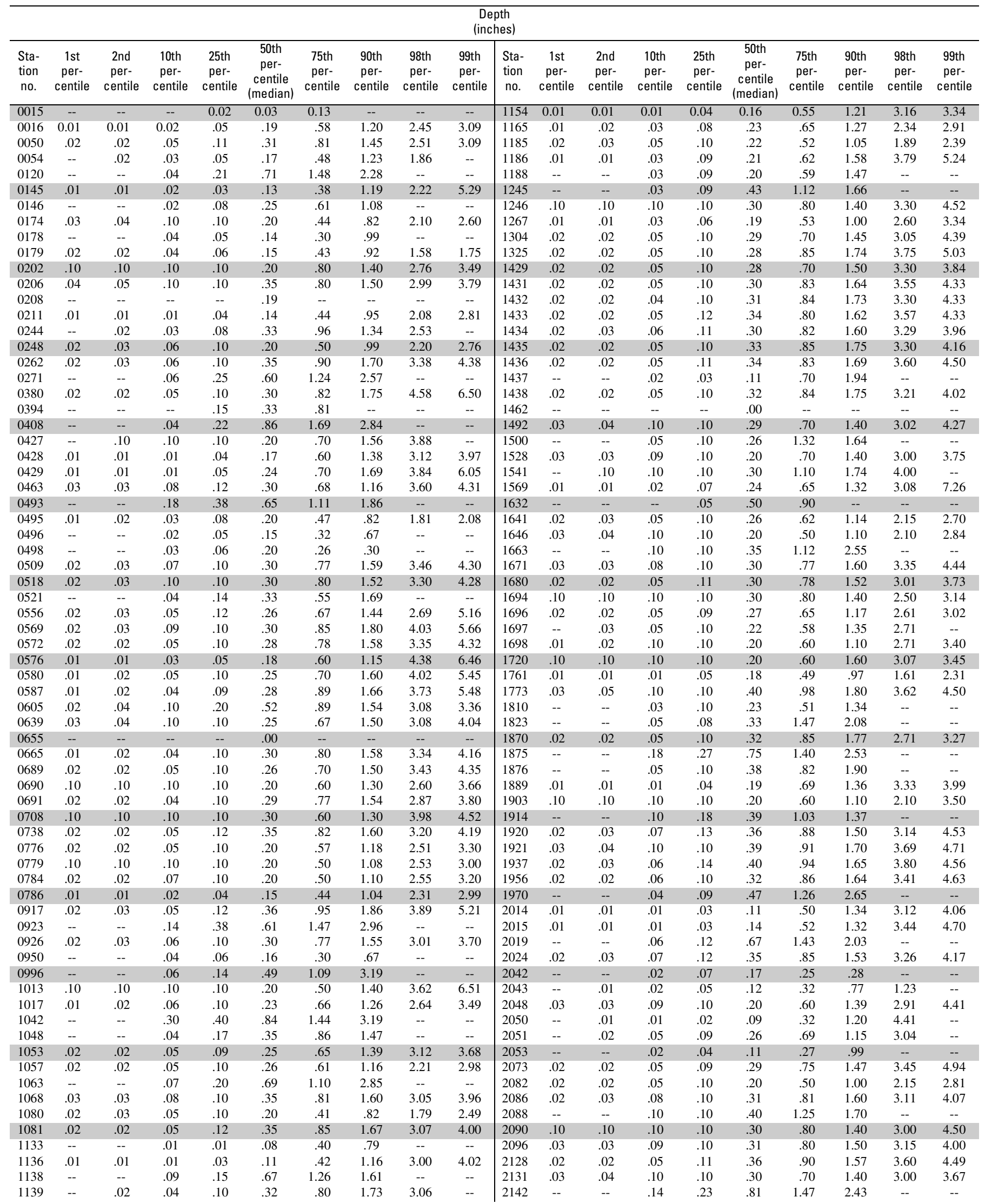


Appendix 4-4.3. Empirical distribution of storm depth defined by 12-hour minimum interevent time for hourly rainfall stations in TexasContinued.

\begin{tabular}{|c|c|c|c|c|c|c|c|c|c|c|c|c|c|c|c|c|c|c|c|}
\hline \multicolumn{20}{|c|}{$\begin{array}{c}\text { Depth } \\
\text { (inches) }\end{array}$} \\
\hline $\begin{array}{l}\text { Sta- } \\
\text { tion } \\
\text { no. }\end{array}$ & $\begin{array}{c}1 \text { st } \\
\text { per- } \\
\text { centile }\end{array}$ & $\begin{array}{c}\text { 2nd } \\
\text { per- } \\
\text { centile }\end{array}$ & $\begin{array}{c}10 \text { th } \\
\text { per- } \\
\text { centile }\end{array}$ & $\begin{array}{c}25 \text { th } \\
\text { per- } \\
\text { centile }\end{array}$ & $\begin{array}{c}\text { 50th } \\
\text { per- } \\
\text { centile } \\
\text { (median) }\end{array}$ & $\begin{array}{c}\text { 75th } \\
\text { per- } \\
\text { centile }\end{array}$ & $\begin{array}{c}\text { 90th } \\
\text { per- } \\
\text { centile }\end{array}$ & $\begin{array}{c}\text { 98th } \\
\text { per- } \\
\text { centile }\end{array}$ & $\begin{array}{c}\text { 99th } \\
\text { per- } \\
\text { centile }\end{array}$ & $\begin{array}{l}\text { Sta- } \\
\text { tion } \\
\text { no. }\end{array}$ & $\begin{array}{c}\text { 1st } \\
\text { per- } \\
\text { centile }\end{array}$ & $\begin{array}{c}\text { 2nd } \\
\text { per- } \\
\text { centile }\end{array}$ & $\begin{array}{c}10 \text { th } \\
\text { per- } \\
\text { centile }\end{array}$ & $\begin{array}{c}\text { 25th } \\
\text { per- } \\
\text { centile }\end{array}$ & $\begin{array}{c}\text { 50th } \\
\text { per- } \\
\text { centile } \\
\text { (median) }\end{array}$ & $\begin{array}{c}\text { 75th } \\
\text { per- } \\
\text { centile }\end{array}$ & $\begin{array}{c}\text { 90th } \\
\text { per- } \\
\text { centile }\end{array}$ & $\begin{array}{c}\text { 98th } \\
\text { per- } \\
\text { centile }\end{array}$ & $\begin{array}{c}\text { 99th } \\
\text { per- } \\
\text { centile }\end{array}$ \\
\hline 2160 & -- & -- & 0.01 & 0.03 & 0.13 & 0.33 & 0.91 & -- & -- & 3463 & -- & -- & 0.03 & 0.07 & 0.28 & 0.89 & 1.85 & -- & -- \\
\hline 2206 & 0.02 & 0.03 & .05 & .11 & .35 & .88 & 1.88 & 3.39 & 5.87 & 3476 & 0.02 & 0.02 & .04 & .10 & .27 & .76 & 1.46 & 2.96 & 3.92 \\
\hline 2238 & .01 & .01 & .02 & .03 & .14 & .44 & 1.02 & 2.05 & 2.32 & 3485 & -- & -- & .10 & .10 & .42 & 1.67 & 2.51 & -- & -- \\
\hline 2240 & -- & .01 & .02 & .04 & .17 & .41 & .98 & 1.95 & -- & 3507 & .06 & .08 & .10 & .10 & .30 & .80 & 1.70 & 3.34 & 3.92 \\
\hline 2242 & .01 & .01 & .02 & .06 & .26 & .78 & 1.48 & 3.05 & 3.66 & 3546 & .02 & .03 & .07 & .11 & .38 & .95 & 1.80 & 3.65 & 4.60 \\
\hline 2244 & .01 & .01 & .03 & .10 & .30 & .77 & 1.60 & 3.20 & 4.34 & 3547 & .02 & .02 & .05 & .12 & .35 & .89 & 1.72 & 3.05 & 3.21 \\
\hline 2247 & -- & .01 & .02 & .05 & .23 & .65 & 1.35 & 3.67 & -- & 3579 & -- & .03 & .06 & .12 & .25 & .97 & 1.67 & 3.35 & -- \\
\hline 2309 & .03 & .04 & .10 & .20 & .47 & 1.00 & 1.71 & 3.57 & 4.71 & 3642 & .02 & .03 & .09 & .10 & .31 & .81 & 1.65 & 3.49 & 4.49 \\
\hline 2312 & .05 & .10 & .10 & .10 & .40 & .90 & 1.60 & 3.00 & 3.92 & 3646 & .02 & .03 & .06 & .12 & .33 & .78 & 1.48 & 3.18 & 4.18 \\
\hline 2334 & -- & .03 & .08 & .21 & .60 & 1.28 & 2.59 & 4.50 & -- & 3668 & -- & -- & .34 & .50 & .89 & 1.81 & 3.26 & -- & -- \\
\hline 2336 & .02 & .03 & .05 & .15 & .37 & .91 & 1.49 & 2.60 & 3.65 & 3673 & -- & -- & .04 & .08 & .40 & 1.58 & 2.27 & -- & -- \\
\hline 2354 & -- & -- & .05 & .08 & .14 & .54 & 1.01 & -- & -- & 3686 & .04 & .07 & .10 & .10 & .30 & .80 & 1.61 & 2.75 & 3.50 \\
\hline 2355 & -- & .01 & .03 & .07 & .25 & .59 & 2.08 & 4.85 & -- & 3691 & .02 & .03 & .10 & .10 & .30 & .80 & 1.50 & 3.09 & 3.90 \\
\hline 2357 & .01 & .01 & .01 & .02 & .09 & .43 & 1.09 & 3.05 & 4.36 & 3734 & -- & -- & .03 & .16 & .55 & 1.75 & 3.54 & -- & -- \\
\hline 2360 & .01 & .01 & .01 & .03 & .11 & .44 & 1.10 & 2.67 & 3.33 & 3771 & .10 & .10 & .10 & .10 & .30 & .80 & 1.60 & 3.50 & 4.18 \\
\hline 2361 & .02 & .02 & .04 & .10 & .20 & .60 & .95 & 2.31 & 6.25 & 3789 & .01 & .01 & .03 & .07 & .11 & .27 & .48 & 1.17 & 2.10 \\
\hline 2394 & .02 & .02 & .05 & .10 & .32 & .83 & 1.60 & 3.40 & 4.70 & 3826 & .01 & .03 & .06 & .15 & .33 & .85 & 1.41 & 2.63 & 3.29 \\
\hline 2404 & .02 & .02 & .05 & .10 & .30 & .80 & 1.55 & 3.15 & 3.80 & 3831 & .03 & .03 & .05 & .14 & .36 & .95 & 1.64 & 3.02 & 5.11 \\
\hline 2415 & .02 & .03 & .07 & .15 & .41 & 1.00 & 1.89 & 3.74 & 4.90 & 3841 & .02 & .02 & .05 & .15 & .30 & .91 & 1.69 & 3.85 & 5.03 \\
\hline 2462 & .03 & .03 & .07 & .13 & .38 & .94 & 1.80 & 3.28 & 4.14 & 3871 & .02 & .03 & .05 & .10 & .30 & .70 & 1.35 & 2.86 & 3.61 \\
\hline 2528 & .03 & .03 & .05 & .10 & .27 & .74 & 1.64 & 2.68 & 2.78 & 3884 & -- & -- & .06 & .10 & .30 & 1.28 & 3.19 & -- & -- \\
\hline 2617 & .01 & .02 & .04 & .08 & .25 & .56 & 1.14 & 3.07 & 3.99 & 3941 & -- & .01 & .04 & .08 & .44 & 1.23 & 2.09 & 4.64 & -- \\
\hline 2619 & .02 & .03 & .05 & .13 & .27 & .65 & 1.13 & 2.29 & 3.82 & 3963 & -- & -- & -- & .03 & .05 & .10 & -- & -- & -- \\
\hline 2621 & .02 & .03 & .06 & .10 & .27 & .63 & 1.30 & 2.46 & 3.08 & 4040 & .02 & .03 & .05 & .11 & .30 & .67 & 1.33 & 2.66 & 3.55 \\
\hline 2675 & .03 & .04 & .10 & .10 & .30 & .80 & 1.50 & 3.40 & 4.29 & 4058 & -- & -- & .06 & .09 & .47 & .88 & 1.76 & -- & -- \\
\hline 2676 & .10 & .10 & .10 & .10 & .30 & .80 & 1.70 & 3.00 & 4.04 & 4098 & .03 & .04 & .10 & .10 & .20 & .50 & .94 & 1.93 & 2.31 \\
\hline 2679 & .02 & .02 & .05 & .10 & .20 & .60 & 1.40 & 3.09 & 4.30 & 4100 & .02 & .02 & .05 & .10 & .25 & .65 & 1.34 & 3.24 & 4.86 \\
\hline 2715 & .02 & .03 & .06 & .10 & .33 & .79 & 1.47 & 2.95 & 3.60 & 4137 & .10 & .10 & .10 & .10 & .30 & .80 & 1.50 & 2.90 & 3.75 \\
\hline 2744 & .02 & .03 & .06 & .10 & .29 & .70 & 1.30 & 2.73 & 3.37 & 4191 & .02 & .03 & .06 & .10 & .24 & .70 & 1.50 & 3.22 & 4.31 \\
\hline 2758 & .01 & .02 & .02 & .05 & .14 & .40 & 1.25 & 4.04 & 4.44 & 4256 & -- & -- & -- & -- & .00 & -- & -- & -- & -- \\
\hline 2794 & -- & -- & .01 & .04 & .16 & .66 & 1.61 & -- & -- & 4257 & .04 & .05 & .10 & .10 & .40 & .97 & 1.84 & 3.79 & 4.50 \\
\hline 2797 & .01 & .01 & .01 & .03 & .08 & .25 & .54 & 1.29 & 1.70 & 4258 & .10 & .10 & .10 & .10 & .30 & .82 & 1.40 & 3.27 & 5.01 \\
\hline 2811 & .02 & .02 & .06 & .10 & .20 & .60 & 1.30 & 2.70 & 3.30 & 4278 & .02 & .02 & .05 & .11 & .31 & .84 & 1.63 & 3.19 & 3.82 \\
\hline 2813 & -- & -- & .05 & .15 & .35 & .60 & 1.58 & -- & -- & 4299 & .02 & .03 & .06 & .10 & .25 & .46 & .81 & 1.24 & 1.46 \\
\hline 2814 & -- & -- & .01 & .03 & .05 & .15 & .96 & -- & -- & 4300 & .01 & .01 & .01 & .05 & .21 & .68 & 1.50 & 3.45 & 4.88 \\
\hline 2815 & .10 & .10 & .10 & .10 & .30 & .60 & 1.30 & 2.72 & 3.82 & 4305 & .01 & .01 & .01 & .04 & .18 & .65 & 1.45 & 3.34 & 4.55 \\
\hline 2818 & .02 & .02 & .04 & .10 & .35 & .78 & 1.49 & 3.29 & 3.78 & 4307 & .01 & .01 & .01 & .04 & .23 & .74 & 1.61 & 3.53 & 5.88 \\
\hline 2986 & .02 & .02 & .07 & .16 & .46 & 1.20 & 2.03 & 3.55 & 4.08 & 4309 & .02 & .02 & .05 & .10 & .32 & .87 & 1.75 & 3.62 & 4.87 \\
\hline 3005 & .02 & .03 & .08 & .10 & .30 & .80 & 1.41 & 2.88 & 3.68 & 4311 & .02 & .02 & .05 & .11 & .35 & .95 & 1.90 & 3.75 & 4.78 \\
\hline 3033 & .02 & .02 & .04 & .08 & .14 & .31 & .62 & 1.30 & 1.88 & 4313 & .02 & .02 & .05 & .11 & .30 & .81 & 1.92 & 4.17 & 6.48 \\
\hline 3034 & -- & -- & -- & -- & .43 & -- & -- & -- & -- & 4319 & -- & .03 & .05 & .12 & .30 & .81 & 1.32 & 3.10 & -- \\
\hline 3047 & -- & -- & .03 & .06 & .34 & .74 & 1.42 & -- & -- & 4329 & .02 & .02 & .05 & .12 & .35 & .85 & 1.80 & 3.90 & 4.88 \\
\hline 3103 & -- & -- & .09 & .14 & .26 & .98 & 2.04 & -- & -- & 4331 & -- & -- & -- & -- & .00 & -- & -- & -- & -- \\
\hline 3133 & .02 & .03 & .07 & .12 & .40 & .87 & 1.63 & 3.40 & 4.40 & 4375 & .10 & .10 & .10 & .10 & .30 & .70 & 1.40 & 3.30 & 4.80 \\
\hline 3156 & .04 & .05 & .10 & .10 & .37 & .88 & 1.84 & 5.47 & 5.93 & 4392 & .02 & .02 & .05 & .13 & .40 & 1.05 & 2.07 & 3.92 & 6.30 \\
\hline 3171 & .02 & .03 & .06 & .11 & .33 & .82 & 1.61 & 3.27 & 4.24 & 4425 & .03 & .03 & .09 & .10 & .20 & .50 & 1.00 & 2.10 & 2.76 \\
\hline 3189 & .01 & .02 & .04 & .09 & .16 & .40 & .84 & 1.65 & 2.46 & 4440 & .02 & .02 & .04 & .09 & .26 & .70 & 1.34 & 2.91 & 3.89 \\
\hline 3260 & .01 & .01 & .03 & .09 & .30 & .79 & 1.35 & 2.55 & 3.65 & 4476 & .03 & .05 & .10 & .10 & .30 & .77 & 1.45 & 2.98 & 3.70 \\
\hline 3267 & .01 & .01 & .03 & .05 & .17 & .58 & 1.09 & 3.70 & 4.13 & 4498 & -- & -- & .02 & .06 & .17 & .38 & .60 & -- & -- \\
\hline 3270 & .05 & .10 & .10 & .10 & .20 & .60 & 1.20 & 2.43 & 3.40 & 4517 & .02 & .02 & .05 & .10 & .31 & .79 & 1.53 & 3.01 & 3.67 \\
\hline 3272 & -- & -- & .02 & .04 & .07 & .13 & .33 & -- & -- & 4520 & .10 & .10 & .10 & .10 & .30 & .70 & 1.50 & 3.11 & 3.65 \\
\hline 3277 & -- & -- & .02 & .02 & .06 & .22 & .58 & -- & -- & 4525 & -- & -- & .04 & .07 & .26 & .78 & 2.63 & -- & -- \\
\hline 3278 & .02 & .03 & .05 & .10 & .20 & .56 & 1.10 & 2.20 & 2.68 & 4563 & -- & -- & .04 & .07 & .34 & .65 & 1.81 & -- & -- \\
\hline 3280 & .01 & .01 & .03 & .05 & .14 & .39 & .85 & 1.91 & 2.48 & 4570 & .02 & .03 & .07 & .10 & .25 & .60 & 1.30 & 2.58 & 3.26 \\
\hline 3281 & -- & -- & .04 & .12 & .25 & .51 & .93 & -- & -- & 4577 & .03 & .03 & .10 & .13 & .40 & .90 & 1.70 & 3.50 & 4.26 \\
\hline 3283 & .01 & .01 & .02 & .05 & .25 & .67 & 1.42 & 3.11 & 4.06 & 4591 & .02 & .03 & .05 & .10 & .33 & .81 & 1.60 & 3.36 & 4.20 \\
\hline 3284 & .01 & .02 & .08 & .10 & .30 & .79 & 1.54 & 2.98 & 3.60 & 4670 & .02 & .02 & .06 & .10 & .21 & .60 & 1.30 & 2.72 & 3.36 \\
\hline 3285 & .03 & .07 & .10 & .10 & .30 & .80 & 1.60 & 3.00 & 3.96 & 4671 & .01 & .01 & .02 & .06 & .20 & .59 & 1.03 & 2.23 & 2.91 \\
\hline 3329 & .01 & .02 & .04 & .10 & .24 & .66 & 1.40 & 3.21 & 4.17 & 4679 & .03 & .04 & .10 & .10 & .30 & .80 & 1.50 & 3.49 & 4.43 \\
\hline 3335 & .02 & .02 & .05 & .16 & .40 & 1.00 & 1.85 & 4.59 & 8.26 & 4696 & -- & -- & .04 & .09 & .26 & .58 & .91 & -- & -- \\
\hline 3370 & .02 & .03 & .05 & .14 & .39 & .94 & 1.75 & 3.49 & 4.37 & 4703 & .02 & .02 & .05 & .10 & .20 & .55 & 1.19 & 2.39 & 3.00 \\
\hline 3410 & .04 & .05 & .10 & .10 & .29 & .60 & 1.13 & 2.50 & 3.04 & 4704 & .02 & .03 & .05 & .12 & .38 & 1.00 & 2.07 & 4.52 & 5.45 \\
\hline 3415 & .02 & .03 & .10 & .10 & .30 & .78 & 1.59 & 3.01 & 4.10 & 4731 & .01 & .01 & .01 & .03 & .17 & .47 & 1.09 & 3.08 & 6.83 \\
\hline 3430 & .01 & .01 & .01 & .05 & .21 & .69 & 1.50 & 3.70 & 4.69 & 4792 & .10 & .10 & .10 & .10 & .30 & .80 & 1.44 & 2.80 & 3.30 \\
\hline 3431 & .01 & .01 & .01 & .04 & .15 & .68 & 1.37 & 4.43 & 5.84 & 4819 & .10 & .10 & .10 & .12 & .40 & 1.00 & 1.61 & 3.70 & 3.84 \\
\hline 3441 & -- & -- & .03 & .10 & .30 & .74 & 1.77 & -- & -- & 4852 & -- & -- & .15 & .35 & .69 & 1.10 & 2.55 & -- & -- \\
\hline 3442 & .02 & .03 & .05 & .09 & .20 & .46 & 1.00 & 2.21 & 2.60 & 4866 & .02 & .04 & .08 & .10 & .36 & .88 & 1.62 & 3.16 & 3.90 \\
\hline 3446 & .03 & .03 & .06 & .10 & .28 & .60 & 1.15 & 2.25 & 2.89 & 4876 & .10 & .10 & .10 & .10 & .40 & 1.00 & 2.30 & 4.55 & 4.95 \\
\hline 3460 & -- & -- & .05 & .10 & .49 & .99 & 1.50 & -- & -- & 4878 & .02 & .02 & .05 & .12 & .38 & .97 & 1.96 & 4.29 & 5.20 \\
\hline 3462 & .02 & .03 & .06 & .11 & .30 & .70 & 1.19 & 2.15 & 3.04 & 4880 & .02 & .03 & .06 & .10 & .22 & .57 & 1.08 & 2.25 & 2.80 \\
\hline
\end{tabular}


Appendix 4-4.3. Empirical distribution of storm depth defined by 12-hour minimum interevent time for hourly rainfall stations in TexasContinued.

\begin{tabular}{|c|c|c|c|c|c|c|c|c|c|c|c|c|c|c|c|c|c|c|c|}
\hline \multicolumn{20}{|c|}{$\begin{array}{c}\text { Depth } \\
\text { (inches) }\end{array}$} \\
\hline $\begin{array}{l}\text { Sta- } \\
\text { tion } \\
\text { no. }\end{array}$ & $\begin{array}{c}1 \text { st } \\
\text { per- } \\
\text { centile }\end{array}$ & $\begin{array}{c}\text { 2nd } \\
\text { per- } \\
\text { centile }\end{array}$ & $\begin{array}{c}10 \text { th } \\
\text { per- } \\
\text { centile }\end{array}$ & $\begin{array}{c}\text { 25th } \\
\text { per- } \\
\text { centile }\end{array}$ & $\begin{array}{c}\text { 50th } \\
\text { per- } \\
\text { centile } \\
\text { (median) }\end{array}$ & $\begin{array}{c}\text { 75th } \\
\text { per- } \\
\text { centile }\end{array}$ & $\begin{array}{c}\text { 90th } \\
\text { per- } \\
\text { centile }\end{array}$ & $\begin{array}{c}\text { 98th } \\
\text { per- } \\
\text { centile }\end{array}$ & $\begin{array}{c}\text { 99th } \\
\text { per- } \\
\text { centile }\end{array}$ & $\begin{array}{l}\text { Sta- } \\
\text { tion } \\
\text { no. }\end{array}$ & $\begin{array}{c}1 \text { st } \\
\text { per- } \\
\text { centile }\end{array}$ & $\begin{array}{c}\text { 2nd } \\
\text { per- } \\
\text { centile }\end{array}$ & $\begin{array}{c}\text { 10th } \\
\text { per- } \\
\text { centile }\end{array}$ & $\begin{array}{c}\text { 25th } \\
\text { per- } \\
\text { centile }\end{array}$ & $\begin{array}{c}\text { 50th } \\
\text { per- } \\
\text { centile } \\
\text { (median) }\end{array}$ & $\begin{array}{c}\text { 75th } \\
\text { per- } \\
\text { centile }\end{array}$ & $\begin{array}{c}\text { 90th } \\
\text { per- } \\
\text { centile }\end{array}$ & $\begin{array}{c}\text { 98th } \\
\text { per- } \\
\text { centile }\end{array}$ & $\begin{array}{c}\text { 99th } \\
\text { per- } \\
\text { centile }\end{array}$ \\
\hline 4920 & 0.02 & 0.03 & 0.07 & 0.10 & 0.22 & 0.70 & 1.42 & 3.18 & 4.08 & 5957 & 0.03 & 0.04 & 0.10 & 0.10 & 0.30 & 0.80 & 1.50 & 3.00 & 3.66 \\
\hline 4934 & -- & -- & -- & .08 & .15 & .36 & -- & -- & -- & 5958 & .01 & .01 & .04 & .10 & .31 & .78 & 1.40 & 2.45 & 3.25 \\
\hline 4972 & .02 & .03 & .08 & .10 & .30 & .71 & 1.40 & 3.00 & 3.90 & 5973 & .01 & .01 & .02 & .05 & .12 & .62 & 1.30 & 3.09 & 4.14 \\
\hline 4973 & .02 & .04 & .09 & .20 & .50 & 1.05 & 1.84 & 3.51 & 3.91 & 5996 & .02 & .03 & .07 & .10 & .30 & .77 & 1.41 & 2.91 & 3.98 \\
\hline 4974 & .02 & .02 & .05 & .10 & .22 & .60 & 1.10 & 2.37 & 3.19 & 6017 & .01 & .01 & .02 & .05 & .17 & .55 & 1.24 & 2.57 & 2.86 \\
\hline 4975 & .10 & .10 & .10 & .10 & .40 & .90 & 1.80 & 3.40 & 4.42 & 6024 & .02 & .02 & .05 & .10 & .45 & 1.16 & 2.20 & 4.86 & 6.27 \\
\hline 4978 & .01 & .02 & .04 & .09 & .24 & .68 & 1.70 & 3.26 & 5.13 & 6050 & -- & -- & .10 & .23 & .38 & 1.01 & 1.72 & -- & -- \\
\hline 4979 & -- & -- & .07 & .24 & .50 & 1.12 & 2.78 & -- & -- & 6104 & .02 & .02 & .05 & .10 & .20 & .41 & .82 & 1.79 & 2.51 \\
\hline 4982 & .02 & .02 & .05 & .10 & .28 & .71 & 1.31 & 2.73 & 3.42 & 6108 & .02 & .03 & .10 & .12 & .40 & 1.00 & 1.89 & 3.70 & 4.80 \\
\hline 5018 & .02 & .03 & .06 & .12 & .34 & .84 & 1.50 & 2.54 & 3.41 & 6136 & .02 & .03 & .10 & .10 & .20 & .50 & .94 & 1.98 & 2.55 \\
\hline 5048 & .02 & .03 & .08 & .10 & .20 & .55 & 1.10 & 2.66 & 3.38 & 6166 & -- & .02 & .04 & .05 & .18 & .46 & 1.16 & 2.05 & -- \\
\hline 5049 & -- & .10 & .10 & .10 & .20 & .50 & .90 & 4.30 & -- & 6176 & .02 & .02 & .05 & .12 & .39 & .82 & 2.00 & 4.14 & 6.00 \\
\hline 5056 & -- & -- & -- & .20 & .37 & .69 & -- & -- & -- & 6177 & .03 & .04 & .10 & .10 & .40 & .90 & 1.80 & 3.74 & 4.90 \\
\hline 5057 & .01 & .01 & .01 & .03 & .10 & .34 & .98 & 2.38 & 3.16 & 6210 & .02 & .03 & .10 & .10 & .30 & .90 & 1.64 & 3.62 & 4.36 \\
\hline 5060 & .01 & .01 & .02 & .05 & .15 & .57 & 1.44 & 4.37 & 6.18 & 6211 & .01 & .01 & .04 & .10 & .28 & .87 & 1.58 & 3.36 & 4.25 \\
\hline 5081 & .02 & .02 & .05 & .12 & .39 & .94 & 1.66 & 3.20 & 3.98 & 6270 & .10 & .10 & .10 & .10 & .40 & .90 & 1.90 & 3.90 & 4.79 \\
\hline 5094 & .02 & .03 & .10 & .10 & .30 & .80 & 1.65 & 3.31 & 4.00 & 6275 & -- & -- & -- & -- & .00 & -- & -- & -- & -- \\
\hline 5113 & .02 & .03 & .07 & .10 & .21 & .66 & 1.45 & 3.48 & 4.40 & 6276 & -- & -- & .09 & .18 & .81 & 1.59 & 2.58 & -- & -- \\
\hline 5114 & -- & -- & -- & -- & .00 & -- & -- & -- & -- & 6335 & .02 & .03 & .06 & .12 & .36 & .90 & 1.61 & 3.03 & 3.70 \\
\hline 5123 & -- & -- & .07 & .18 & .29 & .55 & 2.45 & -- & -- & 6434 & -- & -- & .05 & .13 & .37 & .88 & 1.57 & -- & -- \\
\hline 5192 & .02 & .02 & .07 & .10 & .35 & .86 & 1.70 & 3.35 & 4.40 & 6504 & .02 & .03 & .07 & .10 & .20 & .51 & 1.10 & 2.34 & 3.20 \\
\hline 5193 & .02 & .02 & .06 & .10 & .30 & .80 & 1.56 & 3.25 & 4.16 & 6558 & -- & -- & .05 & .06 & .41 & .97 & 1.80 & -- & -- \\
\hline 5224 & .01 & .02 & .05 & .11 & .38 & 1.05 & 1.85 & 4.25 & 4.95 & 6615 & .08 & .10 & .10 & .10 & .20 & .60 & 1.30 & 2.80 & 4.30 \\
\hline 5228 & .01 & .02 & .04 & .09 & .27 & .81 & 1.60 & 3.33 & 3.62 & 6660 & .02 & .02 & .05 & .10 & .33 & .85 & 1.71 & 3.59 & 3.80 \\
\hline 5235 & -- & -- & .03 & .05 & .32 & .79 & 1.84 & -- & -- & 6663 & .01 & .01 & .03 & .10 & .25 & .65 & 1.41 & 3.05 & 3.54 \\
\hline 5247 & .02 & .03 & .09 & .10 & .21 & .57 & 1.10 & 2.20 & 2.80 & 6734 & .01 & .01 & .04 & .09 & .23 & .57 & 1.11 & 2.52 & 3.20 \\
\hline 5258 & .02 & .03 & .05 & .11 & .33 & .84 & 1.70 & 3.46 & 3.87 & 6736 & .02 & .03 & .09 & .10 & .20 & .60 & 1.20 & 2.50 & 3.43 \\
\hline 5303 & .02 & .03 & .05 & .09 & .25 & .70 & 1.35 & 3.32 & 4.45 & 6740 & -- & -- & .09 & .20 & .82 & 2.15 & 3.49 & -- & - \\
\hline 5312 & .04 & .05 & .10 & .10 & .25 & .70 & 1.31 & 2.80 & 3.87 & 6750 & .01 & .01 & .02 & .05 & .27 & .74 & 1.79 & 4.72 & 9.18 \\
\hline 5341 & -- & -- & .08 & .22 & .41 & 1.24 & 2.24 & -- & -- & 6757 & .01 & .01 & .04 & .10 & .30 & .88 & 1.61 & 3.31 & 4.20 \\
\hline 5342 & -- & -- & -- & -- & .00 & -- & -- & -- & -- & 6775 & .01 & .01 & .03 & .07 & .22 & .55 & 1.08 & 2.50 & 2.98 \\
\hline 5348 & .03 & .05 & .10 & .10 & .40 & 1.00 & 1.80 & 3.40 & 4.30 & 6776 & .02 & .03 & .09 & .10 & .20 & .56 & 1.10 & 2.20 & 2.90 \\
\hline 5358 & .02 & .02 & .05 & .10 & .25 & .65 & 1.30 & 2.60 & 3.18 & 6788 & .02 & .02 & .05 & .15 & .46 & .89 & 1.75 & 3.86 & 4.61 \\
\hline 5398 & .02 & .03 & .06 & .10 & .35 & .87 & 1.74 & 3.45 & 4.43 & 6792 & .02 & .02 & .06 & .10 & .20 & .40 & .90 & 1.70 & 2.10 \\
\hline 5410 & .02 & .02 & .07 & .10 & .20 & .56 & 1.06 & 2.48 & 3.30 & 6794 & -- & -- & .15 & .33 & .80 & 1.88 & 3.05 & -- & -- \\
\hline 5411 & .01 & .01 & .01 & .04 & .14 & .45 & .96 & 2.15 & 2.88 & 6834 & .10 & .10 & .10 & .10 & .40 & 1.00 & 1.80 & 3.55 & 4.40 \\
\hline 5424 & .01 & .01 & .01 & .04 & .25 & .80 & 1.84 & 3.32 & 5.97 & 6893 & .02 & .03 & .08 & .10 & .17 & .40 & .79 & 1.78 & 2.53 \\
\hline 5429 & .02 & .02 & .04 & .08 & .23 & .71 & 1.55 & 3.14 & 4.38 & 6935 & .03 & .04 & .10 & .10 & .20 & .50 & 1.00 & 2.30 & 3.20 \\
\hline 5431 & -- & -- & .13 & .30 & .55 & 1.13 & 2.86 & -- & -- & 6981 & .02 & .03 & .05 & .14 & .37 & .76 & 1.52 & 3.75 & 4.75 \\
\hline 5461 & .02 & .02 & .05 & .13 & .37 & .97 & 1.87 & 3.77 & 5.07 & 7020 & .01 & .02 & .04 & .11 & .34 & .86 & 1.81 & 3.52 & 7.44 \\
\hline 5463 & .10 & .10 & .10 & .10 & .40 & .90 & 1.80 & 3.50 & 4.91 & 7060 & .03 & .04 & .10 & .10 & .27 & .60 & 1.30 & 2.48 & 3.12 \\
\hline 5471 & -- & -- & .02 & .02 & .07 & .10 & .44 & -- & -- & 7066 & .02 & .03 & .10 & .11 & .40 & .96 & 1.85 & 3.49 & 4.50 \\
\hline 5477 & -- & -- & .07 & .15 & .26 & .57 & 1.48 & -- & -- & 7074 & .02 & .02 & .05 & .10 & .20 & .50 & 1.00 & 2.37 & 3.41 \\
\hline 5528 & .02 & .03 & .07 & .15 & .40 & .97 & 1.74 & 3.47 & 4.44 & 7097 & .02 & .02 & .06 & .13 & .30 & .75 & 1.71 & 3.54 & 4.43 \\
\hline 5579 & -- & -- & -- & -- & .55 & -- & -- & - & -- & 7116 & .02 & .02 & .04 & .09 & .24 & .59 & 1.10 & 2.57 & 3.48 \\
\hline 5580 & -- & -- & .05 & .10 & .44 & .86 & 1.48 & -- & -- & 7140 & .01 & .02 & .05 & .10 & .28 & .80 & 1.78 & 4.30 & 5.51 \\
\hline 5589 & .01 & .01 & .02 & .05 & .16 & .41 & .71 & 1.38 & 1.47 & 7173 & .01 & .01 & .02 & .05 & .21 & .78 & 1.87 & 4.91 & 6.55 \\
\hline 5590 & .02 & .02 & .04 & .07 & .17 & .38 & .90 & 1.87 & 2.99 & 7174 & .01 & .01 & .02 & .05 & .25 & .79 & 1.72 & 4.16 & 5.61 \\
\hline 5591 & .02 & .02 & .05 & .10 & .20 & .49 & .85 & 1.49 & 1.76 & 7213 & .02 & .02 & .05 & .11 & .34 & .82 & 1.62 & 3.50 & 4.30 \\
\hline 5592 & .02 & .02 & .04 & .07 & .17 & .40 & .70 & 1.71 & 2.02 & 7243 & .02 & .03 & .10 & .10 & .27 & .70 & 1.30 & 2.70 & 3.70 \\
\hline 5594 & .02 & .02 & .04 & .07 & .16 & .40 & .78 & 1.82 & 2.22 & 7262 & .01 & .01 & .02 & .04 & .10 & .26 & .53 & 1.03 & 1.11 \\
\hline 5595 & -- & -- & -- & -- & .03 & -- & -- & -- & -- & 7274 & .03 & .04 & .10 & .16 & .39 & .86 & 1.49 & 3.59 & 4.30 \\
\hline 5596 & .10 & .10 & .10 & .10 & .20 & .40 & .90 & 1.90 & 2.25 & 7300 & .02 & .03 & .05 & .10 & .30 & .78 & 1.42 & 2.98 & 3.51 \\
\hline 5600 & .01 & .02 & .04 & .09 & .20 & .43 & .85 & 2.06 & 2.66 & 7311 & -- & -- & .10 & .19 & .29 & .77 & 1.77 & -- & -- \\
\hline 5618 & -- & -- & .07 & .11 & .33 & .88 & 1.77 & -- & -- & 7363 & -- & -- & .04 & .09 & .45 & .94 & 1.48 & -- & -- \\
\hline 5650 & -- & -- & .08 & .20 & .45 & .64 & 1.59 & -- & -- & 7422 & .02 & .02 & .06 & .10 & .28 & .71 & 1.50 & 3.28 & 4.20 \\
\hline 5656 & .06 & .10 & .10 & .10 & .20 & .60 & 1.10 & 2.62 & 3.21 & 7431 & .02 & .03 & .05 & .10 & .20 & .54 & 1.05 & 2.33 & 3.01 \\
\hline 5658 & .02 & .02 & .05 & .09 & .23 & .60 & 1.15 & 2.45 & 3.51 & 7481 & .02 & .02 & .05 & .10 & .15 & .40 & .86 & 2.20 & 3.00 \\
\hline 5661 & .10 & .10 & .10 & .10 & .20 & .60 & 1.30 & 5.62 & 6.99 & 7497 & .10 & .10 & .10 & .10 & .30 & .80 & 1.50 & 3.24 & 4.09 \\
\hline 5666 & -- & -- & .05 & .09 & .29 & .60 & 1.34 & -- & -- & 7498 & .07 & .10 & .10 & .10 & .30 & .80 & 1.50 & 2.72 & 5.31 \\
\hline 5695 & .02 & .03 & .06 & .12 & .40 & .90 & 1.60 & 3.40 & 4.15 & 7499 & .04 & .06 & .10 & .10 & .30 & .73 & 1.40 & 2.70 & 3.70 \\
\hline 5742 & -- & -- & .04 & .05 & .18 & .38 & .75 & -- & -- & 7531 & .03 & .03 & .05 & .11 & .35 & .80 & 1.60 & 2.99 & 3.53 \\
\hline 5766 & -- & -- & .06 & .14 & .31 & 1.11 & 2.69 & -- & -- & 7534 & .02 & .03 & .05 & .10 & .27 & .75 & 1.43 & 3.29 & 3.98 \\
\hline 5770 & .02 & .03 & .07 & .10 & .21 & .60 & 1.20 & 2.50 & 3.50 & 7556 & .03 & .04 & .10 & .10 & .30 & .80 & 1.50 & 3.14 & 4.17 \\
\hline 5775 & -- & -- & .05 & .06 & .20 & .71 & .94 & - & -- & 7594 & .02 & .03 & .06 & .13 & .38 & .93 & 1.83 & 3.57 & 4.67 \\
\hline 5779 & -- & -- & .13 & .19 & .48 & .93 & 2.50 & -- & -- & 7596 & .01 & .02 & .04 & .09 & .35 & 1.09 & 1.93 & 3.40 & 4.04 \\
\hline 5840 & .02 & .03 & .05 & .10 & .34 & .80 & 1.75 & 3.07 & 4.43 & 7608 & .01 & .01 & .03 & .07 & .25 & .75 & 1.51 & 3.00 & 3.76 \\
\hline 5890 & .01 & .01 & .01 & .04 & .13 & .39 & .93 & 2.14 & 2.63 & 7622 & -- & -- & .01 & .01 & .04 & .18 & .71 & -- & -- \\
\hline 5891 & .02 & .02 & .05 & .10 & .23 & .55 & .95 & 1.85 & 2.27 & 7700 & .02 & .02 & .04 & .10 & .32 & .85 & 1.76 & 3.52 & 4.30 \\
\hline 5897 & .10 & .10 & .10 & .10 & .30 & .80 & 1.60 & 3.49 & 4.10 & 7706 & .02 & .02 & .06 & .10 & .21 & .60 & 1.26 & 2.70 & 3.60 \\
\hline
\end{tabular}


Appendix 4-4.3. Empirical distribution of storm depth defined by 12-hour minimum interevent time for hourly rainfall stations in TexasContinued.

\begin{tabular}{|c|c|c|c|c|c|c|c|c|c|c|c|c|c|c|c|c|c|c|c|}
\hline \multicolumn{20}{|c|}{$\begin{array}{c}\text { Depth } \\
\text { (inches) }\end{array}$} \\
\hline $\begin{array}{l}\text { Sta- } \\
\text { tion } \\
\text { no. }\end{array}$ & $\begin{array}{c}1 \text { st } \\
\text { per- } \\
\text { centile }\end{array}$ & $\begin{array}{c}\text { 2nd } \\
\text { per- } \\
\text { centile }\end{array}$ & $\begin{array}{c}10 \text { th } \\
\text { per- } \\
\text { centile }\end{array}$ & $\begin{array}{c}25 \text { th } \\
\text { per- } \\
\text { centile }\end{array}$ & $\begin{array}{c}\text { 50th } \\
\text { per- } \\
\text { centile } \\
\text { (median) }\end{array}$ & $\begin{array}{c}\text { 75th } \\
\text { per- } \\
\text { centile }\end{array}$ & $\begin{array}{c}\text { 90th } \\
\text { per- } \\
\text { centile }\end{array}$ & $\begin{array}{c}\text { 98th } \\
\text { per- } \\
\text { centile }\end{array}$ & $\begin{array}{c}\text { 99th } \\
\text { per- } \\
\text { centile }\end{array}$ & $\begin{array}{l}\text { Sta- } \\
\text { tion } \\
\text { no. }\end{array}$ & $\begin{array}{c}\text { 1st } \\
\text { per- } \\
\text { centile }\end{array}$ & $\begin{array}{c}\text { 2nd } \\
\text { per- } \\
\text { centile }\end{array}$ & $\begin{array}{c}10 \text { th } \\
\text { per- } \\
\text { centile }\end{array}$ & $\begin{array}{c}25 \text { th } \\
\text { per- } \\
\text { centile }\end{array}$ & $\begin{array}{c}\text { 50th } \\
\text { per- } \\
\text { centile } \\
\text { (median) }\end{array}$ & $\begin{array}{c}\text { 75th } \\
\text { per- } \\
\text { centile }\end{array}$ & $\begin{array}{c}\text { 90th } \\
\text { per- } \\
\text { centile }\end{array}$ & $\begin{array}{c}\text { 98th } \\
\text { per- } \\
\text { centile }\end{array}$ & $\begin{array}{c}\text { 99th } \\
\text { per- } \\
\text { centile }\end{array}$ \\
\hline 7718 & 0.02 & 0.03 & 0.06 & 0.11 & 0.29 & 0.69 & 1.75 & 3.40 & 4.66 & 8910 & -- & -- & 0.04 & 0.12 & 0.42 & 1.19 & 1.93 & -- & -- \\
\hline 7745 & .01 & .02 & .05 & .10 & .38 & .94 & 1.81 & 3.95 & 5.77 & 8911 & 0.01 & 0.01 & .03 & .07 & .22 & .67 & 1.38 & 3.04 & 3.57 \\
\hline 7922 & .01 & .01 & .02 & .05 & .14 & .30 & .65 & 1.19 & 1.93 & 8924 & .02 & .02 & .05 & .09 & .19 & .39 & .80 & 1.45 & 1.79 \\
\hline 7936 & .03 & .04 & .10 & .10 & .40 & .97 & 1.80 & 3.70 & 5.24 & 8929 & -- & -- & .06 & .14 & .58 & 1.74 & 2.67 & -- & -- \\
\hline 7943 & .01 & .01 & .02 & .04 & .15 & .47 & 1.10 & 2.31 & 3.00 & 8942 & .03 & .06 & .10 & .10 & .40 & .90 & 1.80 & 4.00 & 4.90 \\
\hline 7944 & -- & .01 & .01 & .03 & .14 & .43 & 1.40 & 2.66 & -- & 8944 & .01 & .02 & .03 & .09 & .28 & .80 & 1.59 & 3.26 & 4.22 \\
\hline 7945 & .01 & .01 & .01 & .03 & .14 & .54 & 1.20 & 3.14 & 4.29 & 8996 & .01 & .02 & .04 & .09 & .28 & .76 & 1.57 & 3.23 & 4.08 \\
\hline 7947 & .04 & .05 & .10 & .10 & .30 & .80 & 1.59 & 4.10 & 5.22 & 9014 & -- & -- & .11 & .23 & .50 & .99 & 1.85 & -- & -- \\
\hline 7948 & .01 & .02 & .04 & .08 & .23 & .70 & 1.51 & 3.29 & 4.18 & 9037 & .02 & .02 & .04 & .07 & .16 & .38 & .81 & 1.68 & 2.34 \\
\hline 7951 & .02 & .03 & .06 & .15 & .38 & .91 & 1.65 & 3.04 & 3.51 & 9106 & .02 & .02 & .03 & .05 & .13 & .37 & .72 & 2.23 & 2.49 \\
\hline 7953 & .02 & .02 & .03 & .07 & .20 & .61 & 1.31 & 3.01 & 3.39 & 9107 & -- & -- & .03 & .05 & .18 & .70 & .88 & -- & -- \\
\hline 7981 & .02 & .02 & .04 & .07 & .25 & .67 & 1.66 & 3.00 & 3.56 & 9129 & .01 & .02 & .03 & .05 & .12 & .42 & 1.14 & 3.06 & 3.36 \\
\hline 7990 & .01 & .01 & .02 & .05 & .17 & .53 & 1.37 & 3.77 & 4.70 & 9163 & .02 & .03 & .07 & .10 & .30 & .70 & 1.39 & 2.90 & 3.60 \\
\hline 7992 & -- & -- & .10 & .11 & .40 & 1.39 & 2.03 & -- & -- & 9213 & .01 & .01 & .02 & .07 & .27 & .71 & 1.52 & 3.00 & 4.75 \\
\hline 7997 & .01 & .02 & .04 & .08 & .23 & .57 & 1.51 & 2.68 & 3.10 & 9214 & -- & -- & .13 & .25 & .38 & 1.34 & 1.89 & -- & -- \\
\hline 7999 & -- & -- & .02 & .03 & .16 & .32 & .93 & -- & -- & 9222 & .01 & .01 & .03 & .07 & .23 & .76 & 1.51 & 3.09 & 4.44 \\
\hline 8022 & -- & .01 & .05 & .05 & .19 & .67 & 1.35 & 2.48 & -- & 9248 & .02 & .03 & .05 & .10 & .23 & .61 & 1.20 & 2.11 & 2.94 \\
\hline 8023 & .02 & .03 & .07 & .10 & .20 & .58 & 1.15 & 2.51 & 3.65 & 9266 & -- & .03 & .05 & .12 & .26 & .81 & 1.44 & 2.71 & -- \\
\hline 8047 & .02 & .03 & .07 & .10 & .30 & .80 & 1.50 & 2.83 & 3.54 & 9270 & .03 & .03 & .10 & .10 & .19 & .40 & .81 & 1.70 & 2.20 \\
\hline 8060 & .01 & .02 & .03 & .05 & .15 & .49 & 1.41 & 3.11 & 3.24 & 9295 & .01 & .01 & .02 & .04 & .10 & .27 & .50 & 1.40 & 2.17 \\
\hline 8062 & -- & -- & .02 & .04 & .16 & .72 & 2.60 & -- & -- & 9304 & -- & -- & -- & .10 & .17 & .26 & -- & -- & -- \\
\hline 8068 & -- & -- & .02 & .03 & .11 & .33 & 1.00 & -- & -- & 9307 & .03 & .04 & .10 & .14 & .34 & .72 & 1.43 & 3.19 & 3.41 \\
\hline 8081 & .02 & .03 & .05 & .10 & .22 & .70 & 1.44 & 3.26 & 4.74 & 9328 & .02 & .02 & .05 & .08 & .23 & .61 & 1.19 & 2.12 & 2.91 \\
\hline 8089 & -- & -- & .03 & .09 & .23 & .78 & 1.56 & -- & -- & 9329 & -- & -- & .04 & .07 & .21 & 1.02 & 1.24 & -- & -- \\
\hline 8221 & -- & -- & .11 & .33 & .60 & .99 & 2.30 & -- & -- & 9345 & -- & -- & -- & -- & .75 & -- & -- & -- & -- \\
\hline 8252 & .02 & .03 & .06 & .10 & .20 & .59 & 1.10 & 2.37 & 2.95 & 9363 & .01 & .01 & .01 & .05 & .14 & .53 & 1.14 & 2.98 & 3.73 \\
\hline 8265 & .02 & .02 & .05 & .10 & .30 & .81 & 1.74 & 3.92 & 6.41 & 9364 & .01 & .01 & .01 & .04 & .16 & .58 & 1.38 & 3.42 & 4.70 \\
\hline 8289 & -- & .02 & .04 & .15 & .43 & .78 & 1.07 & 1.53 & -- & 9365 & -- & -- & .04 & .08 & .11 & .30 & .75 & -- & -- \\
\hline 8305 & .02 & .03 & .05 & .10 & .15 & .33 & .70 & 1.50 & 2.00 & 9371 & .03 & .04 & .05 & .10 & .28 & .80 & 1.76 & 2.67 & 3.39 \\
\hline 8335 & .02 & .03 & .06 & .14 & .42 & .99 & 1.87 & 3.66 & 4.44 & 9417 & .01 & .02 & .05 & .10 & .30 & .80 & 1.57 & 3.07 & 3.61 \\
\hline 8400 & .02 & .02 & .04 & .08 & .20 & .41 & .84 & 2.42 & 3.37 & 9419 & .01 & .01 & .02 & .05 & .21 & .66 & 1.42 & 2.78 & 3.44 \\
\hline 8445 & .02 & .02 & .04 & .09 & .27 & .78 & 1.70 & 3.48 & 4.43 & 9435 & -- & .03 & .06 & .10 & .25 & .63 & .99 & 3.68 & -- \\
\hline 8446 & .02 & .03 & .10 & .10 & .30 & .70 & 1.60 & 2.99 & 3.77 & 9491 & .02 & .03 & .06 & .10 & .30 & .82 & 1.65 & 3.20 & 4.28 \\
\hline 8451 & .01 & .02 & .05 & .09 & .23 & .63 & 1.14 & 3.03 & 3.30 & 9499 & .02 & .04 & .10 & .10 & .20 & .61 & 1.30 & 2.60 & 3.30 \\
\hline 8531 & .02 & .03 & .06 & .12 & .33 & .83 & 1.48 & 3.00 & 3.76 & 9522 & -- & -- & .05 & .20 & .42 & 2.33 & 3.07 & -- & -- \\
\hline 8541 & .01 & .01 & .05 & .11 & .34 & .79 & 1.66 & 3.86 & 4.02 & 9527 & .03 & .04 & .10 & .10 & .20 & .50 & 1.10 & 2.30 & 2.64 \\
\hline 8544 & .03 & .05 & .10 & .10 & .29 & .84 & 1.70 & 3.89 & 5.12 & 9532 & .02 & .02 & .08 & .10 & .30 & .78 & 1.47 & 3.00 & 4.10 \\
\hline 8545 & -- & -- & .02 & .06 & .26 & .49 & .62 & -- & -- & 9544 & -- & -- & -- & .11 & .13 & 2.95 & -- & -- & -- \\
\hline 8563 & .10 & .10 & .10 & .10 & .30 & .90 & 1.60 & 3.60 & 4.35 & 9565 & .02 & .03 & .08 & .10 & .29 & .60 & 1.36 & 2.74 & 3.70 \\
\hline 8566 & .01 & .02 & .04 & .08 & .23 & .64 & 1.24 & 2.39 & 2.71 & 9570 & .10 & .10 & .10 & .10 & .20 & .60 & 1.30 & 2.65 & 3.27 \\
\hline 8583 & .10 & .10 & .10 & .10 & .20 & .70 & 1.33 & 2.59 & 3.04 & 9574 & -- & -- & .10 & .10 & .35 & .72 & 1.65 & -- & -- \\
\hline 8584 & .02 & .03 & .09 & .10 & .30 & .80 & 1.45 & 2.99 & 3.41 & 9588 & .01 & .02 & .05 & .10 & .20 & .56 & 1.32 & 3.12 & 3.88 \\
\hline 8623 & .04 & .05 & .10 & .10 & .30 & .80 & 1.50 & 2.81 & 3.61 & 9665 & .02 & .03 & .08 & .10 & .34 & .90 & 1.66 & 3.53 & 4.60 \\
\hline 8625 & .02 & .02 & .05 & .10 & .29 & .76 & 1.39 & 2.94 & 4.05 & 9715 & .02 & .02 & .07 & .10 & .30 & .80 & 1.53 & 3.00 & 3.80 \\
\hline 8630 & .02 & .03 & .07 & .10 & .23 & .60 & 1.25 & 2.30 & 2.58 & 9729 & .01 & .01 & .02 & .05 & .22 & .66 & 1.32 & 2.77 & 3.46 \\
\hline 8631 & .02 & .03 & .05 & .10 & .26 & .62 & 1.36 & 2.94 & 3.80 & 9772 & .01 & .02 & .04 & .08 & .25 & .75 & 1.54 & 3.94 & 5.01 \\
\hline 8646 & .02 & .02 & .05 & .10 & .30 & .78 & 1.53 & 2.96 & 3.53 & 9814 & -- & -- & .04 & .12 & .40 & .94 & 1.69 & -- & -- \\
\hline 8647 & .03 & .03 & .09 & .10 & .20 & .50 & 1.00 & 2.35 & 2.93 & 9815 & .03 & .03 & .06 & .11 & .30 & .79 & 1.60 & 3.14 & 4.26 \\
\hline 8677 & .02 & .02 & .07 & .14 & .30 & .74 & 1.39 & 2.83 & 3.79 & 9816 & -- & .01 & .04 & .07 & .26 & .77 & 1.79 & 4.37 & -- \\
\hline 8696 & -- & -- & .06 & .16 & .43 & 1.20 & 1.72 & -- & -- & 9817 & .04 & .05 & .10 & .10 & .30 & .76 & 1.30 & 2.90 & 3.48 \\
\hline 8743 & .02 & .03 & .07 & .10 & .38 & .93 & 1.80 & 3.90 & 4.71 & 9829 & .02 & .02 & .05 & .10 & .15 & .40 & .90 & 2.00 & 2.52 \\
\hline 8761 & .02 & .03 & .10 & .10 & .20 & .50 & 1.00 & 2.27 & 3.00 & 9830 & .01 & .01 & .03 & .05 & .11 & .39 & .77 & 1.66 & 2.24 \\
\hline 8778 & .03 & .04 & .10 & .10 & .31 & .90 & 1.68 & 3.43 & 3.98 & 9858 & .02 & .02 & .05 & .10 & .25 & .59 & 1.17 & 2.34 & 2.91 \\
\hline 8845 & .02 & .03 & .08 & .10 & .26 & .74 & 1.60 & 3.43 & 4.49 & 9893 & .02 & .03 & .06 & .10 & .30 & .70 & 1.40 & 2.84 & 3.93 \\
\hline 8859 & .02 & .03 & .06 & .15 & .40 & .99 & 1.72 & 3.33 & 4.10 & 9916 & .03 & .05 & .10 & .13 & .40 & 1.00 & 1.90 & 3.65 & 4.24 \\
\hline 8898 & .02 & .03 & .06 & .13 & .38 & .85 & 1.75 & 3.83 & 4.53 & 9976 & .02 & .02 & .05 & .10 & .20 & .60 & 1.30 & 2.86 & 3.61 \\
\hline 8908 & -- & -- & .04 & .10 & .34 & .85 & 1.64 & -- & -- & & & & & & & & & & \\
\hline
\end{tabular}


Appendix 4-4.4. Empirical distribution of storm depth defined by 18-hour minimum interevent time for hourly rainfall stations in Texas.

$[--$, not available $]$

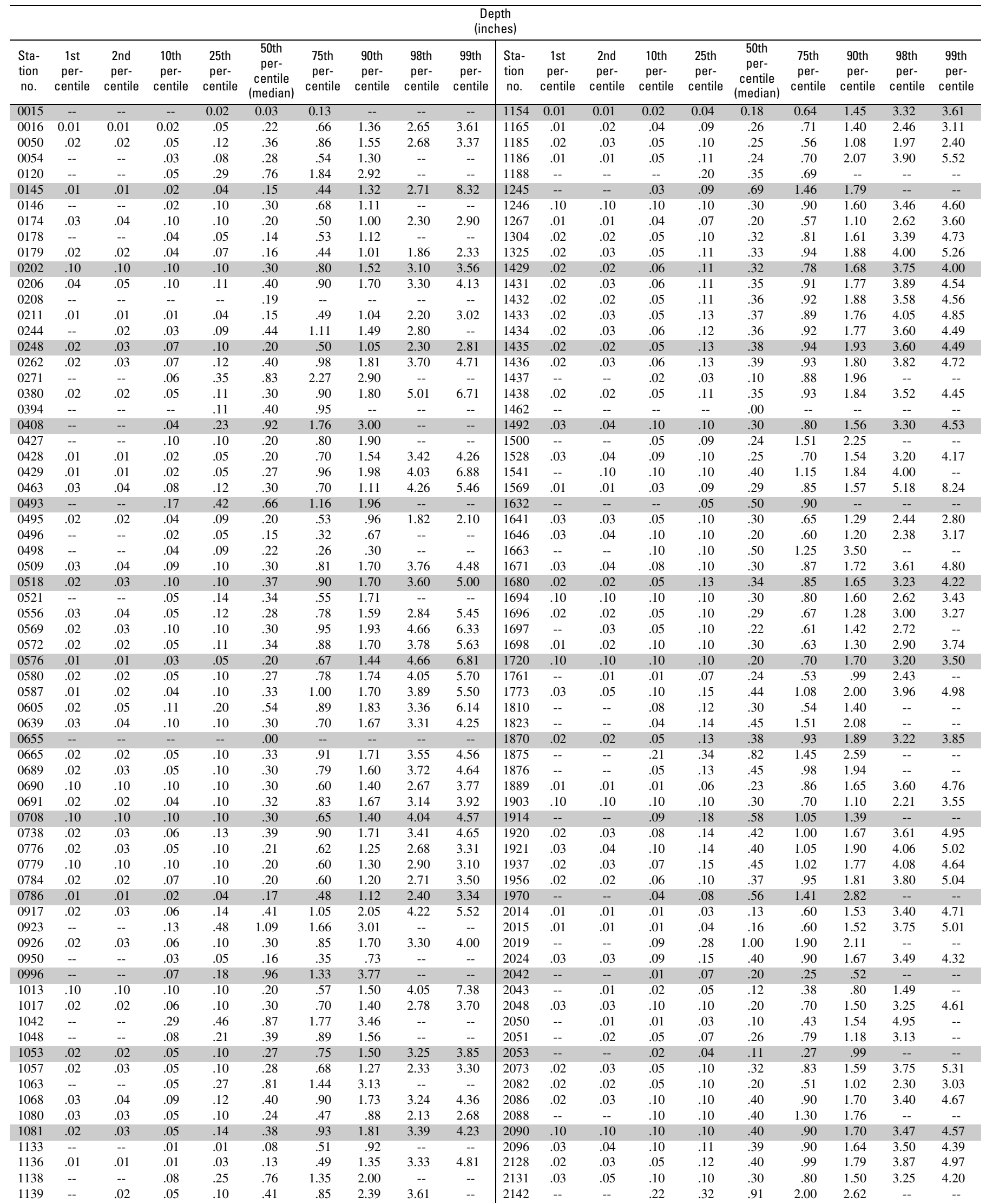


256 Statistical Characteristics of Storm Interevent Time, Depth, and Duration for Eastern New Mexico, Oklahoma, and Texas

Appendix 4-4.4. Empirical distribution of storm depth defined by 18-hour minimum interevent time for hourly rainfall stations in TexasContinued.

\begin{tabular}{|c|c|c|c|c|c|c|c|c|c|c|c|c|c|c|c|c|c|c|c|}
\hline \multicolumn{20}{|c|}{$\begin{array}{c}\text { Depth } \\
\text { (inches) }\end{array}$} \\
\hline $\begin{array}{l}\text { Sta- } \\
\text { tion } \\
\text { no. }\end{array}$ & $\begin{array}{c}\text { 1st } \\
\text { per- } \\
\text { centile }\end{array}$ & $\begin{array}{c}\text { 2nd } \\
\text { per- } \\
\text { centile }\end{array}$ & $\begin{array}{c}10 \text { th } \\
\text { per- } \\
\text { centile }\end{array}$ & $\begin{array}{c}25 \text { th } \\
\text { per- } \\
\text { centile }\end{array}$ & $\begin{array}{c}\text { 50th } \\
\text { per- } \\
\text { centile } \\
\text { (median) }\end{array}$ & $\begin{array}{c}\text { 75th } \\
\text { per- } \\
\text { centile }\end{array}$ & $\begin{array}{c}90 \text { th } \\
\text { per- } \\
\text { centile }\end{array}$ & $\begin{array}{c}\text { 98th } \\
\text { per- } \\
\text { centile }\end{array}$ & $\begin{array}{c}\text { 99th } \\
\text { per- } \\
\text { centile }\end{array}$ & $\begin{array}{l}\text { Sta- } \\
\text { tion } \\
\text { no. }\end{array}$ & $\begin{array}{c}\text { 1st } \\
\text { per- } \\
\text { centile }\end{array}$ & $\begin{array}{c}\text { 2nd } \\
\text { per- } \\
\text { centile }\end{array}$ & $\begin{array}{c}10 \text { th } \\
\text { per- } \\
\text { centile }\end{array}$ & $\begin{array}{c}25 \text { th } \\
\text { per- } \\
\text { centile }\end{array}$ & $\begin{array}{c}\text { 50th } \\
\text { per- } \\
\text { centile } \\
\text { (median) }\end{array}$ & $\begin{array}{c}\text { 75th } \\
\text { per- } \\
\text { centile }\end{array}$ & $\begin{array}{c}\text { 90th } \\
\text { per- } \\
\text { centile }\end{array}$ & $\begin{array}{c}\text { 98th } \\
\text { per- } \\
\text { centile }\end{array}$ & $\begin{array}{c}\text { 99th } \\
\text { per- } \\
\text { centile }\end{array}$ \\
\hline 2160 & -- & -- & 0.02 & 0.04 & 0.14 & 0.35 & 0.97 & -- & -- & 3463 & -- & -- & 0.03 & 0.09 & 0.34 & 0.93 & 2.08 & -- & -- \\
\hline 2206 & 0.02 & 0.03 & .06 & .12 & .38 & .96 & 2.07 & 3.51 & 6.19 & 3476 & 0.02 & 0.02 & .05 & .11 & .31 & .83 & 1.67 & 3.53 & 4.25 \\
\hline 2238 & .01 & .01 & .02 & .04 & .14 & .45 & 1.08 & 2.45 & 2.75 & 3485 & -- & -- & .10 & .10 & .42 & 1.67 & 2.51 & -- & -- \\
\hline 2240 & -- & .01 & .02 & .04 & .20 & .48 & 1.06 & 2.02 & -- & 3507 & .06 & .09 & .10 & .10 & .30 & .90 & 1.80 & 3.60 & 4.32 \\
\hline 2242 & .01 & .01 & .02 & .07 & .30 & .85 & 1.65 & 3.18 & 3.80 & 3546 & .02 & .03 & .08 & .15 & .40 & 1.00 & 1.94 & 3.86 & 5.00 \\
\hline 2244 & .01 & .01 & .03 & .10 & .31 & .85 & 1.73 & 3.65 & 4.64 & 3547 & .02 & .02 & .05 & .12 & .38 & 1.01 & 1.76 & 3.15 & 3.38 \\
\hline 2247 & -- & -- & .02 & .06 & .28 & 1.03 & 1.42 & -- & -- & 3579 & -- & .03 & .08 & .12 & .25 & 1.05 & 1.74 & 3.44 & -- \\
\hline 2309 & .03 & .04 & .10 & .23 & .55 & 1.10 & 1.90 & 3.84 & 5.51 & 3642 & .02 & .03 & .10 & .13 & .40 & .90 & 1.80 & 3.77 & 5.14 \\
\hline 2312 & .07 & .10 & .10 & .20 & .50 & 1.00 & 1.70 & 3.27 & 4.18 & 3646 & .03 & .03 & .06 & .13 & .36 & .86 & 1.61 & 3.62 & 4.72 \\
\hline 2334 & -- & .03 & .09 & .25 & .62 & 1.28 & 3.06 & 4.66 & -- & 3668 & -- & -- & .36 & .69 & 1.03 & 2.06 & 3.38 & -- & -- \\
\hline 2336 & .02 & .03 & .07 & .17 & .42 & 1.00 & 1.56 & 3.10 & 3.69 & 3673 & -- & -- & .04 & .14 & .88 & 1.75 & 2.42 & -- & -- \\
\hline 2354 & -- & -- & .05 & .09 & .14 & .59 & 1.09 & -- & -- & 3686 & .04 & .07 & .10 & .10 & .32 & .90 & 1.74 & 3.18 & 3.70 \\
\hline 2355 & -- & -- & .03 & .05 & .26 & .87 & 1.95 & -- & -- & 3691 & .02 & .03 & .10 & .10 & .33 & .90 & 1.64 & 3.28 & 4.57 \\
\hline 2357 & .01 & .01 & .01 & .02 & .10 & .48 & 1.21 & 3.16 & 4.69 & 3734 & -- & -- & .03 & .15 & .80 & 3.22 & 4.03 & -- & -- \\
\hline 2360 & .01 & .01 & .01 & .04 & .14 & .52 & 1.24 & 2.82 & 3.68 & 3771 & .10 & .10 & .10 & .10 & .30 & .90 & 1.70 & 3.70 & 4.30 \\
\hline 2361 & .02 & .02 & .05 & .10 & .20 & .60 & 1.09 & 2.86 & 8.63 & 3789 & -- & .01 & .02 & .07 & .13 & .30 & .57 & 1.29 & -- \\
\hline 2394 & .02 & .02 & .05 & .11 & .37 & .90 & 1.73 & 3.70 & 4.99 & 3826 & .01 & .03 & .06 & .15 & .35 & .85 & 1.46 & 2.66 & 3.44 \\
\hline 2404 & .02 & .02 & .05 & .10 & .33 & .88 & 1.63 & 3.40 & 4.60 & 3831 & .03 & .03 & .05 & .15 & .39 & 1.02 & 2.06 & 3.32 & 5.17 \\
\hline 2415 & .02 & .03 & .08 & .17 & .47 & 1.09 & 2.05 & 4.02 & 5.21 & 3841 & .02 & .02 & .05 & .15 & .32 & .97 & 1.74 & 4.06 & 5.48 \\
\hline 2462 & .03 & .04 & .09 & .15 & .43 & 1.00 & 1.90 & 3.76 & 4.70 & 3871 & .02 & .03 & .05 & .11 & .34 & .77 & 1.46 & 3.04 & 3.79 \\
\hline 2528 & .03 & .03 & .05 & .10 & .25 & .86 & 1.90 & 2.69 & 2.79 & 3884 & -- & -- & .06 & .09 & .30 & 1.44 & 3.30 & -- & -- \\
\hline 2617 & .02 & .02 & .05 & .10 & .27 & .64 & 1.34 & 3.10 & 4.11 & 3941 & -- & .02 & .05 & .11 & .70 & 1.43 & 2.16 & 4.84 & -- \\
\hline 2619 & .02 & .03 & .06 & .15 & .30 & .71 & 1.16 & 2.77 & 3.86 & 3963 & -- & -- & -- & .03 & .04 & .14 & -- & -- & -- \\
\hline 2621 & .02 & .03 & .06 & .10 & .30 & .69 & 1.39 & 2.77 & 3.30 & 4040 & .02 & .03 & .06 & .12 & .34 & .75 & 1.37 & 3.14 & 3.82 \\
\hline 2675 & .03 & .04 & .10 & .10 & .30 & .85 & 1.70 & 3.94 & 4.87 & 4058 & -- & -- & .06 & .13 & .56 & 1.39 & 2.87 & -- & -- \\
\hline 2676 & .10 & .10 & .10 & .10 & .30 & .90 & 1.88 & 3.36 & 4.13 & 4098 & .03 & .04 & .10 & .10 & .20 & .50 & 1.00 & 2.10 & 2.65 \\
\hline 2679 & .02 & .02 & .05 & .10 & .20 & .65 & 1.50 & 3.50 & 4.48 & 4100 & .02 & .02 & .05 & .10 & .28 & .68 & 1.42 & 3.46 & 4.88 \\
\hline 2715 & .02 & .03 & .07 & .12 & .36 & .83 & 1.60 & 3.31 & 4.29 & 4137 & .10 & .10 & .10 & .10 & .30 & .90 & 1.60 & 3.21 & 4.00 \\
\hline 2744 & .03 & .03 & .07 & .10 & .30 & .75 & 1.36 & 2.98 & 3.69 & 4191 & .03 & .04 & .07 & .10 & .28 & .80 & 1.60 & 3.50 & 4.85 \\
\hline 2758 & .01 & .02 & .03 & .05 & .15 & .49 & 1.53 & 4.31 & 4.79 & 4256 & -- & -- & -- & -- & .00 & -- & -- & -- & -- \\
\hline 2794 & -- & -- & .01 & .04 & .16 & .66 & 1.61 & -- & -- & 4257 & .04 & .05 & .10 & .15 & .47 & 1.09 & 2.02 & 4.15 & 5.00 \\
\hline 2797 & .01 & .01 & .01 & .03 & .10 & .28 & .58 & 1.42 & 1.79 & 4258 & .10 & .10 & .10 & .10 & .40 & .90 & 1.90 & 3.35 & 5.92 \\
\hline 2811 & .02 & .02 & .07 & .10 & .24 & .70 & 1.40 & 3.01 & 3.60 & 4278 & .02 & .02 & .05 & .12 & .34 & .91 & 1.75 & 3.47 & 4.27 \\
\hline 2813 & -- & -- & .06 & .23 & .41 & .80 & 2.16 & -- & -- & 4299 & .02 & .03 & .06 & .11 & .26 & .51 & .84 & 1.24 & 1.48 \\
\hline 2814 & -- & -- & .01 & .03 & .04 & .25 & .99 & -- & -- & 4300 & .01 & .01 & .02 & .06 & .24 & .80 & 1.73 & 4.02 & 5.39 \\
\hline 2815 & .10 & .10 & .10 & .10 & .30 & .60 & 1.39 & 3.18 & 4.51 & 4305 & .01 & .01 & .02 & .05 & .22 & .76 & 1.62 & 3.53 & 5.16 \\
\hline 2818 & .02 & .02 & .05 & .11 & .35 & .79 & 1.49 & 3.82 & 4.79 & 4307 & .01 & .01 & .02 & .05 & .29 & .83 & 1.77 & 4.45 & 7.11 \\
\hline 2986 & .02 & .03 & .08 & .18 & .55 & 1.35 & 2.19 & 3.63 & 4.31 & 4309 & .02 & .02 & .05 & .11 & .35 & .97 & 1.92 & 4.01 & 5.17 \\
\hline 3005 & .02 & .03 & .10 & .10 & .35 & .89 & 1.51 & 3.11 & 3.90 & 4311 & .02 & .02 & .05 & .13 & .39 & 1.05 & 2.04 & 4.16 & 5.28 \\
\hline 3033 & .02 & .02 & .04 & .08 & .15 & .33 & .68 & 1.43 & 1.97 & 4313 & .02 & .03 & .05 & .16 & .40 & 1.08 & 2.30 & 4.23 & 7.48 \\
\hline 3034 & -- & -- & -- & -- & .43 & -- & -- & -- & -- & 4319 & -- & .04 & .07 & .14 & .38 & .86 & 1.39 & 3.41 & -- \\
\hline 3047 & -- & -- & .04 & .10 & .53 & 1.06 & 2.30 & -- & -- & 4329 & .02 & .03 & .06 & .14 & .39 & .98 & 1.94 & 4.15 & 5.34 \\
\hline 3103 & -- & -- & .13 & .19 & .29 & 1.04 & 2.44 & -- & -- & 4331 & -- & -- & -- & -- & .00 & -- & -- & -- & -- \\
\hline 3133 & .02 & .03 & .08 & .15 & .42 & .96 & 1.80 & 3.78 & 4.73 & 4375 & .10 & .10 & .10 & .10 & .30 & .80 & 1.70 & 3.85 & 5.09 \\
\hline 3156 & .04 & .05 & .10 & .10 & .41 & 1.00 & 2.18 & 5.88 & 7.05 & 4392 & .02 & .02 & .05 & .15 & .45 & 1.12 & 2.23 & 4.78 & 7.22 \\
\hline 3171 & .03 & .03 & .07 & .13 & .38 & .92 & 1.76 & 3.49 & 4.68 & 4425 & .03 & .04 & .09 & .10 & .20 & .57 & 1.12 & 2.31 & 2.90 \\
\hline 3189 & .02 & .02 & .04 & .09 & .17 & .43 & .90 & 2.23 & 3.16 & 4440 & .02 & .02 & .05 & .10 & .30 & .75 & 1.44 & 3.28 & 3.93 \\
\hline 3260 & .01 & .01 & .04 & .10 & .31 & .81 & 1.59 & 2.57 & 4.02 & 4476 & .03 & .05 & .10 & .10 & .36 & .83 & 1.54 & 3.20 & 4.11 \\
\hline 3267 & .01 & .02 & .03 & .05 & .17 & .55 & 1.14 & 3.89 & 5.35 & 4498 & -- & -- & .02 & .06 & .17 & .38 & .60 & -- & -- \\
\hline 3270 & .05 & .10 & .10 & .10 & .30 & .70 & 1.30 & 2.54 & 3.60 & 4517 & .02 & .02 & .05 & .12 & .35 & .85 & 1.67 & 3.34 & 3.77 \\
\hline 3272 & -- & -- & .02 & .04 & .12 & .22 & .35 & -- & -- & 4520 & .10 & .10 & .10 & .10 & .30 & .80 & 1.60 & 3.30 & 3.76 \\
\hline 3277 & -- & -- & .01 & .02 & .10 & .23 & .64 & -- & -- & 4525 & -- & -- & .04 & .07 & .27 & .81 & 4.26 & -- & -- \\
\hline 3278 & .02 & .03 & .05 & .10 & .22 & .60 & 1.17 & 2.39 & 3.30 & 4563 & -- & -- & .04 & .06 & .28 & .62 & 1.89 & -- & -- \\
\hline 3280 & .01 & .01 & .03 & .05 & .16 & .41 & .90 & 1.92 & 2.56 & 4570 & .02 & .03 & .08 & .10 & .30 & .70 & 1.40 & 2.90 & 4.10 \\
\hline 3281 & -- & -- & .04 & .10 & .25 & .51 & .99 & -- & -- & 4577 & .03 & .04 & .10 & .17 & .45 & 1.00 & 1.90 & 3.81 & 4.70 \\
\hline 3283 & .01 & .01 & .02 & .07 & .27 & .77 & 1.62 & 3.42 & 4.57 & 4591 & .02 & .03 & .05 & .12 & .39 & .93 & 1.76 & 3.55 & 4.67 \\
\hline 3284 & .01 & .02 & .10 & .10 & .33 & .86 & 1.70 & 3.39 & 4.10 & 4670 & .02 & .02 & .06 & .10 & .29 & .70 & 1.40 & 2.82 & 3.50 \\
\hline 3285 & .03 & .08 & .10 & .10 & .37 & .90 & 1.72 & 3.25 & 4.10 & 4671 & .01 & .01 & .03 & .06 & .27 & .61 & 1.15 & 2.86 & 3.14 \\
\hline 3329 & .01 & .02 & .05 & .10 & .27 & .75 & 1.57 & 3.54 & 4.86 & 4679 & .03 & .04 & .10 & .10 & .30 & .84 & 1.70 & 3.80 & 4.91 \\
\hline 3335 & .02 & .03 & .07 & .20 & .43 & 1.19 & 2.04 & 4.77 & 8.98 & 4696 & -- & -- & .05 & .10 & .28 & .63 & 1.10 & -- & -- \\
\hline 3370 & .02 & .03 & .06 & .15 & .42 & .99 & 1.85 & 3.77 & 4.71 & 4703 & .02 & .02 & .05 & .10 & .20 & .68 & 1.34 & 3.01 & 3.46 \\
\hline 3410 & .04 & .05 & .10 & .10 & .30 & .70 & 1.30 & 2.71 & 3.30 & 4704 & .02 & .03 & .06 & .16 & .40 & 1.06 & 2.55 & 4.64 & 6.58 \\
\hline 3415 & .02 & .03 & .10 & .10 & .37 & .85 & 1.70 & 3.30 & 4.55 & 4731 & .01 & .01 & .01 & .04 & .17 & .53 & 1.14 & 3.45 & 7.60 \\
\hline 3430 & .01 & .01 & .02 & .06 & .25 & .77 & 1.68 & 3.98 & 5.79 & 4792 & .10 & .10 & .10 & .10 & .40 & .90 & 1.70 & 3.00 & 3.80 \\
\hline 3431 & .01 & .01 & .02 & .05 & .21 & .77 & 1.50 & 4.86 & 6.32 & 4819 & .10 & .10 & .10 & .20 & .40 & 1.00 & 1.92 & 3.80 & 4.14 \\
\hline 3441 & -- & -- & .05 & .10 & .30 & .79 & 2.02 & -- & -- & 4852 & -- & -- & .15 & .35 & .69 & 1.10 & 2.55 & -- & -- \\
\hline 3442 & .02 & .03 & .05 & .09 & .21 & .49 & 1.11 & 2.25 & 2.69 & 4866 & .03 & .04 & .09 & .13 & .40 & .91 & 1.79 & 3.40 & 4.06 \\
\hline 3446 & .03 & .03 & .06 & .11 & .30 & .66 & 1.17 & 2.28 & 3.01 & 4876 & .10 & .10 & .10 & .10 & .40 & 1.12 & 2.57 & 4.61 & 6.62 \\
\hline 3460 & -- & -- & .05 & .10 & .55 & 1.15 & 1.64 & -- & -- & 4878 & .02 & .03 & .07 & .15 & .43 & 1.10 & 2.17 & 4.55 & 5.74 \\
\hline 3462 & .02 & .04 & .07 & .12 & .32 & .75 & 1.20 & 2.31 & 4.29 & 4880 & .02 & .03 & .06 & .10 & .25 & .60 & 1.17 & 2.50 & 3.11 \\
\hline
\end{tabular}


Appendix 4-4.4. Empirical distribution of storm depth defined by 18-hour minimum interevent time for hourly rainfall stations in TexasContinued.

\begin{tabular}{|c|c|c|c|c|c|c|c|c|c|c|c|c|c|c|c|c|c|c|c|}
\hline \multicolumn{20}{|c|}{$\begin{array}{c}\text { Depth } \\
\text { (inches) }\end{array}$} \\
\hline $\begin{array}{l}\text { Sta- } \\
\text { tion } \\
\text { no. }\end{array}$ & $\begin{array}{c}1 \text { st } \\
\text { per- } \\
\text { centile }\end{array}$ & $\begin{array}{c}\text { 2nd } \\
\text { per- } \\
\text { centile }\end{array}$ & $\begin{array}{c}10 \text { th } \\
\text { per- } \\
\text { centile }\end{array}$ & $\begin{array}{c}25 \text { th } \\
\text { per- } \\
\text { centile }\end{array}$ & $\begin{array}{c}\text { 50th } \\
\text { per- } \\
\text { centile } \\
\text { (median) }\end{array}$ & $\begin{array}{c}\text { 75th } \\
\text { per- } \\
\text { centile }\end{array}$ & $\begin{array}{c}\text { 90th } \\
\text { per- } \\
\text { centile }\end{array}$ & $\begin{array}{c}\text { 98th } \\
\text { per- } \\
\text { centile }\end{array}$ & $\begin{array}{l}\text { 99th } \\
\text { per- } \\
\text { centile }\end{array}$ & $\begin{array}{l}\text { Sta- } \\
\text { tion } \\
\text { no. }\end{array}$ & $\begin{array}{c}\text { 1st } \\
\text { per- } \\
\text { centile }\end{array}$ & $\begin{array}{c}\text { 2nd } \\
\text { per- } \\
\text { centile }\end{array}$ & $\begin{array}{c}\text { 10th } \\
\text { per- } \\
\text { centile }\end{array}$ & $\begin{array}{c}\text { 25th } \\
\text { per- } \\
\text { centile }\end{array}$ & $\begin{array}{c}\text { 50th } \\
\text { per- } \\
\text { centile } \\
\text { (median) }\end{array}$ & $\begin{array}{c}\text { 75th } \\
\text { per- } \\
\text { centile }\end{array}$ & $\begin{array}{c}\text { 90th } \\
\text { per- } \\
\text { centile }\end{array}$ & $\begin{array}{c}\text { 98th } \\
\text { per- } \\
\text { centile }\end{array}$ & $\begin{array}{c}\text { 99th } \\
\text { per- } \\
\text { centile }\end{array}$ \\
\hline 4920 & 0.02 & 0.03 & 0.08 & 0.10 & 0.30 & 0.80 & 1.53 & 3.49 & 4.50 & 5957 & 0.03 & 0.04 & 0.10 & 0.10 & 0.30 & 0.88 & 1.60 & 3.30 & 4.56 \\
\hline 4934 & -- & -- & -- & .08 & .15 & .36 & -- & - & -- & 5958 & .01 & .01 & .05 & .10 & .34 & .90 & 1.68 & 2.76 & 3.37 \\
\hline 4972 & .03 & .03 & .09 & .10 & .30 & .80 & 1.50 & 3.22 & 4.22 & 5973 & -- & .02 & .03 & .05 & .17 & .67 & 1.61 & 3.33 & -- \\
\hline 4973 & .02 & .05 & .10 & .23 & .58 & 1.15 & 2.01 & 3.70 & 4.04 & 5996 & .03 & .03 & .09 & .10 & .30 & .83 & 1.60 & 3.20 & 4.18 \\
\hline 4974 & .02 & .02 & .05 & .10 & .26 & .63 & 1.19 & 2.57 & 3.46 & 6017 & .01 & .01 & .03 & .05 & .20 & .59 & 1.25 & 2.60 & 2.93 \\
\hline 4975 & .10 & .10 & .10 & .10 & .40 & 1.00 & 1.90 & 4.00 & 4.90 & 6024 & .02 & .03 & .05 & .13 & .47 & 1.26 & 2.40 & 6.08 & 6.54 \\
\hline 4978 & .02 & .02 & .04 & .10 & .35 & .78 & 1.81 & 3.58 & 5.22 & 6050 & -- & -- & .15 & .24 & .40 & 1.03 & 1.73 & -- & -- \\
\hline 4979 & -- & -- & .09 & .34 & .65 & 1.31 & 2.98 & -- & -- & 6104 & .02 & .03 & .05 & .10 & .20 & .48 & .90 & 2.11 & 2.83 \\
\hline 4982 & .02 & .03 & .05 & .11 & .30 & .75 & 1.41 & 2.83 & 3.55 & 6108 & .02 & .04 & .10 & .18 & .50 & 1.10 & 2.00 & 4.31 & 5.10 \\
\hline 5018 & .02 & .03 & .06 & .13 & .38 & .90 & 1.55 & 2.65 & 3.53 & 6136 & .03 & .04 & .10 & .10 & .20 & .52 & 1.00 & 2.24 & 2.76 \\
\hline 5048 & .02 & .03 & .09 & .10 & .20 & .60 & 1.20 & 2.72 & 3.63 & 6166 & -- & .03 & .05 & .07 & .20 & .53 & 1.21 & 2.22 & -- \\
\hline 5049 & -- & .10 & .10 & .10 & .20 & .50 & .90 & 4.41 & -- & 6176 & .02 & .02 & .05 & .14 & .44 & .91 & 2.10 & 4.15 & 6.27 \\
\hline 5056 & -- & -- & -- & .20 & .37 & .69 & -- & -- & -- & 6177 & .03 & .04 & .10 & .15 & .40 & 1.00 & 2.00 & 4.09 & 5.30 \\
\hline 5057 & .01 & .01 & .01 & .03 & .11 & .39 & 1.10 & 2.81 & 3.28 & 6210 & .02 & .03 & .10 & .10 & .40 & .97 & 1.80 & 4.00 & 4.60 \\
\hline 5060 & .01 & .01 & .02 & .05 & .17 & .64 & 1.64 & 5.26 & 6.39 & 6211 & .01 & .01 & .05 & .10 & .31 & .90 & 1.85 & 3.70 & 4.28 \\
\hline 5081 & .02 & .02 & .05 & .14 & .45 & 1.00 & 1.80 & 3.73 & 4.18 & 6270 & .10 & .10 & .10 & .20 & .50 & 1.10 & 2.00 & 4.08 & 4.89 \\
\hline 5094 & .02 & .03 & .10 & .10 & .40 & .90 & 1.80 & 3.70 & 4.31 & 6275 & -- & -- & -- & -- & .00 & -- & -- & -- & -- \\
\hline 5113 & .02 & .03 & .08 & .10 & .29 & .75 & 1.62 & 3.80 & 5.10 & 6276 & -- & -- & .06 & .18 & .91 & 1.73 & 2.75 & -- & -- \\
\hline 5114 & -- & -- & -- & -- & .00 & -- & -- & -- & -- & 6335 & .02 & .03 & .06 & .14 & .40 & .98 & 1.76 & 3.35 & 4.27 \\
\hline 5123 & -- & -- & .07 & .18 & .29 & .55 & 2.45 & -- & -- & 6434 & -- & -- & .09 & .13 & .45 & 1.00 & 1.73 & -- & -- \\
\hline 5192 & .02 & .03 & .08 & .13 & .39 & .94 & 1.85 & 3.51 & 4.58 & 6504 & .02 & .03 & .08 & .10 & .20 & .60 & 1.20 & 2.73 & 3.30 \\
\hline 5193 & .02 & .03 & .06 & .10 & .30 & .90 & 1.70 & 3.54 & 4.60 & 6558 & -- & -- & .05 & .06 & .38 & .97 & 1.90 & -- & -- \\
\hline 5224 & .01 & .02 & .05 & .14 & .45 & 1.11 & 1.98 & 4.49 & 5.08 & 6615 & .07 & .10 & .10 & .10 & .30 & .70 & 1.40 & 3.53 & 4.72 \\
\hline 5228 & .01 & .02 & .04 & .09 & .32 & .91 & 1.76 & 3.44 & 4.23 & 6660 & -- & .02 & .05 & .15 & .39 & .91 & 1.95 & 4.80 & -- \\
\hline 5235 & -- & -- & .03 & .05 & .36 & .81 & 1.91 & -- & -- & 6663 & -- & .01 & .04 & .10 & .27 & .65 & 1.53 & 3.69 & -- \\
\hline 5247 & .02 & .03 & .09 & .10 & .26 & .60 & 1.20 & 2.40 & 3.10 & 6734 & .01 & .02 & .04 & .10 & .25 & .65 & 1.25 & 2.69 & 3.20 \\
\hline 5258 & .02 & .03 & .06 & .14 & .38 & .90 & 1.80 & 3.84 & 4.57 & 6736 & .02 & .03 & .09 & .10 & .21 & .64 & 1.26 & 3.00 & 3.61 \\
\hline 5303 & .02 & .03 & .05 & .10 & .28 & .76 & 1.49 & 3.55 & 5.05 & 6740 & -- & - & .10 & .32 & .82 & 3.35 & 3.56 & -- & -- \\
\hline 5312 & .04 & .05 & .10 & .10 & .30 & .79 & 1.48 & 2.96 & 4.46 & 6750 & .01 & .01 & .02 & .05 & .28 & .87 & 2.12 & 5.56 & 9.47 \\
\hline 5341 & -- & -- & .11 & .27 & .48 & 1.57 & 3.90 & -- & -- & 6757 & .01 & .01 & .05 & .10 & .38 & .97 & 1.80 & 3.60 & 4.69 \\
\hline 5342 & -- & -- & -- & -- & .00 & -- & -- & -- & -- & 6775 & .01 & .01 & .04 & .09 & .25 & .63 & 1.14 & 2.65 & 3.34 \\
\hline 5348 & .03 & .05 & .10 & .14 & .50 & 1.10 & 2.00 & 3.90 & 4.67 & 6776 & .02 & .03 & .09 & .10 & .26 & .60 & 1.15 & 2.46 & 3.10 \\
\hline 5358 & .02 & .02 & .05 & .10 & .29 & .71 & 1.41 & 2.76 & 3.47 & 6788 & .02 & .03 & .06 & .16 & .50 & .93 & 1.80 & 3.99 & 4.62 \\
\hline 5398 & .02 & .03 & .06 & .13 & .40 & .95 & 1.89 & 3.78 & 5.28 & 6792 & .02 & .02 & .06 & .10 & .20 & .47 & .93 & 1.91 & 2.23 \\
\hline 5410 & .02 & .02 & .07 & .10 & .25 & 60 & 1.11 & 2.74 & 3.70 & 6794 & -- & - & .08 & .55 & 1.51 & 2.82 & 3.22 & - & -- \\
\hline 5411 & .01 & .01 & .01 & .04 & .15 & .49 & 1.08 & 2.45 & 3.23 & 6834 & .10 & .10 & .10 & .20 & .50 & 1.10 & 2.06 & 4.00 & 4.90 \\
\hline 5424 & .01 & .01 & .01 & .06 & .34 & .99 & 2.02 & 3.56 & 7.12 & 6893 & .02 & .03 & .08 & .10 & .20 & .40 & .80 & 2.10 & 2.70 \\
\hline 5429 & .02 & .02 & .04 & .09 & .26 & .80 & 1.68 & 3.61 & 4.51 & 6935 & .03 & .04 & .10 & .10 & .20 & .60 & 1.10 & 2.48 & 3.29 \\
\hline 5431 & -- & -- & .14 & .35 & .70 & 1.40 & 3.03 & -- & -- & 6981 & .02 & .04 & .05 & .15 & .40 & .79 & 1.68 & 5.01 & 5.62 \\
\hline 5461 & .02 & .03 & .06 & .15 & .44 & 1.04 & 2.00 & 4.20 & 5.42 & 7020 & .02 & .02 & .05 & .13 & .38 & .99 & 1.92 & 3.87 & 8.06 \\
\hline 5463 & .10 & .10 & .10 & .10 & .40 & 1.00 & 1.90 & 3.88 & 5.46 & 7060 & .03 & .04 & .10 & .10 & .30 & .70 & 1.40 & 2.62 & 3.40 \\
\hline 5471 & -- & -- & .02 & .04 & .07 & .28 & .48 & -- & -- & 7066 & .03 & .03 & .10 & .14 & .45 & 1.06 & 2.00 & 3.93 & 4.93 \\
\hline 5477 & -- & -- & .07 & .15 & .26 & .61 & 1.53 & -- & -- & 7074 & .02 & .02 & .05 & .10 & .20 & .58 & 1.05 & 2.51 & 3.64 \\
\hline 5528 & .03 & .03 & .08 & .17 & .45 & 1.02 & 1.86 & 3.70 & 4.45 & 7097 & .02 & .02 & .08 & .16 & .34 & .83 & 1.75 & 3.58 & 4.51 \\
\hline 5579 & -- & - & -- & - & .55 & -- & -- & -- & -- & 7116 & .02 & .02 & .05 & .10 & .25 & .62 & 1.14 & 2.62 & 3.80 \\
\hline 5580 & -- & -- & .07 & .16 & .44 & .90 & 1.87 & -- & -- & 7140 & .02 & .02 & .05 & .10 & .30 & .89 & 1.97 & 4.72 & 5.98 \\
\hline 5589 & .01 & .01 & .02 & .05 & .18 & .43 & .81 & 1.48 & 1.66 & 7173 & .01 & .01 & .02 & .05 & .27 & .94 & 2.14 & 5.40 & 6.71 \\
\hline 5590 & .02 & .02 & .04 & .08 & .19 & .44 & .94 & 1.92 & 3.10 & 7174 & .01 & .01 & .02 & .06 & .29 & .91 & 1.94 & 4.50 & 5.98 \\
\hline 5591 & .02 & .02 & .05 & .10 & .23 & .50 & .89 & 1.70 & 2.53 & 7213 & .02 & .02 & .05 & .14 & .39 & .92 & 1.85 & 3.71 & 4.50 \\
\hline 5592 & .02 & .02 & .04 & .07 & .18 & .43 & .79 & 1.83 & 2.17 & 7243 & .03 & .04 & .10 & .10 & .30 & .80 & 1.41 & 2.96 & 4.00 \\
\hline 5594 & .02 & .02 & .04 & .08 & .17 & .40 & .78 & 1.90 & 2.23 & 7262 & -- & .01 & .02 & .04 & .12 & .30 & .58 & 1.23 & -- \\
\hline 5595 & -- & - & -- & -- & .03 & -- & -- & -- & -- & 7274 & .04 & .05 & .10 & .19 & .40 & .90 & 1.65 & 3.64 & 4.44 \\
\hline 5596 & .10 & .10 & .10 & .10 & .20 & .50 & 1.00 & 2.10 & 2.67 & 7300 & .02 & .03 & .05 & .11 & .33 & .83 & 1.56 & 3.11 & 3.77 \\
\hline 5600 & .02 & .02 & .04 & .09 & .20 & .48 & .90 & 2.33 & 2.89 & 7311 & -- & -- & .10 & .20 & .29 & 1.05 & 1.88 & -- & -- \\
\hline 5618 & -- & -- & .08 & .15 & .36 & 1.16 & 1.93 & -- & -- & 7363 & -- & -- & .08 & .17 & .71 & 1.25 & 2.11 & -- & -- \\
\hline 5650 & -- & -- & .08 & .20 & .45 & .64 & 1.59 & -- & -- & 7422 & .02 & .02 & .06 & .10 & .30 & .80 & 1.68 & 3.64 & 4.50 \\
\hline 5656 & .10 & .10 & .10 & .10 & .20 & .60 & 1.30 & 2.90 & 3.56 & 7431 & .02 & .03 & .06 & .10 & .21 & .60 & 1.16 & 2.46 & 3.16 \\
\hline 5658 & .02 & .02 & .05 & .10 & .25 & .60 & 1.21 & 3.01 & 4.00 & 7481 & .02 & .02 & .05 & .10 & .17 & .40 & .90 & 2.47 & 3.23 \\
\hline 5661 & .10 & .10 & .10 & .10 & .20 & .60 & 1.50 & 6.51 & 7.24 & 7497 & .10 & .10 & .10 & .10 & .30 & .90 & 1.66 & 3.43 & 4.28 \\
\hline 5666 & -- & -- & .05 & .09 & .29 & .68 & 1.57 & -- & -- & 7498 & .08 & .10 & .10 & .10 & .30 & .90 & 1.63 & 3.26 & 6.06 \\
\hline 5695 & .02 & .04 & .07 & .15 & .42 & .95 & 1.72 & 3.65 & 4.92 & 7499 & .05 & .07 & .10 & .10 & .30 & .80 & 1.52 & 3.11 & 4.02 \\
\hline 5742 & -- & -- & .04 & .05 & .17 & .29 & 1.24 & -- & -- & 7531 & .03 & .03 & .06 & .11 & .36 & 1.02 & 1.92 & 3.52 & 4.06 \\
\hline 5766 & -- & -- & .12 & .30 & .54 & 1.99 & 2.80 & -- & -- & 7534 & .02 & .03 & .05 & .11 & .30 & .85 & 1.57 & 3.48 & 4.41 \\
\hline 5770 & .02 & .03 & .07 & .10 & .26 & .65 & 1.30 & 2.81 & 3.73 & 7556 & .03 & .04 & .10 & .10 & .30 & .85 & 1.60 & 3.50 & 4.89 \\
\hline 5775 & - & - & .05 & .05 & .15 & .73 & .97 & -- & -- & 7594 & .02 & .03 & .06 & .15 & .44 & 1.01 & 1.97 & 3.84 & 5.22 \\
\hline 5779 & -- & -- & .13 & .19 & .48 & .93 & 2.50 & -- & -- & 7596 & .01 & .02 & .04 & .10 & .40 & 1.16 & 2.02 & 3.43 & 4.17 \\
\hline 5840 & .02 & .03 & .05 & .11 & .35 & .85 & 1.75 & 3.33 & 4.74 & 7608 & .01 & .01 & .03 & .08 & .28 & .82 & 1.59 & 3.25 & 4.87 \\
\hline 5890 & .01 & .01 & .02 & .04 & .15 & .44 & 1.02 & 2.25 & 2.85 & 7622 & -- & -- & .01 & .01 & .04 & .23 & .73 & -- & -- \\
\hline 5891 & .02 & .03 & .05 & .10 & .24 & .59 & .99 & 1.86 & 2.30 & 7700 & .02 & .02 & .05 & .11 & .36 & .94 & 1.91 & 3.88 & 4.67 \\
\hline 5897 & .10 & .10 & .10 & .10 & .40 & .90 & 1.80 & 3.70 & 4.30 & 7706 & .02 & .02 & .07 & .10 & .30 & .70 & 1.40 & 2.98 & 4.07 \\
\hline
\end{tabular}


Appendix 4-4.4. Empirical distribution of storm depth defined by 18-hour minimum interevent time for hourly rainfall stations in TexasContinued.

\begin{tabular}{|c|c|c|c|c|c|c|c|c|c|c|c|c|c|c|c|c|c|c|c|}
\hline \multicolumn{20}{|c|}{$\begin{array}{c}\text { Depth } \\
\text { (inches) }\end{array}$} \\
\hline $\begin{array}{l}\text { Sta- } \\
\text { tion } \\
\text { no. }\end{array}$ & $\begin{array}{c}\text { 1st } \\
\text { per- } \\
\text { centile }\end{array}$ & $\begin{array}{c}\text { 2nd } \\
\text { per- } \\
\text { centile }\end{array}$ & $\begin{array}{c}\text { 10th } \\
\text { per- } \\
\text { centile }\end{array}$ & $\begin{array}{c}\text { 25th } \\
\text { per- } \\
\text { centile }\end{array}$ & $\begin{array}{c}\text { 50th } \\
\text { per- } \\
\text { centile } \\
\text { (median) }\end{array}$ & $\begin{array}{c}\text { 75th } \\
\text { per- } \\
\text { centile }\end{array}$ & $\begin{array}{c}\text { 90th } \\
\text { per- } \\
\text { centile }\end{array}$ & $\begin{array}{c}\text { 98th } \\
\text { per- } \\
\text { centile }\end{array}$ & $\begin{array}{c}\text { 99th } \\
\text { per- } \\
\text { centile }\end{array}$ & $\begin{array}{l}\text { Sta- } \\
\text { tion } \\
\text { no. }\end{array}$ & $\begin{array}{c}\text { 1st } \\
\text { per- } \\
\text { centile }\end{array}$ & $\begin{array}{c}\text { 2nd } \\
\text { per- } \\
\text { centile }\end{array}$ & $\begin{array}{c}\text { 10th } \\
\text { per- } \\
\text { centile }\end{array}$ & $\begin{array}{c}25 \text { th } \\
\text { per- } \\
\text { centile }\end{array}$ & $\begin{array}{c}\text { 50th } \\
\text { per- } \\
\text { centile } \\
\text { (median) }\end{array}$ & $\begin{array}{c}\text { 75th } \\
\text { per- } \\
\text { centile }\end{array}$ & $\begin{array}{c}\text { 90th } \\
\text { per- } \\
\text { centile }\end{array}$ & $\begin{array}{c}\text { 98th } \\
\text { per- } \\
\text { centile }\end{array}$ & $\begin{array}{c}\text { 99th } \\
\text { per- } \\
\text { centile }\end{array}$ \\
\hline 7718 & -- & 0.03 & 0.08 & 0.14 & 0.41 & 0.84 & 2.39 & 4.83 & -- & 8910 & -- & -- & 0.04 & 0.12 & 0.42 & 1.19 & 1.93 & -- & -- \\
\hline 7745 & 0.01 & .02 & .06 & .13 & .43 & .96 & 1.91 & 4.68 & 6.48 & 8911 & 0.01 & 0.01 & .03 & .08 & .26 & .77 & 1.52 & 3.19 & 4.10 \\
\hline 7922 & .01 & .01 & .02 & .05 & .15 & .31 & .70 & 1.33 & 1.96 & 8924 & .02 & .02 & .05 & .09 & .20 & .41 & .85 & 1.46 & 1.80 \\
\hline 7936 & .03 & .04 & .10 & .14 & .45 & 1.09 & 1.90 & 4.10 & 5.70 & 8929 & -- & -- & .05 & .14 & 1.14 & 1.85 & 2.81 & -- & -- \\
\hline 7943 & .01 & .01 & .02 & .04 & .17 & .54 & 1.20 & 2.38 & 3.49 & 8942 & .03 & .06 & .10 & .10 & .40 & 1.02 & 2.00 & 4.40 & 5.29 \\
\hline 7944 & -- & .01 & .01 & .03 & .24 & .58 & 1.48 & 2.86 & -- & 8944 & .01 & .01 & .03 & .10 & .33 & .87 & 1.72 & 3.53 & 4.69 \\
\hline 7945 & .01 & .01 & .01 & .04 & .17 & .63 & 1.39 & 3.58 & 4.74 & 8996 & .01 & .02 & .04 & .10 & .31 & .85 & 1.69 & 3.44 & 4.84 \\
\hline 7947 & .03 & .05 & .10 & .10 & .30 & .90 & 1.90 & 5.23 & 5.58 & 9014 & -- & -- & .13 & .26 & .59 & .97 & 2.09 & -- & -- \\
\hline 7948 & .02 & .02 & .04 & .09 & .26 & .75 & 1.67 & 3.50 & 4.23 & 9037 & .02 & .02 & .04 & .07 & .17 & .40 & .86 & 1.75 & 2.61 \\
\hline 7951 & .02 & .03 & .07 & .15 & .40 & 1.01 & 1.85 & 3.25 & 3.74 & 9106 & .02 & .02 & .03 & .05 & .13 & .37 & .77 & 1.92 & 3.82 \\
\hline 7953 & .02 & .02 & .05 & .08 & .23 & .66 & 1.42 & 3.03 & 3.68 & 9107 & -- & -- & .05 & .08 & .20 & .79 & .90 & -- & -- \\
\hline 7981 & .02 & .02 & .04 & .08 & .28 & .79 & 1.80 & 3.08 & 3.63 & 9129 & -- & .02 & .04 & .05 & .13 & .43 & 1.25 & 3.21 & -- \\
\hline 7990 & .01 & .01 & .02 & .06 & .17 & .58 & 1.62 & 4.53 & 4.80 & 9163 & .02 & .03 & .07 & .10 & .31 & .76 & 1.54 & 3.22 & 3.90 \\
\hline 7992 & -- & -- & .10 & .15 & .45 & 1.48 & 2.15 & -- & -- & 9213 & .01 & .01 & .02 & .07 & .28 & .74 & 1.67 & 3.64 & 5.04 \\
\hline 7997 & .02 & .02 & .04 & .10 & .28 & .63 & 1.68 & 3.46 & 3.65 & 9214 & -- & -- & .12 & .22 & .38 & 1.28 & 2.17 & -- & -- \\
\hline 7999 & -- & -- & .02 & .05 & .20 & .45 & 1.14 & -- & -- & 9222 & .01 & .01 & .04 & .10 & .28 & .85 & 1.67 & 3.96 & 4.60 \\
\hline 8022 & -- & .02 & .05 & .07 & .20 & .73 & 1.54 & 2.75 & -- & 9248 & .02 & .03 & .05 & .10 & .27 & .64 & 1.26 & 2.55 & 3.16 \\
\hline 8023 & .03 & .03 & .07 & .10 & .24 & .60 & 1.25 & 2.68 & 3.70 & 9266 & -- & .02 & .05 & .15 & .28 & .95 & 1.47 & 2.92 & -- \\
\hline 8047 & .02 & .03 & .07 & .10 & .32 & .88 & 1.61 & 3.20 & 3.92 & 9270 & .03 & .04 & .10 & .10 & .20 & .42 & .90 & 1.90 & 2.75 \\
\hline 8060 & .01 & .02 & .03 & .05 & .17 & .59 & 1.67 & 3.23 & 3.62 & 9295 & -- & .01 & .02 & .04 & .11 & .27 & .55 & 1.46 & -- \\
\hline 8062 & -- & -- & .02 & .04 & .15 & .79 & 2.69 & -- & -- & 9304 & -- & -- & -- & .10 & .17 & .26 & -- & -- & -- \\
\hline 8068 & -- & -- & .02 & .04 & .11 & .33 & 1.20 & -- & -- & 9307 & .03 & .04 & .10 & .15 & .38 & .79 & 1.45 & 3.20 & 3.45 \\
\hline 8081 & .02 & .03 & .06 & .10 & .26 & .76 & 1.60 & 3.61 & 4.90 & 9328 & .02 & .02 & .05 & .10 & .28 & .64 & 1.27 & 2.82 & 3.10 \\
\hline 8089 & -- & -- & .03 & .10 & .23 & .80 & 1.61 & -- & -- & 9329 & -- & -- & .04 & .06 & .29 & 1.03 & 1.91 & -- & -- \\
\hline 8221 & -- & -- & .11 & .33 & .60 & .99 & 2.30 & -- & -- & 9345 & -- & -- & -- & -- & .75 & -- & -- & -- & -- \\
\hline 8252 & .02 & .03 & .07 & .10 & .23 & .61 & 1.20 & 2.48 & 3.20 & 9363 & .01 & .01 & .01 & .05 & .18 & .60 & 1.37 & 3.47 & 4.35 \\
\hline 8265 & .02 & .02 & .05 & .11 & .35 & .96 & 1.90 & 4.17 & 6.73 & 9364 & .01 & .01 & .02 & .05 & .19 & .65 & 1.53 & 3.72 & 5.40 \\
\hline 8289 & -- & .02 & .05 & .15 & .45 & .83 & 1.12 & 1.55 & -- & 9365 & -- & -- & .05 & .08 & .12 & .30 & .80 & -- & -- \\
\hline 8305 & .02 & .03 & .05 & .10 & .19 & .40 & .77 & 1.71 & 2.17 & 9371 & .03 & .04 & .05 & .10 & .31 & 1.09 & 1.92 & 2.79 & 3.49 \\
\hline 8335 & .03 & .03 & .06 & .16 & .49 & 1.11 & 1.98 & 3.86 & 4.57 & 9417 & .01 & .02 & .05 & .10 & .32 & .91 & 1.70 & 3.17 & 3.96 \\
\hline 8400 & .02 & .02 & .05 & .10 & .20 & .44 & .85 & 2.49 & 3.44 & 9419 & .01 & .01 & .02 & .06 & .25 & .77 & 1.56 & 3.12 & 3.91 \\
\hline 8445 & .02 & .03 & .05 & .11 & .32 & .90 & 1.84 & 3.68 & 4.72 & 9435 & -- & .02 & .06 & .11 & .29 & .68 & 1.04 & 3.97 & -- \\
\hline 8446 & .02 & .03 & .10 & .10 & .30 & .87 & 1.70 & 3.31 & 4.10 & 9491 & .03 & .03 & .07 & .10 & .35 & .94 & 1.80 & 3.50 & 4.60 \\
\hline 8451 & .02 & .02 & .05 & .09 & .24 & .67 & 1.27 & 3.29 & 3.86 & 9499 & .03 & .04 & .10 & .10 & .30 & .70 & 1.40 & 2.74 & 3.39 \\
\hline 8531 & .02 & .03 & .06 & .14 & .38 & .92 & 1.60 & 3.23 & 3.81 & 9522 & -- & -- & .11 & .24 & .45 & 2.33 & 5.26 & -- & -- \\
\hline 8541 & -- & .01 & .05 & .12 & .39 & .94 & 1.86 & 3.92 & -- & 9527 & .03 & .04 & .10 & .10 & .20 & .60 & 1.20 & 2.40 & 3.14 \\
\hline 8544 & .04 & .05 & .10 & .10 & .30 & .95 & 1.71 & 4.27 & 6.13 & 9532 & .02 & .03 & .08 & .10 & .30 & .80 & 1.60 & 3.50 & 4.75 \\
\hline 8545 & -- & -- & .03 & .09 & .30 & .57 & .93 & -- & -- & 9544 & -- & -- & -- & .10 & .24 & 4.34 & -- & -- & -- \\
\hline 8563 & .10 & .10 & .10 & .10 & .40 & .90 & 1.70 & 4.20 & 4.70 & 9565 & .02 & .03 & .09 & .10 & .30 & .70 & 1.50 & 2.94 & 4.00 \\
\hline 8566 & .01 & .02 & .04 & .09 & .25 & .71 & 1.31 & 2.49 & 3.38 & 9570 & .10 & .10 & .10 & .10 & .30 & .60 & 1.50 & 2.70 & 3.36 \\
\hline 8583 & .10 & .10 & .10 & .10 & .30 & .80 & 1.40 & 2.90 & 3.63 & 9574 & -- & -- & .10 & .10 & .35 & .72 & 1.65 & -- & -- \\
\hline 8584 & .03 & .04 & .10 & .10 & .30 & .90 & 1.60 & 3.14 & 4.05 & 9588 & .02 & .02 & .06 & .10 & .20 & .60 & 1.50 & 3.52 & 4.40 \\
\hline 8623 & .05 & .06 & .10 & .11 & .40 & .90 & 1.70 & 3.11 & 3.96 & 9665 & .02 & .03 & .10 & .12 & .40 & 1.00 & 1.80 & 3.96 & 5.00 \\
\hline 8625 & .02 & .02 & .05 & .10 & .33 & .86 & 1.52 & 3.43 & 4.13 & 9715 & .02 & .02 & .08 & .10 & .36 & .90 & 1.65 & 3.20 & 4.21 \\
\hline 8630 & .02 & .03 & .06 & .10 & .26 & .68 & 1.31 & 2.48 & 2.78 & 9729 & .01 & .01 & .02 & .06 & .25 & .73 & 1.45 & 3.00 & 3.93 \\
\hline 8631 & .02 & .03 & .05 & .10 & .30 & .68 & 1.54 & 3.21 & 3.90 & 9772 & .01 & .02 & .04 & .09 & .26 & .80 & 1.65 & 4.74 & 5.86 \\
\hline 8646 & .02 & .02 & .05 & .10 & .34 & .85 & 1.61 & 3.26 & 3.95 & 9814 & -- & -- & .04 & .10 & .36 & .92 & 1.82 & -- & -- \\
\hline 8647 & .03 & .04 & .10 & .10 & .20 & .60 & 1.10 & 2.49 & 3.24 & 9815 & .02 & .03 & .07 & .12 & .34 & .92 & 1.80 & 3.87 & 5.16 \\
\hline 8677 & .01 & .03 & .09 & .15 & .37 & .88 & 1.56 & 3.12 & 4.05 & 9816 & -- & .01 & .04 & .07 & .25 & .78 & 1.92 & 4.41 & -- \\
\hline 8696 & -- & -- & .05 & .10 & .42 & 1.30 & 2.57 & -- & -- & 9817 & .04 & .06 & .10 & .10 & .31 & .80 & 1.50 & 3.29 & 4.33 \\
\hline 8743 & .02 & .03 & .08 & .13 & .41 & 1.02 & 1.97 & 4.16 & 4.83 & 9829 & .02 & .02 & .05 & .10 & .19 & .43 & 1.00 & 2.10 & 2.73 \\
\hline 8761 & .02 & .03 & .10 & .10 & .20 & .57 & 1.20 & 2.40 & 3.03 & 9830 & .01 & .01 & .03 & .06 & .11 & .41 & .85 & 1.72 & 2.28 \\
\hline 8778 & .03 & .05 & .10 & .11 & .40 & 1.00 & 1.90 & 3.70 & 4.40 & 9858 & .02 & .02 & .05 & .10 & .27 & .63 & 1.25 & 2.53 & 3.16 \\
\hline 8845 & .03 & .03 & .08 & .10 & .30 & .83 & 1.76 & 3.97 & 5.24 & 9893 & .02 & .03 & .06 & .10 & .32 & .79 & 1.50 & 3.08 & 4.32 \\
\hline 8859 & .02 & .03 & .07 & .16 & .47 & 1.07 & 1.87 & 3.84 & 4.31 & 9916 & .03 & .05 & .10 & .19 & .41 & 1.10 & 2.00 & 3.93 & 4.90 \\
\hline 8898 & .02 & .03 & .07 & .15 & .42 & .93 & 1.90 & 3.95 & 5.29 & 9976 & .02 & .02 & .06 & .10 & .24 & .70 & 1.40 & 3.06 & 4.04 \\
\hline 8908 & -- & -- & .06 & .12 & .40 & 1.02 & 1.70 & -- & -- & & & & & & & & & & \\
\hline
\end{tabular}


Appendix 4-4.5. Empirical distribution of storm depth defined by 24-hour minimum interevent time for hourly rainfall stations in Texas.

$[--$, not available $]$

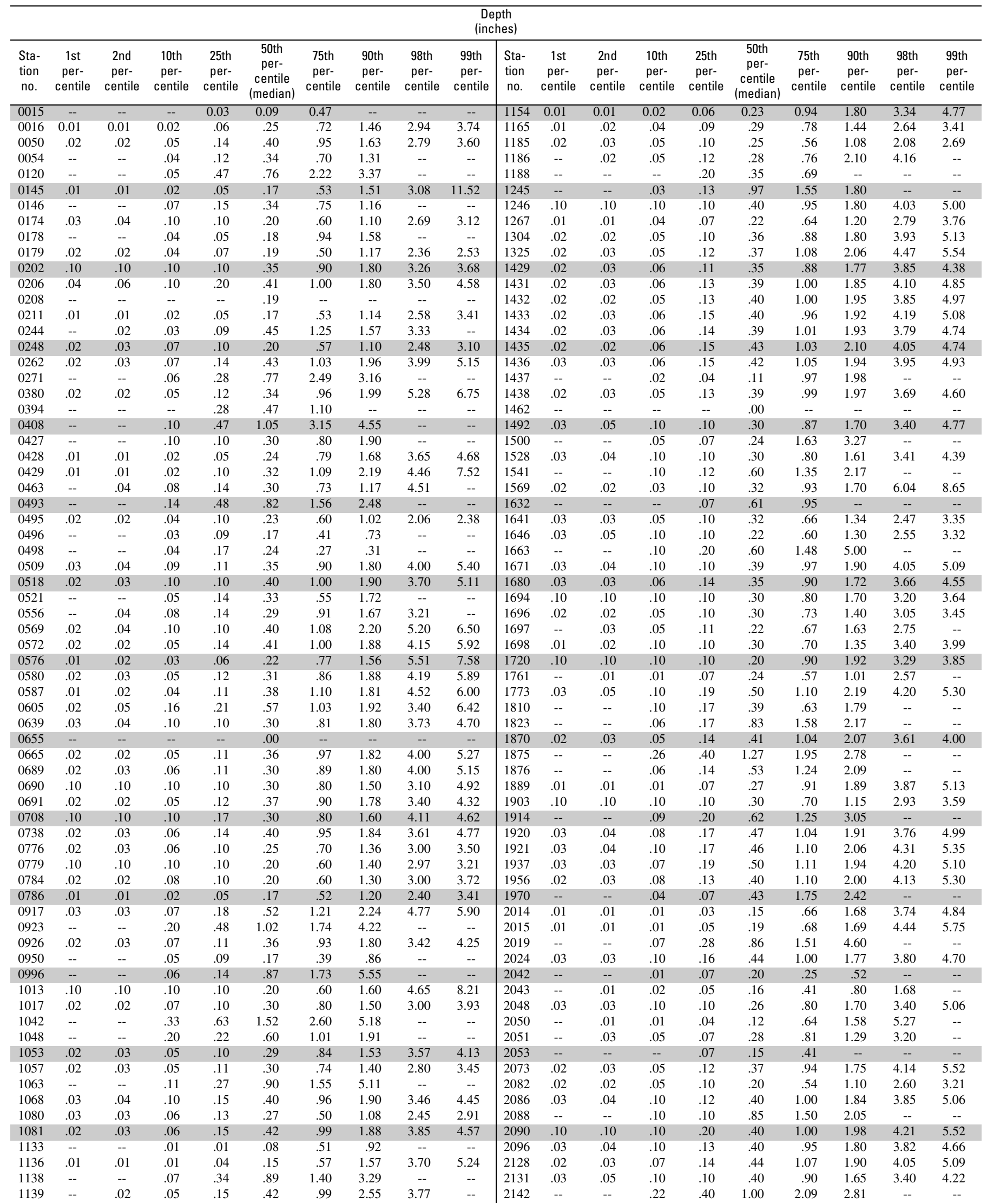


260 Statistical Characteristics of Storm Interevent Time, Depth, and Duration for Eastern New Mexico, Oklahoma, and Texas

Appendix 4-4.5. Empirical distribution of storm depth defined by 24-hour minimum interevent time for hourly rainfall stations in TexasContinued.

\begin{tabular}{|c|c|c|c|c|c|c|c|c|c|c|c|c|c|c|c|c|c|c|c|}
\hline \multicolumn{20}{|c|}{$\begin{array}{c}\text { Depth } \\
\text { (inches) }\end{array}$} \\
\hline $\begin{array}{l}\text { Sta- } \\
\text { tion } \\
\text { no. }\end{array}$ & $\begin{array}{c}\text { 1st } \\
\text { per- } \\
\text { centile }\end{array}$ & $\begin{array}{c}\text { 2nd } \\
\text { per- } \\
\text { centile }\end{array}$ & $\begin{array}{c}10 \text { th } \\
\text { per- } \\
\text { centile }\end{array}$ & $\begin{array}{c}\text { 25th } \\
\text { per- } \\
\text { centile }\end{array}$ & $\begin{array}{c}\text { 50th } \\
\text { per- } \\
\text { centile } \\
\text { (median) }\end{array}$ & $\begin{array}{c}\text { 75th } \\
\text { per- } \\
\text { centile }\end{array}$ & $\begin{array}{c}\text { 90th } \\
\text { per- } \\
\text { centile }\end{array}$ & $\begin{array}{c}\text { 98th } \\
\text { per- } \\
\text { centile }\end{array}$ & $\begin{array}{c}\text { 99th } \\
\text { per- } \\
\text { centile }\end{array}$ & $\begin{array}{l}\text { Sta- } \\
\text { tion } \\
\text { no. }\end{array}$ & $\begin{array}{c}1 \text { st } \\
\text { per- } \\
\text { centile }\end{array}$ & $\begin{array}{c}\text { 2nd } \\
\text { per- } \\
\text { centile }\end{array}$ & $\begin{array}{c}10 \text { th } \\
\text { per- } \\
\text { centile }\end{array}$ & $\begin{array}{c}25 \text { th } \\
\text { per- } \\
\text { centile }\end{array}$ & $\begin{array}{c}\text { 50th } \\
\text { per- } \\
\text { centile } \\
\text { (median) }\end{array}$ & $\begin{array}{c}\text { 75th } \\
\text { per- } \\
\text { centile }\end{array}$ & $\begin{array}{c}90 \text { th } \\
\text { per- } \\
\text { centile }\end{array}$ & $\begin{array}{c}\text { 98th } \\
\text { per- } \\
\text { centile }\end{array}$ & $\begin{array}{c}\text { 99th } \\
\text { per- } \\
\text { centile }\end{array}$ \\
\hline 2160 & -- & -- & 0.02 & 0.03 & 0.26 & 0.41 & 1.14 & -- & -- & 3463 & -- & -- & 0.04 & 0.10 & 0.34 & 1.00 & 2.20 & -- & -- \\
\hline 2206 & 0.02 & 0.03 & .06 & .14 & .45 & 1.10 & 2.18 & 3.93 & 6.52 & 3476 & 0.02 & 0.02 & .05 & .13 & .37 & .90 & 1.80 & 3.90 & 4.32 \\
\hline 2238 & .01 & .01 & .02 & .04 & .17 & .52 & 1.22 & 2.50 & 2.82 & 3485 & -- & -- & .10 & .10 & .49 & 1.82 & 4.92 & -- & -- \\
\hline 2240 & -- & .01 & .02 & .04 & .21 & .52 & 1.27 & 2.20 & -- & 3507 & .06 & .09 & .10 & .10 & .40 & 1.00 & 1.91 & 3.80 & 5.13 \\
\hline 2242 & .01 & .01 & .02 & .08 & .34 & .91 & 1.72 & 3.46 & 3.99 & 3546 & .02 & .03 & .10 & .19 & .47 & 1.14 & 2.12 & 4.30 & 5.30 \\
\hline 2244 & .01 & .01 & .04 & .10 & .38 & .94 & 1.89 & 3.90 & 5.21 & 3547 & .02 & .03 & .06 & .14 & .40 & 1.08 & 1.79 & 3.18 & 3.53 \\
\hline 2247 & -- & -- & .02 & .06 & .37 & 1.19 & 2.15 & -- & -- & 3579 & -- & .05 & .10 & .16 & .36 & 1.43 & 1.89 & 3.70 & -- \\
\hline 2309 & .03 & .04 & .10 & .25 & .57 & 1.18 & 2.02 & 4.46 & 5.86 & 3642 & .03 & .03 & .10 & .15 & .40 & 1.01 & 1.98 & 4.00 & 5.27 \\
\hline 2312 & .08 & .10 & .10 & .20 & .50 & 1.10 & 2.10 & 3.90 & 4.75 & 3646 & .03 & .03 & .06 & .14 & .39 & .94 & 1.70 & 3.87 & 4.98 \\
\hline 2334 & -- & .03 & .08 & .24 & .62 & 1.27 & 3.30 & 5.91 & -- & 3668 & -- & -- & .34 & .69 & 1.20 & 2.24 & 3.74 & -- & -- \\
\hline 2336 & .02 & .03 & .07 & .18 & .47 & 1.10 & 1.70 & 3.18 & 3.78 & 3673 & -- & -- & .04 & .10 & .74 & 2.07 & 2.97 & -- & -- \\
\hline 2354 & -- & -- & .04 & .10 & .17 & .72 & 1.24 & -- & -- & 3686 & .05 & .07 & .10 & .10 & .40 & 1.00 & 1.90 & 3.31 & 4.21 \\
\hline 2355 & -- & -- & .03 & .07 & .34 & .90 & 2.66 & -- & -- & 3691 & .02 & .03 & .10 & .10 & .40 & .91 & 1.80 & 3.50 & 4.69 \\
\hline 2357 & .01 & .01 & .01 & .03 & .13 & .52 & 1.33 & 3.24 & 5.06 & 3734 & -- & - & .03 & .18 & 1.14 & 3.53 & 4.82 & -- & -- \\
\hline 2360 & .01 & .01 & .01 & .04 & .16 & .61 & 1.37 & 3.07 & 4.16 & 3771 & .10 & .10 & .10 & .10 & .40 & 1.00 & 1.82 & 3.80 & 4.50 \\
\hline 2361 & .02 & .02 & .05 & .10 & .20 & .68 & 1.44 & 3.20 & 11.14 & 3789 & -- & .01 & .03 & .07 & .15 & .36 & .67 & 1.51 & -- \\
\hline 2394 & .02 & .02 & .05 & .13 & .40 & 1.00 & 1.91 & 3.90 & 5.32 & 3826 & .01 & .03 & .06 & .17 & .37 & .94 & 1.61 & 3.34 & 4.42 \\
\hline 2404 & .02 & .02 & .06 & .12 & .37 & .92 & 1.76 & 3.64 & 4.81 & 3831 & .03 & .03 & .06 & .16 & .41 & 1.07 & 2.15 & 3.70 & 5.18 \\
\hline 2415 & .03 & .04 & .08 & .19 & .51 & 1.15 & 2.12 & 4.34 & 5.45 & 3841 & .02 & .02 & .06 & .15 & .40 & .99 & 1.74 & 4.96 & 5.63 \\
\hline 2462 & .03 & .04 & .09 & .18 & .48 & 1.10 & 2.05 & 3.90 & 4.87 & 3871 & .02 & .03 & .05 & .13 & .35 & .82 & 1.59 & 3.30 & 3.95 \\
\hline 2528 & - & .03 & .06 & .12 & .29 & .89 & 2.05 & 2.71 & -- & 3884 & -- & -- & .06 & .14 & .30 & 1.86 & 3.50 & - & - \\
\hline 2617 & .02 & .02 & .05 & .10 & .28 & .65 & 1.44 & 3.14 & 4.20 & 3941 & -- & .02 & .05 & .10 & .65 & 1.45 & 2.17 & 5.54 & -- \\
\hline 2619 & .02 & .03 & .05 & .15 & .34 & .79 & 1.26 & 2.98 & 3.94 & 3963 & -- & -- & -- & .03 & .08 & .18 & -- & -- & -- \\
\hline 2621 & .02 & .03 & .07 & .11 & .31 & .73 & 1.50 & 2.93 & 3.67 & 4040 & .03 & .03 & .07 & .13 & .38 & .81 & 1.48 & 3.18 & 4.59 \\
\hline 2675 & .03 & .05 & .10 & .12 & .40 & .90 & 1.90 & 4.27 & 5.47 & 4058 & -- & -- & .08 & .14 & .56 & 1.28 & 3.58 & -- & -- \\
\hline 2676 & .10 & .10 & .10 & .10 & .30 & 1.00 & 1.90 & 3.60 & 4.52 & 4098 & .03 & .04 & .10 & .10 & .20 & .60 & 1.10 & 2.30 & 2.90 \\
\hline 2679 & .02 & .02 & .06 & .10 & .20 & .70 & 1.65 & 3.70 & 5.01 & 4100 & .02 & .04 & .05 & .10 & .31 & .80 & 1.50 & 3.52 & 4.90 \\
\hline 2715 & .02 & .03 & .07 & .14 & .40 & .90 & 1.66 & 3.46 & 4.37 & 4137 & .10 & .10 & .10 & .10 & .40 & 1.00 & 1.70 & 3.60 & 4.40 \\
\hline 2744 & .03 & .03 & .08 & .10 & .30 & .80 & 1.46 & 3.15 & 3.80 & 4191 & .03 & .04 & .08 & .10 & .30 & .83 & 1.80 & 3.78 & 5.29 \\
\hline 2758 & -- & .02 & .03 & .05 & .15 & .56 & 1.62 & 4.41 & -- & 4256 & -- & -- & -- & -- & .00 & -- & -- & -- & -- \\
\hline 2794 & -- & -- & .01 & .04 & .16 & .66 & 1.61 & -- & -- & 4257 & .04 & .05 & .10 & .20 & .50 & 1.17 & 2.20 & 4.41 & 5.59 \\
\hline 2797 & .01 & .01 & .01 & .03 & .11 & .30 & .65 & 1.55 & 1.98 & 4258 & .10 & .10 & .10 & .20 & .50 & 1.10 & 2.05 & 4.19 & 7.07 \\
\hline 2811 & .02 & .03 & .08 & .10 & .30 & .78 & 1.50 & 3.28 & 3.95 & 4278 & .02 & .02 & .05 & .13 & .35 & .96 & 1.83 & 3.63 & 4.52 \\
\hline 2813 & -- & -- & .06 & .22 & .42 & .99 & 2.32 & -- & -- & 4299 & .03 & .04 & .06 & .13 & .27 & .57 & .89 & 1.24 & 1.49 \\
\hline 2814 & -- & -- & .01 & .03 & .07 & .28 & 1.01 & -- & -- & 4300 & .01 & .01 & .02 & .07 & .29 & .93 & 1.92 & 4.29 & 6.57 \\
\hline 2815 & .10 & .10 & .10 & .10 & .30 & .70 & 1.40 & 3.26 & 4.57 & 4305 & .01 & .01 & .02 & .06 & .30 & .92 & 1.94 & 4.45 & 5.56 \\
\hline 2818 & .02 & .02 & .05 & .12 & .37 & .81 & 1.52 & 3.85 & 4.96 & 4307 & .01 & .01 & .02 & .08 & .35 & .93 & 1.99 & 4.86 & 7.56 \\
\hline 2986 & .02 & .04 & .09 & .20 & .62 & 1.39 & 2.17 & 4.42 & 7.61 & 4309 & .02 & .02 & .05 & .13 & .42 & 1.10 & 2.07 & 4.40 & 5.91 \\
\hline 3005 & .02 & .03 & .10 & .12 & .39 & .90 & 1.60 & 3.44 & 4.35 & 4311 & .02 & .02 & .06 & .15 & .44 & 1.18 & 2.20 & 4.55 & 5.71 \\
\hline 3033 & .02 & .02 & .04 & .08 & .15 & .35 & .75 & 1.63 & 2.00 & 4313 & .03 & .04 & .10 & .20 & .45 & 1.20 & 2.55 & 5.02 & 8.24 \\
\hline 3034 & -- & -- & -- & -- & .16 & -- & -- & -- & -- & 4319 & -- & .04 & .10 & .17 & .40 & .85 & 1.85 & 3.63 & -- \\
\hline 3047 & -- & -- & .10 & .13 & .68 & 1.71 & 2.95 & -- & -- & 4329 & .02 & .03 & .07 & .16 & .45 & 1.08 & 2.11 & 4.65 & 5.99 \\
\hline 3103 & -- & -- & .12 & .19 & .29 & 1.10 & 2.74 & -- & -- & 4331 & -- & -- & -- & -- & .00 & -- & -- & -- & -- \\
\hline 3133 & .02 & .03 & .07 & .16 & .46 & 1.06 & 1.95 & 4.03 & 5.25 & 4375 & .10 & .10 & .10 & .10 & .30 & .90 & 1.80 & 4.15 & 5.64 \\
\hline 3156 & .04 & .05 & .10 & .16 & .46 & 1.17 & 2.20 & 5.99 & 9.29 & 4392 & .02 & .03 & .05 & .16 & .50 & 1.20 & 2.24 & 5.99 & 7.26 \\
\hline 3171 & .03 & .03 & .07 & .15 & .41 & 1.00 & 1.88 & 3.79 & 4.92 & 4425 & .03 & .03 & .09 & .10 & .21 & .60 & 1.20 & 2.47 & 3.17 \\
\hline 3189 & .02 & .03 & .05 & .10 & .20 & .50 & 1.00 & 2.54 & 3.40 & 4440 & .02 & .02 & .05 & .11 & .34 & .83 & 1.53 & 3.40 & 4.13 \\
\hline 3260 & .01 & .02 & .04 & .10 & .32 & .85 & 1.81 & 3.33 & 4.10 & 4476 & .04 & .05 & .10 & .14 & .40 & .90 & 1.70 & 3.54 & 4.42 \\
\hline 3267 & -- & .02 & .03 & .05 & .19 & .58 & 1.25 & 5.47 & -- & 4498 & -- & -- & .02 & .06 & .17 & .38 & .60 & -- & -- \\
\hline 3270 & .04 & .10 & .10 & .10 & .30 & .70 & 1.40 & 3.20 & 3.80 & 4517 & .02 & .02 & .05 & .13 & .38 & .94 & 1.80 & 3.52 & 4.06 \\
\hline 3272 & -- & -- & .02 & .03 & .13 & .22 & .37 & -- & -- & 4520 & .10 & .10 & .10 & .10 & .40 & .90 & 1.70 & 3.50 & 4.30 \\
\hline 3277 & -- & -- & .02 & .03 & .13 & .23 & .70 & -- & -- & 4525 & -- & -- & .04 & .07 & .28 & .80 & 5.54 & -- & -- \\
\hline 3278 & .02 & .03 & .06 & .10 & .25 & .69 & 1.30 & 2.65 & 3.38 & 4563 & -- & -- & .04 & .06 & .40 & .64 & 1.92 & -- & -- \\
\hline 3280 & .01 & .01 & .03 & .05 & .16 & .45 & .98 & 1.93 & 2.65 & 4570 & .02 & .03 & .08 & .10 & .30 & .76 & 1.50 & 3.01 & 4.35 \\
\hline 3281 & -- & -- & .04 & .12 & .28 & .52 & 1.00 & -- & -- & 4577 & .03 & .04 & .10 & .20 & .50 & 1.10 & 2.00 & 4.10 & 5.88 \\
\hline 3283 & .01 & .01 & .02 & .08 & .30 & .79 & 1.79 & 3.80 & 4.84 & 4591 & .02 & .03 & .06 & .14 & .42 & 1.00 & 1.90 & 3.85 & 4.91 \\
\hline 3284 & .01 & .02 & .10 & .11 & .40 & .93 & 1.90 & 3.53 & 4.30 & 4670 & .02 & .03 & .07 & .10 & .30 & .79 & 1.50 & 3.07 & 3.62 \\
\hline 3285 & .03 & .08 & .10 & .10 & .40 & 1.00 & 1.90 & 3.71 & 4.50 & 4671 & .01 & .01 & .03 & .06 & .27 & .71 & 1.21 & 3.12 & 3.35 \\
\hline 3329 & .02 & .02 & .05 & .10 & .30 & .81 & 1.75 & 3.67 & 5.11 & 4679 & .03 & .04 & .10 & .10 & .36 & .90 & 1.86 & 4.00 & 5.31 \\
\hline 3335 & .02 & .03 & .10 & .20 & .47 & 1.29 & 2.19 & 4.86 & 9.41 & 4696 & -- & -- & .05 & .09 & .47 & .81 & 1.14 & -- & -- \\
\hline 3370 & .02 & .03 & .06 & .15 & .47 & 1.06 & 2.00 & 4.00 & 5.02 & 4703 & .02 & .02 & .05 & .12 & .24 & .75 & 1.41 & 3.06 & 3.48 \\
\hline 3410 & .04 & .05 & .10 & .10 & .30 & .70 & 1.40 & 2.86 & 3.59 & 4704 & .02 & .03 & .06 & .18 & .48 & 1.24 & 2.79 & 5.00 & 7.09 \\
\hline 3415 & .02 & .03 & .10 & .11 & .40 & .90 & 1.80 & 3.72 & 4.97 & 4731 & -- & .01 & .01 & .05 & .18 & .64 & 1.24 & 4.64 & -- \\
\hline 3430 & .01 & .01 & .02 & .07 & .29 & .86 & 1.90 & 4.31 & 6.46 & 4792 & .10 & .10 & .10 & .10 & .40 & .93 & 1.70 & 3.30 & 4.20 \\
\hline 3431 & .01 & .01 & .02 & .06 & .25 & .81 & 1.67 & 5.41 & 10.33 & 4819 & .10 & .10 & .10 & .20 & .50 & 1.10 & 2.00 & 3.94 & 4.26 \\
\hline 3441 & -- & - & .05 & .19 & .41 & 1.04 & 2.51 & -- & -- & 4852 & - & -- & .14 & .37 & .78 & 1.14 & 2.87 & - & -- \\
\hline 3442 & .02 & .03 & .05 & .10 & .25 & .52 & 1.30 & 2.28 & 2.78 & 4866 & .03 & .04 & .10 & .15 & .44 & 1.00 & 1.86 & 3.61 & 4.37 \\
\hline 3446 & .03 & .04 & .06 & .12 & .30 & .70 & 1.20 & 2.51 & 3.17 & 4876 & .10 & .10 & .10 & .10 & .40 & 1.20 & 2.88 & 5.32 & 7.01 \\
\hline 3460 & -- & -- & .04 & .10 & .82 & 1.35 & 1.90 & -- & -- & 4878 & .02 & .03 & .08 & .18 & .50 & 1.25 & 2.42 & 5.05 & 6.20 \\
\hline 3462 & .05 & .05 & .09 & .15 & .36 & .86 & 1.27 & 2.42 & 4.53 & 4880 & .02 & .03 & .06 & .10 & .27 & .65 & 1.30 & 2.72 & 3.40 \\
\hline
\end{tabular}


Appendix 4-4.5. Empirical distribution of storm depth defined by 24-hour minimum interevent time for hourly rainfall stations in TexasContinued.

\begin{tabular}{|c|c|c|c|c|c|c|c|c|c|c|c|c|c|c|c|c|c|c|c|}
\hline \multicolumn{20}{|c|}{$\begin{array}{c}\text { Depth } \\
\text { (inches) }\end{array}$} \\
\hline $\begin{array}{l}\text { Sta- } \\
\text { tion } \\
\text { no. }\end{array}$ & $\begin{array}{c}\text { 1st } \\
\text { per- } \\
\text { centile }\end{array}$ & $\begin{array}{c}\text { 2nd } \\
\text { per- } \\
\text { centile }\end{array}$ & $\begin{array}{c}\text { 10th } \\
\text { per- } \\
\text { centile }\end{array}$ & $\begin{array}{c}25 \text { th } \\
\text { per- } \\
\text { centile }\end{array}$ & $\begin{array}{c}\text { 50th } \\
\text { per- } \\
\text { centile } \\
\text { (median) }\end{array}$ & $\begin{array}{c}\text { 75th } \\
\text { per- } \\
\text { centile }\end{array}$ & $\begin{array}{c}\text { 90th } \\
\text { per- } \\
\text { centile }\end{array}$ & $\begin{array}{c}\text { 98th } \\
\text { per- } \\
\text { centile }\end{array}$ & $\begin{array}{c}\text { 99th } \\
\text { per- } \\
\text { centile }\end{array}$ & $\begin{array}{l}\text { Sta- } \\
\text { tion } \\
\text { no. }\end{array}$ & $\begin{array}{c}1 \text { st } \\
\text { per- } \\
\text { centile }\end{array}$ & $\begin{array}{c}\text { 2nd } \\
\text { per- } \\
\text { centile }\end{array}$ & $\begin{array}{c}10 \text { th } \\
\text { per- } \\
\text { centile }\end{array}$ & $\begin{array}{c}\text { 25th } \\
\text { per- } \\
\text { centile }\end{array}$ & $\begin{array}{c}\text { 50th } \\
\text { per- } \\
\text { centile } \\
\text { (median) }\end{array}$ & $\begin{array}{c}\text { 75th } \\
\text { per- } \\
\text { centile }\end{array}$ & $\begin{array}{c}\text { 90th } \\
\text { per- } \\
\text { centile }\end{array}$ & $\begin{array}{c}\text { 98th } \\
\text { per- } \\
\text { centile }\end{array}$ & $\begin{array}{l}\text { 99th } \\
\text { per- } \\
\text { centile }\end{array}$ \\
\hline 4920 & 0.03 & 0.03 & 0.09 & 0.10 & 0.30 & 0.90 & 1.70 & 3.73 & 4.80 & 5957 & 0.03 & 0.04 & 0.10 & 0.10 & 0.40 & 0.90 & 1.70 & 3.60 & 4.81 \\
\hline 4934 & -- & -- & -- & .07 & .14 & .66 & -- & -- & -- & 5958 & .01 & .01 & .05 & .13 & .37 & 1.10 & 1.90 & 2.79 & 3.45 \\
\hline 4972 & .03 & .03 & .10 & .11 & .39 & .86 & 1.64 & 3.50 & 4.40 & 5973 & -- & .02 & .03 & .05 & .18 & .66 & 1.75 & 3.88 & -- \\
\hline 4973 & .03 & .05 & .10 & .25 & .60 & 1.20 & 2.12 & 3.76 & 4.39 & 5996 & .03 & .04 & .10 & .10 & .37 & .90 & 1.70 & 3.50 & 4.40 \\
\hline 4974 & .02 & .03 & .05 & .10 & .28 & .69 & 1.31 & 3.05 & 3.85 & 6017 & .01 & .01 & .03 & .06 & .21 & .65 & 1.30 & 2.66 & 2.96 \\
\hline 4975 & .10 & .10 & .10 & .20 & .50 & 1.20 & 2.10 & 4.20 & 5.10 & 6024 & .02 & .03 & .06 & .16 & .60 & 1.44 & 2.55 & 6.17 & 6.60 \\
\hline 4978 & .02 & .02 & .04 & .10 & .35 & .93 & 2.00 & 4.16 & 5.33 & 6050 & -- & -- & .15 & .24 & .40 & 1.03 & 1.73 & -- & -- \\
\hline 4979 & -- & -- & .11 & .35 & .65 & 2.25 & 3.29 & -- & -- & 6104 & .02 & .03 & .05 & .10 & .23 & .57 & 1.11 & 2.56 & 3.31 \\
\hline 4982 & .02 & .03 & .06 & .12 & .33 & .80 & 1.54 & 2.92 & 3.67 & 6108 & .03 & .04 & .10 & .20 & .50 & 1.20 & 2.12 & 4.51 & 5.51 \\
\hline 5018 & .02 & .03 & .07 & .15 & .43 & .97 & 1.66 & 2.87 & 3.99 & 6136 & .03 & .04 & .10 & .10 & .22 & .60 & 1.15 & 2.30 & 3.05 \\
\hline 5048 & .02 & .03 & .10 & .10 & .20 & .70 & 1.30 & 2.95 & 3.73 & 6166 & -- & .03 & .05 & .09 & .21 & .60 & 1.25 & 2.40 & -- \\
\hline 5049 & -- & .10 & .10 & .10 & .25 & .50 & .92 & 4.45 & -- & 6176 & .02 & .02 & .05 & .15 & .49 & .98 & 2.37 & 4.80 & 6.73 \\
\hline 5056 & -- & -- & -- & .20 & .37 & .69 & -- & -- & -- & 6177 & .03 & .05 & .10 & .20 & .50 & 1.11 & 2.10 & 4.45 & 5.80 \\
\hline 5057 & .01 & .01 & .02 & .04 & .14 & .44 & 1.20 & 2.90 & 3.40 & 6210 & .03 & .04 & .10 & .12 & .40 & 1.05 & 1.99 & 4.14 & 4.70 \\
\hline 5060 & .01 & .01 & .02 & .06 & .22 & .77 & 1.92 & 5.90 & 6.74 & 6211 & .01 & .01 & .05 & .12 & .45 & 1.12 & 2.25 & 4.26 & 4.92 \\
\hline 5081 & .02 & .03 & .06 & .17 & .52 & 1.10 & 2.00 & 3.86 & 4.25 & 6270 & .10 & .10 & .10 & .20 & .50 & 1.20 & 2.20 & 4.40 & 5.44 \\
\hline 5094 & .02 & .03 & .10 & .15 & .40 & 1.00 & 1.90 & 3.97 & 4.64 & 6275 & -- & -- & -- & -- & .00 & -- & -- & -- & -- \\
\hline 5113 & .02 & .03 & .08 & .10 & .30 & .80 & 1.80 & 4.16 & 5.38 & 6276 & -- & -- & .06 & .18 & 1.08 & 1.82 & 2.80 & -- & -- \\
\hline 5114 & -- & -- & -- & -- & .00 & -- & -- & -- & -- & 6335 & .02 & .03 & .07 & .16 & .44 & 1.04 & 1.90 & 3.65 & 4.61 \\
\hline 5123 & -- & -- & .07 & .16 & .27 & .89 & 2.54 & -- & -- & 6434 & -- & -- & .09 & .13 & .45 & 1.00 & 1.73 & -- & -- \\
\hline 5192 & .02 & .03 & .09 & .15 & .40 & 1.05 & 1.94 & 3.94 & 5.23 & 6504 & .02 & .03 & .09 & .10 & .25 & .66 & 1.30 & 2.91 & 3.45 \\
\hline 5193 & .02 & .03 & .07 & .10 & .38 & 1.00 & 1.87 & 3.79 & 4.90 & 6558 & -- & -- & .05 & .06 & .49 & .99 & 1.88 & -- & -- \\
\hline 5224 & .01 & .02 & .05 & .14 & .50 & 1.14 & 2.27 & 5.29 & 8.28 & 6615 & .06 & .10 & .10 & .10 & .30 & .70 & 1.50 & 3.64 & 5.02 \\
\hline 5228 & .02 & .02 & .05 & .10 & .40 & 1.03 & 1.82 & 3.71 & 4.32 & 6660 & -- & .03 & .05 & .17 & .40 & .96 & 2.17 & 4.96 & -- \\
\hline 5235 & -- & -- & .04 & .05 & .54 & 1.01 & 1.94 & -- & -- & 6663 & -- & .02 & .10 & .16 & .40 & 1.12 & 1.75 & 4.51 & -- \\
\hline 5247 & .02 & .03 & .10 & .10 & .30 & .70 & 1.30 & 2.52 & 3.18 & 6734 & .01 & .02 & .05 & .10 & .28 & .71 & 1.30 & 3.00 & 3.47 \\
\hline 5258 & .02 & .03 & .07 & .15 & .40 & .94 & 1.89 & 3.89 & 4.70 & 6736 & .03 & .03 & .10 & .10 & .26 & .70 & 1.31 & 3.19 & 3.94 \\
\hline 5303 & .02 & .03 & .05 & .10 & .30 & .89 & 1.67 & 3.87 & 5.18 & 6740 & -- & -- & .09 & .26 & .94 & 3.39 & 4.10 & -- & -- \\
\hline 5312 & .04 & .05 & .10 & .10 & .30 & .80 & 1.60 & 3.29 & 4.99 & 6750 & .01 & .01 & .01 & .05 & .29 & 1.19 & 2.36 & 6.41 & 9.71 \\
\hline 5341 & -- & -- & .10 & .22 & .47 & 1.61 & 5.49 & -- & -- & 6757 & .01 & .02 & .06 & .13 & .41 & 1.07 & 1.96 & 4.00 & 5.06 \\
\hline 5342 & -- & -- & -- & -- & .00 & -- & -- & -- & -- & 6775 & .01 & .01 & .04 & .10 & .26 & .67 & 1.25 & 2.72 & 3.41 \\
\hline 5348 & .03 & .05 & .10 & .20 & .50 & 1.20 & 2.20 & 4.00 & 4.80 & 6776 & .02 & .03 & .10 & .10 & .30 & .66 & 1.23 & 2.70 & 3.40 \\
\hline 5358 & .02 & .02 & .05 & .10 & .30 & .78 & 1.53 & 2.97 & 3.69 & 6788 & .02 & .03 & .06 & .18 & .50 & 1.06 & 1.93 & 4.13 & 5.07 \\
\hline 5398 & .03 & .03 & .08 & .15 & .45 & 1.06 & 2.01 & 4.29 & 5.83 & 6792 & .02 & .02 & .07 & .10 & .20 & .50 & 1.06 & 2.12 & 2.70 \\
\hline 5410 & .02 & .03 & .08 & .10 & .28 & .64 & 1.20 & 3.10 & 4.00 & 6794 & -- & -- & .16 & .56 & 1.38 & 3.15 & 5.07 & -- & -- \\
\hline 5411 & .01 & .01 & .02 & .05 & .17 & .54 & 1.14 & 2.76 & 3.45 & 6834 & .10 & .10 & .10 & .20 & .50 & 1.20 & 2.20 & 4.40 & 5.20 \\
\hline 5424 & .01 & .01 & .02 & .07 & .39 & 1.13 & 2.41 & 4.24 & 7.50 & 6893 & .02 & .03 & .08 & .10 & .20 & .42 & .85 & 2.10 & 2.70 \\
\hline 5429 & .02 & .02 & .04 & .10 & .31 & .92 & 1.84 & 3.94 & 4.79 & 6935 & .03 & .04 & .10 & .10 & .27 & .60 & 1.30 & 2.87 & 3.38 \\
\hline 5431 & -- & -- & .14 & .35 & .70 & 1.40 & 3.03 & -- & -- & 6981 & .02 & .03 & .07 & .16 & .45 & .97 & 1.85 & 5.48 & 5.72 \\
\hline 5461 & .02 & .03 & .06 & .16 & .46 & 1.11 & 2.05 & 4.27 & 5.79 & 7020 & .02 & .02 & .04 & .13 & .38 & 1.07 & 2.07 & 4.15 & 8.42 \\
\hline 5463 & .10 & .10 & .10 & .10 & .50 & 1.10 & 2.00 & 4.30 & 5.70 & 7060 & .03 & .05 & .10 & .10 & .30 & .80 & 1.53 & 2.93 & 3.53 \\
\hline 5471 & -- & -- & .03 & .06 & .10 & .41 & .82 & -- & -- & 7066 & .03 & .03 & .10 & .18 & .50 & 1.13 & 2.14 & 4.09 & 5.09 \\
\hline 5477 & -- & -- & .07 & .15 & .40 & .61 & 3.50 & -- & -- & 7074 & .02 & .02 & .06 & .10 & .23 & .60 & 1.18 & 2.82 & 3.82 \\
\hline 5528 & .03 & .03 & .08 & .18 & .50 & 1.07 & 2.02 & 3.81 & 4.47 & 7097 & -- & .02 & .08 & .17 & .39 & .89 & 1.89 & 3.76 & -- \\
\hline 5579 & -- & -- & -- & -- & .55 & -- & -- & -- & -- & 7116 & .02 & .02 & .05 & .11 & .28 & .69 & 1.28 & 2.64 & 3.87 \\
\hline 5580 & -- & -- & .08 & .30 & .49 & 1.23 & 1.89 & -- & -- & 7140 & .02 & .02 & .05 & .10 & .32 & .98 & 2.17 & 4.99 & 6.50 \\
\hline 5589 & .01 & .01 & .02 & .06 & .20 & .49 & .97 & 1.52 & 1.67 & 7173 & .01 & .01 & .02 & .07 & .38 & 1.10 & 2.49 & 5.98 & 7.56 \\
\hline 5590 & .02 & .02 & .04 & .08 & .20 & .47 & 1.06 & 2.23 & 3.23 & 7174 & .01 & .01 & .02 & .08 & .35 & 1.04 & 2.18 & 5.20 & 7.13 \\
\hline 5591 & .02 & .02 & .05 & .11 & .26 & .55 & .97 & 1.85 & 2.73 & 7213 & .02 & .02 & .06 & .16 & .42 & 1.00 & 1.99 & 3.98 & 4.99 \\
\hline 5592 & .02 & .02 & .04 & .08 & .20 & .47 & .99 & 2.11 & 2.45 & 7243 & .03 & .04 & .10 & .10 & .30 & .81 & 1.57 & 3.02 & 4.09 \\
\hline 5594 & .02 & .03 & .04 & .08 & .20 & .47 & .80 & 1.92 & 2.24 & 7262 & -- & .01 & .01 & .03 & .12 & .34 & .60 & 1.47 & -- \\
\hline 5595 & -- & -- & -- & -- & .03 & -- & -- & -- & -- & 7274 & .04 & .05 & .10 & .20 & .42 & .95 & 1.81 & 3.80 & 5.46 \\
\hline 5596 & .10 & .10 & .10 & .10 & .20 & .50 & 1.10 & 2.60 & 3.37 & 7300 & .02 & .03 & .05 & .13 & .37 & .88 & 1.63 & 3.26 & 4.60 \\
\hline 5600 & .02 & .02 & .05 & .10 & .23 & .53 & 1.01 & 2.59 & 3.71 & 7311 & -- & -- & .10 & .20 & .30 & 1.11 & 1.91 & -- & -- \\
\hline 5618 & -- & -- & .07 & .15 & .36 & 1.21 & 2.38 & -- & -- & 7363 & -- & -- & .07 & .15 & .94 & 1.67 & 2.33 & -- & -- \\
\hline 5650 & -- & -- & .12 & .27 & .45 & .76 & 1.78 & -- & -- & 7422 & .02 & .03 & .07 & .10 & .33 & .90 & 1.81 & 4.00 & 4.92 \\
\hline 5656 & .10 & .10 & .10 & .10 & .30 & .70 & 1.40 & 3.00 & 3.60 & 7431 & .03 & .03 & .06 & .10 & .23 & .60 & 1.22 & 2.67 & 3.37 \\
\hline 5658 & .02 & .02 & .05 & .10 & .26 & .65 & 1.33 & 3.05 & 4.59 & 7481 & .02 & .02 & .05 & .10 & .20 & .45 & .96 & 2.56 & 3.37 \\
\hline 5661 & .10 & .10 & .10 & .10 & .30 & .72 & 1.80 & 6.55 & 8.60 & 7497 & .10 & .10 & .10 & .10 & .40 & 1.00 & 1.72 & 3.70 & 5.09 \\
\hline 5666 & -- & -- & .05 & .09 & .30 & .86 & 1.59 & -- & -- & 7498 & .08 & .10 & .10 & .10 & .40 & 1.05 & 1.70 & 4.24 & 6.66 \\
\hline 5695 & .03 & .04 & .08 & .18 & .48 & 1.03 & 1.84 & 3.83 & 5.07 & 7499 & .05 & .07 & .10 & .10 & .36 & .90 & 1.70 & 3.50 & 4.21 \\
\hline 5742 & -- & -- & .04 & .07 & .20 & .33 & 1.59 & -- & -- & 7531 & .03 & .03 & .06 & .13 & .40 & 1.08 & 1.98 & 3.67 & 4.16 \\
\hline 5766 & -- & -- & .12 & .30 & .54 & 1.99 & 2.80 & -- & -- & 7534 & .02 & .03 & .05 & .11 & .35 & .93 & 1.70 & 3.52 & 5.83 \\
\hline 5770 & .02 & .03 & .07 & .10 & .30 & .70 & 1.40 & 2.96 & 4.00 & 7556 & .03 & .04 & .10 & .10 & .37 & .90 & 1.80 & 3.70 & 5.12 \\
\hline 5775 & -- & -- & .05 & .05 & .15 & .73 & .97 & -- & -- & 7594 & .02 & .03 & .07 & .16 & .48 & 1.10 & 2.10 & 4.33 & 5.70 \\
\hline 5779 & -- & -- & .12 & .19 & .49 & 1.12 & 2.53 & -- & -- & 7596 & .01 & .02 & .04 & .10 & .44 & 1.27 & 2.08 & 3.46 & 4.29 \\
\hline 5840 & .02 & .03 & .05 & .11 & .36 & .90 & 1.91 & 3.93 & 4.82 & 7608 & .01 & .01 & .03 & .10 & .31 & .89 & 1.79 & 3.39 & 4.96 \\
\hline 5890 & .01 & .01 & .02 & .05 & .16 & .47 & 1.07 & 2.49 & 3.06 & 7622 & -- & -- & .01 & .01 & .06 & .34 & .76 & -- & -- \\
\hline 5891 & .02 & .03 & .06 & .10 & .25 & .61 & 1.03 & 1.90 & 2.38 & 7700 & .02 & .02 & .05 & .13 & .44 & 1.09 & 2.08 & 4.20 & 5.03 \\
\hline 5897 & .10 & .10 & .10 & .10 & .40 & 1.00 & 1.90 & 3.80 & 4.60 & 7706 & .02 & .03 & .08 & .10 & .30 & .72 & 1.52 & 3.24 & 4.39 \\
\hline
\end{tabular}


Appendix 4-4.5. Empirical distribution of storm depth defined by 24-hour minimum interevent time for hourly rainfall stations in TexasContinued.

\begin{tabular}{|c|c|c|c|c|c|c|c|c|c|c|c|c|c|c|c|c|c|c|c|}
\hline \multicolumn{20}{|c|}{$\begin{array}{c}\text { Depth } \\
\text { (inches) }\end{array}$} \\
\hline $\begin{array}{l}\text { Sta- } \\
\text { tion } \\
\text { no. }\end{array}$ & $\begin{array}{c}\text { 1st } \\
\text { per- } \\
\text { centile }\end{array}$ & $\begin{array}{c}\text { 2nd } \\
\text { per- } \\
\text { centile }\end{array}$ & $\begin{array}{c}\text { 10th } \\
\text { per- } \\
\text { centile }\end{array}$ & $\begin{array}{c}\text { 25th } \\
\text { per- } \\
\text { centile }\end{array}$ & $\begin{array}{c}\text { 50th } \\
\text { per- } \\
\text { centile } \\
\text { (median) }\end{array}$ & $\begin{array}{c}\text { 75th } \\
\text { per- } \\
\text { centile }\end{array}$ & $\begin{array}{c}\text { 90th } \\
\text { per- } \\
\text { centile }\end{array}$ & $\begin{array}{c}\text { 98th } \\
\text { per- } \\
\text { centile }\end{array}$ & $\begin{array}{c}\text { 99th } \\
\text { per- } \\
\text { centile }\end{array}$ & $\begin{array}{l}\text { Sta- } \\
\text { tion } \\
\text { no. }\end{array}$ & $\begin{array}{c}1 \text { st } \\
\text { per- } \\
\text { centile }\end{array}$ & $\begin{array}{c}\text { 2nd } \\
\text { per- } \\
\text { centile }\end{array}$ & $\begin{array}{c}\text { 10th } \\
\text { per- } \\
\text { centile }\end{array}$ & $\begin{array}{c}25 \text { th } \\
\text { per- } \\
\text { centile }\end{array}$ & $\begin{array}{c}\text { 50th } \\
\text { per- } \\
\text { centile } \\
\text { (median) }\end{array}$ & $\begin{array}{c}\text { 75th } \\
\text { per- } \\
\text { centile }\end{array}$ & $\begin{array}{c}\text { 90th } \\
\text { per- } \\
\text { centile }\end{array}$ & $\begin{array}{c}\text { 98th } \\
\text { per- } \\
\text { centile }\end{array}$ & $\begin{array}{c}\text { 99th } \\
\text { per- } \\
\text { centile }\end{array}$ \\
\hline 7718 & -- & 0.03 & 0.08 & 0.15 & 0.45 & 0.92 & 2.53 & 4.97 & -- & 8910 & -- & -- & 0.04 & 0.10 & 0.42 & 1.41 & 2.37 & -- & -- \\
\hline 7745 & 0.01 & .02 & .06 & .15 & .49 & 1.16 & 2.14 & 5.12 & 7.17 & 8911 & 0.01 & 0.01 & .03 & .09 & .32 & .83 & 1.66 & 3.49 & 4.47 \\
\hline 7922 & .01 & .01 & .02 & .06 & .16 & .34 & .75 & 1.41 & 2.00 & 8924 & .02 & .03 & .05 & .10 & .22 & .47 & .91 & 1.66 & 1.86 \\
\hline 7936 & .03 & .04 & .10 & .20 & .50 & 1.20 & 2.15 & 4.36 & 6.25 & 8929 & -- & -- & .04 & .12 & .83 & 1.60 & 6.25 & -- & -- \\
\hline 7943 & .01 & .01 & .02 & .05 & .19 & .58 & 1.27 & 2.68 & 3.54 & 8942 & .04 & .06 & .10 & .20 & .50 & 1.14 & 2.20 & 4.70 & 5.50 \\
\hline 7944 & -- & .01 & .01 & .04 & .27 & .74 & 1.61 & 3.14 & -- & 8944 & .01 & .02 & .04 & .12 & .38 & .97 & 1.92 & 4.01 & 5.22 \\
\hline 7945 & .01 & .01 & .01 & .05 & .21 & .72 & 1.56 & 3.83 & 5.07 & 8996 & .02 & .02 & .05 & .12 & .37 & .98 & 1.89 & 3.99 & 5.31 \\
\hline 7947 & .05 & .05 & .10 & .10 & .40 & 1.02 & 1.97 & 5.45 & 6.03 & 9014 & -- & -- & .14 & .38 & .74 & 1.40 & 2.42 & -- & -- \\
\hline 7948 & .02 & .02 & .04 & .10 & .30 & .82 & 1.83 & 4.04 & 4.55 & 9037 & .02 & .02 & .05 & .09 & .20 & .43 & .91 & 2.05 & 2.67 \\
\hline 7951 & .02 & .04 & .07 & .19 & .45 & 1.11 & 1.93 & 3.58 & 4.50 & 9106 & .02 & .02 & .03 & .06 & .14 & .42 & .83 & 2.09 & 5.10 \\
\hline 7953 & -- & .02 & .05 & .09 & .23 & .68 & 1.75 & 3.89 & -- & 9107 & -- & -- & .05 & .08 & .20 & .79 & .90 & -- & -- \\
\hline 7981 & .02 & .02 & .05 & .10 & .29 & .84 & 1.99 & 3.69 & 4.01 & 9129 & -- & .02 & .04 & .05 & .16 & .48 & 1.36 & 3.53 & -- \\
\hline 7990 & -- & .01 & .03 & .07 & .20 & .65 & 2.12 & 4.58 & -- & 9163 & .02 & .03 & .07 & .11 & .35 & .80 & 1.70 & 3.30 & 4.19 \\
\hline 7992 & -- & -- & .09 & .12 & .50 & 1.77 & 2.33 & -- & -- & 9213 & .01 & .01 & .02 & .09 & .34 & .88 & 1.72 & 4.33 & 5.10 \\
\hline 7997 & -- & .02 & .04 & .10 & .28 & .67 & 1.60 & 4.00 & -- & 9214 & -- & -- & .14 & .29 & .43 & 1.63 & 4.03 & -- & -- \\
\hline 7999 & -- & -- & .02 & .04 & .18 & .52 & 1.38 & -- & -- & 9222 & .02 & .02 & .04 & .12 & .32 & .98 & 1.83 & 4.35 & 4.89 \\
\hline 8022 & -- & .05 & .05 & .07 & .21 & .92 & 1.58 & 2.86 & -- & 9248 & .02 & .03 & .06 & .10 & .28 & .82 & 1.33 & 2.82 & 3.24 \\
\hline 8023 & .03 & .04 & .08 & .10 & .28 & .70 & 1.36 & 2.85 & 3.74 & 9266 & -- & .02 & .05 & .17 & .28 & 1.00 & 1.56 & 2.93 & -- \\
\hline 8047 & .02 & .03 & .08 & .12 & .40 & .95 & 1.75 & 3.52 & 4.38 & 9270 & .03 & .04 & .10 & .10 & .20 & .50 & 1.00 & 2.20 & 2.93 \\
\hline 8060 & .01 & .02 & .03 & .06 & .18 & .71 & 1.93 & 3.51 & 3.68 & 9295 & -- & .01 & .02 & .04 & .12 & .30 & .68 & 1.57 & -- \\
\hline 8062 & -- & -- & .01 & .04 & .31 & 1.04 & 3.21 & -- & -- & 9304 & -- & -- & -- & -- & .24 & -- & -- & -- & -- \\
\hline 8068 & -- & -- & .02 & .05 & .14 & .40 & 1.23 & -- & -- & 9307 & .03 & .04 & .10 & .15 & .40 & .80 & 1.75 & 3.22 & 3.52 \\
\hline 8081 & .02 & .03 & .07 & .10 & .30 & .85 & 1.70 & 3.87 & 5.60 & 9328 & .01 & .02 & .05 & .10 & .29 & .68 & 1.27 & 2.92 & 3.12 \\
\hline 8089 & -- & -- & .03 & .10 & .30 & .80 & 1.62 & -- & -- & 9329 & -- & -- & .05 & .10 & .65 & 1.09 & 2.10 & -- & -- \\
\hline 8221 & -- & -- & .10 & .27 & .62 & 1.13 & 2.31 & -- & -- & 9345 & -- & -- & -- & -- & .75 & -- & -- & -- & -- \\
\hline 8252 & .02 & .03 & .07 & .10 & .30 & .70 & 1.30 & 2.60 & 3.54 & 9363 & .01 & .01 & .02 & .05 & .21 & .70 & 1.52 & 3.67 & 5.18 \\
\hline 8265 & .02 & .03 & .05 & .14 & .41 & 1.08 & 2.16 & 4.97 & 7.14 & 9364 & .01 & .01 & .02 & .05 & .22 & .78 & 1.73 & 3.96 & 5.92 \\
\hline 8289 & -- & -- & .05 & .15 & .45 & .93 & 1.13 & -- & -- & 9365 & -- & -- & .07 & .10 & .25 & .45 & 1.76 & -- & -- \\
\hline 8305 & .02 & .03 & .05 & .10 & .20 & .40 & .84 & 1.90 & 2.49 & 9371 & .04 & .04 & .07 & .12 & .38 & 1.17 & 2.09 & 3.01 & 3.56 \\
\hline 8335 & .03 & .03 & .07 & .18 & .54 & 1.20 & 2.12 & 4.25 & 4.90 & 9417 & .02 & .02 & .05 & .11 & .37 & 1.00 & 1.85 & 3.33 & 4.31 \\
\hline 8400 & .02 & .02 & .05 & .10 & .25 & .50 & 1.08 & 2.97 & 3.68 & 9419 & .01 & .01 & .02 & .07 & .29 & .85 & 1.66 & 3.27 & 4.25 \\
\hline 8445 & .02 & .03 & .05 & .13 & .39 & 1.03 & 1.93 & 4.12 & 5.40 & 9435 & -- & .02 & .06 & .12 & .36 & .72 & 1.21 & 4.39 & -- \\
\hline 8446 & .02 & .03 & .10 & .10 & .38 & .94 & 1.80 & 3.50 & 4.80 & 9491 & .03 & .03 & .08 & .13 & .40 & 1.04 & 2.00 & 3.74 & 4.80 \\
\hline 8451 & .02 & .02 & .05 & .10 & .25 & .70 & 1.34 & 3.41 & 3.99 & 9499 & .03 & .04 & .10 & .10 & .30 & .80 & 1.50 & 2.82 & 3.40 \\
\hline 8531 & .02 & .03 & .07 & .15 & .40 & 1.00 & 1.77 & 3.40 & 4.05 & 9522 & -- & -- & -- & .21 & .83 & 3.46 & -- & -- & -- \\
\hline 8541 & -- & .01 & .05 & .14 & .41 & .98 & 1.88 & 3.93 & -- & 9527 & .03 & .04 & .10 & .10 & .28 & .60 & 1.30 & 2.69 & 3.60 \\
\hline 8544 & .04 & .05 & .10 & .11 & .32 & 1.01 & 1.90 & 5.19 & 6.60 & 9532 & .02 & .03 & .09 & .10 & .40 & .90 & 1.70 & 3.73 & 4.98 \\
\hline 8545 & -- & -- & .03 & .08 & .38 & .57 & .97 & -- & -- & 9544 & -- & -- & -- & .10 & .24 & 4.34 & -- & -- & -- \\
\hline 8563 & .10 & .10 & .10 & .10 & .40 & 1.00 & 1.90 & 4.30 & 4.85 & 9565 & .02 & .03 & .09 & .10 & .30 & .70 & 1.53 & 3.49 & 4.32 \\
\hline 8566 & .02 & .02 & .05 & .10 & .27 & .78 & 1.48 & 2.73 & 3.45 & 9570 & .10 & .10 & .10 & .10 & .30 & .70 & 1.60 & 2.75 & 3.45 \\
\hline 8583 & .10 & .10 & .10 & .10 & .30 & .90 & 1.50 & 2.99 & 4.08 & 9574 & -- & -- & .10 & .10 & .40 & .90 & 2.20 & -- & -- \\
\hline 8584 & .03 & .04 & .10 & .11 & .37 & .97 & 1.78 & 3.30 & 4.39 & 9588 & .02 & .02 & .06 & .10 & .23 & .72 & 1.70 & 4.05 & 4.86 \\
\hline 8623 & .05 & .06 & .10 & .17 & .40 & 1.00 & 1.80 & 3.34 & 4.44 & 9665 & .03 & .04 & .10 & .15 & .44 & 1.06 & 1.90 & 4.30 & 5.36 \\
\hline 8625 & .02 & .03 & .06 & .12 & .37 & .93 & 1.71 & 3.49 & 4.38 & 9715 & .02 & .03 & .09 & .11 & .40 & .95 & 1.72 & 3.54 & 4.68 \\
\hline 8630 & .02 & .03 & .07 & .10 & .30 & .70 & 1.45 & 2.53 & 2.87 & 9729 & .01 & .01 & .02 & .07 & .28 & .80 & 1.52 & 3.38 & 4.21 \\
\hline 8631 & .02 & .04 & .06 & .11 & .31 & .72 & 1.60 & 3.33 & 4.13 & 9772 & .01 & .02 & .04 & .10 & .31 & .89 & 1.85 & 5.00 & 6.13 \\
\hline 8646 & .02 & .03 & .06 & .12 & .37 & .93 & 1.72 & 3.31 & 4.30 & 9814 & -- & -- & .04 & .10 & .36 & .92 & 1.82 & -- & -- \\
\hline 8647 & .03 & .04 & .10 & .10 & .25 & .62 & 1.20 & 2.67 & 3.53 & 9815 & .03 & .03 & .07 & .15 & .38 & 1.01 & 1.95 & 4.11 & 5.88 \\
\hline 8677 & .01 & .03 & .10 & .17 & .37 & .98 & 1.56 & 3.96 & 5.27 & 9816 & -- & .04 & .05 & .10 & .28 & .81 & 2.11 & 4.51 & -- \\
\hline 8696 & -- & -- & .03 & .18 & .42 & 1.44 & 3.18 & -- & -- & 9817 & .04 & .06 & .10 & .10 & .40 & .81 & 1.60 & 3.40 & 4.40 \\
\hline 8743 & .02 & .04 & .08 & .15 & .49 & 1.10 & 2.10 & 4.49 & 5.42 & 9829 & .02 & .02 & .05 & .10 & .20 & .50 & 1.04 & 2.29 & 2.92 \\
\hline 8761 & .02 & .04 & .10 & .10 & .24 & .60 & 1.30 & 2.49 & 3.51 & 9830 & .01 & .01 & .03 & .07 & .13 & .42 & .92 & 1.88 & 2.42 \\
\hline 8778 & .03 & .05 & .10 & .19 & .43 & 1.01 & 2.00 & 3.80 & 4.60 & 9858 & .02 & .02 & .05 & .11 & .28 & .65 & 1.33 & 2.59 & 3.28 \\
\hline 8845 & .03 & .04 & .09 & .10 & .33 & .96 & 1.99 & 4.20 & 5.56 & 9893 & .02 & .03 & .06 & .10 & .35 & .82 & 1.70 & 3.30 & 4.40 \\
\hline 8859 & .02 & .03 & .08 & .19 & .51 & 1.15 & 2.02 & 4.10 & 4.83 & 9916 & .03 & .05 & .10 & .20 & .50 & 1.20 & 2.18 & 4.00 & 4.90 \\
\hline 8898 & .03 & .03 & .08 & .16 & .45 & 1.03 & 2.09 & 4.02 & 5.61 & 9976 & .02 & .03 & .06 & .10 & .30 & .79 & 1.50 & 3.20 & 4.44 \\
\hline 8908 & -- & -- & .06 & .12 & .46 & 1.05 & 1.71 & -- & -- & & & & & & & & & & \\
\hline
\end{tabular}


Appendix 4-4.6. Empirical distribution of storm depth defined by 48-hour minimum interevent time for hourly rainfall stations in Texas.

$[--$, not available $]$

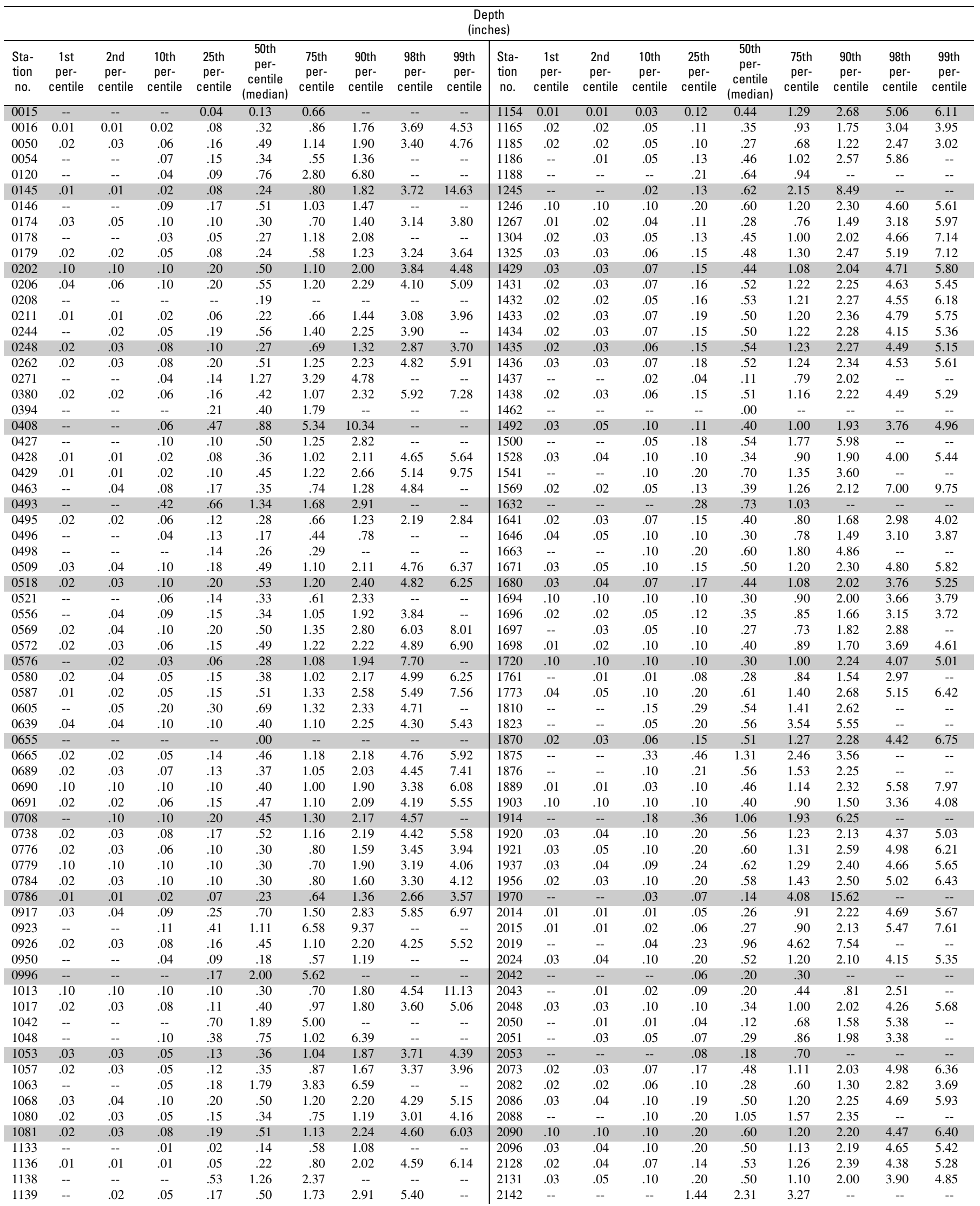


264 Statistical Characteristics of Storm Interevent Time, Depth, and Duration for Eastern New Mexico, Oklahoma, and Texas

Appendix 4-4.6. Empirical distribution of storm depth defined by 48-hour minimum interevent time for hourly rainfall stations in TexasContinued.

\begin{tabular}{|c|c|c|c|c|c|c|c|c|c|c|c|c|c|c|c|c|c|c|c|}
\hline \multicolumn{20}{|c|}{$\begin{array}{c}\text { Depth } \\
\text { (inches) }\end{array}$} \\
\hline $\begin{array}{l}\text { Sta- } \\
\text { tion } \\
\text { no. }\end{array}$ & $\begin{array}{c}1 \text { st } \\
\text { per- } \\
\text { centile }\end{array}$ & $\begin{array}{c}\text { 2nd } \\
\text { per- } \\
\text { centile }\end{array}$ & $\begin{array}{c}10 \text { th } \\
\text { per- } \\
\text { centile }\end{array}$ & $\begin{array}{c}25 \text { th } \\
\text { per- } \\
\text { centile }\end{array}$ & $\begin{array}{c}\text { 50th } \\
\text { per- } \\
\text { centile } \\
\text { (median) }\end{array}$ & $\begin{array}{c}\text { 75th } \\
\text { per- } \\
\text { centile }\end{array}$ & $\begin{array}{c}\text { 90th } \\
\text { per- } \\
\text { centile }\end{array}$ & $\begin{array}{c}\text { 98th } \\
\text { per- } \\
\text { centile }\end{array}$ & $\begin{array}{c}\text { 99th } \\
\text { per- } \\
\text { centile }\end{array}$ & $\begin{array}{l}\text { Sta- } \\
\text { tion } \\
\text { no. }\end{array}$ & $\begin{array}{c}\text { 1st } \\
\text { per- } \\
\text { centile }\end{array}$ & $\begin{array}{c}\text { 2nd } \\
\text { per- } \\
\text { centile }\end{array}$ & $\begin{array}{c}10 \text { th } \\
\text { per- } \\
\text { centile }\end{array}$ & $\begin{array}{c}\text { 25th } \\
\text { per- } \\
\text { centile }\end{array}$ & $\begin{array}{c}\text { 50th } \\
\text { per- } \\
\text { centile } \\
\text { (median) }\end{array}$ & $\begin{array}{c}\text { 75th } \\
\text { per- } \\
\text { centile }\end{array}$ & $\begin{array}{c}\text { 90th } \\
\text { per- } \\
\text { centile }\end{array}$ & $\begin{array}{c}\text { 98th } \\
\text { per- } \\
\text { centile }\end{array}$ & $\begin{array}{c}\text { 99th } \\
\text { per- } \\
\text { centile }\end{array}$ \\
\hline 2160 & -- & -- & 0.02 & 0.08 & 0.29 & 0.88 & 2.65 & -- & -- & 3463 & -- & -- & 0.04 & 0.10 & 0.34 & 1.02 & 2.15 & -- & -- \\
\hline 2206 & 0.03 & 0.03 & .06 & .17 & .55 & 1.48 & 2.59 & 5.92 & 7.94 & 3476 & 0.02 & 0.02 & .06 & .15 & .45 & 1.06 & 2.14 & 4.10 & 6.23 \\
\hline 2238 & .01 & .01 & .02 & .05 & .20 & .61 & 1.56 & 2.72 & 3.32 & 3485 & -- & -- & .09 & .10 & .46 & 2.23 & 6.21 & -- & -- \\
\hline 2240 & -- & .01 & .02 & .09 & .31 & .79 & 1.78 & 2.81 & -- & 3507 & .05 & .07 & .10 & .20 & .50 & 1.30 & 2.50 & 5.00 & 5.60 \\
\hline 2242 & .01 & .01 & .03 & .12 & .47 & 1.11 & 2.02 & 3.98 & 5.18 & 3546 & .03 & .03 & .10 & .20 & .60 & 1.40 & 2.74 & 5.01 & 6.47 \\
\hline 2244 & .01 & .01 & .05 & .15 & .50 & 1.20 & 2.30 & 4.61 & 6.19 & 3547 & .02 & .03 & .07 & .17 & .50 & 1.32 & 2.03 & 3.38 & 5.73 \\
\hline 2247 & -- & -- & .03 & .17 & .47 & 1.34 & 3.04 & -- & -- & 3579 & -- & .05 & .11 & .23 & .60 & 1.61 & 2.29 & 3.76 & -- \\
\hline 2309 & .05 & .05 & .13 & .30 & .72 & 1.44 & 2.42 & 5.64 & 7.40 & 3642 & .03 & .04 & .10 & .20 & .52 & 1.25 & 2.30 & 4.68 & 5.62 \\
\hline 2312 & .10 & .10 & .10 & .30 & .60 & 1.30 & 2.48 & 4.70 & 5.40 & 3646 & .03 & .03 & .07 & .17 & .47 & 1.10 & 2.00 & 4.39 & 5.66 \\
\hline 2334 & -- & -- & .07 & .31 & .89 & 1.78 & 4.00 & -- & -- & 3668 & -- & -- & -- & 1.14 & 2.44 & 3.20 & -- & -- & -- \\
\hline 2336 & .02 & .04 & .10 & .20 & .52 & 1.30 & 2.25 & 3.73 & 3.97 & 3673 & -- & -- & .04 & .09 & .97 & 3.12 & 9.65 & -- & -- \\
\hline 2354 & -- & -- & .04 & .10 & .24 & .85 & 1.47 & -- & -- & 3686 & .05 & .08 & .10 & .20 & .59 & 1.26 & 2.27 & 4.16 & 5.96 \\
\hline 2355 & -- & -- & .04 & .10 & .47 & 1.02 & 3.02 & -- & -- & 3691 & .02 & .04 & .10 & .20 & .50 & 1.14 & 2.20 & 4.30 & 5.40 \\
\hline 2357 & .01 & .01 & .01 & .04 & .20 & .76 & 1.66 & 4.22 & 5.49 & 3734 & -- & -- & .03 & .12 & 1.43 & 4.66 & 16.03 & -- & -- \\
\hline 2360 & .01 & .01 & .02 & .05 & .21 & .73 & 1.59 & 3.71 & 4.83 & 3771 & .10 & .10 & .10 & .20 & .50 & 1.30 & 2.40 & 4.50 & 5.18 \\
\hline 2361 & .02 & .02 & .05 & .10 & .29 & .70 & 1.64 & 3.37 & 17.21 & 3789 & -- & .01 & .04 & .07 & .19 & .45 & .74 & 2.27 & -- \\
\hline 2394 & .02 & .03 & .07 & .17 & .50 & 1.26 & 2.37 & 4.75 & 5.72 & 3826 & .01 & .03 & .10 & .22 & .47 & 1.02 & 1.74 & 4.17 & 5.56 \\
\hline 2404 & .02 & .03 & .07 & .16 & .46 & 1.12 & 2.15 & 4.38 & 5.62 & 3831 & .03 & .04 & .06 & .18 & .51 & 1.40 & 2.45 & 3.98 & 5.65 \\
\hline 2415 & .03 & .04 & .10 & .22 & .61 & 1.40 & 2.66 & 5.15 & 6.24 & 3841 & .02 & .03 & .10 & .20 & .60 & 1.23 & 2.29 & 5.07 & 5.82 \\
\hline 2462 & .04 & .05 & .10 & .22 & .56 & 1.33 & 2.56 & 4.83 & 6.37 & 3871 & .03 & .03 & .06 & .15 & .42 & 1.00 & 1.83 & 3.86 & 4.71 \\
\hline 2528 & -- & .03 & .07 & .15 & .40 & 1.21 & 2.17 & 2.77 & -- & 3884 & -- & -- & .06 & .12 & .94 & 2.88 & 4.19 & -- & -- \\
\hline 2617 & .02 & .02 & .06 & .12 & .38 & .78 & 1.49 & 4.77 & 5.54 & 3941 & -- & -- & .05 & .08 & .92 & 2.02 & 2.42 & -- & -- \\
\hline 2619 & .02 & .03 & .07 & .15 & .39 & .86 & 1.64 & 3.43 & 4.18 & 3963 & -- & -- & -- & .02 & .06 & .30 & -- & -- & -- \\
\hline 2621 & .02 & .04 & .08 & .13 & .36 & .88 & 1.80 & 3.71 & 4.34 & 4040 & .03 & .04 & .07 & .15 & .47 & .96 & 1.76 & 4.16 & 4.79 \\
\hline 2675 & .04 & .05 & .10 & .19 & .50 & 1.10 & 2.30 & 5.35 & 6.48 & 4058 & -- & -- & .08 & .15 & .59 & 1.14 & 9.52 & -- & -- \\
\hline 2676 & .10 & .10 & .10 & .20 & .50 & 1.30 & 2.50 & 4.62 & 7.00 & 4098 & .03 & .04 & .10 & .10 & .30 & .70 & 1.40 & 2.80 & 3.40 \\
\hline 2679 & .02 & .02 & .06 & .10 & .30 & .90 & 2.00 & 4.53 & 6.11 & 4100 & .02 & .04 & .05 & .13 & .39 & .90 & 1.66 & 4.91 & 5.25 \\
\hline 2715 & .03 & .04 & .08 & .19 & .50 & 1.06 & 2.00 & 4.00 & 4.95 & 4137 & .10 & .10 & .10 & .20 & .50 & 1.20 & 2.10 & 4.40 & 5.40 \\
\hline 2744 & .03 & .03 & .09 & .14 & .40 & .95 & 1.75 & 3.57 & 4.47 & 4191 & .03 & .04 & .10 & .12 & .40 & 1.02 & 2.20 & 4.26 & 5.54 \\
\hline 2758 & -- & .02 & .03 & .06 & .20 & .71 & 1.77 & 5.57 & -- & 4256 & -- & -- & -- & -- & .00 & -- & -- & -- & -- \\
\hline 2794 & -- & -- & .01 & .04 & .23 & .84 & 1.65 & -- & -- & 4257 & .05 & .06 & .10 & .20 & .62 & 1.40 & 2.60 & 5.18 & 7.04 \\
\hline 2797 & .01 & .01 & .02 & .04 & .14 & .37 & .77 & 1.84 & 2.34 & 4258 & .10 & .10 & .10 & .20 & .70 & 1.45 & 2.80 & 5.96 & 8.36 \\
\hline 2811 & .02 & .03 & .10 & .10 & .32 & .94 & 1.80 & 3.80 & 5.19 & 4278 & .02 & .03 & .06 & .15 & .45 & 1.14 & 2.24 & 4.37 & 5.63 \\
\hline 2813 & -- & -- & .05 & .20 & .53 & 1.38 & 2.40 & -- & -- & 4299 & .03 & .04 & .06 & .13 & .30 & .63 & .98 & 1.28 & 1.53 \\
\hline 2814 & -- & -- & .01 & .03 & .07 & .44 & 1.93 & -- & -- & 4300 & .01 & .01 & .03 & .12 & .46 & 1.28 & 2.60 & 5.42 & 7.71 \\
\hline 2815 & .10 & .10 & .10 & .20 & .40 & .80 & 2.03 & 3.95 & 5.01 & 4305 & .01 & .01 & .02 & .09 & .45 & 1.23 & 2.56 & 5.55 & 6.90 \\
\hline 2818 & .02 & .02 & .06 & .13 & .40 & 1.01 & 1.96 & 4.97 & 6.28 & 4307 & .01 & .01 & .03 & .11 & .43 & 1.18 & 2.53 & 7.43 & 9.34 \\
\hline 2986 & .05 & .05 & .10 & .25 & .81 & 1.71 & 2.60 & 5.41 & 8.53 & 4309 & .02 & .03 & .06 & .17 & .55 & 1.36 & 2.60 & 5.41 & 7.07 \\
\hline 3005 & .03 & .04 & .10 & .18 & .50 & 1.10 & 1.90 & 4.03 & 4.88 & 4311 & .02 & .03 & .07 & .20 & .59 & 1.47 & 2.74 & 5.31 & 6.79 \\
\hline 3033 & .02 & .02 & .04 & .08 & .19 & .41 & .84 & 1.81 & 2.56 & 4313 & .03 & .04 & .12 & .28 & .67 & 1.60 & 2.79 & 5.50 & 9.51 \\
\hline 3034 & -- & -- & -- & -- & .16 & -- & -- & -- & -- & 4319 & -- & .05 & .12 & .19 & .53 & 1.09 & 3.00 & 3.96 & -- \\
\hline 3047 & -- & -- & .06 & .13 & .77 & 1.58 & 8.84 & -- & -- & 4329 & .03 & .03 & .09 & .20 & .56 & 1.34 & 2.51 & 5.31 & 6.77 \\
\hline 3103 & -- & -- & .12 & .20 & .30 & 1.27 & 3.41 & -- & -- & 4331 & -- & -- & -- & -- & .00 & -- & -- & -- & -- \\
\hline 3133 & .02 & .03 & .09 & .20 & .56 & 1.25 & 2.30 & 4.82 & 5.84 & 4375 & .10 & .10 & .10 & .20 & .40 & 1.10 & 2.13 & 5.00 & 6.78 \\
\hline 3156 & .04 & .05 & .10 & .20 & .56 & 1.23 & 2.44 & 6.23 & 11.53 & 4392 & .03 & .03 & .07 & .21 & .64 & 1.50 & 3.15 & 7.06 & 7.30 \\
\hline 3171 & .03 & .04 & .09 & .19 & .51 & 1.19 & 2.22 & 4.70 & 5.61 & 4425 & .03 & .03 & .10 & .10 & .30 & .70 & 1.47 & 2.74 & 3.40 \\
\hline 3189 & .03 & .04 & .05 & .10 & .22 & .57 & 1.16 & 2.95 & 4.46 & 4440 & .02 & .03 & .05 & .14 & .41 & 1.00 & 1.84 & 4.04 & 4.91 \\
\hline 3260 & .01 & .01 & .05 & .13 & .35 & 1.09 & 2.28 & 3.81 & 4.31 & 4476 & .04 & .06 & .10 & .20 & .50 & 1.10 & 2.10 & 4.20 & 5.00 \\
\hline 3267 & -- & .02 & .04 & .09 & .23 & .68 & 1.69 & 6.00 & -- & 4498 & -- & -- & -- & .09 & .25 & .46 & -- & -- & -- \\
\hline 3270 & .05 & .10 & .10 & .10 & .40 & .90 & 1.60 & 3.60 & 4.79 & 4517 & .02 & .03 & .06 & .16 & .47 & 1.10 & 2.15 & 4.08 & 4.94 \\
\hline 3272 & -- & -- & .01 & .04 & .14 & .36 & .54 & -- & -- & 4520 & .10 & .10 & .10 & .20 & .50 & 1.10 & 2.10 & 4.10 & 4.84 \\
\hline 3277 & -- & -- & .02 & .03 & .14 & .24 & .75 & -- & -- & 4525 & -- & -- & .05 & .10 & .46 & .82 & 10.42 & -- & -- \\
\hline 3278 & .02 & .03 & .07 & .10 & .30 & .80 & 1.58 & 3.23 & 3.80 & 4563 & -- & -- & .04 & .08 & .59 & 1.83 & 3.30 & -- & -- \\
\hline 3280 & .01 & .01 & .03 & .06 & .18 & .56 & 1.21 & 2.19 & 2.94 & 4570 & .02 & .04 & .10 & .13 & .36 & .90 & 1.85 & 4.00 & 4.85 \\
\hline 3281 & -- & -- & .04 & .12 & .28 & .56 & 1.05 & -- & -- & 4577 & .03 & .05 & .10 & .23 & .64 & 1.31 & 2.49 & 4.89 & 6.46 \\
\hline 3283 & .01 & .01 & .02 & .10 & .40 & 1.04 & 2.19 & 4.86 & 5.70 & 4591 & .02 & .03 & .06 & .17 & .51 & 1.22 & 2.35 & 4.46 & 6.06 \\
\hline 3284 & .01 & .02 & .10 & .20 & .50 & 1.15 & 2.17 & 4.35 & 5.56 & 4670 & .02 & .03 & .09 & .10 & .40 & .90 & 1.70 & 3.40 & 4.59 \\
\hline 3285 & .05 & .10 & .10 & .20 & .50 & 1.20 & 2.29 & 4.34 & 5.57 & 4671 & -- & .01 & .03 & .07 & .31 & .90 & 1.76 & 3.21 & -- \\
\hline 3329 & .02 & .03 & .06 & .11 & .40 & 1.02 & 2.04 & 4.16 & 5.89 & 4679 & .04 & .05 & .10 & .19 & .46 & 1.10 & 2.20 & 5.00 & 6.01 \\
\hline 3335 & .03 & .04 & .15 & .26 & .61 & 1.60 & 2.58 & 5.00 & 10.21 & 4696 & -- & -- & -- & .55 & .66 & .97 & -- & -- & -- \\
\hline 3370 & .02 & .03 & .07 & .20 & .57 & 1.25 & 2.35 & 4.85 & 5.69 & 4703 & -- & .02 & .08 & .15 & .34 & .90 & 1.55 & 3.90 & -- \\
\hline 3410 & .04 & .05 & .10 & .14 & .40 & .88 & 1.74 & 3.35 & 3.98 & 4704 & .03 & .03 & .08 & .23 & .65 & 1.64 & 3.21 & 5.38 & 7.95 \\
\hline 3415 & .02 & .04 & .10 & .20 & .50 & 1.20 & 2.20 & 4.79 & 5.69 & 4731 & -- & .01 & .02 & .06 & .27 & .77 & 1.86 & 5.94 & -- \\
\hline 3430 & .01 & .01 & .03 & .10 & .40 & 1.15 & 2.39 & 5.72 & 7.21 & 4792 & .10 & .10 & .10 & .20 & .50 & 1.20 & 2.20 & 4.20 & 4.90 \\
\hline 3431 & .01 & .01 & .04 & .09 & .37 & 1.15 & 2.73 & 7.47 & 12.96 & 4819 & .10 & .10 & .10 & .30 & .80 & 1.50 & 2.70 & 4.30 & 4.46 \\
\hline 3441 & -- & -- & .10 & .22 & .50 & 1.13 & 2.92 & -- & -- & 4852 & -- & -- & .07 & .37 & .80 & 2.33 & 3.66 & -- & -- \\
\hline 3442 & .02 & .03 & .05 & .11 & .29 & .67 & 1.61 & 2.56 & 3.16 & 4866 & .03 & .04 & .10 & .20 & .52 & 1.23 & 2.24 & 4.31 & 5.51 \\
\hline 3446 & .03 & .04 & .06 & .14 & .37 & .81 & 1.37 & 2.73 & 3.48 & 4876 & -- & .10 & .10 & .20 & .70 & 1.30 & 3.30 & 6.58 & -- \\
\hline 3460 & -- & -- & .04 & .12 & 1.07 & 1.48 & 2.06 & -- & -- & 4878 & .03 & .04 & .10 & .25 & 69 & 1.60 & 3.13 & 6.19 & 7.21 \\
\hline 3462 & .05 & .05 & .10 & .18 & .46 & .90 & 1.38 & 4.23 & 5.93 & 4880 & .02 & .03 & .07 & .10 & .30 & .79 & 1.50 & 3.40 & 4.03 \\
\hline
\end{tabular}


Appendix 4-4.6. Empirical distribution of storm depth defined by 48-hour minimum interevent time for hourly rainfall stations in TexasContinued.

\begin{tabular}{|c|c|c|c|c|c|c|c|c|c|c|c|c|c|c|c|c|c|c|c|}
\hline \multicolumn{20}{|c|}{$\begin{array}{c}\text { Depth } \\
\text { (inches) }\end{array}$} \\
\hline $\begin{array}{l}\text { Sta- } \\
\text { tion } \\
\text { no. }\end{array}$ & $\begin{array}{c}\text { 1st } \\
\text { per- } \\
\text { centile }\end{array}$ & $\begin{array}{c}\text { 2nd } \\
\text { per- } \\
\text { centile }\end{array}$ & $\begin{array}{c}\text { 10th } \\
\text { per- } \\
\text { centile }\end{array}$ & $\begin{array}{c}25 \text { th } \\
\text { per- } \\
\text { centile }\end{array}$ & $\begin{array}{c}\text { 50th } \\
\text { per- } \\
\text { centile } \\
\text { (median) }\end{array}$ & $\begin{array}{c}\text { 75th } \\
\text { per- } \\
\text { centile }\end{array}$ & $\begin{array}{c}\text { 90th } \\
\text { per- } \\
\text { centile }\end{array}$ & $\begin{array}{c}\text { 98th } \\
\text { per- } \\
\text { centile }\end{array}$ & $\begin{array}{c}\text { 99th } \\
\text { per- } \\
\text { centile }\end{array}$ & $\begin{array}{l}\text { Sta- } \\
\text { tion } \\
\text { no. }\end{array}$ & $\begin{array}{c}1 \text { st } \\
\text { per- } \\
\text { centile }\end{array}$ & $\begin{array}{c}\text { 2nd } \\
\text { per- } \\
\text { centile }\end{array}$ & $\begin{array}{c}10 \text { th } \\
\text { per- } \\
\text { centile }\end{array}$ & $\begin{array}{c}25 \text { th } \\
\text { per- } \\
\text { centile }\end{array}$ & $\begin{array}{c}\text { 50th } \\
\text { per- } \\
\text { centile } \\
\text { (median) }\end{array}$ & $\begin{array}{c}\text { 75th } \\
\text { per- } \\
\text { centile }\end{array}$ & $\begin{array}{c}\text { 90th } \\
\text { per- } \\
\text { centile }\end{array}$ & $\begin{array}{c}\text { 98th } \\
\text { per- } \\
\text { centile }\end{array}$ & $\begin{array}{c}\text { 99th } \\
\text { per- } \\
\text { centile }\end{array}$ \\
\hline 4920 & 0.03 & 0.03 & 0.10 & 0.11 & 0.38 & 1.00 & 1.90 & 4.39 & 5.47 & 5957 & 0.03 & 0.04 & 0.10 & 0.20 & 0.50 & 1.10 & 2.10 & 4.36 & 5.65 \\
\hline 4934 & -- & -- & -- & .07 & .15 & 1.02 & -- & -- & -- & 5958 & .01 & .02 & .05 & .17 & .52 & 1.14 & 2.19 & 3.42 & 4.19 \\
\hline 4972 & .03 & .03 & .10 & .19 & .45 & 1.00 & 2.00 & 4.12 & 4.92 & 5973 & -- & .01 & .03 & .07 & .20 & .77 & 2.03 & 4.84 & -- \\
\hline 4973 & .03 & .05 & .10 & .30 & .66 & 1.39 & 2.81 & 5.83 & 7.21 & 5996 & .03 & .04 & .10 & .15 & .48 & 1.10 & 2.06 & 4.12 & 5.25 \\
\hline 4974 & .02 & .03 & .06 & .11 & .35 & .79 & 1.61 & 3.45 & 4.34 & 6017 & -- & .02 & .03 & .07 & .27 & .83 & 2.10 & 4.39 & -- \\
\hline 4975 & .10 & .10 & .10 & .30 & .70 & 1.40 & 2.40 & 5.04 & 5.94 & 6024 & .02 & .03 & .08 & .24 & .67 & 1.75 & 2.90 & 6.26 & 8.23 \\
\hline 4978 & .02 & .02 & .04 & .11 & .40 & 1.04 & 2.31 & 4.95 & 5.49 & 6050 & -- & -- & .17 & .24 & .50 & 1.20 & 2.02 & -- & -- \\
\hline 4979 & -- & -- & -- & .32 & 1.85 & 2.69 & -- & -- & -- & 6104 & .02 & .03 & .06 & .10 & .30 & .70 & 1.50 & 3.47 & 4.20 \\
\hline 4982 & .02 & .03 & .06 & .14 & .39 & .95 & 1.87 & 3.55 & 5.09 & 6108 & .03 & .04 & .10 & .20 & .70 & 1.50 & 2.69 & 5.40 & 6.37 \\
\hline 5018 & .02 & .03 & .07 & .17 & .49 & 1.19 & 1.98 & 4.03 & 4.92 & 6136 & .03 & .04 & .10 & .10 & .30 & .70 & 1.40 & 2.86 & 3.40 \\
\hline 5048 & .02 & .04 & .10 & .10 & .30 & .80 & 1.47 & 3.39 & 4.75 & 6166 & -- & .03 & .05 & .10 & .32 & .87 & 1.54 & 2.73 & -- \\
\hline 5049 & -- & .10 & .10 & .10 & .30 & .70 & 1.30 & 4.95 & -- & 6176 & .02 & .02 & .08 & .26 & .58 & 1.19 & 2.63 & 6.68 & 7.26 \\
\hline 5056 & -- & -- & -- & .14 & .39 & .82 & -- & -- & -- & 6177 & .04 & .05 & .10 & .20 & .60 & 1.40 & 2.70 & 5.40 & 7.29 \\
\hline 5057 & .01 & .01 & .02 & .05 & .19 & .59 & 1.56 & 3.17 & 3.85 & 6210 & .03 & .05 & .10 & .20 & .58 & 1.30 & 2.40 & 4.74 & 5.95 \\
\hline 5060 & .01 & .01 & .02 & .07 & .30 & .90 & 2.40 & 6.16 & 7.12 & 6211 & .01 & .02 & .05 & .17 & .60 & 1.43 & 2.61 & 4.70 & 6.25 \\
\hline 5081 & .02 & .03 & .07 & .22 & .64 & 1.39 & 2.40 & 4.72 & 6.42 & 6270 & .10 & .10 & .10 & .30 & .70 & 1.50 & 2.70 & 5.10 & 7.00 \\
\hline 5094 & .02 & .03 & .10 & .20 & .50 & 1.20 & 2.30 & 4.70 & 5.83 & 6275 & -- & -- & -- & -- & .00 & -- & -- & -- & -- \\
\hline 5113 & .03 & .04 & .10 & .11 & .40 & 1.00 & 2.23 & 4.89 & 6.44 & 6276 & -- & -- & .06 & .18 & 1.39 & 3.38 & 8.32 & -- & -- \\
\hline 5114 & -- & -- & -- & -- & .00 & -- & -- & -- & -- & 6335 & .03 & .03 & .08 & .20 & .55 & 1.27 & 2.28 & 4.40 & 5.24 \\
\hline 5123 & -- & -- & .07 & .23 & .33 & 1.62 & 2.70 & -- & -- & 6434 & -- & -- & .10 & .23 & .70 & 1.12 & 1.89 & -- & -- \\
\hline 5192 & .02 & .03 & .10 & .20 & .50 & 1.25 & 2.28 & 4.89 & 5.63 & 6504 & .02 & .03 & .10 & .10 & .30 & .80 & 1.60 & 3.32 & 4.17 \\
\hline 5193 & .03 & .04 & .10 & .15 & .50 & 1.20 & 2.29 & 4.70 & 5.90 & 6558 & -- & -- & .05 & .06 & .60 & .99 & 1.89 & -- & -- \\
\hline 5224 & .01 & .02 & .07 & .23 & .67 & 1.34 & 2.83 & 7.29 & 8.45 & 6615 & .05 & .10 & .10 & .10 & .40 & .87 & 1.70 & 4.66 & 6.08 \\
\hline 5228 & .02 & .03 & .05 & .12 & .49 & 1.24 & 2.20 & 5.00 & 5.31 & 6660 & -- & .03 & .05 & .22 & .52 & 1.17 & 2.39 & 5.08 & -- \\
\hline 5235 & -- & -- & .05 & .14 & .57 & .97 & 2.92 & -- & -- & 6663 & -- & .01 & .10 & .20 & .62 & 1.50 & 1.98 & 5.14 & -- \\
\hline 5247 & .02 & .03 & .10 & .10 & .35 & .80 & 1.60 & 3.10 & 3.70 & 6734 & .01 & .02 & .05 & .12 & .34 & .90 & 1.74 & 3.39 & 4.84 \\
\hline 5258 & .02 & .03 & .08 & .19 & .48 & 1.13 & 2.21 & 4.63 & 5.57 & 6736 & .03 & .04 & .10 & .10 & .30 & .80 & 1.60 & 3.59 & 4.42 \\
\hline 5303 & .02 & .03 & .05 & .13 & .35 & 1.01 & 1.90 & 4.57 & 7.00 & 6740 & -- & -- & .08 & .20 & 1.07 & 3.43 & 4.17 & -- & -- \\
\hline 5312 & .04 & .05 & .10 & .12 & .40 & 1.00 & 2.00 & 4.29 & 6.08 & 6750 & .01 & .01 & .02 & .06 & .31 & 1.29 & 2.78 & 9.57 & 10.27 \\
\hline 5341 & -- & -- & .09 & .27 & .62 & 3.49 & 12.51 & -- & -- & 6757 & .01 & .02 & .07 & .19 & .55 & 1.31 & 2.40 & 4.79 & 5.89 \\
\hline 5342 & -- & -- & -- & -- & .00 & -- & -- & -- & -- & 6775 & .01 & .02 & .05 & .12 & .32 & .85 & 1.58 & 2.89 & 4.58 \\
\hline 5348 & .04 & .06 & .10 & .20 & .70 & 1.50 & 2.79 & 5.00 & 5.60 & 6776 & .02 & .04 & .10 & .10 & .35 & .80 & 1.50 & 3.43 & 4.42 \\
\hline 5358 & .02 & .03 & .06 & .12 & .36 & .90 & 1.79 & 3.46 & 4.19 & 6788 & .02 & .03 & .07 & .24 & .55 & 1.25 & 2.64 & 4.69 & 5.54 \\
\hline 5398 & .03 & .03 & .10 & .20 & .60 & 1.33 & 2.45 & 5.00 & 6.38 & 6792 & .02 & .03 & .08 & .10 & .30 & .60 & 1.20 & 2.30 & 3.37 \\
\hline 5410 & .02 & .03 & .08 & .10 & .30 & .80 & 1.50 & 3.70 & 4.27 & 6794 & -- & -- & -- & .57 & 1.24 & 7.54 & -- & -- & -- \\
\hline 5411 & .01 & .01 & .02 & .06 & .22 & .70 & 1.45 & 3.26 & 4.28 & 6834 & .10 & .10 & .10 & .20 & .70 & 1.40 & 2.70 & 5.34 & 6.40 \\
\hline 5424 & .01 & .01 & .02 & .12 & .59 & 1.66 & 3.29 & 7.15 & 9.40 & 6893 & .03 & .03 & .09 & .10 & .20 & .50 & 1.10 & 2.21 & 3.00 \\
\hline 5429 & .02 & .02 & .05 & .13 & .40 & 1.15 & 2.07 & 4.45 & 6.58 & 6935 & .03 & .04 & .10 & .10 & .30 & .80 & 1.50 & 3.30 & 3.87 \\
\hline 5431 & -- & -- & .16 & .39 & .70 & 2.10 & 3.20 & -- & -- & 6981 & .01 & .03 & .08 & .17 & .54 & 1.33 & 2.23 & 5.66 & 5.74 \\
\hline 5461 & .03 & .04 & .08 & .20 & .57 & 1.32 & 2.49 & 5.38 & 6.70 & 7020 & -- & .02 & .04 & .14 & .42 & 1.12 & 2.44 & 5.82 & -- \\
\hline 5463 & .10 & .10 & .10 & .20 & .60 & 1.30 & 2.50 & 5.10 & 6.10 & 7060 & .03 & .05 & .10 & .13 & .39 & .91 & 1.71 & 3.66 & 5.51 \\
\hline 5471 & -- & -- & .03 & .05 & .11 & .48 & 1.13 & -- & -- & 7066 & .03 & .04 & .10 & .20 & .60 & 1.40 & 2.63 & 5.02 & 6.34 \\
\hline 5477 & -- & -- & .10 & .15 & .44 & .91 & 8.73 & -- & -- & 7074 & .02 & .03 & .06 & .10 & .30 & .70 & 1.40 & 3.40 & 4.30 \\
\hline 5528 & .03 & .04 & .09 & .22 & .58 & 1.26 & 2.35 & 4.48 & 5.98 & 7097 & -- & .02 & .11 & .20 & .60 & 1.48 & 2.90 & 5.03 & -- \\
\hline 5579 & -- & -- & -- & -- & .55 & -- & -- & -- & -- & 7116 & .02 & .02 & .05 & .13 & .36 & .82 & 1.45 & 3.62 & 4.84 \\
\hline 5580 & -- & -- & .07 & .32 & 1.01 & 1.84 & 2.18 & -- & -- & 7140 & .02 & .02 & .06 & .14 & .45 & 1.30 & 2.75 & 6.10 & 7.66 \\
\hline 5589 & .01 & .01 & .02 & .09 & .28 & .70 & 1.10 & 1.64 & 1.68 & 7173 & .01 & .01 & .03 & .13 & .56 & 1.42 & 3.10 & 7.30 & 7.89 \\
\hline 5590 & .02 & .02 & .05 & .10 & .29 & .78 & 1.20 & 3.45 & 4.35 & 7174 & .01 & .01 & .03 & .12 & .51 & 1.42 & 2.91 & 6.76 & 8.95 \\
\hline 5591 & .02 & .03 & .05 & .11 & .30 & .64 & 1.22 & 2.23 & 3.24 & 7213 & .02 & .03 & .08 & .20 & .52 & 1.25 & 2.33 & 4.88 & 5.15 \\
\hline 5592 & .02 & .02 & .04 & .09 & .23 & .53 & 1.11 & 2.38 & 2.63 & 7243 & .03 & .04 & .10 & .16 & .41 & 1.04 & 1.90 & 3.90 & 4.60 \\
\hline 5594 & .02 & .03 & .05 & .10 & .20 & .47 & 1.00 & 2.34 & 3.84 & 7262 & -- & .01 & .02 & .04 & .17 & .37 & .61 & 1.82 & -- \\
\hline 5595 & -- & -- & -- & -- & .03 & -- & -- & -- & -- & 7274 & .03 & .05 & .10 & .21 & .47 & 1.10 & 1.90 & 4.03 & 5.77 \\
\hline 5596 & .10 & .10 & .10 & .10 & .30 & .70 & 1.40 & 3.04 & 4.17 & 7300 & .02 & .03 & .06 & .15 & .45 & 1.07 & 2.00 & 3.71 & 5.44 \\
\hline 5600 & .02 & .03 & .05 & .10 & .26 & .70 & 1.12 & 2.89 & 4.05 & 7311 & -- & -- & .11 & .20 & .38 & 1.40 & 1.99 & -- & -- \\
\hline 5618 & -- & -- & .08 & .23 & .81 & 1.78 & 6.56 & -- & -- & 7363 & -- & -- & .05 & .09 & .24 & 1.90 & 6.83 & -- & -- \\
\hline 5650 & -- & -- & .13 & .36 & .50 & 1.45 & 2.43 & -- & -- & 7422 & .02 & .03 & .09 & .13 & .45 & 1.10 & 2.20 & 4.70 & 6.28 \\
\hline 5656 & .08 & .10 & .10 & .10 & .32 & .80 & 1.70 & 3.61 & 4.60 & 7431 & .03 & .03 & .07 & .10 & .30 & .70 & 1.40 & 2.99 & 4.00 \\
\hline 5658 & .02 & .02 & .05 & .12 & .32 & .76 & 1.56 & 3.67 & 5.18 & 7481 & .02 & .02 & .05 & .10 & .20 & .51 & 1.11 & 2.97 & 3.63 \\
\hline 5661 & .10 & .10 & .10 & .10 & .40 & .90 & 2.31 & 6.73 & 11.01 & 7497 & .10 & .10 & .10 & .20 & .50 & 1.20 & 2.10 & 4.46 & 5.99 \\
\hline 5666 & -- & -- & .04 & .10 & .34 & .97 & 3.14 & -- & -- & 7498 & .08 & .10 & .10 & .17 & .50 & 1.30 & 2.10 & 4.83 & 8.40 \\
\hline 5695 & .03 & .04 & .10 & .20 & .57 & 1.20 & 2.25 & 4.70 & 6.23 & 7499 & .05 & .08 & .10 & .20 & .47 & 1.10 & 2.05 & 4.04 & 4.68 \\
\hline 5742 & -- & -- & .04 & .06 & .21 & .36 & 1.86 & -- & -- & 7531 & -- & .03 & .06 & .23 & .48 & 1.24 & 2.12 & 4.64 & -- \\
\hline 5766 & -- & -- & -- & .49 & 1.14 & 4.25 & -- & -- & -- & 7534 & .02 & .03 & .06 & .15 & .45 & 1.05 & 2.04 & 3.58 & 3.95 \\
\hline 5770 & .02 & .03 & .09 & .10 & .35 & .86 & 1.80 & 3.85 & 4.55 & 7556 & .03 & .05 & .10 & .20 & .47 & 1.10 & 2.30 & 4.62 & 5.49 \\
\hline 5775 & -- & -- & -- & .07 & .41 & 1.00 & -- & -- & -- & 7594 & .03 & .04 & .09 & .21 & .60 & 1.38 & 2.55 & 5.37 & 6.93 \\
\hline 5779 & -- & -- & .12 & .19 & .49 & 1.12 & 2.53 & -- & -- & 7596 & .01 & .02 & .05 & .13 & .58 & 1.48 & 2.72 & 4.69 & 5.47 \\
\hline 5840 & .02 & .03 & .05 & .15 & .43 & 1.06 & 2.06 & 4.72 & 5.95 & 7608 & .01 & .01 & .04 & .12 & .42 & 1.19 & 2.19 & 4.35 & 5.89 \\
\hline 5890 & .01 & .01 & .02 & .06 & .20 & .59 & 1.23 & 2.67 & 3.65 & 7622 & -- & -- & .01 & .01 & .10 & .42 & 1.02 & -- & -- \\
\hline 5891 & .02 & .03 & .07 & .12 & .34 & .74 & 1.32 & 2.12 & 2.44 & 7700 & .02 & .02 & .07 & .20 & .59 & 1.39 & 2.57 & 5.07 & 6.56 \\
\hline 5897 & .10 & .10 & .10 & .20 & .60 & 1.30 & 2.40 & 4.56 & 5.72 & 7706 & .02 & .03 & .09 & .11 & .40 & .90 & 1.80 & 3.90 & 5.67 \\
\hline
\end{tabular}


Appendix 4-4.6. Empirical distribution of storm depth defined by 48-hour minimum interevent time for hourly rainfall stations in TexasContinued.

\begin{tabular}{|c|c|c|c|c|c|c|c|c|c|c|c|c|c|c|c|c|c|c|c|}
\hline \multicolumn{20}{|c|}{$\begin{array}{c}\text { Depth } \\
\text { (inches) }\end{array}$} \\
\hline $\begin{array}{l}\text { Sta- } \\
\text { tion } \\
\text { no. }\end{array}$ & $\begin{array}{c}\text { 1st } \\
\text { per- } \\
\text { centile }\end{array}$ & $\begin{array}{c}\text { 2nd } \\
\text { per- } \\
\text { centile }\end{array}$ & $\begin{array}{c}10 \text { th } \\
\text { per- } \\
\text { centile }\end{array}$ & $\begin{array}{c}25 \text { th } \\
\text { per- } \\
\text { centile }\end{array}$ & $\begin{array}{c}\text { 50th } \\
\text { per- } \\
\text { centile } \\
\text { (median) }\end{array}$ & $\begin{array}{c}\text { 75th } \\
\text { per- } \\
\text { centile }\end{array}$ & $\begin{array}{c}\text { 90th } \\
\text { per- } \\
\text { centile }\end{array}$ & $\begin{array}{c}\text { 98th } \\
\text { per- } \\
\text { centile }\end{array}$ & $\begin{array}{c}\text { 99th } \\
\text { per- } \\
\text { centile }\end{array}$ & $\begin{array}{l}\text { Sta- } \\
\text { tion } \\
\text { no. }\end{array}$ & $\begin{array}{c}\text { 1st } \\
\text { per- } \\
\text { centile }\end{array}$ & $\begin{array}{c}\text { 2nd } \\
\text { per- } \\
\text { centile }\end{array}$ & $\begin{array}{c}10 \text { th } \\
\text { per- } \\
\text { centile }\end{array}$ & $\begin{array}{c}\text { 25th } \\
\text { per- } \\
\text { centile }\end{array}$ & $\begin{array}{c}\text { 50th } \\
\text { per- } \\
\text { centile } \\
\text { (median) }\end{array}$ & $\begin{array}{c}\text { 75th } \\
\text { per- } \\
\text { centile }\end{array}$ & $\begin{array}{c}\text { 90th } \\
\text { per- } \\
\text { centile }\end{array}$ & $\begin{array}{c}\text { 98th } \\
\text { per- } \\
\text { centile }\end{array}$ & $\begin{array}{c}\text { 99th } \\
\text { per- } \\
\text { centile }\end{array}$ \\
\hline 7718 & -- & 0.03 & 0.08 & 0.15 & 0.55 & 1.04 & 2.59 & 5.83 & -- & 8910 & -- & -- & 0.04 & 0.14 & 0.42 & 1.77 & 3.78 & -- & -- \\
\hline 7745 & 0.01 & .02 & .07 & .21 & .61 & 1.41 & 2.77 & 5.73 & 7.97 & 8911 & 0.01 & 0.02 & .04 & .12 & .43 & 1.14 & 2.02 & 4.39 & 4.71 \\
\hline 7922 & .01 & .01 & .03 & .08 & .17 & .42 & .81 & 1.48 & 2.28 & 8924 & .02 & .03 & .06 & .10 & .25 & .55 & 1.00 & 1.83 & 2.22 \\
\hline 7936 & .04 & .05 & .10 & .20 & .70 & 1.50 & 2.85 & 6.10 & 7.25 & 8929 & -- & -- & .06 & .12 & .65 & 1.71 & 10.49 & -- & -- \\
\hline 7943 & .01 & .01 & .02 & .06 & .24 & .71 & 1.59 & 3.31 & 4.23 & 8942 & .05 & .08 & .10 & .20 & 69 & 1.42 & 2.70 & 5.60 & 6.64 \\
\hline 7944 & -- & -- & .02 & .12 & .38 & 1.30 & 2.00 & -- & -- & 8944 & .01 & .02 & .05 & .17 & .50 & 1.22 & 2.38 & 5.47 & 6.74 \\
\hline 7945 & .01 & .01 & .02 & .07 & .30 & .93 & 1.99 & 4.78 & 6.01 & 8996 & .02 & .02 & .06 & .16 & .52 & 1.30 & 2.44 & 4.93 & 6.76 \\
\hline 7947 & .05 & .05 & .10 & .20 & .50 & 1.30 & 2.57 & 5.59 & 10.65 & 9014 & -- & -- & .10 & .41 & 1.14 & 2.23 & 5.99 & -- & -- \\
\hline 7948 & .02 & .02 & .05 & .11 & .40 & 1.04 & 2.03 & 4.56 & 6.62 & 9037 & .02 & .03 & .05 & .09 & .21 & .49 & 1.10 & 2.36 & 2.72 \\
\hline 7951 & .02 & .04 & .09 & .22 & .55 & 1.30 & 2.35 & 4.21 & 5.44 & 9106 & .02 & .02 & .03 & .07 & .14 & .48 & .97 & 2.28 & 7.15 \\
\hline 7953 & -- & .02 & .05 & .10 & .24 & .73 & 2.52 & 4.01 & -- & 9107 & -- & -- & .04 & .07 & .20 & .80 & 1.31 & -- & -- \\
\hline 7981 & .02 & .02 & .05 & .12 & .40 & 1.07 & 2.21 & 3.88 & 5.76 & 9129 & -- & .02 & .05 & .05 & .23 & .74 & 1.98 & 3.81 & -- \\
\hline 7990 & -- & .02 & .04 & .07 & .28 & .85 & 3.21 & 4.77 & -- & 9163 & .03 & .04 & .09 & .15 & .40 & 1.00 & 2.03 & 4.08 & 5.07 \\
\hline 7992 & -- & -- & .08 & .18 & 1.23 & 2.26 & 3.57 & -- & -- & 9213 & .01 & .01 & .03 & .14 & .52 & 1.23 & 2.19 & 5.14 & 5.59 \\
\hline 7997 & -- & .02 & .04 & .10 & .36 & .81 & 1.70 & 4.59 & -- & 9214 & -- & -- & .24 & .31 & 1.06 & 2.03 & 10.71 & -- & -- \\
\hline 7999 & -- & -- & .03 & .07 & .24 & .84 & 1.64 & -- & -- & 9222 & .01 & .02 & .06 & .14 & .42 & 1.08 & 2.24 & 4.62 & 4.95 \\
\hline 8022 & -- & -- & .05 & .09 & .24 & .93 & 1.61 & -- & -- & 9248 & -- & .05 & .07 & .13 & .32 & .90 & 1.56 & 3.33 & -- \\
\hline 8023 & .04 & .05 & .09 & .10 & .30 & .80 & 1.54 & 3.31 & 4.19 & 9266 & -- & .03 & .06 & .18 & .33 & 1.10 & 2.13 & 2.97 & -- \\
\hline 8047 & .03 & .04 & .10 & .18 & .48 & 1.13 & 2.10 & 4.19 & 5.30 & 9270 & .03 & .04 & .10 & .10 & .29 & .60 & 1.23 & 3.19 & 3.73 \\
\hline 8060 & -- & .02 & .03 & .07 & .23 & .96 & 2.53 & 4.02 & -- & 9295 & -- & .01 & .02 & .04 & .12 & .34 & .93 & 1.84 & -- \\
\hline 8062 & -- & -- & .02 & .06 & .34 & 1.22 & 3.43 & -- & -- & 9304 & -- & -- & -- & -- & .24 & -- & -- & -- & -- \\
\hline 8068 & -- & -- & .02 & .07 & .26 & .51 & 1.49 & -- & -- & 9307 & .04 & .04 & .10 & .20 & .53 & .96 & 2.07 & 3.65 & 4.01 \\
\hline 8081 & .03 & .04 & .07 & .10 & .38 & 1.01 & 2.00 & 4.90 & 6.81 & 9328 & .01 & .02 & .06 & .11 & .37 & .78 & 1.73 & 3.31 & 3.48 \\
\hline 8089 & -- & -- & .03 & .12 & .44 & .85 & 1.74 & -- & -- & 9329 & -- & -- & -- & .18 & .93 & 1.15 & -- & -- & -- \\
\hline 8221 & -- & -- & .13 & .63 & .98 & 2.03 & 3.84 & -- & -- & 9345 & -- & -- & -- & -- & .75 & -- & -- & -- & -- \\
\hline 8252 & .02 & .03 & .08 & .10 & .30 & .80 & 1.50 & 3.21 & 4.39 & 9363 & .01 & .01 & .02 & .08 & .30 & .94 & 2.13 & 4.38 & 5.36 \\
\hline 8265 & .02 & .03 & .07 & .19 & .57 & 1.42 & 2.73 & 6.51 & 8.65 & 9364 & .01 & .01 & .03 & .08 & .32 & 1.06 & 2.23 & 5.32 & 7.66 \\
\hline 8289 & -- & -- & .14 & .23 & .65 & 1.00 & 1.17 & -- & -- & 9365 & -- & -- & .07 & .10 & .25 & .64 & 2.03 & -- & -- \\
\hline 8305 & .02 & .03 & .06 & .10 & .20 & .50 & 1.02 & 2.20 & 2.95 & 9371 & -- & .04 & .08 & .18 & .57 & 1.33 & 2.40 & 3.58 & -- \\
\hline 8335 & .03 & .03 & .08 & .22 & .69 & 1.44 & 2.59 & 4.73 & 6.08 & 9417 & .02 & .02 & .06 & .16 & .48 & 1.20 & 2.18 & 4.40 & 5.10 \\
\hline 8400 & .02 & .03 & .07 & .12 & .34 & .68 & 1.35 & 3.31 & 4.17 & 9419 & .01 & .01 & .03 & .10 & .40 & 1.07 & 1.98 & 4.07 & 4.89 \\
\hline 8445 & .03 & .03 & .05 & .15 & .46 & 1.28 & 2.42 & 5.33 & 7.35 & 9435 & -- & .02 & .06 & .17 & .41 & .80 & 1.40 & 5.10 & -- \\
\hline 8446 & .02 & .03 & .10 & .20 & .49 & 1.20 & 2.20 & 4.53 & 5.57 & 9491 & .03 & .03 & .10 & .20 & .55 & 1.30 & 2.40 & 4.97 & 5.92 \\
\hline 8451 & .02 & .02 & .06 & .10 & .31 & .84 & 1.54 & 3.87 & 4.23 & 9499 & .03 & .04 & .10 & .10 & .33 & 1.00 & 1.80 & 3.39 & 4.11 \\
\hline 8531 & .03 & .03 & .08 & .20 & .53 & 1.18 & 2.12 & 3.88 & 5.47 & 9522 & -- & -- & -- & .22 & .40 & 7.87 & -- & -- & -- \\
\hline 8541 & -- & .03 & .07 & .18 & .44 & .99 & 2.29 & 4.05 & -- & 9527 & .04 & .05 & .10 & .10 & .30 & .80 & 1.65 & 3.46 & 4.10 \\
\hline 8544 & .04 & .05 & .10 & .18 & .50 & 1.30 & 2.28 & 5.66 & 8.33 & 9532 & .02 & .03 & .10 & .18 & .50 & 1.10 & 2.00 & 4.60 & 5.69 \\
\hline 8545 & -- & -- & .04 & .18 & .47 & .60 & 1.08 & -- & -- & 9544 & -- & -- & -- & .10 & .24 & 4.34 & -- & -- & -- \\
\hline 8563 & .10 & .10 & .10 & .20 & .60 & 1.30 & 2.40 & 4.91 & 6.20 & 9565 & .02 & .03 & .09 & .11 & .38 & .90 & 1.96 & 4.02 & 4.81 \\
\hline 8566 & .02 & .03 & .05 & .13 & .35 & .97 & 1.78 & 3.37 & 4.22 & 9570 & .10 & .10 & .10 & .20 & .40 & .90 & 1.70 & 3.50 & 4.45 \\
\hline 8583 & .10 & .10 & .10 & .10 & .40 & 1.10 & 1.80 & 4.53 & 5.33 & 9574 & -- & -- & .10 & .10 & .60 & 1.10 & 2.42 & -- & -- \\
\hline 8584 & .03 & .04 & .10 & .16 & .48 & 1.10 & 2.10 & 3.99 & 5.21 & 9588 & .02 & .02 & .07 & .10 & .30 & .90 & 2.00 & 4.40 & 5.50 \\
\hline 8623 & .05 & .07 & .10 & .20 & .50 & 1.20 & 2.20 & 3.90 & 4.84 & 9665 & .03 & .04 & .10 & .20 & .58 & 1.30 & 2.34 & 5.02 & 6.31 \\
\hline 8625 & .02 & .03 & .07 & .16 & .45 & 1.07 & 2.12 & 3.99 & 5.95 & 9715 & .02 & .03 & .10 & .18 & .50 & 1.20 & 2.11 & 4.50 & 5.58 \\
\hline 8630 & .02 & .03 & .07 & .11 & .32 & .84 & 1.62 & 2.86 & 3.86 & 9729 & .01 & .01 & .03 & .09 & .36 & .97 & 1.85 & 3.95 & 4.79 \\
\hline 8631 & .03 & .04 & .06 & .15 & .39 & .90 & 1.75 & 3.76 & 4.77 & 9772 & .02 & .02 & .05 & .14 & .42 & 1.09 & 2.42 & 5.95 & 8.04 \\
\hline 8646 & .02 & .03 & .06 & .15 & .46 & 1.16 & 2.05 & 4.17 & 4.97 & 9814 & -- & -- & .04 & .11 & .36 & 1.09 & 1.86 & -- & -- \\
\hline 8647 & .03 & .04 & .10 & .10 & .30 & .76 & 1.50 & 3.20 & 4.31 & 9815 & .03 & .04 & .09 & .20 & .50 & 1.33 & 2.25 & 5.59 & 7.86 \\
\hline 8677 & .02 & .05 & .10 & .21 & .47 & 1.10 & 2.06 & 4.38 & 5.50 & 9816 & -- & .04 & .05 & .12 & .33 & .86 & 2.34 & 4.74 & -- \\
\hline 8696 & -- & -- & .03 & .16 & 1.18 & 3.56 & 5.63 & -- & -- & 9817 & .04 & .06 & .10 & .14 & .45 & 1.00 & 1.90 & 4.24 & 4.80 \\
\hline 8743 & .03 & .04 & .10 & .20 & .60 & 1.30 & 2.70 & 5.27 & 6.91 & 9829 & .02 & .02 & .06 & .10 & .20 & .59 & 1.20 & 2.63 & 3.40 \\
\hline 8761 & .02 & .03 & .10 & .10 & .30 & .70 & 1.50 & 3.09 & 4.16 & 9830 & .01 & .01 & .03 & .07 & .14 & .50 & .94 & 2.38 & 3.38 \\
\hline 8778 & .04 & .05 & .10 & .20 & .60 & 1.34 & 2.45 & 4.54 & 5.72 & 9858 & .02 & .03 & .06 & .12 & .32 & .79 & 1.54 & 3.28 & 4.05 \\
\hline 8845 & .03 & .04 & .10 & .15 & .43 & 1.20 & 2.33 & 5.11 & 6.10 & 9893 & .02 & .03 & .08 & .14 & .40 & 1.00 & 1.97 & 4.03 & 5.10 \\
\hline 8859 & .03 & .04 & .10 & .22 & .65 & 1.35 & 2.56 & 4.55 & 5.58 & 9916 & .03 & .05 & .10 & .24 & .70 & 1.41 & 2.61 & 4.90 & 5.53 \\
\hline 8898 & .03 & .03 & .09 & .18 & .55 & 1.32 & 2.36 & 5.05 & 6.62 & 9976 & .02 & .03 & .07 & .10 & .32 & .92 & 1.80 & 3.76 & 5.20 \\
\hline 8908 & -- & -- & .07 & .15 & .47 & 1.39 & 2.25 & -- & -- & & & & & & & & & & \\
\hline
\end{tabular}


Appendix 4-4.7. Empirical distribution of storm depth defined by 72-hour minimum interevent time for hourly rainfall stations in Texas.

$[--$, not available $]$

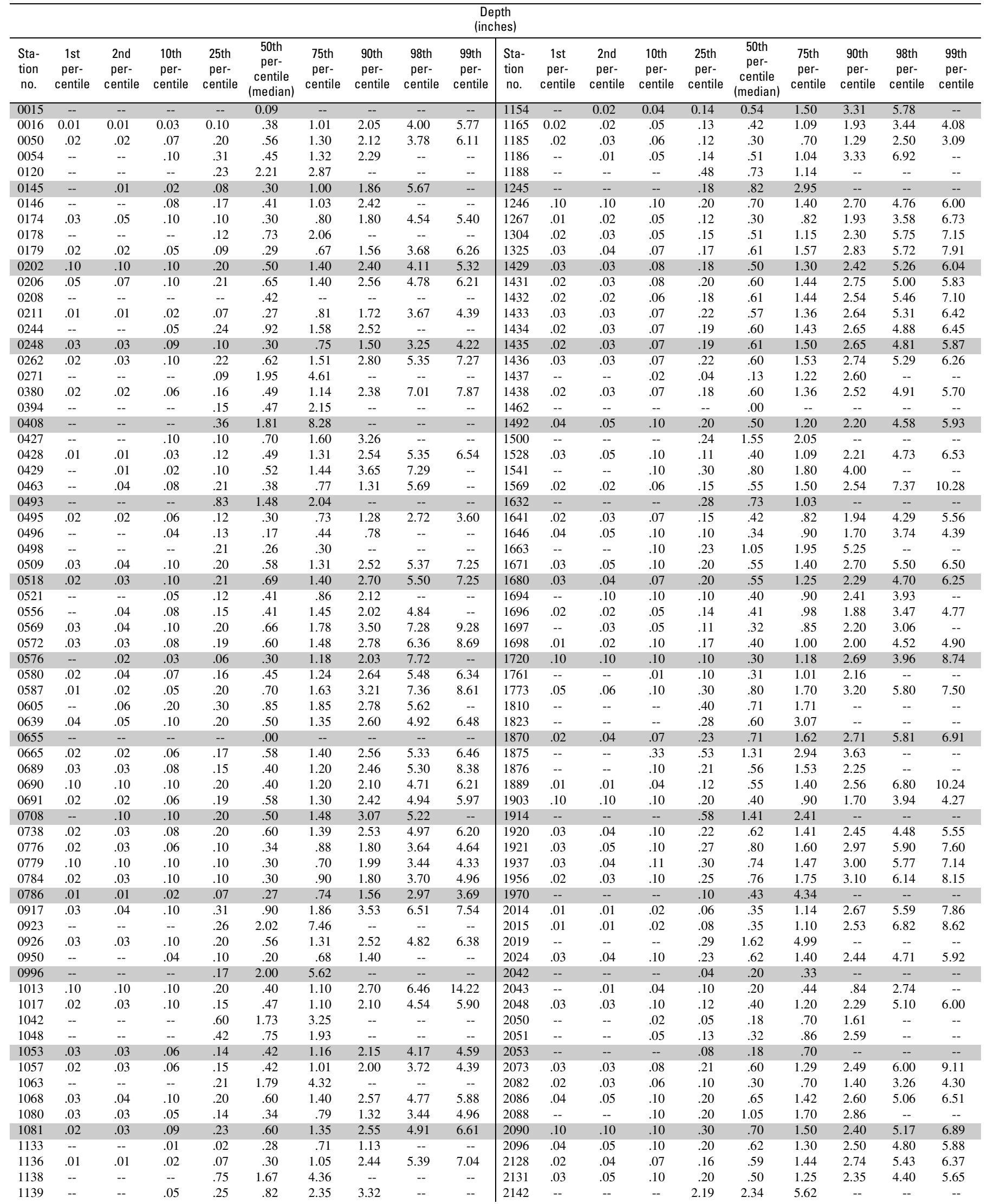


268 Statistical Characteristics of Storm Interevent Time, Depth, and Duration for Eastern New Mexico, Oklahoma, and Texas

Appendix 4-4.7. Empirical distribution of storm depth defined by 72-hour minimum interevent time for hourly rainfall stations in TexasContinued.

\begin{tabular}{|c|c|c|c|c|c|c|c|c|c|c|c|c|c|c|c|c|c|c|c|}
\hline \multicolumn{20}{|c|}{$\begin{array}{c}\text { Depth } \\
\text { (inches) }\end{array}$} \\
\hline $\begin{array}{l}\text { Sta- } \\
\text { tion } \\
\text { no. }\end{array}$ & $\begin{array}{c}1 \text { st } \\
\text { per- } \\
\text { centile }\end{array}$ & $\begin{array}{c}\text { 2nd } \\
\text { per- } \\
\text { centile }\end{array}$ & $\begin{array}{c}10 \text { th } \\
\text { per- } \\
\text { centile }\end{array}$ & $\begin{array}{c}25 \text { th } \\
\text { per- } \\
\text { centile }\end{array}$ & $\begin{array}{c}\text { 50th } \\
\text { per- } \\
\text { centile } \\
\text { (median) }\end{array}$ & $\begin{array}{c}\text { 75th } \\
\text { per- } \\
\text { centile }\end{array}$ & $\begin{array}{c}\text { 90th } \\
\text { per- } \\
\text { centile }\end{array}$ & $\begin{array}{c}\text { 98th } \\
\text { per- } \\
\text { centile }\end{array}$ & $\begin{array}{c}\text { 99th } \\
\text { per- } \\
\text { centile }\end{array}$ & $\begin{array}{l}\text { Sta- } \\
\text { tion } \\
\text { no. }\end{array}$ & $\begin{array}{c}\text { 1st } \\
\text { per- } \\
\text { centile }\end{array}$ & $\begin{array}{c}\text { 2nd } \\
\text { per- } \\
\text { centile }\end{array}$ & $\begin{array}{c}10 \text { th } \\
\text { per- } \\
\text { centile }\end{array}$ & $\begin{array}{c}\text { 25th } \\
\text { per- } \\
\text { centile }\end{array}$ & $\begin{array}{c}\text { 50th } \\
\text { per- } \\
\text { centile } \\
\text { (median) }\end{array}$ & $\begin{array}{c}\text { 75th } \\
\text { per- } \\
\text { centile }\end{array}$ & $\begin{array}{c}\text { 90th } \\
\text { per- } \\
\text { centile }\end{array}$ & $\begin{array}{c}\text { 98th } \\
\text { per- } \\
\text { centile }\end{array}$ & $\begin{array}{l}\text { 99th } \\
\text { per- } \\
\text { centile }\end{array}$ \\
\hline 2160 & -- & -- & 0.02 & 0.09 & 0.35 & 0.90 & 4.00 & -- & -- & 3463 & -- & -- & 0.05 & 0.18 & 0.65 & 1.05 & 2.34 & -- & -- \\
\hline 2206 & 0.02 & 0.03 & .07 & .20 & .67 & 1.83 & 3.06 & 6.23 & 8.14 & 3476 & 0.02 & 0.02 & .06 & .18 & .55 & 1.20 & 2.52 & 4.74 & 7.02 \\
\hline 2238 & .01 & .01 & .02 & .06 & .19 & .69 & 2.07 & 3.24 & 3.79 & 3485 & -- & -- & .08 & .10 & .51 & 3.13 & 9.25 & -- & -- \\
\hline 2240 & -- & -- & .03 & .14 & .41 & .89 & 1.96 & -- & -- & 3507 & .06 & .07 & .10 & .20 & .60 & 1.60 & 3.02 & 5.40 & 6.35 \\
\hline 2242 & .01 & .01 & .04 & .19 & .61 & 1.40 & 2.44 & 4.63 & 6.31 & 3546 & .03 & .04 & .10 & .27 & .77 & 1.80 & 3.29 & 5.95 & 7.68 \\
\hline 2244 & .01 & .01 & .08 & .20 & .60 & 1.50 & 2.76 & 5.55 & 6.82 & 3547 & .02 & .03 & .08 & .21 & .60 & 1.39 & 2.49 & 4.27 & 6.16 \\
\hline 2247 & -- & -- & .04 & .21 & .55 & 1.71 & 3.38 & -- & -- & 3579 & -- & -- & .12 & .24 & .62 & 1.67 & 2.79 & -- & -- \\
\hline 2309 & .05 & .05 & .15 & .35 & .83 & 1.70 & 2.87 & 6.44 & 7.75 & 3642 & .03 & .05 & .10 & .21 & .65 & 1.50 & 2.90 & 5.42 & 6.85 \\
\hline 2312 & .10 & .10 & .10 & .30 & .80 & 1.80 & 3.20 & 5.20 & 6.25 & 3646 & .03 & .04 & .08 & .20 & .56 & 1.25 & 2.29 & 5.12 & 6.14 \\
\hline 2334 & -- & -- & .05 & .25 & 1.01 & 1.90 & 4.14 & -- & -- & 3668 & -- & -- & -- & 1.69 & 2.52 & 5.88 & -- & -- & -- \\
\hline 2336 & .02 & .04 & .10 & .20 & .55 & 1.34 & 2.70 & 3.89 & 4.53 & 3673 & -- & -- & -- & .07 & 1.20 & 3.64 & -- & -- & -- \\
\hline 2354 & -- & -- & .05 & .12 & .49 & 1.10 & 1.69 & -- & -- & 3686 & .07 & .10 & .10 & .27 & .70 & 1.50 & 2.60 & 4.93 & 6.47 \\
\hline 2355 & -- & -- & .04 & .09 & .45 & 1.13 & 3.08 & -- & -- & 3691 & .02 & .04 & .10 & .20 & .60 & 1.38 & 2.56 & 5.19 & 6.29 \\
\hline 2357 & .01 & .01 & .02 & .05 & .27 & .91 & 1.82 & 4.85 & 5.73 & 3734 & -- & -- & -- & .03 & 2.68 & 7.97 & -- & -- & -- \\
\hline 2360 & .01 & .01 & .02 & .06 & .25 & .82 & 1.82 & 4.30 & 5.50 & 3771 & .10 & .10 & .10 & .20 & .70 & 1.50 & 3.04 & 5.34 & 6.54 \\
\hline 2361 & .02 & .02 & .05 & .10 & .30 & .80 & 1.92 & 4.74 & 19.88 & 3789 & -- & .01 & .04 & .10 & .24 & .51 & .97 & 2.55 & -- \\
\hline 2394 & .02 & .03 & .09 & .21 & .62 & 1.50 & 2.84 & 5.54 & 6.60 & 3826 & .02 & .03 & .12 & .23 & .59 & 1.17 & 2.00 & 4.61 & 5.64 \\
\hline 2404 & .02 & .03 & .09 & .20 & .60 & 1.39 & 2.50 & 4.81 & 6.26 & 3831 & .03 & .04 & .07 & .19 & .59 & 1.50 & 2.75 & 5.02 & 6.14 \\
\hline 2415 & .03 & .04 & .10 & .26 & .76 & 1.68 & 3.14 & 6.10 & 7.72 & 3841 & -- & .04 & .12 & .21 & .62 & 1.45 & 2.66 & 5.14 & -- \\
\hline 2462 & .04 & .05 & .10 & .24 & .70 & 1.57 & 2.90 & 5.90 & 7.25 & 3871 & .03 & .04 & .06 & .16 & .48 & 1.09 & 2.06 & 4.39 & 5.15 \\
\hline 2528 & -- & .04 & .08 & .20 & .50 & 1.63 & 2.39 & 3.41 & -- & 3884 & -- & -- & -- & .22 & 1.38 & 3.53 & -- & -- & -- \\
\hline 2617 & .02 & .03 & .06 & .15 & .41 & .84 & 1.82 & 5.25 & 5.65 & 3941 & -- & -- & .06 & .24 & 1.02 & 2.16 & 2.95 & -- & -- \\
\hline 2619 & .02 & .03 & .06 & .15 & .38 & 1.02 & 1.90 & 3.80 & 4.46 & 3963 & -- & -- & -- & .02 & .06 & .30 & -- & -- & -- \\
\hline 2621 & .02 & .04 & .08 & .15 & .40 & .99 & 2.10 & 3.96 & 5.32 & 4040 & .03 & .04 & .07 & .15 & .50 & 1.10 & 2.02 & 4.65 & 5.26 \\
\hline 2675 & .04 & .05 & .10 & .20 & .60 & 1.38 & 2.70 & 5.77 & 8.21 & 4058 & -- & -- & -- & .42 & .94 & 2.38 & -- & -- & -- \\
\hline 2676 & .10 & .10 & .10 & .20 & .60 & 1.60 & 2.84 & 6.51 & 7.62 & 4098 & .03 & .04 & .10 & .10 & .32 & .80 & 1.70 & 3.21 & 3.90 \\
\hline 2679 & .02 & .02 & .07 & .10 & .30 & 1.03 & 2.30 & 5.18 & 6.68 & 4100 & .02 & .04 & .05 & .15 & .41 & .97 & 2.13 & 5.40 & 6.18 \\
\hline 2715 & .02 & .04 & .09 & .20 & .56 & 1.20 & 2.26 & 4.50 & 6.49 & 4137 & .10 & .10 & .10 & .30 & .65 & 1.50 & 2.40 & 5.11 & 7.01 \\
\hline 2744 & .03 & .04 & .10 & .16 & .46 & 1.10 & 2.10 & 3.80 & 5.30 & 4191 & .03 & .04 & .10 & .15 & .45 & 1.20 & 2.50 & 5.07 & 5.87 \\
\hline 2758 & -- & .02 & .03 & .06 & .21 & 1.25 & 2.23 & 6.06 & -- & 4256 & -- & -- & -- & -- & .00 & -- & -- & -- & -- \\
\hline 2794 & -- & -- & -- & .02 & .49 & 1.39 & -- & -- & -- & 4257 & .05 & .06 & .10 & .30 & .80 & 1.70 & 3.20 & 6.20 & 8.20 \\
\hline 2797 & .01 & .01 & .02 & .05 & .16 & .44 & .92 & 2.05 & 2.61 & 4258 & -- & .10 & .10 & .30 & .70 & 1.85 & 3.30 & 7.97 & -- \\
\hline 2811 & .03 & .03 & .10 & .14 & .40 & 1.10 & 2.00 & 4.41 & 5.42 & 4278 & .02 & .03 & .07 & .19 & .52 & 1.32 & 2.46 & 4.85 & 5.96 \\
\hline 2813 & -- & -- & .05 & .21 & .60 & 1.69 & 2.63 & -- & -- & 4299 & -- & .05 & .06 & .13 & .31 & .65 & 1.09 & 1.50 & -- \\
\hline 2814 & -- & -- & -- & .02 & .10 & 1.10 & -- & -- & -- & 4300 & .01 & .01 & .05 & .19 & .63 & 1.70 & 3.34 & 7.19 & 9.27 \\
\hline 2815 & .10 & .10 & .10 & .20 & .40 & .80 & 2.32 & 4.50 & 5.43 & 4305 & .01 & .01 & .03 & .14 & .62 & 1.64 & 3.11 & 6.60 & 8.95 \\
\hline 2818 & .02 & .02 & .07 & .14 & .47 & 1.11 & 1.95 & 4.98 & 8.28 & 4307 & .01 & .01 & .03 & .17 & .65 & 1.68 & 3.24 & 8.42 & 14.86 \\
\hline 2986 & .04 & .05 & .11 & .29 & 1.03 & 1.89 & 3.37 & 7.89 & 8.98 & 4309 & .02 & .03 & .07 & .22 & .75 & 1.66 & 3.15 & 6.17 & 8.38 \\
\hline 3005 & .03 & .04 & .10 & .20 & .60 & 1.30 & 2.34 & 4.49 & 5.51 & 4311 & .02 & .04 & .08 & .23 & .72 & 1.79 & 3.20 & 6.52 & 8.09 \\
\hline 3033 & .02 & .03 & .04 & .08 & .20 & .44 & .89 & 2.24 & 2.96 & 4313 & .02 & .05 & .10 & .29 & .75 & 1.76 & 3.61 & 8.73 & 10.43 \\
\hline 3034 & -- & -- & -- & -- & .16 & -- & -- & -- & -- & 4319 & -- & -- & .13 & .29 & .76 & 1.40 & 3.62 & -- & - \\
\hline 3047 & -- & -- & -- & .12 & .73 & 2.23 & -- & -- & -- & 4329 & .03 & .04 & .10 & .25 & .69 & 1.61 & 3.10 & 6.39 & 8.03 \\
\hline 3103 & -- & -- & .11 & .19 & .30 & 1.46 & 3.97 & -- & -- & 4331 & -- & -- & -- & -- & .00 & -- & -- & -- & -- \\
\hline 3133 & .02 & .03 & .10 & .25 & .70 & 1.50 & 2.78 & 5.34 & 6.47 & 4375 & .10 & .10 & .10 & .20 & .50 & 1.20 & 2.40 & 5.81 & 7.02 \\
\hline 3156 & .04 & .05 & .10 & .20 & .60 & 1.43 & 2.80 & 7.06 & 11.57 & 4392 & .03 & .04 & .08 & .26 & .89 & 2.03 & 3.48 & 7.25 & 8.78 \\
\hline 3171 & .03 & .04 & .10 & .20 & .60 & 1.40 & 2.50 & 5.22 & 6.14 & 4425 & .03 & .03 & .10 & .10 & .30 & .80 & 1.60 & 2.89 & 4.02 \\
\hline 3189 & .03 & .03 & .08 & .11 & .30 & .70 & 1.43 & 3.53 & 4.52 & 4440 & .02 & .03 & .05 & .16 & .51 & 1.17 & 2.09 & 4.19 & 5.76 \\
\hline 3260 & .01 & .02 & .05 & .13 & .39 & 1.14 & 2.36 & 3.96 & 4.32 & 4476 & .04 & .06 & .10 & .22 & .60 & 1.26 & 2.30 & 4.71 & 5.54 \\
\hline 3267 & -- & .03 & .04 & .10 & .33 & .80 & 2.23 & 6.03 & -- & 4498 & -- & -- & -- & .09 & .25 & .46 & -- & -- & -- \\
\hline 3270 & .05 & .10 & .10 & .20 & .40 & 1.00 & 1.88 & 4.25 & 5.30 & 4517 & .02 & .03 & .07 & .19 & .54 & 1.27 & 2.49 & 4.55 & 5.78 \\
\hline 3272 & -- & -- & -- & .07 & .30 & .43 & -- & -- & -- & 4520 & .10 & .10 & .10 & .20 & .60 & 1.25 & 2.50 & 4.70 & 6.22 \\
\hline 3277 & -- & -- & .02 & .03 & .14 & .36 & .82 & -- & -- & 4525 & -- & -- & -- & .27 & .81 & 5.35 & -- & -- & -- \\
\hline 3278 & .03 & .03 & .07 & .11 & .34 & .94 & 1.80 & 3.52 & 4.37 & 4563 & -- & -- & -- & .09 & .60 & 2.83 & -- & -- & -- \\
\hline 3280 & .01 & .01 & .03 & .08 & .20 & .66 & 1.40 & 2.40 & 3.03 & 4570 & .03 & .04 & .10 & .17 & .40 & 1.06 & 2.10 & 4.40 & 5.64 \\
\hline 3281 & -- & -- & .05 & .14 & .33 & .59 & 1.08 & -- & -- & 4577 & .03 & .05 & .10 & .29 & .75 & 1.64 & 2.94 & 5.95 & 7.59 \\
\hline 3283 & .01 & .01 & .03 & .13 & .49 & 1.29 & 2.59 & 5.44 & 6.59 & 4591 & .03 & .03 & .08 & .20 & .61 & 1.48 & 2.70 & 5.69 & 6.29 \\
\hline 3284 & .02 & .03 & .10 & .20 & .60 & 1.37 & 2.59 & 5.10 & 6.10 & 4670 & .02 & .03 & .10 & .15 & .43 & 1.06 & 2.06 & 3.80 & 5.53 \\
\hline 3285 & .06 & .10 & .10 & .20 & .60 & 1.50 & 2.60 & 5.08 & 6.24 & 4671 & -- & .02 & .03 & .09 & .32 & 1.03 & 2.29 & 3.31 & -- \\
\hline 3329 & .02 & .03 & .06 & .14 & .50 & 1.20 & 2.40 & 4.50 & 6.42 & 4679 & .04 & .05 & .10 & .20 & .60 & 1.30 & 2.50 & 5.73 & 7.15 \\
\hline 3335 & .03 & .05 & .18 & .30 & .81 & 1.80 & 3.73 & 6.87 & 11.00 & 4696 & -- & -- & -- & .55 & .66 & .97 & -- & -- & -- \\
\hline 3370 & .03 & .03 & .09 & .25 & .69 & 1.49 & 2.82 & 5.35 & 6.26 & 4703 & -- & .02 & .10 & .18 & .41 & .98 & 1.98 & 5.29 & -- \\
\hline 3410 & .04 & .05 & .10 & .19 & .47 & 1.00 & 2.00 & 3.90 & 4.88 & 4704 & .02 & .03 & .08 & .26 & .81 & 1.97 & 3.93 & 7.06 & 8.75 \\
\hline 3415 & .02 & .04 & .10 & .20 & .60 & 1.40 & 2.64 & 5.30 & 6.56 & 4731 & -- & .01 & .03 & .16 & .35 & 1.01 & 2.18 & 6.89 & -- \\
\hline 3430 & .01 & .01 & .03 & .14 & .53 & 1.49 & 3.09 & 6.59 & 8.53 & 4792 & .10 & .10 & .10 & .20 & .60 & 1.30 & 2.69 & 4.70 & 5.50 \\
\hline 3431 & .01 & .01 & .04 & .15 & .60 & 1.45 & 3.75 & 9.90 & 14.74 & 4819 & .10 & .10 & .10 & .30 & 1.00 & 1.70 & 3.60 & 5.60 & 5.90 \\
\hline 3441 & -- & -- & .09 & .23 & .74 & 1.23 & 2.96 & -- & -- & 4852 & -- & -- & -- & .30 & 1.74 & 3.17 & -- & -- & -- \\
\hline 3442 & .02 & .03 & .06 & .12 & .32 & .82 & 1.73 & 2.98 & 3.59 & 4866 & .03 & .04 & .10 & .20 & .61 & 1.40 & 2.62 & 4.89 & 6.15 \\
\hline 3446 & .03 & .04 & .07 & .15 & .40 & .86 & 1.60 & 2.92 & 4.85 & 4876 & -- & .10 & .10 & .30 & .70 & 1.68 & 3.39 & 9.92 & -- \\
\hline 3460 & -- & -- & .04 & .14 & 1.09 & 1.56 & 2.75 & -- & -- & 4878 & .03 & .04 & .10 & .30 & .83 & 1.95 & 3.86 & 7.25 & 8.35 \\
\hline 3462 & -- & .05 & .10 & .20 & .50 & .98 & 1.60 & 5.70 & -- & 4880 & .02 & .03 & .08 & .13 & .39 & .90 & 1.80 & 3.79 & 4.67 \\
\hline
\end{tabular}


Appendix 4-4.7. Empirical distribution of storm depth defined by 72-hour minimum interevent time for hourly rainfall stations in TexasContinued.

\begin{tabular}{|c|c|c|c|c|c|c|c|c|c|c|c|c|c|c|c|c|c|c|c|}
\hline \multicolumn{20}{|c|}{$\begin{array}{c}\text { Depth } \\
\text { (inches) }\end{array}$} \\
\hline $\begin{array}{l}\text { Sta- } \\
\text { tion } \\
\text { no. }\end{array}$ & $\begin{array}{c}\text { 1st } \\
\text { per- } \\
\text { centile }\end{array}$ & $\begin{array}{c}\text { 2nd } \\
\text { per- } \\
\text { centile }\end{array}$ & $\begin{array}{c}10 \text { th } \\
\text { per- } \\
\text { centile }\end{array}$ & $\begin{array}{c}25 \text { th } \\
\text { per- } \\
\text { centile }\end{array}$ & $\begin{array}{c}\text { 50th } \\
\text { per- } \\
\text { centile } \\
\text { (median) }\end{array}$ & $\begin{array}{c}\text { 75th } \\
\text { per- } \\
\text { centile }\end{array}$ & $\begin{array}{c}\text { 90th } \\
\text { per- } \\
\text { centile }\end{array}$ & $\begin{array}{c}\text { 98th } \\
\text { per- } \\
\text { centile }\end{array}$ & $\begin{array}{c}\text { 99th } \\
\text { per- } \\
\text { centile }\end{array}$ & $\begin{array}{l}\text { Sta- } \\
\text { tion } \\
\text { no. }\end{array}$ & $\begin{array}{c}1 \text { st } \\
\text { per- } \\
\text { centile }\end{array}$ & $\begin{array}{c}\text { 2nd } \\
\text { per- } \\
\text { centile }\end{array}$ & $\begin{array}{c}10 \text { th } \\
\text { per- } \\
\text { centile }\end{array}$ & $\begin{array}{c}25 \text { th } \\
\text { per- } \\
\text { centile }\end{array}$ & $\begin{array}{c}\text { 50th } \\
\text { per- } \\
\text { centile } \\
\text { (median) }\end{array}$ & $\begin{array}{c}\text { 75th } \\
\text { per- } \\
\text { centile }\end{array}$ & $\begin{array}{c}\text { 90th } \\
\text { per- } \\
\text { centile }\end{array}$ & $\begin{array}{c}\text { 98th } \\
\text { per- } \\
\text { centile }\end{array}$ & $\begin{array}{l}\text { 99th } \\
\text { per- } \\
\text { centile }\end{array}$ \\
\hline 4920 & 0.03 & 0.04 & 0.10 & 0.15 & 0.40 & 1.20 & 2.29 & 4.66 & 5.88 & 5957 & 0.03 & 0.04 & 0.10 & 0.20 & 0.60 & 1.30 & 2.38 & 5.20 & 6.91 \\
\hline 4934 & -- & -- & -- & .07 & .15 & 1.02 & -- & -- & -- & 5958 & .01 & .02 & .06 & .19 & .66 & 1.30 & 2.46 & 4.53 & 4.72 \\
\hline 4972 & .03 & .04 & .10 & .20 & .55 & 1.20 & 2.32 & 4.50 & 5.41 & 5973 & -- & .02 & .04 & .08 & .25 & .97 & 2.61 & 5.38 & -- \\
\hline 4973 & .04 & .06 & .13 & .38 & .79 & 1.60 & 3.06 & 6.48 & 8.89 & 5996 & .03 & .04 & .10 & .20 & .57 & 1.29 & 2.38 & 4.52 & 5.43 \\
\hline 4974 & .02 & .03 & .06 & .12 & .38 & .89 & 1.88 & 3.92 & 6.05 & 6017 & -- & .01 & .03 & .10 & .34 & 1.00 & 2.30 & 4.71 & -- \\
\hline 4975 & .10 & .10 & .10 & .30 & .80 & 1.70 & 3.00 & 5.80 & 6.76 & 6024 & .02 & .03 & .10 & .30 & .83 & 2.00 & 3.46 & 6.32 & 10.13 \\
\hline 4978 & -- & .02 & .04 & .11 & .45 & 1.04 & 2.70 & 6.16 & -- & 6050 & -- & -- & .19 & .29 & .57 & 1.45 & 2.29 & -- & -- \\
\hline 4979 & -- & -- & -- & .62 & 1.85 & 5.05 & -- & -- & -- & 6104 & .02 & .03 & .07 & .10 & .32 & .84 & 1.75 & 4.00 & 5.07 \\
\hline 4982 & .02 & .03 & .07 & .16 & .45 & 1.11 & 2.14 & 4.17 & 5.38 & 6108 & .03 & .06 & .10 & .30 & .80 & 1.80 & 3.21 & 5.90 & 7.04 \\
\hline 5018 & .02 & .04 & .07 & .20 & .56 & 1.34 & 2.31 & 4.67 & 7.19 & 6136 & .03 & .04 & .10 & .11 & .32 & .82 & 1.60 & 3.35 & 3.73 \\
\hline 5048 & .03 & .04 & .10 & .10 & .30 & .85 & 1.55 & 3.73 & 5.45 & 6166 & -- & .03 & .06 & .17 & .37 & .89 & 1.74 & 2.88 & -- \\
\hline 5049 & -- & .10 & .10 & .20 & .30 & .80 & 1.56 & 5.16 & -- & 6176 & .02 & .03 & .11 & .30 & .63 & 1.65 & 3.35 & 7.10 & 7.40 \\
\hline 5056 & -- & -- & -- & -- & .67 & -- & -- & -- & -- & 6177 & .04 & .05 & .10 & .30 & .80 & 1.70 & 3.17 & 6.33 & 8.52 \\
\hline 5057 & .01 & .01 & .02 & .06 & .24 & .79 & 1.78 & 3.66 & 4.16 & 6210 & .03 & .05 & .10 & .20 & .70 & 1.50 & 2.80 & 5.60 & 6.69 \\
\hline 5060 & .01 & .01 & .02 & .07 & .43 & 1.09 & 3.05 & 8.06 & 8.82 & 6211 & .01 & .02 & .07 & .23 & .83 & 1.89 & 3.12 & 5.47 & 7.57 \\
\hline 5081 & .02 & .03 & .08 & .25 & .75 & 1.65 & 2.96 & 6.02 & 6.92 & 6270 & .10 & .10 & .10 & .30 & .90 & 1.80 & 3.20 & 6.34 & 7.42 \\
\hline 5094 & .02 & .03 & .10 & .20 & .63 & 1.46 & 2.70 & 5.55 & 6.83 & 6275 & -- & -- & -- & -- & .00 & -- & -- & -- & -- \\
\hline 5113 & .03 & .04 & .10 & .16 & .47 & 1.20 & 2.50 & 5.61 & 7.46 & 6276 & -- & -- & -- & .19 & 1.39 & 4.21 & -- & -- & -- \\
\hline 5114 & -- & -- & -- & -- & .00 & -- & -- & -- & -- & 6335 & .03 & .03 & .10 & .23 & .70 & 1.51 & 2.75 & 4.91 & 5.87 \\
\hline 5123 & -- & -- & .07 & .22 & .49 & 1.92 & 2.78 & -- & -- & 6434 & -- & -- & .14 & .36 & .72 & 1.17 & 1.97 & -- & -- \\
\hline 5192 & .02 & .04 & .10 & .25 & .60 & 1.50 & 2.80 & 5.29 & 6.32 & 6504 & .02 & .03 & .10 & .10 & .36 & .90 & 1.70 & 4.07 & 5.10 \\
\hline 5193 & .03 & .04 & .10 & .20 & .60 & 1.40 & 2.70 & 5.22 & 6.84 & 6558 & -- & -- & .05 & .12 & .71 & .99 & 1.90 & -- & -- \\
\hline 5224 & .01 & .02 & .08 & .30 & .86 & 2.00 & 3.77 & 8.23 & 9.16 & 6615 & .05 & .10 & .10 & .20 & .40 & 1.00 & 1.91 & 5.20 & 6.40 \\
\hline 5228 & .02 & .03 & .06 & .16 & .64 & 1.55 & 2.62 & 5.27 & 6.45 & 6660 & -- & .03 & .08 & .25 & .59 & 1.61 & 2.61 & 5.20 & -- \\
\hline 5235 & -- & -- & .05 & .15 & .54 & 1.15 & 3.58 & -- & -- & 6663 & -- & -- & .11 & .21 & .65 & 1.74 & 4.59 & -- & -- \\
\hline 5247 & .02 & .03 & .10 & .15 & .40 & 1.00 & 1.91 & 3.55 & 4.28 & 6734 & .01 & .02 & .06 & .15 & .41 & 1.13 & 1.94 & 4.47 & 5.79 \\
\hline 5258 & .03 & .03 & .10 & .23 & .60 & 1.30 & 2.50 & 5.32 & 7.50 & 6736 & .03 & .03 & .10 & .12 & .37 & .90 & 1.78 & 3.99 & 4.74 \\
\hline 5303 & .02 & .03 & .05 & .15 & .37 & 1.19 & 2.10 & 5.34 & 8.90 & 6740 & -- & -- & .07 & .37 & 1.70 & 3.46 & 4.25 & -- & -- \\
\hline 5312 & .04 & .05 & .10 & .20 & .50 & 1.20 & 2.30 & 5.42 & 6.51 & 6750 & .01 & .01 & .03 & .12 & .38 & 1.63 & 3.73 & 10.04 & 11.79 \\
\hline 5341 & -- & -- & -- & .47 & .78 & 7.96 & -- & -- & -- & 6757 & .01 & .02 & .10 & .21 & .70 & 1.60 & 3.09 & 5.54 & 6.99 \\
\hline 5342 & -- & -- & -- & -- & .00 & -- & -- & -- & -- & 6775 & .01 & .02 & .05 & .12 & .35 & 1.02 & 1.85 & 4.20 & 5.06 \\
\hline 5348 & .04 & .08 & .10 & .30 & .80 & 1.80 & 3.40 & 5.40 & 5.92 & 6776 & .03 & .04 & .10 & .13 & .40 & 1.00 & 1.90 & 4.09 & 4.87 \\
\hline 5358 & .02 & .03 & .07 & .15 & .41 & 1.07 & 1.99 & 3.98 & 5.14 & 6788 & .02 & .02 & .09 & .29 & .72 & 1.63 & 3.31 & 5.32 & 5.59 \\
\hline 5398 & .03 & .04 & .10 & .28 & .75 & 1.60 & 3.02 & 6.20 & 7.19 & 6792 & .02 & .03 & .10 & .10 & .30 & .70 & 1.40 & 3.23 & 3.95 \\
\hline 5410 & .02 & .03 & .09 & .10 & .37 & .90 & 1.74 & 3.98 & 4.58 & 6794 & -- & -- & -- & 1.20 & 4.96 & 8.37 & -- & -- & -- \\
\hline 5411 & .01 & .01 & .02 & .07 & .27 & .80 & 1.68 & 3.54 & 4.85 & 6834 & .10 & .10 & .10 & .30 & .90 & 1.73 & 3.25 & 6.10 & 7.42 \\
\hline 5424 & .01 & .01 & .03 & .18 & .81 & 2.01 & 3.72 & 8.97 & 11.20 & 6893 & .03 & .04 & .10 & .10 & .27 & .60 & 1.20 & 2.68 & 3.21 \\
\hline 5429 & .02 & .02 & .06 & .16 & .50 & 1.31 & 2.36 & 4.88 & 7.81 & 6935 & .03 & .05 & .10 & .14 & .35 & .87 & 1.80 & 3.63 & 5.00 \\
\hline 5431 & -- & -- & .14 & .38 & .90 & 2.24 & 3.28 & -- & -- & 6981 & .01 & .02 & .10 & .24 & .61 & 1.41 & 2.33 & 5.75 & 6.84 \\
\hline 5461 & .03 & .04 & .10 & .24 & .70 & 1.56 & 2.85 & 5.85 & 6.90 & 7020 & -- & .02 & .04 & .16 & .45 & 1.13 & 2.61 & 7.81 & -- \\
\hline 5463 & .10 & .10 & .10 & .20 & .70 & 1.60 & 2.90 & 5.50 & 6.61 & 7060 & .03 & .05 & .10 & .18 & .40 & 1.10 & 2.10 & 4.39 & 5.70 \\
\hline 5471 & -- & -- & -- & .08 & .41 & .64 & -- & -- & -- & 7066 & .03 & .04 & .10 & .25 & .80 & 1.70 & 3.20 & 5.67 & 7.12 \\
\hline 5477 & -- & -- & .09 & .15 & .46 & 1.42 & 9.73 & -- & -- & 7074 & .02 & .03 & .06 & .10 & .30 & .80 & 1.68 & 3.68 & 4.68 \\
\hline 5528 & .03 & .04 & .10 & .26 & .69 & 1.47 & 2.58 & 5.11 & 6.48 & 7097 & -- & .02 & .11 & .20 & .65 & 1.52 & 3.22 & 5.61 & -- \\
\hline 5579 & -- & -- & -- & -- & .55 & -- & -- & -- & -- & 7116 & .02 & .02 & .05 & .14 & .40 & 1.03 & 1.81 & 3.83 & 5.04 \\
\hline 5580 & -- & -- & -- & .49 & 1.81 & 2.68 & -- & -- & -- & 7140 & .02 & .02 & .07 & .18 & .55 & 1.61 & 3.37 & 7.16 & 9.31 \\
\hline 5589 & -- & .01 & .03 & .09 & .32 & .82 & 1.34 & 1.73 & -- & 7173 & .01 & .01 & .03 & .19 & .73 & 2.09 & 4.14 & 8.01 & 10.07 \\
\hline 5590 & -- & .02 & .05 & .12 & .33 & .93 & 1.53 & 3.81 & -- & 7174 & .01 & .01 & .04 & .19 & .77 & 2.00 & 3.85 & 8.18 & 10.35 \\
\hline 5591 & .02 & .03 & .06 & .12 & .34 & .67 & 1.39 & 2.83 & 3.44 & 7213 & .02 & .03 & .10 & .25 & .67 & 1.47 & 2.69 & 5.11 & 5.93 \\
\hline 5592 & .02 & .02 & .05 & .10 & .27 & .58 & 1.25 & 2.79 & 4.65 & 7243 & .03 & .04 & .10 & .20 & .50 & 1.20 & 2.30 & 4.40 & 5.50 \\
\hline 5594 & .02 & .03 & .06 & .10 & .25 & .61 & 1.10 & 2.50 & 4.12 & 7262 & -- & .01 & .02 & .06 & .17 & .43 & .69 & 2.22 & -- \\
\hline 5595 & -- & -- & -- & -- & .05 & -- & -- & -- & -- & 7274 & .04 & .05 & .10 & .23 & .52 & 1.22 & 2.00 & 5.11 & 5.78 \\
\hline 5596 & .10 & .10 & .10 & .10 & .30 & .80 & 1.70 & 3.67 & 4.66 & 7300 & .02 & .03 & .06 & .18 & .52 & 1.16 & 2.35 & 4.35 & 5.80 \\
\hline 5600 & .02 & .03 & .05 & .12 & .30 & .73 & 1.24 & 3.79 & 5.13 & 7311 & -- & -- & .10 & .20 & .50 & 1.75 & 2.09 & -- & -- \\
\hline 5618 & -- & -- & -- & .17 & 1.13 & 2.52 & -- & -- & -- & 7363 & -- & -- & -- & .14 & .82 & 3.23 & -- & -- & -- \\
\hline 5650 & -- & -- & -- & .45 & .74 & 2.19 & -- & -- & -- & 7422 & .02 & .03 & .10 & .18 & .50 & 1.30 & 2.50 & 5.25 & 6.59 \\
\hline 5656 & .07 & .10 & .10 & .20 & .40 & 1.01 & 1.92 & 4.10 & 5.51 & 7431 & .03 & .03 & .07 & .10 & .30 & .78 & 1.50 & 3.51 & 4.47 \\
\hline 5658 & .02 & .03 & .05 & .13 & .36 & .85 & 1.84 & 4.33 & 5.34 & 7481 & .02 & .03 & .05 & .10 & .23 & .60 & 1.30 & 3.27 & 3.81 \\
\hline 5661 & .10 & .10 & .10 & .17 & .50 & 1.12 & 2.97 & 6.92 & 11.87 & 7497 & .10 & .10 & .10 & .20 & .66 & 1.40 & 2.70 & 5.14 & 7.15 \\
\hline 5666 & -- & -- & .04 & .10 & .30 & 1.01 & 3.38 & -- & -- & 7498 & .07 & .09 & .10 & .20 & .70 & 1.50 & 2.42 & 7.48 & 9.58 \\
\hline 5695 & .04 & .05 & .10 & .28 & .67 & 1.41 & 2.69 & 5.37 & 6.85 & 7499 & .05 & .08 & .10 & .20 & .50 & 1.29 & 2.37 & 4.56 & 6.30 \\
\hline 5742 & -- & -- & .05 & .09 & .21 & .41 & 2.79 & -- & -- & 7531 & -- & .04 & .08 & .26 & .58 & 1.32 & 3.18 & 4.75 & -- \\
\hline 5766 & -- & -- & -- & .69 & 1.35 & 6.34 & -- & -- & -- & 7534 & .03 & .03 & .06 & .18 & .52 & 1.23 & 2.32 & 4.17 & 5.63 \\
\hline 5770 & .03 & .03 & .10 & .15 & .41 & 1.08 & 2.08 & 4.32 & 5.07 & 7556 & .03 & .05 & .10 & .20 & .59 & 1.30 & 2.60 & 5.11 & 6.78 \\
\hline 5775 & -- & -- & -- & .05 & .69 & 1.10 & -- & -- & -- & 7594 & .03 & .04 & .10 & .25 & .73 & 1.68 & 2.98 & 6.29 & 7.48 \\
\hline 5779 & -- & -- & .09 & .22 & .71 & 1.71 & 2.75 & -- & -- & 7596 & .01 & .02 & .05 & .12 & .60 & 1.64 & 3.02 & 4.80 & 5.54 \\
\hline 5840 & .02 & .03 & .05 & .19 & .47 & 1.24 & 2.30 & 5.91 & 6.90 & 7608 & .01 & .02 & .05 & .16 & .60 & 1.48 & 2.59 & 5.50 & 6.87 \\
\hline 5890 & .01 & .01 & .02 & .07 & .24 & .70 & 1.43 & 3.10 & 3.86 & 7622 & -- & -- & .01 & .01 & .10 & .53 & 1.41 & -- & -- \\
\hline 5891 & .02 & .02 & .07 & .14 & .37 & .78 & 1.53 & 2.40 & 3.44 & 7700 & .02 & .03 & .09 & .28 & .76 & 1.70 & 3.10 & 6.03 & 7.80 \\
\hline 5897 & .10 & .10 & .10 & .20 & .70 & 1.60 & 2.90 & 5.40 & 6.49 & 7706 & .02 & .04 & .10 & .15 & .44 & 1.05 & 2.10 & 4.37 & 6.54 \\
\hline
\end{tabular}


Appendix 4-4.7. Empirical distribution of storm depth defined by 72-hour minimum interevent time for hourly rainfall stations in TexasContinued.

\begin{tabular}{|c|c|c|c|c|c|c|c|c|c|c|c|c|c|c|c|c|c|c|c|}
\hline \multicolumn{20}{|c|}{$\begin{array}{c}\text { Depth } \\
\text { (inches) }\end{array}$} \\
\hline $\begin{array}{l}\text { Sta- } \\
\text { tion } \\
\text { no. }\end{array}$ & $\begin{array}{c}\text { 1st } \\
\text { per- } \\
\text { centile }\end{array}$ & $\begin{array}{c}\text { 2nd } \\
\text { per- } \\
\text { centile }\end{array}$ & $\begin{array}{c}\text { 10th } \\
\text { per- } \\
\text { centile }\end{array}$ & $\begin{array}{c}\text { 25th } \\
\text { per- } \\
\text { centile }\end{array}$ & $\begin{array}{c}\text { 50th } \\
\text { per- } \\
\text { centile } \\
\text { (median) }\end{array}$ & $\begin{array}{c}\text { 75th } \\
\text { per- } \\
\text { centile }\end{array}$ & $\begin{array}{c}\text { 90th } \\
\text { per- } \\
\text { centile }\end{array}$ & $\begin{array}{c}\text { 98th } \\
\text { per- } \\
\text { centile }\end{array}$ & $\begin{array}{c}\text { 99th } \\
\text { per- } \\
\text { centile }\end{array}$ & $\begin{array}{l}\text { Sta- } \\
\text { tion } \\
\text { no. }\end{array}$ & $\begin{array}{c}\text { 1st } \\
\text { per- } \\
\text { centile }\end{array}$ & $\begin{array}{c}\text { 2nd } \\
\text { per- } \\
\text { centile }\end{array}$ & $\begin{array}{c}10 \text { th } \\
\text { per- } \\
\text { centile }\end{array}$ & $\begin{array}{c}25 \text { th } \\
\text { per- } \\
\text { centile }\end{array}$ & $\begin{array}{c}\text { 50th } \\
\text { per- } \\
\text { centile } \\
\text { (median) }\end{array}$ & $\begin{array}{c}\text { 75th } \\
\text { per- } \\
\text { centile }\end{array}$ & $\begin{array}{l}\text { 90th } \\
\text { per- } \\
\text { centile }\end{array}$ & $\begin{array}{c}\text { 98th } \\
\text { per- } \\
\text { centile }\end{array}$ & $\begin{array}{c}\text { 99th } \\
\text { per- } \\
\text { centile }\end{array}$ \\
\hline 7718 & -- & 0.03 & 0.09 & 0.21 & 0.60 & 1.07 & 2.68 & 6.67 & -- & 8910 & -- & -- & -- & 0.07 & 0.42 & 2.33 & -- & -- & -- \\
\hline 7745 & 0.01 & .02 & .08 & .24 & .83 & 1.67 & 3.50 & 7.25 & 8.47 & 8911 & 0.01 & 0.02 & 0.05 & .16 & .57 & 1.33 & 2.61 & 5.17 & 5.79 \\
\hline 7922 & .01 & .01 & .04 & .09 & .21 & .49 & .90 & 2.22 & 3.21 & 8924 & .02 & .03 & .06 & .12 & .28 & .65 & 1.16 & 2.06 & 2.62 \\
\hline 7936 & .04 & .05 & .10 & .30 & .85 & 1.90 & 3.30 & 7.15 & 8.45 & 8929 & -- & -- & -- & .13 & .65 & 2.02 & -- & -- & -- \\
\hline 7943 & .01 & .01 & .02 & .07 & .28 & .86 & 1.81 & 3.60 & 4.72 & 8942 & .05 & .08 & .10 & .30 & .80 & 1.80 & 3.40 & 6.10 & 7.70 \\
\hline 7944 & -- & -- & .03 & .15 & .42 & 1.36 & 3.03 & -- & -- & 8944 & .02 & .02 & .06 & .20 & .66 & 1.48 & 3.18 & 6.30 & 7.82 \\
\hline 7945 & .01 & .01 & .02 & .08 & .40 & 1.12 & 2.44 & 5.43 & 7.32 & 8996 & .02 & .03 & .06 & .20 & .64 & 1.57 & 3.01 & 6.06 & 7.53 \\
\hline 7947 & .05 & .05 & .10 & .30 & .70 & 1.60 & 3.30 & 5.64 & 13.07 & 9014 & -- & -- & -- & .81 & 1.46 & 2.26 & -- & -- & -- \\
\hline 7948 & .02 & .02 & .05 & .12 & .45 & 1.23 & 2.29 & 5.00 & 7.16 & 9037 & .02 & .03 & .05 & .10 & .24 & .52 & 1.23 & 2.47 & 2.79 \\
\hline 7951 & .03 & .05 & .10 & .26 & .74 & 1.54 & 2.79 & 5.45 & 6.61 & 9106 & .02 & .02 & .04 & .07 & .17 & .48 & 1.00 & 2.31 & 8.34 \\
\hline 7953 & -- & .02 & .05 & .14 & .30 & .81 & 2.72 & 5.00 & -- & 9107 & -- & -- & .04 & .07 & .20 & .80 & 1.55 & -- & -- \\
\hline 7981 & .02 & .02 & .06 & .13 & .41 & 1.18 & 2.26 & 5.00 & 5.80 & 9129 & -- & .02 & .04 & .07 & .28 & .80 & 2.25 & 3.90 & -- \\
\hline 7990 & -- & .02 & .04 & .10 & .43 & 1.17 & 3.94 & 7.23 & -- & 9163 & .03 & .04 & .09 & .20 & .50 & 1.19 & 2.21 & 5.00 & 5.70 \\
\hline 7992 & -- & -- & -- & 1.15 & 1.75 & 4.62 & -- & -- & -- & 9213 & .01 & .01 & .03 & .16 & .56 & 1.36 & 2.48 & 6.05 & 8.60 \\
\hline 7997 & -- & .02 & .04 & .13 & .38 & .91 & 1.81 & 4.89 & -- & 9214 & -- & -- & -- & 1.29 & 2.35 & 7.64 & -- & -- & -- \\
\hline 7999 & -- & -- & .03 & .07 & .24 & .84 & 1.64 & -- & -- & 9222 & .01 & .02 & .06 & .21 & .54 & 1.25 & 2.45 & 5.46 & 6.48 \\
\hline 8022 & -- & -- & .05 & .10 & .26 & .98 & 1.78 & -- & -- & 9248 & -- & .05 & .07 & .12 & .30 & .94 & 1.56 & 3.78 & -- \\
\hline 8023 & .04 & .05 & .10 & .13 & .35 & .90 & 1.73 & 3.81 & 4.70 & 9266 & -- & .04 & .10 & .19 & .50 & 1.11 & 2.18 & 2.99 & -- \\
\hline 8047 & .03 & .04 & .10 & .20 & .60 & 1.30 & 2.40 & 4.80 & 6.12 & 9270 & .03 & .04 & .10 & .10 & .30 & .72 & 1.54 & 3.59 & 3.97 \\
\hline 8060 & -- & .02 & .03 & .08 & .30 & 1.17 & 3.21 & 4.18 & -- & 9295 & -- & .01 & .02 & .04 & .14 & .51 & 1.14 & 2.32 & -- \\
\hline 8062 & -- & -- & .02 & .11 & .48 & 1.30 & 3.89 & -- & -- & 9304 & -- & -- & -- & -- & .23 & -- & -- & -- & -- \\
\hline 8068 & -- & -- & .03 & .10 & .26 & .54 & 1.53 & -- & -- & 9307 & .05 & .07 & .10 & .25 & .60 & 1.15 & 2.18 & 3.88 & 4.10 \\
\hline 8081 & .03 & .04 & .08 & .15 & .43 & 1.24 & 2.50 & 5.87 & 7.72 & 9328 & .01 & .02 & .06 & .12 & .36 & .91 & 2.50 & 4.15 & 5.07 \\
\hline 8089 & -- & -- & .03 & .14 & .58 & 1.10 & 1.89 & -- & -- & 9329 & -- & -- & -- & .16 & .37 & 2.06 & -- & -- & -- \\
\hline 8221 & -- & -- & -- & .67 & 1.34 & 2.21 & -- & -- & -- & 9345 & -- & -- & -- & -- & .75 & -- & -- & -- & -- \\
\hline 8252 & .03 & .04 & .09 & .12 & .35 & .90 & 1.69 & 3.45 & 4.80 & 9363 & .01 & .01 & .03 & .10 & .43 & 1.32 & 2.59 & 5.31 & 6.18 \\
\hline 8265 & .03 & .03 & .08 & .23 & .71 & 1.69 & 3.45 & 6.72 & 9.30 & 9364 & .01 & .01 & .03 & .11 & .45 & 1.35 & 2.80 & 6.65 & 9.15 \\
\hline 8289 & -- & -- & .14 & .27 & .65 & 1.00 & 1.29 & -- & -- & 9365 & -- & -- & .08 & .14 & .30 & .69 & 2.36 & -- & -- \\
\hline 8305 & .02 & .03 & .07 & .10 & .26 & .58 & 1.18 & 2.60 & 3.27 & 9371 & -- & .04 & .07 & .18 & .74 & 1.76 & 2.52 & 4.18 & -- \\
\hline 8335 & .03 & .04 & .08 & .26 & .80 & 1.69 & 3.22 & 5.42 & 6.71 & 9417 & .02 & .02 & .07 & .18 & .60 & 1.42 & 2.61 & 4.90 & 5.89 \\
\hline 8400 & .02 & .03 & .08 & .14 & .40 & .77 & 1.51 & 3.77 & 4.59 & 9419 & .01 & .01 & .04 & .14 & .53 & 1.35 & 2.41 & 4.85 & 6.00 \\
\hline 8445 & .02 & .03 & .06 & .20 & .58 & 1.55 & 2.88 & 6.08 & 8.49 & 9435 & -- & .05 & .07 & .21 & .52 & .87 & 1.64 & 5.60 & -- \\
\hline 8446 & .02 & .04 & .10 & .20 & .60 & 1.43 & 2.59 & 5.16 & 6.17 & 9491 & .03 & .03 & .10 & .21 & .69 & 1.57 & 3.00 & 5.62 & 6.82 \\
\hline 8451 & .02 & .02 & .06 & .11 & .34 & 1.02 & 1.72 & 4.23 & 4.70 & 9499 & .03 & .04 & .10 & .14 & .40 & 1.10 & 2.00 & 3.70 & 4.84 \\
\hline 8531 & .03 & .03 & .08 & .23 & .62 & 1.40 & 2.49 & 4.20 & 6.13 & 9522 & -- & -- & -- & .21 & 1.28 & 10.64 & -- & -- & -- \\
\hline 8541 & -- & .03 & .08 & .18 & .49 & 1.05 & 2.77 & 5.45 & -- & 9527 & .04 & .05 & .10 & .12 & .36 & .90 & 1.90 & 3.86 & 4.59 \\
\hline 8544 & .05 & .05 & .10 & .20 & .59 & 1.42 & 2.90 & 7.20 & 9.00 & 9532 & .03 & .03 & .10 & .20 & .60 & 1.30 & 2.40 & 5.24 & 6.33 \\
\hline 8545 & -- & -- & .04 & .24 & .54 & .63 & 1.12 & -- & -- & 9544 & -- & -- & -- & .10 & .24 & 4.34 & -- & -- & -- \\
\hline 8563 & .10 & .10 & .10 & .20 & .70 & 1.40 & 2.60 & 5.66 & 7.17 & 9565 & .02 & .03 & .10 & .14 & .40 & 1.09 & 2.23 & 4.87 & 5.38 \\
\hline 8566 & .02 & .03 & .06 & .14 & .40 & 1.04 & 2.04 & 3.51 & 5.14 & 9570 & .10 & .10 & .10 & .20 & .50 & 1.20 & 1.95 & 4.53 & 5.26 \\
\hline 8583 & .10 & .10 & .10 & .20 & .50 & 1.20 & 2.10 & 4.81 & 5.70 & 9574 & -- & -- & .20 & .40 & .90 & 1.40 & 2.54 & -- & -- \\
\hline 8584 & .03 & .04 & .10 & .20 & .54 & 1.24 & 2.37 & 4.70 & 5.40 & 9588 & .02 & .02 & .07 & .10 & .40 & 1.10 & 2.30 & 4.77 & 5.64 \\
\hline 8623 & .05 & .08 & .10 & .20 & .60 & 1.40 & 2.50 & 4.38 & 5.66 & 9665 & .03 & .05 & .10 & .23 & .70 & 1.50 & 2.77 & 5.51 & 6.94 \\
\hline 8625 & .02 & .03 & .08 & .20 & .56 & 1.25 & 2.42 & 4.46 & 6.83 & 9715 & .02 & .03 & .10 & .20 & .60 & 1.30 & 2.50 & 5.06 & 6.36 \\
\hline 8630 & .02 & .03 & .08 & .12 & .37 & .90 & 1.70 & 3.49 & 4.00 & 9729 & .01 & .01 & .03 & .12 & .46 & 1.18 & 2.28 & 4.58 & 5.61 \\
\hline 8631 & .03 & .04 & .06 & .15 & .42 & 1.01 & 1.94 & 4.18 & 5.16 & 9772 & .02 & .03 & .05 & .15 & .54 & 1.47 & 3.03 & 6.67 & 8.96 \\
\hline 8646 & .02 & .03 & .08 & .17 & .55 & 1.34 & 2.47 & 4.72 & 6.04 & 9814 & -- & -- & .04 & .17 & .82 & 1.50 & 2.81 & -- & -- \\
\hline 8647 & .03 & .04 & .10 & .11 & .35 & .90 & 1.90 & 3.88 & 4.82 & 9815 & .03 & .05 & .10 & .23 & .61 & 1.60 & 2.64 & 6.35 & 8.29 \\
\hline 8677 & .02 & .05 & .10 & .22 & .49 & 1.25 & 2.19 & 4.54 & 5.61 & 9816 & -- & .04 & .05 & .12 & .34 & 1.02 & 2.58 & 4.83 & -- \\
\hline 8696 & -- & -- & -- & .23 & 1.18 & 4.10 & -- & -- & -- & 9817 & .04 & .06 & .10 & .20 & .50 & 1.17 & 2.10 & 4.65 & 5.34 \\
\hline 8743 & .03 & .04 & .10 & .27 & .77 & 1.65 & 3.24 & 6.20 & 7.36 & 9829 & .02 & .02 & .06 & .10 & .27 & .67 & 1.40 & 2.92 & 3.55 \\
\hline 8761 & .02 & .03 & .10 & .10 & .30 & .83 & 1.70 & 3.45 & 4.44 & 9830 & .01 & .01 & .03 & .07 & .17 & .67 & 1.12 & 2.44 & 3.54 \\
\hline 8778 & .04 & .06 & .10 & .30 & .80 & 1.60 & 3.00 & 5.01 & 6.29 & 9858 & .02 & .03 & .06 & .14 & .39 & .95 & 1.84 & 3.85 & 4.57 \\
\hline 8845 & .03 & .04 & .10 & .20 & .53 & 1.40 & 2.77 & 5.96 & 6.92 & 9893 & .02 & .03 & .10 & .20 & .50 & 1.17 & 2.24 & 4.51 & 5.45 \\
\hline 8859 & .03 & .04 & .10 & .25 & .80 & 1.66 & 3.00 & 5.31 & 6.41 & 9916 & .03 & .05 & .10 & .30 & .88 & 1.80 & 3.20 & 5.35 & 5.90 \\
\hline 8898 & .03 & .03 & .10 & .24 & .68 & 1.50 & 2.91 & 5.44 & 7.63 & 9976 & .02 & .03 & .08 & .11 & .40 & 1.01 & 2.00 & 4.30 & 6.23 \\
\hline 8908 & -- & -- & .06 & .15 & 1.02 & 2.03 & 3.49 & -- & -- & & & & & & & & & & \\
\hline
\end{tabular}


Appendix 4-5.1. Empirical distribution of storm duration defined by 6-hour minimum interevent time for hourly rainfall stations in Texas.

$[--$, not available $]$

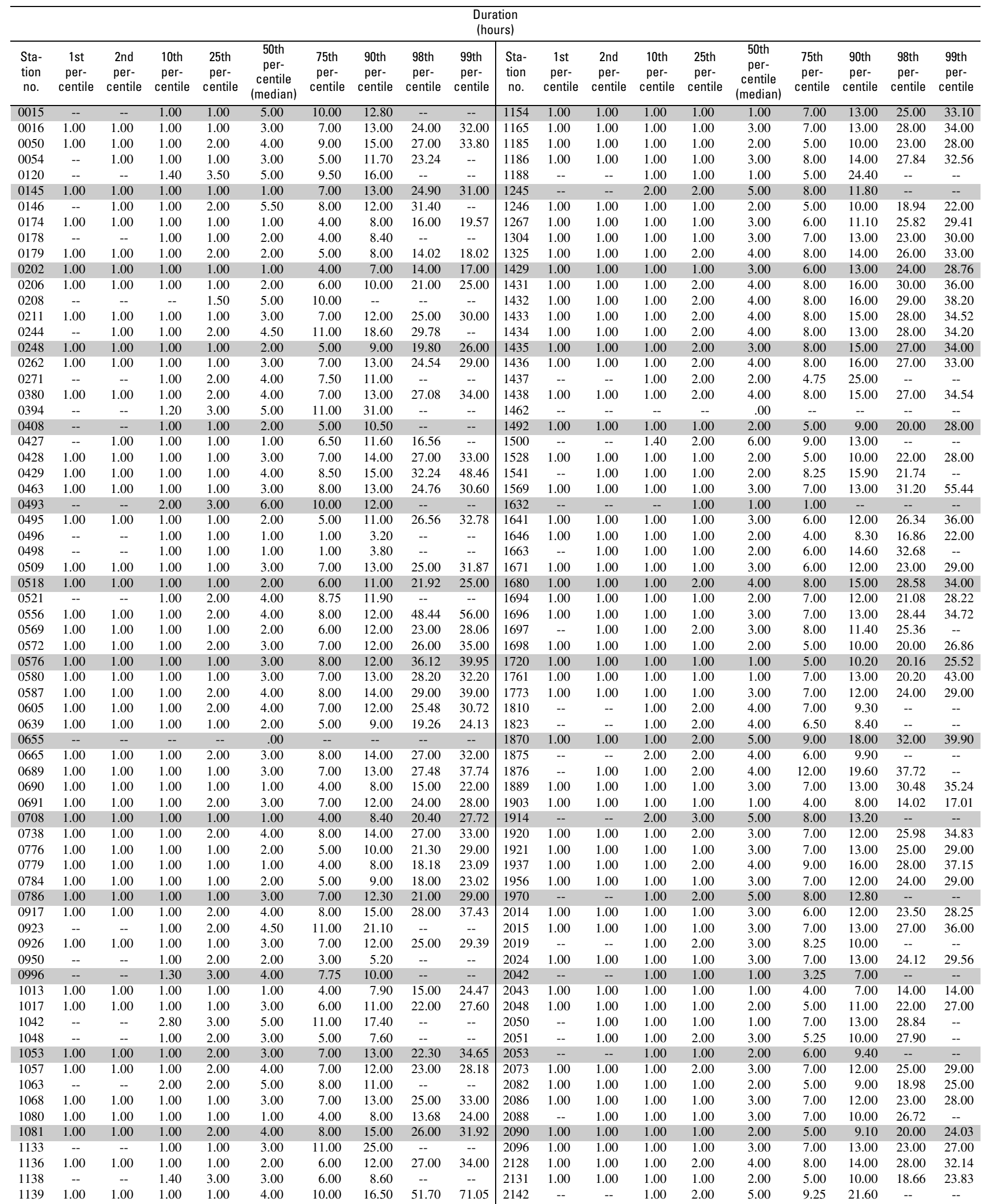


Appendix 4-5.1. Empirical distribution of storm duration defined by 6-hour minimum interevent time for hourly rainfall stations in Texas-Continued.

\begin{tabular}{|c|c|c|c|c|c|c|c|c|c|c|c|c|c|c|c|c|c|c|c|}
\hline \multicolumn{20}{|c|}{$\begin{array}{c}\text { Duration } \\
\text { (hours) }\end{array}$} \\
\hline $\begin{array}{l}\text { Sta- } \\
\text { tion } \\
\text { no. }\end{array}$ & $\begin{array}{c}\text { 1st } \\
\text { per- } \\
\text { centile }\end{array}$ & $\begin{array}{c}\text { 2nd } \\
\text { per- } \\
\text { centile }\end{array}$ & $\begin{array}{c}10 \text { th } \\
\text { per- } \\
\text { centile }\end{array}$ & $\begin{array}{c}25 \text { th } \\
\text { per- } \\
\text { centile }\end{array}$ & $\begin{array}{c}\text { 50th } \\
\text { per- } \\
\text { centile } \\
\text { (median) }\end{array}$ & $\begin{array}{c}\text { 75th } \\
\text { per- } \\
\text { centile }\end{array}$ & $\begin{array}{c}\text { 90th } \\
\text { per- } \\
\text { centile }\end{array}$ & $\begin{array}{c}\text { 98th } \\
\text { per- } \\
\text { centile }\end{array}$ & $\begin{array}{c}\text { 99th } \\
\text { per- } \\
\text { centile }\end{array}$ & $\begin{array}{l}\text { Sta- } \\
\text { tion } \\
\text { no. }\end{array}$ & $\begin{array}{c}1 \text { st } \\
\text { per- } \\
\text { centile }\end{array}$ & $\begin{array}{c}\text { 2nd } \\
\text { per- } \\
\text { centile }\end{array}$ & $\begin{array}{c}\text { 10th } \\
\text { per- } \\
\text { centile }\end{array}$ & $\begin{array}{c}25 \text { th } \\
\text { per- } \\
\text { centile }\end{array}$ & $\begin{array}{c}\text { 50th } \\
\text { per- } \\
\text { centile } \\
\text { (median) }\end{array}$ & $\begin{array}{c}\text { 75th } \\
\text { per- } \\
\text { centile }\end{array}$ & $\begin{array}{c}\text { 90th } \\
\text { per- } \\
\text { centile }\end{array}$ & $\begin{array}{c}\text { 98th } \\
\text { per- } \\
\text { centile }\end{array}$ & $\begin{array}{l}\text { 99th } \\
\text { per- } \\
\text { centile }\end{array}$ \\
\hline 2160 & -- & -- & 1.00 & 1.00 & 7.00 & 13.00 & 25.00 & -- & -- & 3463 & -- & 1.00 & 1.00 & 1.00 & 3.00 & 9.00 & 20.00 & 29.00 & -- \\
\hline 2206 & 1.00 & 1.00 & 1.00 & 2.00 & 3.00 & 7.00 & 12.00 & 26.70 & 30.70 & 3476 & 1.00 & 1.00 & 1.00 & 2.00 & 3.00 & 7.00 & 13.00 & 26.00 & 33.00 \\
\hline 2238 & 1.00 & 1.00 & 1.00 & 1.00 & 3.00 & 6.00 & 12.00 & 26.56 & 32.07 & 3485 & -- & -- & 1.60 & 2.00 & 6.00 & 7.00 & 11.40 & -- & -- \\
\hline 2240 & 1.00 & 1.00 & 1.00 & 1.00 & 1.00 & 7.00 & 13.00 & 19.00 & 19.00 & 3507 & 1.00 & 1.00 & 1.00 & 1.00 & 2.00 & 5.00 & 10.00 & 20.62 & 26.00 \\
\hline 2242 & 1.00 & 1.00 & 1.00 & 2.00 & 3.00 & 8.00 & 13.00 & 25.48 & 33.00 & 3546 & 1.00 & 1.00 & 1.00 & 1.00 & 3.00 & 7.00 & 13.00 & 26.00 & 29.73 \\
\hline 2244 & 1.00 & 1.00 & 1.00 & 1.00 & 3.00 & 7.00 & 13.00 & 25.00 & 30.00 & 3547 & 1.00 & 1.00 & 1.00 & 1.00 & 3.00 & 7.00 & 11.00 & 25.36 & 28.00 \\
\hline 2247 & -- & 1.00 & 1.00 & 2.00 & 4.00 & 7.00 & 11.50 & 20.60 & -- & 3579 & -- & 1.00 & 1.00 & 2.00 & 4.00 & 9.00 & 15.00 & 37.40 & -- \\
\hline 2309 & 1.00 & 1.00 & 1.00 & 2.00 & 4.00 & 9.00 & 14.00 & 25.00 & 28.00 & 3642 & 1.00 & 1.00 & 1.00 & 1.00 & 3.00 & 7.00 & 13.00 & 23.00 & 27.00 \\
\hline 2312 & 1.00 & 1.00 & 1.00 & 1.00 & 2.00 & 6.00 & 11.00 & 19.94 & 21.97 & 3646 & 1.00 & 1.00 & 1.00 & 2.00 & 4.00 & 8.00 & 14.00 & 26.00 & 31.00 \\
\hline 2334 & -- & 1.00 & 1.00 & 1.00 & 3.50 & 9.00 & 17.30 & 27.30 & -- & 3668 & -- & -- & 2.00 & 4.00 & 6.50 & 11.50 & 17.20 & -- & -- \\
\hline 2336 & 1.00 & 1.00 & 1.00 & 1.00 & 3.00 & 6.00 & 11.00 & 20.00 & 32.00 & 3673 & -- & -- & 1.00 & 1.75 & 3.50 & 8.00 & 13.20 & -- & -- \\
\hline 2354 & -- & -- & 1.00 & 1.00 & 3.00 & 6.50 & 13.70 & -- & -- & 3686 & 1.00 & 1.00 & 1.00 & 1.00 & 2.00 & 6.00 & 10.00 & 19.00 & 23.75 \\
\hline 2355 & -- & 1.00 & 1.00 & 2.00 & 4.00 & 7.75 & 19.00 & 26.70 & -- & 3691 & 1.00 & 1.00 & 1.00 & 1.00 & 2.00 & 6.00 & 11.00 & 20.00 & 25.00 \\
\hline 2357 & 1.00 & 1.00 & 1.00 & 1.00 & 3.00 & 7.00 & 15.00 & 28.00 & 35.00 & 3734 & -- & -- & 1.00 & 2.00 & 3.50 & 8.00 & 10.60 & -- & -- \\
\hline 2360 & 1.00 & 1.00 & 1.00 & 1.00 & 2.50 & 6.00 & 12.00 & 23.00 & 31.62 & 3771 & 1.00 & 1.00 & 1.00 & 1.00 & 2.00 & 5.00 & 10.00 & 19.40 & 24.00 \\
\hline 2361 & 1.00 & 1.00 & 1.00 & 1.00 & 2.00 & 4.75 & 9.70 & 17.22 & 27.22 & 3789 & 1.00 & 1.00 & 1.00 & 1.00 & 1.00 & 7.00 & 13.00 & 23.32 & 40.48 \\
\hline 2394 & 1.00 & 1.00 & 1.00 & 2.00 & 4.00 & 8.00 & 14.00 & 25.00 & 32.00 & 3826 & 1.00 & 1.00 & 1.00 & 2.00 & 4.00 & 9.00 & 13.00 & 20.36 & 24.18 \\
\hline 2404 & 1.00 & 1.00 & 1.00 & 1.00 & 3.00 & 7.00 & 13.00 & 24.00 & 31.00 & 3831 & 1.00 & 1.00 & 1.00 & 2.00 & 3.00 & 7.75 & 13.30 & 25.00 & 35.15 \\
\hline 2415 & 1.00 & 1.00 & 1.00 & 2.00 & 4.00 & 8.00 & 14.00 & 27.00 & 33.00 & 3841 & 1.00 & 1.00 & 1.00 & 1.00 & 3.00 & 6.00 & 10.00 & 20.60 & 31.10 \\
\hline 2462 & 1.00 & 1.00 & 1.00 & 1.00 & 3.00 & 8.00 & 14.00 & 24.00 & 29.00 & 3871 & 1.00 & 1.00 & 1.00 & 2.00 & 4.00 & 7.25 & 13.00 & 25.00 & 32.00 \\
\hline 2528 & 1.00 & 1.00 & 1.00 & 1.00 & 4.00 & 8.00 & 14.00 & 45.60 & 54.50 & 3884 & -- & -- & 1.40 & 3.00 & 5.00 & 7.00 & 10.20 & -- & -- \\
\hline 2617 & 1.00 & 1.00 & 1.00 & 2.00 & 4.00 & 8.00 & 15.30 & 28.92 & 36.68 & 3941 & -- & 1.00 & 1.00 & 1.00 & 5.00 & 10.00 & 19.00 & 30.40 & -- \\
\hline 2619 & 1.00 & 1.00 & 1.00 & 1.00 & 4.00 & 7.00 & 12.00 & 25.00 & 33.00 & 3963 & -- & -- & -- & 1.00 & 1.00 & 1.00 & -- & -- & -- \\
\hline 2621 & 1.00 & 1.00 & 1.00 & 1.00 & 3.00 & 6.00 & 12.00 & 23.80 & 30.40 & 4040 & 1.00 & 1.00 & 1.00 & 2.00 & 3.50 & 7.00 & 12.00 & 27.00 & 31.00 \\
\hline 2675 & 1.00 & 1.00 & 1.00 & 1.00 & 3.00 & 6.00 & 12.00 & 21.64 & 27.32 & 4058 & -- & -- & 1.00 & 1.75 & 3.00 & 5.00 & 9.90 & -- & -- \\
\hline 2676 & 1.00 & 1.00 & 1.00 & 1.00 & 1.00 & 5.00 & 9.00 & 18.00 & 23.00 & 4098 & 1.00 & 1.00 & 1.00 & 1.00 & 2.00 & 4.00 & 8.00 & 16.00 & 19.67 \\
\hline 2679 & 1.00 & 1.00 & 1.00 & 1.00 & 2.00 & 6.00 & 10.00 & 20.26 & 26.13 & 4100 & 1.00 & 1.00 & 1.00 & 1.00 & 3.00 & 6.00 & 11.00 & 24.00 & 27.00 \\
\hline 2715 & 1.00 & 1.00 & 1.00 & 2.00 & 3.00 & 7.00 & 13.00 & 25.00 & 31.00 & 4137 & 1.00 & 1.00 & 1.00 & 1.00 & 2.00 & 5.00 & 9.00 & 17.26 & 21.63 \\
\hline 2744 & 1.00 & 1.00 & 1.00 & 1.00 & 3.00 & 6.00 & 11.00 & 23.38 & 31.00 & 4191 & 1.00 & 1.00 & 1.00 & 1.00 & 3.00 & 6.00 & 11.00 & 23.00 & 30.00 \\
\hline 2758 & 1.00 & 1.00 & 1.00 & 1.00 & 3.00 & 7.00 & 16.40 & 33.96 & 56.92 & 4256 & -- & -- & -- & -- & .00 & -- & -- & -- & -- \\
\hline 2794 & -- & -- & 1.00 & 1.00 & 3.00 & 7.00 & 24.80 & -- & -- & 4257 & 1.00 & 1.00 & 1.00 & 1.00 & 3.00 & 6.00 & 12.00 & 24.00 & 29.00 \\
\hline 2797 & 1.00 & 1.00 & 1.00 & 1.00 & 2.00 & 5.00 & 10.00 & 20.00 & 26.00 & 4258 & 1.00 & 1.00 & 1.00 & 1.00 & 2.00 & 6.00 & 10.00 & 18.56 & 23.34 \\
\hline 2811 & 1.00 & 1.00 & 1.00 & 1.00 & 2.00 & 5.00 & 11.00 & 21.00 & 28.00 & 4278 & 1.00 & 1.00 & 1.00 & 2.00 & 3.00 & 7.00 & 13.00 & 25.00 & 32.00 \\
\hline 2813 & -- & -- & 1.00 & 1.00 & 2.50 & 5.00 & 9.70 & -- & -- & 4299 & 1.00 & 1.00 & 1.00 & 1.00 & 2.00 & 5.00 & 9.40 & 17.56 & 19.00 \\
\hline 2814 & -- & -- & 1.00 & 1.00 & 1.00 & 4.00 & 18.00 & -- & -- & 4300 & 1.00 & 1.00 & 1.00 & 1.00 & 3.00 & 7.00 & 13.00 & 27.00 & 34.27 \\
\hline 2815 & 1.00 & 1.00 & 1.00 & 1.00 & 2.00 & 4.00 & 8.00 & 17.00 & 19.16 & 4305 & 1.00 & 1.00 & 1.00 & 1.00 & 3.00 & 7.00 & 13.00 & 27.00 & 35.00 \\
\hline 2818 & 1.00 & 1.00 & 1.00 & 1.00 & 3.00 & 7.00 & 12.00 & 26.60 & 37.00 & 4307 & 1.00 & 1.00 & 1.00 & 1.00 & 3.00 & 7.00 & 13.00 & 30.58 & 33.29 \\
\hline 2986 & 1.00 & 1.00 & 1.00 & 2.00 & 4.00 & 9.00 & 16.00 & 25.00 & 31.08 & 4309 & 1.00 & 1.00 & 1.00 & 2.00 & 3.00 & 7.00 & 13.00 & 25.00 & 31.57 \\
\hline 3005 & 1.00 & 1.00 & 1.00 & 1.00 & 3.00 & 6.00 & 12.00 & 23.00 & 29.00 & 4311 & 1.00 & 1.00 & 1.00 & 2.00 & 3.00 & 8.00 & 13.00 & 26.00 & 32.00 \\
\hline 3033 & 1.00 & 1.00 & 1.00 & 2.00 & 3.00 & 5.00 & 9.00 & 17.00 & 21.99 & 4313 & 1.00 & 1.00 & 1.00 & 1.00 & 3.00 & 7.00 & 15.00 & 27.64 & 38.15 \\
\hline 3034 & -- & -- & -- & -- & 1.00 & -- & -- & -- & -- & 4319 & 1.00 & 1.00 & 1.00 & 2.00 & 4.00 & 7.75 & 13.10 & 31.10 & 32.91 \\
\hline 3047 & -- & -- & 1.00 & 2.00 & 4.00 & 8.00 & 12.90 & -- & -- & 4329 & 1.00 & 1.00 & 1.00 & 2.00 & 3.00 & 8.00 & 14.00 & 27.68 & 32.00 \\
\hline 3103 & -- & -- & 1.00 & 1.00 & 2.00 & 5.50 & 10.40 & -- & -- & 4331 & -- & -- & -- & -- & .00 & -- & -- & -- & -- \\
\hline 3133 & 1.00 & 1.00 & 1.00 & 1.00 & 4.00 & 8.00 & 13.00 & 26.46 & 33.00 & 4375 & 1.00 & 1.00 & 1.00 & 1.00 & 1.00 & 5.00 & 8.70 & 17.00 & 20.07 \\
\hline 3156 & 1.00 & 1.00 & 1.00 & 1.00 & 3.00 & 6.00 & 14.00 & 29.24 & 32.24 & 4392 & 1.00 & 1.00 & 1.00 & 2.00 & 4.00 & 9.00 & 17.00 & 31.00 & 37.00 \\
\hline 3171 & 1.00 & 1.00 & 1.00 & 2.00 & 4.00 & 8.00 & 14.00 & 26.00 & 33.00 & 4425 & 1.00 & 1.00 & 1.00 & 1.00 & 1.00 & 4.00 & 8.80 & 17.00 & 24.18 \\
\hline 3189 & 1.00 & 1.00 & 1.00 & 1.00 & 2.00 & 5.00 & 10.00 & 19.02 & 23.01 & 4440 & 1.00 & 1.00 & 1.00 & 2.00 & 4.00 & 8.00 & 14.00 & 28.10 & 32.00 \\
\hline 3260 & 1.00 & 1.00 & 1.00 & 1.00 & 3.00 & 7.00 & 13.00 & 23.22 & 26.55 & 4476 & 1.00 & 1.00 & 1.00 & 1.00 & 2.00 & 6.00 & 10.00 & 20.00 & 24.90 \\
\hline 3267 & 1.00 & 1.00 & 1.00 & 2.00 & 3.00 & 7.00 & 15.00 & 29.40 & 37.40 & 4498 & -- & -- & 1.00 & 1.00 & 2.00 & 3.25 & 4.50 & -- & -- \\
\hline 3270 & 1.00 & 1.00 & 1.00 & 1.00 & 1.00 & 4.00 & 8.00 & 16.00 & 20.19 & 4517 & 1.00 & 1.00 & 1.00 & 2.00 & 4.00 & 8.00 & 13.00 & 26.00 & 30.00 \\
\hline 3272 & -- & -- & 1.00 & 1.00 & 1.00 & 3.00 & 6.00 & -- & -- & 4520 & 1.00 & 1.00 & 1.00 & 1.00 & 1.00 & 5.00 & 9.00 & 17.00 & 22.00 \\
\hline 3277 & -- & -- & 1.00 & 1.00 & 1.00 & 7.00 & 18.40 & -- & -- & 4525 & -- & -- & 1.60 & 2.00 & 4.00 & 8.00 & 16.40 & -- & -- \\
\hline 3278 & 1.00 & 1.00 & 1.00 & 1.00 & 2.00 & 5.00 & 9.00 & 20.00 & 28.98 & 4563 & -- & -- & 1.00 & 2.00 & 4.00 & 8.00 & 15.80 & -- & -- \\
\hline 3280 & 1.00 & 1.00 & 1.00 & 1.00 & 3.00 & 5.00 & 8.80 & 23.12 & 34.52 & 4570 & 1.00 & 1.00 & 1.00 & 1.00 & 2.00 & 6.00 & 11.00 & 23.00 & 29.66 \\
\hline 3281 & -- & -- & 1.00 & 1.00 & 2.00 & 3.00 & 8.00 & -- & -- & 4577 & 1.00 & 1.00 & 1.00 & 1.00 & 3.00 & 7.00 & 12.00 & 25.00 & 32.64 \\
\hline 3283 & 1.00 & 1.00 & 1.00 & 2.00 & 4.00 & 8.00 & 15.00 & 29.00 & 35.00 & 4591 & 1.00 & 1.00 & 1.00 & 2.00 & 4.00 & 9.00 & 15.00 & 27.54 & 32.77 \\
\hline 3284 & 1.00 & 1.00 & 1.00 & 1.00 & 3.00 & 6.00 & 11.00 & 22.00 & 26.70 & 4670 & 1.00 & 1.00 & 1.00 & 1.00 & 2.00 & 5.00 & 10.00 & 20.00 & 25.00 \\
\hline 3285 & 1.00 & 1.00 & 1.00 & 1.00 & 2.00 & 5.00 & 10.00 & 20.00 & 23.72 & 4671 & 1.00 & 1.00 & 1.00 & 1.00 & 1.00 & 7.00 & 13.00 & 19.00 & 25.00 \\
\hline 3329 & 1.00 & 1.00 & 1.00 & 1.00 & 3.00 & 7.00 & 13.00 & 25.00 & 33.00 & 4679 & 1.00 & 1.00 & 1.00 & 1.00 & 2.00 & 6.00 & 11.00 & 20.00 & 27.00 \\
\hline 3335 & 1.00 & 1.00 & 1.00 & 1.00 & 4.00 & 9.00 & 15.20 & 25.16 & 37.56 & 4696 & -- & -- & 1.00 & 1.00 & 3.00 & 6.00 & 8.20 & -- & -- \\
\hline 3370 & 1.00 & 1.00 & 1.00 & 2.00 & 4.00 & 8.00 & 14.00 & 26.28 & 31.00 & 4703 & 1.00 & 1.00 & 1.00 & 1.00 & 2.00 & 5.00 & 12.00 & 27.50 & 35.55 \\
\hline 3410 & 1.00 & 1.00 & 1.00 & 1.00 & 2.00 & 6.00 & 10.00 & 19.76 & 25.00 & 4704 & 1.00 & 1.00 & 1.00 & 1.00 & 3.00 & 9.00 & 16.00 & 29.00 & 33.78 \\
\hline 3415 & 1.00 & 1.00 & 1.00 & 1.00 & 2.00 & 6.00 & 11.00 & 21.00 & 24.00 & 4731 & 1.00 & 1.00 & 1.00 & 1.00 & 3.00 & 8.00 & 15.00 & 32.00 & 32.53 \\
\hline 3430 & 1.00 & 1.00 & 1.00 & 1.00 & 3.00 & 7.00 & 12.20 & 26.00 & 31.00 & 4792 & 1.00 & 1.00 & 1.00 & 1.00 & 2.00 & 5.00 & 9.00 & 18.00 & 21.97 \\
\hline 3431 & 1.00 & 1.00 & 1.00 & 1.00 & 1.00 & 7.00 & 13.00 & 20.72 & 30.16 & 4819 & 1.00 & 1.00 & 1.00 & 1.00 & 2.00 & 6.00 & 11.00 & 21.00 & 24.12 \\
\hline 3441 & -- & -- & 1.00 & 1.00 & 3.00 & 8.50 & 13.00 & -- & -- & 4852 & -- & -- & 1.00 & 1.00 & 1.00 & 2.50 & 8.60 & -- & -- \\
\hline 3442 & 1.00 & 1.00 & 1.00 & 2.00 & 4.00 & 7.00 & 12.00 & 19.70 & 32.40 & 4866 & 1.00 & 1.00 & 1.00 & 1.00 & 3.00 & 7.00 & 13.00 & 25.32 & 32.00 \\
\hline 3446 & 1.00 & 1.00 & 1.00 & 1.00 & 3.00 & 6.00 & 10.00 & 22.56 & 28.78 & 4876 & 1.00 & 1.00 & 1.00 & 1.00 & 2.00 & 6.00 & 10.40 & 22.88 & 26.64 \\
\hline 3460 & -- & -- & 1.00 & 2.00 & 3.00 & 6.00 & 11.20 & -- & -- & 4878 & 1.00 & 1.00 & 1.00 & 2.00 & 4.00 & 8.00 & 14.00 & 27.00 & 34.00 \\
\hline 3462 & 1.00 & 1.00 & 1.00 & 1.00 & 3.00 & 7.00 & 14.00 & 22.72 & 24.08 & 4880 & 1.00 & 1.00 & 1.00 & 1.00 & 3.00 & 6.00 & 11.00 & 22.00 & 27.76 \\
\hline
\end{tabular}


Appendix 4-5.1. Empirical distribution of storm duration defined by 6-hour minimum interevent time for hourly rainfall stations in Texas-Continued.

\begin{tabular}{|c|c|c|c|c|c|c|c|c|c|c|c|c|c|c|c|c|c|c|c|}
\hline \multicolumn{20}{|c|}{$\begin{array}{c}\text { Duration } \\
\text { (hours) }\end{array}$} \\
\hline $\begin{array}{l}\text { Sta- } \\
\text { tion } \\
\text { no. }\end{array}$ & $\begin{array}{c}1 \text { st } \\
\text { per- } \\
\text { centile }\end{array}$ & $\begin{array}{c}\text { 2nd } \\
\text { per- } \\
\text { centile }\end{array}$ & $\begin{array}{c}\text { 10th } \\
\text { per- } \\
\text { centile }\end{array}$ & $\begin{array}{c}25 \text { th } \\
\text { per- } \\
\text { centile }\end{array}$ & $\begin{array}{c}\text { 50th } \\
\text { per- } \\
\text { centile } \\
\text { (median) }\end{array}$ & $\begin{array}{c}\text { 75th } \\
\text { per- } \\
\text { centile }\end{array}$ & $\begin{array}{c}\text { 90th } \\
\text { per- } \\
\text { centile }\end{array}$ & $\begin{array}{c}\text { 98th } \\
\text { per- } \\
\text { centile }\end{array}$ & $\begin{array}{c}\text { 99th } \\
\text { per- } \\
\text { centile }\end{array}$ & $\begin{array}{l}\text { Sta- } \\
\text { tion } \\
\text { no. }\end{array}$ & $\begin{array}{c}\text { 1st } \\
\text { per- } \\
\text { centile }\end{array}$ & $\begin{array}{l}\text { 2nd } \\
\text { per- } \\
\text { centile }\end{array}$ & $\begin{array}{c}10 \text { th } \\
\text { per- } \\
\text { centile }\end{array}$ & $\begin{array}{c}25 \text { th } \\
\text { per- } \\
\text { centile }\end{array}$ & $\begin{array}{c}\text { 50th } \\
\text { per- } \\
\text { centile } \\
\text { (median) }\end{array}$ & $\begin{array}{c}\text { 75th } \\
\text { per- } \\
\text { centile }\end{array}$ & $\begin{array}{c}\text { 90th } \\
\text { per- } \\
\text { centile }\end{array}$ & $\begin{array}{c}\text { 98th } \\
\text { per- } \\
\text { centile }\end{array}$ & $\begin{array}{c}\text { 99th } \\
\text { per- } \\
\text { centile }\end{array}$ \\
\hline 4920 & 1.00 & 1.00 & 1.00 & 1.00 & 2.00 & 6.00 & 11.00 & 22.00 & 27.00 & 5957 & 1.00 & 1.00 & 1.00 & 1.00 & 2.00 & 6.00 & 10.00 & 21.66 & 27.00 \\
\hline 4934 & -- & -- & -- & 1.00 & 2.00 & 3.50 & -- & -- & -- & 5958 & 1.00 & 1.00 & 1.00 & 2.00 & 3.00 & 6.00 & 13.00 & 19.34 & 23.34 \\
\hline 4972 & 1.00 & 1.00 & 1.00 & 1.00 & 3.00 & 6.00 & 11.00 & 22.12 & 29.00 & 5973 & 1.00 & 1.00 & 1.00 & 2.00 & 4.00 & 7.00 & 13.40 & 44.76 & 55.44 \\
\hline 4973 & 1.00 & 1.00 & 1.00 & 2.00 & 4.00 & 8.50 & 14.00 & 25.00 & 29.00 & 5996 & 1.00 & 1.00 & 1.00 & 1.00 & 3.00 & 7.00 & 13.00 & 24.00 & 29.00 \\
\hline 4974 & 1.00 & 1.00 & 1.00 & 1.00 & 3.00 & 6.00 & 10.00 & 21.00 & 24.16 & 6017 & 1.00 & 1.00 & 1.00 & 1.00 & 3.00 & 6.00 & 11.00 & 18.96 & 23.36 \\
\hline 4975 & 1.00 & 1.00 & 1.00 & 1.00 & 2.00 & 6.00 & 11.00 & 20.00 & 26.46 & 6024 & 1.00 & 1.00 & 1.00 & 1.25 & 4.00 & 8.00 & 17.00 & 34.48 & 46.21 \\
\hline 4978 & 1.00 & 1.00 & 1.00 & 1.00 & 2.00 & 6.00 & 10.00 & 24.40 & 30.10 & 6050 & -- & -- & 1.00 & 2.00 & 5.00 & 10.00 & 13.00 & -- & -- \\
\hline 4979 & -- & -- & 1.50 & 3.00 & 6.50 & 13.50 & 26.50 & -- & -- & 6104 & 1.00 & 1.00 & 1.00 & 1.00 & 2.00 & 4.00 & 8.00 & 17.14 & 24.00 \\
\hline 4982 & 1.00 & 1.00 & 1.00 & 2.00 & 3.00 & 7.00 & 12.00 & 24.00 & 29.25 & 6108 & 1.00 & 1.00 & 1.00 & 1.00 & 3.00 & 7.00 & 12.00 & 24.00 & 29.94 \\
\hline 5018 & 1.00 & 1.00 & 1.00 & 2.00 & 4.00 & 7.00 & 13.00 & 22.00 & 32.00 & 6136 & 1.00 & 1.00 & 1.00 & 1.00 & 2.00 & 4.00 & 8.00 & 16.00 & 20.00 \\
\hline 5048 & 1.00 & 1.00 & 1.00 & 1.00 & 2.00 & 5.00 & 9.00 & 20.00 & 26.00 & 6166 & 1.00 & 1.00 & 1.00 & 1.75 & 3.00 & 6.00 & 10.00 & 21.00 & 27.51 \\
\hline 5049 & 1.00 & 1.00 & 1.00 & 1.00 & 1.00 & 4.00 & 7.00 & 11.80 & 12.00 & 6176 & 1.00 & 1.00 & 1.00 & 2.00 & 3.00 & 9.00 & 16.00 & 28.04 & 38.51 \\
\hline 5056 & -- & -- & -- & 1.00 & 4.00 & 12.50 & -- & -- & -- & 6177 & 1.00 & 1.00 & 1.00 & 1.00 & 3.00 & 7.00 & 12.00 & 25.00 & 31.00 \\
\hline 5057 & 1.00 & 1.00 & 1.00 & 1.00 & 2.00 & 6.00 & 13.00 & 28.00 & 37.00 & 6210 & 1.00 & 1.00 & 1.00 & 1.00 & 3.00 & 6.00 & 12.00 & 22.00 & 27.00 \\
\hline 5060 & 1.00 & 1.00 & 1.00 & 2.00 & 3.00 & 7.00 & 13.00 & 36.68 & 50.26 & 6211 & 1.00 & 1.00 & 1.00 & 1.00 & 2.00 & 6.00 & 12.00 & 24.70 & 34.00 \\
\hline 5081 & 1.00 & 1.00 & 1.00 & 2.00 & 4.00 & 9.00 & 16.00 & 28.16 & 32.08 & 6270 & 1.00 & 1.00 & 1.00 & 1.00 & 2.00 & 6.00 & 11.00 & 21.00 & 28.00 \\
\hline 5094 & 1.00 & 1.00 & 1.00 & 1.00 & 2.00 & 6.00 & 11.00 & 21.00 & 26.00 & 6275 & -- & -- & -- & -- & .00 & -- & -- & -- & -- \\
\hline 5113 & 1.00 & 1.00 & 1.00 & 1.00 & 2.00 & 6.00 & 12.00 & 23.00 & 28.00 & 6276 & -- & -- & 1.00 & 3.00 & 5.00 & 10.00 & 17.00 & -- & -- \\
\hline 5114 & -- & -- & -- & -- & .00 & -- & -- & -- & -- & 6335 & 1.00 & 1.00 & 1.00 & 2.00 & 4.00 & 8.00 & 14.00 & 26.00 & 31.00 \\
\hline 5123 & -- & -- & 1.00 & 1.50 & 3.00 & 5.50 & 13.00 & -- & -- & 6434 & -- & -- & 1.00 & 2.00 & 5.00 & 8.00 & 18.40 & -- & -- \\
\hline 5192 & 1.00 & 1.00 & 1.00 & 1.00 & 3.00 & 7.00 & 13.00 & 23.52 & 29.00 & 6504 & 1.00 & 1.00 & 1.00 & 1.00 & 2.00 & 5.00 & 9.00 & 19.00 & 24.49 \\
\hline 5193 & 1.00 & 1.00 & 1.00 & 1.00 & 3.00 & 7.00 & 13.00 & 25.00 & 29.85 & 6558 & -- & -- & 1.00 & 2.00 & 4.50 & 9.00 & 13.00 & -- & -- \\
\hline 5224 & 1.00 & 1.00 & 1.00 & 1.00 & 4.00 & 8.00 & 13.00 & 22.68 & 27.68 & 6615 & 1.00 & 1.00 & 1.00 & 1.00 & 1.00 & 4.00 & 8.00 & 16.00 & 21.46 \\
\hline 5228 & 1.00 & 1.00 & 1.00 & 1.00 & 3.00 & 7.00 & 12.00 & 28.62 & 35.00 & 6660 & 1.00 & 1.00 & 1.00 & 2.00 & 4.00 & 8.00 & 13.00 & 32.76 & 43.34 \\
\hline 5235 & -- & -- & 1.00 & 2.00 & 4.00 & 11.00 & 19.40 & -- & -- & 6663 & 1.00 & 1.00 & 1.00 & 1.00 & 1.00 & 4.00 & 10.00 & 18.80 & 28.00 \\
\hline 5247 & 1.00 & 1.00 & 1.00 & 1.00 & 2.00 & 5.00 & 9.00 & 19.94 & 26.00 & 6734 & 1.00 & 1.00 & 1.00 & 1.00 & 3.00 & 7.00 & 13.00 & 20.48 & 25.86 \\
\hline 5258 & 1.00 & 1.00 & 1.00 & 2.00 & 4.00 & 8.00 & 14.00 & 28.78 & 35.89 & 6736 & 1.00 & 1.00 & 1.00 & 1.00 & 2.00 & 5.00 & 9.00 & 20.00 & 27.00 \\
\hline 5303 & 1.00 & 1.00 & 1.00 & 2.00 & 3.00 & 7.00 & 13.00 & 23.00 & 27.00 & 6740 & -- & -- & 1.20 & 2.50 & 7.00 & 12.50 & 21.60 & -- & -- \\
\hline 5312 & 1.00 & 1.00 & 1.00 & 1.00 & 2.00 & 5.00 & 9.00 & 19.00 & 24.86 & 6750 & 1.00 & 1.00 & 1.00 & 1.00 & 1.00 & 7.00 & 13.00 & 28.08 & 43.00 \\
\hline 5341 & -- & -- & 1.00 & 2.00 & 4.00 & 6.50 & 18.80 & -- & -- & 6757 & 1.00 & 1.00 & 1.00 & 1.00 & 3.00 & 7.00 & 13.00 & 26.00 & 31.00 \\
\hline 5342 & -- & -- & -- & -- & .00 & -- & -- & -- & -- & 6775 & 1.00 & 1.00 & 1.00 & 1.00 & 3.00 & 7.00 & 13.00 & 27.40 & 35.00 \\
\hline 5348 & 1.00 & 1.00 & 1.00 & 1.00 & 3.00 & 6.00 & 11.30 & 23.00 & 25.23 & 6776 & 1.00 & 1.00 & 1.00 & 1.00 & 2.00 & 5.00 & 9.80 & 21.00 & 26.00 \\
\hline 5358 & 1.00 & 1.00 & 1.00 & 1.50 & 3.00 & 6.00 & 10.00 & 21.00 & 28.00 & 6788 & 1.00 & 1.00 & 1.00 & 2.00 & 4.00 & 7.00 & 12.70 & 18.00 & 25.82 \\
\hline 5398 & 1.00 & 1.00 & 1.00 & 2.00 & 4.00 & 8.00 & 15.00 & 27.00 & 35.00 & 6792 & 1.00 & 1.00 & 1.00 & 1.00 & 2.00 & 4.00 & 7.00 & 16.00 & 19.87 \\
\hline 5410 & 1.00 & 1.00 & 1.00 & 1.00 & 2.00 & 5.00 & 9.00 & 20.00 & 26.17 & 6794 & -- & -- & 1.00 & 3.00 & 6.50 & 11.50 & 19.90 & -- & -- \\
\hline 5411 & 1.00 & 1.00 & 1.00 & 1.00 & 3.00 & 6.00 & 12.00 & 25.00 & 30.14 & 6834 & 1.00 & 1.00 & 1.00 & 1.00 & 2.00 & 6.00 & 11.00 & 20.08 & 25.00 \\
\hline 5424 & 1.00 & 1.00 & 1.00 & 1.00 & 3.00 & 7.00 & 15.60 & 34.12 & 41.24 & 6893 & 1.00 & 1.00 & 1.00 & 1.00 & 2.00 & 4.00 & 8.00 & 16.00 & 20.85 \\
\hline 5429 & 1.00 & 1.00 & 1.00 & 1.00 & 3.00 & 6.00 & 12.00 & 24.54 & 29.27 & 6935 & 1.00 & 1.00 & 1.00 & 1.00 & 2.00 & 4.00 & 8.00 & 16.00 & 20.00 \\
\hline 5431 & -- & -- & 1.00 & 3.75 & 10.00 & 15.25 & 23.50 & -- & -- & 6981 & 1.00 & 1.00 & 1.00 & 2.00 & 3.00 & 7.00 & 13.00 & 27.32 & 34.83 \\
\hline 5461 & 1.00 & 1.00 & 1.00 & 2.00 & 4.00 & 9.00 & 15.00 & 30.00 & 37.00 & 7020 & 1.00 & 1.00 & 1.00 & 2.00 & 4.00 & 11.00 & 19.00 & 29.80 & 38.40 \\
\hline 5463 & 1.00 & 1.00 & 1.00 & 1.00 & 2.00 & 5.00 & 10.00 & 21.48 & 24.00 & 7060 & 1.00 & 1.00 & 1.00 & 1.00 & 2.00 & 5.00 & 9.00 & 18.00 & 22.12 \\
\hline 5471 & -- & -- & 1.00 & 1.00 & 1.00 & 1.75 & 4.10 & -- & -- & 7066 & 1.00 & 1.00 & 1.00 & 1.00 & 3.00 & 7.00 & 13.00 & 24.28 & 31.92 \\
\hline 5477 & -- & -- & 1.00 & 1.00 & 2.50 & 7.00 & 17.30 & -- & -- & 7074 & 1.00 & 1.00 & 1.00 & 1.00 & 2.00 & 5.00 & 9.00 & 18.00 & 23.00 \\
\hline 5528 & 1.00 & 1.00 & 1.00 & 2.00 & 3.00 & 7.00 & 13.00 & 25.00 & 29.00 & 7097 & 1.00 & 1.00 & 1.00 & 2.00 & 3.00 & 7.00 & 12.00 & 24.88 & 30.00 \\
\hline 5579 & -- & -- & -- & -- & 4.00 & -- & -- & -- & -- & 7116 & 1.00 & 1.00 & 1.00 & 2.00 & 3.00 & 6.00 & 12.00 & 23.00 & 34.00 \\
\hline 5580 & -- & -- & 1.00 & 2.00 & 4.00 & 5.50 & 13.00 & -- & -- & 7140 & 1.00 & 1.00 & 1.00 & 1.00 & 3.00 & 7.00 & 13.00 & 24.24 & 31.00 \\
\hline 5589 & 1.00 & 1.00 & 1.00 & 1.00 & 2.00 & 4.00 & 8.00 & 13.40 & 19.10 & 7173 & 1.00 & 1.00 & 1.00 & 1.00 & 3.00 & 7.00 & 14.00 & 29.00 & 34.08 \\
\hline 5590 & 1.00 & 1.00 & 1.00 & 1.00 & 2.50 & 5.00 & 12.50 & 26.30 & 35.40 & 7174 & 1.00 & 1.00 & 1.00 & 1.00 & 3.00 & 7.00 & 13.00 & 26.10 & 33.05 \\
\hline 5591 & 1.00 & 1.00 & 1.00 & 1.00 & 2.00 & 5.00 & 8.00 & 18.00 & 20.00 & 7213 & 1.00 & 1.00 & 1.00 & 2.00 & 3.00 & 8.00 & 14.00 & 28.00 & 35.00 \\
\hline 5592 & 1.00 & 1.00 & 1.00 & 2.00 & 3.00 & 5.00 & 9.00 & 16.74 & 20.87 & 7243 & 1.00 & 1.00 & 1.00 & 1.00 & 2.00 & 5.00 & 11.00 & 23.00 & 29.00 \\
\hline 5594 & 1.00 & 1.00 & 1.00 & 1.00 & 2.00 & 4.00 & 7.00 & 17.00 & 21.00 & 7262 & 1.00 & 1.00 & 1.00 & 1.00 & 1.00 & 1.00 & 7.00 & 19.00 & 30.16 \\
\hline 5595 & -- & -- & -- & -- & 1.50 & -- & -- & -- & -- & 7274 & 1.00 & 1.00 & 1.00 & 1.00 & 3.00 & 7.00 & 11.00 & 22.56 & 27.56 \\
\hline 5596 & 1.00 & 1.00 & 1.00 & 1.00 & 1.00 & 3.00 & 6.00 & 14.00 & 15.00 & 7300 & 1.00 & 1.00 & 1.00 & 2.00 & 3.00 & 7.00 & 12.00 & 22.00 & 27.35 \\
\hline 5600 & 1.00 & 1.00 & 1.00 & 2.00 & 3.00 & 5.00 & 11.00 & 25.64 & 31.56 & 7311 & -- & -- & 1.00 & 1.00 & 3.00 & 6.75 & 9.70 & -- & -- \\
\hline 5618 & -- & -- & 2.00 & 2.00 & 4.00 & 8.50 & 12.40 & -- & -- & 7363 & -- & -- & 1.00 & 2.00 & 4.00 & 9.75 & 16.20 & -- & -- \\
\hline 5650 & -- & -- & 1.30 & 2.00 & 3.00 & 6.25 & 10.40 & -- & -- & 7422 & 1.00 & 1.00 & 1.00 & 1.00 & 2.00 & 6.00 & 12.00 & 24.00 & 29.00 \\
\hline 5656 & 1.00 & 1.00 & 1.00 & 1.00 & 1.00 & 4.00 & 8.00 & 17.72 & 23.00 & 7431 & 1.00 & 1.00 & 1.00 & 1.00 & 2.00 & 5.00 & 11.00 & 23.00 & 31.61 \\
\hline 5658 & 1.00 & 1.00 & 1.00 & 2.00 & 3.00 & 7.00 & 12.00 & 28.16 & 36.04 & 7481 & 1.00 & 1.00 & 1.00 & 1.00 & 2.00 & 5.00 & 10.00 & 20.00 & 23.00 \\
\hline 5661 & 1.00 & 1.00 & 1.00 & 1.00 & 1.00 & 5.00 & 10.00 & 21.00 & 30.60 & 7497 & 1.00 & 1.00 & 1.00 & 1.00 & 2.00 & 5.00 & 10.00 & 20.00 & 26.00 \\
\hline 5666 & -- & -- & 1.00 & 1.75 & 3.00 & 8.25 & 11.00 & -- & -- & 7498 & 1.00 & 1.00 & 1.00 & 1.00 & 2.00 & 5.00 & 10.00 & 24.28 & 27.00 \\
\hline 5695 & 1.00 & 1.00 & 1.00 & 1.00 & 3.00 & 7.00 & 13.00 & 24.00 & 28.93 & 7499 & 1.00 & 1.00 & 1.00 & 1.00 & 2.00 & 6.00 & 10.00 & 19.00 & 24.00 \\
\hline 5742 & -- & -- & 1.00 & 1.00 & 5.00 & 8.00 & 11.60 & -- & -- & 7531 & 1.00 & 1.00 & 1.00 & 2.00 & 3.00 & 9.00 & 15.40 & 32.36 & 38.76 \\
\hline 5766 & -- & -- & 1.00 & 2.00 & 3.00 & 9.00 & 11.80 & -- & -- & 7534 & 1.00 & 1.00 & 1.00 & 1.00 & 3.00 & 7.00 & 12.70 & 26.00 & 32.74 \\
\hline 5770 & 1.00 & 1.00 & 1.00 & 1.00 & 2.00 & 6.00 & 11.00 & 21.00 & 27.00 & 7556 & 1.00 & 1.00 & 1.00 & 1.00 & 2.00 & 6.00 & 11.00 & 22.00 & 25.00 \\
\hline 5775 & -- & -- & 1.00 & 1.00 & 3.00 & 7.25 & 16.50 & -- & -- & 7594 & 1.00 & 1.00 & 1.00 & 2.00 & 3.00 & 7.00 & 13.00 & 26.00 & 31.29 \\
\hline 5779 & -- & -- & 1.00 & 1.00 & 5.00 & 9.00 & 11.80 & -- & -- & 7596 & 1.00 & 1.00 & 1.00 & 2.00 & 3.00 & 7.00 & 13.00 & 35.44 & 54.20 \\
\hline 5840 & 1.00 & 1.00 & 1.00 & 2.00 & 4.00 & 8.00 & 15.00 & 28.40 & 36.00 & 7608 & 1.00 & 1.00 & 1.00 & 1.00 & 3.00 & 7.00 & 13.00 & 24.00 & 29.34 \\
\hline 5890 & 1.00 & 1.00 & 1.00 & 1.00 & 3.00 & 6.00 & 11.00 & 24.00 & 31.00 & 7622 & -- & -- & 1.00 & 1.00 & 2.00 & 5.00 & 14.60 & -- & -- \\
\hline 5891 & 1.00 & 1.00 & 1.00 & 1.00 & 3.00 & 5.00 & 10.80 & 24.00 & 25.36 & 7700 & 1.00 & 1.00 & 1.00 & 2.00 & 4.00 & 8.00 & 15.00 & 28.00 & 34.36 \\
\hline 5897 & 1.00 & 1.00 & 1.00 & 1.00 & 2.00 & 5.00 & 10.00 & 19.00 & 24.00 & 7706 & 1.00 & 1.00 & 1.00 & 1.00 & 2.00 & 5.00 & 10.00 & 20.00 & 24.72 \\
\hline
\end{tabular}


Appendix 4-5.1. Empirical distribution of storm duration defined by 6-hour minimum interevent time for hourly rainfall stations in Texas-Continued.

\begin{tabular}{|c|c|c|c|c|c|c|c|c|c|c|c|c|c|c|c|c|c|c|c|}
\hline \multicolumn{20}{|c|}{$\begin{array}{c}\text { Duration } \\
\text { (hours) }\end{array}$} \\
\hline $\begin{array}{l}\text { Sta- } \\
\text { tion } \\
\text { no. }\end{array}$ & $\begin{array}{c}\text { 1st } \\
\text { per- } \\
\text { centile }\end{array}$ & $\begin{array}{c}\text { 2nd } \\
\text { per- } \\
\text { centile }\end{array}$ & $\begin{array}{c}10 \text { th } \\
\text { per- } \\
\text { centile }\end{array}$ & $\begin{array}{c}25 \text { th } \\
\text { per- } \\
\text { centile }\end{array}$ & $\begin{array}{c}\text { 50th } \\
\text { per- } \\
\text { centile } \\
\text { (median) }\end{array}$ & $\begin{array}{c}\text { 75th } \\
\text { per- } \\
\text { centile }\end{array}$ & $\begin{array}{c}\text { 90th } \\
\text { per- } \\
\text { centile }\end{array}$ & $\begin{array}{c}\text { 98th } \\
\text { per- } \\
\text { centile }\end{array}$ & $\begin{array}{c}\text { 99th } \\
\text { per- } \\
\text { centile }\end{array}$ & $\begin{array}{l}\text { Sta- } \\
\text { tion } \\
\text { no. }\end{array}$ & $\begin{array}{c}1 \text { st } \\
\text { per- } \\
\text { centile }\end{array}$ & $\begin{array}{c}\text { 2nd } \\
\text { per- } \\
\text { centile }\end{array}$ & $\begin{array}{c}10 \text { th } \\
\text { per- } \\
\text { centile }\end{array}$ & $\begin{array}{c}\text { 25th } \\
\text { per- } \\
\text { centile }\end{array}$ & $\begin{array}{c}\text { 50th } \\
\text { per- } \\
\text { centile } \\
\text { (median) }\end{array}$ & $\begin{array}{c}\text { 75th } \\
\text { per- } \\
\text { centile }\end{array}$ & $\begin{array}{c}\text { 90th } \\
\text { per- } \\
\text { centile }\end{array}$ & $\begin{array}{c}\text { 98th } \\
\text { per- } \\
\text { centile }\end{array}$ & $\begin{array}{c}\text { 99th } \\
\text { per- } \\
\text { centile }\end{array}$ \\
\hline 7718 & 1.00 & 1.00 & 1.00 & 1.00 & 4.00 & 8.00 & 13.00 & 34.44 & 39.81 & 8910 & -- & -- & 1.40 & 2.00 & 4.00 & 5.00 & 9.20 & -- & -- \\
\hline 7745 & 1.00 & 1.00 & 1.00 & 2.00 & 5.00 & 10.00 & 16.90 & 31.38 & 46.76 & 8911 & 1.00 & 1.00 & 1.00 & 1.00 & 3.00 & 6.00 & 12.00 & 22.00 & 25.95 \\
\hline 7922 & 1.00 & 1.00 & 1.00 & 1.00 & 2.00 & 5.00 & 10.00 & 19.02 & 21.51 & 8924 & 1.00 & 1.00 & 1.00 & 1.00 & 2.00 & 4.00 & 8.00 & 15.44 & 23.32 \\
\hline 7936 & 1.00 & 1.00 & 1.00 & 1.00 & 3.00 & 6.00 & 12.00 & 23.00 & 28.00 & 8929 & -- & -- & 1.00 & 1.00 & 3.00 & 8.00 & 17.00 & -- & -- \\
\hline 7943 & 1.00 & 1.00 & 1.00 & 1.00 & 3.00 & 6.00 & 11.00 & 23.00 & 31.00 & 8942 & 1.00 & 1.00 & 1.00 & 1.00 & 2.00 & 7.00 & 12.00 & 23.38 & 28.00 \\
\hline 7944 & -- & 1.00 & 1.00 & 1.00 & 3.00 & 8.00 & 14.00 & 26.12 & -- & 8944 & 1.00 & 1.00 & 1.00 & 1.00 & 3.00 & 7.00 & 13.00 & 24.52 & 30.00 \\
\hline 7945 & 1.00 & 1.00 & 1.00 & 1.00 & 3.00 & 7.00 & 14.00 & 28.00 & 35.00 & 8996 & 1.00 & 1.00 & 1.00 & 2.00 & 3.00 & 7.00 & 13.00 & 26.00 & 33.00 \\
\hline 7947 & 1.00 & 1.00 & 1.00 & 1.00 & 2.00 & 6.00 & 10.00 & 25.18 & 44.24 & 9014 & -- & -- & 1.60 & 3.00 & 6.00 & 9.00 & 15.60 & -- & -- \\
\hline 7948 & 1.00 & 1.00 & 1.00 & 1.00 & 3.00 & 6.00 & 12.00 & 25.00 & 29.85 & 9037 & 1.00 & 1.00 & 1.00 & 1.00 & 3.00 & 6.00 & 11.00 & 23.00 & 31.00 \\
\hline 7951 & 1.00 & 1.00 & 1.00 & 2.00 & 4.00 & 8.00 & 14.00 & 26.00 & 33.00 & 9106 & 1.00 & 1.00 & 1.00 & 1.00 & 3.00 & 5.00 & 10.00 & 21.20 & 35.90 \\
\hline 7953 & 1.00 & 1.00 & 1.00 & 2.00 & 3.00 & 7.00 & 12.80 & 37.00 & 38.16 & 9107 & -- & -- & 1.00 & 1.00 & 1.00 & 3.00 & 8.80 & -- & -- \\
\hline 7981 & 1.00 & 1.00 & 1.00 & 1.00 & 3.00 & 7.00 & 12.00 & 25.78 & 31.39 & 9129 & 1.00 & 1.00 & 1.00 & 1.00 & 3.00 & 6.00 & 11.00 & 28.80 & 32.10 \\
\hline 7990 & 1.00 & 1.00 & 1.00 & 1.00 & 3.00 & 7.00 & 13.00 & 30.30 & 39.01 & 9163 & 1.00 & 1.00 & 1.00 & 1.00 & 3.00 & 6.00 & 12.00 & 24.00 & 29.00 \\
\hline 7992 & -- & -- & 1.90 & 3.00 & 4.50 & 7.75 & 11.10 & -- & -- & 9213 & 1.00 & 1.00 & 1.00 & 1.00 & 1.00 & 7.00 & 13.00 & 25.00 & 31.84 \\
\hline 7997 & 1.00 & 1.00 & 1.00 & 2.00 & 3.00 & 6.00 & 10.80 & 19.16 & 26.40 & 9214 & -- & -- & 1.60 & 2.00 & 4.00 & 6.00 & 15.40 & -- & -- \\
\hline 7999 & -- & -- & 1.00 & 1.00 & 2.00 & 7.00 & 13.60 & -- & -- & 9222 & 1.00 & 1.00 & 1.00 & 1.00 & 3.00 & 9.00 & 15.00 & 28.68 & 33.00 \\
\hline 8022 & -- & 1.00 & 1.00 & 1.00 & 3.00 & 6.25 & 13.00 & 37.04 & -- & 9248 & 1.00 & 1.00 & 1.00 & 1.00 & 3.00 & 7.00 & 17.00 & 31.24 & 34.12 \\
\hline 8023 & 1.00 & 1.00 & 1.00 & 1.00 & 2.00 & 5.00 & 10.00 & 23.00 & 29.00 & 9266 & -- & 1.00 & 1.00 & 2.00 & 4.00 & 8.25 & 14.00 & 31.26 & -- \\
\hline 8047 & 1.00 & 1.00 & 1.00 & 1.00 & 3.00 & 6.00 & 11.00 & 23.00 & 29.00 & 9270 & 1.00 & 1.00 & 1.00 & 1.00 & 2.00 & 4.00 & 7.00 & 15.00 & 18.00 \\
\hline 8060 & 1.00 & 1.00 & 1.00 & 1.00 & 3.00 & 7.00 & 13.00 & 30.00 & 35.80 & 9295 & 1.00 & 1.00 & 1.00 & 1.00 & 1.00 & 7.00 & 7.00 & 25.00 & 29.98 \\
\hline 8062 & -- & -- & 1.00 & 2.00 & 4.00 & 10.00 & 14.60 & -- & -- & 9304 & -- & -- & -- & 1.50 & 4.00 & 5.75 & -- & -- & -- \\
\hline 8068 & -- & -- & 1.00 & 1.00 & 2.00 & 6.00 & 10.60 & -- & -- & 9307 & 1.00 & 1.00 & 1.00 & 1.00 & 3.00 & 6.75 & 12.00 & 26.56 & 31.21 \\
\hline 8081 & 1.00 & 1.00 & 1.00 & 1.00 & 3.00 & 6.00 & 12.00 & 26.00 & 31.00 & 9328 & 1.00 & 1.00 & 1.00 & 1.00 & 3.00 & 6.00 & 14.00 & 35.10 & 45.17 \\
\hline 8089 & -- & -- & 1.00 & 1.00 & 4.00 & 6.25 & 13.00 & -- & -- & 9329 & -- & -- & 1.00 & 1.00 & 5.00 & 11.50 & 28.30 & -- & -- \\
\hline 8221 & -- & -- & 1.00 & 3.00 & 6.00 & 10.00 & 19.00 & -- & -- & 9345 & -- & -- & -- & -- & 8.00 & -- & -- & -- & -- \\
\hline 8252 & 1.00 & 1.00 & 1.00 & 1.00 & 2.00 & 5.00 & 10.00 & 23.00 & 30.00 & 9363 & 1.00 & 1.00 & 1.00 & 1.00 & 3.00 & 7.00 & 12.00 & 23.00 & 29.70 \\
\hline 8265 & 1.00 & 1.00 & 1.00 & 2.00 & 4.00 & 8.00 & 16.00 & 31.00 & 37.11 & 9364 & 1.00 & 1.00 & 1.00 & 1.00 & 3.00 & 6.00 & 12.00 & 27.00 & 33.51 \\
\hline 8289 & -- & 1.00 & 1.00 & 1.00 & 3.00 & 7.00 & 12.00 & 30.04 & -- & 9365 & -- & -- & 1.00 & 1.00 & 3.50 & 5.00 & 11.90 & -- & -- \\
\hline 8305 & 1.00 & 1.00 & 1.00 & 1.00 & 2.00 & 4.00 & 8.00 & 15.00 & 19.00 & 9371 & 1.00 & 1.00 & 1.00 & 2.00 & 3.50 & 8.75 & 13.70 & 28.16 & 31.54 \\
\hline 8335 & 1.00 & 1.00 & 1.00 & 2.00 & 4.00 & 9.00 & 16.00 & 30.00 & 38.00 & 9417 & 1.00 & 1.00 & 1.00 & 2.00 & 3.00 & 7.00 & 13.00 & 25.00 & 32.21 \\
\hline 8400 & 1.00 & 1.00 & 1.00 & 1.00 & 2.00 & 4.00 & 8.00 & 20.46 & 26.23 & 9419 & 1.00 & 1.00 & 1.00 & 1.00 & 3.00 & 7.00 & 14.00 & 28.00 & 34.00 \\
\hline 8445 & 1.00 & 1.00 & 1.00 & 2.00 & 4.00 & 8.00 & 15.00 & 30.00 & 35.00 & 9435 & -- & 1.00 & 1.00 & 1.00 & 3.00 & 6.00 & 12.40 & 39.76 & -- \\
\hline 8446 & 1.00 & 1.00 & 1.00 & 1.00 & 2.00 & 6.00 & 11.00 & 22.00 & 25.13 & 9491 & 1.00 & 1.00 & 1.00 & 1.00 & 3.00 & 7.00 & 13.00 & 24.00 & 29.00 \\
\hline 8451 & 1.00 & 1.00 & 1.00 & 2.00 & 3.00 & 7.00 & 13.00 & 20.00 & 26.91 & 9499 & 1.00 & 1.00 & 1.00 & 1.00 & 2.00 & 5.00 & 9.30 & 20.00 & 27.00 \\
\hline 8531 & 1.00 & 1.00 & 1.00 & 2.00 & 4.00 & 8.00 & 14.00 & 27.00 & 34.00 & 9522 & -- & -- & 2.00 & 3.00 & 5.00 & 10.00 & 16.00 & -- & -- \\
\hline 8541 & 1.00 & 1.00 & 1.00 & 2.00 & 4.00 & 7.00 & 11.70 & 21.00 & 24.85 & 9527 & 1.00 & 1.00 & 1.00 & 1.00 & 2.00 & 4.00 & 8.00 & 18.00 & 23.00 \\
\hline 8544 & 1.00 & 1.00 & 1.00 & 1.00 & 3.00 & 7.00 & 14.00 & 26.00 & 30.00 & 9532 & 1.00 & 1.00 & 1.00 & 1.00 & 3.00 & 6.00 & 11.00 & 22.00 & 30.00 \\
\hline 8545 & -- & -- & 1.00 & 1.00 & 4.00 & 10.00 & 19.80 & -- & -- & 9544 & -- & -- & -- & 1.00 & 1.00 & 6.00 & -- & -- & -- \\
\hline 8563 & 1.00 & 1.00 & 1.00 & 1.00 & 2.00 & 6.00 & 9.00 & 19.78 & 21.39 & 9565 & 1.00 & 1.00 & 1.00 & 1.00 & 2.00 & 6.00 & 10.00 & 19.00 & 23.17 \\
\hline 8566 & 1.00 & 1.00 & 1.00 & 1.00 & 2.00 & 5.00 & 10.00 & 20.00 & 26.61 & 9570 & 1.00 & 1.00 & 1.00 & 1.00 & 1.00 & 4.00 & 8.00 & 18.00 & 22.00 \\
\hline 8583 & 1.00 & 1.00 & 1.00 & 1.00 & 1.00 & 5.00 & 8.00 & 17.00 & 21.78 & 9574 & -- & -- & 1.00 & 1.00 & 2.00 & 3.00 & 5.20 & -- & -- \\
\hline 8584 & 1.00 & 1.00 & 1.00 & 1.00 & 2.00 & 6.00 & 11.00 & 22.00 & 29.39 & 9588 & 1.00 & 1.00 & 1.00 & 1.00 & 2.00 & 6.00 & 11.00 & 24.00 & 29.00 \\
\hline 8623 & 1.00 & 1.00 & 1.00 & 1.00 & 2.00 & 6.00 & 10.00 & 21.62 & 29.00 & 9665 & 1.00 & 1.00 & 1.00 & 1.00 & 3.00 & 7.00 & 12.50 & 24.00 & 29.00 \\
\hline 8625 & 1.00 & 1.00 & 1.00 & 2.00 & 4.00 & 8.00 & 13.10 & 27.00 & 33.44 & 9715 & 1.00 & 1.00 & 1.00 & 1.00 & 3.00 & 7.00 & 13.00 & 24.00 & 29.00 \\
\hline 8630 & 1.00 & 1.00 & 1.00 & 1.00 & 3.00 & 6.00 & 11.00 & 20.76 & 27.00 & 9729 & 1.00 & 1.00 & 1.00 & 1.00 & 3.00 & 7.00 & 13.00 & 26.00 & 31.00 \\
\hline 8631 & 1.00 & 1.00 & 1.00 & 2.00 & 3.00 & 7.00 & 12.00 & 25.14 & 36.07 & 9772 & 1.00 & 1.00 & 1.00 & 2.00 & 3.00 & 7.00 & 13.00 & 27.00 & 33.50 \\
\hline 8646 & 1.00 & 1.00 & 1.00 & 2.00 & 4.00 & 8.00 & 14.00 & 25.00 & 31.00 & 9814 & -- & -- & 1.00 & 2.00 & 4.00 & 10.50 & 16.80 & -- & -- \\
\hline 8647 & 1.00 & 1.00 & 1.00 & 1.00 & 2.00 & 5.00 & 9.00 & 18.34 & 23.00 & 9815 & 1.00 & 1.00 & 1.00 & 2.00 & 3.00 & 8.00 & 14.00 & 27.02 & 33.00 \\
\hline 8677 & 1.00 & 1.00 & 1.00 & 2.00 & 4.00 & 7.50 & 12.20 & 23.04 & 29.26 & 9816 & -- & 1.00 & 1.00 & 1.00 & 3.00 & 8.00 & 12.00 & 30.44 & -- \\
\hline 8696 & -- & -- & 1.00 & 2.00 & 3.50 & 7.25 & 9.90 & -- & -- & 9817 & 1.00 & 1.00 & 1.00 & 1.00 & 2.00 & 6.00 & 10.00 & 20.00 & 25.00 \\
\hline 8743 & 1.00 & 1.00 & 1.00 & 1.00 & 4.00 & 8.00 & 14.00 & 26.00 & 32.00 & 9829 & 1.00 & 1.00 & 1.00 & 1.00 & 2.00 & 5.00 & 10.00 & 21.00 & 27.00 \\
\hline 8761 & 1.00 & 1.00 & 1.00 & 1.00 & 2.00 & 4.00 & 8.00 & 18.00 & 23.81 & 9830 & 1.00 & 1.00 & 1.00 & 1.00 & 2.00 & 5.00 & 8.40 & 17.48 & 20.16 \\
\hline 8778 & 1.00 & 1.00 & 1.00 & 1.00 & 3.00 & 6.00 & 12.00 & 23.00 & 28.00 & 9858 & 1.00 & 1.00 & 1.00 & 2.00 & 3.00 & 7.00 & 12.00 & 24.70 & 31.70 \\
\hline 8845 & 1.00 & 1.00 & 1.00 & 1.00 & 3.00 & 7.00 & 12.00 & 24.00 & 29.44 & 9893 & 1.00 & 1.00 & 1.00 & 1.00 & 3.00 & 6.00 & 12.00 & 24.00 & 31.00 \\
\hline 8859 & 1.00 & 1.00 & 1.00 & 2.00 & 4.00 & 9.00 & 15.00 & 30.00 & 34.00 & 9916 & 1.00 & 1.00 & 1.00 & 1.00 & 3.00 & 7.00 & 13.00 & 22.08 & 28.08 \\
\hline 8898 & 1.00 & 1.00 & 1.00 & 2.00 & 4.00 & 9.00 & 15.00 & 27.58 & 34.00 & 9976 & 1.00 & 1.00 & 1.00 & 1.00 & 2.00 & 6.00 & 11.00 & 25.00 & 31.28 \\
\hline 8908 & -- & -- & 1.00 & 2.00 & 4.00 & 7.00 & 11.30 & -- & -- & & & & & & & & & & \\
\hline
\end{tabular}


Appendix 4-5.2. Empirical distribution of storm duration defined by 8-hour minimum interevent time for hourly rainfall stations in Texas.

$[--$, not available $]$

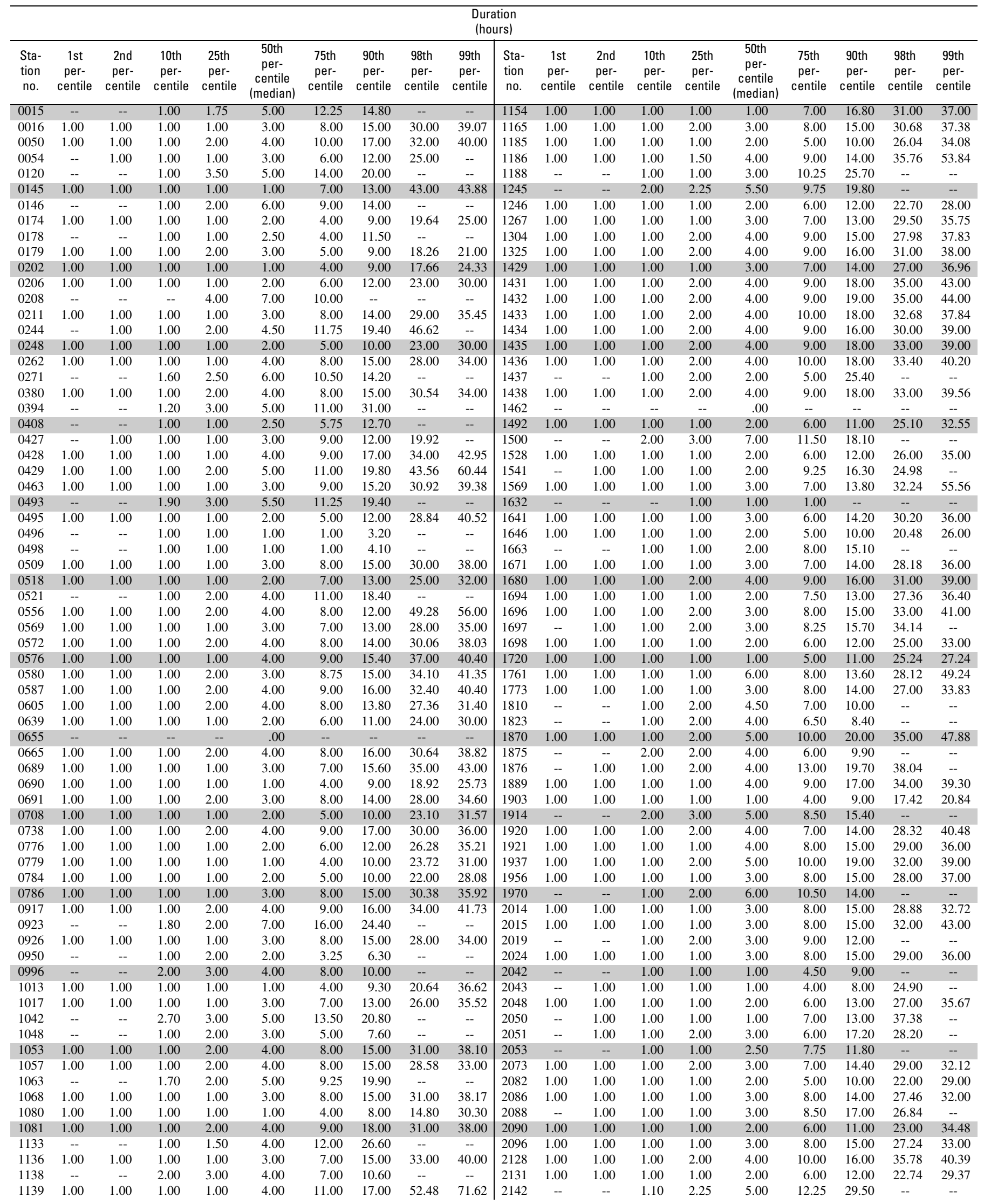


Appendix 4-5.2. Empirical distribution of storm duration defined by 8-hour minimum interevent time for hourly rainfall stations in Texas-Continued.

\begin{tabular}{|c|c|c|c|c|c|c|c|c|c|c|c|c|c|c|c|c|c|c|c|}
\hline \multicolumn{20}{|c|}{$\begin{array}{c}\text { Duration } \\
\text { (hours) }\end{array}$} \\
\hline $\begin{array}{l}\text { Sta- } \\
\text { tion } \\
\text { no. }\end{array}$ & $\begin{array}{c}\text { 1st } \\
\text { per- } \\
\text { centile }\end{array}$ & $\begin{array}{c}\text { 2nd } \\
\text { per- } \\
\text { centile }\end{array}$ & $\begin{array}{c}10 \text { th } \\
\text { per- } \\
\text { centile }\end{array}$ & $\begin{array}{c}25 \text { th } \\
\text { per- } \\
\text { centile }\end{array}$ & $\begin{array}{c}\text { 50th } \\
\text { per- } \\
\text { centile } \\
\text { (median) }\end{array}$ & $\begin{array}{c}\text { 75th } \\
\text { per- } \\
\text { centile }\end{array}$ & $\begin{array}{c}\text { 90th } \\
\text { per- } \\
\text { centile }\end{array}$ & $\begin{array}{c}\text { 98th } \\
\text { per- } \\
\text { centile }\end{array}$ & $\begin{array}{c}\text { 99th } \\
\text { per- } \\
\text { centile }\end{array}$ & $\begin{array}{l}\text { Sta- } \\
\text { tion } \\
\text { no. }\end{array}$ & $\begin{array}{c}\text { 1st } \\
\text { per- } \\
\text { centile }\end{array}$ & $\begin{array}{c}\text { 2nd } \\
\text { per- } \\
\text { centile }\end{array}$ & $\begin{array}{c}10 \text { th } \\
\text { per- } \\
\text { centile }\end{array}$ & $\begin{array}{c}25 \text { th } \\
\text { per- } \\
\text { centile }\end{array}$ & $\begin{array}{c}\text { 50th } \\
\text { per- } \\
\text { centile } \\
\text { (median) }\end{array}$ & $\begin{array}{c}\text { 75th } \\
\text { per- } \\
\text { centile }\end{array}$ & $\begin{array}{c}\text { 90th } \\
\text { per- } \\
\text { centile }\end{array}$ & $\begin{array}{c}\text { 98th } \\
\text { per- } \\
\text { centile }\end{array}$ & $\begin{array}{c}\text { 99th } \\
\text { per- } \\
\text { centile }\end{array}$ \\
\hline 2160 & -- & -- & 1.00 & 1.00 & 7.00 & 13.00 & 25.00 & -- & -- & 3463 & -- & -- & 1.00 & 2.00 & 5.00 & 11.00 & 23.00 & -- & -- \\
\hline 2206 & 1.00 & 1.00 & 1.00 & 2.00 & 3.00 & 8.00 & 15.00 & 28.00 & 34.00 & 3476 & 1.00 & 1.00 & 1.00 & 2.00 & 4.00 & 8.00 & 14.00 & 28.00 & 35.38 \\
\hline 2238 & 1.00 & 1.00 & 1.00 & 1.00 & 3.00 & 7.00 & 13.00 & 34.04 & 40.04 & 3485 & -- & -- & 1.60 & 2.00 & 6.00 & 7.00 & 11.40 & -- & -- \\
\hline 2240 & 1.00 & 1.00 & 1.00 & 1.00 & 1.00 & 7.00 & 13.00 & 19.00 & 19.00 & 3507 & 1.00 & 1.00 & 1.00 & 1.00 & 2.00 & 6.25 & 13.00 & 26.00 & 31.00 \\
\hline 2242 & 1.00 & 1.00 & 1.00 & 2.00 & 4.00 & 9.00 & 16.00 & 31.26 & 36.00 & 3546 & 1.00 & 1.00 & 1.00 & 1.00 & 3.00 & 8.00 & 16.00 & 30.00 & 37.00 \\
\hline 2244 & 1.00 & 1.00 & 1.00 & 1.00 & 3.00 & 8.00 & 16.00 & 30.00 & 35.00 & 3547 & 1.00 & 1.00 & 1.00 & 1.00 & 3.00 & 8.00 & 14.00 & 32.80 & 40.40 \\
\hline 2247 & -- & 1.00 & 1.00 & 2.00 & 5.00 & 7.00 & 11.70 & 26.84 & -- & 3579 & -- & 1.00 & 1.00 & 2.00 & 4.00 & 10.00 & 21.00 & 49.80 & -- \\
\hline 2309 & 1.00 & 1.00 & 1.00 & 2.00 & 4.00 & 10.00 & 17.00 & 31.56 & 41.12 & 3642 & 1.00 & 1.00 & 1.00 & 1.00 & 4.00 & 8.00 & 15.00 & 28.00 & 33.25 \\
\hline 2312 & 1.00 & 1.00 & 1.00 & 1.00 & 3.00 & 8.00 & 13.00 & 22.00 & 27.53 & 3646 & 1.00 & 1.00 & 1.00 & 2.00 & 4.00 & 9.00 & 16.00 & 29.00 & 37.00 \\
\hline 2334 & -- & 1.00 & 1.00 & 1.00 & 4.00 & 9.00 & 18.40 & 27.40 & -- & 3668 & -- & -- & 2.00 & 4.00 & 7.00 & 14.75 & 23.00 & -- & -- \\
\hline 2336 & 1.00 & 1.00 & 1.00 & 1.00 & 3.00 & 7.75 & 13.30 & 24.56 & 34.63 & 3673 & -- & -- & 1.00 & 3.00 & 5.00 & 9.00 & 21.80 & -- & -- \\
\hline 2354 & -- & -- & 1.00 & 1.00 & 3.00 & 7.00 & 16.40 & -- & -- & 3686 & 1.00 & 1.00 & 1.00 & 1.00 & 2.00 & 7.00 & 12.00 & 25.00 & 27.63 \\
\hline 2355 & -- & 1.00 & 1.00 & 2.00 & 4.00 & 9.00 & 22.00 & 39.00 & -- & 3691 & 1.00 & 1.00 & 1.00 & 1.00 & 3.00 & 7.00 & 12.00 & 25.00 & 31.00 \\
\hline 2357 & 1.00 & 1.00 & 1.00 & 1.00 & 3.00 & 7.75 & 16.00 & 30.00 & 40.00 & 3734 & -- & -- & 1.00 & 2.00 & 4.00 & 8.00 & 18.40 & -- & -- \\
\hline 2360 & 1.00 & 1.00 & 1.00 & 1.00 & 3.00 & 7.00 & 13.00 & 28.84 & 37.42 & 3771 & 1.00 & 1.00 & 1.00 & 1.00 & 2.00 & 6.00 & 12.00 & 24.00 & 28.42 \\
\hline 2361 & 1.00 & 1.00 & 1.00 & 1.00 & 2.00 & 5.00 & 10.00 & 22.92 & 34.64 & 3789 & 1.00 & 1.00 & 1.00 & 1.00 & 1.00 & 7.00 & 13.00 & 23.32 & 40.48 \\
\hline 2394 & 1.00 & 1.00 & 1.00 & 2.00 & 4.00 & 9.00 & 16.00 & 31.00 & 37.00 & 3826 & 1.00 & 1.00 & 1.00 & 2.00 & 4.00 & 10.00 & 14.70 & 23.02 & 35.35 \\
\hline 2404 & 1.00 & 1.00 & 1.00 & 1.00 & 3.00 & 8.00 & 15.00 & 29.00 & 33.00 & 3831 & 1.00 & 1.00 & 1.00 & 2.00 & 4.00 & 8.00 & 14.00 & 28.00 & 41.15 \\
\hline 2415 & 1.00 & 1.00 & 1.00 & 2.00 & 4.00 & 9.00 & 16.00 & 30.00 & 37.00 & 3841 & 1.00 & 1.00 & 1.00 & 1.00 & 3.00 & 6.00 & 11.80 & 21.00 & 31.38 \\
\hline 2462 & 1.00 & 1.00 & 1.00 & 1.00 & 4.00 & 8.25 & 15.00 & 29.00 & 36.00 & 3871 & 1.00 & 1.00 & 1.00 & 2.00 & 4.00 & 8.00 & 16.00 & 30.68 & 36.00 \\
\hline 2528 & 1.00 & 1.00 & 1.00 & 1.00 & 4.00 & 8.50 & 14.40 & 46.32 & 54.70 & 3884 & -- & -- & 1.40 & 3.00 & 5.00 & 7.00 & 10.20 & -- & -- \\
\hline 2617 & 1.00 & 1.00 & 1.00 & 2.00 & 4.00 & 9.00 & 17.00 & 29.70 & 40.35 & 3941 & -- & 1.00 & 1.00 & 1.00 & 5.00 & 12.25 & 23.00 & 37.50 & -- \\
\hline 2619 & 1.00 & 1.00 & 1.00 & 1.00 & 4.00 & 8.00 & 14.00 & 31.28 & 39.56 & 3963 & -- & -- & -- & 1.00 & 1.00 & 1.00 & -- & -- & -- \\
\hline 2621 & 1.00 & 1.00 & 1.00 & 1.00 & 3.00 & 7.00 & 13.00 & 25.00 & 32.02 & 4040 & 1.00 & 1.00 & 1.00 & 2.00 & 4.00 & 8.00 & 14.80 & 30.00 & 33.56 \\
\hline 2675 & 1.00 & 1.00 & 1.00 & 1.00 & 3.00 & 7.00 & 14.00 & 30.10 & 34.00 & 4058 & -- & -- & 1.00 & 1.75 & 3.00 & 5.00 & 9.90 & -- & -- \\
\hline 2676 & 1.00 & 1.00 & 1.00 & 1.00 & 2.00 & 6.00 & 11.00 & 23.00 & 27.57 & 4098 & 1.00 & 1.00 & 1.00 & 1.00 & 2.00 & 4.00 & 9.00 & 19.00 & 23.00 \\
\hline 2679 & 1.00 & 1.00 & 1.00 & 1.00 & 2.00 & 6.00 & 12.00 & 26.00 & 32.49 & 4100 & 1.00 & 1.00 & 1.00 & 2.00 & 3.00 & 7.00 & 13.00 & 27.56 & 33.68 \\
\hline 2715 & 1.00 & 1.00 & 1.00 & 2.00 & 4.00 & 8.00 & 15.00 & 28.90 & 34.00 & 4137 & 1.00 & 1.00 & 1.00 & 1.00 & 2.00 & 6.00 & 11.00 & 20.00 & 23.00 \\
\hline 2744 & 1.00 & 1.00 & 1.00 & 1.00 & 3.00 & 6.00 & 13.00 & 26.24 & 35.00 & 4191 & 1.00 & 1.00 & 1.00 & 1.00 & 3.00 & 7.00 & 14.00 & 28.00 & 36.48 \\
\hline 2758 & 1.00 & 1.00 & 1.00 & 2.00 & 3.00 & 9.00 & 18.00 & 36.12 & 60.28 & 4256 & -- & -- & -- & -- & .00 & -- & -- & -- & -- \\
\hline 2794 & -- & -- & 1.00 & 1.00 & 3.00 & 7.00 & 24.80 & -- & -- & 4257 & 1.00 & 1.00 & 1.00 & 1.00 & 3.00 & 7.00 & 14.00 & 27.80 & 34.00 \\
\hline 2797 & 1.00 & 1.00 & 1.00 & 1.00 & 2.00 & 6.00 & 11.00 & 23.00 & 30.02 & 4258 & 1.00 & 1.00 & 1.00 & 1.00 & 2.00 & 7.00 & 12.50 & 23.70 & 37.35 \\
\hline 2811 & 1.00 & 1.00 & 1.00 & 1.00 & 2.00 & 6.00 & 12.00 & 26.50 & 33.25 & 4278 & 1.00 & 1.00 & 1.00 & 2.00 & 4.00 & 8.00 & 15.00 & 29.00 & 36.84 \\
\hline 2813 & -- & -- & 1.00 & 1.00 & 2.50 & 5.00 & 9.70 & -- & -- & 4299 & 1.00 & 1.00 & 1.00 & 1.00 & 2.00 & 5.00 & 12.00 & 22.20 & 29.80 \\
\hline 2814 & -- & -- & 1.00 & 1.00 & 1.00 & 5.50 & 25.00 & -- & -- & 4300 & 1.00 & 1.00 & 1.00 & 1.00 & 3.00 & 8.00 & 16.00 & 33.00 & 41.44 \\
\hline 2815 & 1.00 & 1.00 & 1.00 & 1.00 & 2.00 & 5.00 & 10.00 & 19.00 & 21.75 & 4305 & 1.00 & 1.00 & 1.00 & 1.00 & 3.00 & 8.00 & 16.00 & 31.00 & 38.91 \\
\hline 2818 & 1.00 & 1.00 & 1.00 & 1.00 & 3.00 & 8.00 & 16.00 & 29.16 & 37.00 & 4307 & 1.00 & 1.00 & 1.00 & 1.00 & 3.00 & 8.00 & 17.40 & 37.08 & 49.12 \\
\hline 2986 & 1.00 & 1.00 & 1.00 & 2.00 & 5.00 & 11.00 & 19.00 & 38.42 & 50.28 & 4309 & 1.00 & 1.00 & 1.00 & 2.00 & 3.00 & 8.00 & 15.00 & 30.00 & 36.00 \\
\hline 3005 & 1.00 & 1.00 & 1.00 & 1.00 & 3.00 & 7.00 & 13.00 & 28.00 & 33.00 & 4311 & 1.00 & 1.00 & 1.00 & 2.00 & 4.00 & 8.00 & 15.00 & 30.00 & 38.99 \\
\hline 3033 & 1.00 & 1.00 & 1.00 & 2.00 & 3.00 & 5.00 & 10.00 & 21.00 & 26.00 & 4313 & 1.00 & 1.00 & 1.00 & 1.00 & 4.00 & 8.00 & 16.90 & 30.98 & 41.97 \\
\hline 3034 & -- & -- & -- & -- & 1.00 & -- & -- & -- & -- & 4319 & 1.00 & 1.00 & 1.00 & 2.00 & 4.00 & 8.00 & 14.40 & 31.40 & 32.94 \\
\hline 3047 & -- & -- & 1.00 & 2.00 & 4.00 & 8.00 & 13.20 & -- & -- & 4329 & 1.00 & 1.00 & 1.00 & 2.00 & 3.00 & 8.00 & 16.00 & 31.00 & 38.92 \\
\hline 3103 & -- & -- & 1.00 & 1.00 & 2.50 & 8.00 & 12.50 & -- & -- & 4331 & -- & -- & -- & -- & .00 & -- & -- & -- & -- \\
\hline 3133 & 1.00 & 1.00 & 1.00 & 2.00 & 4.00 & 9.00 & 16.00 & 31.00 & 37.00 & 4375 & 1.00 & 1.00 & 1.00 & 1.00 & 2.00 & 5.00 & 10.00 & 21.00 & 28.00 \\
\hline 3156 & 1.00 & 1.00 & 1.00 & 1.00 & 3.00 & 7.50 & 15.00 & 30.64 & 34.64 & 4392 & 1.00 & 1.00 & 1.00 & 2.00 & 5.00 & 11.00 & 20.00 & 43.10 & 49.91 \\
\hline 3171 & 1.00 & 1.00 & 1.00 & 2.00 & 4.00 & 9.00 & 16.00 & 29.00 & 37.00 & 4425 & 1.00 & 1.00 & 1.00 & 1.00 & 2.00 & 5.00 & 10.00 & 24.00 & 33.73 \\
\hline 3189 & 1.00 & 1.00 & 1.00 & 1.00 & 2.00 & 6.00 & 11.00 & 20.42 & 34.15 & 4440 & 1.00 & 1.00 & 1.00 & 2.00 & 4.00 & 9.00 & 16.00 & 31.00 & 37.20 \\
\hline 3260 & 1.00 & 1.00 & 1.00 & 1.00 & 3.00 & 7.00 & 14.00 & 24.64 & 26.80 & 4476 & 1.00 & 1.00 & 1.00 & 1.00 & 3.00 & 7.00 & 12.00 & 25.00 & 31.00 \\
\hline 3267 & 1.00 & 1.00 & 1.00 & 2.00 & 4.00 & 7.00 & 16.40 & 41.22 & 62.01 & 4498 & -- & -- & 1.00 & 1.00 & 2.00 & 4.00 & 8.00 & -- & -- \\
\hline 3270 & 1.00 & 1.00 & 1.00 & 1.00 & 2.00 & 5.00 & 9.00 & 20.00 & 26.08 & 4517 & 1.00 & 1.00 & 1.00 & 2.00 & 4.00 & 8.00 & 15.00 & 31.00 & 38.00 \\
\hline 3272 & -- & -- & 1.00 & 1.00 & 1.50 & 3.00 & 8.50 & -- & -- & 4520 & 1.00 & 1.00 & 1.00 & 1.00 & 2.00 & 6.00 & 11.00 & 21.64 & 26.32 \\
\hline 3277 & -- & -- & 1.00 & 1.00 & 1.00 & 7.00 & 18.40 & -- & -- & 4525 & -- & -- & 1.60 & 2.00 & 4.00 & 8.00 & 16.40 & -- & -- \\
\hline 3278 & 1.00 & 1.00 & 1.00 & 1.00 & 2.00 & 5.00 & 9.00 & 25.76 & 33.52 & 4563 & -- & -- & 1.00 & 1.75 & 3.50 & 7.25 & 10.90 & -- & -- \\
\hline 3280 & 1.00 & 1.00 & 1.00 & 1.00 & 3.00 & 5.00 & 11.00 & 24.66 & 34.97 & 4570 & 1.00 & 1.00 & 1.00 & 1.00 & 2.00 & 6.00 & 13.00 & 28.00 & 37.00 \\
\hline 3281 & -- & -- & 1.00 & 1.00 & 2.00 & 3.25 & 8.60 & -- & -- & 4577 & 1.00 & 1.00 & 1.00 & 1.00 & 3.00 & 8.00 & 14.00 & 28.80 & 35.00 \\
\hline 3283 & 1.00 & 1.00 & 1.00 & 2.00 & 4.00 & 10.00 & 17.00 & 35.00 & 44.48 & 4591 & 1.00 & 1.00 & 1.00 & 2.00 & 5.00 & 10.00 & 18.00 & 31.00 & 38.00 \\
\hline 3284 & 1.00 & 1.00 & 1.00 & 1.00 & 3.00 & 7.00 & 14.00 & 26.00 & 32.00 & 4670 & 1.00 & 1.00 & 1.00 & 1.00 & 2.00 & 6.00 & 11.00 & 24.00 & 30.95 \\
\hline 3285 & 1.00 & 1.00 & 1.00 & 1.00 & 2.00 & 6.00 & 11.00 & 23.00 & 28.00 & 4671 & 1.00 & 1.00 & 1.00 & 1.00 & 1.00 & 7.00 & 13.00 & 22.60 & 26.80 \\
\hline 3329 & 1.00 & 1.00 & 1.00 & 1.00 & 3.00 & 8.00 & 16.00 & 30.18 & 36.00 & 4679 & 1.00 & 1.00 & 1.00 & 1.00 & 3.00 & 7.00 & 13.00 & 24.00 & 31.00 \\
\hline 3335 & 1.00 & 1.00 & 1.00 & 2.00 & 4.00 & 10.00 & 17.00 & 33.36 & 45.03 & 4696 & -- & -- & 1.00 & 1.00 & 3.00 & 6.00 & 8.20 & -- & -- \\
\hline 3370 & 1.00 & 1.00 & 1.00 & 2.00 & 4.00 & 9.00 & 16.00 & 30.00 & 37.00 & 4703 & 1.00 & 1.00 & 1.00 & 1.00 & 3.00 & 7.25 & 12.00 & 28.30 & 41.22 \\
\hline 3410 & 1.00 & 1.00 & 1.00 & 1.00 & 3.00 & 6.00 & 12.00 & 25.00 & 30.00 & 4704 & 1.00 & 1.00 & 1.00 & 2.00 & 4.00 & 10.00 & 18.00 & 32.30 & 42.95 \\
\hline 3415 & 1.00 & 1.00 & 1.00 & 1.00 & 3.00 & 7.00 & 13.00 & 24.00 & 31.00 & 4731 & 1.00 & 1.00 & 1.00 & 1.00 & 3.00 & 10.00 & 17.20 & 32.00 & 32.62 \\
\hline 3430 & 1.00 & 1.00 & 1.00 & 1.00 & 3.00 & 8.00 & 15.00 & 31.00 & 38.09 & 4792 & 1.00 & 1.00 & 1.00 & 1.00 & 2.00 & 6.00 & 10.00 & 22.00 & 27.89 \\
\hline 3431 & 1.00 & 1.00 & 1.00 & 1.00 & 1.00 & 7.00 & 18.70 & 31.00 & 36.07 & 4819 & 1.00 & 1.00 & 1.00 & 1.00 & 3.00 & 8.00 & 14.00 & 24.68 & 26.00 \\
\hline 3441 & -- & -- & 1.00 & 1.00 & 3.00 & 8.75 & 13.00 & -- & -- & 4852 & -- & -- & 1.00 & 1.00 & 1.00 & 2.50 & 8.60 & -- & -- \\
\hline 3442 & 1.00 & 1.00 & 1.00 & 2.00 & 4.00 & 8.00 & 13.00 & 26.20 & 35.00 & 4866 & 1.00 & 1.00 & 1.00 & 1.00 & 3.00 & 8.00 & 16.00 & 29.24 & 38.00 \\
\hline 3446 & 1.00 & 1.00 & 1.00 & 1.00 & 3.00 & 6.00 & 11.00 & 26.02 & 32.02 & 4876 & 1.00 & 1.00 & 1.00 & 1.00 & 3.00 & 7.00 & 12.00 & 25.30 & 32.12 \\
\hline 3460 & -- & -- & 1.00 & 2.00 & 4.00 & 7.75 & 13.00 & -- & -- & 4878 & 1.00 & 1.00 & 1.00 & 2.00 & 4.00 & 9.00 & 17.00 & 33.00 & 41.00 \\
\hline 3462 & 1.00 & 1.00 & 1.00 & 1.25 & 4.00 & 7.75 & 16.70 & 28.82 & 35.70 & 4880 & 1.00 & 1.00 & 1.00 & 1.00 & 3.00 & 7.00 & 13.00 & 26.00 & 31.11 \\
\hline
\end{tabular}


Appendix 4-5.2. Empirical distribution of storm duration defined by 8-hour minimum interevent time for hourly rainfall stations in Texas-Continued.

\begin{tabular}{|c|c|c|c|c|c|c|c|c|c|c|c|c|c|c|c|c|c|c|c|}
\hline \multicolumn{20}{|c|}{$\begin{array}{c}\text { Duration } \\
\text { (hours) }\end{array}$} \\
\hline $\begin{array}{l}\text { Sta- } \\
\text { tion } \\
\text { no. }\end{array}$ & $\begin{array}{c}\text { 1st } \\
\text { per- } \\
\text { centile }\end{array}$ & $\begin{array}{c}\text { 2nd } \\
\text { per- } \\
\text { centile }\end{array}$ & $\begin{array}{c}\text { 10th } \\
\text { per- } \\
\text { centile }\end{array}$ & $\begin{array}{c}25 \text { th } \\
\text { per- } \\
\text { centile }\end{array}$ & $\begin{array}{c}\text { 50th } \\
\text { per- } \\
\text { centile } \\
\text { (median) }\end{array}$ & $\begin{array}{l}\text { 75th } \\
\text { per- } \\
\text { centile }\end{array}$ & $\begin{array}{c}\text { 90th } \\
\text { per- } \\
\text { centile }\end{array}$ & $\begin{array}{c}\text { 98th } \\
\text { per- } \\
\text { centile }\end{array}$ & $\begin{array}{c}\text { 99th } \\
\text { per- } \\
\text { centile }\end{array}$ & $\begin{array}{l}\text { Sta- } \\
\text { tion } \\
\text { no. }\end{array}$ & $\begin{array}{c}\text { 1st } \\
\text { per- } \\
\text { centile }\end{array}$ & $\begin{array}{c}\text { 2nd } \\
\text { per- } \\
\text { centile }\end{array}$ & $\begin{array}{c}\text { 10th } \\
\text { per- } \\
\text { centile }\end{array}$ & $\begin{array}{c}\text { 25th } \\
\text { per- } \\
\text { centile }\end{array}$ & $\begin{array}{c}\text { 50th } \\
\text { per- } \\
\text { centile } \\
\text { (median) }\end{array}$ & $\begin{array}{c}\text { 75th } \\
\text { per- } \\
\text { centile }\end{array}$ & $\begin{array}{c}\text { 90th } \\
\text { per- } \\
\text { centile }\end{array}$ & $\begin{array}{c}\text { 98th } \\
\text { per- } \\
\text { centile }\end{array}$ & $\begin{array}{c}\text { 99th } \\
\text { per- } \\
\text { centile }\end{array}$ \\
\hline 4920 & 1.00 & 1.00 & 1.00 & 1.00 & 2.00 & 6.00 & 12.00 & 26.00 & 32.27 & 5957 & 1.00 & 1.00 & 1.00 & 1.00 & 3.00 & 6.00 & 12.00 & 25.00 & 31.60 \\
\hline 4934 & -- & -- & -- & 1.00 & 2.00 & 9.75 & -- & -- & -- & 5958 & 1.00 & 1.00 & 1.00 & 2.00 & 3.00 & 7.00 & 15.00 & 26.80 & 32.40 \\
\hline 4972 & 1.00 & 1.00 & 1.00 & 1.00 & 3.00 & 7.00 & 14.00 & 27.54 & 33.27 & 5973 & 1.00 & 1.00 & 1.00 & 2.00 & 5.00 & 8.00 & 16.00 & 46.44 & 65.58 \\
\hline 4973 & 1.00 & 1.00 & 1.00 & 2.00 & 5.00 & 9.00 & 17.00 & 28.00 & 33.13 & 5996 & 1.00 & 1.00 & 1.00 & 1.00 & 3.00 & 8.00 & 15.00 & 28.00 & 31.80 \\
\hline 4974 & 1.00 & 1.00 & 1.00 & 1.00 & 3.00 & 7.00 & 12.00 & 24.04 & 32.00 & 6017 & 1.00 & 1.00 & 1.00 & 1.00 & 3.00 & 8.00 & 13.00 & 24.54 & 27.59 \\
\hline 4975 & 1.00 & 1.00 & 1.00 & 1.00 & 3.00 & 7.00 & 13.00 & 24.38 & 30.69 & 6024 & 1.00 & 1.00 & 1.00 & 2.00 & 4.00 & 9.50 & 18.00 & 42.72 & 49.72 \\
\hline 4978 & 1.00 & 1.00 & 1.00 & 1.00 & 2.00 & 7.00 & 13.40 & 28.00 & 32.68 & 6050 & -- & -- & 1.00 & 5.00 & 8.00 & 12.50 & 13.90 & -- & -- \\
\hline 4979 & -- & -- & 1.30 & 3.75 & 7.00 & 14.25 & 27.10 & -- & -- & 6104 & 1.00 & 1.00 & 1.00 & 1.00 & 2.00 & 5.00 & 10.00 & 21.00 & 29.00 \\
\hline 4982 & 1.00 & 1.00 & 1.00 & 2.00 & 3.00 & 8.00 & 14.00 & 27.46 & 34.00 & 6108 & 1.00 & 1.00 & 1.00 & 1.00 & 3.00 & 8.00 & 15.00 & 29.00 & 35.00 \\
\hline 5018 & 1.00 & 1.00 & 1.00 & 2.00 & 4.00 & 8.00 & 15.00 & 31.44 & 41.44 & 6136 & 1.00 & 1.00 & 1.00 & 1.00 & 2.00 & 4.00 & 9.00 & 19.00 & 26.13 \\
\hline 5048 & 1.00 & 1.00 & 1.00 & 1.00 & 2.00 & 5.00 & 11.00 & 24.14 & 30.00 & 6166 & -- & 1.00 & 1.00 & 2.00 & 3.00 & 7.00 & 11.40 & 32.12 & -- \\
\hline 5049 & 1.00 & 1.00 & 1.00 & 1.00 & 1.00 & 5.00 & 9.60 & 12.92 & 15.88 & 6176 & 1.00 & 1.00 & 1.00 & 2.00 & 4.00 & 10.00 & 18.00 & 36.58 & 42.16 \\
\hline 5056 & -- & -- & -- & 1.00 & 4.00 & 12.50 & -- & -- & -- & 6177 & 1.00 & 1.00 & 1.00 & 1.00 & 3.00 & 8.00 & 14.00 & 28.00 & 36.00 \\
\hline 5057 & 1.00 & 1.00 & 1.00 & 1.00 & 2.00 & 7.00 & 16.00 & 36.60 & 43.00 & 6210 & 1.00 & 1.00 & 1.00 & 1.00 & 3.00 & 7.00 & 13.00 & 25.00 & 31.31 \\
\hline 5060 & 1.00 & 1.00 & 1.00 & 2.00 & 3.00 & 7.50 & 16.00 & 37.88 & 51.16 & 6211 & 1.00 & 1.00 & 1.00 & 1.00 & 2.00 & 7.00 & 15.00 & 29.56 & 39.92 \\
\hline 5081 & 1.00 & 1.00 & 1.00 & 2.00 & 5.00 & 11.00 & 18.00 & 32.00 & 40.16 & 6270 & 1.00 & 1.00 & 1.00 & 1.00 & 3.00 & 7.00 & 13.00 & 25.60 & 29.90 \\
\hline 5094 & 1.00 & 1.00 & 1.00 & 1.00 & 3.00 & 7.00 & 13.00 & 25.00 & 30.46 & 6275 & -- & -- & -- & -- & .00 & -- & -- & -- & -- \\
\hline 5113 & 1.00 & 1.00 & 1.00 & 1.00 & 3.00 & 7.00 & 13.10 & 28.00 & 35.00 & 6276 & -- & -- & 1.00 & 3.25 & 5.50 & 10.50 & 17.60 & -- & -- \\
\hline 5114 & -- & -- & -- & -- & .00 & -- & -- & -- & -- & 6335 & 1.00 & 1.00 & 1.00 & 2.00 & 4.00 & 9.00 & 16.00 & 29.28 & 35.00 \\
\hline 5123 & -- & -- & 1.00 & 2.00 & 3.00 & 7.50 & 14.50 & -- & -- & 6434 & -- & -- & 1.00 & 2.00 & 5.00 & 8.00 & 18.40 & -- & -- \\
\hline 5192 & 1.00 & 1.00 & 1.00 & 1.00 & 3.00 & 8.00 & 15.00 & 27.00 & 33.00 & 6504 & 1.00 & 1.00 & 1.00 & 1.00 & 2.00 & 5.00 & 11.00 & 23.00 & 30.74 \\
\hline 5193 & 1.00 & 1.00 & 1.00 & 1.00 & 3.00 & 8.00 & 15.00 & 28.00 & 35.00 & 6558 & -- & -- & 1.00 & 2.00 & 3.50 & 8.75 & 19.30 & -- & -- \\
\hline 5224 & 1.00 & 1.00 & 1.00 & 1.00 & 4.00 & 8.00 & 16.00 & 28.20 & 36.40 & 6615 & 1.00 & 1.00 & 1.00 & 1.00 & 2.00 & 5.00 & 9.00 & 19.86 & 27.72 \\
\hline 5228 & 1.00 & 1.00 & 1.00 & 1.00 & 3.00 & 8.00 & 14.20 & 29.08 & 44.04 & 6660 & 1.00 & 1.00 & 1.00 & 1.00 & 4.00 & 9.00 & 18.80 & 40.56 & 43.52 \\
\hline 5235 & -- & -- & 1.00 & 2.00 & 4.00 & 13.00 & 20.60 & -- & -- & 6663 & 1.00 & 1.00 & 1.00 & 1.00 & 2.00 & 5.00 & 12.60 & 27.20 & 35.16 \\
\hline 5247 & 1.00 & 1.00 & 1.00 & 1.00 & 2.00 & 6.00 & 12.00 & 24.00 & 32.00 & 6734 & 1.00 & 1.00 & 1.00 & 1.00 & 3.00 & 8.00 & 15.00 & 27.00 & 34.00 \\
\hline 5258 & 1.00 & 1.00 & 1.00 & 2.00 & 4.00 & 9.00 & 16.00 & 32.00 & 37.72 & 6736 & 1.00 & 1.00 & 1.00 & 1.00 & 2.00 & 5.00 & 11.00 & 24.02 & 31.00 \\
\hline 5303 & 1.00 & 1.00 & 1.00 & 2.00 & 3.00 & 8.00 & 15.00 & 27.00 & 37.89 & 6740 & -- & -- & 1.80 & 4.00 & 8.00 & 14.50 & 35.40 & -- & -- \\
\hline 5312 & 1.00 & 1.00 & 1.00 & 1.00 & 2.00 & 6.00 & 11.00 & 24.00 & 30.00 & 6750 & 1.00 & 1.00 & 1.00 & 1.00 & 1.00 & 7.00 & 13.80 & 39.16 & 46.78 \\
\hline 5341 & -- & -- & 1.00 & 2.00 & 4.00 & 7.00 & 26.00 & -- & -- & 6757 & 1.00 & 1.00 & 1.00 & 1.00 & 4.00 & 8.00 & 15.50 & 30.00 & 35.00 \\
\hline 5342 & -- & -- & -- & -- & .00 & -- & -- & -- & -- & 6775 & 1.00 & 1.00 & 1.00 & 1.00 & 3.00 & 8.00 & 15.00 & 30.80 & 35.00 \\
\hline 5348 & 1.00 & 1.00 & 1.00 & 1.00 & 3.00 & 7.00 & 14.00 & 25.00 & 30.86 & 6776 & 1.00 & 1.00 & 1.00 & 1.00 & 2.00 & 6.00 & 11.00 & 23.00 & 28.00 \\
\hline 5358 & 1.00 & 1.00 & 1.00 & 2.00 & 3.00 & 7.00 & 13.00 & 25.78 & 32.39 & 6788 & 1.00 & 1.00 & 1.00 & 2.00 & 4.00 & 9.00 & 13.00 & 25.16 & 29.80 \\
\hline 5398 & 1.00 & 1.00 & 1.00 & 2.00 & 4.00 & 9.00 & 17.00 & 32.26 & 38.26 & 6792 & 1.00 & 1.00 & 1.00 & 1.00 & 2.00 & 4.00 & 8.00 & 19.00 & 22.00 \\
\hline 5410 & 1.00 & 1.00 & 1.00 & 1.00 & 2.00 & 5.00 & 11.00 & 24.78 & 30.00 & 6794 & -- & -- & 1.00 & 3.50 & 7.00 & 12.50 & 21.00 & -- & -- \\
\hline 5411 & 1.00 & 1.00 & 1.00 & 1.00 & 3.00 & 7.00 & 14.00 & 29.00 & 35.00 & 6834 & 1.00 & 1.00 & 1.00 & 1.00 & 3.00 & 7.00 & 13.00 & 24.00 & 30.00 \\
\hline 5424 & 1.00 & 1.00 & 1.00 & 1.00 & 5.00 & 10.25 & 19.00 & 40.04 & 64.91 & 6893 & 1.00 & 1.00 & 1.00 & 1.00 & 2.00 & 4.00 & 9.00 & 19.04 & 26.00 \\
\hline 5429 & 1.00 & 1.00 & 1.00 & 1.00 & 3.00 & 7.00 & 15.00 & 29.00 & 36.00 & 6935 & 1.00 & 1.00 & 1.00 & 1.00 & 2.00 & 5.00 & 9.00 & 21.00 & 26.61 \\
\hline 5431 & -- & -- & 1.00 & 3.75 & 10.00 & 15.25 & 23.50 & -- & -- & 6981 & 1.00 & 1.00 & 1.00 & 2.00 & 3.00 & 8.00 & 16.20 & 33.04 & 38.68 \\
\hline 5461 & 1.00 & 1.00 & 1.00 & 2.00 & 4.00 & 10.00 & 17.00 & 35.00 & 41.00 & 7020 & 1.00 & 1.00 & 1.00 & 2.00 & 5.00 & 12.00 & 19.30 & 41.18 & 44.53 \\
\hline 5463 & 1.00 & 1.00 & 1.00 & 1.00 & 2.00 & 7.00 & 12.00 & 24.00 & 29.00 & 7060 & 1.00 & 1.00 & 1.00 & 1.00 & 2.00 & 5.00 & 11.00 & 19.00 & 27.00 \\
\hline 5471 & -- & -- & 1.00 & 1.00 & 1.00 & 1.00 & 6.40 & -- & -- & 7066 & 1.00 & 1.00 & 1.00 & 1.00 & 4.00 & 8.00 & 15.00 & 28.02 & 36.00 \\
\hline 5477 & -- & -- & 1.00 & 1.00 & 2.00 & 5.00 & 17.40 & -- & -- & 7074 & 1.00 & 1.00 & 1.00 & 1.00 & 2.00 & 5.00 & 10.00 & 21.00 & 28.00 \\
\hline 5528 & 1.00 & 1.00 & 1.00 & 2.00 & 4.00 & 8.00 & 15.00 & 27.64 & 32.00 & 7097 & 1.00 & 1.00 & 1.00 & 2.00 & 3.00 & 8.00 & 15.80 & 29.56 & 48.72 \\
\hline 5579 & -- & -- & -- & -- & 15.00 & -- & -- & -- & -- & 7116 & 1.00 & 1.00 & 1.00 & 2.00 & 3.00 & 7.00 & 13.00 & 29.00 & 35.00 \\
\hline 5580 & -- & -- & 1.00 & 2.00 & 4.00 & 5.50 & 13.00 & -- & -- & 7140 & 1.00 & 1.00 & 1.00 & 1.00 & 3.00 & 7.00 & 14.00 & 30.00 & 35.39 \\
\hline 5589 & 1.00 & 1.00 & 1.00 & 1.00 & 2.00 & 5.00 & 8.00 & 20.00 & 24.08 & 7173 & 1.00 & 1.00 & 1.00 & 1.00 & 3.00 & 8.00 & 16.00 & 34.78 & 45.89 \\
\hline 5590 & 1.00 & 1.00 & 1.00 & 1.00 & 3.00 & 6.00 & 16.00 & 30.40 & 43.08 & 7174 & 1.00 & 1.00 & 1.00 & 1.00 & 3.00 & 8.00 & 16.00 & 31.00 & 40.57 \\
\hline 5591 & 1.00 & 1.00 & 1.00 & 2.00 & 3.00 & 5.00 & 9.00 & 20.00 & 26.12 & 7213 & 1.00 & 1.00 & 1.00 & 2.00 & 3.00 & 8.00 & 16.00 & 30.22 & 36.61 \\
\hline 5592 & 1.00 & 1.00 & 1.00 & 2.00 & 3.00 & 5.00 & 9.00 & 21.00 & 33.00 & 7243 & 1.00 & 1.00 & 1.00 & 1.00 & 2.00 & 6.00 & 12.00 & 27.00 & 35.00 \\
\hline 5594 & 1.00 & 1.00 & 1.00 & 1.00 & 2.00 & 4.00 & 8.20 & 21.88 & 29.42 & 7262 & 1.00 & 1.00 & 1.00 & 1.00 & 1.00 & 1.00 & 7.00 & 19.00 & 30.28 \\
\hline 5595 & -- & -- & -- & -- & 1.50 & -- & -- & -- & -- & 7274 & 1.00 & 1.00 & 1.00 & 1.00 & 3.00 & 8.00 & 11.00 & 23.80 & 29.40 \\
\hline 5596 & 1.00 & 1.00 & 1.00 & 1.00 & 1.00 & 3.00 & 8.00 & 15.00 & 21.00 & 7300 & 1.00 & 1.00 & 1.00 & 2.00 & 3.00 & 7.00 & 14.00 & 24.94 & 31.00 \\
\hline 5600 & 1.00 & 1.00 & 1.00 & 2.00 & 3.00 & 5.00 & 11.00 & 28.94 & 38.47 & 7311 & -- & -- & 1.00 & 1.00 & 3.00 & 6.75 & 9.70 & -- & -- \\
\hline 5618 & -- & -- & 2.00 & 2.00 & 4.00 & 9.50 & 12.40 & -- & -- & 7363 & -- & -- & 1.00 & 2.00 & 4.00 & 9.75 & 16.20 & -- & -- \\
\hline 5650 & -- & -- & 1.10 & 2.25 & 5.50 & 9.00 & 11.90 & -- & -- & 7422 & 1.00 & 1.00 & 1.00 & 1.00 & 3.00 & 7.00 & 13.00 & 27.00 & 34.30 \\
\hline 5656 & 1.00 & 1.00 & 1.00 & 1.00 & 1.00 & 4.00 & 10.00 & 22.00 & 27.46 & 7431 & 1.00 & 1.00 & 1.00 & 1.00 & 3.00 & 6.00 & 12.00 & 29.74 & 34.00 \\
\hline 5658 & 1.00 & 1.00 & 1.00 & 2.00 & 4.00 & 8.00 & 15.00 & 32.00 & 42.16 & 7481 & 1.00 & 1.00 & 1.00 & 1.00 & 2.00 & 5.00 & 11.00 & 23.00 & 33.55 \\
\hline 5661 & 1.00 & 1.00 & 1.00 & 1.00 & 1.00 & 5.00 & 10.90 & 26.98 & 38.95 & 7497 & 1.00 & 1.00 & 1.00 & 1.00 & 2.00 & 6.00 & 12.00 & 24.84 & 30.00 \\
\hline 5666 & -- & -- & 1.00 & 2.00 & 3.50 & 8.75 & 18.30 & -- & -- & 7498 & 1.00 & 1.00 & 1.00 & 1.00 & 2.00 & 7.00 & 12.00 & 26.00 & 29.43 \\
\hline 5695 & 1.00 & 1.00 & 1.00 & 1.00 & 3.00 & 9.00 & 15.00 & 29.94 & 36.47 & 7499 & 1.00 & 1.00 & 1.00 & 1.00 & 2.00 & 7.00 & 12.00 & 23.00 & 28.23 \\
\hline 5742 & -- & -- & 1.00 & 1.00 & 5.00 & 8.50 & 16.60 & -- & -- & 7531 & 1.00 & 1.00 & 1.00 & 2.00 & 4.00 & 10.00 & 19.90 & 47.66 & 55.88 \\
\hline 5766 & -- & -- & 1.00 & 2.00 & 4.00 & 10.00 & 16.00 & -- & -- & 7534 & 1.00 & 1.00 & 1.00 & 1.00 & 3.00 & 7.00 & 14.00 & 33.02 & 34.00 \\
\hline 5770 & 1.00 & 1.00 & 1.00 & 1.00 & 3.00 & 7.00 & 13.00 & 25.00 & 33.00 & 7556 & 1.00 & 1.00 & 1.00 & 1.00 & 3.00 & 7.00 & 13.00 & 24.00 & 29.00 \\
\hline 5775 & -- & -- & 1.00 & 1.00 & 3.00 & 7.25 & 16.50 & -- & -- & 7594 & 1.00 & 1.00 & 1.00 & 2.00 & 4.00 & 8.00 & 15.00 & 32.90 & 41.00 \\
\hline 5779 & -- & -- & 1.00 & 1.00 & 6.50 & 10.25 & 25.10 & -- & -- & 7596 & 1.00 & 1.00 & 1.00 & 2.00 & 3.00 & 7.00 & 14.50 & 35.50 & 54.50 \\
\hline 5840 & 1.00 & 1.00 & 1.00 & 2.00 & 4.00 & 9.00 & 17.00 & 36.10 & 42.40 & 7608 & 1.00 & 1.00 & 1.00 & 1.00 & 3.00 & 8.00 & 14.00 & 26.00 & 31.17 \\
\hline 5890 & 1.00 & 1.00 & 1.00 & 1.00 & 3.00 & 7.00 & 13.00 & 28.00 & 37.00 & 7622 & -- & -- & 1.00 & 1.00 & 2.00 & 5.00 & 15.20 & -- & -- \\
\hline 5891 & 1.00 & 1.00 & 1.00 & 1.00 & 3.00 & 7.00 & 12.90 & 24.00 & 27.61 & 7700 & 1.00 & 1.00 & 1.00 & 2.00 & 4.00 & 9.00 & 16.70 & 32.00 & 40.67 \\
\hline 5897 & 1.00 & 1.00 & 1.00 & 1.00 & 2.00 & 6.00 & 12.00 & 24.00 & 27.00 & 7706 & 1.00 & 1.00 & 1.00 & 1.00 & 2.00 & 6.00 & 13.00 & 24.00 & 28.11 \\
\hline
\end{tabular}


Appendix 4-5.2. Empirical distribution of storm duration defined by 8-hour minimum interevent time for hourly rainfall stations in Texas-Continued.

\begin{tabular}{|c|c|c|c|c|c|c|c|c|c|c|c|c|c|c|c|c|c|c|c|}
\hline \multicolumn{20}{|c|}{$\begin{array}{c}\text { Duration } \\
\text { (hours) }\end{array}$} \\
\hline $\begin{array}{l}\text { Sta- } \\
\text { tion } \\
\text { no. }\end{array}$ & $\begin{array}{c}1 \text { st } \\
\text { per- } \\
\text { centile }\end{array}$ & $\begin{array}{c}\text { 2nd } \\
\text { per- } \\
\text { centile }\end{array}$ & $\begin{array}{c}10 \text { th } \\
\text { per- } \\
\text { centile }\end{array}$ & $\begin{array}{c}25 \text { th } \\
\text { per- } \\
\text { centile }\end{array}$ & $\begin{array}{c}\text { 50th } \\
\text { per- } \\
\text { centile } \\
\text { (median) }\end{array}$ & $\begin{array}{c}\text { 75th } \\
\text { per- } \\
\text { centile }\end{array}$ & $\begin{array}{c}\text { 90th } \\
\text { per- } \\
\text { centile }\end{array}$ & $\begin{array}{c}\text { 98th } \\
\text { per- } \\
\text { centile }\end{array}$ & $\begin{array}{c}\text { 99th } \\
\text { per- } \\
\text { centile }\end{array}$ & $\begin{array}{l}\text { Sta- } \\
\text { tion } \\
\text { no. }\end{array}$ & $\begin{array}{c}\text { 1st } \\
\text { per- } \\
\text { centile }\end{array}$ & $\begin{array}{c}\text { 2nd } \\
\text { per- } \\
\text { centile }\end{array}$ & $\begin{array}{c}10 \text { th } \\
\text { per- } \\
\text { centile }\end{array}$ & $\begin{array}{c}25 \text { th } \\
\text { per- } \\
\text { centile }\end{array}$ & $\begin{array}{c}\text { 50th } \\
\text { per- } \\
\text { centile } \\
\text { (median) }\end{array}$ & $\begin{array}{c}\text { 75th } \\
\text { per- } \\
\text { centile }\end{array}$ & $\begin{array}{c}\text { 90th } \\
\text { per- } \\
\text { centile }\end{array}$ & $\begin{array}{c}\text { 98th } \\
\text { per- } \\
\text { centile }\end{array}$ & $\begin{array}{c}\text { 99th } \\
\text { per- } \\
\text { centile }\end{array}$ \\
\hline 7718 & 1.00 & 1.00 & 1.00 & 1.50 & 4.00 & 9.00 & 13.00 & 38.16 & 39.86 & 8910 & -- & -- & 1.30 & 2.00 & 4.00 & 5.75 & 10.00 & -- & -- \\
\hline 7745 & 1.00 & 1.00 & 1.00 & 2.00 & 5.00 & 11.00 & 20.00 & 41.54 & 48.36 & 8911 & 1.00 & 1.00 & 1.00 & 1.00 & 3.00 & 6.00 & 14.00 & 25.78 & 35.39 \\
\hline 7922 & 1.00 & 1.00 & 1.00 & 1.00 & 2.00 & 5.00 & 11.00 & 22.20 & 26.00 & 8924 & 1.00 & 1.00 & 1.00 & 1.00 & 2.00 & 4.00 & 9.00 & 19.00 & 28.34 \\
\hline 7936 & 1.00 & 1.00 & 1.00 & 1.00 & 3.00 & 7.00 & 13.00 & 26.00 & 36.00 & 8929 & -- & -- & 1.00 & 1.00 & 4.50 & 10.00 & 20.30 & -- & -- \\
\hline 7943 & 1.00 & 1.00 & 1.00 & 1.00 & 3.00 & 7.00 & 14.00 & 30.00 & 39.00 & 8942 & 1.00 & 1.00 & 1.00 & 1.00 & 3.00 & 8.00 & 14.00 & 25.00 & 32.64 \\
\hline 7944 & -- & 1.00 & 1.00 & 1.00 & 3.00 & 9.00 & 15.50 & 41.90 & -- & 8944 & 1.00 & 1.00 & 1.00 & 1.00 & 3.00 & 8.00 & 14.00 & 30.00 & 38.90 \\
\hline 7945 & 1.00 & 1.00 & 1.00 & 1.00 & 3.00 & 9.00 & 17.00 & 32.00 & 41.00 & 8996 & 1.00 & 1.00 & 1.00 & 2.00 & 3.00 & 8.00 & 15.00 & 29.00 & 37.70 \\
\hline 7947 & 1.00 & 1.00 & 1.00 & 1.00 & 2.00 & 7.00 & 13.00 & 36.12 & 61.29 & 9014 & -- & -- & 2.00 & 3.00 & 7.00 & 11.00 & 22.20 & -- & -- \\
\hline 7948 & 1.00 & 1.00 & 1.00 & 1.00 & 3.00 & 7.00 & 14.00 & 27.06 & 35.06 & 9037 & 1.00 & 1.00 & 1.00 & 1.00 & 3.00 & 7.00 & 13.00 & 28.32 & 38.66 \\
\hline 7951 & 1.00 & 1.00 & 1.00 & 2.00 & 4.00 & 9.00 & 16.00 & 27.00 & 36.94 & 9106 & 1.00 & 1.00 & 1.00 & 1.00 & 3.00 & 6.00 & 12.00 & 34.84 & 38.00 \\
\hline 7953 & 1.00 & 1.00 & 1.00 & 2.00 & 3.00 & 7.50 & 17.20 & 37.00 & 43.60 & 9107 & -- & -- & 1.00 & 1.00 & 1.00 & 3.00 & 8.80 & -- & -- \\
\hline 7981 & 1.00 & 1.00 & 1.00 & 1.00 & 3.00 & 7.25 & 15.00 & 26.96 & 32.49 & 9129 & 1.00 & 1.00 & 1.00 & 1.00 & 3.00 & 7.00 & 12.00 & 29.12 & 40.92 \\
\hline 7990 & 1.00 & 1.00 & 1.00 & 1.00 & 3.00 & 8.00 & 15.00 & 36.84 & 52.52 & 9163 & 1.00 & 1.00 & 1.00 & 1.00 & 3.00 & 7.00 & 14.00 & 26.24 & 34.00 \\
\hline 7992 & -- & -- & 1.90 & 3.00 & 4.50 & 7.75 & 11.10 & -- & -- & 9213 & 1.00 & 1.00 & 1.00 & 1.00 & 1.00 & 8.00 & 14.00 & 36.92 & 55.30 \\
\hline 7997 & 1.00 & 1.00 & 1.00 & 2.00 & 4.00 & 7.00 & 12.00 & 21.96 & 26.70 & 9214 & -- & -- & 1.40 & 3.00 & 4.00 & 6.50 & 16.60 & -- & -- \\
\hline 7999 & -- & -- & 1.00 & 1.00 & 4.00 & 8.00 & 17.00 & -- & -- & 9222 & 1.00 & 1.00 & 1.00 & 1.00 & 4.00 & 10.00 & 17.60 & 33.24 & 35.56 \\
\hline 8022 & -- & 1.00 & 1.00 & 1.00 & 3.00 & 7.00 & 13.50 & 47.50 & -- & 9248 & 1.00 & 1.00 & 1.00 & 1.00 & 3.00 & 9.00 & 18.20 & 31.48 & 46.02 \\
\hline 8023 & 1.00 & 1.00 & 1.00 & 1.00 & 2.00 & 5.00 & 11.00 & 25.68 & 32.84 & 9266 & -- & 1.00 & 1.00 & 2.00 & 4.00 & 9.00 & 18.00 & 31.32 & -- \\
\hline 8047 & 1.00 & 1.00 & 1.00 & 1.00 & 3.00 & 7.00 & 13.00 & 27.00 & 32.75 & 9270 & 1.00 & 1.00 & 1.00 & 1.00 & 2.00 & 4.00 & 9.00 & 18.00 & 25.00 \\
\hline 8060 & 1.00 & 1.00 & 1.00 & 2.00 & 3.00 & 9.00 & 16.00 & 38.00 & 40.95 & 9295 & 1.00 & 1.00 & 1.00 & 1.00 & 1.00 & 7.00 & 7.40 & 25.00 & 30.04 \\
\hline 8062 & -- & -- & 1.00 & 2.00 & 3.00 & 10.50 & 15.20 & -- & -- & 9304 & -- & -- & -- & 1.50 & 4.00 & 5.75 & -- & -- & -- \\
\hline 8068 & -- & -- & 1.00 & 1.00 & 2.00 & 6.00 & 14.00 & -- & -- & 9307 & 1.00 & 1.00 & 1.00 & 1.00 & 4.00 & 8.00 & 15.00 & 27.92 & 35.68 \\
\hline 8081 & 1.00 & 1.00 & 1.00 & 1.00 & 3.00 & 7.00 & 14.00 & 30.00 & 38.00 & 9328 & 1.00 & 1.00 & 1.00 & 1.00 & 3.00 & 7.00 & 16.00 & 41.20 & 47.04 \\
\hline 8089 & -- & -- & 1.00 & 1.00 & 4.00 & 11.00 & 14.20 & -- & -- & 9329 & -- & -- & 1.00 & 1.00 & 5.00 & 11.50 & 28.30 & -- & -- \\
\hline 8221 & -- & -- & 1.20 & 4.00 & 7.00 & 15.00 & 27.40 & -- & -- & 9345 & -- & -- & -- & -- & 8.00 & -- & -- & -- & -- \\
\hline 8252 & 1.00 & 1.00 & 1.00 & 1.00 & 2.00 & 6.00 & 11.00 & 25.00 & 34.59 & 9363 & 1.00 & 1.00 & 1.00 & 1.00 & 3.00 & 7.00 & 14.00 & 29.18 & 37.00 \\
\hline 8265 & 1.00 & 1.00 & 1.00 & 2.00 & 4.00 & 10.00 & 18.00 & 33.00 & 41.36 & 9364 & 1.00 & 1.00 & 1.00 & 1.00 & 3.00 & 7.00 & 15.00 & 32.00 & 41.00 \\
\hline 8289 & -- & 1.00 & 1.00 & 1.00 & 3.00 & 8.00 & 23.80 & 40.04 & -- & 9365 & -- & -- & 1.00 & 1.75 & 4.00 & 6.25 & 12.00 & -- & -- \\
\hline 8305 & 1.00 & 1.00 & 1.00 & 1.00 & 2.00 & 4.00 & 9.00 & 17.90 & 23.95 & 9371 & 1.00 & 1.00 & 1.00 & 2.00 & 4.00 & 9.00 & 14.90 & 42.24 & 49.29 \\
\hline 8335 & 1.00 & 1.00 & 1.00 & 2.00 & 4.50 & 10.00 & 17.00 & 36.00 & 47.93 & 9417 & 1.00 & 1.00 & 1.00 & 2.00 & 4.00 & 8.00 & 15.00 & 28.72 & 35.00 \\
\hline 8400 & 1.00 & 1.00 & 1.00 & 1.00 & 2.00 & 5.00 & 10.00 & 23.20 & 46.00 & 9419 & 1.00 & 1.00 & 1.00 & 2.00 & 4.00 & 9.00 & 17.00 & 31.86 & 39.00 \\
\hline 8445 & 1.00 & 1.00 & 1.00 & 2.00 & 4.00 & 9.00 & 16.00 & 33.00 & 44.90 & 9435 & -- & 1.00 & 1.00 & 1.00 & 3.00 & 6.00 & 16.00 & 40.44 & -- \\
\hline 8446 & 1.00 & 1.00 & 1.00 & 1.00 & 3.00 & 7.00 & 12.20 & 25.00 & 30.00 & 9491 & 1.00 & 1.00 & 1.00 & 1.00 & 3.00 & 8.00 & 14.00 & 27.00 & 31.32 \\
\hline 8451 & 1.00 & 1.00 & 1.00 & 2.00 & 3.00 & 8.00 & 15.00 & 32.02 & 38.36 & 9499 & 1.00 & 1.00 & 1.00 & 1.00 & 2.00 & 6.00 & 11.00 & 24.04 & 31.52 \\
\hline 8531 & 1.00 & 1.00 & 1.00 & 2.00 & 4.00 & 9.00 & 16.00 & 30.00 & 35.82 & 9522 & -- & -- & 1.80 & 3.00 & 5.00 & 13.50 & 18.60 & -- & -- \\
\hline 8541 & 1.00 & 1.00 & 1.00 & 2.00 & 4.00 & 8.00 & 18.00 & 26.00 & 36.56 & 9527 & 1.00 & 1.00 & 1.00 & 1.00 & 2.00 & 5.00 & 10.00 & 21.00 & 27.28 \\
\hline 8544 & 1.00 & 1.00 & 1.00 & 1.00 & 3.00 & 8.00 & 15.00 & 31.00 & 36.00 & 9532 & 1.00 & 1.00 & 1.00 & 1.00 & 3.00 & 7.00 & 12.00 & 25.00 & 33.75 \\
\hline 8545 & -- & -- & 1.00 & 1.75 & 4.50 & 11.25 & 20.10 & -- & -- & 9544 & -- & -- & -- & 1.00 & 1.00 & 6.00 & -- & -- & -- \\
\hline 8563 & 1.00 & 1.00 & 1.00 & 1.00 & 2.00 & 7.00 & 11.00 & 21.00 & 27.00 & 9565 & 1.00 & 1.00 & 1.00 & 1.00 & 3.00 & 6.00 & 12.00 & 23.00 & 30.08 \\
\hline 8566 & 1.00 & 1.00 & 1.00 & 1.00 & 2.00 & 5.00 & 12.00 & 24.32 & 27.16 & 9570 & 1.00 & 1.00 & 1.00 & 1.00 & 2.00 & 5.00 & 9.00 & 22.00 & 26.98 \\
\hline 8583 & 1.00 & 1.00 & 1.00 & 1.00 & 2.00 & 6.00 & 11.00 & 21.00 & 26.00 & 9574 & -- & -- & 1.00 & 1.00 & 2.00 & 5.00 & 11.00 & -- & -- \\
\hline 8584 & 1.00 & 1.00 & 1.00 & 1.00 & 3.00 & 7.00 & 13.00 & 27.00 & 36.42 & 9588 & 1.00 & 1.00 & 1.00 & 1.00 & 2.00 & 7.00 & 14.00 & 28.62 & 35.00 \\
\hline 8623 & 1.00 & 1.00 & 1.00 & 1.00 & 3.00 & 7.00 & 12.00 & 24.00 & 31.46 & 9665 & 1.00 & 1.00 & 1.00 & 1.00 & 3.00 & 8.00 & 15.00 & 29.00 & 35.00 \\
\hline 8625 & 1.00 & 1.00 & 1.00 & 2.00 & 4.00 & 9.00 & 16.00 & 30.00 & 40.00 & 9715 & 1.00 & 1.00 & 1.00 & 1.00 & 3.00 & 8.00 & 14.00 & 26.00 & 32.00 \\
\hline 8630 & 1.00 & 1.00 & 1.00 & 1.00 & 3.00 & 7.00 & 13.00 & 25.56 & 30.56 & 9729 & 1.00 & 1.00 & 1.00 & 2.00 & 4.00 & 9.00 & 16.00 & 30.16 & 39.00 \\
\hline 8631 & 1.00 & 1.00 & 1.00 & 2.00 & 3.00 & 8.00 & 13.00 & 29.00 & 39.60 & 9772 & 1.00 & 1.00 & 1.00 & 2.00 & 3.00 & 7.00 & 14.00 & 30.12 & 40.06 \\
\hline 8646 & 1.00 & 1.00 & 1.00 & 2.00 & 4.00 & 9.00 & 15.00 & 28.00 & 34.20 & 9814 & -- & -- & 1.00 & 2.00 & 4.00 & 11.00 & 16.80 & -- & -- \\
\hline 8647 & 1.00 & 1.00 & 1.00 & 1.00 & 2.00 & 5.00 & 10.00 & 21.00 & 27.00 & 9815 & 1.00 & 1.00 & 1.00 & 2.00 & 4.00 & 9.00 & 16.00 & 31.28 & 36.28 \\
\hline 8677 & 1.00 & 1.00 & 1.00 & 3.00 & 5.00 & 10.00 & 17.00 & 24.32 & 31.08 & 9816 & -- & 1.00 & 1.00 & 1.00 & 3.00 & 8.00 & 12.90 & 31.46 & -- \\
\hline 8696 & -- & -- & 1.00 & 2.00 & 3.00 & 7.50 & 10.00 & -- & -- & 9817 & 1.00 & 1.00 & 1.00 & 1.00 & 2.00 & 6.00 & 11.00 & 23.02 & 31.00 \\
\hline 8743 & 1.00 & 1.00 & 1.00 & 2.00 & 4.00 & 9.00 & 16.00 & 30.00 & 36.00 & 9829 & 1.00 & 1.00 & 1.00 & 1.00 & 2.00 & 5.00 & 12.00 & 24.80 & 33.90 \\
\hline 8761 & 1.00 & 1.00 & 1.00 & 1.00 & 2.00 & 5.00 & 10.00 & 19.46 & 26.69 & 9830 & 1.00 & 1.00 & 1.00 & 1.00 & 2.00 & 6.00 & 10.00 & 23.72 & 28.70 \\
\hline 8778 & 1.00 & 1.00 & 1.00 & 1.00 & 3.00 & 7.00 & 14.00 & 26.00 & 33.00 & 9858 & 1.00 & 1.00 & 1.00 & 2.00 & 3.00 & 7.00 & 13.00 & 28.00 & 35.00 \\
\hline 8845 & 1.00 & 1.00 & 1.00 & 1.00 & 3.00 & 7.00 & 14.00 & 28.00 & 34.00 & 9893 & 1.00 & 1.00 & 1.00 & 1.00 & 3.00 & 7.00 & 13.00 & 27.00 & 35.09 \\
\hline 8859 & 1.00 & 1.00 & 1.00 & 2.00 & 4.00 & 10.00 & 17.00 & 33.00 & 38.00 & 9916 & 1.00 & 1.00 & 1.00 & 1.00 & 3.00 & 9.00 & 15.00 & 28.32 & 36.00 \\
\hline 8898 & 1.00 & 1.00 & 1.00 & 2.00 & 4.00 & 9.00 & 16.00 & 32.64 & 41.66 & 9976 & 1.00 & 1.00 & 1.00 & 1.00 & 3.00 & 6.00 & 14.00 & 30.00 & 39.00 \\
\hline 8908 & -- & -- & 1.00 & 2.00 & 4.00 & 7.00 & 16.60 & -- & -- & & & & & & & & & & \\
\hline
\end{tabular}


Appendix 4-5.3. Empirical distribution of storm duration defined by 12-hour minimum interevent time for hourly rainfall stations in Texas.

[--, not available]

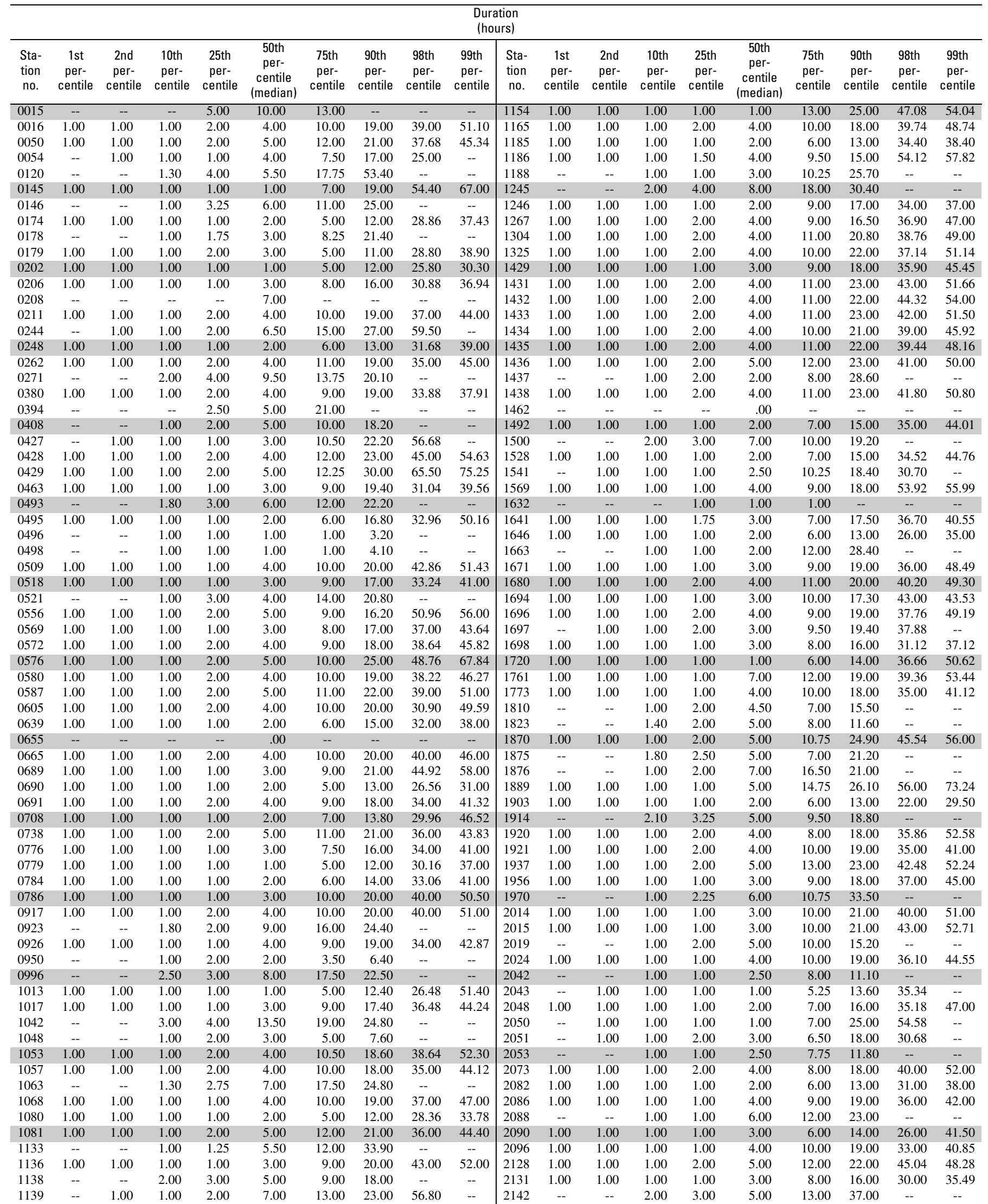


Appendix 4-5.3. Empirical distribution of storm duration defined by 12-hour minimum interevent time for hourly rainfall stations in Texas-Continued.

\begin{tabular}{|c|c|c|c|c|c|c|c|c|c|c|c|c|c|c|c|c|c|c|c|}
\hline \multicolumn{20}{|c|}{$\begin{array}{c}\text { Duration } \\
\text { (hours) }\end{array}$} \\
\hline $\begin{array}{l}\text { Sta- } \\
\text { tion } \\
\text { no. }\end{array}$ & $\begin{array}{c}\text { 1st } \\
\text { per- } \\
\text { centile }\end{array}$ & $\begin{array}{c}\text { 2nd } \\
\text { per- } \\
\text { centile }\end{array}$ & $\begin{array}{c}10 \text { th } \\
\text { per- } \\
\text { centile }\end{array}$ & $\begin{array}{c}25 \text { th } \\
\text { per- } \\
\text { centile }\end{array}$ & $\begin{array}{c}\text { 50th } \\
\text { per- } \\
\text { centile } \\
\text { (median) }\end{array}$ & $\begin{array}{c}\text { 75th } \\
\text { per- } \\
\text { centile }\end{array}$ & $\begin{array}{c}\text { 90th } \\
\text { per- } \\
\text { centile }\end{array}$ & $\begin{array}{c}\text { 98th } \\
\text { per- } \\
\text { centile }\end{array}$ & $\begin{array}{c}\text { 99th } \\
\text { per- } \\
\text { centile }\end{array}$ & $\begin{array}{c}\text { Sta- } \\
\text { tion } \\
\text { no. }\end{array}$ & $\begin{array}{c}1 \text { st } \\
\text { per- } \\
\text { centile }\end{array}$ & $\begin{array}{c}\text { 2nd } \\
\text { per- } \\
\text { centile }\end{array}$ & $\begin{array}{c}10 \text { th } \\
\text { per- } \\
\text { centile }\end{array}$ & $\begin{array}{c}25 \text { th } \\
\text { per- } \\
\text { centile }\end{array}$ & $\begin{array}{c}\text { 50th } \\
\text { per- } \\
\text { centile } \\
\text { (median) }\end{array}$ & $\begin{array}{c}\text { 75th } \\
\text { per- } \\
\text { centile }\end{array}$ & $\begin{array}{c}\text { 90th } \\
\text { per- } \\
\text { centile }\end{array}$ & $\begin{array}{c}\text { 98th } \\
\text { per- } \\
\text { centile }\end{array}$ & $\begin{array}{c}\text { 99th } \\
\text { per- } \\
\text { centile }\end{array}$ \\
\hline 2160 & -- & -- & 1.00 & 1.00 & 7.00 & 25.00 & 46.20 & -- & -- & 3463 & -- & -- & 1.00 & 2.00 & 4.50 & 11.00 & 25.20 & -- & -- \\
\hline 2206 & 1.00 & 1.00 & 1.00 & 2.00 & 4.00 & 9.00 & 17.00 & 40.00 & 49.00 & 3476 & 1.00 & 1.00 & 1.00 & 2.00 & 4.00 & 9.50 & 18.00 & 37.00 & 42.00 \\
\hline 2238 & 1.00 & 1.00 & 1.00 & 1.00 & 3.00 & 8.00 & 17.70 & 42.76 & 55.29 & 3485 & -- & -- & 1.30 & 3.75 & 7.00 & 12.25 & 20.40 & -- & -- \\
\hline 2240 & -- & 1.00 & 1.00 & 1.00 & 1.00 & 7.00 & 19.00 & 26.20 & -- & 3507 & 1.00 & 1.00 & 1.00 & 1.00 & 2.00 & 8.00 & 18.00 & 31.42 & 36.21 \\
\hline 2242 & 1.00 & 1.00 & 1.00 & 2.00 & 4.00 & 11.00 & 23.00 & 39.00 & 47.54 & 3546 & 1.00 & 1.00 & 1.00 & 1.00 & 4.00 & 10.00 & 20.00 & 38.00 & 45.00 \\
\hline 2244 & 1.00 & 1.00 & 1.00 & 1.00 & 4.00 & 10.00 & 20.00 & 38.00 & 46.00 & 3547 & 1.00 & 1.00 & 1.00 & 1.00 & 3.00 & 8.00 & 20.00 & 37.88 & 50.61 \\
\hline 2247 & -- & 1.00 & 1.00 & 2.00 & 5.00 & 9.00 & 16.00 & 30.20 & -- & 3579 & -- & 1.00 & 1.00 & 2.00 & 4.00 & 10.00 & 21.40 & 50.08 & -- \\
\hline 2309 & 1.00 & 1.00 & 1.00 & 2.00 & 5.00 & 12.00 & 22.00 & 40.22 & 45.37 & 3642 & 1.00 & 1.00 & 1.00 & 1.00 & 4.00 & 10.00 & 19.00 & 34.00 & 41.84 \\
\hline 2312 & 1.00 & 1.00 & 1.00 & 1.00 & 3.00 & 10.00 & 17.00 & 30.80 & 40.20 & 3646 & 1.00 & 1.00 & 1.00 & 2.00 & 5.00 & 10.00 & 20.00 & 34.00 & 43.00 \\
\hline 2334 & -- & 1.00 & 1.00 & 1.00 & 5.00 & 12.25 & 21.00 & 31.34 & -- & 3668 & -- & -- & 2.10 & 4.00 & 11.00 & 18.75 & 29.60 & -- & -- \\
\hline 2336 & 1.00 & 1.00 & 1.00 & 1.00 & 3.00 & 9.00 & 16.30 & 30.00 & 34.73 & 3673 & -- & -- & 1.60 & 3.00 & 6.00 & 19.50 & 41.00 & -- & -- \\
\hline 2354 & -- & -- & 1.00 & 1.00 & 3.00 & 12.00 & 23.20 & -- & -- & 3686 & 1.00 & 1.00 & 1.00 & 1.00 & 3.00 & 9.00 & 15.00 & 32.54 & 40.54 \\
\hline 2355 & -- & 1.00 & 1.00 & 2.00 & 4.00 & 9.50 & 25.00 & 54.10 & -- & 3691 & 1.00 & 1.00 & 1.00 & 1.00 & 3.00 & 8.00 & 16.00 & 31.70 & 38.35 \\
\hline 2357 & 1.00 & 1.00 & 1.00 & 1.00 & 3.00 & 10.00 & 21.00 & 40.00 & 55.83 & 3734 & -- & -- & 1.00 & 2.00 & 5.50 & 11.50 & 31.40 & -- & -- \\
\hline 2360 & 1.00 & 1.00 & 1.00 & 1.00 & 3.00 & 9.00 & 18.00 & 35.00 & 45.09 & 3771 & 1.00 & 1.00 & 1.00 & 1.00 & 2.00 & 8.00 & 15.00 & 29.00 & 36.81 \\
\hline 2361 & 1.00 & 1.00 & 1.00 & 1.00 & 3.00 & 6.00 & 14.30 & 30.56 & 41.65 & 3789 & 1.00 & 1.00 & 1.00 & 1.00 & 1.00 & 7.00 & 13.00 & 40.84 & 54.28 \\
\hline 2394 & 1.00 & 1.00 & 1.00 & 2.00 & 4.00 & 11.00 & 20.00 & 38.00 & 46.07 & 3826 & 1.00 & 1.00 & 1.00 & 2.00 & 4.00 & 11.00 & 18.00 & 34.92 & 52.18 \\
\hline 2404 & 1.00 & 1.00 & 1.00 & 1.00 & 4.00 & 10.00 & 19.00 & 34.00 & 41.00 & 3831 & 1.00 & 1.00 & 1.00 & 2.00 & 4.00 & 10.00 & 17.00 & 42.82 & 53.82 \\
\hline 2415 & 1.00 & 1.00 & 1.00 & 2.00 & 5.00 & 11.00 & 21.00 & 38.36 & 47.00 & 3841 & 1.00 & 1.00 & 1.00 & 1.00 & 3.00 & 8.00 & 16.00 & 33.70 & 48.75 \\
\hline 2462 & 1.00 & 1.00 & 1.00 & 1.00 & 4.00 & 10.00 & 18.00 & 34.80 & 40.20 & 3871 & 1.00 & 1.00 & 1.00 & 2.00 & 4.00 & 10.00 & 20.20 & 41.32 & 50.22 \\
\hline 2528 & 1.00 & 1.00 & 1.00 & 1.00 & 4.00 & 10.00 & 24.30 & 52.32 & 55.49 & 3884 & -- & -- & 1.20 & 3.50 & 6.00 & 8.50 & 14.00 & -- & -- \\
\hline 2617 & 1.00 & 1.00 & 1.00 & 2.00 & 5.00 & 10.00 & 18.00 & 45.60 & 53.55 & 3941 & -- & 1.00 & 1.00 & 2.00 & 7.00 & 15.00 & 25.00 & 75.80 & -- \\
\hline 2619 & 1.00 & 1.00 & 1.00 & 1.00 & 4.00 & 10.00 & 19.90 & 37.90 & 67.49 & 3963 & -- & -- & -- & 1.00 & 1.00 & 1.00 & -- & -- & -- \\
\hline 2621 & 1.00 & 1.00 & 1.00 & 1.00 & 3.00 & 8.00 & 17.00 & 33.00 & 41.74 & 4040 & 1.00 & 1.00 & 1.00 & 2.00 & 4.00 & 8.00 & 18.00 & 32.02 & 43.02 \\
\hline 2675 & 1.00 & 1.00 & 1.00 & 1.00 & 3.00 & 8.50 & 17.00 & 34.00 & 41.00 & 4058 & -- & -- & 1.00 & 2.00 & 3.00 & 6.00 & 21.00 & -- & -- \\
\hline 2676 & 1.00 & 1.00 & 1.00 & 1.00 & 2.00 & 7.00 & 14.00 & 30.00 & 38.00 & 4098 & 1.00 & 1.00 & 1.00 & 1.00 & 2.00 & 6.00 & 13.00 & 26.00 & 31.00 \\
\hline 2679 & 1.00 & 1.00 & 1.00 & 1.00 & 3.00 & 8.00 & 17.00 & 36.00 & 44.00 & 4100 & 1.00 & 1.00 & 1.00 & 2.00 & 3.00 & 9.00 & 16.00 & 36.30 & 46.55 \\
\hline 2715 & 1.00 & 1.00 & 1.00 & 2.00 & 4.00 & 10.00 & 19.00 & 33.00 & 41.82 & 4137 & 1.00 & 1.00 & 1.00 & 1.00 & 2.00 & 7.00 & 15.00 & 30.00 & 35.50 \\
\hline 2744 & 1.00 & 1.00 & 1.00 & 1.00 & 3.00 & 8.00 & 18.00 & 36.00 & 46.50 & 4191 & 1.00 & 1.00 & 1.00 & 1.00 & 3.00 & 9.00 & 19.00 & 37.00 & 45.00 \\
\hline 2758 & 1.00 & 1.00 & 1.00 & 2.00 & 4.00 & 13.25 & 23.70 & 59.58 & 78.91 & 4256 & -- & -- & -- & -- & .00 & -- & -- & -- & -- \\
\hline 2794 & -- & -- & 1.00 & 1.00 & 4.00 & 10.00 & 24.80 & -- & -- & 4257 & 1.00 & 1.00 & 1.00 & 1.00 & 3.00 & 9.00 & 19.00 & 34.00 & 41.00 \\
\hline 2797 & 1.00 & 1.00 & 1.00 & 1.00 & 3.00 & 7.00 & 15.00 & 31.00 & 38.00 & 4258 & 1.00 & 1.00 & 1.00 & 1.00 & 3.00 & 9.25 & 19.00 & 33.02 & 40.21 \\
\hline 2811 & 1.00 & 1.00 & 1.00 & 1.00 & 3.00 & 7.00 & 16.00 & 33.00 & 40.00 & 4278 & 1.00 & 1.00 & 1.00 & 2.00 & 4.00 & 9.00 & 18.00 & 36.00 & 48.76 \\
\hline 2813 & -- & -- & 1.00 & 1.00 & 3.00 & 7.00 & 14.80 & -- & -- & 4299 & 1.00 & 1.00 & 1.00 & 1.00 & 3.00 & 5.00 & 13.40 & 28.92 & 34.70 \\
\hline 2814 & -- & -- & 1.00 & 1.00 & 1.00 & 5.50 & 25.00 & -- & -- & 4300 & 1.00 & 1.00 & 1.00 & 2.00 & 4.00 & 11.00 & 21.00 & 44.00 & 51.00 \\
\hline 2815 & 1.00 & 1.00 & 1.00 & 1.00 & 2.00 & 7.00 & 13.00 & 24.04 & 28.08 & 4305 & 1.00 & 1.00 & 1.00 & 1.00 & 4.00 & 11.00 & 21.00 & 43.00 & 55.00 \\
\hline 2818 & 1.00 & 1.00 & 1.00 & 1.00 & 3.00 & 10.00 & 19.50 & 43.30 & 51.60 & 4307 & 1.00 & 1.00 & 1.00 & 1.00 & 4.00 & 12.00 & 25.00 & 50.80 & 66.50 \\
\hline 2986 & 1.00 & 1.00 & 1.00 & 2.00 & 5.00 & 14.00 & 26.00 & 50.00 & 54.32 & 4309 & 1.00 & 1.00 & 1.00 & 2.00 & 3.00 & 9.00 & 19.00 & 39.00 & 49.00 \\
\hline 3005 & 1.00 & 1.00 & 1.00 & 1.00 & 3.00 & 9.00 & 17.00 & 33.00 & 39.76 & 4311 & 1.00 & 1.00 & 1.00 & 2.00 & 4.00 & 10.00 & 20.00 & 41.00 & 49.00 \\
\hline 3033 & 1.00 & 1.00 & 1.00 & 2.00 & 3.00 & 6.00 & 12.00 & 25.06 & 30.06 & 4313 & 1.00 & 1.00 & 1.00 & 2.00 & 4.50 & 11.00 & 20.30 & 42.66 & 55.33 \\
\hline 3034 & -- & -- & -- & -- & 1.00 & -- & -- & -- & -- & 4319 & -- & 1.00 & 1.00 & 2.00 & 4.50 & 8.25 & 20.30 & 34.16 & -- \\
\hline 3047 & -- & -- & 1.00 & 2.00 & 4.50 & 9.25 & 15.00 & -- & -- & 4329 & 1.00 & 1.00 & 1.00 & 2.00 & 4.00 & 10.00 & 21.00 & 40.00 & 50.00 \\
\hline 3103 & -- & -- & 1.00 & 1.00 & 2.00 & 8.00 & 13.60 & -- & -- & 4331 & -- & -- & -- & -- & .00 & -- & -- & -- & -- \\
\hline 3133 & 1.00 & 1.00 & 1.00 & 2.00 & 4.00 & 11.00 & 20.00 & 37.00 & 44.00 & 4375 & 1.00 & 1.00 & 1.00 & 1.00 & 2.00 & 7.00 & 14.00 & 31.24 & 39.48 \\
\hline 3156 & 1.00 & 1.00 & 1.00 & 1.00 & 4.00 & 11.00 & 19.40 & 36.56 & 41.68 & 4392 & 1.00 & 1.00 & 1.00 & 2.00 & 6.00 & 15.00 & 26.10 & 54.82 & 61.82 \\
\hline 3171 & 1.00 & 1.00 & 1.00 & 2.00 & 4.00 & 10.00 & 20.00 & 38.00 & 46.00 & 4425 & 1.00 & 1.00 & 1.00 & 1.00 & 2.00 & 6.00 & 14.00 & 31.86 & 42.72 \\
\hline 3189 & 1.00 & 1.00 & 1.00 & 1.00 & 2.00 & 8.00 & 15.00 & 33.16 & 49.24 & 4440 & 1.00 & 1.00 & 1.00 & 2.00 & 4.00 & 11.00 & 21.00 & 36.00 & 49.10 \\
\hline 3260 & 1.00 & 1.00 & 1.00 & 1.00 & 3.00 & 8.00 & 17.00 & 33.20 & 46.90 & 4476 & 1.00 & 1.00 & 1.00 & 1.00 & 3.00 & 9.00 & 17.00 & 31.00 & 35.94 \\
\hline 3267 & 1.00 & 1.00 & 1.00 & 2.00 & 4.00 & 8.00 & 17.00 & 41.52 & 77.88 & 4498 & -- & -- & 1.00 & 1.00 & 2.00 & 4.00 & 25.20 & -- & -- \\
\hline 3270 & 1.00 & 1.00 & 1.00 & 1.00 & 2.00 & 6.00 & 13.70 & 27.00 & 32.47 & 4517 & 1.00 & 1.00 & 1.00 & 2.00 & 4.00 & 10.00 & 19.00 & 39.00 & 45.28 \\
\hline 3272 & -- & -- & 1.00 & 1.00 & 2.00 & 4.00 & 11.60 & -- & -- & 4520 & 1.00 & 1.00 & 1.00 & 1.00 & 2.00 & 8.00 & 15.50 & 28.00 & 36.20 \\
\hline 3277 & -- & -- & 1.00 & 1.00 & 1.00 & 7.00 & 27.40 & -- & -- & 4525 & -- & -- & 1.10 & 2.00 & 5.00 & 11.75 & 31.40 & -- & -- \\
\hline 3278 & 1.00 & 1.00 & 1.00 & 1.00 & 2.00 & 5.00 & 12.00 & 30.00 & 41.96 & 4563 & -- & -- & 1.00 & 2.00 & 5.50 & 8.00 & 18.30 & -- & -- \\
\hline 3280 & 1.00 & 1.00 & 1.00 & 1.00 & 3.00 & 6.00 & 13.00 & 34.46 & 38.05 & 4570 & 1.00 & 1.00 & 1.00 & 1.00 & 3.00 & 8.00 & 16.00 & 36.00 & 45.30 \\
\hline 3281 & -- & -- & 1.00 & 1.00 & 2.00 & 3.50 & 8.80 & -- & -- & 4577 & 1.00 & 1.00 & 1.00 & 1.00 & 4.00 & 9.00 & 19.00 & 35.00 & 46.02 \\
\hline 3283 & 1.00 & 1.00 & 1.00 & 2.00 & 5.00 & 12.00 & 23.00 & 42.56 & 53.28 & 4591 & 1.00 & 1.00 & 1.00 & 2.00 & 5.00 & 12.00 & 22.00 & 40.00 & 49.92 \\
\hline 3284 & 1.00 & 1.00 & 1.00 & 1.00 & 3.00 & 9.00 & 18.00 & 35.86 & 42.00 & 4670 & 1.00 & 1.00 & 1.00 & 1.00 & 3.00 & 8.00 & 16.00 & 32.00 & 39.00 \\
\hline 3285 & 1.00 & 1.00 & 1.00 & 1.00 & 3.00 & 8.00 & 16.00 & 30.00 & 36.56 & 4671 & 1.00 & 1.00 & 1.00 & 1.00 & 1.00 & 8.00 & 19.00 & 37.36 & 61.12 \\
\hline 3329 & 1.00 & 1.00 & 1.00 & 1.00 & 4.00 & 10.00 & 20.00 & 40.00 & 47.04 & 4679 & 1.00 & 1.00 & 1.00 & 1.00 & 3.00 & 9.00 & 17.00 & 34.00 & 41.94 \\
\hline 3335 & 1.00 & 1.00 & 1.00 & 2.00 & 4.00 & 11.00 & 24.00 & 42.28 & 47.73 & 4696 & -- & -- & 1.00 & 1.75 & 4.00 & 6.25 & 8.50 & -- & -- \\
\hline 3370 & 1.00 & 1.00 & 1.00 & 2.00 & 4.00 & 11.00 & 20.00 & 38.00 & 44.00 & 4703 & 1.00 & 1.00 & 1.00 & 1.00 & 3.00 & 8.50 & 15.60 & 34.36 & 43.24 \\
\hline 3410 & 1.00 & 1.00 & 1.00 & 1.00 & 3.00 & 8.00 & 16.00 & 34.00 & 41.00 & 4704 & 1.00 & 1.00 & 1.00 & 2.00 & 5.00 & 12.00 & 24.20 & 41.08 & 45.14 \\
\hline 3415 & 1.00 & 1.00 & 1.00 & 1.00 & 3.00 & 9.00 & 17.00 & 34.00 & 41.15 & 4731 & 1.00 & 1.00 & 1.00 & 1.00 & 3.50 & 11.00 & 24.00 & 37.50 & 38.00 \\
\hline 3430 & 1.00 & 1.00 & 1.00 & 1.00 & 4.00 & 11.00 & 20.00 & 41.00 & 51.00 & 4792 & 1.00 & 1.00 & 1.00 & 1.00 & 3.00 & 8.00 & 16.00 & 28.00 & 34.20 \\
\hline 3431 & 1.00 & 1.00 & 1.00 & 1.00 & 6.00 & 13.00 & 31.00 & 49.36 & 64.18 & 4819 & 1.00 & 1.00 & 1.00 & 1.00 & 3.50 & 10.00 & 18.00 & 33.88 & 38.11 \\
\hline 3441 & -- & -- & 1.00 & 1.00 & 3.00 & 9.00 & 23.00 & -- & -- & 4852 & -- & -- & 1.00 & 1.00 & 1.00 & 2.75 & 13.20 & -- & -- \\
\hline 3442 & 1.00 & 1.00 & 1.00 & 2.00 & 4.00 & 9.00 & 17.00 & 34.00 & 48.00 & 4866 & 1.00 & 1.00 & 1.00 & 1.00 & 4.00 & 10.00 & 19.00 & 37.00 & 44.00 \\
\hline 3446 & 1.00 & 1.00 & 1.00 & 2.00 & 3.00 & 7.00 & 15.00 & 32.00 & 43.90 & 4876 & 1.00 & 1.00 & 1.00 & 1.00 & 3.00 & 8.00 & 14.00 & 33.24 & 35.24 \\
\hline 3460 & -- & -- & 1.00 & 2.00 & 4.00 & 9.50 & 19.00 & -- & -- & 4878 & 1.00 & 1.00 & 1.00 & 2.00 & 4.00 & 11.00 & 21.00 & 42.66 & 50.83 \\
\hline 3462 & 1.00 & 1.00 & 1.00 & 2.00 & 4.00 & 10.00 & 17.00 & 41.30 & 52.80 & 4880 & 1.00 & 1.00 & 1.00 & 1.00 & 3.00 & 7.00 & 16.00 & 33.08 & 42.08 \\
\hline
\end{tabular}


Appendix 4-5.3. Empirical distribution of storm duration defined by 12-hour minimum interevent time for hourly rainfall stations in Texas-Continued.

\begin{tabular}{|c|c|c|c|c|c|c|c|c|c|c|c|c|c|c|c|c|c|c|c|}
\hline \multicolumn{20}{|c|}{$\begin{array}{c}\text { Duration } \\
\text { (hours) }\end{array}$} \\
\hline $\begin{array}{l}\text { Sta- } \\
\text { tion } \\
\text { no. }\end{array}$ & $\begin{array}{c}1 \text { st } \\
\text { per- } \\
\text { centile }\end{array}$ & $\begin{array}{c}\text { 2nd } \\
\text { per- } \\
\text { centile }\end{array}$ & $\begin{array}{c}10 \text { th } \\
\text { per- } \\
\text { centile }\end{array}$ & $\begin{array}{c}25 \text { th } \\
\text { per- } \\
\text { centile }\end{array}$ & $\begin{array}{c}\text { 50th } \\
\text { per- } \\
\text { centile } \\
\text { (median) }\end{array}$ & $\begin{array}{c}\text { 75th } \\
\text { per- } \\
\text { centile }\end{array}$ & $\begin{array}{c}\text { 90th } \\
\text { per- } \\
\text { centile }\end{array}$ & $\begin{array}{c}\text { 98th } \\
\text { per- } \\
\text { centile }\end{array}$ & $\begin{array}{c}\text { 99th } \\
\text { per- } \\
\text { centile }\end{array}$ & $\begin{array}{l}\text { Sta- } \\
\text { tion } \\
\text { no. }\end{array}$ & $\begin{array}{c}\text { 1st } \\
\text { per- } \\
\text { centile }\end{array}$ & $\begin{array}{c}\text { 2nd } \\
\text { per- } \\
\text { centile }\end{array}$ & $\begin{array}{c}10 \text { th } \\
\text { per- } \\
\text { centile }\end{array}$ & $\begin{array}{c}25 \text { th } \\
\text { per- } \\
\text { centile }\end{array}$ & $\begin{array}{c}\text { 50th } \\
\text { per- } \\
\text { centile } \\
\text { (median) }\end{array}$ & $\begin{array}{c}\text { 75th } \\
\text { per- } \\
\text { centile }\end{array}$ & $\begin{array}{c}\text { 90th } \\
\text { per- } \\
\text { centile }\end{array}$ & $\begin{array}{c}\text { 98th } \\
\text { per- } \\
\text { centile }\end{array}$ & $\begin{array}{l}\text { 99th } \\
\text { per- } \\
\text { centile }\end{array}$ \\
\hline 4920 & 1.00 & 1.00 & 1.00 & 1.00 & 3.00 & 7.00 & 15.00 & 32.00 & 38.63 & 5957 & 1.00 & 1.00 & 1.00 & 1.00 & 3.00 & 8.00 & 16.00 & 31.00 & 38.00 \\
\hline 4934 & -- & -- & -- & 1.00 & 3.00 & 15.00 & -- & -- & -- & 5958 & 1.00 & 1.00 & 1.00 & 2.00 & 4.00 & 12.00 & 18.00 & 30.00 & 49.00 \\
\hline 4972 & 1.00 & 1.00 & 1.00 & 1.00 & 4.00 & 9.00 & 18.00 & 33.00 & 39.47 & 5973 & 1.00 & 1.00 & 1.00 & 2.00 & 5.00 & 13.00 & 22.30 & 63.94 & 84.74 \\
\hline 4973 & 1.00 & 1.00 & 1.00 & 2.00 & 5.00 & 11.00 & 21.00 & 33.00 & 35.53 & 5996 & 1.00 & 1.00 & 1.00 & 1.00 & 4.00 & 10.00 & 19.00 & 37.36 & 45.00 \\
\hline 4974 & 1.00 & 1.00 & 1.00 & 1.00 & 4.00 & 9.00 & 16.00 & 33.00 & 41.10 & 6017 & 1.00 & 1.00 & 1.00 & 1.00 & 3.00 & 9.00 & 17.00 & 29.08 & 37.12 \\
\hline 4975 & 1.00 & 1.00 & 1.00 & 1.00 & 3.00 & 9.00 & 17.00 & 31.00 & 36.25 & 6024 & 1.00 & 1.00 & 1.00 & 2.00 & 5.00 & 12.25 & 21.00 & 45.64 & 56.79 \\
\hline 4978 & 1.00 & 1.00 & 1.00 & 1.00 & 3.00 & 9.00 & 17.20 & 35.24 & 39.24 & 6050 & -- & -- & 1.00 & 5.00 & 8.00 & 12.50 & 13.90 & -- & -- \\
\hline 4979 & -- & -- & 1.20 & 4.50 & 7.00 & 17.50 & 27.40 & -- & -- & 6104 & 1.00 & 1.00 & 1.00 & 1.00 & 2.00 & 5.00 & 13.00 & 31.00 & 40.00 \\
\hline 4982 & 1.00 & 1.00 & 1.00 & 2.00 & 4.00 & 9.00 & 17.00 & 33.88 & 44.35 & 6108 & 1.00 & 1.00 & 1.00 & 1.00 & 4.00 & 10.00 & 18.00 & 35.00 & 48.13 \\
\hline 5018 & 1.00 & 1.00 & 1.00 & 2.00 & 4.00 & 9.00 & 20.00 & 35.00 & 43.80 & 6136 & 1.00 & 1.00 & 1.00 & 1.00 & 2.00 & 6.00 & 13.00 & 26.00 & 35.88 \\
\hline 5048 & 1.00 & 1.00 & 1.00 & 1.00 & 2.00 & 6.00 & 14.00 & 32.00 & 43.85 & 6166 & -- & 1.00 & 1.00 & 2.00 & 3.00 & 8.00 & 17.00 & 42.12 & -- \\
\hline 5049 & -- & 1.00 & 1.00 & 1.00 & 2.00 & 6.00 & 11.00 & 16.00 & -- & 6176 & 1.00 & 1.00 & 1.00 & 2.00 & 4.00 & 12.00 & 20.90 & 43.54 & 55.09 \\
\hline 5056 & -- & -- & -- & 1.00 & 4.00 & 12.50 & -- & -- & -- & 6177 & 1.00 & 1.00 & 1.00 & 1.00 & 4.00 & 9.00 & 18.00 & 37.00 & 47.30 \\
\hline 5057 & 1.00 & 1.00 & 1.00 & 1.00 & 3.00 & 9.00 & 21.00 & 46.00 & 54.58 & 6210 & 1.00 & 1.00 & 1.00 & 1.00 & 4.00 & 9.00 & 17.00 & 32.00 & 40.00 \\
\hline 5060 & 1.00 & 1.00 & 1.00 & 2.00 & 3.00 & 8.00 & 21.00 & 46.00 & 58.75 & 6211 & 1.00 & 1.00 & 1.00 & 1.00 & 3.00 & 9.00 & 19.00 & 44.00 & 48.76 \\
\hline 5081 & 1.00 & 1.00 & 1.00 & 2.00 & 6.00 & 13.00 & 23.00 & 41.00 & 52.29 & 6270 & 1.00 & 1.00 & 1.00 & 1.00 & 3.00 & 9.00 & 17.00 & 33.00 & 39.90 \\
\hline 5094 & 1.00 & 1.00 & 1.00 & 1.00 & 3.00 & 9.00 & 17.00 & 32.00 & 38.00 & 6275 & -- & -- & -- & -- & .00 & -- & -- & -- & -- \\
\hline 5113 & 1.00 & 1.00 & 1.00 & 1.00 & 3.00 & 9.00 & 19.00 & 39.00 & 52.40 & 6276 & -- & -- & 1.00 & 4.00 & 6.00 & 17.75 & 29.00 & -- & -- \\
\hline 5114 & -- & -- & -- & -- & .00 & -- & -- & -- & -- & 6335 & 1.00 & 1.00 & 1.00 & 2.00 & 4.00 & 11.00 & 20.00 & 35.00 & 43.00 \\
\hline 5123 & -- & -- & 1.00 & 2.00 & 3.00 & 10.00 & 20.20 & -- & -- & 6434 & -- & -- & 1.00 & 3.00 & 6.00 & 16.00 & 28.00 & -- & -- \\
\hline 5192 & 1.00 & 1.00 & 1.00 & 1.00 & 4.00 & 10.00 & 19.00 & 35.00 & 42.75 & 6504 & 1.00 & 1.00 & 1.00 & 1.00 & 2.00 & 7.00 & 15.00 & 31.00 & 37.00 \\
\hline 5193 & 1.00 & 1.00 & 1.00 & 1.00 & 4.00 & 10.00 & 19.40 & 38.00 & 48.00 & 6558 & -- & -- & 1.00 & 1.75 & 5.50 & 12.25 & 21.40 & -- & -- \\
\hline 5224 & 1.00 & 1.00 & 1.00 & 1.00 & 4.00 & 10.75 & 20.00 & 43.10 & 56.51 & 6615 & 1.00 & 1.00 & 1.00 & 1.00 & 2.00 & 6.00 & 13.00 & 33.02 & 41.51 \\
\hline 5228 & 1.00 & 1.00 & 1.00 & 1.00 & 3.00 & 8.00 & 17.00 & 35.00 & 45.66 & 6660 & 1.00 & 1.00 & 1.00 & 1.00 & 5.00 & 11.00 & 24.00 & 48.48 & 55.96 \\
\hline 5235 & -- & -- & 1.00 & 2.00 & 6.50 & 14.00 & 21.90 & -- & -- & 6663 & 1.00 & 1.00 & 1.00 & 1.00 & 1.00 & 6.00 & 14.60 & 43.36 & 44.96 \\
\hline 5247 & 1.00 & 1.00 & 1.00 & 1.00 & 3.00 & 7.00 & 15.30 & 32.00 & 41.43 & 6734 & 1.00 & 1.00 & 1.00 & 2.00 & 4.00 & 10.00 & 20.00 & 35.00 & 48.75 \\
\hline 5258 & 1.00 & 1.00 & 1.00 & 2.00 & 5.00 & 11.00 & 21.00 & 37.64 & 45.00 & 6736 & 1.00 & 1.00 & 1.00 & 1.00 & 2.00 & 6.00 & 14.00 & 32.70 & 43.35 \\
\hline 5303 & 1.00 & 1.00 & 1.00 & 2.00 & 4.00 & 10.00 & 19.00 & 38.96 & 52.48 & 6740 & -- & -- & 1.60 & 3.00 & 7.00 & 13.00 & 56.40 & -- & -- \\
\hline 5312 & 1.00 & 1.00 & 1.00 & 1.00 & 2.00 & 7.00 & 16.00 & 31.46 & 39.00 & 6750 & 1.00 & 1.00 & 1.00 & 1.00 & 4.00 & 12.00 & 25.00 & 44.68 & 66.08 \\
\hline 5341 & -- & -- & 1.00 & 2.00 & 4.00 & 15.00 & 26.20 & -- & -- & 6757 & 1.00 & 1.00 & 1.00 & 1.25 & 4.00 & 10.00 & 20.00 & 36.00 & 47.00 \\
\hline 5342 & -- & -- & -- & -- & .00 & -- & -- & -- & -- & 6775 & 1.00 & 1.00 & 1.00 & 2.00 & 4.00 & 9.00 & 20.00 & 37.90 & 47.45 \\
\hline 5348 & 1.00 & 1.00 & 1.00 & 1.00 & 3.00 & 10.00 & 18.00 & 32.00 & 39.12 & 6776 & 1.00 & 1.00 & 1.00 & 1.00 & 3.00 & 7.00 & 15.00 & 29.00 & 36.00 \\
\hline 5358 & 1.00 & 1.00 & 1.00 & 2.00 & 4.00 & 8.00 & 16.00 & 33.00 & 44.43 & 6788 & 1.00 & 1.00 & 1.00 & 2.00 & 5.00 & 10.50 & 18.00 & 31.52 & 35.30 \\
\hline 5398 & 1.00 & 1.00 & 1.00 & 2.00 & 4.00 & 11.00 & 21.40 & 40.00 & 46.34 & 6792 & 1.00 & 1.00 & 1.00 & 1.00 & 2.00 & 5.00 & 10.00 & 24.00 & 26.49 \\
\hline 5410 & 1.00 & 1.00 & 1.00 & 1.00 & 2.00 & 6.00 & 14.00 & 31.00 & 42.00 & 6794 & -- & -- & 2.20 & 5.00 & 9.00 & 19.50 & 33.40 & -- & -- \\
\hline 5411 & 1.00 & 1.00 & 1.00 & 1.00 & 3.00 & 9.00 & 18.00 & 36.00 & 49.00 & 6834 & 1.00 & 1.00 & 1.00 & 1.00 & 3.00 & 9.00 & 16.00 & 31.00 & 36.77 \\
\hline 5424 & 1.00 & 1.00 & 1.00 & 1.00 & 6.50 & 13.00 & 25.00 & 69.00 & 73.73 & 6893 & 1.00 & 1.00 & 1.00 & 1.00 & 2.00 & 5.00 & 12.00 & 26.00 & 35.31 \\
\hline 5429 & 1.00 & 1.00 & 1.00 & 1.00 & 3.00 & 9.00 & 19.00 & 38.00 & 48.68 & 6935 & 1.00 & 1.00 & 1.00 & 1.00 & 2.00 & 6.00 & 13.00 & 26.00 & 33.96 \\
\hline 5431 & -- & -- & 1.70 & 4.50 & 10.50 & 22.75 & 29.50 & -- & -- & 6981 & 1.00 & 1.00 & 1.00 & 2.00 & 3.00 & 9.00 & 18.70 & 41.00 & 46.21 \\
\hline 5461 & 1.00 & 1.00 & 1.00 & 2.00 & 5.00 & 12.00 & 22.00 & 46.24 & 55.00 & 7020 & 1.00 & 1.00 & 1.00 & 3.00 & 6.00 & 16.00 & 28.00 & 43.44 & 72.08 \\
\hline 5463 & 1.00 & 1.00 & 1.00 & 1.00 & 3.00 & 8.00 & 16.00 & 29.16 & 33.00 & 7060 & 1.00 & 1.00 & 1.00 & 1.00 & 2.00 & 7.00 & 15.00 & 28.82 & 34.41 \\
\hline 5471 & -- & -- & 1.00 & 1.00 & 1.00 & 4.50 & 12.00 & -- & -- & 7066 & 1.00 & 1.00 & 1.00 & 2.00 & 4.00 & 11.00 & 20.00 & 37.94 & 44.47 \\
\hline 5477 & -- & -- & 1.00 & 1.00 & 3.00 & 8.25 & 19.00 & -- & -- & 7074 & 1.00 & 1.00 & 1.00 & 1.00 & 2.00 & 6.00 & 14.00 & 30.00 & 35.22 \\
\hline 5528 & 1.00 & 1.00 & 1.00 & 2.00 & 4.00 & 10.00 & 18.10 & 33.00 & 42.13 & 7097 & 1.00 & 1.00 & 1.00 & 2.00 & 4.00 & 8.00 & 18.80 & 49.44 & 75.12 \\
\hline 5579 & -- & -- & -- & -- & 15.00 & -- & -- & -- & -- & 7116 & 1.00 & 1.00 & 1.00 & 2.00 & 4.00 & 8.00 & 17.00 & 33.82 & 40.91 \\
\hline 5580 & -- & -- & 1.00 & 2.00 & 4.00 & 11.00 & 15.80 & -- & -- & 7140 & 1.00 & 1.00 & 1.00 & 1.00 & 3.00 & 9.00 & 18.00 & 38.14 & 46.57 \\
\hline 5589 & 1.00 & 1.00 & 1.00 & 1.00 & 2.00 & 6.00 & 13.00 & 20.54 & 24.62 & 7173 & 1.00 & 1.00 & 1.00 & 1.00 & 3.00 & 10.00 & 21.00 & 46.00 & 57.98 \\
\hline 5590 & 1.00 & 1.00 & 1.00 & 2.00 & 3.00 & 8.00 & 18.90 & 39.58 & 48.51 & 7174 & 1.00 & 1.00 & 1.00 & 1.00 & 4.00 & 11.00 & 21.00 & 43.00 & 54.81 \\
\hline 5591 & 1.00 & 1.00 & 1.00 & 2.00 & 3.00 & 6.00 & 12.00 & 28.00 & 37.43 & 7213 & 1.00 & 1.00 & 1.00 & 2.00 & 3.00 & 10.00 & 20.00 & 46.00 & 51.23 \\
\hline 5592 & 1.00 & 1.00 & 1.00 & 2.00 & 3.00 & 6.00 & 13.00 & 34.24 & 42.54 & 7243 & 1.00 & 1.00 & 1.00 & 1.00 & 3.00 & 8.00 & 17.00 & 35.00 & 44.00 \\
\hline 5594 & 1.00 & 1.00 & 1.00 & 1.00 & 2.00 & 4.00 & 8.80 & 34.24 & 39.96 & 7262 & 1.00 & 1.00 & 1.00 & 1.00 & 1.00 & 1.00 & 13.00 & 24.64 & 30.82 \\
\hline 5595 & -- & -- & -- & -- & 1.50 & -- & -- & -- & -- & 7274 & 1.00 & 1.00 & 1.00 & 1.00 & 4.00 & 9.00 & 15.80 & 29.00 & 37.68 \\
\hline 5596 & 1.00 & 1.00 & 1.00 & 1.00 & 1.00 & 5.00 & 11.00 & 25.00 & 29.17 & 7300 & 1.00 & 1.00 & 1.00 & 2.00 & 4.00 & 9.00 & 17.00 & 30.00 & 37.00 \\
\hline 5600 & 1.00 & 1.00 & 1.00 & 2.00 & 3.00 & 6.00 & 14.00 & 39.00 & 43.75 & 7311 & -- & -- & 1.00 & 1.00 & 4.00 & 8.25 & 10.00 & -- & -- \\
\hline 5618 & -- & -- & 2.00 & 3.00 & 7.00 & 11.50 & 15.60 & -- & -- & 7363 & -- & -- & 1.00 & 2.00 & 4.00 & 10.00 & 18.80 & -- & -- \\
\hline 5650 & -- & -- & 1.00 & 3.00 & 6.00 & 11.00 & 16.00 & -- & -- & 7422 & 1.00 & 1.00 & 1.00 & 1.00 & 3.00 & 9.00 & 18.00 & 35.00 & 43.00 \\
\hline 5656 & 1.00 & 1.00 & 1.00 & 1.00 & 2.00 & 6.00 & 14.00 & 29.00 & 35.00 & 7431 & 1.00 & 1.00 & 1.00 & 1.00 & 3.00 & 7.00 & 15.00 & 32.00 & 42.00 \\
\hline 5658 & 1.00 & 1.00 & 1.00 & 2.00 & 4.00 & 9.00 & 19.00 & 40.00 & 51.00 & 7481 & 1.00 & 1.00 & 1.00 & 1.00 & 2.00 & 6.00 & 14.00 & 34.02 & 44.00 \\
\hline 5661 & 1.00 & 1.00 & 1.00 & 1.00 & 2.00 & 7.00 & 13.00 & 42.12 & 53.12 & 7497 & 1.00 & 1.00 & 1.00 & 1.00 & 3.00 & 8.00 & 17.00 & 33.00 & 41.65 \\
\hline 5666 & -- & -- & 1.00 & 2.00 & 4.00 & 9.00 & 20.20 & -- & -- & 7498 & 1.00 & 1.00 & 1.00 & 1.00 & 2.00 & 9.00 & 15.00 & 30.48 & 42.20 \\
\hline 5695 & 1.00 & 1.00 & 1.00 & 1.00 & 4.00 & 10.00 & 18.00 & 37.00 & 44.00 & 7499 & 1.00 & 1.00 & 1.00 & 1.00 & 3.00 & 8.00 & 16.00 & 30.00 & 38.50 \\
\hline 5742 & -- & -- & 1.00 & 1.00 & 5.00 & 11.00 & 20.00 & -- & -- & 7531 & 1.00 & 1.00 & 1.00 & 2.00 & 5.00 & 12.00 & 24.00 & 48.40 & 57.20 \\
\hline 5766 & -- & -- & 1.00 & 2.00 & 5.00 & 13.00 & 21.70 & -- & -- & 7534 & 1.00 & 1.00 & 1.00 & 1.00 & 3.00 & 10.00 & 17.00 & 34.00 & 43.58 \\
\hline 5770 & 1.00 & 1.00 & 1.00 & 1.00 & 3.00 & 8.00 & 16.00 & 33.00 & 42.00 & 7556 & 1.00 & 1.00 & 1.00 & 1.00 & 3.00 & 8.00 & 17.00 & 32.00 & 39.70 \\
\hline 5775 & -- & -- & 1.00 & 1.00 & 4.00 & 11.00 & 19.40 & -- & -- & 7594 & 1.00 & 1.00 & 1.00 & 2.00 & 4.00 & 9.00 & 19.00 & 40.00 & 48.00 \\
\hline 5779 & -- & -- & 1.00 & 1.00 & 7.00 & 16.00 & 29.00 & -- & -- & 7596 & 1.00 & 1.00 & 1.00 & 2.00 & 3.00 & 8.00 & 18.00 & 35.64 & 69.04 \\
\hline 5840 & 1.00 & 1.00 & 1.00 & 2.00 & 5.00 & 11.00 & 22.00 & 49.88 & 55.44 & 7608 & 1.00 & 1.00 & 1.00 & 1.00 & 3.00 & 9.00 & 18.00 & 33.00 & 46.00 \\
\hline 5890 & 1.00 & 1.00 & 1.00 & 1.00 & 3.00 & 9.00 & 17.00 & 36.00 & 47.00 & 7622 & -- & -- & 1.00 & 1.00 & 2.00 & 5.00 & 15.20 & -- & -- \\
\hline 5891 & 1.00 & 1.00 & 1.00 & 1.00 & 3.00 & 8.00 & 15.00 & 28.80 & 40.80 & 7700 & 1.00 & 1.00 & 1.00 & 2.00 & 5.00 & 12.00 & 22.00 & 40.00 & 50.88 \\
\hline 5897 & 1.00 & 1.00 & 1.00 & 1.00 & 3.00 & 8.00 & 16.00 & 29.88 & 35.00 & 7706 & 1.00 & 1.00 & 1.00 & 1.00 & 3.00 & 8.00 & 16.00 & 32.26 & 43.26 \\
\hline
\end{tabular}


Appendix 4-5.3. Empirical distribution of storm duration defined by 12-hour minimum interevent time for hourly rainfall stations in Texas-Continued.

\begin{tabular}{|c|c|c|c|c|c|c|c|c|c|c|c|c|c|c|c|c|c|c|c|}
\hline \multicolumn{20}{|c|}{$\begin{array}{c}\text { Duration } \\
\text { (hours) }\end{array}$} \\
\hline $\begin{array}{l}\text { Sta- } \\
\text { tion } \\
\text { no. }\end{array}$ & $\begin{array}{c}\text { 1st } \\
\text { per- } \\
\text { centile }\end{array}$ & $\begin{array}{c}\text { 2nd } \\
\text { per- } \\
\text { centile }\end{array}$ & $\begin{array}{c}10 \text { th } \\
\text { per- } \\
\text { centile }\end{array}$ & $\begin{array}{c}25 \text { th } \\
\text { per- } \\
\text { centile }\end{array}$ & $\begin{array}{c}\text { 50th } \\
\text { per- } \\
\text { centile } \\
\text { (median) }\end{array}$ & $\begin{array}{c}\text { 75th } \\
\text { per- } \\
\text { centile }\end{array}$ & $\begin{array}{c}\text { 90th } \\
\text { per- } \\
\text { centile }\end{array}$ & $\begin{array}{c}\text { 98th } \\
\text { per- } \\
\text { centile }\end{array}$ & $\begin{array}{c}\text { 99th } \\
\text { per- } \\
\text { centile }\end{array}$ & $\begin{array}{c}\text { Sta- } \\
\text { tion } \\
\text { no. }\end{array}$ & $\begin{array}{c}\text { 1st } \\
\text { per- } \\
\text { centile }\end{array}$ & $\begin{array}{c}\text { 2nd } \\
\text { per- } \\
\text { centile }\end{array}$ & $\begin{array}{c}10 \text { th } \\
\text { per- } \\
\text { centile }\end{array}$ & $\begin{array}{c}\text { 25th } \\
\text { per- } \\
\text { centile }\end{array}$ & $\begin{array}{c}\text { 50th } \\
\text { per- } \\
\text { centile } \\
\text { (median) }\end{array}$ & $\begin{array}{c}\text { 75th } \\
\text { per- } \\
\text { centile }\end{array}$ & $\begin{array}{c}\text { 90th } \\
\text { per- } \\
\text { centile }\end{array}$ & $\begin{array}{c}\text { 98th } \\
\text { per- } \\
\text { centile }\end{array}$ & $\begin{array}{l}\text { 99th } \\
\text { per- } \\
\text { centile }\end{array}$ \\
\hline 7718 & 1.00 & 1.00 & 1.00 & 2.00 & 4.00 & 10.00 & 23.10 & 39.00 & 39.97 & 8910 & -- & -- & 2.00 & 2.25 & 4.50 & 8.00 & 15.40 & -- & -- \\
\hline 7745 & 1.00 & 1.00 & 1.00 & 2.00 & 6.00 & 14.00 & 27.40 & 50.00 & 57.12 & 8911 & 1.00 & 1.00 & 1.00 & 1.00 & 3.00 & 7.00 & 16.00 & 35.00 & 42.95 \\
\hline 7922 & 1.00 & 1.00 & 1.00 & 1.00 & 2.00 & 7.00 & 13.00 & 31.00 & 36.80 & 8924 & 1.00 & 1.00 & 1.00 & 1.00 & 2.00 & 5.00 & 11.00 & 27.20 & 38.20 \\
\hline 7936 & 1.00 & 1.00 & 1.00 & 1.00 & 3.00 & 9.00 & 17.00 & 35.00 & 45.00 & 8929 & -- & -- & 1.00 & 1.75 & 5.00 & 14.00 & 37.20 & -- & -- \\
\hline 7943 & 1.00 & 1.00 & 1.00 & 2.00 & 3.00 & 9.00 & 18.00 & 38.86 & 46.93 & 8942 & 1.00 & 1.00 & 1.00 & 1.00 & 3.00 & 9.00 & 18.00 & 36.00 & 43.68 \\
\hline 7944 & -- & 1.00 & 1.00 & 1.00 & 3.00 & 11.00 & 24.30 & 53.08 & -- & 8944 & 1.00 & 1.00 & 1.00 & 1.00 & 4.00 & 9.00 & 18.00 & 40.00 & 46.94 \\
\hline 7945 & 1.00 & 1.00 & 1.00 & 1.00 & 4.00 & 11.00 & 23.00 & 44.00 & 50.17 & 8996 & 1.00 & 1.00 & 1.00 & 2.00 & 4.00 & 9.00 & 18.00 & 37.50 & 46.75 \\
\hline 7947 & 1.00 & 1.00 & 1.00 & 1.00 & 3.00 & 8.00 & 17.00 & 49.00 & 63.50 & 9014 & -- & -- & 2.00 & 3.00 & 7.00 & 11.00 & 22.20 & -- & -- \\
\hline 7948 & 1.00 & 1.00 & 1.00 & 1.00 & 3.00 & 9.00 & 18.00 & 36.78 & 42.00 & 9037 & 1.00 & 1.00 & 1.00 & 1.00 & 3.00 & 7.00 & 14.00 & 40.00 & 49.12 \\
\hline 7951 & 1.00 & 1.00 & 1.00 & 2.00 & 4.00 & 10.00 & 19.10 & 36.00 & 42.00 & 9106 & 1.00 & 1.00 & 1.00 & 1.00 & 3.00 & 8.00 & 15.80 & 38.00 & 40.94 \\
\hline 7953 & 1.00 & 1.00 & 1.00 & 2.00 & 4.00 & 9.00 & 24.60 & 56.56 & 69.56 & 9107 & -- & -- & 1.00 & 1.00 & 1.00 & 7.00 & 14.40 & -- & -- \\
\hline 7981 & 1.00 & 1.00 & 1.00 & 1.00 & 4.00 & 10.00 & 20.90 & 32.38 & 40.45 & 9129 & 1.00 & 1.00 & 1.00 & 1.00 & 3.00 & 10.00 & 21.00 & 33.20 & 43.00 \\
\hline 7990 & 1.00 & 1.00 & 1.00 & 1.00 & 3.00 & 11.00 & 20.90 & 39.90 & 54.73 & 9163 & 1.00 & 1.00 & 1.00 & 1.00 & 3.00 & 9.00 & 17.00 & 33.96 & 42.98 \\
\hline 7992 & -- & -- & 1.40 & 4.00 & 7.00 & 13.00 & 19.20 & -- & -- & & & & & & & & & & \\
\hline 7997 & 1.00 & 1.00 & 1.00 & 2.00 & 3.50 & 7.00 & 13.80 & 25.90 & 43.01 & 9213 & 1.00 & 1.00 & 1.00 & 1.00 & 7.00 & 13.00 & 25.00 & 55.00 & 70.00 \\
\hline 7999 & -- & -- & 1.00 & 1.00 & 4.00 & 8.00 & 17.00 & -- & -- & 9214 & -- & -- & 1.10 & 2.75 & 4.00 & 7.50 & 16.90 & -- & -- \\
\hline 8022 & -- & 1.00 & 1.00 & 1.00 & 2.50 & 7.00 & 21.00 & 64.24 & -- & 9222 & 1.00 & 1.00 & 1.00 & 2.00 & 5.00 & 14.00 & 26.90 & 45.34 & 52.89 \\
\hline 8023 & 1.00 & 1.00 & 1.00 & 1.00 & 2.00 & 7.00 & 15.00 & 33.14 & 42.00 & 9248 & 1.00 & 1.00 & 1.00 & 1.00 & 3.00 & 10.75 & 20.00 & 47.04 & 56.13 \\
\hline 8047 & 1.00 & 1.00 & 1.00 & 1.00 & 3.00 & 8.00 & 17.00 & 33.00 & 42.83 & 9266 & -- & 1.00 & 1.00 & 2.00 & 4.00 & 12.00 & 19.50 & 54.50 & -- \\
\hline 8060 & 1.00 & 1.00 & 1.00 & 2.00 & 3.00 & 11.00 & 23.00 & 42.00 & 42.71 & 9270 & 1.00 & 1.00 & 1.00 & 1.00 & 2.00 & 5.00 & 12.00 & 26.00 & 31.00 \\
\hline 8062 & -- & -- & 1.00 & 2.00 & 3.00 & 11.00 & 30.60 & -- & -- & 9295 & 1.00 & 1.00 & 1.00 & 1.00 & 1.00 & 7.00 & 13.00 & 35.56 & 65.20 \\
\hline 8068 & -- & -- & 1.00 & 1.00 & 3.00 & 7.75 & 17.50 & -- & -- & 9304 & -- & -- & -- & 3.50 & 5.50 & 10.50 & -- & -- & -- \\
\hline 8081 & 1.00 & 1.00 & 1.00 & 1.00 & 3.00 & 8.00 & 18.50 & 40.00 & 53.30 & 9307 & 1.00 & 1.00 & 1.00 & 2.00 & 4.00 & 10.00 & 19.00 & 37.60 & 44.60 \\
\hline 8089 & -- & -- & 1.00 & 2.00 & 4.00 & 12.00 & 16.80 & -- & -- & 9328 & 1.00 & 1.00 & 1.00 & 1.00 & 3.00 & 9.00 & 17.10 & 49.20 & 63.05 \\
\hline 8221 & -- & -- & 1.00 & 4.00 & 7.00 & 21.00 & 34.00 & -- & -- & 9329 & -- & -- & 1.00 & 3.00 & 6.00 & 13.00 & 28.40 & -- & -- \\
\hline 8252 & 1.00 & 1.00 & 1.00 & 1.00 & 3.00 & 6.00 & 16.00 & 34.00 & 37.00 & 9345 & -- & -- & -- & -- & 10.00 & -- & -- & -- & -- \\
\hline 8265 & 1.00 & 1.00 & 1.00 & 2.00 & 4.00 & 12.00 & 23.00 & 41.24 & 53.00 & 9363 & 1.00 & 1.00 & 1.00 & 1.00 & 3.00 & 10.00 & 20.00 & 41.50 & 53.00 \\
\hline 8289 & -- & 1.00 & 1.00 & 1.00 & 3.00 & 8.50 & 24.20 & 51.12 & -- & 9364 & 1.00 & 1.00 & 1.00 & 1.00 & 3.00 & 9.00 & 20.00 & 44.00 & 53.90 \\
\hline 8305 & 1.00 & 1.00 & 1.00 & 1.00 & 2.00 & 5.00 & 12.00 & 25.00 & 31.02 & 9365 & -- & -- & 1.00 & 1.75 & 4.00 & 7.25 & 17.50 & -- & -- \\
\hline 8335 & 1.00 & 1.00 & 1.00 & 2.00 & 5.00 & 13.00 & 21.00 & 44.00 & 54.35 & 9371 & 1.00 & 1.00 & 1.00 & 2.00 & 4.00 & 11.00 & 20.20 & 44.04 & 50.72 \\
\hline 8400 & 1.00 & 1.00 & 1.00 & 1.00 & 2.00 & 6.00 & 16.00 & 34.00 & 72.28 & 9417 & 1.00 & 1.00 & 1.00 & 2.00 & 4.00 & 10.00 & 19.00 & 37.00 & 45.00 \\
\hline 8445 & 1.00 & 1.00 & 1.00 & 2.00 & 4.00 & 10.00 & 20.00 & 41.00 & 51.55 & 9419 & 1.00 & 1.00 & 1.00 & 2.00 & 4.00 & 11.00 & 21.00 & 39.00 & 47.79 \\
\hline 8446 & 1.00 & 1.00 & 1.00 & 1.00 & 3.00 & 8.00 & 16.30 & 34.00 & 41.46 & 9435 & -- & 1.00 & 1.00 & 1.00 & 3.00 & 6.00 & 18.20 & 49.76 & -- \\
\hline 8451 & 1.00 & 1.00 & 1.00 & 2.00 & 4.00 & 9.00 & 18.00 & 41.60 & 48.60 & 9491 & 1.00 & 1.00 & 1.00 & 1.00 & 4.00 & 9.00 & 19.00 & 37.00 & 43.83 \\
\hline 8531 & 1.00 & 1.00 & 1.00 & 2.00 & 4.00 & 10.00 & 20.00 & 38.00 & 47.00 & 9499 & 1.00 & 1.00 & 1.00 & 1.00 & 2.00 & 7.00 & 15.00 & 32.30 & 38.30 \\
\hline 8541 & 1.00 & 1.00 & 1.00 & 2.00 & 4.00 & 10.00 & 21.00 & 35.76 & 56.37 & 9522 & -- & -- & 2.20 & 4.00 & 8.00 & 16.00 & 26.00 & -- & -- \\
\hline 8544 & 1.00 & 1.00 & 1.00 & 2.00 & 4.00 & 10.00 & 20.00 & 36.00 & 43.50 & 9527 & 1.00 & 1.00 & 1.00 & 1.00 & 2.00 & 7.00 & 14.00 & 29.00 & 38.00 \\
\hline 8545 & -- & -- & 1.00 & 1.25 & 4.50 & 16.50 & 27.30 & -- & -- & 9532 & 1.00 & 1.00 & 1.00 & 1.00 & 3.00 & 8.00 & 17.00 & 33.00 & 40.11 \\
\hline 8563 & 1.00 & 1.00 & 1.00 & 1.00 & 3.00 & 9.00 & 15.00 & 31.00 & 34.54 & 9544 & -- & -- & -- & 1.00 & 1.00 & 6.00 & -- & -- & -- \\
\hline 8566 & 1.00 & 1.00 & 1.00 & 1.00 & 2.00 & 6.00 & 14.00 & 26.80 & 35.80 & 9565 & 1.00 & 1.00 & 1.00 & 1.00 & 3.00 & 7.00 & 16.00 & 31.52 & 39.00 \\
\hline 8583 & 1.00 & 1.00 & 1.00 & 1.00 & 2.00 & 8.00 & 16.00 & 29.00 & 33.00 & 9570 & 1.00 & 1.00 & 1.00 & 1.00 & 2.00 & 6.00 & 13.00 & 25.74 & 29.87 \\
\hline 8584 & 1.00 & 1.00 & 1.00 & 1.00 & 3.00 & 8.00 & 18.00 & 36.00 & 42.64 & 9574 & -- & -- & 1.00 & 1.00 & 2.50 & 11.00 & 15.40 & -- & -- \\
\hline 8623 & 1.00 & 1.00 & 1.00 & 1.00 & 3.00 & 9.00 & 17.40 & 30.88 & 39.88 & 9588 & 1.00 & 1.00 & 1.00 & 1.00 & 3.00 & 9.00 & 18.00 & 36.00 & 50.69 \\
\hline 8625 & 1.00 & 1.00 & 1.00 & 2.00 & 5.00 & 11.00 & 20.00 & 40.00 & 46.02 & 9665 & 1.00 & 1.00 & 1.00 & 1.00 & 4.00 & 9.00 & 19.00 & 37.00 & 44.00 \\
\hline 8630 & 1.00 & 1.00 & 1.00 & 1.00 & 3.00 & 8.00 & 15.00 & 30.00 & 34.34 & 9715 & 1.00 & 1.00 & 1.00 & 1.00 & 4.00 & 9.00 & 18.00 & 34.00 & 39.00 \\
\hline 8631 & 1.00 & 1.00 & 1.00 & 2.00 & 4.00 & 9.00 & 17.90 & 44.56 & 55.39 & 9729 & 1.00 & 1.00 & 1.00 & 2.00 & 4.00 & 11.00 & 20.00 & 39.00 & 47.00 \\
\hline 8646 & 1.00 & 1.00 & 1.00 & 2.00 & 4.00 & 10.00 & 18.00 & 35.00 & 43.00 & 9772 & 1.00 & 1.00 & 1.00 & 2.00 & 3.00 & 9.00 & 18.30 & 37.66 & 47.83 \\
\hline 8647 & 1.00 & 1.00 & 1.00 & 1.00 & 2.00 & 7.00 & 15.00 & 29.28 & 35.00 & 9814 & -- & -- & 1.60 & 2.00 & 4.00 & 14.00 & 24.40 & -- & -- \\
\hline 8677 & 1.00 & 1.00 & 1.00 & 3.00 & 5.00 & 11.00 & 21.00 & 41.64 & 42.64 & 9815 & 1.00 & 1.00 & 1.00 & 2.00 & 4.00 & 10.00 & 20.00 & 35.42 & 45.71 \\
\hline 8696 & -- & -- & 1.00 & 2.00 & 5.50 & 10.50 & 16.30 & -- & -- & 9816 & -- & 1.00 & 1.00 & 1.00 & 3.00 & 11.00 & 16.00 & 41.50 & -- \\
\hline 8743 & 1.00 & 1.00 & 1.00 & 2.00 & 4.00 & 10.00 & 21.00 & 39.00 & 48.33 & 9817 & 1.00 & 1.00 & 1.00 & 1.00 & 3.00 & 8.00 & 15.00 & 31.00 & 36.00 \\
\hline 8761 & 1.00 & 1.00 & 1.00 & 1.00 & 2.00 & 6.00 & 13.00 & 28.00 & 33.00 & 9829 & 1.00 & 1.00 & 1.00 & 1.00 & 2.00 & 6.00 & 14.00 & 36.18 & 46.18 \\
\hline 8778 & 1.00 & 1.00 & 1.00 & 1.00 & 4.00 & 10.00 & 18.00 & 33.00 & 44.00 & 9830 & 1.00 & 1.00 & 1.00 & 1.00 & 3.00 & 7.00 & 12.30 & 26.72 & 56.80 \\
\hline 8845 & 1.00 & 1.00 & 1.00 & 1.00 & 3.00 & 10.00 & 20.00 & 38.00 & 49.14 & 9858 & 1.00 & 1.00 & 1.00 & 2.00 & 3.00 & 8.00 & 16.00 & 35.00 & 40.26 \\
\hline 8859 & 1.00 & 1.00 & 1.00 & 2.00 & 5.00 & 11.00 & 22.00 & 41.00 & 50.00 & 9893 & 1.00 & 1.00 & 1.00 & 1.00 & 4.00 & 9.00 & 18.00 & 34.00 & 45.19 \\
\hline 8898 & 1.00 & 1.00 & 1.00 & 2.00 & 5.00 & 11.25 & 22.00 & 39.00 & 46.00 & 9916 & 1.00 & 1.00 & 1.00 & 1.00 & 4.00 & 11.00 & 19.00 & 36.00 & 41.00 \\
\hline 8908 & -- & -- & 1.00 & 2.00 & 4.00 & 9.75 & 20.50 & -- & -- & 9976 & 1.00 & 1.00 & 1.00 & 1.00 & 3.00 & 8.00 & 17.00 & 39.00 & 45.00 \\
\hline
\end{tabular}


Appendix 4-5.4. Empirical distribution of storm duration defined by 18-hour minimum interevent time for hourly rainfall stations in Texas.

[--, not available]

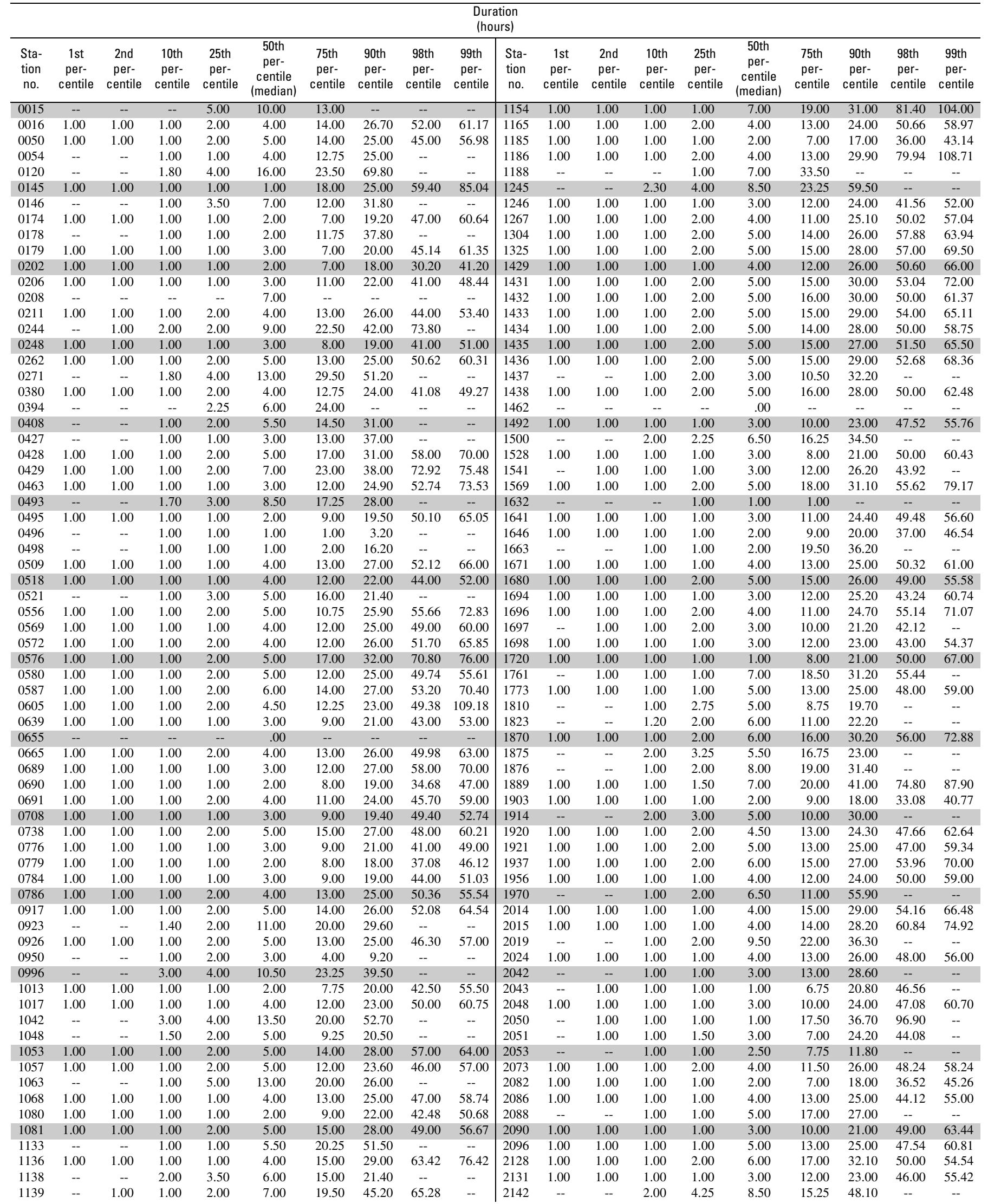


Appendix 4-5.4. Empirical distribution of storm duration defined by 18-hour minimum interevent time for hourly rainfall stations in Texas-Continued.

\begin{tabular}{|c|c|c|c|c|c|c|c|c|c|c|c|c|c|c|c|c|c|c|c|}
\hline \multicolumn{20}{|c|}{$\begin{array}{c}\text { Duration } \\
\text { (hours) }\end{array}$} \\
\hline $\begin{array}{l}\text { Sta- } \\
\text { tion } \\
\text { no. }\end{array}$ & $\begin{array}{c}\text { 1st } \\
\text { per- } \\
\text { centile }\end{array}$ & $\begin{array}{c}\text { 2nd } \\
\text { per- } \\
\text { centile }\end{array}$ & $\begin{array}{c}10 \text { th } \\
\text { per- } \\
\text { centile }\end{array}$ & $\begin{array}{c}25 \text { th } \\
\text { per- } \\
\text { centile }\end{array}$ & $\begin{array}{c}\text { 50th } \\
\text { per- } \\
\text { centile } \\
\text { (median) }\end{array}$ & $\begin{array}{c}\text { 75th } \\
\text { per- } \\
\text { centile }\end{array}$ & $\begin{array}{c}\text { 90th } \\
\text { per- } \\
\text { centile }\end{array}$ & $\begin{array}{c}\text { 98th } \\
\text { per- } \\
\text { centile }\end{array}$ & $\begin{array}{c}99 \text { th } \\
\text { per- } \\
\text { centile }\end{array}$ & $\begin{array}{l}\text { Sta- } \\
\text { tion } \\
\text { no. }\end{array}$ & $\begin{array}{c}1 \text { st } \\
\text { per- } \\
\text { centile }\end{array}$ & $\begin{array}{c}\text { 2nd } \\
\text { per- } \\
\text { centile }\end{array}$ & $\begin{array}{c}10 \text { th } \\
\text { per- } \\
\text { centile }\end{array}$ & $\begin{array}{c}25 \text { th } \\
\text { per- } \\
\text { centile }\end{array}$ & $\begin{array}{c}\text { 50th } \\
\text { per- } \\
\text { centile } \\
\text { (median) }\end{array}$ & $\begin{array}{c}\text { 75th } \\
\text { per- } \\
\text { centile }\end{array}$ & $\begin{array}{c}\text { 90th } \\
\text { per- } \\
\text { centile }\end{array}$ & $\begin{array}{c}\text { 98th } \\
\text { per- } \\
\text { centile }\end{array}$ & $\begin{array}{c}\text { 99th } \\
\text { per- } \\
\text { centile }\end{array}$ \\
\hline 2160 & -- & -- & 1.00 & 1.00 & 7.00 & 25.00 & 46.90 & -- & -- & 3463 & -- & -- & 1.00 & 2.00 & 6.00 & 16.50 & 27.50 & -- & -- \\
\hline 2206 & 1.00 & 1.00 & 1.00 & 2.00 & 4.00 & 12.00 & 24.00 & 46.00 & 64.02 & 3476 & 1.00 & 1.00 & 1.00 & 2.00 & 4.00 & 13.00 & 26.60 & 44.00 & 56.68 \\
\hline 2238 & 1.00 & 1.00 & 1.00 & 1.00 & 4.00 & 13.00 & 26.00 & 67.20 & 79.20 & 3485 & -- & -- & 1.30 & 3.75 & 7.00 & 12.25 & 20.40 & -- & -- \\
\hline 2240 & -- & 1.00 & 1.00 & 1.00 & 1.00 & 19.00 & 25.00 & 51.64 & -- & 3507 & 1.00 & 1.00 & 1.00 & 1.00 & 3.00 & 12.00 & 23.00 & 45.48 & 55.72 \\
\hline 2242 & 1.00 & 1.00 & 1.00 & 2.00 & 5.00 & 15.00 & 28.70 & 49.00 & 57.34 & 3546 & 1.00 & 1.00 & 1.00 & 1.00 & 5.00 & 14.00 & 26.00 & 48.56 & 58.78 \\
\hline 2244 & 1.00 & 1.00 & 1.00 & 1.00 & 5.00 & 15.00 & 27.00 & 51.00 & 62.08 & 3547 & 1.00 & 1.00 & 1.00 & 1.00 & 4.00 & 10.00 & 21.00 & 52.26 & 62.75 \\
\hline 2247 & -- & -- & 1.00 & 3.25 & 6.50 & 14.25 & 33.30 & -- & -- & 3579 & -- & 1.00 & 1.00 & 2.00 & 5.00 & 15.00 & 27.80 & 50.92 & -- \\
\hline 2309 & 1.00 & 1.00 & 1.00 & 2.00 & 6.00 & 15.00 & 30.00 & 50.00 & 58.18 & 3642 & 1.00 & 1.00 & 1.00 & 2.00 & 5.00 & 13.00 & 25.00 & 46.24 & 54.62 \\
\hline 2312 & 1.00 & 1.00 & 1.00 & 1.00 & 4.00 & 13.00 & 22.20 & 44.68 & 61.52 & 3646 & 1.00 & 1.00 & 1.00 & 2.00 & 5.00 & 13.00 & 25.00 & 46.08 & 62.68 \\
\hline 2334 & -- & 1.00 & 1.00 & 1.00 & 5.00 & 19.00 & 27.80 & 34.88 & -- & 3668 & -- & -- & 2.90 & 4.00 & 11.00 & 20.00 & 47.80 & -- & -- \\
\hline 2336 & 1.00 & 1.00 & 1.00 & 1.00 & 3.00 & 11.00 & 20.10 & 38.28 & 52.01 & 3673 & -- & -- & 1.30 & 3.75 & 11.00 & 23.00 & 49.10 & -- & -- \\
\hline 2354 & -- & -- & 1.00 & 1.50 & 3.00 & 12.50 & 22.80 & -- & -- & 3686 & 1.00 & 1.00 & 1.00 & 1.00 & 4.00 & 11.00 & 24.00 & 45.00 & 50.87 \\
\hline 2355 & -- & -- & 1.00 & 2.00 & 4.00 & 15.75 & 43.50 & -- & -- & 3691 & 1.00 & 1.00 & 1.00 & 1.00 & 4.00 & 11.00 & 23.00 & 42.80 & 52.00 \\
\hline 2357 & 1.00 & 1.00 & 1.00 & 1.00 & 3.00 & 13.00 & 28.00 & 53.00 & 63.98 & 3734 & -- & -- & 1.00 & 2.00 & 9.00 & 21.00 & 36.20 & -- & -- \\
\hline 2360 & 1.00 & 1.00 & 1.00 & 1.00 & 4.00 & 13.00 & 27.00 & 53.00 & 67.00 & 3771 & 1.00 & 1.00 & 1.00 & 1.00 & 3.00 & 10.00 & 21.00 & 40.00 & 49.00 \\
\hline 2361 & 1.00 & 1.00 & 1.00 & 1.00 & 3.00 & 8.25 & 23.00 & 39.32 & 53.54 & 3789 & -- & 1.00 & 1.00 & 1.00 & 1.00 & 7.00 & 19.00 & 57.16 & -- \\
\hline 2394 & 1.00 & 1.00 & 1.00 & 2.00 & 5.00 & 13.00 & 26.00 & 47.00 & 57.64 & 3826 & 1.00 & 1.00 & 1.00 & 2.00 & 4.00 & 11.00 & 19.00 & 51.36 & 69.32 \\
\hline 2404 & 1.00 & 1.00 & 1.00 & 2.00 & 5.00 & 13.00 & 25.00 & 44.24 & 54.00 & 3831 & 1.00 & 1.00 & 1.00 & 2.00 & 5.00 & 15.00 & 27.00 & 47.78 & 60.17 \\
\hline 2415 & 1.00 & 1.00 & 1.00 & 2.00 & 5.00 & 14.00 & 27.00 & 51.00 & 60.70 & 3841 & 1.00 & 1.00 & 1.00 & 1.00 & 3.00 & 9.50 & 19.80 & 42.68 & 74.18 \\
\hline 2462 & 1.00 & 1.00 & 1.00 & 2.00 & 4.00 & 13.00 & 27.00 & 49.00 & 54.11 & 3871 & 1.00 & 1.00 & 1.00 & 2.00 & 5.00 & 14.00 & 28.00 & 52.00 & 65.00 \\
\hline 2528 & 1.00 & 1.00 & 1.00 & 1.00 & 5.00 & 11.25 & 34.30 & 55.58 & 78.32 & 3884 & -- & -- & 1.10 & 3.25 & 6.50 & 10.50 & 15.80 & -- & -- \\
\hline 2617 & 1.00 & 1.00 & 1.00 & 2.00 & 5.00 & 13.00 & 27.40 & 53.44 & 56.72 & 3941 & -- & 1.00 & 1.00 & 3.00 & 9.00 & 16.75 & 43.20 & 81.24 & -- \\
\hline 2619 & 1.00 & 1.00 & 1.00 & 1.00 & 4.00 & 12.00 & 25.00 & 57.76 & 77.68 & 3963 & -- & -- & -- & 1.00 & 1.00 & 4.50 & -- & -- & -- \\
\hline 2621 & 1.00 & 1.00 & 1.00 & 1.00 & 3.00 & 12.00 & 23.00 & 42.00 & 49.84 & 4040 & 1.00 & 1.00 & 1.00 & 2.00 & 4.00 & 11.00 & 23.00 & 39.66 & 53.00 \\
\hline 2675 & 1.00 & 1.00 & 1.00 & 1.00 & 4.00 & 11.00 & 23.00 & 50.00 & 58.91 & 4058 & -- & -- & 1.00 & 3.00 & 4.50 & 20.50 & 40.80 & -- & -- \\
\hline 2676 & 1.00 & 1.00 & 1.00 & 1.00 & 2.00 & 9.00 & 21.00 & 43.24 & 55.00 & 4098 & 1.00 & 1.00 & 1.00 & 1.00 & 2.00 & 8.00 & 19.00 & 37.00 & 47.00 \\
\hline 2679 & 1.00 & 1.00 & 1.00 & 1.00 & 3.00 & 11.00 & 24.00 & 47.22 & 57.61 & 4100 & 1.00 & 1.00 & 1.00 & 2.00 & 4.00 & 10.00 & 21.00 & 46.50 & 54.50 \\
\hline 2715 & 1.00 & 1.00 & 1.00 & 2.00 & 5.00 & 13.00 & 24.00 & 44.00 & 52.18 & 4137 & 1.00 & 1.00 & 1.00 & 1.00 & 3.00 & 11.00 & 21.00 & 37.00 & 44.53 \\
\hline 2744 & 1.00 & 1.00 & 1.00 & 1.00 & 3.00 & 10.00 & 23.00 & 47.00 & 58.95 & 4191 & 1.00 & 1.00 & 1.00 & 1.00 & 4.00 & 12.00 & 24.00 & 47.30 & 60.00 \\
\hline 2758 & 1.00 & 1.00 & 1.00 & 2.00 & 4.00 & 13.50 & 30.20 & 93.36 & 96.94 & 4256 & -- & -- & -- & -- & .00 & -- & -- & -- & -- \\
\hline 2794 & -- & -- & 1.00 & 1.00 & 4.00 & 10.00 & 24.80 & -- & -- & 4257 & 1.00 & 1.00 & 1.00 & 1.00 & 4.00 & 14.00 & 25.00 & 44.00 & 54.26 \\
\hline 2797 & 1.00 & 1.00 & 1.00 & 1.00 & 3.00 & 9.00 & 22.00 & 39.00 & 47.29 & 4258 & 1.00 & 1.00 & 1.00 & 1.00 & 3.00 & 15.00 & 25.00 & 49.40 & 60.70 \\
\hline 2811 & 1.00 & 1.00 & 1.00 & 1.00 & 3.00 & 10.00 & 23.00 & 47.00 & 57.00 & 4278 & 1.00 & 1.00 & 1.00 & 2.00 & 4.00 & 11.25 & 24.00 & 51.00 & 63.53 \\
\hline 2813 & -- & -- & 1.00 & 1.00 & 3.50 & 12.00 & 32.30 & -- & -- & 4299 & 1.00 & 1.00 & 1.00 & 1.00 & 2.50 & 5.25 & 17.10 & 36.62 & 48.34 \\
\hline 2814 & -- & -- & 1.00 & 1.00 & 1.00 & 11.75 & 31.30 & -- & -- & 4300 & 1.00 & 1.00 & 1.00 & 2.00 & 5.00 & 16.00 & 31.00 & 57.04 & 72.76 \\
\hline 2815 & 1.00 & 1.00 & 1.00 & 1.00 & 3.00 & 9.00 & 19.00 & 30.76 & 36.38 & 4305 & 1.00 & 1.00 & 1.00 & 2.00 & 5.00 & 16.00 & 29.00 & 61.02 & 74.00 \\
\hline 2818 & 1.00 & 1.00 & 1.00 & 1.00 & 4.00 & 11.00 & 23.70 & 46.00 & 77.46 & 4307 & 1.00 & 1.00 & 1.00 & 1.00 & 5.00 & 19.00 & 37.00 & 70.80 & 85.50 \\
\hline 2986 & 1.00 & 1.00 & 1.00 & 2.00 & 7.00 & 17.00 & 30.00 & 55.50 & 66.95 & 4309 & 1.00 & 1.00 & 1.00 & 2.00 & 4.00 & 12.00 & 24.00 & 51.00 & 62.36 \\
\hline 3005 & 1.00 & 1.00 & 1.00 & 1.00 & 4.00 & 12.00 & 23.00 & 42.00 & 52.00 & 4311 & 1.00 & 1.00 & 1.00 & 2.00 & 4.00 & 12.00 & 26.00 & 51.54 & 63.00 \\
\hline 3033 & 1.00 & 1.00 & 1.00 & 2.00 & 3.00 & 6.00 & 16.00 & 31.00 & 36.56 & 4313 & 1.00 & 1.00 & 1.00 & 2.00 & 5.00 & 18.00 & 34.00 & 48.52 & 55.63 \\
\hline 3034 & -- & -- & -- & -- & 1.00 & -- & -- & -- & -- & 4319 & -- & 1.00 & 1.00 & 2.50 & 6.00 & 16.50 & 27.00 & 47.24 & -- \\
\hline 3047 & -- & -- & 1.40 & 3.00 & 6.00 & 23.00 & 46.80 & -- & -- & 4329 & 1.00 & 1.00 & 1.00 & 2.00 & 4.00 & 13.00 & 26.00 & 53.00 & 62.66 \\
\hline 3103 & -- & -- & 1.00 & 1.00 & 4.00 & 12.50 & 24.80 & -- & -- & 4331 & -- & -- & -- & -- & .00 & -- & -- & -- & -- \\
\hline 3133 & 1.00 & 1.00 & 1.00 & 2.00 & 5.00 & 13.00 & 25.00 & 47.00 & 53.31 & 4375 & 1.00 & 1.00 & 1.00 & 1.00 & 3.00 & 10.00 & 21.00 & 45.52 & 63.38 \\
\hline 3156 & 1.00 & 1.00 & 1.00 & 1.00 & 5.00 & 15.75 & 30.00 & 63.28 & 72.94 & 4392 & 1.00 & 1.00 & 1.00 & 3.00 & 6.00 & 19.00 & 31.00 & 60.64 & 87.02 \\
\hline 3171 & 1.00 & 1.00 & 1.00 & 2.00 & 5.00 & 14.00 & 25.00 & 47.60 & 54.30 & 4425 & 1.00 & 1.00 & 1.00 & 1.00 & 2.00 & 9.00 & 21.00 & 42.00 & 46.00 \\
\hline 3189 & 1.00 & 1.00 & 1.00 & 1.00 & 2.00 & 9.00 & 21.00 & 50.40 & 73.20 & 4440 & 1.00 & 1.00 & 1.00 & 2.00 & 5.00 & 14.00 & 26.00 & 50.00 & 69.00 \\
\hline 3260 & 1.00 & 1.00 & 1.00 & 1.00 & 5.00 & 12.00 & 24.40 & 36.64 & 52.08 & 4476 & 1.00 & 1.00 & 1.00 & 1.00 & 4.00 & 12.00 & 22.00 & 40.00 & 50.54 \\
\hline 3267 & 1.00 & 1.00 & 1.00 & 2.00 & 4.00 & 9.75 & 27.90 & 41.94 & 102.39 & 4498 & -- & -- & 1.00 & 1.00 & 2.00 & 4.00 & 25.20 & -- & -- \\
\hline 3270 & 1.00 & 1.00 & 1.00 & 1.00 & 2.00 & 8.00 & 19.00 & 34.36 & 44.36 & 4517 & 1.00 & 1.00 & 1.00 & 2.00 & 5.00 & 14.00 & 27.00 & 50.44 & 63.00 \\
\hline 3272 & -- & -- & 1.00 & 1.00 & 2.00 & 12.00 & 31.00 & -- & -- & 4520 & 1.00 & 1.00 & 1.00 & 1.00 & 3.00 & 11.00 & 23.00 & 39.00 & 46.28 \\
\hline 3277 & -- & -- & 1.00 & 1.00 & 1.00 & 8.50 & 28.00 & -- & -- & 4525 & -- & -- & 1.00 & 2.00 & 5.00 & 30.50 & 68.40 & -- & -- \\
\hline 3278 & 1.00 & 1.00 & 1.00 & 1.00 & 3.00 & 7.00 & 19.00 & 42.36 & 49.00 & 4563 & -- & -- & 1.00 & 2.00 & 4.50 & 8.50 & 23.40 & -- & -- \\
\hline 3280 & 1.00 & 1.00 & 1.00 & 1.00 & 3.00 & 6.00 & 18.90 & 35.98 & 54.39 & 4570 & 1.00 & 1.00 & 1.00 & 1.00 & 3.00 & 12.00 & 23.00 & 46.00 & 55.29 \\
\hline 3281 & -- & -- & 1.00 & 1.00 & 2.00 & 5.00 & 20.20 & -- & -- & 4577 & 1.00 & 1.00 & 1.00 & 1.00 & 4.00 & 12.00 & 24.00 & 47.00 & 58.38 \\
\hline 3283 & 1.00 & 1.00 & 1.00 & 2.00 & 6.00 & 17.00 & 31.00 & 57.24 & 67.36 & 4591 & 1.00 & 1.00 & 1.00 & 2.00 & 6.00 & 15.00 & 28.40 & 53.08 & 66.08 \\
\hline 3284 & 1.00 & 1.00 & 1.00 & 1.00 & 4.00 & 12.00 & 24.40 & 47.00 & 60.00 & 4670 & 1.00 & 1.00 & 1.00 & 1.00 & 3.00 & 11.00 & 22.00 & 43.88 & 55.32 \\
\hline 3285 & 1.00 & 1.00 & 1.00 & 1.00 & 3.00 & 11.00 & 23.00 & 41.00 & 50.74 & 4671 & 1.00 & 1.00 & 1.00 & 1.00 & 7.00 & 13.00 & 25.00 & 50.80 & 78.40 \\
\hline 3329 & 1.00 & 1.00 & 1.00 & 2.00 & 5.00 & 14.00 & 27.00 & 52.00 & 66.36 & 4679 & 1.00 & 1.00 & 1.00 & 1.00 & 4.00 & 13.00 & 24.00 & 43.00 & 54.00 \\
\hline 3335 & 1.00 & 1.00 & 1.00 & 2.00 & 5.00 & 17.00 & 33.40 & 54.00 & 62.00 & 4696 & -- & -- & 1.00 & 1.25 & 4.00 & 6.75 & 35.60 & -- & -- \\
\hline 3370 & 1.00 & 1.00 & 1.00 & 2.00 & 5.00 & 13.00 & 24.00 & 44.00 & 50.30 & 4703 & 1.00 & 1.00 & 1.00 & 1.00 & 3.00 & 10.50 & 23.00 & 46.80 & 109.10 \\
\hline 3410 & 1.00 & 1.00 & 1.00 & 1.00 & 3.00 & 11.00 & 22.00 & 43.00 & 54.48 & 4704 & 1.00 & 1.00 & 1.00 & 2.00 & 6.00 & 18.00 & 28.00 & 55.00 & 66.98 \\
\hline 3415 & 1.00 & 1.00 & 1.00 & 1.00 & 4.00 & 12.00 & 23.00 & 45.00 & 55.03 & 4731 & 1.00 & 1.00 & 1.00 & 1.00 & 5.00 & 17.50 & 31.00 & 64.00 & 99.40 \\
\hline 3430 & 1.00 & 1.00 & 1.00 & 2.00 & 5.00 & 15.00 & 28.00 & 57.90 & 69.00 & 4792 & 1.00 & 1.00 & 1.00 & 1.00 & 3.00 & 10.00 & 22.00 & 45.00 & 51.00 \\
\hline 3431 & 1.00 & 1.00 & 1.00 & 1.00 & 7.00 & 25.00 & 37.00 & 67.00 & 73.00 & 4819 & 1.00 & 1.00 & 1.00 & 1.00 & 4.00 & 12.00 & 23.60 & 46.44 & 57.32 \\
\hline 3441 & -- & -- & 1.00 & 1.00 & 3.00 & 10.50 & 29.20 & -- & -- & 4852 & -- & -- & 1.00 & 1.00 & 1.00 & 2.75 & 13.20 & -- & -- \\
\hline 3442 & 1.00 & 1.00 & 1.00 & 2.00 & 5.00 & 11.00 & 22.00 & 51.44 & 59.08 & 4866 & 1.00 & 1.00 & 1.00 & 2.00 & 4.00 & 13.00 & 25.00 & 46.00 & 57.00 \\
\hline 3446 & 1.00 & 1.00 & 1.00 & 2.00 & 3.00 & 8.00 & 19.00 & 38.00 & 48.04 & 4876 & 1.00 & 1.00 & 1.00 & 1.00 & 4.00 & 11.25 & 23.00 & 49.54 & 74.64 \\
\hline 3460 & -- & -- & 1.00 & 2.00 & 6.00 & 16.50 & 25.30 & -- & -- & 4878 & 1.00 & 1.00 & 1.00 & 2.00 & 5.00 & 16.00 & 28.00 & 55.00 & 67.00 \\
\hline 3462 & 1.00 & 1.00 & 1.00 & 2.00 & 5.00 & 14.00 & 24.20 & 50.72 & 64.97 & 4880 & 1.00 & 1.00 & 1.00 & 1.00 & 3.00 & 10.00 & 22.00 & 44.16 & 57.00 \\
\hline
\end{tabular}


Appendix 4-5.4. Empirical distribution of storm duration defined by 18-hour minimum interevent time for hourly rainfall stations in Texas-Continued.

\begin{tabular}{|c|c|c|c|c|c|c|c|c|c|c|c|c|c|c|c|c|c|c|c|}
\hline \multicolumn{20}{|c|}{$\begin{array}{l}\text { Duration } \\
\text { (hours) }\end{array}$} \\
\hline $\begin{array}{l}\text { Sta- } \\
\text { tion } \\
\text { no. }\end{array}$ & $\begin{array}{c}1 \text { st } \\
\text { per- } \\
\text { centile }\end{array}$ & $\begin{array}{l}\text { 2nd } \\
\text { per- } \\
\text { centile }\end{array}$ & $\begin{array}{c}\text { 10th } \\
\text { per- } \\
\text { centile }\end{array}$ & $\begin{array}{c}25 \text { th } \\
\text { per- } \\
\text { centile }\end{array}$ & $\begin{array}{c}\text { 50th } \\
\text { per- } \\
\text { centile } \\
\text { (median) }\end{array}$ & $\begin{array}{c}\text { 75th } \\
\text { per- } \\
\text { centile }\end{array}$ & $\begin{array}{c}\text { 90th } \\
\text { per- } \\
\text { centile }\end{array}$ & $\begin{array}{c}\text { 98th } \\
\text { per- } \\
\text { centile }\end{array}$ & $\begin{array}{l}\text { 99th } \\
\text { per- } \\
\text { centile }\end{array}$ & $\begin{array}{l}\text { Sta- } \\
\text { tion } \\
\text { no. }\end{array}$ & $\begin{array}{c}1 \text { st } \\
\text { per- } \\
\text { centile }\end{array}$ & $\begin{array}{c}\text { 2nd } \\
\text { per- } \\
\text { centile }\end{array}$ & $\begin{array}{c}10 \text { th } \\
\text { per- } \\
\text { centile }\end{array}$ & $\begin{array}{c}25 \text { th } \\
\text { per- } \\
\text { centile }\end{array}$ & $\begin{array}{c}\text { 50th } \\
\text { per- } \\
\text { centile } \\
\text { (median) }\end{array}$ & $\begin{array}{c}\text { 75th } \\
\text { per- } \\
\text { centile }\end{array}$ & $\begin{array}{c}\text { 90th } \\
\text { per- } \\
\text { centile }\end{array}$ & $\begin{array}{c}\text { 98th } \\
\text { per- } \\
\text { centile }\end{array}$ & $\begin{array}{c}\text { 99th } \\
\text { per- } \\
\text { centile }\end{array}$ \\
\hline 4920 & 1.00 & 1.00 & 1.00 & 1.00 & 3.00 & 10.00 & 22.40 & 47.00 & 59.00 & 5957 & 1.00 & 1.00 & 1.00 & 1.00 & 3.00 & 10.00 & 21.00 & 40.44 & 52.00 \\
\hline 4934 & -- & -- & -- & 1.00 & 3.00 & 15.00 & -- & -- & -- & 5958 & 1.00 & 1.00 & 1.00 & 2.00 & 4.00 & 15.00 & 28.30 & 62.46 & 67.00 \\
\hline 4972 & 1.00 & 1.00 & 1.00 & 1.00 & 4.00 & 12.00 & 25.00 & 44.00 & 54.00 & 5973 & -- & 1.00 & 1.00 & 2.75 & 7.00 & 17.25 & 35.70 & 71.24 & -- \\
\hline 4973 & 1.00 & 1.00 & 1.00 & 2.50 & 6.00 & 17.00 & 28.00 & 43.96 & 53.86 & 5996 & 1.00 & 1.00 & 1.00 & 1.00 & 4.00 & 13.00 & 25.00 & 47.00 & 57.38 \\
\hline 4974 & 1.00 & 1.00 & 1.00 & 1.00 & 4.00 & 11.00 & 23.00 & 44.96 & 57.00 & 6017 & 1.00 & 1.00 & 1.00 & 1.00 & 3.00 & 10.00 & 25.00 & 46.16 & 56.48 \\
\hline 4975 & 1.00 & 1.00 & 1.00 & 1.00 & 4.00 & 12.00 & 23.00 & 42.00 & 48.00 & 6024 & 1.00 & 1.00 & 1.00 & 2.00 & 5.00 & 17.00 & 28.00 & 60.40 & 69.95 \\
\hline 4978 & 1.00 & 1.00 & 1.00 & 1.00 & 3.00 & 12.00 & 22.60 & 39.64 & 62.78 & 6050 & -- & -- & 1.00 & 5.00 & 8.00 & 13.00 & 19.00 & -- & -- \\
\hline 4979 & -- & -- & 1.00 & 4.75 & 7.00 & 18.25 & 53.50 & -- & -- & 6104 & 1.00 & 1.00 & 1.00 & 1.00 & 2.00 & 7.00 & 20.00 & 42.00 & 54.00 \\
\hline 4982 & 1.00 & 1.00 & 1.00 & 2.00 & 4.00 & 12.00 & 22.00 & 41.00 & 51.20 & 6108 & 1.00 & 1.00 & 1.00 & 1.00 & 4.00 & 13.00 & 25.00 & 49.00 & 55.00 \\
\hline 5018 & 1.00 & 1.00 & 1.00 & 2.00 & 5.00 & 12.00 & 23.00 & 41.66 & 48.33 & 6136 & 1.00 & 1.00 & 1.00 & 1.00 & 2.00 & 8.00 & 19.00 & 40.00 & 47.00 \\
\hline 5048 & 1.00 & 1.00 & 1.00 & 1.00 & 2.00 & 9.00 & 21.00 & 43.00 & 53.00 & 6166 & -- & 1.00 & 1.00 & 2.00 & 4.00 & 10.50 & 23.00 & 43.24 & -- \\
\hline 5049 & -- & 1.00 & 1.00 & 1.00 & 2.00 & 8.00 & 12.80 & 30.52 & -- & 6176 & 1.00 & 1.00 & 1.00 & 2.00 & 5.00 & 14.25 & 26.00 & 55.00 & 77.05 \\
\hline 5056 & -- & -- & -- & 1.00 & 4.00 & 12.50 & -- & -- & -- & 6177 & 1.00 & 1.00 & 1.00 & 1.00 & 4.00 & 13.00 & 25.00 & 48.00 & 59.66 \\
\hline 5057 & 1.00 & 1.00 & 1.00 & 1.00 & 3.00 & 12.00 & 27.00 & 61.00 & 71.56 & 6210 & 1.00 & 1.00 & 1.00 & 1.00 & 4.00 & 12.00 & 23.00 & 43.34 & 51.34 \\
\hline 5060 & 1.00 & 1.00 & 1.00 & 2.00 & 3.00 & 10.00 & 26.00 & 57.58 & 92.87 & 6211 & 1.00 & 1.00 & 1.00 & 1.00 & 3.00 & 12.00 & 25.90 & 50.44 & 61.18 \\
\hline 5081 & 1.00 & 1.00 & 1.00 & 2.00 & 6.00 & 16.00 & 28.50 & 56.00 & 78.00 & 6270 & 1.00 & 1.00 & 1.00 & 1.00 & 4.00 & 13.00 & 24.00 & 45.76 & 55.76 \\
\hline 5094 & 1.00 & 1.00 & 1.00 & 1.00 & 4.00 & 12.00 & 23.00 & 43.00 & 54.48 & 6275 & -- & -- & -- & -- & .00 & -- & -- & -- & -- \\
\hline 5113 & 1.00 & 1.00 & 1.00 & 1.00 & 4.00 & 13.00 & 26.00 & 54.00 & 68.04 & 6276 & -- & -- & 1.00 & 3.50 & 6.00 & 25.00 & 41.60 & -- & -- \\
\hline 5114 & -- & -- & -- & -- & .00 & -- & -- & -- & -- & 6335 & 1.00 & 1.00 & 1.00 & 2.00 & 5.00 & 13.00 & 25.00 & 47.00 & 55.42 \\
\hline 5123 & -- & -- & 1.00 & 2.00 & 3.00 & 10.00 & 20.20 & -- & -- & 6434 & -- & -- & 2.60 & 5.00 & 8.00 & 18.00 & 33.80 & -- & -- \\
\hline 5192 & 1.00 & 1.00 & 1.00 & 2.00 & 4.00 & 12.00 & 24.00 & 43.14 & 52.07 & 6504 & 1.00 & 1.00 & 1.00 & 1.00 & 3.00 & 10.00 & 21.00 & 40.00 & 56.00 \\
\hline 5193 & 1.00 & 1.00 & 1.00 & 2.00 & 4.00 & 14.00 & 26.00 & 49.00 & 58.54 & 6558 & -- & -- & 1.00 & 1.50 & 6.00 & 13.00 & 24.00 & -- & -- \\
\hline 5224 & 1.00 & 1.00 & 1.00 & 1.50 & 5.00 & 15.00 & 27.40 & 51.92 & 56.82 & 6615 & 1.00 & 1.00 & 1.00 & 1.00 & 2.00 & 8.00 & 21.00 & 39.68 & 48.21 \\
\hline 5228 & 1.00 & 1.00 & 1.00 & 1.00 & 3.00 & 10.00 & 23.00 & 49.24 & 53.00 & 6660 & -- & 1.00 & 1.00 & 2.00 & 6.00 & 18.00 & 35.60 & 62.20 & -- \\
\hline 5235 & -- & -- & 1.00 & 2.00 & 11.00 & 21.00 & 29.00 & -- & -- & 6663 & -- & 1.00 & 1.00 & 1.00 & 1.00 & 8.00 & 17.40 & 46.40 & -- \\
\hline 5247 & 1.00 & 1.00 & 1.00 & 1.00 & 3.00 & 10.00 & 22.00 & 45.48 & 56.74 & 6734 & 1.00 & 1.00 & 1.00 & 2.00 & 4.00 & 13.00 & 22.20 & 47.04 & 55.56 \\
\hline 5258 & 1.00 & 1.00 & 1.00 & 2.00 & 5.00 & 15.00 & 28.00 & 48.00 & 58.00 & 6736 & 1.00 & 1.00 & 1.00 & 1.00 & 3.00 & 8.00 & 19.00 & 44.00 & 56.00 \\
\hline 5303 & 1.00 & 1.00 & 1.00 & 2.00 & 4.00 & 13.00 & 26.00 & 52.06 & 59.06 & 6740 & -- & -- & 1.40 & 2.50 & 7.00 & 34.50 & 72.00 & -- & -- \\
\hline 5312 & 1.00 & 1.00 & 1.00 & 1.00 & 3.00 & 9.00 & 22.00 & 45.00 & 55.60 & 6750 & 1.00 & 1.00 & 1.00 & 1.00 & 6.00 & 14.50 & 36.00 & 64.64 & 67.00 \\
\hline 5341 & -- & -- & 1.30 & 2.75 & 5.50 & 27.00 & 37.40 & -- & -- & 6757 & 1.00 & 1.00 & 1.00 & 2.00 & 5.00 & 14.00 & 26.00 & 52.06 & 62.00 \\
\hline 5342 & -- & -- & -- & -- & .00 & -- & -- & -- & -- & 6775 & 1.00 & 1.00 & 1.00 & 2.00 & 5.00 & 15.00 & 25.00 & 48.00 & 57.84 \\
\hline 5348 & 1.00 & 1.00 & 1.00 & 1.00 & 4.00 & 13.00 & 24.00 & 47.32 & 58.66 & 6776 & 1.00 & 1.00 & 1.00 & 1.00 & 3.00 & 9.00 & 21.00 & 40.00 & 49.00 \\
\hline 5358 & 1.00 & 1.00 & 1.00 & 2.00 & 4.00 & 10.00 & 23.00 & 46.00 & 54.63 & 6788 & 1.00 & 1.00 & 1.00 & 3.00 & 6.00 & 13.00 & 23.70 & 33.48 & 44.55 \\
\hline 5398 & 1.00 & 1.00 & 1.00 & 2.00 & 5.00 & 14.00 & 26.00 & 51.00 & 63.40 & 6792 & 1.00 & 1.00 & 1.00 & 1.00 & 2.00 & 6.00 & 17.00 & 36.38 & 45.69 \\
\hline 5410 & 1.00 & 1.00 & 1.00 & 1.00 & 3.00 & 8.50 & 21.00 & 42.00 & 54.94 & 6794 & -- & -- & 1.00 & 6.00 & 19.00 & 31.00 & 39.00 & -- & -- \\
\hline 5411 & 1.00 & 1.00 & 1.00 & 2.00 & 4.00 & 13.00 & 25.00 & 54.42 & 67.00 & 6834 & 1.00 & 1.00 & 1.00 & 1.00 & 4.00 & 12.00 & 23.00 & 43.00 & 50.96 \\
\hline 5424 & 1.00 & 1.00 & 1.00 & 1.00 & 7.00 & 19.00 & 43.00 & 73.68 & 81.34 & 6893 & 1.00 & 1.00 & 1.00 & 1.00 & 2.00 & 7.00 & 16.00 & 34.42 & 52.63 \\
\hline 5429 & 1.00 & 1.00 & 1.00 & 1.00 & 4.00 & 12.00 & 25.50 & 48.30 & 62.30 & 6935 & 1.00 & 1.00 & 1.00 & 1.00 & 2.00 & 7.25 & 18.00 & 36.82 & 47.41 \\
\hline 5431 & -- & -- & 1.50 & 3.75 & 13.50 & 26.50 & 43.50 & -- & -- & 6981 & 1.00 & 1.00 & 1.00 & 2.00 & 4.00 & 11.75 & 29.00 & 47.38 & 57.60 \\
\hline 5461 & 1.00 & 1.00 & 1.00 & 2.00 & 5.00 & 14.00 & 29.00 & 56.08 & 64.54 & 7020 & 1.00 & 1.00 & 1.00 & 3.00 & 7.00 & 19.00 & 31.80 & 62.80 & 80.28 \\
\hline 5463 & 1.00 & 1.00 & 1.00 & 1.00 & 3.00 & 11.00 & 23.00 & 44.40 & 53.00 & 7060 & 1.00 & 1.00 & 1.00 & 1.00 & 3.00 & 10.00 & 21.00 & 35.46 & 47.23 \\
\hline 5471 & -- & -- & 1.00 & 1.00 & 1.00 & 11.75 & 28.50 & -- & -- & 7066 & 1.00 & 1.00 & 1.00 & 2.00 & 5.00 & 14.00 & 27.00 & 48.68 & 60.34 \\
\hline 5477 & -- & -- & 1.00 & 1.00 & 3.00 & 9.00 & 24.20 & -- & -- & 7074 & 1.00 & 1.00 & 1.00 & 1.00 & 3.00 & 8.00 & 19.00 & 42.00 & 48.00 \\
\hline 5528 & 1.00 & 1.00 & 1.00 & 2.00 & 4.00 & 11.00 & 23.00 & 42.94 & 49.00 & 7097 & 1.00 & 1.00 & 1.00 & 2.00 & 4.00 & 14.00 & 25.70 & 53.88 & 77.28 \\
\hline 5579 & -- & -- & -- & -- & 15.00 & -- & -- & -- & -- & 7116 & 1.00 & 1.00 & 1.00 & 2.00 & 4.00 & 10.00 & 22.90 & 41.00 & 51.17 \\
\hline 5580 & -- & -- & 1.50 & 3.00 & 5.00 & 13.75 & 23.00 & -- & -- & 7140 & 1.00 & 1.00 & 1.00 & 1.00 & 4.00 & 12.00 & 25.00 & 49.00 & 62.86 \\
\hline 5589 & 1.00 & 1.00 & 1.00 & 1.00 & 2.00 & 7.00 & 19.00 & 37.72 & 61.90 & 7173 & 1.00 & 1.00 & 1.00 & 2.00 & 4.00 & 16.00 & 32.30 & 60.86 & 68.43 \\
\hline 5590 & 1.00 & 1.00 & 1.00 & 2.00 & 3.00 & 13.00 & 22.20 & 47.20 & 57.36 & 7174 & 1.00 & 1.00 & 1.00 & 2.00 & 5.00 & 16.00 & 30.00 & 57.00 & 72.00 \\
\hline 5591 & 1.00 & 1.00 & 1.00 & 2.00 & 3.00 & 6.00 & 19.00 & 41.00 & 55.79 & 7213 & 1.00 & 1.00 & 1.00 & 2.00 & 4.00 & 14.00 & 25.00 & 53.98 & 60.00 \\
\hline 5592 & 1.00 & 1.00 & 1.00 & 2.00 & 3.00 & 7.00 & 18.00 & 41.16 & 51.58 & 7243 & 1.00 & 1.00 & 1.00 & 1.00 & 3.00 & 12.00 & 24.00 & 47.00 & 60.24 \\
\hline 5594 & 1.00 & 1.00 & 1.00 & 1.00 & 2.00 & 4.00 & 16.00 & 37.48 & 50.18 & 7262 & -- & 1.00 & 1.00 & 1.00 & 1.00 & 7.00 & 19.00 & 52.20 & -- \\
\hline 5595 & -- & -- & -- & -- & 1.50 & -- & -- & -- & -- & 7274 & 1.00 & 1.00 & 1.00 & 1.00 & 4.00 & 10.00 & 21.40 & 36.76 & 49.00 \\
\hline 5596 & 1.00 & 1.00 & 1.00 & 1.00 & 2.00 & 6.00 & 18.00 & 38.74 & 47.37 & 7300 & 1.00 & 1.00 & 1.00 & 2.00 & 4.00 & 10.00 & 21.00 & 39.16 & 50.00 \\
\hline 5600 & 1.00 & 1.00 & 1.00 & 2.00 & 3.00 & 7.00 & 23.00 & 47.66 & 70.88 & 7311 & -- & -- & 1.00 & 1.00 & 5.00 & 10.00 & 25.50 & -- & -- \\
\hline 5618 & -- & -- & 2.00 & 2.25 & 7.50 & 19.00 & 35.20 & -- & -- & 7363 & -- & -- & 1.10 & 3.25 & 7.00 & 20.25 & 34.00 & -- & -- \\
\hline 5650 & -- & -- & 1.00 & 3.00 & 6.00 & 11.00 & 16.00 & -- & -- & 7422 & 1.00 & 1.00 & 1.00 & 1.00 & 4.00 & 12.00 & 25.00 & 50.04 & 60.02 \\
\hline 5656 & 1.00 & 1.00 & 1.00 & 1.00 & 2.00 & 10.00 & 20.70 & 40.14 & 51.00 & 7431 & 1.00 & 1.00 & 1.00 & 1.00 & 3.00 & 9.00 & 20.00 & 39.34 & 51.00 \\
\hline 5658 & 1.00 & 1.00 & 1.00 & 2.00 & 4.00 & 10.50 & 23.00 & 51.00 & 68.66 & 7481 & 1.00 & 1.00 & 1.00 & 1.00 & 3.00 & 8.00 & 18.80 & 44.00 & 49.48 \\
\hline 5661 & 1.00 & 1.00 & 1.00 & 1.00 & 2.00 & 10.00 & 23.30 & 48.12 & 79.11 & 7497 & 1.00 & 1.00 & 1.00 & 1.00 & 3.00 & 12.00 & 23.00 & 44.60 & 61.60 \\
\hline 5666 & -- & -- & 1.00 & 2.00 & 4.00 & 16.25 & 25.70 & -- & -- & 7498 & 1.00 & 1.00 & 1.00 & 1.00 & 3.00 & 11.00 & 23.00 & 46.00 & 48.32 \\
\hline 5695 & 1.00 & 1.00 & 1.00 & 1.00 & 4.00 & 13.00 & 25.00 & 44.28 & 55.56 & 7499 & 1.00 & 1.00 & 1.00 & 1.00 & 3.00 & 11.00 & 23.60 & 42.00 & 52.00 \\
\hline 5742 & -- & -- & 1.00 & 1.00 & 5.00 & 19.25 & 34.70 & -- & -- & 7531 & 1.00 & 1.00 & 1.00 & 2.00 & 6.00 & 16.50 & 34.80 & 56.80 & 68.66 \\
\hline 5766 & -- & -- & 2.00 & 5.00 & 17.00 & 22.00 & 28.00 & -- & -- & 7534 & 1.00 & 1.00 & 1.00 & 1.00 & 3.00 & 12.25 & 24.50 & 53.10 & 67.20 \\
\hline 5770 & 1.00 & 1.00 & 1.00 & 1.00 & 3.00 & 11.00 & 22.00 & 45.82 & 55.00 & 7556 & 1.00 & 1.00 & 1.00 & 1.00 & 4.00 & 11.00 & 22.00 & 40.98 & 49.99 \\
\hline 5775 & -- & -- & 1.00 & 1.00 & 3.00 & 6.00 & 16.70 & -- & -- & 7594 & 1.00 & 1.00 & 1.00 & 2.00 & 4.00 & 12.00 & 25.00 & 50.00 & 60.00 \\
\hline 5779 & -- & -- & 1.00 & 1.00 & 7.00 & 16.00 & 29.00 & -- & -- & 7596 & 1.00 & 1.00 & 1.00 & 1.50 & 3.00 & 9.50 & 24.00 & 49.08 & 71.24 \\
\hline 5840 & 1.00 & 1.00 & 1.00 & 2.00 & 5.00 & 14.75 & 28.00 & 55.34 & 79.05 & 7608 & 1.00 & 1.00 & 1.00 & 1.00 & 4.00 & 12.00 & 23.00 & 46.00 & 52.96 \\
\hline 5890 & 1.00 & 1.00 & 1.00 & 2.00 & 4.00 & 12.00 & 25.00 & 48.00 & 56.59 & 7622 & -- & -- & 1.00 & 1.00 & 2.00 & 8.50 & 20.00 & -- & -- \\
\hline 5891 & 1.00 & 1.00 & 1.00 & 2.00 & 3.00 & 10.00 & 16.70 & 40.34 & 58.63 & 7700 & 1.00 & 1.00 & 1.00 & 2.00 & 5.00 & 15.00 & 28.00 & 54.92 & 66.96 \\
\hline 5897 & 1.00 & 1.00 & 1.00 & 1.00 & 3.00 & 12.00 & 23.00 & 43.00 & 49.90 & 7706 & 1.00 & 1.00 & 1.00 & 1.00 & 3.00 & 12.00 & 23.00 & 47.00 & 55.10 \\
\hline
\end{tabular}


Appendix 4-5.4. Empirical distribution of storm duration defined by 18-hour minimum interevent time for hourly rainfall stations in Texas-Continued.

\begin{tabular}{|c|c|c|c|c|c|c|c|c|c|c|c|c|c|c|c|c|c|c|c|}
\hline \multicolumn{20}{|c|}{$\begin{array}{c}\text { Duration } \\
\text { (hours) }\end{array}$} \\
\hline $\begin{array}{l}\text { Sta- } \\
\text { tion } \\
\text { no. }\end{array}$ & $\begin{array}{c}\text { 1st } \\
\text { per- } \\
\text { centile }\end{array}$ & $\begin{array}{c}\text { 2nd } \\
\text { per- } \\
\text { centile }\end{array}$ & $\begin{array}{c}10 \text { th } \\
\text { per- } \\
\text { centile }\end{array}$ & $\begin{array}{c}25 \text { th } \\
\text { per- } \\
\text { centile }\end{array}$ & $\begin{array}{c}\text { 50th } \\
\text { per- } \\
\text { centile } \\
\text { (median) }\end{array}$ & $\begin{array}{c}\text { 75th } \\
\text { per- } \\
\text { centile }\end{array}$ & $\begin{array}{c}\text { 90th } \\
\text { per- } \\
\text { centile }\end{array}$ & $\begin{array}{c}\text { 98th } \\
\text { per- } \\
\text { centile }\end{array}$ & $\begin{array}{c}\text { 99th } \\
\text { per- } \\
\text { centile }\end{array}$ & $\begin{array}{l}\text { Sta- } \\
\text { tion } \\
\text { no. }\end{array}$ & $\begin{array}{c}1 \text { st } \\
\text { per- } \\
\text { centile }\end{array}$ & $\begin{array}{c}\text { 2nd } \\
\text { per- } \\
\text { centile }\end{array}$ & $\begin{array}{c}10 \text { th } \\
\text { per- } \\
\text { centile }\end{array}$ & $\begin{array}{c}25 \text { th } \\
\text { per- } \\
\text { centile }\end{array}$ & $\begin{array}{c}\text { 50th } \\
\text { per- } \\
\text { centile } \\
\text { (median) }\end{array}$ & $\begin{array}{c}\text { 75th } \\
\text { per- } \\
\text { centile }\end{array}$ & $\begin{array}{c}\text { 90th } \\
\text { per- } \\
\text { centile }\end{array}$ & $\begin{array}{c}\text { 98th } \\
\text { per- } \\
\text { centile }\end{array}$ & $\begin{array}{c}\text { 99th } \\
\text { per- } \\
\text { centile }\end{array}$ \\
\hline 7718 & -- & 1.00 & 1.00 & 2.00 & 7.00 & 21.00 & 35.70 & 79.42 & -- & 8910 & -- & -- & 2.00 & 3.00 & 5.00 & 9.50 & 15.80 & -- & -- \\
\hline 7745 & 1.00 & 1.00 & 1.00 & 2.00 & 7.00 & 20.00 & 33.60 & 56.72 & 67.87 & 8911 & 1.00 & 1.00 & 1.00 & 1.00 & 4.00 & 10.00 & 21.40 & 42.00 & 53.74 \\
\hline 7922 & 1.00 & 1.00 & 1.00 & 1.00 & 2.00 & 7.00 & 18.00 & 36.92 & 48.76 & 8924 & 1.00 & 1.00 & 1.00 & 1.00 & 2.00 & 5.00 & 16.00 & 34.24 & 39.81 \\
\hline 7936 & 1.00 & 1.00 & 1.00 & 1.00 & 4.00 & 12.00 & 24.00 & 45.86 & 58.43 & 8929 & -- & -- & 1.00 & 1.75 & 7.00 & 26.75 & 43.20 & -- & -- \\
\hline 7943 & 1.00 & 1.00 & 1.00 & 2.00 & 4.00 & 13.00 & 26.00 & 51.00 & 58.45 & 8942 & 1.00 & 1.00 & 1.00 & 1.00 & 4.00 & 14.00 & 25.00 & 45.00 & 55.00 \\
\hline 7944 & -- & 1.00 & 1.00 & 1.00 & 4.00 & 15.00 & 31.00 & 76.84 & -- & 8944 & 1.00 & 1.00 & 1.00 & 1.00 & 4.00 & 13.00 & 25.00 & 46.02 & 65.01 \\
\hline 7945 & 1.00 & 1.00 & 1.00 & 2.00 & 5.00 & 17.00 & 30.00 & 64.26 & 76.00 & 8996 & 1.00 & 1.00 & 1.00 & 2.00 & 4.00 & 12.00 & 25.00 & 49.92 & 61.46 \\
\hline 7947 & 1.00 & 1.00 & 1.00 & 1.00 & 4.00 & 12.00 & 27.00 & 60.92 & 67.46 & 9014 & -- & -- & 2.00 & 4.25 & 8.00 & 13.75 & 26.20 & -- & -- \\
\hline 7948 & 1.00 & 1.00 & 1.00 & 1.00 & 4.00 & 12.00 & 25.60 & 45.00 & 52.78 & 9037 & 1.00 & 1.00 & 1.00 & 1.00 & 3.00 & 8.00 & 19.00 & 47.96 & 69.00 \\
\hline 7951 & 1.00 & 1.00 & 1.00 & 2.00 & 5.00 & 13.00 & 24.00 & 46.02 & 61.53 & 9106 & 1.00 & 1.00 & 1.00 & 2.00 & 3.00 & 9.00 & 25.80 & 53.44 & 88.26 \\
\hline 7953 & 1.00 & 1.00 & 1.00 & 2.00 & 4.00 & 13.25 & 29.90 & 63.38 & 97.37 & 9107 & -- & -- & 1.00 & 1.00 & 1.00 & 7.00 & 37.20 & -- & -- \\
\hline 7981 & 1.00 & 1.00 & 1.00 & 1.00 & 4.00 & 12.00 & 26.30 & 65.02 & 80.37 & 9129 & -- & 1.00 & 1.00 & 1.00 & 3.00 & 17.00 & 28.40 & 45.52 & -- \\
\hline 7990 & 1.00 & 1.00 & 1.00 & 1.25 & 5.00 & 18.00 & 36.00 & 80.56 & 138.42 & 9163 & 1.00 & 1.00 & 1.00 & 1.00 & 4.00 & 12.00 & 24.00 & 44.84 & 53.00 \\
\hline 7992 & -- & -- & 3.00 & 4.00 & 7.00 & 15.25 & 39.00 & -- & -- & 9213 & 1.00 & 1.00 & 1.00 & 1.00 & 7.00 & 13.75 & 37.00 & 73.00 & 86.00 \\
\hline 7997 & 1.00 & 1.00 & 1.00 & 2.00 & 4.00 & 10.00 & 21.00 & 57.28 & 76.24 & 9214 & -- & -- & 1.00 & 2.25 & 4.00 & 8.50 & 35.20 & -- & -- \\
\hline 7999 & -- & -- & 1.00 & 1.00 & 4.00 & 13.00 & 21.40 & -- & -- & 9222 & 1.00 & 1.00 & 1.00 & 2.00 & 6.00 & 18.00 & 33.20 & 59.68 & 75.88 \\
\hline 8022 & -- & 1.00 & 1.00 & 1.00 & 2.50 & 16.75 & 29.90 & 74.54 & -- & 9248 & 1.00 & 1.00 & 1.00 & 1.00 & 3.00 & 16.00 & 28.60 & 52.30 & 56.49 \\
\hline 8023 & 1.00 & 1.00 & 1.00 & 1.00 & 3.00 & 9.00 & 21.00 & 44.76 & 55.38 & 9266 & -- & 1.00 & 1.00 & 2.00 & 5.00 & 15.00 & 28.00 & 58.80 & -- \\
\hline 8047 & 1.00 & 1.00 & 1.00 & 1.00 & 4.00 & 11.00 & 23.00 & 47.00 & 55.00 & 9270 & 1.00 & 1.00 & 1.00 & 1.00 & 2.00 & 7.00 & 19.00 & 41.00 & 50.22 \\
\hline 8060 & 1.00 & 1.00 & 1.00 & 2.00 & 4.00 & 13.50 & 26.40 & 62.58 & 92.73 & 9295 & -- & 1.00 & 1.00 & 1.00 & 1.00 & 7.00 & 25.00 & 38.20 & -- \\
\hline 8062 & -- & -- & 1.00 & 2.00 & 3.00 & 12.00 & 30.80 & -- & -- & 9304 & -- & -- & -- & 3.50 & 5.50 & 10.50 & -- & -- & -- \\
\hline 8068 & -- & -- & 1.00 & 1.00 & 3.00 & 11.25 & 22.50 & -- & -- & 9307 & 1.00 & 1.00 & 1.00 & 1.75 & 4.00 & 11.00 & 24.40 & 41.42 & 52.99 \\
\hline 8081 & 1.00 & 1.00 & 1.00 & 1.00 & 4.00 & 12.00 & 26.00 & 55.00 & 72.28 & 9328 & 1.00 & 1.00 & 1.00 & 1.00 & 3.00 & 13.00 & 29.20 & 66.10 & 90.55 \\
\hline 8089 & -- & -- & 1.00 & 1.75 & 4.00 & 13.00 & 21.00 & -- & -- & 9329 & -- & -- & 1.00 & 3.25 & 8.50 & 21.50 & 57.80 & -- & -- \\
\hline 8221 & -- & -- & 1.00 & 4.00 & 7.00 & 21.00 & 34.00 & -- & -- & 9345 & -- & -- & -- & -- & 10.00 & -- & -- & -- & -- \\
\hline 8252 & 1.00 & 1.00 & 1.00 & 1.00 & 3.00 & 9.00 & 21.00 & 41.70 & 54.00 & 9363 & 1.00 & 1.00 & 1.00 & 1.00 & 4.00 & 15.00 & 30.00 & 56.00 & 78.24 \\
\hline 8265 & 1.00 & 1.00 & 1.00 & 2.00 & 5.00 & 16.00 & 31.00 & 55.88 & 65.32 & 9364 & 1.00 & 1.00 & 1.00 & 1.00 & 4.00 & 13.00 & 27.00 & 54.00 & 67.00 \\
\hline 8289 & -- & 1.00 & 1.00 & 1.00 & 4.00 & 12.00 & 24.80 & 51.78 & -- & 9365 & -- & -- & 1.00 & 2.00 & 4.00 & 7.00 & 25.00 & -- & -- \\
\hline 8305 & 1.00 & 1.00 & 1.00 & 1.00 & 2.00 & 7.00 & 20.00 & 35.00 & 43.00 & 9371 & 1.00 & 1.00 & 1.00 & 2.00 & 4.00 & 13.00 & 32.70 & 54.02 & 67.39 \\
\hline 8335 & 1.00 & 1.00 & 1.00 & 2.00 & 6.00 & 16.00 & 29.00 & 56.68 & 66.52 & 9417 & 1.00 & 1.00 & 1.00 & 2.00 & 5.00 & 13.00 & 24.00 & 48.24 & 59.00 \\
\hline 8400 & 1.00 & 1.00 & 1.00 & 1.00 & 2.00 & 7.00 & 19.30 & 46.72 & 73.88 & 9419 & 1.00 & 1.00 & 1.00 & 2.00 & 5.00 & 16.00 & 30.00 & 54.00 & 66.00 \\
\hline 8445 & 1.00 & 1.00 & 1.00 & 2.00 & 5.00 & 14.00 & 28.00 & 51.16 & 67.08 & 9435 & -- & 1.00 & 1.00 & 1.00 & 3.00 & 7.00 & 25.60 & 57.72 & -- \\
\hline 8446 & 1.00 & 1.00 & 1.00 & 1.00 & 4.00 & 12.00 & 24.00 & 45.00 & 53.63 & 9491 & 1.00 & 1.00 & 1.00 & 2.00 & 4.00 & 13.00 & 26.00 & 49.92 & 55.38 \\
\hline 8451 & 1.00 & 1.00 & 1.00 & 2.00 & 4.00 & 12.00 & 30.00 & 54.28 & 61.12 & 9499 & 1.00 & 1.00 & 1.00 & 1.00 & 3.00 & 10.00 & 22.00 & 42.00 & 51.38 \\
\hline 8531 & 1.00 & 1.00 & 1.00 & 2.00 & 5.00 & 13.00 & 25.00 & 49.74 & 56.48 & 9522 & -- & -- & 3.20 & 5.00 & 16.00 & 24.00 & 61.00 & -- & -- \\
\hline 8541 & -- & 1.00 & 1.00 & 2.75 & 4.50 & 17.00 & 26.00 & 48.00 & -- & 9527 & 1.00 & 1.00 & 1.00 & 1.00 & 3.00 & 9.00 & 21.00 & 41.00 & 50.00 \\
\hline 8544 & 1.00 & 1.00 & 1.00 & 2.00 & 4.00 & 14.00 & 26.00 & 55.92 & 73.92 & 9532 & 1.00 & 1.00 & 1.00 & 1.00 & 4.00 & 11.00 & 23.00 & 44.00 & 53.00 \\
\hline 8545 & -- & -- & 1.00 & 1.50 & 11.50 & 25.50 & 41.20 & -- & -- & 9544 & -- & -- & -- & 1.00 & 3.00 & 17.00 & -- & -- & -- \\
\hline 8563 & 1.00 & 1.00 & 1.00 & 1.00 & 4.00 & 12.00 & 22.00 & 37.00 & 51.76 & 9565 & 1.00 & 1.00 & 1.00 & 1.00 & 3.00 & 10.00 & 22.00 & 42.20 & 57.60 \\
\hline 8566 & 1.00 & 1.00 & 1.00 & 1.00 & 3.00 & 7.00 & 19.00 & 40.36 & 59.12 & 9570 & 1.00 & 1.00 & 1.00 & 1.00 & 2.00 & 8.00 & 21.00 & 36.28 & 48.60 \\
\hline 8583 & 1.00 & 1.00 & 1.00 & 1.00 & 3.00 & 11.75 & 22.00 & 43.62 & 53.31 & 9574 & -- & -- & 1.00 & 1.00 & 2.50 & 11.00 & 15.40 & -- & -- \\
\hline 8584 & 1.00 & 1.00 & 1.00 & 1.00 & 3.00 & 11.00 & 24.00 & 44.38 & 55.69 & 9588 & 1.00 & 1.00 & 1.00 & 1.00 & 3.00 & 11.00 & 27.00 & 52.00 & 66.00 \\
\hline 8623 & 1.00 & 1.00 & 1.00 & 1.00 & 4.00 & 12.00 & 23.00 & 46.00 & 56.00 & 9665 & 1.00 & 1.00 & 1.00 & 2.00 & 4.00 & 13.00 & 26.00 & 49.66 & 58.83 \\
\hline 8625 & 1.00 & 1.00 & 1.00 & 2.00 & 5.00 & 13.00 & 26.00 & 48.14 & 58.28 & 9715 & 1.00 & 1.00 & 1.00 & 2.00 & 4.00 & 12.00 & 23.00 & 43.00 & 50.00 \\
\hline 8630 & 1.00 & 1.00 & 1.00 & 1.00 & 3.00 & 10.00 & 20.00 & 36.80 & 43.70 & 9729 & 1.00 & 1.00 & 1.00 & 2.00 & 5.00 & 14.00 & 26.00 & 50.00 & 60.00 \\
\hline 8631 & 1.00 & 1.00 & 1.00 & 2.00 & 4.00 & 11.00 & 25.00 & 52.48 & 66.00 & 9772 & 1.00 & 1.00 & 1.00 & 2.00 & 4.00 & 12.00 & 24.00 & 52.92 & 73.48 \\
\hline 8646 & 1.00 & 1.00 & 1.00 & 2.00 & 5.00 & 12.00 & 23.00 & 48.00 & 54.78 & 9814 & -- & -- & 1.30 & 2.25 & 5.50 & 15.75 & 25.70 & -- & -- \\
\hline 8647 & 1.00 & 1.00 & 1.00 & 1.00 & 3.00 & 10.00 & 20.00 & 38.38 & 47.57 & 9815 & 1.00 & 1.00 & 1.00 & 2.00 & 5.00 & 14.00 & 29.00 & 60.00 & 73.32 \\
\hline 8677 & 1.00 & 1.00 & 1.00 & 3.00 & 7.00 & 18.00 & 29.40 & 57.08 & 68.26 & 9816 & -- & 1.00 & 1.00 & 1.00 & 3.00 & 12.00 & 19.00 & 41.56 & -- \\
\hline 8696 & -- & -- & 1.00 & 2.00 & 8.00 & 15.00 & 21.60 & -- & -- & 9817 & 1.00 & 1.00 & 1.00 & 1.00 & 3.00 & 11.00 & 22.00 & 42.00 & 54.14 \\
\hline 8743 & 1.00 & 1.00 & 1.00 & 2.00 & 5.00 & 15.00 & 26.00 & 53.00 & 62.53 & 9829 & 1.00 & 1.00 & 1.00 & 1.00 & 3.00 & 8.00 & 20.00 & 49.00 & 58.00 \\
\hline 8761 & 1.00 & 1.00 & 1.00 & 1.00 & 3.00 & 8.00 & 18.00 & 37.00 & 47.75 & 9830 & 1.00 & 1.00 & 1.00 & 1.00 & 3.00 & 7.25 & 17.30 & 35.90 & 71.73 \\
\hline 8778 & 1.00 & 1.00 & 1.00 & 1.00 & 4.00 & 13.00 & 24.00 & 47.00 & 57.02 & 9858 & 1.00 & 1.00 & 1.00 & 2.00 & 4.00 & 9.00 & 22.00 & 44.00 & 51.00 \\
\hline 8845 & 1.00 & 1.00 & 1.00 & 1.00 & 4.00 & 14.00 & 27.00 & 55.56 & 70.28 & 9893 & 1.00 & 1.00 & 1.00 & 1.00 & 4.00 & 12.00 & 24.00 & 46.00 & 58.34 \\
\hline 8859 & 1.00 & 1.00 & 1.00 & 2.00 & 5.00 & 15.00 & 30.00 & 55.00 & 69.20 & 9916 & 1.00 & 1.00 & 1.00 & 1.00 & 5.00 & 14.00 & 25.00 & 42.34 & 52.34 \\
\hline 8898 & 1.00 & 1.00 & 1.00 & 2.00 & 6.00 & 15.00 & 27.00 & 54.00 & 70.78 & 9976 & 1.00 & 1.00 & 1.00 & 1.00 & 3.00 & 10.00 & 24.00 & 45.86 & 57.86 \\
\hline 8908 & -- & -- & 1.00 & 2.00 & 6.00 & 20.00 & 31.40 & -- & -- & & & & & & & & & & \\
\hline
\end{tabular}


Appendix 4-5.5. Empirical distribution of storm duration defined by 24-hour minimum interevent time for hourly rainfall stations in Texas.

[--, not available]

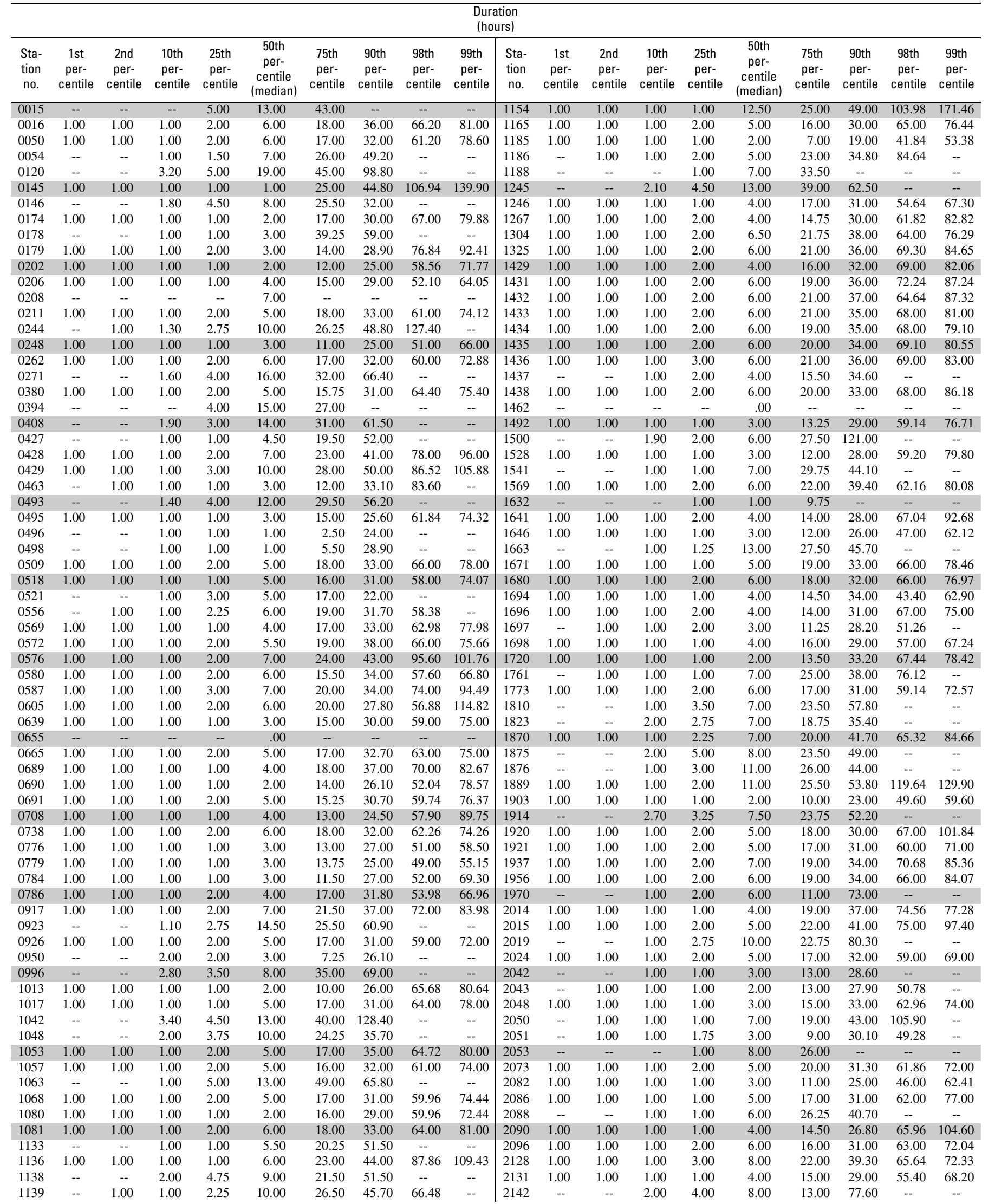


Appendix 4-5.5. Empirical distribution of storm duration defined by 24-hour minimum interevent time for hourly rainfall stations in Texas-Continued.

\begin{tabular}{|c|c|c|c|c|c|c|c|c|c|c|c|c|c|c|c|c|c|c|c|}
\hline \multicolumn{20}{|c|}{$\begin{array}{c}\text { Duration } \\
\text { (hours) }\end{array}$} \\
\hline $\begin{array}{l}\text { Sta- } \\
\text { tion } \\
\text { no. }\end{array}$ & $\begin{array}{c}\text { 1st } \\
\text { per- } \\
\text { centile }\end{array}$ & $\begin{array}{c}\text { 2nd } \\
\text { per- } \\
\text { centile }\end{array}$ & $\begin{array}{c}10 \text { th } \\
\text { per- } \\
\text { centile }\end{array}$ & $\begin{array}{c}\text { 25th } \\
\text { per- } \\
\text { centile }\end{array}$ & $\begin{array}{c}\text { 50th } \\
\text { per- } \\
\text { centile } \\
\text { (median) }\end{array}$ & $\begin{array}{c}\text { 75th } \\
\text { per- } \\
\text { centile }\end{array}$ & $\begin{array}{c}\text { 90th } \\
\text { per- } \\
\text { centile }\end{array}$ & $\begin{array}{l}\text { 98th } \\
\text { per- } \\
\text { centile }\end{array}$ & $\begin{array}{c}\text { 99th } \\
\text { per- } \\
\text { centile }\end{array}$ & $\begin{array}{l}\text { Sta- } \\
\text { tion } \\
\text { no. }\end{array}$ & $\begin{array}{c}\text { 1st } \\
\text { per- } \\
\text { centile }\end{array}$ & $\begin{array}{c}\text { 2nd } \\
\text { per- } \\
\text { centile }\end{array}$ & $\begin{array}{c}\text { 10th } \\
\text { per- } \\
\text { centile }\end{array}$ & $\begin{array}{l}\text { 25th } \\
\text { per- } \\
\text { centile }\end{array}$ & $\begin{array}{c}\text { 50th } \\
\text { per- } \\
\text { centile } \\
\text { (median) }\end{array}$ & $\begin{array}{c}\text { 75th } \\
\text { per- } \\
\text { centile }\end{array}$ & $\begin{array}{c}\text { 90th } \\
\text { per- } \\
\text { centile }\end{array}$ & $\begin{array}{c}\text { 98th } \\
\text { per- } \\
\text { centile }\end{array}$ & $\begin{array}{c}\text { 99th } \\
\text { per- } \\
\text { centile }\end{array}$ \\
\hline 2160 & -- & -- & 1.00 & 1.00 & 7.00 & 42.00 & 55.00 & -- & -- & 3463 & -- & -- & 1.00 & 2.00 & 6.00 & 18.00 & 29.00 & -- & -- \\
\hline 2206 & 1.00 & 1.00 & 1.00 & 2.00 & 5.00 & 18.00 & 34.00 & 64.42 & 72.71 & 3476 & 1.00 & 1.00 & 1.00 & 2.00 & 5.00 & 15.75 & 34.00 & 61.36 & 77.00 \\
\hline 2238 & 1.00 & 1.00 & 1.00 & 1.00 & 5.00 & 19.25 & 34.10 & 82.06 & 89.09 & 3485 & -- & -- & 1.80 & 6.50 & 11.00 & 23.50 & 49.80 & -- & -- \\
\hline 2240 & -- & 1.00 & 1.00 & 1.00 & 7.00 & 19.00 & 41.80 & 77.80 & -- & 3507 & 1.00 & 1.00 & 1.00 & 1.00 & 4.00 & 16.00 & 31.00 & 58.84 & 71.40 \\
\hline 2242 & 1.00 & 1.00 & 1.00 & 2.00 & 7.00 & 20.00 & 35.00 & 61.00 & 72.43 & 3546 & 1.00 & 1.00 & 1.00 & 2.00 & 6.00 & 18.00 & 33.30 & 66.86 & 80.43 \\
\hline 2244 & 1.00 & 1.00 & 1.00 & 2.00 & 6.00 & 19.00 & 35.00 & 65.00 & 80.00 & 3547 & 1.00 & 1.00 & 1.00 & 1.00 & 4.00 & 12.00 & 27.80 & 60.36 & 88.52 \\
\hline 2247 & -- & -- & 1.00 & 3.50 & 7.00 & 27.00 & 40.20 & -- & -- & 3579 & -- & 1.00 & 1.00 & 2.00 & 6.50 & 21.50 & 47.00 & 101.88 & -- \\
\hline 2309 & 1.00 & 1.00 & 1.00 & 2.00 & 6.00 & 17.00 & 35.40 & 68.88 & 81.52 & 3642 & 1.00 & 1.00 & 1.00 & 2.00 & 6.00 & 17.00 & 31.00 & 60.00 & 75.00 \\
\hline 2312 & 1.00 & 1.00 & 1.00 & 1.00 & 6.00 & 17.00 & 30.00 & 62.90 & 90.10 & 3646 & 1.00 & 1.00 & 1.00 & 2.00 & 6.00 & 16.00 & 31.00 & 63.86 & 74.93 \\
\hline 2334 & -- & 1.00 & 1.00 & 1.00 & 6.00 & 21.00 & 28.60 & 34.92 & -- & 3668 & -- & -- & 2.70 & 4.00 & 8.00 & 36.50 & 63.40 & -- & -- \\
\hline 2336 & 1.00 & 1.00 & 1.00 & 1.00 & 4.00 & 14.00 & 28.50 & 53.30 & 70.25 & 3673 & -- & -- & 1.00 & 4.00 & 12.00 & 47.00 & 51.00 & -- & -- \\
\hline 2354 & -- & -- & 1.00 & 2.00 & 4.50 & 20.50 & 43.10 & -- & -- & 3686 & 1.00 & 1.00 & 1.00 & 1.00 & 5.00 & 15.00 & 30.00 & 56.00 & 69.73 \\
\hline 2355 & -- & -- & 1.00 & 2.00 & 4.00 & 29.00 & 57.00 & -- & -- & 3691 & 1.00 & 1.00 & 1.00 & 1.00 & 5.00 & 16.00 & 29.00 & 56.00 & 67.56 \\
\hline 2357 & 1.00 & 1.00 & 1.00 & 1.00 & 4.00 & 19.00 & 39.80 & 68.00 & 75.78 & 3734 & -- & -- & 1.00 & 2.00 & 9.00 & 29.00 & 77.00 & -- & -- \\
\hline 2360 & 1.00 & 1.00 & 1.00 & 2.00 & 5.00 & 20.00 & 34.00 & 67.00 & 84.18 & 3771 & 1.00 & 1.00 & 1.00 & 1.00 & 4.00 & 16.00 & 31.00 & 52.00 & 58.42 \\
\hline 2361 & 1.00 & 1.00 & 1.00 & 1.00 & 3.00 & 15.00 & 28.00 & 62.72 & 92.64 & 3789 & -- & 1.00 & 1.00 & 1.00 & 1.00 & 13.00 & 37.00 & 106.60 & -- \\
\hline 2394 & 1.00 & 1.00 & 1.00 & 2.00 & 6.00 & 18.00 & 33.00 & 61.00 & 73.85 & 3826 & 1.00 & 1.00 & 1.00 & 2.00 & 5.00 & 14.00 & 30.60 & 70.96 & 75.12 \\
\hline 2404 & 1.00 & 1.00 & 1.00 & 2.00 & 5.00 & 17.00 & 31.80 & 55.00 & 66.16 & 3831 & 1.00 & 1.00 & 1.00 & 2.00 & 5.00 & 17.25 & 38.00 & 67.38 & 73.38 \\
\hline 2415 & 1.00 & 1.00 & 1.00 & 2.00 & 6.00 & 17.00 & 31.00 & 57.58 & 72.16 & 3841 & 1.00 & 1.00 & 1.00 & 1.00 & 4.00 & 14.00 & 26.60 & 47.04 & 75.86 \\
\hline 2462 & 1.00 & 1.00 & 1.00 & 2.00 & 5.00 & 16.00 & 32.00 & 65.60 & 73.60 & 3871 & 1.00 & 1.00 & 1.00 & 2.00 & 5.00 & 18.00 & 34.00 & 64.52 & 80.26 \\
\hline 2528 & -- & 1.00 & 1.00 & 1.00 & 5.00 & 16.50 & 47.20 & 63.68 & -- & 3884 & -- & -- & 1.00 & 3.75 & 7.00 & 14.50 & 28.00 & -- & -- \\
\hline 2617 & 1.00 & 1.00 & 1.00 & 2.00 & 6.00 & 16.00 & 33.40 & 53.68 & 56.84 & 3941 & -- & 1.00 & 1.00 & 3.00 & 9.00 & 17.00 & 43.60 & 102.16 & -- \\
\hline 2619 & 1.00 & 1.00 & 1.00 & 1.00 & 5.00 & 15.00 & 30.70 & 74.56 & 86.75 & 3963 & -- & -- & -- & 1.00 & 1.00 & 18.00 & -- & -- & -- \\
\hline 2621 & 1.00 & 1.00 & 1.00 & 1.00 & 4.00 & 15.00 & 29.00 & 55.32 & 65.32 & 4040 & 1.00 & 1.00 & 1.00 & 2.00 & 5.00 & 15.00 & 27.00 & 53.14 & 67.10 \\
\hline 2675 & 1.00 & 1.00 & 1.00 & 2.00 & 5.00 & 17.00 & 30.00 & 67.00 & 87.84 & 4058 & -- & -- & 1.00 & 3.00 & 5.00 & 27.00 & 47.20 & -- & -- \\
\hline 2676 & 1.00 & 1.00 & 1.00 & 1.00 & 3.00 & 16.00 & 30.00 & 66.00 & 85.44 & 4098 & 1.00 & 1.00 & 1.00 & 1.00 & 3.00 & 11.00 & 26.00 & 51.00 & 59.44 \\
\hline 2679 & 1.00 & 1.00 & 1.00 & 1.00 & 4.00 & 15.00 & 29.00 & 58.12 & 74.06 & 4100 & 1.00 & 1.00 & 1.00 & 2.00 & 5.00 & 14.00 & 26.00 & 50.00 & 55.00 \\
\hline 2715 & 1.00 & 1.00 & 1.00 & 2.00 & 5.00 & 16.00 & 28.40 & 54.00 & 68.14 & 4137 & 1.00 & 1.00 & 1.00 & 1.00 & 4.00 & 16.00 & 28.00 & 48.80 & 62.70 \\
\hline 2744 & 1.00 & 1.00 & 1.00 & 1.00 & 4.00 & 14.00 & 29.00 & 63.00 & 79.22 & 4191 & 1.00 & 1.00 & 1.00 & 1.00 & 4.00 & 17.00 & 30.80 & 60.76 & 75.00 \\
\hline 2758 & -- & 1.00 & 1.00 & 2.00 & 4.00 & 17.00 & 47.00 & 94.30 & -- & 4256 & -- & -- & -- & -- & .00 & -- & -- & -- & -- \\
\hline 2794 & -- & -- & 1.00 & 1.00 & 4.00 & 10.00 & 24.80 & -- & -- & 4257 & 1.00 & 1.00 & 1.00 & 1.00 & 6.00 & 18.00 & 32.00 & 59.64 & 72.00 \\
\hline 2797 & 1.00 & 1.00 & 1.00 & 1.00 & 4.00 & 16.00 & 29.00 & 53.00 & 66.72 & 4258 & 1.00 & 1.00 & 1.00 & 1.00 & 8.00 & 24.00 & 42.50 & 76.90 & 100.15 \\
\hline 2811 & 1.00 & 1.00 & 1.00 & 1.00 & 3.00 & 15.00 & 30.00 & 60.48 & 75.24 & 4278 & 1.00 & 1.00 & 1.00 & 2.00 & 5.00 & 15.00 & 29.80 & 59.76 & 67.76 \\
\hline 2813 & -- & -- & 1.00 & 1.00 & 4.00 & 14.00 & 33.20 & -- & -- & 4299 & 1.00 & 1.00 & 1.00 & 1.00 & 3.00 & 6.75 & 25.40 & 43.66 & 50.35 \\
\hline 2814 & -- & -- & 1.00 & 1.00 & 1.00 & 21.00 & 33.40 & -- & -- & 4300 & 1.00 & 1.00 & 1.00 & 2.00 & 7.00 & 23.00 & 43.00 & 83.24 & 99.00 \\
\hline 2815 & 1.00 & 1.00 & 1.00 & 1.00 & 3.00 & 13.00 & 25.00 & 44.34 & 58.78 & 4305 & 1.00 & 1.00 & 1.00 & 2.00 & 8.00 & 24.00 & 45.00 & 81.00 & 99.68 \\
\hline 2818 & 1.00 & 1.00 & 1.00 & 1.00 & 4.00 & 11.50 & 25.40 & 53.72 & 130.52 & 4307 & 1.00 & 1.00 & 1.00 & 1.00 & 7.00 & 25.00 & 49.00 & 91.68 & 111.73 \\
\hline 2986 & 1.00 & 1.00 & 1.00 & 2.00 & 7.00 & 21.00 & 41.00 & 65.50 & 94.50 & 4309 & 1.00 & 1.00 & 1.00 & 2.00 & 5.00 & 19.00 & 34.70 & 66.00 & 76.00 \\
\hline 3005 & 1.00 & 1.00 & 1.00 & 1.00 & 4.00 & 14.00 & 28.00 & 55.00 & 68.52 & 4311 & 1.00 & 1.00 & 1.00 & 2.00 & 6.00 & 20.00 & 34.00 & 70.00 & 77.00 \\
\hline 3033 & 1.00 & 1.00 & 1.00 & 2.00 & 3.00 & 8.00 & 24.60 & 39.64 & 49.16 & 4313 & 1.00 & 1.00 & 1.00 & 3.00 & 8.00 & 23.00 & 39.60 & 58.44 & 64.72 \\
\hline 3034 & -- & -- & -- & -- & 1.00 & -- & -- & -- & -- & 4319 & -- & 1.00 & 1.00 & 3.00 & 6.50 & 23.00 & 42.50 & 83.50 & -- \\
\hline 3047 & -- & -- & 1.90 & 4.50 & 10.50 & 39.00 & 97.00 & -- & -- & 4329 & 1.00 & 1.00 & 1.00 & 2.00 & 5.00 & 19.00 & 35.00 & 71.00 & 80.00 \\
\hline 3103 & -- & -- & 1.00 & 1.00 & 5.00 & 13.00 & 33.60 & -- & -- & 4331 & -- & -- & -- & -- & .00 & -- & -- & -- & -- \\
\hline 3133 & 1.00 & 1.00 & 1.00 & 2.00 & 6.00 & 17.00 & 33.90 & 63.00 & 74.09 & 4375 & 1.00 & 1.00 & 1.00 & 1.00 & 3.00 & 14.00 & 27.00 & 61.26 & 74.39 \\
\hline 3156 & 1.00 & 1.00 & 1.00 & 1.00 & 6.00 & 18.75 & 32.00 & 72.68 & 93.39 & 4392 & 1.00 & 1.00 & 1.00 & 3.00 & 8.00 & 22.00 & 35.00 & 79.00 & 112.20 \\
\hline 3171 & 1.00 & 1.00 & 1.00 & 2.00 & 6.00 & 17.00 & 33.00 & 60.64 & 72.00 & 4425 & 1.00 & 1.00 & 1.00 & 1.00 & 2.00 & 12.00 & 26.00 & 49.00 & 65.72 \\
\hline 3189 & 1.00 & 1.00 & 1.00 & 1.00 & 4.00 & 17.00 & 32.00 & 72.84 & 101.00 & 4440 & 1.00 & 1.00 & 1.00 & 2.00 & 6.00 & 19.00 & 32.00 & 67.00 & 82.28 \\
\hline 3260 & 1.00 & 1.00 & 1.00 & 2.00 & 5.00 & 17.00 & 31.00 & 58.08 & 68.24 & 4476 & 1.00 & 1.00 & 1.00 & 1.00 & 4.00 & 16.00 & 30.00 & 53.00 & 65.00 \\
\hline 3267 & -- & 1.00 & 1.00 & 2.00 & 4.00 & 18.75 & 34.10 & 76.48 & -- & 4498 & -- & -- & 1.00 & 1.00 & 3.00 & 10.00 & 27.80 & -- & -- \\
\hline 3270 & 1.00 & 1.00 & 1.00 & 1.00 & 3.00 & 12.00 & 25.00 & 47.26 & 61.63 & 4517 & 1.00 & 1.00 & 1.00 & 2.00 & 5.00 & 18.00 & 33.00 & 62.60 & 69.60 \\
\hline 3272 & -- & -- & 1.00 & 1.00 & 4.00 & 18.00 & 31.40 & -- & -- & 4520 & 1.00 & 1.00 & 1.00 & 1.00 & 4.00 & 16.00 & 29.00 & 48.00 & 60.52 \\
\hline 3277 & -- & -- & 1.00 & 1.00 & 7.00 & 19.00 & 39.40 & -- & -- & 4525 & -- & -- & 1.00 & 2.00 & 6.00 & 30.00 & 128.60 & -- & -- \\
\hline 3278 & 1.00 & 1.00 & 1.00 & 1.00 & 3.00 & 10.00 & 26.00 & 54.00 & 74.54 & 4563 & -- & -- & 1.00 & 2.00 & 5.00 & 9.00 & 24.20 & -- & -- \\
\hline 3280 & 1.00 & 1.00 & 1.00 & 1.00 & 3.00 & 7.00 & 23.40 & 60.56 & 69.13 & 4570 & 1.00 & 1.00 & 1.00 & 1.00 & 4.00 & 15.00 & 29.00 & 58.28 & 74.46 \\
\hline 3281 & -- & -- & 1.00 & 1.00 & 2.00 & 5.00 & 20.40 & -- & -- & 4577 & 1.00 & 1.00 & 1.00 & 2.00 & 5.00 & 17.00 & 31.00 & 68.00 & 76.00 \\
\hline 3283 & 1.00 & 1.00 & 1.00 & 2.00 & 7.00 & 21.00 & 38.00 & 76.00 & 85.25 & 4591 & 1.00 & 1.00 & 1.00 & 2.00 & 7.00 & 20.00 & 34.20 & 71.84 & 86.42 \\
\hline 3284 & 1.00 & 1.00 & 1.00 & 1.00 & 5.00 & 17.00 & 32.00 & 63.00 & 78.65 & 4670 & 1.00 & 1.00 & 1.00 & 1.00 & 4.00 & 15.00 & 28.00 & 56.00 & 69.00 \\
\hline 3285 & 1.00 & 1.00 & 1.00 & 1.00 & 5.00 & 16.00 & 29.20 & 55.04 & 67.08 & 4671 & 1.00 & 1.00 & 1.00 & 1.00 & 7.00 & 19.00 & 35.50 & 70.24 & 86.86 \\
\hline 3329 & 1.00 & 1.00 & 1.00 & 2.00 & 6.00 & 19.00 & 33.00 & 68.56 & 80.78 & 4679 & 1.00 & 1.00 & 1.00 & 1.00 & 5.00 & 17.00 & 31.00 & 53.26 & 67.26 \\
\hline 3335 & 1.00 & 1.00 & 1.00 & 2.75 & 7.00 & 21.00 & 35.00 & 77.50 & 87.75 & 4696 & -- & -- & 1.00 & 1.00 & 3.50 & 27.50 & 50.00 & -- & -- \\
\hline 3370 & 1.00 & 1.00 & 1.00 & 2.00 & 5.00 & 16.00 & 30.00 & 52.00 & 67.47 & 4703 & 1.00 & 1.00 & 1.00 & 1.00 & 4.00 & 13.00 & 26.00 & 46.92 & 113.24 \\
\hline 3410 & 1.00 & 1.00 & 1.00 & 1.00 & 4.00 & 14.00 & 28.00 & 55.00 & 63.00 & 4704 & 1.00 & 1.00 & 1.00 & 2.00 & 8.00 & 22.00 & 42.00 & 74.76 & 90.00 \\
\hline 3415 & 1.00 & 1.00 & 1.00 & 1.00 & 5.00 & 16.00 & 30.00 & 56.00 & 70.00 & 4731 & -- & 1.00 & 1.00 & 1.25 & 11.00 & 25.00 & 36.30 & 77.62 & -- \\
\hline 3430 & 1.00 & 1.00 & 1.00 & 2.00 & 7.00 & 21.00 & 39.00 & 73.00 & 86.88 & 4792 & 1.00 & 1.00 & 1.00 & 1.00 & 4.00 & 15.00 & 28.00 & 54.00 & 69.00 \\
\hline 3431 & 1.00 & 1.00 & 1.00 & 1.00 & 7.00 & 28.75 & 55.00 & 98.80 & 153.70 & 4819 & 1.00 & 1.00 & 1.00 & 1.00 & 6.00 & 17.00 & 32.00 & 65.00 & 73.00 \\
\hline 3441 & -- & -- & 1.00 & 1.00 & 5.50 & 21.00 & 48.90 & -- & -- & 4852 & -- & -- & 1.00 & 1.00 & 1.00 & 6.00 & 29.40 & -- & -- \\
\hline 3442 & 1.00 & 1.00 & 1.00 & 2.00 & 6.00 & 15.00 & 31.00 & 56.46 & 64.65 & 4866 & 1.00 & 1.00 & 1.00 & 2.00 & 5.00 & 17.00 & 32.00 & 60.00 & 76.00 \\
\hline 3446 & 1.00 & 1.00 & 1.00 & 2.00 & 4.00 & 10.00 & 25.30 & 51.92 & 61.53 & 4876 & 1.00 & 1.00 & 1.00 & 1.00 & 5.50 & 17.25 & 30.00 & 69.04 & 81.01 \\
\hline 3460 & -- & -- & 1.00 & 2.00 & 9.00 & 24.00 & 32.20 & -- & -- & 4878 & 1.00 & 1.00 & 1.00 & 2.00 & 7.00 & 22.00 & 39.00 & 73.66 & 90.33 \\
\hline 3462 & 1.00 & 1.00 & 1.00 & 2.00 & 6.00 & 17.00 & 32.80 & 62.48 & 66.43 & 4880 & 1.00 & 1.00 & 1.00 & 1.00 & 4.00 & 13.00 & 29.00 & 58.00 & 70.01 \\
\hline
\end{tabular}


Appendix 4-5.5. Empirical distribution of storm duration defined by 24-hour minimum interevent time for hourly rainfall stations in Texas-Continued.

\begin{tabular}{|c|c|c|c|c|c|c|c|c|c|c|c|c|c|c|c|c|c|c|c|}
\hline \multicolumn{20}{|c|}{$\begin{array}{c}\text { Duration } \\
\text { (hours) }\end{array}$} \\
\hline $\begin{array}{l}\text { Sta- } \\
\text { tion } \\
\text { no. }\end{array}$ & $\begin{array}{c}1 \text { st } \\
\text { per- } \\
\text { centile }\end{array}$ & $\begin{array}{c}\text { 2nd } \\
\text { per- } \\
\text { centile }\end{array}$ & $\begin{array}{c}\text { 10th } \\
\text { per- } \\
\text { centile }\end{array}$ & $\begin{array}{c}25 \text { th } \\
\text { per- } \\
\text { centile }\end{array}$ & $\begin{array}{c}\text { 50th } \\
\text { per- } \\
\text { centile } \\
\text { (median) }\end{array}$ & $\begin{array}{l}\text { 75th } \\
\text { per- } \\
\text { centile }\end{array}$ & $\begin{array}{c}\text { 90th } \\
\text { per- } \\
\text { centile }\end{array}$ & $\begin{array}{c}\text { 98th } \\
\text { per- } \\
\text { centile }\end{array}$ & $\begin{array}{c}\text { 99th } \\
\text { per- } \\
\text { centile }\end{array}$ & $\begin{array}{l}\text { Sta- } \\
\text { tion } \\
\text { no. }\end{array}$ & $\begin{array}{c}\text { 1st } \\
\text { per- } \\
\text { centile }\end{array}$ & $\begin{array}{c}\text { 2nd } \\
\text { per- } \\
\text { centile }\end{array}$ & $\begin{array}{c}\text { 10th } \\
\text { per- } \\
\text { centile }\end{array}$ & $\begin{array}{c}25 \text { th } \\
\text { per- } \\
\text { centile }\end{array}$ & $\begin{array}{c}\text { 50th } \\
\text { per- } \\
\text { centile } \\
\text { (median) }\end{array}$ & $\begin{array}{c}\text { 75th } \\
\text { per- } \\
\text { centile }\end{array}$ & $\begin{array}{c}\text { 90th } \\
\text { per- } \\
\text { centile }\end{array}$ & $\begin{array}{c}\text { 98th } \\
\text { per- } \\
\text { centile }\end{array}$ & $\begin{array}{c}\text { 99th } \\
\text { per- } \\
\text { centile }\end{array}$ \\
\hline 4920 & 1.00 & 1.00 & 1.00 & 1.00 & 4.00 & 15.00 & 29.00 & 59.42 & 70.63 & 5957 & 1.00 & 1.00 & 1.00 & 1.00 & 4.00 & 14.00 & 28.00 & 55.00 & 66.89 \\
\hline 4934 & -- & -- & -- & 1.00 & 6.00 & 17.00 & -- & -- & -- & 5958 & 1.00 & 1.00 & 1.00 & 2.00 & 5.00 & 21.00 & 33.00 & 66.04 & 71.56 \\
\hline 4972 & 1.00 & 1.00 & 1.00 & 2.00 & 5.00 & 15.00 & 31.00 & 54.82 & 68.00 & 5973 & -- & 1.00 & 1.00 & 3.00 & 7.00 & 18.50 & 41.80 & 87.32 & -- \\
\hline 4973 & 1.00 & 1.00 & 1.00 & 3.00 & 6.00 & 19.00 & 30.00 & 55.76 & 72.47 & 5996 & 1.00 & 1.00 & 1.00 & 2.00 & 5.00 & 16.00 & 31.00 & 57.00 & 74.00 \\
\hline 4974 & 1.00 & 1.00 & 1.00 & 2.00 & 4.00 & 14.75 & 30.00 & 60.00 & 77.92 & 6017 & 1.00 & 1.00 & 1.00 & 2.00 & 3.00 & 13.75 & 28.80 & 56.14 & 94.67 \\
\hline 4975 & 1.00 & 1.00 & 1.00 & 1.00 & 5.00 & 16.00 & 31.00 & 52.00 & 62.00 & 6024 & 1.00 & 1.00 & 1.00 & 2.00 & 9.00 & 21.00 & 34.00 & 70.22 & 72.00 \\
\hline 4978 & 1.00 & 1.00 & 1.00 & 1.00 & 4.00 & 15.00 & 29.80 & 77.28 & 141.67 & 6050 & -- & -- & 1.00 & 5.00 & 8.00 & 13.00 & 19.00 & -- & -- \\
\hline 4979 & -- & -- & 2.40 & 5.00 & 8.00 & 24.75 & 82.90 & -- & -- & 6104 & 1.00 & 1.00 & 1.00 & 1.00 & 3.00 & 18.00 & 33.00 & 73.00 & 82.52 \\
\hline 4982 & 1.00 & 1.00 & 1.00 & 2.00 & 5.00 & 16.00 & 27.00 & 51.80 & 61.90 & 6108 & 1.00 & 1.00 & 1.00 & 1.00 & 5.00 & 17.00 & 31.00 & 59.20 & 72.00 \\
\hline 5018 & 1.00 & 1.00 & 1.00 & 2.00 & 5.00 & 17.00 & 31.00 & 57.56 & 70.67 & 6136 & 1.00 & 1.00 & 1.00 & 1.00 & 3.00 & 13.00 & 27.00 & 53.00 & 61.73 \\
\hline 5048 & 1.00 & 1.00 & 1.00 & 1.00 & 3.00 & 13.00 & 26.00 & 53.00 & 67.75 & 6166 & -- & 1.00 & 1.00 & 2.00 & 5.00 & 21.00 & 31.20 & 56.48 & -- \\
\hline 5049 & -- & 1.00 & 1.00 & 1.00 & 2.00 & 8.00 & 17.30 & 38.86 & -- & 6176 & 1.00 & 1.00 & 1.00 & 2.00 & 7.00 & 20.00 & 31.30 & 75.14 & 97.65 \\
\hline 5056 & -- & -- & -- & 2.50 & 8.00 & 18.50 & -- & -- & -- & 6177 & 1.00 & 1.00 & 1.00 & 2.00 & 5.00 & 17.00 & 32.00 & 69.48 & 84.00 \\
\hline 5057 & 1.00 & 1.00 & 1.00 & 1.00 & 5.00 & 21.00 & 39.00 & 70.00 & 93.00 & 6210 & 1.00 & 1.00 & 1.00 & 1.00 & 5.00 & 16.00 & 31.00 & 53.34 & 62.67 \\
\hline 5060 & 1.00 & 1.00 & 1.00 & 2.00 & 5.00 & 22.00 & 37.90 & 105.56 & 123.38 & 6211 & 1.00 & 1.00 & 1.00 & 2.00 & 6.00 & 23.00 & 42.50 & 82.30 & 94.40 \\
\hline 5081 & 1.00 & 1.00 & 1.00 & 3.00 & 8.00 & 21.00 & 37.00 & 76.00 & 83.50 & 6270 & 1.00 & 1.00 & 1.00 & 1.00 & 6.00 & 18.00 & 32.00 & 61.00 & 71.38 \\
\hline 5094 & 1.00 & 1.00 & 1.00 & 1.00 & 5.00 & 16.00 & 29.00 & 54.00 & 67.60 & 6275 & -- & -- & -- & -- & .00 & -- & -- & -- & -- \\
\hline 5113 & 1.00 & 1.00 & 1.00 & 1.00 & 4.00 & 17.00 & 33.00 & 66.54 & 77.54 & 6276 & -- & -- & 1.00 & 3.25 & 7.00 & 26.00 & 56.60 & -- & -- \\
\hline 5114 & -- & -- & -- & -- & .00 & -- & -- & -- & -- & 6335 & 1.00 & 1.00 & 1.00 & 2.00 & 6.00 & 18.00 & 31.00 & 61.00 & 75.42 \\
\hline 5123 & -- & -- & 1.00 & 1.75 & 3.00 & 11.75 & 28.00 & -- & -- & 6434 & -- & -- & 2.60 & 5.00 & 8.00 & 18.00 & 33.80 & -- & -- \\
\hline 5192 & 1.00 & 1.00 & 1.00 & 2.00 & 5.00 & 16.00 & 30.00 & 53.38 & 63.19 & 6504 & 1.00 & 1.00 & 1.00 & 1.00 & 3.00 & 14.00 & 28.00 & 55.08 & 68.00 \\
\hline 5193 & 1.00 & 1.00 & 1.00 & 2.00 & 5.00 & 18.00 & 34.00 & 65.00 & 78.14 & 6558 & -- & -- & 1.00 & 1.00 & 8.00 & 28.00 & 40.60 & -- & -- \\
\hline 5224 & 1.00 & 1.00 & 1.00 & 2.00 & 6.00 & 19.00 & 34.00 & 75.72 & 107.68 & 6615 & 1.00 & 1.00 & 1.00 & 1.00 & 3.00 & 12.00 & 27.00 & 57.20 & 69.55 \\
\hline 5228 & 1.00 & 1.00 & 1.00 & 1.00 & 4.00 & 16.00 & 33.00 & 53.90 & 90.90 & 6660 & -- & 1.00 & 1.00 & 2.00 & 7.00 & 24.00 & 50.20 & 67.84 & -- \\
\hline 5235 & -- & -- & 1.00 & 2.00 & 12.00 & 29.00 & 45.40 & -- & -- & 6663 & -- & 1.00 & 1.00 & 1.00 & 10.00 & 32.50 & 49.00 & 83.40 & -- \\
\hline 5247 & 1.00 & 1.00 & 1.00 & 1.00 & 4.00 & 14.00 & 27.00 & 56.00 & 68.22 & 6734 & 1.00 & 1.00 & 1.00 & 2.00 & 5.00 & 17.00 & 30.80 & 58.12 & 75.04 \\
\hline 5258 & 1.00 & 1.00 & 1.00 & 2.00 & 6.00 & 18.00 & 31.00 & 62.94 & 77.47 & 6736 & 1.00 & 1.00 & 1.00 & 1.00 & 3.00 & 11.00 & 27.00 & 51.50 & 59.75 \\
\hline 5303 & 1.00 & 1.00 & 1.00 & 2.00 & 5.00 & 17.75 & 34.00 & 66.02 & 80.51 & 6740 & -- & -- & 1.30 & 3.50 & 7.50 & 34.75 & 100.30 & -- & -- \\
\hline 5312 & 1.00 & 1.00 & 1.00 & 1.00 & 3.00 & 13.00 & 27.00 & 57.88 & 71.44 & 6750 & 1.00 & 1.00 & 1.00 & 1.00 & 7.00 & 25.00 & 44.80 & 69.16 & 119.98 \\
\hline 5341 & -- & -- & 1.00 & 3.00 & 5.00 & 26.00 & 81.00 & -- & -- & 6757 & 1.00 & 1.00 & 1.00 & 2.00 & 6.00 & 19.00 & 35.00 & 67.00 & 81.60 \\
\hline 5342 & -- & -- & -- & -- & .00 & -- & -- & -- & -- & 6775 & 1.00 & 1.00 & 1.00 & 2.00 & 5.00 & 19.00 & 31.30 & 67.94 & 75.31 \\
\hline 5348 & 1.00 & 1.00 & 1.00 & 1.00 & 5.00 & 17.00 & 30.00 & 61.00 & 77.80 & 6776 & 1.00 & 1.00 & 1.00 & 1.00 & 4.00 & 13.00 & 28.00 & 56.00 & 65.89 \\
\hline 5358 & 1.00 & 1.00 & 1.00 & 2.00 & 4.00 & 12.75 & 29.00 & 54.94 & 64.00 & 6788 & 1.00 & 1.00 & 1.00 & 3.00 & 6.00 & 16.00 & 28.60 & 53.60 & 61.36 \\
\hline 5398 & 1.00 & 1.00 & 1.00 & 2.00 & 6.00 & 20.00 & 36.00 & 69.52 & 89.26 & 6792 & 1.00 & 1.00 & 1.00 & 1.00 & 2.00 & 10.00 & 25.00 & 48.00 & 63.00 \\
\hline 5410 & 1.00 & 1.00 & 1.00 & 1.00 & 3.00 & 12.00 & 27.00 & 53.66 & 66.00 & 6794 & -- & -- & 1.00 & 6.75 & 20.00 & 46.00 & 93.90 & -- & -- \\
\hline 5411 & 1.00 & 1.00 & 1.00 & 2.00 & 5.00 & 18.00 & 32.00 & 70.64 & 80.32 & 6834 & 1.00 & 1.00 & 1.00 & 1.00 & 5.00 & 16.00 & 29.00 & 54.70 & 61.35 \\
\hline 5424 & 1.00 & 1.00 & 1.00 & 1.00 & 9.00 & 25.00 & 58.20 & 113.84 & 146.96 & 6893 & 1.00 & 1.00 & 1.00 & 1.00 & 2.00 & 9.00 & 23.00 & 50.88 & 67.88 \\
\hline 5429 & 1.00 & 1.00 & 1.00 & 2.00 & 5.00 & 19.00 & 35.20 & 66.00 & 79.16 & 6935 & 1.00 & 1.00 & 1.00 & 1.00 & 3.00 & 11.00 & 26.00 & 50.68 & 60.84 \\
\hline 5431 & -- & -- & 1.50 & 3.75 & 13.50 & 26.50 & 43.50 & -- & -- & 6981 & 1.00 & 1.00 & 1.00 & 2.00 & 4.00 & 18.00 & 34.10 & 67.86 & 107.03 \\
\hline 5461 & 1.00 & 1.00 & 1.00 & 2.00 & 6.00 & 16.00 & 34.50 & 63.90 & 82.35 & 7020 & 1.00 & 1.00 & 1.00 & 3.00 & 8.00 & 22.00 & 46.20 & 75.52 & 82.44 \\
\hline 5463 & 1.00 & 1.00 & 1.00 & 1.00 & 4.00 & 15.00 & 29.00 & 52.00 & 59.99 & 7060 & 1.00 & 1.00 & 1.00 & 1.00 & 3.00 & 13.00 & 28.00 & 51.68 & 63.00 \\
\hline 5471 & -- & -- & 1.00 & 1.00 & 2.00 & 28.75 & 46.20 & -- & -- & 7066 & 1.00 & 1.00 & 1.00 & 2.00 & 6.00 & 18.00 & 33.00 & 61.00 & 73.00 \\
\hline 5477 & -- & -- & 1.00 & 1.00 & 3.00 & 17.50 & 68.80 & -- & -- & 7074 & 1.00 & 1.00 & 1.00 & 1.00 & 3.00 & 11.00 & 26.00 & 52.00 & 62.00 \\
\hline 5528 & 1.00 & 1.00 & 1.00 & 2.00 & 5.00 & 15.00 & 29.50 & 51.00 & 59.00 & 7097 & -- & 1.00 & 1.00 & 2.00 & 5.50 & 21.00 & 29.70 & 62.72 & -- \\
\hline 5579 & -- & -- & -- & -- & 15.00 & -- & -- & -- & -- & 7116 & 1.00 & 1.00 & 1.00 & 2.00 & 5.00 & 16.00 & 29.60 & 49.72 & 61.44 \\
\hline 5580 & -- & -- & 1.10 & 2.25 & 5.50 & 31.00 & 50.80 & -- & -- & 7140 & 1.00 & 1.00 & 1.00 & 2.00 & 4.00 & 18.00 & 33.00 & 68.00 & 79.00 \\
\hline 5589 & 1.00 & 1.00 & 1.00 & 1.00 & 3.00 & 13.00 & 23.80 & 52.28 & 70.60 & 7173 & 1.00 & 1.00 & 1.00 & 2.00 & 8.00 & 25.00 & 47.00 & 73.00 & 84.04 \\
\hline 5590 & 1.00 & 1.00 & 1.00 & 2.00 & 4.00 & 18.00 & 31.60 & 64.28 & 72.16 & 7174 & 1.00 & 1.00 & 1.00 & 2.00 & 7.00 & 23.00 & 43.00 & 79.00 & 98.00 \\
\hline 5591 & 1.00 & 1.00 & 1.00 & 2.00 & 3.00 & 12.00 & 27.00 & 57.24 & 78.02 & 7213 & 1.00 & 1.00 & 1.00 & 2.00 & 5.00 & 18.00 & 34.00 & 64.00 & 84.04 \\
\hline 5592 & 1.00 & 1.00 & 1.00 & 2.00 & 3.00 & 12.00 & 28.00 & 55.58 & 75.72 & 7243 & 1.00 & 1.00 & 1.00 & 1.00 & 4.00 & 16.00 & 29.00 & 57.00 & 70.59 \\
\hline 5594 & 1.00 & 1.00 & 1.00 & 1.00 & 2.00 & 6.00 & 23.80 & 44.96 & 54.84 & 7262 & -- & 1.00 & 1.00 & 1.00 & 1.00 & 11.50 & 30.40 & 73.84 & -- \\
\hline 5595 & -- & -- & -- & -- & 1.50 & -- & -- & -- & -- & 7274 & 1.00 & 1.00 & 1.00 & 1.00 & 4.00 & 11.00 & 25.00 & 52.32 & 69.00 \\
\hline 5596 & 1.00 & 1.00 & 1.00 & 1.00 & 2.00 & 13.00 & 26.40 & 56.92 & 74.74 & 7300 & 1.00 & 1.00 & 1.00 & 2.00 & 4.00 & 15.00 & 27.00 & 52.00 & 69.00 \\
\hline 5600 & 1.00 & 1.00 & 1.00 & 2.00 & 3.00 & 12.00 & 34.20 & 78.36 & 95.00 & 7311 & -- & -- & 1.00 & 1.00 & 5.00 & 14.50 & 31.80 & -- & -- \\
\hline 5618 & -- & -- & 2.00 & 2.25 & 10.00 & 20.75 & 48.50 & -- & -- & 7363 & -- & -- & 1.00 & 3.50 & 9.00 & 27.50 & 83.20 & -- & -- \\
\hline 5650 & -- & -- & 1.00 & 4.00 & 8.00 & 11.50 & 28.40 & -- & -- & 7422 & 1.00 & 1.00 & 1.00 & 1.00 & 5.00 & 18.00 & 33.00 & 65.76 & 79.76 \\
\hline 5656 & 1.00 & 1.00 & 1.00 & 1.00 & 3.00 & 13.00 & 27.00 & 51.52 & 61.00 & 7431 & 1.00 & 1.00 & 1.00 & 1.00 & 3.00 & 11.00 & 25.00 & 50.00 & 60.00 \\
\hline 5658 & 1.00 & 1.00 & 1.00 & 2.00 & 5.00 & 15.00 & 30.00 & 60.86 & 74.31 & 7481 & 1.00 & 1.00 & 1.00 & 1.00 & 3.00 & 10.00 & 25.00 & 50.00 & 61.70 \\
\hline 5661 & 1.00 & 1.00 & 1.00 & 1.00 & 2.00 & 16.25 & 37.00 & 82.50 & 100.75 & 7497 & 1.00 & 1.00 & 1.00 & 1.00 & 4.00 & 16.25 & 29.00 & 62.64 & 73.41 \\
\hline 5666 & -- & -- & 1.00 & 2.00 & 4.00 & 21.25 & 44.90 & -- & -- & 7498 & 1.00 & 1.00 & 1.00 & 1.00 & 3.00 & 15.00 & 30.40 & 51.48 & 79.96 \\
\hline 5695 & 1.00 & 1.00 & 1.00 & 2.00 & 5.00 & 16.50 & 31.00 & 59.96 & 76.22 & 7499 & 1.00 & 1.00 & 1.00 & 1.00 & 4.00 & 15.00 & 29.00 & 55.00 & 66.19 \\
\hline 5742 & -- & -- & 1.00 & 2.50 & 13.00 & 26.00 & 53.50 & -- & -- & 7531 & 1.00 & 1.00 & 1.00 & 2.00 & 6.50 & 20.00 & 43.60 & 68.96 & 72.66 \\
\hline 5766 & -- & -- & 2.00 & 5.00 & 17.00 & 22.00 & 28.00 & -- & -- & 7534 & 1.00 & 1.00 & 1.00 & 1.00 & 4.00 & 14.00 & 30.00 & 63.50 & 71.50 \\
\hline 5770 & 1.00 & 1.00 & 1.00 & 1.00 & 4.00 & 15.00 & 29.00 & 54.32 & 63.16 & 7556 & 1.00 & 1.00 & 1.00 & 1.00 & 4.00 & 15.00 & 28.00 & 52.00 & 60.00 \\
\hline 5775 & -- & -- & 1.00 & 1.00 & 3.00 & 6.00 & 16.70 & -- & -- & 7594 & 1.00 & 1.00 & 1.00 & 2.00 & 5.00 & 16.00 & 32.00 & 59.36 & 74.00 \\
\hline 5779 & -- & -- & 1.00 & 1.00 & 6.50 & 20.00 & 46.20 & -- & -- & 7596 & 1.00 & 1.00 & 1.00 & 2.00 & 4.00 & 14.75 & 28.00 & 62.16 & 77.14 \\
\hline 5840 & 1.00 & 1.00 & 1.00 & 2.00 & 5.00 & 18.00 & 34.00 & 60.76 & 81.60 & 7608 & 1.00 & 1.00 & 1.00 & 1.00 & 5.00 & 17.00 & 32.30 & 64.98 & 75.33 \\
\hline 5890 & 1.00 & 1.00 & 1.00 & 2.00 & 4.00 & 16.00 & 30.40 & 58.00 & 72.14 & 7622 & -- & -- & 1.00 & 1.00 & 2.00 & 17.00 & 27.00 & -- & -- \\
\hline 5891 & 1.00 & 1.00 & 1.00 & 2.00 & 3.00 & 12.00 & 24.60 & 52.32 & 68.72 & 7700 & 1.00 & 1.00 & 1.00 & 3.00 & 7.00 & 22.00 & 40.00 & 69.00 & 79.24 \\
\hline 5897 & 1.00 & 1.00 & 1.00 & 1.00 & 4.00 & 17.00 & 31.00 & 53.00 & 69.16 & 7706 & 1.00 & 1.00 & 1.00 & 1.00 & 4.00 & 16.00 & 30.00 & 60.46 & 74.46 \\
\hline
\end{tabular}


Appendix 4-5.5. Empirical distribution of storm duration defined by 24-hour minimum interevent time for hourly rainfall stations in Texas-Continued.

\begin{tabular}{|c|c|c|c|c|c|c|c|c|c|c|c|c|c|c|c|c|c|c|c|}
\hline \multicolumn{20}{|c|}{$\begin{array}{c}\text { Duration } \\
\text { (hours) }\end{array}$} \\
\hline $\begin{array}{l}\text { Sta- } \\
\text { tion } \\
\text { no. }\end{array}$ & $\begin{array}{c}\text { 1st } \\
\text { per- } \\
\text { centile }\end{array}$ & $\begin{array}{c}\text { 2nd } \\
\text { per- } \\
\text { centile }\end{array}$ & $\begin{array}{c}10 \text { th } \\
\text { per- } \\
\text { centile }\end{array}$ & $\begin{array}{c}25 \text { th } \\
\text { per- } \\
\text { centile }\end{array}$ & $\begin{array}{c}\text { 50th } \\
\text { per- } \\
\text { centile } \\
\text { (median) }\end{array}$ & $\begin{array}{c}\text { 75th } \\
\text { per- } \\
\text { centile }\end{array}$ & $\begin{array}{c}\text { 90th } \\
\text { per- } \\
\text { centile }\end{array}$ & $\begin{array}{c}\text { 98th } \\
\text { per- } \\
\text { centile }\end{array}$ & $\begin{array}{c}\text { 99th } \\
\text { per- } \\
\text { centile }\end{array}$ & $\begin{array}{l}\text { Sta- } \\
\text { tion } \\
\text { no. }\end{array}$ & $\begin{array}{c}\text { 1st } \\
\text { per- } \\
\text { centile }\end{array}$ & $\begin{array}{c}\text { 2nd } \\
\text { per- } \\
\text { centile }\end{array}$ & $\begin{array}{c}10 \text { th } \\
\text { per- } \\
\text { centile }\end{array}$ & $\begin{array}{c}25 \text { th } \\
\text { per- } \\
\text { centile }\end{array}$ & $\begin{array}{c}\text { 50th } \\
\text { per- } \\
\text { centile } \\
\text { (median) }\end{array}$ & $\begin{array}{c}\text { 75th } \\
\text { per- } \\
\text { centile }\end{array}$ & $\begin{array}{c}\text { 90th } \\
\text { per- } \\
\text { centile }\end{array}$ & $\begin{array}{c}\text { 98th } \\
\text { per- } \\
\text { centile }\end{array}$ & $\begin{array}{c}\text { 99th } \\
\text { per- } \\
\text { centile }\end{array}$ \\
\hline 7718 & -- & 1.00 & 1.00 & 2.00 & 8.00 & 24.00 & 39.00 & 80.20 & -- & 8910 & -- & -- & 1.80 & 3.00 & 5.00 & 15.00 & 36.80 & -- & -- \\
\hline 7745 & 1.00 & 1.00 & 1.00 & 2.50 & 10.00 & 24.50 & 42.80 & 75.80 & 101.70 & 8911 & 1.00 & 1.00 & 1.00 & 1.00 & 4.00 & 16.00 & 31.30 & 60.24 & 76.18 \\
\hline 7922 & 1.00 & 1.00 & 1.00 & 1.00 & 2.00 & 9.00 & 24.00 & 49.36 & 55.80 & 8924 & 1.00 & 1.00 & 1.00 & 1.00 & 3.00 & 8.00 & 25.00 & 43.30 & 50.60 \\
\hline 7936 & 1.00 & 1.00 & 1.00 & 2.00 & 6.00 & 18.00 & 31.00 & 70.00 & 82.00 & 8929 & -- & -- & 1.00 & 1.00 & 6.50 & 29.75 & 128.00 & -- & -- \\
\hline 7943 & 1.00 & 1.00 & 1.00 & 2.00 & 5.00 & 16.00 & 31.00 & 58.12 & 71.00 & 8942 & 1.00 & 1.00 & 1.00 & 1.00 & 6.00 & 17.00 & 32.00 & 61.00 & 76.10 \\
\hline 7944 & -- & 1.00 & 1.00 & 2.00 & 9.00 & 26.50 & 50.40 & 78.04 & -- & 8944 & 1.00 & 1.00 & 1.00 & 2.00 & 6.00 & 18.00 & 36.00 & 66.22 & 79.22 \\
\hline 7945 & 1.00 & 1.00 & 1.00 & 2.00 & 7.00 & 23.00 & 41.00 & 80.00 & 98.00 & 8996 & 1.00 & 1.00 & 1.00 & 2.00 & 5.00 & 19.00 & 35.00 & 70.92 & 86.00 \\
\hline 7947 & 1.00 & 1.00 & 1.00 & 1.00 & 6.00 & 18.00 & 44.00 & 62.24 & 87.96 & 9014 & -- & -- & 1.80 & 4.50 & 10.00 & 29.00 & 58.00 & -- & -- \\
\hline 7948 & 1.00 & 1.00 & 1.00 & 1.00 & 4.00 & 16.00 & 31.90 & 63.18 & 73.27 & 9037 & 1.00 & 1.00 & 1.00 & 2.00 & 3.00 & 11.25 & 26.00 & 52.86 & 76.51 \\
\hline 7951 & 1.00 & 1.00 & 1.00 & 2.00 & 6.00 & 18.00 & 32.00 & 63.00 & 82.04 & 9106 & 1.00 & 1.00 & 1.00 & 2.00 & 4.00 & 16.00 & 31.00 & 64.58 & 118.38 \\
\hline 7953 & -- & 1.00 & 1.00 & 2.00 & 4.00 & 21.00 & 47.00 & 118.54 & -- & 9107 & -- & -- & 1.00 & 1.00 & 1.00 & 7.00 & 37.20 & -- & -- \\
\hline 7981 & 1.00 & 1.00 & 1.00 & 1.00 & 4.00 & 16.75 & 33.40 & 72.66 & 83.98 & 9129 & -- & 1.00 & 1.00 & 1.00 & 3.00 & 21.00 & 47.80 & 97.40 & -- \\
\hline 7990 & -- & 1.00 & 1.00 & 2.00 & 6.00 & 22.75 & 42.50 & 126.90 & -- & 9163 & 1.00 & 1.00 & 1.00 & 2.00 & 5.00 & 16.00 & 30.00 & 58.00 & 72.00 \\
\hline 7992 & -- & -- & 2.60 & 4.00 & 9.00 & 38.00 & 45.80 & -- & -- & 9213 & 1.00 & 1.00 & 1.00 & 1.00 & 7.00 & 19.00 & 43.00 & 97.48 & 141.54 \\
\hline 7997 & -- & 1.00 & 1.00 & 2.00 & 5.00 & 17.00 & 32.70 & 62.90 & -- & 9214 & -- & -- & 2.00 & 3.00 & 4.50 & 14.50 & 83.90 & -- & -- \\
\hline 7999 & -- & -- & 1.00 & 1.00 & 4.50 & 13.50 & 36.90 & -- & -- & 9222 & 1.00 & 1.00 & 1.00 & 3.00 & 10.00 & 25.00 & 41.40 & 73.00 & 98.70 \\
\hline 8022 & -- & 1.00 & 1.00 & 2.00 & 3.50 & 23.50 & 33.50 & 82.90 & -- & 9248 & 1.00 & 1.00 & 1.00 & 1.00 & 3.00 & 19.00 & 32.20 & 67.52 & 74.44 \\
\hline 8023 & 1.00 & 1.00 & 1.00 & 1.00 & 3.00 & 12.00 & 27.00 & 50.00 & 59.31 & 9266 & -- & 1.00 & 1.00 & 2.00 & 6.00 & 17.00 & 31.20 & 59.32 & -- \\
\hline 8047 & 1.00 & 1.00 & 1.00 & 1.00 & 5.00 & 16.00 & 30.00 & 60.20 & 75.60 & 9270 & 1.00 & 1.00 & 1.00 & 1.00 & 3.00 & 14.00 & 27.30 & 53.00 & 75.84 \\
\hline 8060 & 1.00 & 1.00 & 1.00 & 2.00 & 5.00 & 19.00 & 38.30 & 97.60 & 102.72 & 9295 & -- & 1.00 & 1.00 & 1.00 & 3.50 & 9.25 & 25.00 & 57.16 & -- \\
\hline 8062 & -- & -- & 1.00 & 2.00 & 4.00 & 31.00 & 38.60 & -- & -- & 9304 & -- & -- & -- & -- & 12.00 & -- & -- & -- & -- \\
\hline 8068 & -- & -- & 1.00 & 1.00 & 5.50 & 17.25 & 28.80 & -- & -- & 9307 & 1.00 & 1.00 & 1.00 & 2.00 & 5.00 & 17.00 & 33.20 & 51.52 & 59.80 \\
\hline 8081 & 1.00 & 1.00 & 1.00 & 1.00 & 4.00 & 18.00 & 32.10 & 67.00 & 79.61 & 9328 & 1.00 & 1.00 & 1.00 & 1.00 & 5.00 & 16.00 & 33.20 & 70.20 & 124.56 \\
\hline 8089 & -- & -- & 1.00 & 2.00 & 4.00 & 13.00 & 32.00 & -- & -- & 9329 & -- & -- & 1.00 & 2.50 & 15.00 & 38.75 & 75.50 & -- & -- \\
\hline 8221 & -- & -- & 1.00 & 4.00 & 7.00 & 19.00 & 74.40 & -- & -- & 9345 & -- & -- & -- & -- & 10.00 & -- & -- & -- & -- \\
\hline 8252 & 1.00 & 1.00 & 1.00 & 1.00 & 3.00 & 13.00 & 27.00 & 53.28 & 65.56 & 9363 & 1.00 & 1.00 & 1.00 & 2.00 & 6.00 & 23.00 & 42.00 & 80.62 & 104.86 \\
\hline 8265 & 1.00 & 1.00 & 1.00 & 3.00 & 7.50 & 23.00 & 41.00 & 72.02 & 88.00 & 9364 & 1.00 & 1.00 & 1.00 & 2.00 & 5.00 & 22.00 & 41.00 & 75.36 & 88.68 \\
\hline 8289 & -- & -- & 1.00 & 1.00 & 4.50 & 16.50 & 32.70 & -- & -- & 9365 & -- & -- & 1.40 & 2.00 & 5.00 & 27.00 & 60.40 & -- & -- \\
\hline 8305 & 1.00 & 1.00 & 1.00 & 1.00 & 3.00 & 10.75 & 26.00 & 51.58 & 62.58 & 9371 & 1.00 & 1.00 & 1.00 & 2.00 & 6.00 & 22.00 & 38.80 & 57.68 & 69.92 \\
\hline 8335 & 1.00 & 1.00 & 1.00 & 2.00 & 7.00 & 18.00 & 35.00 & 64.00 & 77.84 & 9417 & 1.00 & 1.00 & 1.00 & 2.00 & 6.00 & 18.00 & 33.00 & 61.00 & 77.00 \\
\hline 8400 & 1.00 & 1.00 & 1.00 & 1.00 & 2.00 & 16.00 & 27.00 & 62.84 & 75.32 & 9419 & 1.00 & 1.00 & 1.00 & 2.00 & 7.00 & 21.00 & 38.00 & 70.00 & 84.00 \\
\hline 8445 & 1.00 & 1.00 & 1.00 & 2.00 & 6.00 & 19.00 & 37.00 & 69.44 & 77.22 & 9435 & -- & 1.00 & 1.00 & 1.00 & 3.00 & 16.00 & 31.80 & 59.04 & -- \\
\hline 8446 & 1.00 & 1.00 & 1.00 & 1.00 & 5.00 & 17.00 & 32.00 & 57.14 & 67.00 & 9491 & 1.00 & 1.00 & 1.00 & 2.00 & 6.00 & 19.00 & 34.00 & 62.78 & 75.89 \\
\hline 8451 & 1.00 & 1.00 & 1.00 & 2.00 & 4.00 & 16.00 & 33.70 & 54.54 & 62.16 & 9499 & 1.00 & 1.00 & 1.00 & 1.00 & 3.00 & 12.00 & 27.00 & 52.00 & 66.30 \\
\hline 8531 & 1.00 & 1.00 & 1.00 & 2.00 & 5.00 & 18.00 & 33.70 & 59.00 & 80.47 & 9522 & -- & -- & -- & 5.00 & 15.50 & 71.75 & -- & -- & -- \\
\hline 8541 & -- & 1.00 & 1.00 & 3.00 & 5.00 & 17.00 & 30.80 & 56.28 & -- & 9527 & 1.00 & 1.00 & 1.00 & 1.00 & 3.00 & 13.00 & 27.00 & 54.00 & 66.00 \\
\hline 8544 & 1.00 & 1.00 & 1.00 & 2.00 & 5.00 & 16.00 & 33.00 & 72.00 & 85.85 & 9532 & 1.00 & 1.00 & 1.00 & 1.00 & 5.00 & 15.00 & 29.00 & 57.00 & 69.52 \\
\hline 8545 & -- & -- & 1.00 & 1.00 & 11.00 & 28.00 & 52.00 & -- & -- & 9544 & -- & -- & -- & 1.00 & 3.00 & 17.00 & -- & -- & -- \\
\hline 8563 & 1.00 & 1.00 & 1.00 & 1.00 & 5.00 & 15.00 & 28.00 & 52.00 & 61.51 & 9565 & 1.00 & 1.00 & 1.00 & 1.00 & 4.00 & 13.00 & 26.90 & 55.56 & 71.39 \\
\hline 8566 & 1.00 & 1.00 & 1.00 & 1.00 & 4.00 & 13.00 & 26.00 & 60.00 & 71.60 & 9570 & 1.00 & 1.00 & 1.00 & 1.00 & 3.00 & 14.00 & 29.00 & 49.00 & 63.70 \\
\hline 8583 & 1.00 & 1.00 & 1.00 & 1.00 & 4.00 & 16.00 & 29.00 & 54.00 & 62.86 & 9574 & -- & -- & 1.00 & 1.00 & 4.00 & 14.00 & 31.00 & -- & -- \\
\hline 8584 & 1.00 & 1.00 & 1.00 & 1.00 & 4.00 & 15.00 & 31.00 & 60.96 & 73.00 & 9588 & 1.00 & 1.00 & 1.00 & 1.00 & 4.00 & 18.00 & 35.00 & 79.00 & 98.00 \\
\hline 8623 & 1.00 & 1.00 & 1.00 & 1.00 & 4.00 & 15.00 & 30.00 & 60.00 & 74.60 & 9665 & 1.00 & 1.00 & 1.00 & 2.00 & 5.00 & 17.00 & 32.00 & 64.00 & 76.24 \\
\hline 8625 & 1.00 & 1.00 & 1.00 & 2.00 & 6.00 & 18.00 & 33.00 & 59.00 & 73.10 & 9715 & 1.00 & 1.00 & 1.00 & 2.00 & 5.00 & 16.00 & 30.00 & 54.00 & 72.00 \\
\hline 8630 & 1.00 & 1.00 & 1.00 & 1.00 & 4.00 & 12.00 & 26.00 & 45.24 & 61.24 & 9729 & 1.00 & 1.00 & 1.00 & 2.00 & 6.00 & 19.00 & 33.00 & 64.90 & 78.00 \\
\hline 8631 & 1.00 & 1.00 & 1.00 & 2.00 & 4.00 & 14.00 & 31.00 & 66.18 & 81.77 & 9772 & 1.00 & 1.00 & 1.00 & 2.00 & 5.00 & 19.00 & 32.90 & 75.16 & 92.58 \\
\hline 8646 & 1.00 & 1.00 & 1.00 & 2.00 & 5.00 & 16.00 & 30.00 & 57.00 & 70.00 & 9814 & -- & -- & 1.30 & 2.25 & 5.50 & 15.75 & 25.70 & -- & -- \\
\hline 8647 & 1.00 & 1.00 & 1.00 & 1.00 & 4.00 & 15.00 & 27.00 & 56.84 & 63.63 & 9815 & 1.00 & 1.00 & 1.00 & 2.00 & 6.00 & 18.00 & 34.00 & 82.12 & 101.24 \\
\hline 8677 & 1.00 & 1.00 & 1.00 & 3.00 & 7.00 & 20.00 & 34.00 & 72.32 & 109.04 & 9816 & -- & 1.00 & 1.00 & 1.00 & 3.00 & 13.50 & 30.80 & 41.68 & -- \\
\hline 8696 & -- & -- & 1.00 & 4.00 & 8.00 & 24.00 & 52.00 & -- & -- & 9817 & 1.00 & 1.00 & 1.00 & 1.00 & 4.00 & 14.00 & 27.00 & 53.86 & 72.79 \\
\hline 8743 & 1.00 & 1.00 & 1.00 & 2.00 & 6.00 & 18.00 & 32.00 & 63.00 & 75.65 & 9829 & 1.00 & 1.00 & 1.00 & 1.00 & 3.00 & 13.00 & 27.00 & 53.50 & 62.00 \\
\hline 8761 & 1.00 & 1.00 & 1.00 & 1.00 & 3.00 & 13.00 & 27.00 & 51.12 & 58.00 & 9830 & 1.00 & 1.00 & 1.00 & 1.00 & 4.00 & 11.00 & 26.00 & 46.40 & 76.24 \\
\hline 8778 & 1.00 & 1.00 & 1.00 & 1.00 & 5.00 & 17.00 & 31.00 & 62.00 & 71.77 & 9858 & 1.00 & 1.00 & 1.00 & 2.00 & 4.00 & 12.00 & 26.00 & 51.00 & 57.00 \\
\hline 8845 & 1.00 & 1.00 & 1.00 & 1.00 & 5.00 & 19.00 & 34.00 & 73.00 & 87.00 & 9893 & 1.00 & 1.00 & 1.00 & 1.00 & 4.00 & 15.00 & 30.00 & 61.00 & 76.00 \\
\hline 8859 & 1.00 & 1.00 & 1.00 & 2.00 & 6.00 & 18.00 & 37.00 & 69.00 & 80.62 & 9916 & 1.00 & 1.00 & 1.00 & 2.00 & 6.00 & 17.00 & 29.20 & 54.64 & 64.82 \\
\hline 8898 & 1.00 & 1.00 & 1.00 & 2.25 & 6.00 & 19.00 & 31.90 & 64.14 & 76.57 & 9976 & 1.00 & 1.00 & 1.00 & 1.00 & 4.00 & 16.00 & 31.70 & 59.00 & 77.28 \\
\hline 8908 & -- & -- & 1.00 & 2.00 & 7.50 & 21.25 & 32.60 & -- & -- & & & & & & & & & & \\
\hline
\end{tabular}


Appendix 4-5.6. Empirical distribution of storm duration defined by 48-hour minimum interevent time for hourly rainfall stations in Texas.

[--, not available]

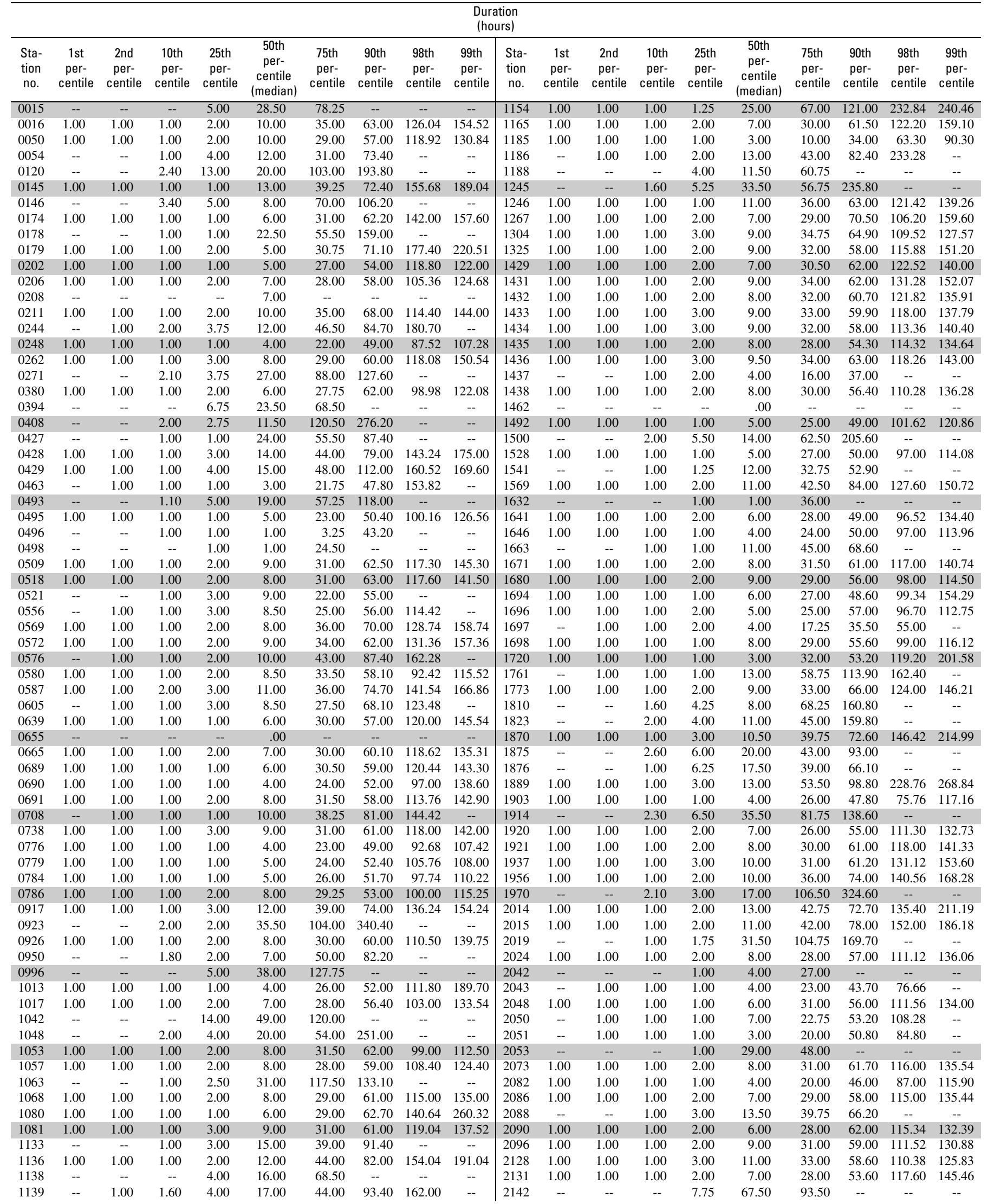


Appendix 4-5.6. Empirical distribution of storm duration defined by 48-hour minimum interevent time for hourly rainfall stations in Texas-Continued.

\begin{tabular}{|c|c|c|c|c|c|c|c|c|c|c|c|c|c|c|c|c|c|c|c|}
\hline \multicolumn{20}{|c|}{$\begin{array}{c}\text { Duration } \\
\text { (hours) }\end{array}$} \\
\hline $\begin{array}{l}\text { Sta- } \\
\text { tion } \\
\text { no. }\end{array}$ & $\begin{array}{c}\text { 1st } \\
\text { per- } \\
\text { centile }\end{array}$ & $\begin{array}{c}\text { 2nd } \\
\text { per- } \\
\text { centile }\end{array}$ & $\begin{array}{c}10 \text { th } \\
\text { per- } \\
\text { centile }\end{array}$ & $\begin{array}{c}\text { 25th } \\
\text { per- } \\
\text { centile }\end{array}$ & $\begin{array}{c}\text { 50th } \\
\text { per- } \\
\text { centile } \\
\text { (median) }\end{array}$ & $\begin{array}{c}\text { 75th } \\
\text { per- } \\
\text { centile }\end{array}$ & $\begin{array}{c}\text { 90th } \\
\text { per- } \\
\text { centile }\end{array}$ & $\begin{array}{c}\text { 98th } \\
\text { per- } \\
\text { centile }\end{array}$ & $\begin{array}{c}\text { 99th } \\
\text { per- } \\
\text { centile }\end{array}$ & $\begin{array}{l}\text { Sta- } \\
\text { tion } \\
\text { no. }\end{array}$ & $\begin{array}{c}1 \text { st } \\
\text { per- } \\
\text { centile }\end{array}$ & $\begin{array}{c}\text { 2nd } \\
\text { per- } \\
\text { centile }\end{array}$ & $\begin{array}{c}\text { 10th } \\
\text { per- } \\
\text { centile }\end{array}$ & $\begin{array}{c}25 \text { th } \\
\text { per- } \\
\text { centile }\end{array}$ & $\begin{array}{c}\text { 50th } \\
\text { per- } \\
\text { centile } \\
\text { (median) }\end{array}$ & $\begin{array}{c}\text { 75th } \\
\text { per- } \\
\text { centile }\end{array}$ & $\begin{array}{c}\text { 90th } \\
\text { per- } \\
\text { centile }\end{array}$ & $\begin{array}{c}\text { 98th } \\
\text { per- } \\
\text { centile }\end{array}$ & $\begin{array}{c}\text { 99th } \\
\text { per- } \\
\text { centile }\end{array}$ \\
\hline 2160 & -- & -- & 1.00 & 1.00 & 25.00 & 55.00 & 110.20 & -- & -- & 3463 & -- & -- & 1.00 & 2.00 & 8.00 & 25.00 & 65.00 & $\overline{--}$ & -- \\
\hline 2206 & 1.00 & 1.00 & 1.00 & 2.00 & 8.00 & 33.50 & 64.80 & 147.04 & 180.82 & 3476 & 1.00 & 1.00 & 1.00 & 2.00 & 7.00 & 30.75 & 65.10 & 126.28 & 156.42 \\
\hline 2238 & 1.00 & 1.00 & 1.00 & 2.00 & 9.00 & 33.50 & 63.60 & 117.72 & 171.76 & 3485 & -- & -- & 1.50 & 6.75 & 11.50 & 30.75 & 110.00 & -- & -- \\
\hline 2240 & -- & 1.00 & 1.00 & 1.00 & 19.00 & 49.00 & 91.00 & 120.04 & -- & 3507 & 1.00 & 1.00 & 1.00 & 1.00 & 8.00 & 32.00 & 67.00 & 117.00 & 168.00 \\
\hline 2242 & 1.00 & 1.00 & 1.00 & 3.00 & 11.00 & 35.00 & 63.70 & 133.54 & 164.27 & 3546 & 1.00 & 1.00 & 1.00 & 2.00 & 10.00 & 35.00 & 67.00 & 124.28 & 147.92 \\
\hline 2244 & 1.00 & 1.00 & 1.00 & 2.00 & 10.00 & 35.00 & 66.00 & 127.04 & 151.52 & 3547 & 1.00 & 1.00 & 1.00 & 1.00 & 7.00 & 27.50 & 52.80 & 98.56 & 132.94 \\
\hline 2247 & -- & -- & 1.20 & 5.00 & 12.00 & 41.00 & 109.60 & -- & -- & 3579 & -- & 1.00 & 2.00 & 3.00 & 14.00 & 47.00 & 64.80 & 117.92 & -- \\
\hline 2309 & 1.00 & 1.00 & 1.00 & 3.00 & 10.00 & 35.00 & 71.00 & 119.90 & 140.00 & 3642 & 1.00 & 1.00 & 1.00 & 2.00 & 9.00 & 30.00 & 62.00 & 113.00 & 146.96 \\
\hline 2312 & 1.00 & 1.00 & 1.00 & 2.00 & 11.00 & 33.00 & 64.00 & 116.08 & 139.08 & 3646 & 1.00 & 1.00 & 1.00 & 3.00 & 9.00 & 29.00 & 57.00 & 113.66 & 138.49 \\
\hline 2334 & -- & -- & 1.00 & 1.00 & 14.00 & 32.25 & 58.00 & -- & -- & 3668 & -- & -- & -- & 10.00 & 74.50 & 113.50 & -- & -- & -- \\
\hline 2336 & 1.00 & 1.00 & 1.00 & 2.00 & 5.50 & 25.75 & 53.70 & 129.62 & 151.85 & 3673 & -- & -- & 1.30 & 4.75 & 17.00 & 77.75 & 347.90 & -- & -- \\
\hline 2354 & -- & -- & 1.00 & 1.75 & 3.50 & 48.00 & 73.80 & -- & -- & 3686 & 1.00 & 1.00 & 1.00 & 2.00 & 9.00 & 34.00 & 59.00 & 117.00 & 145.80 \\
\hline 2355 & -- & -- & 1.00 & 2.00 & 15.00 & 53.50 & 80.60 & -- & -- & 3691 & 1.00 & 1.00 & 1.00 & 2.00 & 7.00 & 29.00 & 56.00 & 122.00 & 151.12 \\
\hline 2357 & 1.00 & 1.00 & 1.00 & 2.00 & 8.50 & 39.00 & 73.00 & 118.50 & 154.20 & 3734 & -- & -- & 1.10 & 2.75 & 23.00 & 93.00 & 343.20 & -- & -- \\
\hline 2360 & 1.00 & 1.00 & 1.00 & 2.00 & 7.00 & 31.00 & 63.00 & 119.00 & 135.75 & 3771 & 1.00 & 1.00 & 1.00 & 1.00 & 9.00 & 34.00 & 62.00 & 114.00 & 126.52 \\
\hline 2361 & 1.00 & 1.00 & 1.00 & 1.00 & 4.00 & 23.00 & 54.10 & 141.70 & 210.28 & 3789 & -- & 1.00 & 1.00 & 1.00 & 7.00 & 31.00 & 76.60 & 144.52 & -- \\
\hline 2394 & 1.00 & 1.00 & 1.00 & 3.00 & 10.00 & 33.00 & 65.00 & 121.00 & 148.81 & 3826 & 1.00 & 1.00 & 1.00 & 3.00 & 8.00 & 27.00 & 48.00 & 118.60 & 166.80 \\
\hline 2404 & 1.00 & 1.00 & 1.00 & 2.00 & 8.00 & 30.00 & 57.00 & 112.76 & 149.19 & 3831 & 1.00 & 1.00 & 1.00 & 2.00 & 7.00 & 32.00 & 68.00 & 92.20 & 103.80 \\
\hline 2415 & 1.00 & 1.00 & 1.00 & 3.00 & 9.00 & 32.00 & 64.00 & 118.54 & 142.08 & 3841 & 1.00 & 1.00 & 1.00 & 2.00 & 6.00 & 28.00 & 62.00 & 101.20 & 119.20 \\
\hline 2462 & 1.00 & 1.00 & 1.00 & 2.00 & 8.00 & 30.00 & 60.80 & 147.12 & 183.00 & 3871 & 1.00 & 1.00 & 1.00 & 2.00 & 8.00 & 30.50 & 58.00 & 97.08 & 144.02 \\
\hline 2528 & -- & 1.00 & 1.00 & 2.00 & 8.00 & 35.00 & 63.60 & 87.24 & -- & 3884 & -- & -- & 1.40 & 5.00 & 11.00 & 52.00 & 90.80 & -- & -- \\
\hline 2617 & 1.00 & 1.00 & 1.00 & 2.75 & 9.00 & 34.00 & 57.20 & 105.84 & 122.30 & 3941 & -- & -- & 1.00 & 3.00 & 10.00 & 41.00 & 59.80 & -- & -- \\
\hline 2619 & 1.00 & 1.00 & 1.00 & 1.00 & 6.00 & 26.00 & 53.10 & 97.72 & 123.02 & 3963 & -- & -- & -- & 1.00 & 11.00 & 35.25 & -- & -- & -- \\
\hline 2621 & 1.00 & 1.00 & 1.00 & 2.00 & 5.00 & 25.00 & 54.00 & 99.76 & 117.71 & 4040 & 1.00 & 1.00 & 1.00 & 2.00 & 7.00 & 27.00 & 53.20 & 113.88 & 141.00 \\
\hline 2675 & 1.00 & 1.00 & 1.00 & 2.00 & 8.00 & 28.00 & 64.20 & 126.12 & 151.08 & 4058 & -- & -- & 1.60 & 3.25 & 4.50 & 27.00 & 205.80 & -- & -- \\
\hline 2676 & 1.00 & 1.00 & 1.00 & 1.00 & 8.50 & 37.25 & 66.10 & 136.08 & 150.09 & 4098 & 1.00 & 1.00 & 1.00 & 1.00 & 4.00 & 23.00 & 49.00 & 96.96 & 112.98 \\
\hline 2679 & 1.00 & 1.00 & 1.00 & 1.00 & 6.00 & 28.00 & 55.00 & 103.16 & 132.24 & 4100 & 1.00 & 1.00 & 1.00 & 2.00 & 7.00 & 26.00 & 55.00 & 111.08 & 117.04 \\
\hline 2715 & 1.00 & 1.00 & 1.00 & 2.00 & 8.00 & 27.00 & 56.00 & 105.00 & 133.54 & 4137 & 1.00 & 1.00 & 1.00 & 2.00 & 8.00 & 30.00 & 56.00 & 124.60 & 153.00 \\
\hline 2744 & 1.00 & 1.00 & 1.00 & 2.00 & 6.00 & 26.00 & 52.60 & 101.00 & 118.36 & 4191 & 1.00 & 1.00 & 1.00 & 2.00 & 7.00 & 30.00 & 57.00 & 112.00 & 131.00 \\
\hline 2758 & -- & 1.00 & 1.00 & 2.25 & 12.50 & 45.00 & 94.70 & 125.16 & -- & 4256 & -- & -- & -- & -- & .00 & -- & -- & -- & -- \\
\hline 2794 & -- & -- & 1.00 & 1.00 & 6.00 & 16.00 & 43.30 & -- & -- & 4257 & 1.00 & 1.00 & 1.00 & 2.00 & 9.00 & 31.00 & 64.20 & 120.84 & 148.84 \\
\hline 2797 & 1.00 & 1.00 & 1.00 & 2.00 & 6.00 & 27.00 & 57.70 & 113.62 & 144.39 & 4258 & 1.00 & 1.00 & 1.00 & 2.00 & 12.00 & 33.00 & 92.40 & 187.32 & 196.70 \\
\hline 2811 & 1.00 & 1.00 & 1.00 & 1.00 & 5.00 & 27.00 & 56.00 & 107.00 & 118.08 & 4278 & 1.00 & 1.00 & 1.00 & 2.00 & 7.00 & 29.00 & 59.00 & 117.76 & 134.00 \\
\hline 2813 & -- & -- & 1.00 & 1.00 & 5.00 & 18.00 & 52.00 & -- & -- & 4299 & 1.00 & 1.00 & 1.00 & 2.00 & 4.00 & 14.75 & 38.80 & 75.94 & 77.98 \\
\hline 2814 & -- & -- & 1.00 & 1.00 & 11.00 & 72.00 & 81.00 & -- & -- & 4300 & 1.00 & 1.00 & 1.00 & 3.00 & 15.00 & 47.00 & 88.00 & 179.38 & 219.76 \\
\hline 2815 & 1.00 & 1.00 & 1.00 & 1.00 & 5.00 & 26.00 & 56.30 & 90.76 & 115.57 & 4305 & 1.00 & 1.00 & 1.00 & 3.00 & 15.00 & 46.00 & 93.70 & 173.36 & 211.68 \\
\hline 2818 & 1.00 & 1.00 & 1.00 & 1.00 & 5.00 & 20.00 & 54.40 & 98.64 & 173.44 & 4307 & 1.00 & 1.00 & 1.00 & 2.00 & 15.00 & 43.00 & 90.20 & 216.40 & 247.76 \\
\hline 2986 & 1.00 & 1.00 & 1.00 & 3.00 & 10.00 & 36.00 & 64.80 & 149.48 & 176.96 & 4309 & 1.00 & 1.00 & 1.00 & 2.00 & 10.00 & 37.00 & 70.00 & 131.88 & 162.00 \\
\hline 3005 & 1.00 & 1.00 & 1.00 & 2.00 & 7.00 & 27.00 & 57.00 & 111.00 & 136.84 & 4311 & 1.00 & 1.00 & 1.00 & 3.00 & 9.00 & 36.00 & 70.00 & 132.00 & 154.52 \\
\hline 3033 & 1.00 & 1.00 & 1.00 & 2.00 & 4.00 & 20.00 & 49.00 & 93.30 & 103.50 & 4313 & 1.00 & 1.00 & 2.00 & 4.00 & 18.00 & 40.00 & 68.80 & 115.12 & 143.76 \\
\hline 3034 & -- & -- & -- & -- & 1.00 & -- & -- & -- & -- & 4319 & -- & 1.00 & 2.00 & 4.00 & 12.00 & 40.00 & 86.20 & 144.96 & -- \\
\hline 3047 & -- & -- & 1.20 & 2.00 & 38.00 & 62.00 & 279.20 & -- & -- & 4329 & 1.00 & 1.00 & 1.00 & 2.00 & 10.00 & 33.50 & 67.00 & 126.44 & 141.66 \\
\hline 3103 & -- & -- & 1.00 & 1.00 & 3.00 & 19.00 & 47.00 & -- & -- & 4331 & -- & -- & -- & -- & .00 & -- & -- & -- & -- \\
\hline 3133 & 1.00 & 1.00 & 1.00 & 2.00 & 8.00 & 30.00 & 58.00 & 118.00 & 139.16 & 4375 & 1.00 & 1.00 & 1.00 & 1.00 & 6.00 & 28.00 & 56.30 & 119.88 & 136.93 \\
\hline 3156 & 1.00 & 1.00 & 1.00 & 2.00 & 8.00 & 31.00 & 65.20 & 99.52 & 159.40 & 4392 & 1.00 & 1.00 & 1.00 & 3.00 & 14.00 & 37.25 & 81.00 & 121.00 & 165.48 \\
\hline 3171 & 1.00 & 1.00 & 1.00 & 2.25 & 8.00 & 30.00 & 57.00 & 111.22 & 135.00 & 4425 & 1.00 & 1.00 & 1.00 & 1.00 & 3.00 & 19.00 & 46.90 & 106.00 & 131.28 \\
\hline 3189 & 1.00 & 1.00 & 1.00 & 1.00 & 5.00 & 29.50 & 59.00 & 130.20 & 180.10 & 4440 & 1.00 & 1.00 & 2.00 & 3.00 & 9.00 & 31.25 & 60.00 & 101.00 & 135.88 \\
\hline 3260 & 1.00 & 1.00 & 1.00 & 2.00 & 7.00 & 25.00 & 54.90 & 137.40 & 159.48 & 4476 & 1.00 & 1.00 & 1.00 & 2.00 & 9.00 & 31.00 & 57.00 & 101.00 & 123.57 \\
\hline 3267 & -- & 1.00 & 1.00 & 3.00 & 11.00 & 35.50 & 70.00 & 125.80 & -- & 4498 & -- & -- & -- & 1.50 & 4.00 & 26.00 & -- & -- & -- \\
\hline 3270 & 1.00 & 1.00 & 1.00 & 1.00 & 5.00 & 24.00 & 49.00 & 101.56 & 120.90 & 4517 & 1.00 & 1.00 & 1.00 & 2.00 & 8.00 & 31.00 & 61.00 & 119.00 & 136.50 \\
\hline 3272 & -- & -- & 1.00 & 1.25 & 13.50 & 55.50 & 123.00 & -- & -- & 4520 & 1.00 & 1.00 & 1.00 & 1.00 & 8.00 & 31.00 & 53.40 & 112.48 & 151.48 \\
\hline 3277 & -- & -- & 1.00 & 1.00 & 7.00 & 25.00 & 45.40 & -- & -- & 4525 & -- & -- & 1.00 & 2.00 & 10.00 & 72.75 & 284.80 & -- & -- \\
\hline 3278 & 1.00 & 1.00 & 1.00 & 1.00 & 4.00 & 23.00 & 49.90 & 111.02 & 178.24 & 4563 & -- & -- & 1.60 & 3.00 & 7.00 & 40.00 & 149.20 & -- & -- \\
\hline 3280 & 1.00 & 1.00 & 1.00 & 1.00 & 3.00 & 13.75 & 53.00 & 84.50 & 140.25 & 4570 & 1.00 & 1.00 & 1.00 & 1.00 & 6.00 & 27.00 & 54.00 & 103.62 & 125.86 \\
\hline 3281 & -- & -- & 1.00 & 1.00 & 2.00 & 5.50 & 42.20 & -- & -- & 4577 & 1.00 & 1.00 & 1.00 & 2.00 & 8.00 & 31.00 & 68.00 & 130.00 & 157.00 \\
\hline 3283 & 1.00 & 1.00 & 1.00 & 3.00 & 11.00 & 39.00 & 78.00 & 134.00 & 157.27 & 4591 & 1.00 & 1.00 & 1.00 & 3.00 & 9.00 & 30.00 & 71.00 & 129.00 & 149.00 \\
\hline 3284 & 1.00 & 1.00 & 1.00 & 2.00 & 8.00 & 30.00 & 62.00 & 124.76 & 157.76 & 4670 & 1.00 & 1.00 & 1.00 & 2.00 & 7.00 & 28.00 & 58.00 & 101.00 & 117.13 \\
\hline 3285 & 1.00 & 1.00 & 1.00 & 1.00 & 8.00 & 30.00 & 57.00 & 120.74 & 143.00 & 4671 & -- & 1.00 & 1.00 & 1.00 & 10.00 & 37.00 & 62.00 & 132.00 & -- \\
\hline 3329 & 1.00 & 1.00 & 1.00 & 2.00 & 10.00 & 32.00 & 64.00 & 108.96 & 131.96 & 4679 & 1.00 & 1.00 & 1.00 & 2.00 & 8.00 & 32.00 & 62.00 & 114.04 & 147.08 \\
\hline 3335 & 1.00 & 1.00 & 1.00 & 3.00 & 10.00 & 29.00 & 55.80 & 118.88 & 124.41 & 4696 & -- & -- & -- & 5.00 & 33.00 & 51.00 & -- & -- & -- \\
\hline 3370 & 1.00 & 1.00 & 1.00 & 2.00 & 7.00 & 27.75 & 54.00 & 121.14 & 154.00 & 4703 & -- & 1.00 & 1.00 & 1.00 & 9.00 & 37.00 & 70.00 & 146.00 & -- \\
\hline 3410 & 1.00 & 1.00 & 1.00 & 1.00 & 6.00 & 27.00 & 55.00 & 100.50 & 119.25 & 4704 & 1.00 & 1.00 & 1.00 & 3.00 & 14.00 & 37.00 & 64.40 & 139.56 & 56.70 \\
\hline 3415 & 1.00 & 1.00 & 1.00 & 2.00 & 8.00 & 29.25 & 61.00 & 117.00 & 143.23 & 4731 & -- & 1.00 & 1.00 & 1.00 & 16.00 & 34.00 & 76.30 & 169.38 & -- \\
\hline 3430 & 1.00 & 1.00 & 1.00 & 3.00 & 12.00 & 42.00 & 77.00 & 143.92 & 170.46 & 4792 & 1.00 & 1.00 & 1.00 & 1.00 & 6.00 & 29.00 & 60.00 & 120.00 & 152.00 \\
\hline 3431 & 1.00 & 1.00 & 1.00 & 1.00 & 19.00 & 55.00 & 97.00 & 239.40 & 276.05 & 4819 & 1.00 & 1.00 & 1.00 & 2.00 & 10.00 & 37.50 & 75.50 & 123.00 & 163.80 \\
\hline 3441 & -- & -- & 1.00 & 1.00 & 10.00 & 39.50 & 99.80 & -- & -- & 4852 & -- & -- & 1.00 & 1.00 & 1.00 & 21.00 & 107.00 & -- & -- \\
\hline 3442 & 1.00 & 1.00 & 1.00 & 3.00 & 8.00 & 28.00 & 54.00 & 90.20 & 107.93 & 4866 & 1.00 & 1.00 & 1.00 & 2.00 & 8.00 & 32.00 & 58.00 & 119.00 & 133.10 \\
\hline 3446 & 1.00 & 1.00 & 1.00 & 2.00 & 5.00 & 22.25 & 49.00 & 89.88 & 94.97 & 4876 & -- & 1.00 & 1.00 & 2.00 & 9.00 & 30.00 & 56.00 & 126.20 & -- \\
\hline 3460 & -- & -- & 1.00 & 2.00 & 11.00 & 26.00 & 67.00 & -- & -- & 4878 & 1.00 & 1.00 & 1.00 & 3.00 & 12.00 & 42.00 & 79.00 & 142.64 & 155.82 \\
\hline 3462 & 1.00 & 1.00 & 1.00 & 2.00 & 7.00 & 24.50 & 63.20 & 160.92 & 169.86 & 4880 & 1.00 & 1.00 & 1.00 & 2.00 & 6.00 & 27.00 & 55.00 & 106.56 & 139.00 \\
\hline
\end{tabular}


Appendix 4-5.6. Empirical distribution of storm duration defined by 48-hour minimum interevent time for hourly rainfall stations in Texas-Continued.

\begin{tabular}{|c|c|c|c|c|c|c|c|c|c|c|c|c|c|c|c|c|c|c|c|}
\hline \multicolumn{20}{|c|}{$\begin{array}{c}\text { Duration } \\
\text { (hours) }\end{array}$} \\
\hline $\begin{array}{l}\text { Sta- } \\
\text { tion } \\
\text { no. }\end{array}$ & $\begin{array}{c}1 \text { st } \\
\text { per- } \\
\text { centile }\end{array}$ & $\begin{array}{c}\text { 2nd } \\
\text { per- } \\
\text { centile }\end{array}$ & $\begin{array}{c}10 \text { th } \\
\text { per- } \\
\text { centile }\end{array}$ & $\begin{array}{c}\text { 25th } \\
\text { per- } \\
\text { centile }\end{array}$ & $\begin{array}{c}\text { 50th } \\
\text { per- } \\
\text { centile } \\
\text { (median) }\end{array}$ & $\begin{array}{c}\text { 75th } \\
\text { per- } \\
\text { centile }\end{array}$ & $\begin{array}{c}\text { 90th } \\
\text { per- } \\
\text { centile }\end{array}$ & $\begin{array}{c}\text { 98th } \\
\text { per- } \\
\text { centile }\end{array}$ & $\begin{array}{c}\text { 99th } \\
\text { per- } \\
\text { centile }\end{array}$ & $\begin{array}{l}\text { Sta- } \\
\text { tion } \\
\text { no. }\end{array}$ & $\begin{array}{c}\text { 1st } \\
\text { per- } \\
\text { centile }\end{array}$ & $\begin{array}{c}\text { 2nd } \\
\text { per- } \\
\text { centile }\end{array}$ & $\begin{array}{c}10 \text { th } \\
\text { per- } \\
\text { centile }\end{array}$ & $\begin{array}{c}25 \text { th } \\
\text { per- } \\
\text { centile }\end{array}$ & $\begin{array}{c}\text { 50th } \\
\text { per- } \\
\text { centile } \\
\text { (median) }\end{array}$ & $\begin{array}{c}\text { 75th } \\
\text { per- } \\
\text { centile }\end{array}$ & $\begin{array}{c}\text { 90th } \\
\text { per- } \\
\text { centile }\end{array}$ & $\begin{array}{c}\text { 98th } \\
\text { per- } \\
\text { centile }\end{array}$ & $\begin{array}{c}\text { 99th } \\
\text { per- } \\
\text { centile }\end{array}$ \\
\hline 4920 & 1.00 & 1.00 & 1.00 & 1.00 & 6.00 & 26.00 & 53.90 & 101.38 & 120.69 & 5957 & 1.00 & 1.00 & 1.00 & 2.00 & 6.00 & 28.00 & 57.70 & 104.00 & 128.85 \\
\hline 4934 & -- & -- & -- & 1.00 & 2.00 & 39.00 & -- & -- & -- & 5958 & 1.00 & 1.00 & 1.00 & 2.00 & 9.50 & 29.75 & 62.00 & 123.38 & 135.52 \\
\hline 4972 & 1.00 & 1.00 & 1.00 & 2.00 & 8.00 & 30.00 & 55.00 & 103.00 & 124.00 & 5973 & -- & 1.00 & 1.00 & 2.75 & 13.00 & 37.25 & 81.10 & 131.54 & -- \\
\hline 4973 & 1.00 & 1.00 & 1.00 & 3.00 & 9.00 & 27.25 & 59.00 & 122.30 & 175.00 & 5996 & 1.00 & 1.00 & 1.00 & 2.00 & 8.00 & 31.00 & 63.00 & 116.00 & 130.48 \\
\hline 4974 & 1.00 & 1.00 & 1.00 & 2.00 & 6.00 & 29.00 & 57.00 & 101.74 & 121.48 & 6017 & -- & 1.00 & 1.00 & 2.00 & 8.50 & 35.75 & 86.00 & 130.00 & -- \\
\hline 4975 & 1.00 & 1.00 & 1.00 & 2.00 & 9.00 & 32.00 & 57.00 & 120.00 & 147.10 & 6024 & 1.00 & 1.00 & 1.00 & 4.00 & 13.00 & 28.00 & 63.00 & 108.52 & 127.56 \\
\hline 4978 & 1.00 & 1.00 & 1.00 & 1.00 & 6.50 & 23.00 & 61.50 & 104.70 & 154.50 & 6050 & -- & -- & 1.00 & 5.50 & 11.00 & 16.50 & 44.20 & -- & -- \\
\hline 4979 & -- & -- & -- & 8.00 & 70.00 & 127.75 & -- & -- & -- & 6104 & 1.00 & 1.00 & 1.00 & 2.00 & 7.00 & 33.00 & 77.00 & 145.00 & 189.80 \\
\hline 4982 & 1.00 & 1.00 & 1.00 & 2.00 & 7.00 & 25.00 & 53.70 & 100.54 & 119.27 & 6108 & 1.00 & 1.00 & 1.00 & 2.00 & 9.00 & 32.00 & 62.30 & 119.00 & 138.00 \\
\hline 5018 & 1.00 & 1.00 & 1.00 & 3.00 & 7.00 & 25.00 & 55.00 & 124.70 & 179.65 & 6136 & 1.00 & 1.00 & 1.00 & 1.00 & 5.00 & 25.00 & 51.00 & 103.00 & 127.00 \\
\hline 5048 & 1.00 & 1.00 & 1.00 & 1.00 & 4.00 & 23.00 & 45.90 & 83.18 & 98.81 & 6166 & -- & 1.00 & 1.40 & 3.00 & 10.00 & 32.00 & 59.20 & 98.72 & -- \\
\hline 5049 & -- & 1.00 & 1.00 & 1.00 & 4.50 & 21.50 & 44.10 & 97.16 & -- & 6176 & 1.00 & 1.00 & 1.00 & 2.50 & 12.00 & 32.00 & 71.00 & 125.08 & 147.36 \\
\hline 5056 & -- & -- & -- & 5.00 & 12.50 & 19.25 & -- & -- & -- & 6177 & 1.00 & 1.00 & 1.00 & 2.00 & 9.00 & 32.00 & 64.00 & 117.82 & 143.82 \\
\hline 5057 & 1.00 & 1.00 & 1.00 & 2.00 & 8.00 & 35.00 & 69.00 & 138.72 & 160.90 & 6210 & 1.00 & 1.00 & 1.00 & 2.00 & 8.00 & 32.00 & 61.00 & 116.00 & 138.15 \\
\hline 5060 & 1.00 & 1.00 & 1.00 & 2.00 & 7.00 & 32.50 & 70.50 & 124.80 & 143.00 & 6211 & 1.00 & 1.00 & 1.00 & 3.00 & 13.00 & 45.00 & 73.00 & 146.12 & 194.92 \\
\hline 5081 & 1.00 & 1.00 & 1.00 & 4.00 & 11.00 & 37.00 & 72.00 & 131.90 & 146.05 & 6270 & 1.00 & 1.00 & 1.00 & 2.00 & 10.00 & 34.25 & 65.00 & 113.68 & 145.17 \\
\hline 5094 & 1.00 & 1.00 & 1.00 & 2.00 & 8.00 & 29.00 & 59.00 & 118.00 & 142.55 & 6275 & -- & -- & -- & -- & .00 & -- & -- & -- & -- \\
\hline 5113 & 1.00 & 1.00 & 1.00 & 2.00 & 8.00 & 32.00 & 63.90 & 115.18 & 135.18 & 6276 & -- & -- & 1.00 & 3.25 & 12.50 & 81.75 & 379.80 & -- & -- \\
\hline 5114 & -- & -- & -- & -- & .00 & -- & -- & -- & -- & 6335 & 1.00 & 1.00 & 1.00 & 3.00 & 9.00 & 29.00 & 61.00 & 116.60 & 135.80 \\
\hline 5123 & -- & -- & 1.00 & 2.25 & 10.00 & 27.25 & 31.70 & -- & -- & 6434 & -- & -- & 2.20 & 5.00 & 8.00 & 33.00 & 50.60 & -- & -- \\
\hline 5192 & 1.00 & 1.00 & 1.00 & 2.00 & 8.00 & 30.00 & 57.30 & 111.00 & 125.46 & 6504 & 1.00 & 1.00 & 1.00 & 1.00 & 5.00 & 25.00 & 56.00 & 105.34 & 127.35 \\
\hline 5193 & 1.00 & 1.00 & 1.00 & 2.00 & 9.00 & 33.00 & 68.00 & 125.36 & 146.36 & 6558 & -- & -- & 1.00 & 1.00 & 7.00 & 28.25 & 42.10 & -- & -- \\
\hline 5224 & 1.00 & 1.00 & 1.00 & 3.00 & 13.00 & 43.00 & 74.80 & 136.00 & 169.48 & 6615 & 1.00 & 1.00 & 1.00 & 1.00 & 4.00 & 25.50 & 50.60 & 104.84 & 151.64 \\
\hline 5228 & 1.00 & 1.00 & 1.00 & 1.00 & 6.00 & 32.50 & 61.00 & 144.40 & 154.10 & 6660 & -- & 1.00 & 1.00 & 3.00 & 10.00 & 41.75 & 63.20 & 100.28 & -- \\
\hline 5235 & -- & -- & 1.00 & 2.00 & 13.00 & 46.00 & 75.90 & -- & -- & 6663 & -- & 1.00 & 1.00 & 2.00 & 15.00 & 50.00 & 118.60 & 218.92 & -- \\
\hline 5247 & 1.00 & 1.00 & 1.00 & 2.00 & 7.00 & 27.00 & 56.60 & 108.00 & 128.16 & 6734 & 1.00 & 1.00 & 1.00 & 2.00 & 9.00 & 31.50 & 63.40 & 104.80 & 126.90 \\
\hline 5258 & 1.00 & 1.00 & 1.00 & 3.00 & 8.00 & 29.00 & 62.00 & 110.00 & 132.69 & 6736 & 1.00 & 1.00 & 1.00 & 1.00 & 4.00 & 22.00 & 48.40 & 97.76 & 121.88 \\
\hline 5303 & 1.00 & 1.00 & 1.00 & 2.00 & 7.00 & 30.00 & 58.20 & 99.48 & 115.64 & 6740 & -- & -- & 1.20 & 5.00 & 8.00 & 35.00 & 118.00 & -- & -- \\
\hline 5312 & 1.00 & 1.00 & 1.00 & 1.00 & 6.00 & 30.00 & 57.10 & 108.00 & 125.00 & 6750 & 1.00 & 1.00 & 1.00 & 1.00 & 12.00 & 43.00 & 88.60 & 208.84 & 231.96 \\
\hline 5341 & -- & -- & 1.10 & 2.75 & 5.50 & 80.00 & 401.60 & -- & -- & 6757 & 1.00 & 1.00 & 1.00 & 2.00 & 10.00 & 35.00 & 70.00 & 128.90 & 152.45 \\
\hline 5342 & -- & -- & -- & -- & .00 & -- & -- & -- & -- & 6775 & 1.00 & 1.00 & 1.00 & 2.00 & 10.00 & 32.00 & 63.80 & 104.60 & 114.16 \\
\hline 5348 & 1.00 & 1.00 & 1.00 & 2.00 & 10.00 & 34.25 & 61.00 & 111.66 & 144.33 & 6776 & 1.00 & 1.00 & 1.00 & 1.00 & 6.00 & 29.00 & 57.00 & 107.76 & 148.19 \\
\hline 5358 & 1.00 & 1.00 & 1.00 & 2.00 & 6.00 & 28.00 & 53.00 & 98.86 & 118.00 & 6788 & 1.00 & 1.00 & 1.20 & 3.00 & 8.00 & 26.00 & 50.80 & 103.64 & 122.40 \\
\hline 5398 & 1.00 & 1.00 & 1.00 & 3.00 & 10.00 & 34.00 & 70.00 & 126.00 & 147.00 & 6792 & 1.00 & 1.00 & 1.00 & 1.00 & 4.00 & 24.00 & 50.00 & 101.00 & 130.35 \\
\hline 5410 & 1.00 & 1.00 & 1.00 & 1.00 & 4.00 & 26.00 & 52.00 & 96.48 & 138.74 & 6794 & -- & -- & -- & 9.00 & 21.00 & 112.50 & -- & -- & -- \\
\hline 5411 & 1.00 & 1.00 & 1.00 & 2.00 & 9.00 & 34.00 & 66.90 & 126.96 & 155.43 & 6834 & 1.00 & 1.00 & 1.00 & 2.00 & 8.00 & 30.00 & 59.00 & 115.00 & 139.00 \\
\hline 5424 & 1.00 & 1.00 & 1.00 & 4.00 & 19.00 & 64.75 & 116.00 & 201.68 & 262.28 & 6893 & 1.00 & 1.00 & 1.00 & 1.00 & 3.00 & 18.00 & 44.00 & 92.00 & 104.92 \\
\hline 5429 & 1.00 & 1.00 & 1.00 & 2.00 & 8.00 & 33.50 & 66.00 & 132.88 & 145.88 & 6935 & 1.00 & 1.00 & 1.00 & 1.00 & 5.00 & 26.00 & 52.00 & 98.74 & 123.96 \\
\hline 5431 & -- & -- & 1.30 & 5.25 & 13.50 & 26.75 & 97.10 & -- & -- & 6981 & 1.00 & 1.00 & 1.00 & 2.00 & 9.50 & 30.75 & 51.10 & 119.16 & 149.07 \\
\hline 5461 & 1.00 & 1.00 & 1.00 & 2.00 & 8.00 & 31.00 & 62.00 & 117.00 & 141.00 & 7020 & -- & 1.00 & 1.00 & 3.25 & 14.50 & 34.50 & 73.10 & 117.52 & -- \\
\hline 5463 & 1.00 & 1.00 & 1.00 & 1.00 & 8.00 & 32.00 & 58.70 & 105.48 & 119.74 & 7060 & 1.00 & 1.00 & 1.00 & 1.00 & 5.00 & 24.00 & 50.10 & 109.44 & 128.33 \\
\hline 5471 & -- & -- & 1.00 & 1.00 & 2.00 & 52.50 & 86.80 & -- & -- & 7066 & 1.00 & 1.00 & 1.00 & 3.00 & 9.00 & 33.00 & 63.80 & 120.16 & 146.08 \\
\hline 5477 & -- & -- & 1.00 & 1.00 & 3.00 & 24.50 & 245.80 & -- & -- & 7074 & 1.00 & 1.00 & 1.00 & 1.00 & 4.00 & 23.50 & 52.00 & 101.04 & 125.00 \\
\hline 5528 & 1.00 & 1.00 & 1.00 & 2.00 & 7.00 & 28.00 & 53.00 & 109.98 & 132.98 & 7097 & -- & 1.00 & 1.00 & 2.00 & 10.00 & 47.00 & 92.30 & 253.12 & -- \\
\hline 5579 & -- & -- & -- & -- & 15.00 & -- & -- & -- & -- & 7116 & 1.00 & 1.00 & 1.00 & 2.00 & 7.00 & 28.00 & 53.00 & 111.60 & 142.50 \\
\hline 5580 & -- & -- & 1.60 & 3.00 & 19.00 & 52.00 & 76.40 & -- & -- & 7140 & 1.00 & 1.00 & 1.00 & 2.00 & 9.00 & 35.00 & 70.00 & 130.60 & 156.16 \\
\hline 5589 & 1.00 & 1.00 & 1.00 & 1.00 & 6.00 & 31.00 & 53.60 & 116.76 & 233.16 & 7173 & 1.00 & 1.00 & 1.00 & 3.00 & 16.00 & 47.00 & 81.80 & 162.52 & 218.76 \\
\hline 5590 & 1.00 & 1.00 & 1.00 & 2.00 & 14.50 & 42.50 & 64.50 & 151.30 & 169.37 & 7174 & 1.00 & 1.00 & 1.00 & 3.00 & 15.00 & 46.00 & 86.00 & 174.00 & 209.46 \\
\hline 5591 & 1.00 & 1.00 & 1.00 & 2.00 & 4.00 & 21.00 & 54.00 & 116.72 & 145.68 & 7213 & 1.00 & 1.00 & 1.00 & 2.00 & 8.00 & 32.00 & 60.40 & 118.28 & 139.84 \\
\hline 5592 & 1.00 & 1.00 & 1.00 & 2.00 & 4.00 & 25.00 & 49.00 & 109.26 & 155.52 & 7243 & 1.00 & 1.00 & 1.00 & 2.00 & 8.00 & 31.00 & 59.10 & 113.02 & 134.01 \\
\hline 5594 & 1.00 & 1.00 & 1.00 & 2.00 & 3.00 & 13.50 & 44.00 & 92.80 & 158.60 & 7262 & -- & 1.00 & 1.00 & 1.00 & 1.00 & 26.50 & 49.00 & 176.80 & -- \\
\hline 5595 & -- & -- & -- & -- & 1.50 & -- & -- & -- & -- & 7274 & 1.00 & 1.00 & 1.00 & 1.00 & 5.00 & 18.75 & 37.10 & 91.06 & 109.12 \\
\hline 5596 & 1.00 & 1.00 & 1.00 & 1.00 & 5.00 & 28.00 & 57.00 & 113.00 & 140.72 & 7300 & 1.00 & 1.00 & 1.00 & 2.00 & 7.00 & 25.00 & 50.00 & 100.00 & 118.00 \\
\hline 5600 & 1.00 & 1.00 & 1.00 & 2.00 & 4.00 & 28.75 & 52.00 & 145.06 & 165.24 & 7311 & -- & -- & 1.00 & 1.00 & 5.00 & 24.00 & 40.00 & -- & -- \\
\hline 5618 & -- & -- & 2.40 & 10.00 & 35.00 & 79.00 & 247.40 & -- & -- & 7363 & -- & -- & 1.20 & 3.00 & 9.00 & 62.00 & 284.80 & -- & -- \\
\hline 5650 & -- & -- & 1.60 & 6.50 & 13.50 & 45.75 & 84.60 & -- & -- & 7422 & 1.00 & 1.00 & 1.00 & 2.00 & 8.00 & 32.00 & 60.00 & 118.18 & 139.59 \\
\hline 5656 & 1.00 & 1.00 & 1.00 & 1.00 & 6.00 & 28.00 & 52.00 & 96.60 & 121.10 & 7431 & 1.00 & 1.00 & 1.00 & 1.00 & 4.00 & 20.00 & 48.00 & 93.64 & 120.30 \\
\hline 5658 & 1.00 & 1.00 & 1.00 & 2.00 & 7.00 & 28.00 & 57.00 & 104.00 & 118.53 & 7481 & 1.00 & 1.00 & 1.00 & 1.00 & 4.00 & 20.00 & 48.00 & 86.00 & 100.96 \\
\hline 5661 & 1.00 & 1.00 & 1.00 & 1.00 & 9.00 & 38.00 & 69.60 & 131.52 & 181.51 & 7497 & 1.00 & 1.00 & 1.00 & 1.00 & 10.00 & 32.50 & 65.80 & 126.12 & 142.56 \\
\hline 5666 & -- & -- & 1.00 & 2.00 & 14.50 & 48.25 & 58.60 & -- & -- & 7498 & 1.00 & 1.00 & 1.00 & 1.00 & 7.00 & 30.50 & 56.20 & 137.16 & 157.82 \\
\hline 5695 & 1.00 & 1.00 & 1.00 & 2.00 & 8.00 & 29.00 & 59.00 & 108.00 & 125.87 & 7499 & 1.00 & 1.00 & 1.00 & 1.00 & 7.00 & 30.00 & 57.00 & 109.54 & 142.54 \\
\hline 5742 & -- & -- & 1.00 & 2.00 & 9.00 & 23.00 & 101.80 & -- & -- & 7531 & -- & 1.00 & 1.00 & 2.25 & 10.00 & 44.50 & 70.00 & 107.84 & -- \\
\hline 5766 & -- & -- & -- & 30.50 & 57.00 & 120.50 & -- & -- & -- & 7534 & 1.00 & 1.00 & 1.00 & 2.00 & 8.00 & 31.50 & 56.60 & 95.32 & 108.22 \\
\hline 5770 & 1.00 & 1.00 & 1.00 & 2.00 & 8.00 & 31.00 & 60.00 & 114.00 & 141.31 & 7556 & 1.00 & 1.00 & 1.00 & 2.00 & 7.00 & 28.00 & 56.00 & 112.80 & 129.70 \\
\hline 5775 & -- & -- & -- & 1.00 & 23.00 & 46.25 & -- & -- & -- & 7594 & 1.00 & 1.00 & 1.00 & 2.00 & 8.00 & 32.00 & 66.00 & 121.20 & 145.10 \\
\hline 5779 & -- & -- & 1.00 & 1.00 & 6.50 & 20.00 & 46.20 & -- & -- & 7596 & 1.00 & 1.00 & 1.00 & 2.00 & 6.50 & 28.00 & 61.70 & 91.58 & 110.16 \\
\hline 5840 & 1.00 & 1.00 & 1.00 & 2.00 & 7.00 & 25.00 & 63.00 & 114.36 & 134.56 & 7608 & 1.00 & 1.00 & 1.00 & 2.00 & 9.00 & 36.00 & 65.40 & 118.08 & 151.08 \\
\hline 5890 & 1.00 & 1.00 & 1.00 & 2.00 & 7.00 & 28.00 & 56.00 & 108.68 & 138.68 & 7622 & -- & -- & 1.00 & 1.00 & 2.00 & 23.50 & 48.20 & -- & -- \\
\hline 5891 & 1.00 & 1.00 & 1.00 & 2.00 & 5.00 & 24.50 & 46.50 & 76.00 & 104.00 & 7700 & 1.00 & 1.00 & 1.00 & 3.00 & 13.00 & 42.00 & 76.00 & 135.76 & 150.76 \\
\hline 5897 & 1.00 & 1.00 & 1.00 & 1.00 & 10.00 & 32.00 & 58.20 & 113.28 & 129.00 & 7706 & 1.00 & 1.00 & 1.00 & 1.00 & 6.00 & 27.00 & 56.00 & 106.40 & 129.90 \\
\hline
\end{tabular}


Appendix 4-5.6. Empirical distribution of storm duration defined by 48-hour minimum interevent time for hourly rainfall stations in Texas-Continued.

\begin{tabular}{|c|c|c|c|c|c|c|c|c|c|c|c|c|c|c|c|c|c|c|c|}
\hline \multicolumn{20}{|c|}{$\begin{array}{c}\text { Duration } \\
\text { (hours) }\end{array}$} \\
\hline $\begin{array}{l}\text { Sta- } \\
\text { tion } \\
\text { no. }\end{array}$ & $\begin{array}{c}\text { 1st } \\
\text { per- } \\
\text { centile }\end{array}$ & $\begin{array}{c}\text { 2nd } \\
\text { per- } \\
\text { centile }\end{array}$ & $\begin{array}{c}\text { 10th } \\
\text { per- } \\
\text { centile }\end{array}$ & $\begin{array}{c}\text { 25th } \\
\text { per- } \\
\text { centile }\end{array}$ & $\begin{array}{c}\text { 50th } \\
\text { per- } \\
\text { centile } \\
\text { (median) }\end{array}$ & $\begin{array}{c}\text { 75th } \\
\text { per- } \\
\text { centile }\end{array}$ & $\begin{array}{c}\text { 90th } \\
\text { per- } \\
\text { centile }\end{array}$ & $\begin{array}{c}\text { 98th } \\
\text { per- } \\
\text { centile }\end{array}$ & $\begin{array}{c}\text { 99th } \\
\text { per- } \\
\text { centile }\end{array}$ & $\begin{array}{l}\text { Sta- } \\
\text { tion } \\
\text { no. }\end{array}$ & $\begin{array}{c}\text { 1st } \\
\text { per- } \\
\text { centile }\end{array}$ & $\begin{array}{c}\text { 2nd } \\
\text { per- } \\
\text { centile }\end{array}$ & $\begin{array}{c}\text { 10th } \\
\text { per- } \\
\text { centile }\end{array}$ & $\begin{array}{c}25 \text { th } \\
\text { per- } \\
\text { centile }\end{array}$ & $\begin{array}{c}\text { 50th } \\
\text { per- } \\
\text { centile } \\
\text { (median) }\end{array}$ & $\begin{array}{c}\text { 75th } \\
\text { per- } \\
\text { centile }\end{array}$ & $\begin{array}{c}\text { 90th } \\
\text { per- } \\
\text { centile }\end{array}$ & $\begin{array}{c}\text { 98th } \\
\text { per- } \\
\text { centile }\end{array}$ & $\begin{array}{c}\text { 99th } \\
\text { per- } \\
\text { centile }\end{array}$ \\
\hline 7718 & -- & 1.00 & 1.00 & 2.00 & 9.00 & 29.00 & 52.20 & 175.00 & -- & 8910 & -- & -- & 1.40 & 4.50 & 10.00 & 45.50 & 91.20 & -- & -- \\
\hline 7745 & 1.00 & 1.00 & 1.00 & 3.00 & 13.00 & 40.50 & 72.00 & 116.80 & 134.70 & 8911 & 1.00 & 1.00 & 1.00 & 2.00 & 7.00 & 32.00 & 66.00 & 147.54 & 162.18 \\
\hline 7922 & 1.00 & 1.00 & 1.00 & 1.00 & 4.00 & 20.00 & 43.00 & 97.12 & 116.60 & 8924 & 1.00 & 1.00 & 1.00 & 2.00 & 3.00 & 23.00 & 44.00 & 98.60 & 162.80 \\
\hline 7936 & 1.00 & 1.00 & 1.00 & 3.00 & 10.00 & 36.00 & 72.00 & 141.08 & 163.56 & 8929 & -- & -- & 1.00 & 1.00 & 5.00 & 36.00 & 278.40 & -- & -- \\
\hline 7943 & 1.00 & 1.00 & 1.00 & 2.00 & 7.00 & 30.00 & 60.00 & 114.92 & 142.96 & 8942 & 1.00 & 1.00 & 1.00 & 2.00 & 10.00 & 35.00 & 68.00 & 126.00 & 154.21 \\
\hline 7944 & -- & -- & 1.00 & 4.00 & 20.00 & 50.00 & 90.40 & -- & -- & 8944 & 1.00 & 1.00 & 1.00 & 2.00 & 9.00 & 39.00 & 74.00 & 123.96 & 142.68 \\
\hline 7945 & 1.00 & 1.00 & 1.00 & 2.00 & 14.00 & 42.00 & 77.00 & 148.78 & 181.39 & 8996 & 1.00 & 1.00 & 1.00 & 3.00 & 10.00 & 38.00 & 73.90 & 133.00 & 155.00 \\
\hline 7947 & 1.00 & 1.00 & 1.00 & 2.00 & 9.00 & 44.00 & 66.70 & 140.40 & 253.00 & 9014 & -- & -- & 2.40 & 8.25 & 34.00 & 101.50 & 202.00 & -- & -- \\
\hline 7948 & 1.00 & 1.00 & 1.00 & 2.00 & 6.00 & 29.00 & 58.00 & 119.24 & 144.00 & 9037 & 1.00 & 1.00 & 1.00 & 2.00 & 4.00 & 19.00 & 47.10 & 90.76 & 105.83 \\
\hline 7951 & 1.00 & 1.00 & 1.00 & 3.00 & 9.00 & 29.00 & 64.00 & 126.34 & 151.05 & 9106 & 1.00 & 1.00 & 1.00 & 2.00 & 4.00 & 25.00 & 47.20 & 115.44 & 201.04 \\
\hline 7953 & -- & 1.00 & 1.20 & 2.00 & 7.00 & 43.50 & 70.00 & 144.28 & -- & 9107 & -- & -- & 1.00 & 1.00 & 2.00 & 9.00 & 74.20 & -- & -- \\
\hline 7981 & 1.00 & 1.00 & 1.00 & 2.00 & 7.00 & 29.25 & 62.60 & 127.86 & 181.01 & 9129 & -- & 1.00 & 1.00 & 2.00 & 6.00 & 41.00 & 86.00 & 133.00 & -- \\
\hline 7990 & -- & 1.00 & 1.00 & 2.00 & 7.00 & 40.75 & 94.50 & 222.30 & -- & 9163 & 1.00 & 1.00 & 1.00 & 2.00 & 7.00 & 28.00 & 55.40 & 104.48 & 130.22 \\
\hline 7992 & -- & -- & 1.60 & 4.00 & 14.50 & 60.50 & 163.60 & -- & -- & 9213 & 1.00 & 1.00 & 1.00 & 1.00 & 13.00 & 49.00 & 89.20 & 155.00 & 176.00 \\
\hline 7997 & -- & 1.00 & 1.00 & 2.00 & 7.00 & 29.50 & 49.00 & 81.84 & -- & 9214 & -- & -- & 3.00 & 3.00 & 25.00 & 100.50 & 228.70 & -- & -- \\
\hline 7999 & -- & -- & 1.00 & 2.50 & 7.00 & 37.50 & 54.20 & -- & -- & 9222 & 1.00 & 1.00 & 1.00 & 4.00 & 16.00 & 33.00 & 56.00 & 187.00 & 224.20 \\
\hline 8022 & -- & -- & 1.00 & 2.00 & 4.00 & 29.00 & 43.00 & -- & -- & 9248 & -- & 1.00 & 1.00 & 2.00 & 10.50 & 29.75 & 62.60 & 114.20 & -- \\
\hline 8023 & 1.00 & 1.00 & 1.00 & 1.00 & 4.00 & 23.00 & 49.70 & 97.08 & 126.81 & 9266 & -- & 1.00 & 1.00 & 2.00 & 8.00 & 28.00 & 64.00 & 142.16 & -- \\
\hline 8047 & 1.00 & 1.00 & 1.00 & 2.00 & 7.50 & 29.00 & 59.00 & 107.66 & 138.00 & 9270 & 1.00 & 1.00 & 1.00 & 1.00 & 5.00 & 28.00 & 66.00 & 139.40 & 165.30 \\
\hline 8060 & -- & 1.00 & 1.00 & 2.00 & 6.00 & 42.00 & 85.20 & 205.68 & -- & 9295 & -- & 1.00 & 1.00 & 1.00 & 7.00 & 13.00 & 37.00 & 191.68 & -- \\
\hline 8062 & -- & -- & 1.00 & 2.00 & 14.50 & 34.50 & 47.70 & -- & -- & 9304 & -- & -- & -- & -- & 12.00 & -- & -- & -- & -- \\
\hline 8068 & -- & -- & 1.00 & 2.50 & 18.50 & 44.75 & 68.30 & -- & -- & 9307 & 1.00 & 1.00 & 1.00 & 2.00 & 6.00 & 25.75 & 49.70 & 100.68 & 172.91 \\
\hline 8081 & 1.00 & 1.00 & 1.00 & 2.00 & 7.00 & 29.00 & 58.00 & 120.42 & 147.05 & 9328 & 1.00 & 1.00 & 1.00 & 2.00 & 6.00 & 33.00 & 68.00 & 120.48 & 125.37 \\
\hline 8089 & -- & -- & 1.00 & 2.00 & 6.00 & 31.00 & 58.40 & -- & -- & 9329 & -- & -- & -- & 3.50 & 17.00 & 59.50 & -- & -- & -- \\
\hline 8221 & -- & -- & 7.30 & 10.00 & 29.50 & 107.25 & 148.80 & -- & -- & 9345 & -- & -- & -- & -- & 10.00 & -- & -- & -- & -- \\
\hline 8252 & 1.00 & 1.00 & 1.00 & 1.00 & 4.00 & 24.00 & 50.40 & 96.48 & 118.22 & 9363 & 1.00 & 1.00 & 1.00 & 2.00 & 15.00 & 46.00 & 79.40 & 155.24 & 173.64 \\
\hline 8265 & 1.00 & 1.00 & 2.00 & 3.00 & 13.00 & 43.00 & 77.00 & 139.00 & 160.80 & 9364 & 1.00 & 1.00 & 1.00 & 2.00 & 13.00 & 43.00 & 79.00 & 157.08 & 186.00 \\
\hline 8289 & -- & -- & 1.00 & 3.00 & 18.00 & 33.50 & 61.00 & -- & -- & 9365 & -- & -- & 1.20 & 2.00 & 5.00 & 42.00 & 67.20 & -- & -- \\
\hline 8305 & 1.00 & 1.00 & 1.00 & 1.00 & 4.00 & 23.00 & 53.00 & 103.00 & 123.00 & 9371 & -- & 1.00 & 1.00 & 2.00 & 13.00 & 41.50 & 68.20 & 111.24 & -- \\
\hline 8335 & 1.00 & 1.00 & 1.00 & 3.00 & 11.00 & 34.00 & 69.00 & 127.00 & 151.00 & 9417 & 1.00 & 1.00 & 1.00 & 2.00 & 9.00 & 33.00 & 62.30 & 119.46 & 148.92 \\
\hline 8400 & 1.00 & 1.00 & 1.00 & 1.00 & 6.00 & 31.50 & 71.00 & 112.32 & 139.22 & 9419 & 1.00 & 1.00 & 1.00 & 3.00 & 12.00 & 38.00 & 72.90 & 136.58 & 152.29 \\
\hline 8445 & 1.00 & 1.00 & 1.00 & 3.00 & 10.00 & 37.00 & 68.00 & 126.00 & 152.25 & 9435 & -- & 1.00 & 1.00 & 1.00 & 4.00 & 24.50 & 63.20 & 108.40 & -- \\
\hline 8446 & 1.00 & 1.00 & 1.00 & 2.00 & 8.00 & 32.00 & 63.00 & 113.66 & 146.32 & 9491 & 1.00 & 1.00 & 1.00 & 2.00 & 10.00 & 35.00 & 68.00 & 130.00 & 149.00 \\
\hline 8451 & 1.00 & 1.00 & 1.00 & 2.00 & 5.50 & 28.25 & 55.00 & 97.76 & 133.08 & 9499 & 1.00 & 1.00 & 1.00 & 1.00 & 5.00 & 26.00 & 51.00 & 101.00 & 121.14 \\
\hline 8531 & 1.00 & 1.00 & 1.00 & 2.00 & 8.00 & 32.00 & 60.70 & 123.08 & 140.77 & 9522 & -- & -- & -- & 3.50 & 8.00 & 188.00 & -- & -- & -- \\
\hline 8541 & -- & 1.00 & 2.00 & 3.00 & 6.00 & 23.50 & 59.20 & 127.96 & -- & 9527 & 1.00 & 1.00 & 1.00 & 1.00 & 5.00 & 28.00 & 60.00 & 107.28 & 142.00 \\
\hline 8544 & 1.00 & 1.00 & 1.00 & 3.00 & 10.00 & 35.00 & 66.80 & 110.80 & 129.76 & 9532 & 1.00 & 1.00 & 1.00 & 2.00 & 8.00 & 29.00 & 56.00 & 104.00 & 139.68 \\
\hline 8545 & -- & -- & 1.00 & 2.50 & 12.00 & 34.00 & 101.20 & -- & -- & 9544 & -- & -- & -- & 1.00 & 3.00 & 17.00 & -- & -- & -- \\
\hline 8563 & 1.00 & 1.00 & 1.00 & 2.00 & 9.00 & 30.00 & 57.40 & 116.08 & 146.94 & 9565 & 1.00 & 1.00 & 1.00 & 2.00 & 6.00 & 25.00 & 57.00 & 113.06 & 130.53 \\
\hline 8566 & 1.00 & 1.00 & 1.00 & 1.00 & 5.00 & 26.00 & 61.20 & 116.24 & 136.08 & 9570 & 1.00 & 1.00 & 1.00 & 1.00 & 4.00 & 29.00 & 56.40 & 104.36 & 139.92 \\
\hline 8583 & 1.00 & 1.00 & 1.00 & 1.00 & 9.00 & 30.00 & 57.30 & 115.98 & 154.65 & 9574 & -- & -- & 1.00 & 1.00 & 16.00 & 39.00 & 53.20 & -- & -- \\
\hline 8584 & 1.00 & 1.00 & 1.00 & 1.00 & 6.00 & 30.00 & 56.20 & 101.00 & 111.00 & 9588 & 1.00 & 1.00 & 1.00 & 1.00 & 6.00 & 30.00 & 63.00 & 136.00 & 160.88 \\
\hline 8623 & 1.00 & 1.00 & 1.00 & 2.00 & 7.00 & 29.50 & 57.00 & 116.80 & 138.90 & 9665 & 1.00 & 1.00 & 1.00 & 2.00 & 8.00 & 33.00 & 67.00 & 125.00 & 148.36 \\
\hline 8625 & 1.00 & 1.00 & 1.00 & 3.00 & 10.00 & 31.00 & 60.00 & 115.04 & 140.04 & 9715 & 1.00 & 1.00 & 1.00 & 2.00 & 8.00 & 31.00 & 57.00 & 115.48 & 140.62 \\
\hline 8630 & 1.00 & 1.00 & 1.00 & 2.00 & 5.00 & 22.00 & 48.00 & 89.28 & 110.35 & 9729 & 1.00 & 1.00 & 1.00 & 3.00 & 10.00 & 33.00 & 63.00 & 118.00 & 144.00 \\
\hline 8631 & 1.00 & 1.00 & 1.00 & 2.00 & 6.00 & 25.00 & 51.00 & 100.00 & 120.65 & 9772 & 1.00 & 1.00 & 1.00 & 2.00 & 9.00 & 30.50 & 62.40 & 146.08 & 169.68 \\
\hline 8646 & 1.00 & 1.00 & 1.00 & 2.00 & 7.00 & 28.00 & 58.20 & 119.44 & 137.44 & 9814 & -- & -- & 1.00 & 2.50 & 9.00 & 19.00 & 49.00 & -- & -- \\
\hline 8647 & 1.00 & 1.00 & 1.00 & 1.00 & 6.00 & 25.00 & 56.00 & 121.24 & 150.09 & 9815 & 1.00 & 1.00 & 1.00 & 3.00 & 9.00 & 33.00 & 71.00 & 127.04 & 147.17 \\
\hline 8677 & 1.00 & 1.00 & 1.40 & 3.50 & 8.00 & 33.00 & 69.40 & 142.16 & 161.18 & 9816 & -- & 1.00 & 1.00 & 1.00 & 3.50 & 15.00 & 43.40 & 152.22 & -- \\
\hline 8696 & -- & -- & 2.20 & 7.00 & 32.50 & 109.75 & 201.10 & -- & -- & 9817 & 1.00 & 1.00 & 1.00 & 1.00 & 6.00 & 25.00 & 54.00 & 106.10 & 125.85 \\
\hline 8743 & 1.00 & 1.00 & 1.00 & 3.00 & 9.00 & 34.00 & 65.00 & 119.32 & 143.16 & 9829 & 1.00 & 1.00 & 1.00 & 1.00 & 4.00 & 23.00 & 50.00 & 91.00 & 114.00 \\
\hline 8761 & 1.00 & 1.00 & 1.00 & 1.00 & 4.00 & 23.00 & 48.00 & 101.00 & 134.85 & 9830 & 1.00 & 1.00 & 1.00 & 1.00 & 4.00 & 21.00 & 43.00 & 98.60 & 186.20 \\
\hline 8778 & 1.00 & 1.00 & 1.00 & 2.00 & 10.00 & 35.00 & 68.00 & 123.00 & 147.21 & 9858 & 1.00 & 1.00 & 1.00 & 2.00 & 6.00 & 22.00 & 54.00 & 95.68 & 109.00 \\
\hline 8845 & 1.00 & 1.00 & 1.00 & 2.00 & 9.00 & 33.00 & 64.00 & 112.72 & 140.86 & 9893 & 1.00 & 1.00 & 1.00 & 2.00 & 7.00 & 28.00 & 56.00 & 101.98 & 112.98 \\
\hline 8859 & 1.00 & 1.00 & 1.00 & 2.00 & 8.00 & 33.00 & 63.40 & 126.56 & 158.12 & 9916 & 1.00 & 1.00 & 1.00 & 3.00 & 11.00 & 34.00 & 62.60 & 118.92 & 154.86 \\
\hline 8898 & 1.00 & 1.00 & 1.00 & 3.00 & 9.00 & 29.75 & 60.10 & 120.22 & 151.33 & 9976 & 1.00 & 1.00 & 1.00 & 2.00 & 6.00 & 30.00 & 57.00 & 100.00 & 124.60 \\
\hline 8908 & -- & -- & 1.00 & 2.25 & 9.50 & 23.50 & 107.50 & -- & -- & & & & & & & & & & \\
\hline
\end{tabular}


Appendix 4-5.7. Empirical distribution of storm duration defined by 72-hour minimum interevent time for hourly rainfall stations in Texas.

[--, not available]

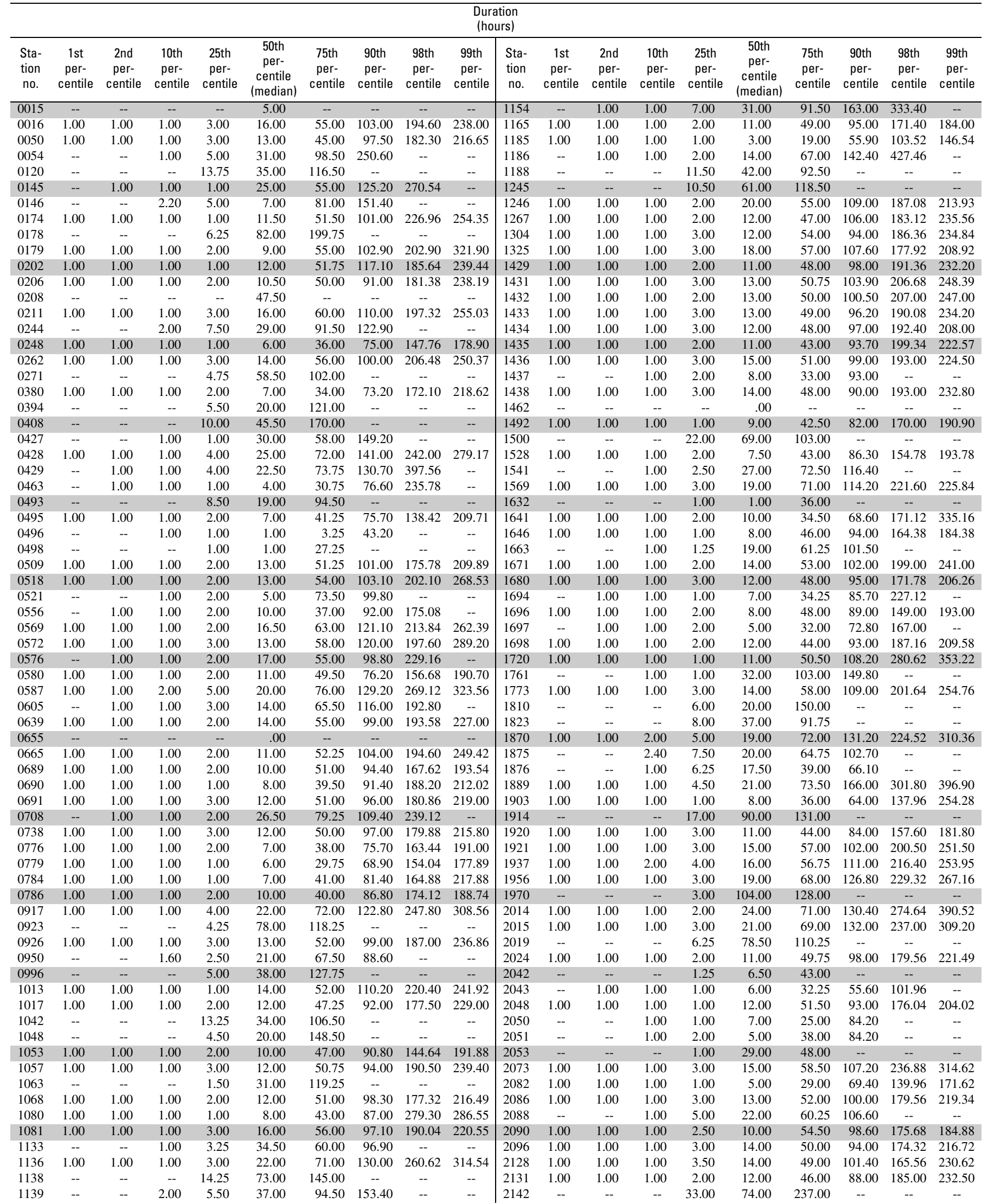


Appendix 4-5.7. Empirical distribution of storm duration defined by 72-hour minimum interevent time for hourly rainfall stations in Texas-Continued.

\begin{tabular}{|c|c|c|c|c|c|c|c|c|c|c|c|c|c|c|c|c|c|c|c|}
\hline \multicolumn{20}{|c|}{$\begin{array}{c}\text { Duration } \\
\text { (hours) }\end{array}$} \\
\hline $\begin{array}{l}\text { Sta- } \\
\text { tion } \\
\text { no. }\end{array}$ & $\begin{array}{c}\text { 1st } \\
\text { per- } \\
\text { centile }\end{array}$ & $\begin{array}{c}\text { 2nd } \\
\text { per- } \\
\text { centile }\end{array}$ & $\begin{array}{c}\text { 10th } \\
\text { per- } \\
\text { centile }\end{array}$ & $\begin{array}{c}25 \text { th } \\
\text { per- } \\
\text { centile }\end{array}$ & $\begin{array}{c}\text { 50th } \\
\text { per- } \\
\text { centile } \\
\text { (median) }\end{array}$ & $\begin{array}{c}\text { 75th } \\
\text { per- } \\
\text { centile }\end{array}$ & $\begin{array}{c}\text { 90th } \\
\text { per- } \\
\text { centile }\end{array}$ & $\begin{array}{c}\text { 98th } \\
\text { per- } \\
\text { centile }\end{array}$ & $\begin{array}{c}\text { 99th } \\
\text { per- } \\
\text { centile }\end{array}$ & $\begin{array}{l}\text { Sta- } \\
\text { tion } \\
\text { no. }\end{array}$ & $\begin{array}{c}1 \text { st } \\
\text { per- } \\
\text { centile }\end{array}$ & $\begin{array}{c}\text { 2nd } \\
\text { per- } \\
\text { centile }\end{array}$ & $\begin{array}{c}10 \text { th } \\
\text { per- } \\
\text { centile }\end{array}$ & $\begin{array}{c}25 \text { th } \\
\text { per- } \\
\text { centile }\end{array}$ & $\begin{array}{c}\text { 50th } \\
\text { per- } \\
\text { centile } \\
\text { (median) }\end{array}$ & $\begin{array}{c}\text { 75th } \\
\text { per- } \\
\text { centile }\end{array}$ & $\begin{array}{c}\text { 90th } \\
\text { per- } \\
\text { centile }\end{array}$ & $\begin{array}{c}\text { 98th } \\
\text { per- } \\
\text { centile }\end{array}$ & $\begin{array}{c}\text { 99th } \\
\text { per- } \\
\text { centile }\end{array}$ \\
\hline 2160 & -- & -- & 1.00 & 2.50 & 34.00 & 109.00 & 185.20 & -- & -- & 3463 & -- & -- & 1.00 & 2.75 & 8.50 & 71.75 & 115.80 & -- & -- \\
\hline 2206 & 1.00 & 1.00 & 1.00 & 2.00 & 12.00 & 57.00 & 117.00 & 203.88 & 229.18 & 3476 & 1.00 & 1.00 & 1.00 & 2.00 & 11.00 & 54.00 & 99.00 & 171.64 & 229.60 \\
\hline 2238 & 1.00 & 1.00 & 1.00 & 2.25 & 14.00 & 48.50 & 92.50 & 214.50 & 317.35 & 3485 & -- & -- & 1.10 & 5.75 & 12.50 & 84.75 & 253.50 & -- & -- \\
\hline 2240 & -- & -- & 1.00 & 5.50 & 19.00 & 56.50 & 108.40 & -- & -- & 3507 & 1.00 & 1.00 & 1.00 & 2.00 & 16.00 & 58.00 & 103.90 & 197.52 & 277.18 \\
\hline 2242 & 1.00 & 1.00 & 1.00 & 4.00 & 22.00 & 62.00 & 117.70 & 221.76 & 283.00 & 3546 & 1.00 & 1.00 & 1.00 & 3.00 & 17.50 & 64.00 & 118.00 & 209.82 & 254.69 \\
\hline 2244 & 1.00 & 1.00 & 1.00 & 3.00 & 19.00 & 65.00 & 124.00 & 218.76 & 257.88 & 3547 & 1.00 & 1.00 & 1.00 & 1.00 & 8.00 & 47.00 & 77.80 & 159.60 & 226.48 \\
\hline 2247 & -- & -- & 1.00 & 4.75 & 13.50 & 78.75 & 147.50 & -- & -- & 3579 & -- & -- & 2.00 & 2.75 & 20.50 & 49.50 & 116.00 & -- & -- \\
\hline 2309 & 1.00 & 1.00 & 1.00 & 3.00 & 12.00 & 65.00 & 104.00 & 221.44 & 248.44 & 3642 & 1.00 & 1.00 & 1.00 & 3.00 & 14.00 & 57.00 & 102.60 & 196.72 & 239.00 \\
\hline 2312 & 1.00 & 1.00 & 1.00 & 4.00 & 19.00 & 69.50 & 124.00 & 251.00 & 289.00 & 3646 & 1.00 & 1.00 & 1.00 & 3.00 & 11.00 & 44.75 & 94.00 & 170.00 & 212.52 \\
\hline 2334 & -- & -- & 1.00 & 1.00 & 16.00 & 55.75 & 95.30 & -- & -- & 3668 & -- & -- & -- & 7.00 & 76.00 & 180.75 & -- & -- & -- \\
\hline 2336 & 1.00 & 1.00 & 1.00 & 2.00 & 7.00 & 33.00 & 72.40 & 154.80 & 179.56 & 3673 & -- & -- & -- & 7.50 & 22.00 & 107.50 & -- & -- & -- \\
\hline 2354 & -- & -- & 1.00 & 3.00 & 47.00 & 67.00 & 98.40 & -- & -- & 3686 & 1.00 & 1.00 & 1.00 & 3.00 & 14.00 & 53.00 & 100.00 & 181.34 & 235.38 \\
\hline 2355 & -- & -- & 1.00 & 2.00 & 17.50 & 56.75 & 87.30 & -- & -- & 3691 & 1.00 & 1.00 & 1.00 & 2.00 & 12.00 & 52.00 & 99.00 & 186.80 & 220.80 \\
\hline 2357 & 1.00 & 1.00 & 1.00 & 2.00 & 22.00 & 60.00 & 107.80 & 180.96 & 230.46 & 3734 & -- & -- & -- & 8.00 & 81.00 & 161.00 & -- & -- & -- \\
\hline 2360 & 1.00 & 1.00 & 1.00 & 2.00 & 12.00 & 45.25 & 95.00 & 199.68 & 234.17 & 3771 & 1.00 & 1.00 & 1.00 & 2.00 & 20.00 & 57.00 & 100.80 & 202.40 & 238.44 \\
\hline 2361 & 1.00 & 1.00 & 1.00 & 1.00 & 6.00 & 29.00 & 92.40 & 241.48 & 289.94 & 3789 & -- & 1.00 & 1.00 & 1.00 & 13.00 & 67.00 & 140.20 & 241.00 & -- \\
\hline 2394 & 1.00 & 1.00 & 1.00 & 4.00 & 17.00 & 58.00 & 109.00 & 205.50 & 249.75 & 3826 & 1.00 & 1.00 & 1.00 & 3.50 & 11.00 & 40.50 & 87.20 & 196.16 & 262.08 \\
\hline 2404 & 1.00 & 1.00 & 1.00 & 3.00 & 15.00 & 54.00 & 101.00 & 179.84 & 231.64 & 3831 & 1.00 & 1.00 & 1.00 & 2.00 & 11.00 & 47.50 & 77.00 & 180.64 & 215.05 \\
\hline 2415 & 1.00 & 1.00 & 1.00 & 3.00 & 14.00 & 56.25 & 107.10 & 209.06 & 251.03 & 3841 & -- & 1.00 & 1.00 & 2.00 & 8.00 & 40.75 & 87.70 & 159.42 & -- \\
\hline 2462 & 1.00 & 1.00 & 1.00 & 2.00 & 13.00 & 46.50 & 94.80 & 194.60 & 246.68 & 3871 & 1.00 & 1.00 & 1.00 & 3.00 & 11.00 & 47.00 & 91.00 & 168.36 & 207.18 \\
\hline 2528 & -- & 1.00 & 1.00 & 4.00 & 22.00 & 64.00 & 85.00 & 127.12 & -- & 3884 & -- & -- & -- & 6.00 & 47.50 & 99.75 & -- & -- & -- \\
\hline 2617 & 1.00 & 1.00 & 1.00 & 3.00 & 11.50 & 45.00 & 87.70 & 179.74 & 222.73 & 3941 & -- & -- & 1.00 & 5.00 & 41.00 & 74.00 & 103.00 & -- & -- \\
\hline 2619 & 1.00 & 1.00 & 1.00 & 1.00 & 8.00 & 42.50 & 94.00 & 202.48 & 216.10 & 3963 & -- & -- & -- & 1.00 & 11.00 & 35.25 & -- & -- & -- \\
\hline 2621 & 1.00 & 1.00 & 1.00 & 2.00 & 9.00 & 38.50 & 79.40 & 164.56 & 189.96 & 4040 & 1.00 & 1.00 & 1.00 & 2.00 & 8.00 & 39.00 & 81.80 & 168.04 & 216.28 \\
\hline 2675 & 1.00 & 1.00 & 1.00 & 2.00 & 13.00 & 60.50 & 119.80 & 198.12 & 234.92 & 4058 & -- & -- & -- & 3.50 & 18.00 & 101.00 & -- & -- & -- \\
\hline 2676 & 1.00 & 1.00 & 1.00 & 2.00 & 18.00 & 63.00 & 120.80 & 209.88 & 238.60 & 4098 & 1.00 & 1.00 & 1.00 & 1.00 & 7.00 & 42.00 & 86.50 & 160.60 & 210.95 \\
\hline 2679 & 1.00 & 1.00 & 1.00 & 2.00 & 9.00 & 45.00 & 79.00 & 166.04 & 220.52 & 4100 & 1.00 & 1.00 & 1.00 & 2.00 & 8.50 & 43.00 & 88.20 & 173.10 & 189.86 \\
\hline 2715 & 1.00 & 1.00 & 1.00 & 3.00 & 10.00 & 42.00 & 81.30 & 176.90 & 253.03 & 4137 & 1.00 & 1.00 & 1.00 & 2.00 & 16.00 & 54.00 & 101.00 & 196.40 & 276.00 \\
\hline 2744 & 1.00 & 1.00 & 1.00 & 2.00 & 8.00 & 44.00 & 84.00 & 167.94 & 216.94 & 4191 & 1.00 & 1.00 & 1.00 & 2.00 & 11.00 & 49.00 & 93.80 & 178.56 & 209.90 \\
\hline 2758 & -- & 1.00 & 1.00 & 2.00 & 17.00 & 69.50 & 110.20 & 524.60 & -- & 4256 & -- & -- & -- & -- & .00 & -- & -- & -- & -- \\
\hline 2794 & -- & -- & -- & 10.00 & 45.00 & 71.00 & -- & -- & -- & 4257 & 1.00 & 1.00 & 1.00 & 3.00 & 14.00 & 58.00 & 103.80 & 203.12 & 257.56 \\
\hline 2797 & 1.00 & 1.00 & 1.00 & 2.00 & 10.00 & 43.50 & 88.80 & 202.00 & 241.62 & 4258 & -- & 1.00 & 1.00 & 2.25 & 14.50 & 74.00 & 167.00 & 348.60 & -- \\
\hline 2811 & 1.00 & 1.00 & 1.00 & 2.00 & 9.00 & 47.00 & 93.00 & 165.00 & 189.25 & 4278 & 1.00 & 1.00 & 1.00 & 3.00 & 9.00 & 46.00 & 89.70 & 160.14 & 191.49 \\
\hline 2813 & -- & -- & 1.00 & 1.25 & 5.00 & 39.00 & 109.40 & -- & -- & 4299 & -- & 1.00 & 1.00 & 2.00 & 4.00 & 23.00 & 69.00 & 124.56 & -- \\
\hline 2814 & -- & -- & -- & 1.00 & 72.00 & 82.00 & -- & -- & -- & 4300 & 1.00 & 1.00 & 1.00 & 5.00 & 28.00 & 84.00 & 169.00 & 301.30 & 347.55 \\
\hline 2815 & 1.00 & 1.00 & 1.00 & 1.00 & 5.00 & 33.00 & 65.80 & 227.68 & 254.68 & 4305 & 1.00 & 1.00 & 1.00 & 5.00 & 28.00 & 92.00 & 152.00 & 284.28 & 340.48 \\
\hline 2818 & 1.00 & 1.00 & 1.00 & 2.00 & 7.00 & 38.50 & 88.00 & 147.00 & 287.25 & 4307 & 1.00 & 1.00 & 1.00 & 4.00 & 30.50 & 92.75 & 160.70 & 310.86 & 416.54 \\
\hline 2986 & 1.00 & 1.00 & 1.00 & 4.00 & 17.00 & 61.75 & 125.50 & 204.40 & 239.76 & 4309 & 1.00 & 1.00 & 1.00 & 3.00 & 17.00 & 65.00 & 121.00 & 215.68 & 272.84 \\
\hline 3005 & 1.00 & 1.00 & 1.00 & 2.00 & 11.00 & 50.75 & 96.70 & 199.70 & 234.08 & 4311 & 1.00 & 1.00 & 1.00 & 3.00 & 15.00 & 61.00 & 118.60 & 207.00 & 262.30 \\
\hline 3033 & 1.00 & 1.00 & 1.00 & 2.00 & 4.00 & 30.00 & 71.00 & 143.40 & 153.90 & 4313 & 1.00 & 1.00 & 2.00 & 5.00 & 22.00 & 54.50 & 112.60 & 248.20 & 309.30 \\
\hline 3034 & -- & -- & -- & -- & 1.00 & -- & -- & -- & -- & 4319 & -- & -- & 2.00 & 5.00 & 23.00 & 94.50 & 149.60 & -- & -- \\
\hline 3047 & -- & -- & -- & 2.00 & 61.00 & 120.00 & -- & -- & -- & 4329 & 1.00 & 1.00 & 1.00 & 3.00 & 15.00 & 56.00 & 109.00 & 215.40 & 262.20 \\
\hline 3103 & -- & -- & 1.00 & 1.00 & 5.00 & 24.00 & 79.40 & -- & -- & 4331 & -- & -- & -- & -- & .00 & -- & -- & -- & -- \\
\hline 3133 & 1.00 & 1.00 & 1.00 & 3.00 & 13.00 & 54.50 & 98.60 & 164.04 & 201.52 & 4375 & 1.00 & 1.00 & 1.00 & 1.25 & 12.00 & 48.75 & 87.00 & 174.54 & 217.08 \\
\hline 3156 & 1.00 & 1.00 & 1.00 & 2.00 & 11.00 & 42.50 & 89.00 & 148.56 & 189.08 & 4392 & 1.00 & 1.00 & 2.00 & 5.00 & 26.50 & 74.50 & 120.00 & 225.28 & 254.93 \\
\hline 3171 & 1.00 & 1.00 & 1.00 & 3.00 & 12.00 & 49.00 & 92.00 & 179.40 & 225.80 & 4425 & 1.00 & 1.00 & 1.00 & 1.00 & 5.00 & 33.00 & 72.00 & 169.72 & 209.48 \\
\hline 3189 & 1.00 & 1.00 & 1.00 & 1.00 & 13.00 & 52.00 & 85.00 & 192.00 & 273.00 & 4440 & 1.00 & 1.00 & 2.00 & 3.00 & 14.00 & 51.00 & 96.00 & 171.84 & 221.10 \\
\hline 3260 & 1.00 & 1.00 & 1.00 & 2.00 & 7.50 & 31.00 & 73.10 & 166.44 & 172.35 & 4476 & 1.00 & 1.00 & 1.00 & 3.00 & 13.00 & 47.00 & 95.00 & 184.00 & 218.54 \\
\hline 3267 & -- & 1.00 & 1.00 & 3.25 & 15.50 & 55.50 & 108.00 & 178.02 & -- & 4498 & -- & -- & -- & 1.50 & 4.00 & 26.00 & -- & -- & -- \\
\hline 3270 & 1.00 & 1.00 & 1.00 & 1.00 & 7.00 & 43.00 & 81.60 & 148.00 & 184.64 & 4517 & 1.00 & 1.00 & 1.00 & 2.00 & 13.00 & 50.00 & 91.00 & 180.80 & 225.20 \\
\hline 3272 & -- & -- & -- & 1.50 & 11.00 & 78.00 & -- & -- & -- & 4520 & 1.00 & 1.00 & 1.00 & 2.00 & 14.00 & 51.00 & 99.00 & 201.16 & 261.34 \\
\hline 3277 & -- & -- & 1.00 & 1.00 & 13.00 & 37.00 & 53.00 & -- & -- & 4525 & -- & -- & -- & 3.00 & 77.00 & 128.00 & -- & -- & -- \\
\hline 3278 & 1.00 & 1.00 & 1.00 & 1.00 & 5.00 & 40.00 & 92.40 & 201.44 & 247.20 & 4563 & -- & -- & -- & 2.50 & 7.00 & 200.00 & -- & -- & -- \\
\hline 3280 & 1.00 & 1.00 & 1.00 & 2.00 & 4.00 & 28.50 & 66.00 & 129.96 & 206.38 & 4570 & 1.00 & 1.00 & 1.00 & 2.00 & 10.00 & 47.00 & 87.00 & 165.00 & 200.70 \\
\hline 3281 & -- & -- & 1.00 & 1.00 & 2.00 & 7.75 & 52.60 & -- & -- & 4577 & 1.00 & 1.00 & 1.00 & 3.00 & 13.00 & 58.00 & 114.00 & 211.48 & 278.40 \\
\hline 3283 & 1.00 & 1.00 & 1.00 & 4.00 & 21.00 & 70.00 & 123.80 & 226.56 & 324.04 & 4591 & 1.00 & 1.00 & 1.00 & 3.00 & 14.00 & 62.00 & 112.90 & 208.58 & 274.45 \\
\hline 3284 & 1.00 & 1.00 & 1.00 & 3.00 & 14.50 & 55.00 & 107.00 & 196.78 & 253.34 & 4670 & 1.00 & 1.00 & 1.00 & 2.00 & 9.00 & 46.00 & 87.00 & 169.62 & 211.29 \\
\hline 3285 & 1.00 & 1.00 & 1.00 & 2.00 & 12.00 & 50.00 & 102.00 & 213.40 & 262.80 & 4671 & -- & 1.00 & 1.00 & 1.00 & 19.00 & 55.00 & 97.00 & 280.36 & -- \\
\hline 3329 & 1.00 & 1.00 & 1.00 & 3.00 & 13.00 & 51.25 & 102.00 & 175.80 & 232.75 & 4679 & 1.00 & 1.00 & 1.00 & 2.00 & 15.00 & 55.25 & 98.00 & 184.90 & 226.95 \\
\hline 3335 & 1.00 & 1.00 & 2.00 & 4.00 & 20.00 & 54.25 & 103.20 & 181.76 & 239.75 & 4696 & -- & -- & -- & 5.00 & 33.00 & 51.00 & -- & -- & -- \\
\hline 3370 & 1.00 & 1.00 & 1.00 & 3.00 & 11.00 & 47.00 & 102.90 & 192.58 & 231.58 & 4703 & -- & 1.00 & 1.00 & 1.75 & 11.50 & 66.25 & 130.10 & 236.52 & -- \\
\hline 3410 & 1.00 & 1.00 & 1.00 & 2.00 & 9.00 & 45.00 & 89.40 & 166.04 & 196.08 & 4704 & 1.00 & 1.00 & 1.00 & 5.00 & 20.50 & 57.50 & 113.80 & 207.44 & 299.03 \\
\hline 3415 & 1.00 & 1.00 & 1.00 & 3.00 & 15.00 & 54.00 & 104.20 & 193.22 & 240.83 & 4731 & -- & 1.00 & 1.00 & 2.00 & 20.00 & 65.00 & 117.00 & 275.32 & -- \\
\hline 3430 & 1.00 & 1.00 & 1.00 & 4.00 & 23.00 & 74.00 & 139.00 & 257.22 & 305.00 & 4792 & 1.00 & 1.00 & 1.00 & 2.00 & 10.00 & 46.00 & 98.00 & 191.96 & 242.76 \\
\hline 3431 & 1.00 & 1.00 & 1.00 & 7.00 & 34.00 & 97.00 & 171.00 & 281.80 & 304.30 & 4819 & 1.00 & 1.00 & 1.00 & 3.00 & 14.00 & 71.50 & 129.00 & 242.00 & 321.50 \\
\hline 3441 & -- & -- & 1.00 & 1.00 & 13.00 & 66.00 & 123.10 & -- & -- & 4852 & -- & -- & -- & 3.75 & 38.50 & 68.50 & -- & -- & -- \\
\hline 3442 & 1.00 & 1.00 & 1.00 & 3.00 & 11.50 & 45.25 & 83.70 & 173.80 & 188.08 & 4866 & 1.00 & 1.00 & 1.00 & 3.00 & 11.00 & 45.00 & 91.00 & 184.64 & 219.35 \\
\hline 3446 & 1.00 & 1.00 & 1.00 & 2.00 & 6.00 & 37.25 & 71.50 & 117.70 & 145.90 & 4876 & -- & 1.00 & 1.00 & 3.00 & 20.50 & 48.00 & 88.80 & 229.52 & -- \\
\hline 3460 & -- & -- & 1.00 & 2.25 & 11.50 & 36.50 & 112.00 & -- & -- & 4878 & 1.00 & 1.00 & 1.00 & 4.00 & 21.00 & 72.00 & 124.00 & 240.96 & 285.97 \\
\hline 3462 & -- & 1.00 & 1.00 & 3.00 & 11.00 & 43.00 & 84.60 & 235.16 & -- & 4880 & 1.00 & 1.00 & 1.00 & 2.00 & 9.00 & 45.00 & 91.00 & 169.00 & 202.88 \\
\hline
\end{tabular}


Appendix 4-5.7. Empirical distribution of storm duration defined by 72-hour minimum interevent time for hourly rainfall stations in Texas-Continued.

\begin{tabular}{|c|c|c|c|c|c|c|c|c|c|c|c|c|c|c|c|c|c|c|c|}
\hline \multicolumn{20}{|c|}{$\begin{array}{c}\text { Duration } \\
\text { (hours) }\end{array}$} \\
\hline $\begin{array}{l}\text { Sta- } \\
\text { tion } \\
\text { no. }\end{array}$ & $\begin{array}{c}1 \text { st } \\
\text { per- } \\
\text { centile }\end{array}$ & $\begin{array}{l}\text { 2nd } \\
\text { per- } \\
\text { centile }\end{array}$ & $\begin{array}{c}10 \text { th } \\
\text { per- } \\
\text { centile }\end{array}$ & $\begin{array}{c}\text { 25th } \\
\text { per- } \\
\text { centile }\end{array}$ & $\begin{array}{c}\text { 50th } \\
\text { per- } \\
\text { centile } \\
\text { (median) }\end{array}$ & $\begin{array}{c}\text { 75th } \\
\text { per- } \\
\text { centile }\end{array}$ & $\begin{array}{c}\text { 90th } \\
\text { per- } \\
\text { centile }\end{array}$ & $\begin{array}{c}\text { 98th } \\
\text { per- } \\
\text { centile }\end{array}$ & $\begin{array}{c}\text { 99th } \\
\text { per- } \\
\text { centile }\end{array}$ & $\begin{array}{l}\text { Sta- } \\
\text { tion } \\
\text { no. }\end{array}$ & $\begin{array}{c}1 \text { st } \\
\text { per- } \\
\text { centile }\end{array}$ & $\begin{array}{c}\text { 2nd } \\
\text { per- } \\
\text { centile }\end{array}$ & $\begin{array}{c}10 \text { th } \\
\text { per- } \\
\text { centile }\end{array}$ & $\begin{array}{c}\text { 25th } \\
\text { per- } \\
\text { centile }\end{array}$ & $\begin{array}{c}\text { 50th } \\
\text { per- } \\
\text { centile } \\
\text { (median) }\end{array}$ & $\begin{array}{c}\text { 75th } \\
\text { per- } \\
\text { centile }\end{array}$ & $\begin{array}{c}\text { 90th } \\
\text { per- } \\
\text { centile }\end{array}$ & $\begin{array}{c}\text { 98th } \\
\text { per- } \\
\text { centile }\end{array}$ & $\begin{array}{c}\text { 99th } \\
\text { per- } \\
\text { centile }\end{array}$ \\
\hline 4920 & 1.00 & 1.00 & 1.00 & 2.00 & 9.00 & 43.00 & 84.60 & 162.72 & 189.08 & 5957 & 1.00 & 1.00 & 1.00 & 2.00 & 11.00 & 48.00 & 94.00 & 183.24 & 242.00 \\
\hline 4934 & -- & -- & -- & 1.00 & 2.00 & 39.00 & -- & -- & -- & 5958 & 1.00 & 1.00 & 1.00 & 3.00 & 17.00 & 55.75 & 112.30 & 152.62 & 162.72 \\
\hline 4972 & 1.00 & 1.00 & 1.00 & 3.00 & 11.00 & 49.00 & 95.70 & 173.42 & 200.14 & 5973 & -- & 1.00 & 1.00 & 3.00 & 17.50 & 65.50 & 127.90 & 246.38 & -- \\
\hline 4973 & 1.00 & 1.00 & 1.20 & 4.00 & 14.00 & 50.00 & 89.80 & 196.88 & 319.24 & 5996 & 1.00 & 1.00 & 1.00 & 2.00 & 12.00 & 51.00 & 97.00 & 179.00 & 228.63 \\
\hline 4974 & 1.00 & 1.00 & 1.00 & 2.00 & 9.00 & 43.00 & 81.10 & 172.10 & 208.00 & 6017 & -- & 1.00 & 1.00 & 2.00 & 18.50 & 76.75 & 113.80 & 210.08 & -- \\
\hline 4975 & 1.00 & 1.00 & 1.00 & 3.00 & 17.00 & 58.00 & 109.50 & 196.30 & 233.30 & 6024 & 1.00 & 1.00 & 1.00 & 4.00 & 18.00 & 48.00 & 96.00 & 164.36 & 190.52 \\
\hline 4978 & -- & 1.00 & 1.00 & 2.00 & 10.00 & 46.25 & 77.20 & 308.14 & -- & 6050 & -- & -- & 1.00 & 6.00 & 11.00 & 24.00 & 99.60 & -- & -- \\
\hline 4979 & -- & -- & -- & 11.50 & 75.50 & 193.25 & -- & -- & -- & 6104 & 1.00 & 1.00 & 1.00 & 2.00 & 12.00 & 58.00 & 122.80 & 239.16 & 288.08 \\
\hline 4982 & 1.00 & 1.00 & 1.00 & 3.00 & 10.00 & 43.00 & 89.00 & 165.48 & 203.28 & 6108 & 1.00 & 1.00 & 1.00 & 3.00 & 14.00 & 54.25 & 106.00 & 197.42 & 241.00 \\
\hline 5018 & 1.00 & 1.00 & 1.70 & 3.00 & 10.00 & 44.00 & 99.00 & 212.82 & 260.26 & 6136 & 1.00 & 1.00 & 1.00 & 1.00 & 8.00 & 44.00 & 88.60 & 174.60 & 206.52 \\
\hline 5048 & 1.00 & 1.00 & 1.00 & 1.00 & 7.00 & 31.00 & 69.40 & 136.56 & 174.56 & 6166 & -- & 1.00 & 2.00 & 3.25 & 23.00 & 51.50 & 92.60 & 128.02 & -- \\
\hline 5049 & -- & 1.00 & 1.00 & 1.00 & 6.00 & 40.00 & 78.20 & 181.72 & -- & 6176 & 1.00 & 1.00 & 2.00 & 3.00 & 16.00 & 65.00 & 119.00 & 213.00 & 322.00 \\
\hline 5056 & -- & -- & -- & -- & 42.50 & -- & -- & -- & -- & 6177 & 1.00 & 1.00 & 1.00 & 3.00 & 16.00 & 62.00 & 116.00 & 215.52 & 239.39 \\
\hline 5057 & 1.00 & 1.00 & 1.00 & 2.00 & 15.00 & 61.00 & 116.60 & 198.36 & 222.24 & 6210 & 1.00 & 1.00 & 1.00 & 3.00 & 13.00 & 53.00 & 100.00 & 208.80 & 230.00 \\
\hline 5060 & 1.00 & 1.00 & 1.00 & 2.00 & 13.00 & 73.50 & 128.60 & 225.80 & 290.40 & 6211 & 1.00 & 1.00 & 1.00 & 3.50 & 24.00 & 69.00 & 121.80 & 260.44 & 321.88 \\
\hline 5081 & 1.00 & 1.00 & 2.00 & 4.00 & 18.00 & 67.00 & 118.00 & 218.20 & 298.80 & 6270 & 1.00 & 1.00 & 1.00 & 3.00 & 16.00 & 61.25 & 109.10 & 206.68 & 255.05 \\
\hline 5094 & 1.00 & 1.00 & 1.00 & 2.00 & 12.00 & 52.00 & 101.30 & 183.66 & 221.98 & 6275 & -- & -- & -- & -- & .00 & -- & -- & -- & -- \\
\hline 5113 & 1.00 & 1.00 & 1.00 & 2.00 & 14.00 & 53.00 & 100.00 & 190.40 & 229.05 & 6276 & -- & -- & -- & 1.75 & 27.00 & 87.25 & -- & -- & -- \\
\hline 5114 & -- & -- & -- & -- & .00 & -- & -- & -- & -- & 6335 & 1.00 & 1.00 & 1.00 & 4.00 & 14.00 & 57.00 & 107.00 & 204.80 & 231.45 \\
\hline 5123 & -- & -- & 1.00 & 2.00 & 17.00 & 31.00 & 63.20 & -- & -- & 6434 & -- & -- & 4.00 & 5.75 & 18.00 & 34.00 & 73.00 & -- & -- \\
\hline 5192 & 1.00 & 1.00 & 1.00 & 3.00 & 14.00 & 52.25 & 101.00 & 196.72 & 239.48 & 6504 & 1.00 & 1.00 & 1.00 & 1.00 & 9.00 & 40.00 & 90.50 & 179.10 & 211.55 \\
\hline 5193 & 1.00 & 1.00 & 1.00 & 3.00 & 15.00 & 57.00 & 112.00 & 204.80 & 239.48 & 6558 & -- & -- & 1.00 & 2.25 & 14.50 & 35.75 & 60.10 & -- & -- \\
\hline 5224 & 1.00 & 1.00 & 1.00 & 4.00 & 21.50 & 73.75 & 126.60 & 254.12 & 356.15 & 6615 & 1.00 & 1.00 & 1.00 & 1.00 & 7.00 & 38.25 & 83.30 & 183.34 & 212.10 \\
\hline 5228 & 1.00 & 1.00 & 1.00 & 2.00 & 12.00 & 57.00 & 106.00 & 233.20 & 301.40 & 6660 & -- & 1.00 & 1.00 & 4.00 & 17.00 & 57.00 & 93.40 & 149.52 & -- \\
\hline 5235 & -- & -- & 1.00 & 2.00 & 22.00 & 54.50 & 113.60 & -- & -- & 6663 & -- & -- & 1.00 & 2.25 & 26.00 & 107.50 & 160.40 & -- & -- \\
\hline 5247 & 1.00 & 1.00 & 1.00 & 2.00 & 10.00 & 47.00 & 93.00 & 177.00 & 225.00 & 6734 & 1.00 & 1.00 & 1.00 & 3.00 & 17.50 & 59.00 & 116.50 & 204.10 & 220.05 \\
\hline 5258 & 1.00 & 1.00 & 1.00 & 3.00 & 14.00 & 53.50 & 103.60 & 184.56 & 276.52 & 6736 & 1.00 & 1.00 & 1.00 & 1.00 & 6.00 & 36.00 & 79.00 & 150.76 & 189.00 \\
\hline 5303 & 1.00 & 1.00 & 1.00 & 3.00 & 11.00 & 43.50 & 80.20 & 162.92 & 201.34 & 6740 & -- & -- & 1.10 & 4.25 & 15.00 & 49.75 & 121.00 & -- & -- \\
\hline 5312 & 1.00 & 1.00 & 1.00 & 1.00 & 9.00 & 46.00 & 97.00 & 182.32 & 218.56 & 6750 & 1.00 & 1.00 & 1.00 & 1.00 & 31.00 & 67.00 & 160.00 & 270.50 & 308.25 \\
\hline 5341 & -- & -- & -- & 3.00 & 55.00 & 83.00 & -- & -- & -- & 6757 & 1.00 & 1.00 & 1.00 & 3.00 & 17.00 & 66.00 & 120.10 & 221.00 & 248.55 \\
\hline 5342 & -- & -- & -- & -- & .00 & -- & -- & -- & -- & 6775 & 1.00 & 1.00 & 1.00 & 3.00 & 15.00 & 56.50 & 99.00 & 185.70 & 198.39 \\
\hline 5348 & 1.00 & 1.00 & 1.00 & 3.00 & 17.00 & 61.75 & 112.60 & 215.00 & 248.99 & 6776 & 1.00 & 1.00 & 1.00 & 2.00 & 10.00 & 51.00 & 96.80 & 194.96 & 257.40 \\
\hline 5358 & 1.00 & 1.00 & 1.00 & 2.00 & 9.00 & 43.00 & 80.20 & 160.72 & 189.86 & 6788 & 1.00 & 1.00 & 1.00 & 4.00 & 14.50 & 59.50 & 110.90 & 156.86 & 185.83 \\
\hline 5398 & 1.00 & 1.00 & 1.00 & 4.00 & 18.00 & 68.00 & 117.00 & 212.44 & 251.72 & 6792 & 1.00 & 1.00 & 1.00 & 1.00 & 6.00 & 41.00 & 77.00 & 156.64 & 222.96 \\
\hline 5410 & 1.00 & 1.00 & 1.00 & 1.00 & 7.00 & 41.00 & 82.00 & 192.00 & 238.00 & 6794 & -- & -- & -- & 76.25 & 102.00 & 171.25 & -- & -- & -- \\
\hline 5411 & 1.00 & 1.00 & 1.00 & 3.00 & 15.00 & 55.00 & 109.80 & 210.36 & 243.62 & 6834 & 1.00 & 1.00 & 1.00 & 2.00 & 15.00 & 58.25 & 110.50 & 198.50 & 233.50 \\
\hline 5424 & 1.00 & 1.00 & 1.00 & 7.00 & 35.00 & 97.00 & 186.40 & 380.00 & 469.00 & 6893 & 1.00 & 1.00 & 1.00 & 1.00 & 4.00 & 30.25 & 71.50 & 147.00 & 180.45 \\
\hline 5429 & 1.00 & 1.00 & 1.00 & 3.00 & 13.00 & 53.00 & 113.40 & 185.44 & 256.88 & 6935 & 1.00 & 1.00 & 1.00 & 1.00 & 7.00 & 40.00 & 86.00 & 169.76 & 208.00 \\
\hline 5431 & -- & -- & 1.20 & 4.00 & 22.00 & 67.00 & 103.60 & -- & -- & 6981 & 1.00 & 1.00 & 1.00 & 3.00 & 17.50 & 46.00 & 101.00 & 168.50 & 198.05 \\
\hline 5461 & 1.00 & 1.00 & 1.00 & 3.00 & 11.00 & 52.00 & 103.00 & 187.58 & 221.79 & 7020 & -- & 1.00 & 1.00 & 4.00 & 16.00 & 49.00 & 91.80 & 174.52 & -- \\
\hline 5463 & 1.00 & 1.00 & 1.00 & 2.00 & 13.00 & 51.00 & 101.80 & 177.16 & 197.56 & 7060 & 1.00 & 1.00 & 1.00 & 1.00 & 7.00 & 41.00 & 91.80 & 179.18 & 216.06 \\
\hline 5471 & -- & -- & -- & 1.00 & 33.00 & 78.00 & -- & -- & -- & 7066 & 1.00 & 1.00 & 1.00 & 3.00 & 15.00 & 59.00 & 110.00 & 211.54 & 260.59 \\
\hline 5477 & -- & -- & 1.00 & 1.00 & 4.00 & 27.00 & 285.60 & -- & -- & 7074 & 1.00 & 1.00 & 1.00 & 1.00 & 6.00 & 41.00 & 82.00 & 155.00 & 196.76 \\
\hline 5528 & 1.00 & 1.00 & 1.00 & 2.00 & 10.00 & 43.00 & 85.00 & 157.36 & 208.16 & 7097 & -- & 1.00 & 1.00 & 2.00 & 14.00 & 56.00 & 120.00 & 301.20 & -- \\
\hline 5579 & -- & -- & -- & -- & 15.00 & -- & -- & -- & -- & 7116 & 1.00 & 1.00 & 1.00 & 2.00 & 10.00 & 40.75 & 99.10 & 188.72 & 238.93 \\
\hline 5580 & -- & -- & -- & 12.25 & 52.00 & 214.00 & -- & -- & -- & 7140 & 1.00 & 1.00 & 1.00 & 3.00 & 15.00 & 57.75 & 111.50 & 212.70 & 278.65 \\
\hline 5589 & -- & 1.00 & 1.00 & 2.00 & 10.00 & 51.00 & 87.70 & 188.84 & -- & 7173 & 1.00 & 1.00 & 1.00 & 4.00 & 27.00 & 81.00 & 156.80 & 349.80 & 387.14 \\
\hline 5590 & -- & 1.00 & 1.00 & 2.00 & 19.00 & 65.25 & 120.70 & 220.60 & -- & 7174 & 1.00 & 1.00 & 1.00 & 5.00 & 27.00 & 86.00 & 170.00 & 311.16 & 362.08 \\
\hline 5591 & 1.00 & 1.00 & 1.00 & 2.00 & 5.00 & 40.00 & 86.60 & 157.72 & 172.86 & 7213 & 1.00 & 1.00 & 1.00 & 2.00 & 14.00 & 53.50 & 98.30 & 205.44 & 240.02 \\
\hline 5592 & 1.00 & 1.00 & 1.00 & 2.00 & 6.00 & 35.75 & 83.60 & 178.42 & 218.26 & 7243 & 1.00 & 1.00 & 1.00 & 2.00 & 13.00 & 52.00 & 98.00 & 187.64 & 223.10 \\
\hline 5594 & 1.00 & 1.00 & 1.00 & 2.00 & 3.50 & 27.25 & 73.50 & 157.90 & 181.70 & 7262 & -- & 1.00 & 1.00 & 1.00 & 7.00 & 49.00 & 67.00 & 224.44 & -- \\
\hline 5595 & -- & -- & -- & -- & 71.00 & -- & -- & -- & -- & 7274 & 1.00 & 1.00 & 1.00 & 2.00 & 6.00 & 26.00 & 65.00 & 103.62 & 119.86 \\
\hline 5596 & 1.00 & 1.00 & 1.00 & 1.00 & 10.00 & 51.00 & 97.20 & 189.36 & 215.76 & 7300 & 1.00 & 1.00 & 1.00 & 2.00 & 9.00 & 39.00 & 83.40 & 170.44 & 216.76 \\
\hline 5600 & 1.00 & 1.00 & 1.00 & 2.00 & 5.00 & 41.50 & 75.00 & 187.20 & 229.00 & 7311 & -- & -- & 1.00 & 1.25 & 7.00 & 37.25 & 101.00 & -- & -- \\
\hline 5618 & -- & -- & -- & 7.00 & 14.00 & 147.50 & -- & -- & -- & 7363 & -- & -- & -- & 4.50 & 67.50 & 113.00 & -- & -- & -- \\
\hline 5650 & -- & -- & -- & 6.50 & 12.50 & 109.50 & -- & -- & -- & 7422 & 1.00 & 1.00 & 1.00 & 2.00 & 13.00 & 50.00 & 97.40 & 187.12 & 218.28 \\
\hline 5656 & 1.00 & 1.00 & 1.00 & 1.00 & 11.00 & 47.00 & 94.00 & 177.84 & 218.55 & 7431 & 1.00 & 1.00 & 1.00 & 2.00 & 5.00 & 30.00 & 73.00 & 145.62 & 173.39 \\
\hline 5658 & 1.00 & 1.00 & 1.00 & 3.00 & 10.00 & 43.00 & 91.70 & 152.00 & 176.43 & 7481 & 1.00 & 1.00 & 1.00 & 2.00 & 6.00 & 38.00 & 75.00 & 127.90 & 147.60 \\
\hline 5661 & 1.00 & 1.00 & 1.00 & 1.00 & 13.00 & 49.25 & 94.40 & 194.08 & 206.55 & 7497 & 1.00 & 1.00 & 1.00 & 2.00 & 17.00 & 58.00 & 104.00 & 205.38 & 238.30 \\
\hline 5666 & -- & -- & 1.00 & 2.00 & 10.00 & 52.50 & 106.60 & -- & -- & 7498 & 1.00 & 1.00 & 1.00 & 2.00 & 10.00 & 55.00 & 131.00 & 213.16 & 255.64 \\
\hline 5695 & 1.00 & 1.00 & 1.00 & 2.50 & 12.00 & 52.00 & 97.00 & 171.08 & 201.72 & 7499 & 1.00 & 1.00 & 1.00 & 2.00 & 12.00 & 48.00 & 93.00 & 172.48 & 207.00 \\
\hline 5742 & -- & -- & 1.20 & 3.75 & 17.00 & 37.75 & 211.60 & -- & -- & 7531 & -- & 1.00 & 1.00 & 4.00 & 16.50 & 59.25 & 104.10 & 186.68 & -- \\
\hline 5766 & -- & -- & -- & 56.00 & 109.00 & 201.00 & -- & -- & -- & 7534 & 1.00 & 1.00 & 1.00 & 2.00 & 11.00 & 51.50 & 93.70 & 186.68 & 189.98 \\
\hline 5770 & 1.00 & 1.00 & 1.00 & 2.00 & 13.00 & 53.00 & 101.00 & 177.64 & 224.30 & 7556 & 1.00 & 1.00 & 1.00 & 2.00 & 12.00 & 50.00 & 95.00 & 178.80 & 233.34 \\
\hline 5775 & -- & -- & -- & 1.00 & 30.00 & 48.00 & -- & -- & -- & 7594 & 1.00 & 1.00 & 1.00 & 3.00 & 11.00 & 55.00 & 112.50 & 204.50 & 245.75 \\
\hline 5779 & -- & -- & 1.00 & 4.00 & 15.00 & 60.00 & 81.40 & -- & -- & 7596 & 1.00 & 1.00 & 1.00 & 2.00 & 9.50 & 34.75 & 74.00 & 142.70 & 181.41 \\
\hline 5840 & 1.00 & 1.00 & 1.00 & 3.00 & 9.00 & 38.00 & 84.00 & 205.60 & 263.80 & 7608 & 1.00 & 1.00 & 1.00 & 3.00 & 18.00 & 67.00 & 115.00 & 213.40 & 249.30 \\
\hline 5890 & 1.00 & 1.00 & 1.00 & 2.00 & 10.00 & 46.00 & 92.00 & 184.86 & 207.72 & 7622 & -- & -- & 1.00 & 1.00 & 2.00 & 30.75 & 158.50 & -- & -- \\
\hline 5891 & 1.00 & 1.00 & 1.00 & 2.00 & 8.00 & 33.00 & 56.80 & 166.48 & 179.92 & 7700 & 1.00 & 1.00 & 2.00 & 5.00 & 23.00 & 74.00 & 127.00 & 266.40 & 295.10 \\
\hline 5897 & 1.00 & 1.00 & 1.00 & 3.00 & 17.00 & 54.00 & 106.00 & 183.20 & 247.30 & 7706 & 1.00 & 1.00 & 1.00 & 2.00 & 11.00 & 50.00 & 90.00 & 167.74 & 210.70 \\
\hline
\end{tabular}


Appendix 4-5.7. Empirical distribution of storm duration defined by 72-hour minimum interevent time for hourly rainfall stations in Texas-Continued.

\begin{tabular}{|c|c|c|c|c|c|c|c|c|c|c|c|c|c|c|c|c|c|c|c|}
\hline \multicolumn{20}{|c|}{$\begin{array}{c}\text { Duration } \\
\text { (hours) }\end{array}$} \\
\hline $\begin{array}{l}\text { Sta- } \\
\text { tion } \\
\text { no. }\end{array}$ & $\begin{array}{c}\text { 1st } \\
\text { per- } \\
\text { centile }\end{array}$ & $\begin{array}{c}\text { 2nd } \\
\text { per- } \\
\text { centile }\end{array}$ & $\begin{array}{c}\text { 10th } \\
\text { per- } \\
\text { centile }\end{array}$ & $\begin{array}{c}\text { 25th } \\
\text { per- } \\
\text { centile }\end{array}$ & $\begin{array}{c}\text { 50th } \\
\text { per- } \\
\text { centile } \\
\text { (median) }\end{array}$ & $\begin{array}{c}\text { 75th } \\
\text { per- } \\
\text { centile }\end{array}$ & $\begin{array}{c}\text { 90th } \\
\text { per- } \\
\text { centile }\end{array}$ & $\begin{array}{c}\text { 98th } \\
\text { per- } \\
\text { centile }\end{array}$ & $\begin{array}{c}\text { 99th } \\
\text { per- } \\
\text { centile }\end{array}$ & $\begin{array}{l}\text { Sta- } \\
\text { tion } \\
\text { no. }\end{array}$ & $\begin{array}{c}\text { 1st } \\
\text { per- } \\
\text { centile }\end{array}$ & $\begin{array}{c}\text { 2nd } \\
\text { per- } \\
\text { centile }\end{array}$ & $\begin{array}{c}10 \text { th } \\
\text { per- } \\
\text { centile }\end{array}$ & $\begin{array}{c}\text { 25th } \\
\text { per- } \\
\text { centile }\end{array}$ & $\begin{array}{c}\text { 50th } \\
\text { per- } \\
\text { centile } \\
\text { (median) }\end{array}$ & $\begin{array}{c}\text { 75th } \\
\text { per- } \\
\text { centile }\end{array}$ & $\begin{array}{c}\text { 90th } \\
\text { per- } \\
\text { centile }\end{array}$ & $\begin{array}{c}\text { 98th } \\
\text { per- } \\
\text { centile }\end{array}$ & $\begin{array}{c}\text { 99th } \\
\text { per- } \\
\text { centile }\end{array}$ \\
\hline 7718 & -- & 1.00 & 1.00 & 2.00 & 10.00 & 40.00 & 96.00 & 265.88 & -- & 8910 & -- & -- & -- & 3.00 & 5.00 & 106.50 & -- & -- & -- \\
\hline 7745 & 1.00 & 1.00 & 1.00 & 4.00 & 26.00 & 73.50 & 121.60 & 232.16 & 254.58 & 8911 & 1.00 & 1.00 & 1.00 & 2.00 & 12.00 & 65.00 & 120.00 & 249.60 & 302.00 \\
\hline 7922 & 1.00 & 1.00 & 1.00 & 2.00 & 6.50 & 32.50 & 77.80 & 172.12 & 231.26 & 8924 & 1.00 & 1.00 & 1.00 & 2.00 & 4.00 & 28.00 & 67.00 & 139.44 & 206.58 \\
\hline 7936 & 1.00 & 1.00 & 1.00 & 4.00 & 18.00 & 66.00 & 123.00 & 239.12 & 298.26 & 8929 & -- & -- & -- & 1.00 & 5.00 & 63.00 & -- & -- & -- \\
\hline 7943 & 1.00 & 1.00 & 1.00 & 2.00 & 11.00 & 50.00 & 96.00 & 178.20 & 204.30 & 8942 & 1.00 & 1.00 & 1.00 & 3.00 & 16.00 & 63.00 & 118.00 & 225.80 & 288.70 \\
\hline 7944 & -- & -- & 1.00 & 8.50 & 34.00 & 107.00 & 154.80 & -- & -- & 8944 & 1.00 & 1.00 & 1.00 & 3.00 & 17.00 & 67.00 & 125.00 & 267.60 & 315.08 \\
\hline 7945 & 1.00 & 1.00 & 1.00 & 3.00 & 23.00 & 69.00 & 129.50 & 246.00 & 286.95 & 8996 & 1.00 & 1.00 & 1.00 & 3.00 & 19.00 & 68.50 & 124.00 & 224.00 & 290.74 \\
\hline 7947 & 1.00 & 1.00 & 1.00 & 2.50 & 20.00 & 59.00 & 117.00 & 296.60 & 349.10 & 9014 & -- & -- & -- & 13.00 & 47.00 & 121.00 & -- & -- & -- \\
\hline 7948 & 1.00 & 1.00 & 1.00 & 2.00 & 10.00 & 44.00 & 86.20 & 177.88 & 216.68 & 9037 & 1.00 & 1.00 & 1.00 & 2.00 & 6.00 & 30.00 & 72.10 & 134.66 & 148.22 \\
\hline 7951 & 1.00 & 1.00 & 1.00 & 3.00 & 14.00 & 63.50 & 119.40 & 219.16 & 269.14 & 9106 & 1.00 & 1.00 & 1.00 & 2.00 & 4.00 & 31.00 & 75.00 & 203.70 & 225.30 \\
\hline 7953 & -- & 1.00 & 2.00 & 2.00 & 7.00 & 56.25 & 103.40 & 200.80 & -- & 9107 & -- & -- & 1.00 & 1.00 & 2.00 & 32.25 & 102.00 & -- & -- \\
\hline 7981 & 1.00 & 1.00 & 1.00 & 2.00 & 8.00 & 45.50 & 80.00 & 178.56 & 197.96 & 9129 & -- & 1.00 & 1.00 & 3.00 & 9.00 & 52.00 & 97.80 & 173.48 & -- \\
\hline 7990 & -- & 1.00 & 1.00 & 3.00 & 25.50 & 81.75 & 196.10 & 295.84 & -- & 9163 & 1.00 & 1.00 & 1.00 & 2.00 & 11.00 & 48.00 & 94.20 & 187.08 & 212.02 \\
\hline 7992 & -- & -- & -- & 10.50 & 71.00 & 229.25 & -- & -- & -- & 9213 & 1.00 & 1.00 & 1.00 & 1.00 & 25.00 & 73.00 & 147.40 & 299.48 & 370.06 \\
\hline 7997 & -- & 1.00 & 1.00 & 2.00 & 7.50 & 34.50 & 59.50 & 112.00 & -- & 9214 & -- & -- & -- & 59.25 & 117.00 & 233.00 & -- & -- & -- \\
\hline 7999 & -- & -- & 1.00 & 2.50 & 7.00 & 37.50 & 54.20 & -- & -- & 9222 & 1.00 & 1.00 & 1.00 & 5.00 & 23.00 & 56.00 & 117.20 & 231.28 & 241.00 \\
\hline 8022 & -- & -- & 1.00 & 2.00 & 4.50 & 30.00 & 72.10 & -- & -- & 9248 & -- & 1.00 & 1.00 & 2.00 & 12.50 & 30.25 & 68.30 & 133.34 & -- \\
\hline 8023 & 1.00 & 1.00 & 1.00 & 2.00 & 6.00 & 37.00 & 76.70 & 156.28 & 187.28 & 9266 & -- & 1.00 & 1.00 & 2.25 & 10.50 & 37.25 & 74.50 & 148.08 & -- \\
\hline 8047 & 1.00 & 1.00 & 1.00 & 2.00 & 11.00 & 49.75 & 94.00 & 170.20 & 216.10 & 9270 & 1.00 & 1.00 & 1.00 & 1.00 & 8.00 & 52.00 & 101.80 & 211.12 & 240.76 \\
\hline 8060 & -- & 1.00 & 1.00 & 2.00 & 7.50 & 56.75 & 118.80 & 270.66 & -- & 9295 & -- & 1.00 & 1.00 & 1.00 & 7.00 & 70.00 & 129.40 & 212.92 & -- \\
\hline 8062 & -- & -- & 1.00 & 3.75 & 31.00 & 41.25 & 81.20 & -- & -- & 9304 & -- & -- & -- & -- & 20.50 & -- & -- & -- & -- \\
\hline 8068 & -- & -- & 1.00 & 5.50 & 24.00 & 48.00 & 72.60 & -- & -- & 9307 & 1.00 & 1.00 & 1.00 & 2.00 & 8.00 & 38.00 & 85.80 & 161.88 & 214.40 \\
\hline 8081 & 1.00 & 1.00 & 1.00 & 2.00 & 12.00 & 52.00 & 97.00 & 204.44 & 254.72 & 9328 & 1.00 & 1.00 & 1.00 & 2.00 & 8.50 & 52.75 & 121.20 & 233.94 & 256.40 \\
\hline 8089 & -- & -- & 1.00 & 2.00 & 6.00 & 64.75 & 112.40 & -- & -- & 9329 & -- & -- & -- & 3.00 & 23.00 & 95.00 & -- & -- & -- \\
\hline 8221 & -- & -- & -- & 10.00 & 29.50 & 115.75 & -- & -- & -- & 9345 & -- & -- & -- & -- & 10.00 & -- & -- & -- & -- \\
\hline 8252 & 1.00 & 1.00 & 1.00 & 2.00 & 6.00 & 36.00 & 73.50 & 149.10 & 187.15 & 9363 & 1.00 & 1.00 & 1.00 & 3.00 & 27.00 & 80.00 & 145.40 & 266.92 & 303.84 \\
\hline 8265 & 1.00 & 1.00 & 2.00 & 4.00 & 22.00 & 72.00 & 127.30 & 243.06 & 300.53 & 9364 & 1.00 & 1.00 & 1.00 & 3.00 & 25.00 & 74.00 & 144.00 & 261.00 & 295.28 \\
\hline 8289 & -- & -- & 1.00 & 3.00 & 19.00 & 52.00 & 74.80 & -- & -- & 9365 & -- & -- & 1.90 & 2.00 & 27.00 & 66.50 & 102.10 & -- & -- \\
\hline 8305 & 1.00 & 1.00 & 1.00 & 1.00 & 6.00 & 39.00 & 77.20 & 164.00 & 205.00 & 9371 & -- & 1.00 & 1.00 & 2.75 & 23.50 & 68.25 & 115.20 & 207.36 & -- \\
\hline 8335 & 1.00 & 1.00 & 1.00 & 3.00 & 15.00 & 60.00 & 104.60 & 210.64 & 243.44 & 9417 & 1.00 & 1.00 & 1.00 & 3.00 & 13.00 & 52.00 & 104.10 & 199.00 & 257.86 \\
\hline 8400 & 1.00 & 1.00 & 1.00 & 1.00 & 10.00 & 52.00 & 92.60 & 186.08 & 210.88 & 9419 & 1.00 & 1.00 & 1.00 & 4.00 & 21.00 & 66.25 & 126.00 & 222.62 & 283.31 \\
\hline 8445 & 1.00 & 1.00 & 2.00 & 4.00 & 18.00 & 65.00 & 114.50 & 208.00 & 270.00 & 9435 & -- & 1.00 & 1.00 & 1.00 & 6.50 & 50.50 & 73.00 & 215.82 & -- \\
\hline 8446 & 1.00 & 1.00 & 1.00 & 3.00 & 15.00 & 61.00 & 112.00 & 215.08 & 250.04 & 9491 & 1.00 & 1.00 & 1.00 & 3.00 & 16.00 & 58.75 & 114.50 & 222.30 & 257.30 \\
\hline 8451 & 1.00 & 1.00 & 1.00 & 2.00 & 9.50 & 47.00 & 84.10 & 139.62 & 144.31 & 9499 & 1.00 & 1.00 & 1.00 & 1.00 & 8.00 & 42.00 & 81.00 & 162.40 & 184.60 \\
\hline 8531 & 1.00 & 1.00 & 1.00 & 2.00 & 11.00 & 46.00 & 101.20 & 193.28 & 262.84 & 9522 & -- & -- & -- & 3.25 & 11.00 & 273.00 & -- & -- & -- \\
\hline 8541 & -- & 1.00 & 2.00 & 4.00 & 9.50 & 42.00 & 75.10 & 223.04 & -- & 9527 & 1.00 & 1.00 & 1.00 & 1.00 & 8.00 & 47.00 & 89.90 & 183.74 & 242.00 \\
\hline 8544 & 1.00 & 1.00 & 1.00 & 3.00 & 16.00 & 58.00 & 102.00 & 202.00 & 243.00 & 9532 & 1.00 & 1.00 & 1.00 & 3.00 & 12.00 & 51.50 & 97.00 & 172.24 & 217.00 \\
\hline 8545 & -- & -- & 1.90 & 10.25 & 20.50 & 64.75 & 103.90 & -- & -- & 9544 & -- & -- & -- & 1.00 & 3.00 & 17.00 & -- & -- & -- \\
\hline 8563 & 1.00 & 1.00 & 1.00 & 2.00 & 14.00 & 49.00 & 87.40 & 177.64 & 231.10 & 9565 & 1.00 & 1.00 & 1.00 & 2.00 & 8.00 & 49.00 & 99.00 & 156.00 & 199.40 \\
\hline 8566 & 1.00 & 1.00 & 1.00 & 2.00 & 7.50 & 47.00 & 96.00 & 177.94 & 209.26 & 9570 & 1.00 & 1.00 & 1.00 & 1.00 & 13.00 & 55.00 & 107.00 & 193.10 & 267.50 \\
\hline 8583 & 1.00 & 1.00 & 1.00 & 1.00 & 13.50 & 49.75 & 92.60 & 174.06 & 185.21 & 9574 & -- & -- & 7.20 & 31.00 & 50.00 & 82.00 & 113.00 & -- & -- \\
\hline 8584 & 1.00 & 1.00 & 1.00 & 2.00 & 9.00 & 44.00 & 84.00 & 161.24 & 194.08 & 9588 & 1.00 & 1.00 & 1.00 & 2.00 & 10.00 & 49.00 & 98.80 & 197.00 & 220.18 \\
\hline 8623 & 1.00 & 1.00 & 1.00 & 2.00 & 12.00 & 48.75 & 91.00 & 160.72 & 203.93 & 9665 & 1.00 & 1.00 & 1.00 & 3.00 & 13.00 & 53.00 & 99.00 & 187.28 & 225.07 \\
\hline 8625 & 1.00 & 1.00 & 2.00 & 4.00 & 13.00 & 54.00 & 99.00 & 170.20 & 209.70 & 9715 & 1.00 & 1.00 & 1.00 & 3.00 & 12.00 & 52.00 & 103.60 & 199.96 & 234.88 \\
\hline 8630 & 1.00 & 1.00 & 1.00 & 2.00 & 7.00 & 32.00 & 71.00 & 154.30 & 179.55 & 9729 & 1.00 & 1.00 & 1.00 & 4.00 & 17.00 & 58.00 & 111.00 & 205.00 & 256.92 \\
\hline 8631 & 1.00 & 1.00 & 1.00 & 2.00 & 7.00 & 39.75 & 79.80 & 152.04 & 190.66 & 9772 & 1.00 & 1.00 & 1.00 & 3.00 & 16.00 & 66.00 & 130.60 & 257.32 & 296.84 \\
\hline 8646 & 1.00 & 1.00 & 1.00 & 3.00 & 12.00 & 49.00 & 100.00 & 187.00 & 216.60 & 9814 & -- & -- & 2.00 & 3.00 & 13.00 & 86.00 & 138.90 & -- & -- \\
\hline 8647 & 1.00 & 1.00 & 1.00 & 1.00 & 10.00 & 50.00 & 92.10 & 199.28 & 238.28 & 9815 & 1.00 & 1.00 & 1.00 & 3.00 & 16.00 & 60.25 & 107.80 & 217.40 & 247.70 \\
\hline 8677 & 1.00 & 1.00 & 2.00 & 4.00 & 10.00 & 41.00 & 88.40 & 162.52 & 200.44 & 9816 & -- & 1.00 & 1.00 & 1.00 & 4.00 & 26.00 & 79.70 & 222.06 & -- \\
\hline 8696 & -- & -- & -- & 5.00 & 32.50 & 123.00 & -- & -- & -- & 9817 & 1.00 & 1.00 & 1.00 & 2.00 & 9.00 & 42.00 & 83.00 & 177.12 & 229.28 \\
\hline 8743 & 1.00 & 1.00 & 1.00 & 4.00 & 17.00 & 61.00 & 111.00 & 208.28 & 251.32 & 9829 & 1.00 & 1.00 & 1.00 & 1.00 & 6.00 & 37.50 & 75.00 & 141.44 & 186.54 \\
\hline 8761 & 1.00 & 1.00 & 1.00 & 1.00 & 6.00 & 35.00 & 84.00 & 161.40 & 179.80 & 9830 & 1.00 & 1.00 & 1.00 & 2.00 & 5.00 & 27.50 & 74.00 & 179.64 & 202.56 \\
\hline 8778 & 1.00 & 1.00 & 1.00 & 3.00 & 16.00 & 60.50 & 115.00 & 202.00 & 232.00 & 9858 & 1.00 & 1.00 & 1.00 & 2.00 & 8.00 & 44.00 & 85.00 & 183.18 & 207.72 \\
\hline 8845 & 1.00 & 1.00 & 1.00 & 2.00 & 15.00 & 55.00 & 104.00 & 204.00 & 249.45 & 9893 & 1.00 & 1.00 & 1.00 & 2.00 & 11.00 & 49.00 & 91.00 & 170.10 & 200.25 \\
\hline 8859 & 1.00 & 1.00 & 1.00 & 3.00 & 14.00 & 58.00 & 112.80 & 198.76 & 226.38 & 9916 & 1.00 & 1.00 & 1.00 & 4.00 & 18.00 & 56.00 & 101.10 & 229.74 & 254.73 \\
\hline 8898 & 1.00 & 1.00 & 2.00 & 4.00 & 15.00 & 48.50 & 106.20 & 182.20 & 225.74 & 9976 & 1.00 & 1.00 & 1.00 & 2.00 & 9.00 & 46.00 & 87.00 & 178.82 & 201.41 \\
\hline 8908 & -- & -- & 1.80 & 3.00 & 17.00 & 101.00 & 172.00 & -- & -- & & & & & & & & & & \\
\hline
\end{tabular}




\section{Glossary}

Best-management practice (BMP)—A practice, inclusive of structures, or combination of practices that controls or otherwise mitigates stormwater runoff and associated contaminants (for example, a detention pond).

Drawdown time-Time required for a structure that stores water to completely drain.

$\mathrm{L}-\mathrm{CV}$ - The coefficient of L-variation, which is the ratio of L-scale divided by the mean. $\mathrm{L}-\mathrm{CV}$ is a measure of the dimensionless variability of the distribution or sample data.

L-kurtosis-The fourth L-moment ratio, which is the ratio of the fourth L-moment divided by the L-scale. L-kurtosis measures the peakness of the distribution.

L-moments-Summary statistics for probability distributions and data samples. L-moments are analogous to ordinary or product moments. L-moments provide measures of location, dispersion, skewness, kurtosis, and other characteristics of probability distributions or data samples.

L-moment ratio diagram-L-moment ratio diagrams are used for graphical evaluation of the goodness of fit of candidate distributions to the sample L-moments. Specifically, the theoretical values of L-skew and L-kurtosis of each distribution are compared to those of the data.

L-scale - The second L-moment, which measures the variability of the distribution or the sample data.

L-skew-The third L-moment ratio, which is the ratio of the third L-moment divided by the L-scale. L-skew measures the skewness or asymmetry of the distribution or sample data.

Memoryless-Refers to expected absence of storage in a BMP before runoff from the next storm arrives. For example, the 48-hour minimum interevent time, by definition, ensures that storage in the BMP is zero before runoff from the next storm arrives.

Minimum interevent time (MIT) - A minimum time during which no rainfall occurs. Storms are defined by specifying minimum interevent time.

Tau5-The fifth L-moment ratio, which is the ratio of the fifth L-moment divided by the L-scale. Tau5 has no general interpretation, unlike L-skew or L-kurtosis.

$F$-Nonexceedence probability.

$x$-Dimensionless variable. Dimension is restored by multiplying curve ordinates $(x)$ by the mean storm depth or mean storm duration to produce quantile functions of storm depth and duration.

$X$ - Random variable of either storm depth or duration.

$x(F)$-Dimensionless quantile function (a dimensionless frequency curve) for nonexceedance probability $F$.

$X(F)$-Quantile function for nonexceedance probability $F$.

$\Lambda$-Exponential mean interevent time, in days. The $\Lambda$ parameter is used in conjunction with MIT to obtain storm interevent time distribution.

$\mu-$ Generic symbol for arithmetic mean.

$\theta$ and $\beta$-Dimensionless parameters of the cumulative distribution function of the gamma distribution.

$\Gamma(\theta)$ Complete gamma function of $\theta$.

$\xi$-Dimensionless location parameter of the cumulative distribution function of the kappa distribution.

$\alpha$-Dimensionless scale parameter of the kappa distribution.

$\kappa$ and $h$-Dimensionless shape parameters of the kappa distribution. 
Prepared by the USGS Texas Water Science Center:

8027 Exchange Drive

Austin, TX 78754-4733

Information regarding water resources in Texas is available at http: //tx.usgs.gov/ 

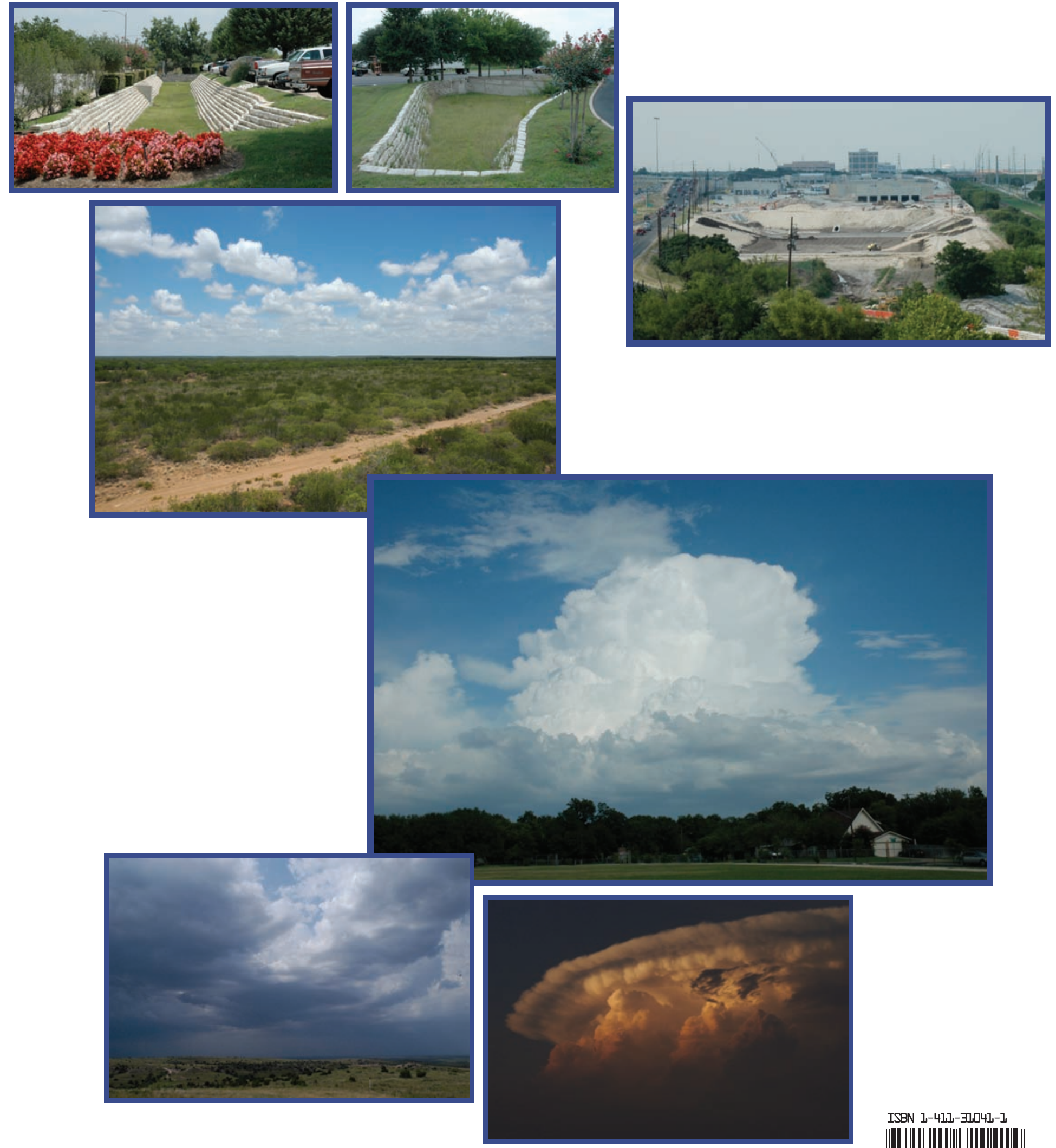

ISBN 1-41]-31041]

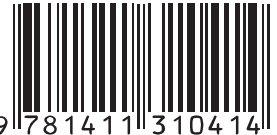

Printed on recycled paper 